Comparative Effectiveness Review

Number 198

\title{
Treatments for
} Schizophrenia in Adults: A Systematic Review 


\section{Comparative Effectiveness Review}

Number 198

\section{Treatments for Schizophrenia in Adults: A Systematic Review}

\section{Prepared for:}

Agency for Healthcare Research and Quality

U.S. Department of Health and Human Services

5600 Fishers Lane

Rockville, MD 20857

www.ahrq.gov

Contract No. 290-2015-00009-I

Prepared by:

Pacific Northwest Evidence-based Practice Center

Portland, OR

Investigators:

Marian S. McDonagh, Pharm.D.

Tracy Dana, M.L.S.

Shelley Selph, M.D., M.P.H.

Emily B. Devine, Pharm.D., Ph.D., M.B.A.

Amy Cantor, M.D., M.P.H.

Christina Bougatsos, M.P.H.

Ian Blazina, M.P.H.

Sara Grusing, B.A.

Rochelle Fu, Ph.D.

Sarah L. Kopelovich, Ph.D.

Maria Monroe-DeVita, Ph.D.

Daniel W. Haupt, M.D.

AHRQ Publication No. 17(18)-EHC031-EF

October 2017 


\section{Key Messages}

\section{Purpose of Review}

To evaluate treatments for schizophrenia.

Key Messages

- Olanzapine, aripiprazole, risperidone, quetiapine, and ziprasidone were similar in function, quality of life, mortality, and overall adverse events. Core illness symptoms were better with olanzapine and risperidone than asenapine, quetiapine, and ziprasidone, and with paliperidone than lurasidone and iloperidone.

- Haloperidol had similar benefits but more adverse events than olanzapine and risperidone.

- Psychosocial treatments improved outcomes versus usual care: assertive community care (core illness symptoms, function), cognitive behavioral therapy (core illness symptoms, function, quality of life), cognitive remediation (core illness symptoms), family interventions (core illness symptoms, function, relapse), illness self-management (core illness symptoms), psychoeducation (core illness symptoms, function, relapse), social skills training (core illness symptoms, function), and supported employment (core illness symptoms, employment). 
This report is based on research conducted by the Pacific Northwest Evidence-based Practice Center (EPC) under contract to the Agency for Healthcare Research and Quality (AHRQ), Rockville, MD (Contract No. 290-2015-00009-I). The findings and conclusions in this document are those of the authors, who are responsible for its contents; the findings and conclusions do not necessarily represent the views of AHRQ. Therefore, no statement in this report should be construed as an official position of AHRQ or of the U.S. Department of Health and Human Services.

\section{None of the investigators have any affiliations or financial involvement that conflicts with the material presented in this report.}

The information in this report is intended to help health care decisionmakers - patients and clinicians, health system leaders, and policymakers, among others-make well-informed decisions and thereby improve the quality of health care services. This report is not intended to be a substitute for the application of clinical judgment. Anyone who makes decisions concerning the provision of clinical care should consider this report in the same way as any medical reference and in conjunction with all other pertinent information, i.e., in the context of available resources and circumstances presented by individual patients.

This report is made available to the public under the terms of a licensing agreement between the author and the Agency for Healthcare Research and Quality. This report may be used and reprinted without permission except those copyrighted materials that are clearly noted in the report. Further reproduction of those copyrighted materials is prohibited without the express permission of copyright holders.

AHRQ or U.S. Department of Health and Human Services endorsement of any derivative products that may be developed from this report, such as clinical practice guidelines, other quality enhancement tools, or reimbursement or coverage policies, may not be stated or implied. This report may periodically be assessed for the currency of conclusions. If an assessment is done, the resulting surveillance report describing the methodology and findings will be found on the Effective Health Care Program Web site at www.effectivehealthcare.ahrq.gov. Search on the title of the report.

Persons using assistive technology may not be able to fully access information in this report. For assistance contact epc@ahrq.hhs.gov.

Suggested citation: McDonagh MS, Dana T, Selph S, Devine EB, Cantor A, Bougatsos C, Blazina I, Grusing S, Fu R, Kopelovich SL, Monroe-DeVita M, Haupt DW. Treatments for Schizophrenia in Adults: A Systematic Review. Comparative Effectiveness Review No. 198. (Prepared by the Pacific Northwest Evidence-based Practice Center under Contract No. 2902015-00009-I.) AHRQ Publication No. 17(18)-EHC031-EF. Rockville, MD: Agency for Healthcare Research and Quality; October 2017. www.effectivehealthcare.ahrq.gov/reports/final.cfm. DOI: https://doi.org/10.23970/AHRQEPCCER198. 


\section{Preface}

The Agency for Healthcare Research and Quality (AHRQ), through its Evidence-based Practice Centers (EPCs), sponsors the development of systematic reviews to assist public- and private-sector organizations in their efforts to improve the quality of health care in the United States. These reviews provide comprehensive, science-based information on common, costly medical conditions, and new health care technologies and strategies.

Systematic reviews are the building blocks underlying evidence-based practice; they focus attention on the strength and limits of evidence from research studies about the effectiveness and safety of a clinical intervention. In the context of developing recommendations for practice, systematic reviews can help clarify whether assertions about the value of the intervention are based on strong evidence from clinical studies. For more information about AHRQ EPC systematic reviews, see www.effectivehealthcare.ahrq.gov/reference/purpose.cfm.

AHRQ expects that these systematic reviews will be helpful to health plans, providers, purchasers, government programs, and the health care system as a whole. Transparency and stakeholder input are essential to the Effective Health Care Program. Please visit the Web site (www.effectivehealthcare.ahrq.gov) to see draft research questions and reports or to join an email list to learn about new program products and opportunities for input.

If you have comments on this evidence report, they may be sent by mail to the Task Order Officers named below at: Agency for Healthcare Research and Quality, 5600 Fishers Lane, Rockville, MD 20857, or by email to epc@ahrq.hhs.gov.

Gopal Khanna, M.B.A.

Director

Agency for Healthcare Research and Quality

Stephanie Chang, M.D., M.P.H.

Director

Evidence-based Practice Center Program

Center for Evidence and Practice

Improvement

Agency for Healthcare Research and Quality
Arlene S. Bierman, M.D., M.S.

Director

Center for Evidence and Practice

Improvement

Agency for Healthcare Research and Quality

David W. Niebuhr, M.D., M.P.H., M.Sc.

Aysegul Gozu, M.D., M.P.H.

Task Order Officers

Center for Evidence and Practice

Improvement

Agency for Healthcare Research and Quality 


\section{Acknowledgments}

The authors gratefully acknowledge the following individuals for their contributions to this project: Elaine Graham, M.L.S.; Andrew Hamilton, M.L.S., M.S.; Leah Williams, B.S.; Ryan Stoner, Ph.D.; Liev Miller, B.S.; and Brittany H. Lazur, M.P.H.

\section{Key Informants}

In designing the study questions, the EPC consulted several Key Informants who represent the end-users of research. The EPC sought the Key Informant input on the priority areas for research and synthesis. Key Informants are not involved in the analysis of the evidence or the writing of the report. Therefore, in the end, study questions, design, methodological approaches, and/or conclusions do not necessarily represent the views of individual Key Informants.

Key Informants must disclose any financial conflicts of interest greater than $\$ 10,000$ and any other relevant business or professional conflicts of interest. Because of their role as end-users, individuals with potential conflicts may be retained. The TOO and the EPC work to balance, manage, or mitigate any conflicts of interest.

The list of Key Informants who provided input to this report follows:

Bob Correia, R.Ph., Pharm.D.

New York Medicaid Pharmacy Agency

New York State Department of Health

New York, NY

Lisa B. Dixon, M.D., M.P.H.*

Columbia University

New York, NY

Laura J. Fochtmann, M.D., M.B.I.*

Departments of Psychiatry, Pharmacological

Sciences and Biomedical Informatics

Stony Brook University School of Medicine

Stony Brook, NY

*Provided input on Draft Report
Anthony F. Lehman, M.D., M.S.P.H.

University of Maryland

College Park, MD

Janie Marsh, M.P.H.:H.A.

Mental Health America of Oregon

Portland, OR

Piper Meyer-Kalos, Ph.D., L.P.

University of Minnesota

Minneapolis, MN

Joyce M. Shea, D.N.Sc., A.P.R.N.,

P.M.H.C.N.S.-B.C.

American Psychiatric Nurses Association

Falls Church, VA 


\section{Technical Expert Panel}

In designing the study questions and methodology at the outset of this report, the EPC consulted several technical and content experts. Broad expertise and perspectives were sought. Divergent and conflicting opinions are common and perceived as healthy scientific discourse that results in a thoughtful, relevant systematic review. Therefore, in the end, study questions, design, methodologic approaches, and/or conclusions do not necessarily represent the views of individual technical and content experts.

Technical Experts must disclose any financial conflicts of interest greater than $\$ 10,000$ and any other relevant business or professional conflicts of interest. Because of their unique clinical or content expertise, individuals with potential conflicts may be retained. The TOO and the EPC work to balance, manage, or mitigate any potential conflicts of interest identified.

The list of Technical Experts who provided input to this report follows:

Austin R. Campbell, Pharm.D.

University of Missouri

Columbia, $\mathrm{MO}$

Lisa B. Dixon, M.D., M.P.H.*

Columbia University

New York, NY

Laura J. Fochtmann, M.D., M.B.I*.

Departments of Psychiatry, Pharmacological

Sciences and Biomedical Informatics

Stony Brook University School of Medicine

Stony Brook, NY

Eric Granholm, Ph.D.*

Department of Psychiatry, University of

California, San Diego

*Provided input on Draft Report
VA San Diego Healthcare System San Diego, CA

Lisa Hartling, M.D.

Alberta Research Centre for Health

Evidence

Edmonton, Alberta, Canada

Piper Meyer-Kalos, Ph.D., L.P.

University of Minnesota

Minneapolis, MN

Matthew V. Rudorfer, M.D.*

National Institutes of Health

National Institute of Mental Health

Bethesda, MD 


\section{Peer Reviewers}

Prior to publication of the final evidence report, EPCs sought input from independent Peer reviewers without financial conflicts of interest. However, the conclusions and synthesis of the scientific literature presented in this report do not necessarily represent the views of individual reviewers.

Peer Reviewers must disclose any financial conflicts of interest greater than $\$ 10,000$ and any other relevant business or professional conflicts of interest. Because of their unique clinical or content expertise, individuals with potential nonfinancial conflicts may be retained. The TOO and the EPC work to balance, manage, or mitigate any potential nonfinancial conflicts of interest identified.

The list of Peer Reviewers follows:

Lynn Bufka, Ph.D.

American Psychological Association

Washington, DC

Kim Mueser, Ph.D.

Boston University

Boston, MA
Scott Stroup, M.D., M.P.H.

Columbia University

New York, NY

Ann Wheeler, Pharm. D., B.C.P.P.

United Healthcare

Oregon Health \& Science University

Portland, OR 


\section{Treatments for Schizophrenia in Adults: A Systematic Review}

\section{Structured Abstract}

Objectives. This systematic review (SR) provides evidence on pharmacological and psychosocial treatments for schizophrenia.

Data sources. MEDLINE ${ }^{\circledR}$, the Cochrane Library databases, PsycINFO ${ }^{\circledR}$, and included studies through February 2017.

Study selection. We included studies comparing second-generation antipsychotics (SGA) with each other or with a first-generation antipsychotic (FGA) and studies comparing psychosocial interventions with usual care in adults with schizophrenia.

Data extraction. We extracted study design, year, setting, country, sample size, eligibility criteria, population, clinical and intervention characteristics, results, and funding source.

Results. We included 1 SR of 138 trials $(\mathrm{N}=47,189)$ and 24 trials $(\mathrm{N}=6,672)$ for SGAs versus SGAs, 1 SR of 111 trials $(\mathrm{N}=118,503)$ and 5 trials $(\mathrm{N}=1,055)$ for FGAs versus SGAs, and 13 SRs of 271 trials $(\mathrm{N}=25,050)$ and 27 trials $(n=6,404)$ for psychosocial interventions. Trials were mostly fair quality and strength of evidence was low or moderate. For drug therapy, the majority of the head-to-head evidence was on older SGAs, with sparse data on SGAs approved in the last 10 years (asenapine, lurasidone, iloperidone, cariprazine, brexpiprazole) and recent long-acting injection (LAI) formulations of aripiprazole and paliperidone. Older SGAs were similar in measures of function, quality of life, mortality, and overall adverse events, except that risperidone LAI had better social function than quetiapine. Core illness symptoms were improved more with olanzapine and risperidone than asenapine, quetiapine, and ziprasidone, and more with paliperidone than lurasidone and iloperidone; all were superior to placebo.

Risperidone LAI and olanzapine had less withdrawal due to adverse events. Compared with olanzapine and risperidone, haloperidol, the most studied FGA, had similar improvement in core illness symptoms, negative symptoms, symptom response, and remission but greater incidence of adverse event outcomes. In comparison with usual care, most psychosocial interventions reviewed were more effective in improving intervention-targeted outcomes, including core illness symptoms. Various functional outcomes were improved more with assertive community treatment, cognitive behavioral therapy, family interventions, psychoeducation, social skills training, supported employment, and early interventions for first episode psychosis (FEP) than with usual care. Quality of life was improved more with cognitive behavioral therapy and early interventions for FEP than usual care. Relapse was reduced with family interventions, psychoeducation, illness self-management, family interventions, and early interventions for FEP.

Conclusions. Most comparative evidence on pharmacotherapy relates to the older drugs, with clozapine, olanzapine, and risperidone superior on more outcomes than other SGAs. Older SGAs were similar to haloperidol on benefit outcomes but had fewer adverse event outcomes. Most psychosocial interventions improved functional outcomes, quality of life, and core illness symptoms, and several reduced relapse compared with usual care. 


\section{Contents}

Evidence Summary .................................................................................................................... ES-1

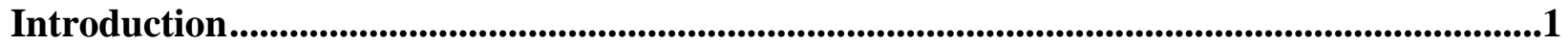

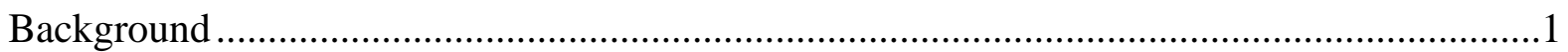

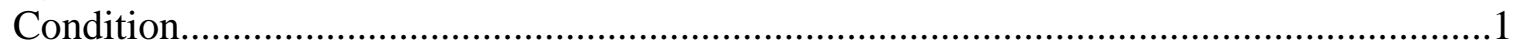

Treatment Strategies ........................................................................................................

Scope and Key Questions ..........................................................................................

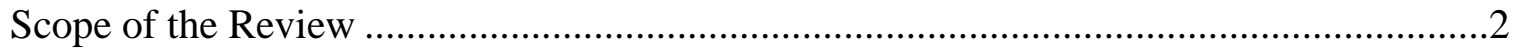

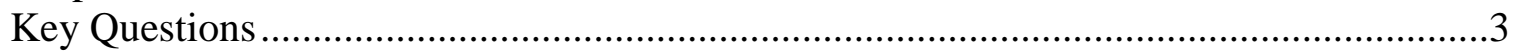

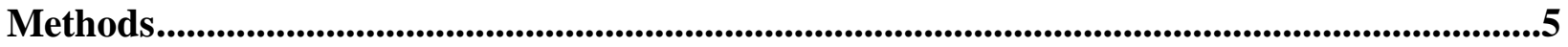

Topic Refinement and Review Protocol .........................................................................

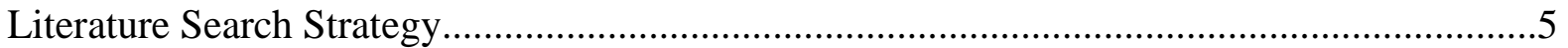

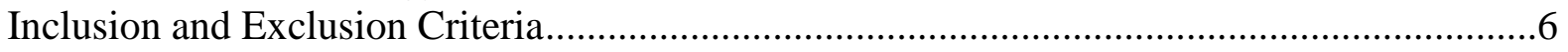

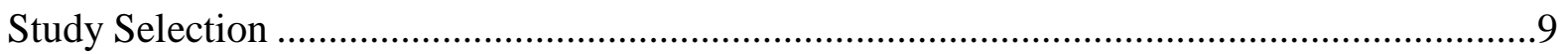

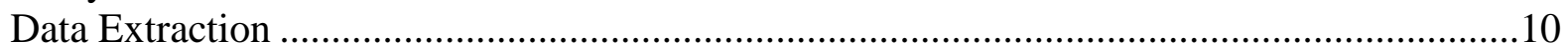

Quality Assessment of Individual Studies .........................................................................10

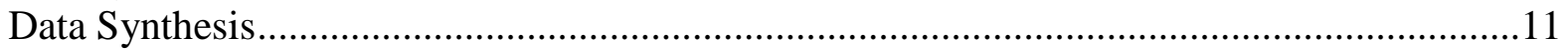

Strength of the Body of Evidence........................................................................................12

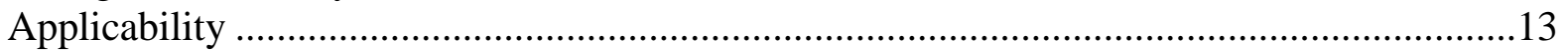

Peer Review and Public Commentary ……………….....................................................14

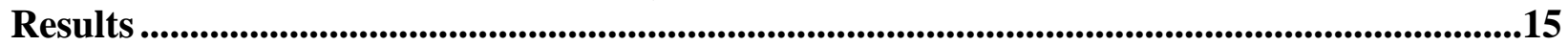

Results of Literature Searches .........................................................................................15

Key Question 1a. Comparative Benefits and Harms of Pharmacological Treatments for

Adults With Schizophrenia ..................................................................................................22

Second-Generation Antipsychotic Versus Second-Generation Antipsychotic...................20

First-Generation Antipsychotic Versus Second-Generation Antipsychotic ........................37

Key Question 1b. Variation of Benefits and Harms of Pharmacological Treatments

for Adults With Schizophrenia by Patient Characteristics ......................................................4

Second-Generation Antipsychotic Versus Second-Generation Antipsychotic....................43

First-Generation Antipsychotic Versus Second-Generation Antipsychotic ........................50

Key Question 2a. Benefits and Harms of Psychosocial and Other Nonpharmacological

Treatments for Adults With Schizophrenia Compared With Usual Care..................................53

Assertive Community Treatment Versus Usual Care ...........................................................53

Cognitive Adaptation Training Versus Usual Care ...........................................................56

Cognitive Behavioral Therapy Versus Usual Care .............................................................56

Cognitive Remediation/Training Versus Usual Care .....................................................66

Family Interventions Versus Usual Care ...........................................................................69

Intensive Case Management Versus Usual Care ................................................................72

Illness Self-Management and Recovery Versus Usual Care ...............................................74

Patient Psychoeducation (Individual or Group) Versus Usual Care....................................76

Social Skills Training Versus Usual Care...................................................................78

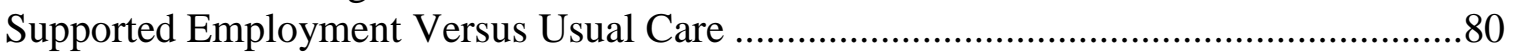

Supportive Therapy Versus Usual Care..............................................................................83 
Key Question 2b. Variation of Benefits and Harms of Psychosocial and Other Nonpharmacological Treatments Versus Usual Care for Adults with Schizophrenia by

Patient Characteristics.

Key Points

Detailed Synthesis

Discussion.

Key Findings and Strength of Evidence

Key Questions 1a and 1b: Comparative Evidence Regarding Antipsychotic Drugs

Key Questions 2a and 2b: Evidence on Psychosocial and Other Nonpharmacological Interventions Versus Usual Care

Findings in Relationship to What Is Already Known

Cognitive Behavioral Therapy

Cognitive Remediation

Family Interventions

Social Skills Training

Supported Employment

Applicability

Key Question 1. Comparative Effectiveness of Pharmacological Treatments

Key Question 2. Psychosocial and Other Nonpharmacological Interventions

Versus Usual Care

Implications for Clinical and Policy Decisionmaking.....

Limitations of the Systematic Review Process

Limitations of the Evidence Base

Research Recommendations ....

Pharmacological Interventions Versus Each Other

Psychosocial and Other Nonpharmacological Interventions Versus Usual Care

Conclusions.

References.

Abbreviations

Tables

Table A. Summary of key findings and strength of evidence for Key Question 1: SGA versus SGA

Table B. Summary of key findings and strength of evidence for Key Question 1: FGA versus SGA

Table C. Summary of key findings and strength of evidence for Key Question 2: nonpharmacological interventions versus usual care

Table 1. Characteristics of included systematic reviews for Key Question 1 .......................... 18

Table 2. Characteristics of new included trials for Key Question 1 ......................................... 18

Table 3. Characteristics of included systematic reviews for Key Question 2 ........................... 19

Table 4. Characteristics of included trials for Key Question 2 ............................................. 19

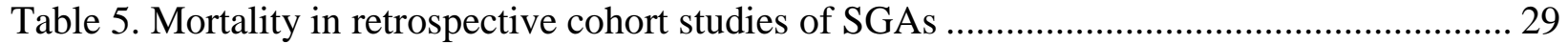

Table 6. Overall adverse events in trials of SGAs versus SGAs ............................................ 32

Table 7. Extrapyramidal symptoms: significant differences in trials of SGAs .......................... 36

Table 8. Studies of SGAs in patients treated for first episode of schizophrenia ........................ 45

Table 9. Functional outcomes in randomized controlled trials of cognitive behavioral therapy versus usual care 
Table 10. Summary of evidence for pharmacological treatments

\section{Figures}

Figure A. Analytic framework

Figure 1. Analytic framework.

Figure 2. Key Question 1 literature flow diagram ................................................................ 16

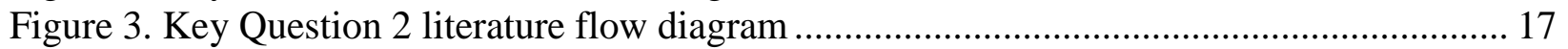

Figure 4. Network meta-analysis of response rates in trials of SGAs .................................... 28

Figure 5. Network meta-analysis of withdrawals due to adverse events in trials of SGAs......... 33

\section{Appendixes}

Appendix A. Search Strategies

Appendix B. Included Studies

Appendix C. Excluded Studies With Reasons

Appendix D. Scale Abbreviations and Scoring

Appendix E. Data Abstraction

Appendix F. Quality Assessment

Appendix G. Forest Plots for Pooled Analyses and Matrixes of Results for Network Meta-

Analyses

Appendix H. Strength of Evidence

Appendix I. Strength of Evidence-Drug Comparisons 


\section{Evidence Summary}

\section{Condition and Treatment Strategies}

Schizophrenia is a chronic mental health condition that most often presents in early adulthood and can lead to disabling outcomes. The most recent version of the American Psychiatric Association's Diagnostic and Statistical Manual of Mental Disorders, 5th edition, (DSM-5), ${ }^{1}$ defines schizophrenia as: the presence of two or more of the five core symptoms (delusions, hallucinations, disorganized speech, grossly disorganized or catatonic behavior, and negative symptoms), with at least one of the symptoms being delusions, hallucinations, or disorganized speech, and the presence of symptoms for at least 6 months. Differential diagnosis is broad, and includes delineation from mood disorders (bipolar disorder or major depressive disorder) with psychotic features and substance/medication-induced psychotic disorders. The course of schizophrenia varies. Approximately 20 percent of individuals may experience significant improvement including, in some cases, full recovery; however, the majority tend to experience some degree of social and occupational difficulty as well as need for daily living supports. ${ }^{2}$ That said, more recent research and practice has focused on early intervention with first episode psychosis, demonstrating promise toward improving outcomes sooner and reducing longer-term disability.

Antipsychotic medications and nonpharmacological treatments are typically used together when treating individuals with schizophrenia. Both pharmacological and nonpharmacological treatments for schizophrenia can result in meaningful improvements in a variety of outcome areas, including psychiatric symptoms, functioning (e.g., employment, social), service utilization (e.g., hospitalization, crisis services), legal system involvement, quality of life, self-harm and aggressive behaviors, treatment engagement and retention, and co-occurring substance abuse. Ideally, improvements in symptoms translate to long-term, clinically relevant, positive changes in other outcome areas, with limited and manageable adverse effects.

Older, first-generation antipsychotics (FGAs), such as haloperidol, have proven efficacy but adverse effects, such as extrapyramidal symptoms and in some cases tardive dyskinesia, often limit long-term adherence. Second-generation antipsychotics (SGAs), beginning with clozapine, were introduced as having equal or better efficacy, particularly with negative symptoms, and lower risk of extrapyramidal symptoms and tardive dyskinesia. SGAs have potentially serious adverse effects (e.g., cardiovascular and endocrinologic effects) that make their overall risk/benefit profile less clear-cut than anticipated.

Although there are a large number of treatments for schizophrenia, it is not clear whether they afford long-term benefits on employment and social relationships and increase the likelihood of recovery, or what the most effective duration of treatment is. Equally important in selecting among competing interventions for a specific patient is consideration of patient-level characteristics that may affect the outcomes across a diverse group of possible interventions.

\section{Scope and Key Questions}

\section{Scope of the Review}

This systematic review provides a comprehensive review of current evidence that can help in determining how to treat individuals with schizophrenia. The review synthesizes evidence on pharmacological treatments compared with each other and the general effectiveness of 
psychosocial and other nonpharmacological strategies compared with usual care for treating individuals with schizophrenia, and highlights areas of controversy and areas for future research. The analytic framework (Figure A) illustrates the population, interventions, and outcomes considered. Due to a very large body of research literature, the review has been focused in several ways (see Methods).

Figure A. Analytic framework

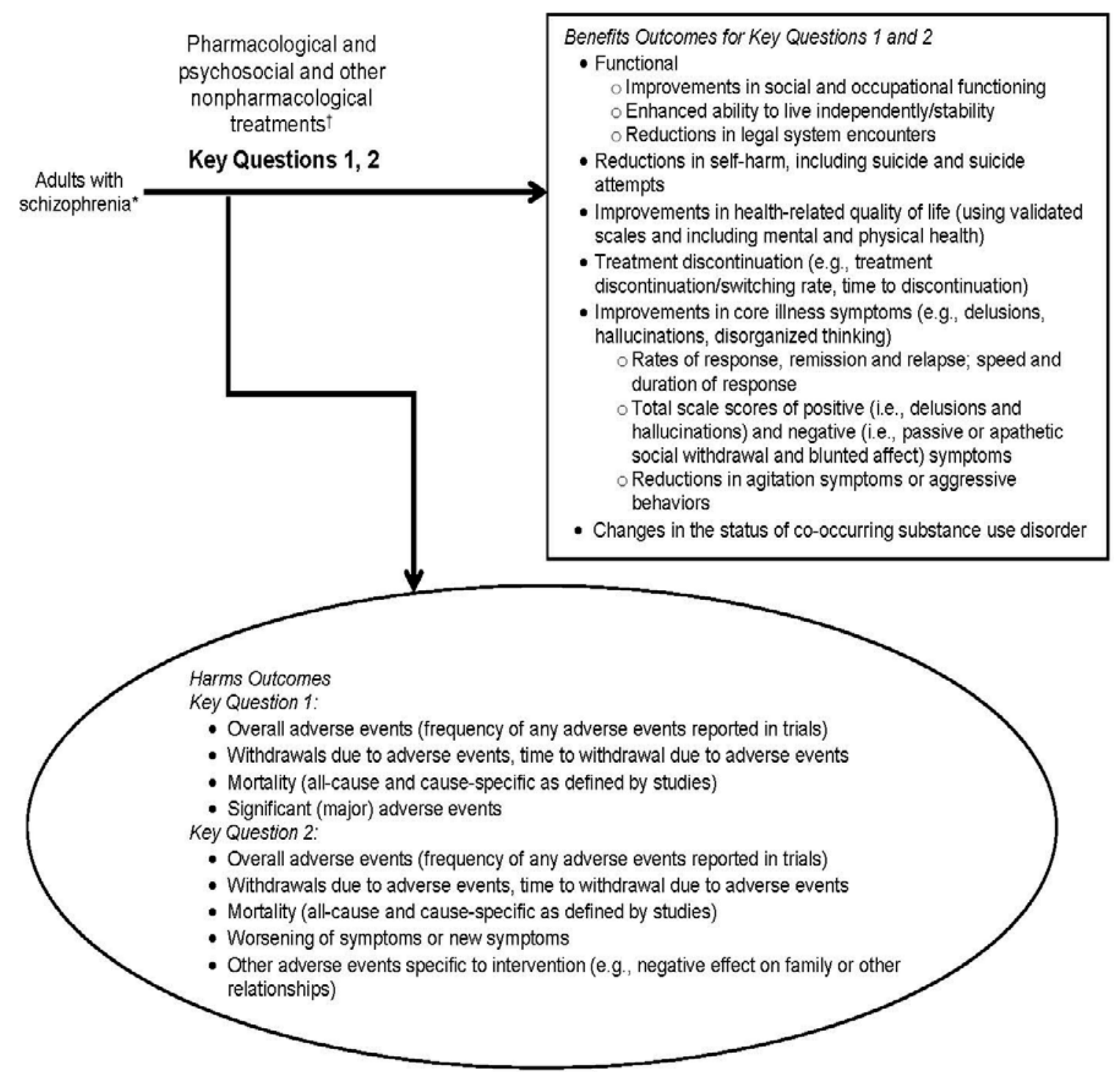

* Adults with a diagnosis of schizophrenia, including those with co-occurring substance use disorders, and including those experiencing a first episode of schizophrenia (including those with schizophreniform disorder).

1. Pharmacological treatments:

a. At least 90 percent of patients must have been diagnosed with schizophrenia.

b. For studies specifically on harms of antipsychotic drugs, populations can be mixed-diagnoses, as the harms are not diagnosis-specific

2. Psychosocial and other nonpharmacological treatments: 50 percent of patients must have been diagnosed with a schizophrenia spectrum disorder diagnosis (i.e., schizophrenia, schizoaffective disorder, or schizophreniform disorder) 
${ }^{\dagger}$ Pharmacological treatments include US Food and Drug Administration-approved second-generation and selected firstgeneration antipsychotics. Psychosocial and other nonpharmacological treatments include: assertive community treatment, cognitive adaptive training, cognitive behavioral therapy, cognitive remediation/training, co-occurring substance use and schizophrenia interventions, early interventions for first episode psychosis, family interventions, intensive case management, illness self-management training, psychoeducation, social skills training, supported employment, and supportive therapy.

\section{Key Questions}

1a. What are the comparative benefits and harms of pharmacological treatments for adults with schizophrenia?

1b. How do the benefits and harms of pharmacological treatments for adults with schizophrenia vary by patient characteristics? ${ }^{\mathrm{a}}$

2a. What are the benefits and harms of psychosocial and other nonpharmacological treatments for adults with schizophrenia?

2b. How do the benefits and harms of psychosocial and other nonpharmacological treatments for adults with schizophrenia vary by patient characteristics? ${ }^{\mathrm{a}}$

\section{Methods}

The methods for this systematic review follow the Methods Guide for Effectiveness and Comparative Effectiveness Reviews ${ }^{5}$ and are reported according to the Preferred Reporting Items for Systematic Review and Meta-Analysis (PRISMA) checklist. ${ }^{6}$ The scope of the report was developed with consultation with a group of key informants. The details of the inclusion criteria, including the prioritized list of outcomes, were developed with input from a group of technical experts. See the full report and the review protocol (http://effectivehealthcare.ahrq.gov/index.cfm) for additional details on methods.

\section{Literature Search Strategy and Inclusion Criteria}

A research librarian searched Ovid MEDLINE ${ }^{\circledR}$, the Cochrane Library, and PsycINFO ${ }^{\circledR}$. For Key Question 1, recent high-quality systematic reviews were used as the starting point, such that our searches began in 2011 for FGA versus SGA drugs and in 2013 for SGA versus SGA drugs. For Key Question 2, search dates were not restricted. Searches were conducted through February 1, 2017. Other standard search methods were also applied. Only English-language articles were included. A summary of the eligibility criteria and review methods are described below, and further details are in the full report.

${ }^{a}$ Patient characteristics include age, sex, race, ethnicity, socioeconomic status, time since illness onset, prior treatment history, cooccurring psychiatric disorders, pregnancy, etc. 


\section{Key Eligibility Criteria}

Population(s): Adults with a diagnosis of schizophrenia Interventions:

- Key Question 1: Antipsychotic medications

o First-generation antipsychotic drugs (FGAs)

- $\quad$ Fluphenazine $\left(\right.$ Prolixin $^{\circledR}$, Permitil ${ }^{\circledR}$ )

- Haloperidol (Haldol ${ }^{\circledR}$ )

- Perphenazine (Trilafon ${ }^{\circledR}$ )

o Second-generation antipsychotic drugs (SGAs)

- Aripiprazole (Abilify ${ }^{\circledR}$, Aristada $^{\mathrm{TM}}$ )

- Asenapine (Saphris ${ }^{\circledR}$ ),

- Brexpiprazole (Rexulti ${ }^{\circledR}$ )

- Cariprazine (Vraylar ${ }^{\mathrm{TM}}$ )

- Clozapine $^{\mathrm{b}}$ (Clozaril $^{\circledR}$, Fazaclo $^{\circledR}$ ODT, Versacloz ${ }^{\mathrm{TM}}$ )

- Iloperidone (Fanapt ${ }^{\circledR}$ )

- Lurasidone (Latuda ${ }^{\circledR}$ )

- Olanzapine $^{\mathrm{b}}$ (Zyprexa $^{\circledR}$, Zyprexa Zydis $\left.{ }^{\circledR}\right)$,

- Olanzapine Pamoate (Zyprexa ${ }^{\circledR}$ Relprevv $^{\mathrm{TM}}$ )

- Paliperidone $^{\mathrm{b}}$ (Invega ${ }^{\circledR}$ ) and Paliperidone palmitate (Invega ${ }^{\circledR}$ Sustenna ${ }^{\circledR}$, Invega Trinza ${ }^{\mathrm{TM}}$ )

- Oral paliperidone is marketed only as an extended-release product, and will be noted as paliperidone in the report because there is no immediate-release formulation.

- $\quad$ Quetiapine ${ }^{\mathrm{b}}$ (Seroquel $^{\circledR}$, Seroquel $\mathrm{XR}^{\circledR}$ )

- The extended-release formulation is noted as quetiapine ER in this report; the immediate-release formulation is not noted by a suffix to be consistent with the other immediate release formulations of SGAs.

- $\quad$ Risperidone ${ }^{\mathrm{b}}$ (Risperdal ${ }^{\circledR}$, Risperdal ${ }^{\circledR}$ M-TAB ${ }^{\circledR}$ ODT (oral dissolving tablet), Risperdal $^{\circledR}$ Consta $\left.^{\circledR}\right)$

- Ziprasidone ${ }^{\mathrm{b}}\left(\right.$ Geodon $\left.^{\circledR}\right)$

- Key Question 2: Psychosocial and other nonpharmacological interventions ${ }^{\mathrm{c}}$

0 Assertive community treatment

o Cognitive adaptive training

o Cognitive behavioral therapy

o Cognitive remediation/training

o Co-occurring substance use and schizophrenia interventions

o Early interventions for first episode psychosis

o Family interventions

o Intensive case management

o Illness self-management training

b،Older” SGAs; approved up through 2001 and included in the Clinical Antipsychotic Trials of Intervention Effectiveness (CATIE) trials.

${ }^{\mathrm{c}}$ Limited to the most commonly used interventions relevant to U.S. practices. 
o Psychoeducation

o Social skills training

o Supported employment

o Supportive therapy

\section{Comparators:}

- Key Question 1: Head-to-head comparisons: FGAs versus SGAs, and SGAs versus SGAs.

- Key Question 2: Usual care/standard care/treatment as usual/waitlist, as defined in the trials.

o Usual care can consist of elements of medication treatment, medication management, case management, rehabilitation services, and psychotherapy. Both groups (treatment and usual care) received usual care, including drug treatment throughout the study.

o Evidence with active controls (other interventions with expected benefit, or attention controls which have minimal or no benefit but similar patient participation time) was considered where the evidence base with usual care comparisons for a given intervention is too small to draw conclusions (i.e., one or two trials, no systematic reviews).

\section{Outcomes for each question (see also outcomes in Figure A):}

We limited the outcomes to those that are patient centered health outcomes (rather than intermediate outcomes), which were arranged according to their priority from the perspective of the patient, their family, and their clinicians. We considered advice from our experts in selecting and prioritizing this list of outcomes.

- For each Key Question, eight outcomes were prioritized as most important.

o Key Question 1: Functional outcomes, quality of life, response and/or remission rate, mortality, reductions in self-harm, overall/any adverse events, improvements in core illness symptoms, and withdrawal due to adverse events.

o Key Question 2: Functional outcomes (including social, occupational and other types of function), quality of life, reductions in self-harm, response and/or remission rate, improvements in core illness symptoms, treatment discontinuation (for any reason; may be reported as loss to followup or leaving study early), relapse rate, and adverse events.

- Rehospitalization was not included as an outcome because: (1) there is important variation in the indications for and length of psychiatric hospitalizations across time, in different localities, and with different financial contexts, and (2) there is important variation across trials in how rehospitalization is measured/evaluated, which may confound study interpretation. However, it was reported in addition to the prioritized outcomes for assertive community treatment because it is the target of this

\section{Timing:} intervention for patients with a history of frequent hospitalization.

- Minimum duration of followup: 12 weeks. 


\section{Settings:}

- United States-relevant, such as countries listed as "high" or "very high" on the United Nations International Human Development Index (HDI), and applicable to United States practices.

- Excluded: inpatient setting.

Study designs:

- Recent, comprehensive, good- or fair-quality systematic reviews, as well as randomized controlled trials (RCTs) published since the systematic reviews.

- $\quad$ Sample size of >50 for Key Question 2.

\section{Study Inclusion Decisions}

Two independent reviewers assessed study eligibility and extracted data from included studies, with discrepancies resolved by consensus and involvement of a third reviewer, if necessary. Only English-language articles were included. We included trials with study populations of mostly outpatients and duration of at least 12 weeks, and systematic reviews that assessed the comparisons in Key Questions 1 and 2 that were deemed to be good or fair quality (see below). Whenever possible, systematic reviews were used as the primary evidence, with trials not included in reviews also fully evaluated and synthesized with the review evidence.

\section{Risk of Bias Assessment of Individual Studies}

Two investigators independently rated the risk of bias (quality) of each included study based on predefined criteria. Disagreements were resolved by consensus. Randomized controlled trials were evaluated with criteria developed by the Drug Effectiveness Review Project. ${ }^{7}$ The quality of systematic reviews was assessed using the Assessing the Methodological Quality of Systematic Reviews quality (AMSTAR)-rating instrument. ${ }^{8}$ These methods were used in accordance with the approach recommended in the chapter, "Assessing the Risk of Bias of Individual Studies When Comparing Medical Interventions” in the Methods Guide for Effectiveness and Comparative Effectiveness Reviews. ${ }^{5}$ Studies were rated as "good," "fair," or "poor."

\section{Data Synthesis}

We synthesized results by summarizing study characteristics and investigating whether there were important differences in the distribution in characteristics that modified the treatment effects. Synthesis focused on the better-quality studies. Meta-analyses were conducted when studies were homogeneous enough to provide a meaningful combined estimate. We conducted pairwise meta-analyses, using the DerSimonian and Laird random-effects model. Statistical heterogeneity was assessed using the $\mathrm{I}^{2}$ statistic or the Q-statistic chi-square. Network metaanalyses were conducted using a Bayesian hierarchical model.

\section{Strength of the Body of Evidence}

The strength of evidence (SOE) for each prioritized outcome was assessed by two reviewers using the approach described in the Methods Guide for Effectiveness and Comparative Effectiveness Reviews. ${ }^{5,9}$ We assigned an SOE grade of High, Moderate, Low, or Insufficient for the body of evidence for each outcome, based on evaluation of four domains: study limitations, consistency, directness, and precision. High, Moderate and Low ratings reflect our confidence in 
the accuracy and validity of the findings and whether future studies might alter these findings (magnitude or direction). We gave a rating of insufficient when we were unable to draw conclusions due to serious inconsistency, serious methodological limitations, or sparseness of evidence.

\section{Peer Review and Public Commentary}

Experts in treatments for schizophrenia were invited to provide external peer review of this systematic review; the Agency for Healthcare Research and Quality (AHRQ) and an associate editor also provided comments. In addition, the draft report was posted on the AHRQ Web site for 4 weeks to elicit public comment. We addressed the reviewer comments and revised the text as appropriate.

\section{Results Summary}

\section{Summary of Results of Literature Searches}

For Key Question 1 on the benefits and harms of pharmacological interventions for schizophrenia, we reviewed 698 titles and abstracts and included one systematic review of 138 trials and 24 additional trials for SGAs versus SGAs, and one systematic review of 111 trials and five additional trials for FGAs versus SGAs. Some studies included comparisons of both intervention areas (SGA vs. SGA and SGA vs. FGA). The majority of new trials (71\%) were fair quality, with 21 percent rated poor quality and 8 percent good quality.

For Key Question 2 on the benefits and harms of psychosocial and other nonpharmacological interventions for schizophrenia, we reviewed 2,766 titles and abstracts and included 13 systematic reviews of 271 trials and 32 additional trials. The included studies investigated 13 main intervention areas. Of these new trials, 20 were fair quality, four were good quality, and three were poor quality.

For each intervention area, we reported on the available evidence for prioritized outcomes, as described in the Methods section. Prioritized outcomes for which the evidence was insufficient or unavailable are not included in the Results Summary.

\section{Summary of Results by Key Question}

\section{Key Question 1: Comparative Evidence Regarding Antipsychotic Drugs}

The findings on antipsychotic drugs came from one systematic review of 138 trials $(\mathrm{N}=47,189)$ and 24 additional trials $(\mathrm{N}=6,672)$ for SGAs versus SGAs, and one systematic review of 111 trials $(\mathrm{N}=118,503)$ and five additional trials $(\mathrm{N}=1,055)$ for FGAs versus SGAs. In our review, we examined the prioritized outcomes: measures of functional abilities, quality of life, response and/or remission, mortality, self-harm, core illness symptoms, overall adverse events, and withdrawal from treatment due to adverse events. Overall, no drug intervention had high-strength evidence for any outcome of interest, but we found moderate-strength evidence for some outcomes. The evidence is divided into SGA versus SGA and FGA versus SGA according to traditional categorization of the drugs used in the two systematic reviews, although the drugs could be considered as one group with variations in effects associated with individual drugs. 


\section{Second-Generation Antipsychotics Versus Second-Generation Antipsychotics}

We found the most evidence about the older SGAs (clozapine, risperidone, olanzapine, quetiapine, and ziprasidone). We also found some evidence on the most commonly reported outcomes (e.g., core illness symptom improvement) for oral aripiprazole and paliperidone. Evidence for the newer drugs (asenapine, brexpiprazole, cariprazine, iloperidone, lurasidone, paliperidone, and long-acting injection [LAI] formulations of aripiprazole and paliperidone) is limited, with few studies, none finding a newer drug superior to an older SGA or each other on any outcome. Similarly, quetiapine and ziprasidone (older SGAs) were not found superior to any other SGA on any outcome.

\section{Benefits Outcomes}

Although functional outcomes were prioritized as most important, few studies of SGA versus SGA reported these outcomes. Very few differences were found among the older SGAs regarding effects on social, occupational, or global functioning (low SOE). A single study found risperidone LAI to result in greater improvements in social function over 24 months compared with quetiapine. None of the studies of the newer SGAs reported on any type of functional outcomes. Findings on quality of life showed that there was no difference between olanzapine and risperidone or ziprasidone (moderate SOE); olanzapine or risperidone oral or LAI and quetiapine; or oral aripiprazole and aripiprazole monthly LAI (low SOE) in studies with up to 2 years of followup.

Symptom response and remission are dichotomous outcomes, which are measured as response or no response, remission or no remission. By definition, response and remission are outcomes that are meant to reflect clinically relevant improvement in core illness symptoms. However, response was defined in varying ways in the trials, although the most common definition was 20 percent improvement on a core illness symptoms scale, such as the Positive and Negative Symptoms Scale (PANSS). A network meta-analysis of 46 head-to-head trials found that olanzapine and risperidone were significantly more likely to result in response than quetiapine (low SOE). Other comparisons and meta-regressions examining the influence of study duration, dose-level, populations (either treatment-resistant or first-episode status), and category of response definition did not result in any statistically significant differences between the SGAs (low SOE). Remission was reported too infrequently to assess comparatively, except in the group of studies on patients with a first episode of schizophrenia.

Improvement in core illness symptoms is a continuous outcome measured as the mean change in symptoms using a scale. A published network meta-analysis of 212 trials found that clozapine was superior to other oral SGAs except for olanzapine in improving core illness symptoms (low SOE). Olanzapine and risperidone were not significantly different compared with each other, and both were superior to the other SGAs, except for paliperidone and clozapine (low SOE). Paliperidone also improved core illness symptoms more than lurasidone and iloperidone (low SOE). This analysis found that all of the drugs included were superior to placebo. In treatment-resistant patients, olanzapine improved core illness symptoms more than quetiapine. These findings are based on two published network meta-analyses (low SOE).

While infrequent, self-harm, including suicide, is a major cause of death among individuals with schizophrenia that antipsychotics, along with other interventions, are intended to help prevent. Although clozapine is often reserved for treatment-resistant patients, due to the serious adverse event profile and required monitoring, evidence supports its superiority over the other SGAs (primarily the older ones) in preventing self-harm (suicide-related outcomes) in both 
patients at risk for suicide-related outcomes (versus olanzapine) and in patients with unknown or mixed risk for these outcomes (versus olanzapine, risperidone, quetiapine, ziprasidone, and aripiprazole) (low SOE).

\section{Harms Outcomes}

Although SGAs have somewhat differing adverse event profiles, the evidence indicates no difference in the overall risk for adverse events between asenapine and olanzapine (moderate SOE). Differences were also not found between quetiapine extended release (ER) versus quetiapine and risperidone; risperidone versus clozapine and aripiprazole; olanzapine versus paliperidone; risperidone LAI versus paliperidone and paliperidone palmitate monthly LAI; and aripiprazole versus aripiprazole monthly LAI (all low SOE). Given the variation in specific adverse event profiles across the SGAs, withdrawals due to adverse events is an outcome measure that has the advantage of measuring the seriousness and tolerability of adverse events experienced, including those that might be treated with another drug or dose reduction. Our network meta-analysis of 90 trials indicates that risperidone LAI had significantly lower risk of withdrawal due to adverse events than five other SGAs: clozapine, lurasidone, quetiapine ER, risperidone and ziprasidone (low SOE). Olanzapine had lower risk than five other SGAs: clozapine, lurasidone, quetiapine, risperidone, and ziprasidone (low SOE). Aripiprazole had lower risk than two SGAs: clozapine and ziprasidone, and cariprazine and iloperidone had lower risk of withdrawal due to adverse events than clozapine (low SOE). Comparative evidence on extrapyramidal symptoms, cardiovascular events, diabetes, weight gain, metabolic syndrome, and sexual function is summarized in the full report. Although these were secondary outcomes in this report, in general the evidence is not able to identify differences between drugs studied in cardiovascular adverse events, metabolic syndrome, and sexual function. Risk of diabetes and weight gain is greater with olanzapine, with increased risk of weight gain also found with clozapine and quetiapine. Findings on extrapyramidal symptoms are more mixed.

All-cause mortality is a rare event, but it is still an important outcome to evaluate as SGAs continue to be developed, approved, and marketed, and particularly as all SGAs carry an FDA Boxed Warning against their use in older patients with dementia due to increased risk of mortality. The mortality rate is low in SGA trials and cohort studies (0 to $1.17 \%$ ), and there were no differences in mortality rates between olanzapine and risperidone or asenapine, risperidone and quetiapine, or paliperidone palmitate monthly LAI and risperidone LAI. There were also no differences in cardiovascular mortality among risperidone, olanzapine, and quetiapine (low SOE). Comparative evidence on the risk of cardiovascular or all-cause mortality was not available for the other SGA drugs.

\section{Subgroups}

There are few differences among the SGAs in effects on several important outcomes, but in some cases the superior drug has serious adverse effects (e.g., clozapine's risk of agranulocytosis [severe neutropenia] and olanzapine's risk of weight gain and new onset diabetes). Therefore, it is especially important to consider how patient characteristics may affect outcomes. Evidence in subgroups was low strength.

In patients experiencing their first episode of schizophrenia, response and remission were not significantly different among olanzapine, quetiapine, risperidone, ziprasidone, aripiprazole, or paliperidone. Most studies also reported no difference in improvement in core illness symptoms, measured by symptoms scales, except that core illness symptoms were more improved with paliperidone than ziprasidone or aripiprazole, but response rates did not differ significantly. 
Response rates with olanzapine and risperidone were similar in patients with first-episode schizophrenia compared with patients with multiple previous episodes. These findings did not differ according to the duration of study, the specific drugs compared, in women, or whether or not studies were blinded. Evidence on SGA treatment discontinuation was more limited, with conflicting findings from five trials. An included systematic review reports that the incidence of clinically important weight gain is significant in first-episode patients, who have little previous exposure to antipsychotics, but differences among the SGA drugs has not been shown. These studies did not find a difference in benefits outcomes between risperidone and olanzapine over the first 3 years of treatment, but they found that that risperidone had higher risk of some specific adverse events (worsening akathisia, sexual dysfunction, and amenorrhea). Aripiprazole had either lower rates of or longer time to discontinuation due to adverse events than ziprasidone or quetiapine. Core illness symptoms were improved more with paliperidone than ziprasidone or aripiprazole, but response rates did not differ significantly.

In treatment-resistant patients (most commonly defined as having received an adequate course of at least two prior antipsychotics without achieving symptom response), a network meta-analysis of 40 trials indicated that olanzapine resulted in greater improvement in core illness symptoms, although the difference in mean change (-6 points) in the PANSS may not meet minimal clinically important difference criteria (-11.5 points for more severe symptoms), depending on the severity of the patient's symptoms at baseline. A network meta-analysis of negative symptoms also found olanzapine significantly better than the other older SGAs, whereas response rates and all-cause discontinuations indicated no significant differences among the older SGAs. Clozapine had fewer discontinuations due to lack of efficacy than risperidone and quetiapine.

The evidence on other subgroups of patients is limited. Analysis of age subgroups did not find differences for comparisons of olanzapine with risperidone. Women had greater improvements than men in core illness symptoms with clozapine and in quality of life with olanzapine. Improvement in core illness symptoms was similar in Asian patients, compared with overall study populations for comparisons of aripiprazole and paliperidone with olanzapine, quetiapine, and risperidone. Among illicit drug users, differences between older SGAs were not found in rate or time to drug discontinuation. Response rates with olanzapine and risperidone were similar in patients with a history of cannabis use disorders and in those without such history.

\section{First-Generation Antipsychotics Versus Second-Generation Antipsychotics}

Although the SGAs were initially marketed as having multiple advantages over the FGAs, there has been concern that the evidence on first-generation versus second-generation antipsychotics was biased toward the SGAs in various ways (e.g., using higher than typical doses of the first-generation drugs). The Clinical Antipsychotic Trials of Intervention Effectiveness (CATIE) trial included one FGA along with five SGAs to test this theory. The trial found perphenazine to be noninferior to the other drugs, with the exception of olanzapine. However, the CATIE trial did not resolve the questions around the use of FGAs in current practice. The findings of the comprehensive systematic review of FGAs versus SGAs published in 2012 are not substantially changed with the additional consideration of five newer studies ( 2 good quality, 2 fair quality, and 1 poor quality). The 111 trials included in the previously published systematic review were rated as mainly fair quality (70 studies), with 41 rated as poor quality, and none rated as good quality. The FGA evidence was largely about haloperidol, with 108 studies, and 
only 7 of perphenazine and 4 of fluphenazine. The most common comparisons were risperidone (37 trials) and olanzapine (34 trials) versus haloperidol.

\section{Benefits Outcomes}

Quality of life, a highly prioritized outcome, was not different between the FGAs and SGAs, quetiapine and risperidone (low SOE), and olanzapine (moderate SOE). Only ziprasidone was found better than haloperidol (low SOE). Evidence on functional outcomes was insufficient to draw conclusions. Risperidone is not different from haloperidol in response rates (moderate SOE). Symptom response and remission were better with olanzapine than haloperidol, but no differences were found in response between haloperidol and aripiprazole, quetiapine and ziprasidone, or in remission between haloperidol and ziprasidone (low SOE).

Comparative evidence on core illness symptoms is only available for haloperidol versus older SGAs. Core illness symptoms were improved significantly more with olanzapine and risperidone than haloperidol (moderate SOE), but evidence on other comparisons did not show significant differences (low SOE). Olanzapine improved negative symptoms significantly more than haloperidol (moderate SOE), and risperidone and aripiprazole improved negative symptoms significantly more than haloperidol (low SOE).

\section{Harms Outcomes}

Overall rates of patients reporting adverse events were 11 to 20 percent higher with haloperidol versus aripiprazole (moderate SOE), risperidone, and ziprasidone (low SOE). Similarly, evidence indicates a higher rate of withdrawal from study (and treatment) due to adverse events with haloperidol versus aripiprazole, olanzapine, risperidone, and ziprasidone (moderate SOE). There were no differences in withdrawal due to adverse events between haloperidol and clozapine or quetiapine (low SOE).

\section{Subgroups}

Evidence comparing FGAs to SGAs in population subgroups is fairly limited, with unclear implications. In general, differences in outcomes were not found between FGAs and SGAs in patients with a first episode of schizophrenia. In treatment-resistant patients the effects on total core illness symptoms and negative symptoms mirrored the findings in the overall population. Response and core illness symptom improvement was similar in Asian populations and the overall study populations. In patients with co-occurring substance use disorder, core illness symptoms were improved more with olanzapine than haloperidol, but not with risperidone.

\section{Key Question 2: Evidence on Psychosocial and Other Nonpharmacological Interventions}

The studies included in our review reported that psychosocial and other nonpharmacological interventions were administered in addition to usual care, which typically includes treatment with antipsychotics, but could include other treatments. Therefore, the studies that make up the evidence base for this question compared (a) psychosocial and other nonpharmacological interventions plus usual care with (b) usual care alone. With usual care as the comparator, we did not include studies that provided direct evidence about head-to-head comparisons and therefore do not consider this a comparative effectiveness review. The evidence base is comprised of 13 systematic reviews (11 good quality, 2 fair quality) that included 271 trials $(\mathrm{N}=25,050)$ relevant to this report. In addition, we included 27 trials that were not included in these reviews $(\mathrm{N}=6,404)$. Of these new trials, 4 were good, 20 were fair, and 3 were poor quality. Overall, no 
psychosocial intervention had high-strength evidence for any outcome of interest, but we found moderate-strength evidence for some outcomes.

\section{Benefit Outcomes}

Patients receiving assertive community treatment were more likely to be living independently and to be employed, and they were less likely to be homeless or to discontinue treatment compared with patients assigned to usual care (moderate SOE). There were no significant differences in the degree of improvement in core illness symptoms or social functioning, and there were no differences in arrests, imprisonment, or police contacts compared with usual care (low SOE).

Cognitive behavioral therapy (CBT) resulted in improvements in global function and quality of life (low SOE), and overall core illness symptoms (moderate SOE) compared with usual care during treatment and with up to 6 months of followup. In studies with longer-term followup after CBT ended, these differences were not significant, although there were few studies with a usual care control group. Low-strength evidence suggests that improvement in negative symptoms was not different between CBT and usual care.

Cognitive remediation resulted in small positive effects on social, occupational, and global function, core illness symptoms (low SOE), and negative symptoms (moderate SOE) compared with usual care over 15 to 16 weeks of treatment.

Supported employment, specifically the individual placement and support model intervention, resulted in significantly better employment outcomes over 2 years compared with usual care. More patients gained either employment (competitive or any job), had more hours worked, were employed longer, and earned more money than those receiving usual care. Evidence with comparisons with other vocational training confirmed these findings.

Family interventions resulted in significantly lower relapse rates than usual care with up to 24 months treatment and at 5 years post-treatment followup; differences in relapse rates were not found from 25 to 36 months. Family interventions improved core illness symptoms, including negative symptoms. Unemployment, independent living, social functioning, or reduction in selfharm were not found to be different between groups (low SOE, except for reduced relapse from 7 to 12 months [moderate SOE]).

Intensive case management was not found to improve global function, quality of life, or core illness symptoms more than usual care.

Illness self-management training interventions reduced symptom severity (moderate SOE) and relapse rates (low SOE). No significant difference was found for negative symptoms (low SOE). Fidelity to intervention was associated with better effects.

Psychoeducation had a greater effect than usual care on global function at 1 year and resulted in lower relapse rates at 9 to 18 months (moderate SOE).

Social skills training improved social function at 6 months, 1 year, and 2 years, compared with usual care. Core illness symptoms and negative symptoms were also improved more with social skills training than usual care.

Supportive therapy was not significantly different from usual care in improving global or social function (low SOE). 


\section{Subgroups}

\section{Clinical Subgroups}

Early team-based multi-component treatment programs for patients with first episode psychosis resulted in significant improvements in global function with up to 2 years of treatment compared with usual care, but there were no significant differences in housing status (moderate SOE). Quality of life was improved and participants in team-based multi-component treatment programs were less likely to relapse (moderate SOE), but there was no difference in total PANSS scores or rates of self-harm compared with usual care (low SOE).

In patients with co-occurring substance use disorder, there was low-strength evidence that assertive community treatment was not different from usual care in function, mortality, and substance use.

\section{Demographic Subgroups}

We found limited subgroup analyses across all psychosocial and nonpharmacological interventions to identify potential patient characteristics that might predict outcomes. Limited evidence on social skills training from one trial of a mixed population (about $50 \%$ diagnosed with schizophrenia or schizoaffective disorder) suggested that the intervention may be more effective in men than women for improving social function and core illness symptoms.

\section{Harms Outcomes}

Four trials and seven systematic reviews assessed or reported any type of harms associated with psychosocial or other nondrug interventions. The few that did (e.g., studies of family interventions) resulted in insufficient evidence.

\section{Discussion}

\section{Key Findings and Strength of Evidence}

This systematic review evaluated the evidence on treatments for schizophrenia, comparing drug treatments with each other and psychosocial and other nonpharmacological interventions with usual care. The purpose was to inform clinicians, patients and their families, and guideline authors with the ultimate goal of improving patient care. In the summary of the key findings and strength of evidence tables (Tables A, B, and C), we do not include findings where the evidence was insufficient to draw conclusions. (The full report presents additional detail on the findings.) There were no instances of high-strength evidence. This was primarily due to specific intervention comparisons having only fair-quality trials with few studies contributing evidence for a particular outcome, leaving moderate- and low-strength evidence. Tables showing the summary results for each drug, indicating magnitude, direction, and strength of evidence for an effect across all seven prioritized, patient-important, outcomes are included in Appendix I of the full report. 
Table A. Summary of key findings and strength of evidence for Key Question 1: SGA versus SGA*

\begin{tabular}{|c|c|c|}
\hline Outcome & Moderate Strength of Evidence & Low Strength of Evidence \\
\hline $\begin{array}{r}\text { Function: } \\
\text { Improvements in } \\
\text { Social Function }\end{array}$ & & $\begin{array}{l}\text { - Risperidone LAI significantly better than quetiapine } \\
\text { in social function over } 24 \text { months } \\
\text { - No difference between paliperidone palmitate LAI } \\
\text { (monthly) and risperidone LAI (every } 2 \text { weeks) }\end{array}$ \\
\hline $\begin{array}{r}\text { Function: } \\
\text { Improvements in } \\
\text { Occupational } \\
\text { Function }\end{array}$ & & $\begin{array}{l}\text { No significant differences between risperidone, } \\
\text { olanzapine, quetiapine, and ziprasidone at } 18 \\
\text { months (CATIE) }\end{array}$ \\
\hline $\begin{array}{r}\text { Function: } \\
\text { Improvements in } \\
\text { Global Functioning }\end{array}$ & & $\begin{array}{l}\text { - Global functioning was not different between } \\
\text { olanzapine and either risperidone or quetiapine }\end{array}$ \\
\hline $\begin{array}{l}\text { Improvements in } \\
\text { Quality of Life }\end{array}$ & $\begin{array}{l}\text { - Olanzapine was not found } \\
\text { significantly different than } \\
\text { risperidone or ziprasidone }\end{array}$ & $\begin{array}{l}\text { With up to } 2 \text { years of followup: } \\
\text { - Olanzapine and risperidone were not found } \\
\text { different from quetiapine } \\
\text { - Risperidone LAI was not found different from } \\
\text { quetiapine } \\
\text { - Oral aripiprazole was not found different from } \\
\text { aripiprazole monthly LAI }\end{array}$ \\
\hline Response & & $\begin{array}{l}\text { - Significantly more likely with olanzapine and } \\
\text { risperidone than quetiapine based on a network } \\
\text { meta-analysis of } 46 \text { trials }\end{array}$ \\
\hline Mortality & & $\begin{array}{l}\text { No difference between: } \\
\text { - Asenapine and olanzapine } \\
\text { - Quetiapine and risperidone } \\
\text { - Paliperidone palmitate LAI (monthly) and } \\
\text { risperidone LAI } \\
\text { - Risperidone, olanzapine, and quetiapine (including } \\
\text { cardiovascular mortality) }\end{array}$ \\
\hline Self-Harm & $\begin{array}{l}\text { - Clozapine was found superior to } \\
\text { olanzapine in preventing significant } \\
\text { suicide attempts or hospitalization to } \\
\text { prevent suicide in high-risk patients }\end{array}$ & $\begin{array}{l}\text { Clozapine was associated with lower risk of suicide } \\
\text { or suicide attempts than olanzapine, quetiapine, } \\
\text { and ziprasidone in unselected patients }\end{array}$ \\
\hline $\begin{array}{r}\text { Core IIIness } \\
\text { Symptoms: } \\
\text { Improvements in } \\
\text { Total Scale Scores }\end{array}$ & & $\begin{array}{l}\text { - Clozapine improved core illness symptoms more } \\
\text { than the other SGAs, except for olanzapine } \\
\text { - Olanzapine and risperidone improved core illness } \\
\text { symptoms more than the other SGAs, except for } \\
\text { each other and paliperidone } \\
\text { - Paliperidone improved core illness symptoms more } \\
\text { than lurasidone and iloperidone } \\
\text { - In treatment-resistant patients, olanzapine } \\
\text { improved core illness symptoms more than } \\
\text { quetiapine }\end{array}$ \\
\hline $\begin{array}{r}\text { Overall Adverse } \\
\text { Events }\end{array}$ & $\begin{array}{l}\text { - No significant difference in overall } \\
\text { adverse events between olanzapine } \\
\text { and asenapine }\end{array}$ & $\begin{array}{l}\text { No differences between: Quetiapine ER vs. } \\
\text { quetiapine and risperidone; risperidone vs. } \\
\text { clozapine and aripiprazole; olanzapine vs. } \\
\text { paliperidone; risperidone LAI vs. paliperidone and } \\
\text { paliperidone palmitate monthly LAI; and } \\
\text { aripiprazole vs. aripiprazole monthly LAI }\end{array}$ \\
\hline
\end{tabular}




\begin{tabular}{|r|c|l|}
\hline \multicolumn{1}{|c|}{ Outcome } & Moderate Strength of Evidence & \multicolumn{1}{c|}{ Low Strength of Evidence } \\
\hline $\begin{array}{r}\text { Withdrawal Due to } \\
\text { Adverse Events }\end{array}$ & & $\begin{array}{l}\text { Based on a network meta-analysis of } 90 \text { trials: } \\
\text { - Risperidone LAl had significantly lower risk than } \\
\text { clozapine, lurasidone, quetiapine ER, risperidone, } \\
\text { and ziprasidone } \\
\text { Olanzapine had lower risk than clozapine, } \\
\text { lurasidone, quetiapine, risperidone, and ziprasidone } \\
\end{array}$ \\
& $\begin{array}{l}\text { Aripiprazole had lower risk than clozapine and } \\
\text { ziprasidone } \\
\text { Cariprazine and Iloperidone had lower risk than } \\
\text { clozapine }\end{array}$ \\
\hline
\end{tabular}

CATIE = Clinical Antipsychotic Trials of Intervention Effectiveness; ER = extended release; IR = immediate release; LAI = long-acting injectable; SGA = second-generation antipsychotic

*No interventions met high strength of evidence criteria for any outcome

Table B. Summary of key findings and strength of evidence for Key Question 1: FGA versus SGA*

\begin{tabular}{|c|c|c|}
\hline Outcome & Moderate Strength of Evidence & Low Strength of Evidence \\
\hline Quality of Life & $\begin{array}{l}\text { - No differences between haloperidol } \\
\text { and olanzapine }\end{array}$ & $\begin{array}{l}\text { - Quality of life was better with ziprasidone than } \\
\text { haloperidol } \\
\text { - No differences between perphenazine and } \\
\text { olanzapine, quetiapine, risperidone, or ziprasidone }\end{array}$ \\
\hline Response/Remission & $\begin{array}{l}\text { - No difference in response rates } \\
\text { between haloperidol and risperidone }\end{array}$ & $\begin{array}{l}\text { - Response was better with olanzapine than } \\
\text { haloperidol } \\
\text { - No difference in response between haloperidol and } \\
\text { aripiprazole, quetiapine, and ziprasidone } \\
\text { - Remission was greater with olanzapine than with } \\
\text { haloperidol } \\
\text { - No difference in remission rates between } \\
\text { haloperidol and ziprasidone }\end{array}$ \\
\hline $\begin{array}{r}\text { Core Illness } \\
\text { Symptoms: } \\
\text { Improvements in } \\
\text { Total Scale Scores }\end{array}$ & $\begin{array}{l}\text { - Olanzapine and risperidone } \\
\text { improved PANSS total more than } \\
\text { haloperidol }\end{array}$ & $\begin{array}{l}\text { - No differences in total PANSS, BPRS, CGI-S, and } \\
\text { CGI-I scores for other FGA vs. SGA comparisons }\end{array}$ \\
\hline $\begin{array}{r}\text { Core Illness } \\
\text { Symptoms: } \\
\text { Improvements in } \\
\text { Negative Scale } \\
\text { Scores }\end{array}$ & $\begin{array}{l}\text { - Olanzapine was more effective than } \\
\text { haloperidol at improving negative } \\
\text { symptoms based on SANS scores }\end{array}$ & $\begin{array}{l}\text { - SGAs had significant, but small, improvements in } \\
\text { PANSS negative subscale scores over haloperidol } \\
\text { (aripiprazole, olanzapine, and risperidone) } \\
\text { - No differences in PANSS negative or SANS scores } \\
\text { for other FGA vs. SGA comparisons }\end{array}$ \\
\hline $\begin{array}{r}\text { Overall Adverse } \\
\text { Events }\end{array}$ & $\begin{array}{l}\text { - Overall adverse event rates favored } \\
\text { SGAs when comparing haloperidol } \\
\text { with aripiprazole }\end{array}$ & $\begin{array}{l}\text { - Overall adverse event rates favored SGAs when } \\
\text { comparing haloperidol with risperidone and } \\
\text { ziprasidone }\end{array}$ \\
\hline $\begin{array}{l}\text { Withdrawal Due to } \\
\text { Adverse Events }\end{array}$ & $\begin{array}{l}\text { - Withdrawals due to adverse events } \\
\text { were significantly higher with } \\
\text { haloperidol use compared with } \\
\text { aripiprazole, olanzapine, } \\
\text { risperidone, and ziprasidone }\end{array}$ & $\begin{array}{l}\text { No differences in withdrawal due to adverse events } \\
\text { between haloperidol and clozapine or quetiapine }\end{array}$ \\
\hline
\end{tabular}

BPRS = Brief Psychiatric Rating Scale; CI = confidence interval; CGI-S = Clinical Global Impressions-Severity scale; CGI-I = Clinical Global Impressions-Improvement scale; FGA = first-generation antipsychotic; PANSS = Positive and Negative Syndrome Scale; RCT = randomized controlled trial; RR = risk ratio; SANS = Scale for Assessment of Negative Symptoms;

SGA = second-generation antipsychotic

*No interventions met high strength of evidence criteria for any outcome 
Table C. Summary of key findings and strength of evidence for Key Question 2:

nonpharmacological interventions versus usual care*

\begin{tabular}{|c|c|c|}
\hline Outcome & Moderate Strength of Evidence & Low Strength of Evidence \\
\hline $\begin{array}{r}\text { Function: } \\
\text { Improvements in } \\
\text { Global Function }\end{array}$ & $\begin{array}{l}\text { - CBT: benefit over usual care over } 6 \\
\text { months; not during } 6 \text { to } 12 \text { months } \\
\text { of treatment } \\
\text { - Early team-based multi-component } \\
\text { treatment programs for first-episode } \\
\text { psychosis: Beneficial with treatment } \\
\text { duration up to } 2 \text { years } \\
\text { - Psychoeducation x } 3 \text { months; } \\
\text { beneficial at 1-year followup }\end{array}$ & $\begin{array}{l}\text { - Social skills training: Beneficial at end of treatment } \\
\text { (6 months to } 2 \text { years treatment duration) versus } \\
\text { usual care } \\
\text { - Cognitive remediation resulted in a small positive } \\
\text { effect on social, occupational, living situation, and } \\
\text { global function versus usual care, based on six } \\
\text { RCTs (effect sizes ranged from } 0.16 \text { to } 0.40 \text { ) } \\
\text { - ICM: Not different from usual care } \\
\text { - Supportive therapy: Not different from usual care }\end{array}$ \\
\hline $\begin{array}{r}\text { Function: } \\
\text { Improvements in } \\
\text { Social Function }\end{array}$ & $\begin{array}{l}\text { - CBT: Benefit over usual care over } 6 \\
\text { months; not during } 6 \text { to } 12 \text { months } \\
\text { treatment } \\
\text { - Early team-based multi-component } \\
\text { treatment programs for first-episode } \\
\text { psychosis: Beneficial with treatment } \\
\text { duration up to } 2 \text { years }\end{array}$ & $\begin{array}{l}\text { - ACT: Not different from usual care in social function } \\
\text { or criminal justice system events } \\
\text { - ICM: Not different from usual care in rate of } \\
\text { imprisonment } \\
\text { - Family Intervention: Not different from usual care }\end{array}$ \\
\hline $\begin{array}{r}\text { Function: } \\
\text { Improvements in } \\
\text { Occupational Function }\end{array}$ & $\begin{array}{l}\text { - ACT: beneficial versus usual care } \\
\text { with intervention duration up to } 2 \\
\text { years } \\
\text { - Supported employment, using the } \\
\text { individual placement and support } \\
\text { (IPS) model is beneficial versus } \\
\text { usual care with intervention } \\
\text { duration up to } 2 \text { years (more } \\
\text { patients employed, worked more, } \\
\text { for longer, and earned more) }\end{array}$ & - Family Interventions: Not different from usual care \\
\hline $\begin{array}{r}\text { Function: } \\
\text { Improvements in } \\
\text { Living Situation }\end{array}$ & $\begin{array}{l}\text { - ACT: beneficial with treatment } \\
\text { duration up to } 2 \text { years }\end{array}$ & - Family Interventions: Not different from usual care \\
\hline $\begin{array}{l}\text { Improvements in } \\
\text { Quality of Life }\end{array}$ & & $\begin{array}{l}\text { - CBT: Benefit over usual care over } 6 \text { months } \\
\text { treatment; difference not found with longer followup } \\
\text { versus usual care (up to } 18 \text { months followup) } \\
\text { - Early team-based multi-component treatment } \\
\text { programs for first-episode psychosis: Beneficial } \\
\text { with treatment duration up to } 2 \text { years }\end{array}$ \\
\hline $\begin{array}{r}\text { Core Illness } \\
\text { Symptoms: } \\
\text { Improvements in Total } \\
\text { Scale Scores }\end{array}$ & $\begin{array}{l}\text { - CBT: Benefit over usual care during } \\
\text { treatment (8 weeks to } 5 \text { years); } \\
\text { effect not maintained after } \\
\text { treatment end } \\
\text { - Illness self-management: Benefit } \\
\text { over usual care during treatment } \\
\text { (12-48 sessions) }\end{array}$ & $\begin{array}{l}\text { - Cognitive remediation: Small improvements in core } \\
\text { illness symptoms versus usual care, based on } 2 \\
\text { trials } \\
\text { - Early team-based multi-component treatment } \\
\text { programs for first-episode psychosis: Not different } \\
\text { from usual care } \\
\text { - Family Interventions: Improved core illness } \\
\text { symptoms } \\
\text { - ICM: Not different from usual care } \\
\text { - Social skills training: Greater improvement than } \\
\text { with usual care during } 6 \text { months and } 2 \text { years of } \\
\text { treatment } \\
\text { - ACT: Not different from usual care }\end{array}$ \\
\hline
\end{tabular}




\begin{tabular}{|c|c|c|}
\hline Outcome & Moderate Strength of Evidence & Low Strength of Evidence \\
\hline $\begin{array}{r}\text { Core Illness } \\
\text { Symptoms: } \\
\text { Improvements in } \\
\text { Negative Scale Scores }\end{array}$ & $\begin{array}{l}\text { - Cognitive remediation: Beneficial } \\
\text { compared with usual care (1 SR of } \\
18 \text { RCTs, effect size }-0.36,95 \% \mathrm{Cl} \\
-0.52 \text { to }-0.20) \text {. }\end{array}$ & $\begin{array}{l}\text { - CBT: Not different from usual care (treatment } \\
\text { duration } 8 \text { weeks to } 5 \text { years) } \\
\text { - Illness self-management: Not different from usual } \\
\text { care (treatment duration } 16-48 \text { sessions) } \\
\text { - Social skills training: Greater improvement than } \\
\text { with usual care during } 6 \text { months and } 2 \text { years of } \\
\text { treatment } \\
\text { - Family interventions: Improved negative symptoms } \\
\text { based on } 3 \text { RCTs }\end{array}$ \\
\hline $\begin{array}{l}\text { Improvements in } \\
\text { Rates of Relapse }\end{array}$ & $\begin{array}{l}\text { - Early team-based multi-component } \\
\text { treatment programs for first-episode } \\
\text { psychosis: Lower relapse rate than } \\
\text { usual care with treatment duration } \\
\text { up to } 2 \text { years } \\
\text { - Psychoeducation x } 3 \text { months; lower } \\
\text { relapse rate than usual care at } 9 \text { to } \\
18 \text { months of followup } \\
\text { - Family Interventions: Lower relapse } \\
\text { rates than usual care from } 7 \text { to } 12 \\
\text { months }\end{array}$ & $\begin{array}{l}\text { - Family interventions: Lower than usual care } 0 \text { to } 6 \\
\text { months, } 13 \text { to } 24 \text { months, } 5 \text { years; not different } \\
\text { from usual care at } 25 \text { to } 36 \text { months } \\
\text { - Illness self-management: Lower relapse with }>10 \\
\text { sessions, not different from usual care with } \leq 10 \\
\text { sessions }\end{array}$ \\
\hline
\end{tabular}

ACT = assertive community treatment; CBT = cognitive behavioral therapy; ICM = intensive case management; IPS = individual placement and support; PANSS = Positive and Negative Syndrome Scale

*No interventions met high strength of evidence criteria for any outcome

\section{Findings in Relationship to What Is Already Known}

With regard to drug therapy, the findings of our review are generally consistent with prior systematic reviews that make comparisons among the SGAs and between SGAs and FGAs. ${ }^{10-15}$ Although we incorporated the most relevant of these systematic reviews in our report, our findings differ to some extent from previous reviews because we consider outcomes prioritized with input from technical experts, incorporate newer evidence and the most recently approved drugs, and include three updated network meta-analyses. For example, in comparing SGAs, our network meta-analyses of response, withdrawal due to adverse events, and all-cause treatment discontinuation of treatment incorporate evidence on brexpiprazole and cariprazine, the two most recently approved oral drugs, and all of the long-acting injection SGAs, whereas the previously published network meta-analyses are limited to older oral drugs, included drugs not approved in the United States, and did not control for important potential effect modifiers. ${ }^{10,11,13,15-18}$ Therefore, there are no existing reviews that cover the same scope as this report.

Our review is consistent with other reviews in the findings on the older SGAs. Clozapine, risperidone, and olanzapine have the most consistent evidence of superiority for specific outcomes (e.g., symptom improvement, response, self-harm, all-cause treatment discontinuations, and time to discontinuation), or populations (first-episode and treatmentresistant). ${ }^{14,17,19-21}$ Other findings in this review are new, such as the finding that risperidone LAI and olanzapine result in significantly lower withdrawals due to adverse events than most other SGAs. Previous reviews did not assess key effectiveness outcomes, such as function, quality of life, and mortality.

A single comprehensive review on FGAs versus SGAs is available and serves as the basis of our review of FGAs versus SGAs, with nine new trials included. ${ }^{22,23}$ Our findings are generally consistent with this review, which concluded that there were few differences of clinical 
importance for effectiveness outcomes, and that evidence on patient-important outcomes and adverse events were not well-studied. In adding new evidence, we found moderate-strength evidence of specific SGAs resulting in better symptom improvement (olanzapine and risperidone) and lower rates of overall adverse events (aripiprazole) and withdrawal due to adverse events (aripiprazole, olanzapine, risperidone, and ziprasidone) than haloperidol.

For the psychosocial interventions, our findings are consistent with some prior review findings and discordant with others. Key reasons for differing findings can be attributed to study eligibility criteria, outcomes included, inclusion of additional, newer studies, and review methodology. For example, we included trials with a usual care comparison group and excluded studies with sample sizes $<50$ patients and studies conducted in countries that were not United States-relevant (primarily studies conducted in China for certain interventions). Each of these criterion eliminated studies that were included in some other reviews.

The decision to focus our review of psychosocial interventions on comparisons with usual care was made as part of a set of decisions required to reduce the scope of the project. After identifying a large body of evidence for Key Question 2, we determined that the funding and timeline required a reduction in scope. We first decided to use systematic reviews as the primary evidence, with subsequently published trials included as well. Examining those, we saw a large amount of heterogeneity in how control groups were defined and handled. In some reviews, all controls were lumped together, while in others "active" and usual care controls were assessed separately. Controls described as "active” varied widely, from competing interventions to attention controls, and these were not handled consistently across reviews. Interventions categorized as "active" in one review were evaluated separately as "passive" in another review. Many, however, reviewed usual care comparisons separately or exclusively. Therefore, within the systematic reviews, usual care was the most commonly reported comparison group. In the end we included well over 200 studies of the 12 psychosocial interventions that made comparisons with usual care. The implications of this choice certainly have been contemplated in the literature before ${ }^{24-27}$ with no clear conclusion, although some have found little difference in analyses limiting to usual care comparisons and those including other comparisons. ${ }^{24}$ The potential bias introduced by this decision depends on the usual care actually received by patients in the control group. For example, if no difference was found between an intervention and usual care controls, it could be attributed to better usual care; but where a difference was found it could be due to the intervention, lower quality usual care, or a combination of factors. In addition, the magnitude of difference could be affected. The difference in usual care received could occur at the patient level, at the study level, or at the body of evidence level for a given intervention.

The decision to eliminate studies conducted in China mainly affected the body of studies for family psychoeducation interventions. In this case, both a prior Cochrane review ${ }^{28}$ and our own analysis indicate that the studies from China very likely overestimate treatment effects, which is consistent with the findings of other researchers in other clinical areas. Our decision to exclude rehospitalization as one of the prioritized outcomes was made after considering input from our technical expert panel, reflecting the lack of confidence that the findings are meaningful across time and different health care systems or settings. While studies of a few interventions regularly report this outcome, primarily as a proxy for relapse, we found that only assertive community treatment formally targets reducing rehospitalization. Hence, we reported rehospitalization as an outcome only for that intervention in the full report.

The other potential reasons for differences are to be expected-our searches are more recent, adding new evidence that could alter the prior findings, and we used the most up-to-date 
systematic review methodology, including assessing the strength of the body of evidence. Our finding that the strength of evidence for psychosocial interventions was moderate or low is consistent with our findings for antipsychotic drugs and with numerous reviews across other populations and interventions. This system of assessing the strength of evidence helps to make clear where future studies could alter findings, either in direction or magnitude, inform future research, and identify outcomes for which a given intervention is not effective. It does not, however, determine whether the intervention is useful or not in a broader sense, since the ratings are made on an outcome-by-outcome basis.

Below we summarize our findings in the context of key prior reviews for selected interventions for which differences in findings may be of particular interest. The Schizophrenia Patient Outcomes Research Team (PORT) 2009 publication is a highly regarded resource that assessed evidence and made recommendations on using several psychosocial interventions, and we discuss their findings as well as individual reviews of these specific interventions. ${ }^{29}$

\section{Cognitive Behavioral Therapy}

Overall, our findings on CBT are consistent with prior findings, except that we found additional outcomes where CBT showed benefit over usual care and we did not find strong evidence regarding duration of effects. Consistent with other reviews, we found CBT to be effective at improving core illness symptoms with treatment durations of 8 weeks to 5 years and additionally for outcomes other than symptoms (e.g., functional outcomes), even when those outcomes were not the focus of the CBT. ${ }^{29-31}$ With respect to the durability of these effects after CBT ends, there is less clarity. A 2011 meta-analysis found that the effects on symptoms were greater at followup that at the end of treatment, but only with comparisons to a diverse group of comparators, and with no specified duration of followup. Their findings for CBT compared with usual care are not statistically significant, so are similar to ours. ${ }^{32}$ Results related to durability of treatment from individual trials with longer post-treatment followup have been mixed. One trial ${ }^{33}$ of 9 months of CBT versus befriending found sustained benefit on overall and negative symptoms at 5-year followup with CBT, while a second trial ${ }^{34}$ of 6 months of intensive CBT versus leisure activities found no difference between groups in negative symptoms after 5 years. Both studies had methodological limitations, which makes generalizable interpretation of these results difficult.

CBT in schizophrenia typically targets positive symptoms, with few studies targeting negative symptoms specifically. ${ }^{30,35}$ Our findings regarding negative symptoms, based on two good-quality systematic reviews, ${ }^{24,36}$ are somewhat in contrast with a 2008 review by Wykes et al. that found CBT associated with significant improvements in negative symptoms. ${ }^{30}$ The Velthorst 2015 review found that studies published prior to 2003 reported larger and more positive effect sizes than studies published later. All three reviews found higher study quality to be associated with lower effect sizes, resulting in a nonsignificant effect on negative symptoms in favor of CBT.

\section{Cognitive Remediation}

Although the direct focus of cognitive remediation is on improving cognitive functioning, an outcome that is outside the scope of our review, there is some evidence that improvements in cognition can lead to improved global functioning. ${ }^{37}$ Our review found that cognitive remediation improved functional outcomes, overall symptoms, and negative symptoms. Our findings differ from the conclusions of the 2009 PORT publication, which determined that the 
evidence base was inadequate to make recommendations, primarily due to a paucity of goodquality trials. Our findings are based on more than 39 trials included in two good-quality systematic reviews. $^{29}$

\section{Family Interventions}

Previous systematic reviews ${ }^{38}$ and other reviews ${ }^{39}$ and the 2009 PORT publication ${ }^{29}$ report findings similar to our review. The 2001 systematic review by Pitschel-Walz and colleagues found that both short- and long-term family interventions are superior to usual care in prevention of relapse. ${ }^{38}$ They also found that the effect remained regardless of the length of the followup period, but that the type of intervention (psychoeducation or therapeutic) made little difference in treatment effect (both better than usual care). These results are largely consistent with our findings. The Dixon update on family psychoeducation ${ }^{39}$ concludes that family psychoeducation should be included as part of best practice guidelines for schizophrenia. The 2009 PORT publication recommends that family interventions should last between 6 and 9 months to reduce rates of relapse and hospitalization. ${ }^{40}$ Similarly, we found the strongest evidence for interventions lasting 7 to 12 months. In addition, we found that the number of sessions was more predictive of reduction in relapse than was duration of treatment. The two studies with family interventions consisting of 10 or fewer sessions at 7 to 12 months were not different from usual care on risk of relapse. Pooled estimates for relapse in trials of 11 to 20 sessions, 21 to 50 sessions, and greater than 50 sessions were all statistically superior to treatment as usual. One difference between our review and some others is that we excluded trials conducted in China as we are not confident that the findings from Chinese studies are applicable to the United States population. Our review, and two other reviews, conducted sensitivity analyses (two analyses, one including the Chinese studies and a second excluding them) and found pooled effect estimates were reduced when Chinese studies were excluded. ${ }^{41,42}$

\section{Social Skills Training}

Our inclusion criteria were considerably stricter than those of other recent reviews ${ }^{43,44}$ in that we limited to larger trials $(\mathrm{N}>50)$ with longer duration ( $>12$ weeks) that utilized a usual care control group. Still, our findings for function, one of the primary targets of social skills training, were consistent with other reviews that found significant improvements in measures of function with social skills training. ${ }^{43-45}$ Our findings for relapse, another target of social skills training, were also consistent with other reviews ${ }^{43,45}$ that found social skills training reduced relapse; however, our estimates did not reach statistical significance, likely due to the low number of events and because the analysis in the other reviews included rehospitalizations as a surrogate for relapse. Our review also found social skills training significantly reduced negative symptoms, a finding that is consistent with one of these other reviews. ${ }^{43}$ The addition of new trials provided information on additional outcomes or durations of followup, but did not change the prior findings. In 2009, the PORT publication reported that evidence for skills training supported benefits in community functioning, but that the studies were not adequate to show positive effects on symptoms or relapse. ${ }^{29}$ Our findings are consistent with these findings.

\section{Supported Employment}

Our findings on supported employment are consistent with other reviews, such as the 2009 PORT recommendations and a review by Marshall, et al. ${ }^{29,46}$ We found that supported employment, specifically the individual placement and support model intervention, resulted in 
significantly better employment outcomes over 2 years compared with usual care. More patients either gained employment (competitive or any job), had more hours worked, were employed longer, or earned more money than those receiving usual care. Because we found only one trial that met our criteria for inclusion in this review, we included a review and a study that included other comparison groups besides usual care. ${ }^{47,48}$ In using this evidence, our findings are similar to PORT and Marshall, with the exception that our strength of evidence rating is moderate, while the Marshall rating is high. Our lower strength of evidence rating is due to our comparison group, i.e., usual care, where Marshall did not specify a comparison group. We note also, that the good quality Cochrane review ${ }^{47}$ that we included rated the evidence as very low quality according to the Grading of Recommendations, Assessment, Development, and Evaluation working group (GRADE) ${ }^{49-55}$ criteria for multiple reasons, including large amounts of missing data due to higher dropout rates in the control groups, skewed data for some outcomes, and concerns over the lack of blinding of outcome assessors.

\section{Applicability}

The applicability of the evidence in this review is limited to adult outpatients in United States-relevant settings. Applicability specific to the Key Questions is summarized in terms of the populations, interventions, comparisons, outcomes, timing, and study designs/settings (PICOTS).

\section{Key Question 1: Comparative Effectiveness of Pharmacological Treatments}

\section{Populations}

Findings are applicable to adults (mean age 25 to 50 years), with mainly moderate and moderate-to-severe disease. There is heterogeneity in the relative predominance of specific symptoms of patients enrolled. For comparisons of SGAs, there is fairly robust evidence on firstepisode patients, but less on treatment-resistant patients. The evidence is not clearly applicable to adolescents, older adults, patients with severe disease, or patients with multiple comorbidities.

\section{Interventions/Comparisons}

For the SGAs versus each other, the majority of the evidence is relevant to comparisons of the older SGAs, with very little evidence regarding drugs approved in the last 10 years. For the FGAs versus the SGAs, the evidence is almost entirely applicable to comparisons of the older SGAs and haloperidol. The evidence is less applicable to newer SGAs (i.e., brexpiprazole, cariprazine, iloperidone, lurasidone, and LAIs of paliperidone and aripiprazole). Evidence on clozapine may be less generalizable due to the potential effects of the required monitoring, which in essence insures adherence to treatment and may provide nonspecific support, encouragement, and even structure to the daily or weekly schedule through consistent interaction with a provider.

\section{Outcomes}

For the SGAs versus each other, there is evidence for all of the prioritized outcomes; however, again the majority of the evidence on effectiveness (long-term health outcomes) is mainly limited to the older drugs. The newer drugs primarily have evidence only for symptombased outcomes and adverse events. For FGAs versus SGAs, the outcomes are more limited, with little good evidence on effectiveness outcomes. The evidence is less applicable to long-term 
outcomes, such as function, long-term quality of life, self-harm, and mortality, particularly for the comparison of FGAs versus SGAs and newer SGAs.

\section{Timing}

For all of the drug interventions, whereas the range of study durations was less than 1 day to 22 years, more studies were short term (6 to 12 weeks) than longer term (1 to 2 years). The evidence is not applicable to long-term followup (greater than 2 years).

\section{Setting}

For SGAs versus each other, the evidence applies only to outpatients. In the systematic review we included on FGAs versus SGAs, almost half the studies were in inpatients.

\section{Key Question 2: Psychosocial and Other Nonpharmacological Interventions}

Similar to the issues noted in Key Question 1, the evidence base is limited in part by the scope identified for this review. For example, for Key Question 2 we added criteria that studies had to have at least 50 percent of patients diagnosed with schizophrenia, to reflect the fact that many of these interventions are aimed at patients with serious mental illness, as a group, rather than at specific diagnoses. Similar to our limiting FGAs to only the three drugs most commonly used today, we limited the Key Question 2 interventions also to those that are used commonly in clinical practice. We also limited to studies with a comparator of usual care across the 13 interventions included. Thus, this is not a traditional comparison of two active interventions.

\section{Populations}

Findings are applicable to adults ranging in age from 16 to 80 years (adolescents to older adults), mostly with a diagnosis of schizophrenia or a related disorder. The specific characteristics of patients varied somewhat by intervention category. For example, supportive therapy is most applicable to middle-aged men with schizophrenia and related conditions who were experiencing long-standing hallucinations and/or delusions. The evidence is not clearly applicable to patients with treatment resistance, or multiple comorbidities. Across the interventions it is not clear what level of disease severity was addressed.

\section{Interventions/Comparisons}

The evidence in this review, by design, applies only to the comparisons with usual care, and the 13 intervention categories identified here. The evidence is not applicable to comparative effectiveness questions. For some interventions, such as family interventions and supportive therapy, a key limitation of the ability to understand the applicability of the evidence is varying or unclear definitions and descriptions of the elements of interventions and poor reporting of intervention and usual care details. As a result, specific description of the intervention applicability is limited. The evidence is less applicable to variations of these interventions, or emerging interventions.

\section{Outcomes}

The evidence is applicable only to a select group of outcomes that vary by intervention. Not all prioritized outcomes were reported consistently across studies. The evidence generally does not apply to long-term effectiveness outcomes that were highly prioritized (e.g., function, quality 
of life, mortality). For some interventions, outcomes reported were common, standard outcomes used in assessing individuals with schizophrenia, whereas for others there was wide variety and introduction of unique outcome measures.

\section{Timing}

Most of the interventions do not have evidence that is applicable to long-term followup (greater than 2 years).

\section{Setting}

The settings were mostly applicable to the United States, as evidence clearly not applicable was excluded from our review. The evidence is not exclusively applicable to the outpatient setting. Although the criteria for this review stipulated an outpatient setting, several of the systematic reviews used to provide evidence for Key Question 2 included inpatient studies as well, limiting the applicability based on setting.

\section{Research Recommendations}

Based on the research gaps and limitations identified in this review (see the full report for a more extensive discussion of limitations of the review and of the evidence base), we recommend the following:

\section{Pharmacological Interventions}

Trials should:

- Involve multiple newer SGA drugs (approved in the last 10 years), in comparison with one of the older SGAs (e.g., clozapine, olanzapine, risperidone LAI) and haloperidol and compare fluphenazine and perphenazine with both older and newer SGAs.

- Ensure comparable dosing with the best dosing titration methods for all drugs included.

- Measure key health outcomes, using agreed-upon direct measures. For example, measuring functional outcomes using not only valid and reliable scales, but also actual measures of patient functioning. These measures need to be agreed upon by clinical and research experts and then used consistently across trials.

- Study durations must reflect real-life practice. Minimum study duration should be 1 year, with 3- to 5-year followup in order to measure the durability of effects, and truly longterm outcomes, including harms (e.g., metabolic changes and tardive dyskinesia). Longterm harms are not assessable in short-term studies, and relying on observational evidence has limitations.

- The concept of recovery should be incorporated into study designs, with testing of duration of effect and discontinuation of drug treatment following remission.

- Enroll subjects who reflect real populations. Studies exclusively of older patients, with multiple comorbidities and concomitant medications, and patients with severe disease, including treatment-resistance are needed. To better study other subgroups, such as minorities and women, specification and planning of subgroup analyses a priori and use of randomization methods that insure adequate distribution of these characteristics are needed to examine differences.

- Inpatients need to be studied separately from outpatients. Future reviews should evaluate treatments for inpatients. 


\section{Psychosocial and Other Nonpharmacological Interventions}

The issues may vary by the specific intervention, but below are several key recommendations:

- Trials should have adequate sample sizes to address important health outcomes, rather than intermediate or surrogate outcomes and should adhere to the current standards for reporting, such as the Consolidated Standards of Reporting Trials (CONSORT) criteria. ${ }^{56}$

- Studies need to be conducted in broader, but better-defined populations, with either separate studies of subpopulations or large enough sample sizes to allow meaningful subgroup analysis.

o Future studies might consider using the National Institutes of Mental Health Research Domain Criteria ${ }^{57}$ approach to categorizing patients.

o Future reviews should evaluate treatments for inpatients.

- Interventions should be clearly defined and described, including required components. Some interventions, such as cognitive remediation, have used expert groups to refine definitions and required components of interventions. Measurements of fidelity to the intervention model should be undertaken where possible.

- Trials need to evaluate and report patient-important health outcomes such as function, quality of life, self-harm, and adverse effects using standardized and easily interpretable methods. Studies should identify what constitutes clinically meaningful change in scale scores.

- Studies are needed to address the heterogeneity in usual care control groups. Usual care is highly variable; so studies using a usual care control group must report on the specific services and treatments received and standardize the comparison or control for attention effects.

- Studies should measure both intensity and duration of intervention required to achieve the best result and the duration of effect in relation to these.

- Additional well-designed long-term studies are needed. The long-term benefits versus risks and costs of treatments remain unclear, particular for individuals whose illness is resistant or only partially responsive to treatment.

- Future systematic review research should:

o Include an evaluation of comparative effectiveness of psychosocial interventions compared with each other. Emerging methods of evaluating complex interventions may be helpful in such future studies. ${ }^{58,59}$

o Include other nonpharmacological, device-based somatic treatments, such as electroconvulsive therapy and transcranial magnetic stimulation.

o Organize the evidence according to the patient characteristics that the intervention focuses on.

\section{Conclusions}

The majority of the comparative evidence on pharmacotherapy to treat schizophrenia relates to the older SGAs (mainly clozapine, olanzapine, risperidone, quetiapine, and ziprasidone), with some evidence on paliperidone and aripiprazole, and the LAIs of risperidone, aripiprazole, and paliperidone. There is very little comparative evidence on newer SGAs (drugs approved in the last 10 years: asenapine, brexpiprazole, cariprazine, iloperidone, and lurasidone). Although there are some differences among the older SGAs on specific outcomes, no single drug was superior 
on multiple high-priority outcomes. However, clozapine, olanzapine, and risperidone oral and LAI did have superiority on more outcomes than other SGAs and quetiapine and ziprasidone were not superior to other SGAs on any outcome. No evidence found a newer SGA superior to older SGAs on any outcome. Evidence on FGAs versus SGAs indicates that olanzapine, risperidone, ziprasidone, and aripiprazole were similar to haloperidol on some outcomes of benefit, and were superior on overall adverse events and withdrawal due to adverse events.

In comparison with usual care, most of the psychosocial interventions to treat schizophrenia reviewed were more effective in improving two or more outcomes, including nontargeted but patient-important outcomes. Various functional outcomes were improved more with assertive community treatment, CBT, psychoeducation, social skills training, supported employment, and early team-based multi-component treatment programs for patients with first-episode psychosis than with usual care. Quality of life was improved more with CBT and early team-based multicomponent treatment programs for first-episode psychosis than usual care. Core illness symptoms were improved with assertive community treatment, CBT, cognitive remediation, illness self-management, psychoeducation, social skills training, and early team-based multicomponent treatment programs for patients with first-episode psychosis. Relapse was reduced with psychoeducation, illness self-management, family interventions, and early team-based multi-component treatment programs for patients with first-episode psychosis. Self-harm, response and/or remission, and adverse events were rarely reported. 


\section{References}

1. American Psychiatric Association.

Diagnostic and Statistical Manual of Mental

Disorders, 5th ed. Arlington, VA: American

Psychiatric Publishing; 2013.

2. Harding CM. Changes in Schizophrenia

Across Time: Pardoxes, Patterns and

Predictors. In: Cohen CI, ed Schizophrenia

into later life: Treatment, research and

policy. Arlington, VA: American Psychiatric

Publishing, Inc.; 2003:19-41.

3. Wils RS, Gotfredsen DR, Hjorthøj C, et al. Antipsychotic medication and remission of psychotic symptoms 10 years after a firstepisode psychosis. Schizophr Res.

2017;182:42-8. doi:

10.1016/j.schres.2016.10.030. PMID: 28277310 .

4. Wunderink L, Nieboer RM, Wiersma D, et al. Recovery in remitted first-episode psychosis at 7 years of follow-up of an early dose reduction/discontinuation or maintenance treatment strategy: long-term follow-up of a 2-year randomized clinical trial. JAMA Psychiatry. 2013

Sep;70(9):913-20. doi:

10.1001/jamapsychiatry.2013.19. PMID: 23824214.

5. Agency for Healthcare Research and Quality. Methods Guide for Effectiveness and Comparative Effectiveness Reviews. AHRQ Publication No. 10(14)-EHC063-EF. Rockville MD: January 2014. Chapters available at:

www.effectivehealthcare.ahrq.gov Accessed April 22, 2017.

6. Moher D, Liberati A, Tetzlaff J, et al. Preferred reporting items for systematic reviews and meta-analyses: the PRISMA statement. BMJ. 2009 Jul 21;339:b2535. doi: 10.1136/bmj.b2535. PMID: 19622551.

7. McDonagh MS, Jonas DE, Gartlehner G, et al. Methods for the drug effectiveness review project. BMC Med Res Methodol. 2012;12(1):140. doi: 10.1186/1471-228812-140. PMID: 22970848.
8. Shea BJ, Grimshaw JM, Wells GA, et al. Development of AMSTAR: a measurement tool to assess the methodological quality of systematic reviews. BMC Med Res Methodol. 2007 Feb 15;7:10. doi: 10.1186/1471-2288-7-10. PMID: 17302989.

9. Berkman ND, Lohr KN, Ansari MT, et al. Grading the strength of a body of evidence when assessing health care interventions: an EPC update. J Clin Epidemiol. 2015 11//;68(11):1312-24. doi: 10.1016/j.jclinepi.2014.11.023. PMID: 25721570.

10. Kishimoto T, Robenzadeh A, Leucht C, et al. Long-acting injectable vs oral antipsychotics for relapse prevention in schizophrenia: a meta-analysis of randomized trials. Schizophr Bull. 2014 Jan;40(1):192-213. doi: 10.1093/schbul/sbs150. PMID: 23256986.

11. Leucht S, Cipriani A, Spineli L, et al. Comparative efficacy and tolerability of 15 antipsychotic drugs in schizophrenia: a multiple-treatments meta-analysis. Lancet. 2013 Sep 14;382(9896):951-62. doi: 10.1016/S0140-6736(13)60733-3. PMID: 23810019.

12. Ranasinghe I, Sin J. A systematic review of evidence-based treatment for individuals with treatment-resistant schizophrenia and a suboptimal response to clozapine monotherapy. Psychosis. 2014 Jul;6(3):25365. doi: 10.1080/17522439.2013.830319.

13. Samara MT, Dold M, Gianatsi M, et al. Efficacy, acceptability, and tolerability of antipsychotics in treatment-resistant schizophrenia: a network meta-analysis. JAMA Psychiatry. 2016 Mar;73(3):199-210. doi: 10.1001/jamapsychiatry.2015.2955. PMID: 26842482.

14. Souza JS, Kayo M, Tassell I, et al. Efficacy of olanzapine in comparison with clozapine for treatment-resistant schizophrenia: evidence from a systematic review and meta-analyses. CNS Spectr. 2013 Apr;18(2):82-9. doi: 10.1017/S1092852912000806. PMID: 23253621. 
15. Zhang J-P, Gallego JA, Robinson DG, et al. Efficacy and safety of individual secondgeneration vs. first generation antipsychotics in first-episode psychosis: a systematic review and meta-analysis. Int $\mathrm{J}$

Neuropsychopharmacol. 2013

Jul;16(6):1205-18. doi:

10.1017/S1461145712001277. PMID: 23199972.

16. Fusar-Poli P, Kempton MJ, Rosenheck RA. Efficacy and safety of second-generation long-acting injections in schizophrenia: a meta-analysis of randomized-controlled trials. Int Clin Psychopharmacol. 2013 Mar;28(2):57-66. doi: 10.1097/YIC.0b013e32835b091f. PMID: 23165366.

17. Soares-Weiser K, Bechard-Evans L, Lawson $\mathrm{AH}$, et al. Time to all-cause treatment discontinuation of olanzapine compared to other antipsychotics in the treatment of schizophrenia: a systematic review and meta-analysis. Eur Neuropsychopharmacol. 2013 Feb;23(2):118-25. doi: 10.1016/j.euroneuro.2012.05.001. PMID: 22633617.

18. Tonin FS, Piazza T, Wiens A, et al. Adverse events and treatment failure leading to discontinuation of recently approved antipsychotic drugs in schizophrenia: a network meta-analysis. Schizophr Res. 2015 Dec;169(1-3):483-5. doi: 10.1016/j.schres.2015.09.019. PMID: 26516102.

19. Asenjo Lobos C, Komossa K, RummelKluge C, et al. Clozapine versus other atypical antipsychotics for schizophrenia. Cochrane Database Syst Rev. 2010 Nov 10(11):Cd006633. doi: 10.1002/14651858.CD006633.pub2. PMID: 21069690.

20. Asmal L, Flegar SJ, Wang J, et al. Quetiapine versus other atypical antipsychotics for schizophrenia. Cochrane Database Syst Rev. 2013 Nov 18(11):Cd006625. doi: 10.1002/14651858.CD006625.pub3. PMID: 24249315.
21. Ayub M, Saeed K, Munshi TA, et al. Clozapine for psychotic disorders in adults with intellectual disabilities. Cochrane Database Syst Rev. 2015 Sep 23(9):Cd010625. doi: 10.1002/14651858.CD010625.pub2. PMID: 26397173.

22. Abou-Setta AM, Mousavi SS, Spooner C, et al. First-generation versus second-generation antipsychotics in adults: comparative effectiveness. Comparative effectiveness review No. 63. (Prepared by the University of Alberta Evidence-based Practice Center under Contract No. 290-200-10021.) AHRQ Publication No. 12-EHC054-EF. Rockville, MD: Agency for Healthcare Research and Quality: 2012.

www.effectivehealthcare.ahrq.gov/reports/fi nal.cfm Accessed April 22, 2017. PMID: 23035275.

23. Hartling L, Abou-Setta AM, Dursun S, et al. Antipsychotics in adults with schizophrenia: comparative effectiveness of first-generation versus second-generation medications: a systematic review and meta-analysis. Ann Intern Med. 2012 Oct 2;157(7):498-511. PMID: 22893011.

24. Jauhar S, McKenna PJ, Radua J, et al. Cognitive-behavioural therapy for the symptoms of schizophrenia: systematic review and meta-analysis with examination of potential bias. Br J Psychiatry. 2014 Jan;204(1):20-9. doi: 10.1192/bjp.bp.112.116285. PMID: 24385461.

25. Kirsch I. Placebo psychotherapy: synonym or oxymoron? J Clin Psychol. 2005 Jul;61(7):791-803. doi: 10.1002/jclp.20126. PMID: 15827992.

26. Chambless DL, Hollon SD. Defining empirically supported therapies. J Consult Clin Psychol. 1998 Feb;66(1):7-18. PMID: 9489259.

27. Jensen PS, Weersing R, Hoagwood KE, et al. What is the evidence for evidence-based treatments? A hard look at our soft underbelly. Ment Health Serv Res. 2005 Mar;7(1):53-74. PMID: 15832693. 
28. Vickers A, Goyal N, Harland R, et al. Do certain countries produce only positive results? A systematic review of controlled trials. Control Clin Trials. 1998;19(2):15966. doi: 10.1016/S0197-2456(97)00150-5. PMID: 9551280.

29. Dixon LB, Dickerson F, Bellack AS, et al. The 2009 schizophrenia PORT psychosocial treatment recommendations and summary statements. Schizophr Bull. 2010 Jan;36(1):48-70. doi: 10.1093/schbul/sbp115. PMID: 19955389.

30. Wykes T, Steel C, Everitt B, et al. Cognitive behavior therapy for schizophrenia: effect sizes, clinical models, and methodological rigor. Schizophr Bull. 2008 May;34(3):52337. PMID: 17962231.

31. Jones C, Hacker D, Cormac I, et al. Cognitive behavioural therapy versus other psychosocial treatments for schizophrenia. Cochrane Database Syst Rev. 2012 Apr 18(4):Cd008712. doi: 10.1002/14651858.CD008712.pub2. PMID: 22513966.

32. Sarin F, Wallin L, Widerlov B. Cognitive behavior therapy for schizophrenia: a metaanalytical review of randomized controlled trials. Nord J Psychiatry. 2011

Jun;65(3):162-74. doi: 10.3109/08039488.2011.577188. PMID: 21563994.

33. Turkington D, Sensky T, Scott J, et al. A randomized controlled trial of cognitivebehavior therapy for persistent symptoms in schizophrenia: a five-year follow-up. Schizophr Res. 2008 Jan;98(1-3):1-7. PMID: 17936590.

34. Drury V, Birchwood M, Cochrane R. Cognitive therapy and recovery from acute psychosis: a controlled trial. 3. Five-year follow-up. Br J Psychiatry. 2000 Jul;177:814. PMID: 10945081.

35. Rector NA, Beck AT. Cognitive behavioral therapy for schizophrenia: An empirical review. J Nerv Ment Dis. 2001

May;189(5):278-87. doi: 10.1097/00005053-200105000-00002. PMID: 11379970.
36. Velthorst E, Koeter M, van der Gaag M, et al. Adapted cognitive-behavioural therapy required for targeting negative symptoms in schizophrenia: meta-analysis and metaregression. Psychol Med. 2015 Feb;45(3):453-65. doi: 10.1017/S0033291714001147. PMID: 24993642.

37. Cella M, Preti A, Edwards C, et al. Cognitive remediation for negative symptoms of schizophrenia: A network meta-analysis. Clin Psychol Rev. 2017 Mar;52:43-51. doi: 10.1016/j.cpr.2016.11.009. PMID: 27930934.

38. Pitschel-Walz G, Leucht S, Bauml J, et al. The effect of family interventions on relapse and rehospitalization in schizophrenia--a meta-analysis. Schizophr Bull. 2001;27(1):73-92. PMID: 11215551.

39. Dixon L, Adams C, Lucksted A. Update on family psychoeducation for schizophrenia. Schizophr Bull. 2000;26(1):5-20. PMID: 10755667.

40. Dixon L, Duncan DC, Johnson P, et al. Occupational therapy for patients with Parkinson's disease. Cochrane Database Syst Rev. 2009(4) PMID: 17636709.

41. Pharoah F, Mari J, Rathbone J, et al. Family intervention for schizophrenia. Cochrane Database Syst Rev. 2010 Dec 08(12):CD000088. doi: 10.1002/14651858.CD000088.pub2. PMID: 21154340.

42. Xia J, Merinder BL, Belgamwar MR. Psychoeducation for schizophrenia. Cochrane Database Syst Rev. 2013(1) PMID: 21678337.

43. Kurtz MM, Mueser KT. A meta-analysis of controlled research on social skills training for schizophrenia. J Consult Clin Psychol. 2008 Jun;76(3):491-504. doi: 10.1037/0022006X.76.3.491. PMID: 18540742.

44. Almerie MQ, Okba Al Marhi M, Jawoosh $\mathrm{M}$, et al. Social skills programmes for schizophrenia. Cochrane Database Syst Rev. 2015 Jun 09(6):Cd009006. doi: 10.1002/14651858.CD009006.pub2. PMID: 26059249. 
45. Pfammatter M, Junghan UM, Brenner HD. Efficacy of psychological therapy in schizophrenia: conclusions from metaanalyses. Schizophr Bull. 2006;32(suppl_1):S64-S80. doi: 10.1093/schbul/sbl030. PMID: 16905634.

46. Marshall T, Goldberg RW, Braude L, et al. Supported employment: assessing the evidence. Psychiatr Serv. 2014 Jan 01;65(1):16-23. doi: 10.1176/appi.ps.201300262. PMID: 24247197.

47. Kinoshita Y, Furukawa TA, Kinoshita K, et al. Supported employment for adults with severe mental illness. Cochrane Database Syst Rev. 2013 Sep 13(9):Cd008297. doi: 10.1002/14651858.CD008297.pub2. PMID: 24030739.

48. Cook JA, Leff HS, Blyler CR, et al. Results of a multisite randomized trial of supported employment interventions for individuals with severe mental illness. Arch Gen Psychiatry. 2005 May;62(5):505-12. PMID: 15867103.

49. Balshem $\mathrm{H}$, Helfand $\mathrm{M}$, Schünemann HJ, et al. GRADE guidelines: 3 . Rating the quality of evidence. J Clin Epidemiol. 2011;64(4):401-6. PMID: 21208779.

50. Guyatt GH, Oxman AD, Kunz R, et al. GRADE guidelines: 2. Framing the question and deciding on important outcomes. J Clin Epidemiol. 2011;64(4):395-400. PMID: 21194891.

51. Guyatt GH, Oxman AD, Kunz R, et al. GRADE guidelines: 6 . Rating the quality of evidence-imprecision. J Clin Epidemiol. 2011;64(12):1283-93. PMID: 21839614.

52. Guyatt GH, Oxman AD, Kunz R, et al. GRADE guidelines: 8. Rating the quality of evidence-indirectness. J Clin Epidemiol. 2011;64(12):1303-10. PMID: 21802903.
53. Guyatt GH, Oxman AD, Kunz R, et al. GRADE guidelines: 7. Rating the quality of evidence-inconsistency. J Clin Epidemiol. 2011;64(12):1294-302. PMID: 21803546.

54. Guyatt GH, Oxman AD, Montori V, et al. GRADE guidelines: 5 . Rating the quality of evidence-publication bias. J Clin Epidemiol. 2011;64(12):1277-82. PMID: 29802904.

55. Guyatt GH, Oxman AD, Vist G, et al. GRADE guidelines: 4 . Rating the quality of evidence - study limitations (risk of bias). J Clin Epidemiol. 2011;64(4):407-15. PMID: 21247734.

56. Schulz KF, Altman DG, Moher D. CONSORT 2010 Statement: updated guidelines for reporting parallel group randomised trials. BMJ. 2010;340. doi: 10.1136/bmj.c332. PMID: 20332509.

57. National Institute of Mental Health. Research Domain Criteria. https://www.nimh.nih.gov/researchpriorities/rdoc/index.shtml. Accessed July 172017.

58. Guise JM, Chang C, Viswanathan M, et al. Agency for Healthcare Research and Quality Evidence-based Practice Center methods for systematically reviewing complex multicomponent health care interventions. J Clin Epidemiol. 2014 Nov;67(11):1181-91. doi: 10.1016/j.jclinepi.2014.06.010. PMID: 25438663.

59. Guise JM, Chang C, Viswanathan M, et al. AHRQ Methods for Effective Health Care Agency for Healthcare Research and Quality (US). Rockville (MD): 2014. PMID: 24696890. 


\section{Introduction}

\section{Background}

\section{Condition}

Schizophrenia is a chronic mental health condition that most often presents in early adulthood and can lead to disabling outcomes. The most recent version of the American Psychiatric Association's Diagnostic and Statistical Manual of Mental Disorders, 5th Edition (DSM-5), ${ }^{1}$ has continued the trend of clarifying and simplifying the diagnostic criteria for schizophrenia from the American Psychiatric Association's Diagnostic and Statistical Manual of Mental Disorders, 3rd Edition (DSM-III) through DSM-IV without changing the defined patient population among the editions. ${ }^{2}$ Currently, DSM-5 defines schizophrenia as: the presence of two or more of the five core symptoms (delusions, hallucinations, disorganized speech, grossly disorganized or catatonic behavior, and negative symptoms); at least one of the symptoms being delusions, hallucinations, or disorganized speech; and, symptoms being present for at least 6 months. Lifetime prevalence is approximately 0.3 to 0.7 percent, with onset most commonly between late adolescence through the third decade. ${ }^{3}$ Differential diagnosis is broad and includes delineation from mood disorders (bipolar disorder or major depressive disorder) with psychotic features and substance/medication-induced psychotic disorders. The course of schizophrenia varies. Approximately 20 percent of individuals may experience significant improvement including, in some cases, full recovery; ${ }^{4}$ however, the majority tend to experience some degree of social and occupational difficulty as well as need for daily living supports. That said, more recent research and practice has focused on early intervention with first episode psychosis, demonstrating promise toward improving outcomes sooner and reducing longer-term disability. ${ }^{5,6}$

\section{Treatment Strategies}

Antipsychotic medications (primarily effective via dopaminergic antagonism) and nonpharmacological treatments are typically used together when treating individuals with schizophrenia. Both approaches can result in meaningful improvements in a variety of outcome areas, including psychiatric symptoms, functioning (e.g., employment, social), service utilization (e.g., hospitalization, crisis services), legal system involvement, quality of life, self-harm and aggressive behaviors, treatment engagement and retention, and co-occurring substance abuse. Ideally, improvements in symptoms translate to long-term, clinically relevant, positive changes in other outcome areas, with limited and manageable adverse effects. While pivotal trials of antipsychotic efficacy are limited to measurement of symptom reduction, measurement of other important recovery-oriented outcomes that reflect improvement in social and occupational functioning are necessary to describe benefits of treatments on overall quality of life and functional ability.

Historically, the wide array of antipsychotic drug treatments has had uncertain impact on long-term patient-centered outcomes, such as the ability to have consistent employment and successful interpersonal relationships, as well as maintenance in independent living, and includes serious concerns about adverse effects (e.g., tardive dyskinesia, weight gain, diabetes, and dyslipidemia) for some treatments. Many patients prescribed an antipsychotic discontinue it. Discontinuation rates and time to discontinuation vary by treatment and patient characteristics. 
Older, first-generation antipsychotics (FGAs), such as haloperidol, have proven efficacy but adverse effects such as extrapyramidal symptoms (EPS) and in some cases tardive dyskinesia, often limit long-term adherence. Second-generation antipsychotics (SGAs), beginning with clozapine, were introduced as having equal or better efficacy, specifically potentially better effects on negative symptoms, and the hope of fewer EPS and lower risk of tardive dyskinesia. But SGAs also have potentially serious adverse effects (e.g., cardiovascular and endocrinologic adverse effects) that make their overall risk/benefit profile less clear-cut than anticipated.

Although there are a large number of treatments for schizophrenia, it is not clear whether they afford long-term benefits on employment and social relationships and improve the likelihood of recovery, and the most effective duration of treatment is unclear. Equally important in selecting among competing interventions for a specific patient is consideration of patient-level characteristics that may affect the outcomes (including age, duration of symptoms, severity, and other psychiatric or medical comorbidities) across a diverse group of possible interventions. For example, treatment of negative symptoms (e.g., diminished emotional response and lack of interest) may differ from treatment of positive symptoms (e.g., hallucinations and delusions). Most psychosocial interventions have specific targets for the patient population and outcome measure. Patients and providers are also interested in other patient-centered health outcomes.

While the success of any given treatment depends on the balance of benefit and harm, specific treatment considerations will vary across the lifespan. Treating patients aggressively early in the disease is thought to improve long-term outcomes although overly aggressive treatments, particularly drug treatments, may result in adverse effects. Additionally, substance abuse (e.g., tobacco, alcohol, illicit drugs) may begin early on for individuals with schizophrenia, and in these patients successful treatment of schizophrenia involves consideration of this comorbidity as well. ${ }^{7-10}$ The metabolic effects related to both the disease and drug treatments are concerning across age groups, starting in adolescence or early adulthood. ${ }^{11}$ Epidemiologic studies have found an association with obesity, elevated lipids, and shorter lifespan, and several antipsychotic drugs are known to increase these risks. ${ }^{12-18}$ Older patients may have increased risk of mortality ${ }^{19-22}$ and require antipsychotic dosing changes with age. ${ }^{23,24}$ Across all age groups, primary goals include diminishing core illness symptoms and reducing relapses of acute psychosis; however, physical health comorbidities can present challenges requiring modification of the treatment plan.

\section{Scope and Key Questions}

\section{Scope of the Review}

In this systematic review, we evaluate the current evidence to inform treatment options for individuals with schizophrenia. The review synthesizes evidence on the comparative effectiveness of pharmacological treatments and the effectiveness of common psychosocial and other nonpharmacological treatment strategies for individuals with schizophrenia, points out areas of controversy, and highlights future research needed.

Due to a very large number of studies and interventions, the scope of the review focuses on specific types of evidence. For pharmacological interventions, we limited to the most commonly used FGA drugs (fluphenazine, haloperidol, and perphenazine), but included all United States Food and Drug Administration (FDA)-approved SGA drugs. For the pharmacological interventions, the focus was on comparative evidence, directly comparing drugs to each other. This decision was based in part on the availability of sufficient evidence on general efficacy of 
the drugs to treat schizophrenia in the form of studies used to gain FDA approval, that is, placebo-controlled trials. For psychosocial and nonpharmacological interventions, we limited to the most commonly-used interventions relevant to United States practices. In doing this, we excluded single studies of unique interventions, and studies conducted in countries with cultural, social, and health care environments that are very different from the United States. We limited to studies comparing a psychosocial or other nonpharmacological intervention to "usual care" as a common comparator. This decision was made largely due to the heterogeneity of interventions and comparators across studies, the existence of many studies comparing these interventions to usual care, and to provide a common comparator across the interventions.

We limited the outcomes to those that are patient centered health outcomes (rather than intermediate outcomes), which were arranged according to their priority from the perspective of the patient, their family, and their clinicians. We considered advice from our experts in selecting and prioritizing the outcomes. We did not include two outcomes that are sometimes evaluated. First, we excluded rehospitalization, because: (1) there is important variation in the indications for and length of psychiatric hospitalizations across time, in different localities, and with different financial contexts; and (2) there is important variation across trials in how rehospitalization is measured/evaluated, which may confound study interpretation. For assertive community treatment, where decreasing rehospitalization is the target of the intervention, we included rehospitalization as an outcome. Second, we excluded changes in neurocognitive test results, as these were viewed as an intermediate outcome. Instead, we have prioritized measures of functioning that include neurocognition as part of a set of broader patient-centered health outcomes. The Analytic Framework for the review appears in Figure 1.

\section{Key Questions}

\section{1a. What are the comparative benefits and harms of pharmacological treatments for adults with schizophrenia?}

\section{1b. How do the benefits and harms of pharmacological treatments for adults with schizophrenia vary by patient characteristics? ${ }^{\mathrm{a}}$}

\section{2a. What are the benefits and harms of psychosocial and other nonpharmacological treatments for adults with schizophrenia?}

\section{$2 \mathrm{~b}$. How do the benefits and harms of psychosocial and other nonpharmacological treatments for adults with schizophrenia vary by patient characteristics? ${ }^{\mathrm{a}}$}

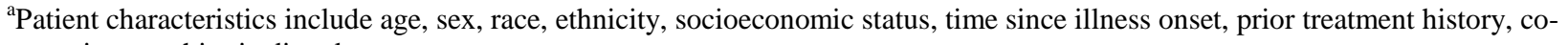
occurring psychiatric disorders, pregnancy, etc.
} 
Figure 1. Analytic framework

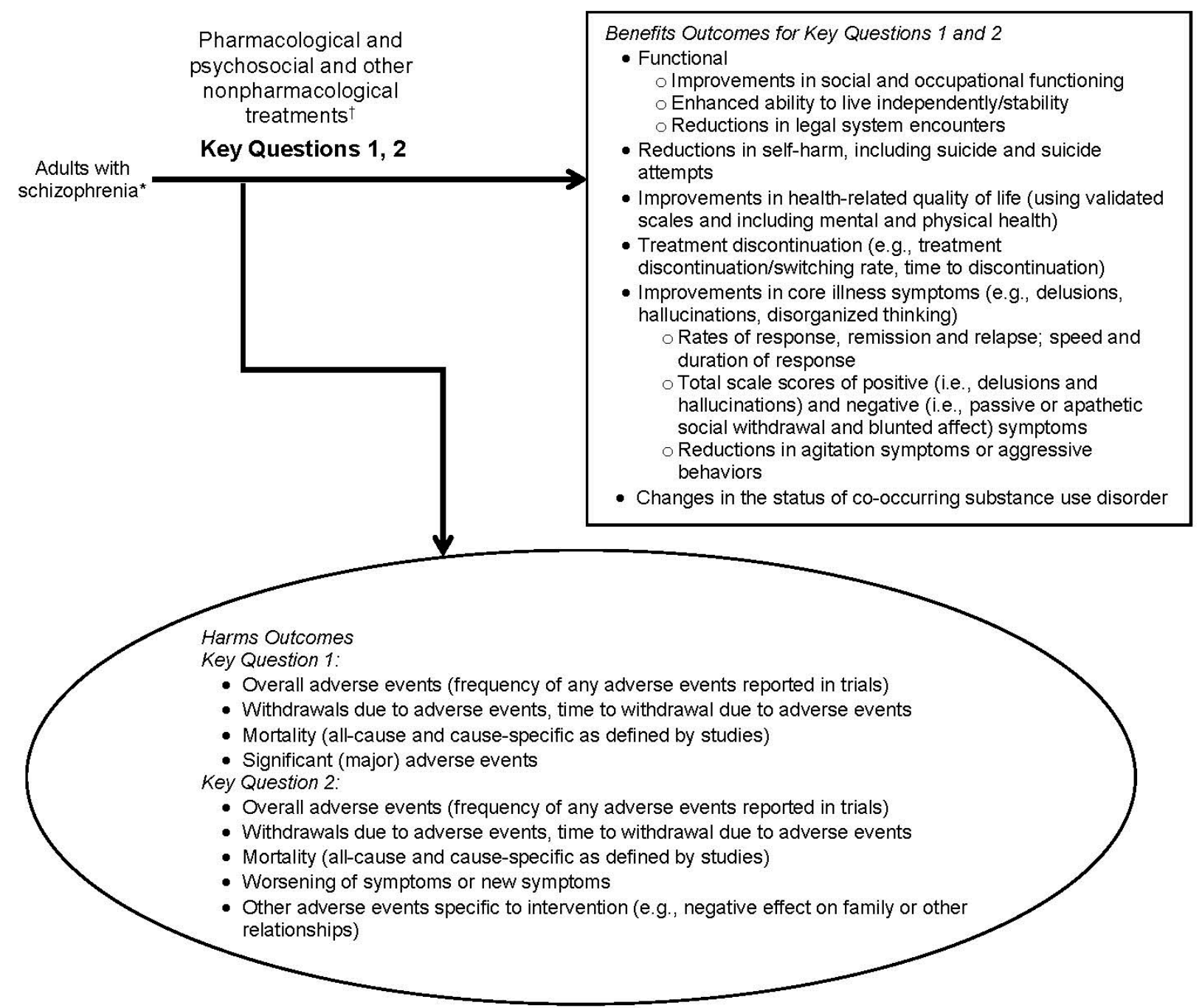

* Adults with a diagnosis of schizophrenia, including those with co-occurring substance use disorders, and including those experiencing a first episode of schizophrenia (including those with schizophreniform disorder).

1. Pharmacological treatments:

a. At least 90 percent of patients must have been diagnosed with schizophrenia.

b. For studies specifically on harms of antipsychotic drugs, populations can be mixed-diagnoses, as the harms are not diagnosis-specific

2. Psychosocial and other nonpharmacological treatments: 50 percent of patients must have been diagnosed with a

schizophrenia spectrum disorder diagnosis (i.e., schizophrenia, schizoaffective disorder, or schizophreniform disorder)

${ }^{\dagger}$ Pharmacological treatments include US Food and Drug Administration-approved second-generation and selected firstgeneration antipsychotics. Psychosocial and other nonpharmacological treatments include: assertive community treatment, cognitive adaptive training, cognitive behavioral therapy, cognitive remediation/training, co-occurring substance use and schizophrenia interventions, early interventions for first episode psychosis, family interventions, intensive case management, illness self-management training, psychoeducation, social skills training, supported employment, and supportive therapy. 


\section{Methods}

The methods for this systematic review follow the Agency for Healthcare Research \& Quality (AHRQ) Methods Guide for Effectiveness and Comparative Effectiveness Reviews and the Preferred Reporting Items for Systematic Reviews and Meta-Analyses (PRISMA) checklist. ${ }^{25,26}$ See the review protocol (http://effectivehealthcare.ahrq.gov/index.cfm) for full details.

\section{Topic Refinement and Review Protocol}

Initially a panel of key informants gave input on the key questions to be examined; these key questions were posted on AHRQ's Effective Health Care (EHC) Web site for public comment in May 2016 for 3 weeks and revised in response to comments. We then drafted a protocol for the systematic review and recruited a panel of technical experts to provide high-level content and methodological expertise throughout the development of the review. The finalized protocol is posted on the EHC Web site at http://effectivehealthcare.ahrq.gov/index.cfm/search-for-guidesreviews-and-reports/?pageaction=displayproduct\&productid=2279. The International Prospective Register of Systematic Reviews (PROSPERO) registration is PROSPERO 2016:CRD42016048403.

\section{Literature Search Strategy}

Publication date range. For Key Question 1 on pharmacological interventions, recent highquality systematic reviews directly addressing large portions of the key questions in the current review have been published and were used as the starting point for the review. ${ }^{27-29}$ Based on the search dates in these reviews, searches for trials and systematic reviews began in 2011 for first generation antipsychotic (FGA) versus second generation antipsychotic (SGA) drugs and in 2013 for SGA versus SGA drugs. Starting the searches in January of 2011 and 2013, respectively, allows for multiple months of overlap of the new search dates with the search dates in the prior reviews.

For Key Question 2 on nonpharmacological interventions, search dates were not restricted. Within these searches we first identified the most recent, good-quality systematic reviews for particular interventions. Any trials identified in our searches that were published since the search dates in these reviews were also included to update the included reviews.

Library searches were updated through February 1, 2017 during which the draft report was posted for public comment and peer review to capture any new publications. Literature identified during the updated search, or through other methods described below, was assessed by following the same process of dual review as all other trials considered for inclusion in the report.

Literature databases. A research librarian searched Ovid MEDLINE ${ }^{\circledR}$, the Cochrane Central Register of Controlled Trials, the Cochrane Database of Systematic Reviews, and PsycINFO ${ }^{\circledR}$ to capture published literature (Appendix A.).

Scientific information packets. The AHRQ Evidence-based Practice Center (EPC) Scientific Resource Center sent email notification to relevant stakeholders about the opportunity to submit scientific information packets (SIPs) via the EHC Web site for the pharmaceutical interventions listed in Key Question 1.

Hand searching. Reference lists of included articles were reviewed for includable literature. 
Grey literature. Searches for grey (unpublished) literature include the SIPs submitted for pharmacological interventions and the ClinicalTrials.gov trial registry to identify trials that have been completed but not yet published.

\section{Inclusion and Exclusion Criteria}

\section{Population(s):}

- Adults with a diagnosis of schizophrenia, including those with co-occurring substance use disorders (SUDs), and including those experiencing a first episode of schizophrenia (including those with schizophreniform disorder)

o Key Question 1:

- At least 90 percent of patients must have been diagnosed with schizophrenia

- For studies specifically on harms of antipsychotic drugs, populations can be mixed-diagnoses, as the harms are not diagnosis-specific

o Key Question 2:

- 50 percent of patients must have been diagnosed with a schizophrenia spectrum disorder (i.e., schizophrenia, schizoaffective disorder, or

Interventions: schizophreniform disorder). ${ }^{30}$

- Key Question 1: Antipsychotic medications

o Key first-generation antipsychotic drugs

- Fluphenazine (Prolixin ${ }^{\circledR}$, Permitil ${ }^{\circledR}$ )

- Haloperidol (Haldol ${ }^{\circledR}$ )

- Perphenazine $\left(\right.$ Trilafon ${ }^{\circledR}$ )

o Second-generation antipsychotic drugs

- Aripiprazole (Abilify ${ }^{\circledR}$, Aristada $^{\mathrm{TM}}$ )

- Asenapine (Saphris ${ }^{\circledR}$ ),

- Brexpiprazole (Rexulti ${ }^{\circledR}$ )

- Cariprazine (Vraylar ${ }^{\mathrm{TM}}$ )

- Clozapine $^{\mathrm{b}}\left(\right.$ Clozaril $^{\circledR}$, Fazaclo $^{\circledR}$ oral dissolving tablet [ODT], Versacloz ${ }^{\mathrm{TM}}$ )

- Iloperidone (Fanapt ${ }^{\circledR}$ )

- Lurasidone (Latuda ${ }^{\circledR}$ )

- Olanzapine $^{\mathrm{b}}\left(\right.$ Zyprexa $^{\circledR}$, Zyprexa Zydis $\left.^{\circledR}\right)$, Olanzapine Pamoate (Zyprexa ${ }^{\circledR}$ Relprevv $\left.{ }^{\mathrm{TM}}\right)$

- $\quad$ Paliperidone ${ }^{\mathrm{b}}$ (Invega ${ }^{\circledR}$ ) and Paliperidone palmitate (Invega ${ }^{\circledR}$ Sustenna ${ }^{\circledR}$, Invega Trinza ${ }^{\mathrm{TM}}$ )

- Oral paliperidone is marketed only as an extended-release product, and will be noted as paliperidone in the report because there is no immediaterelease formulation.

\footnotetext{
b،Older” SGAs; approved up through 2001 and included in the Clinical Antipsychotic Trials of Intervention Effectiveness (CATIE) trials.
} 
- Quetiapine $^{\mathrm{b}}$ (Seroquel $^{\circledR}$, Seroquel XR ${ }^{\circledR}$ )

- The extended release formulation is noted as quetiapine extended release (ER) in this report; the immediate-release formulation is not noted by a suffix, to be consistent with the other immediate release formulations of SGAs.

- Risperidone ${ }^{\mathrm{b}}$ (Risperdal ${ }^{\circledR}$, Risperdal ${ }^{\circledR}$ M-TAB ${ }^{\circledR}$ ODT, Risperdal ${ }^{\circledR}$ Consta ${ }^{\circledR}$ )

- Ziprasidone ${ }^{\mathrm{b}}\left(\right.$ Geodon $\left.^{\circledR}\right)$

Excluded: Short-acting injectable drugs, as they are generally only used in emergent, acute conditions and on a short-term basis (hours to days)

- Key Question 2: Psychosocial and other nonpharmacological interventions ${ }^{c}$

o Assertive community treatment

o Cognitive adaptive training

o Cognitive behavioral therapy

o Cognitive remediation/training

o Co-occurring substance use and schizophrenia interventions (reported in Key Question 2b)

o Early interventions for first episode psychosis (reported in Key Question 2b)

o Family interventions

0 Intensive case management

o Illness self-management training

o Psychoeducation

o Social skills training

o Supported employment

o Supportive therapy

\section{Comparators:}

- Key Question 1:

o Head-to-head comparisons: three FGAs (listed above) and all FDA-approved SGAs

- Exclude: FGA versus FGA drug comparisons

- Key Question 2:

o Antipsychotic drugs (alone)

o Usual care/standard care/treatment as usual/waitlist, as defined in the trials

- Usual care can consist of elements of medication treatment, medication management, case management, rehabilitation services, and psychotherapy. We assumed that randomization would balance the specific elements of usual care interventions between treatment and control arms within each randomized controlled trial (RCT). Both groups (treatment and usual care) received usual care, including drug treatment throughout the study.

\footnotetext{
b، Older” SGAs; approved up through 2001 and included in the Clinical Antipsychotic Trials of Intervention Effectiveness (CATIE) trials.

${ }^{\mathrm{C}}$ Limited to the most commonly-used interventions relevant to United States practices.
} 
- Evidence with active controls (other interventions with expected benefit, or attention controls which have minimal or no benefit but similar patient participation time) was considered where the evidence base with usual care comparisons for a given intervention is too small to draw conclusions (i.e., one or two trials, no systematic reviews).

\section{Outcomes for each question: ${ }^{\mathrm{d}}$}

- Benefits outcomes

o Key Questions 1 and 2

- Functional

- Improvements in social and occupational/educational functioning

- Enhanced level of independent or stable living situation

- Reductions in legal system encounters

- Global functioning

- Reductions in self-harm, including suicide and suicide attempts

- Improvements in health-related quality of life (using validated scales and including mental and physical health)

- Treatment discontinuation (e.g., treatment discontinuation/switching rate, time to discontinuation for any reason including lack of efficacy or intolerable adverse effects)

- Improvements in core illness symptoms (e.g., delusions, hallucinations, disorganized thinking)

- Rates of response, remission, and relapse; speed and duration of response

- Total scale scores of positive (i.e., delusions and hallucinations) and negative (i.e., passive or apathetic social withdrawal and blunted affect) symptoms

- Reductions in agitation symptoms or aggressive behaviors

- Changes in the status of co-occurring SUD (Key Question 2b).

- Exclusions:

o Rehospitalization was not included as an outcome because: (1) there is important variation in the indications for and length of psychiatric hospitalizations across time, in different localities, and with different financial contexts, and (2) there is important variation across trials in how rehospitalization is measured/evaluated, which may confound study interpretation. However, it was reported in addition to the prioritized outcomes for assertive community treatment because it is the target of this intervention for patients with a history of frequent hospitalization.

o Neurocognitive testing is an intermediate outcome, rather than a patient-centered health outcome, and is excluded in favor of improvements in functioning that reflect cognition.

\footnotetext{
${ }^{\mathrm{d}}$ Intervention patient-outcome targets are highlighted in the results; most are included among the prioritized outcomes listed above.
} 
- Harms outcomes

o Key Question 1

- Overall adverse events (frequency of any adverse events reported in trials)

- Withdrawals due to adverse events, time to withdrawal due to adverse events

- Mortality (all-cause and cause-specific as defined by studies)

- Significant (major) adverse events (e.g., life threatening, results in long-term morbidity, or require medical intervention to treat, such as cerebrovascular or cardiovascular disease and related events, development of diabetes mellitus, diabetic ketoacidosis, neuroleptic malignant syndrome, seizures, extrapyramidal symptoms (EPS), tardive dyskinesia, clinically important weight changes, dyslipidemia, incidence and severity of sexual dysfunction, galactorrhea, amenorrhea, orthostatic hypotension, and agranulocytosis/severe neutropenia)

o Key Question 2

- Overall adverse events (frequency of any adverse events reported in trials)

- Withdrawals due to adverse events, time to withdrawal due to adverse events

- Mortality (all-cause and cause-specific as defined by trials)

- Outcomes reported as adverse events related to the intervention, such as:

- New or worsening symptoms (e.g., anxiety or depression) using validated scales

Timing:

- Negative effect on family or other relationships.

- Minimum duration of followup: 12 weeks.

\section{Settings:}

- United States-relevant, for example such as countries listed as "high" or "very high" on the United Nations International Human Development Index (HDI), and applicable to United States practices.

- Excluded: inpatient setting

\section{Study designs:}

- Key Questions 1 and 2:

o Best-evidence approach: $^{31}$

- Recent, comprehensive, good- or fair-quality systematic reviews to be used as primary evidence, as well as RCTs published since the systematic reviews

- For benefits of any included intervention, systematic reviews of RCTs will be included

- For harms of any included intervention, systematic reviews of observational trials to evaluate harms will be included in addition to reviews of trials

- Note: We included some systematic reviews that included a portion of trials with inpatients, otherwise, most evidence would have had to be excluded. However, we did not include individual trials that included inpatients.

- If no systematic reviews available for particular interventions, RCTs will be included 
- Key Question 2: Studies must have a sample size of $>50$.

\section{Study Selection}

The pre-established criteria (described above) was used to determine eligibility for inclusion and exclusion of abstracts in accordance with the Methods Guide for Effectiveness and Comparative Effectiveness Reviews. ${ }^{26}$ All citations deemed appropriate for inclusion by at least one of the reviewers was retrieved; excluded abstracts were dual reviewed. Each full-text article was independently reviewed for eligibility by at least two team members. Any disagreements were resolved by consensus.

A list of included studies is available in Appendix B, and a list of excluded studies with reasons for exclusion is available in Appendix C.

\section{Data Extraction}

After studies were selected for inclusion, the following data was abstracted into predetermined table templates: study design, year, setting, country, sample size, eligibility criteria, population and clinical characteristics, intervention characteristics, and results relevant to each key question, as well as other information (e.g., funding source). When results were reported as scale scores, we included the scale abbreviation in our tables; full names of scales used, score ranges, and direction of effect are available in Appendix D. Abstracted information that is relevant for assessing applicability included the number of patients randomized relative to the number of patients enrolled, use of run-in or wash-out periods (for drug studies), and characteristics of the population, intervention, and care settings. All study data has been verified for accuracy and completeness by a second team member. Data extraction tables are available in Appendix E.

\section{Quality Assessment of Individual Studies}

The quality of individual controlled trials and systematic reviews were assessed by using clearly predefined criteria. RCTs were evaluated with appropriate criteria and methods developed by the Drug Effectiveness Review Project (DERP). ${ }^{32}$ Systematic reviews were assessed using the Assessing the Methodological Quality of Systematic Reviews (AMSTAR) quality-rating instrument. ${ }^{33}$ These criteria and methods were used in conjunction with the approach recommended in the chapter, "Assessing the Risk of Bias of Individual Studies When Comparing Medical Interventions" in the Methods Guide for Effectiveness and Comparative Effectiveness Reviews. ${ }^{26}$ Studies were rated as "good," "fair," or "poor." Observational studies that were included in systematic reviews included in this review must have been assessed for quality or risk of bias using a design-appropriate tool.

Studies rated "good" quality were considered to have the least risk of bias, and their results will be considered valid. Good-quality studies include clear descriptions of the population, setting, interventions, and comparison groups; a valid method for allocation of patients to treatment; low dropout rates and clear reporting of dropouts; appropriate means for preventing bias; and appropriate measurement of outcomes.

Studies rated "fair" quality were susceptible to some bias, although not enough to invalidate the results. These studies may not meet all the criteria for a rating of good quality, but no flaw is likely to cause major bias. The study may be missing information, making it difficult to assess 
limitations and potential problems. The fair-quality category is broad, and studies with this rating will vary in their strengths and weaknesses.

Studies rated "poor" quality have significant flaws that imply biases of various types that may invalidate the results. They will have a serious or "fatal" flaw in design, analysis, or reporting; large amounts of missing information; discrepancies in reporting; or serious problems in the delivery of the intervention. The results of these studies will be as likely to reflect flaws in the study as the true difference between the compared interventions. We will not exclude studies rated as being poor in quality a priori, but poor-quality studies will be considered to be less reliable than higher-quality studies when synthesizing the evidence, particularly if discrepancies between studies are present (see strength of evidence [SOE] rating system below).

This approach to evaluating the internal validity of studies is similar to the "risk of bias" method, although the direction of the scale for ratings is inverse to the good, fair, and poor ratings used here. Specifically, low, moderate, or high risk of bias correlates with good, fair, and poor quality, respectively. Each study was dual-reviewed for quality by two team members. Any disagreements were resolved by consensus. Quality assessment tables are available in Appendix F.

\section{Data Synthesis}

We constructed evidence tables identifying the study characteristics (as discussed above), results for outcomes of interest, and quality ratings for all included studies, and, when appropriate, summary tables to highlight the main findings. Good- and fair-quality systematic reviews and trials were the focus of the results for each key question. Results from systematic reviews were presented first, followed by synthesis of the findings from trials not included in the reviews. To the extent possible, meta-analyses in included systematic reviews were updated with data from trials not included in the reviews. For the remainder of the included trials (those not included in the included systematic reviews and with evidence not conducive to meta-analysis), we summarized the trials' findings in the context of the included systematic review findings; we identified both consistent and discordant findings and evaluated reasons for any discordant findings.

Qualitative data was summarized as ranges and descriptive analysis, and interpretation of the results was provided. We compiled and summarized study characteristics and investigated whether there were important differences in the distribution in characteristics that modified the treatment effects.

Meta-analyses were conducted to summarize data and obtain more precise estimates on outcomes for which studies were homogeneous enough to provide a meaningful combined estimate. To determine whether meta-analysis could be meaningfully performed, we considered the quality of the studies and the heterogeneity among studies in design, patient population, interventions, and outcomes. We examined the possibility for conducting network meta-analyses to provide estimates of comparative effect across the interventions for specific outcomes, according to key question.

We conducted pairwise meta-analyses to calculate relative risks for dichotomous outcomes. For continuous outcomes, we used endpoint scores where baseline scores were similar between groups (within study), and changed scores where they were the only data provided. We used standard deviations where reported and performed calculations when standard errors or 95\% confidence intervals (CIs) were reported instead. We used the DerSimonian and Laird randomeffects model with Review Manager Version 5.3 software, ${ }^{34}$ StatsDirect, version 3.0.167, ${ }^{35}$ or 
Stata $14 .{ }^{36}$ Statistical heterogeneity was assessed using the $I^{2}$ statistic for three or more studies and the Q-statistic chi square test where only two studies could be pooled.

Network meta-analyses were conducted for key effectiveness binary outcomes with adequate data to support a network using a Bayesian hierarchical model for exploratory purpose. ${ }^{37,38}$ The appropriateness of combining direct and indirect evidence and the consistency of the network was assessed by using the loop-specific approach to examine inconsistency separately in every closed loop of a network, the node-splitting method and overall comparison of consistency and inconsistency models. $^{39,40}$ Inconsistency was explored if detected. We used vague priors in the Bayesian model, and posterior inference was based on Markov Chain Monte Carlo (MCMC) Sampling using two chains. Trace plot and the Gelman-Rubin statistic, as modified by Brooks and Gelman (1998) was used to check convergence of Markov Chains. ${ }^{41}$ Posterior point and interval estimates of model parameters were obtained based on 200,000 MCMC iterations thinned at every $100^{\text {th }}$ value after initial burn-in of 200,000 iterations (i.e., 2000 iterations per chain). Comparative effectiveness was measured by using odds ratio (OR), and estimated by controlling for variation in study duration, dose levels (low, medium, and high), and whether studies enrolled patients with a first episode of schizophrenia or whose symptoms were resistant to prior treatment in all models. For Response, the Bayesian model also included assessment of the definition of response, grouped into three categories: 20 percent improvement in Positive and Negative Syndrome Scale (PANSS); other scales (i.e., Clinical Global Impression - Improvement scale [CGI-I]) or thresholds; and combined 20 percent improvement in PANSS and other measures. Controlling the study-level variables also allowed us to evaluate whether the drug effectiveness varied by these variables. The construction of network plot and evaluation of inconsistency was done using Stata/SE 14.1 (StataCorp LP, College Station, TX 77845), and the Bayesian model was implemented using OpenBUGS 3.2.3

(http://www.openbugs.net/w/FrontPage). Forest plots for pooled analyses and matrixes of results for network analyses are available in Appendix G.

\section{Strength of the Body of Evidence}

The SOE for each key outcome per intervention area was assessed by using the approach described in the AHRQ EPC Methods Guide. ${ }^{26,42}$ Key outcomes were clinical, patient-centered (i.e., health outcomes) and were selected based on input from the Technical Expert Panel (TEP), a panel of experts convened to review the appropriateness and relevance of the report at the scoping phase. The prioritized outcomes are listed below, per intervention area.

\section{Pharmacological interventions:}

1. Functional outcomes (e.g., social, occupational)

2. Health-related quality of life (including physical)

3. Rates of response and/or remission

4. Mortality (all-cause and/or specific)

5. Reductions in self-harm, suicide, and suicide attempts

6. Improvements in core illness symptoms scale score changes

7. Overall/any adverse events (rate or proportion)

8. Withdrawal due to adverse events 
Psychosocial and other nonpharmacological interventions:

1. Functional (e.g., social, occupational)

2. Health-related quality of life

3. Reductions in self-harm, suicide, and suicide attempts

4. Rates of response and/or remission

5. Improvements in core illness symptoms scale score changes

6. Treatment discontinuation (typically reported as the number of patients lost to followup or leaving study early)

7. Rates of relapse

8. Outcomes reported as adverse events related to the intervention

SOE was initially assessed by one researcher, and to ensure consistency and validity of the evaluation, a senior reviewer reviewed the grades and any disagreements were resolved through consensus. SOE was based on the following domains ${ }^{\mathrm{e}}$ :

1. Study limitations (low, medium, or high level of study limitations based on the quality/risk of bias of individual studies)

2. Consistency (consistent, inconsistent, or unknown/not applicable)

3. Directness (direct or indirect)

4. Precision (precise or imprecise)

The SOE was assigned an overall grade of high, moderate, low, or insufficient according to a four-level scale by evaluating and weighing the combined results of the above domains:

High - We are very confident that the estimate of effect lies close to the true effect for this outcome. The body of evidence has few or no deficiencies. We believe that the findings are stable (i.e., another study would not change the conclusions).

Moderate - We are moderately confident that the estimate of effect lies close to the true effect for this outcome. The body of evidence has some deficiencies. We believe that the findings are likely to be stable, but some doubt remains.

Low - We have limited confidence that the estimate of effect lies close to the true effect for this outcome. The body of evidence has major or numerous deficiencies (or both). We believe that additional evidence is needed before concluding either that the findings are stable or that the estimate of effect is close to the true effect.

Insufficient - We have no evidence, we are unable to estimate an effect, or we have no confidence in the estimate of effect for this outcome. No evidence is available or the body of evidence has unacceptable deficiencies, precluding reaching a conclusion.

The details of the SOE are available in Appendix H. If the entire body of evidence was deemed insufficient for an intervention category, we presented tables of the main study characteristics and quality, descriptive summary of findings, funding source and reasons for being deemed insufficient. These bodies of evidence did not undergo further synthesis.

\footnotetext{
${ }^{\mathrm{e}}$ Reporting bias is also a domain listed in the AHRQ EPC Methods guidance on SOE, but as none of the included reviews assessed this domain we were unable to fully assess this item. If obvious problems with reporting bias were found, they are noted under the study limitations domain.
} 
Similarly, the evidence for subgroups in Key Questions $1 \mathrm{~b}$ and 2b was only assessed for SOE if an intervention was studied in a specific subgroup, but subgroup analyses of the larger evidence base were not assessed as they represent hypothesis generating research and would not meet the criteria for SOE.

\section{Applicability}

Applicability was assessed according to the approach described in the Methods Guide for Effectiveness and Comparative Effectiveness Reviews. ${ }^{26}$ We used the population, intervention, comparison, outcomes, timing, study design/setting (PICOTS) framework to consider the applicability of the evidence base for each key question and intervention area, for example examining the characteristics of the patient populations (e.g., age, severity and duration of illness).

\section{Peer Review and Public Commentary}

Experts in treatments for schizophrenia were invited to provide external peer review of this systematic review; AHRQ and an associate editor also provided comments. In addition, the draft report was posted on the AHRQ Web site for 4 weeks to elicit public comment. We addressed the reviewer comments and revised the text as appropriate. 


\section{Results}

\section{Results of Literature Searches}

For Key Question 1 on the benefits and harms of pharmacological interventions for schizophrenia, we reviewed 698 titles and abstracts and included one systematic review ${ }^{29}$ of 138 trials $(\mathrm{N}=47,189)$ and 24 additional trials ${ }^{43-66}(\mathrm{~N}=6,672)$ for second-generation antipsychotics (SGAs) versus other SGAs, and one systematic review of 111 trials $^{27,28}(\mathrm{~N}=118,503)$ and five additional trials $^{57,67-70}(\mathrm{~N}=1,055)$ for first-generation antipsychotics (FGAs) versus SGAs. There was significant overlap of studies between the two sections.

For Key Question 2 on the benefits and harms of psychosocial and other nonpharmacological interventions for schizophrenia, we reviewed 2,766 titles and abstracts and included 13 systematic reviews of 271 trials $(\mathrm{N}=25,050)$ and 27 additional trials $(\mathrm{n}=6,404)$ across 13 main intervention areas: assertive community treatment ( 1 systematic review ${ }^{71}$ and 1 trial $^{72}$ ), cognitive adaptive training (CAT; 3 trials $^{73-76}$ ), cognitive-behavioral therapy (CBT; 3 systematic reviews ${ }^{77-}$ 79 and 5 trials ${ }^{76,80-83}$ ), cognitive remediation/training ( 2 systematic reviews ${ }^{84,85}$ and 4 trials), ${ }^{86-90}$ family interventions ( 1 systematic review ${ }^{91}$ and 6 trials $^{92-97}$ ), intensive case management (ICM; 1 systematic review $^{98}$ and 1 trial $^{99}$ ), illness self-management and recovery ( 1 systematic review ${ }^{100}$ and 1 trial $^{101}$ ), psychoeducation ( 1 systematic review ${ }^{102}$ ), social skills training (3 trials ${ }^{97,103-105}$ ), supported employment (1 systematic review ${ }^{106}$ and 2 trials $^{107-109}$ ) supportive therapy (1 systematic review $^{110}$, early interventions for first episode psychosis (4 trials ${ }^{111-119}$ ), and cooccurring substance use disorders (SUDs) and schizophrenia (1 systematic review ${ }^{120}$ ). For each intervention area, we reported on the available evidence for prioritized, patient centered, outcomes, as described in the Methods. Prioritized outcomes for which there was no evidence available are not included in the Results. Direct comparisons were to usual care, which may include pharmacologic as well as other nonpharmacologic treatments.

The literature flow diagrams are shown in Figures 2 and 3. Tables 1-4 show the main characteristics of the included studies for the systematic reviews and trials for Key Questions 1 and 2 by intervention area. Detailed evidence tables of included studies are available in Appendix E, and details on quality assessment are available in Appendix F.

As described in the methods, for Key Question 2 we excluded unique psychosocial and nonpharmacological interventions, as well as interventions that are not commonly used in the United States (see Appendix C). 


\section{Figure 2. Key Question 1 literature flow diagram}

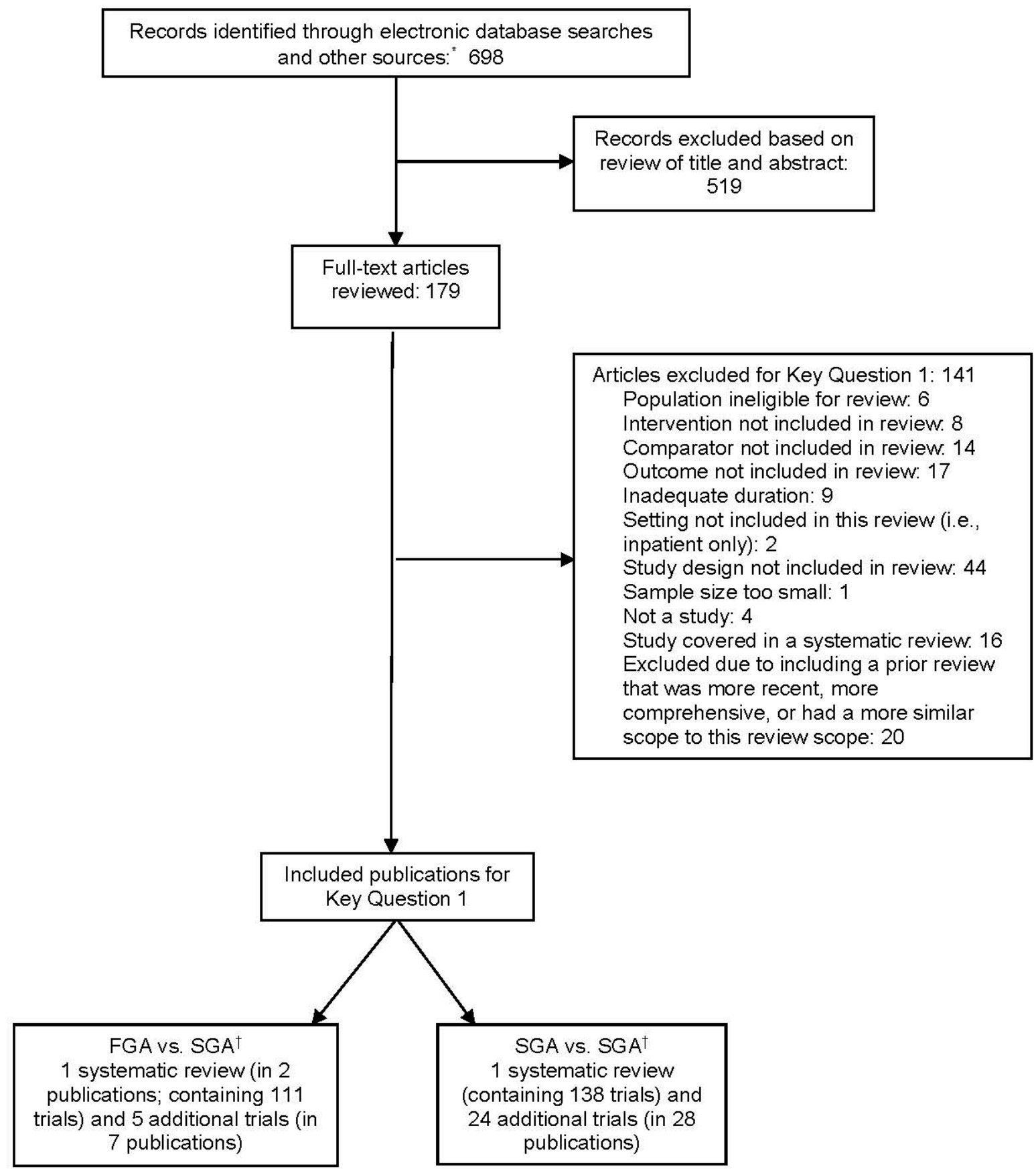

FGA = first-generation antipsychotic; SGA = second-generation antipsychotic

* Other sources include prior reports, references lists, referrals from experts, and grey literature.

${ }^{\dagger}$ Some studies were included for both FGA vs. SGA and SGA vs. SGA sections. 


\section{Figure 2. Key Question 2 literature flow diagram}

Records identified through electronic database searches and other sources: ${ }^{*} 2,766$

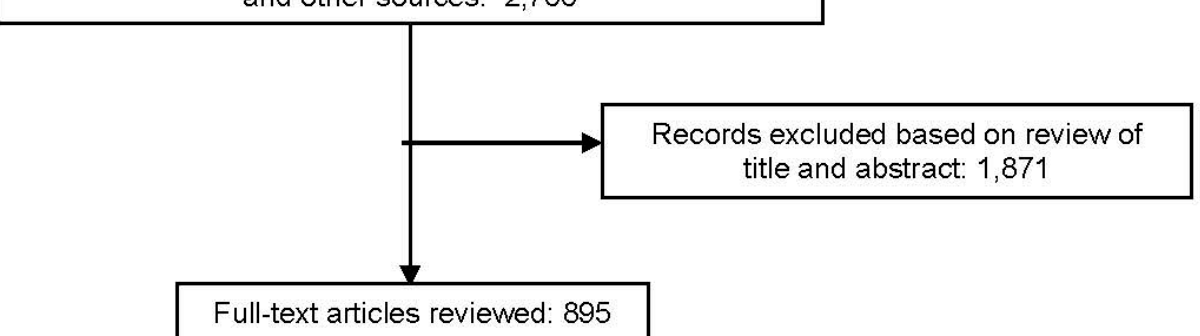

Articles excluded for Key Question 2: 842

Population ineligible for review. 17

Intervention not included in review. 153

Comparator not included in review: 74

Outcome not included in review. 96

Inadequate duration ( $<12$ weeks): 18

Setting ineligible for review (i.e., inpatient only): 26

Country not eligible: 28

Study design not included in review: 102

Not a study: 78

Not English language: 8

Sample size too small $(<50): 17$

Interventions evaluated in a single study: 42

Systematic review/meta-analysis used as a source

document to identify individual studies: 10

Study covered in a systematic review. 136

Excluded due to including a review that was more

recent, more comprehensive, or had a more similar scope to this review scope: 37

Included publications for Key Question 2

Assertive community treatment: 1 systematic review (containing 14 studies) and 1 trial

Cognitive adaptive training: 3 trials (in 4 publications)

Cognitive behavioral therapy: 3 systematic reviews (containing 89 studies) and 5 trials (in 6 publications)

Cognitive remediation/training: 2 systematic reviews (containing 57 studies) and 4 trials (in 5 publications)

Co-occurring substance use and schizophrenia interventions: 1 systematic review (containing 32 studies)

Early interventions for first episode psychosis: 4 trials (in 9 publications)

Family interventions: 1 systematic review (containing 27 studies) and 6 trials (in 8 publications)

Intensive case management: 1 systematic review (containing 10 studies) and 1 trial

Illness self-management: 1 systematic review (containing 13 studies) and 1 trial

Psychoeducation: 1 systematic review (containing 10 studies)

Social skills training: 3 trials (in 4 publications)

Supported employment: 1 systematic review (containing 14 studies) and 2 trials (in 3 publications)

Supportive therapy: 1 systematic review (containing 5 studies)

*Other sources include prior reports, reference lists, referrals from experts, and grey literature. 
Table 1. Characteristics of included systematic reviews for Key Question 1

\begin{tabular}{|l|c|c|}
\hline \multicolumn{1}{|c|}{ Study Characteristic } & FGA Versus SGA & SGA Versus SGA \\
\hline Study quality (number of SRs) & Good $(1)$ & Good $(1)$ \\
\hline Number of included studies & $\begin{array}{c}113 \text { (111 RCTs and 2 observational } \\
\text { studies) }\end{array}$ & $\begin{array}{c}169 \text { (138 RCTs and 31 observational } \\
\text { studies) }\end{array}$ \\
\hline Sample size & 118,503 & $\begin{array}{c}138 \text { RCTs }(\mathrm{N}=47,189) \\
31 \text { observational studies }(\mathrm{N}=602,547)\end{array}$ \\
\hline $\begin{array}{l}\text { Duration of Intervention } \\
\text { (range) }\end{array}$ & $\begin{array}{c}<1 \text { day-22 years } \\
\text { (median } 8 \text { weeks) }\end{array}$ & $6-104$ weeks \\
\hline
\end{tabular}

FGA = first-generation antipsychotic; RCT = randomized controlled trial; SGA = second-generation antipsychotic; $\mathrm{SR}=$ systematic review

Table 2. Characteristics of new included trials for Key Question 1

\begin{tabular}{|l|c|c|c|}
\hline \multicolumn{1}{|c|}{ Study Characteristic } & Category & FGA Versus SGA & SGA Versus SGA \\
\hline \multirow{3}{*}{ Study quality } & Good & 2 & 2 \\
\cline { 2 - 4 } & Fair & 2 & 17 \\
\cline { 2 - 4 } & Poor & 1 & 5 \\
\hline Sample size & Total & 1,055 & 672 \\
\hline $\begin{array}{l}\text { Duration of intervention / } \\
\text { followup }\end{array}$ & Range & 12 weeks-3 years & 6 weeks-3 years \\
\hline \multirow{2}{*}{ Location } & U.S. only & 1 & 3 \\
\cline { 2 - 4 } & Other & 4 & 21 \\
\hline
\end{tabular}

FGA = first-generation antipsychotic; SGA = second-generation antipsychotic 
Table 3. Characteristics of included systematic reviews for Key Question 2

\begin{tabular}{|c|c|c|c|c|c|c|c|c|c|c|}
\hline $\begin{array}{c}\text { Study } \\
\text { Characteristic }\end{array}$ & ACT & CBT & $\begin{array}{c}\text { Cognitive } \\
\text { Remediation/ } \\
\text { Training }\end{array}$ & $\begin{array}{c}\text { Co- } \\
\text { occurring } \\
\text { Substance } \\
\text { Use } \\
\text { Disorder }\end{array}$ & Family & ICM & $\begin{array}{l}\text { Illness Self- } \\
\text { Management }\end{array}$ & $\begin{array}{l}\text { Psycho- } \\
\text { education }\end{array}$ & \begin{tabular}{|c|c|} 
Supported \\
Employment
\end{tabular} & \begin{tabular}{|c} 
Supportive \\
Therapy
\end{tabular} \\
\hline $\begin{array}{l}\text { Study quality } \\
\text { (number of } \\
\text { SRs) }\end{array}$ & Good (1) & Good (3) & Good (2) & Good (1) & Fair (1) & Good (1) & Fair (1) & Good (1) & Good (1) & Good (1) \\
\hline $\begin{array}{l}\text { Number of } \\
\text { included } \\
\text { studies }\end{array}$ & 14 & 89 & 57 & 32 & 27 & 10 & 13 & 10 & 14 & 5 \\
\hline Sample size & 2,281 & 7,154 & 2,885 & 3,165 & 2,297 & 1,652 & 1,404 & 1,125 & 2,265 & 822 \\
\hline $\begin{array}{l}\text { Duration of } \\
\text { intervention }\end{array}$ & $\begin{array}{c}\text { Not } \\
\text { reported }\end{array}$ & $\begin{array}{l}8 \text { weeks-5 } \\
\text { years }\end{array}$ & $\begin{array}{c}2 \text { weeks-2 } \\
\text { years }\end{array}$ & $\begin{array}{l}1 \text { month-3 } \\
\text { years }\end{array}$ & $\begin{array}{c}6 \text { weeks-3 } \\
\text { years }\end{array}$ & $\begin{array}{l}\text { Not } \\
\text { reported }\end{array}$ & $\begin{array}{c}\text { 7-49 sessions, } \\
45-90 \text { minutes } \\
\text { each }\end{array}$ & $\begin{array}{c}1-18 \\
\text { months }\end{array}$ & $\begin{array}{l}12-24 \\
\text { months }\end{array}$ & $\begin{array}{c}7 \text { months-1 } \\
\text { year }\end{array}$ \\
\hline $\begin{array}{l}\text { Duration of } \\
\text { followup }\end{array}$ & $\begin{array}{l}1 \text { month- } \\
2 \text { years }\end{array}$ & $\begin{array}{l}8 \text { weeks-5 } \\
\text { years }\end{array}$ & $\begin{array}{l}2 \text { weeks-2 } \\
\text { years }\end{array}$ & $\begin{array}{l}1 \text { month-3 } \\
\text { years }\end{array}$ & $\begin{array}{c}6 \text { weeks-8 } \\
\text { years }\end{array}$ & $\begin{array}{l}1 \text { month- } \\
4 \text { years }\end{array}$ & $\begin{array}{l}\text { Up to } 24 \\
\text { months post- } \\
\text { treatment }\end{array}$ & $\begin{array}{l}2 \text { months- } \\
5 \text { years }\end{array}$ & 2 years & $\begin{array}{c}7 \text { months-2 } \\
\text { years }\end{array}$ \\
\hline
\end{tabular}

ACT = assertive community treatment; CBT = cognitive behavioral therapy; ICM = intensive case management; $\mathrm{SR}=$ systematic review

Table 4. Characteristics of included trials for Key Question 2

\begin{tabular}{|c|c|c|c|c|c|c|c|c|c|c|c|}
\hline $\begin{array}{c}\text { Study } \\
\text { Characteristic }\end{array}$ & Category & Overall & ACT & CBT & $\begin{array}{c}\text { Cognitive } \\
\text { Remediation/ } \\
\text { Training }\end{array}$ & $\begin{array}{c}\text { Early } \\
\text { Interventions } \\
\text { for First } \\
\text { Episode } \\
\end{array}$ & Family & ICM & $\begin{array}{l}\text { Illness Self- } \\
\text { Management }\end{array}$ & $\begin{array}{l}\text { Social } \\
\text { Skills } \\
\text { Training }\end{array}$ & $\begin{array}{c}\text { Supported } \\
\text { Employment }\end{array}$ \\
\hline \multirow{3}{*}{$\begin{array}{l}\text { Study quality } \\
\text { and number of } \\
\text { trials }\end{array}$} & Good & 4 & 0 & 1 & 1 & 1 & 1 & 0 & 0 & 0 & 0 \\
\hline & Fair & 20 & 1 & 4 & 3 & 2 & 3 & 1 & 1 & 3 & 2 \\
\hline & Poor & 3 & 0 & 0 & 0 & 1 & 2 & 0 & 0 & 0 & 0 \\
\hline Sample size & Total & 6,404 & 118 & 823 & 341 & 2,363 & 562 & 77 & 210 & 433 & 1477 \\
\hline $\begin{array}{l}\text { Duration of } \\
\text { intervention }\end{array}$ & Range & $\begin{array}{c}8 \text { weeks-2 } \\
\text { years }\end{array}$ & 1 year & $\begin{array}{c}8 \text { weeks-9 } \\
\text { months }\end{array}$ & $\begin{array}{c}12 \text { weeks-6 } \\
\text { months }\end{array}$ & $1-2$ years & $\begin{array}{c}6-12 \\
\text { months }\end{array}$ & 18 months & 8 months & $\begin{array}{c}6 \text { months-2 } \\
\text { years }\end{array}$ & 2 years \\
\hline $\begin{array}{l}\text { Duration of } \\
\text { followup }\end{array}$ & Range & $\begin{array}{c}6 \text { months-3 } \\
\text { years }\end{array}$ & 2 years & $\begin{array}{c}24 \text { weeks-2 } \\
\text { years }\end{array}$ & 6 months-1 year & $1-10$ years & $1-2$ years & 3 years & 8 months & $\begin{array}{c}6 \text { months-3 } \\
\text { years }\end{array}$ & 2 years \\
\hline \multirow{2}{*}{ Location } & U.S. & 8 & 0 & 2 & 1 & 1 & 2 & 0 & 0 & 1 & 2 \\
\hline & Other & 18 & 1 & 3 & 3 & 3 & 4 & 1 & 1 & 2 & 0 \\
\hline
\end{tabular}

ACT = assertive community treatment; CBT = cognitive-behavioral therapy; ICM = intensive case management 


\title{
Key Question 1a. Comparative Benefits and Harms of Pharmacological Treatments for Adults With Schizophrenia
}

\author{
Second-Generation Antipsychotic Versus Second-Generation \\ Antipsychotic
}

\section{Key Points}

- The comparative evidence on SGAs came from one good-quality systematic review that included 138 head-to-head randomized controlled trials (RCTs) $(\mathrm{N}=47,189), 31$ observational studies $(\mathrm{N}=602,547)$, and 24 newer RCTs $(\mathrm{N}=6,672)$. The studies were mostly fair quality.

- SGAs did not differ in effects on social, occupational, or global functioning.

o No difference in social functioning was found between paliperidone palmitate monthly long-acting injection (LAI) and risperidone bi-weekly LAI (Personal and Social Performance [PSP] scale mean change from baseline 16.8 and 18.6, respectively; least squares mean [LSM] difference 0.5, 95\% confidence interval [CI] -2.14 to 3.12) based on one RCT ( $\mathrm{N}=452)$ (strength of evidence [SOE]: low).

o A single study ( $\mathrm{N}=666)$ found risperidone LAI to result in greater improvements in social function over 24 months compared with quetiapine (change at endpoint 6.6 vs. $1.1 ; \mathrm{p}<0.0001)$ (SOE: low).

o Although both groups improved significantly from baseline, risperidone LAI resulted in greater improvements than quetiapine on the Social and Occupational Functioning Assessment Scale (SOFAS) at 6 months (6.1 vs. 2.7, p=0.02) and 12 months (9.5 vs. 6.1; $\mathrm{p}=0.009)$ (SOE: low).

o Clinical Antipsychotic Trials of Intervention Effectiveness (CATIE) Phase 1 found no significant differences in rates of employment between risperidone, olanzapine, quetiapine, perphenazine, and ziprasidone at 18 months (SOE: low)

o Global functioning was not different (based on the Global Assessment of Functioning [GAF] scale) between olanzapine and either risperidone (four cohort studies; weighted mean difference [WMD] 0.61, 95\% CI -1.78 to 2.99) or quetiapine (two RCTs; WMD 1.14, 95\% CI -4.75 to 7.02) (SOE: low).

- Older SGAs (clozapine, risperidone oral and LAI, olanzapine, quetiapine, and ziprasidone) were not found different from one another on a variety of quality of life measures, although small but significant improvements were seen from baseline.

o Olanzapine was not found significantly different than risperidone (two RCTs; moderate SOE), ziprasidone (two RCTs; moderate SOE), or quetiapine (one RCT; low SOE) at 12 months using the Heinrich Carpenter Quality of Life Scale (QLS) (change in scores ranged from 0.09 to 0.26 ).

o Risperidone was not found significantly different from quetiapine or ziprasidone at 12 months using the QLS scale (one RCT each; range of change in scores 0.19 to 0.26) (SOE: low). Risperidone LAI was not found different from quetiapine on the 12-Item Short Form Health Survey (SF-12) or Schizophrenia Quality of Life Scale-Revision 4 (SQLS-R4) at 24 months (SOE: low). 
- Response was significantly more likely with olanzapine (odds ratio [OR] 1.71. 95\% CI 1.11 to 2.68 ) and risperidone (OR 1.41, 95\% CI 1.01 to 2.00) than quetiapine, based on a network meta-analysis of 46 head-to-head RCTs (SOE: low)

o The rate of response in individual study arms ranged from 20 to 80 percent.

o Meta-regression examining the influence of study duration, dose-level, population (either treatment-resistant or first-episode status), and category of response definition did not result in any significant findings.

o Due to few studies, the findings for newer SGAs (e.g., the 3-month paliperidone palmitate LAI, lurasidone, iloperidone, brexpiprazole, and cariprazine) should be interpreted with caution.

- All-cause mortality was not different between the SGAs in incidental reports in RCTs or retrospective cohort studies, but evidence was not available for the newest SGAs.

0 With mortality rates of 0 to 1.17 percent, significant differences in mortality were not found in two RCTs (4 to 24 months duration) of asenapine with olanzapine (relative risk [RR] 2.49, 95\% CI 0.54 to 11.5), quetiapine and risperidone (RR 3.24, 95\% CI 0.72 to 14.6), and two RCTS (also 4 to 24 months duration) of paliperidone palmitate monthly LAI versus risperidone LAI (RR 1.26, 95\% CI 0.21 to 7.49 ) (SOE: low).

o Retrospective cohort studies found no significant difference in the risk of allcause (one study, $\mathrm{N}=48,595$ ) or cardiovascular mortality (two studies, $\mathrm{N}=55,582$ ) between risperidone, olanzapine, and quetiapine (SOE: low).

- Clozapine was found superior to olanzapine in preventing significant suicide attempts or hospitalization to prevent suicide (hazard ratio [HR] 0.76, 95\% CI 0.58 to 0.97) and Clinical Global Impression of Severity-Suicidality Scale (CGI-SS) ratings of "much worse" or "very much worse" (HR 0.78, 95\% CI 0.61 to 0.99); number needed to treat [NNT] of 12) among patients at high risk (SOE: low). Observational studies confirm these findings in broader populations.

- There were no significant differences between the SGAs in the proportions of patients reporting overall adverse events, based on 72 RCTs and 31 drug comparisons.

- Clozapine was found to improve core illness symptoms significantly more than the other SGAs, except for olanzapine (network meta-analysis of 212 RCTs; standard mean differences [SMDs] on Positive and Negative Syndrome Scale [PANSS] or Brief Psychiatric Rating Scale [BPRS]. All of the SGAs were superior to placebo (SMDs -0.33 to $-0 \cdot 88$ ) (SOE: low).

- Olanzapine and risperidone were found to result in similar core illness symptom improvements, which were greater in these drugs than in the other SGAs, except for paliperidone (SMDs -0.13 to -0.26) (SOE: low). Paliperidone was found to improve core illness symptoms more than lurasidone and iloperidone (SMDs -0.17) (SOE: low).

- In a separate network analysis of 40 RCTs of clozapine, risperidone, olanzapine, quetiapine, and ziprasidone in patients who were resistant to treatment, the only significant difference was that the mean change in the PANSS was greater with olanzapine than quetiapine (SMD -0.29, 95\% CI -0.56 to -0.13) (SOE: low).

- Based on a network meta-analysis of 90 head-to-head RCTs withdrawal due to adverse events were significantly lower with four SGAs compared with the others (SOE: low).

o Risperidone LAI had significantly lower risk than clozapine (OR 0.27, 95\% CI 0.10 to 0.71 ); lurasidone (OR 0.39, $95 \%$ CI 0.18 to 0.84 ); quetiapine extended 
release (ER) (OR 0.43, 95\% CI 0.22 to 0.81); risperidone (OR 0.50, 95\% CI 0.25 to 0.99 ); and ziprasidone (OR $0.40,95 \%$ CI 0.20 to 0.82 )

o Olanzapine had lower risk than clozapine (OR 0.39, 95\% CI 0.19 to 0.79); lurasidone (OR 0.57, 95\% CI 0.34 to 0.94 ); quetiapine (OR 0.62, 95\% CI 0.44 to 0.87 ); risperidone (OR 0.72, 95\% CI 0.55 to 0.96); and ziprasidone (OR 0.58, 95\% CI 0.41 to 0.82 )

o Aripiprazole had lower risk than ziprasidone (OR 0.64, 95\% CI 0.44 to 0.94) and clozapine (OR 0.43, 95\% CI 0.21 to 0.88 )

o Cariprazine (OR 0.40, 95\% CI 0.17 to 0.95) and iloperidone (OR 0.34, 95\% CI 0.13 to 0.91 ) had lower risk than clozapine

o Meta-regression examining the influence of study duration, dose level, and either treatment-resistant or first-episode status did not result in any significant findings.

- There are fewer data available for the newer drugs; results for these drugs should be interpreted with caution.

\section{Detailed Synthesis}

\section{Description of Included Studies}

A recent large, good-quality systematic review compared the benefits and harms of SGAs, including oral and LAI drugs (Appendix Tables E-1 and F-1). ${ }^{29}$ The review included 138 RCTs of at least 6 weeks duration, including 47,189 patients with schizophrenia or related psychosis (includes 3 trials of adolescents with 315 patients). Of these, 17.5 percent were rated as poor quality, and 9 percent as good quality. Reasons for trials being rated as poor quality were unclear methods of randomization and/or allocation concealment, lack of blinding of outcome assessors and incomplete reporting of outcome data or high attrition (missing data). Patients enrolled in the included trials of adults not experiencing a first episode of schizophrenia had a mean age of 39 years, and in trials of adults with a first episode the mean age was 26 years. These trials made comparisons of the older SGAs (clozapine, risperidone, olanzapine, quetiapine, ziprasidone and aripiprazole) with few studies or only placebo-controlled trials of newer SGAs (iloperidone, lurasidone, paliperidone, and the LAI products). The duration of followup among the included studies ranged widely from 6 weeks to 3 years, with most being 8 to 12 weeks. The review also included 31 observational studies (cohort and case-control) with at least 6 months of followup to evaluate harms and selected effectiveness outcomes (e.g., functioning, employment). These studies included 602,547 patients, mostly diagnosed with schizophrenia or related psychosis. Twenty-one percent of these studies were rated poor quality, and 5 percent were good quality. Reasons for observational studies being rated as poor quality were potentially biased selection of patients, unclear description of methods for ascertaining exposure or outcomes, and lack of blinding of, or inadequate, control for confounding. Mean doses reported for the observational studies tended to be lower than those used in the trials noted above. Mean doses of olanzapine in particular were 10 to $12 \mathrm{mg}$ daily in the observational studies, whereas across 54 trials reporting a mean olanzapine dose, the mean was $17 \mathrm{mg}$ daily. For risperidone, the observational studies reported doses of 3 to $4 \mathrm{mg}$ daily, whereas the mean across 55 trials was $5.7 \mathrm{mg}$ daily. ${ }^{29}$ Evidence on dosing of other SGAs was limited. The reasons for this apparent difference in dosing between the observational studies and trials were not clear, primarily because data on patient characteristics were poorly reported in the observational studies. A number of these studies were poor quality for a variety of reasons, but primarily unclear population selection 
criteria and methods (potential for biased selection), lack of blinding outcome assessors, short durations of followup, small sample sizes, and little or no statistical analysis of potential confounding factors. $^{29}$

Since the inclusion dates of the systematic review above, we have included 24 newer RCTs directly comparing SGAs ${ }^{43-66}$ (Appendix Tables E-2 and F-2). The comparisons in these newer trials included seven studies of a LAI drug; four of oral versus LAI of the same drugs (aripiprazole and risperidone, two studies each), and three comparing different LAI formulations or drugs (aripiprazole and paliperidone monthly injections, risperidone 2-week and paliperidone monthly, and paliperidone monthly and 3-month injections). Additionally, there were eight new trials comparing aripiprazole to other SGAs, including brexpiprazole, olanzapine, paliperidone, quetiapine, risperidone and ziprasidone; three new trials comparing either immediate or extended-release quetiapine to risperidone; one trial of olanzapine and ziprasidone, and two of cariprazine and risperidone. The duration of treatment in these studies was six to 52 weeks, median 28 weeks with six being 52 weeks long. Mean age of patients in these trials was 36 years. Five trials (21\%) were deemed to be poor quality, based on unclear reporting on randomization and concealment of allocation procedures, lack of blinding of outcome assessors, some differences in prognostic factors at baseline, and high attrition combined with unclear handling of missing data. $44,53,58,60,64$ Two trials were good quality ${ }^{56,61}$ and the rest were fair quality.

In comparing SGAs with each other (and to one FGA), the CATIE trial, a large, federally funded effectiveness trial with three phases, is a major contribution to the evidence base, and deserves introduction. ${ }^{121-125}$ In Phase 1 patients were randomized to olanzapine, quetiapine, risperidone, ziprasidone, or perphenazine (however, patients with tardive dyskinesia at baseline were not randomized to perphenazine; this group=Phase 1A). Ziprasidone was added to the trial partway through enrollment, after it received FDA approval. As a result, the numbers of patients randomized to ziprasidone were fewer (183 vs. 329 to 333 in other SGA groups), limiting statistical power. The mean modal dose of each SGA was within the typical dosing range for each drug. ${ }^{29}$ The study was planned to enroll patients from a broad range of settings, excluding patients with treatment resistance. However, the large number of study sites at major academic centers drew criticism that the results may not be generalizable to other setting. The study was funded by the National Institute of Mental Health and is a good-quality study.

In Phase 1B those patients who were randomized to perphenazine in Phase 1 but discontinued the drug prior to 18 months were then randomized to one of the four SGAs. In Phase 2E patients who discontinued the originally assigned drug in Phase 1 due to inadequate efficacy were randomized to open-label clozapine or to a blinded trial of olanzapine, risperidone, or quetiapine. In Phase 2T patients who discontinued the originally assigned drug in Phase 1 due to poor tolerability were randomized to ziprasidone or one of olanzapine, risperidone, or quetiapine with no one receiving the same drug assigned in Phase 1 during Phase 2. It has been noted, however, that some patients who discontinued drug during Phase 1 due to lack of efficacy opted to be enrolled in Phase 2T. Fifty-eight percent (184 of 318) of those enrolling had discontinued treatment in Phase 1 due to lack of efficacy, most likely due to patients wanting to avoid randomization to clozapine. The authors noted "patients who were assigned to olanzapine during Phase 2 had the lowest rates of Phase 1 discontinuation because of intolerable side effects and the lowest rates of discontinuation due to weight gain or metabolic side effects.” In Phase 3 , 270 patients who discontinued the Phase 2 drug (or discontinued Phase 1 drug and did not wish to be rerandomized to another treatment) were offered enrollment in an open-label treatment chosen by the patient, clinician, and research staff from among nine treatments: aripiprazole, 
clozapine, fluphenazine decanoate, olanzapine, perphenazine, quetiapine, risperidone, ziprasidone, or two of these combined. ${ }^{125}$ In addition to the results from the main analyses of each of these phases, numerous subgroup analyses and modeling studies have been published using data from this study.

The primary outcome measure in CATIE, all-cause treatment discontinuation, was selected for two reasons: first, because it was a discrete, common outcome that is easily understood; and second, because it encompassed lack of efficacy and/or intolerable side effects. Although this was an important outcome measure, it was an indirect measure of effectiveness and there appeared to be lack of agreement about its value to patients. ${ }^{126-128}$ Direct measures of effectiveness would include ability to work and to maintain successful social relationships. Hence, this outcome was not prioritized in the current review.

\section{Findings}

\section{Function}

\section{Social Function}

Across five RCTs (four included in the systematic review) and one observational study, assessments of social function resulted in mixed findings and insufficient evidence for the comparison of the older SGAs (clozapine, olanzapine, risperidone and quetiapine; Appendix Tables E-1 and E-2). ${ }^{29,50}$ Two fair-quality, open-label randomized trials with 12 months of followup came to different conclusions comparing olanzapine and risperidone, using different scales. In the first trial $(\mathrm{N}=108)$ no significant differences were seen between olanzapine and risperidone based on the Role Functioning Scale (RFS) or the Social Adjustment Scale (SAS) Severely Mentally Ill version. ${ }^{129}$ In contrast, in the second trial $(\mathrm{N}=235)$, improvement on the Social Function Scale (SFS) was greater with olanzapine $(+7.75)$ than risperidone $(-0.92$; $\mathrm{p}=0.0028$ ) after controlling for baseline scores. ${ }^{130}$ These changes from baseline are very small, given the range of the scale (0 to 226). Data were not presented in a way that would allow pooling these findings, and the difference may be attributable to differences in patient characteristics. In the study finding a significant difference, patients had predominantly negative symptoms and SFS scores correlated with improvements in negative symptoms. In the study not finding a significant difference, patients had two or more psychiatric hospitalizations in the past 12 months and were known to be nonadherent to treatments. A large $(\mathrm{N}=10,972)$ prospective cohort study reported 84.6 percent of patients taking olanzapine to be socially active (self-report) at 6 months. ${ }^{131}$ This was significantly more than with risperidone (82.4\%, OR 1.27, 95\% CI 1.05 to 1.54 ) or quetiapine (78.9\%, OR $1.67,95 \%$ CI 1.29 to 2.16). Clozapine was not found significantly different from olanzapine (81.6\%; OR 1.25, 95\% CI 0.87 to 1.80). Evidence on comparisons of older SGAs on social functioning is insufficient to draw conclusions. Three short-term (8- and 10-week) trials found no differences in various social function scales (Social Skills Performance Assessment [SSPA], the Penn Emotional Acuity Test [PEAT], and the SFS) between risperidone and either quetiapine or clozapine. ${ }^{132-134}$

For newer SGAs, the review found that evidence from a pooled analysis of patient-level data from three, 6-week placebo-controlled trials of paliperidone and a small group assigned to olanzapine was insufficient to draw conclusions. ${ }^{135}$ Although the publication states that there were no significant differences on the PSP scale, statistical results were not reported and reporting of baseline characteristic of the olanzapine group were inadequately reported. 
For the comparison of paliperidone palmitate monthly LAI and risperidone biweekly LAI, the review found low strength evidence that there is no difference in improvements in social functioning. This finding is based on a single fair-quality trial conducted in China $(\mathrm{N}=452)$ that found no difference at 13 weeks on the clinician rated PSP scale (mean change from baseline 16.8 with paliperidone and 18.6 with risperidone; LSM difference 0.5 [95\% CI -2.14 to 3.12]). ${ }^{136}$ Since the review, another very small $(\mathrm{N}=30)$ fair-quality 6-month trial of paliperidone palmitate LAI and risperidone LAI, conducted in Japan, found paliperidone palmitate to result in a significant improvement if SFS scores compared with risperidone (14.60 vs. -1.64; $\mathrm{p}=0.038){ }^{50,137}$ The study also assessed functional capacity using the San Diego Performance Based Skills Assessment- Brief (UPSA-B) tool, but found no difference between groups on this measure. This study should be interpreted cautiously due to the very small sample size.

A secondary publication from a trial of risperidone LAI and quetiapine that was originally included in the systematic review reported on social function using the SOFAS scale. ${ }^{138}$ Although both groups improved significantly from baseline, risperidone LAI resulted in greater improvements in SOFA at 6 months (6.1 vs. 2.7; $\mathrm{p}=0.02$ ), 12 months (9.5 vs. 6.1; $\mathrm{p}=0.009$ ), and endpoint (6.6 vs. $1.1 ; \mathrm{p}<0.0001)$. This evidence was low strength.

A single fair-quality trial of cariprazine and risperidone $(\mathrm{N}=456)$ that enrolled patients with stable schizophrenia and predominantly negative symptoms found no difference between the drugs on the SAS after 26 weeks of treatment. ${ }^{56}$

\section{Employment and Residential Status}

The systematic review included two RCTs and three observational studies that reported employment outcomes with the older SGAs (olanzapine, risperidone, quetiapine and ziprasidone) and found low strength evidence of no differences between the drugs (Appendix Table E-1). ${ }^{29}$ Results from Phase 1 of the CATIE study $(\mathrm{N}=1,121)$ did not indicate differences in employment at 18 months followup among olanzapine, quetiapine, risperidone, or ziprasidone. ${ }^{139}$ The threshold for "employment" was low-1 day in the last 30 days or an average of 1 hour a week over the last 30 days, with a mean of 18 percent reporting employment. The other smaller 12month, open-label trial $(\mathrm{N}=235)$ only reported on the subscale occupation/employment item of the SFS, and found olanzapine to result in an improvement while risperidone resulted in a decrease in score $(\mathrm{p}=0.0024) .{ }^{130}$ Three observational studies did not show significant differences in employment outcomes between older SGAs. ${ }^{140-142}$

One fair-quality trial, comparing quetiapine with risperidone $(\mathrm{N}=771)$, assessed combined occupational and residential status using a "modified vocational status index" and a "modified location code index". 55 They defined "real functional improvement" as better status in both at 12 months than at baseline, and stable status if the status for both was unchanged from baseline. Using these definitions, they found that 3.8 and 3.1 percent, respectively, had real improved status, and that 75.5 and 75.3 percent had stable status, with no significant differences between groups. This evidence is insufficient to draw conclusions due to imprecision, unknown consistency, and study limitations.

\section{Global Assessment of Functioning}

The systematic review found that several studies have reported on the comparative effects of older SGAs using the GAF scale (score 0 to 100), but that very small differences ( $<4$ points) were found favoring olanzapine compared with risperidone, quetiapine, and ziprasidone in three trials, otherwise differences were not found among drugs in nine studies (Appendix Table E-1). ${ }^{29}$ 
Such small differences are unlikely to have clinical importance as the minimal clinically important difference has been suggested to be 10 points. ${ }^{143}$

In the review, four observational studies reported on GAF scores in patients taking olanzapine and risperidone with followup of 6 months to 2 years. The pooled estimate of change from baseline in these studies was not significant (pooled WMD 0.61, 95\% CI -1.78 to 2.99; $\mathrm{I}^{2}=43 \%$ ). The range in change of score from baseline was 6.0 to 18.34 with olanzapine and 6.0 to 16.13 with risperidone. In comparing olanzapine and quetiapine, the pooled estimate for difference in change in GAF score was not significant (2 RCTs, N=363; pooled WMD 1.14, 95\% $\mathrm{CI}-4.75$ to $7.02 ; \mathrm{Q}=3.99, \mathrm{df}=1 ; \mathrm{p}=0.045)$. One of these studies found a small but significant difference, favoring olanzapine in patients with predominantly negative symptoms. This difference was correlated with improvements in negative symptoms (difference of 3.8 points; $\mathrm{p}=0.007) .{ }^{144}$ The evidence for indicating no difference in global function between olanzapine and risperidone or quetiapine was low strength. Evidence on comparisons of quetiapine and risperidone (one RCT), olanzapine and ziprasidone (one RCT), and clozapine and multiple other antipsychotics (one retrospective cohort study, one RCT) was insufficient to draw conclusions due to study limitations, unknown consistency and imprecision.

\section{Quality of Life}

Quality of life is a major consideration for choice of antipsychotic medication and is affected by both effectiveness and adverse events. There are multiple methods of measuring quality of life, many of which are intended for use in any population, such as the EuroQol five dimensions questionnaire (EQ-5D) or 36-Item Short Form Health Survey (SF-36), whereas a few are specifically designed for people with schizophrenia, such as the QLS. These methods measure different aspects of quality of life, and the data have been reported with too much variation to allow statistical pooling (e.g., not reporting standard deviations or standard errors, not reporting total scale scores, reporting endpoint only). The systematic review included five RCTs $(\mathrm{N}=3,443)$, including the CATIE trial, and five prospective cohort studies $(\mathrm{N}=5,728)$. Since then three additional trials of SGAs have reported quality of life. ${ }^{49,54,55,138}$ Using specific and nonspecific tools, 14 studies evaluated quality of life with seven oral and two injectable SGAs, with the majority finding no statistical or clinically meaningful differences (Appendix Tables E-1 and $\mathrm{E}-2)$.

Comparisons of olanzapine with risperidone and ziprasidone resulted in moderate-strength evidence of no significant difference in quality of life at 6 and 12 months. Low-strength evidence (due to smaller sample sizes) of no difference was found for comparisons of olanzapine with quetiapine, risperidone compared with quetiapine, and oral aripiprazole and aripiprazole monthly LAI. Evidence for the comparisons of olanzapine and asenapine, quetiapine ER and risperidone, and monthly LAIs of aripiprazole and paliperidone palmitate was insufficient to draw conclusions due to methodological limitations, unknown consistency (single trials), and imprecision in estimates (inadequate sample sizes). A secondary publication from a trial of risperidone LAI and quetiapine that was originally included in the systematic review reported on quality of life using the SF-12 physical and mental components and the SQLF-R4. ${ }^{138}$ Although both groups improved significantly from baseline, there was not a significant difference between groups at end point (24 months). This evidence was low strength.

The observational studies included in the systematic review support these findings for olanzapine, risperidone, and quetiapine with two exceptions. First, in the European Schizophrenia Outpatient Health Outcome (SOHO) study $(\mathrm{N}=919)$ whereas there were no differences in quality of life based on the EQ-5D at 6 or 36 months in the overall group, in the 
subgroup who were treatment-naïve at entry, patients taking olanzapine had significantly higher scores at 6 months than risperidone (adjusted mean difference 3.73, 95\% CI -1.48 to 5.97). ${ }^{145}$ In another prospective cohort study ( $\mathrm{N}=903 ; 612$ with schizophrenia), olanzapine oral dissolving tablet (ODT) was found to have greater improvement on the Psychological General Well-being Index (scale scores 0 to 110 ) at 12 months than standard oral olanzapine (22.3 vs. 12.2, $\mathrm{p}<0.001) .{ }^{146}$ Because these are single observational studies, this evidence is insufficient to draw conclusions but may be useful in planning future RCTs.

\section{Response and Remission}

Response rates varied somewhat across trials due to differences in patient populations, timing of measurement, and definition of response (Appendix Tables E-1 and E-2). The most common definition of response was $\geq 20$ percent improvement on the PANSS. Other definitions included the Kane criteria (improvement of $\geq 20 \%$ on BPRS and either Clinical Global Impression Severity scale (CGI-S) $\leq 3$ or BPRS $\leq 35$ ); ${ }^{147} 30,40$, and 50 percent improvements in PANSS or BPRS; and $\leq 3$ on all PANSS items and $\leq 3$ on the CGI-S. Across the trials, significant differences in response rates were very rare, and generally were not confirmed in other trials, if available. Remission was rarely reported in these RCTs.

We conducted a network meta-analysis of response rates, controlling for duration of study, category of dose (low, mid-level, high), treatment status (first-episode and treatment-resistant) and definition of response (Figure 4). We grouped the response definition into three categories: $>20$ percent improvement on PANSS or BPRS scale, definition based on a scale with any threshold (20\%, 30\% 40\%, etc.) and composite definitions and subjective definitions (e.g., Kane criteria plus one other element like hospitalization). Forty-six trials (40 two-arm studies and six three-arm studies; $\mathrm{N}=12,536),{ }^{43,46,47,51,55,59,130,132-135,148-182}$ including 10 oral drugs (aripiprazole, asenapine, brexpiprazole, cariprazine, clozapine, olanzapine, paliperidone, quetiapine, risperidone, and ziprasidone) and aripiprazole monthly LAI were eligible for the analysis. Trials of the LAIs of paliperidone palmitate (monthly or 3-month formulations) and risperidone did not have a comparator drug in common with anything else in the network, and could not be included. Aripiprazole 4 to 6 week LAI, iloperidone, and lurasidone had no response data in head-to-head trials and could also not be included. The network analysis found only two significant differences between the drugs; both olanzapine (OR 1.71. 95\% CI 1.11 to 2.68) and risperidone (OR 1.41, 95\% CI 1.01 to 2.00) were significantly more likely to result in response than quetiapine. The rate of response in individual study arms ranged from 20 to 80 percent (data not shown, available upon request). The matrix of results for response can be found in Appendix G1. Multiple methods were used to assess the model for inconsistency. Although the data available to test for inconsistency was limited, inconsistency in the model was not found. Taking this lack of inconsistency into account, and considering the indirectness of comparisons in the network, and the limited evidence for some comparisons, we found the results of the network metaanalysis to be low-strength evidence. Meta-regression examining the influence of study duration, dose-level, population (either treatment-resistant or first-episode status), and category of response definition did not result in any significant findings. There are fewer data available for the newer drugs; particularly all of the LAI drugs (e.g., the 3-month paliperidone palmitate injection), lurasidone, iloperidone, brexpiprazole, and cariprazine. Results for these drugs should be interpreted with caution.

A fair-quality published network analysis of patients with treatment-resistant symptoms assessed response. ${ }^{183}$ Significant differences were not found in comparisons of clozapine, risperidone, olanzapine quetiapine, and ziprasidone. 
Figure 3. Network meta-analysis of response rates in trials of SGAs

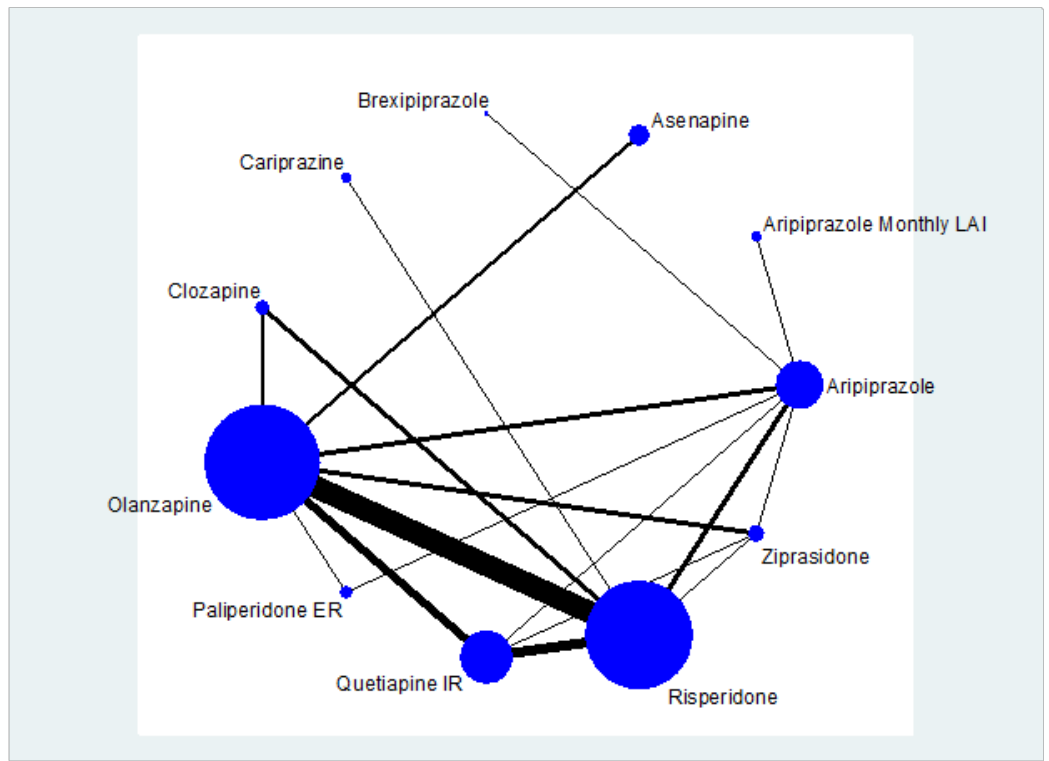

ER = extended-release; IR = immediate-release; LAI = long-acting injection; SGAs = second-generation antipsychotic

Legend: Circles represent relative numbers of studies including each drug. Line thickness represents number of studies making specific comparison for this outcome.

\section{Mortality}

In April 2005, the United States Food and Drug Administration (FDA) issued a public health advisory regarding increased risk of all-cause mortality associated with the use of all SGAs in elderly patients with dementia-related psychosis (www.fda.gov/cder/drug/advisory/antipsychotics.htm). Evidence on the risk of death associated with the SGAs specifically in patients with schizophrenia is limited by the lack of RCTs with adequate duration, sample sizes, and broadness of eligibility criteria as well as the lack of specific methodology for identifying and categorizing cases. Evaluating the incidental reports of mortality in RCTs included in the systematic review indicates that over 4 months to 2 years of treatment the rate of all-cause deaths was 0 to 1.17 percent in trials of quetiapine compared with quetiapine ER (one RCT), quetiapine immediate-release or ER versus risperidone (three RCTs), asenapine versus olanzapine (two RCTs), paliperidone versus risperidone LAI (one RCT), paliperidone monthly LAI versus risperidone LAI (one RCT), paliperidone 3-month versus monthly LAIs (one RCT), and brexpiprazole versus aripiprazole (one RCT) (Appendix Tables E1 and E-2). ${ }^{43,55,136,154,175,184-188}$ None of the comparisons were significant. For comparisons of asenapine with olanzapine (RR 2.49, 95\% CI 0.54 to 11.5), quetiapine and risperidone (RR 3.24, 95\% CI 0.72 to 14.6), and paliperidone palmitate monthly LAI versus risperidone LAI (RR 1.26, 95\% CI 0.21 to 7.49), there was low-strength evidence of no difference in mortality over 4 to 24 months, but the evidence for the other comparisons is insufficient to draw conclusions.

The systematic review included three observational studies to address the risk of mortality with SGAs in patients with schizophrenia (Table 5). Only older SGAs were included in these studies. There was low-strength evidence of no significant difference in the risk of all-cause or cardiovascular mortality between risperidone and olanzapine or quetiapine (Appendix $\mathrm{H}$ ). Evidence on the association of clozapine, risperidone, olanzapine and quetiapine and all-cause 
mortality versus no treatment is insufficient to draw conclusions due to the risk of bias of the study designs, and the lack of confirmatory studies (i.e., unknown consistency). Similarly, the evidence on cardiovascular mortality with risperidone compared with clozapine is insufficient. This study reported no differences in an analysis of older (starting drug at age 55 or older) versus younger patients, and found no association, however the mortality rates were very similar between drugs in the younger group (e.g., 2.7\% and 2.8\%) but the absolute difference was larger in the older group (e.g., 16.0\% clozapine and 5.7\% risperidone); the very small sample size in the older group may have prevented finding a significant difference.

Table 5. Mortality in retrospective cohort studies of SGAs

\begin{tabular}{|c|c|c|c|c|}
\hline Study, Year & $\begin{array}{l}\text { Study } \\
\text { Population } \\
\text { N, Mean Age } \\
\text { (Quality) }\end{array}$ & $\begin{array}{l}\text { Drugs and } \\
\text { Comparison }\end{array}$ & $\begin{array}{l}\text { All-Cause Mortality } \\
(95 \% \mathrm{Cl})\end{array}$ & $\begin{array}{l}\text { Cardiovascular } \\
\text { Mortality }\end{array}$ \\
\hline $\begin{array}{l}\text { Pasternak, } \\
2014^{189}\end{array}$ & $\begin{array}{l}\text { Starting SGA } \\
N=48,595 \\
39 \text { years } \\
\text { (Good) }\end{array}$ & $\begin{array}{l}\text { Risperidone versus } \\
\text { olanzapine, or } \\
\text { quetiapine } \\
1 \text { year followup }\end{array}$ & $\begin{array}{l}\text { Risperidone versus: } \\
\text { Olanzapine } \\
\text { HR 1.09, (0.79 - 1.49) } \\
\text { Quetiapine } \\
\text { HR 0.75, (0.53 -1.07) }\end{array}$ & $\begin{array}{l}\text { Risperidone versus: } \\
\text { Olanzapine } \\
\text { HR } 0.99(0.37-2.67) \\
\text { Quetiapine } \\
\text { HR } 0.76(0.25-2.28)\end{array}$ \\
\hline $\begin{array}{l}\text { Kiviniemi, } \\
2013^{190}\end{array}$ & $\begin{array}{l}\text { First episode } \\
\mathrm{N}=6,987 \\
39 \text { years } \\
\text { (Good) }\end{array}$ & $\begin{array}{l}\text { Clozapine, risperidone, } \\
\text { olanzapine and } \\
\text { quetiapine versus no } \\
\text { treatment a } \\
5 \text { years followup }\end{array}$ & $\begin{array}{l}\text { Clozapine } \\
\text { OR } 0.35(0.21-0.58) \\
\text { Quetiapine } \\
\text { OR } 0.46(0.30-0.72) \\
\text { Risperidone } \\
\text { OR } 1.0(0.75-1.43) \\
\text { Olanzapine } \\
\text { OR } 0.73(0.54-1.00)\end{array}$ & $\begin{array}{l}\text { Risperidone } \\
\text { OR } 0.82(0.41-1.66) \\
\text { Clozapine } \\
\text { OR } 0.23(0.05-.02) \\
\text { Olanzapine } \\
\text { OR } 0.89(0.46-1.72) \\
\text { Quetiapine } \\
\text { OR } 0.72(0.30-1.73)\end{array}$ \\
\hline $\begin{array}{l}\text { Kelly, } \\
2010^{191}\end{array}$ & $\begin{array}{l}\text { Starting SGA } \\
\mathrm{N}=1686 \\
40 \text { years } \\
\text { (Fair) }\end{array}$ & $\begin{array}{l}\text { Clozapine versus } \\
\text { risperidone } \\
10 \text { years followup }\end{array}$ & $\mathrm{NR}$ & $\begin{array}{l}4.8 \% \text { and } 2.5 \% ; \text { RR } 1.39 \\
(0.61 \text { to } 2.53)\end{array}$ \\
\hline
\end{tabular}

$\mathrm{CI}$ = confidence interval; $\mathrm{HR}$ = hazard ratio; $\mathrm{OR}$ = odds ratio; $\mathrm{RR}$ = risk ratio; SGA = second-generation antipsychotic

\section{Reduction in Self-Harm}

The best evidence on comparative effectiveness of SGAs in preventing suicide and suiciderelated behaviors comes from the good-quality InterSePT trial ${ }^{192}$ of clozapine and olanzapine, that was included in the systematic review (Appendix Table E-1). ${ }^{29}$ This pragmatic, open-label RCT (N=980) of patients with schizophrenia or schizoaffective disorder who were at high risk of suicide behaviors was conducted in 11 countries and had the specific aim of assessing suicidal behaviors over 2 years. Clozapine was found superior to olanzapine in preventing significant suicide attempts or hospitalization to prevent suicide (HR 0.76, 95\% CI 0.58 to 0.97) and CGISS ratings of "much worse" or "very much worse" (HR 0.78, 95\% CI 0.61 to 0.99). Additional analyses that controlled for drug treatment, prior suicide attempts, active substance or alcohol misuse, country, sex, and age also found clozapine superior (HR 0.74, 95\% CI 0.57 to 0.96), and indicated that the olanzapine group had significantly higher rates of using antidepressants, anxiolytics and rescue interventions to prevent suicide. The Kaplan-Meier life-table estimates indicated a significant reduction in the 2-year event rate in the clozapine group $(\mathrm{p}=0.02)$ with a NNT of 12. There was not a significant difference in suicide deaths (5 for clozapine and 3 for olanzapine). The strength of this evidence is moderate for serious attempts/hospitalizations and deaths, moderate for worsening of CGI-SS.

There were no other trials of SGAs that reported suicidal attempts, suicide deaths or other self-harm as a primary outcome measure, using explicit methods for ascertaining the outcome. 
Six fair-quality trials reported suicide-related outcomes as adverse events, all with very low event rates and no clear differences between treatments. Studies that did not prespecify or report methods were used for ascertaining and verifying the outcomes are open to misclassification and missing events. Patients were not selected for the trial based on risk for suicidal behavior, and there were no apparent differences between study groups in baseline severity of illness. A 52week fair-quality efficacy trial of asenapine compared with olanzapine $(\mathrm{N}=1,225)$ reported 1.8 and 2.3 percent suicides attempts, respectively. ${ }^{187}$ A 13 -week trial of risperidone LAI compared with paliperidone palmitate monthly LAI $(\mathrm{N}=452)$ reported that there were three suicidal behavior-related adverse events in the risperidone group (1.4\%) and none in the paliperidone palmitate injection group ( $0 \%)$, with one suicide death in a patient with no prior history of suicidal behavior (0.5\%). ${ }^{136}$ A 24-month trial of risperidone and quetiapine $(\mathrm{N}=1,098)$ initially designed to assess risk of ocular adverse events, reported two suicide deaths in the quetiapine group $(0.34 \%)$ and one in the risperidone group $(0.2 \%) .{ }^{184}$ Similarly, a 12 -month trial of quetiapine ER and risperidone $(\mathrm{N}=798)$ reported a single death in the risperidone group (0.25\%). ${ }^{55}$ In a RCT of asenapine and olanzapine, conducted at sites around the world, results were reported according to the hemisphere of study site. In the Eastern hemisphere results, there was one suicide death in each group (0.41\% and $0.42 \%),{ }^{154}$ and none in the Western hemisphere analysis. This evidence is insufficient to draw conclusions due to lack of confirmatory studies, study limitations (i.e., ascertainment techniques), and small numbers of events (imprecision).

The systematic review also included four observational studies that used adequate ascertainment methods to assess the risk of suicide or suicide attempts in patients taking SGAs (not limited to those at high risk). Low-strength evidence from two retrospective cohort studies (combined $\mathrm{N}=16,584$ ) found clozapine to be associated with lower risk of death by suicide. At 6 months, compared with no treatment, the risk with clozapine was OR 0.29, 95\% CI 0.14 to 0.63. Risperidone, olanzapine, and quetiapine were not different from no treatment. ${ }^{190}$ In the other study, patients with schizophrenia newly starting on clozapine had a lower rate of suicide at 1 year $(1.1 \%)$ than the other drugs studied or than the 6 months prior to treatment $(2.2 \%) .{ }^{193}$ Rates for the other drugs studied were risperidone 51 (2.1\%), aripiprazole 13 (2.2\%), risperidone LAI 26 (2.4\%), quetiapine 49 (3.1\%), olanzapine 57 (3.5\%), and ziprasidone 17 (3.7\%).

The other two studies, one assessing risk of suicide attempts in the fair-quality European SOHO prospective cohort study $(\mathrm{N}=10,204)$ of olanzapine, risperidone, quetiapine, and clozapine and the other assessing the risk of suicide attempts or death by suicide with aripiprazole compared with older SGAs provide only insufficient evidence due to methodological limitations of the study designs and lack of confirmatory studies for the specific outcomes and comparisons.

\section{Improvement in Core IIIness Symptoms}

Three good-quality network meta-analyses compared several of the SGAs with each other on improvements in core illness symptoms. ${ }^{183,194}$ A network analysis published in 2013 included 212 head-to-head and placebo-controlled trials of 15 oral antipsychotics (both FGAs and SGAs). ${ }^{194}$ Cariprazine was not included, and the analysis includes drugs not available in the United States. This analysis pooled changes in the PANSS or BPRS, using SMDs. Clozapine was found significantly superior to all the other drugs in the network, except olanzapine, on this measure with SMDs ranging from -0.32 to -0.55 (small to medium differences). Olanzapine and risperidone were superior to the other drugs, except for each other and paliperidone, with effect sizes ranging from -0.13 to -0.26 (generally small differences). Using only indirect comparisons in the network, paliperidone was found superior to lurasidone and iloperidone, with SMDs of 0.17 (a small difference). All other comparisons were not significant, but all of the drugs in the 
network were found superior to placebo (SMDs -0.33 to -0.88). The network did not include injectable drugs, and it is possible that some of these findings would change, particularly for newer drugs, with new head-to-head studies. This evidence was low strength.

An analysis of six oral SGAs (clozapine, risperidone, olanzapine, quetiapine, and ziprasidone) in 40 RCTs of patients with treatment-resistant schizophrenia found that the mean change in the PANSS was greater with olanzapine than quetiapine (SMD -0.29, 95\% CI -0.56 to $-0.13){ }^{183}$ The authors noted that this corresponds to a difference in points on the PANSS of -6.08 (scale scores range from 30 to 210; 180 possible points). There is some evidence that in patients with more severe disease a minimal clinically important difference on the PANSS is 11.5 points, indicating that a difference of six points may not be clinically important, although statistically significant. ${ }^{195}$ The newer oral drugs (aripiprazole, iloperidone, lurasidone, asenapine, cariprazine, brexpiprazole) were not included. This was low-strength evidence.

A single fair-quality 6-week trial of brexpiprazole and aripiprazole ( $\mathrm{N}=97)$ found that both drugs improved symptoms using the PANSS scale (-22.9 vs. -19.4 from baseline mean of 93.7; $\mathrm{p}<0.0001$ for each drug vs. baseline). ${ }^{43}$ Comparisons across the drugs were not made, although the absolute difference was very small. This study was not included in published network metaanalyses, and alone this evidence is insufficient.

\section{Overall Adverse Events}

We identified 64 RCTs, making 34 comparisons of SGAs that reported the numbers of patients reporting an adverse event during the study by group. ${ }^{45-47,49-51,54-}$

56,61,123,125,133,136,148,150,151,154,155,161,163,167,169,171,172,174-178,184,186-188,196-227 The majority of the trials did not find significant differences between the drugs compared. The rates of patients reporting adverse events varied widely across the studies, although the majority was above 60 percent for all SGAs, and the variability does not correlate with duration of study or mean or range of doses.

There was moderate-strength evidence of no significant difference in overall adverse event reporting between asenapine and olanzapine (five RCTs; $\mathrm{N}=2189)^{154,176,187,223}$, and quetiapine and risperidone (seven RCTs; N=3,254; Table 6). ${ }^{123,133,169,171,172,184,186}$

Similarly, the following comparisons had two to three RCTs each (6 weeks to 12 month; $\mathrm{N}=7810$ ) that again found no significant differences in proportions of patients reporting adverse events: Quetiapine ER versus quetiapine and risperidone; risperidone versus clozapine and aripiprazole; olanzapine versus paliperidone; risperidone LAI versus paliperidone and paliperidone palmitate monthly LAI; and aripiprazole versus aripiprazole monthly LAI. This was low-strength evidence for these comparisons.

Single trials ( 3 weeks to 2 years; $N=6700$ ) of oral aripiprazole versus brexpiprazole, olanzapine, paliperidone, and risperidone LAI; ziprasidone versus clozapine, risperidone, iloperidone and lurasidone; risperidone versus asenapine, cariprazine and risperidone and risperidone LAI; clozapine versus quetiapine, quetiapine versus risperidone LAI; olanzapine versus olanzapine LAI and lurasidone; aripiprazole monthly LAI versus paliperidone; and paliperidone palmitate monthly LAI versus 3-monthly LAI found similar rates of overall adverse events with no significant differences. This evidence is insufficient to draw conclusions due to lack of confirmatory studies and imprecision for each comparison's estimate of effect. 
Table 6. Overall adverse events in trials of SGAs versus SGAs

\begin{tabular}{|c|c|c|}
\hline $\begin{array}{l}\text { SGA Comparison } \\
\text { Number Studies/Number Patients }\end{array}$ & $\begin{array}{l}\text { Percentage of Patients } \\
\text { Reporting Adverse Events }\end{array}$ & Pooled Relative Risk \\
\hline $\begin{array}{l}\text { Asenapine vs. olanzapine } \\
5 \text { RCTs (4 publications); N=2189) }{ }^{154,176,187,225}\end{array}$ & $\begin{array}{l}\text { Asenapine } 68 \% \text { to } 82 \% \\
\text { Olanzapine } 69 \% \text { to } 82 \%\end{array}$ & $\begin{array}{l}1.00(95 \% \mathrm{Cl} 0.96 \text { to } \\
1.05) ; I^{2} 9 \%\end{array}$ \\
\hline $\begin{array}{l}\text { Quetiapine vs. risperidone } \\
7 \text { RCTs }(\mathrm{N}=3254)^{123,133,169,171,172,184,186}\end{array}$ & $\begin{array}{l}\text { Quetiapine } 67 \% \text { to } 93 \% \\
\text { Risperidone } 42 \% \text { to } 88 \%\end{array}$ & $\begin{array}{l}1.04(95 \% \mathrm{Cl} 0.97 \text { to } \\
1.12) ; I^{2} 56 \%\end{array}$ \\
\hline Clozapine vs. olanzapine $(\mathrm{N}=182)^{169,214}$ & $\begin{array}{l}\text { Clozapine } 77 \% \text { to } 91 \% \\
\text { Olanzapine } 73 \% \text { to } 77 \%\end{array}$ & $\begin{array}{l}\text { RR } 1.15(95 \% \mathrm{Cl} 1.00 \text { to } \\
1.33) ; \mathrm{I}^{2} 0 \%\end{array}$ \\
\hline $\begin{array}{l}\text { Risperidone vs. olanzapine } \\
5 \text { RCTs }(\mathrm{N}=873)^{123,149,163,169,171}\end{array}$ & $\begin{array}{l}\text { Risperidone } 42 \% \text { to } 71 \% \\
\text { Olanzapine } 47 \% \text { to } 74 \%\end{array}$ & $\begin{array}{l}\text { RR } 1.02(95 \% \mathrm{Cl} 0.81 \text { to } \\
1.29) ;\left.\right|^{2}=77 \%\end{array}$ \\
\hline $\begin{array}{l}\text { Olanzapine vs. ziprasidone } \\
5 \text { RCTs ( } N=1097 ; 6 \text { weeks to } 6 \text { months } \\
\text { durations) }\end{array}$ & $\begin{array}{l}\text { Olanzapine } 30 \% \text { to } 94 \% \\
\text { Ziprasidone } 28 \% \text { to } 92 \%\end{array}$ & $\begin{array}{l}\text { RR } 1.00(95 \% \mathrm{Cl} 0.86 \text { to } \\
1.16) ; l^{2}=80 \%\end{array}$ \\
\hline $\begin{array}{l}\text { Olanzapine vs. quetiapine } \\
3 \text { RCTs }(\mathrm{N}=448)^{123,169,171}\end{array}$ & $\begin{array}{l}\text { Olanzapine } 47 \% \text { to } 74 \% \\
\text { Quetiapine } 60 \% \text { to } 68 \%\end{array}$ & $\begin{array}{l}\text { RR } 0.90(95 \% \mathrm{Cl} 0.74 \text { to } \\
1.11) ;\left.\right|^{2}=30 \%\end{array}$ \\
\hline
\end{tabular}

CATIE = Clinical Antipsychotic Trials of Intervention Effectiveness; CI = confidence interval; RCT = randomized controlled trial; RR = risk ratio; SGA = second-generation antipsychotic

\section{Withdrawals Due to Adverse Events}

Adverse events that are intolerable lead to discontinuation from studies, although some may take longer to result in discontinuation. Such discontinuations take into account the patient's evaluation of the degree to which the adverse event is tolerable. The CATIE trials included these discontinuations as a secondary outcome measure and found significant differences among the drugs. In CATIE Phase 1, discontinuations due to adverse events were highest among patients taking olanzapine (primarily due to weight gain or other metabolic effects, $18 \%$ ) and lowest among those taking risperidone ( $10 \%$; $\mathrm{p}=0.04$ across groups). Time to discontinuation for adverse events did not differ among the groups. In Phases 1B, 2T, and 2E, differences were not seen between groups for rate of discontinuations or time to discontinuation due to adverse events (intolerability).

A network meta-analysis assessed discontinuation rates due to adverse events using data from 90 head-to-head trials of greater than 6-weeks duration (77 two arm studies, 8 three arm studies, 3 four arm studies and 2 five arm studies; $N=29,678)$. 43,45-47,49-51,54-57,59,61,63,66,121-124,132134,136,144,148,150-156,158,160,161,163-166,168-172,175-178,180,184,186-188,192,197,200,202,204-206,209,211,213,214,216,218-

221,223,224,226-243 (Figure 5). This analysis used direct and indirect comparisons based on the headto-head trials and found that risperidone LAI had significantly lower risk of withdrawals due to adverse events than clozapine (OR 0.27, 95\% CI 0.10 to 0.71); lurasidone (OR 0.39, 95\% CI 0.18 to 0.84 ); quetiapine ER (OR $0.43,95 \%$ CI 0.22 to 0.81 ); risperidone (OR $0.50,95 \%$ CI 0.25 to 0.99 ); and ziprasidone (OR 0.40, 95\% CI 0.20 to 0.82 ). Olanzapine had lower risk than clozapine (OR $0.39,95 \%$ CI 0.19 to 0.79 ); lurasidone (OR $0.57,95 \%$ CI 0.34 to 0.94 ); quetiapine (OR 0.62, 95\% CI 0.44 to 0.87 ); risperidone (OR 0.72, 95\% CI 0.55 to 0.96 ); and ziprasidone (OR $0.58,95 \%$ CI 0.41 to 0.82 ). Aripiprazole had lower risk than clozapine (OR $0.43,95 \%$ CI 0.21 to 0.88 ) and ziprasidone (OR $0.64,95 \%$ CI 0.44 to 0.94 ). Cariprazine (OR $0.40,95 \%$ CI 0.17 to 0.95 ) and iloperidone (OR $0.34,95 \%$ CI 0.13 to 0.91 ) had lower risk than clozapine. The matrix of results for withdrawals can be found in Appendix G-2. Multiple methods were used to assess the model for inconsistency, and although the data available to test for inconsistency was limited, inconsistency was not found.

Meta-regression examining the influence of study duration, dose-level, and either treatmentresistant or first-episode status did not result in any significant findings. There are fewer data available for the newer drugs; particularly all of the LAI drugs (e.g., the 3-month paliperidone 
palmitate injection), lurasidone, iloperidone, brexpiprazole, and cariprazine. Results for these drugs should be interpreted with caution.

Figure 4. Network meta-analysis of withdrawals due to adverse events in trials of SGAs

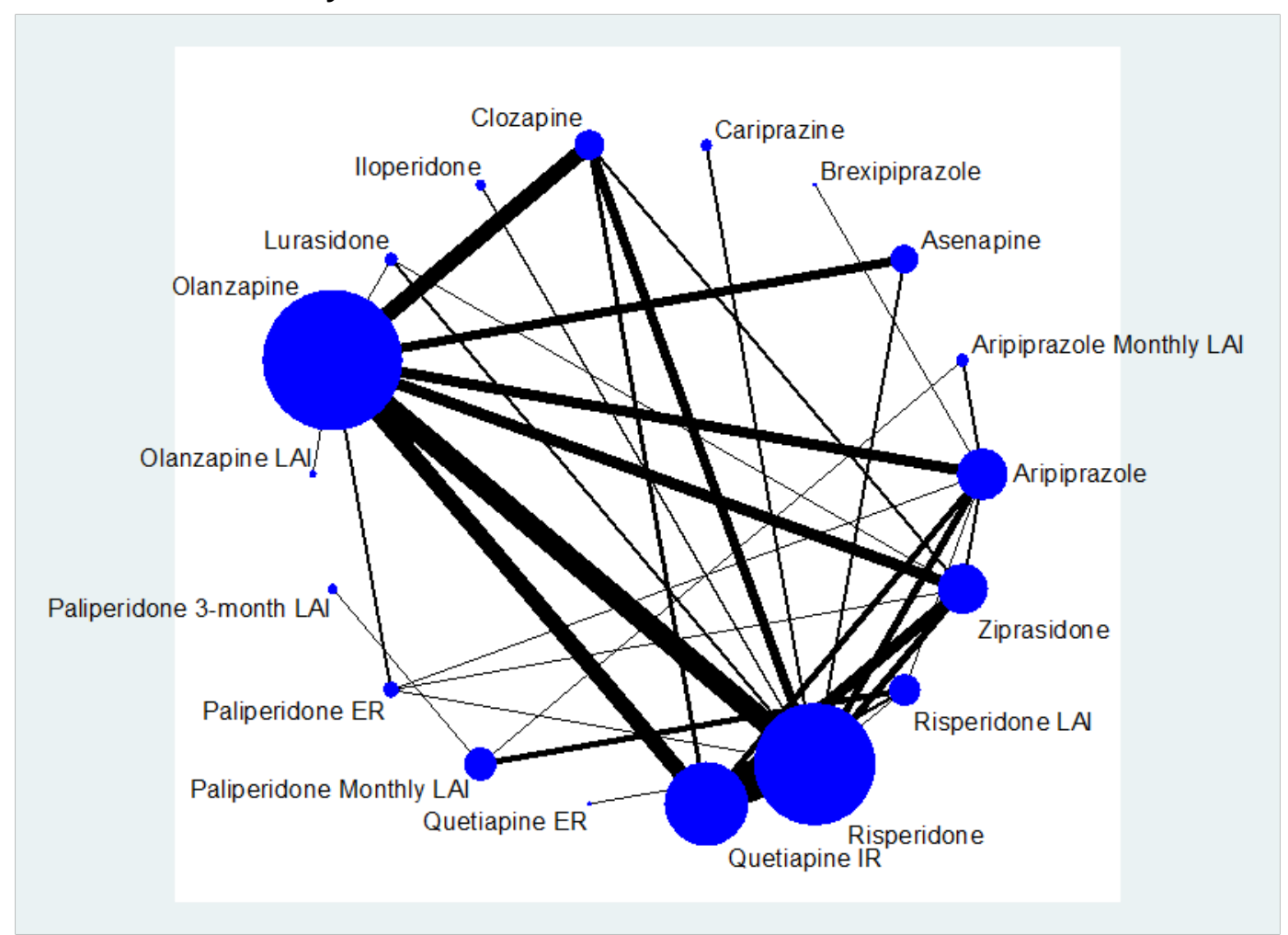

ER = extended-release; IR = immediate-release; LAI = long-acting injection; SGAs = second-generation antipsychotic

Legend: Circles represent relative numbers of studies including each drug. Line thickness represents number of studies making specific comparison for this outcome.

\section{Other Outcomes}

There were several outcomes that were important to the evaluation of the evidence on SGAs, but that were lower priority and were not rated for their SOE. However, the Drug Effectiveness Review Project (DERP) systematic review ${ }^{29}$ included evidence synthesis on these outcomes, which is summarized below; complete details can be found in the review report.

\section{Relapse}

The systematic review found that the evidence on relapse suffers from methodological issues that could affect the findings, mainly lack of blinding, high dropout rates, and that no two studies used the same definition of relapse. ${ }^{29}$ Evidence on the comparison of olanzapine with risperidone and quetiapine was inconsistent, and conclusions of differences could not be drawn. Comparisons of risperidone and quetiapine to each other or to clozapine and lurasidone were based on very few studies but generally did not indicate significant differences. Single studies found risperidone LAI had lower relapse rates than oral risperidone (5\% to $18 \%$ vs. $33 \%$ to $50 \%$ at 1 year; $\mathrm{p}<0.01)$ or quetiapine $(16.5 \%$ vs. $31.3 \%$; $\mathrm{p}<0.0001$ at 1 year). No differences in relapse rates were found for comparisons of lurasidone and quetiapine ER or risperidone; aripiprazole LAI or risperidone LAI, or oral olanzapine and oral aripiprazole; or risperidone and quetiapine ER. 


\section{Drug Discontinuation and Time to Discontinuation}

The rate of drug discontinuation and time to discontinuation were summary values representing the net effect of the two main causes of discontinuations: lack of efficacy and adverse events. Patients who withdraw from a study are also counted as having discontinued the study treatment drug. Based on a network analysis of 111 studies (95 two-arm studies, 11 threearm studies, three four-arm studies, and two five-arm studies; $\mathrm{N}=32,096$ ), there were several significant findings (the matrix of results for discontinuation can be found in Appendix G-3). ${ }^{43,45-}$ 47,49-51,54-57,59,61,63,66,121-124,132-134,136,144,148,150-156,158,160,161,163-166,168-172,175-178,180,184,186-

188,192,197,200,202,204-206,209,211,213,214,216,218-221,223,224,226-243 Olanzapine and clozapine had significantly lower discontinuation rates than asenapine, cariprazine, iloperidone, lurasidone, olanzapine LAI, quetiapine, risperidone, and ziprasidone (ORs range from 0.42 for clozapine vs. iloperidone to 0.69 for clozapine vs. risperidone). Clozapine was found to also have lower risk than paliperidone palmitate monthly LAI (OR 0.56, 95\% CI 0.33 to 0.96 ) and olanzapine had lower risk than paliperidone (OR 0.67, 95\% CI 0.50 to 0.89). Quetiapine ER had lower risk of discontinuing study than iloperidone, olanzapine LAI, or quetiapine (ORs 0.26 to 0.35), and both risperidone and aripiprazole had lower risk than iloperidone or quetiapine (ORs 0.61 to 0.77). Both risperidone and aripiprazole monthly LAI had lower risk than iloperidone (ORs 0.52 and 0.62, respectively). Few studies of newer drugs exist, suggesting that these findings should be interpreted cautiously. Meta-regression examining the influence of study duration, dose level, and either treatment-resistant or first-episode status did not result in any significant findings.

Olanzapine was found to have longer time to discontinuation than quetiapine, risperidone, and ziprasidone (4 months longer based on trial data; 46 to 66 days longer based on observational data). Based on a single small trial, Phase 2E of the CATIE study, clozapine may have longer time to discontinuation (10.5 months) than olanzapine (2.7 months), risperidone (2.8 months) or quetiapine (3.3 months). Evidence did not differentiate aripiprazole, olanzapine, risperidone and quetiapine or ziprasidone and olanzapine or risperidone. A single fair-quality retrospective study found risperidone LAI to have significantly longer duration of treatment than aripiprazole, clozapine, olanzapine, quetiapine, or ziprasidone (79 to 120 days longer). These findings need confirmation.

\section{Cardiac and Cardiovascular Risk}

The DERP systematic review ${ }^{29}$ included evidence synthesis on these outcomes, although the evidence on cardiovascular risks is limited largely to observational studies of the older SGAs.

Coronary heart disease. A large, good-quality retrospective cohort study found no significant differences in the risk of cardiovascular death, acute coronary syndrome or ischemic stroke between risperidone and olanzapine or quetiapine in patients age 18 to 64 years within the first year of starting the drug. Based on data from CATIE, the estimated 10-year risk of coronary heart disease was increased with olanzapine compared with risperidone, and the highest risk increases occurred among those with higher baseline risk.

Myocarditis and cardiomyopathy. A large adverse event database study found that clozapine was significantly associated with myocarditis or cardiomyopathy, whereas olanzapine, quetiapine, and risperidone were not. Limited evidence suggested an increased risk of cardiac arrest and arrhythmia with risperidone compared with clozapine. Comparisons of secondgeneration to conventional antipsychotics showed lower odds of cardiomyopathy or coronary heart disease with aripiprazole, and increased odds of hypertension with ziprasidone. 
Diabetes mellitus and ketoacidosis. The systematic review reported that evidence on diabetes mellitus and ketoacidosis was limited, and the studies did not control for several important potentially confounding factors such as weight or family history of diabetes. ${ }^{29}$ The absolute increase in risk was not clear based on this evidence. In children and adolescents, the risk of diabetes increased with antipsychotic exposure based on one good-quality systematic review of observational studies when compared with healthy controls (OR 2.58, 95\% CI 1.56 to 4.24) or nonexposed psychiatric controls (OR 2.09, 95\% CI 1.50 to 52.90). In children and adolescents, treatment with aripiprazole was associated with increased risk of diabetes when compared with risperidone based on one large observational study (OR 1.58, 95\% CI 1.21 to 2.07). In adults, observational evidence indicated an increased risk of new-onset diabetes with olanzapine compared with risperidone (OR 1.16, 95\% CI 1.03 to 1.31). Limited evidence did not consistently support a significant difference between clozapine and risperidone or between quetiapine and olanzapine, risperidone, or clozapine.

Diabetic ketoacidosis was significantly increased with olanzapine compared with risperidone (OR 3.5, 95\% CI 1.7 to 7.9) in a single study; a second study found no difference in a composite outcome of diabetic ketoacidosis, hyperglycemia, or hyperglycemic hyperosmolar state between risperidone and olanzapine, regardless of age group, but a significantly lower risk with quetiapine compared with risperidone in older patients (adjusted HR 0.69, 95\% CI 0.53 to 0.90).

Tardive dyskinesia. Comparative observational evidence suggested a significantly increased risk of new-onset tardive dyskinesia with risperidone compared with olanzapine (OR 1.70, 95\% CI 1.35 to 2.14). Similar increases were not seen with clozapine or quetiapine. Rates of newonset tardive dyskinesia were low overall: 3 percent with risperidone and 1 to 2 percent for others.

\section{Extrapyramidal Symptoms}

The systematic review reported that the best evidence suggested that the rates of patients experiencing extrapyramidal side effects (prevalent or incident), measures of severity of symptoms were mostly not different among the drugs, although use of anticholinergic medications did differ in some comparisons. ${ }^{29}$ Differences found, mainly in single studies, are summarized in Table 7. 
Table 7. Extrapyramidal symptoms: significant differences in trials of SGAs

\begin{tabular}{|c|c|}
\hline $\begin{array}{l}\text { Primary SGA } \\
\text { Comparison }\end{array}$ & Findings \\
\hline \multirow[t]{4}{*}{ Risperidone } & $\begin{array}{l}\text { Quetiapine and ziprasidone had lower use of anticholinergic medications to treat EPS and lower } \\
\text { rates of withdrawal due to EPS than risperidone. }\end{array}$ \\
\hline & $\begin{array}{l}\text { EPS adverse events were more frequent with risperidone LAI than with oral olanzapine or } \\
\text { quetiapine }\end{array}$ \\
\hline & $\begin{array}{l}\text { A single fair-quality trial suggested that aripiprazole may cause worse akathisia in early weeks of } \\
\text { treatment but not with longer treatment. }\end{array}$ \\
\hline & $\begin{array}{l}\text { Differences were not found between risperidone and cariprazine over } 6 \text { weeks on EPS outcomes in } \\
\text { a fair-quality trial. }\end{array}$ \\
\hline \multirow[t]{2}{*}{ Ziprasidone } & $\begin{array}{l}\text { Ziprasidone was associated with lower risk of withdrawal due to EPS adverse events than } \\
\text { quetiapine, but quetiapine had lower use of anticholinergic medications to treat EPS. }\end{array}$ \\
\hline & $\begin{array}{l}\text { EPS adverse events were significantly more frequent with ziprasidone (9\%) than with iloperidone } \\
(3 \%) \text { in a fair-quality 3-week trial. }\end{array}$ \\
\hline \multirow[t]{2}{*}{ Olanzapine } & $\begin{array}{l}\text { Based on the CATIE trial, quetiapine had lower risk of patients using anticholinergic medications } \\
\text { than olanzapine. }\end{array}$ \\
\hline & $\begin{array}{l}\text { Evidence suggested that paliperidone and asenapine cause more EPS adverse events and worse } \\
\text { severity of symptoms than olanzapine, and that asenapine results in more patients using an } \\
\text { anticholinergic medication ( } 6 \% \text { vs. } 2 \% \text { ). }\end{array}$ \\
\hline \multirow{3}{*}{$\begin{array}{r}\text { Long-Acting } \\
\text { Injections }\end{array}$} & Aripiprazole monthly LAI resulted in greater incidence of EPS adverse events (RR $1.88,95 \% \mathrm{Cl}$ \\
\hline & $\begin{array}{l}1.26 \text { to } 2.81) \text { and worse akathisia symptoms ( }+0.06 \text { vs. }-0.05 \text { on a } 0 \text { to } 5 \text { scale; } p=0.0184 \text { ) than oral } \\
\text { aripiprazole in a short-term study, but differences were not found in a year-long study. }\end{array}$ \\
\hline & $\begin{array}{l}\text { Differences in EPS adverse events were not found in a } 28 \text {-week trial of aripiprazole and } \\
\text { paliperidone palmitate monthly injections, or in a network meta-analysis comparing the monthly and } \\
\text { 4- to 6-week injections of aripiprazole. }\end{array}$ \\
\hline
\end{tabular}

CATIE = Clinical Antipsychotic Trials of Intervention Effectiveness; CI = confidence interval; EPS = extrapyramidal symptoms; IR = immediate-release; LAI = long-acting injection; RR = risk ratio; SGA = second-generation antipsychotic

Source: McDonagh, $2013^{29}$

\section{Weight Gain}

The systematic review reported that the rate of clinically important weight gain (defined as a $7 \%$ or more increase from baseline) in clinical trials was greater with olanzapine than with aripiprazole (RR 2.31), asenapine (RR 2.59), clozapine (RR 1.71), quetiapine (RR 1.82), risperidone (RR 1.81), and particularly ziprasidone (RR 5.76) across 3.7 to 24 months. ${ }^{29}$ The analysis of risk of important weight gain for olanzapine compared with risperidone appeared to vary by duration of study, whereas the others did not. The RR of 1.81 represents studies of 6 to 7 months duration, whereas the CATIE Phase 1 results indicated much higher risk (RR 7.49, 95\% CI 4.25 to 13.33 ) at 18 months.

The review reports that single studies of olanzapine compared with olanzapine ER, olanzapine ODT, and paliperidone palmitate injection did not find significant differences in risk of weight gain. ${ }^{29}$ Data for other SGAs compared with olanzapine were insufficient.

Observational evidence generally agreed with trial evidence, but resulted in somewhat lower estimates of increased risk with olanzapine. Risperidone was found to have greater risk of weight gain (in single studies) compared with aripiprazole (12\% vs. 3\%; $\mathrm{p}=0.018)$, or cariprazine (calculated RR 1.98, 95\% CI 1.03 to 3.80 for any dose cariprazine vs. risperidone). There was not a significant difference in the proportion of patients with weight gain between paliperidone and aripiprazole at 6 months in a single study. An open-label RCT of medication-naïve patients with first-episode schizophrenia (not included in the review) found aripiprazole to result in greater absolute weight gain and more patients with $\geq 7$ percent gain than with ziprasidone or quetiapine. $^{244}$ 


\section{Metabolic Syndrome}

The systematic review found that olanzapine had a significantly greater risk of metabolic syndrome than risperidone with followup of 6 weeks to 3 months (pooled OR 1.60, 95\% CI 1.10 to $\left.2.21, \mathrm{I}^{2}=0 \%\right) .{ }^{29}$ Olanzapine also had significantly greater risk of metabolic syndrome than aripiprazole (Evidence-based Practice Center [EPC] pooled OR 2.50, 95\% CI 1.32 to 4.76; $\mathrm{I}^{2}=0 \%$ ) with followup of 3.5 to 12 months. Evidence for other comparisons was too limited to draw conclusions.

\section{Sexual Function}

Evidence on the comparative effect of SGAs on sexual function reported in the systematic review $^{29}$ was inconsistent or limited by single-study bodies of evidence, inadequate sample sizes or lack of explicit methodology to measure symptoms. Based on four very small trials, evidence on risperidone compared with quetiapine was inconclusive. A single study comparing risperidone and quetiapine ER $(\mathrm{N}=798)$ found significantly more men had sexual adverse effects at 6 months $(13 \%$ vs. $6 \%$; $p<0.05)$, but the difference was not significant at 12 months. Individual trials found no significant differences between olanzapine and paliperidone, risperidone, or ziprasidone or between risperidone and paliperidone or aripiprazole.

\section{Agranulocytosis (Severe Neutropenia)}

Although the incidence of agranulocytosis and neutropenia are known serious adverse events with clozapine that require regular monitoring, evidence on the risk with other SGAs is very limited and does not indicate a serious concern with the other drugs currently available. ${ }^{29}$

\section{First-Generation Antipsychotic Versus Second-Generation Antipsychotic}

\section{Key Points}

- Evidence comparing FGA versus SGA is available from a good-quality systematic review (111 RCTs and two cohort studies; $\mathrm{N}=118,503)$ and five RCTs $(\mathrm{N}=1,055)$ not included in the systematic review.

- There was little evidence of a differential effect between FGAs and SGAs in quality of life outcomes using various measures based on a good-quality systematic review, with one trial comparing haloperidol with ziprasidone finding a positive effect favoring ziprasidone (effect estimate -12.12, 95\% CI -22.06 to -2.17) and no difference between groups reported in another trial (SOE: low)

o There were no differences between haloperidol and olanzapine (SOE: moderate), or perphenazine and olanzapine, quetiapine, risperidone, or ziprasidone (SOE: low).

- For response and remission, olanzapine was found superior to haloperidol.

o Pooled results from 14 RCTs comparing haloperidol with olanzapine found a significant effect on response rate favoring olanzapine ( $N=4,099$; RR 0.86, 95\% CI 0.78 to 0.96 ) (SOE: low). Three trials comparing haloperidol with olanzapine found a significant difference in remission rates favoring olanzapine (RR 0.64, $95 \%$ CI 0.45 to 0.94 ) (SOE: low). 
o There was no difference in response rates between haloperidol and aripiprazole (five RCTs, N=2,185; RR 1.01; 95\% CI 0.76 to 1.34), quetiapine (six RCTs, $\mathrm{N}=1,421$; RR 0.99, 95\% CI 0.76 to 1.30), risperidone (16 RCTs, $\mathrm{N}=3,452$; RR 0.94, 95\% CI 0.87 to 1.02), and ziprasidone (six RCTs, N=1,283; RR 0.98, 95\% CI 0.74 to 1.30) (SOE: moderate for haloperidol versus risperidone; low for other comparisons).

o There was no difference in remission rates between haloperidol and ziprasidone based on three trials (RR 0.89, 95\% CI 0.71 to 1.12) (SOE: low).

- Reductions in core illness symptoms were greater with older SGAs than with haloperidol

o There were significant difference in total PANSS between haloperidol and olanzapine (15 RCTs, N=4,209; mean difference [MD] 2.31, 95\% CI 0.44 to 4.18) and risperidone (21 RCTs, $\mathrm{N}=4,020$; MD 3.24, 95\% CI 1.62 to 4.86), both favoring the SGA over haloperidol (SOE: moderate).

o There were no differences in total PANSS, BPRS, CGI-S, and Clinical Global Impression - Improvement scale (CGI-I) scores for other FGA versus SGA comparisons (SOE: low).

- SGAs improved negative symptoms more than haloperidol

o Olanzapine was more effective than haloperidol at improving negative symptoms based on Scale for Assessment of Negative Symptoms (SANS) scores (five RCTs, $\mathrm{N}=535$; MD 2.56, 95\% CI 0.94 to 4.18) (SOE: moderate).

o Using the negative symptoms subscale of the PANSS scale, MDs (although small) between haloperidol and aripiprazole (three RCTs, N=1,701; MD 0.80, 95\% CI 0.14 to 1.46), olanzapine (14 RCTs, $\mathrm{N}=3,742$; MD 1.06, 95\% CI 0.46 to 1.67), and risperidone (22 RCTs, N=4,142; MD 0.80, 95\% CI 0.14 to 1.46) all favored the SGA (SOE: low).

o There were no differences in negative PANSS or SANS scores for other FGA versus SGA comparisons (SOE: low).

- Overall adverse event rates were lower with SGAs when comparing haloperidol with aripiprazole (three RCTs, N=1,713; RR 1.11; 95 \% CI 1.06 to 1.17), risperidone (eight RCTs, N=1,313; RR 1.20, 95\% CI 1.01 to 1.42), and ziprasidone (six RCTs, N=1,448, RR 1.13, 95\% CI 1.03 to 1.23) (SOE: moderate).

- Withdrawals due to adverse events were significantly higher with haloperidol than with SGAs: compared with aripiprazole (eight RCTs, N=3,232; RR 1.25, 95\% CI 1.07 to 1.47), olanzapine (24 RCTs, N=5,708; RR 1.89; 95\% CI 1.57 to 2.27), risperidone (25 RCTs, N=4,581; RR 1.32; 95\% CI 1.09 to 1.60), and ziprasidone (seven RCTs, N=1,597; RR 1.68, 95\% CI 1.26 to 2.23) (SOE: moderate).

\section{Detailed Synthesis}

\section{Description of Included Studies}

A recent large, good-quality systematic review compared the benefits and harms of FGAs versus SGAs (Appendix Table E-1). ${ }^{27,28}$ The review included 111 RCTs and 2 cohort studies conducted in people with schizophrenia or related psychosis $(n=118,503)$. Patients enrolled in the included studies had a mean age of 37 years; half of the included studies were conducted in inpatient populations. The included studies compared: oral fluphenazine with olanzapine (two RCTs), quetiapine (one RCT) and risperidone (one RCT); haloperidol with aripiprazole (eight 
RCTs), asenapine (one RCT), clozapine (10 RCTs, one cohort study), olanzapine (34 RCTs, one cohort study), paliperidone (one RCT), quetiapine (10 RCTs, one cohort study), risperidone (37 RCTs, one nonrandomized trial, one cohort study) and ziprasidone (eight RCTs, one nonrandomized trial); and perphenazine with aripiprazole (one RCT), olanzapine (two RCTs), quetiapine (one RCT), risperidone (two RCTs) and ziprasidone (one RCT). No studies compared a FGA with brexpiprazole, cariprazine, iloperidone, or lurasidone. Doses of FGAs and SGAs varied widely among the studies included in the systematic review. For FGAs, doses of fluphenazine ranged from 6 to 21 milligrams per day (mg/day), haloperidol ranged from 1 to 30 $\mathrm{mg} /$ day and perphenazine ranged from 8 to $64 \mathrm{mg} / \mathrm{day}$. SGA doses ranged from 1 to $45 \mathrm{mg} /$ day for aripiprazole, 5 to $10 \mathrm{mg} /$ day for asenapine, 200 to $800 \mathrm{mg} /$ day for clozapine, 1 to $40 \mathrm{mg} /$ day for olanzapine, 200 to $1200 \mathrm{mg} /$ day for quetiapine 1 to $6 \mathrm{mg} /$ day for risperidone and 4 to 240 $\mathrm{mg} /$ day for ziprasidone. The duration of followup among the included studies ranged widely from less than 1 day to 22 years, although median followup was eight weeks. None of the included studies were rated as having low risk of bias. Reasons for unclear or high risk of bias among the studies were unclear methods of randomization and/or allocation concealment, unclear or lack of blinding of outcome assessors and incomplete reporting of outcome data (Appendix Table F-1).

We also included five additional trials not in the systematic review comparing FGAs with SGAs (N=1,055; Appendix Table E-2). ${ }^{57,67-70}$ These trials enrolled between 78 and 300 participants, mean age ranged from 26 to 45 years and the proportion of female participants ranged from 25 to 42 percent. Race was reported in one trial, enrolling predominantly black patients (57\%); this was also the only trial conducted in the United States. ${ }^{70}$ Two trials limited enrollment to participants with first-episode psychosis. ${ }^{67,69}$ The FGA used in all of the trials was haloperidol, compared with olanzapine (three RCTs), quetiapine (two RCTs), aripiprazole, risperidone and ziprasidone (one RCT each). Two trials were rated good quality, ${ }^{68,70}$ one was rated poor quality, ${ }^{69}$ and the others were rated fair quality (Appendix Table F-2). The primary limitation in the fair-quality trials was lack of blinding of clinicians and patients.

\section{Findings}

\section{Function}

Outcomes related to function were rarely reported in the systematic review, with no significant differences between FGAs and SGAs for any measure of function (Appendix Table E-1). ${ }^{28}$ No significant differences were found in global function, based on GAF score, between haloperidol and olanzapine (one RCT, $\mathrm{N}=208$; effect estimate -4.00 ; 95\% CI -13.70 to 5.70), quetiapine (one RCT, $\mathrm{N}=207$; effect estimate $0.20,95 \%$ CI -9.60 to 9.80), or ziprasidone (two RCTs, one non-RCT, $\mathrm{N}=1,085$; effect estimate $0.30,95 \% \mathrm{CI}-1.58$ to $2.19 ; \mathrm{I}^{2}=0 \%$ ), or in encounters with the legal system (one RCT, $\mathrm{N}=31$; RR 3.20, 95\% CI 0.76 to 13.46). There were also no differences in proportion of patients with paid employment for comparisons of perphenazine versus olanzapine ( $\mathrm{N}=597$; RR 1.29; 95\% CI 0.70 to 2.38), quetiapine ( $\mathrm{N}=598$; $\mathrm{RR}$ 1.75; 95\% CI 0.90 to 3.43), risperidone (N=602; RR 1.38; 95\% CI 0.74 to 2.57) and ziprasidone ( $\mathrm{N}=446$; RR 1.22; 95\% CI 0.60 to 2.51) based on one trial each. The number of patients with economic independence was also not different in one trial of haloperidol versus risperidone ( $\mathrm{N}=100$; RR 0.94; 95\% CI 0.68 to 1.29). This SOE was insufficient due to limited evidence for each comparison. 


\section{Quality of Life}

The systematic review found little evidence of a differential effect between FGAs and SGAs in quality of life outcomes (Appendix Table E-1). ${ }^{28}$ One trial comparing haloperidol with ziprasidone found a positive effect on QLS score favoring ziprasidone ( $\mathrm{N}=599$; effect estimate 12.12, 95\% CI -22.06 to -2.17). There was no difference between haloperidol and ziprasidone in one other trial based on the Manchester Short Assessment of Quality of Life (MANSA) score ( $\mathrm{N}=185$; effect estimate $-0.10,95 \%$ CI -1.48 to 1.28 ). This was low SOE. There was no difference in quality of life outcomes in trials comparing haloperidol with olanzapine (five RCTs, N=816; effect sizes ranged from -3.62 to 0 ). This SOE was moderate. There was also no difference for haloperidol versus quetiapine (one RCT, N=207; effect estimate 0.00, 95\% CI 1.38 to 1.38 ) and risperidone (two RCTs, $\mathrm{N}=352$; effect estimates ranged from -0.10 to 0.10 ) using various quality of life measures; this evidence was of insufficient strength. Based on one trial each, no significant differences in quality of life measures were found when comparing perphenazine with aripiprazole ( $\mathrm{N}=300$; $\mathrm{RR} 4.74,95 \%$ CI 2.58 to 8.69), olanzapine $(\mathrm{N}=597$; effect estimate 0.00 ; $95 \%$ CI -0.16 to 0.16 ), quetiapine ( $\mathrm{N}=598$; effect estimate 0.10 , $95 \%$ CI 0.07 to 0.27 ), risperidone ( $\mathrm{N}=602$; effect estimate $-0.07,95 \% \mathrm{CI}-0.24$ to 0.10 ), or ziprasidone ( $\mathrm{N}=446$; effect estimate $-0.07,95 \% \mathrm{CI}-0.27$ to 0.13 ). Due to limited evidence and the heterogeneity of measures used to assess quality of life, this SOE was low to insufficient (for perphenazine versus aripiprazole).

\section{Response and Remission}

\section{Response}

Evidence on response rate was mixed in the systematic review and heterogeneity was moderate to high for most risk estimates (Appendix Table E-1) ${ }^{28}$ Pooled results from 14 RCTs comparing haloperidol with olanzapine found a significant effect favoring olanzapine $(\mathrm{N}=4,099$; RR $0.86,95 \%$ CI 0.78 to 0.96 ; $\mathrm{I}^{2}=55 \%$ ). However, the review found no difference in response rates between haloperidol and aripiprazole (five RCTs, N=2,185; RR 1.01, 95\% CI 0.76 to 1.34; $\mathrm{I}^{2}=83 \%$ ), quetiapine (six RCTs, $\mathrm{N}=1,421$; RR $0.99 ; 95 \%$ CI 0.76 to $1.30 ; \mathrm{I}^{2}=77 \%$ ), risperidone (16 RCTs, N=3,452; RR 0.94, 95\% CI 0.87 to $1.02 ; \mathrm{I}^{2}=29 \%$ ) and ziprasidone (six RCTs, $\mathrm{N}=1,283$; RR 0.98 , 95\% CI 0.74 to $1.30 ; \mathrm{I}^{2}=80 \%$ ). This was low to moderate (for haloperidol versus risperidone) SOE. There was no difference between groups for other comparisons, including fluphenazine versus olanzapine, quetiapine and risperidone and for perphenazine versus aripiprazole based on one trial each. This evidence was insufficient for all comparisons.

\section{Remission}

Pooled evidence from three trials comparing haloperidol with olanzapine found a significant difference in remission rates favoring olanzapine (RR 0.64; $95 \%$ CI 0.45 to 0.94 ) with moderate heterogeneity $\left(\mathrm{I}^{2}=54 \%\right) .{ }^{28}$ Evidence from a trial not included in the systematic review was consistent, though not significant, finding a greater number of patients taking olanzapine experienced remission compared with the haloperidol group $33 \%$ versus $25 \%$; $\mathrm{p}=0.5$ ) ${ }^{67}$ This was low SOE. There were no differences between haloperidol and clozapine (one RCT, N=71; RR 0.16, $95 \%$ CI 0.02 to 1.20 ), quetiapine (one RCT, N=207; RR 0.72, 95\% CI 0.41 to 1.25 ), risperidone (two RCTs, $\mathrm{N}=179$; RR $0.84,95 \%$ CI 0.56 to 1.24 ; $\mathrm{I}^{2}=0 \%$ ), or ziprasidone (three RCTs, N=1,085; RR 0.89, 95\% CI 0.71 to 1.12 ; $\mathrm{I}^{2}=12 \%$ ). This was low (for ziprasidone) and insufficient (for clozapine, quetiapine and risperidone) SOE. 


\section{Mortality}

The systematic review did not include any trials of outpatients reporting mortality rates. ${ }^{28}$ One retrospective cohort study was included, reporting higher risk of mortality in patients taking haloperidol versus clozapine or risperidone. These results are difficult to interpret, as patient characteristics such as age were not reported in patients taking antipsychotic medications, and the controls in the study were taking antipsychotic mediations for reasons other than schizophrenia or other psychotic disorders. ${ }^{245}$

\section{Reduction in Self-Harm}

Most RCTs excluded patients at risk for suicide. In trials of haloperidol versus olanzapine $(\mathrm{N}=182)$ and perphenazine versus olanzapine $(\mathrm{N}=597)$ there were no differences in incidence of attempted suicide (RR 3.13, 95\% CI 0.13 to 76; and 0.64, 95\% CI 0.06 to 7.06) or suicide deaths (RR 3.13, 95\% CI 0.13 to 76; and 3.86, 95\% CI 0.40 to 37). ${ }^{28}$ One trial not included in the systematic review reported no difference between LAI paliperidone and LAI haloperidol in incidence of suicidal or homicidal ideation (RR 1.10, 95\% CI 0.63 to 1.89 ). ${ }^{70}$ This was insufficient SOE.

\section{Improvement in Core Illness Symptoms}

The systematic review assessed total and negative symptoms based on a number of scale measures, including total PANSS, BPRS, CGI-S and CGI-I for total symptoms and PANSS and SANS for negative symptoms (Appendix Table E-1). ${ }^{27,28}$ Comparative evidence was only available for haloperidol versus SGAs. The review did not combine results across different scales.

\section{Total Symptoms}

\section{PANSS}

Based on pooled analysis, there were significant difference in total PANSS between haloperidol and olanzapine (15 RCTs, N=4,209; MD 2.31, 95\% 0.44 to 4.18) and risperidone (21 RCTs, N=4,020; MD 3.24, 95\% CI 1.62 to 4.86), both favoring the SGA over haloperidol. This was moderate SOE, and the differences are small enough to possibly not meet a minimal clinically important difference threshold. There were no differences between haloperidol and clozapine (four RCTs, N=607; MD 2.69, 95\% CI -1.28 to 6.65), quetiapine (five RCTs, $\mathrm{N}=1,013$; MD 0.31, 95\% CI -2.34 to 2.96) and ziprasidone (four RCTs, N=1,105; MD 1.22, 95\% CI -0.62 to 3.07). This evidence was low strength.

\section{BPRS}

There were no differences in BPRS scores between haloperidol and aripiprazole (three RCTs, $\mathrm{N}=779$; MD -0.01, 95\% CI -2.82 to 2.81), clozapine (four RCTs, N=268; MD2.16, 95\% CI -0.56 to 4.87), olanzapine (13 RCTs, N=4,014; MD 0.19, 95\% CI -2.09 to 2.47), quetiapine (four RCTs, N=756; MD 1.23, 95\% CI -0.50 to 2.96), risperidone (14 RCTs, N=2,659; MD 0.67, 95\% CI -0.53 to 1.88 ) and ziprasidone (four RCTs, $\mathrm{N}=1,143$; MD $0.24,95 \% \mathrm{CI}-0.57$ to 1.06 ). This was low-strength evidence.

\section{CGI-S}

CGI-S scores, as a measure of illness severity, were marginally better with in olanzapine (MD 0.16, 95\% CI 0.01 to 0.31) when compared with haloperidol, and better with haloperidol 
when compared with quetiapine (MD -0.23, 95\% CI -0.42 to -0.04) based on analysis of eight $(\mathrm{N}=3,564)$ and four $(\mathrm{N}=1,253)$ trials each. Though these estimates were significant, they were considered not clinically meaningful. ${ }^{27}$ The SOE was moderate for both comparisons. MD in scores for haloperidol and aripiprazole (five RCTs, N=1,366; $-0.03,95 \%-0.20$ to 0.14 ) risperidone (eight RCTs, $\mathrm{N}=2,348 ; 0.07,95 \% \mathrm{CI}-0.11$ to 0.25 ) and ziprasidone (four RCTs, $\mathrm{N}=1,143$; -0.00 , 95\% CI -0.26 to 0.26 ) was not significantly different between groups, with low SOE for all comparisons.

\section{CGI-I}

There was no difference in CGI-I scores, as a measure of improvement since beginning treatment, between haloperidol and olanzapine (two RCTs, N=281; MD 0.11, 95\% -0.30 to 0.51 ), quetiapine (three RCTs, $\mathrm{N}=623$; MD 0.02, $95 \% \mathrm{CI}-0.24$ to 0.27 ) and risperidone (three RCTs, N=657; -0.02, 95 \% CI -0.39 to 0.36) based on pooled analysis. This was low SOE.

\section{Negative Symptoms}

\section{PANSS Negative}

There was moderate-strength evidence that some SGAs were more effective than haloperidol at improving PANSS negative symptom scores. Analysis of MD in score between haloperidol and aripiprazole (three RCTs, $\mathrm{N}=1,701 ; 0.80,95 \%$ CI 0.14 to 1.46), olanzapine (14 RCTs, $\mathrm{N}=3,742 ; 1.06$, 95\% CI 0.46 to 1.67), and risperidone (22 RCTs, $\mathrm{N}=4,142 ; 0.80,95 \%$ CI 0.14 to 1.46) all favored the SGA. Evidence was not able to identify a difference in PANSS negative symptoms scores when comparing haloperidol and clozapine (three RCTs, N=184; MD 0.28, 95\% CI -0.96 to 1.51), quetiapine (three RCTs, $\mathrm{N}=358$; MD0.53, 95\% CI -0.81 to 1.87 ) and ziprasidone (two RCTs, N=900; MD 0.56, 95\% CI -0.30 to 1.42). This evidence is low strength, primarily due to the small sample sizes. Whether significant or not, the differences found were very small.

\section{SANS}

Olanzapine was found to be more effective than haloperidol at improving SANS scores based on analysis of five RCTs ( $\mathrm{N}=535$; MD 2.56, 95\% CI 0.94 to 4.18). The SOE was moderate. Evidence was not able to identify a difference between haloperidol and clozapine (two RCTs, $\mathrm{N}=157$; MD 0.94, 95\% CI -2.60 to 4.48) or risperidone (4 RCTs, N=508; MD 0.30, 95\% CI 2.79 to 3.38). This was low-strength evidence.

\section{Overall Adverse Events}

Overall adverse event rates were reported for each FGA and select SGAs (Appendix Table E$1){ }^{28}$ When compared with haloperidol, there were significant differences favoring aripiprazole (three RCTs, $\mathrm{N}=1,713$; RR 1.11, 95\% CI 1.06 to 1.17; $\mathrm{I}^{2}=0 \%$ ), risperidone (eight RCTs, $\mathrm{N}=1,313$; RR 1.20 , 95\% CI 1.01 to $1.42 ; \mathrm{I}^{2}=84 \%$ ), and ziprasidone (six RCTs, N=1,448; RR 1.13, $95 \%$ CI 1.03 to $\left.1.23 ; \mathrm{I}^{2}=31 \%\right)$. Due to limited evidence and heterogeneity for the risperidone and ziprasidone comparisons, SOE is low. No significant differences overall adverse event rates for other comparisons, primarily based on single studies, comparing: fluphenazine with olanzapine (one RCT, N=60; RR 9.00, 95\% CI 0.51 to 160); haloperidol with asenapine (one RCT, N=335; RR 1.09, 95\% CI 0.96 to 1.25), clozapine (one RCT, N=423; RR 0.81, 95\% CI 0.49 to 1.34), olanzapine (one RCT, $\mathrm{N}=119$; RR 10.47, 95\% 0.59 to 185), or quetiapine (three RCTs, N=859; RR 1.08, 95\% CI 0.93 to 1.25; $\mathrm{I}^{2}=25 \%$ ); perphenazine with olanzapine (one RCT, 
$\mathrm{N}=597$; RR 0.93, 95\% CI 0.83 to 1.04), quetiapine (one RCT, N=598; RR 1.00, 95\% CI 0.89 to 1.12), risperidone (one RCT, N=602; RR 0.96, 95\% CI 0.85 to 1.07), or ziprasidone (one RCT, $\mathrm{N}=446$; RR 1.01; 95\% CI 0.88 to 1.16). SOE for all of these comparisons was insufficient.

\section{Withdrawal Due to Adverse Events}

Evidence on withdrawals due to adverse events comes from the systematic review ${ }^{28}$ and four additional RCTs ${ }^{57,67-69}$ not included in the systematic review (Appendix Tables E-1 and E-2). When comparing haloperidol with specific SGAs, withdrawals due to adverse events were significantly higher with haloperidol use compared with aripiprazole (eight RCTs, N=3,232; RR 1.25, 1.07 to 1.47; I²=0\%; Appendix G-4), olanzapine (24 RCTs, N=5,708; RR 1.89, 95\% 1.57 to 2.27; $\mathrm{I}^{2}=0 \%$; Appendix G-5), risperidone (25 RCTs, N=4,581; RR 1.32, $95 \%$ CI 1.09 to 1.60; $\mathrm{I}^{2}=0 \%$; Appendix G-6), and ziprasidone (seven RCTs, N=1,597; RR 1.68, 95\% 1.26 to 2.23; $\mathrm{I}^{2}=0$ $\%$; Appendix G-7). This was moderate SOE. There is low SOE of no difference in withdrawal due to adverse events when comparing haloperidol and clozapine (5 RCTs, N=719; RR 1.00, 95\% CI 0.66 to $1.50 ; \mathrm{I}^{2}=0 \%$ ) or quetiapine (10 RCTs, $\mathrm{N}=1,759$; RR 1.97, 95\% CI 0.96 to 4.01; $\mathrm{I}^{2}=62 \%$; Appendix G-8). Based on single studies for each comparison, there was no significant differences when comparing: haloperidol to asenapine (N=335; $\mathrm{RR} 1.53$, 95\% 0.74 to 3.16); fluphenazine to olanzapine ( $\mathrm{N}=60$; RR 0.74 , 95\% CI 0.51 to 1.07 ) or quetiapine ( $\mathrm{N}=25$; RR 0.19 , 95\% CI 0.01 to 3.52); or perphenazine to aripiprazole (N=300; RR 0.53, 95\% CI 0.27 to 1.05 ), olanzapine ( $\mathrm{N}=597$; RR 0.83, 95\% CI 0.58 to 1.19), quetiapine ( $\mathrm{N}=598$; RR 1.05, 95\% CI 0.72 to 1.55$)$, risperidone ( $\mathrm{N}=602$; RR 1.54 , 95\% CI 1.00 to 2.36), or ziprasidone ( $N=446$; RR 1.01, 95\% CI 0.65 to 1.58). SOE for these comparisons was insufficient.

\section{Key Question 1b. Variation of Benefits and Harms of Pharmacological Treatments for Adults With Schizophrenia by Patient Characteristics}

\section{Second-Generation Antipsychotic Versus Second-Generation Antipsychotic}

\section{Key Points}

- Evidence on SGAs versus each other in population subgroups was limited.

- In 17 trials in patients with first-episode psychosis evidence did not indicate significant differences between oral olanzapine, quetiapine, risperidone, ziprasidone, aripiprazole, or paliperidone in rates of response or remission.

o Most studies also reported no difference in core illness symptom measures. These findings did not differ according to the duration of study, the specific drugs compared, in adolescents or women, or whether or not studies were blinded.

o Evidence on study medication discontinuation was more limited, with conflicting findings from five trials. Olanzapine was not found to have fewer discontinuations and longer time to discontinuation consistently across the studies.

o Single-trial evidence suggests that fewer patients taking aripiprazole discontinue medication than ziprasidone or quetiapine but patients receiving aripiprazole had more weight gain than those receiving ziprasidone. 
- In treatment-resistant patients, network meta-analyses indicated a small benefit with olanzapine over other older SGAs in core illness symptom improvement, negative symptoms, whereas response rates and all-cause treatment discontinuations were not different. Clozapine had fewer discontinuations due to lack of efficacy.

- In subgroups based on age and sex:

o Subgroup analyses did not find differences based on age between olanzapine and risperidone in symptom measures, quality of life, or persistence ( $>60$ years or 50 to 65 years vs. younger populations).

o Subgroup analyses indicated that women improved more than men on the CGI scale with clozapine and the EQ-5D visual analog scale score with olanzapine, compared with men.

0 Women and younger patients ( $<40$ years) were at higher risk of new onset diabetes than older, male patients with olanzapine and risperidone compared with FGAs.

- Asian patients: Comparisons of aripiprazole and paliperidone with olanzapine, and quetiapine and risperidone in Asian patients were similar to the overall conclusions for these comparisons.

- In subgroups based on comorbidities and concomitant drug use:

o Users of illicit drugs did not have different findings on any outcome compared with the overall study population in CATIE Phase 1 (olanzapine, risperidone, quetiapine and ziprasidone).

o Response rates were also similar for olanzapine and risperidone in patients with first-episode schizophrenia and a history of cannabis use disorders.

\section{Detailed Synthesis}

\section{Description of Included Studies}

Very limited direct comparative evidence addressed SGAs used for the treatment of schizophrenia in subgroups of the population. Five studies assessed the impact of age, ${ }^{163,240,246-248}$ two assessed the impact of race, ${ }^{249,250}$ and three evaluated the impact of SGAs in patients with comorbid substance use or alcohol use disorders. ${ }^{251-253}$ Most trials did not report ethnicity of enrolled patients and although three trials reported that a substantial number of patients were of African ancestry, none stratified results to examine differences in response or adverse events. ${ }^{152,192,254}$ Three trials assessed the effects of these drugs on depressive symptoms, but the patients were not selected for the trial based on depressive symptoms. ${ }^{255-257}$ The results of these trials were discussed above.

In a subgroup analysis, 100 patients who had been randomly assigned to aripiprazole or risperidone were tested for the rs2514218 genotype. The authors then correlated symptom response and adverse events with genotype status. They found that "homozygotes for the risk (C) allele at rs2514218 had significantly greater reduction in positive symptoms during 12 weeks of treatment compared to the $\mathrm{T}$ allele carriers. In the aripiprazole group, $\mathrm{C} / \mathrm{C}$ homozygotes also reported more akathisia than the $\mathrm{T}$ allele carriers, whereas in the risperidone group, male $\mathrm{T}$ allele carriers demonstrated greater prolactin elevations compared to male C/C homozygotes."258 These findings suggest further development of genotyping may help with selection of SGA medications, but is preliminary at this point. 


\section{Findings}

\section{Clinical Subgroups}

\section{First-Episode Schizophrenia}

The systematic review identified 17 trials (in 22 publications) of oral SGAs in patients experiencing their first episode of symptoms of schizophrenia. ${ }^{29}$ The studies were mainly fair quality (12 of 17), with nine open label trials (Table 8). Six trials had at least 12 months of followup and sample sizes ranged from 50 to 498. One trial included only women. And the mean age of patients was early 20s to late 30s.

Differences among the SGAs on at least one outcome were found in five of 12 fair-quality trials. Olanzapine was found to have lower risk and longer time to all-cause treatment discontinuation than risperidone (one of three RCTs), quetiapine (two of two RCTs), and ziprasidone (one of three RCTs), but differences in other benefit outcomes were not found. One trial that did not find differences in benefit outcomes at 1 year also found no differences at 3 years of followup. This trial also reported that patients taking risperidone had higher incidence of increasing severity of akathisia, sexual dysfunction (in men) and amenorrhea, whereas more patients reported daytime drowsiness with olanzapine. In a single study, aripiprazole was found to have lower risk of all-cause treatment discontinuation than quetiapine or ziprasidone, lower risk of discontinuing due to adverse events than ziprasidone, longer time to discontinuing than quetiapine, and greater risk of important weight gain than ziprasidone, but differences in other benefit outcomes were not found. A single trial found that core illness symptoms (based on the PANSS) were better with paliperidone than ziprasidone or aripiprazole, but that response rates did not differ significantly.

Table 8. Studies of SGAs in patients treated for first episode of schizophrenia

\begin{tabular}{|c|c|c|c|}
\hline Study & $\begin{array}{l}\mathbf{N} \\
\text { Duration } \\
\text { Blinding }\end{array}$ & Comparison & Results \\
\hline $\begin{array}{l}\text { Crespo-Facorro, } \\
2011^{67}\end{array}$ & $\begin{array}{l}\mathrm{N}=174 \\
1 \text { year with } \\
3 \text { year } \\
\text { followup } \\
\text { Open }\end{array}$ & $\begin{array}{l}\text { Olanzapine vs. } \\
\text { Risperidone }\end{array}$ & $\begin{array}{l}\text { NSD relapse, time to relapse or remission at } 1 \text { year } \\
\text { NSD Symptoms or global functioning ( } 1 \text { and } 3 \text { years) } \\
\text { NSD in discontinuation for any reason ( } 1 \text { and } 3 \text { years). } \\
\text { NSD \% reporting weight gain, change in EPS or new } \\
\text { parkinsonism ( } 3 \text { years) } \\
\text { NSD \% akathisia, but increase in severity greater with } \\
\text { risperidone vs. olanzapine ( } p=0.042) \text {. } \\
\text { Daytime drowsiness greater with olanzapine } \\
\text { Sexual dysfunction in men and amenorrhea in women greater } \\
\text { with risperidone. }\end{array}$ \\
\hline $\begin{array}{l}\text { Crespo-Facorro, } \\
2006^{159}\end{array}$ & $\begin{array}{l}\mathrm{N}=182 \\
6 \text { weeks } \\
\text { Open }\end{array}$ & $\begin{array}{l}\text { Olanzapine vs. } \\
\text { Risperidone }\end{array}$ & NSD symptoms \\
\hline Robinson, $2006^{173}$ & $\begin{array}{l}\mathrm{N}=112 \\
16 \text { weeks } \\
\text { Open }\end{array}$ & $\begin{array}{l}\text { Olanzapine vs. } \\
\text { Risperidone }\end{array}$ & NSD response, negative symptoms \\
\hline $\begin{array}{l}\text { McEvoy, } 2007^{169} \\
\text { (CAFÉ study) }\end{array}$ & $\begin{array}{l}\mathrm{N}=400 \\
1 \text { year } \\
\mathrm{DB}\end{array}$ & $\begin{array}{l}\text { Olanzapine vs. } \\
\text { Quetiapine vs. } \\
\text { Risperidone }\end{array}$ & $\begin{array}{l}\text { NSD in all-cause treatment discontinuations and core illness } \\
\text { symptoms }\end{array}$ \\
\hline Li, $2012^{167}$ & $\begin{array}{l}\mathrm{N}=80 \\
6 \text { weeks } \\
\text { Open }\end{array}$ & $\begin{array}{l}\text { Olanzapine vs. } \\
\text { Ziprasidone }\end{array}$ & NSD symptoms \\
\hline
\end{tabular}




\begin{tabular}{|c|c|c|c|}
\hline Study & $\begin{array}{l}\mathbf{N} \\
\text { Duration } \\
\text { Blinding }\end{array}$ & Comparison & Results \\
\hline $\begin{array}{l}\text { Kahn, } 2008^{231,259} \\
\text { (EUFEST study) }\end{array}$ & $\begin{array}{l}\mathrm{N}=498 \\
12 \text { months } \\
\text { Open }\end{array}$ & $\begin{array}{l}\text { Olanzapine vs. } \\
\text { Quetiapine vs. } \\
\text { Ziprasidone }\end{array}$ & $\begin{array}{l}\text { NSD response or remission. } \\
\text { NSD all-cause treatment discontinuations for ziprasidone vs. } \\
\text { quetiapine and olanzapine } \\
\text { Quetiapine results in significantly higher risk of discontinuation } \\
\text { than olanzapine. (OR } 2.41,95 \% \mathrm{Cl} 1.31 \text { to } 4.45 \text { ) }\end{array}$ \\
\hline San, $2012^{68}$ & $\begin{array}{l}\mathrm{N}=114 \\
12 \text { months } \\
\text { Open }\end{array}$ & $\begin{array}{l}\text { Olanzapine vs. } \\
\text { Quetiapine vs. } \\
\text { Risperidone vs. } \\
\text { Ziprasidone }\end{array}$ & $\begin{array}{l}\text { All-cause treatment discontinuation lower with olanzapine } \\
(40 \%) \text { than quetiapine }(56.5 \%) \text {, risperidone }(64 \%) \text {, or } \\
\text { ziprasidone ( } 80 \%) \text {. } \\
\text { Mean time to discontinuation significantly longer with } \\
\text { olanzapine ( } 260 \text { days) than the other drugs (range } 142 \text { days } \\
\text { ziprasidone to } 206 \text { days risperidone; } p=0.005) \text {. }\end{array}$ \\
\hline Liu, $2014^{52}$ & $\begin{array}{l}\mathrm{N}=80 \\
12 \text { months } \\
\text { Open } \\
\text { Women }\end{array}$ & $\begin{array}{l}\text { Quetiapine vs. } \\
\text { Risperidone }\end{array}$ & $\begin{array}{l}\text { NSD Core illness symptoms at } 9 \text { and } 12 \text { months (lower with } \\
\text { risperidone at } 3 \text { and } 6 \text { months). }\end{array}$ \\
\hline Gafoor, $2010^{260}$ & $\begin{array}{l}\mathrm{N}=72 \\
12 \text { weeks } \\
\mathrm{SB}\end{array}$ & $\begin{array}{l}\text { Quetiapine vs. } \\
\text { Risperidone }\end{array}$ & $\begin{array}{l}\text { NSD all-cause treatment discontinuation, time to } \\
\text { discontinuation, symptoms }\end{array}$ \\
\hline Robinson, $2015^{59}$ & $\begin{array}{l}\mathrm{N}=209 \\
12 \text { weeks } \\
\mathrm{DB}\end{array}$ & $\begin{array}{l}\text { Aripiprazole vs. } \\
\text { Risperidone }\end{array}$ & NSD in response, core illness symptoms \\
\hline $\begin{array}{l}\text { Crespo-Facorro, } \\
2013^{261}\end{array}$ & $\begin{array}{l}\mathrm{N}=249 \\
12 \text { months } \\
\text { Open }\end{array}$ & $\begin{array}{l}\text { Aripiprazole vs. } \\
\text { Ziprasidone vs. } \\
\text { Quetiapine }\end{array}$ & $\begin{array}{l}\text { NSD relapse or remission, core illness symptoms, adverse } \\
\text { events, EPS, akathisia } \\
\text { Treatment discontinuation: } \\
\text { Quetiapine } 82.3 \% \text {, ziprasidone } 66.1 \% \text {, aripiprazole } 43.6 \% \\
(p<0.001) \\
\text { Time to D/C (mean days): } \\
\text { Quetiapine } 77.24 \text {, ziprasidone } 129.88 \text {, aripiprazole } 106.71 \\
(p<0.001) \\
\text { D/C due to adverse events: } \\
\text { Quetiapine } 11.3 \% \text {, ziprasidone } 29 \% \text {, aripiprazole } 10.3 \% \\
(p=0.005) \\
\geq 7 \% \text { weight gain: } \\
\text { Aripiprazole vs. ziprasidone: } 45.6 \% \text { vs. } 23.5 \%(p=0.02)\end{array}$ \\
\hline Zhang, $2012^{242}$ & $\begin{array}{l}\mathrm{N}=254 \\
52 \text { weeks } \\
\text { Open }\end{array}$ & $\begin{array}{l}\text { Paliperidone } \\
\text { vs. } \\
\text { Ziprasidone vs. } \\
\text { Aripiprazole }\end{array}$ & $\begin{array}{l}\text { Core illness symptoms significantly lower with paliperidone } \\
\text { than ziprasidone or aripiprazole at } 13,26 \text { and } 52 \text { weeks } \\
\text { NSD response rate }\end{array}$ \\
\hline
\end{tabular}

CGI = Clinical Global Impressions scale; CGI-S = Clinical Global Impressions-Severity scale; $\mathrm{CI}$ = confidence interval; $\mathrm{N}=$ sample size; NSD = no significant difference; OR = odds ratio; PANSS = Positive and Negative Syndrome Scale; SGA = secondgeneration antipsychotic; SS = statistically significant

\section{Treatment-Resistant Patients}

Treatment resistance, defined as at least two adequate trials of an antipsychotic medication with failure to respond, is a challenge in approximately 20 to 30 percent of patients with schizophrenia. ${ }^{262}$ Although clozapine has been considered the treatment of choice, comparisons to other SGAs are needed since clozapine has a serious adverse event profile, the need for regular laboratory monitoring, and not all patients respond.

An analysis of six oral SGAs (clozapine, risperidone, olanzapine, quetiapine, and ziprasidone) in 40 RCTS of patients with treatment-resistant schizophrenia found that the mean change in the PANSS was greater with olanzapine than quetiapine (SMD -0.29, 95\% CI -0.56 to $-0.13){ }^{183}$ The authors note that this corresponds to a difference in points on the PANSS of -6.08 (scale scores range from 30 to 210; 180 possible points). There is some evidence that in patients with more severe disease a minimal clinically important difference on the PANSS is 11.5 points, 
indicating that a difference of six points may not be clinically important, although statistically significant. ${ }^{195}$ The newer oral drugs (aripiprazole, iloperidone, lurasidone, asenapine, cariprazine, brexpiprazole) were not included. This was low-strength evidence.

There are three systematic reviews published since 2013 that address the comparative benefits and harms of SGAs for treatment-resistant patients. ${ }^{183,263,264}$ The most recent and comprehensive of these uses network meta-analysis of 40 RCTS to analyze clozapine, risperidone, olanzapine, quetiapine, and ziprasidone (as well as three FGAs and an SGA not available in the United States). ${ }^{183}$ The newer oral drugs (aripiprazole, iloperidone, lurasidone, asenapine, cariprazine, brexpiprazole) and injectable SGAs were not included. The mean change in the PANSS was greater with olanzapine than quetiapine (SMD -0.29, 95\% CI -0.56 to $0.13){ }^{183}$ The authors note that this corresponds to a difference in points on the PANSS of -6.08 (scale scores range from 30 to 210; 180 possible points). There is some evidence that in patients with more severe disease a minimal clinically important difference on the PANSS is 11.5 points, indicating that a difference of six points may not be clinically important, although statistically significant. ${ }^{195}$ Network analysis of negative symptoms found olanzapine significantly better than the other SGAs (and two FGAs), whereas response rates and all-cause treatment discontinuations indicated no significant differences among the SGAs (although SGAs were better than haloperidol). Analysis of discontinuations due to lack of efficacy showed clozapine to be better than risperidone and quetiapine (and two FGAs). One of the other two reviews compared only olanzapine and clozapine, and had consistent findings (no differences on discontinuation rates or improvement in PANSS total scores; clozapine superior on positive and negative symptoms). ${ }^{263}$ The third review evaluated strategies when clozapine fails, but was not able to come to a conclusion on adjunctive drug therapy including adding a second SGA. ${ }^{264}$

\section{Special Populations}

Age. Two fair-quality studies were specifically designed to compare the effects of olanzapine with risperidone in older patients ( $\geq 60$ years) with schizophrenia or schizoaffective disorder. ${ }^{163,240,265}$ In an 8-week trial, no between-group differences were found in response rates (20\% improvement on PANSS) or change in PANSS, CGI, or Hamilton Depression Scale (HAM-D) scores. In a smaller study ( $\mathrm{N}=66$ ), during the initial 6 months of followup there were no significant differences in efficacy outcomes (BPRS, SANS, Montgomery-Asberg Depression Scale [MADRS]) between the drugs. However, patients taking olanzapine were seen to have better quality of life at 6 months as assessed using the World Health Organization Quality of Life tool ( $\mathrm{p}=0.040$ for overall quality of life, $\mathrm{p}=0.031$ for satisfaction with health), with better physical health and social relationships. Differences were not seen on the psychological or environmental domains. These outcomes are similar to outcomes found in younger populations, reported above.

Post hoc subgroup analyses of the Tran trial, which compared olanzapine with risperidone, reported outcomes for the subgroup of patients 50 to 65 years old. ${ }^{181,247,266}$ Out of a total study population of 339 patients, 39 were between 50 and 65 years old. The split between sexes was not evenly distributed across the two drug groups. The risperidone group was 42 percent male, whereas the olanzapine group was 70 percent male. Another difference at baseline was the duration of the current episode, a mean of 61 days in the olanzapine group and 120 days in the risperidone group (although not significant). The mean modal dose in the olanzapine group was $18 \mathrm{mg}$ (within midrange) and in the risperidone group $8 \mathrm{mg}$ (above midrange). In general, because the size of the subgroup was small and the age range covered only up to 65 years, the implications of the findings of this subanalysis for older patients with schizophrenia were 
difficult to interpret. However, the analysis did indicate that results were probably not different in this older population.

A retrospective study from the US Department of Veterans Affairs database, conducted to evaluate the risk of new onset diabetes among new users of SGAs, found a differential effect with analysis by age. ${ }^{246}$ Higher risk was found with olanzapine $(\mathrm{p}=0.05)$ and risperidone $(p=0.03$ ) for patients less than 45 years old, whereas the risk with quetiapine in this group was not significant.

Race. A retrospective study of Texas Medicaid claims data analyzing the mean number of days patients continued to take their prescribed SGA drug found that patients who were Mexican American or African American had significantly fewer days on drug than white patients, although the difference in days was small (18 and 19, respectively). ${ }^{250}$ The analysis did not indicate a difference among these groups when stratified by which SGA they were taking (olanzapine or risperidone).

Subgroup analyses of a 26-week trial of aripiprazole and olanzapine ( $N=314)$ evaluated the risk of metabolic syndrome in white patients and black or Hispanic patients. In comparing the drugs, the results across the subgroups were similar to the overall findings (that aripiprazole resulted in lower risk), although the point estimate was lower for white patients than for black and Hispanic patients and the comparison for the smaller black/Hispanic group did not reach significance. ${ }^{267}$ The ORs were 0.33 (95\% CI 0.19 to 0.55 ) for all patients, 0.20 (95\% CI 0.10 to 0.41 ) for white patients, and 0.53 (95\% CI 0.25 to 1.12) for black and Hispanic patients. Analyses of effects of ethnicity within each drug group found that white patients had lower risk than black and Hispanic patients taking aripiprazole, but that there was no difference between these groups among patients taking olanzapine.

Aripiprazole's effect in Japanese patients, compared with other drugs, was evaluated in metaanalyses using both published and unpublished information in a good-quality systematic review. ${ }^{268}$ Although the overall analysis combined results from multiple different comparator drugs in a simple way (i.e., not an indirect comparison or network meta-analysis), the publication also reported pair-wise comparisons for aripiprazole compared with risperidone, olanzapine and quetiapine, based on a single study that included all four drugs. ${ }^{230}$ This study found no differences between the drugs on the PANSS total scores or subscale scores, but did find aripiprazole to result in a higher risk of discontinuation due to lack of efficacy compared with olanzapine (OR 6.25, 95\% CI 1.14 to 34.12) and risperidone (OR 4.52, 95\% CI 1.30 to 15.73), but no difference compared with quetiapine (OR $0.68,95 \%$ CI 0.20 to 2.30). The confidence intervals are wide, as these results are based on a single, small $(\mathrm{N}=80)$, 8-week study and the results should be interpreted with caution. Future studies could overturn these findings. These results are consistent with the findings of all trials comparing these drugs. Another trial, reported above in Key Questions 1 and 2, included 455 Japanese, Taiwanese, Malaysian and Filipino patients randomized to oral or aripiprazole LAI, finding the injectable drug to be noninferior to the oral drug in "non-exacerbation of psychotic symptoms/non-relapse" as the primary outcome measure. ${ }^{49}$ There were no significant differences on secondary outcomes as well, including extrapyramidal adverse events.

Two trials compared aripiprazole and risperidone in Asian patients; one in Taiwan ${ }^{155}$ and one in mainland China. ${ }^{51,193}$ Both studies found no significant differences in efficacy outcomes at 4 and 6 weeks, consistent with the findings of the overall analysis in Key Question 1 above. These studies come to different conclusions on extrapyramidal symptoms (EPS)-related adverse events, 
with the small 4-week study conducted in Taiwan $(\mathrm{N}=83)$ reporting more EPS adverse events, particularly akathisia with aripiprazole, ${ }^{155}$ and the larger 6-week study conducted in China $(\mathrm{N}=279)$ reporting no differences between the drugs on EPS outcomes. A third study, conducted in North America in patients with first-episode schizophrenia, found aripiprazole to be significantly associated with higher akathisia scores on the Barnes Akathisia Scale in the early months of the trial, but not at 12 months. ${ }^{59}$ Other measures, Parkinsonism and EPS severity, were not found different when akathisia was not considered part of EPS. Based on these studies, it is not clear that there is a difference in effects, benefits or harms, of aripiprazole and risperidone in Asian patients.

A fair-quality systematic review evaluated paliperidone and paliperidone palmitate monthly LAI in Chinese patients with schizophrenia. ${ }^{269}$ The review included 53 studies of the oral paliperidone and 9 of the injection that were conducted in China, including pharmacokinetic studies, single-arm studies, and studies with olanzapine, quetiapine, risperidone or aripiprazole. The review concludes that few differences were found between the drugs and that the findings are consistent with study results in non-Asian patients.

Sex. Analysis of differences in effect by sex in the European SOHO study found that compared with women, men had lower odds of response (based on the CGI scale; OR 0.56, 95\% CI 0.34 to 0.93) with clozapine and smaller improvement in quality of life (based on EQ-5D visual analog score; OR -1.52, 95\% CI -2.53 to -0.50). ${ }^{248}$ Risperidone did not result in any differences between men and women.

\section{Substance Use}

In a post-hoc analysis of the CATIE Phase 1 trial data, outcomes were compared between users and nonusers of illicit substances. ${ }^{252}$ Based on the primary outcome measure of overall discontinuation (rate and time to), the results were consistent with the overall trial results for those who were nonusers (olanzapine superior to quetiapine and risperidone, ziprasidone not significantly different). However, significant differences were not found for any of the comparisons among users of illicit drugs. Further analyses compared olanzapine to the combined group of antipsychotic drugs in the trial and were not useful for the purposes of this report.

A subgroup analysis from a fair-quality trial of 49 patients with first-episode schizophrenia and a lifetime history of cannabis use disorders found no significant difference between olanzapine and risperidone in rate of response at 16 weeks, defined as (1) mild or better on all the Schedule for Affective Disorders and Schizophrenia - Change Version with Psychosis and Disorganization (SADS-C + PD) items severity of delusions, severity of hallucinations, impaired understandability, derailment, illogical thinking, and bizarre behavior; and (2) a concurrent rating of very much improved or much improved on the CGI ( $45 \%$ vs. $54 \%$; $p=0.68) .{ }^{270}$ These results were consistent with results for the trial population as a whole $(\mathrm{N}=112){ }^{173}$

Three additional studies addressed substance misuse subgroups, but we rated them poorquality and they did not contribute to our overall conclusions. ${ }^{251,253}$ A small study of 29 patients with comorbid schizophrenia and cocaine or marijuana abuse or dependence that compared olanzapine with risperidone was rated poor-quality based on unclear randomization and allocation concealment procedures with resulting imbalances in baseline characteristics among the groups, unclear analyses, and differential discontinuation. ${ }^{253}$ A small cohort study $(\mathrm{N}=67)$ of patients with comorbid alcohol use disorder that compared rehospitalization rates with risperidone or clozapine was rated poor-quality due to unclear methods of patient selection. Nine percent of patients were removed from analysis because they discontinued drug due to adverse 
events and potentially important differences at baseline were not controlled for in analyses. ${ }^{251}$ We also gave a poor-quality rating to a randomized trial of 139 patients with schizophrenia and nicotine dependence because of unclear methods of randomization, allocation concealment, and blinding and unclear reporting about attrition and completeness of the analysis dataset. ${ }^{271}$

\section{Obesity}

An exploratory analysis of treatment effect across baseline body mass index categories (normal: $<25 \mathrm{~kg} / \mathrm{m}^{2}$; overweight: $\geq 25$ to $<30 \mathrm{~kg} / \mathrm{m}^{2}$; obese: $\geq 30 \mathrm{~kg} / \mathrm{m}^{2}$ ) from a 53-week, fair-quality RCT of 749 patients found that the difference in mean change in PANSS total score indicated noninferiority for paliperidone palmitate injection $63.5 \mathrm{mg}$ (mean dose) compared with risperidone long-acting injectable $32.4 \mathrm{mg}$ (mean dose) for the normal and overweight subgroup (difference in least-squared means $-0.5,95 \%$ CI -4.01 to +3.08 ), but not for the obese subgroup $(-7.5,95 \%$ CI -12.1 to -2.82$){ }^{188}$ The findings of this study may be affected by the rate of dose initiation and location of injections used for paliperidone palmitate injection, which was lower than currently recommended.

\section{First-Generation Antipsychotic Versus Second-Generation Antipsychotic}

\section{Key Points}

- In patients with a first episode of schizophrenia, the evidence comparing FGAs to SGAs is less robust than SGAs versus SGAs, but did not find significant differences between the drugs.

- In treatment-resistant patients, response was significantly better with ziprasidone than haloperidol ( $\mathrm{N}=120$; RR 1.54, 95\% CI 1.19 to 2.00), whereas there was no significant effect in nontreatment-resistant patients. Negative symptoms were significantly reduced with olanzapine versus haloperidol ( $\mathrm{N}=2,207$; MD 1.28, 95\% CI 0.11 to 2.44, whereas in mixed populations the difference was not significant.

- There was not a clear impact of dose on response or core illness symptom improvement based on subgroup analyses of aripiprazole, olanzapine, quetiapine, and risperidone versus haloperidol.

- Asian patients: Although response rates were similar between Asian and non-Asian subgroups, core illness symptoms were significantly improved in Asian patients with olanzapine versus haloperidol (one RCT; MD 4.40; 95\% CI 0.33 to 8.47), and not in other races.

- In patients with co-occurring SUD, core illness symptoms improved significantly more with olanzapine than haloperidol, whereas the subgroup of studies excluding these patients showed no difference. With risperidone, the results were opposite: there was no significant difference in studies that included patients with SUD, whereas risperidone was better than haloperidol in studies that excluded such patients. 


\section{Detailed Synthesis}

\section{Description of Included Studies}

The 2012 systematic review reported a number of subgroup analyses according to demographic and clinical patient characteristics. Analyses for which there was a difference between patient groups are reported below. ${ }^{28}$

\section{Findings}

\section{Clinical Subgroups}

\section{First-Episode Schizophrenia}

There was no difference between groups in total PANSS score in studies of haloperidol versus risperidone in patients with first-episode psychosis (one RCT, N=183; MD 1.60, 95\% CI 5.61 to 8.81) and those with multiple psychotic episodes (seven RCTs, N=1,984; MD -0.56, 95\% CI -3.98 to 2.86 ; $\left.\mathrm{I}^{2}=65 \%\right)$. ${ }^{28}$ For other comparisons, outcomes were significantly better for the SGA only for the subgroup that included multiple episodes (Appendix Table E-1).

\section{Treatment-Resistant Schizophrenia}

Analysis of studies of haloperidol versus olanzapine stratifying results according to treatment resistance found mixed and unclear effects on total symptom scores (Appendix Table E-1). ${ }^{28}$ Using BPRS as a measure, a mixed population treatment and nontreatment-resistant patients fared better with olanzapine than haloperidol (nine RCTs, N=1,809; MD 1.10, 95 \% CI 0.62 to $1.58 ; \mathrm{I}^{2}=0 \%$ ), whereas in the treatment-resistant only populations there was no difference between the drugs, (four RCTs, N=2,205; MD -5.50, 95\% CI -14.1 to 3.07) although heterogeneity was so high as to render this estimate unreliable $\left(\mathrm{I}^{2}=95 \%\right)$. Using CGI-S as a measure, in treatment-resistant patients olanzapine resulted in a significant benefit (two RCTs, $\mathrm{N}=2,059$; MD 0.24 , $95 \%$ CI 0.01 to $\left.0.47 ; \mathrm{I}^{2}=32 \%\right)$, although the difference is so small ( 0.24 on a 0 to 7 scale) that clinical relevance is unlikely. The analysis of mixed populations showed no significant benefit (five RCTs, $\mathrm{N}=1,297$; MD $0.07,95 \%$ CI -0.10 to 0.24 ; $\mathrm{I}^{2}=71 \%$ ).

Negative symptoms were reduced with olanzapine use in treatment-resistant patients based on five trials ( $\mathrm{N}=2,207$; MD 1.28, $95 \%$ CI 0.11 to $2.44 ; \mathrm{I}^{2}=40 \%$ ) with no significant effect in patients with no treatment resistance based on one small trial $(\mathrm{N}=44$; MD 1.02, 95\% CI -2.39 to 4.43; Appendix Table E-1).

Treatment-resistant patients taking ziprasidone were twice as likely to respond to treatment versus haloperidol based on one trial ( $\mathrm{N}=120$; RR 1.54, 95\% CI 1.19 to 2.00) whereas no such effect was found in three trials of patients without treatment resistance $(\mathrm{N}=1,053$; RR 0.79, 95\% CI 0.56 to 1.13; Appendix Table E-1).

\section{Impact of Dose}

Analyses according to FGA dose were limited to studies comparing haloperidol with aripiprazole, olanzapine, quetiapine and ziprasidone with response being the only outcome available for all comparisons (Appendix Table E-1). ${ }^{28}$ The subgroup analyses divided the haloperidol dosing into $<20 \mathrm{mg} /$ day and $>20 \mathrm{mg} /$ day. The findings are not consistent across the SGAs, and not consistent with the theory that lower doses of FGAs would have better results. The findings of the overall and lower-haloperidol dose subgroup analyses were consistent; olanzapine was significantly better. The higher-haloperidol dose subgroup analysis 
was not significantly different, but there were only two studies, limiting the statistical power to find a difference, and the relative risk was similar to the other analyses. For aripiprazole and quetiapine, again the overall and lower-haloperidol dose analyses were similar (no difference in these cases), but the higher-haloperidol dose subgroup analyses showed the FGA to be better. With ziprasidone, finally, the lower-dose haloperidol subgroup analysis showed ziprasidone superior, whereas the other analyses found no difference. This evidence is difficult to interpret in due to high heterogeneity and the small numbers of studies using higher doses of haloperidol (1-2 studies of the higher doses for each comparison).

\section{Special Populations}

\section{Race}

When comparing haloperidol and olanzapine, total BPRS scores were significantly better in the olanzapine group when stratified according to Asian race (one RCT; MD 4.40; 95\% CI 0.33 to 8.47) but not significant in a subgroup of studies in other races (12 RCTs; MD 0.28; 95\% CI 1.48 to 2.04). For the same comparison, response rates were similar for Asian (one RCT; RR 0.87; 95\% CI 0.76 to 1.00) versus other races (13 RCTs; RR 0.86; 95\% CI 0.76 to 0.97) favoring olanzapine, although the estimate was significant for other races but not Asians (Appendix Table E-1). ${ }^{28}$

\section{Substance Use}

Evidence on the comparative effectiveness of haloperidol versus SGAs on core illness total symptom scores in patients with co-occurring SUD were mixed (Appendix Table E-1). ${ }^{28}$ Although the systematic review did not have data to evaluate only a subgroup of patients with SUD, in studies of mixed populations olanzapine had significantly better total PANSS (12 RCTs, $\mathrm{N}=3,726$; MD 2.71, 95\% CI 0.75 to 4.67; I²=40\%), BRPS (seven RCTs, N=2,890; MD 2.05; 95\% CI 0.55 to 3.55; $\mathrm{I}^{2}=90 \%$ ) and CGI-S (three RCTs, N=2,467; MD 0.28, 95\% CI 0.19 to $0.38 ; \mathrm{I}^{2}=0 \%$ ) scores than haloperidol. In studies that excluded people with SUD the difference was not significant, possibly due to smaller sample sizes (PANSS: three RCTs, N=483; MD 0.73, 95\% CI -5.83 to 4.38; $\mathrm{I}^{2}=0$ \%; BPRS: six RCTs, N=1,124; MD -2.37, 95\% CI -6.19 to 1.44; $\mathrm{I}^{2}=36 \%$; and CGI-S: five RCTs, N=1,097; MD 0.30, 95\% CI -0.13 to 0.20). Conversely, risperidone use resulted in better symptom scores in the studies that excluded those with SUD based on total PANSS (14 RCTs, N=3,188; MD 2.56, 95\% CI 0.65 to 4.47; $\mathrm{I}^{2}=18 \%$ ) and BPRS (seven RCTs, N=1,875; MD $0.84,95 \%$ CI 0.36 to 1.32 ; $\mathrm{I}^{2}=0 \%$ ), whereas there was no significant effect in studies of mixed populations (six RCTs, N=833; MD 1.95, 95\% CI -3.14 to 7.04; $\mathrm{I}^{2}=82 \%$; and six RCTs, $\mathrm{N}=717$; MD 0.23 , 95\% CI -1.44 to $1.90 ; \mathrm{I}^{2}=29 \%$ ).

Haloperidol use resulted in significantly better negative symptom scores in patients with cooccurring SUD based on one small ( $\mathrm{N}=31) \mathrm{RCT}$ versus olanzapine (MD -3.20, 95\% CI -6.03 to 0.37), whereas in nine trials $(\mathrm{N}=3,184)$ enrolling a mixed population, olanzapine use resulted in better negative symptom scores compared with haloperidol (MD 1.27, 95\% CI 0.82 to 1.72; $\left.\mathrm{I}^{2}=2 \%\right)$. 


\title{
Key Question 2a. Benefits and Harms of Psychosocial and Other Nonpharmacological Treatments for Adults With Schizophrenia Compared With Usual Care
}

\author{
Assertive Community Treatment Versus Usual Care
}

\section{Key Points}

- Evidence on assertive community treatment comes from one good-quality systematic review (14 RCTs; $\mathrm{N}=2,281)$ and one other RCT $(\mathrm{N}=118)$ not included in the review. The primary outcome target of this intervention is reduction in rehospitalization (not a prioritized outcome for this review).

- Assertive community treatment did not improve social function more than usual care, based on pooled analysis of three studies (MD 0.03; 95\% CI -0.28 to 0.34); an additional trial also found no difference (SOE: low).

- Compared with usual care, there were no significant differences in arrests (two trials, total $\mathrm{N}=604$; OR 1.17, 95\% CI 0.60 to 2.29), imprisonment (four trials, total $\mathrm{N}=471$; OR $1.19,95 \%$ CI 0.70 to 2.01 ), or police contacts (two trials, total $\mathrm{N}=149$; OR $0.76,95 \% \mathrm{CI}$ 0.32 to 1.79 ) with assertive community treatment (SOE: low).

- Assertive community treatment resulted in a lower likelihood of not living independently (four trials; OR 0.52, 95\% CI 0.35 to 0.79 ), being homeless (four trials, OR 0.20, 95\% CI 0.09 to 0.47 ) and being unemployed (three trials; OR 0.46, 95\% CI 0.21 to 0.99 ) compared with usual care (SOE: moderate).

- Core illness symptoms improved to a similar degree in both assertive community treatment and usual care groups (three trials, MD -0.14; 95\% CI -0.36 to 0.08). (SOE: moderate).

- Assertive community treatment reduced the likelihood of patients being admitted to a hospital, compared with usual care ( $\mathrm{N}=$ six RCTs; OR 0.59, 95\% CI 0.41 to 0.85, $\left.\mathrm{I}^{2}=73 \%\right)$.

\section{Detailed Synthesis}

\section{Description of Included Studies}

A good-quality systematic review of 14 RCTs $(\mathrm{N}=2,281)$ assessed assertive community treatment compared with usual care (Appendix Tables E-3 and F-3). ${ }^{71}$ The review defines assertive community treatment as a multi-disciplinary team-based approach to caring for patients with severe mental illness who are described as reluctant and uncooperative. The care is provided through "assertive outreach," offering services at home or work, with team members sharing responsibility for patients. Key goals of assertive community treatment are to maintain the patient's contact with services, reduce the incidence and duration of hospitalizations, and improve social functioning and quality of life. ${ }^{71} \mathrm{~A}$ more recent review that combined evidence for assertive community treatment and intensive case management was not used, as we considered these to be distinct interventions. ${ }^{272}$ However, all of the trials of assertive community treatment included in the newer review are included here. The earlier systematic review included 
trials of interventions explicitly described as assertive community treatment, assertive case management, or PACT (Program of Assertive Community Treatment), or as being based on acknowledged models of assertive community treatment. Participants in the included trials had been diagnosed with schizophrenia or schizophrenia-like disorders, bipolar disorder, or depression with psychotic features. Most studies used eligibility criteria aimed at enrolling patients with frequent hospitalizations over the past 2 to 5 years, or recently hospitalized patients, but not all. Mean age ranged from 29 to 48 years. Among trials reporting other patient demographics, the proportion of female participants ranged from 0 to 56 percent and blacks were 18 to 72 percent of included populations. Quality of included studies ranged from fair to good; poor-quality studies were excluded, and the review notes that one of the flaws in the evidence based is the lack of measurements of program fidelity. Study duration ranged from 6 months to 2 years.

We identified one additional fair-quality RCT $(\mathrm{N}=118)$ of assertive community treatment, conducted in the Netherlands and published since the systematic review described above that enrolled participants with severe mental illness, including schizophrenia-spectrum or psychotic disorders (75\%), bipolar disorder (4\%), major depressive disorder (14\%), and other mental illness (7\%). ${ }^{72}$ (Appendix Table E-4). The mean age was 42 years, 31 percent were females; duration of illness was about 8 years; ethnicity was not reported and patients were followed for 15 to 24 months. This study measured fidelity to the assertive community treatment program using the Dartmouth Assertive Community Treatment Scale (DACT), and found that the study treatment achieved scores ranging from 3.8 to 4.1 on a scale of 1 to 5 .

\section{Findings}

\section{Function}

\section{Social Function}

Based on a systematic review of three RCTs and one additional trial not included in the RCT, there was low-strength evidence that assertive community treatment does not improve social function more than usual care after 1 to 2 years. The systematic review of assertive community treatment found no significant effect on social function, based on pooled analysis of three trials $(\mathrm{N}=206)$ using different measures of social function (MD 0.03; 95\% CI -0.28 to $0.34 ; \mathrm{I}^{2}=7 \%$; Appendix Table E-3). ${ }^{71}$ Using the SFS scale, the additional RCT $(\mathrm{N}=118)$ found no impact of assertive community treatment compared with usual care at 12 months followup. ${ }^{72}$ (Appendix Table E-4). The SOE for function was low due to study limitations and imprecision.

\section{Legal System Encounters}

The systematic review reported no significant differences in arrests (two trials, total $\mathrm{N}=604$; OR 1.17, 95\% CI 0.60 to 2.29; $\mathrm{I}^{2}=0 \%$ ), imprisonment (four trials, total N=471; OR 1.19, 95\% CI 0.70 to $2.01 ; \mathrm{I}^{2}=27 \%$ ), or police contacts (two trials, total $\mathrm{N}=149$; OR $0.76,95 \%$ CI 0.32 to 1.79 ; $\mathrm{I}^{2}=84 \%$; Appendix Table E-3). This was low-strength evidence due to study limitations and imprecision.

\section{Living Situation}

The systematic review reported that assertive community treatment patients were less likely to not be living independently (i.e., more likely to be living independently) at the end of the trials compared with those receiving usual care (three trials, total N=362; OR 0.46, 95\% CI 0.29 to 
0.74; $\mathrm{I}^{2}=0 \%$ ) (Appendix Table E-3). The additional trial reported that the change in the number of days in "sheltered homes" was not different between assertive community treatment and usual care. $^{72}$ In our analysis, assertive community treatment resulted in a lower likelihood of not living independently (four trials; OR 0.52, 95\% CI 0.35 to 0.79; Appendix G-9) compared with usual care. This SOE is moderate.

The review also found that patients assigned to assertive community treatment were less likely to be homeless during or at the end of study compared to patients assigned to usual care (three trials, total $\mathrm{N}=374$; OR 0.24 , 95\% CI 0.11 to $0.51 ; \mathrm{I}^{2}=52 \%$ ). The additional study $(\mathrm{N}=118$ ) also found a reduction in homelessness in the assertive community treatment group. Combining this evidence, the pooled odds ratio is 0.20 (95\% CI 0.09 to 0.47; Appendix G-10). This was low-strength evidence due to the small numbers of events, leading to imprecise estimates.

\section{Employment}

The systematic review reported significantly less unemployment in the assertive community treatment group compared to the usual care group based on two trials (total $\mathrm{N}=604$; OR 0.31 , 95\% CI 0.19 to $0.50 ; \mathrm{I}^{2}=34 \%$ ). In our analysis, assertive community treatment resulted in a lower likelihood of being unemployed (three trials; OR 0.46, 95\% CI 0.21 to 0.99; Appendix G-11) compared with usual care. The strength of this evidence is moderate.

\section{Health-Related Quality of Life}

Only one trial included in the systematic review reported quality of life, finding assertive community treatment associated with a significant but very small difference in quality of life score relative to usual care (MD -0.52, 95\% CI -0.99 to -0.05; Appendix Table E-3). The additional trial also reported quality of life, using the MANSA scale, but found no impact with assertive community treatment relative to usual care. This evidence is insufficient due to study limitations, inconsistency between the trials, and imprecision.

\section{Improvement in Core Illness Symptoms}

The systematic review combined a wide range of symptom measures, including the BPRS, Brief Symptom Inventory, and Colorado Symptom Index and reported no significant differences between groups in symptoms based on three trials ( $\mathrm{N}=255$; MD -0.14 ; 95\% CI -0.36 to 0.08 ) (Appendix Table E-3). ${ }^{71}$

The other RCT $(\mathrm{N}=118)$ not included in the systematic review reported total symptom scores using the BPRS symptom scale and found that, whereas symptoms improved in both assertive community treatment and usual care groups over time, there was no difference between interventions at 1 year (Appendix Table E-4). ${ }^{72}$ This was moderate SOE.

\section{Treatment Discontinuation}

The systematic review reported significantly less loss to followup with assertive community treatment compared to usual care based on 10 trials (total N=1,597; OR 0.51, 95\% CI 0.40 to $0.65 ; \mathrm{I}^{2}=0 \%$ ) (Appendix Table E-3). The RCT reported the number of patients who were "out-ofcare" during the last 12 months of the study, and found assertive community treatment to result in significantly lower rates (OR $0.10,95 \%$ CI 0.03 to 0.33 ). ${ }^{72}$ In our analysis, fewer patients receiving assertive community treatment were lost to followup (discontinued treatment) than those in usual care (12 trials; OR 0.51, 95\% CI 0.41 to 0.63; Appendix G-12). This was moderate SOE. 


\section{Other Outcomes}

Rehospitalization. The systematic review found that assertive community treatment reduced the likelihood of patients being admitted to a hospital, compared with usual care (OR 0.59, 95\% CI 0.41 to $\left.0.85, \mathrm{I}^{2}=73 \%\right){ }^{71}$ The statistical heterogeneity was high in this analysis, and examination of the forest plot reveals that inclusion criteria and country of study may have influenced these findings - specifically, those studies enrolling a broader population, not limited to those with a history of frequent hospitalizations, had a smaller and not significant effect. The review also found that the duration of hospitalizations was reduced for those receiving assertive community treatment compared with usual care, in seven of eight trials reporting this outcome but was statistically significant in five. The data were reported in varying ways, such that a mean or range of difference in duration was not clear, and the authors of the review note that not all the data were analyzed correctly.

The additional RCT that was not included in this review did not find that assertive community treatment significantly reduced inpatient days per month or the number of admissions per month. ${ }^{72}$ This difference in findings may be related to the setting, which was the Netherlands, whereas all of the studies finding a significant difference in the review were conducted in the United States. None of the studies of assertive community treatment that were excluded from this review (Appendix C) were excluded solely because they reported hospitalization as an outcome.

\section{Cognitive Adaptation Training Versus Usual Care}

\section{Key Points}

- Evidence from three RCTs of cognitive adaptation training was insufficient due to the limited number of studies and participants.

\section{Detailed Synthesis}

We identified three RCTs (in four publications) comparing cognitive adaptive training with usual care (Appendix Table E-5). ${ }^{73-76}$ Due to the limited number of studies and participants, we were not able to reliably rate the SOE for this intervention. However, among the three trials, all found that cognitive adaptation training led to greater improvements in function (based on various scale measures) relative to usual care, and one trial found cognitive adaptation training reduced risk of relapse.

\section{Cognitive Behavioral Therapy Versus Usual Care}

\section{Key Points}

- Three good-quality systematic reviews (9 to 50 trials each; $\mathrm{N}=895$ to 3,947) and five RCTs ( $N=66$ to 422) provided evidence for cognitive behavioral therapy (CBT). Most trials were designed to assess the effect of CBT on symptoms.

- $\quad$ CBT resulted in consistent improved short-term global function based on GAF and other measures, compared with usual care. Differences were not seen in long-term followup (>1 year; seven RCTs) (SOE: moderate and low, respectively).

- Social and occupational function was better with CBT than usual care in trials of $\leq 6$ months duration (three trials; MD on SOFAS 9.11; 95\% CI 6.31 to 11.91); evidence on longer-term effects was inconsistent (SOE: low). 
- CBT improved quality of life more than usual care in the short term (12 to 24 weeks followup) based on two trials. Differences were not seen with longer followup (18 to 24 months; two trials) (SOE: low).

- CBT had a greater effect than usual care on overall core illness symptoms based on one good-quality systematic review of 34 trials (SMD -0.33, 95\% CI -0.47 to -0.19) (SOE: moderate).

- Based on two systematic reviews, there is no meaningful difference in negative symptom improvement with CBT compared with usual care (SOE: low).

\section{Detailed Synthesis}

\section{Description of Included Studies}

We identified three good-quality systematic reviews ${ }^{77-79}$ comparing CBT with usual care (Appendix Tables E-6 and F-3). The systematic reviews included nine to 50 RCTs each ( $N=895$ to 3,947) and followup among the included studies ranged widely from 8 weeks to 5 years. Studies of both individual and group CBT were included and combined in all three reviews, though one review conducted separate analyses for individual and group CBT. ${ }^{79}$ In all three reviews, the majority of included studies were focused on the use of CBT to control positive symptoms and were no necessarily designed to address other outcomes. Control groups among the trials included in the reviews varied. In addition to usual care, other controls included waitlists and nonactive interventions such as befriending and psychosocial interventions designed to control for the nonspecific effects of psychotherapy. Patient demographics were not well reported in the reviews. Participants in the included studies ranged from 18 to 65 years in one review ${ }^{78}$ and 22 to 40 years in another. Duration of illness also varied, from 6 months to 30 years across all studies. The proportion of female participants also varied widely, from 8 to 67 percent. Two reviews ${ }^{77,78}$ included studies conducted in people with schizophrenia, schizoaffective disorder and/or psychosis, while the third included studies conducted exclusively in people with schizophrenia. ${ }^{79}$ Though two of the reviews were recently published, ${ }^{77,79}$ the third, a Cochrane review, was last updated in $2010 .^{78}$

The three systematic reviews were all rated good quality, however, they have some important limitations regarding applicability to our review. Presumably all three reviews included some inpatient studies, though this was only reported in one of the reviews, which included 11 (of 30) studies that enrolled exclusively inpatients or mixed inpatient and outpatient populations. ${ }^{79}$ The reviews all noted that there was considerable variability among the CBT interventions, and the specific methods and timing of treatment delivery were not always clearly described. Finally, studies included in these reviews utilized a wide range of outcome measures and scales, making meaningful synthesis and judgment regarding clinical applicability challenging.

Literature searches identified five additional RCTs not included in the systematic reviews (Appendix Table E-7) ${ }^{76,80-83}$ Searches identified one trial ${ }^{95}$ of family-based CBT, which is included in the Family Interventions section. The number of patients enrolled in these trials ranged from 66 to 422 . Mean age ranged from 30 to 47 years, and the proportion of female patients ranged from 14 to 54 percent. In four trials reporting race, white patients predominated in two trials, while blacks were the majority in the one and Hispanics were the majority in the other. ${ }^{76,80-82}$ Baseline symptom severity varied among the trials based on study inclusion criteria and baseline measures. Three trials enrolled patients with persistent and ongoing symptoms and/or risk of relapse. ${ }^{76,80,82}$ Baseline symptom scores (total PANSS) were 76 and 82 in two 
trials, ${ }^{80,81}$ approximately corresponding to moderate illness. ${ }^{273}$ At baseline, patients ranged from minimal to serious functional impairment, based on three trials using various measures. ${ }^{76,80,82}$ The mean duration of illness, reported in three trials, ranged from 15 to 20 years in two trials, ${ }^{81,83}$ while a third study reported time elapsed since first contact with services of $\geq 10$ years for about half of the participants. ${ }^{80}$

As with RCTs included in the above systematic reviews, the additional RCTs that met our inclusion criteria varied with regard to modality (group vs. individual), duration (brief vs. longerterm), and treatment target (e.g., role functioning, symptoms, and medication adherence). Studies were inconsistent in reporting therapist qualifications to provide CBT and few addressed fidelity. In four trials, CBT was delivered in individual sessions, ${ }^{76,80,82,83}$ and one trial ${ }^{81}$ used a combination of individual and group CBT as part of a manualized intervention targeting occupational functioning. Both brief and longer-term CBT interventions were included, with duration of CBT ranging from 8 weeks to 9 months. Four trials included post-treatment followup ranging from 4 to 19 months; total followup in these studies ranged from 6 to 24 months. ${ }^{76,80,82}$ One trial was rated good quality ${ }^{80}$ and the remaining four were rated fair quality (Appendix Table F-4). ${ }^{76,81-83}$ Methodological limitations in the fair-quality trials included unclear group allocation concealment and high attrition rates.

As the two more recent systematic reviews ${ }^{77,79}$ only assessed the effect of CBT on total and/or negative symptoms, we reviewed the included studies of both reviews for reporting of other outcomes of interest. Based on this review, we identified seven additional RCTs reporting function, relapse, and/or quality of life. ${ }^{93,274-279}$ We acknowledge that many of these studies were not specifically designed to assess these outcome measures, but we included evidence on these secondary outcomes when reported. Study characteristics, symptom outcomes and risk of bias assessments are presented in the existing good-quality systematic reviews, and we abstracted additional information only on relevant outcomes.

\section{Findings}

\section{Global Function}

\section{Short Term}

The effects of CBT on short-term ( $\leq 6$ months since CBT initiation) function was reported in four RCTs ${ }^{83,274,278,279}$ and one systematic review ${ }^{78}$ that included two RCTs ${ }^{280,281}$ reporting functional outcomes (Table 9). Results from the single study that focused on global function found a higher proportion of CBT patients had normal functioning after 6 months treatment versus usual care patients (28\% vs. 14\%, RR 2.21, 95\% CI 1.25 to 3.93). ${ }^{279}$

In total, six trials reported a common measure of global function (GAF score), that when pooled found delivery of CBT resulted in significantly improved function regardless of CBT focus or treatment modality (MD 5.35, 95\% CI 1.05 to 9.65, $\mathrm{I}^{2}=77 \%$ ) (Appendix G13). ${ }^{83,274,278,280,281}$ The single study of group CBT (focused on positive symptoms) is also the only study that did not find a positive effect of CBT on short-term function. ${ }^{274}$ Removing this study from the pooled analysis resulted in a pooled MD of 6.62 (95\% CI 4.47 to 8.78), and eliminated statistical heterogeneity $\left(\mathrm{I}^{2}=0 \%\right)$. This SOE is moderate.

\section{Medium Term}

The effect of CBT on medium term function ( $>6$ months to 1 year since CBT initiation) was reported in one RCT of group CBT versus usual care focused on positive symptoms, reporting no 
difference between CBT and usual care in Global Assessment Scale (GAS) scores (Table 9). ${ }^{274}$ However, it should be noted that this trial evaluated the effect of a group-delivered CBT primarily intended to target positive symptoms of psychosis with functioning assessed as a secondary outcome. This SOE is insufficient to draw conclusions.

\section{Long Term}

The single trial designed to assess long-term global function reported better adjusted mean GAS scores in the CBT group after 18 months treatment (58.3 vs. 47.9 ; $\mathrm{p}=0.03$; Table 9 ). ${ }^{276}$ Patients enrolled in this study had chronic schizophrenia (mean duration of illness was $>15$ years) and were low-functioning at baseline. Two other trials not specifically focused on function reported no significant difference between CBT and usual care at 6-months and 1-year posttreatment followup. ${ }^{76,279}$ A systematic review pooled three RCTs and found no statistically significant difference in GAF between CBT and usual care (MD 4.20; 95\% CI -0.63 to 9.03) (Appendix Table E-6). ${ }^{78}$

\section{Social and Occupational Function}

\section{Short Term}

The effects of CBT on short-term ( $\leq 6$ months since CBT initiation) social and/or occupational function were reported in two RCTs ${ }^{83,282}$ (Table 9). Improvement in function was the CBT focus in one of the trials, which found significant improvement GAF, SFS and SOFAS. ${ }^{83}$ Significant improvement in SOFAS was also found when results from both trials were pooled (MD 9.11; 95\% CI 6.31 to 11.91; Appendix G-13). One other trial found a significantly higher proportion of study participants in the CBT group had normal function, based on an SFS score within 95 percent of a normal population, when compared with usual care (RR 2.21; 95\% CI 1.25 to 3.93). ${ }^{279}$ This SOE is low.

\section{Medium Term}

A trial of 6 months of vocational-focused CBT (both group and individual sessions) found a significant effect favoring CBT for hours worked and Work Behavior Inventory (WBI) score. ${ }^{81}$ This evidence is insufficient to draw conclusions due to lack of corroborating evidence. There was no clear effect of CBT on medium term social and combined social and/or occupational function (>6 months to 1 year since CBT initiation) based on one additional RCT (Table 9). ${ }^{93}$ Neither trial identified functional improvement as the primary outcome. In one of the trials, SOFAS scores were assessed at the conclusion of 12-20 sessions over 9 months of individual CBT aimed at preventing relapse, finding no difference between CBT and usual care groups. ${ }^{93}$ This SOE is low.

\section{Long Term}

Three trials ${ }^{78,82,279}$ with long-term post-treatment followup (9 to 18 months after treatment cessation) did not find sustained benefits in social and/or occupational function, though none of these studies were designed to assess functional outcomes and one study ${ }^{279}$ used a novel cotherapy approach to CBT (Table 9; Appendix Table E-6). This evidence is low strength. 


\section{Basic Living Skills}

A single trial reported the effect of CBT on measures of basic living skills after 6 months treatment and followup, found improvement in basic living skills, based on the Independent Living Skills Survey (ILSS), (Table 9). ${ }^{275}$ This evidence is insufficient to draw conclusions.

\section{Overall Findings on Functional Outcomes}

Although many trials reported functional outcomes, few were specifically designed to assess measures of function as a primary outcome (Table 9). Short-term, individual CBT (treatment $\leq 6$ months) consistently resulted in greater functional improvements versus usual care, even if function was not the primary focus of CBT. Studies that employed CBT targeted at improving functioning consistently led to functional improvements. Longer-term effects of CBT focused on functioning were less consistent with CBT focused on other outcomes (e.g., positive symptoms) or with lengthy post-treatment followup, suggesting that CBT was more effective for primary treatment targets than other targets of interest.

Evidence of differences between individual and group CBT and functional outcomes was limited. Only one trial utilized a group CBT approach (directed at improving positive symptoms) finding no difference between CBT and usual care in functional outcomes. ${ }^{274}$ One trial that included both individual and group CBT focused on vocational outcomes found significantly better outcomes with $\mathrm{CBT}^{81}$ 
Table 9. Functional outcomes in randomized controlled trials of cognitive behavioral therapy versus usual care

\begin{tabular}{|c|c|c|c|c|c|}
\hline $\begin{array}{l}\text { Timing of } \\
\text { Assessment }\end{array}$ & Outcome & Author, Year & $\begin{array}{l}\text { Study Characteristics } \\
\text { Intervention/Control } \\
\text { CBT Focus/Primary Outcome } \\
\text { Total N } \\
\text { Duration of Treatment } \\
\text { Duration of Followup }\end{array}$ & CBT Versus Usual Care Results & $\begin{array}{l}\text { CBT Versus Usual } \\
\text { Care Effect }\end{array}$ \\
\hline \multirow[t]{4}{*}{$\begin{array}{l}\text { Short Term } \\
\text { ( } \leq 6 \text { months) }\end{array}$} & $\begin{array}{l}\text { Global } \\
\text { Function }\end{array}$ & $\begin{array}{l}\text { van der Gaag } \\
2011^{279}\end{array}$ & $\begin{array}{l}\text { CBT vs. usual care } \\
\text { Global function } \\
\mathrm{N}=216 \\
\text { Treatment: } 6 \text { months } \\
\text { Followup: } 18 \text { months }\end{array}$ & $\begin{array}{l}\text { 6-month outcomes } \\
\text { Participants with normal functioning; } 28 \% \\
\text { (31/109) vs. } 14 \%(14 / 97) ; \mathrm{RR} 2.21 \text { (95\% Cl } \\
1.25 \text { to } 3.93)\end{array}$ & Favors CBT \\
\hline & $\begin{array}{l}\text { Global } \\
\text { Function }\end{array}$ & $\begin{array}{l}\text { Barrowclough } \\
2006^{274}\end{array}$ & $\begin{array}{l}\text { Group CBT vs. usual care } \\
\text { Positive symptoms } \\
\mathrm{N}=113 \\
\text { Treatment: } 6 \text { months } \\
\text { Followup: } 12 \text { months }\end{array}$ & $\begin{array}{l}\text { 6-month outcomes } \\
\text { GAF - Disability mean score }(\mathrm{N}=54 \text { vs. } 45) \text { : } \\
38.11 \text { vs. } 39.98 ; \text { mean difference }-1.87(95 \% \\
\mathrm{Cl}-5.47 \text { to } 1.73)\end{array}$ & No difference \\
\hline & $\begin{array}{l}\text { Global } \\
\text { Function }\end{array}$ & Lincoln $2012^{278}$ & $\begin{array}{l}\text { CBT vs. waitlist } \\
\text { Positive symptoms } \\
\mathrm{N}=80 \\
\text { Treatment: } 4 \text { months } \\
\text { Followup: } 4 \text { months }\end{array}$ & $\begin{array}{l}\text { GAF mean score: } 54.5 \text { vs. } 47.0 ; \text { mean } \\
\text { difference } 7.50 \text { ( } 95 \% \mathrm{Cl} 1.80 \text { to } 13.20)\end{array}$ & Favors CBT \\
\hline & $\begin{array}{l}\text { Global and } \\
\text { Sociall } \\
\text { Occupational } \\
\text { Function }\end{array}$ & Zimmer $2007^{83}$ & $\begin{array}{l}\text { Integrated psychological CBT vs. } \\
\text { usual care } \\
\text { Social function } \\
\mathrm{N}=66 \\
\text { Treatment: } 12 \text { weeks } \\
\text { Followup: } 12 \text { weeks }\end{array}$ & $\begin{array}{l}\text { GAF mean score: } 39.50 \text { vs. } 33.81 \text {; mean } \\
\text { difference } 5.69 \text { ( } 95 \% \text { CI } 2.05 \text { to } 10.97) \\
\text { SOFAS mean score: } 43.25 \text { vs. } 34.14 \text {; mean } \\
\text { difference } 9.11 \text { ( } 95 \% \text { CI } 6.31 \text { to } 11.91) \\
\text { SAS mean score: } 1.86 \text { vs. } 2.27 ; p=0.04\end{array}$ & $\begin{array}{l}\text { All scales favor } \\
\text { CBT }\end{array}$ \\
\hline \multirow[t]{2}{*}{$\begin{array}{l}\text { Medium } \\
\text { Term (6-12 } \\
\text { months) }\end{array}$} & $\begin{array}{l}\text { Global } \\
\text { Function }\end{array}$ & $\begin{array}{l}\text { Barrowclough } \\
2006^{274}\end{array}$ & $\begin{array}{l}\text { Group CBT vs. usual care } \\
\text { Positive symptoms } \\
\mathrm{N}=113 \\
\text { Treatment: } 6 \text { months } \\
\text { Followup: } 12 \text { months } \\
\end{array}$ & $\begin{array}{l}\text { 12-month outcomes } \\
\text { GAF - Disability mean score ( } N=52 \text { vs. } 46) \text { : } \\
39.04 \text { vs. } 40.74 ; \text { mean difference }-1.70(95 \% \\
\mathrm{Cl}-6.00 \text { to } 2.60)\end{array}$ & No difference \\
\hline & $\begin{array}{l}\text { Sociall } \\
\text { Occupational } \\
\text { Function }\end{array}$ & Garety $2008^{93}$ & $\begin{array}{l}\text { CBT vs. usual care } \\
\text { Relapse } \\
\mathrm{N}=273 \\
\text { Treatment: } 12 \text { months } \\
\text { Followup: } 24 \text { months } \\
\end{array}$ & $\begin{array}{l}\text { 12-month outcomes } \\
\text { SOFAS, treatment effect: } 2.77(95 \% \mathrm{Cl}-1.02 \\
\text { to } 6.55)\end{array}$ & No difference \\
\hline
\end{tabular}




\begin{tabular}{|c|c|c|c|c|c|}
\hline \multirow[t]{3}{*}{$\begin{array}{l}\text { Timing of } \\
\text { Assessment }\end{array}$} & Outcome & Author, Year & $\begin{array}{l}\text { Study Characteristics } \\
\text { Intervention/Control } \\
\text { CBT Focus/Primary Outcome } \\
\text { Total N } \\
\text { Duration of Treatment } \\
\text { Duration of Followup }\end{array}$ & CBT Versus Usual Care Results & $\begin{array}{l}\text { CBT Versus Usual } \\
\text { Care Effect }\end{array}$ \\
\hline & $\begin{array}{l}\text { Occupational } \\
\text { function }\end{array}$ & Lysaker $2009^{81}$ & $\begin{array}{l}\text { Indianapolis Vocational Intervention } \\
\text { Program (group and individual } \\
\text { sessions) vs. social support } \\
\text { Employment } \\
\mathrm{N}=100 \\
\text { Treatment: } 26 \text { weeks } \\
\text { Followup: } 26 \text { weeks }\end{array}$ & $\begin{array}{l}\text { Mean total hours worked: } 360.86 \text { vs. } 228.82 ; \\
p<0.01 \\
\text { Mean number of weeks at least } 1 \text { hour } \\
\text { worked: } 18.64 \text { vs. } 14.46 ; p<0.05 \\
\text { Mean WBI: } 113.34 \text { vs. } 105.43 ; p<0.05\end{array}$ & Favors CBT \\
\hline & $\begin{array}{l}\text { Basic Living } \\
\text { Skills }\end{array}$ & $\begin{array}{l}\text { Granholm } \\
2005^{275}\end{array}$ & $\begin{array}{l}\text { CBT }+ \text { social skills training vs. usual } \\
\text { care } \\
\text { Social function } \\
\text { N=76 } \\
\text { Treatment: } 6 \text { months } \\
\text { Followup: } 6 \text { months }\end{array}$ & $\begin{array}{l}\text { ILSS marginal mean score: } 0.72 \text { vs. } 0.67 \\
p<0.05\end{array}$ & ILSS: favors CBT \\
\hline \multirow[t]{4}{*}{$\begin{array}{l}\text { Long Term } \\
\text { (>1 year) }\end{array}$} & $\begin{array}{l}\text { Global } \\
\text { Function }\end{array}$ & Grant $2012^{276}$ & $\begin{array}{l}\text { CBT vs. usual care } \\
\text { Global function } \\
\mathrm{N}=60 \\
\text { Treatment: } 18 \text { months } \\
\text { Followup: } 18 \text { months }\end{array}$ & $\begin{array}{l}\text { GAS adjusted mean score } 58.3 \text { vs. } 47.9 ; \\
p=0.03\end{array}$ & Favors CBT \\
\hline & $\begin{array}{l}\text { Global } \\
\text { Function }\end{array}$ & $\begin{array}{l}\text { van der Gaag } \\
2011^{279}\end{array}$ & $\begin{array}{l}\text { CBT vs. usual care } \\
\text { Global function } \\
\mathrm{N}=216 \\
\text { Treatment: } 6 \text { months } \\
\text { Followup: } 18 \text { months } \\
\end{array}$ & $\begin{array}{l}\text { 18-month outcomes } \\
\text { Participants with normal functioning (based } \\
\text { on SFS score } 95 \% \text { of normal population); } \\
38 \%(39 / 109) \text { vs. } 26 \%(25 / 97) ; \text { RR } 1.39(95 \% \\
\text { Cl } 0.79 \text { to } 1.27)\end{array}$ & No difference \\
\hline & $\begin{array}{l}\text { Global } \\
\text { Function }\end{array}$ & $\begin{array}{l}\text { Velligan } \\
2015 b^{76}\end{array}$ & $\begin{array}{l}\text { CBT vs. usual care } \\
\text { Positive symptoms and global } \\
\text { function } \\
\mathrm{N}=85 \\
\text { Treatment: } 9 \text { months } \\
\text { Followup: } 15 \text { months } \\
\end{array}$ & $\begin{array}{l}\text { Normatively reported a nonsignificant } \\
\text { treatment effect on function with CBT }\end{array}$ & No difference \\
\hline & $\begin{array}{l}\text { Social/Occup } \\
\text { ational } \\
\text { Function }\end{array}$ & Garety $2008^{93}$ & $\begin{array}{l}\text { CBT vs. usual care } \\
\text { Relapse and positive function } \\
\mathrm{N}=273 \\
\text { Treatment: } 12 \text { months } \\
\text { Followup: } 24 \text { months }\end{array}$ & $\begin{array}{l}\text { 24-month outcomes } \\
\text { SOFAS, treatment effect: } 2.42(95 \% \mathrm{Cl}-1.42 \\
\text { to } 6.26)\end{array}$ & No difference \\
\hline
\end{tabular}




\begin{tabular}{|c|c|c|c|c|c|}
\hline $\begin{array}{l}\text { Timing of } \\
\text { Assessment }\end{array}$ & Outcome & Author, Year & $\begin{array}{l}\text { Study Characteristics } \\
\text { Intervention/Control } \\
\text { CBT Focus/Primary Outcome } \\
\text { Total N } \\
\text { Duration of Treatment } \\
\text { Duration of Followup }\end{array}$ & CBT Versus Usual Care Results & $\begin{array}{l}\text { CBT Versus Usual } \\
\text { Care Effect }\end{array}$ \\
\hline & $\begin{array}{l}\text { Occupational } \\
\text { function }\end{array}$ & Malik $2009^{82}$ & $\begin{array}{l}\text { Brief CBT vs. usual care } \\
\text { Relapse } \\
\mathrm{N}=422 \\
\text { Treatment: } 5 \text { months } \\
\text { Followup: } 2 \text { years }\end{array}$ & $\begin{array}{l}\text { Proportion of patients with occupational } \\
\text { recovery: } 10 \%(21 / 205) \text { vs. } 14 \%(17 / 125) \text {; RR } \\
0.75 \text { (95\% Cl } 0.41 \text { to } 1.37)\end{array}$ & No difference \\
\hline
\end{tabular}

CBT = cognitive behavioral therapy; CI = confidence interval; GAF = Global Assessment of Functioning; GAS = Global Assessment Scale; ILSS = Independent Living Skills Survey; MCID = minimally clinical important difference; NS = not significant; RR = relative risk; SFS = Social Functioning Scale; SOFAS = Social and Occupational Functioning Assessment Scale; WBI = Work Behavior Inventory 


\section{Health-Related Quality of Life}

Quality of life is challenging to assess in people with schizophrenia, and the reliability of such assessments is tied to clinical factors, including the patient's level of insight. ${ }^{283}$ Only one small study ( $\mathrm{N}=66$ ) included in this review assessed quality of life as a primary outcome, ${ }^{83}$ while three other trials ( $\mathrm{N}=66$ to 273) reported on the effect of CBT on quality of life as a tertiary outcome (Appendix Table E-7) ${ }^{80,93,279}$ All four trials employed an individual CBT approach. Patient populations were similar across the four trials, but duration of treatment ranged from 8 weeks to 1 year. Quality of life was assessed using different scales, and measured at time points ranging from the cessation of treatment to 1 year after treatment cessation. CBT was associated with better quality of life than usual care in two trials ${ }^{80,83}$ with short-term followup (12 weeks and 24 weeks), including the single trial designed to assess quality of life. ${ }^{83}$ The two trials with quality of life assessments up to 1 year after cessation of treatment (total followup 18 and 24 months), found no difference between CBT and usual care. ${ }^{93,279}$ This evidence is low strength.

\section{Reduction in Self-Harm}

No studies reviewed evaluated the effect of CBT on self-harm as a primary outcome. A single trial, included in one systematic review, reported incidental suicide, finding no difference in risk between CBT and nonactive psychological therapies (3\% [2/78] versus 4\% [3/79]; RR $0.68,95 \%$ CI 0.12 to 3.93). ${ }^{284}$ An additional trial, not included in any of the systematic reviews, found no difference between CBT and usual care in suicide attempts (3\% [2/73] versus $5 \%$ [4/77]; RR 0.53 , 95\% CI 0.12 to 2.79 ) or serious violent incidents (3\% [2/73] versus $1 \%$ [1/77]; RR 2.11, 95\% CI 0.20 to 23). ${ }^{80}$ This evidence is insufficient to draw conclusions.

\section{Improvement in Core IIIness Symptoms}

\section{Overall Symptoms}

A recent systematic review of 34 RCTs found CBT more effective than usual care at improving overall symptoms (SMD -0.33 , 95\% CI -0.47 to $-0.19 ; \mathrm{I}^{2}=68 \%$ [a negative estimate favors CBT]; Appendix Table E-6) based on a number of scales (PANSS, BPRS, Comprehensive Psychopathology Rating Scale [CPRS] and the Hopkins Psychiatric Rating Scale). ${ }^{77}$ As noted above, the majority of the included studies in this review were designed to address positive symptoms. The review combined studies of both individual and group CBT, and brief and longer duration CBT, but did not stratify results according to treatment modality or duration. Limiting the analysis to 20 trials in which outcome assessors were blinded to intervention group greatly reduced the effect size $(-0.15)$. Although it remained significant (95\% CI -0.27 to -0.03 ), it is unclear that this difference is clinically meaningful. One trial not included in the systematic review found similar improvements in total PANSS with CBT (Appendix Table E-7) ${ }^{80}$ This evidence is moderate.

\section{Negative Symptoms}

The effect of CBT on negative symptoms, based on PANSS-negative subscale, BPRSnegative subscale and SANS scales, was mixed based on two reviews. Study inclusion criteria for the two reviews differed slightly in that the Jauhar review included any study of CBT that reported symptom outcomes (positive, negative or overall) regardless of the CBT focus and the Velthorst review only included studies of CBT specifically targeted at either positive or negative symptom reduction. It should be noted that, of the 30 RCTs included in the Velthorst review, 
only two studies identified negative symptoms as the primary treatment target. The Jauhar review found a small, marginally significant effect in favor of CBT (34 trials; SMD -0.13, 95\% CI -0.25 to $-0.01 ; \mathrm{I}^{2}=48 \%$ [a negative estimate favors CBT]), ${ }^{77}$ while the Velthorst review found no difference between CBT and usual care based on 28 trials (SMD 0.09, 95\% CI -0.03 to 0.21; $\mathrm{I}^{2}=63 \%$ [a positive estimate favors CBT]). ${ }^{79}$ Pooled results from the two trials that specifically targeted negative symptoms as a primary outcome resulted in an improved (but not significant) effect size favoring CBT: SMD 0.16, 95\% CI -0.10 to 0.41. Estimates from this review were consistent when trials were limited to those that enrolled outpatients (10 trials; SMD 0.12, 95\% CI -0.08 to 0.31). Estimates were also similar when stratified according to short-term (3-6 months after treatment; 13 trials; SMD 0.21 , 95\% CI -0.05 to 0.46) and long-term (9-12 months after treatment; 10 trials; SMD 0.01, 95\% CI -0.02 to 0.18) treatment effects. The same review found individual (SMD 0.21, 95\% CI 0.05 to 0.37) more effective than group CBT (SMD -0.17, 95\% CI -0.44 to 0.09.) Trials published more recently (since 2009) and higher-quality trials reported smaller effect sizes (Appendix Table E-6).

Because the difference found in the Jauhar review was so small and the Velthorst review found consistent results with multiple sensitivity analyses, we find low strength evidence for negative symptom improvement.

\section{Treatment Discontinuation}

In addition to one outpatient study included in the systematic reviews, ${ }^{285}$ we identified 11 other RCTs not included in the systematic review reporting on the proportion of patients remaining in treatment. ${ }^{76,80,82,83,93,274-279}$ Treatment duration among the trials ranged from 8 weeks to 18 months. When pooled (Appendix G-14), there was no difference between CBT and usual care (86\% vs. 82\%; RR 1.02, 95\% CI 0.95 to 1.10). The risk of discontinuing treatment was not affected by the intended duration of treatment; $\leq 6$ months or $>6$ months did not affect the risk estimates (RR 1.07, 95\% CI 0.97 to 1.17 versus RR $0.98,95 \%$ CI 0.88 to 1.09 ). This was low strength evidence. One other study reported that the mean number of sessions attended was higher for employment-focused CBT compared with general social support (17.34 vs. 12.78 sessions; $\mathrm{p}<0.05)$. $^{81}$

\section{Relapse}

We identified five RCTs (in six publications) reporting relapse rates in patients receiving CBT or usual care in the outpatient setting. ${ }^{82,93,274,277,286,287}$ One of these trials ${ }^{286,287}$ had been included in a good-quality systematic review that pooled data on inpatients with data on outpatients. ${ }^{78}$ In these five trials $(\mathrm{N}=1,013)$ duration of treatment ranged widely, from 10 weeks to 2 years. In all of the trials, relapse prevention was a focus of the CBT intervention. Relapse was variably defined among these trials, though all but one included hospitalization as a criterion for relapse (Appendix Table E-7). When results from these five trials were pooled, there was no difference in relapse rate ( $29 \%$ vs. $30 \%$; RR 0.93 , 95\% CI 0.61 to 1.42 ), though heterogeneity was high ( $\mathrm{I}^{2}=73 \%$; Appendix G-15). When the analysis was limited to three trials ${ }^{82,277,286,287}$ that used only hospitalization as criteria for defining relapse, there was a significant effect in favor of CBT and statistical heterogeneity was eliminated (RR 0.70, 95\% CI 0.54 to $0.91 ; \mathrm{I}^{2}=0 \%$ ).

In subgroup of two trials that used criteria related to increasing symptoms and/or hospitalization as criteria for relapse, neither found a significant difference between CBT and usual care. $^{93,274}$ Due to the inconsistency of these risk estimates, this evidence is insufficient to draw conclusions. As with other bodies of evidence in this report, the reasons likely lie in the variability in definition of relapse. 


\section{Harms}

Harms of treatment were infrequently reported in trials of CBT; only one trial identified harms of treatment a priori as an outcome of interest. ${ }^{80}$ This trial found no difference between CBT and usual care in incidence of mortality (no deaths in either group). This evidence is insufficient to draw conclusions.

\section{Cognitive Remediation/Training Versus Usual Care}

\section{Key Points}

- Two good-quality systematic reviews and one good- and three fair-quality trials ( $\mathrm{N}=56$ to 156) provided evidence for cognitive remediation. The reviews stratified results for studies with usual care comparators.

- Compared with usual care, cognitive remediation resulted in a small positive effect on social, occupational, living situation, and global function, based six RCTs (effect sizes ranged from 0.16 to 0.40 ) (SOE: low).

- Cognitive remediation resulted in small improvements in core illness symptoms, based on two trials (N=153, SMD -0.62 (95\% CI -1.01 to -0.24) (SOE: low).

- Negative symptoms were significantly improved with cognitive remediation compared to usual care based on a good-quality systematic review of 18 RCTs (SOE: moderate).

\section{Detailed Synthesis}

\section{Description of Included Studies}

Studies of cognitive remediation focus on improving cognitive function. Two good-quality systematic reviews of 39 (Wykes 2011, N=2,104) and 18 (Cella 2017, N=781) trials reported on the effect of cognitive remediation versus active or passive (usual care) controls, with subgroup analyses of studies with usual care comparisons (Appendix Tables E-8 and F-3). ${ }^{84,85}$ Studies were included based on cognitive remediation as defined by the Cognitive Remediation Experts Workshop $^{288}$ or based on standard cognitive remediation principles. Inclusion criteria in the Wykes review ${ }^{84}$ required that at least 70 percent of the study population had a diagnosis of schizophrenia, while the Cella review used a 75 percent threshold. ${ }^{85}$

In the Wykes review, 19 studies were conducted in inpatient, 18 in outpatient, and 2 in mixed in and outpatient populations. Mean age across all studies was 36 years (range 18-49; one other study enrolled adolescents); men made up 67 percent of the study populations. Baseline symptom severity, reported in 26 trials, was characterized as mild to moderate. The review included trials that reported cognitive (outside the scope of our review) or functional outcomes, including 31 studies of individual and nine studies of group cognitive remediation. The review's inclusion criteria required a usual care control group, although an active comparator was also included in most studies. Separate analyses according to control group were conducted for measures of function. Cognitive remediation was delivered using drill and practice methods in 21 trials, and drill plus strategy in 19 trials. The mean number of sessions per week was 2.2 (range 0.6 to five), for a mean total of 32 hours (range four to 130) over 17 weeks (range 2 to 104 weeks). Study quality among the included studies ranged from poor to fair. The review noted that inadequate method of randomization and allocation concealment, and lack of treatment fidelity were frequent contributors to diminished study quality. 
The Cella review provided few details about the 18 included studies comparing cognitive remediation with usual care. ${ }^{85}$ The mean age across all studies was 35 years, and men made up 71 percent of the population. Baseline symptom severity, treatment setting (inpatient or outpatient), and description of cognitive remediation delivery were not reported. Seven studies had treatment duration $\geq 12$ weeks. Negative symptoms were primarily assessed using the PANSS negative subscale, although BPRS and SANS were also used. As with the Wykes review, studies with both passive (usual care) and active control groups were included in the review, but sub-group analyses were conducted according to control group. The studies were judged to have medium to high risk of bias.

We identified four other RCTs (in five publications) of cognitive remediation not included in one or both systematic reviews above (Appendix Table E-9) ${ }^{86-90}$ The trials enrolled between 56 and 156 participants, mean age ranged from 31 to 41 years and $<60$ percent of participants were female across all studies. Whites comprised 60 percent of the population in the only study reporting race. ${ }^{89}$ Three of the trials required a diagnosis of schizophrenia for study inclusion, ${ }^{86-}$ ${ }^{88,90}$ and in the fourth trial ${ }^{89}$ the vast majority (97\%) of the study population had a diagnosis of schizophrenia or schizoaffective disorder. Duration of illness ranged from 10 to 18 years. All four trials provided biweekly, group-based cognitive remediation; duration of treatment ranged from 4 to 6 months. The trials used both computer-based and manual cognitive remediation, often in combination. One study was rated good quality, ${ }^{86,90}$ and the other three were rated fair quality due to unclear method of randomization or allocation concealment and lack of intention to treat analysis (Appendix Table F-4).

\section{Findings}

\section{Function}

When limiting analyses to three trials that used a passive control group (usual care), the Wykes systematic review of cognitive remediation found a small positive effect that was not statistically significant (effect size $0.16,95 \%$ CI -0.16 to 0.49 ). This lack of significance may be due to the paucity of evidence rather than a true reflection of the effect of cognitive remediation on function. For context, when the analysis included 19 trials with either usual care or active control groups $(\mathrm{N}=1,036)$ a larger, significant, effect size was found (effect size 0.42 ; $95 \% \mathrm{CI}$ 0.21 to 0.62; Appendix Table E-8), and the effect was sustained in 12 trials that provided posttreatment followup (6 to 24 months; effect size 0.37 ; $95 \%$ CI 0.11 to 0.66 ). ${ }^{84}$ These analyses combined 16 different measures of social, occupational, living situation and global function, and used the effect size measure to standardize across the assessments of function.

Results from three other RCTs, not included in the systematic review, also reported on functional outcomes. ${ }^{87-89}$ The trials used different measures of function at different timepoints, but all found delivery of cognitive remediation improved function relative to usual care, although results were not always significant (Appendix Table E-9). For example, one study found significantly better global function after 15 weeks of cognitive remediation therapy versus usual care based on GAF score (SMD 0.56; $95 \%$ CI 0.34 to 0.88 ), ${ }^{88}$ while the difference between groups was similar in magnitude, though not significant, in another study measuring function and disability with the Life Skills Profile (LSP) after 16 weeks of treatment (SMD 0.41; 95\% CI 0.10 to 0.91$).{ }^{87}$ Combined, this evidence is low strength. 


\section{Health-Related Quality of Life}

One study reported on the effect of cognitive remediation on quality of life (Appendix Table E-9). ${ }^{89}$ There was no difference in Subjective Quality of Life (QOLI) scores after 12 weeks of treatment (effect estimate, adjusted for group and time 0.52; $\mathrm{p}=0.17$ ), but QOLI score was significantly better in the cognitive remediation group at 3-months post-treatment followup (effect estimate, adjusted for group and time 1.15; $\mathrm{p}=0.002$ ). This evidence is insufficient due to study limitations, lack of confirmatory trials, and imprecision.

\section{Improvement in Core Illness Symptoms}

\section{Overall Symptoms}

Results were mixed in two RCTs ( $\mathrm{N}=153)$, not included in either of the systematic reviews, reporting total symptom scores (Appendix Table E-9). In one trial comparing 6 months of cognitive remediation to usual care, improvements in total PANSS were greater in the cognitive remediation group at the end of treatment (SMD -0.90; 95\% CI -1.50 to -0.31) and at 1-year followup (SMD -0.68; 95\% CI -1.26 to -0.09) ${ }^{86}$ The other study did not find the difference to be statistically significant at 16 (SMD -0.42 ; $95 \%$ CI -0.92 to 0.09 ) and 40 weeks (SMD - 0.04 ; 95\% CI -0.54 to 0.46$).{ }^{87}$ Pooling these provides a combined point SMD of -0.62 (95\% CI-1.01 to $0.24)$.

The Wykes systematic review combined a wide range of symptom measures, including the Scale for Assessment of Positive Symptoms (SAPS), SANS, PANSS and BPRS, among others (Appendix Table E-8). The review found that cognitive remediation improved symptoms, based on 20 trials (effect size 0.18 ; 95\% CI 0.03 to 0.32 ) ${ }^{84}$ Symptom improvement was not sustained following treatment removal (8 RCTs, effect size $0.17,95 \%$ CI -0.03 to 0.48 ). The review did not report results for the subgroup of studies utilizing a passive control group, but the effect sizes for the four studies reporting symptom effects versus usual care ranged from 0.05 to 0.45 . Combined, this evidence is low strength.

\section{Negative Symptoms}

The Cella systematic review (18 RCTs; N=781) conducted a network meta-analysis on the effect of cognitive remediation versus usual care on negative symptoms. ${ }^{85}$ The review found cognitive remediation led to significant improvement in negative symptoms, with an effect size of -0.36 ( $95 \%$ CI -0.52 to -0.20 ; a negative effect size favors cognitive remediation). These results were consistent when compared to all control interventions (usual care alone and usual care plus an active treatment, excluding three outliers; effect size -0.30 , 95\% CI -0.36 to -0.22 ) and at post-treatment followup (effect size $-0.36,95 \%$ CI -0.51 to -0.21 ). One other RCT not included in the systematic review and focused on executive function and metacognition, also found negative symptoms, based on PANSS-negative scores, were significantly improved after 4 months treatment (effect size $0.36,95 \%$ CI 0.01 to 0.70 ). ${ }^{87}$ There was no difference between groups at 6 month post-treatment followup.

\section{Treatment Discontinuation}

Treatment discontinuation was not different between cognitive remediation and usual care groups in two RCTs not included in the systematic review (Appendix Table E-9). Relative risk was 1.01 (95\% CI 0.91 to 1.12) in one trial, and the other trial normatively reported no difference between groups in the proportion of patients able to maintain treatment $(\mathrm{p}=0.08) .{ }^{87,88}$ The Cella systematic review found no difference between cognitive remediation and usual care in the 
proportion of patients discontinuing treatment (21 trials; RR 0.82, 95\% CI 0.67 to 1.01; Appendix Table E-8), but did not conduct subgroup analyses of only trials comparing to usual care. $^{85}$ This was moderate strength evidence.

\section{Family Interventions Versus Usual Care}

\section{Key Points}

- Trials of family interventions differ in type of interventions included, methods of intervention delivery, number of treatment sessions, duration of intervention, level of patient participation, and target of the intervention. Most family interventions are targeted at reducing relapse.

- $\quad$ One fair-quality systematic review provided evidence from 27 trials ( $N=2,297)$; six trials not in the systematic review were also included $(\mathrm{N}=562)$.

- Family interventions did not affect social function, including employment and housing situation, more than usual care at 1 year (SOE: low).

- Suicide rates were similar in family intervention participants and those who received usual care, but events were few (SOE: low).

- Improvement in core illness symptoms was found with family intervention compared with usual care (four RCTs, N=223, SMD -0.46, 95\% CI -0.73 to -0.20) compared with usual care (SOE: low).

- Family interventions resulted in significantly lower relapse rates than usual care when measured at:

o 0 to 6 months (three RCTs; N=244, 23\% vs. 37\%, RR 0.62, 95\% CI 0.41 to 0.92 )

o 7-12 months (19 RCTs; N=1118, 30\% vs. 44\%, RR 0.67; 95\% CI 0.54 to 0.83)

o 13-24 months (nine RCTs; N=517; 49\% vs. 61\%; RR 0.75, 95\% CI 0.58 to 0.99 )

o 5 years post-treatment (two RCTs; N=140,78\% vs. 94\%, RR 0.82; 95\% CI 0.72 to $0.94)$

o Evidence was not adequate to show a difference for 25 to 36 months (SOE: moderate for 7-12 months, low for all others)

o Evidence suggests that relapse is lower with $>10$ treatment sessions.

\section{Detailed Synthesis}

\section{Description of Included Studies}

One fair-quality Cochrane systematic review included 53 trials that enrolled patients with schizophrenia and/or schizoaffective disorder (Appendix Table E-10). ${ }^{91}$ Approximately half of the included trials were conducted in China. As applicability to United States populations was a concern, we excluded these studies and, where necessary, performed our own analyses using the remaining 27 studies ( $\mathrm{N}=2,297)$. We also included six additional trials (in eight publications, $\mathrm{N}=562$ ) that were not included in the systematic review. ${ }^{92-97,289,290}$ and pooled results where appropriate. Including all evidence, participant age ranged from 15 to 65 years, and the proportion of men to women was 66.5 to 33.5 percent, although six trials did not report gender distribution. Length of treatment ranged from 6 weeks to 3 years and consisted of any familyoriented psychosocial intervention that required at least five sessions compared with usual care. One trial met all quality criteria. ${ }^{93}$ Relatively few trials reported methods of randomization and allocation concealment or reported blinded outcome assessors. Study attrition and reasons for 
attrition were also infrequently reported. Information on harms was rare. Most studies were small in size with fewer than 100 participants.

In addition to duration of treatment, trials of family intervention are heterogeneous in what is actually included in the family intervention (Appendix Tables E-11 and E-12). In some instances psychoeducation is included but may consist of one or two brief education sessions or several entire days. Psychoeducation may be the sole part of the intervention or, more frequently, part of a package that may include motivational interviewing, behavioral family therapy, support groups, communication training, stress management, goal setting, and development of social networks. Families may experience the intervention as a single family or in multiple family groups. The patient may be present for some sessions and not others. The target of the intervention may be to prevent relapse (most often) or hospitalization, promote treatment or medication adherence, or improve social or global function, for example. In pooled analyses of trials with relapse as an outcome, we have stratified these trials by number of treatment sessions, duration of treatment, and an estimate of the degree psychoeducation is included in the family intervention, in order to explore the degree to which each factor may influence patient relapse.

\section{Findings}

\section{Function}

\section{Social Functioning}

One small study $(\mathrm{N}=69)$ reported patient change scores from baseline on the SFS. ${ }^{95}$ This trial provided 6 months of treatment and assessed social functioning at 6 months and 12 months. Family intervention included motivational interviewing, individual cognitive behavior therapy, along with a family intervention based on carer's needs. This trial provide insufficient evidence of no difference in social function between family intervention and usual care at either 12 or 24 months ( $\mathrm{p}>0.05)$.

\section{Unemployment}

Four trials provided low-strength evidence of no difference in unemployment with family interventions compared with usual care after 6-12 months of followup ( $\mathrm{N}=230 ; 75 \%$ vs. 66\%; RR 1.09; 95\% CI 0.91 to 1.29, $\mathrm{I}^{2}=0 \%$; Appendix G-16). ${ }^{91}$ Although unemployment rates at 2 years ( $\mathrm{N}=51$; $69 \%$ vs. 52\%; RR 1.33; 95\% CI 0.84 to 2.10$)$ and at 3 years of followup ( $\mathrm{N}=99$; 82\% vs. 69\%; RR 1.19; 95\% CI 0.92 to 1.55) were also not different between family intervention and usual care, the evidence is insufficient to draw conclusions due to methodological limitations, lack of confirmatory trials, and imprecision.

\section{Unable To Live Independently}

Based on three RCTs, there was low-strength evidence of no differences between family interventions and usual care in the inability to live independently at 1 year ( $N=164 ; 57 \%$ vs. $63 \%$; RR 0.83; 95\% CI 0.66 to 1.03). ${ }^{91}$ A single trial included in the included systematic review reported independent living at 3 years post intervention (N=99; 54\% vs. 66\%; RR 0.82; 95\% CI 0.59 to 1.14$)$, but this evidence is insufficient. On small trial $(\mathrm{N}=97)$ reported data from 63 participants (65\%) at a 5-year followup of a 15-month intervention and found family intervention associated with living fewer months in an institution for psychiatric patients (10.87 months vs. 21.18 months, $\mathrm{p}=0.04$ ) compared with usual care. ${ }^{291}$ Evidence at 5 years was considered 
insufficient from which to draw meaningful conclusions regarding the effect of family intervention in ability to live independently.

\section{Health-Related Quality of Life}

One study ( $\mathrm{N}=50)$ reported mean endpoint change in quality of life scores based on the Heinrichs QLS scale (21-item scale, high score=good). ${ }^{91}$ An additional study $(\mathrm{N}=55)$ reported that a 9-month family intervention improved overall quality of life based on the EuroQol scale by 7.38 points over treatment as usual at 24 months but this difference was not statistically significant (-7.38, 95\% CI -22.07 to 7.31). ${ }^{93}$ Values on the EuroQol were not reported prior to the 24-month followup. Evidence was considered insufficient to draw meaningful conclusions on the effect of family interventions versus usual care on quality of life.

\section{Reduction in Self-Harm}

There was low-strength evidence, from six trials, of no difference in risk of suicide for participants who received family interventions compared with usual care, but events were few (N=314, $4 \%$ vs. $6 \%$, RR 0.85; 95\% CI 0.24 to 3.02, $\mathrm{I}^{2}=23 \%$; Appendix G-17). ${ }^{91}$

\section{Improvement in Core IIIness Symptoms}

\section{Overall Symptoms}

Combining the scores on the BPRS from three trials $(\mathrm{N}=190) \cdot{ }^{91,96}$ with PANSS scores from one trial ${ }^{93}(\mathrm{~N}=55)$ provided low strength evidence of improvement in core illness symptoms with family interventions (four RCTs, $\mathrm{N}=223$, SMD -0.46, 95\% CI -0.73 to $-0.20, \mathrm{I}^{2}=0 \%$; Appendix G-18). ${ }^{91,93,96}$ The data from one small trial $(\mathrm{N}=64)$ could not be pooled with the other trials, but reported a significant improvement with family intervention in an analysis of covariance $(\mathrm{p}=0.017){ }^{95}$

\section{Negative Symptoms}

Three trials ( $\mathrm{N}=163)^{92,93,96}$ also reported scores on the negative subscale of the BPRS, the, PANSS, and the Modified Scale for the Assessment of Negative Symptoms. A pooled analysis found lower risk of negative symptoms with family intervention (SMD -0.38, 95\% CI -0.69 to 0.07; Appendix G-19).

\section{Treatment Discontinuation}

Six trials provided low strength evidence of no difference between family intervention and usual care on leaving the study early between 3 and 6 months (N=504; 13\% vs. 14\%; RR 0.86; 95\% CI 0.51 to $1.45, \mathrm{I}^{2}=19 \%$; Appendix G-20). ${ }^{91}$ However, between 7 and 12 months participants receiving usual care were more likely to leave the study early when compared with participants receiving a family intervention (13 RCTs; N=953, 24\% vs. 26\%, RR 0.77; 95\% CI 0.64 to $0.93, \mathrm{I}^{2}=0 \%$; Appendix G-21 $)^{91,93,94,97}$ based on low-strength evidence. Results for leaving the study early were not different between 13 months and 2 years (six RCTs, $\mathrm{N}=362$, $22 \%$ vs. $27 \%$, RR 0.82 , $95 \%$ CI 0.57 to 1.16 , $\left.\mathrm{I}^{2}=0 \%\right)$, ${ }^{91}$ based on low-strength evidence. Evidence was insufficient to determine the benefit of family therapy compared with usual care on study departures between 25 and 36 months and after 3 years. 


\section{Relapse}

Consistent with the included systematic review, ${ }^{91}$ we considered participants missing data for relapse as having relapsed. Three trials provided low-strength evidence of lower risk of relapse between 0 and 6 months with family interventions compared with usual care, $(\mathrm{N}=244,23 \%$ vs. $37 \%$, RR 0.62; 95\% CI 0.41 to $0.92, \mathrm{I}^{2}=0 \%$, Appendix G-22). ${ }^{91,290}$ In the trial with a greater number of sessions (11 to 20 ) relapse rates were slightly lower than in the two trials with a family intervention of 0 to 10 sessions (RR 0.51 vs. RR 0.69 ).

Nineteen trials $(\mathrm{N}=1118)$ provided moderate-strength evidence of lower risk of relapse between 7 and 12 months with family interventions compared with usual care (30\% vs. 44\%, RR 0.67; 95\% CI 0.54 to 0.83; $\mathrm{I}^{2}=44 \%$; Appendix G-23). ${ }^{91,93,96,97,290}$ As above, studies with greater number of sessions (i.e., greater than 10) were more likely to favor family intervention, while duration of the intervention (Appendix G-24) favored trials up to 12 months, and trials including psychoeducation (Appendix G-25) as all or part of the treatment package were slightly less likely to show a treatment effect (RR 0.78; 95\% CI 0.60 to 1.02).

The SOE was considered low for relapse rates beyond 12 months. Relapse rates at 13 to 24 months also favored family intervention (nine RCTs; N=517; 49\% vs. 61\%; RR 0.75, 95\% CI 0.58 to $0.99 ; \mathrm{I}^{2}=57 \%$; Appendix G-26). ${ }^{91}$ There were no difference in the statistical significance of the treatment effect based on number of sessions within the family intervention or the duration of the intervention (Appendix G-27). Almost all trials included psychoeducation. For 25 to 36 months, there was low-strength evidence of no difference between family intervention and usual care on relapse rates (two RCTs; $\mathrm{N}=147 ; 79 \%$ vs. 73\%; RR 1.05; 95\% CI 0.79 to 1.39; $\mathrm{I}^{2}=45 \%$; Appendix G-28).

Two small studies $(\mathrm{N}=140)$ provided relapse data at 5 years followup (after 15 months of intervention). ${ }^{91,289}$ Pooled analysis provided low-strength evidence that the benefit of family interventions on the risk of relapse may be retained at 5 years (78\% vs. 94\%, RR 0.82; 95\% CI 0.72 to $0.94, \mathrm{I}^{2}=0 \%$; Appendix $\left.\mathrm{G}-29\right)$. One small, study $(\mathrm{N}=63)$ provided data at 8 years and found no difference in risk of relapse between family intervention and usual care (81\% vs. 94\%, RR 0.86; 95\% CI 0.71 to 1.05 ). ${ }^{91}$ This evidence at 8 years is insufficient for which to draw conclusions.

\section{Harms}

One small study $(\mathrm{N}=51)$ provided insufficient evidence on measures of family burden to determine whether family interventions reduce family burden when compared with usual care. ${ }^{91}$

There was insufficient evidence of no difference in nonsuicide mortality based on three trials (N=113, 3\% vs. 4\%, RR 0.96; 95\% CI 0.17 to 5.33, $\mathrm{I}^{2}=0 \%$; Appendix G-30). ${ }^{91}$

\section{Intensive Case Management Versus Usual Care}

\section{Key Points}

- ICM was assessed in one good-quality systematic review (10 trials; $\mathrm{N}=1,652)$ and one RCT (N=77).

- ICM was not different to usual care in improvements in social function, based on pooled analysis of three trials (MD 0.46; 95\% CI -0.34 to 1.26) (SOE: low).

- There was no difference in rates of imprisonment with ICM versus usual care, based on pooled analysis of five trials (OR 0.90 , 95\% CI 0.45 to 1.82) (SOE: low).

- ICM did not improve quality of life more than usual care in two trials (SOE: low). 
- ICM did not improve core illness symptoms more than usual care, based on pooled analysis of two trials (MD, 0.46; 95\% CI -3.67 to 4.60). One subsequent trial also reported no difference in symptoms using a different scale (SOE: low).

\section{Detailed Synthesis}

\section{Description of Included Studies}

A good-quality systematic review of 10 trials $(\mathrm{N}=1,652)$ assessed ICM compared with usual care (Appendix Tables E-13 and F-3). ${ }^{98}$ Another review that combined evidence for assertive community treatment and ICM was not used, as we considered these to be distinct interventions. ${ }^{272}$ However, all of the studies included in the second review are included here. Included studies enrolled patients with schizophrenia or schizophrenia-like disorders, bipolar disorder, or depression with psychotic features. Mean age of participants enrolled in the studies ranged from 35 to 49 years, 0 to 59 percent were female, and 0 to 91 percent were nonwhite. Included interventions were explicitly described as case management; studies (or arms of studies) of assertive community treatment and home-based care were excluded. Study quality of included trials ranged from fair to good; poor-quality studies were excluded.

We identified one other RCT of ICM published since the systematic review described above (Appendix Tables E-14 and F-4). ${ }^{99}$ The fair-quality study (N=77) enrolled participants with a mean age of 37 years, and the proportion of female participants was 53 percent; race/ethnicity was not reported. The trial enrolled Swedish patients with diagnosed mental illness and serious functional impairment. Patients were followed for 3 years.

\section{Findings}

\section{Function}

\section{Global and Social Function}

The systematic review of ICM found no significant effect on social function, based on pooled analysis of three trials ( $\mathrm{N}=197$ ) (MD0.46, 95\% CI -0.34 to 0.1.26; $\mathrm{I}^{2}=48 \%$; Appendix Table E13). ${ }^{98}$

One trial published since the systematic review also reported on functional outcomes. ${ }^{99}$ Consistent with the findings of the systematic review, the study reported no difference between groups in function assessed using the Strauss Carpenter scale (Appendix Table E-14). The SOE for this comparison is low due to study limitations and imprecision.

\section{Encounters With the Legal System}

The systematic review reported no significant differences in imprisonment based on five trials ( $\mathrm{N}=757$; OR $0.90,95 \%$ CI 0.45 to $\left.1.82 ; \mathrm{I}^{2}=0 \%\right) .{ }^{98}$ This was low-strength evidence due to study limitations and imprecision.

\section{Health-Related Quality of Life}

The systematic review of ICM identified two trials reporting quality of life (Appendix Table E-13). ${ }^{98}$ ICM was not associated with differences in quality of life scores on the Quality of Life Scale (MD, 0.09 , 95\% CI -0.23 to 0.42 ). One subsequent trial, ${ }^{99}$ which assessed quality of life using the Lancashire Quality of Life Profile, also found no difference between groups in quality of life. This evidence is low strength due to study limitations and imprecision. 


\section{Improvement in Core IIIness Symptoms}

The systematic review reported no significant differences between groups in symptoms based on BPRS scores (two trials; N=126; MD, 0.46; 95\% CI -3.67 to 4.60). ${ }^{98}$ One subsequent trial assessed symptoms using the Hopkins Symptom Check List and also reported no difference between groups (Appendix Table E-14). ${ }^{99}$ This evidence is low strength due to study limitations and imprecision.

\section{Treatment Discontinuation}

The systematic review reported significantly less loss to followup with ICM compared to usual care based on seven trials ( $\mathrm{N}=1,210$; OR $0.70,95 \%$ CI 0.54 to $0.90 ; \mathrm{I}^{2}=36 \%$; Appendix Table E-13) ${ }^{98}$ The addition of data from the single study published since the systematic review did not substantially alter these results (eight trials; N=1,287; OR 0.71, 95\% CI 0.54 to 0.95 ; $\mathrm{I}^{2}=11 \%$; Appendix G-32). This was moderate-strength evidence.

\section{Illness Self-Management and Recovery Versus Usual Care}

\section{Key Points}

- One fair-quality systematic review provided evidence from 13 trials $(\mathrm{N}=1,404)$; one other trial not included in the review provided additional evidence.

- Participants receiving a self-management education intervention were significantly more likely to demonstrate a reduction in severity of symptoms based on the BPRS (five RCTs, WMD -4.19, 95\% CI -5.84 to -2.54) (SOE: moderate), but no change in negative symptoms based PANSS - negative subscale (SOE: low).

- Patients receiving more than 10 self-management intervention sessions had a greater reduction in the likelihood of experiencing relapse compared with usual care (OR 0.41; $95 \%$ CI 0.21 to 0.79 ), whereas those receiving 10 or fewer sessions had a smaller, nonsignificant, reduction in the risk of relapse (OR 0.67, 95\% CI 0.39 to 1.15) (SOE: low).

\section{Detailed Synthesis}

\section{Description of Included Studies}

Illness self-management training programs are designed to improve knowledge, management of symptoms, social and occupational functioning, with a primary goal of reducing the risk of relapse by focusing on medication management, recognizing signs or relapse, and developing a relapse prevention plan and coping skills for persistent symptoms. Given these, the primary target of this intervention is reducing the risk of relapse.

We identified one fair-quality systematic review ${ }^{100}$ that examined the effect of selfmanagement education interventions compared with usual care, which was not clearly defined (Appendix Tables E-15 and F-3). This review included 13 trials ( $\mathrm{N}=1404$; range 23 to 125) with three trials from United States populations $(\mathrm{N}=211)$. Only three to five trials $(\mathrm{N}=257$ to 534$)$ reported results for each outcome of interest. The proportion of female participants ranged from 27 to 58 percent in 12 trials; one study enrolled exclusively male participants. Mean age ranged from 30 to 40 years. All interventions were delivered in a group setting and the number of intervention sessions ranged from 7 to 48 sessions lasting 45 minutes to 90 minutes each. Duration of followup ranged from the time of treatment cessation to 24 months post-treatment. 
We included one other fair-quality RCT comparing a specific illness self-management training program for schizophrenia, the Illness Management and Recovery program compared with usual care. ${ }^{101}$ This Israeli study included 210 people with severe mental illness and measured efficacy using the Coping Efficacy Scale and the Illness Management and Recovery scale. The percentage of participants with schizophrenia was 80 and 89 percent in the intervention and control groups, respectively. Fidelity to the program was measured via the Illness Management and Recovery Fidelity scale after 4 to 5 months and ranged from 2.66 to 4.77 (means) on a scale of 1 to 5 . There were no significant differences between groups in any of the sociodemographic variables (Appendix Tables E-16 and F-4).

\section{Findings}

\section{Function}

Functional outcomes were reported in 10 RCTs included in the systematic review, ${ }^{100}$ and the single RCT that was published after the review, ${ }^{101}$ but none of the trials used the same method to measure the outcomes. Overall, five trials found benefit with self-management interventions on global, social, and occupational outcomes whereas the other six trials did not. This evidence is insufficient to draw conclusions.

\section{Improvement in Core Illness Symptoms}

\section{Overall Symptoms (BPRS)}

The systematic review ${ }^{100}$ reported the effect of self-management education interventions on psychiatric symptoms (Appendix Table E-15). Five trials (N=409) reported mean data for psychiatric symptoms using the BPRS, with meta-analysis of these data demonstrating that participants in the intervention group were significantly more likely to demonstrate a reduction in the severity of symptoms (WMD -4.19, 95\% CI -5.84 to -2.54). A measure of statistical heterogeneity was not reported, and it is not clear that a difference of 4.19 points is clinically meaningful. This evidence was moderate strength. A meta-analysis of 3 of 13 trials $(\mathrm{N}=257)$ showed a significant reduction in the severity of positive symptoms (WMD -2.12, 95\% CI -3.04 to -1.20) as measured by the PANSS, but total scores were not assessed.

\section{Negative Symptoms (PANSS-Negative)}

The effect of self-management education on negative symptoms, based on PANSS Negative symptoms subscale, was reported in one systematic review that included three RCTs for this outcome $(\mathrm{N}=257) .{ }^{100}$ Results demonstrate a significant reduction in severity of negative symptoms reported favoring the intervention group (WMD -4.01, 95\% CI -5.23 to -2.79; Appendix Table E-15). This evidence was low strength due to study limitations and imprecision.

\section{Relapse}

The systematic review included five trials $(\mathrm{N}=534)$ that reported outcome data on relapse and stratified the analysis by the number of self-management education sessions (Appendix Table E15). ${ }^{100}$ Three trials had more than 10 sessions and two trials had less than 10 sessions. Patients receiving more than 10 sessions were 59 percent less likely to experience relapse than those receiving usual care (OR $0.41,95 \%$ CI 0.21 to 0.79 ) whereas those receiving 10 sessions or less were 33 percent less likely to experience relapse than those receiving usual care (OR 0.67, 95\% CI 0.39-1.15). This was low-strength evidence. 


\section{Other Outcomes}

The fair-quality RCT published after the systematic review included the Illness Management and Recovery Scale as the primary outcome measure (Appendix Table E-16). ${ }^{101}$ The scale is a composite scale, measuring personal goals, knowledge of mental illness, involvement with significant others, functioning, symptoms, stress, coping, relapse prevention, hospitalization, medication, and use of drugs and alcohol (range to 5; higher scores better). The intervention resulted in significantly greater improvement on this scale, although the absolute improvements were small ( 0.18 vs. 0.03 ; $\mathrm{p}<0.01$ ). The study also reported the Coping Efficacy Scale, which assesses symptoms, substance use and sleep problems experienced, strategies used to cope, and the patient's assessment of the efficacy of these strategies, with scores ranging from 1 to 5 . On this scale, the intervention also resulted in small but statistically significant improvements compared with usual care (final scores 3.25 versus 3.09; $\mathrm{p}<0.05$ ). In post hoc analyses, this study found better results with higher intervention fidelity scores.

\section{Patient Psychoeducation (Individual or Group) Versus Usual Care}

\section{Key Points}

- One good-quality systematic review provided evidence from 10 trials $(\mathrm{N}=1,125)$.

- Psychoeducation had a greater effect than usual care on global functional outcomes at 1 year of followup based on one good-quality systematic review of three trials (MD -5.23, 95\% CI -8.76 to -1.71) (SOE: low).

- Psychoeducation had a greater effect than usual care on relapse rates (with or without readmission) at 9 to 18 months of followup based on one good-quality systematic review of six trials (RR 0.80, 95\% CI 0.70 to 0.92) (SOE: moderate).

\section{Detailed Synthesis}

\section{Description of Included Studies}

We identified one good-quality systematic review ${ }^{102}$ comparing formalized psychoeducation to usual care (Appendix Tables E-17 and F-3). This review included 10 RCTs (total N=1,125), eight of which included stabilized outpatients, with duration of followup as long as 5 years. Subjects among the included studies had various diagnoses, including schizophrenia, schizophreniform disorder, schizoaffective disorder, and schizotypal disorder (with inclusion of individuals with multiple diagnoses). Studies were conducted primarily in North America and northern Europe (with 15\% of subjects from the United States). Psychoeducational interventions were diverse, including variations of brief, usual length, individual, and group techniques. Usual care was also diversely defined, including elements of medication management, psychosocial rehabilitation, and supportive psychotherapy.

Psychoeducation itself is related to the informed consent process, which is an integral part of usual management of any illness. This could be hypothesized to account for some difficulty in observing differences in outcomes between psychoeducation and usual care. Heterogeneity in study designs further complicates the synthesis of available data into overall assessments of the effect of psychoeducation. 


\section{Findings}

\section{Function}

Functional outcomes at 1 year of followup as measured by use of either the GAF or GAS scales in three trials were pooled, showing a significant beneficial effect of psychoeducation (MD -5.23, 95\% CI -8.76 to $-1.71 ; \mathrm{I}^{2}=79 \%$ ). ${ }^{102}$ Nevertheless, the SOE for this effect was low related to study limitations mentioned above and small number of observations. Individual trials reported the GAS or GAF at the end of treatment, 6 months, 18 months, and 2 and 5 years after treatment, but this evidence was insufficient to draw conclusions due to study limitations, lack of confirmatory studies, and small sample sizes. Within this evidence, two RCTs suggested the possibility of improved functional outcomes with psychoeducation; one study finding a significant benefit of psychoeducation at 2 years, but not 5 years of followup, and the other finding improvement in psychosocial functioning immediately after the intervention, with no observable difference at 6 or 18 months of followup using the SAS scale (Appendix Table E-17).

\section{Health-Related Quality of Life}

Although a single small RCT $(\mathrm{N}=114)$ observed a nonsignificant finding of improved quality of life as measured by Heinrich's Scale at the end of treatment and at 3 months, the SOE evidence was insufficient due to study limitations and small number of observations and inability to assess consistency in this single RCT. ${ }^{102}$

\section{Improvement in Core Illness Symptoms}

Differences in BPRS total scores were compared in two RCTs (one observing a small but nonsignificant benefit, one showing no effect), with overall SOE insufficient related to study design, imprecision, and unknown consistency. ${ }^{102}$

\section{Relapse}

A combined relapse and readmission analysis was conducted in the systematic review in order to increase the amount of extractable data (Appendix Table E-17). Based on six trials $(\mathrm{N}=720)$, psychoeducation had a greater effect than usual care on relapse rates (with or without readmission) at 9 to 18 months of followup (RR $0.80,95 \%$ CI 0.70 to 0.92 ; $\mathrm{I}^{2}=54 \%$ ) ${ }^{102}$ SOE for this finding is moderate. Other analyses of the available RCTs restricted to relapse without readmission showed no significant effects of psychoeducation, and had low SOE related to study methodologies and lack of precision. One group included in this $\mathrm{SR}^{292}$ has reported data suggesting benefit from psychoeducation in reducing readmission rates after 7 years of followup.

\section{Harms}

Potential harms that might be related to a psychoeducation intervention that were analyzed in the systematic review ${ }^{102}$ included death and all-cause dropouts of subjects exposed to the psychoeducation intervention (Appendix Table E-17). Only two RCTs reported on deaths, with no indication that deaths varied between groups, but the SOE was low primarily related to imprecision and the infrequency of death during the trials. No difference was detected in rates of subjects who left trials or were lost to followup in eight trials (RR 1.13, 95\% CI 0.89 to 1.44; $\mathrm{I}^{2}=15 \%$ ) 


\section{Social Skills Training Versus Usual Care}

\section{Key Points}

- Three fair-quality RCTs $(\mathrm{N}=384)$ provided evidence for social skills training.

- Global function was significantly better in patients receiving 6 months (SMD on the GAF scale 1.60, 95\% CI 1.19 to 2.02) and 1 year (SMD 2.02; 95\% CI 1.53 to 2.52) of social skills training. Social function was significantly better in patients receiving 2 years (SMD on The Multnomah Community Ability Scale (MCAS) 0.65, 95\% CI 0.36 to 0.95) of social skills training in three trials (SOE: low).

- In patients with schizophrenia, symptoms, based on the PANSS, improved more with social skills training at 6 months (SMD -1.50, 95\% CI -1.92 to -1.09) and 2 years (SMD $0.81,95 \%$ CI -1.22 to -0.40 ) (SOE: low).

- Negative symptoms were consistently and significantly improved with social skills training relative to usual care in three trials (SMD range -0.45 to -1.30 ) (SOE: low).

\section{Detailed Synthesis}

\section{Description of Included Studies}

We identified two systematic reviews of social skills training (Kurtz $2008^{293}$ and Almerie $2015^{294}$ ), however both were subsequently excluded due to the inclusion of a high number of inpatients and short-term studies (i.e., less than 12 weeks duration), and/or studies that used an active control group with no separate analysis according to active or passive controls. Although these reviews did not meet our inclusion criteria, for contextual purposes we have included the findings from the Kurtz review below for function, negative symptoms and relapse. We did not report results from the Almerie review due to the inclusion of a high proportion of studies conducted in China, which have been shown to overestimate treatment effects and are of questionable relevance to United States clinical practice. ${ }^{295}$

Review of the reference lists of these reviews and literature searches identified three RCTs (in 4 publications) meeting inclusion criteria comparing social skills training and usual care (Appendix Table E-18). ${ }^{97,103-105}$ One other RCT of cognitive behavioral social skills training is included in the CBT section of this report. ${ }^{275}$

The three RCTs of social skills training enrolled between 98 and 183 study participants. In these trials, social skills training consisted weekly sessions (ranging from 24 weeks to 1 year; total followup 6 month to 3 years) of specific, progressive intervention modules that generally included management of symptoms and medication, improving social and family relationships, and increasing functional skills such as money management, among others. In all three trials, social skills training was specifically focused on improving psychosocial function and reducing relapse and need for hospitalization. One study was conducted in the United States, ${ }^{103,104}$ and the other two were conducted in Mexico. ${ }^{97,105}$ Mean age was about 30 years in two trials. ${ }^{97,105}$ In the other study, which specifically enrolled older adults, the mean age was 60 years. ${ }^{103,104}$ In the same study, more than half of study participants were female, and $>80$ percent were white. In the other two trials, women made up about 30 percent of participants and race was not reported. ${ }^{97,105}$ Two trials required a diagnosis of schizophrenia for study inclusion, but in the other, only about 55 percent of participants had schizophrenia or schizoaffective disorder. Duration of illness was 8 and 9 years in two trials, ${ }^{97,105}$ and not reported in the other. All three trials were rated fair quality (Appendix Table F-4). Methodological limitations among the studies were inadequate 
reporting of methods of randomization and allocation concealment, dissimilarities of baseline groups (one study) and no intention to treat analysis (two studies).

\section{Findings}

\section{Function}

Functional improvement is a primary focus of social skills training. All three trials reported significant improvements functional outcomes with social skills training, based on different measures (Appendix Table E-18). ${ }^{97,103-105}$ Since functional outcomes were reported in various ways in the trials, we converted scale scores to SMDs here for comparison purposes. In two trials, GAF scores were significantly better in social skills training groups compared with usual care after 6 months ( $\mathrm{N}=119$; SMD $1.60,95 \%$ CI 1.19 to 2.02$)^{105}$ and 1 year of treatment $(\mathrm{N}=98$; SMD 2.02, 95\% CI 1.53 to 2.52$).{ }^{97}$ The third study, which included a mix of patients with schizophrenia and mood disorders, reported improvement in function based on MCAS score after 2 years of social skills training ( $\mathrm{N}=183$; SMD 0.65 , 95\% CI 0.36 to 0.95$)$ but these results were not sustained at 3 years followup (1 year after treatment was stopped; SMD 0.24; 95\% CI 0.05 to 0.53$).{ }^{103,104}$ These results are consistent with those in the Kurtz 2008 systematic review described above, which also reported a positive effect of social skills training on psychosocial function (seven trials, $\mathrm{N}=371$ ); effect size 0.52 , $95 \%$ CI 0.31 to 0.73 ). ${ }^{293}$ The evidence on improvements during treatment was low strength; evidence on maintenance of effect after discontinuing treatment was limited to one small study and is therefore insufficient to draw conclusions.

\section{Improvement in Core IIIness Symptoms}

\section{Overall Symptoms}

Significantly greater reduction in overall symptoms was found following completion of social skills training compared with usual care in the two trials ( $\mathrm{N}=119$ and 98) that exclusively enrolled patients with schizophrenia (Appendix Table E-18). ${ }^{97,105}$ SMD in total PANSS scores was -1.50 (95\% CI -1.92 to -1.09$)$ and -0.81 (95\% CI -1.22 to -0.40) after 6 months and 1 year, respectively. This evidence was low strength. In the third study, there was a small, nonsignificant effect in favor of social skills training based on BPRS score (N=183; SMD -0.04, 95\% CI -0.33 to 0.25$)$. This study enrolled a combination of patients with schizophrenia/schizoaffective disorder (56\%), depression (24\%), and bipolar disorder (20\%), potentially limiting the effect on symptom scores. ${ }^{103,104}$

\section{Negative Symptoms}

Negative symptoms were consistently improved following 6 months (SMD -1.30, 95\% CI 1.70 to -0.90 ), ${ }^{97} 1$ year (SMD -0.82; $95 \%$ CI - 1.23 to -1.40 ), ${ }^{105}$ and 2 years (SMD -0.45; 95\% CI -0.74 to -0.15$)^{104}$ of social skills training based on the SANS (1 study) and PANSS (two trials) scores (low-strength evidence). Results from these trials are consistent with the findings in the Kurtz systematic review, which also found that social skills training significantly improved negative symptom scores ( 6 trials, $\mathrm{N}=363$; effect size $0.40,95 \%$ CI 0.19 to 0.61 ). ${ }^{293}$ In the only study meeting inclusion criteria to follow patients after the cessation of treatment, negative symptom scores were still significantly better in social skills training patients 1 year after treatment had ended (SMD -0.45, 95\% CI -0.74 to -0.15 ). ${ }^{103}$ Evidence on maintenance of effect 
after treatment discontinuation is insufficient due to study limitations, lack of confirmatory studies, and imprecision.

\section{Treatment Discontinuation}

There was no difference in treatment discontinuation, based on two trials with 1- (88\% [43/49] vs. 80\% [39/49]; RR 1.10, 95\% CI 0.92 to 1.31 ) and 2-year (82\% [76/93] vs. 81\% [73/90]; RR 1.01; 95\% CI 0.88 to 1.16) followup (Appendix Table E-18). ${ }^{97,104}$ This was lowstrength evidence.

\section{Relapse}

In one study with 1-year followup reporting undefined relapse, patients in the social skills training group were half as likely to relapse compared with those in the usual care group (5\% vs. $10 \%)$, but absolute number of patients relapsing was small (5/49 vs. 10/49) and the risk estimate was not significant (RR 0.50; 95\% CI 0.18 to 1.36$).{ }^{97}$ Due to the lack of confirmatory studies in relevant populations, and small sample size, this evidence is insufficient. The 2008 Kurtz review reported a combined effect size for relapse and hospitalization, indicating a small (0.23) but significant finding (95\% CI 0.04 to 0.41 ) in favor of social skills training based on nine trials $(\mathrm{N}=485)^{293}$

\section{Supported Employment Versus Usual Care}

\section{Key Points}

- Supported employment, using the individual placement and support (IPS) model, results in better employment outcomes than usual care with 2 years of followup. The evidence is based on one fair-quality trial $(\mathrm{N}=204)$, and is supported by evidence from a systematic review of 14 RCTs with vocational training comparisons, and a large RCT $(\mathrm{N}=1,273)$ with both usual care and vocational training comparisons.

- Patients receiving IPS were significantly more likely to obtain competitive work than those receiving usual care (75\% vs. $27.5 \%$, $\mathrm{p}=0.001)$. These findings are consistent with findings of a large trial of various supported employment interventions that included other comparison groups (SOE: moderate). The time to obtaining first competitive employment was 22 days shorter with IPS than with usual care $(\mathrm{p}<0.001$, SOE: low).

- IPS resulted in more patients working more than 20 hours per week (13\% vs. 34\%; $\mathrm{p}=0.00$ ), having more weeks of employment overall (24 more weeks competitive and 11 more weeks any employment; $\mathrm{p}<0.001$ ), and longer tenure per individual job (4 weeks; $\mathrm{p}=0.048$ ) than those in either usual care, other vocational interventions, or both. These findings are consistent with findings of a large trial and a systematic review of 14 trials that included other comparison groups (SOE: moderate).

- Patients receiving IPS earned more money than those in usual care $(\$ 2,078 /$ month vs $\$ 617.59 /$ month; $\mathrm{p}<0.001$ ). These findings are consistent with findings of a large trial of supported employment interventions that included other comparison groups, except that the overall earnings and the magnitude of difference between groups was smaller. ( $\$ 122 /$ month vs $\$ 99 /$ month; $p=0.04$, SOE: moderate) 


\section{Detailed Synthesis}

\section{Description of Included Studies}

In our review, we included studies that compared supported employment with usual care as direct evidence. We identified one fair-quality trial $(\mathrm{N}=204)$ that examined the effect of the IPS model of supported employment compared with standard services offered off-site from the mental health center (usual care) and a psychosocial rehabilitation intervention (Appendix Tables E-19 and F-4). ${ }^{109}$ The target population included patients with severe mental illness receiving care at a state mental health department with an Axis I diagnosis and severe impairment in psychosocial functioning or self-care; lacking competitive employment; desire for competitive work; capable of providing informed consent. Comprehensive employment data were collected and interviews were conducted at baseline and every 6 months for the 2-year study duration. Fidelity to the IPS model was evaluated yearly, with the IPS group achieving high fidelity scores (using the IPS Fidelity Scale). Study participants received services at the lead community mental health center in Hartford, Connecticut. The study was conducted in a population that was mainly African American and Latino; the proportion of eligible patients enrolled was highest among Latinos (86\%) and African Americans (81\%) with a lower rate among white patients (66\%). Participants in the study were mostly men in their 40s (mean age 41 years, 63\% male).

A number of employment outcomes were collected, with the two main outcomes categorized as competitive work, "jobs paying competitive wages in integrated settings, contracted by clients, and not reserved for persons with disabilities," and any paid work, which could include jobs set aside for persons with disabilities. Nonvocational outcomes were evaluated using standardized scales, but were reported as "trends" because of the large number of tests conducted. Although intention-to-treat analysis was undertaken, the impact of missing data was a concern, as less than 40 percent of patients assigned to usual care contributed data in the second year versus 90 percent for the IPS group.

Because there was only one eligible study comparing supported employment with usual care, we included evidence, comparing supported employment with other vocational interventions. This evidence included a good quality systematic review of 14 RCTs with other vocational training interventions as controls $(\mathrm{N}=2,265)^{106}$ (Appendix Tables E-20 and F-3). The psychosocial rehabilitation intervention arm of the study described above was also included. Thirteen of the 14 included studies used IPS as the model for supported employment. Duration of followup was 12 to 24 months. We also included a large, United States-based, fair quality RCT of supported employment conducted in eight states $(\mathrm{N}=1,273)$ which made comparisons to usual care and active vocational interventions, depending on the study site ${ }^{108}$ (Appendix Tables E-19 and F-4). Approximately half of the study sites used a usual care comparison group, and three of eight states explicitly used the IPS model. Duration of followup was two years. An analysis of results in patients with and without a schizophrenia diagnosis was also published. ${ }^{107}$ The review and the trial reported similar outcome measures as the RCT described above.

\section{Findings}

\section{Occupational Function}

Based on intention-to-treat analysis, the trial of IPS versus usual care described above found that patients in the IPS group were significantly more likely to obtain competitive work (75\% vs. $27.5 \% \mathrm{p}<0.001) .{ }^{109}$ The other RCT, with both usual care and vocational intervention 
comparators, also found that more patients obtained competitive employment while receiving supported employment interventions than other interventions, including usual care (55\% vs 34\%; $\mathrm{p}<0.001) .{ }^{108}$ Subgroup analysis of only patients diagnosed with schizophrenia showed a similar pattern although the overall success in obtaining competitive employment was lower (22\% vs. $12 \% ; \mathrm{p}<0.001$ with mixed effects logistic regression). ${ }^{107}$ Overall, this evidence was moderate strength. Based on the trial data comparing IPS to usual care, the mean time for patient to obtain their first competitive employment was 22 days $(\mathrm{p}<0.001)$ shorter with IPS than with usual care. ${ }^{109}$ This was low-strength evidence.

Patients receiving IPS were also significantly more likely to obtain any work than those receiving usual care (75\% vs. 53.6\%, $\mathrm{p}=0.001) .{ }^{109}$ This finding is supported by the systematic review findings, where the risk ratio, based on seven RCTs ( $\mathrm{N}=951)$ for finding any employment was 2.62 (95\% CI 2.18 to 3.16) with IPS over vocational training interventions. ${ }^{106}$ Combined, this was moderate-strength evidence.

The measures of how much a patient worked, how consistently they were employed and for how long varied, but all showed that supported employment interventions were beneficial over either usual care or other vocational interventions. Compared with usual care, the RCT found that significantly more patients receiving IPS worked more than 20 hours per week (33.8\% vs. 13\%; $\mathrm{p}=0.001){ }^{109}$ This evidence is consistent with evidence that finds that more patients receiving supported employment interventions work 40 or more hours per month than those receiving other vocational training or usual care (51\% vs. 39\%; $\mathrm{p}<0.001) .{ }^{107,108}$ Patients receiving IPS worked more weeks in competitive employment than those in usual care (mean 29.72 vs 5.45 weeks; $\mathrm{p}<0.001$ ). ${ }^{109}$ The systematic review also found that IPS results in more days of competitive employment than other interventions (MD 70.63 days, 95\% CI 43.22 to 98.04). ${ }^{106}$ Similarly, evidence with both usual care and active controls find that patients receiving IPS have more time in any employment than those receiving usual care (30.18 weeks vs. 19.08 weeks; $\mathrm{p}<0.001$ for IPS versus usual care, ${ }^{109}$ and MD of 84.94 days more [95\% CI 51.99 to 117.89] versus other vocational interventions). The tenure on any individual job was also longer with IPS. Compared with usual care, IPS resulted in a mean 19.75 weeks compared with 15.56 weeks per job with usual care $(\mathrm{p}<0.001) .{ }^{109}$ Findings from the systematic review, based on two trials, were very similar with a MD of 3.86 weeks (95\% CI -5.66 to 13.38). ${ }^{106}$ These findings were moderate SOE.

Patients receiving supported employment interventions earned more money than those in usual care (\$2,078/month with IPS vs \$617.59/month with usual care; $\mathrm{p}<0.001) .{ }^{109}$ Findings from a trial with both vocational training and usual care comparison groups supports this finding, although the amount earned per month and the relative difference was smaller (\$122/month vs \$99/month; $\mathrm{p}=0.04) .{ }^{107,108}$ Combined, this evidence was moderate strength for the direction of effect. The variation in magnitude of effect was lower strength.

\section{Improvement in Core Illness Symptoms}

Differences in nonvocational outcomes were reported as not being significant between IPS and usual care. ${ }^{109}$ This outcome was either not significantly different or not reported in the other studies.

\section{Treatment Discontinuation}

Treatment discontinuation (withdrawal from study) was much higher in the usual care group compared with the IPS group; rates of retention in the study were less than 40 percent for standard services in the second year versus 90 percent for IPS. ${ }^{109}$ 


\section{Supportive Therapy Versus Usual Care}

\section{Key Points}

- A good-quality systematic review evaluated five trials of supportive therapy versus usual care and found no differences in global functioning (SOE: low).

\section{Detailed Synthesis}

\section{Description of Included Studies}

We identified one good-quality systematic review comparing supportive therapy or supportive care that included 24 RCTs (total N=2126, range 12 to 315 per study) and 10 weeks to 3 years of followup (Appendix Tables E-21 and F-3). ${ }^{110}$ Only five of the trials used a usual care control group ( $\mathrm{N}=822)$. Three of these were conducted in the outpatient setting and two were conducted in the United States. Most of the patients in the trials had been diagnosed with schizophrenia and the ages ranged from 16 to 72 . Four of the trials specified that the patients had to be suffering from on-going hallucinations and/or delusions. Four of the trials enrolled patients with long-standing disease, but one enrolled majority experiencing their first episode and included inpatients. ${ }^{284}$ This study also had a lower median age (27 years) than the mean in other trials (36 to 47 years). All studies enrolled more men than women.

Definitions of the interventions were not consistent, but generally the study interventions were aimed at maintaining current functioning or to assist the patients with pre-existing coping abilities. Specific treatments received in the usual care group were not reported. The frequency and duration of treatments varied, ranging from twice a week for 3 months with monthly booster sessions for 4 months, to sessions every 2 weeks for a total of 10 sessions, with no booster sessions. Although this review was good quality, the trials themselves were mostly fair or even poor quality (only 1 of the 5 was good quality).

\section{Findings}

\section{Function}

The effect of supportive therapy on function was reported in two trials in the systematic review, but differences were not found at 9 months in either global or social functioning (Appendix Table E-21). ${ }^{110}$ Although two trials used similar scales to measure global functioning, the results were not pooled GAF (modified version), $\mathrm{N}=29$, MD 1.40, 95\% CI -5.09 to 7.89; GAS, N=260, MD -2.66, 95\% CI -6.20 to 0.88). Only one of these trials also measured social functioning (using the SFS), and found no difference between the groups. ( $\mathrm{N}=260, \mathrm{MD}-0.67$, 95\% CI -7.05 to 5.71). The trials were small, such that this evidence is imprecise. The evidence on global functioning was low strength, whereas the evidence on social functioning is insufficient.

\section{Health-Related Quality of Life}

In the systematic review, a single study reported outcomes that were categorized as quality of life, although the three scales reported do not measure global health-related quality of life (Appendix Table E-21). The scales used were the Rosenberg Self-Esteem Scale (RSES) noted to measure positive aspects of psychological functioning, the Well-being Scale (WBS) Global 
health questionnaire (GHQ) noted to measure nonpsychotic psychiatric symptoms. No differences were found on these measures and the evidence is insufficient.

\section{Improvement in Core IIIness Symptoms}

\section{Overall Symptoms}

The systematic review identified one study reporting general symptomatology using the PANSS, one in the short term and one in the long term, with neither study finding significant differences between groups: short term (13 to 26 weeks; N=131, MD -4.42 (95\% CI -10.13 to 1.29); long term (more than 26 weeks; $\mathrm{N}=36$, MD 4.70 (95\% CI -6.71 to 16.11; Appendix Table E-21). This evidence is insufficient.

\section{Negative Symptoms}

The systematic review found only one study that reported negative symptoms, using the SANS, but determined that the data were skewed and did not analyze the significance of the findings. In the short term the endpoint scores were very similar (10.19 and 10.73 for treatment and control), although in the long term the scores were more different (9.90 and 11.46, respectively). The study was very small $(\mathrm{N}=47)$ so this evidence is insufficient.

\section{Treatment Discontinuation}

Leaving the study early was reported in four RCTs $(\mathrm{N}=354)$ in the systematic review, resulting in a pooled estimate that indicated no significant difference between groups (RR 0.86, 95\% CI 0.53 to 1.40). This was low-strength evidence.

\section{Relapse}

Only one study reported on relapse, but defined it as readmission to hospital for clinical deterioration that lased at least 5 days and resulted in functional impairment (Appendix Table E21). The systematic review found no significant difference in relapse rates between supportive therapy and usual care at either medium-term followup (13 to 26 weeks, N=54, RR 0.12, 95\% CI 0.01 to 2.11), or long-term followup (more than 26 weeks, $\mathrm{N}=54$, RR 0.96, 95\% CI 0.44 to 2.11). This evidence is insufficient.

\section{Key Question 2b. Variation of Benefits and Harms of Psychosocial and Other Nonpharmacological Treatments Versus Usual Care for Adults With Schizophrenia by Patient Characteristics}

\section{Key Points}

\section{Clinical Subgroups}

- Patients experiencing a first episode of psychosis: Pooled results found that the teambased multi-component interventions resulted in higher global functioning, based on GAF and GAS scores after up to 2 years of treatment (three RCTs; WMD: 3.88; 95\% CI 0.91 to 6.85) (SOE: moderate). 
o Team-based multi-component interventions resulted in significantly more people (22\%) working or in school after up to 2 years of treatment (three RCTs; RR 1.22; 95\% CI 1.01 to 1.47) (SOE: moderate).

o There were no significant differences between multi-component treatment programs and usual care on housing status for up to 2-year treatment duration based on two RCTs (SOE: low).

o Two RCTs found significant differences between multi-component programs and usual care on quality of life scores for up to 2-year treatment duration (effect size 0.84; 95\% CI 0.14 to 1.55 ) (SOE: moderate).

o There was no difference in reduction in self-harm in two trials of multicomponent programs versus usual care (SOE: low).

0 There was no difference between multi-component programs and usual care in total PANSS scores based on three RCTs (WMD -2.53; 95\% CI -5.45 to 0.39). (SOE: low). Removal of one study with between-group baseline differences resulted in a small but significant estimate (-1.40, 95\% CI -2.25 to -0.55) (SOE: low).

o Team-based multi-component program participants were significantly less likely to relapse compared with those in usual care based on two RCTs (RR 0.64; 95\% CI 0.52 to 0.79) (SOE: moderate).

- Comorbidities:

o Substance use: One good-quality systematic review of 32 trials $(\mathrm{N}=3,165)$ of cooccurring SUD and schizophrenia found no differences between integrated assertive community treatment and usual care in function at 12 months and mortality, and substance use at 36 months in people with co-occurring SUD (SOE: low for all outcomes).

\section{Demographic Subgroups}

- Evidence from systematic reviews and RCTs of CBT, cognitive remediation, and social skills training found no difference for any outcome when results were stratified according to patient age, or when comparing results from trials conducted in younger versus older adults.

0 A systematic review of CBT found no difference in negative symptom outcomes when results were stratified according to sex. Limited evidence from one RCT of a mixed population (about 50\% diagnosed with schizophrenia or schizoaffective disorder) of social skills training suggested that the intervention may be more effective in men than women for function and symptoms. 


\section{Detailed Synthesis}

\section{Findings}

\section{Clinical Subgroups}

\section{Early Interventions for Patients With a First Episode of Psychosis Versus Usual Care}

We identified one fair-quality systematic review comparing 12 early intervention programs for treating first-episode psychosis with usual care. ${ }^{296}$ This review was subsequently excluded, as 11 of the included studies did not meet our inclusion criteria, either due to small sample size, enrollment of inpatients, lack of applicability to the United States population, and/or use of a one-off intervention. One trial (the Danish OPUS trial) of team-based multi-component treatment programs included in the systematic review met our inclusion criteria (Appendix Table E-22). ${ }^{111}$

Literature searches identified three additional RCTs (in seven publications) not included in the systematic review reporting on the effect of team-based multi-component treatment compared to usual care (Appendix Table E-22). ${ }^{112-117,119}$ We identified one other study reporting 10 -year outcomes from the OPUS trial. ${ }^{118}$ The number of participants enrolled in these studies ranged from 99 to 1,268. Mean age ranged from 23 to 27 years, and 32 to 46 percent of participants were female. In two trials reporting race, Blacks comprised about 50 percent of the study population in both. Baseline psychotic symptom severity was similar in two studies (mean PANSS total score ranging from 67.4 to 77.5 ), ${ }^{113,116}$ but was lower in a third study (mean total PANSS 44.6). ${ }^{115}$ Mean duration of untreated psychosis, was 50 weeks ${ }^{118}$ to over 3 years ${ }^{116}$ in two trials. Duration of followup ranged from 1 to 10 years. Two trials were rated good quality, ${ }^{111,112}$ one was rated fair quality, ${ }^{115}$ and one was rated poor quality. ${ }^{116}$ Methodological limitations in the fair- and poor-quality trials included unclear randomization method and high attrition rates (Appendix Table F-4).

\section{Function}

Global. The effect of team-based multi-component treatment programs on global function was reported in three RCTs. ${ }^{111,113,114}$ Pooled results found that the multi-component programs resulted in higher functioning, based on GAF and GAS scores during up to 2 years of intervention (three RCTs; WMD 3.88, 95\% CI 0.91 to 6.85, $\mathrm{I}^{2}=64 \%$ ). This evidence was moderate strength. Treatment effects were not sustained at $5^{111}$ and 10 years ${ }^{118}$ after treatment removal.

Employment or school attendance. The effect of multi-component programs versus usual care on participation in work and school was reported in three RCTs. ${ }^{111,113,114}$ Pooled results found that multi-component programs resulted in significantly more people working or in school for the (up to) 2-year intervention period (three RCTs; pooled RR 1.22, 95\% CI 1.01 to 1.47). This evidence was moderate strength. These effects were not sustained at $5^{111}$ and 10 years ${ }^{118}$ after treatment removal.

Living situation. Pooled results from two RCTs found no significant differences between the team-based multi-component interventions and usual care during the up to 2-year intervention period (two RCTs; pooled RR 1.06, 95\% CI 0.86 to 1.30 ). ${ }^{111,113}$ This SOE was low. Five-year 
followup data suggest findings in favor of the team-based approach, with significantly more participants living in noninstitutional supported housing ( $\mathrm{N}=547$, RR $0.42 \mathrm{CI} 0.21$ to 0.83 ). ${ }^{111}$ This result was not significant at 10 -year followup. ${ }^{118}$

\section{Health-Related Quality of Life}

Team-based multi-component programs resulted in greater quality of life ratings after 18 months to 2 years treatment, based on results from two trials (pooled effect size $0.84,95 \%$ CI 0.14 to 1.55 , $\mathrm{p}=0.02){ }^{113,116}$ This evidence was moderate strength.

\section{Reduction in Self-Harm}

One trial, included in the systematic review, reported suicide incidence, finding no difference in risk between multi-component treatment programs and usual care (N=506, RR, CI 0.930 .06 to $14.81)^{111}$ A second trial found that the proportion of participants who, at 10-year followup, had experienced thoughts of suicide within the preceding 2 years was similar within multicomponent programs (39.4\%) and in usual care (379\%, $\mathrm{p}=0.77) .{ }^{118}$ This was low SOE.

\section{Improvement in Core Illness Symptoms}

The effect of team-based multi-component programs on psychotic symptoms, based on PANSS scores, was reported in three trials. ${ }^{113,114,116}$ Pooled results found no difference between groups in scores (WMD -2.53; 95\% CI -5.45 to 0.39 ; $\mathrm{I}^{2}=55 \%$ ). Sensitivity analysis removing a study with a 5.9-point difference at baseline results in a very small but significant difference and no heterogeneity (WMD -1.40; 95\% CI -2.25 to -0.55; Cochran Q for heterogeneity 0.0014 , $\mathrm{df}=1) .{ }^{114,116}$ This evidence was low strength. There was no effect on depressive symptoms, based on the Calgary Depression Scale (two RCTs; WMD -0.44; 95\% CI -1.08 to 0.20). This was low strength evidence.

\section{Treatment Discontinuation}

Results from two trials found participants in team-based multi-component treatment programs had a significantly greater rate of treatment retention compared with usual care after 12 to 18 months treatment (RR 1.27; 95\% CI 1.16 to 1.38 ; Cochran $\mathrm{Q}=0.03$, $\mathrm{df}=1$ ). ${ }^{112,114}$ This evidence was moderate strength. When rates were adjusted for baseline differences in sex, previous psychotic episode, and ethnicity, drop-out rates remained significant based on one trial (RR 0.28, CI 0.12 to 0.73 ). ${ }^{112}$ While a third $\mathrm{RCT}^{116}$ found significant differences in treatment retention, favoring team-based programs, this study had a 49 percent attrition rate, so those findings are not included here.

\section{Relapse}

Pooled analysis from two trials found that participants in team-based multi-component treatment programs were significantly less likely to relapse (defined as worsening of psychotic symptoms and/or hospitalization) than those in usual care (RR 0.64; 95\% CI 0.52 to 0.79 ; Cochran $\mathrm{Q}=0.024, \mathrm{df}=1) .{ }^{112,114}$ This evidence was moderate strength.

\section{Harms}

One trial found no significant differences between team-based multi-component care and usual care in rates of accidental death (RR 0.31; 95\% CI 0.01 to 7.59) or unexplained death (RR 0.31; 95\% CI 0.01 to 7.56). ${ }^{111}$ Ten-year followup from the same trial found no difference in between-group mortality (RR 0.92; 95\% CI 0.45 to 1.88 ). ${ }^{118}$ 


\section{Comorbidities: Substance Use Disorder and Schizophrenia}

One good-quality Cochrane systematic review included 32 randomized trials that enrolled a total of 3,165 patients (aged 18 years to 65 years) with severe mental illness and comorbid substance misuse (Appendix Table E-23). ${ }^{120}$ Most trial participants were diagnosed with a schizophrenia spectrum disorder or psychosis with a concurrent diagnosis of substance misuse (e.g., cannabis, cocaine, opioids) and were diagnosed using American Psychiatric Association's Diagnostic and Statistical Manual of Mental Disorders (DSM) criteria. Studies that enrolled only patients with major depressive disorder, bipolar disorder, or patients who only misused tobacco were excluded. Interventions were more intense (e.g., integrated models of care with assertive community treatment) or were stand-alone treatments (e.g., cognitive behavioral therapy, social skills training). Three trials were conducted solely in the hospital (10\% of patients), 19 in community, and the remaining were a combination of settings. Function, mortality, relapse rates, and treatment maintenance (reported as loss to treatment) were among the reported outcomes. None of the included trials met all quality criteria. Three trials met most criteria, with the majority of trials reporting adequate randomization techniques. However, few trials reported appropriate allocation concealment or blinding of participants. Most SOE was rated as low strength or insufficient.

\section{Long-Term Intervention: Integrated Models of Care Versus Usual Care}

Integrated models of care refer to the coordination of care at the provider (or team of providers) level for both the mental health and substance misuse diagnoses and the actual services provided vary according to the needs of the patient. Within the Cochrane review, four RCTs provided data for 735 participants. ${ }^{120}$ SOE was low for all outcomes.

\section{Function}

One trial ( $\mathrm{N}=198)$ provided data on global functioning using the GAF and found lowstrength evidence of no difference between assertive community treatment compared with usual care at 12 months ( 40.4 vs. 39.7, MD 0.70; 95\% CI -2.07 to 3.47). ${ }^{120}$ Findings were similar at multiple timepoints, beginning at 6 months through 36 months and continued to show no difference between treatments based on low-strength evidence (Appendix Table E-23).

\section{Treatment Discontinuation}

Pooled analysis of three trials ( $\mathrm{N}=603$ ) indicated no difference in likelihood of being lost to treatment between integrated treatment and usual care at 36 months ( $24 \%$ vs. $21 \%$, RR 1.09 , $95 \%$ CI 0.82 to $1.45, \mathrm{I}^{2}=0 \%$ ) based on low-strength evidence. ${ }^{120}$

\section{Substance Use}

One trial $(\mathrm{N}=143)$ found low-strength evidence of no difference between integrated assertive community treatment compared with standard case management at 36 months for not being in remission for alcohol use ( $57 \%$ vs. $50 \%$, RR $1.15,95 \%$ CI 0.84 to 1.56 ) and for drug use (58\% vs. $65 \%$, RR $0.89,95 \%$ CI 0.63 to 1.25$).{ }^{120}$

\section{Harms}

Two trials ( $\mathrm{N}=421)$ reported all-cause mortality and found low strength evidence of no difference between assertive community treatment and usual care through 36 months (3\% vs. $3 \%$, RR 1.18, $95 \%$ CI 0.39 to $\left.3.57, \mathrm{I}^{2}=0 \%\right) .{ }^{120}$ 


\section{Long-Term Intervention: Nonintegrated Models of Care Versus Usual Care}

Nonintegrated models of care describe interventions where care teams do not coordinate care. ICM with higher skilled case managers with smaller caseloads is included as a nonintegrated model. However, models still had to address the patient's substance misuse. Within the Cochrane review four trials $(\mathrm{N}=163)$ met criteria for nonintegrated models of care. ${ }^{120}$ Included interventions were nonintegrated assertive community treatment ( 2 trials, $\mathrm{N}=84$ ) and intense case management (two trials, $\mathrm{N}=79$ ). Trials were small and of lower quality and provided insufficient strength evidence to compare patient function and loss to treatment between nonintegrated models of care and usual care.

\section{Short-Term, Patient-Focused Interventions: Cognitive-Behavioral Therapy Versus Usual Care \\ CBT was an included intervention to address substance misuse in patients with a dual diagnosis of schizophrenia and substance misuse disorder. ${ }^{120}$}

Treatment discontinuation. Pooled analysis from two trials $(\mathrm{N}=152)$ found no difference between treatment with CBT and usual care at 3 months in risk of discontinuing treatment (12\% vs. $10 \%$, RR 1.12; $95 \%$ CI 0.44 to $2.86, \mathrm{I}^{2}=0 \%$ ) based on low-strength evidence. ${ }^{120}$

\section{Short-Term, Patient-Focused Interventions: Social Skills Training Versus Usual Care \\ Social skills training was included help patients improve interpersonal skills and relationships and manage conflict in social situations involving substance misuse. ${ }^{120}$ Evidence for loss to treatment was considered insufficient to draw meaningful conclusions based on data from two trials $(\mathrm{N}=94)$.}

\section{Demographic Characteristics}

\section{Age}

Evidence of the effect of nonpharmacological interventions on schizophrenia outcomes from subgroup analyses based on patient age, whereas very limited, suggests that age is not a moderating factor. Systematic reviews of $\mathrm{CBT}^{79}$ and cognitive remediation ${ }^{84}$ review found no difference between interventions and usual care when trials were stratified according to age. In individual trials of cognitive remediation ${ }^{89}$ and social skills training ${ }^{103,104}$ enrolling older adults (over age 50), there was also no difference in outcomes when compared with trials enrolling a younger population. ${ }^{86-88,90,97,105}$ Age-based subgroup analyses were not reported for other nonpharmacological interventions.

\section{Sex}

Evidence on differences in outcomes based on sex is extremely limited. One systematic review of 30 RCTs of CBT found no difference in negative symptom outcomes when results were stratified according to sex. ${ }^{79}$

One study of social skills training that exclusively enrolled older adults (mean age 60 years) had an effect size for men that was consistently better than that for women for outcomes related to function and symptoms, suggesting that social skills training was more effective in men compared with women. ${ }^{103,104}$ Of note is the fact that only about half of participants in this study had a diagnosis of schizophrenia or schizoaffective disorder, whereas the remaining participants 
had various mood disorders. This potentially limits the applicability of these findings, as additional subgroup analysis found consistently lower benefit of social skills training for all outcomes when compared with participants with mood disorders.

Other trials of nonpharmacological interventions did not report subgroup analyses according to sex. 


\section{Discussion}

\section{Key Findings and Strength of Evidence}

This systematic review evaluated the evidence on treatments for schizophrenia, comparing drug treatments to each other and psychosocial and other nonpharmacological interventions to usual care. The purpose was to inform clinicians, patients and their families, and guideline authors with the ultimate goal of improving patient care. The key findings and strength of evidence (SOE) for these findings are summarized in the summary of evidence tables (Tables 10 and 11). The complete assessments of strength of the evidence, according to comparisons and outcomes, are in Appendix H. In this summary, we do not include findings where the evidence was insufficient to draw conclusions. Generally, these were situations where the evidence was limited to a single study, with inadequate sample size, and only fair quality. Unfortunately, even with the large volume of studies available, there were no instances of high-strength evidence. This was primarily due to specific intervention comparisons having only a few fair-quality trials with few studies contributing evidence for a particular outcome, resulting in moderate and lowstrength evidence. The key findings are presented below by key question and prioritized outcomes. The findings are then discussed in relation to what is already known about these interventions, applicability of the findings, implications of the findings for policy and decisionmaking, limitations of the review and the evidence, and finally our research recommendations based on gaps in the evidence,

\section{Key Questions 1a and 1b: Comparative Evidence Regarding Antipsychotic Drugs}

The findings on antipsychotic drugs come from two large systematic reviews ${ }^{27-29}$ and a total of 28 additional, newer trials ${ }^{43-70}$ (One trial is included in both the first-generation antipsychotic (FGA) versus second generation antipsychotic (SGA) and SGA versus SGA sections. ${ }^{57}$ ) The prioritized outcomes were function, quality of life, response and/or remission, mortality, selfharm, core illness symptoms, overall adverse events, and withdrawal from treatment due to adverse events. The evidence is divided into SGA versus SGA and FGA versus SGA according to traditional categorization of the drugs used in the two systematic reviews, although the drugs could be considered as one group with variations in effects associated with individual drugs.

\section{Second-Generation Drugs: SGA Versus SGA}

We found the most evidence about the older SGAs (clozapine, risperidone, olanzapine, quetiapine, and ziprasidone). We also found some evidence on the most commonly reported outcomes (e.g., core illness symptom improvement) for oral aripiprazole and paliperidone. Evidence for the newer drugs (asenapine, brexpiprazole, cariprazine, iloperidone, lurasidone, paliperidone, and long-acting injectable (LAI) formulations of aripiprazole and paliperidone) is limited, with few studies, none finding a newer drug superior to an older SGA on any outcome. Similarly, quetiapine and ziprasidone were not found superior to another SGA on any outcome (Table 10). Tables showing the summary results for each drug, indicating magnitude, direction, and strength of evidence for an effect across all seven prioritized outcomes are included in Appendix I. 


\section{Benefits Outcomes}

Although functional outcomes were prioritized as most important, few studies of SGA versus SGA reported these outcomes. Very few differences were found among the older SGAs regarding effects on social, occupational, or global functioning. Low strength evidence from a single study found risperidone LAI to result in greater improvements in social function over 24 months compared with quetiapine. None of the studies of the newer SGAs reported on functional outcomes. Findings on quality of life show that there was no difference between olanzapine and risperidone or ziprasidone (moderate-strength evidence); olanzapine or risperidone oral or LAI and quetiapine; oral aripiprazole and aripiprazole monthly LAI (low-strength evidence) in studies with up to 2 years of followup.

Response and remission are dichotomous outcomes, which are measured as response or no response, remission or no remission. By definition, response and remission are outcomes that are meant to reflect clinically relevant improvement in core illness symptoms. However, response was defined in varying ways in the randomized controlled trials (RCTs), although the most common definition was 20 percent improvement on a core illness symptoms scale, such as the Positive and Negative Syndrome Scale (PANSS). Low-strength evidence from a network metaanalysis of 46 head-to-head trials found that olanzapine and risperidone were significantly more likely to result in response than quetiapine. Other comparisons and meta-regression examining the influence of study duration, dose-level, population (either treatment-resistant or first-episode status), and category of response definition did not result in any statistically significant differences between the SGAs (low-strength evidence). Remission was reported too infrequently to assess comparatively.

Improvement in core illness symptoms is a continuous outcome measured as the mean change in symptoms using a scale. A published network meta-analysis found that clozapine was superior to other oral SGAs except for olanzapine in improving core illness symptoms. Olanzapine and risperidone were not significantly different in treating core illness symptoms compared with each other, and both were superior to the other SGAs, except for paliperidone and clozapine. Paliperidone also improved core illness symptoms more than lurasidone and iloperidone. This network analysis also found that all of the drugs included were superior to placebo. These findings are low strength evidence. In treatment-resistant patients, olanzapine improved core illness symptoms more than quetiapine. These findings are based on two published network meta-analyses (low-strength evidence).

All-cause mortality is a rare event, but it is still an important outcome to evaluate as SGAs continue to be developed, approved, and marketed, and particularly as all SGAs carry a United States Food and Drug Administration (FDA) Boxed Warning against their use in older patients with dementia due to increased risk of mortality. Low-strength evidence suggests that the mortality rate is low in SGA trials and cohort studies (0 to 1.17\%), and that there were no differences in mortality rates between olanzapine and risperidone or asenapine, risperidone and quetiapine, or paliperidone palmitate monthly LAI and risperidone LAI. There were also no differences in cardiovascular mortality among risperidone, olanzapine, and quetiapine. Comparative evidence on the risk of cardiovascular or all-cause mortality was not available for the other SGA drugs. Self-harm, including suicide deaths, while infrequent is a major cause of death among individuals with schizophrenia that antipsychotics, along with other interventions, are intended to help prevent. Although clozapine is often reserved for treatment-resistant patients, due to the serious adverse event profile and required monitoring, there is moderate strength evidence supporting its superiority over the other SGAs (primarily the older ones) in 
preventing self-harm (suicide-related outcomes) in both patients at risk for suicide-related outcomes (vs. olanzapine) and in patients with unknown or mixed risk for these outcomes (vs. olanzapine, risperidone, quetiapine, ziprasidone, and aripiprazole).

\section{Harms Outcomes}

Although SGAs have somewhat differing adverse event profiles, the evidence indicates no difference in the overall risk for adverse events between asenapine and olanzapine (moderatestrength evidence) or quetiapine extended release (ER) versus quetiapine and risperidone; risperidone versus clozapine and aripiprazole; olanzapine versus paliperidone; risperidone LAI versus paliperidone and paliperidone palmitate monthly LAI; and aripiprazole versus aripiprazole monthly LAI (all low strength evidence). Given the variation in specific adverse event profiles across the SGAs, withdrawals due to adverse events is an outcome measure that has the advantage of measuring the seriousness and tolerability of adverse events experienced, including those that might be treated with another drug or dose reduction. Network meta-analysis of 90 trials indicates that risperidone LAI had significantly lower risk of withdrawal due to adverse events than five other SGAs: clozapine, lurasidone, quetiapine ER, risperidone and ziprasidone. Olanzapine had lower risk than five other SGAs: clozapine, lurasidone, quetiapine, risperidone, and ziprasidone. Aripiprazole had lower risk than clozapine and ziprasidone, and cariprazine and iloperidone had lower risk than clozapine. Comparative evidence on extrapyramidal symptoms (EPS), cardiovascular events, diabetes, weight gain, metabolic syndrome, and sexual function is summarized in the Drug Effectiveness Review Project report. ${ }^{29}$ Although these were secondary outcomes in this report, in general the evidence is not able to identify differences between drugs studied in cardiovascular adverse events, metabolic syndrome, and sexual function. Risk of diabetes and weight gain is greater with olanzapine, with increased risk of weight gain also found with clozapine and quetiapine. Findings on EPS are more mixed.

\section{Subgroups}

Evidence in subgroups was sparse and low strength. In patients experiencing their first episode of schizophrenia, response and remission were not significantly different among olanzapine, quetiapine, risperidone, ziprasidone, aripiprazole, or paliperidone. Most studies also reported no difference in improvement in core illness symptoms, measured by symptoms scales. Although core illness symptoms were more improved with paliperidone than ziprasidone or aripiprazole, response rates did not differ significantly. Response rates with olanzapine and risperidone were similar in patients with first-episode schizophrenia compared with patients with multiple previous episodes. Findings on core illness symptoms or response did not differ according to the duration of study, the specific drugs compared, in women, or whether or not studies were blinded. Evidence on SGA treatment discontinuation was more limited, with conflicting findings from five trials. An included systematic review reports that the incidence of clinically important weight gain is significant in first episode patients, who have little previous exposure to antipsychotics, but differences among the SGA drugs has not been shown. These studies did not find a difference in benefits outcomes between risperidone and olanzapine over the first 3 years of treatment, but they found that that risperidone had higher risk of some specific adverse events (worsening akathisia, sexual dysfunction, or amenorrhea). Aripiprazole had either lower rates of or longer time to discontinuation due to adverse events than ziprasidone or quetiapine. 
In treatment-resistant patients, a network meta-analysis of 40 RCTs indicated that olanzapine resulted in greater improvement in core illness symptoms, although the difference in mean change (-6 points) in the PANSS may not meet minimal clinically important difference criteria (11.5 points for more severe symptoms), depending on the severity of the patients symptoms at baseline. A network meta-analysis of negative symptoms also found olanzapine significantly better than the other older SGAs, whereas response rates and all-cause treatment discontinuations indicated no significant differences among the older SGAs. Clozapine had fewer discontinuations due to lack of efficacy than risperidone and quetiapine.

Across the 46 trials reporting response, the rates ranged from 20 to 80 percent across SGA trial arms. The variation appears be associated with prior drug exposure of patients enrolled; patients resistant to previous treatment had lower rates of response and those with a first episode had greater rates.

The evidence on other subgroups of patients is limited. Analysis of age subgroups did not find differences for comparisons of olanzapine with risperidone. Women had greater improvements than men in core illness symptoms with clozapine and in quality of life with olanzapine. Improvement in core illness symptoms was similar in Asian patients, compared with overall study populations for comparisons of aripiprazole and paliperidone with olanzapine, quetiapine, and risperidone. Among illicit drug users, differences between older SGAs were not found in rate or time to drug discontinuation. Response rates with olanzapine and risperidone were similar in patients with a history of cannabis use disorders and in those without such history.

\section{First-Generation Antipsychotic Versus Second-Generation Antipsychotic}

Although the SGAs were initially marketed as having multiple advantages over the FGAs, there has been concern that the evidence on first-generation versus second-generation antipsychotics was biased toward the SGAs in various ways (e.g., using higher than typical doses of the first-generation drugs). The Clinical Antipsychotic Trials of Intervention Effectiveness (CATIE) trial included one FGA along with five SGAs to test this theory. The trial did not find perphenazine to be inferior to the other drugs, with the exception of olanzapine. However, the CATIE trial did not resolve the questions around the use of FGAs in current practice, such that a thorough review of the comparative evidence is still necessary. The findings of the comprehensive systematic review of FGAs versus SGAs published in 2012 are not substantially changed with the additional consideration of five newer studies (two good quality, three fair quality). The 111 trials included in the previously published systematic review were rated as mainly fair quality (70 studies), with 41 rated as poor quality, and none rated as good quality. The FGA evidence is largely about haloperidol, with 108 studies, and only 7 of perphenazine and 4 of fluphenazine. Olanzapine was the most commonly compared SGA.

\section{Benefits Outcomes}

Quality of life, a highly prioritized outcome, was not different between the FGAs and SGAs, based on low (quetiapine and risperidone) to moderate (olanzapine) strength evidence. Only ziprasidone was found better than haloperidol with low strength evidence. Evidence on functional outcomes was insufficient to draw conclusions. Moderate strength evidence suggests that risperidone is not different from haloperidol in response rates. Low strength evidence finds that response and remission were better with olanzapine than haloperidol, but no differences 
were found in response between haloperidol and aripiprazole, quetiapine and ziprasidone or in remission between haloperidol and ziprasidone.

Comparative evidence on core illness symptoms is only available for haloperidol versus older SGAs. Moderate strength evidence suggests that core illness symptoms were improved significantly more with olanzapine and risperidone than haloperidol, but evidence on other comparisons did not show significant differences (low strength evidence). Olanzapine improved negative symptoms significantly more than haloperidol (moderate strength evidence), and risperidone and aripiprazole improved negative symptoms significantly more than haloperidol (low strength evidence).

\section{Harms Outcomes}

Overall rates of patients reporting adverse events were 11 to 20 percent higher with haloperidol versus aripiprazole (moderate strength evidence), risperidone, and ziprasidone (low strength evidence). Similarly, moderate strength evidence indicates a higher rate of withdrawal from study (and treatment) due to adverse events with haloperidol, versus aripiprazole, olanzapine, risperidone, and ziprasidone. There were no differences in withdrawal due to adverse events between haloperidol and clozapine or quetiapine.

\section{Subgroups}

Evidence comparing FGAs to SGAs in population subgroups is fairly limited, with unclear implications. In general, differences in outcomes were not found between FGAs and SGAs in patients with a first episode of schizophrenia. In treatment-resistant patients the effects on total core illness symptoms and negative symptoms mirrored the findings in the overall population. Response and core illness symptom improvement was similar in Asian populations and the overall study populations. In patients with co-occurring substance use disorder, core illness symptoms were improved more with olanzapine than haloperidol, but improvement in core illness symptoms was comparable for risperidone and haloperidol.

Evidence on pharmacological treatments is summarized in Table 10. 
Table 10. Summary of evidence for pharmacological treatments

\begin{tabular}{|c|c|c|c|}
\hline Comparison & Outcome & $\begin{array}{l}\text { Strength of } \\
\text { Evidence }\end{array}$ & Conclusions \\
\hline \multirow[t]{6}{*}{ SGA vs. SGA } & Function & Low & 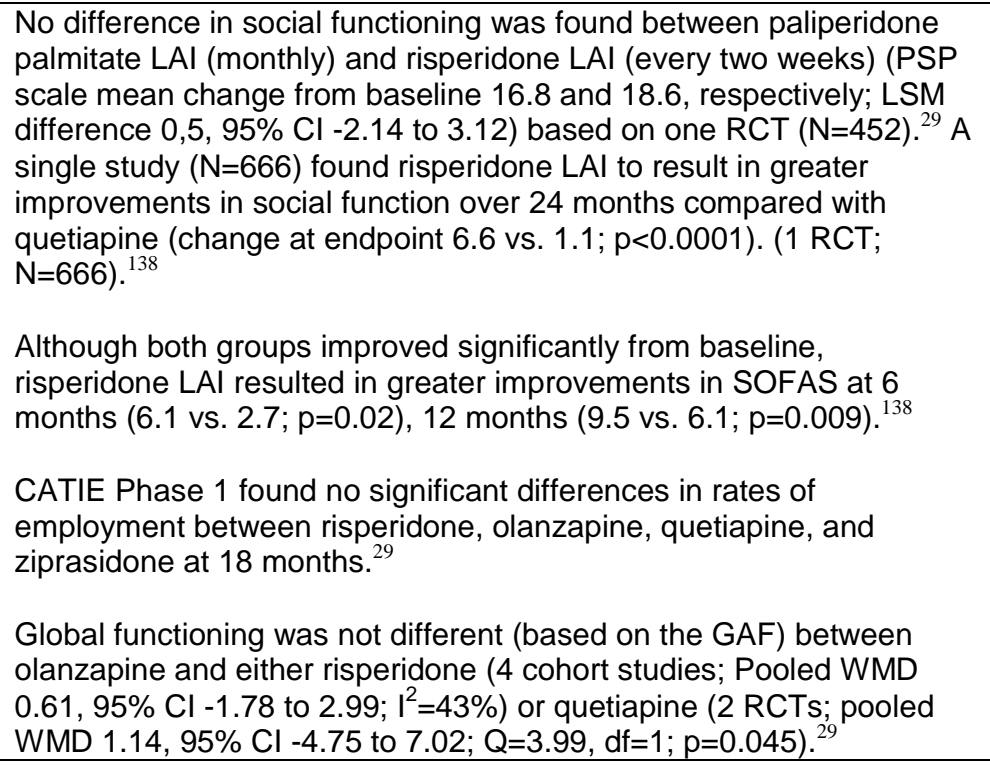 \\
\hline & Quality of life & Moderate & $\begin{array}{l}\text { Olanzapine was not found significantly different than risperidone ( } 2 \\
\text { RCTs) or ziprasidone (2 RCTs) at } 12 \text { months using the QLS scale } \\
\text { (change in scores ranged from } 0.19 \text { to } 0.26) .{ }^{29}\end{array}$ \\
\hline & Quality of life & Low & $\begin{array}{l}\text { Olanzapine was not found significantly different than quetiapine (1 } \\
\text { RCT) at } 12 \text { months using the QLS scale. }{ }^{29} \\
\text { Risperidone was not found significantly different from quetiapine or } \\
\text { ziprasidone at } 12 \text { months using the QLS scale (1 RCT each; range of } \\
\text { change in scores } 0.19 \text { to } 0.26){ }^{29} \\
\text { Risperidone LAI was not found different from quetiapine on the SF-12 } \\
\text { or SQLS-R4 at } 24 \text { months. }{ }^{138}\end{array}$ \\
\hline & $\begin{array}{l}\text { Response/ } \\
\text { remission }\end{array}$ & Low & $\begin{array}{l}\text { Response was significantly more likely with olanzapine (OR } 1.71 \text {, } \\
95 \% \mathrm{Cl} 1.11 \text { to } 2.68 \text { ) and risperidone (OR } 1.41,5 \% \mathrm{Cl} 1.01 \text { to } 2.00) \\
\text { than quetiapine, based on a network meta-analysis of } 46 \text { head-to- } \\
\text { head RCTs. }\end{array}$ \\
\hline & Mortality & Low & $\begin{array}{l}\text { With incidence rates of } 0 \text { to } 1.17 \% \text {, significant differences in mortality } \\
\text { were not found in two RCTs each ( } 4 \text { to } 24 \text { months duration) of } \\
\text { asenapine with olanzapine (RR } 2.49,95 \% \mathrm{Cl} 0.54 \text { to } 11.5){ }^{154,187} \\
\text { quetiapine and risperidone (RR } 3.24,95 \% \mathrm{Cl} 0.72 \text { to } 14.6){ }^{184,186} \text { and } \\
\text { paliperidone palmitate } \mathrm{LA} \text { (monthly) versus risperidone LAl (RR } 1.26 \text {, } \\
95 \% \mathrm{Cl} 0.21 \text { to } 7.49) .{ }^{136,188} \\
\text { Retrospective cohort studies found no significant difference in the risk } \\
\text { of all-cause (1 study, } \mathrm{N}=48,595) \text { or cardiovascular mortality ( } 2 \\
\text { studies, } \mathrm{N}=55,582) \text { between risperidone, olanzapine, and } \\
\text { quetiapine. }\end{array}$ \\
\hline & $\begin{array}{l}\text { Reduction in } \\
\text { self-harm }\end{array}$ & Low & $\begin{array}{l}\text { Clozapine was found superior to olanzapine in preventing significant } \\
\text { suicide attempts or hospitalization to prevent suicide (HR 0.76, } 95 \% \\
\mathrm{Cl} 0.58 \text { to } 0.97 \text { ) and CGI-Suicide Severity (SS) ratings of "much } \\
\text { worse" or "very much worse" (HR } 0.78,95 \% \mathrm{Cl} 0.61 \text { to } 0.99) ; \\
\text { NNT=12) among patients at high risk. Observational studies confirm } \\
\text { these findings in broader populations. }{ }^{29}\end{array}$ \\
\hline
\end{tabular}




\begin{tabular}{|c|c|c|c|}
\hline Comparison & Outcome & $\begin{array}{l}\text { Strength of } \\
\text { Evidence }\end{array}$ & Conclusions \\
\hline \multirow[t]{4}{*}{ SGA vS. SGA } & $\begin{array}{l}\text { Core illness } \\
\text { symptoms: } \\
\text { Total } \\
\text { symptoms }\end{array}$ & Low & $\begin{array}{l}\text { Clozapine was found to improve core illness symptoms significantly } \\
\text { more than the other SGAs, except for olanzapine (network meta- } \\
\text { analysis of } 212 \text { RCTs; SMDs on PANSS or BPRS -0.32 to -0.55). }{ }^{194} \\
\text { All of the SGAs were superior to placebo (SMDs }-0.33 \text { to -0.88). } \\
\text { Olanzapine and risperidone were found to improve symptoms more } \\
\text { than the other SGAs, except for each other and paliperidone (SMDs - } \\
0.13 \text { to -0.26). } \\
\text { Paliperidone was found to improve symptoms more than lurasidone } \\
\text { and iloperidone (SMDs=-0.17). }{ }^{194} \\
\text { In a separate network analysis of } 40 \text { RCTs of clozapine, risperidone, } \\
\text { olanzapine, quetiapine, and ziprasidone in patients who were } \\
\text { resistant to treatment, the only significant difference was that the } \\
\text { mean change in the PANSS was greater with olanzapine than } \\
\text { quetiapine (SMD -0.29, } 95 \% \text { Cl -0.56 to -0.13). }{ }^{183}\end{array}$ \\
\hline & $\begin{array}{l}\text { Overall/any } \\
\text { adverse } \\
\text { events }\end{array}$ & Moderate & $\begin{array}{l}\text { There was no significant difference in overall adverse event reporting } \\
\text { between asenapine and olanzapine in five RCTs (in } 4 \\
\text { publications). }{ }^{154,176,187,225}\end{array}$ \\
\hline & $\begin{array}{l}\text { Overall/any } \\
\text { adverse } \\
\text { events }\end{array}$ & Low & $\begin{array}{l}\text { There was no difference between groups based on two to three trials } \\
\text { each comparing: quetiapine ER versus quetiapine and risperidone; } \\
\text { risperidone versus clozapine and aripiprazole; olanzapine versus } \\
\text { paliperidone; risperidone LAl versus paliperidone and paliperidone } \\
\text { palmitate monthly LAI; and aripiprazole versus aripiprazole monthly } \\
\text { LAl. } 45-47,49-51,54-56,61,123,125,133,136,148,150,151,154,155,161,163,167,169,171,172,174-178,184,186- \\
188,196-227\end{array}$ \\
\hline & $\begin{array}{l}\text { Withdrawal } \\
\text { due to } \\
\text { adverse } \\
\text { events }\end{array}$ & Low & 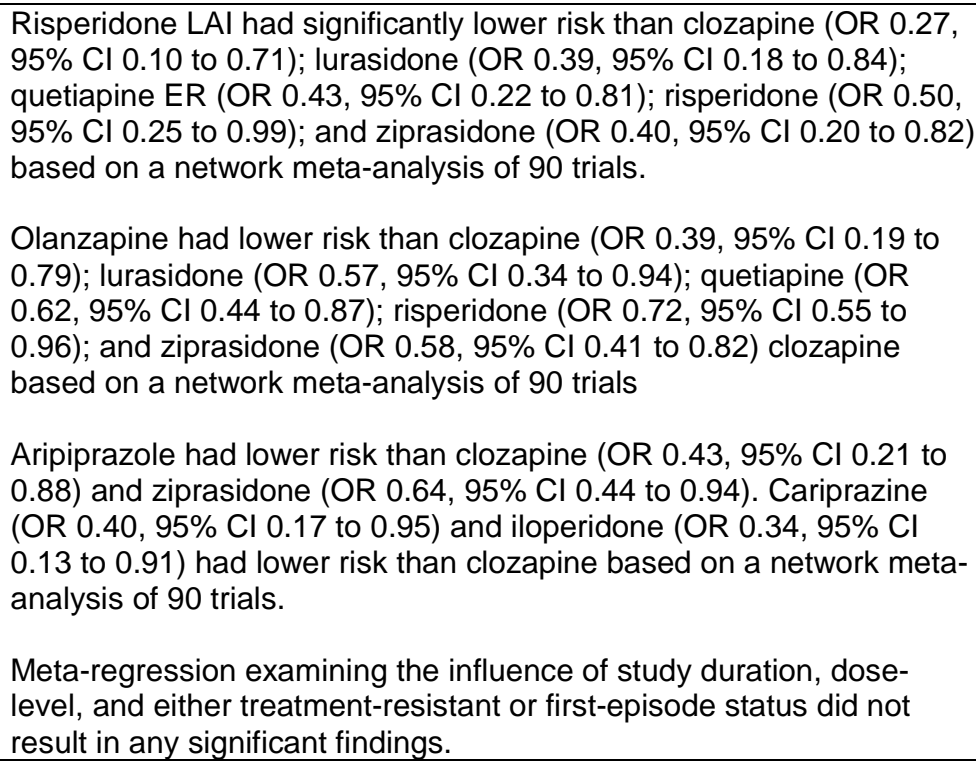 \\
\hline \multirow[t]{3}{*}{ FGA vS. SGA } & Quality of life & Moderate & $\begin{array}{l}\text { There were no differences between haloperidol and olanzapine in } \\
\text { quality of life scores. (SOE: Moderate); or perphenazine and } \\
\text { olanzapine, quetiapine, risperidone or ziprasidone. }{ }^{28}\end{array}$ \\
\hline & Quality of life & Low & $\begin{array}{l}\text { One trial comparing haloperidol with ziprasidone found a positive } \\
\text { effect favoring ziprasidone (effect estimate }-12.12 ; 95 \% \mathrm{Cl}-22.06 \text { to - } \\
\text { 2.17) with no difference between groups in another trial. }{ }^{28} \\
\text { There were no differences between haloperidol and olanzapine; or } \\
\text { perphenazine and quetiapine, risperidone or ziprasidone. }{ }^{28}\end{array}$ \\
\hline & $\begin{array}{l}\text { Response/ } \\
\text { Remission }\end{array}$ & Moderate & $\begin{array}{l}\text { There was no difference in response rates between haloperidol and } \\
\text { risperidone (16 RCTs, N=3,452; RR } 0.94 ; 95 \% \mathrm{Cl} 0.87 \text { to } 1.02){ }^{28}\end{array}$ \\
\hline
\end{tabular}




\begin{tabular}{|c|c|c|c|}
\hline Comparison & Outcome & $\begin{array}{l}\text { Strength of } \\
\text { Evidence }\end{array}$ & Conclusions \\
\hline \multirow[t]{7}{*}{ FGA vs. SGA } & $\begin{array}{l}\text { Response/ } \\
\text { Remission }\end{array}$ & Low & 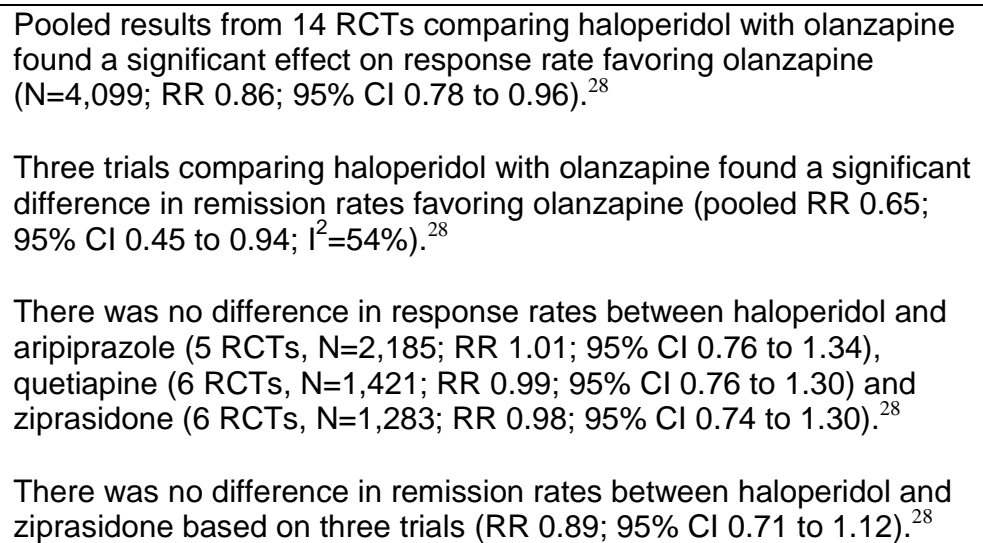 \\
\hline & $\begin{array}{l}\text { Core illness } \\
\text { symptoms: } \\
\text { Total } \\
\text { symptom } \\
\text { score }\end{array}$ & Moderate & $\begin{array}{l}\text { There were significant difference in total PANSS between haloperidol } \\
\text { and olanzapine ( } 15 \mathrm{RCTs}, \mathrm{N}=4,209 ; \text { mean difference } 2.31 ; 95 \% 0.44 \\
\text { to } 4.18) \text { and risperidone ( } 21 \mathrm{RCTs}, \mathrm{N}=4,020 ; \text { mean difference } 3.24 \text {; } \\
95 \% \mathrm{Cl} 1.62 \text { to } 4.86) \text {, both favoring the SGA over haloperidol. }{ }^{28}\end{array}$ \\
\hline & $\begin{array}{l}\text { Core illness } \\
\text { symptoms: } \\
\text { Total } \\
\text { symptom } \\
\text { score }\end{array}$ & Low & $\begin{array}{l}\text { There were no differences in total PANSS, BPRS, CGI-S and CGI-I } \\
\text { scores for other FGA versus SGA comparisons. }{ }^{28}\end{array}$ \\
\hline & $\begin{array}{l}\text { Core illness } \\
\text { symptoms: } \\
\text { Negative } \\
\text { symptom } \\
\text { score }\end{array}$ & Moderate & $\begin{array}{l}\text { Olanzapine was more effective than haloperidol at improving negative } \\
\text { symptoms based on SANS scores ( } 5 \text { RCTs, } N=535 \text {; mean difference } \\
2.56 ; 95 \% \mathrm{Cl} 0.94 \text { to } 4.18 \text { ). }{ }^{28}\end{array}$ \\
\hline & $\begin{array}{l}\text { Core illness } \\
\text { symptoms: } \\
\text { Negative } \\
\text { symptom } \\
\text { score }\end{array}$ & Low & $\begin{array}{l}\text { Using the negative symptoms subscale of the PANSS scale, mean } \\
\text { differences (although small) between haloperidol and aripiprazole ( } 3 \\
\mathrm{RCTs}, \mathrm{N}=1,701 ; 0.80 ; 95 \% \mathrm{Cl} 0.14 \text { to } 1.46 \text { ), olanzapine ( } 14 \mathrm{RCTs} \text {, } \\
\mathrm{N}=3,742 ; 1.06 ; 95 \% \mathrm{Cl} 0.46 \text { to } 1.67) \text {, and risperidone ( } 22 \mathrm{RCTS} \text {, } \\
\mathrm{N}=4,142 ; 0.80 ; 95 \% \mathrm{Cl} 0.14 \text { to } 1.46 \text { ) all favored the SGA.8 } \\
\text { There were no differences in negative PANSS or SANS scores for } \\
\text { other FGA versus SGA comparisons. }\end{array}$ \\
\hline & $\begin{array}{l}\text { Overall/any } \\
\text { adverse } \\
\text { events }\end{array}$ & Moderate & $\begin{array}{l}\text { Overall adverse event rates favored SGAs when comparing } \\
\text { haloperidol with aripiprazole (3 RCTs, N=1,713; RR } 1.11 ; 95 \% \mathrm{Cl} \\
1.06 \text { to } 1.17 ; I^{2}=0 \% \text { ), risperidone (8 RCTs, N=1313; RR } 1.20 ; 95 \% \mathrm{Cl} \\
1.01 \text { to } 1.42 ; I^{2}=84 \% \text { ), and ziprasidone (6 RCTs, N=1448; RR } 1.13 \text {; } \\
\left.95 \% \text { Cl } 1.03 \text { to } 1.23 ; I^{2}=31 \%\right) .{ }^{28}\end{array}$ \\
\hline & $\begin{array}{l}\text { Withdrawal } \\
\text { due to } \\
\text { adverse } \\
\text { events }\end{array}$ & Moderate & 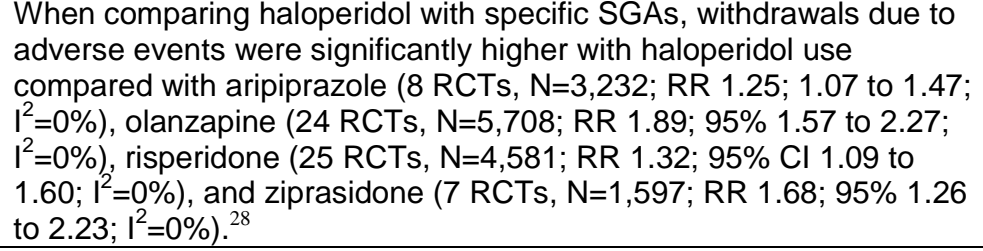 \\
\hline
\end{tabular}

BPRS = Brief Psychiatric Rating Scale; CATIE = Clinical Antipsychotic Trials of Intervention Effectiveness; CGI = Clinical Global Impressions scale; $\mathrm{CI}$ = confidence interval; $\mathrm{df}$ = degrees of freedom; ER = extended release; FGA = first-generation antipsychotic; GAF = Global Assessment of Functioning; HR = hazard ratio; IR = immediate release; LAI = long-acting injectable; LSM = least squares mean; NNT = number needed to treat; OR = odds ratio; PANSS = Positive and Negative Syndrome Scale; PSP = Personal and Social Performance Scale; RCTs = randomized controlled trials; RR = risk ratio; SANS = Scale for the Assessment of Negative Symptoms; SF-12 = 12-item Short Form Health Survey; SGA = second-generation antipsychotic; SMD = standardized mean difference, SOE = strength of evidence; SOFAS = Social and Occupational Functioning Assessment Scale; SQLS-R4 = Schizophrenia Quality of Life Scale- Revision 4; WMD = weighted mean difference 


\section{Key Questions 2a and 2b: Evidence on Psychosocial and Other Nonpharmacological Interventions Versus Usual Care}

The studies included in our review evaluated 13 discrete psychosocial interventions in comparison with usual care. The purpose was not to evaluate head-to-head comparisons of active interventions to each other. The evidence base is comprised of 13 systematic reviews (11 good quality, 2 fair quality) that included 271 trials relevant to this report. In addition, we included 27 trials that were not included in these reviews. Of these new trials, four were good, 20 were fair, and three were poor quality. The strength of the body of evidence for each intervention-outcome pair was moderate and low strength overall (Table 11).

\section{Benefit Outcomes}

Patients receiving assertive community treatment were more likely to be living independently and employed and were less likely to be homeless or to discontinue treatment compared with patients assigned to usual care (moderate SOE). There were no significant difference in the degree of improvement in core illness symptoms or social functioning, and there were no differences in arrests, imprisonment, or police contacts compared with usual care (low SOE). Rehospitalization, the target of this intervention, was also significantly lower with assertive community treatment than usual care, as well as the duration of hospital stay.

Cognitive behavioral therapy (CBT) resulted in improvements in global function and quality of life (low SOE), and overall core illness symptoms (moderate SOE) compared with usual care during treatment and with up to 6 months followup. In studies with longer-term followup after CBT ended, these differences were not significant, although there were few studies with a usual care control group. Low strength evidence suggests that improvement in negative symptoms was not different between CBT and usual care.

Cognitive remediation resulted in small positive effects on social, occupational, and global function, core illness symptoms (low SOE) and negative symptoms (moderate SOE) compared with usual care over 15 to 16 weeks of treatment.

Family interventions resulted in significantly lower relapse rates than usual care at up to 24 months treatment and at 5 years followup, although differences were not found from 25 to 36 months. Family interventions were also associated with improved core illness symptoms. Differences were not noted in social function, including employment and housing situation, reduction in self-harm (moderate SOE for reduced relapse from 7 to 12 months, low SOE for all others).

Intensive case management (ICM) was not found to improve global function, quality of life, or core illness symptoms more than usual care (low SOE).

Illness self-management training interventions reduced symptom severity (moderate SOE) and relapse rates (low SOE). No significant difference was found for negative symptoms (low SOE). Greater fidelity to intervention was associated with better effects.

Psychoeducation had a greater effect than usual care on global function at 1 year and resulted in lower relapse rates at nine to 18 months (moderate SOE).

Social skills training improved social function at 6 months, 1 year, and 2 years, compared with usual care. Core illness symptoms and negative symptoms were also improved more with social skills training than usual care.

Supported employment, specifically the individual placement and support (IPS) model intervention, resulted in significantly better employment outcomes over 2 years compared with usual care. More patients gained either employment (competitive or any job), had worked more 
hours, were employed longer, and earned more money than those receiving usual care. Evidence with comparisons with other vocational training confirmed these findings (moderate SOE).

Supportive therapy was not significantly different from usual care in improving global or social function (low SOE).

\section{Subgroups}

\section{Demographic Subgroups}

We found limited subgroup analyses across psychosocial interventions to identify potential patient characteristics that might predict outcomes. Limited evidence on social skills training from one RCT of a mixed population (about 50\% diagnosed with schizophrenia or schizoaffective disorder) suggested that the intervention may be more effective in men than women for improving social function and core illness symptoms.

\section{Clinical Subgroups}

\section{Early team-based multi-component treatment programs for first-episode psychosis} resulted in significant improvements in global function with up to 2 years of treatment compared with usual care; participants were more likely to be working or in school, but there were no significant differences in housing status (moderate SOE). Quality of life was improved and participants in team-based multi-component treatment programs were less likely to relapse (moderate SOE), but there was no difference in total PANSS scores or rates of self-harm compared with usual care (low SOE).

In patients with co-occurring substance use disorder and schizophrenia, long-term integrated models of care did not result in different improvements in function, mortality, or substance abuse than usual care.

\section{Harms Outcomes}

Four trials and seven systematic reviews assessed or reported any type of harms associated with psychosocial or other nondrug interventions. The few that did (e.g., studies of family interventions) resulted in insufficient evidence.

Evidence for Key Question 2 is summarized in Table 11.

Table 11. Summary of evidence for psychosocial and nonpharmacological interventions

\begin{tabular}{|c|c|c|c|}
\hline $\begin{array}{l}\text { Intervention vs. } \\
\text { Usual Care }\end{array}$ & Outcome & $\begin{array}{l}\text { Strength } \\
\text { of } \\
\text { Evidence }\end{array}$ & Conclusions \\
\hline $\begin{array}{l}\text { Assertive } \\
\text { community } \\
\text { treatment }(A C T)\end{array}$ & Social Function & Low & $\begin{array}{l}\text { ACT did not improve improved social function more than } \\
\text { usual care, based on pooled analysis of } 3 \text { studies (MD } 0.03 \text {; } \\
95 \% \mathrm{Cl}-0.28 \text { to } 0.34 \text { ); an additional trial also found no } \\
\text { difference. } \\
\text { Compared with usual care, there were no significant } \\
\text { differences in arrests ( } 2 \text { trials, total } \mathrm{N}=604 \text {; OR } 1.17,95 \% \mathrm{Cl} \\
0.60 \text { to } 2.29 ; \mathrm{I}^{2}=0 \% \text { ), imprisonment ( } 4 \text { trials, total } \mathrm{N}=471 ; \mathrm{OR} \\
1.19,95 \% \mathrm{Cl} 0.70 \text { to } 2.01 ; I^{2}=27 \% \text { ), or police contacts }(2 \\
\left.\text { trials, total } \mathrm{N}=149 ; \mathrm{OR} 0.76,95 \% \mathrm{Cl} 0.32 \text { to } 1.79 ; \mathrm{I}^{2}=84 \%\right) .{ }^{71}\end{array}$ \\
\hline$A C T$ & $\begin{array}{l}\text { Housing } \\
\text { Function }\end{array}$ & Moderate & $\begin{array}{l}\text { Patients receiving ACT had lower likelihood of not living } \\
\text { independently ( } 4 \text { trials; OR } 0.52,95 \% \mathrm{Cl} 0.35 \text { to } 0.79),{ }^{71} \text { or } \\
\text { being homeless ( } 4 \text { trials, OR } 0.24,95 \% \mathrm{Cl} 0.12 \text { to } 0.48 \text { ) } \\
\text { compared with usual care. }{ }^{71,72}\end{array}$ \\
\hline
\end{tabular}




\begin{tabular}{|c|c|c|c|}
\hline $\begin{array}{l}\text { Intervention vs. } \\
\text { Usual Care }\end{array}$ & Outcome & $\begin{array}{l}\text { Strength } \\
\text { of } \\
\text { Evidence }\end{array}$ & Conclusions \\
\hline & Employment & Moderate & $\begin{array}{l}\text { Patients receiving ACT had lower likelihood of being } \\
\text { unemployed than those receiving usual care ( } 3 \text { trials; OR } \\
0.46,95 \% \mathrm{Cl} 0.21 \text { to } 0.99) .^{71}\end{array}$ \\
\hline & $\begin{array}{l}\text { Core illness } \\
\text { symptoms: } \\
\text { symptoms }\end{array}$ & Moderate & $\begin{array}{l}\text { Core illness symptoms improved with both } \mathrm{ACT} \text { and usual } \\
\text { care, with no differences between groups (MD, }-0.14 ; 95 \% \\
\mathrm{Cl}-0.36 \text { to } 0.08 \text { ); one additional trial also found no difference } \\
\text { in symptom improvement. }\end{array}$ \\
\hline \multirow[t]{5}{*}{$\begin{array}{l}\text { Cognitive } \\
\text { behavioral therapy } \\
(C B T)\end{array}$} & $\begin{array}{l}\text { Global, Social } \\
\text { and } \\
\text { Occupational } \\
\text { Function } \\
\text { Short-term ( } \leq 6 \\
\text { months since } \\
\text { initiation) }\end{array}$ & Moderate & $\begin{array}{l}\text { CBT improved global function more than usual care in the } \\
\text { short-term (GAF scale, } 6 \text { trials; MD } 5.49 ; 95 \% \mathrm{Cl} 1.85 \text { to } \\
9.14 \text { ). } \\
\text { CBT improved social and occupational function more than } \\
\text { usual care in the short-term (SOFAS scores, } 2 \text { trials; MD } \\
9.11 ; 95 \% \mathrm{Cl} 6.31 \text { to } 11.91)^{78,83,95,274,278,279}\end{array}$ \\
\hline & $\begin{array}{l}\text { Global, Social } \\
\text { and } \\
\text { Occupational } \\
\text { Function } \\
\text { Long-term (> } 1 \\
\text { year since } \\
\text { initiation) }\end{array}$ & Low & $\begin{array}{l}\text { Global and social/occupational function were not different to } \\
\text { usual care in longer-term followup }(>1 \text { year, GAF and } \\
\text { SOFAS scores in one systematic review and three RCTs; } \\
\text { one other RCT found a positive effect in favor of } \\
\text { CBT). }{ }^{76,78,93,276,279}\end{array}$ \\
\hline & Quality of life & Low & $\begin{array}{l}\text { CBT improved quality of life more than usual care in the } \\
\text { short term (12 to } 24 \text { weeks followup) based on two trials, }{ }^{80,83} \\
\text { but this difference was not found in two trials with longer } \\
\text { followup (18 to } 24 \text { months). }{ }^{93,279}\end{array}$ \\
\hline & $\begin{array}{l}\text { Core Illness } \\
\text { symptoms }\end{array}$ & Moderate & $\begin{array}{l}\text { CBT had a greater effect on core illness symptoms than } \\
\text { usual care during treatment ( } 8 \text { weeks to } 5 \text { years) based on a } \\
\text { good-quality systematic review of } 34 \text { studies (SMD }-0.33 \text {, } \\
95 \% \mathrm{Cl}-0.47 \text { to }-0.19) .^{77}\end{array}$ \\
\hline & $\begin{array}{l}\text { Negative } \\
\text { symptoms }\end{array}$ & Low & $\begin{array}{l}\text { No meaningful difference between CBT and usual care in } \\
\text { negative symptom improvement based on two systematic } \\
\text { reviews. }{ }^{77,79} \text { The target of the CBT was varied in the studies } \\
\text { included, with few targeting negative symptoms. }\end{array}$ \\
\hline \multirow[t]{3}{*}{$\begin{array}{l}\text { Cognitive } \\
\text { Remediation }\end{array}$} & $\begin{array}{l}\text { Global, Social, } \\
\text { Occupational } \\
\text { Function }\end{array}$ & Low & $\begin{array}{l}\text { Compared with usual care, cognitive remediation resulted in } \\
\text { a small positive effect on social, occupational, living situation } \\
\text { and global function, based six RCTs (effect sizes ranged } \\
\text { from } 0.16 \text { to } 0.40) .{ }^{87-89}\end{array}$ \\
\hline & $\begin{array}{l}\text { Core Illness } \\
\text { symptoms }\end{array}$ & Moderate & $\begin{array}{l}\text { Cognitive remediation resulted in small improvements in } \\
\text { core illness symptoms, based on } 2 \text { trials (SMD - } 0.62(95 \% \\
\text { Cl-1.01 to }-0.24) .{ }^{86,89}\end{array}$ \\
\hline & $\begin{array}{l}\text { Negative } \\
\text { symptoms }\end{array}$ & Moderate & $\begin{array}{l}\text { Negative symptoms were significantly improved with } \\
\text { cognitive remediation compared with usual care (1SR of } 18 \\
\text { RCTs, effect size }-0.36,95 \% \mathrm{Cl}-0.52 \text { to }-0.20) .{ }^{85}\end{array}$ \\
\hline \multirow[t]{2}{*}{$\begin{array}{l}\text { Early Interventions } \\
\text { for First-Episode } \\
\text { Psychosis }\end{array}$} & Global Function & Moderate & $\begin{array}{l}\text { Pooled results found that the early team-based multi- } \\
\text { component treatment programs resulted in higher } \\
\text { functioning, based on GAF and GAS scores after up to } 2 \\
\text { years of treatment ( } 3 \text { RCTs; WMD: } 3.88 ; 95 \% \text { Cl } 0.91 \text { to } \\
\left.6.85 ; I^{2}=64 \%\right) .111,113,114,296\end{array}$ \\
\hline & Social Function & Moderate & $\begin{array}{l}\text { Early team-based multi-component treatment programs } \\
\text { resulted in significantly more people }(22 \%) \text { working or in } \\
\text { school after up to } 2 \text { years of treatment (3 RCTs; RR } 1.22 \text {; } \\
95 \% \mathrm{Cl} 1.01 \text { to } 1.47) .111,113,114,296\end{array}$ \\
\hline $\begin{array}{l}\text { Early Interventions } \\
\text { for First-Episode } \\
\text { Psychosis }\end{array}$ & $\begin{array}{l}\text { Housing } \\
\text { Function }\end{array}$ & Low & $\begin{array}{l}\text { There was no significant differences between early team- } \\
\text { based multi-component treatment programs and usual care } \\
\text { on housing status for up to 2-year treatment duration based } \\
\text { on two RCTs. }{ }^{111,113,296}\end{array}$ \\
\hline
\end{tabular}




\begin{tabular}{|c|c|c|c|}
\hline $\begin{array}{l}\text { Intervention vs. } \\
\text { Usual Care }\end{array}$ & Outcome & $\begin{array}{l}\text { Strength } \\
\text { of } \\
\text { Evidence }\end{array}$ & Conclusions \\
\hline & Quality of life & Moderate & $\begin{array}{l}\text { Two RCTs found significant differences between early team- } \\
\text { based multi-component treatment programs and usual care } \\
\text { on quality of life scores for up to } 2 \text {-year treatment duration } \\
\text { (pooled effect size } 0.84 ; 95 \% \mathrm{Cl} 0.14 \text { to } 1.55) .^{113,117}\end{array}$ \\
\hline & $\begin{array}{l}\text { Reduction in self- } \\
\text { harm }\end{array}$ & Low & $\begin{array}{l}\text { There was no difference in reduction in self-harm in two } \\
\text { trials of early team-based multi-component treatment } \\
\text { programs versus usual care. }\end{array}$ \\
\hline & $\begin{array}{l}\text { Core Illness } \\
\text { symptoms }\end{array}$ & Low & $\begin{array}{l}\text { There was no difference between early team-based multi- } \\
\text { component treatment programs and usual care in core } \\
\text { illness symptoms, based on three RCTs (WMD on PANSS - } \\
\left.2.53 ; 95 \% \mathrm{Cl}-5.45 \text { to } 0.39 ; I^{2}=55 \%\right){ }^{113,114,117}\end{array}$ \\
\hline & Relapse & Moderate & $\begin{array}{l}\text { Early team-based multi-component treatment program } \\
\text { participants were significantly less likely to relapse } \\
\text { compared with those in usual care based on two RCTs (RR } \\
0.64 ; 95 \% \mathrm{Cl} 0.52 \text { to } 0.79){ }^{112,114}\end{array}$ \\
\hline $\begin{array}{l}\text { Supported } \\
\text { Employment }\end{array}$ & $\begin{array}{l}\text { Occupational } \\
\text { Function }\end{array}$ & Low & $\begin{array}{l}\text { Supported employment, using the individual placement and } \\
\text { support (IPS) model resulted in significantly better } \\
\text { employment outcomes over } 2 \text { years compared with usual } \\
\text { care (more patients were employed, competitive or any job, } \\
\text { worked more hours, were employed longer, and earned } \\
\text { more money). } 109 \text { Evidence with vocational training control } \\
\text { groups supports these findings. }\end{array}$ \\
\hline \multirow[t]{6}{*}{$\begin{array}{l}\text { Family } \\
\text { Interventions }\end{array}$} & Social Function & Low & $\begin{array}{l}\text { There were no differences in social functioning scale scores } \\
\text { or not being able to live independently between family } \\
\text { intervention and usual care based on one RCT. }{ }^{95}\end{array}$ \\
\hline & $\begin{array}{l}\text { Occupational } \\
\text { Function }\end{array}$ & Low & $\begin{array}{l}\text { There were no differences in unemployment rates between } \\
\text { participants in family interventions and usual care at } 1 \text { year } \\
\text { based on one systematic review. }{ }^{91}\end{array}$ \\
\hline & $\begin{array}{l}\text { Reduction in self- } \\
\text { harm }\end{array}$ & Low & $\begin{array}{l}\text { Suicide rates were similar in family intervention participants } \\
\text { and those who received usual care in one SR, but events } \\
\text { were few. }{ }^{91}\end{array}$ \\
\hline & $\begin{array}{l}\text { Core Illness } \\
\text { symptoms }\end{array}$ & Low & $\begin{array}{l}\text { Family interventions reduced core illness symptoms based } \\
\text { on } 4 \text { trials (SMD }-0.46,05 \% \mathrm{Cl}-0.73 \text { to }-0.20) .{ }_{91,93,96} \text { but } \\
\text { there was no difference in core illness symptoms. }\end{array}$ \\
\hline & \multirow[t]{2}{*}{ Relapse } & Moderate & $\begin{array}{l}\text { Family interventions resulted in significantly lower relapse } \\
\text { rates at } 7 \text { to } 12 \text { months based on one systematic review } \\
\text { and four additional studies } \\
95 \% \mathrm{Cl} 0.54 \text { to } 0.83 \text { ). }\end{array}$ \\
\hline & & Low & $\begin{array}{l}\text { Family interventions resulted in lower relapse rates at } 0 \text { to } 6 \\
\text { months compared with usual care based on one systematic } \\
\text { review }^{91} \text { plus one additional trial }{ }^{290} \text { ( } 23 \% \text { vs. } 37 \% \text {, RR0.62; } \\
95 \% \mathrm{Cl} 0.41 \text { to } 0.92) \text {, at } 13-24 \text { months based on a } \\
\text { systematic review }{ }^{91} \text { ( } 49 \% \text { vs. } 61 \% \text {; RR } 0.75,95 \% \mathrm{Cl} 0.58 \text { to } \\
0.99 \text { ) and at } 5 \text { years followup based on a systematic } \\
\text { review } \text { pl }^{91} \text { plus one additional trial }{ }^{289} \text { ( } 78 \% \text { vs. } 94 \% \text {, RR } 0.82 ; \\
95 \% \mathrm{Cl} 0.72 \text { to } 0.94) \text {. The difference at } 25 \text { to } 36 \text { months was } \\
\text { not significant. }\end{array}$ \\
\hline $\begin{array}{l}\text { Intensive Case } \\
\text { Management } \\
\text { (ICM) }\end{array}$ & Global Function & Low & $\begin{array}{l}\text { ICM did not differ significantly from usual care in change in } \\
\text { global function, based on pooled analysis of } 3 \text { studies (MD } \\
0.46 ; 95 \% \mathrm{Cl}-0.34 \text { to } 1.26 \text { ). }{ }^{98,99} \\
\text { There was no difference in rates of imprisonment based on } \\
\text { pooled analysis of } 5 \text { trials (OR } 0.90,95 \% \mathrm{Cl} 0.45 \text { to } 1.82 \text { ). }{ }^{98}\end{array}$ \\
\hline$I C M$ & $\begin{array}{l}\text { Core Illness } \\
\text { symptoms }\end{array}$ & Low & $\begin{array}{l}\text { ICM was not significantly different to usual care in } \\
\text { improvement in core illness symptoms, based on pooled } \\
\text { analysis of } 2 \text { studies (MD, } 0.46 ; 95 \% \mathrm{Cl}-3.67 \text { to } 4.60) \text {. One } \\
\text { subsequent trial also reported no difference in symptoms } \\
\text { using a different scale. }\end{array}$ \\
\hline
\end{tabular}




\begin{tabular}{|c|c|c|c|}
\hline $\begin{array}{l}\text { Intervention vs. } \\
\text { Usual Care }\end{array}$ & Outcome & $\begin{array}{l}\text { Strength } \\
\text { of } \\
\text { Evidence }\end{array}$ & Conclusions \\
\hline \multirow[t]{3}{*}{$\begin{array}{l}\text { Illness self- } \\
\text { management }\end{array}$} & $\begin{array}{l}\text { Core Illness } \\
\text { symptoms }\end{array}$ & Moderate & $\begin{array}{l}\text { Participants receiving a self-management education } \\
\text { intervention were significantly more likely to demonstrate a } \\
\text { reduction in severity of core illness symptoms based on the } \\
\text { BPRS (5 RCTs; pooled WMD=-4.19, } 95 \% \mathrm{Cl}-5.84 \text { to - } \\
2.54) . .^{100}\end{array}$ \\
\hline & $\begin{array}{l}\text { Negative } \\
\text { symptoms }\end{array}$ & Low & $\begin{array}{l}\text { There was no change in negative symptoms based PANSS } \\
- \text { negative subscale based on five RCTs. }{ }^{100}\end{array}$ \\
\hline & Relapse & Low & $\begin{array}{l}\text { Patients receiving more than } 10 \text { self-management } \\
\text { intervention sessions had a greater reduction in the } \\
\text { likelihood of experiencing relapse compared with usual care } \\
\text { (OR } 0.41 ; 95 \% \mathrm{Cl} 0.21-0.79 \text { ), whereas those receiving } 10 \text { or } \\
\text { fewer sessions had a smaller, nonsignificant, reduction in } \\
\text { the risk of relapse (OR } 0.67,95 \% \mathrm{Cl} 0.39 \text { to } 1.15 \text { ) based on } \\
\text { one SR. }\end{array}$ \\
\hline \multirow[t]{3}{*}{ Psychoeducation } & Global Function & Low & $\begin{array}{l}\text { Psychoeducation had a greater effect than usual care on } \\
\text { global functional outcomes at } 1 \text { year of followup based on } \\
\text { one good-quality systematic review of three studies (MD - } \\
\left.5.23,95 \% \mathrm{Cl}-8.76 \text { to }-1.71 ; \mathrm{I}^{2}=79 \%\right) .{ }^{102}\end{array}$ \\
\hline & Relapse & Moderate & $\begin{array}{l}\text { Psychoeducation had a greater effect than usual care on } \\
\text { relapse rates (with or without readmission) at nine to } 18 \\
\text { months of followup based on one good-quality systematic } \\
\text { review of six studies (RR } 0.80,95 \% \mathrm{Cl} 0.70 \text { to } 0.92 ; \\
\left.\mathrm{I}^{2}=54 \%\right)^{102}\end{array}$ \\
\hline & Harms & Low & $\begin{array}{l}\text { There was no difference between psychoeducation and } \\
\text { usual care in rate of harms based on } 10 \text { RCTs. }{ }^{102}\end{array}$ \\
\hline \multirow[t]{3}{*}{$\begin{array}{l}\text { Social Skills } \\
\text { Training }\end{array}$} & Social Function & Low & $\begin{array}{l}\text { Social Function was significantly better in patients receiving } \\
6 \text { months (SMD } 1.60 ; 95 \% \mathrm{Cl} 1.19 \text { to } 2.02), 1 \text { year (SMD } \\
2.02 ; 95 \% \mathrm{Cl} 1.53 \text { to } 2.52 \text { ) and } 2 \text { years (SMD } 0.65 ; 95 \% \mathrm{Cl} \\
0.36 \text { to } 0.95) \text { of social skills training in three studies (in four } \\
\text { publications). }{ }^{97,103-105}\end{array}$ \\
\hline & $\begin{array}{l}\text { Core Illness } \\
\text { symptoms }\end{array}$ & Low & $\begin{array}{l}\text { Core illness symptoms improved more with social skills } \\
\text { training vs. usual care at } 6 \text { months based on two RCTs } \\
\text { (SMD on PANSS }-1.50(95 \% \mathrm{Cl}-1.92 \text { to }-1.09 \text { and } 2 \text { years - } \\
0.81(95 \% \mathrm{Cl}-1.22 \text { to }-0.40) .{ }^{97,105}\end{array}$ \\
\hline & $\begin{array}{l}\text { Negative } \\
\text { symptoms }\end{array}$ & Low & $\begin{array}{l}\text { Negative symptoms were consistently and significantly } \\
\text { improved with social skills training relative to usual care in } \\
\text { three studies (SMD range }-0.45 \text { to }-1.30 \text {; in four } \\
\text { publications). }\end{array}$ \\
\hline $\begin{array}{l}\text { Supportive } \\
\text { Therapy }\end{array}$ & $\begin{array}{l}\text { Global and } \\
\text { Social Function }\end{array}$ & Low & $\begin{array}{l}\text { There was no difference between supportive therapy and } \\
\text { usual care for global or social function based on two studies } \\
\text { in a systematic review. }\end{array}$ \\
\hline
\end{tabular}

ACT $=$ assertive community treatment; BPRS $=$ Brief Psychiatric Rating Scale; $\mathrm{CBT}=$ cognitive behavioral therapy; $\mathrm{CI}=$ confidence interval; GAF = Global Assessment of Functioning; GAS = Global Assessment Scale; ICM = intensive case management; $\mathrm{MD}=$ mean difference; $\mathrm{OR}$ = odds ratio; PANSS = Positive and Negative Syndrome Scale; RCT = randomized controlled trial; RR = risk ratio; SMD = standardized mean difference; SOFAS = Social and Occupational Functioning Assessment Scale; $\mathrm{SR}$ = systematic review; $\mathrm{WMD}$ = weighted mean difference

\section{Findings in Relationship to What Is Already Known}

With regard to drug therapy, the findings of our review are generally consistent with prior systematic reviews that make comparisons among the SGAs and between SGAs and FGAs. ${ }^{183,194,263,264,297,298}$ Although we incorporated the most relevant of these systematic reviews in our report, our findings differ to some extent from previous reviews because we consider outcomes prioritized by technical experts, incorporate newer evidence and the most recently approved drugs, and include three updated network meta-analyses. For example, in comparing SGAs, our network meta-analyses of response, withdrawal due to adverse events, and all-cause 
treatment discontinuation of treatment incorporate evidence on brexpiprazole and cariprazine, the two most recently approved oral drugs, and all of the long-acting injection SGAs, whereas the previously published network meta-analyses are limited to older oral drugs, included drugs not approved in the United States, and did not control for important potential effect modifiers. ${ }^{183,194,297-301}$ Therefore, there are no existing reviews that cover the same scope as this report.

Our review is consistent with other reviews in the findings on the older SGAs. Clozapine, risperidone, and olanzapine have the most consistent evidence of superiority for specific outcomes (e.g., symptom improvement, response, self-harm, all-cause treatment discontinuations, and time to discontinuation), or populations (first-episode and treatmentresistant). ${ }^{263,300,302-304}$ Other findings in this review are new; such as the finding that risperidone LAI and olanzapine result in significantly lower withdrawals due to adverse events than several other SGAs. Previous reviews did not assess key effectiveness outcomes, such as function, quality of life, and mortality.

A single comprehensive review on FGAs versus SGAs is available and serves as the basis of our report, with nine new trials included. ${ }^{27,28}$ Our findings are generally consistent with this review, which concluded that there were few differences of clinical importance for effectiveness outcomes, and that evidence on patient-important outcomes and adverse events were not well studied. In adding new evidence, we found moderate-strength evidence of specific SGAs resulting in better symptom improvement (olanzapine and risperidone) and lower rates of overall adverse events (aripiprazole) and withdrawal due to adverse events (aripiprazole, olanzapine, risperidone, and ziprasidone) than haloperidol.

For the psychosocial interventions, our findings are consistent with some prior review findings, and discordant with others. Key reasons for differing findings can be attributed to study eligibility criteria, outcomes included, inclusion of additional, newer, studies, and review methodology. For example, we included only trials with a usual care comparison group and excluded studies with sample sizes $<50$ patients and studies conducted in countries that were not United States relevant (in this case, primarily studies conducted in China). Each of these criterion eliminated studies that were included in some other reviews.

The decision to focus our review of psychosocial interventions on comparisons with usual care was made as part of a set of decisions required to reduce the scope of the project. After identifying a large body of evidence for Key Question 2, we determined that the funding and timeline required a reduction in scope. We first decided to use systematic reviews as the primary evidence, with subsequently published trials included as well. Examining those, we saw a large amount of heterogeneity in how control groups were defined and handled. In some reviews, all controls were lumped together, while in others "active" and usual care controls were assessed separately. Controls described as "active" varied widely, from competing interventions to attention controls, and were not handled consistently across reviews. For example, interventions categorized as "active" in one review were evaluated separately as "passive" in another review. Many, however, reviewed usual care comparisons separately, or exclusively. Therefore, within the systematic reviews, usual care was the most commonly reported comparison group. In the end we included well over 200 studies of psychosocial interventions that made comparisons to usual care. The implications of this choice certainly have been contemplated in the literature before $^{77,305-307}$ with no clear conclusion, although some have found little difference in analyses limiting to usual care comparisons and those including other comparisons. ${ }^{77}$ The potential bias introduced by this decision depends on the usual care actually received by patients in the control 
group. For example, if no difference was found between an intervention and usual care controls, it could be attributed to better usual care; but where a difference was found it could be due to the intervention, lower quality usual care, or a combination of factors. In addition, the magnitude of difference could be affected. The difference in usual care received could occur at the patient level, the study level or at the body of evidence level for a given intervention.

The decision to eliminate studies conducted in China mainly affected the body of studies for family interventions. In this case, both a prior Cochrane review and our own analysis indicate that the studies from China very likely overestimate treatment effects, which is consistent with the findings of other researchers in other clinical areas. ${ }^{308}$ Our decision to exclude rehospitalization as one of the prioritized outcomes was made after considering input from our technical expert panel, reflecting the lack of confidence that the findings are meaningful across time and different healthcare systems or settings. While studies of a few interventions regularly report this outcome, primarily as a proxy for relapse, we found that only assertive community treatment formally targets reducing rehospitalization. Hence, we reported rehospitalization as an outcome only for that intervention in the full report.

The other potential reasons for differences are to be expected - our searches are more recent, adding new evidence that could alter the prior findings, and we used the most up-to-date systematic review methodology, including assessing the strength of the body of evidence, based on domains including study methodological limitations, directness and consistency of evidence, and precision of estimates of effect. Our finding that the SOE for psychosocial interventions was moderate and low is consistent with our findings for antipsychotic drugs, and with numerous reviews across other populations and interventions. This system helps to make clear where future studies could alter findings, either in direction or magnitude, inform future research, and identify outcomes for which a given intervention is not effective. It does not, however, determine whether the intervention is useful or not in a broader sense, since the ratings are made on an outcome-by-outcome basis.

Below we summarize our findings in the context of key prior reviews for selected interventions, where there may be concern over how our and why findings differ. Because The Schizophrenia Patient Outcomes Research Team (PORT) 2009 publication is a highly regarded resource that assessed evidence and made recommendations on using several psychosocial interventions, we include their findings as well as individual reviews of these specific interventions. $^{295}$

\section{Cognitive Behavioral Therapy}

Overall, our findings are consistent with prior findings, except that we find additional outcomes where CBT showed benefit over usual care, and we did not find strong evidence regarding duration of effects. The use of CBT in schizophrenia has historically been focused on reducing positive symptoms. ${ }^{309,310}$ Consistent with other reviews, we found CBT to be effective at improving core illness symptoms with treatment durations of 8 weeks to 5 years and additionally for outcomes other than symptoms, even when those outcomes were not the focus of the CBT. ${ }^{78,309}$ Specifically, we found short-term measures of global, social and occupational function, quality of life and relapse requiring hospitalization to be significantly better with CBT based on low to moderate SOE. These findings build on the findings of prior reviews and the PORT publication, which noted that there was evidence that CBT improved symptoms (positive, negative, and overall) and social function - but that there were also studies that did not find these effects, and that there was no good evidence on relapse and suicidality. ${ }^{295}$ With respect to the 
durability of these effects after CBT ends, there is less clarity. A 2011 meta-analysis finds that the effects on symptoms were greater at followup that at the end of treatment, but only with comparisons to a diverse group of comparators, and with no specified duration of followup. Their findings for CBT compared with usual care are not statistically significant, so are similar to ours. ${ }^{311}$ Results related to durability of treatment from individual trials with longer posttreatment followup have been mixed. One trial ${ }^{312}$ of 9 months of CBT versus befriending found sustained benefit on overall and negative symptoms at 5-year followup with CBT, while a second trial ${ }^{313}$ of 6 months of intensive CBT versus leisure activities found no difference between groups in negative symptoms after 5 years. Both studies had methodological limitations which makes generalizable interpretation of these results difficult.

As with a previous meta-analysis, ${ }^{309}$ the studies we included rarely targeted negative symptoms. Our findings regarding negative symptoms, based on two good-quality systematic reviews, ${ }^{77,79}$ are somewhat in contrast with a 2008 review by Wykes et al. that found CBT resulted in significant improvements in negative symptoms. ${ }^{309}$ The Velthorst 2015 review found study year to be correlated with effect size, in that studies published prior to 2003 reported larger and more positive effect sizes than studies published in 2004 and later. Although based on limited evidence, this may partially explain the disparity in negative symptom pooled effect sizes between the two more recent reviews and the older review. All three reviews found higher study quality to be associated with lower effect sizes, and when limited to high quality studies (based on established risk of bias measures), they were in accord in finding a nonsignificant effect on negative symptoms in favor of CBT.

Evidence assessing how individual versus group CBT effects outcomes was limited. One review $^{79}$ found individual CBT more effective than group CBT on negative symptom reduction, but Wykes $^{309}$ reported no difference in effectiveness between individual and group CBT directed at symptoms as the primary outcome. The reason for this disparity may be due to the focus of the CBT. The Velthorst review noted that of 30 included trials, only two had negative symptoms as the primary treatment target, whereas the Wykes review assessed the effect of individual and group CBT only on studies’ primary treatment target.

\section{Cognitive Remediation}

The direct focus of cognitive remediation is on improving cognitive functioning, an outcome that is outside the scope of our review. There is some evidence that improvements in cognition can lead to improved global functioning, ${ }^{85}$ and outcomes related to function and symptoms were commonly reported in trials of cognitive remediation. Our review found that cognitive remediation improved functional outcomes, overall symptoms and negative symptoms. Our findings on function and total symptoms are based on the 2011 Wykes review $^{84}$ that combined both passive and active control groups. This review was an update and expansion of an older review, which also found cognitive remediation had a positive effect on function and symptoms. ${ }^{314}$ Our findings on negative symptoms are based on the Cella review ${ }^{85}$ and limited to studies with a passive usual care control group. These findings differ from the conclusions of the 2009 PORT publication, which determined that the evidence base was inadequate to make recommendations, primarily due to a paucity of good-quality RCTs. Our findings are based on more than 39 trials included in two good-quality systematic reviews. ${ }^{295}$ 


\section{Family Interventions}

Previous systematic reviews ${ }^{315}$ and other reviews ${ }^{316}$ and the 2009 PORT publication. ${ }^{295}$ report findings similar to our review. The 2001 systematic review by Pitschel-Walz and colleagues found that both short- and long-term family interventions are superior to usual care in prevention of relapse. ${ }^{315}$ They also found that the effect remained regardless of the length of the followup period, but that the type of intervention (psychoeducation or therapeutic) made little difference in treatment effect, both better than usual care. These results are largely consistent with our findings. The Dixon update on family psychoeducation ${ }^{316}$ concludes that family psychoeducation should be included as part of best practice guidelines for schizophrenia. The 2009 PORT publication recommends that family interventions should last between 6 and 9 months to reduce rates of relapse and hospitalization. ${ }^{317}$ Similarly, we found the strongest evidence for interventions lasting seven to 12 months. In addition, we found that the number of sessions was more predictive of reduction in relapse than duration of treatment. The two studies with family interventions consisting of 10 or fewer sessions at 7 to 12 months were not different from usual care on risk of relapse. Pooled estimates for relapse in trials of 11 to 20 sessions, 21 to 50 sessions, and greater than 50 sessions were all statistically superior to treatment as usual. One difference between our review and others is that we excluded trials conducted in China as we are not confident that the findings from Chinese studies are applicable to a United States population. Two reviews have conducted sensitivity analyses removing Chinese studies and found pooled effect estimates were reduced when Chinese studies were excluded. ${ }^{91,318}$

\section{Social Skills Training}

Our inclusion criteria were considerably stricter than those of other recent reviews ${ }^{293,294}$ in that we limited to larger trials $(\mathrm{N}>50)$, with longer duration $(>12$ weeks) that utilized a usual care control group. Still, our findings for function, one of the primary targets of social skills training, were consistent with other reviews that found significant improvements in measures of function with social skills training. ${ }^{293,294,319}$ Our findings for relapse, another target of social skills training, were also consistent with other reviews ${ }^{293,319}$ that found social skills training reduced relapse, although our estimates did not reach statistical significance, likely due to the low number of events and because the analysis in the other reviews included rehospitalizations as a surrogate for relapse. Our review also found social skills training significantly reduced negative symptoms, a finding that is consistent with one of these other reviews. ${ }^{293}$ The addition of new trials provided information on additional outcomes or durations of followup, but did not change the prior findings. In 2009, the PORT publication reported that evidence for skills training supported benefits in community functioning, but that the studies were not adequate to show positive effects on symptoms or relapse. ${ }^{295}$ Our findings are consistent with these findings.

\section{Supported Employment}

Our findings on supported employment are consistent with other reviews, such as the 2009 PORT recommendations and a review by Marshall, et al. ${ }^{295,320}$ We found that supported employment, specifically the IPS model intervention, resulted in significantly better employment outcomes over 2 years compared with usual care. More patients gained either employment (competitive or any job), had worked more hours, were employed longer, and earned more money than those receiving usual care. Because we found only one RCT that met our criteria for this review, we included other evidence; a review and a study that included other comparison 
groups. ${ }^{106,108}$ In using this evidence, our findings are similar to PORT and Marshall, with the exception that our SOE rating is moderate, while the Marshall rating is high. Our lower SOE rating is due to our comparison group, i.e. usual care, where Marshall did not specify a comparison group. We note also that the good quality Cochrane review ${ }^{106}$ that we included in our evidence rated the evidence as very low quality (according to the Grading of Recommendations Assessment, Development and Evaluation [GRADE] ${ }^{321-327}$ criteria) for multiple reasons, including large amounts of missing data due to higher dropout rates in the control groups, skewed data for some outcomes, and concerns over the lack of blinding of outcome assessors.

\section{Applicability}

\section{Key Question 1. Comparative Effectiveness of Pharmacological Treatments}

The applicability of the evidence in this review is limited in part by the scope of the criteria we set (e.g., adults, outpatients, etc.), and the studies themselves. As a result the evidence in our report is applicable to the following:

- Populations: Adults; mean age 25 to 50 years with mainly moderate and moderate to severe disease. There is heterogeneity in the relative predominance of specific symptoms of patients enrolled. For comparisons of SGAs, there is fairly robust evidence on firstepisode patients, but less on treatment-resistant patients.

o The evidence is not clearly applicable to adolescents, older adults, patients with severe disease, or patients with multiple comorbidities.

- Interventions/Comparisons: For the SGAs versus each other, the majority of the evidence is relevant to comparisons of the older SGAs, with very little evidence regarding newer drugs (those approved in the last 10 years). For the FGAs versus the SGAs, the evidence is almost entirely applicable to comparisons of the older SGAs and haloperidol, with a wide range of dosing.

o The evidence is less applicable to the newest SGAs (i.e., brexpiprazole, cariprazine, iloperidone, lurasidone, and the newest LAIs of paliperidone and aripiprazole).

o Evidence on clozapine may be less generalizable due to the potential effects of the required monitoring, which in essence insures adherence to treatment and may provide nonspecific support, encouragement, and even structure to the daily or weekly schedule, through consistent interaction with a provider.

- Outcomes: The evidence is less applicable to long-term effectiveness outcomes, such as function, long-term quality of life, self-harm, and mortality, particularly for the comparison of FGAs versus SGAs and newer SGAs.

- Timing: For all of the drug interventions, more studies were short-term (6 to 12 weeks) than longer-term (1 to 2 years). The evidence is not applicable to long-term followup (greater than 3 years).

- Setting: For SGAs versus each other, the evidence only applies to outpatients, whereas in the systematic review we included on FGAs versus SGAs almost half of the studies were in inpatients. 


\section{Key Question 2. Psychosocial and Other Nonpharmacological Interventions Versus Usual Care}

Similar to the issues noted in Key Question 1, the evidence base is limited in part by the scope identified for this review. For example, for Key Question 2 we added criteria that studies had to have at least 50 percent of patients diagnosed with schizophrenia, to reflect the fact that many of these interventions are aimed at patients with serious mental illness, as a group, rather than at specific diagnoses. We also applied sample size, duration, country, and comparison group (usual care) limits. As a result the evidence in our report is applicable to the following:

- Populations: Adults; age range 16 to 80 (teens to older adults), mostly with a diagnosis of schizophrenia or related disorder. The specific characteristics of patients varied somewhat by intervention category, for example:

o Evidence on supportive therapy is most applicable to middle aged men with schizophrenia and related diseases, who were experiencing long-standing hallucinations and/or delusions.

o Evidence on assertive community treatment is most applicable to patients with a history of severe mental illness and frequent hospitalizations over the past 2 to 5 years (e.g., 2 to 5 instances).

o The evidence is not clearly applicable to patients with treatment resistance, or multiple comorbidities. Across the interventions, the severity of the disease of the patients included in the studies is unclear.

- Interventions/Comparisons: The evidence in this review, by prespecified design, applies to comparisons with usual care, and the 13 intervention categories identified here. The purpose was not to evaluate head-to-head comparisons of active interventions to each other.

o Interventions were poorly defined or described for some intervention categories, such as family interventions and supportive therapy, seriously limiting the generalizability.

o Similarly, what was considered usual care and what specific interventions or services patients received as part of usual care was infrequently described. As a result, the evidence is less applicable to variations in level or quality of these interventions, or emerging interventions.

o The evidence is not applicable to comparative effectiveness questions and the review findings apply only to comparisons with usual care. The evidence may apply to other comparisons, as our analysis of evidence for supported employment indicated little difference in findings or conclusions between usual care and active control-group evidence.

- Outcomes: The evidence is applicable only to a select group of outcomes that vary by intervention. Not all prioritized outcomes were reported consistently across studies.

- Timing: Most of the interventions do not have evidence that is applicable to long-term followup (greater than 3 years).

- Setting: The settings were mostly applicable to the United States, as evidence clearly not applicable was excluded from our review.

- Although applicability is separate from the strength of the evidence, the overall SOE does impact why applicability of the included studies may be limited. Importantly, the 
heterogeneity of interventions and treatment effects, including both clinical and methodological heterogeneity, detracts from the overall applicability of the findings.

\section{Implications for Clinical and Policy Decisionmaking}

Our findings have implications for clinical and policy decisionmaking. The American Psychiatric Association's (APA's) most recent guideline for treating adults with schizophrenia was published in 2004, ${ }^{328}$ with a focused update in $2009 .{ }^{329}$ Also from 2009, are the Schizophrenia PORT recommendations for psychosocial treatments for individuals with schizophrenia. ${ }^{295}$ Since these publications, important developments have occurred both in the pharmacological and nonpharmacological treatment interventions for schizophrenia. There are five new antipsychotics and five new LAI formulations approved in the United States since the 2009 APA guideline update. Similarly, many psychosocial and other nonpharmacological treatments have been developed, refined or expanded in recent years. ${ }^{295,329,330}$ Given the everevolving nature of schizophrenia treatments and their potential for meaningful benefits and significant harms, this review will allow the APA to update their guideline, and for clinicians and patients to have a single source to access up-to-date evidence synthesis. Similarly, the review may allow policymakers to use the evidence in making decisions, depending on their priorities.

\section{Limitations of the Systematic Review Process}

As with other types of research, the limitations of this systematic review are important to recognize. The generalizability of the results is limited by the scope of the review, based on the inclusion criteria, which determines the studies included and the specific methodology we applied to the review process. In terms of the review process, potential limitations are that we limited inclusion to studies with at least an abstract published in English (i.e., publications not available in an English form were not included) and had limitations on the number of databases we searched (Ovid MEDLINE ${ }^{\circledR}$, the Cochrane Central Register of Controlled Trials, the Cochrane Database of Systematic Reviews, and PsycINFO ${ }^{\circledR}$ ). As we were limiting the study characteristics to those relevant to a United States setting, and because we reviewed Englishlanguage abstracts of studies whose full publication was in another language, we feel that the risk of this limitation is low. We have found that searching these key databases has been adequate, and in this case we also have the advantage of using systematic reviews in our review that had access to other sources to search for literature, and also depend on the scientific information packet (SIP) process and involvement of our Technical Expert Panel (TEP) to help identify any missing literature.

With respect to the limitations of the scope identified a priori for this review, there are several potential limitations. For pharmacologic interventions, the scope of this review is on direct, head-to-head comparisons of the drugs and did not evaluate comparisons to placebo, no treatment or older antipsychotic drugs. This may have introduced some biases or gaps in the conclusions particularly for newer drugs that have minimal or no comparative evidence.

For psychosocial interventions, due to the large volume and heterogeneous nature of studies, we made inclusion decisions to narrow the scope to the most commonly used interventions and to the most common control (usual care). These scope restrictions were made based on the evidence available and consultation with experts and were intended to allow us to review the most pertinent evidence as in-depth as possible, within the given time frame and budget. Clearly they could have impacted some of the findings and conclusions. We excluded, for example, assisted outpatient treatment (also known as outpatient commitment therapy and compulsory 
community treatment orders), because our clinical expert advisors felt these were mechanisms to get patients into treatment, rather than a treatment themselves. There is a review of these and other interventions aimed at reducing hospitalization in patients with serious mental illness. ${ }^{331}$

For both key questions, we limited our review to RCTs with a followup of at least 12 weeks, and for Key Question 2 we also required a patient population of at least 50 participants. For Key Question 2 on psychosocial interventions, these criteria resulted in excluding 35 trials (Figure 3). Limiting to usual care controls resulted in excluding 74 studies (compared with 271 studies included with usual care controls). These were primarily comparisons to other competing interventions (i.e. head to head comparisons) and comparisons of different intensities of the same intervention. In reviewing our findings in comparison with findings of reviews including active comparators, however, we did not find strong differences in direction or magnitude of effect. For example, our own findings on supported employment were consistent between usual care and the active comparator of pre-vocational training.

We limited the outcomes to those that are patient centered health outcomes (rather than intermediate outcomes), which were arranged according to their priority from the perspective of the patient, their family, and their clinicians. We considered advice from our experts in selecting and prioritizing this list of outcomes, and as a result outcomes such as positive symptoms, rehospitalization and cognitive outcomes were generally not included in our review. For Key Question 2, we ultimately excluded 96 studies because no included outcome was reported. Rehospitalization was excluded because there is important variation in the indications for and length of psychiatric hospitalizations across time, in different localities, and with different financial contexts, and there is important variation across trials in how rehospitalization is measured/evaluated, which may confound study interpretation. However, it should be noted that other outcomes may also suffer from similar issues. For example, employment may be influenced by local economic conditions, and outcomes related to interactions with law enforcement can also vary over time and by location. Most of the psychosocial interventions have target patient-outcome pairs, for example family interventions were targeted at patients with a history of, or at high risk of, frequent relapses. These targeted outcomes were generally included in our list of included outcomes, but were not prioritized on an intervention-byintervention basis. In the example of family interventions, the target outcome of relapse prevention is outcome number seven in priority order. Certainly some highly prioritized, and patient-important, outcomes such as function and quality of life (outcomes number one and two in priority order) were not the target of most interventions and may not be greatly improved by these interventions. However, across the interventions, it is important to consider the evidence for both of these highly prioritized outcomes, as well as the targeted outcomes.

A final potential limitation is that we considered any intervention for schizophrenia without explicit consideration of the patient characteristics that the intervention focuses on. Because several interventions have a specific population and outcome focus, this may have limited our ability to determine for whom each intervention is best.

\section{Limitations of the Evidence Base}

For the pharmacological interventions, the limitations of the evidence base are fairly clear; there are few studies of the newer SGAs, few studies reporting the most important outcomes (i.e., function and quality of life), inadequate information on key subgroups such as older patients and those with multiple or serious comorbidities, and patients with severe illness, including treatment-resistance, clearly separated out. Study quality was not a key limitation, but 
funding source may have been, given that over 80 percent of studies were funded by the manufacturer of one of the drugs in the trial.

Other issues making it more difficult to draw firm conclusions were the variability in which drugs compared across studies, outcomes reported and how they are measured, and of course, variability in patient characteristics, that are often poorly reported on. Consensus is needed regarding outcomes and measures used to assess outcomes; for example the primary outcome measure in the CATIE trials, which was well publicized as to how and why it was selected, was not given high priority by our TEP. Although many of the older studies suffered from problems with generalizability to the real-life practice setting because either they used doses that were higher or lower than those used in practice today or made unfair dose-comparisons (e.g., low dose of one drug versus high dose of a comparator drug); our analysis of these issues as part of our network analyses indicates that more recent studies have fewer issues with dosing. In the drug comparisons, an important limitation is the change in dosing over time, particularly with lower doses in more recent studies for several older drugs, and the prior treatment experience of patients enrolled (fewer patients with prior FGA exposure or resistance in more recent studies). The systematic review we included on this comparison attempted multiple sensitivity analyses to explore these issues, but no clear conclusions were drawn.

Although we excluded individual RCTs that enrolled inpatient populations, we included systematic reviews that included some studies conducted in inpatients. A few reviews conducted sensitivity analysis and found similar results regardless of setting.

Limitations for studies of psychosocial and nonpharmacological interventions included problems with reporting definitions of interventions and usual care groups, poor description of treatment versus followup periods, and variability in outcome reporting and methodological issues relating to small sample sizes, lack of power calculations, blinding of outcome assessors, post-treatment followup, and inadequate handling of missing data.

Many interventions were not clearly defined or described in the manuscripts and therefore difficult to categorize. In many studies the exact period of the intervention was unclear. Some reported a specific number of sessions but not the duration in weeks or months, while reporting outcomes at a specific time (e.g., 6 months). This was apparent in the CBT and family intervention studies, for example. There was also considerable variation in which how outcomes are reported, even when the same outcome measure was used. This was particularly true for symptom, functional and other outcomes measured using continuous scale scores. In many studies, there were small but significant improvements in scale scores with use of psychosocial interventions, but the clinical importance of these changes is largely unclear. This is an important limitation and is also related to the previous point about method of outcome reporting and to the small sample sizes in the majority of studies. For example, for those scales with known thresholds for clinical significance, we could not adequately assess if the threshold for clinical significance has been met if a study only reported effect size. For this review, the outcome of treatment discontinuation was included as a high priority outcome, but it was difficult to operationalize, and unclear what the meaning was for some interventions. In terms of study participant dropout, while only about a quarter of studies had rates higher than 30 percent, approximately 40 percent either did not conduct, or were unclear on conducting, an intention to treat analysis.

Few studies of psychosocial interventions reported on the effects of treatment after treatment cessation, while none of the included studies of antipsychotic drugs did so. For those studies that did report posttreatment followup, there tended to be a loss of effect after treatment withdrawal, 
but this evidence is insufficient to draw conclusions. Evidence to inform the best duration or schedule of treatment is not available for either category of intervention. For many interventionoutcome pairs, evidence was too limited to draw conclusions about the effect of treatment, resulting in insufficient SOE (Appendix $\mathrm{H}$ ). For both key questions, evidence on subgroups is limited by sample sizes and that most are post hoc subgroup analyses of trials rather than either preplanned analyses or trials designed to address these questions as the primary objective.

\section{Research Recommendations}

Based on the gaps and limitations identified in this review, we recommend the following future research on comparative effectiveness of pharmacological interventions and general effectiveness of psychosocial and other nonpharmacological interventions. In general, these recommendations are for RCTs.

\section{Pharmacological Interventions Versus Each Other}

Trials should:

- Involve multiple newer SGA drugs (approved in the last 10 years), in comparison with one of the older SGAs (e.g., clozapine, olanzapine, risperidone LAI) and haloperidol and compare fluphenazine and perphenazine with both older and newer SGAs.

- Ensure comparable dosing with the best dosing titration methods for all drugs included.

- Measure key health outcomes, using agreed-upon direct measures. For example, measuring functional outcomes using not only valid and reliable scales, but also direct, objective measures of patient functioning. These measures need to be agreed upon by clinical and research experts and then used consistently across trials.

- Study durations must reflect real-life practice. Minimum study duration should be 1 year, with 3- to 5-year followup in order to measure the durability of effects, and truly longterm outcomes, including harms (e.g., metabolic changes and tardive dyskinesia). Longterm harms are not assessable in short-term studies, and relying on observational evidence has limitations.

- The concept of recovery should be incorporated into study designs, with testing of duration of effect and discontinuation of drug treatment following remission.

- Enroll subjects who reflect real populations. Studies exclusively of older patients, with multiple comorbidities and concomitant medications, and patients with severe disease, including treatment-resistance are needed. To better study other subgroups, such as minorities and women, specification and planning of subgroup analyses a priori and use of randomization methods that insure adequate distribution of these characteristics are needed to examine differences.

- Clearly inpatients need to be studied separately from outpatients, but future reviews should evaluate treatments for inpatients.

\section{Psychosocial and Other Nonpharmacological Interventions Versus Usual Care}

Issues may vary by the specific intervention, but there are several key recommendations that are relevant to all of the interventions: 
- Trials should have adequate sample sizes to address important health outcomes, rather than intermediate or surrogate outcomes, and should adhere to the current standards for reporting, such as the Consolidated Standards of Reporting Trials (CONSORT) criteria 332

- Studies need to be conducted in broader, but better-defined populations, with either separate studies of subpopulations or large enough sample sizes to allow meaningful subgroup analysis.

o Future studies might consider using the National Institutes of Mental Health Research Domain Criteria approach to categorizing patients.

o Future reviews should evaluate treatments for inpatients.

- Interventions should be clearly defined and described, including required components. Some interventions, such as cognitive remediation, have used expert groups to refine definitions and required components of interventions. Measurements of fidelity to the intervention model should be undertaken where possible.

- Trials need to evaluate and report patient-important health outcomes such as function, quality of life, self-harm, and adverse effects using standardized and easily interpretable methods. Studies should identify what constitutes clinically meaningful change in scale scores.

- Studies are needed to address the heterogeneity in usual care control groups. Usual care is highly variable, so studies using a usual care control group must report on the specific services and treatments received, and standardize the comparison or control for attention effects.

- Studies should measure both the intensity and duration of the intervention required to achieve the best results.

- Additional, well-designed long-term studies are needed. The long-term benefits versus risks and costs of treatments remain unclear, in particular for individuals whose illness is resistant or only partially responsive to treatment.

- Future systematic review research should:

o Include other nonpharmacological treatments, such as device-based somatic treatments (e.g., electroconvulsive therapy and transcranial magnetic stimulation).

o Include an evaluation of comparative effectiveness of psychosocial interventions compared with each other.

o Incorporate the concepts of complex interventions into the methods for reviewing the evidence for some of the psychosocial interventions. ${ }^{333,334}$

o Organize the evidence according to the patient characteristics that the intervention focuses on. 


\section{Conclusions}

The majority of the comparative evidence on pharmacotherapy to treat schizophrenia relates to the older SGAs (mainly clozapine, olanzapine, risperidone, quetiapine, and ziprasidone), with some evidence on paliperidone and aripiprazole, and the LAIs of risperidone, aripiprazole, and paliperidone. There is very little comparative evidence on newer SGAs (drugs approved in the last 10 years; asenapine, brexpiprazole, cariprazine, iloperidone, and lurasidone). Although there are some differences among the older SGA on specific outcomes, no single drug was superior on multiple high-priority outcomes. However, clozapine, olanzapine, and risperidone oral and LAI, did have superiority on more outcomes than other SGAs and quetiapine and ziprasidone were not superior to other SGAs on any outcome. No evidence found a newer SGA superior to older SGAs on any outcome. Evidence on FGAs versus SGAs indicates that olanzapine, risperidone, ziprasidone, and aripiprazole were similar to haloperidol on some outcomes of benefit, and were superior on overall adverse events and withdrawal due to adverse events.

In comparison with usual care, most of the psychosocial interventions to treat schizophrenia reviewed were more effective in improving two or more outcomes, including nontargeted but patient-important outcomes. Various functional outcomes were improved more with assertive community treatment, CBT, psychoeducation, social skills training, supported employment, and early team-based multi-component treatment programs for first episode psychosis than with usual care. Quality of life was improved more with CBT and early team-based multi-component treatment programs for first episode psychosis than usual care. Core illness symptoms were improved with assertive community treatment, CBT, cognitive remediation, illness selfmanagement, psychoeducation, social skills training, and early team-based multi-component treatment programs for first episode psychosis. Relapse was reduced with psychoeducation, illness self-management, family interventions, and early team-based multi-component treatment programs for first episode psychosis. Self harm, response and/or remission, and adverse events were rarely reported. 


\section{References}

1. American Psychiatric Association. Diagnostic and Statistical Manual of Mental Disorders, 5th ed. Arlington, VA: American Psychiatric Publishing; 2013.

2. Tandon R, Gaebel W, Barch DM, et al. Definition and description of schizophrenia in the DSM-5. Schizophr Res. 2013 Oct;150(1):3-10. doi: 10.1016/j.schres.2013.05.028. PMID: 23800613.

3. McGrath J, Saha S, Chant D, et al. Schizophrenia: a concise overview of incidence, prevalence, and mortality. Epidemiol Rev. 2008;30:67-76. PMID: 18480098.

4. Harding CM. Changes in Schizophrenia Across Time: Pardoxes, Patterns and Predictors. In: Cohen CI, ed Schizophrenia into later life: Treatment, research and policy. Arlington, VA: American Psychiatric Publishing, Inc.; 2003:19-41.

5. Wils RS, Gotfredsen DR, Hjorthøj C, et al. Antipsychotic medication and remission of psychotic symptoms 10 years after a firstepisode psychosis. Schizophr Res.

2017;182:42-8. doi:

10.1016/j.schres.2016.10.030. PMID: 28277310.

6. Frese FJ, 3rd, Knight EL, Saks E. Recovery from schizophrenia: with views of psychiatrists, psychologists, and others diagnosed with this disorder. Schizophr Bull. 2009 Mar;35(2):370-80. doi: 10.1093/schbul/sbn175. PMID: 19304812.

7. Buchy L, Cadenhead KS, Cannon TD, et al. Substance use in individuals at clinical high risk of psychosis. Psychol Med. 2015;45(11):2275-84. PMID: 25727300.

8. Goerke D, Kumra S. Substance abuse and psychosis. Psychiatr Clin North Am. 2013;22(4):643-54. PMID: 24012078.

9. Mesholam-Gately RI, Gibson LE, Seidman LJ, et al. Schizophrenia and co-occurring substance use disorder: reward, olfaction and clozapine. Schizophr Res. 2014;155(13):45-51. PMID: 24685823.
10. De Witte NA, Crunelle CL, Sabbe B, et al. Treatment for outpatients with comorbid schizophrenia and substance use disorders: a review. Eur Addict Res. 2014;20(3):105-14. doi: 10.1159/000355267. PMID: 24192558.

11. American Diabetes Association, American Psychiatric Association, American Association of Clinical Endocrinologists, et al. Consensus development conference on antipsychotic drugs and obesity and diabetes. Diabetes Care. 2004 Feb;27(2):596-601. PMID: 14747245.

12. Newcomer JW, Hennekens CH. Severe mental illness and risk of cardiovascular disease. JAMA. 2007;298(15):1794-6. PMID: 17940236.

13. Henderson D, Vincenzi B, Andrea NV, et al. Pathophysiological mechanisms of increased cardiometabolic risk in people with schizophrenia and other severe mental illnesses. Lancet Psychiatry. 2015;2(5):45264. PMID: 26360288.

14. Marder SR, Essock SM, Miller AL, et al. Physical health monitoring of patients with schizophrenia. Am J Psychiatry. 2004;161(8):1334-49. PMID: 15285957.

15. McDonagh MS, Peterson K, Carson S, et al. Drug class review of atypical antipsychotic drugs; update 3. Portland, OR: Oregon Health \& Science University Drug Effectiveness Review Project; 2010. http://www.ohsu.edu/xd/research/centersinstitutes/evidence-based-practicecenter/drug-effectiveness-reviewproject/current-past-reports.cfm Accessed April 22, 2017.

16. Mayo Clinic. Diseases and conditions: schizophrenia.

http://www.mayoclinic.org/diseasesconditions/schizophrenia/basics/definition/C ON-20021077. Accessed April 22, 2017.

17. Centers for Disease Control. Burden of mental illness. 2013.

http://www.cdc.gov/mentalhealth/basics/bur den.htm. Accessed April 22, 2017. 
18. National Institute of Mental Health. Schizophrenia.

http://www.nimh.nih.gov/health/topics/schiz ophrenia/index.shtml. Accessed April 22, 2017.

19. Gareri P, De Fazio P, Manfredi VG, et al. Use and safety of antipsychotics in behavioral disorders in elderly people with dementia. J Clin Psychopharmacol. 2014;34(1):109-23. PMID: 24158020.

20. Kales HC, Kim HM, Zivin K, et al. Risk of mortality among individual antipsychotics in patients with dementia. Am J Psychiatry. 2012;169(1):71-9. PMID: 22193526.

21. Schneider LS, Dagerman KS, Insel P. Risk of death with atypical antipsychotic drug treatment for dementia: meta-analysis of randomized placebo-controlled trials. JAMA. 2005 Oct 19;294(15):1934-43. doi: 10.1001/jama.294.15.1934. PMID: 16234500 .

22. Gill SS, Bronskill SE, Normand SL, et al. Antipsychotic drug use and mortality in older adults with dementia. Ann Intern Med. 2007 Jun 5;146(11):775-86. PMID: 17548409 .

23. Alexopoulos GS, Jeste DV, Chung H, et al. The expert consensus guideline series. Treatment of dementia and its behavioral disturbances. Introduction: methods, commentary, and summary. Postgrad Med. 2005 Jan;Spec No:6-22. PMID: 17203561.

24. Jeste DV, Rockwell E, Harris MJ, et al. Conventional vs. newer antipsychotics in elderly patients. Am J Geriatr Psychiatry. 1999 Winter;7(1):70-6. PMID: 9919323.

25. Moher D, Liberati A, Tetzlaff J, et al. Preferred reporting items for systematic reviews and meta-analyses: the PRISMA statement. BMJ. 2009 Jul 21;339:b2535. doi: 10.1136/bmj.b2535. PMID: 19622551.

26. Agency for Healthcare Research and Quality. Methods Guide for Effectiveness and Comparative Effectiveness Reviews. AHRQ Publication No. 10(14)-EHC063-EF. Rockville MD: January 2014. Chapters available at: www.effectivehealthcare.ahrq.gov Accessed April 22, 2017.
27. Hartling L, Abou-Setta AM, Dursun S, et al. Antipsychotics in adults with schizophrenia: comparative effectiveness of first-generation versus second-generation medications: a systematic review and meta-analysis. Ann Intern Med. 2012 Oct 2;157(7):498-511. PMID: 22893011.

28. Abou-Setta AM, Mousavi SS, Spooner C, et al. First-generation versus second-generation antipsychotics in adults: comparative effectiveness. Comparative effectiveness review No. 63. (Prepared by the University of Alberta Evidence-based Practice Center under Contract No. 290-200-10021.) AHRQ Publication No. 12-EHC054-EF. Rockville, MD: Agency for Healthcare Research and Quality: 2012.

www.effectivehealthcare.ahrq.gov/reports/fi nal.cfm Accessed April 22, 2017. PMID: 23035275.

29. McDonagh M, Peterson K, Fu R, et al. Second Generation Antipsychotic Drugs. Final Update 4 Report prepared by the Pacific Northwest Evidence-based Practice Center for the Drug Effectiveness Review Project. Oregon Health \& Science University, Portland, Oregon, November 2013. 2013. Available with membership in the Drug Effectiveness Review Project. http://www.ohsu.edu/xd/research/centersinstitutes/evidence-based-practicecenter/drug-effectiveness-reviewproject/upload/SGA final-update-4report_Nov-2013_version-2.pdf. Accessed May 23, 2017.

30. Kreyenbuhl J, Buchanan RW, Dickerson FB, et al. The Schizophrenia Patient Outcomes Research Team (PORT): updated treatment recommendations 2009. Schizophr Res. 2010 Jan;36(1):94-103. doi: 10.1093/schbul/sbp130. PMID: 19955388.

31. Treadwell J, Singh S, Talati R, et al. A Framework for "Best Evidence" Approaches in Systematic Reviews. Methods Research Report. (Prepared by the ECRI Institute Evidence-based Practice Center under Contract No. HHSA 290-2007-10063-I.) AHRQ Publication No. 11-EHC046-EF. Rockville, MD: Agency for Healthcare Research and Quality. June 2011. Available at: www.effectivehealthcare.ahrq.gov/reports/fi nal.cfm. Accessed May 19, 2017. 2011. 
32. McDonagh MS, Jonas DE, Gartlehner G, et al. Methods for the drug effectiveness review project. BMC Med Res Methodol. 2012;12(1):140. doi: 10.1186/1471-228812-140. PMID: 22970848.

33. Shea BJ, Grimshaw JM, Wells GA, et al. Development of AMSTAR: a measurement tool to assess the methodological quality of systematic reviews. BMC Med Res Methodol. 2007 Feb 15;7:10. doi: 10.1186/1471-2288-7-10. PMID: 17302989.

34. Review Manager (RevMan), Version 5.3. Copenhagen: The Nordic Cochrane Centre; 2014.

35. StatsDirect Statistical Software. Chesire, UK: StatsDirect Ltd.; 2013.

36. Stata Statistical Software, Release 14. College Station, TX: StataCorp LP; 2015.

37. Lu G, Ades AE. Combination of direct and indirect evidence in mixed treatment comparisons. Stat Med. 2004;23(20):310524. doi: 10.1002/sim.1875 PMID: 15449338

38. Lu X, Cheng H, Song S, et al. A study of quetiapine and risperidone and clozapine in the treatment of schizophrenia. China and Foreign Medical Journal 2006;4 (6):62-4.; 2006.

39. Bucher HC, Guyatt GH, Griffith LE, et al. The results of direct and indirect treatment comparisons in meta-analysis of randomized controlled trials. J Clin Epidemiol. 1997 Jun;50(6):683-91. PMID: 9250266.

40. Dias S, Welton NJ, Caldwell DM, et al. Checking consistency in mixed treatment comparison meta-analysis. Stat Med. 2010 Mar 30;29(7-8):932-44. doi: 10.1002/sim.3767. PMID: 20213715.

41. Brooks SP, Gelman A. General Methods for Monitoring Convergence of Iterative Simulations. J Comp Graph Stat. 1998;7(4):434-55. doi: 10.1080/10618600.1998.10474787.

42. Berkman ND, Lohr KN, Ansari MT, et al. Grading the strength of a body of evidence when assessing health care interventions: an EPC update. J Clin Epidemiol. 2015 11//;68(11):1312-24. doi: 10.1016/j.jclinepi.2014.11.023. PMID: 25721570 .
43. Citrome L, Ota A, Nagamizu K, et al. The effect of brexpiprazole (OPC-34712) and aripiprazole in adult patients with acute schizophrenia: results from a randomized, exploratory study. Int Clin

Psychopharmacol. 2016 Jul;31(4):192-201. doi: 10.1097/yic.0000000000000123. PMID: 26963842.

44. Detke HC, Weiden PJ, Llorca PM, et al. Comparison of olanzapine long-acting injection and oral olanzapine: a 2-year, randomized, open-label study in outpatients with schizophrenia. J Clin Psychopharmacol. 2014 Aug;34(4):426-34. doi: $10.1097 /$ JCP.0000000000000140. PMID: 24781441.

45. Di Fiorino M, Montagnani G, Trespi G, et al. Extended-release quetiapine fumarate (quetiapine $\mathrm{XR}$ ) versus risperidone in the treatment of depressive symptoms in patients with schizoaffective disorder or schizophrenia: a randomized, open-label, parallel-group, flexible-dose study. Int Clin Psychopharmacol. 2014 May;29(3):166-76. doi: 10.1097/YIC.0000000000000017. PMID: 24681810.

46. Durgam S, Starace A, Li D, et al. An evaluation of the safety and efficacy of cariprazine in patients with acute exacerbation of schizophrenia: a phase II, randomized clinical trial. Schizophr Res. 2014 Feb;152(2-3):450-7. doi: 10.1016/j.schres.2013.11.041. PMID: 24412468.

47. Fleischhacker WW, Sanchez R, Perry PP, et al. Aripiprazole once-monthly for treatment of schizophrenia: double-blind, randomised, non-inferiority study. Br J Psychol. 2014 Aug;205(2):135-44. doi: 10.1192/bjp.bp.113.134213. PMID: 24925984.

48. Green AI, Brunette MF, Dawson R, et al. Long-acting injectable vs oral risperidone for schizophrenia and co-occurring alcohol use disorder: a randomized trial. J Clin Psychiatry. 2015 Oct;76(10):1359-65. doi: 10.4088/JCP.13m08838. PMID: 26302441. 
49. Ishigooka J, Nakamura J, Fujii Y, et al. Efficacy and safety of aripiprazole oncemonthly in Asian patients with schizophrenia: a multicenter, randomized, double-blind, non-inferiority study versus oral aripiprazole. Schizophr Res. 2015 Feb;161(2-3):421-8. doi: 10.1016/j.schres.2014.12.013. PMID: 25556976.

50. Koshikawa Y, Takekita Y, Kato M, et al. The comparative effects of risperidone longacting injection and paliperidone palmitate on social functioning in schizophrenia: a 6month, open-label, randomized controlled pilot trial. Neuropsychobiology. 2016;73(1):35-42. doi: 10.1159/000442209. PMID: 26812618

51. Li H, Luo J, Wang C, et al. Efficacy and safety of aripiprazole in Chinese Han schizophrenia subjects: a randomized, double-blind, active parallel-controlled, multicedinter clinical trial. Schizophr Res. 2014 Aug;157(1-3):112-9. doi: 10.1016/j.schres.2014.05.040. PMID: 24994555.

52. Liu J, Sun J, Shen X, et al. Randomized controlled trial comparing changes in serum prolactin and weight among female patients with first-episode schizophrenia over 12 months of treatment with risperidone or quetiapine. Shanghai Arch Psychiatry. 2014;26(2):88-94. PMID: 25092954.

53. Maat A, Cahn W, Gijsman HJ, et al. Open, randomized trial of the effects of aripiprazole versus risperidone on social cognition in schizophrenia. Eur Neuropsychopharmacol. 2014 Apr;24(4):575-84. PMID: 24418213.

54. Naber D, Hansen K, Forray C, et al. Qualify: a randomized head-to-head study of aripiprazole once-monthly and paliperidone palmitate in the treatment of schizophrenia. Schizophr Res. 2015;168(1-2):498-504. PMID: 26232241

55. Naber D, Peuskens J, Schwarzmann N, et al. Subjective well-being in schizophrenia: a randomised controlled open-label 12-month non-inferiority study comparing quetiapine XR with risperidone (RECOVER). Eur Neuropsychopharmacol. 2013 Oct;23(10):1257-69. doi: 10.1016/j.euroneuro.2013.07.006. PMID: 23953270.
56. Nemeth G, Laszlovszky I, Czobor P, et al. Cariprazine versus risperidone monotherapy for treatment of predominant negative symptoms in patients with schizophrenia: a randomised, double-blind, controlled trial. Lancet. 2017 Feb 07doi: 10.1016/s01406736(17)30060-0. PMID: 28185672.

57. Parabiaghi A, Tettamanti M, D'Avanzo B, et al. Metabolic syndrome and drug discontinuation in schizophrenia: a randomized trial comparing aripiprazole olanzapine and haloperidol. Acta Psychiatr Scand. 2016;133(1):63-75. PMID: 26252780.

58. Park S, Yi KK, Kim MS, et al. Effects of ziprasidone and olanzapine on body composition and metabolic parameters: an open-label comparative pilot study. Behav Brain Funct. 2013;9:27. doi: 10.1186/17449081-9-27. PMID: 23866300.

59. Robinson DG, Gallego JA, John M, et al. A randomized comparison of aripiprazole and risperidone for the acute treatment of firstepisode schizophrenia and related disorders: 3-month outcomes. Schizophr Bull. 2015;41(6):1227-36. PMID: 26338693.

60. Sanz-Fuentenebro J, Taboada D, Palomo T, et al. Randomized trial of clozapine vs. risperidone in treatment-naive first-episode schizophrenia: results after one year. Schizophr Res. 2013 Sep;149(1-3):156-61. doi: 10.1016/j.schres.2013.07.003. PMID: 23870807.

61. Savitz AJ, Xu H, Gopal S, et al. Efficacy and safety of paliperidone palmitate 3month formulation for patients with schizophrenia: a randomized, multicenter, double-blind, noninferiority study. Int $\mathrm{J}$ Neuropsychopharmacol. 2016 Jul;19(7)doi: 10.1093/ijnp/pyw018. PMID: 26902950.

62. Shoja Shafti S, Kaviani H. Quetiapine versus aripiprazole in the management of schizophrenia. Ther Adv Psychopharmacol. 2015;5(3):166-71. PMID: 26199719. 
63. Subotnik KL, Casaus LR, Ventura J, et al. Long-acting injectable risperidone for relapse prevention and control of breakthrough symptoms after a recent first episode of schizophrenia. A randomized clinical trial. JAMA Psychiatry. 2015 Aug;72(8):822-9. doi: 10.1001/jamapsychiatry.2015.0270. PMID: 26107752.

64. Tybura P, Mak M, Samochowiec A, et al. The influence of antipsychotic therapy on the cognitive functions of schizophrenic patients. Psychiatr Pol. 2013;47(4):567-76. PMID: 24946464.

65. Tybura P, Trzesniowska-Drukala B, Bienkowski P, et al. Pharmacogenetics of adverse events in schizophrenia treatment: comparison study of ziprasidone, olanzapine and perazine. Psychiatry Res. 2014 Oct 30;219(2):261-7. doi: 10.1016/j.psychres.2014.05.039. PMID: 24930580.

66. Wani RA, Dar MA, Chandel RK, et al. Effects of switching from olanzapine to aripiprazole on the metabolic profiles of patients with schizophrenia and metabolic syndrome: a double-blind, randomized, open-label study. Neuropsychiatr Dis Treat. 2015;11:685-93. PMID: 25792838.

67. Crespo-Facorro B, Perez-Iglesias R, Mata I, et al. Effectiveness of haloperidol, risperidone and olanzapine in the treatment of first-episode non-affective psychosis: results of a randomized, flexible-dose, openlabel 1-year follow-up comparison. J Psychopharmacol. 2011 Jun;25(6):744-54. PMID: 21292922.

68. San L, Arranz B, Perez V, et al. One-year, randomized, open trial comparing olanzapine, quetiapine, risperidone and ziprasidone effectiveness in antipsychoticnaive patients with a first-episode psychosis. Psychiatry Res. 2012 Dec 30;200(2-3):693701. doi: 10.1016/j.psychres.2012.07.005. PMID: 22954905.

69. Amr M, Lakhan SE, Sanhan S, et al. Efficacy and tolerability of quetiapine versus haloperidol in first-episode schizophrenia: a randomized clinical trial. Int Arch Med. 2013;6(1) PMID: 24308507.
70. McEvoy JP, Byerly M, Hamer RM, et al. Effectiveness of paliperidone palmitate vs haloperidol decanoate for maintenance treatment of schizophrenia: a randomized clinical trial.[Erratum appears in JAMA. 2014 Oct 8;312(14):1473]. JAMA. 2014 May 21;311(19):1978-87. doi: 10.1001/jama.2014.4310. PMID: 24846035.

71. Marshall M, Lockwood A. Assertive community treatment for people with severe mental disorders. Cochrane Database Syst Rev. 2000a(2):Cd001089. doi: 10.1002/14651858.cd001089. PMID: 10796415.

72. Sytema S, Wunderink L, Bloemers W, et al. Assertive community treatment in the Netherlands: a randomized controlled trial. Acta Psychiatr Scand. 2007 Aug;116(2):105-12. PMID: 17650271.

73. Velligan DI, Diamond P, Mueller J, et al. The short-term impact of generic versus individualized environmental supports on functional outcomes and target behaviors in schizophrenia. Psychiatry Res. 2009 Jul 30;168(2):94-101. doi: 10.1016/j.psychres.2008.03.016. PMID: 19523690.

74. Velligan DI, Diamond PM, Maples NJ, et al. Comparing the efficacy of interventions that use environmental supports to improve outcomes in patients with schizophrenia. Schizophr Res. 2008 Jul;102(1-3):312-9. doi: 10.1016/j.schres.2008.02.005. PMID: 18374542.

75. Velligan DI, Diamond PM, Mintz J, et al. The use of individually tailored environmental supports to improve medication adherence and outcomes in schizophrenia. Schizophr Bull. 2008 May;34(3):483-93. PMID: 17932089.

76. Velligan DI, Tai S, Roberts DL, et al. A randomized controlled trial comparing cognitive behavior therapy, cognitive adaptation training, their combination and treatment as usual in chronic schizophrenia. Schizophr Bull. 2015 May;41(3):597-603. doi: 10.1093/schbul/sbu127. PMID: 25193976. 
77. Jauhar S, McKenna PJ, Radua J, et al. Cognitive-behavioural therapy for the symptoms of schizophrenia: systematic review and meta-analysis with examination of potential bias. Br J Psychiatry. 2014 Jan;204(1):20-9. doi: 10.1192/bjp.bp.112.116285. PMID: 24385461.

78. Jones C, Hacker D, Cormac I, et al. Cognitive behavioural therapy versus other psychosocial treatments for schizophrenia. Cochrane Database Syst Rev. 2012 Apr 18(4):Cd008712. doi: 10.1002/14651858.CD008712.pub2. PMID: 22513966 .

79. Velthorst E, Koeter M, van der Gaag M, et al. Adapted cognitive-behavioural therapy required for targeting negative symptoms in schizophrenia: meta-analysis and metaregression. Psychol Med. 2015 Feb;45(3):453-65. doi: 10.1017/S0033291714001147. PMID: 24993642.

80. Freeman D, Dunn G, Startup H, et al. Effects of cognitive behaviour therapy for worry on persecutory delusions in patients with psychosis (WIT): a parallel, singleblind, randomised controlled trial with a mediation analysis. Lancet Psychiatry. 2015;2(4):305-13. PMID: 26360083.

81. Lysaker PH, Davis LW, Bryson GJ, et al. Effects of cognitive behavioral therapy on work outcomes in vocational rehabilitation for participants with schizophrenia spectrum disorders. Schizophr Res. 2009 Feb;107(23):186-91. doi: 10.1016/j.schres.2008.10.018. PMID: 19046856.

82. Malik N, Kingdon D, Pelton J, et al. Effectiveness of brief cognitive-behavioral therapy for schizophrenia delivered by mental health nurses: relapse and recovery at 24 months. J Clin Psychiatry. 2009 Feb;70(2):201-7. PMID: 19210949.

83. Zimmer M, Duncan AV, Laitano D, et al. A twelve-week randomized controlled study of the cognitive-behavioral integrated psychological therapy program: positive effect on the social functioning of schizophrenic patients. Rev Bras Psiquiatr. 2007 Jun;29(2):140-7. PMID: 17650536.
84. Wykes T, Huddy V, Cellard C, et al. A meta-analysis of cognitive remediation for schizophrenia: methodology and effect sizes. Am J Psychiatry. 2011 May;168(5):472-85. doi: 10.1176/appi.ajp.2010.10060855. PMID: 21406461.

85. Cella M, Preti A, Edwards C, et al. Cognitive remediation for negative symptoms of schizophrenia: A network meta-analysis. Clin Psychol Rev. 2017 Mar;52:43-51. doi: 10.1016/j.cpr.2016.11.009. PMID: 27930934.

86. Deste G, Barlati S, Cacciani P, et al. Persistence of effectiveness of cognitive remediation interventions in schizophrenia: a 1-year follow-up study. Schizophr Res. 2015 Feb;161(2-3):403-6. doi: 10.1016/j.schres.2014.12.004. PMID: 25533593.

87. Farreny A, Aguado J, Ochoa S, et al. REPYFLEC cognitive remediation group training in schizophrenia: looking for an integrative approach. Schizophr Res. 2012 Dec;142(1-3):137-44. doi: 10.1016/j.schres.2012.08.035. PMID: 23017827.

88. Mueller DR, Schmidt SJ, Roder V. One-year randomized controlled trial and follow-up of integrated neurocognitive therapy for schizophrenia outpatients. Schizophr Bull. 2015 May;41(3):604-16. doi: 10.1093/schbul/sbu223. PMID: 25713462.

89. Twamley EW, Vella L, Burton CZ, et al. Compensatory cognitive training for psychosis: effects in a randomized controlled trial. J Clin Psychiatry. 2012 Sep;73(9):1212-9. doi: 10.4088/JCP.12m07686. PMID: 22939029.

90. Vita A, De Peri L, Barlati S, et al. Effectiveness of different modalities of cognitive remediation on symptomatological, neuropsychological, and functional outcome domains in schizophrenia: a prospective study in a realworld setting. Schizophr Res. 2011

Dec;133(1-3):223-31. doi: 10.1016/j.schres.2011.08.010. PMID: 21907544. 
91. Pharoah F, Mari J, Rathbone J, et al. Family intervention for schizophrenia. Cochrane Database Syst Rev. 2010 Dec

08(12):CD000088. doi: 10.1002/14651858.CD000088.pub2. PMID: 21154340.

92. Dyck DG, Short RA, Hendryx MS, et al. Management of negative symptoms among patients with schizophrenia attending multiple-family groups. Psychiatr Serv. 2000 Apr;51(4):513-9. PMID: 10737828.

93. Garety PA, Fowler DG, Freeman D, et al. Cognitive--behavioural therapy and family intervention for relapse prevention and symptom reduction in psychosis: randomised controlled trial. Br J Psychiatry. 2008 Jun;192(6):412-23. doi: 10.1192/bjp.bp.107.043570. PMID: 18515890.

94. Kopelowicz A, Zarate R, Wallace CJ, et al. The ability of multifamily groups to improve treatment adherence in Mexican Americans with schizophrenia. Arch Gen Psychiatry. 2012 Mar;69(3):265-73. doi: 10.1001/archgenpsychiatry.2011.135. PMID: 22393219.

95. Sellwood W, Barrowclough C, Tarrier N, et al. Needs-based cognitive-behavioural family intervention for carers of patients suffering from schizophrenia: 12-month follow-up. Acta Psychiatr Scand. 2001 Nov;104(5):346-55. PMID: 11722315.

96. Mayoral F, Berrozpe A, de la Higuera J, et al. Efficacy of a family intervention program for prevention of hospitalization in patients with schizophrenia. A naturalistic multicenter controlled and randomized study in Spain. Rev Psiquiatr Salud Ment. 2015 Apr-Jun;8(2):83-91. doi: 10.1016/j.rpsm.2013.11.001. PMID: 25017624.

97. Valencia M, Rascon ML, Juarez F, et al. A psychosocial skills training approach in Mexican out-patients with schizophrenia. Psychol Med. 2007 Oct;37(10):1393-402. PMID: 17472761.

98. Marshall M, Gray A, Lockwood A, et al. Case management for people with severe mental disorders. Cochrane Database Syst Rev. 2000b(2):Cd000050. doi: 10.1002/14651858.cd000050. PMID: 10796288 .
99. Bjorkman T, Hansson L, Sandlund M. Outcome of case management based on the strengths model compared to standard care. A randomised controlled trial. Soc Psychiatry Psychiatr Epidemiol. 2002 Apr;37(4):147-52. PMID: 12027240.

100. Zou H, Li Z, Nolan MT, et al. Selfmanagement education interventions for persons with schizophrenia: a meta-analysis. Int J Ment Health Nurs. 2013 Jun;22(3):25671. doi: 10.1111/j.1447-0349.2012.00863.x. PMID: 22882803.

101. Hasson-Ohayon I, Roe D, Kravetz S. A randomized controlled trial of the effectiveness of the illness management and recovery program. Psychiatr Serv. 2007 Nov;58(11):1461-6. doi: 10.1176/ps.2007.58.11.1461. PMID: 17978257.

102. Pekkala E, Merinder L. Psychoeducation for schizophrenia. Cochrane Database Syst Rev. 2002(2):Cd002831. doi: 10.1002/14651858.cd002831. PMID: 12076455.

103. Bartels SJ, Pratt SI, Mueser KT, et al. Longterm outcomes of a randomized trial of integrated skills training and preventive healthcare for older adults with serious mental illness. Am J Geriatr Psychiatry. 2014 Nov;22(11):1251-61. doi: 10.1016/j.jagp.2013.04.013. PMID: 23954039.

104. Mueser KT, Pratt SI, Bartels SJ, et al. Randomized trial of social rehabilitation and integrated health care for older people with severe mental illness. J Consult Clin Psychol. 2010 Aug;78(4):561-73. doi: 10.1037/a0019629. PMID: 20658812.

105. Valencia M, Fresan A, Juarez F, et al. The beneficial effects of combining pharmacological and psychosocial treatment on remission and functional outcome in outpatients with schizophrenia. J Psychiatr Res. 2013 Dec;47(12):1886-92. doi: 10.1016/j.jpsychires.2013.09.006. PMID: 24112947.

106. Kinoshita Y, Furukawa TA, Kinoshita K, et al. Supported employment for adults with severe mental illness. Cochrane Database Syst Rev. 2013 Sep 13(9):Cd008297. doi: 10.1002/14651858.CD008297.pub2. PMID: 24030739 . 
107. Cook JA, Blyler CR, Burke-Miller JK, et al. Effectiveness of supported employment for individuals with schizophrenia: results of a multi-site, randomized trial. Clin Schizophr Relat Psychoses. 2008;2(1):37-46.

108. Cook JA, Leff HS, Blyler CR, et al. Results of a multisite randomized trial of supported employment interventions for individuals with severe mental illness. Arch Gen Psychiatry. 2005 May;62(5):505-12. PMID: 15867103.

109. Mueser KT, Clark RE, Haines M, et al. The Hartford study of supported employment for persons with severe mental illness. J Consult Clin Psychol. 2004 Jun;72(3):479-90. doi: 10.1037/0022-006x.72.3.479. PMID: 15279531.

110. Buckley LA, Maayan N, Soares-Weiser K, et al. Supportive therapy for schizophrenia. Cochrane Database Syst Rev. 2015 Apr 14(4):Cd004716. doi:

10.1002/14651858.CD004716.pub4. PMID: 25871462 .

111. Bertelsen M, Jeppesen P, Petersen L, et al. Course of illness in a sample of 265 patients with first-episode psychosis-five-year follow-up of the Danish OPUS trial. Schizophr Res. 2009;107(2-3):173-8. doi: 10.1016/j.schres.2008.09.018. PMID: 18945593.

112. Craig TK, Garety P, Power P, et al. The Lambeth Early Onset (LEO) team: randomised controlled trial of the effectiveness of specialised care for early psychosis. BMJ. 2004 Nov

6;329(7474):1067. doi: 10.1136/bmj.38246.594873.7C. PMID: 15485934.

113. Garety PA, Craig TK, Dunn G, et al. Specialised care for early psychosis: symptoms, social functioning and patient satisfaction: randomised controlled trial. $\mathrm{Br}$ J Psychiatry. 2006 Jan;188:37-45. doi: 10.1192/bjp.bp.104.007286. PMID: 16388068.
114. Guo X, Zhai J, Liu Z, et al. Effect of antipsychotic medication alone vs combined with psychosocial intervention on outcomes of early-stage schizophrenia: a randomized, 1-year study. Arch Gen Psychiatry. 2010 Sep;67(9):895-904. doi: 10.1001/archgenpsychiatry.2010.105. PMID: 20819983.

115. Guo X, Zhao J, Liu Z, et al. Antipsychotic combination with psychosocial intervention on outcome of schizophrenia (acpios): rationale and design of the clinical trial. Clin Schizophr Relat Psychoses. 2007;1(2):18592.

116. Kane JM, Robinson DG, Schooler NR, et al. Comprehensive versus usual community care for first-episode psychosis: 2-year outcomes from the NIMH RAISE early treatment program. Am J Psychiatry. 2016 Apr 1;173(4):362-72. doi: 10.1176/appi.ajp.2015.15050632. PMID: 26481174.

117. Kane JM, Schooler NR, Marcy P, et al. The RAISE early treatment program for firstepisode psychosis: background, rationale, and study design. J Clin Psychiatry. 2015 Mar;76(3):240-6. doi: 10.4088/JCP.14m09289. PMID: 25830446.

118. Secher RG, Hjorthoj CR, Austin SF, et al. Ten-year follow-up of the OPUS specialized early intervention trial for patients with a first episode of psychosis. Schizophr Bull. 2015 May;41(3):617-26. doi: 10.1093/schbul/sbu155. PMID: 25381449.

119. Tempier R, Balbuena L, Garety P, et al. Does assertive community outreach improve social support? Results from the Lambeth study of early-episode psychosis. Psychiatr Serv. 2012 Mar;63(3):216-22. doi: 10.1176/appi.ps.20110013. PMID: 22388528.

120. Hunt GE, Siegfried N, Morley K, et al. Psychosocial interventions for people with both severe mental illness and substance misuse. Cochrane Database Syst Rev. 2013 Oct 03(10):Cd001088. doi: 10.1002/14651858.CD001088.pub3. PMID: 24092525. 
121. Lieberman JA, Stroup TS, McEvoy JP, et al. Effectiveness of antipsychotic drugs in patients with chronic schizophrenia. N Engl J Med. 2005 Sep 22;353(12):1209-23. doi: 10.1056/NEJMoa051688. PMID: 16172203.

122. McEvoy JP, Lieberman JA, Stroup TS, et al. Effectiveness of clozapine versus olanzapine, quetiapine, and risperidone in patients with chronic schizophrenia who did not respond to prior atypical antipsychotic treatment. Am J Psychiatry. 2006 Apr;163(4):600-10. PMID: 16585434.

123. Stroup TS, Lieberman JA, McEvoy JP, et al. Effectiveness of olanzapine, quetiapine, and risperidone in patients with chronic schizophrenia after discontinuing perphenazine: a CATIE study. Am J Psychiatry. 2007 Mar;164(3):415-27. PMID: 17329466.

124. Stroup TS, Lieberman JA, McEvoy JP, et al. Effectiveness of olanzapine, quetiapine, risperidone, and ziprasidone in patients with chronic schizophrenia following discontinuation of a previous atypical antipsychotic. Am J Psychiatry. 2006 Apr;163(4):611-22. PMID: 16585435.

125. Stroup TS, Lieberman JA, McEvoy JP, et al. Results of phase 3 of the CATIE schizophrenia trial. Schizophr Res. 2009 Jan;107(1):1-12. PMID: 19027269.

126. Manschreck TC, Boshes RA. The CATIE schizophrenia trial: results, impact, controversy. Harv Rev Psychiatry. 2007 Sep-Oct;15(5):245-58. PMID: 17924259.

127. Teich J. The CATIE study. Am J Psychiatry. 2006 Mar;163(3):554-5; author reply 5-6. PMID: 16513894.

128. Weiden PJ. Discontinuing and switching antipsychotic medications: understanding the CATIE schizophrenia trial. J Clin Psychiatry. 2007;68(Suppl1):12-9. PMID: 17286523.

129. Jerrell JM. Cost-effectiveness of risperidone, olanzapine, and conventional antipsychotic medications. Schizophr Bull. 2002;28(4):589-605. PMID: 12795493
130. Ciudad A, Olivares JM, Bousono M, et al. Improvement in social functioning in outpatients with schizophrenia with prominent negative symptoms treated with olanzapine or risperidone in a 1 year randomized, open-label trial. Prog Neuropsychopharmacol Biol Psychiatry. 2006 Dec 30;30(8):1515-22. PMID: 16820255.

131. Haro J, al. E. Effectiveness of antipsychotic treatment for schizophrenia: 6-month results of the Pan-European Schizophrenia Outpatient Health Outcomes (SOHO) study. Acta Psychiatr Scand. 2005;111:220-31. doi: 10.1111/j.1600-0447.2004.00450.x PMID: 15701107

132. Harvey PD, Patterson TL, Potter LS, et al. Improvement in social competence with short-term atypical antipsychotic treatment: a randomized, double-blind comparison of quetiapine versus risperidone for social competence, social cognition, and neuropsychological functioning. Am J Psychiatry. 2006 Nov;163(11):1918-25. PMID: 17074943.

133. Zhong KX, Sweitzer DE, Hamer RM, et al. Comparison of quetiapine and risperidone in the treatment of schizophrenia: a randomized, double-blind, flexible-dose, 8week study. J Clin Psychiatry. 2006 Jul;67(7):1093-103. PMID: 16889453.

134. Wahlbeck K, Cheine M, Tuisku K, et al. Risperidone versus clozapine in treatmentresistant schizophrenia: a randomized pilot study. Prog Neuropsychopharmacol Biol Psychiatry. 2000a;24(6):911-22. PMID: 11041534.

135. Meltzer HY, Bobo WV, Nuamah IF, et al. Efficacy and tolerability of oral paliperidone extended-release tablets in the treatment of acute schizophrenia: pooled data from three 6-week, placebo-controlled studies. J Clin Psychiatry. 2008 May;69(5):817-29. PMID: 18466043.

136. Li H, Rui Q, Ning X, et al. A comparative study of paliperidone palmitate and risperidone long-acting injectable therapy in schizophrenia. Prog Neuropsychopharmacol Biol Psychiatry. 2011 Jun 1;35(4):1002-8. doi: 10.1016/j.pnpbp.2011.02.001. PMID: 21315787. 
137. Takekita Y, Koshikawa Y, Fabbri C, et al. Cognitive function and risperidone longacting injection vs. paliperidone palmitate in schizophrenia: a 6-month, open-label, randomized, pilot trial. BMC Psychiatry. 2016 May 29;16:172. doi: 10.1186/s12888016-0883-9. PMID: 27236412.

138. Rouillon F, Eriksson L, Burba B, et al. Functional recovery results from the risperidone long-acting injectable versus quetiapine relapse prevention trial (ConstaTRE). Acta Neuropsychiatr. 2013;25(5):297-306. doi: 10.1017/neu.2013.7 PMID: 25287730.

139. Resnick SG, Rosenheck RA, Canive JM, et al. Employment outcomes in a randomized trial of second-generation antipsychotics and perphenazine in the treatment of individuals with schizophrenia. J Behav Health Serv Res. 2008 Apr;35(2):215-25. PMID: 18246429.

140. Alonso J, Croudace T, Brown J, et al. Health-related quality of life (HRQL) and continuous antipsychotic treatment: 3-year results from the Schizophrenia Health Outcomes (SOHO) study. Value Health. 2009 Jun;12(4):536-43. PMID: 19900255.

141. Ritsner M, Gibel A, Perelroyzen G, et al. Quality of life outcomes of risperidone, olanzapine, and typical antipsychotics among schizophrenia patients treated in routine clinical practice: a naturalistic comparative study. J Clin Psychopharmacol. 2004;24(6):582-91. PMID: 15538118.

142. Bond GR, Kim HW, Meyer PS, et al. Response to vocational rehabilitation during treatment with first- or second-generation antipsychotics. Psychiatr Serv. 2004;55(1):59-66. doi: 10.1176/appi.ps.55.1.59 PMID: 14699202.

143. Miller DD, Caroff SN, Davis SM, et al. Extrapyramidal side-effects of antipsychotics in a randomised trial. Br J Psychol. 2008 Oct;193(4):279-88. PMID: 18827289.
144. Kinon BJ, Noordsy DL, Liu-Seifert H, et al. Randomized, double-blind 6-month comparison of olanzapine and quetiapine in patients with schizophrenia or schizoaffective disorder with prominent negative symptoms and poor functioning. J Clin Psychopharmacol. 2006 Oct;26(5):45361. PMID: 16974184.

145. Gasquet I, al. E. Pharmacological treatment and other predictors of treatment outcomes in previously untreated patients with schizophrenia: results from the European Schizophrenia Outpatient Health Outcomes (SOHO) study. Int Clin Psychopharmacol. 2005 Jul;20:199-205. PMID: 15933480

146. Kraemer S, Chartier F, Augendre-Ferrante $\mathrm{B}$, et al. Effectiveness of two formulations of oral olanzapine in patients with schizophrenia or bipolar disorder in a natural setting: results from a 1-year European observational study. Hum Psychopharmacol. 2012 May;27(3):284-94. doi: 10.1002/hup.2224. PMID: 22473831.

147. Kane J, Honigfeld G, Singer J, et al. Clozapine for the treatment-resistant schizophrenic. Arch Gen Psychiatry. 1988;45(9):789-96. PMID: 3046553

148. Addington DE, Pantelis C, Dineen M, et al. Efficacy and tolerability of ziprasidone versus risperidone in patients with acute exacerbation of schizophrenia or schizoaffective disorder: an 8-week, doubleblind, multicenter trial. J Clin Psychiatry. 2004 Dec;65(12):1624-33. PMID: 15641867

149. Alvarez E, Ciudad A, Olivares JM, et al. A randomized, 1-year follow-up study of olanzapine and risperidone in the treatment of negative symptoms in outpatients with schizophrenia. J Clin Psychopharmacol. 2006 Jun;26(3):238-49. PMID: 16702888.

150. Apiquian R, Fresan A, Herrera K, et al. Minimum effective doses of haloperidol for the treatment of first psychotic episode: a comparative study with risperidone and olanzapine. Int J Neuropsychopharmacol. 2003 Dec;6(4):403-8. PMID: 14604455. 
151. Azorin JM, Spiegel R, Remington G, et al. A double-blind comparative study of clozapine and risperidone in the management of severe chronic schizophrenia. Am J Psychiatry. 2001;158(8):1305-13. doi: 10.1176/appi.ajp.158.8.1305 PMID: 11481167

152. Bitter I, Dossenbach MR, Brook S, et al. Olanzapine versus clozapine in treatmentresistant or treatment-intolerant schizophrenia. Prog Neuropsychopharmacol Biol Psychiatry. 2004 Jan;28(1):173-80. doi: 10.1016/j.pnpbp.2003.09.033 PMID: 14687871.

153. Bondolfi G, Dufour H, Patris M, et al. Risperidone versus clozapine in treatmentresistant chronic schizophrenia: a randomized double-blind study. The Risperidone study group. Am J Psychiatry. 1998;155(4):499-504. doi: 10.1176/ajp.155.4.499 PMID: 9545995

154. Buchanan RW, Panagides J, Zhao J, et al. Asenapine versus olanzapine in people with persistent negative symptoms of schizophrenia. J Clin Psychopharmacol. 2012 Feb;32(1):36-45. doi: 10.1097/JCP.0b013e31823f880a. PMID: 22198451.

155. Chan H-Y, Lin W-W, Lin S-K, et al. Efficacy and safety of aripiprazole in the acute treatment of schizophrenia in Chinese patients with risperidone as an active control: a randomized trial. J Clin Psychiatry. 2007 Jan;68(1):29-36. PMID: 17284127.

156. Chowdhury AN, Mukherjee A, Ghosh K, et al. Horizon of a new hope: recovery of schizophrenia in India. IMJ. 1999;6(3):1815.

157. Conley RR, Mahmoud R. A randomized double-blind study of risperidone and olanzapine in the treatment of schizophrenia or schizoaffective disorder.[erratum appears in Am J Psychiatry 2001 Oct;158(10):1759]. Am J Psychiatry. 2001;158(5):765-74. doi: 10.1176/appi.ajp.158.5.765 PMID: 11329400.
158. Conley RR, Kelly DL, Nelson MW, et al. Risperidone, quetiapine, and fluphenazine in the treatment of patients with therapyrefractory schizophrenia. Clin Neuropharmacol. 2005 Jul-Aug;28(4):163-8. PMID: 16062094

159. Crespo-Facorro B, Perez-Iglesias R, Ramirez-Bonilla M, et al. A practical clinical trial comparing haloperidol, risperidone, and olanzapine for the acute treatment of first-episode nonaffective psychosis. J Clin Psychiatry. 2006 11/21/2007;67(10):1511-21. PMID: 17107241 .

160. Fleischhacker WW, McQuade RD, Marcus $\mathrm{RN}$, et al. A double-blind, randomized comparative study of aripiprazole and olanzapine in patients with schizophrenia. Biol Psychiatry. 2009 Mar 15;65(6):510-7. PMID: 18986646.

161. Grootens KP, van Veelen NMJ, Peuskens J, et al. Ziprasidone vs olanzapine in recentonset schizophrenia and schizoaffective disorder: results of an 8-week double-blind randomized controlled trial. Schizophr Res. 2011 Mar;37(2):352-61. doi: 10.1093/schbul/sbp037. PMID: 19542525.

162. Gureje O, Miles W, Keks N, et al. Olanzapine vs risperidone in the management of schizophrenia: a randomized double-blind trial in Australia and New Zealand. Schizophr Res. 2003;61(2-3):30314. PMID: 12729882

163. Jeste DV, Barak Y, Madhusoodanan S, et al. International multisite double-blind trial of the atypical antipsychotics risperidone and olanzapine in 175 elderly patients with chronic schizophrenia. Am J Geriatr Psychiatry. 2003;11(6):638-47. PMID: 14609804.

164. Kane JM, Osuntokun O, Kryzhanovskaya LA, et al. A 28-week, randomized, doubleblind study of olanzapine versus aripiprazole in the treatment of schizophrenia. J Clin Psychiatry. 2009 Apr;70(4):572-81. PMID: 19323965.

165. Keefe R, al. E. One-year double-blind study of the neurocognitive efficacy of olanzapine, risperidone, and haloperidol in schizophrenia. Schizophr Res. 2006;81:1-15. doi: 10.1016/j.schres.2005.07.038 PMID: 16202565. 
166. Keks NA, Ingham M, Khan A, et al. Longacting injectable risperidone v. olanzapine tablets for schizophrenia or schizoaffective disorder. Randomised, controlled, openlabel study. Br J Psychol. 2007;191:131-9. doi: 10.1192/bjp.bp.105.017020 PMID: 17666497.

167. Li YM, Zhao JP, Ou JJ, et al. Efficacy and tolerability of ziprasidone vs. olanzapine in naive first-episode schizophrenia: a 6-week, randomized, open-label, flexible-dose study. Pharmacopsychiatry. 2012 Jul;45(5):177-81. doi: 10.1055/s-0031-1299769. PMID: 22290206.

168. McCue RE, Waheed R, Urcuyo L, et al. Comparative effectiveness of secondgeneration antipsychotics and haloperidol in acute schizophrenia. Br J Psychol. 2006 Nov;189:433-40. PMID: 17077434.

169. McEvoy JP, Lieberman JA, Perkins DO, et al. Efficacy and tolerability of olanzapine, quetiapine, and risperidone in the treatment of early psychosis: a randomized, doubleblind 52-week comparison. Am J Psychiatry. 2007 Jul;164(7):1050-60. PMID: 17606657.

170. McQuade RD, Stock E, Marcus R, et al. A comparison of weight change during treatment with olanzapine or aripiprazole: results from a randomized, double-blind study. J Clin Psychiatry. 2004;65 Suppl 18:47-56. PMID: 15600384.

171. Newcomer JW, Ratner RE, Eriksson JW, et al. A 24-week, multicenter, open-label, randomized study to compare changes in glucose metabolism in patients with schizophrenia receiving treatment with olanzapine, quetiapine, or risperidone. J Clin Psychiatry. 2009 Apr;70(4):487-99. PMID: 19358783.

172. Potkin SG, Gharabawi GM, Greenspan AJ, et al. A double-blind comparison of risperidone, quetiapine and placebo in patients with schizophrenia experiencing an acute exacerbation requiring hospitalization. Schizophr Res. 2006 Jul;85(1-3):254-65. PMID: 16797162.
173. Robinson DG, Woerner MG, Napolitano B, et al. Randomized comparison of olanzapine versus risperidone for the treatment of firstepisode schizophrenia: 4-month outcomes. Am J Psychiatry. 2006 Dec;163(12):2096102. PMID: 17151160.

174. Sacchetti E, Valsecchi P, Parrinello G, et al. A randomized, flexible-dose, quasinaturalistic comparison of quetiapine, risperidone, and olanzapine in the short-term treatment of schizophrenia: the QUERISOLA trial. Schizophr Res. 2008 Jan;98(1-3):55-65. PMID: 17933497.

175. Savitz AJ, Lane R, Nuamah I, et al. Efficacy and safety of paliperidone extended release in adolescents with schizophrenia: a randomized, double-blind study. J Am Acad Child Adolesc Psychiatry. 2015;54(2):12637.e1. PMID: 25617253.

176. Schoemaker J, Stet L, Vrijland P, et al. Long-term efficacy and safety of asenapine or olanzapine in patients with schizophrenia or schizoaffective disorder: an extension study. Pharmacopsychiatry. 2012 Jul;45(5):196-203. doi: 10.1055/s-00311301310. PMID: 22454251.

177. Schreiner A, Niehaus D, Shuriquie NA, et al. Metabolic effects of paliperidone extended release versus oral olanzapine in patients with schizophrenia: a prospective, randomized, controlled trial. J Clin Psychopharmacol. 2012 Aug;32(4):449-57. doi: 10.1097/JCP.0b013e31825cccad. PMID: 22722501.

178. Simpson GM, Glick ID, Weiden PJ, et al. Randomized, controlled, double-blind multicenter comparison of the efficacy and tolerability of ziprasidone and olanzapine in acutely ill inpatients with schizophrenia or schizoaffective disorder. Am J Psychiatry. 2004 Oct;161(10):1837-47. doi: 10.1176/ajp.161.10.1837 PMID: 15465981.

179. Suzuki T, Uchida H, Watanabe K, et al. How effective is it to sequentially switch among olanzapine, quetiapine and risperidone?--A randomized, open-label study of algorithm-based antipsychotic treatment to patients with symptomatic schizophrenia in the real-world clinical setting. Psychopharmacology (Berl). 2007 Dec;195(2):285-95. PMID: 17701027. 
180. Tollefson GD, Birkett MA, Kiesler GM, et al. Double-blind comparison of olanzapine versus clozapine in schizophrenic patients clinically eligible for treatment with clozapine. Biol Psychiatry. 2001;49(1):5263. PMID: 11163780

181. Tran PV, Hamilton SH, Kuntz AJ, et al. Double-blind comparison of olanzapine versus risperidone in the treatment of schizophrenia and other psychotic disorders. J Clin Psychopharmacol. 1997;17(5):40718. PMID: 9315992.

182. van Bruggen J, Tijssen J, Dingemans P, et al. Symptom response and side-effects of olanzapine and risperidone in young adults with recent onset schizophrenia. Int Clin Psychopharmacol. 2003;18(6):341-6. doi: 10.1097/01.yic.0000097037.25861.df PMID: 14571154.

183. Samara MT, Dold M, Gianatsi M, et al. Efficacy, acceptability, and tolerability of antipsychotics in treatment-resistant schizophrenia: a network meta-analysis. JAMA Psychiatry. 2016 Mar;73(3):199-210. doi: 10.1001/jamapsychiatry.2015.2955. PMID: 26842482.

184. AstraZeneca. A multicenter, open-label, flexible-dose, parallel-group evaluation of the cataractogenic potential of quetiapine fumarate (Seroquel) and risperidone (Risperdal) in the Long-term treatment of patients with schizophrenia or schizoaffective disorder [CLEARS]. Clinical Study Report Synopsis. 2010.

185. AstraZeneca. An 8-week, multicenter, double-blind, randomized, parallel-group, placebo-controlled study of the efficacy and safety of quetiapine fumarate (SEROQUEL) extended-release in children and adolescent subjects with bipolar depression. Clinical Study Report Synopsis. 201131 May 2011;D144AC00001.

186. Mullen J, Jibson MD, Sweitzer D. A comparison of the relative safety, efficacy, and tolerability of quetiapine and risperidone in outpatients with schizophrenia and other psychotic disorders: the quetiapine experience with safety and tolerability (QUEST) study. Clin Ther. 2001;23(11):1839-54. PMID: 11768836.
187. Schoemaker J, Naber D, Vrijland P, et al. Long-term assessment of asenapine vs. olanzapine in patients with schizophrenia or schizoaffective disorder.[Erratum appears in Pharmacopsychiatry. 2011 Nov;44(7):343]. Pharmacopsychiatry. 2010 Jun;43(4):13846. doi: 10.1055/s-0030-1248313. PMID: 20205074.

188. Fleischhacker W, Gopal S, Lane R, et al. A randomized trial of paliperidone palmitate and risperidone long-acting injectable in schizophrenia. Int J Neuropsychopharmacol. 2012 Feb;15(1):107-18. doi:

10.1017/S1461145711001076. PMID: 21777507.

189. Pasternak B, Svanstrom H, Ranthe MF, et al. Atypical antipsychotics olanzapine, quetiapine, and risperidone and risk of acute major cardiovascular events in young and middle-aged adults: a nationwide registerbased cohort study in Denmark. CNS Drugs. 2014 Oct;28(10):963-73. doi: 10.1007/s40263-014-0176-0. PMID: 24895158.

190. Kiviniemi M, Suvisaari J, KoivumaaHonkanen H, et al. Antipsychotics and mortality in first-onset schizophrenia: prospective Finnish register study with 5year follow-up. Schizophr Res. 2013 Oct;150(1):274-80. doi: 10.1016/j.schres.2013.07.043. PMID: 23953217.

191. Kelly DL, McMahon RP, Liu F, et al. Cardiovascular disease mortality in patients with chronic schizophrenia treated with clozapine: a retrospective cohort study. J Clin Psychiatry. 2010 Mar;71(3):304-11. doi: 10.4088/JCP.08m04718yel. PMID: 20079332.

192. Meltzer HY, Alphs L, Green AI, et al. Clozapine treatment for suicidality in schizophrenia: International Suicide Prevention Trial (InterSePT). Arch Gen Psychiatry. 2003;60(1):82-91. PMID: 12511175

193. Bitter I, Katona L, Zambori J, et al. Comparative effectiveness of depot and oral second generation antipsychotic drugs in schizophrenia: a nationwide study in Hungary. Eur Neuropsychopharmacol. 2013 Nov;23(11):1383-90. doi: 10.1016/j.euroneuro.2013.02.003. PMID: 23477752. 
194. Leucht S, Cipriani A, Spineli L, et al. Comparative efficacy and tolerability of 15 antipsychotic drugs in schizophrenia: a multiple-treatments meta-analysis. Lancet. 2013 Sep 14;382(9896):951-62. doi: 10.1016/S0140-6736(13)60733-3. PMID: 23810019.

195. Hermes E, Rosenheck R. Choice of randomization to clozapine versus other second generation antipsychotics in the CATIE schizophrenia trial. J Clin Psychopharmacol. 2012 Sep;26(9):1194200. doi: $10.1177 / 0269881112443744$. PMID: 22516668.

196. AstraZeneca. (D1444C00132-EMBOLDEN II) A 6-week, international, multicenter, double-blind, doubledummy, randomized comparison of the efficacy and safety of sustained-release formulation quetiapine fumarate (SEROQUEL ${ }^{\mathrm{TM}}$ ) and placebo in the treatment of acutely ill patients with schizophrenia. 2007.

197. Breier A, al e. Olanzapine versus ziprasidone: results of a 28-week doubleblind study in patients with schizophrenia. Am J Psychiatry. 2005 Oct;162:1879-87. doi: 10.1176/appi.ajp.162.10.1879 PMID: 16199834.

198. Canuso CM, Dirks B, Carothers J, et al. Randomized, double-blind, placebocontrolled study of paliperidone extendedrelease and quetiapine in inpatients with recently exacerbated schizophrenia. Am J Psychiatry. 2009 Jun;166(6):691-701. PMID: 19411369.

199. Chue P, Eerdekens M, Augustyns I, et al. Comparative efficacy and safety of longacting risperidone and risperidone oral tablets. Eur Neuropsychopharmacol. 2005 Jan;15(1):111-7. doi:

10.1016/j.euroneuro.2004.07.003 PMID: 15572280

200. Citrome L, Cucchiaro J, Sarma K, et al. Long-term safety and tolerability of lurasidone in schizophrenia: a 12-month, double-blind, active-controlled study. Int Clin Psychopharmacol. 2012

May;27(3):165-76. doi: 10.1097/YIC.0b013e32835281ef. PMID: 22395527.
201. Cutler AJ, Kalali AH, Weiden PJ, et al. Four-week, double-blind, placebo- and ziprasidone-controlled trial of iloperidone in patients with acute exacerbations of schizophrenia. J Clin Psychopharmacol. 2008 Apr;28(2 Suppl 1):S20-8. PMID: 18334909.

202. Davidson M, Emsley R, Kramer M, et al. Efficacy, safety and early response of paliperidone extended-release tablets (paliperidone ER): results of a 6-week, randomized, placebo-controlled study. Schizophr Res. 2007 Jul;93(1-3):117-30. PMID: 17466492.

203. Emsley R, Lambert T, McGrath J, et al. Risperidone in the treatment of first-episode psychotic patients: a double-blind multicenter study. Schizophr Bull. 1999;25(4):721-9. PMID: 10667742.

204. Gaebel W, Schreiner A, Bergmans P, et al. Relapse prevention in schizophrenia and schizoaffective disorder with risperidone long-acting injectable vs quetiapine: results of a long-term, open-label, randomized clinical trial. Neuropsychopharmacology. 2010 Nov;35(12):2367-77. doi: 10.1038/npp.2010.111. PMID: 20686456.

205. Kane J, Canas F, Kramer M, et al. Treatment of schizophrenia with paliperidone extended-release tablets: a 6-week placebocontrolled trial. Schizophr Res. 2007 Feb;90(1-3):147-61. PMID: 17092691.

206. Kim S-W, Chung Y-C, Lee Y-H, et al. Paliperidone ER versus risperidone for neurocognitive function in patients with schizophrenia: a randomized, open-label, controlled trial. Int Clin Psychopharmacol. 2012 Sep;27(5):267-74. doi: 10.1097/YIC.0b013e328356acad. PMID: 22809972.

207. Kluge M, Himmerich H, Wehmeier PM, et al. Sleep propensity at daytime as assessed by Multiple Sleep Latency Tests (MSLT) in patients with schizophrenia increases with clozapine and olanzapine. Schizophr Res. 2012 Mar;135(1-3):123-7. doi: 10.1016/j.schres.2011.12.017. PMID: 22257975. 
208. Kluge M, Schuld A, Himmerich H, et al. Clozapine and olanzapine are associated with food craving and binge eating: results from a randomized double-blind study. J Clin Psychopharmacol. 2007 Dec;27(6):6626. PMID: 18004133.

209. Kane JM, Detke HC, Naber D, et al. Olanzapine long-acting injection: a 24week, randomized, double-blind trial of maintenance treatment in patients with schizophrenia. Am J Psychiatry. 2010 Feb;167(2):181-9. PMID: 20008947.

210. Lindenmayer JP. The efficacy and tolerability of once-daily extended release quetiapine fumarate in hospitalized patients with acute schizophrenia: a 6-week randomized, double-blind, placebocontrolled study. Psychopharmacol Bull. 2008;41(3):11-35. PMID: 18779774.

211. Macfadden W, Ma Y-W, Haskins J, et al. A prospective study comparing the long-term effectiveness of injectable risperidone longacting therapy and oral aripiprazole in patients with schizophrenia. Psychiatry. 2010 Nov;7(11):23-31. PMID: 21191530.

212. McDonnell DP, Kryzhanovskaya LA, Zhao $\mathrm{F}$, et al. Comparison of metabolic changes in patients with schizophrenia during randomized treatment with intramuscular olanzapine long-acting injection versus oral olanzapine. Hum Psychopharmacol. 2011 Aug;26(6):422-33. doi: 10.1002/hup.1225 PMID: 21823172.

213. Meltzer HY, Cucchiaro J, Silva R, et al. Lurasidone in the treatment of schizophrenia: a randomized, double-blind, placebo- and olanzapine-controlled study. Am J Psychiatry. 2011 Sep;168(9):957-67. doi: 10.1176/appi.ajp.2011.10060907. PMID: 21676992.

214. Naber D, Riedel M, Klimke A, et al. Randomized double blind comparison of olanzapine vs. clozapine on subjective wellbeing and clinical outcome in patients with schizophrenia. Acta Psychiatr Scand. 2005 Feb;111(2):106-15. PMID: 15667429.
215. Nakajima S, Takeuchi H, Fervaha G, et al. Comparative efficacy between clozapine and other atypical antipsychotics on depressive symptoms in patients with schizophrenia: analysis of the CATIE phase 2E data. Schizophr Res. 2015 Feb;161(2-3):429-33. PMID: 25556080.

216. Newcomer JW, Campos JA, Marcus RN, et al. A multicenter, randomized, double-blind study of the effects of aripiprazole in overweight subjects with schizophrenia or schizoaffective disorder switched from olanzapine. J Clin Psychiatry. 2008 Jul;69(7):1046-56. PMID: 18605811.

217. Alphs L, Bossie CA, Sliwa JK, et al. Paliperidone palmitate and risperidone longacting injectable in subjects with schizophrenia recently treated with oral risperidone or other oral antipsychotics. Neuropsychiatr Dis Treat. 2013 Mar;9:341 50. PMID: 23493643.

218. Pandina G, Lane Rd, Gopal S, et al. A double-blind study of paliperidone palmitate and risperidone long-acting injectable in adults with schizophrenia. Prog Neuropsychopharmacol Biol Psychiatry. 2011 Jan 15;35(1):218-26. doi: 10.1016/j.pnpbp.2010.11.008. PMID: 21092748.

219. Potkin SG, Cohen M, Panagides J. Efficacy and tolerability of asenapine in acute schizophrenia: a placebo- and risperidonecontrolled trial. J Clin Psychiatry. 2007 Oct;68(10):1492-500. PMID: 17960962.

220. Potkin SG, Ogasa M, Cucchiaro J, et al. Double-blind comparison of the safety and efficacy of lurasidone and ziprasidone in clinically stable outpatients with schizophrenia or schizoaffective disorder. Schizophr Res. 2011 Nov;132(2-3):101-7. doi: 10.1016/j.schres.2011.04.008. PMID: 21889878.

221. Potkin SG, Saha AR, Kujawa MJ, et al. Aripiprazole, an antipsychotic iith a novel mechanism of action, and risperidone vs placebo in patients with schizophrenia and schizoaffective disorder. Arch Gen Psychiatry. 2003b;60(7):681-90. doi: 10.1001/archpsyc.60.7.681 PMID: 12860772. 
222. Sacchetti E, Galluzzo A, Valsecchi P, et al. Ziprasidone vs clozapine in schizophrenia patients refractory to multiple antipsychotic treatments: the MOZART study. Schizophr Res. 2009 May;110(1-3):80-9. PMID: 19269791.

223. Schering Plough. Data on file. (Unpublished Study 25543) A multicenter, double-blind, flexible-dose, 6-month trial comparing the efficacy and safeaty of asenapine with olanzapnie in stable subjects with predominant, persistent negative symptoms of schizophrenia. 2006.

224. Schering Plough. Data on file. (Unpublished Study 25517) Long-term assessment of asenapine versus olanzapine in patients with schizophrenia or schizoaffective disorder. 2006.

225. Schering Plough. (Study 041022) A multicenter, randomized, double-blind, flexible-dose, 6-week trial of the efficacy and safety of asenapine compared with placebo using olanzapine positive control in subjects with an acute exacerbation of schizophrenia. 2006.

226. Simpson G, al. E. Six-month, blinded, multicenter continuation study of ziprasidone versus olanzapine in schizophrenia. Am J Psychiatry. 2005 Aug;162:1535-8. doi: 10.1176/appi.ajp.162.8.1535 PMID: 16055779.

227. Sirota P, Pannet I, Koren A, et al. Quetiapine versus olanzapine for the treatment of negative symptoms in patients with schizophrenia. Hum Psychopharmacol. 2006 Jun;21(4):227-34. PMID: 16783811.

228. Conley RR, Kelly DL, Richardson CM, et al. The efficacy of high-dose olanzapine versus clozapine in treatment-resistant schizophrenia: A double-blind, crossover study. J Clin Psychopharmacol. 2003 Dec;23(6):668-71. PMID: 14624201.

229. Daniel DG, Goldberg TE, Weinberger DR, et al. Different side effect profiles of risperidone and clozapine in 20 outpatients with schizophrenia or schizoaffective disorder: a pilot study. Am J Psychiatry. 1996 Mar;153(3):417-9. doi: 10.1176/ajp.153.3.417 PMID: 8610833.
230. Hatta K, Sato K, Hamakawa H, et al. Effectiveness of second-generation antipsychotics with acute-phase schizophrenia. Schizophr Res. 2009;113(1):49-55. doi: 10.1016/j.schres.2009.05.030 PMID: 19553086.

231. Kahn RS. Effectiveness of antipsychotic drugs in first-episode schizophrenia and schizophreniform disorder: an open randomized clinical trial. World Psychiatry. 2009;8(Suppl 1):44-5.

232. Krakowski MI, Czobor P, Citrome L, et al. Atypical antipsychotic agents in the treatment of violent patients with schizophrenia and schizoaffective disorder. Arch Gen Psychiatry. 2006 Jun;63(6):622-9. PMID: 16754835.

233. Lindenmayer JP, Iskander A, Park M, et al. Clinical and neurocognitive effects of clozapine and risperidone in treatmentrefractory schizophrenic patients: a prospective study. J Clin Psychiatry. 1998 Oct;59(10):521-7. PMID: 9818633.

234. Lublin H, Haug H-J, Koponen H, et al. Ziprasidone versus olanzapine, risperidone or quetiapine in patients with chronic schizophrenia: a 12-week open-label, multicentre clinical trial. World J Biol Psychiatry. 2009;10(4 Pt 3):710-8. doi: 10.1080/15622970802269589. PMID: 18803070.

235. Perez-Iglesias R, Crespo-Facorro B, Amado JA, et al. A 12-week randomized clinical trial to evaluate metabolic changes in drugnaive, first-episode psychosis patients treated with haloperidol, olanzapine, or risperidone. J Clin Psychiatry. 2007 Nov;68(11):1733-40. PMID: 18052567.

236. Potkin SG, Alphs L, Hsu C, et al. Predicting suicidal risk in schizophrenic and schizoaffective patients in a prospective two-year trial. Biol Psychiatry. 2003;54(4):444-52. PMID: 12915289.

237. Potkin SG, Litman RE, Torres R, et al. Efficacy of iloperidone in the treatment of schizophrenia: initial phase 3 studies. J Clin Psychopharmacol. 2008 Apr;28(2 Suppl 1):S4-11. PMID: 18334911. 
238. Purdon SE, Jones BD, Stip E, et al. Neuropsychological change in early phase schizophrenia during 12 months of treatment with olanzapine, risperidone, or haloperidol. The Canadian Collaborative Group for research in schizophrenia. Arch Gen Psychiatry. 2000 Mar;57(3):249-58. PMID: 10711911.

239. Riedel M, al e. Quetiapine has equivalent efficacy and superior tolerability to risperidone in the treatment of schizophrenia with predominantly negative symptoms. Eur Arch Psychiatry Clin Neurosci. 2005 Nov;255:432-7. doi: 10.1007/s00406-0050622-6 PMID: 16267634.

240. Ritchie C, al. E. A comparison of the efficacy and safety of olanzapine and risperidone in the treatment of elderly patients with schizophrenia: an open study of six months duration. Int J Geriatr Psychiatry. 2006;21(2):171-9. doi: 10.1002/gps.1446 PMID: 16416458

241. Volavka J, Czobor P, Nolan K, et al. Overt aggression and psychotic symptoms in patients with schizophrenia treated with clozapine, olanzapine, risperidone, or haloperidol. J Clin Psychopharmacol. 2004 Apr;24(2):225-8. PMID: 15206671.

242. Zhang Y, Dai G. Efficacy and metabolic influence of paliperidone ER, aripiprazole and ziprasidone to patients with first-episode schizophrenia through 52 weeks follow-up in China. Hum Psychopharmacol. 2012 Nov;27(6):605-14. doi: 10.1002/hup.2270. PMID: 24446539.

243. Chrzanowski WK, Marcus RN, Torbeyns A, et al. Effectiveness of long-term aripiprazole therapy in patients with acutely relapsing or chronic, stable schizophrenia: a 52-week, open-label comparison with olanzapine. Psychopharmacology (Berl). 2006 Dec;189(2):259-66. PMID: 17058105.

244. Perez-Iglesias R, Ortiz-Garcia de la Foz V, Martinez Garcia O, et al. Comparison of metabolic effects of aripiprazole, quetiapine and ziprasidone after 12 weeks of treatment in first treated episode of psychosis.

Schizophr Res. 2014 Oct;159(1):90-4. doi: 10.1016/j.schres.2014.07.045. PMID: 25151200 .
245. Hennessy S, Bilker WB, Knauss JS, et al. Cardiac arrest and ventricular arrhythmia in patients taking antipsychotic drugs: cohort study using administrative data. BMJ. 2002 Nov 09;325(7372):1070. PMID: 12424166.

246. Lambert BL, Cunningham FE, Miller DR, et al. Diabetes risk associated with use of olanzapine, quetiapine, and risperidone in veterans health administration patients with schizophrenia. Am J Epidemiol. 2006 Oct 1;164(7):672-81. PMID: 16943266.

247. Feldman PD, Kaiser CJ, Kennedy JS, et al. Comparison of risperidone and olanzapine in the control of negative symptoms of chronic schizophrenia and related psychotic disorders in patients aged 50 to 65 years. $\mathrm{J}$ Clin Psychiatry. 2003;64(9):998-1004.

248. Usall J, Suarez D, Haro JM, et al. Gender differences in response to antipsychotic treatment in outpatients with schizophrenia. Psychiatry Res. 2007 Dec 3;153(3):225-31. PMID: 17681611.

249. Ciliberto N, Bossie CA, Urioste R, et al. Lack of impact of race on the efficacy and safety of long-acting risperidone versus placebo in patients with schizophrenia or schizoaffective disorder. Int Clin Psychopharmacol. 2005 Jul;20(4):207-12. PMID: 15933481.

250. Opolka J, al. E. Role of ethnicity in predicting antipsychotic medication adherence. Ann Pharmacother. 2003;37:62530. PMID: 12708934.

251. Kim JH, Kim D, Marder SR. Time to rehospitalization of clozapine versus risperidone in the naturalistic treatment of comorbid alcohol use disorder and schizophrenia. Prog Neuropsychopharmacol Biol Psychiatry. 2008 May 15;32(4):984-8. PMID: 18262321.

252. Swartz MS, Wagner HR, Swanson JW, et al. The effectiveness of antipsychotic medications in patients who use or avoid illicit substances: results from the CATIE study. Schizophr Res. 2008 Mar;100(13):39-52. PMID: 18191383.

253. Akerele E, Levin FR. Comparison of olanzapine to risperidone in substanceabusing individuals with schizophrenia. Am J Addict. 2007 Jul-Aug;16(4):260-8. PMID: 17661193. 
254. Kinon B, ed. Improvement of comorbid depression with olanzapine versus ziprasidone treatment in patients with schizophrenia or schizoaffective disorder. Eleventh Biennial Winter Workshop on Schizophrenia; 2004 Feb 7-14; Davos, Switzerland.

255. Addington DE, Labelle A, Kulkarni J, et al. A comparison of ziprasidone and risperidone in the long-term treatment of schizophrenia: a 44-week, double-blind, continuation study. Can J Psychiatry. 2009 Jan;54(1):46-54. PMID: 19175979.

256. Tollefson GD, Andersen SW. Should we consider mood disturbance in schizophrenia as an important determinant of quality of life? J Clin Psychiatry. 1999b;60(5):23-9. PMID: 10192404

257. Sajatovic M, Mullen JA, Sweitzer DE. Efficacy of quetiapine and risperidone against depressive symptoms in outpatients with psychosis. J Clin Psychiatry. 2002;63(12):1156-63. PMID: 12523876.

258. Zhang J-P, Robinson DG, Gallego JA, et al. Association of a schizophrenia risk variant at the DRD2 locus with antipsychotic treatment response in first-episode psychosis. Schizophr Bull. 2015 Nov;41(6):1248-55. doi: 10.1093/schbul/sbv116. PMID: 26320194.

259. Boter H, Derks EM, Fleischhacker WW, et al. Generalizability of the results of efficacy trials in first-episode schizophrenia: comparisons between subgroups of participants of the European First Episode Schizophrenia Trial (EUFEST). J Clin Psychiatry. 2010 Jan;71(1):58-65. PMID: 19852905.

260. Gafoor R, Landau S, Craig TKJ, et al. Esquire trial: efficacy and adverse effects of quetiapine versus risperidone in first-episode schizophrenia. J Clin Psychopharmacol. 2010 Oct;30(5):600-6. doi: 10.1097/JCP.0b013e3181f198da. PMID: 20814318.
261. Crespo-Facorro B, Ortiz-Garcia de la Foz V, Mata I, et al. Aripiprazole, ziprasidone and quetiapine in the treatment of first-episode nonaffective psychosis: a 12-week randomized, flexible-dose, open-label trial. Schizophr Res. 2013 Jul;147(2-3):375-82. doi: 10.1016/j.schres.2013.04.014. PMID: 23643328.

262. Lieberman JA. Pathophysiologic mechanisms in the pathogenesis and clinical course of schizophrenia. J Clin Psychiatry. 1999;60 Suppl 12:9-12. PMID: 10372603.

263. Souza JS, Kayo M, Tassell I, et al. Efficacy of olanzapine in comparison with clozapine for treatment-resistant schizophrenia: evidence from a systematic review and meta-analyses. CNS Spectr. 2013 Apr;18(2):82-9. doi: 10.1017/S1092852912000806. PMID: 23253621.

264. Ranasinghe I, Sin J. A systematic review of evidence-based treatment for individuals with treatment-resistant schizophrenia and a suboptimal response to clozapine monotherapy. Psychosis. 2014 Jul;6(3):25365. doi: 10.1080/17522439.2013.830319.

265. Ritchie CW, Harrigan S, Mastwyk M, et al. Predictors of adherence to atypical antipsychotics (risperidone or olanzapine) in older patients with schizophrenia: an open study of 3(1/2) years duration. Int J Geriatr Psychiatry. 2010 Apr;25(4):411-8. doi: 10.1002/gps.2354. PMID: 19946860.

266. Sutton VK, Street JS, Kennedy JS, et al. Superiority of olanzapine over risperidone in the control of negative symptoms of schizophrenia and related psychotic disorders in older patients. Eur Neuropsychopharmacol. 2001;11:276.

267. Meyer JM, Rosenblatt LC, Kim E, et al. The moderating impact of ethnicity on metabolic outcomes during treatment with olanzapine and aripiprazole in patients with schizophrenia. J Clin Psychiatry. 2009 Mar;70(3):318-25. PMID: 19192469.

268. Kishi T, Matsuda Y, Matsunaga S, et al. Aripiprazole for the management of schizophrenia in the Japanese population: a systematic review and meta-analysis of randomized controlled trials. Neuropsychiatr Dis Treat. 2015 Feb;11:419-34. doi: 10.2147/NDT.S78977 PMID: 25759585. 
269. Zhang H, Li H, Liu Y, et al. Safety and efficacy of paliperidone extended-release in Chinese patients with schizophrenia: A 24week, open-label extension of a randomized, double-blind, placebo-controlled study. Neuropsychiatr Dis Treat 2016 Jan;12:6977. doi: 10.2147/NDT.S88875 PMID: 26811679

270. Sevy S, Robinson DG, Sunday S, et al. Olanzapine vs. risperidone in patients with first-episode schizophrenia and a lifetime history of cannabis use disorders: 16-week clinical and substance use outcomes. Psychiatry Res. 2011 Aug 15;188(3):310-4. doi: 10.1016/j.psychres.2011.05.001. PMID: 21636134.

271. Kim SH, Han DH, Joo SY, et al. The effect of dopamine partial agonists on the nicotine dependency in patients with schizophrenia. Hum Psychopharmacol. 2010 Mar;25(2):187-90. doi: 10.1002/hup.1089. PMID: 20033907.

272. Dieterich M, Irving CB, Park B, et al. Intensive case management for severe mental illness. Cochrane Database Syst Rev. 2010 Oct 06(10):Cd007906. doi: 10.1002/14651858.CD007906.pub2. PMID: 20927766.

273. Leucht S, Kane JM, Kissling W, et al. What does the PANSS mean? Schizophr Res. 2005 Nov 15;79(2-3):231-8. doi: 10.1016/j.schres.2005.04.008. PMID: 15982856.

274. Barrowclough C, Haddock G, Lobban F, et al. Group cognitive-behavioural therapy for schizophrenia. Randomised controlled trial. Br J Psychiatry. 2006 Dec;189:527-32. PMID: 17139037.

275. Granholm E, McQuaid JR, McClure FS, et al. A randomized, controlled trial of cognitive behavioral social skills training for middle-aged and older outpatients with chronic schizophrenia. Am J Psychiatry. 2005 Mar;162(3):520-9. PMID: 15741469.

276. Grant PM, Huh GA, Perivoliotis D, et al. Randomized trial to evaluate the efficacy of cognitive therapy for low-functioning patients with schizophrenia. Arch Gen Psychiatry. 2012 Feb;69(2):121-7. doi: 10.1001/archgenpsychiatry.2011.129. PMID: 21969420.
277. Gumley A, O'Grady M, McNay L, et al. Early intervention for relapse in schizophrenia: results of a 12-month randomized controlled trial of cognitive behavioural therapy. Psychol Med. 2003 Apr;33(3):419-31. PMID: 12701663.

278. Lincoln TM, Ziegler M, Mehl S, et al. Moving from efficacy to effectiveness in cognitive behavioral therapy for psychosis: a randomized clinical practice trial. J Consult Clin Psychol. 2012 Aug;80(4):674-86. doi: 10.1037/a0028665. PMID: 22663901.

279. van der Gaag M, Stant AD, Wolters KJ, et al. Cognitive-behavioural therapy for persistent and recurrent psychosis in people with schizophrenia-spectrum disorder: costeffectiveness analysis. Br J Psychiatry. 2011 Jan;198(1):59-65, sup 1. doi: 10.1192/bjp.bp.109.071522. PMID: 21200078.

280. Haddock G, Barrowclough C, Shaw JJ, et al. Cognitive-behavioural therapy v. social activity therapy for people with psychosis and a history of violence: randomised controlled trial. Br J Psychiatry. 2009 Feb;194(2):152-7. doi: 10.1192/bjp.bp.107.039859. PMID: 19182178.

281. Kemp R, Kirov G, Everitt B, et al. Randomised controlled trial of compliance therapy. 18-month follow-up. Br J Psychiatry. 1998 May;172:413-9. PMID: 9747403.

282. Jackson HJ, McGorry PD, Killackey E, et al. Acute-phase and 1-year follow-up results of a randomized controlled trial of CBT versus befriending for first-episode psychosis: the ACE project. Psychol Med. 2008 May;38(5):725-35. PMID: 18005494.

283. Karow A, Wittmann L, Schottle D, et al. The assessment of quality of life in clinical practice in patients with schizophrenia. Dialogues Clin Neurosci. 2014 Jun;16(2):185-95. PMID: 25152657.

284. Lewis S, Tarrier N, Haddock G, et al. Randomised controlled trial of cognitivebehavioural therapy in early schizophrenia: acute-phase outcomes. Br J Psychiatry Suppl. 2002 Sep;43:s91-7. PMID: 12271807. 
285. Sensky T, Turkington D, Kingdon D, et al. A randomized controlled trial of cognitivebehavioral therapy for persistent symptoms in schizophrenia resistant to medication. Arch Gen Psychiatry. 2000 Feb;57(2):16572. PMID: 10665619.

286. Tarrier N, Wittkowski A, Kinney C, et al. Durability of the effects of cognitivebehavioural therapy in the treatment of chronic schizophrenia: 12-month follow-up. Br J Psychiatry. 1999 Jun;174:500-4. doi: 10.1192/bjp.174.6.500. PMID: 10616627.

287. Tarrier N, Yusupoff L, Kinney C, et al. Randomised controlled trial of intensive cognitive behaviour therapy for patients with chronic schizophrenia. BMJ. 1998 Aug 1;317(7154):303-7. PMID: 9685273.

288. Saperstein AM, Medalia A. The empirical basis for the practice of cognitive remediation for schizophrenia. Neuropsychiatry. 2012;2(2):101-9. doi: 10.2217/npy.12.10.

289. Sellwood W, Wittkowski A, Tarrier N, et al. Needs-based cognitive-behavioural family intervention for patients suffering from schizophrenia: 5-year follow-up of a randomized controlled effectiveness trial. Acta Psychiatr Scand. 2007 Dec;116(6):44752. PMID: 17961200.

290. Barrowclough C, Tarrier N, Lewis S, et al. Randomised controlled effectiveness trial of a needs-based psychosocial intervention service for carers of people with schizophrenia. Br J Psychiatry. 1999 Jun;174:505-11. PMID: 10616628.

291. Lenior ME, Dingemans PM, Linszen DH, et al. Social functioning and the course of early-onset schizophrenia: five-year followup of a psychosocial intervention. Br J Psychiatry. 2001;179:53-8. PMID: 11435269.

292. Bauml J, Pitschel-Walz G, Volz A, et al. Psychoeducation in schizophrenia: 7-year follow-up concerning rehospitalization and days in hospital in the Munich Psychosis Information Project Study. J Clin Psychiatry. 2007 Jun;68(6):854-61. PMID: 17592908.
293. Kurtz MM, Mueser KT. A meta-analysis of controlled research on social skills training for schizophrenia. J Consult Clin Psychol. 2008 Jun;76(3):491-504. doi: 10.1037/0022006X.76.3.491. PMID: 18540742.

294. Almerie MQ, Okba Al Marhi M, Jawoosh $\mathrm{M}$, et al. Social skills programmes for schizophrenia. Cochrane Database Syst Rev. 2015 Jun 09(6):Cd009006. doi: 10.1002/14651858.CD009006.pub2. PMID: 26059249.

295. Dixon LB, Dickerson F, Bellack AS, et al. The 2009 schizophrenia PORT psychosocial treatment recommendations and summary statements. Schizophr Bull. 2010 Jan;36(1):48-70. doi: 10.1093/schbul/sbp115. PMID: 19955389.

296. Marshall M, Rathbone J. Early intervention for psychosis. Cochrane Database Syst Rev. 2011 Jun 15(6):Cd004718. doi: 10.1002/14651858.CD004718.pub3. PMID: 21678345.

297. Kishimoto T, Robenzadeh A, Leucht C, et al. Long-acting injectable vs oral antipsychotics for relapse prevention in schizophrenia: a meta-analysis of randomized trials. Schizophr Bull. 2014 Jan;40(1):192-213. doi: 10.1093/schbul/sbs150. PMID: 23256986.

298. Zhang J-P, Gallego JA, Robinson DG, et al. Efficacy and safety of individual secondgeneration vs. first generation antipsychotics in first-episode psychosis: a systematic review and meta-analysis. Int $\mathrm{J}$ Neuropsychopharmacol. 2013 Jul;16(6):1205-18. doi: 10.1017/S1461145712001277. PMID: 23199972.

299. Fusar-Poli P, Kempton MJ, Rosenheck RA. Efficacy and safety of second-generation long-acting injections in schizophrenia: a meta-analysis of randomized-controlled trials. Int Clin Psychopharmacol. 2013 Mar;28(2):57-66. doi: 10.1097/YIC.0b013e32835b091f. PMID: 23165366. 
300. Soares-Weiser K, Bechard-Evans L, Lawson $\mathrm{AH}$, et al. Time to all-cause treatment discontinuation of olanzapine compared to other antipsychotics in the treatment of schizophrenia: a systematic review and meta-analysis. Eur Neuropsychopharmacol. 2013 Feb;23(2):118-25. doi: 10.1016/j.euroneuro.2012.05.001. PMID: 22633617.

301. Tonin FS, Piazza T, Wiens A, et al. Adverse events and treatment failure leading to discontinuation of recently approved antipsychotic drugs in schizophrenia: a network meta-analysis. Schizophr Res. 2015 Dec;169(1-3):483-5. doi: 10.1016/j.schres.2015.09.019. PMID: 26516102.

302. Asenjo Lobos C, Komossa K, RummelKluge C, et al. Clozapine versus other atypical antipsychotics for schizophrenia. Cochrane Database Syst Rev. 2010 Nov 10(11):Cd006633. doi: 10.1002/14651858.CD006633.pub2. PMID: 21069690.

303. Asmal L, Flegar SJ, Wang J, et al. Quetiapine versus other atypical antipsychotics for schizophrenia. Cochrane Database Syst Rev. 2013 Nov

18(11):Cd006625. doi: 10.1002/14651858.CD006625.pub3. PMID: 24249315.

304. Ayub M, Saeed K, Munshi TA, et al. Clozapine for psychotic disorders in adults with intellectual disabilities. Cochrane Database Syst Rev. 2015 Sep

23(9):Cd010625. doi: 10.1002/14651858.CD010625.pub2. PMID: 26397173.

305. Kirsch I. Placebo psychotherapy: synonym or oxymoron? J Clin Psychol. 2005

Jul;61(7):791-803. doi: 10.1002/jclp.20126. PMID: 15827992.

306. Chambless DL, Hollon SD. Defining empirically supported therapies. J Consult Clin Psychol. 1998 Feb;66(1):7-18. PMID: 9489259.

307. Jensen PS, Weersing R, Hoagwood KE, et al. What is the evidence for evidence-based treatments? A hard look at our soft underbelly. Ment Health Serv Res. 2005 Mar;7(1):53-74. PMID: 15832693.
308. Vickers A, Goyal N, Harland R, et al. Do certain countries produce only positive results? A systematic review of controlled trials. Control Clin Trials. 1998;19(2):15966. doi: 10.1016/S0197-2456(97)00150-5. PMID: 9551280.

309. Wykes T, Steel C, Everitt B, et al. Cognitive behavior therapy for schizophrenia: effect sizes, clinical models, and methodological rigor. Schizophr Bull. 2008 May;34(3):52337. PMID: 17962231.

310. Rector NA, Beck AT. Cognitive behavioral therapy for schizophrenia: An empirical review. J Nerv Ment Dis. 2001

May;189(5):278-87. doi: 10.1097/00005053-200105000-00002. PMID: 11379970.

311. Sarin F, Wallin L, Widerlov B. Cognitive behavior therapy for schizophrenia: a metaanalytical review of randomized controlled trials. Nord J Psychiatry. 2011

Jun;65(3):162-74. doi: 10.3109/08039488.2011.577188. PMID: 21563994.

312. Turkington D, Sensky T, Scott J, et al. A randomized controlled trial of cognitivebehavior therapy for persistent symptoms in schizophrenia: a five-year follow-up. Schizophr Res. 2008 Jan;98(1-3):1-7. PMID: 17936590.

313. Drury V, Birchwood M, Cochrane R. Cognitive therapy and recovery from acute psychosis: a controlled trial. 3. Five-year follow-up. Br J Psychiatry. 2000 Jul;177:814. PMID: 10945081.

314. McGurk SR, Twamley EW, Sitzer DI, et al. A meta-analysis of cognitive remediation in schizophrenia. Am J Psychiatry. 2007 Dec;164(12):1791-802. PMID: 18056233.

315. Pitschel-Walz G, Leucht S, Bauml J, et al. The effect of family interventions on relapse and rehospitalization in schizophrenia--a meta-analysis. Schizophr Bull. 2001;27(1):73-92. PMID: 11215551.

316. Dixon L, Adams C, Lucksted A. Update on family psychoeducation for schizophrenia. Schizophr Bull. 2000;26(1):5-20. PMID: 10755667. 
317. Dixon L, Duncan DC, Johnson P, et al. Occupational therapy for patients with Parkinson's disease. Cochrane Database Syst Rev. 2009(4) PMID: 17636709.

318. Xia J, Merinder BL, Belgamwar MR. Psychoeducation for schizophrenia. Cochrane Database Syst Rev. 2013(1) PMID: 21678337.

319. Pfammatter M, Junghan UM, Brenner HD. Efficacy of psychological therapy in schizophrenia: conclusions from metaanalyses. Schizophr Bull. 2006;32(suppl_1):S64-S80. doi: 10.1093/schbul/sbl030. PMID: 16905634.

320. Marshall T, Goldberg RW, Braude L, et al. Supported employment: assessing the evidence. Psychiatr Serv. 2014 Jan 01;65(1):16-23. doi: 10.1176/appi.ps.201300262. PMID: 24247197.

321. Balshem H, Helfand $M$, Schünemann HJ, et al. GRADE guidelines: 3 . Rating the quality of evidence. J Clin Epidemiol. 2011;64(4):401-6. PMID: 21208779.

322. Guyatt GH, Oxman AD, Kunz R, et al. GRADE guidelines: 2. Framing the question and deciding on important outcomes. J Clin Epidemiol. 2011;64(4):395-400. PMID: 21194891.

323. Guyatt GH, Oxman AD, Kunz R, et al. GRADE guidelines: 6 . Rating the quality of evidence-imprecision. J Clin Epidemiol. 2011;64(12):1283-93. PMID: 21839614.

324. Guyatt GH, Oxman AD, Kunz R, et al. GRADE guidelines: 8. Rating the quality of evidence-indirectness. J Clin Epidemiol. 2011;64(12):1303-10. PMID: 21802903.

325. Guyatt GH, Oxman AD, Kunz R, et al. GRADE guidelines: 7. Rating the quality of evidence-inconsistency. J Clin Epidemiol. 2011;64(12):1294-302. PMID: 21803546.

326. Guyatt GH, Oxman AD, Montori V, et al. GRADE guidelines: 5 . Rating the quality of evidence-publication bias. J Clin Epidemiol. 2011;64(12):1277-82. PMID: 29802904.
327. Guyatt GH, Oxman AD, Vist G, et al. GRADE guidelines: 4 . Rating the quality of evidence - study limitations (risk of bias). J Clin Epidemiol. 2011;64(4):407-15. PMID: 21247734.

328. American Psychiatric Association. Work group on Schizophrenia; Lehman AF, Lieberman JA, Dixon LB, et al. Practice guideline for the treatment of patients with schizophrenia, second edition. Am J Psychiatry. 2004;161:1-56. PMID: 15000267.

329. American Psychiatric Association. Dixon L, Perkins D, Calmes C. Guideline watch: practice guideline for the treatment of patients with schizophrenia. Arlington, VA: American Psychiatric Association; September 2009. http://psychiatryonline.org/pb/assets/raw/site wide/practice guidelines/guidelines/schizop hrenia-watch.pdf. Accessed April 22, 2017.

330. Ben-Zeev D, Davis KE, Kaiser S, et al. Mobile technologies among people with serious mental illness: opportunities for future services. Adm Policy Ment Health. 2013 Jul;40(4):340-3. doi: 10.1007/s10488012-0424-x. PMID: 22648635.

331. Gaynes BN, Brown C, Lux LJ, et al. Management Strategies To Reduce Psychiatric Readmissions. Rockville (MD): Agency for Healthcare Research and Quality (US); 2015.

332. Schulz KF, Altman DG, Moher D. CONSORT 2010 Statement: updated guidelines for reporting parallel group randomised trials. BMJ. 2010;340. doi: 10.1136/bmj.c332. PMID: 20332509.

333. Guise JM, Chang C, Viswanathan M, et al. AHRQ Methods for Effective Health Care Agency for Healthcare Research and Quality (US). Rockville (MD): 2014. PMID: 24696890.

334. Guise JM, Chang C, Viswanathan M, et al. Agency for Healthcare Research and Quality Evidence-based Practice Center methods for systematically reviewing complex multicomponent health care interventions. J Clin Epidemiol. 2014 Nov;67(11):1181-91. doi: 10.1016/j.jclinepi.2014.06.010. PMID: 25438663. 


\section{Abbreviations}

\begin{tabular}{|c|c|}
\hline Abbreviation & Definition \\
\hline ACT & assertive community treatment \\
\hline AHRQ & Agency for Healthcare Research \& Quality \\
\hline APA & American Psychiatric Association \\
\hline BPRS & Brief Psychiatric Rating Scale \\
\hline CAT & cognitive adaption training \\
\hline CATIE & Clinical Antipsychotic Trials of Intervention Effectiveness \\
\hline CBT & cognitive behavioral therapy \\
\hline CGI & Clinical Global Impression Scale \\
\hline CGI-I & Clinical Global Impression - Improvement scale \\
\hline CGI-S & Clinical Global Impression - Severity scale \\
\hline CGI-SS & Clinical Global Impression of severity - Suicidality Scale \\
\hline $\mathrm{Cl}$ & confidence interval \\
\hline CPRS & Comprehensive Psychopathology Rating Scale \\
\hline DACT & Dartmouth Assertive Community Treatment Scale \\
\hline DERP & Drug Effectiveness Review Project \\
\hline df & degrees of freedom \\
\hline DSM & American Psychiatric Association's Diagnostic and Statistical Manual of Mental Disorders \\
\hline DSM-III & American Psychiatric Association's Diagnostic and Statistical Manual of Mental Disorders, version 3 \\
\hline DSM-IV & American Psychiatric Association's Diagnostic and Statistical Manual of Mental Disorders, version 4 \\
\hline DSM-5 & American Psychiatric Association's Diagnostic and Statistical Manual of Mental Disorders, version 5 \\
\hline EHC & Effective Health Care \\
\hline EPC & Evidence-based Practice Center \\
\hline EPS & extrapyramidal symptoms \\
\hline EQ-5D & EuroQol five dimensions questionnaire \\
\hline ER & extended release \\
\hline FDA & U.S. Food and Drug Administration \\
\hline FEP & first-episode psychosis \\
\hline FGA & first-generation antipsychotic \\
\hline GAF & Global Assessment of Functioning \\
\hline GAF-M & Global Assessment of Functioning - Modified version \\
\hline GAS & Global Assessment Scale \\
\hline GHQ & Global Health Questionnaire \\
\hline HAM-D & Hamilton Depression Scale \\
\hline $\mathrm{HDI}$ & United Nations International Human Development Index \\
\hline $\mathrm{HR}$ & hazard ratio \\
\hline ICM & intensive case management \\
\hline ILSS & Independent Living Skills Survey \\
\hline IPS & individual placement and support \\
\hline IR & immediate release \\
\hline $\mathrm{KQ}$ & key question \\
\hline LAl & long-acting injectable \\
\hline LSM & least squares mean \\
\hline MADRS & Montgomery-Asberg Depression Scale \\
\hline MCAS & The Multnomah Community Ability Scale \\
\hline MD & mean difference \\
\hline $\mathrm{N}$ & Number \\
\hline NNT & number needed to treat \\
\hline ODT & oral dissolving tablet \\
\hline OR & odds ratio \\
\hline PACT & Program of Assertive Community Treatment \\
\hline PANSS & Positive and Negative Syndrome Scale \\
\hline PEAT & Penn Emotional Acuity Test \\
\hline PICOT & population, intervention, comparison, outcomes, timing \\
\hline PSP & Personal and Social Performance Scale \\
\hline QLS & Heinrich Carpenter Quality of Life Scale \\
\hline QOLI & Subjective Quaity of Life \\
\hline
\end{tabular}




\begin{tabular}{|l|l|}
\hline Abbreviation & Definition \\
\hline RCT & randomized controlled trial \\
\hline RFS & Role Functioning Scale \\
\hline RR & relative risk \\
\hline RSES & Rosenberg Self-Esteem Scale \\
\hline SAS & Social Adjustment Scale - Severely Mentally Ill version \\
\hline SADS-C +PD & Schedule for Affective Disorders and Schizophrenia - Change Version with psychosis and disorganization \\
\hline SANS & Scale for Assessment of Negative Symptoms \\
\hline SAPS & Scale for the Assessment of Positive Symptoms \\
\hline SF-12 & 12-Item Short Form Health Survey \\
\hline SFS & Social Function Scale \\
\hline SGA & Second-generation antipsychotic \\
\hline SIP & Scientific Information Packet \\
\hline SMD & standardized mean difference \\
\hline SOE & strength of evidence \\
\hline SOFAS & Social and Occupational Functioning Assessment Scale \\
\hline SOHO & Schizophrenia Outpatient Health Outcome \\
\hline SQLS-R4 & Schizophrenia Quality of Life Scale - Revision 4 \\
\hline SR & systematic review \\
\hline SSPA & Social Skills Performance Assessment \\
\hline SUD & substance use disorder \\
\hline TEP & Technical Expert Panel \\
\hline UPSA-B & San Diego Performance Based Skills Assessment - Brief \\
\hline VPRS & Seven point verbal rating scale \\
\hline vS. & Versus \\
\hline WBI & Work Behavior Inventory \\
\hline WBS & Well-being Scale \\
\hline WMD & weighted mean difference \\
\hline
\end{tabular}




\title{
Appendix A. Search Strategies
}

\section{Key Question 1}

\section{FGA Versus SGA}

\author{
Database: Ovid MEDLINE \\ 1 exp Schizophrenia/dt [Drug Therapy] \\ disease supplementary concept word, unique identifier] \\ concept word, unique identifier] \\ 41 and 2 and 3 \\ 5 limit 4 to english language \\ 6 limit 5 to (meta analysis or systematic reviews) \\ 7 limit 5 to randomized controlled trial \\ 8 limit 5 to controlled clinical trial \\ 96 or 7 or 8 \\ 10 (201* not 2010*).ed,dp. \\ 119 and 10
}

2 (Aripiprazole or Asenapine or Brexpiprazole or Cariprazine or Clozapine or Iloperidone or Lurasidone or Olanzapine or Paliperidone or Quetiapine or Risperidone or Ziprasidone).mp. [mp=title, abstract, original title, name of substance word, subject heading word, keyword heading word, protocol supplementary concept word, rare

3 (Fluphenazine or Haloperidol or Perphenazine).mp. [mp=title, abstract, original title, name of substance word, subject heading word, keyword heading word, protocol supplementary concept word, rare disease supplementary

Database: EBM Reviews - Cochrane Database of Systematic Reviews

1 schizophren*.mp. [mp=title, abstract, full text, keywords, caption text]

2 (Aripiprazole or Asenapine or Brexpiprazole or Cariprazine or Clozapine or Iloperidone or Lurasidone or Olanzapine or Paliperidone or Quetiapine or Risperidone or Ziprasidone).mp. [mp=title, abstract, full text, keywords, caption text]

3 (Fluphenazine or Haloperidol or Perphenazine).mp. [mp=title, abstract, full text, keywords, caption text] 41 and 2 and 3

5 limit 4 to $y r=" 2011$-Current"

\section{Database: EBM Reviews - Cochrane Central Register of Controlled Trials}

1 schizophren*.mp. [mp=title, original title, abstract, mesh headings, heading words, keyword]

2 (Aripiprazole or Asenapine or Brexpiprazole or Cariprazine or Clozapine or Iloperidone or Lurasidone or Olanzapine or Paliperidone or Quetiapine or Risperidone or Ziprasidone).mp. [mp=title, original title, abstract, mesh headings, heading words, keyword]

3 (Fluphenazine or Haloperidol or Perphenazine).mp. [mp=title, original title, abstract, mesh headings, heading words, keyword]

41 and 2 and 3

5 limit 4 to $y r=" 2011$-Current"

\section{Database: PsycINFO}

1 exp Schizophrenia/

2 (Aripiprazole or Asenapine or Brexpiprazole or Cariprazine or Clozapine or Iloperidone or Lurasidone or Olanzapine or Paliperidone or Quetiapine or Risperidone or Ziprasidone).mp. [mp=title, abstract, heading word, table of contents, key concepts, original title, tests \& measures]

3 (Fluphenazine or Haloperidol or Perphenazine).mp. [mp=title, abstract, heading word, table of contents, key concepts, original title, tests \& measures]

41 and 2 and 3

5 limit 4 to english language

6 limit 5 to systematic reviews

7 limit 5 to "2000 treatment outcome/clinical trial"

8 random*.mp. 
9 (clinical* adj5 (trial* or study or protocol*)).mp.

108 and 9

11 ((random* or control*) adj5 (trial* or study or protocol*)).mp.

125 and 10

135 and 11

146 or 7 or 12 or 13

15 (201* not 2010*).dp,up.

1614 and 15

\section{SGA Versus SGA}

\section{Database: Ovid MEDLINE}

1 exp Schizophrenia/dt [Drug Therapy]

2 ((compar* or contrast* or evaluat* or analy* or measur* or quanti* or judg*) adj7 (((second or 2nd) adj2 generation) or atypical*) adj3 (antipsychotic* or anti-psychotic*)).mp.

3 (Aripiprazole and (Asenapine or Brexpiprazole or Cariprazine or Clozapine or Iloperidone or Lurasidone or Olanzapine or Paliperidone or Quetiapine or Risperidone or Ziprasidone)).mp. [mp=title, abstract, original title, name of substance word, subject heading word, keyword heading word, protocol supplementary concept word, rare disease supplementary concept word, unique identifier]

4 (Asenapine and (Aripiprazole or Brexpiprazole or Cariprazine or Clozapine or Iloperidone or Lurasidone or Olanzapine or Paliperidone or Quetiapine or Risperidone or Ziprasidone)).mp. [mp=title, abstract, original title, name of substance word, subject heading word, keyword heading word, protocol supplementary concept word, rare disease supplementary concept word, unique identifier]

5 (Brexpiprazole and (Aripiprazole or Asenapine or Cariprazine or Clozapine or Iloperidone or Lurasidone or Olanzapine or Paliperidone or Quetiapine or Risperidone or Ziprasidone)).mp.

6 (Cariprazine and (Aripiprazole or Asenapine or Brexpiprazole or Clozapine or Iloperidone or Lurasidone or Olanzapine or Paliperidone or Quetiapine or Risperidone or Ziprasidone)).mp. [mp=title, abstract, original title, name of substance word, subject heading word, keyword heading word, protocol supplementary concept word, rare disease supplementary concept word, unique identifier]

7 (Clozapine and (Aripiprazole or Asenapine or Brexpiprazole or Cariprazine or Iloperidone or Lurasidone or Olanzapine or Paliperidone or Quetiapine or Risperidone or Ziprasidone)).mp. [mp=title, abstract, original title, name of substance word, subject heading word, keyword heading word, protocol supplementary concept word, rare disease supplementary concept word, unique identifier]

8 (Iloperidone and (Aripiprazole or Asenapine or Brexpiprazole or Cariprazine or Clozapine or Lurasidone or Olanzapine or Paliperidone or Quetiapine or Risperidone or Ziprasidone)).mp. [mp=title, abstract, original title, name of substance word, subject heading word, keyword heading word, protocol supplementary concept word, rare disease supplementary concept word, unique identifier]

9 (Lurasidone and (Aripiprazole or Asenapine or Brexpiprazole or Cariprazine or Clozapine or Iloperidone or Olanzapine or Paliperidone or Quetiapine or Risperidone or Ziprasidone)).mp. [mp=title, abstract, original title, name of substance word, subject heading word, keyword heading word, protocol supplementary concept word, rare disease supplementary concept word, unique identifier]

10 (Olanzapine and (Aripiprazole or Asenapine or Brexpiprazole or Cariprazine or Clozapine or Iloperidone or Lurasidone or Paliperidone or Quetiapine or Risperidone or Ziprasidone)).mp. [mp=title, abstract, original title, name of substance word, subject heading word, keyword heading word, protocol supplementary concept word, rare disease supplementary concept word, unique identifier]

11 (Paliperidone and (Aripiprazole or Asenapine or Brexpiprazole or Cariprazine or Clozapine or Iloperidone or Lurasidone or Olanzapine or Quetiapine or Risperidone or Ziprasidone)).mp. [mp=title, abstract, original title, name of substance word, subject heading word, keyword heading word, protocol supplementary concept word, rare disease supplementary concept word, unique identifier]

12 (Quetiapine and (Aripiprazole or Asenapine or Brexpiprazole or Cariprazine or Clozapine or Iloperidone or Lurasidone or Olanzapine or Paliperidone or Risperidone or Ziprasidone)).mp. [mp=title, abstract, original title, name of substance word, subject heading word, keyword heading word, protocol supplementary concept word, rare disease supplementary concept word, unique identifier]

13 (Risperidone and (Aripiprazole or Asenapine or Brexpiprazole or Cariprazine or Clozapine or Iloperidone or Lurasidone or Olanzapine or Paliperidone or Quetiapine or Ziprasidone)).mp. [mp=title, abstract, original title, name 
of substance word, subject heading word, keyword heading word, protocol supplementary concept word, rare disease supplementary concept word, unique identifier]

14 (Ziprasidone and (Aripiprazole or Asenapine or Brexpiprazole or Cariprazine or Clozapine or Iloperidone or Lurasidone or Olanzapine or Paliperidone or Quetiapine or Risperidone)).mp. [mp=title, abstract, original title, name of substance word, subject heading word, keyword heading word, protocol supplementary concept word, rare disease supplementary concept word, unique identifier]

152 or 3 or 4 or 5 or 6 or 7 or 8 or 9 or 10 or 11 or 12 or 13 or 14

161 and 15

17 limit 16 to english language

18 limit 17 to systematic reviews

19 limit 17 to randomized controlled trial

20 limit 17 to controlled clinical trial

2118 or 19 or 20

22 (2013* or $2014 *$ or $2015 *$ or $2016 *$ ).ed,dp.

2321 and 22

\section{Database: EBM Reviews - Cochrane Database of Systematic Reviews}

1 Schizophren*.mp. [mp=title, abstract, full text, keywords, caption text]

2 ((compar* or contrast* or evaluat* or analy* or measur* or quanti* or judg*) adj7 (((second or 2nd) adj2 generation) or atypical*) adj3 (antipsychotic* or anti-psychotic*)).mp.

3 (Aripiprazole and (Asenapine or Brexpiprazole or Cariprazine or Clozapine or Iloperidone or Lurasidone or Olanzapine or Paliperidone or Quetiapine or Risperidone or Ziprasidone)).mp. [mp=title, abstract, full text, keywords, caption text]

4 (Asenapine and (Aripiprazole or Brexpiprazole or Cariprazine or Clozapine or Iloperidone or Lurasidone or Olanzapine or Paliperidone or Quetiapine or Risperidone or Ziprasidone)).mp. [mp=title, abstract, full text, keywords, caption text]

5 (Brexpiprazole and (Aripiprazole or Asenapine or Cariprazine or Clozapine or Iloperidone or Lurasidone or Olanzapine or Paliperidone or Quetiapine or Risperidone or Ziprasidone)).mp.

6 (Cariprazine and (Aripiprazole or Asenapine or Brexpiprazole or Clozapine or Iloperidone or Lurasidone or Olanzapine or Paliperidone or Quetiapine or Risperidone or Ziprasidone)).mp. [mp=title, abstract, full text, keywords, caption text]

7 (Clozapine and (Aripiprazole or Asenapine or Brexpiprazole or Cariprazine or Iloperidone or Lurasidone or Olanzapine or Paliperidone or Quetiapine or Risperidone or Ziprasidone)).mp. [mp=title, abstract, full text, keywords, caption text]

8 (Iloperidone and (Aripiprazole or Asenapine or Brexpiprazole or Cariprazine or Clozapine or Lurasidone or Olanzapine or Paliperidone or Quetiapine or Risperidone or Ziprasidone)).mp. [mp=title, abstract, full text, keywords, caption text]

9 (Lurasidone and (Aripiprazole or Asenapine or Brexpiprazole or Cariprazine or Clozapine or Iloperidone or Olanzapine or Paliperidone or Quetiapine or Risperidone or Ziprasidone)).mp. [mp=title, abstract, full text, keywords, caption text]

10 (Olanzapine and (Aripiprazole or Asenapine or Brexpiprazole or Cariprazine or Clozapine or Iloperidone or Lurasidone or Paliperidone or Quetiapine or Risperidone or Ziprasidone)).mp. [mp=title, abstract, full text, keywords, caption text]

11 (Paliperidone and (Aripiprazole or Asenapine or Brexpiprazole or Cariprazine or Clozapine or Iloperidone or Lurasidone or Olanzapine or Quetiapine or Risperidone or Ziprasidone)).mp. [mp=title, abstract, full text, keywords, caption text]

12 (Quetiapine and (Aripiprazole or Asenapine or Brexpiprazole or Cariprazine or Clozapine or Iloperidone or Lurasidone or Olanzapine or Paliperidone or Risperidone or Ziprasidone)).mp. [mp=title, abstract, full text, keywords, caption text]

13 (Risperidone and (Aripiprazole or Asenapine or Brexpiprazole or Cariprazine or Clozapine or Iloperidone or Lurasidone or Olanzapine or Paliperidone or Quetiapine or Ziprasidone)).mp. [mp=title, abstract, full text, keywords, caption text]

14 (Ziprasidone and (Aripiprazole or Asenapine or Brexpiprazole or Cariprazine or Clozapine or Iloperidone or Lurasidone or Olanzapine or Paliperidone or Quetiapine or Risperidone)).mp. [mp=title, abstract, full text, keywords, caption text]

152 or 3 or 4 or 5 or 6 or 7 or 8 or 9 or 10 or 11 or 12 or 13 or 14 
161 and 15

17 limit 16 to english language [Limit not valid; records were retained]

18 (2013* or $2014^{*}$ or $2015^{*}$ or $2016^{*}$ ).up.

1917 and 18

\section{Database: EBM Reviews - Cochrane Central Register of Controlled Trials}

1 Schizophren*.mp. [mp=title, original title, abstract, mesh headings, heading words, keyword]

2 ((compar* or contrast* or evaluat* or analy* or measur* or quanti* or judg*) adj7 (((second or 2nd) adj2 generation) or atypical*) adj3 (antipsychotic* or anti-psychotic*)).mp.

3 (Aripiprazole and (Asenapine or Brexpiprazole or Cariprazine or Clozapine or Iloperidone or Lurasidone or Olanzapine or Paliperidone or Quetiapine or Risperidone or Ziprasidone)).mp. [mp=title, original title, abstract, mesh headings, heading words, keyword]

4 (Asenapine and (Aripiprazole or Brexpiprazole or Cariprazine or Clozapine or Iloperidone or Lurasidone or Olanzapine or Paliperidone or Quetiapine or Risperidone or Ziprasidone)).mp. [mp=title, original title, abstract, mesh headings, heading words, keyword]

5 (Brexpiprazole and (Aripiprazole or Asenapine or Cariprazine or Clozapine or Iloperidone or Lurasidone or Olanzapine or Paliperidone or Quetiapine or Risperidone or Ziprasidone)).mp.

6 (Cariprazine and (Aripiprazole or Asenapine or Brexpiprazole or Clozapine or Iloperidone or Lurasidone or Olanzapine or Paliperidone or Quetiapine or Risperidone or Ziprasidone)).mp. [mp=title, original title, abstract, mesh headings, heading words, keyword]

7 (Clozapine and (Aripiprazole or Asenapine or Brexpiprazole or Cariprazine or Iloperidone or Lurasidone or Olanzapine or Paliperidone or Quetiapine or Risperidone or Ziprasidone)).mp. [mp=title, original title, abstract, mesh headings, heading words, keyword]

8 (Iloperidone and (Aripiprazole or Asenapine or Brexpiprazole or Cariprazine or Clozapine or Lurasidone or Olanzapine or Paliperidone or Quetiapine or Risperidone or Ziprasidone)).mp. [mp=title, original title, abstract, mesh headings, heading words, keyword]

9 (Lurasidone and (Aripiprazole or Asenapine or Brexpiprazole or Cariprazine or Clozapine or Iloperidone or Olanzapine or Paliperidone or Quetiapine or Risperidone or Ziprasidone)).mp. [mp=title, original title, abstract, mesh headings, heading words, keyword]

10 (Olanzapine and (Aripiprazole or Asenapine or Brexpiprazole or Cariprazine or Clozapine or Iloperidone or Lurasidone or Paliperidone or Quetiapine or Risperidone or Ziprasidone)).mp. [mp=title, original title, abstract, mesh headings, heading words, keyword]

11 (Paliperidone and (Aripiprazole or Asenapine or Brexpiprazole or Cariprazine or Clozapine or Iloperidone or Lurasidone or Olanzapine or Quetiapine or Risperidone or Ziprasidone)).mp. [mp=title, original title, abstract, mesh headings, heading words, keyword]

12 (Quetiapine and (Aripiprazole or Asenapine or Brexpiprazole or Cariprazine or Clozapine or Iloperidone or Lurasidone or Olanzapine or Paliperidone or Risperidone or Ziprasidone)).mp. [mp=title, original title, abstract, mesh headings, heading words, keyword]

13 (Risperidone and (Aripiprazole or Asenapine or Brexpiprazole or Cariprazine or Clozapine or Iloperidone or Lurasidone or Olanzapine or Paliperidone or Quetiapine or Ziprasidone)).mp. [mp=title, original title, abstract, mesh headings, heading words, keyword]

14 (Ziprasidone and (Aripiprazole or Asenapine or Brexpiprazole or Cariprazine or Clozapine or Iloperidone or Lurasidone or Olanzapine or Paliperidone or Quetiapine or Risperidone)).mp. [mp=title, original title, abstract, mesh headings, heading words, keyword]

152 or 3 or 4 or 5 or 6 or 7 or 8 or 9 or 10 or 11 or 12 or 13 or 14

161 and 15

17 limit 16 to english language

18 (2013* or $2014^{*}$ or $2015^{*}$ or $\left.2016^{*}\right)$.up.

1917 and 18

\section{Database: PsycINFO}

1 exp Schizophrenia/

2 ((compar* or contrast* or evaluat* or analy* or measur* or quanti* or judg*) adj7 (((second or 2nd) adj2 generation) or atypical*) adj3 (antipsychotic* or anti-psychotic*)).mp. 
3 (Aripiprazole and (Asenapine or Brexpiprazole or Cariprazine or Clozapine or Iloperidone or Lurasidone or Olanzapine or Paliperidone or Quetiapine or Risperidone or Ziprasidone)).mp. [mp=title, abstract, heading word, table of contents, key concepts, original title, tests \& measures]

4 (Asenapine and (Aripiprazole or Brexpiprazole or Cariprazine or Clozapine or Iloperidone or Lurasidone or Olanzapine or Paliperidone or Quetiapine or Risperidone or Ziprasidone)).mp. [mp=title, abstract, heading word, table of contents, key concepts, original title, tests \& measures]

5 (Brexpiprazole and (Aripiprazole or Asenapine or Cariprazine or Clozapine or Iloperidone or Lurasidone or Olanzapine or Paliperidone or Quetiapine or Risperidone or Ziprasidone)).mp.

6 (Cariprazine and (Aripiprazole or Asenapine or Brexpiprazole or Clozapine or Iloperidone or Lurasidone or Olanzapine or Paliperidone or Quetiapine or Risperidone or Ziprasidone)).mp. [mp=title, abstract, heading word, table of contents, key concepts, original title, tests \& measures]

7 (Clozapine and (Aripiprazole or Asenapine or Brexpiprazole or Cariprazine or Iloperidone or Lurasidone or Olanzapine or Paliperidone or Quetiapine or Risperidone or Ziprasidone)).mp. [mp=title, abstract, heading word, table of contents, key concepts, original title, tests \& measures]

8 (Iloperidone and (Aripiprazole or Asenapine or Brexpiprazole or Cariprazine or Clozapine or Lurasidone or Olanzapine or Paliperidone or Quetiapine or Risperidone or Ziprasidone)).mp. [mp=title, abstract, heading word, table of contents, key concepts, original title, tests \& measures]

9 (Lurasidone and (Aripiprazole or Asenapine or Brexpiprazole or Cariprazine or Clozapine or Iloperidone or Olanzapine or Paliperidone or Quetiapine or Risperidone or Ziprasidone)).mp. [mp=title, abstract, heading word, table of contents, key concepts, original title, tests \& measures]

10 (Olanzapine and (Aripiprazole or Asenapine or Brexpiprazole or Cariprazine or Clozapine or Iloperidone or Lurasidone or Paliperidone or Quetiapine or Risperidone or Ziprasidone)).mp. [mp=title, abstract, heading word, table of contents, key concepts, original title, tests \& measures]

11 (Paliperidone and (Aripiprazole or Asenapine or Brexpiprazole or Cariprazine or Clozapine or Iloperidone or Lurasidone or Olanzapine or Quetiapine or Risperidone or Ziprasidone)).mp. [mp=title, abstract, heading word, table of contents, key concepts, original title, tests \& measures]

12 (Quetiapine and (Aripiprazole or Asenapine or Brexpiprazole or Cariprazine or Clozapine or Iloperidone or Lurasidone or Olanzapine or Paliperidone or Risperidone or Ziprasidone)).mp. [mp=title, abstract, heading word, table of contents, key concepts, original title, tests \& measures]

13 (Risperidone and (Aripiprazole or Asenapine or Brexpiprazole or Cariprazine or Clozapine or Iloperidone or Lurasidone or Olanzapine or Paliperidone or Quetiapine or Ziprasidone)).mp. [mp=title, abstract, heading word, table of contents, key concepts, original title, tests \& measures]

14 (Ziprasidone and (Aripiprazole or Asenapine or Brexpiprazole or Cariprazine or Clozapine or Iloperidone or Lurasidone or Olanzapine or Paliperidone or Quetiapine or Risperidone)).mp. [mp=title, abstract, heading word, table of contents, key concepts, original title, tests \& measures]

152 or 3 or 4 or 5 or 6 or 7 or 8 or 9 or 10 or 11 or 12 or 13 or 14

161 and 15

17 limit 16 to english language

18 limit 17 to systematic reviews

19 limit 17 to "2000 treatment outcome/clinical trial"

20 random*.mp.

2117 and 20

22 ((random* or control*) adj5 (trial* or study or protocol*)).mp.

2317 and 22

24 (clinical* adj5 (trial* or study or protocol*)).mp. [mp=title, abstract, heading word, table of contents, key concepts, original title, tests \& measures]

2521 and 24

2618 or 19 or 23 or 25

27 (2013* or $2014 *$ or $2015 *$ or $2016 *)$.up,dp.

2826 and 27

\section{Key Question 2}

\section{Broad Search}




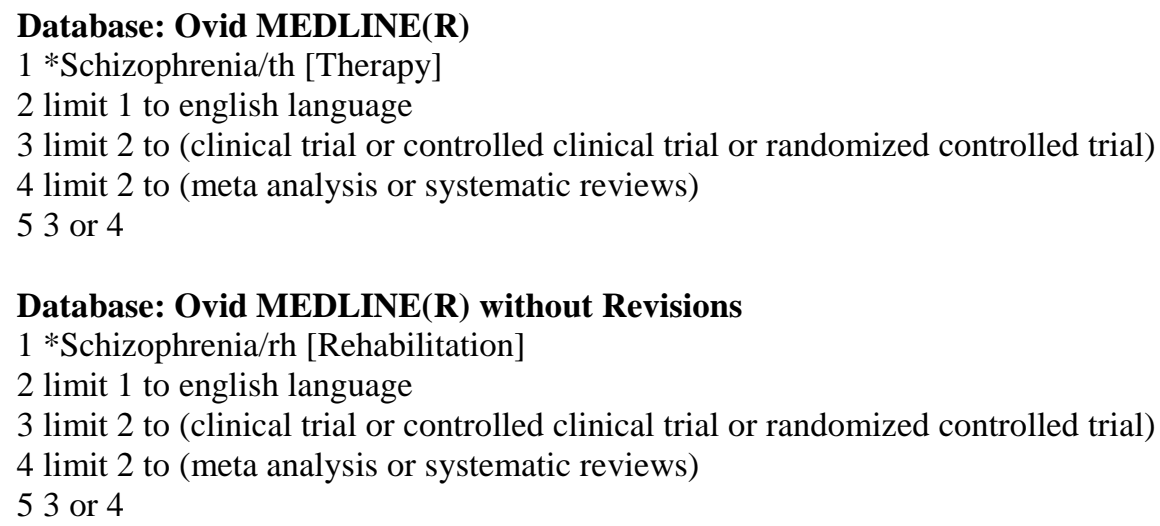

Database: EBM Reviews - Cochrane Central Register of Controlled Trials $1 *$ Schizophrenia/th [Therapy]

2 Schizophrenia/

3 schizophrenia.ti,ab.

4 (non-pharmacolog* or nonpharmacolog* or psychosocial or psycho-social).mp.

5 (2 or 3$)$ and 4

61 or 5

7 limit 6 to english language

\section{Database: PsycINFO}

1 Schizophrenia/

2 schizophrenia.ti,ab.

3 (non-pharmacolog* or nonpharmacolog* or psychosocial or psycho-social).mp.

4 (1 or 2$)$ and 3

5 ((systematic* adj3 (review* or overview*)) or (methodologic* adj3 (review* or overview*))).ti,ab.

6 (meta-analy* or metaanaly* or systematic review*).mp. [mp=title, abstract, heading word, table of contents, key concepts, original title, tests \& measures]

74 and (5 or 6)

\section{Database: PsycINFO}

1 Schizophrenia/

2 schizophrenia.ti,ab.

3 (non-pharmacolog* or nonpharmacolog* or psychosocial or psycho-social).mp.

4 (1 or 2$)$ and 3

5 ("randomi*ed controlled trial" or "RCT").mp. [mp=title, abstract, heading word, table of contents, key concepts, original title, tests \& measures]

6 ((random* or control*) adj5 (trial or study)).mp.

74 and (5 or 6$)$

8 limit 7 to english language

Database: ClinicalTrials.gov

NOT NOTEXT [FIRST-RECEIVED-RESULTS-DATE] AND EXACT "Interventional" [STUDY-TYPES] AND ( Schizophrenia OR Schizoaffective Disorder ) [DISEASE] AND Behavioral [TREATMENT] AND EXACT Adult [AGE-GROUP]

\section{Narrow Search}

\section{Database: Ovid MEDLINE(R)}

1 exp schizophrenia/

2 exp Community Mental Health Services/

31 and 2 
4 exp Cognitive Therapy/

51 and 4

6 (Cognit* adj5 Remediat*).mp.

71 and 6

8 exp Family Therapy/

9 (((family or families) adj5 psychoeducat*) or ((family or families) adj3 (therap* or counsel*))).mp.

108 or 9

111 and 10

12 exp Peer Group/

131 and 12

14 (peer* adj5 (support* or group*)).mp.

151 and 14

16 (Intensiv* adj3 ((case or cases) adj3 manag*)).mp.

171 and 16

18 exp Case Management/

191 and 18

20 Skills Training.mp.

21 ((skill* or job or jobs or employment or vocation*) adj5 train*).mp.

221 and 21

23 exp Employment, Supported/

24 ((support* or subsidiz*) adj2 educat*).mp.

25 exp Rehabilitation, Vocational/

26 exp Sheltered Workshops/

2723 or 24 or 25 or 26

281 and 27

29 exp residential facilities/

30 ((support* or subsidiz*) adj2 hous*).mp.

3129 or 30

321 and 31

33 (("Illness Management and Recovery" or IMR) adj3 (toolkit* or tool kit*)).mp.

341 and 33

353 or 5 or 7 or 11 or 13 or 15 or 17 or 19 or 22 or 28 or 32 or 34

36 limit 35 to english language

37 limit 36 to systematic reviews

38 limit 36 to randomized controlled trial

39 limit 36 to controlled clinical trial

4037 or 38 or 39

41 limit 40 to yr="1902 - 1995"

\section{Database: Ovid MEDLINE(R) without Revisions}

1 exp schizophrenia/

2 exp Community Mental Health Services/

31 and 2

4 exp Cognitive Therapy/

51 and 4

6 (Cognit* adj5 Remediat*).mp. [mp=title, abstract, original title, name of substance word, subject heading word, keyword heading word, protocol supplementary concept word, rare disease supplementary concept word, unique identifier]

71 and 6

8 exp Family Therapy/

9 (((family or families) adj5 psychoeducat*) or ((family or families) adj3 (therap* or counsel*))).mp. [mp=title, abstract, original title, name of substance word, subject heading word, keyword heading word, protocol supplementary concept word, rare disease supplementary concept word, unique identifier]

108 or 9

111 and 10

12 exp Peer Group/ 


\section{1 and 12}

14 (peer* adj5 (support* or group*)).mp. [mp=title, abstract, original title, name of substance word, subject heading word, keyword heading word, protocol supplementary concept word, rare disease supplementary concept word, unique identifier]

151 and 14

16 (Intensiv* adj3 ((case or cases) adj3 manag*)).mp. [mp=title, abstract, original title, name of substance word, subject heading word, keyword heading word, protocol supplementary concept word, rare disease supplementary concept word, unique identifier]

171 and 16

18 exp Case Management/

191 and 18

20 Skills Training.mp.

21 ((skill* or job or jobs or employment or vocation*) adj5 train*).mp. [mp=title, abstract, original title, name of substance word, subject heading word, keyword heading word, protocol supplementary concept word, rare disease supplementary concept word, unique identifier]

221 and 21

23 exp Employment, Supported/

24 ((support* or subsidiz*) adj2 educat*).mp. [mp=title, abstract, original title, name of substance word, subject heading word, keyword heading word, protocol supplementary concept word, rare disease supplementary concept word, unique identifier]

25 exp Rehabilitation, Vocational/

26 exp Sheltered Workshops/

2723 or 24 or 25 or 26

281 and 27

29 exp residential facilities/

30 ((support* or subsidiz*) adj2 hous*).mp. [mp=title, abstract, original title, name of substance word, subject heading word, keyword heading word, protocol supplementary concept word, rare disease supplementary concept word, unique identifier]

3129 or 30

321 and 31

33 (("Illness Management and Recovery" or IMR) adj3 (toolkit* or tool kit*)).mp. [mp=title, abstract, original title, name of substance word, subject heading word, keyword heading word, protocol supplementary concept word, rare disease supplementary concept word, unique identifier]

341 and 33

353 or 5 or 7 or 11 or 13 or 15 or 17 or 19 or 22 or 28 or 32 or 34

36 limit 35 to english

37 limit 36 to systematic reviews

38 limit 36 to randomized controlled trial

39 limit 36 to controlled clinical trial

4037 or 38 or 39

\section{Database: EBM Reviews - Cochrane Database of Systematic Reviews}

1 schizophren*.mp. [mp=title, abstract, full text, keywords, caption text]

2 (Communit* adj7 (mental* or psych*) adj5 (health* or servic* or clinic*)).mp. [mp=title, abstract, full text, keywords, caption text]

31 and 2

4 (Cognit* adj3 (therap* or treat*)).mp. [mp=title, abstract, full text, keywords, caption text]

51 and 4

6 (Cognit* adj5 Remediat*).mp. [mp=title, abstract, full text, keywords, caption text]

71 and 6

8 (((family or families) adj5 psychoeducat*) or ((family or families) adj3 (therap* or counsel*))).mp. [mp=title, abstract, full text, keywords, caption text]

91 and 8

10 (peer* adj5 (support* or group* or interact* or relation*)).mp. [mp=title, abstract, full text, keywords, caption text]

111 and 10 
12 (Intensiv* adj3 ((case or cases) adj3 manag*)).mp. [mp=title, abstract, full text, keywords, caption text]

131 and 12

14 (case* adj2 manag*).mp. [mp=title, abstract, full text, keywords, caption text]

151 and 14

16 ((skill* or job or jobs or employment or vocation*) adj5 train*).mp. [mp=title, abstract, full text, keywords, caption text]

171 and 16

18 (support* adj3 (employ* or job* or occupation* or workplac* or vocation*)).mp. [mp=title, abstract, full text, keywords, caption text]

19 ((support* or subsidiz*) adj2 educat*).mp. [mp=title, abstract, full text, keywords, caption text]

20 (rehab* adj3 (vocational* or occupation* or workplac* or employment)).mp. [mp=title, abstract, full text, keywords, caption text]

21 (shelter* adj3 (Workshop* or workplac*)).mp. [mp=title, abstract, full text, keywords, caption text]

2218 or 19 or 20 or 21

231 and 22

24 ((support* or subsidiz*) adj2 hous*).mp. [mp=title, abstract, full text, keywords, caption text]

251 and 24

26 (("Illness Management and Recovery" or IMR) adj3 (toolkit* or tool kit*)).mp. [mp=title, abstract, full text, keywords, caption text]

271 and 26

283 or 5 or 7 or 9 or 11 or 13 or 15 or 17 or 23 or 25 or 27

29 limit 28 to English

\section{Database: EBM Reviews - Cochrane Central Register of Controlled Trials}

1 schizophren*.mp. [mp=title, original title, abstract, mesh headings, heading words, keyword]

2 (Communit* adj7 (mental* or psych*) adj5 (health* or servic* or clinic*)).mp. [mp=title, original title, abstract, mesh headings, heading words, keyword]

31 and 2

4 (Cognit* adj3 (therap* or treat* or train* or interven*)).mp. [mp=title, original title, abstract, mesh headings, heading words, keyword]

51 and 4

6 (Cognit* adj5 Remediat*).mp. [mp=title, original title, abstract, mesh headings, heading words, keyword]

71 and 6

8 (((family or families) adj5 psychoeducat*) or ((family or families) adj3 (therap* or counsel*))).mp. [mp=title, original title, abstract, mesh headings, heading words, keyword]

91 and 8

10 (peer* adj5 (support* or group* or interact* or relation*)).mp. [mp=title, original title, abstract, mesh headings, heading words, keyword]

111 and 10

12 (Intensiv* adj3 ((case or cases) adj3 manag*)).mp. [mp=title, original title, abstract, mesh headings, heading words, keyword]

131 and 12

14 (case* adj2 manag*).mp. [mp=title, original title, abstract, mesh headings, heading words, keyword]

151 and 14

16 ((skill* or job or jobs or employment or vocation*) adj5 train*).mp. [mp=title, original title, abstract, mesh headings, heading words, keyword]

171 and 16

18 (support* adj3 (employ* or job* or occupation* or workplac* or vocation*)).mp. [mp=title, original title, abstract, mesh headings, heading words, keyword]

19 ((support* or subsidiz*) adj2 educat*).mp. [mp=title, original title, abstract, mesh headings, heading words, keyword]

20 (rehab* adj3 (vocational* or occupation* or workplac* or employment)).mp. [mp=title, original title, abstract, mesh headings, heading words, keyword]

21 (shelter* adj3 (Workshop* or workplac*)).mp. [mp=title, original title, abstract, mesh headings, heading words, keyword]

2218 or 19 or 20 or 21 
231 and 22

24 ((support* or subsidiz*) adj2 hous*).mp. [mp=title, original title, abstract, mesh headings, heading words, keyword]

251 and 24

26 (("Illness Management and Recovery" or IMR) adj3 (toolkit* or tool kit*)).mp. [mp=title, original title, abstract, mesh headings, heading words, keyword]

271 and 26

283 or 5 or 7 or 9 or 11 or 13 or 15 or 17 or 23 or 25 or 27

29 limit 28 to english

30 limit 29 to $\mathrm{yr}=" 2011$-Current"

31 limit 29 to $\mathrm{yr}=" 1898$ - 2010"

\section{Database: EBM Reviews - Cochrane Central Register of Controlled Trials}

1 schizophren*.mp. [mp=title, original title, abstract, mesh headings, heading words, keyword]

2 (Communit* adj7 (mental* or psych*) adj5 (health* or servic* or clinic*)).mp. [mp=title, original title, abstract, mesh headings, heading words, keyword]

31 and 2

4 (Cognit* adj3 (therap* or treat* or train* or interven*)).mp. [mp=title, original title, abstract, mesh headings, heading words, keyword]

51 and 4

6 (Cognit* adj5 Remediat*).mp. [mp=title, original title, abstract, mesh headings, heading words, keyword]

71 and 6

8 (((family or families) adj5 psychoeducat*) or ((family or families) adj3 (therap* or counsel*))).mp. [mp=title, original title, abstract, mesh headings, heading words, keyword]

91 and 8

10 (peer* adj5 (support* or group* or interact* or relation*)).mp. [mp=title, original title, abstract, mesh headings, heading words, keyword]

111 and 10

12 (Intensiv* adj3 ((case or cases) adj3 manag*)).mp. [mp=title, original title, abstract, mesh headings, heading words, keyword]

131 and 12

14 (case* adj2 manag*).mp. [mp=title, original title, abstract, mesh headings, heading words, keyword]

151 and 14

16 ((skill* or job or jobs or employment or vocation*) adj5 train*).mp. [mp=title, original title, abstract, mesh headings, heading words, keyword]

171 and 16

18 (support* adj3 (employ* or job* or occupation* or workplac* or vocation*)).mp. [mp=title, original title, abstract, mesh headings, heading words, keyword]

19 ((support* or subsidiz*) adj2 educat*).mp. [mp=title, original title, abstract, mesh headings, heading words, keyword]

20 (rehab* adj3 (vocational* or occupation* or workplac* or employment)).mp. [mp=title, original title, abstract, mesh headings, heading words, keyword]

21 (shelter* adj3 (Workshop* or workplac*)).mp. [mp=title, original title, abstract, mesh headings, heading words, keyword]

2218 or 19 or 20 or 21

231 and 22

24 ((support* or subsidiz*) adj2 hous*).mp. [mp=title, original title, abstract, mesh headings, heading words, keyword]

251 and 24

26 (("Illness Management and Recovery" or IMR) adj3 (toolkit* or tool kit*)).mp. [mp=title, original title, abstract, mesh headings, heading words, keyword]

271 and 26

283 or 5 or 7 or 9 or 11 or 13 or 15 or 17 or 23 or 25 or 27

29 limit 28 to english

30 limit 29 to yr="2011 -Current"

31 limit 29 to $\mathrm{yr}=" 1898$ - 2010" 


\section{Database: PsycINFO}

1 exp schizophrenia/

2 exp Community Mental Health Services/

31 and 2

4 exp Cognitive Therapy/

51 and 4

6 (Cognit* adj5 Remediat*).mp. [mp=title, abstract, heading word, table of contents, key concepts, original title, tests \& measures]

71 and 6

8 exp Family Therapy/

9 (((family or families) adj5 psychoeducat*) or ((family or families) adj3 (therap* or counsel*))).mp. [mp=title, abstract, heading word, table of contents, key concepts, original title, tests \& measures]

108 or 9

111 and 10

12 exp Peer Group/

131 and 12

14 (peer* adj5 (support* or group*)).mp. [mp=title, abstract, heading word, table of contents, key concepts, original title, tests \& measures]

151 and 14

16 (Intensiv* adj3 ((case or cases) adj3 manag*)).mp. [mp=title, abstract, heading word, table of contents, key concepts, original title, tests \& measures]

171 and 16

18 exp Case Management/

191 and 18

20 Skills Training.mp.

21 ((skill* or job or jobs or employment or vocation*) adj5 train*).mp. [mp=title, abstract, heading word, table of contents, key concepts, original title, tests \& measures]

221 and 21

23 exp Employment, Supported/

24 ((support* or subsidiz*) adj2 educat*).mp. [mp=title, abstract, heading word, table of contents, key concepts, original title, tests \& measures]

25 exp Rehabilitation, Vocational/

26 exp Sheltered Workshops/

2723 or 24 or 25 or 26

281 and 27

29 exp residential facilities/

30 ((support* or subsidiz*) adj2 hous*).mp. [mp=title, abstract, heading word, table of contents, key concepts, original title, tests $\&$ measures]

3129 or 30

321 and 31

33 (("Illness Management and Recovery" or IMR) adj3 (toolkit* or tool kit*)).mp. [mp=title, abstract, heading word, table of contents, key concepts, original title, tests \& measures]

341 and 33

353 or 5 or 7 or 11 or 13 or 15 or 17 or 19 or 22 or 28 or 32 or 34

36 limit 35 to english

37 limit 36 to systematic reviews

38 random*.mp.

39 (clinical* adj5 (trial* or study or protocol*)).mp.

4038 and 39

41 ((random* or control*) adj5 (trial* or study or protocol*)).mp.

4240 or 41

4336 and 42

4437 or 43

45 limit 36 to "2000 treatment outcome/clinical trial"

4644 or 45 


\section{Database: PsycINFO}

1 exp schizophrenia/

2 exp Community Mental Health Services/

31 and 2

4 exp Cognitive Therapy/

51 and 4

6 (Cognit* adj5 Remediat*).mp. [mp=title, abstract, heading word, table of contents, key concepts, original title, tests \& measures]

71 and 6

8 exp Family Therapy/

9 (((family or families) adj5 psychoeducat*) or ((family or families) adj3 (therap* or counsel*))).mp. [mp=title, abstract, heading word, table of contents, key concepts, original title, tests \& measures]

108 or 9

111 and 10

12 exp Peers/ or exp peer relations/

131 and 12

14 (peer* adj5 (support* or group*)).mp. [mp=title, abstract, heading word, table of contents, key concepts, original title, tests \& measures]

151 and 14

16 (Intensiv* adj3 ((case or cases) adj3 manag*)).mp. [mp=title, abstract, heading word, table of contents, key concepts, original title, tests \& measures]

171 and 16

18 exp Case Management/

191 and 18

20 Skills Training.mp.

21 ((skill* or job or jobs or employment or vocation*) adj5 train*).mp. [mp=title, abstract, heading word, table of contents, key concepts, original title, tests \& measures]

221 and 21

23 exp supported employment/

24 ((support* or subsidiz*) adj2 educat*).mp. [mp=title, abstract, heading word, table of contents, key concepts, original title, tests \& measures]

25 exp Rehabilitation, Vocational/

26 exp Sheltered Workshops/

2723 or 24 or 25 or 26

281 and 27

29 ((support* or subsidiz*) adj2 hous*).mp. [mp=title, abstract, heading word, table of contents, key concepts, original title, tests \& measures]

301 and 29

31 (("Illness Management and Recovery" or IMR) adj3 (toolkit* or tool kit*)).mp. [mp=title, abstract, heading word, table of contents, key concepts, original title, tests \& measures]

321 and 31

333 or 5 or 7 or 11 or 13 or 15 or 17 or 19 or 22 or 28 or 30 or 32

34 limit 33 to english

35 limit 34 to systematic reviews

36 random*.mp.

37 (clinical* adj5 (trial* or study or protocol*)).mp.

3836 and 37

39 ((random* or control*) adj5 (trial* or study or protocol*)).mp.

4038 or 39

4134 and 40

4235 or 41

43 limit 34 to "2000 treatment outcome/clinical trial"

4442 or 43 


\section{Appendix B. Included Studies}

1. Abou-Setta AM, Mousavi SS, Spooner C, et al. First-generation versus second-generation antipsychotics in adults: comparative effectiveness. Comparative effectiveness review No. 63. (Prepared by the University of Alberta Evidence-based Practice Center under Contract No. 290-200-10021.) AHRQ Publication No. 12EHC054-EF. Rockville, MD: Agency for Healthcare Research and Quality: 2012. www.effectivehealthcare.ahrq.gov/reports/final.cf m Accessed April 22, 2017. PMID: 23035275.

2. Amr M, Lakhan SE, Sanhan S, et al. Efficacy and tolerability of quetiapine versus haloperidol in first-episode schizophrenia: a randomized clinical trial. Int Arch Med. 2013;6(1) PMID: 24308507.

3. Arnold JG, Miller AL, Canive JM, et al. Comparison of outcomes for African Americans, Hispanics, and non-Hispanic whites in the CATIE study. Psychiatr Serv. 2013;64(6):570-8. PMID: 23494108.

4. Barrowclough C, Tarrier N, Lewis S, et al. Randomised controlled effectiveness trial of a needs-based psychosocial intervention service for carers of people with schizophrenia. $\mathrm{Br} \mathrm{J}$ Psychiatry. 1999 Jun;174:505-11. PMID: 10616628.

5. Bartels SJ, Pratt SI, Mueser KT, et al. Long-term outcomes of a randomized trial of integrated skills training and preventive healthcare for older adults with serious mental illness. Am J Geriatr Psychiatry. 2014 Nov;22(11):1251-61. doi: 10.1016/j.jagp.2013.04.013. PMID: 23954039.

6. Bertelsen M, Jeppesen P, Petersen L, et al. Course of illness in a sample of 265 patients with first-episode psychosis-five-year follow-up of the Danish OPUS trial. Schizophr Res. 2009;107(2-3):173-8. doi: 10.1016/j.schres.2008.09.018. PMID: 18945593.

7. Bjorkman T, Hansson L, Sandlund M. Outcome of case management based on the strengths model compared to standard care. A randomised controlled trial. Soc Psychiatry Psychiatr Epidemiol. 2002 Apr;37(4):147-52. PMID: 12027240.

8. Buckley LA, Maayan N, Soares-Weiser K, et al. Supportive therapy for schizophrenia. Cochrane Database Syst Rev. 2015 Apr 14(4):Cd004716. doi: 10.1002/14651858.CD004716.pub4. PMID: 25871462

9. Caroff SN, Davis VG, Miller DD, et al. Treatment outcomes of patients with tardive dyskinesia and chronic schizophrenia. J Clin Psychiatry. 2011 Mar;72(3):295-303. doi: 10.4088/JCP.09m05793yel. PMID: 20816031.
10. Cella M, Preti A, Edwards C, et al. Cognitive remediation for negative symptoms of schizophrenia: A network meta-analysis. Clin Psychol Rev. 2017 Mar;52:43-51. doi: 10.1016/j.cpr.2016.11.009. PMID: 27930934.

11. Citrome L, Ota A, Nagamizu K, et al. The effect of brexpiprazole (OPC-34712) and aripiprazole in adult patients with acute schizophrenia: results from a randomized, exploratory study. Int Clin Psychopharmacol. 2016 Jul;31(4):192-201. doi: 10.1097/yic.0000000000000123. PMID: 26963842.

12. Cook JA, Blyler CR, Burke-Miller JK, et al. Effectiveness of supported employment for individuals with schizophrenia: results of a multisite, randomized trial. Clin Schizophr Relat Psychoses. 2008;2(1):37-46.

13. Cook JA, Leff HS, Blyler CR, et al. Results of a multisite randomized trial of supported employment interventions for individuals with severe mental illness. Arch Gen Psychiatry. 2005 May;62(5):505-12. PMID: 15867103.

14. Craig TK, Garety P, Power P, et al. The Lambeth Early Onset (LEO) team: randomised controlled trial of the effectiveness of specialised care for early psychosis. BMJ. 2004 Nov 6;329(7474):1067. doi: 10.1136/bmj.38246.594873.7C. PMID: 15485934.16.

15. Crespo-Facorro B, Perez-Iglesias R, Mata I, et al. Effectiveness of haloperidol, risperidone and olanzapine in the treatment of first-episode nonaffective psychosis: results of a randomized, flexible-dose, open-label 1-year follow-up comparison. J Psychopharmacol. 2011 Jun;25(6):744-54. PMID: 21292922.

16. Deste G, Barlati S, Cacciani P, et al. Persistence of effectiveness of cognitive remediation interventions in schizophrenia: a 1-year followup study. Schizophr Res. 2015 Feb;161(2-3):4036. doi: 10.1016/j.schres.2014.12.004. PMID: 25533593.

17. Detke HC, Weiden PJ, Llorca PM, et al. Comparison of olanzapine long-acting injection and oral olanzapine: a 2-year, randomized, openlabel study in outpatients with schizophrenia. J Clin Psychopharmacol. 2014 Aug;34(4):426-34. doi: 10.1097/JCP.0000000000000140. PMID: 24781441. 
18. Di Fiorino M, Montagnani G, Trespi G, et al. Extended-release quetiapine fumarate (quetiapine $\mathrm{XR}$ ) versus risperidone in the treatment of depressive symptoms in patients with schizoaffective disorder or schizophrenia: a randomized, open-label, parallel-group, flexibledose study. Int Clin Psychopharmacol. 2014 May;29(3):166-76. doi: 10.1097/YIC.0000000000000017. PMID: 24681810.

19. Durgam S, Starace A, Li D, et al. An evaluation of the safety and efficacy of cariprazine in patients with acute exacerbation of schizophrenia: a phase II, randomized clinical trial. Schizophr Res. 2014 Feb;152(2-3):450-7. doi: 10.1016/j.schres.2013.11.041. PMID: 24412468.

20. Dyck DG, Short RA, Hendryx MS, et al. Management of negative symptoms among patients with schizophrenia attending multiplefamily groups. Psychiatr Serv. 2000 Apr;51(4):513-9. PMID: 10737828.

21. Farreny A, Aguado J, Ochoa S, et al. REPYFLEC cognitive remediation group training in schizophrenia: looking for an integrative approach. Schizophr Res. 2012 Dec;142(13):137-44. doi: 10.1016/j.schres.2012.08.035. PMID: 23017827.

22. Fervaha G, Agid O, Takeuchi H, et al. Effect of antipsychotic medication on overall life satisfaction among individuals with chronic schizophrenia: findings from the NIMH CATIE study. Eur Neuropsychopharmacol. 2014 Jul;24(7):1078-85. doi: 10.1016/j.euroneuro.2014.03.001. PMID: 24726579.

23. Fleischhacker WW, Sanchez R, Perry PP, et al. Aripiprazole once-monthly for treatment of schizophrenia: double-blind, randomised, noninferiority study. Br J Psychol. 2014 Aug;205(2):135-44. doi: 10.1192/bjp.bp.113.134213. PMID: 24925984.

24. Freeman D, Dunn G, Startup H, et al. Effects of cognitive behaviour therapy for worry on persecutory delusions in patients with psychosis (WIT): a parallel, single-blind, randomised controlled trial with a mediation analysis. Lancet Psychiatry. 2015;2(4):305-13. PMID: 26360083.

25. Garety PA, Craig TK, Dunn G, et al. Specialised care for early psychosis: symptoms, social functioning and patient satisfaction: randomised controlled trial. Br J Psychiatry. 2006 Jan;188:3745. doi: 10.1192/bjp.bp.104.007286. PMID: 16388068.
26. Garety PA, Fowler DG, Freeman D, et al. Cognitive--behavioural therapy and family intervention for relapse prevention and symptom reduction in psychosis: randomised controlled trial. Br J Psychiatry. 2008 Jun;192(6):412-23. doi: 10.1192/bjp.bp.107.043570. PMID: 18515890.

27. Green AI, Brunette MF, Dawson R, et al. Longacting injectable vs oral risperidone for schizophrenia and co-occurring alcohol use disorder: a randomized trial. J Clin Psychiatry. 2015 Oct;76(10):1359-65. doi: 10.4088/JCP.13m08838. PMID: 26302441.

28. Guo X, Zhai J, Liu Z, et al. Effect of antipsychotic medication alone vs combined with psychosocial intervention on outcomes of earlystage schizophrenia: a randomized, 1-year study. Arch Gen Psychiatry. 2010 Sep;67(9):895-904. doi: 10.1001/archgenpsychiatry.2010.105. PMID: 20819983.

29. Guo X, Zhao J, Liu Z, et al. Antipsychotic combination with psychosocial intervention on outcome of schizophrenia (acpios): rationale and design of the clinical trial. Clin Schizophr Relat Psychoses. 2007;1(2):185-92.

30. Hartling L, Abou-Setta AM, Dursun S, et al. Antipsychotics in adults with schizophrenia: comparative effectiveness of first-generation versus second-generation medications: a systematic review and meta-analysis. Ann Intern Med. 2012 Oct 2;157(7):498-511. PMID: 22893011.

31. Hasson-Ohayon I, Roe D, Kravetz S. A randomized controlled trial of the effectiveness of the illness management and recovery program. Psychiatr Serv. 2007 Nov;58(11):1461-6. doi: 10.1176/ps.2007.58.11.1461. PMID: 17978257.

32. Hunt GE, Siegfried N, Morley K, et al. Psychosocial interventions for people with both severe mental illness and substance misuse. Cochrane Database Syst Rev. 2013 Oct 03(10):Cd001088. doi: 10.1002/14651858.CD001088.pub3. PMID: 24092525.

33. Ishigooka J, Nakamura J, Fujii Y, et al. Efficacy and safety of aripiprazole once-monthly in Asian patients with schizophrenia: a multicenter, randomized, double-blind, non-inferiority study versus oral aripiprazole. Schizophr Res. 2015 Feb;161(2-3):421-8. doi: 10.1016/j.schres.2014.12.013. PMID: 25556976.

34. Jauhar S, McKenna PJ, Radua J, et al. Cognitivebehavioural therapy for the symptoms of schizophrenia: systematic review and metaanalysis with examination of potential bias. $\mathrm{Br} \mathrm{J}$ Psychiatry. 2014 Jan;204(1):20-9. doi: 10.1192/bjp.bp.112.116285. PMID: 24385461. 
35. Jones C, Hacker D, Cormac I, et al. Cognitive behavioural therapy versus other psychosocial treatments for schizophrenia. Cochrane Database Syst Rev. 2012 Apr 18(4):Cd008712. doi: 10.1002/14651858.CD008712.pub2. PMID: 22513966.

36. Kane JM, Robinson DG, Schooler NR, et al. Comprehensive versus usual community care for first-episode psychosis: 2-year outcomes from the NIMH RAISE early treatment program. Am J Psychiatry. 2016 Apr 1;173(4):362-72. doi: 10.1176/appi.ajp.2015.15050632. PMID: 26481174.

37. Kane JM, Schooler NR, Marcy P, et al. The RAISE early treatment program for first-episode psychosis: background, rationale, and study design. J Clin Psychiatry. 2015 Mar;76(3):240-6. doi: 10.4088/JCP.14m09289. PMID: 25830446.

38. Kinoshita Y, Furukawa TA, Kinoshita K, et al. Supported employment for adults with severe mental illness. Cochrane Database Syst Rev. 2013 Sep 13(9):Cd008297. doi: 10.1002/14651858.CD008297.pub2. PMID: 24030739.

39. Kopelowicz A, Zarate R, Wallace CJ, et al. The ability of multifamily groups to improve treatment adherence in Mexican Americans with schizophrenia. Arch Gen Psychiatry. 2012 Mar;69(3):265-73. doi: 10.1001/archgenpsychiatry.2011.135. PMID: 22393219.

40. Koshikawa Y, Takekita Y, Kato M, et al. The comparative effects of risperidone long-acting injection and paliperidone palmitate on social functioning in schizophrenia: a 6-month, openlabel, randomized controlled pilot trial.

Neuropsychobiology. 2016;73(1):35-42. doi: 10.1159/000442209. PMID: 26812618

41. Li H, Luo J, Wang C, et al. Efficacy and safety of aripiprazole in Chinese Han schizophrenia subjects: a randomized, double-blind, active parallel-controlled, multicedinter clinical trial. Schizophr Res. 2014 Aug;157(1-3):112-9. doi: 10.1016/j.schres.2014.05.040. PMID: 24994555.

42. Lieberman JA, Stroup TS, McEvoy JP, et al. Effectiveness of antipsychotic drugs in patients with chronic schizophrenia. N Engl J Med. 2005 Sep 22;353(12):1209-23. doi: 10.1056/NEJMoa051688. PMID: 16172203.

43. Liu J, Sun J, Shen X, et al. Randomized controlled trial comparing changes in serum prolactin and weight among female patients with first-episode schizophrenia over 12 months of treatment with risperidone or quetiapine. Shanghai Arch Psychiatry. 2014;26(2):88-94. PMID: 25092954.
44. Lysaker PH, Davis LW, Bryson GJ, et al. Effects of cognitive behavioral therapy on work outcomes in vocational rehabilitation for participants with schizophrenia spectrum disorders. Schizophr Res. 2009 Feb;107(23):186-91. doi: 10.1016/j.schres.2008.10.018. PMID: 19046856.

45. Maat A, Cahn W, Gijsman HJ, et al. Open, randomized trial of the effects of aripiprazole versus risperidone on social cognition in schizophrenia. Eur Neuropsychopharmacol. 2014 Apr;24(4):575-84. PMID: 24418213.

46. Malik N, Kingdon D, Pelton J, et al. Effectiveness of brief cognitive-behavioral therapy for schizophrenia delivered by mental health nurses: relapse and recovery at 24 months. J Clin Psychiatry. 2009 Feb;70(2):201-7. PMID: 19210949.

47. Marshall M, Gray A, Lockwood A, et al. Case management for people with severe mental disorders. Cochrane Database Syst Rev. 2000b(2):Cd000050. doi: 10.1002/14651858.cd000050. PMID: 10796288.

48. Marshall M, Lockwood A. Assertive community treatment for people with severe mental disorders. Cochrane Database Syst Rev. 2000a(2):Cd001089. doi: 10.1002/14651858.cd001089. PMID: 10796415.

49. Mayoral F, Berrozpe A, de la Higuera J, et al. Efficacy of a family intervention program for prevention of hospitalization in patients with schizophrenia. A naturalistic multicenter controlled and randomized study in Spain. Revista de Psiquiatria y Salud Mental. 2015 AprJun;8(2):83-91. doi: 10.1016/j.rpsm.2013.11.001. PMID: 25017624.

50. McDonagh M, Peterson K, Fu R, et al. Second Generation Antipsychotic Drugs. Final Update 4 Report prepared by the Pacific Northwest Evidence-based Practice Center for the Drug Effectiveness Review Project. Oregon Health \& Science University, Portland, Oregon, November 2013. 2013. Available with membership in the Drug Effectiveness Review Project. http://www.ohsu.edu/xd/research/centersinstitutes/evidence-based-practice-center/drugeffectiveness-review-project/upload/SGA_finalupdate-4-report_Nov-2013_version-2.pdf. Accessed May 23, 2017.

51. McEvoy JP, Byerly M, Hamer RM, et al. Effectiveness of paliperidone palmitate vs haloperidol decanoate for maintenance treatment of schizophrenia: a randomized clinical trial. JAMA. 2014 May 21;311(19):1978-87. doi: 10.1001/jama.2014.4310. PMID: 24846035. 
52. Mueller DR, Schmidt SJ, Roder V. One-year randomized controlled trial and follow-up of integrated neurocognitive therapy for schizophrenia outpatients. Schizophr Bull. 2015 May;41(3):604-16. doi: 10.1093/schbul/sbu223. PMID: 25713462.

53. Mueser KT, Clark RE, Haines M, et al. The Hartford study of supported employment for persons with severe mental illness. J Consult Clin Psychol. 2004 Jun;72(3):479-90. doi: 10.1037/0022-006x.72.3.479. PMID: 15279531.

54. Mueser KT, Pratt SI, Bartels SJ, et al. Randomized trial of social rehabilitation and integrated health care for older people with severe mental illness. J Consult Clin Psychol. 2010 Aug;78(4):561-73. doi: 10.1037/a0019629. PMID: 20658812.

55. Naber D, Hansen K, Forray C, et al. Qualify: a randomized head-to-head study of aripiprazole once-monthly and paliperidone palmitate in the treatment of schizophrenia. Schizophr Res. 2015;168(1-2):498-504. PMID: 26232241

56. Naber D, Peuskens J, Schwarzmann N, et al. Subjective well-being in schizophrenia: a randomised controlled open-label 12-month noninferiority study comparing quetiapine $\mathrm{XR}$ with risperidone (RECOVER). Eur

Neuropsychopharmacol. 2013 Oct;23(10):125769. doi: 10.1016/j.euroneuro.2013.07.006. PMID: 23953270 .

57. Nemeth G, Laszlovszky I, Czobor P, et al. Cariprazine versus risperidone monotherapy for treatment of predominant negative symptoms in patients with schizophrenia: a randomised, double-blind, controlled trial. Lancet. 2017 Feb 07doi: 10.1016/s0140-6736(17)30060-0. PMID: 28185672.

58. Parabiaghi A, Tettamanti M, D'Avanzo B, et al. Metabolic syndrome and drug discontinuation in schizophrenia: a randomized trial comparing aripiprazole olanzapine and haloperidol. Acta Psychiatr Scand. 2016;133(1):63-75. PMID: 26252780.

59. Park S, Yi KK, Kim MS, et al. Effects of ziprasidone and olanzapine on body composition and metabolic parameters: an open-label comparative pilot study. Behav Brain Funct. 2013;9:27. doi: 10.1186/1744-9081-9-27. PMID: 23866300 .

60. Pekkala E, Merinder L. Psychoeducation for schizophrenia. Cochrane Database Syst Rev. 2002(2):Cd002831. doi: 10.1002/14651858.cd002831. PMID: 12076455.
61. Pharoah F, Mari J, Rathbone J, et al. Family intervention for schizophrenia. Cochrane Database Syst Rev. 2010 Dec 08(12):CD000088. doi: 10.1002/14651858.CD000088.pub2. PMID: 21154340 .

62. Robinson DG, Gallego JA, John M, et al. A randomized comparison of aripiprazole and risperidone for the acute treatment of firstepisode schizophrenia and related disorders: 3month outcomes. Schizophr Bull. 2015;41(6):1227-36. PMID: 26338693.

63. Rosenheck R, Lin H. Noninferiority of perphenazine vs. three second-generation antipsychotics in chronic schizophrenia. J Nerv Ment Dis. 2014 Jan;202(1):18-24. doi: 10.1097/NMD.0000000000000065. PMID: 24375207.

64. San L, Arranz B, Perez V, et al. One-year, randomized, open trial comparing olanzapine, quetiapine, risperidone and ziprasidone effectiveness in antipsychotic-naive patients with a first-episode psychosis. Psychiatry Res. 2012 Dec 30;200(2-3):693-701. doi: 10.1016/j.psychres.2012.07.005. PMID: 22954905.

65. Sanz-Fuentenebro J, Taboada D, Palomo T, et al. Randomized trial of clozapine vs. risperidone in treatment-naive first-episode schizophrenia: results after one year. Schizophr Res. 2013 Sep;149(1-3):156-61. doi: 10.1016/j.schres.2013.07.003. PMID: 23870807.

66. Savitz AJ, Xu H, Gopal S, et al. Efficacy and safety of paliperidone palmitate 3-month formulation for patients with schizophrenia: a randomized, multicenter, double-blind, noninferiority study. Int $\mathrm{J}$ Neuropsychopharmacol. 2016 Jul;19(7)doi: 10.1093/ijnp/pyw018. PMID: 26902950.

67. Secher RG, Hjorthoj CR, Austin SF, et al. Tenyear follow-up of the OPUS specialized early intervention trial for patients with a first episode of psychosis. Schizophr Bull. 2015

May;41(3):617-26. doi: 10.1093/schbul/sbu155. PMID: 25381449.

68. Sellwood W, Barrowclough C, Tarrier N, et al. Needs-based cognitive-behavioural family intervention for carers of patients suffering from schizophrenia: 12-month follow-up. Acta Psychiatr Scand. 2001 Nov;104(5):346-55. PMID: 11722315.

69. Sellwood W, Wittkowski A, Tarrier N, et al. Needs-based cognitive-behavioural family intervention for patients suffering from schizophrenia: 5-year follow-up of a randomized controlled effectiveness trial. Acta Psychiatr Scand. 2007 Dec;116(6):447-52. PMID: 17961200 . 
70. Shoja Shafti S, Kaviani H. Quetiapine versus aripiprazole in the management of schizophrenia. Ther Adv Psychopharmacol. 2015;5(3):166-71.

PMID: 26199719.

71. Subotnik KL, Casaus LR, Ventura J, et al. Longacting injectable risperidone for relapse prevention and control of breakthrough symptoms after a recent first episode of schizophrenia. A randomized clinical trial. JAMA Psychiatry. 2015 Aug;72(8):822-9. doi: 10.1001/jamapsychiatry.2015.0270. PMID: 26107752.

72. Sytema S, Wunderink L, Bloemers W, et al. Assertive community treatment in the Netherlands: a randomized controlled trial. Acta Psychiatr Scand. 2007 Aug;116(2):105-12. PMID: 17650271.

73. Takekita Y, Koshikawa Y, Fabbri C, et al. Cognitive function and risperidone long-acting injection vs. paliperidone palmitate in schizophrenia: a 6-month, open-label, randomized, pilot trial. BMC Psychiatry. 2016 May 29;16:172. doi: 10.1186/s12888-016-08839. PMID: 27236412.

74. Tempier R, Balbuena L, Garety P, et al. Does assertive community outreach improve social support? Results from the Lambeth study of early-episode psychosis. Psychiatr Serv. 2012 Mar;63(3):216-22. doi:

10.1176/appi.ps.20110013. PMID: 22388528.

75. Turkington D, Kingdon D, Turner T, et al. Effectiveness of a brief cognitive-behavioural therapy intervention in the treatment of schizophrenia. Br J Psychiatry. 2002 Jun;180:523-7. PMID: 12042231.

76. Twamley EW, Vella L, Burton CZ, et al. Compensatory cognitive training for psychosis: effects in a randomized controlled trial. J Clin Psychiatry. 2012 Sep;73(9):1212-9. doi: 10.4088/JCP.12m07686. PMID: 22939029.

77. Tybura P, Mak M, Samochowiec A, et al. The influence of antipsychotic therapy on the cognitive functions of schizophrenic patients. Psychiatr Pol. 2013;47(4):567-76. PMID: 24946464.

78. Tybura P, Trzesniowska-Drukala B, Bienkowski $\mathrm{P}$, et al. Pharmacogenetics of adverse events in schizophrenia treatment: comparison study of ziprasidone, olanzapine and perazine. Psychiatry Res. 2014 Oct 30;219(2):261-7. doi: 10.1016/j.psychres.2014.05.039. PMID: 24930580.
79. Valencia M, Fresan A, Juarez F, et al. The beneficial effects of combining pharmacological and psychosocial treatment on remission and functional outcome in outpatients with schizophrenia. J Psychiatr Res. 2013 Dec;47(12):1886-92. doi: 10.1016/j.jpsychires.2013.09.006. PMID: 24112947.

80. Valencia M, Rascon ML, Juarez F, et al. A psychosocial skills training approach in Mexican out-patients with schizophrenia. Psychol Med. 2007 Oct;37(10):1393-402. PMID: 17472761.

81. Velligan DI, Diamond P, Mueller J, et al. The short-term impact of generic versus individualized environmental supports on functional outcomes and target behaviors in schizophrenia. Psychiatry Res. 2009 Jul 30;168(2):94-101. doi: 10.1016/j.psychres.2008.03.016. PMID: 19523690.

82. Velligan DI, Diamond PM, Maples NJ, et al. Comparing the efficacy of interventions that use environmental supports to improve outcomes in patients with schizophrenia. Schizophr Res. 2008 Jul;102(1-3):312-9. doi: 10.1016/j.schres.2008.02.005. PMID: 18374542.

83. Velligan DI, Diamond PM, Mintz J, et al. The use of individually tailored environmental supports to improve medication adherence and outcomes in schizophrenia. Schizophr Bull. 2008 May;34(3):483-93. PMID: 17932089.

84. Velligan DI, Tai S, Roberts DL, et al. A randomized controlled trial comparing cognitive behavior therapy, cognitive adaptation training, their combination and treatment as usual in chronic schizophrenia. Schizophr Bull. 2015 May;41(3):597-603. doi: 10.1093/schbul/sbu127. PMID: 25193976.

85. Velthorst E, Koeter M, van der Gaag M, et al. Adapted cognitive-behavioural therapy required for targeting negative symptoms in schizophrenia: meta-analysis and metaregression. Psychol Med. 2015 Feb;45(3):453-65. doi: 10.1017/S0033291714001147. PMID: 24993642.

86. Vita A, De Peri L, Barlati S, et al. Effectiveness of different modalities of cognitive remediation on symptomatological, neuropsychological, and functional outcome domains in schizophrenia: a prospective study in a real-world setting. Schizophr Res. 2011 Dec;133(1-3):223-31. doi: 10.1016/j.schres.2011.08.010. PMID: 21907544. 
87. Wani RA, Dar MA, Chandel RK, et al. Effects of switching from olanzapine to aripiprazole on the metabolic profiles of patients with schizophrenia and metabolic syndrome: a double-blind, randomized, open-label study. Neuropsychiatr Dis Treat. 2015;11:685-93. PMID: 25792838.

88. Wykes T, Huddy V, Cellard C, et al. A metaanalysis of cognitive remediation for schizophrenia: methodology and effect sizes. Am J Psychiatry. 2011 May;168(5):472-85. doi: 10.1176/appi.ajp.2010.10060855. PMID: 21406461.

89. Zhang J-P, Robinson DG, Gallego JA, et al. Association of a schizophrenia risk variant at the DRD2 locus with antipsychotic treatment response in first-episode psychosis. Schizophr Bull. 2015 Nov;41(6):1248-55. doi: 10.1093/schbul/sbv116. PMID: 26320194.

90. Zimmer M, Duncan AV, Laitano D, et al. A twelve-week randomized controlled study of the cognitive-behavioral integrated psychological therapy program: positive effect on the social functioning of schizophrenic patients. Rev Bras Psiquiatr. 2007 Jun;29(2):140-7. PMID: 17650536.

91. Zou H, Li Z, Nolan MT, et al. Self-management education interventions for persons with schizophrenia: a meta-analysis. Int J Ment Health Nurs. 2013 Jun;22(3):256-71. doi: 10.1111/j.1447-0349.2012.00863.x. PMID: 22882803. 


\section{Appendix C. Excluded Studies With Reasons}

Aberg-Wistedt A, Cressell T, Lidberg Y, et al. Two-year outcome of team-based intensive case management for patients with schizophrenia. Psychiatr Serv. 1995 Dec;46(12):1263-6. PMID: 8590112. Study covered in a systematic review.

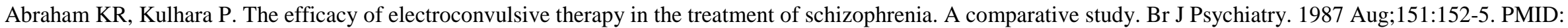
3318990. Intervention not included in review.

Addington DE, McKenzie E, Norman R, et al. Essential evidence-based components of first-episode psychosis services. Psychiatr Serv. 2013 May 1;64(5):452-7. doi: 10.1176/appi.ps.201200156. PMID: 23370444. Systematic review/meta-analysis used as a source document to identify individual studies.

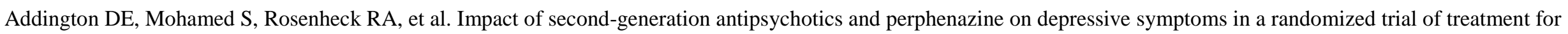
chronic schizophrenia. J Clin Psychiatry. 2011 Jan;72(1):75-80. doi: 10.4088/JCP.09m05258gre. PMID: 20868641. Outcome not included in review.

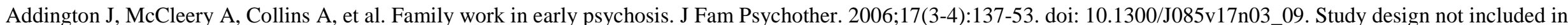
review.

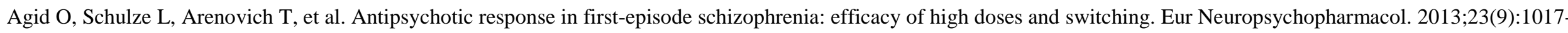
22. PMID: 23706529. Study design not included in review.

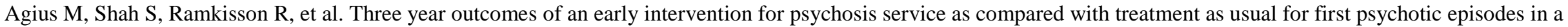
standard community mental health team. Preliminary results. Psychiatr Danub. 2007 Jun;19(1-2):10-9. PMID: 17603411. Study design not included in review.

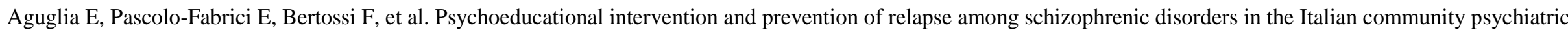
network. Clin Pract Epidemiol Ment Health. 2007;3:7. doi: 10.1186/1745-0179-3-7. PMID: 17593299. Not a study.

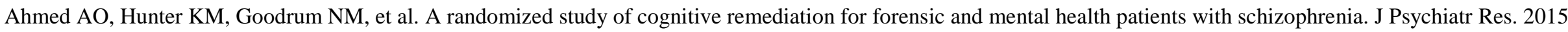
Sep;68:8-18. doi: 10.1016/j.jpsychires.2015.05.013. PMID: 26228394. Setting ineligible for review (i.e., inpatient only).

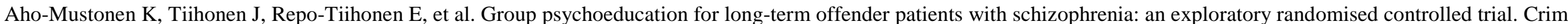
Behav Ment Health. 2011 Jul;21(3):163-76. doi: 10.1002/cbm.788. PMID: 20859932. Population ineligible for review.

Almerie MQ, Okba Al Marhi M, Jawoosh M, et al. Social skills programmes for schizophrenia. Cochrane Database Syst Rev. 2015 Jun 09(6):Cd009006. doi:

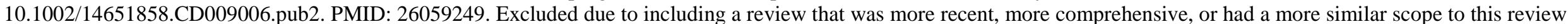
scope.

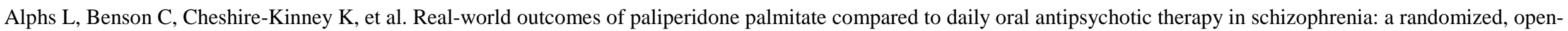

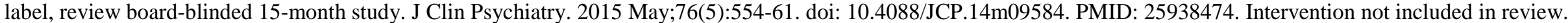

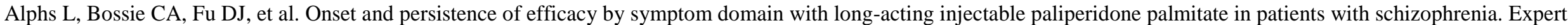
Opin Pharmacother. 2014 May;15(7):1029-42. doi: 10.1517/14656566.2014.909409. PMID: 24754314. Study design not included in review.

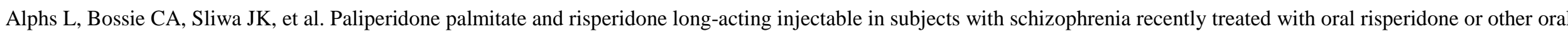
antipsychotics. Neuropsychiatr Dis Treat. 2013 Mar;9:341 - 50. PMID: 23493643. Not a study.

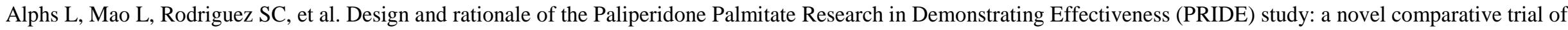

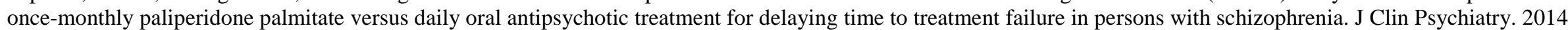
Dec;75(12):1388-93. PMID: 25375367. Not a study. 
Anaya C, Martinez Aran A, Ayuso-Mateos JL, et al. A systematic review of cognitive remediation for schizo-affective and affective disorders. J Affect Disord. 2012 Dec;142(13):13-21. doi: 10.1016/j.jad.2012.04.020. PMID: 22840620. Excluded due to including a review that was more recent, more comprehensive, or had a more similar scope to this review scope.

Andres K, Pfammatter M, Fries A, et al. The significance of coping as a therapeutic variable for the outcome of psychological therapy in schizophrenia. Eur Psychiatry. 2003 Jun;18(4):149-54. PMID: 12814846. Intervention not included in review.

Andres K, Pfammatter M, Garst F, et al. Effects of a coping-orientated group therapy for schizophrenia and schizoaffective patients: a pilot study. Acta Psychiatr Scand. 2000 Apr;101(4):318-22. PMID: 10782553. Intervention not included in review.

Anthony WA. Cognitive remediation and psychiatric rehabilitation. Psychiatr Rehabil J. 2008 Fall;32(2):87-8. doi: 10.2975/32.2.2008.87.88. PMID: 18840561. Not a study.

Anttinen E, Stenij P. The care and rehabilitation of schizophrenic out-patients with home-care. Psychiatria Fennica. 1974;145-154:100. Study design not included in review.

Armando M, Pontillo M, Vicari S. Psychosocial interventions for very early and early-onset schizophrenia: a review of treatment efficacy. Curr Opin Psychiatry. 2015 Jul;28(4):312-23. doi: 10.1097/YCO.0000000000000165. PMID: 26001923. Study design not included in review.

Armijo J, Mendez E, Morales R, et al. Efficacy of community treatments for schizophrenia and other psychotic disorders: a literature review. Front Psychiatry. 2013 Oct;4:116. doi: 10.3389/fpsyt.2013.00116. PMID: 24130534. Not a study.

Arslan M, Kurt E, Eryildiz D, et al. Effects of a psychosocial rehabilitation program in addition to medication in schizophrenic patients: a controlled study. Klinik Psikofarmakol Bulteni. 2014;24(4):360-7. doi: 10.5455/bcp.20140701074403. Study design not included in review.

Arslan M, Yazici A, Yilmaz T, et al. Long-term effects of a rehabilitation program on the clinical outcomes, social functionality, and life quality of schizophrenic patients-a follow-up study. Anadolu Psikiyatri Dergisi. 2015 Dec;16(4):238-46. Not English language.

Asenjo Lobos C, Komossa K, Rummel-Kluge C, et al. Clozapine versus other atypical antipsychotics for schizophrenia. Cochrane Database Syst Rev. 2010 Nov $10(11)$ :Cd006633. doi: 10.1002/14651858.CD006633.pub2. PMID: 21069690. Study covered in a systematic review.

Askey R, Gamble C, Gray R. Family work in first-onset psychosis: a literature review. J Psychiatr Ment Health Nurs. 2007 Jun;14(4):356-65. doi: 10.1111/j.1365-

2850.2007.01093.x. PMID: 17517026. Excluded due to including a review that was more recent, more comprehensive, or had a more similar scope to this review scope.

Asmal L, Flegar SJ, Wang J, et al. Quetiapine versus other atypical antipsychotics for schizophrenia. Cochrane Database Syst Rev. 2013 Nov 18(11):Cd006625. doi: 10.1002/14651858.CD006625.pub3. PMID: 24249315. Excluded due to including a review that was more recent, more comprehensive, or had a more similar scope to this review scope.

Atkinson JM, Coia DA, Gilmour WH, et al. The impact of education groups for people with schizophrenia on social functioning and quality of life. Br J Psychiatry. 1996 Feb;168(2):199-204. PMID: 8837910. Intervention not included in review.

Au DW, Tsang HW, So WW, et al. Effects of integrated supported employment plus cognitive remediation training for people with schizophrenia and schizoaffective disorders. Schizophr Res. 2015 Aug;166(1-3):297-303. doi: 10.1016/j.schres.2015.05.013. PMID: 26044114. Country not eligible.

Audini B, Marks IM, Lawrence RE, et al. Home-based versus out-patient/in-patient care for people with serious mental illness. Phase II of a controlled study. Br J Psychiatry. 1994 Aug;165(2):204-10. PMID: 7953033. Comparator not included in review.

Awad G, Ng-Mak D, Rajagopalan K, et al. Long-term health-related quality of life improvements among patients treated with lurasidone: results from the open-label extension of a switch trial in schizophrenia. BMC Psychiatry. 2016 Jun 01;16:176. doi: 10.1186/s12888-016-0879-5. PMID: 27245981. Comparator not included in review.

Ayesa-Arriola R, Rodriguez-Sanchez JM, Perez-Iglesias R, et al. Long-term (3-year) neurocognitive effectiveness of antipsychotic medications in first-episode non-affective psychosis: a randomized comparison of haloperidol, olanzapine, and risperidone. Psychopharmacology (Berl). 2013 Jun;227(4):615-25. doi: 10.1007/s00213-013-2994-z. PMID: 23455591. Outcome not included in review. 
Ayub M, Saeed K, Munshi TA, et al. Clozapine for psychotic disorders in adults with intellectual disabilities. Cochrane Database Syst Rev. 2015 Sep 23(9):Cd010625. doi: 10.1002/14651858.CD010625.pub2. PMID: 26397173. Population ineligible for review.

Azrin NH, Teichner G. Evaluation of an instructional program for improving medication compliance for chronically mentally ill outpatients. Behav Res Ther. 1998;36:849-61. PMID: 9701860. Intervention not included in review.

Bademli K, Cetinkaya Duman Z. Family to family support programs for the caregivers of schizophrenia patients: a systematic review. Turk Psikiyatri Derg. 2011;22(4):255-65. PMID: 22143951. Systematic review/meta-analysis used as a source document to identify individual studies.

Bai Z, Wang G, Cai S, et al. Efficacy, acceptability and tolerability of 8 atypical antipsychotics in chinese patients with acute schizophrenia: A network meta-analysis. Schizophr Res. 2017 Jandoi: 10.1016/j.schres.2017.01.002. PMID: 28108226. Excluded due to including a review that was more recent, more comprehensive, or had a more similar scope to this review scope.

Bais L, Vercammen A, Stewart R, et al. Short and long term effects of left and bilateral repetitive transcranial magnetic stimulation in schizophrenia patients with auditory verbal hallucinations: a randomized controlled trial. PLoS ONE. 2014;9(10):e108828. doi: 10.1371/journal.pone.0108828. PMID: 25329799. Intervention not included in review.

Bak M, Fransen A, Janssen J, et al. Almost all antipsychotics result in weight gain: a meta-analysis. PLoS One. 2014;9(4):e94112. doi: 10.1371/journal.pone.0094112. PMID: 24763306. Comparator not included in review.

Bak M, van Os J, Delespaul P, et al. An observational, "real life" trial of the introduction of assertive community treatment in a geographically defined area using clinical rather than service use outcome criteria. Soc Psychiatry Psychiatr Epidemiol. 2007 Feb;42(2):125-30. PMID: 17235445. Study design not included in review.

Baker A, Bucci S, Lewin TJ, et al. Cognitive-behavioural therapy for substance use disorders in people with psychotic disorders: randomised controlled trial. Br J Psychiatry. 2006 May;188:439-48. doi: 10.1192/bjp.188.5.439. PMID: 16648530. Study covered in a systematic review.

Balhara Y, Verma R. Schizophrenia and suicide. East Asian Arch Psychiatry. 2012 Sep;22(3):126-33. PMID: 23019287. Not a study.

Barak Y, Savorai O, Mavashev S, et al. Animal-assisted therapy for elderly schizophrenic patients: a one-year controlled trial. Am J Geriatr Psychiatry. 2001;9(4):439-42. PMID: 11739071. Intervention not included in review.

Barbato A, D'Avanzo B. Family interventions in schizophrenia and related disorders: a critical review of clinical trials. Acta Psychiatr Scand. 2000 Aug;102(2):81-97. PMID: 10937780. Excluded due to including a review that was more recent, more comprehensive, or had a more similar scope to this review scope.

Barbui C, Accordini S, Nose M, et al. Aripiprazole versus haloperidol in combination with clozapine for treatment-resistant schizophrenia in routine clinical care: a randomized, controlled trial. J Clin Psychopharmacol. 2011 Jun;31(3):266-73. doi: 10.1097/JCP.0b013e318219cba3. PMID: 21508849. Intervention not included in review.

Bark N, Revheim N, Huq F, et al. The impact of cognitive remediation on psychiatric symptoms of schizophrenia. Schizophr Res. 2003 Oct 1;63(3):229-35. PMID: 12957702. Setting ineligible for review (i.e., inpatient only).

Barkhof E, Meijer CJ, de Sonneville LM, et al. The effect of motivational interviewing on medication adherence and hospitalization rates in nonadherent patients with multiepisode schizophrenia. Schizophr Bull. 2013 Nov;39(6):1242-51. doi: 10.1093/schbul/sbt138. PMID: 24072808. Comparator not included in review.

Barlati S, De Peri L, Deste G, et al. Cognitive remediation in the early course of schizophrenia: a critical review. Curr Pharm Des. 2012;18(4):534-41. PMID: 22239585. Excluded due to including a review that was more recent, more comprehensive, or had a more similar scope to this review scope.

Barnes TRE, Drake RJ, Dunn G, et al. Effect of prior treatment with antipsychotic long-acting injection on randomised clinical trial treatment outcomes. Br J Psychiatry. 2013;203(3):215-20. PMID: 23888001. Study design not included in review.

Barretto EM, Kayo M, Avrichir BS, et al. A preliminary controlled trial of cognitive behavioral therapy in clozapine-resistant schizophrenia. J Nerv Ment Dis. 2009 Nov;197(11):865-8. doi: 10.1097/NMD.0b013e3181be7422. PMID: 19996727. Study design not included in review. 
Barrio C, Yamada A-M. Culturally based intervention development: the case of Latino families dealing with schizophrenia. Res Soc Work Pract. 2010 Sep;20(5):483-92. doi: 10.1177/1049731510361613. PMID: 22121328. Outcome not included in review.

Barrowclough C, Haddock G, Tarrier N, et al. Randomized controlled trial of motivational interviewing, cognitive behavior therapy, and family intervention for patients with comorbid schizophrenia and substance use disorders. Am J Psychiatry. 2001 Oct;158(10):1706-13. PMID: 11579006. Study covered in a systematic review.

Barrowclough C, Haddock G, Wykes T, et al. Integrated motivational interviewing and cognitive behavioural therapy for people with psychosis and comorbid substance misuse: randomised controlled trial. BMJ. 2010;341 PMID: 21106618. Study covered in a systematic review.

Barry SJ, Gaughan TM, Hunter R. Schizophrenia. BMJ Clin Evid. 2012 PMID: 23870705. Excluded due to including a review that was more recent, more comprehensive, or had a more similar scope to this review scope.

Bateman K, Hansen L, Turkington D, et al. Cognitive behavioral therapy reduces suicidal ideation in schizophrenia: results from a randomized controlled trial. Suicide Life Threat Behav. 2007 Jun;37(3):284-90. PMID: 17579541. Study covered in a systematic review.

Bauml J, Frobose T, Kraemer S, et al. Psychoeducation: a basic psychotherapeutic intervention for patients with schizophrenia and their families. Schizophr Bull. 2006 Oct;32(Suppl1):S1-S9. doi: 10.1093/schbul/sbl017. PMID: 16920788. Outcome not included in review.

Bauml J, Pitschel-Walz G, Volz A, et al. Psychoeducation in schizophrenia: 7-year follow-up concerning rehospitalization and days in hospital in the Munich Psychosis Information Project Study. J Clin Psychiatry. 2007 Jun;68(6):854-61. PMID: 17592908. Outcome not included in review.

Bechdolf A, Knost B, Kuntermann C, et al. A randomized comparison of group cognitive-behavioural therapy and group psychoeducation in patients with schizophrenia.[Erratum appears in Acta Psychiatr Scand. 2004 Dec;110(6):483]. Acta Psychiatr Scand. 2004 Jul;110(1):21-8. PMID: 15180776. Comparator not included in review.

Bechdolf A, Knost B, Nelson B, et al. Randomized comparison of group cognitive behaviour therapy and group psychoeducation in acute patients with schizophrenia: effects on subjective quality of life. Aust N Z J Psychiatry. 2010 Feb;44(2):144-50. doi: 10.3109/00048670903393571. PMID: 20113303. Population ineligible for review.

Bechdolf A, Kohn D, Knost B, et al. A randomized comparison of group cognitive-behavioural therapy and group psychoeducation in acute patients with schizophrenia: outcome at 24 months. Acta Psychiatr Scand. 2005 Sep;112(3):173-9. PMID: 16095471. Comparator not included in review.

Bechdolf A, Phillips LJ, Francey SM, et al. Recent approaches to psychological interventions for people at risk of psychosis. Eur Arch Psychiatry Clin Neurosci. 2006 Apr;256(3):159-73. PMID: 16639521. Not a study.

Bechi M, Spangaro M, Bosia M, et al. Theory of mind intervention for outpatients with schizophrenia. Neuropsychol Rehabil. 2013;23(3):383-400. PMID: 23379271. Outcome not included in review.

Beck K, McCutcheon R, Bloomfield MA, et al. The practical management of refractory schizophrenia--the Maudsley Treatment REview and Assessment Team service approach. Acta Psychiatrica Scandinavica. 2014 Dec;130(6):427-38. doi: 10.1111/acps.12327. PMID: 25201058. Study design not included in review.

Becker DR, Drake RE, Bond GR, et al. Job terminations among persons with severe mental illness participating in supported employment. Community Ment Health J. 1998 Feb;34(1):71-82. PMID: 9559241. Comparator not included in review.

Beebe LH. Community nursing support for clients with schizophrenia. Arch Psychiatr Nurs. 2001 Oct;15(5):214-22. doi: 10.1053/apnu.2001.27018. PMID: 11584350. Outcome not included in review.

Behere RV, Arasappa R, Jagannathan A, et al. Effect of yoga therapy on facial emotion recognition deficits, symptoms and functioning in patients with schizophrenia. Acta Psychiatr Scand. 2011 Feb;123(2):147-53. doi: 10.1111/j.1600-0447.2010.01605.x. PMID: 20846271. Intervention not included in review.

Bell M, Bryson G, Greig T, et al. Neurocognitive enhancement therapy with work therapy: effects on neuropsychological test performance. Arch Gen Psychiatry. 2001

Aug;58(8):763-8. PMID: 11483142. Comparator not included in review. 
Bell M, Bryson G, Wexler BE. Cognitive remediation of working memory deficits: durability of training effects in severely impaired and less severely impaired schizophrenia. Acta Psychiatr Scand. 2003 Aug;108(2):101-9. PMID: 12823166. Outcome not included in review.

Bell M, Fiszdon J, Greig T, et al. Neurocognitive enhancement therapy with work therapy in schizophrenia: 6-month follow-up of neuropsychological performance. J Rehabil Res Dev. 2007;44(5):761-70. PMID: 17943687. Outcome not included in review.

Bell M, Lysaker P, Bryson G. A behavioral intervention to improve work performance in schizophrenia: work behavior inventory feedback. J Vocat Rehabil. 2003;18(1):43-50. Intervention not included in review.

Bell MD, Bryson GJ, Greig TC, et al. Neurocognitive enhancement therapy with work therapy: productivity outcomes at 6- and 12-month follow-ups. J Rehabil Res Dev. 2005 Nov-Dec;42(6):829-38. PMID: 16680620. Study covered in a systematic review.

Bell MD, Choi KH, Dyer C, et al. Benefits of cognitive remediation and supported employment for schizophrenia patients with poor community functioning. Psychiatr Serv. 2014 Apr 1;65(4):469-75. doi: 10.1176/appi.ps.201200505. PMID: 24382594. Study design not included in review.

Bell MD, Lysaker PH. Clinical benefits of paid work activity in schizophrenia: 1-year followup. Schizophr Bull. 1997;23(2):317-28. PMID: 9165640. Intervention not included in review.

Bell MD, Lysaker PH, Milstein RM. Clinical benefits of paid work activity in schizophrenia. Schizophr Bull. 1996;22(1):51-67. PMID: 8685664. Study covered in a systematic review.

Bell MD, Zito W, Greig T, et al. Neurocognitive enhancement therapy with vocational services: work outcomes at two-year follow-up. Schizophr Res. 2008 Oct;105(1-3):18-29. doi: 10.1016/j.schres.2008.06.026. PMID: 18715755. Comparator not included in review.

Bender S, Dittmann-Balcar A, Schall U, et al. Influence of atypical neuroleptics on executive functioning in patients with schizophrenia: a randomized, double-blind comparison of olanzapine vs. clozapine. Int J Neuropsychopharmacol. 2006 Apr;9(2):135-45. PMID: 16174427. Outcome not included in review.

Benton MK, Schroeder HE. Social skills training with schizophrenics: a meta-analytic evaluation. J Consult Clin Psychol. 1990 Dec;58(6):741-7. PMID: 2149858. Excluded due to including a review that was more recent, more comprehensive, or had a more similar scope to this review scope.

Bhugra D, Ayonrinde O, Butler G, et al. A randomised controlled trial of assertive outreach vs. treatment as usual for black people with severe mental illness. Epidemiol Psychiatr Sci. 2011 Mar;20(1):83-9. PMID: 21657119. Country not eligible.

Bio DS, Gattaz WF. Vocational rehabilitation improves cognition and negative symptoms in schizophrenia. Schizophr Res. 2011 Mar;126(1-3):265-9. doi:

10.1016/j.schres.2010.08.003. PMID: 20800453. Intervention not included in review.

Birchwood M, Michail M, Meaden A, et al. The MRC command trial: results of a multi-centre, randomised controlled trial of cognitive therapy to prevent harmful compliance with command hallucinations. Schizophr Res. 2014;153(5)doi: 10.1016/S0920-9964(14)70239-7. Not a study.

Birchwood M, Trower P. Cognitive therapy for command hallucinations: not a quasi-neuroleptic. J Contemp Psychother. 2006 Mar;36(1):1-7. doi: 10.1007/s10879-005-9000-y. Outcome not included in review.

Boczkowski JA, Zeichner A, DeSanto N. Neuroleptic compliance among chronic schizophrenic outpeople: an intervention outcome report. J Consult Clin Psychol. 1985;53:66671. PMID: 2865281. Outcome not included in review.

Bola JR, Lehtinen K, Cullberg J, et al. Psychosocial treatment, antipsychotic postponement, and low-dose medication strategies in first-episode psychosis: a review of the literature. Psychosis. 2009 Feb;1(1):4-18. Not a study.

Bola JR, Mosher LR. Treatment of acute psychosis without neuroleptics: two-year outcomes from the Soteria project. J Nerv Ment Dis. 2003 Apr;191(4):219-29. PMID:

12695732. Comparator not included in review. 
Bond GR, Miller LD, Krumwied RD, et al. Assertive case management in three CMHCs: a controlled study. Hosp Community Psychiatry. 1988 Apr;39(4):411-8. PMID: 2836295. Study covered in a systematic review.

Bond GR, Salyers MP, Dincin J, et al. A randomized controlled trial comparing two vocational models for persons with severe mental illness. J Consult Clin Psychol. 2007 Dec;75(6):968-82. doi: 10.1037/0022-006x.75.6.968. PMID: 18085913. Study covered in a systematic review.

Bond GR, Witheridge TF, Dincin J, et al. Assertive community treatment for frequent users of psychiatric hospitals in a large city: a controlled study. Am J Community Psychol. 1990 Dec;18(6):865-91. PMID: 2091459. Study covered in a systematic review.

Borlido C, Remington G, Graff-Guerrero A, et al. Switching From 2 antipsychotics to 1 Antipsychotic in schizophrenia: a randomized, double-blind, placebo-controlled study. J Clin Psychiatry. 2016;77(1):e14-e20. PMID: 26845273. Study design not included in review.

Bosch P, van den Noort M, Staudte H, et al. Schizophrenia and depression: a systematic review of the effectiveness and the working mechanisms behind acupuncture. Explore (NY) 2015 Jul-Aug;11(4):281-91. doi: 10.1016/j.explore.2015.04.004. PMID: 26007331. Excluded due to including a review that was more recent, more comprehensive, or had a more similar scope to this review scope.

Bosch P, van den Noort M, Yeo S, et al. The effect of acupuncture on mood and working memory in patients with depression and schizophrenia. J Integr Med. 2015 Nov;13(6):380-90. doi: 10.1016/S2095-4964(15)60204-7. PMID: 26559363. Intervention not included in review.

Boter H, Derks EM, Fleischhacker WW, et al. Generalizability of the results of efficacy trials in first-episode schizophrenia: comparisons between subgroups of participants of the European First Episode Schizophrenia Trial (EUFEST). J Clin Psychiatry. 2010 Jan;71(1):58-65. PMID: 19852905. Study design not included in review.

Bourque J, Lakis N, Champagne J, et al. Clozapine and visuospatial processing in treatment-resistant schizophrenia. Cogn Neuropsychiatry. 2013;18(6):615-30. PMID: 23343453. Outcome not included in review.

Bowie CR, Grossman M, Gupta M, et al. Cognitive remediation in schizophrenia: efficacy and effectiveness in patients with early versus long-term course of illness. Early Interv Psychiatry. 2014 Feb;8(1):32-8. doi: 10.1111/eip.12029. PMID: 23343011. Comparator not included in review.

Bowie CR, McGurk SR, Mausbach B, et al. Combined cognitive remediation and functional skills training for schizophrenia: effects on cognition, functional competence, and realworld behavior. Am J Psychiatry. 2012 Jul;169(7):710-8. doi: 10.1176/appi.ajp.2012.11091337. PMID: 22581070. Comparator not included in review.

Boyer L, Lancon C, Baumstarck K, et al. Evaluating the impact of a quality of life assessment with feedback to clinicians in patients with schizophrenia: randomised controlled trial. Br J Psychiatry. 2013;202:447-53. PMID: 23661768. Interventions evaluated in a single study.

Brabban A, Tai S, Turkington D. Predictors of outcome in brief cognitive behavior therapy for schizophrenia. Schizophr Bull. 2009;35(5):859-64. doi: 10.1093/schbul/sbp065. PMID: 19571248. Study design not included in review.

Bradley GM, Couchman GM, Perlesz A, et al. Multiple-family group treatment for English- and Vietnamese-speaking families living with schizophrenia. Psychiatr Serv. 2006 Apr;57(4):521-30. doi: 10.1176/appi.ps.57.4.521. PMID: 16603748. Study covered in a systematic review.

Bradshaw W. Structured group work for individuals with schizophrenia: a coping skills approach. Res Soc Work Pract. 1996 Apr;6(2):139-54. doi: 10.1177/104973159600600201. Outcome not included in review.

Bradshaw W. Integrating cognitive-behavioral psychotherapy for persons with schizophrenia into a psychiatric rehabilitation program: results of a three year trial. Community Ment Health J. 2000 Oct;36(5):491-500. PMID: 10994682. Not a study.

Bradshaw WH. Coping-skills training versus a problem-solving approach with schizophrenic patients. Hosp Community Psychiatry. 1993 Nov;44(11):1102-4. PMID: 8288183. Intervention not included in review.

Brandon S, Cowley P, McDonald C, et al. Leicester ECT trial: results in schizophrenia. Br J Psychiatry. 1985 Feb;146:177-83. PMID: 3884080. Intervention not included in review. 
Bressi C, Manenti S, Frongia P, et al. Systemic family therapy in schizophrenia: a randomized clinical trial of effectiveness. Psychother Psychosom. 2008;77(1):43-9. PMID: 18087207. Study design not included in review.

Briand C, Belanger R, Hamel V, et al. [Implementation of the multi-site Integrated Psychological Treatment (IPT) program for people with schizophrenia. Elaboration of renewed version]. Sante Ment Que. 2005 Spring;30(1):73-95. PMID: 16170424. Not English language.

Briand C, Lesage A, Lalonde P, et al. The integrated psychological treatment (IPT) for patients with schizophrenia: evidence of effectiveness during program implementation in various sites in Quebec, Canada. Schizophr Res. 2003;60(1, Supplement):320. doi: 10.1016/S0920-9964(03)80275-X. Not English language.

Briki M, Monnin J, Haffen E, et al. Metacognitive training for schizophrenia: a multicentre randomised controlled trial. Schizophr Res. 2014 Aug;157(1-3):99-106. doi: 10.1016/j.schres.2014.06.005. PMID: 24972754. Study covered in a systematic review.

Broderick J, Knowles A, Chadwick J, et al. Yoga versus standard care for schizophrenia. Cochrane Database Syst Rev. 2015 Oct 21(10):Cd010554. doi:

10.1002/14651858.CD010554.pub2. PMID: 26488850. Intervention not included in review.

Brooker C, Falloon I, Butterworth A, et al. The outcome of training community psychiatric nurses to deliver psychosocial intervention. Br J Psychiatry. 1994 Aug;165(2):222-30. PMID: 7953037. Not a study.

Bryson G, Lysaker P, Bell M. Quality of life benefits of paid work activity in schizophrenia. Schizophr Bull. 2002;28(2):249-57. PMID: 12693431. Comparator not included in review.

Bucci P, Piegari G, Mucci A, et al. Neurocognitive individualized training versus social skills individualized training: a randomized trial in patients with schizophrenia. Schizophr Res. 2013 Oct;150(1):69-75. doi: 10.1016/j.schres.2013.07.053. PMID: 23962828. Comparator not included in review.

Buchain PC, Vizzotto AD, Henna Neto J, et al. Randomized controlled trial of occupational therapy in patients with treatment-resistant schizophrenia. Rev Bras Psiquiatr. 2003 Mar;1999) 25(1):26-30. PMID: 12975676. Intervention not included in review.

Buchkremer G, Klingberg S, Holle R, et al. Psychoeducational psychotherapy for schizophrenic patients and their key relatives or care-givers: results of a 2-year follow-up. Acta Psychiatr Scand. 1997 Dec;96(6):483-91. PMID: 9421346. Study covered in a systematic review.

Burns T, Catty J, Becker T, et al. The effectiveness of supported employment for people with severe mental illness: a randomised controlled trial. Lancet. 2007 Sep 29;370(9593):1146-52. doi: 10.1016/s0140-6736(07)61516-5. PMID: 17905167. Study covered in a systematic review.

Burns T, Catty J, White S, et al. The impact of supported employment and working on clinical and social functioning: results of an international study of individual placement and support. Schizophr Bull. 2009 Sep;35(5):949-58. doi: 10.1093/schbul/sbn024. PMID: 18403375. Intervention not included in review.

Burns T, White SJ, Catty J. Individual placement and support in Europe: the EQOLISE trial. Int Rev Psychiatry. 2008;20(6):498-502. PMID: 19085404. Study covered in a systematic review.

Bustillo JR, Lauriello J, Horan WP, et al. The psychosocial treatment of schizophrenia: an update. Am J Psychiatry. 2001 Feb;158(2):163-75. doi: 10.1176/appi.ajp.158.2.163. PMID: 11156795. Not a study.

Byerly MJ, Fisher R, Carmody T, et al. A trial of compliance therapy in outpatients with schizophrenia or schizoaffective disorder. J Clin Psychiatry. 2005 Aug;66(8):997-1001. PMID: 16086614. Study design not included in review.

Cacciotti-Saija C, Langdon R, Ward PB, et al. A double-blind randomized controlled trial of oxytocin nasal spray and social cognition training for young people with early psychosis. Schizophr Bull. 2015 Mar;41(2):483-93. PMID: 24962607. Intervention not included in review.

Caforio G, Di Giorgio A, Rampino A, et al. Mirtazapine add-on improves olanzapine effect on negative symptoms of schizophrenia. J Clin Psychopharmacol. 2013 Dec;33(6):810-

2. PMID: 24113675. Intervention not included in review. 
Calvo P, Fortuny JR, Guzman S, et al. Animal assisted therapy (AAT) program as a useful adjunct to conventional psychosocial rehabilitation for patients with schizophrenia: Results of a small-scale randomized controlled trial. Frontiers in Psychology Vol 7 2016, ArtID 631. 2016 May;7 PMID: 27199859. Intervention not included in review.

Campbell K, Bond GR, Drake RE. Who benefits from supported employment: a meta-analytic study. Schizophr Bull. 2011 Mar;37(2):370-80. doi: 10.1093/schbul/sbp066. PMID: 19661196. Comparator not included in review.

Campbell LA, Kisely SR. Advance treatment directives for people with severe mental illness. Cochrane Database Syst Rev. 2009 Jan 21(8): Cd005963. doi: 10.1002/14651858.CD005963.pub2. PMID: 19160260. Intervention not included in review.

Caplan B, Schutt RK, Turner WM, et al. Change in neurocognition by housing type and substance abuse among formerly homeless seriously mentally ill persons. Schizophr Res. 2006 Mar;83(1):77-86. PMID: 16504484. Outcome not included in review.

Carpenter WT, Heinrichs DW, Hanlon TE. A comparative trial of pharmacologic strategies in schizophrenia. Am J Psychiatry. 1987 Nov;144(11):1466-70. PMID: 2890307. Intervention not included in review.

Carra G, Montomoli C, Clerici M, et al. Family interventions for schizophrenia in Italy: randomized controlled trial. Eur Arch Psychiatry Clin Neurosci. 2007 Feb;257(1):23-30. PMID: 16917681. Study covered in a systematic review.

Castelein S, Bruggeman R, van Busschbach JT, et al. The effectiveness of peer support groups in psychosis: a randomized controlled trial. Acta Psychiatr Scand. 2008 Jul;118(1):64-72. PMID: 18595176. Interventions evaluated in a single study.

Castelein S, Knegtering H, van Meijel B, et al. Dutch guideline on schizophrenia 2012: basic care within the areas of psychosocial interventions and nursing care. Tijdschr Psychiatr. 2013;55(9):707-14. PMID: 24046249. Not English language.

Cather C, Penn D, Otto MW, et al. A pilot study of functional Cognitive Behavioral Therapy (fCBT) for schizophrenia. Schizophr Res. 2005 May 1;74(2-3):201-9. PMID: 15722000. Study covered in a systematic review.

Catty J, Lissouba P, White S, et al. Predictors of employment for people with severe mental illness: results of an international six-centre randomised controlled trial. Br J Psychiatry. 2008 Mar;192(3):224-31. doi: 10.1192/bjp.bp.107.041475. PMID: 18310585. Study covered in a systematic review.

Catty SJ, Burns T, Comas A, et al. Day centres for severe mental illness. Cochrane Database Syst Rev. 2007 Jan 24(1):Cd001710. doi: 10.1002/14651858.CD001710.pub2. PMID: 17253463. Intervention not included in review.

Cavallaro R, Anselmetti S, Poletti S, et al. Computer-aided neurocognitive remediation as an enhancing strategy for schizophrenia rehabilitation. Psychiatry Res. 2009 Oct 30;169(3):191-6. doi: 10.1016/j.psychres.2008.06.027. PMID: 19740550. Study covered in a systematic review.

Cazorla P, Mackle M, Zhao J, et al. Safety and tolerability of switching to asenapine from other antipsychotic agents: Pooled results from two randomized multicenter trials in stable patients with persistent negative symptoms in schizophrenia. Neuropsychiatr Dis Treat. 2012;8:247-57. PMID: 22745558. Study design not included in review.

Cella M, Bishara AJ, Medin E, et al. Identifying cognitive remediation change through computational modelling-effects on reinforcement learning in schizophrenia. Schizophr Bull. 2014 Nov;40(6):1422-32. doi: 10.1093/schbul/sbt152. PMID: 24214932. Study design not included in review.

Cella M, Reeder C, Wykes T. It is all in the factors: effects of cognitive remediation on symptom dimensions. Schizophr Res. 2014 Jun;156(1):60-2. doi: 10.1016/j.schres.2014.03.032. PMID: 24768130. Outcome not included in review.

Ceskova E, Prikryl R, Libiger J, et al. Gender differences in the treatment of first-episode schizophrenia: results from the European first episode schizophrenia trial. Schizophr Res. 2015 Dec;169(1-3):303-7. doi: 10.1016/j.schres.2015.10.013. PMID: 26545298. Study covered in a systematic review.

Chabannes JP, Bazin N, Leguay D, et al. Two-year study of relapse prevention by a new education program in schizophrenic patients treated with the same antipsychotic drug. Eur Psychiatry. 2008 Jan;23(1):8-13. PMID: 17964764. Comparator not included in review. 
Chadwick P, Hughes S, Russell D, et al. Mindfulness groups for distressing voices and paranoia: a replication and randomized feasibility trial. Behav Cogn Psychother. 2009 Jul;37(4):403-12. doi: 10.1017/S1352465809990166. PMID: 19545481. Inadequate duration ( $<12$ weeks).

Chafetz L, White M, Collins-Bride G, et al. Clinical trial of wellness training: health promotion for severely mentally ill adults. J Nerv Ment Dis. 2008 Jun;196(6):475-83. doi: 10.1097/NMD.0b013e31817738de. PMID: 18552625. Intervention not included in review.

Challis S, Nielssen O, Harris A, et al. Systematic meta-analysis of the risk factors for deliberate self-harm before and after treatment for first-episode psychosis. Acta Psychiatrica Scandinavica. 2013 Jun;127(6):442-54. doi: 10.1111/acps.12074. PMID: 23298325. Outcome not included in review.

Chan GHK, Jim OTT, AuYang CWS, et al. Effects of extended case management on functioning in people with early psychosis-preliminary findings of the easy3 randomised controlled study. Schizophr Res. 2012;136(14). Not a study.

Chan JY, Hirai HW, Tsoi KK. Can computer-assisted cognitive remediation improve employment and productivity outcomes of patients with severe mental illness? A metaanalysis of prospective controlled trials. J Psychiatr Res. 2015 Sep;68:293-300. doi: 10.1016/j.jpsychires.2015.05.010. PMID: 26028551. Comparator not included in review.

Chan SH, Lee SW, Chan IW. TRIP: a psycho-educational programme in Hong Kong for people with schizophrenia. Occup Ther Int. 2007;14(2):86-98. PMID: 17623381. Country not eligible.

Chan SW, Yip B, Tso S, et al. Evaluation of a psychoeducation program for Chinese clients with schizophrenia and their family caregivers. Patient Educ Couns. 2009 Apr;75(1):67-76. PMID: 18963721. Study covered in a systematic review.

Chandler D, Hu TW, Meisel J, et al. Mental health costs, other public costs, and family burden among mental health clients in capitated integrated service agencies. J Ment Health Adm. 1997 Spring;24(2):178-88. PMID: 9110521. Not a study.

Chandler D, Meisel J, Hu T, et al. A capitated model for a cross-section of severely mentally ill clients: employment outcomes. Community Ment Health J. 1997 Dec;33(6):50116. PMID: 9435997. Intervention not included in review.

Chandler D, Meisel J, Hu TW, et al. Client outcomes in a three-year controlled study of an integrated service agency model. Psychiatr Serv. 1996 Dec;47(12):1337-43. PMID: 9117472. Study covered in a systematic review.

Chanpattana W, Chakrabhand ML, Sackeim HA, et al. Continuation ECT in treatment-resistant schizophrenia: a controlled study. J ECT. 1999 Sep;15(3):178-92. PMID: 10492856. Intervention not included in review.

Chen JJ, Chan HY, Chen CH, et al. Risperidone and olanzapine versus another first generation antipsychotic in patients with schizophrenia inadequately responsive to first generation antipsychotics. Pharmacopsychiatry. 2012 Mar;45(2):64-71. doi: 10.1055/s-0031-1291293. PMID: 22086749. Inadequate duration (<12 weeks).

Chen L-f, Liu J, Zhang J, et al. Non-pharmacological interventions for caregivers of patients with schizophrenia: a meta-analysis. Psychiatry Res. 2015 Novdoi: 10.1016/j.psychres.2015.11.037. PMID: 26639649. Outcome not included in review.

Chien WT, Chan S, Morrissey J, et al. Effectiveness of a mutual support group for families of patients with schizophrenia. J Adv Nurs. 2005 Sep;51(6):595-608. doi: 10.1111/j.1365-2648.2005.03545.x. PMID: 16129010. Country not eligible.

Chien WT, Chan SW. One-year follow-up of a multiple-family-group intervention for Chinese families of patients with schizophrenia. Psychiatr Serv. 2004 Nov;55(11):1276-84. PMID: 15534017. Country not eligible.

Chien WT, Chan SW, Thompson DR. Effects of a mutual support group for families of Chinese people with schizophrenia: 18-month follow-up. Br J Psychiatry. 2006 Jul;189:419. doi: 10.1192/bjp.bp.105.008375. PMID: 16816305. Country not eligible.

Chien WT, Lee IY. The schizophrenia care management program for family caregivers of Chinese patients with schizophrenia. Psychiatr Serv. 2010 Mar;61(3):317-20. PMID:

20194413. Country not eligible. 
Chien WT, Lee IY. The mindfulness-based psychoeducation program for Chinese patients with schizophrenia. Psychiatr Serv. 2013 Apr 1;64(4):376-9. doi: 10.1176/appi.ps.002092012. PMID: 23412024. Country not eligible.

Chien WT, Mui J, Gray R, et al. Adherence therapy versus routine psychiatric care for people with schizophrenia spectrum disorders: a randomised controlled trial. BMC Psychiatry. 2016;16(1)doi: 10.1186/s12888-016-0744-6. PMID: 26911397. Country not eligible.

Chien WT, Mui JHC, Cheung EFC, et al. Effects of motivational interviewing-based adherence therapy for schizophrenia spectrum disorders: a randomized controlled trial. Trials. 2015;16(1)doi: 10.1186/s13063-015-0785-z. PMID: 26072311. Country not eligible.

Chien WT, Thompson DR. An RCT with three-year follow-up of peer support groups for Chinese families of persons with schizophrenia. Psychiatr Serv. 2013 Oct;64(10):9971005. doi: 10.1176/appi.ps.201200243. PMID: 23820670. Country not eligible.

Chien WT, Thompson DR. Effects of a mindfulness-based psychoeducation programme for Chinese patients with schizophrenia: 2-year follow-up. Br J Psychiatry. 2014 Jul;205(1):52-9. doi: 10.1192/bjp.bp.113.134635. PMID: 24809397. Country not eligible.

Chien WT, Thompson DR, Norman I. Evaluation of a peer-led mutual support group for Chinese families of people with schizophrenia. Am J Community Psychol. 2008 Sep;42(12):122-34. doi: 10.1007/s10464-008-9178-8. PMID: 18584319. Country not eligible.

Chien WT, Wong KF. A family psychoeducation group program for chinese people with schizophrenia in Hong Kong. Psychiatr Serv. 2007 Jul;58(7):1003-6. PMID: 17602019. Country not eligible.

Chien W-T, Chan SWC. One-year follow-up of a multiple-family-group intervention for Chinese families of patients with schizophrenia. Psychiatr Serv. 2004;55(11):1276-84. doi: 10.1176/appi.ps.55.11.1276. PMID: 15534017. Country not eligible.

Chien W-T, Norman I. The effectiveness and active ingredients of mutual support groups for family caregivers of people with psychotic disorders: a literature review. Int J Nurs Stud. 2009 Dec;46(12):1604-23. doi: 10.1016/j.ijnurstu.2009.04.003. PMID: 19481205. Study design not included in review.

Chien W-T, Norman I, Thompson DR. A randomized controlled trial of a mutual support group for family caregivers of patients with schizophrenia. Int J Nurs Stud. 2004;41(6):637-49. doi: 10.1016/j.ijnurstu.2004.01.010. PMID: 15240088. Study covered in a systematic review.

Chilvers R, Macdonald GM, Hayes AA. Supported housing for people with severe mental disorders. Cochrane Database Syst Rev. 2006 Oct 18(4):Cd000453. doi: 10.1002/14651858.CD000453.pub2. PMID: 17054130. Not a study.

Choi KH, Kwon JH. Social cognition enhancement training for schizophrenia: a preliminary randomized controlled trial. Community Ment Health J. 2006 Apr;42(2):177-87. PMID: 16732472. Outcome not included in review.

Cipriani A, Accordini S, Nose M, et al. Aripiprazole versus haloperidol in combination with clozapine for treatment-resistant schizophrenia: a 12-month, randomized, naturalistic trial. J Clin Psychopharmacol. 2013 Aug;33(4):533-7. PMID: 23775051. Intervention not included in review.

Citrome L, Weiden PJ, Alva G, et al. Switching to iloperidone: an omnibus of clinically relevant observations from a 12-week, open-label, randomized clinical trial in 500 persons with schizophrenia. Clin Schizophr Relat Psychoses. 2015 Jan;8(4):183-95. doi: 10.3371/CSRP.CIWE.103114. PMID: 25367165. Study design not included in review.

Citrome L, Weiden PJ, McEvoy JP, et al. Effectiveness of lurasidone in schizophrenia or schizoaffective patients switched from other antipsychotics: a 6-month, open-label, extension study. CNS Spectr. 2014;19(4):330-9. PMID: 24330868. Study design not included in review.

Ciudad A, Anand E, Berggren L, et al. Switching to olanzapine long-acting injection from either oral olanzapine or any other antipsychotic: comparative post hoc analyses. Neuropsychiatr Dis Treat. 2013;9:1737-50. PMID: 24235837. Study design not included in review.

Clarke GN, Herinckx HA, Kinney RF, et al. Psychiatric hospitalizations, arrests, emergency room visits, and homelessness of clients with serious and persistent mental illness: findings from a randomized trial of two ACT programs vs. usual care. Ment Health Serv Res. 2000 Sep;2(3):155-64. PMID: 11256724. Study covered in a systematic review. 
Cohen LJ, Test MA, Brown RL. Suicide and schizophrenia: data from a prospective community treatment study. Am J Psychiatry. 1990 May;147(5):602-7. doi: 10.1176/ajp.147.5.602. PMID: 2327487. Study design not included in review.

Cook JA, Copeland ME, Jonikas JA, et al. Results of a randomized controlled trial of mental illness self-management using wellness recovery action planning. Schizophr Bull. 2012 Jun;38(4):881-91. doi: 10.1093/schbul/sbr012. PMID: 21402724. Intervention not included in review.

Correll CU, Cucchiaro J, Silva R, et al. Long-term safety and effectiveness of lurasidone in schizophrenia: a 22-month, open-label extension study. CNS Spectr. 2016 Oct;21(5):393-402. doi: 10.1017/s1092852915000917. PMID: 27048911. Comparator not included in review.

Covell NH, McEvoy JP, Schooler NR, et al. Effectiveness of switching from long-acting injectable fluphenazine or haloperidol decanoate to long-acting injectable risperidone microspheres: an open-label, randomized controlled trial. J Clin Psychiatry. 2012 May;73(5):669-75. doi: 10.4088/JCP.11m07074. PMID: 22480442. Study design not included in review.

Craig TK, Johnson S, McCrone P, et al. Integrated care for co-occurring disorders: psychiatric symptoms, social functioning, and service costs at 18 months. Psychiatr Serv. 2008 Mar;59(3):276-82. doi: 10.1176/appi.ps.59.3.276. PMID: 18308908. Study design not included in review.

Cramer H, Lauche R, Klose P, et al. Yoga for schizophrenia: a systematic review and meta-analysis. BMC Psychiatry. 2013;13:32. doi: 10.1186/1471-244X-13-32. PMID: 23327116. Intervention not included in review.

Crawford MJ, Killaspy H, Barnes TR, et al. Group art therapy as an adjunctive treatment for people with schizophrenia: multicentre pragmatic randomised trial. BMJ. 2012;344:e846. doi: 10.1136/bmj.e846. PMID: 22374932. Intervention not included in review.

Crawford-Walker CJ, King A, Chan S. Distraction techniques for schizophrenia. Cochrane Database Syst Rev. 2009(4) PMID: 15674963. Systematic review/meta-analysis used as a source document to identify individual studies.

Crespo-Facorro B, de la Foz VO, Mata I, et al. Treatment of first-episode non-affective psychosis: a randomized comparison of aripiprazole, quetiapine and ziprasidone over 1 year. Psychopharmacology (Berl). 2014 Jan;231(2):357-66. PMID: 23958945. Study covered in a systematic review.

Crespo-Facorro B, Perez-Iglesias R, Mata I, et al. Long-term (3-year) effectiveness of haloperidol, risperidone and olanzapine: results of a randomized, flexible-dose, open-label comparison in first-episode nonaffective psychosis. Psychopharmacology (Berl). 2012 Jan;219(1):225-33. PMID: 21735072. Study covered in a systematic review.

Crespo-Facorro B, Perez-Iglesias R, Mata I, et al. Aripiprazole, ziprasidone, and quetiapine in the treatment of first-episode nonaffective psychosis: results of a 6-week, randomized, flexible-dose, open-label comparison. J Clin Psychopharmacol. 2013 Apr;33(2):215-20. doi: 10.1097/JCP.0b013e3182825c1e. PMID: 23422371. Inadequate duration ( $<12$ weeks).

Crowther R, Marshall M, Bond G, et al. Vocational rehabilitation for people with severe mental illness. Cochrane Database Syst Rev. 2001(2):Cd003080. doi: 10.1002/14651858.cd003080. PMID: 11406069. Intervention not included in review.

Cuijpers P, Hollon SD, van Straten A, et al. Does cognitive behaviour therapy have an enduring effect that is superior to keeping patients on continuation pharmacotherapy? A meta-analysis. BMJ Open. 2013;3(4)doi: 10.1136/bmjopen-2012-002542. PMID: 23624992. Comparator not included in review.

Cunningham Owens DG, Carroll A, Fattah S, et al. A randomized, controlled trial of a brief interventional package for schizophrenic out-patients. Acta Psychiatr Scand. 2001 May;103(5):362-9. PMID: 11380306. Interventions evaluated in a single study.

Curtis JL, Millman EJ, Struening E, et al. Deaths among former psychiatric inpatients in an outreach case management program. Psychiatr Serv. 1996 Apr;47(4):398-402. doi: 10.1176/ps.47.4.398. PMID: 8689371. Study covered in a systematic review.

Curtis JL, Millman EJ, Struening EL, et al. Does outreach case management improve patients' quality of life? Psychiatr Serv. 1998 Mar;49(3):351-4. doi: 10.1176/ps.49.3.351. PMID: 9525795. Study covered in a systematic review.

Cutler AJ, Kalali AH, Mattingly GW, et al. Long-term safety and tolerability of iloperidone: results from a 25-week, open-label extension trial. CNS Spectr. 2013 Feb;18(1):43-54. doi: 10.1017/S1092852912000764. PMID: 23312567. Study design not included in review. 
Dalum HS, Korsbek L, Mikkelsen JH, et al. Illness management and recovery (IMR) in Danish community mental health centres. Trials. 2011;12(195) PMID: 21849024. Study design not included in review.

d'Amato T, Bation R, Cochet A, et al. A randomized, controlled trial of computer-assisted cognitive remediation for schizophrenia. Schizophr Res. 2011 Feb;125(2-3):284-90. doi: 10.1016/j.schres.2010.10.023. PMID: 21094025. Intervention not included in review.

Dang J, Zhang J, Guo Z, et al. A pilot study of iPad-assisted cognitive training for schizophrenia. Arch Psychiatr Nurs. 2014 Jun;28(3):197-9. PMID: 24856273. Outcome not included in review.

Davidson L, Chinman M, Sells D, et al. Peer support among adults with serious mental illness: a report from the field. Schizophr Bull. 2006 Jul;32(3):443-50. PMID: 16461576. Not a study.

Davis LW, Lysaker PH, Kristeller JL, et al. Effect of mindfulness on vocational rehabilitation outcomes in stable phase schizophrenia. Psychol Serv. 2015 Aug;12(3):303-12. doi: 10.1037/ser0000028. PMID: 25938855. Intervention not included in review.

Davis MC, Green MF, Lee J, et al. Oxytocin-augmented social cognitive skills training in schizophrenia. Neuropsychopharmacology. 2014 Aug;39(9):2070-7. doi: 10.1038/npp.2014.68. PMID: 24637803. Intervention not included in review.

Davis MC, Lee J, Horan WP, et al. Effects of single dose intranasal oxytocin on social cognition in schizophrenia. Schizophr Res. 2013 Jul;147(2-3):393-7. PMID: 23676253. Intervention not included in review.

De Giacomo P, Pierri G, Santoni Rugiu A, et al. Schizophrenia: a study comparing a family therapy group following a paradoxical model plus psychodrugs and a group treated by the conventional clinical approach. Acta Psychiatr Scand. 1997 Mar;95(3):183-8. PMID: 9111850. Study covered in a systematic review.

de Jesus DR, Gil A, Barbosa L, et al. A pilot double-blind sham-controlled trial of repetitive transcranial magnetic stimulation for patients with refractory schizophrenia treated with clozapine. Psychiatry Res. 2011 Jul 30;188(2):203-7. doi: 10.1016/j.psychres.2010.11.022. PMID: 21186062. Intervention not included in review.

de Jesus Mari J, Streiner DL. An overview of family interventions and relapse on schizophrenia: meta-analysis of research findings. Psychol Med. 1994 Aug;24(3):565-78. doi: 10.1017/S0033291700027720. PMID: 7991739. Excluded due to including a review that was more recent, more comprehensive, or had a more similar scope to this review scope.

De Silva MJ, Cooper S, Li HL, et al. Effect of psychosocial interventions on social functioning in depression and schizophrenia: meta-analysis. Br J Psychiatry. 2013 Apr;202(4):253-60. doi: 10.1192/bjp.bp.112.118018. PMID: 23549941. Excluded due to including a review that was more recent, more comprehensive, or had a more similar scope to this review scope.

de Weijer AD, Sommer IE, Lotte Meijering A, et al. High frequency rTMS; a more effective treatment for auditory verbal hallucinations? Psychiatry Res. 2014 Dec 30;224(3):204-10. doi: 10.1016/j.pscychresns.2014.10.007. PMID: 25453990. Inadequate duration (<12 weeks).

Dean M, Weston AR, Osborn DP, et al. Activity groups for people with schizophrenia: a randomized controlled trial. J Ment Health. 2014 Aug;23(4):171-5. doi: 10.3109/09638237.2014.889285. PMID: 24660971. Interventions evaluated in a single study.

Dekker J, Wijdenes W, Koning YA, et al. Assertive community treatment in Amsterdam. Community Ment Health J. 2002 Oct;38(5):425-34. PMID: 12236412. Intervention not included in review.

Demily C, Cavezian C, Desmurget M, et al. The game of chess enhances cognitive abilities in schizophrenia. Schizophr Res. 2009 Jan;107(1):112-3. doi: 10.1016/j.schres.2008.09.024. PMID: 18995990. Intervention not included in review.

Depp CA, Mausbach B, Granholm E, et al. Mobile interventions for severe mental illness: design and preliminary data from three approaches. J Nerv Ment Dis. 2010 Oct;198(10):715-21. doi: 10.1097/NMD.0b013e3181f49ea3. PMID: 20921861. Study design not included in review.

D'Ercole A, Struening E, Curtis JL, et al. Effects of diagnosis, demographic characteristics, and case management on rehospitalization. Psychiatr Serv. 1997 May;48(5):682-8. PMID: 9144824. Outcome not included in review. 


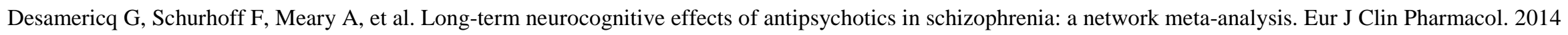
Feb;70(2):127-34. doi: 10.1007/s00228-013-1600-y. PMID: 24145817. Outcome not included in review.

Dickerson FB, Lehman AF. Evidence-based psychotherapy for schizophrenia. J Nerv Ment Dis. 2006 Jan;194(1):3-9. PMID: 16462548. Not a study.

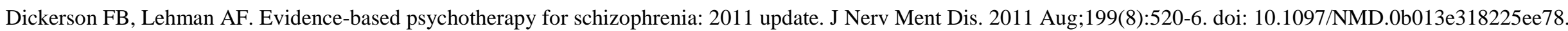
PMID: 21814072. Not a study.

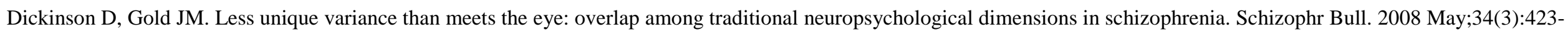
34. PMID: 17702991. Outcome not included in review.

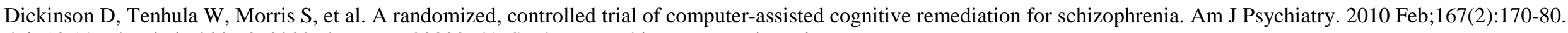
doi: 10.1176/appi.ajp.2009.09020264. PMID: 20008941. Study covered in a systematic review.

Dieterich M, Irving CB, Park B, et al. Intensive case management for severe mental illness. Cochrane Database Syst Rev. 2010 Oct 06(10):Cd007906. doi: 10.1002/14651858.CD007906.pub2. PMID: 20927766. Intervention not included in review.

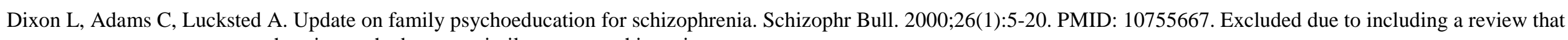
was more recent, more comprehensive, or had a more similar scope to this review scope.

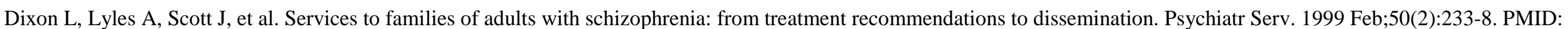
10030482. Outcome not included in review.

Dixon L, Stewart B, Burland J, et al. Pilot study of the effectiveness of the family-to-family education program. Psychiatr Serv. 2001 Jul;52(7):965-7. doi:

10.1176/appi.ps.52.7.965. PMID: 11433116. Study design not included in review.

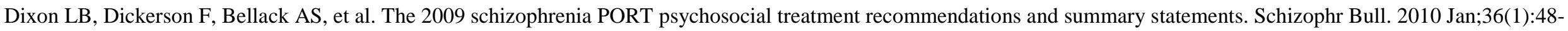

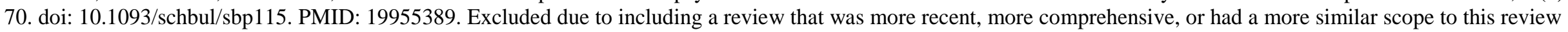
scope.

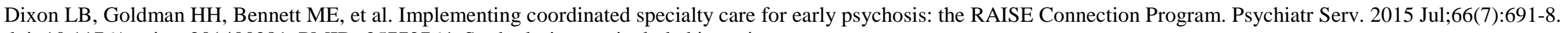
doi: 10.1176/appi.ps.201400281. PMID: 25772764. Study design not included in review.

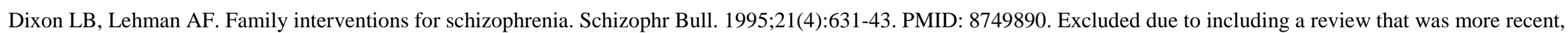
more comprehensive, or had a more similar scope to this review scope.

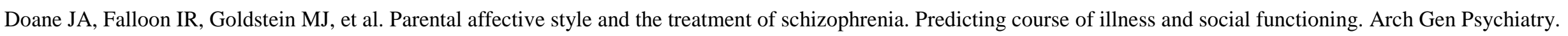
1985 Jan;42(1):34-42. PMID: 3966851. Study design not included in review.

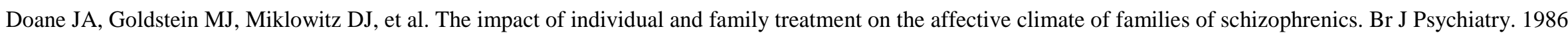
Mar;148:279-87. doi: 10.1192/bjp.148.3.279. PMID: 3719220. Outcome not included in review.

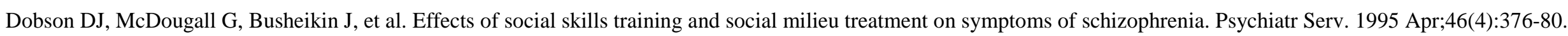
PMID: 7788460. Inadequate duration (<12 weeks).

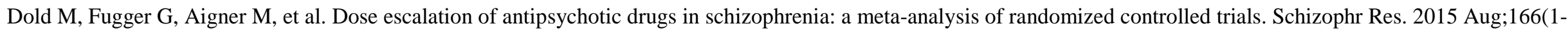
3):187-93. doi: 10.1016/j.schres.2015.04.024. PMID: 26008883. Study design not included in review.

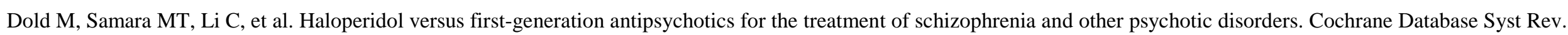
2015(1) PMID: 25592299. Excluded due to including a review that was more recent, more comprehensive, or had a more similar scope to this review scope. 


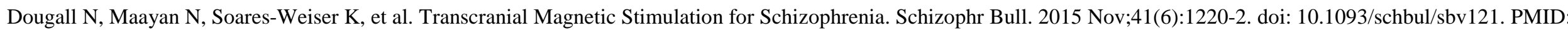
26392626. Excluded due to including a review that was more recent, more comprehensive, or had a more similar scope to this review scope.

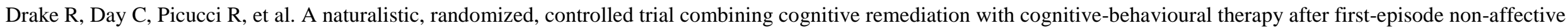
psychosis. Psychol Med. 2014 Jul;44(9):1889-99. doi: 10.1017/S0033291713002559. PMID: 24172842. Outcome not included in review.

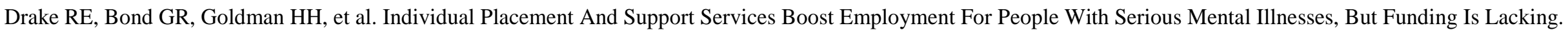
Health Aff (Millwood). 2016 Jun 01;35(6):1098-105. doi: 10.1377/hlthaff.2016.0001. PMID: 27269028. Not a study.

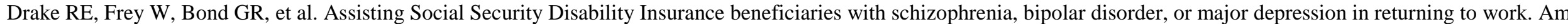
J Psychiatry. 2013 Dec;170(12):1433-41. doi: 10.1176/appi.ajp.2013.13020214. PMID: 23929355. Intervention not included in review.

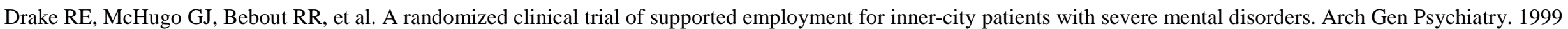
Jul;56(7):627-33. PMID: 10401508. Study covered in a systematic review.

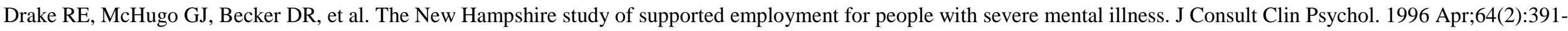
9. PMID: 8871423. Study covered in a systematic review.

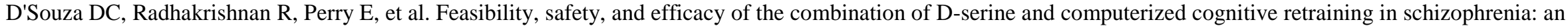

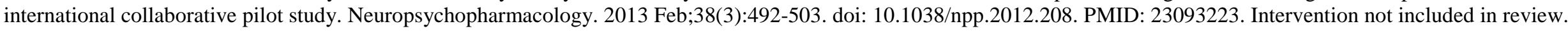

Duckworth K, Halpern L. Peer support and peer-led family support for persons living with schizophrenia. Curr Opin Psychiatry. 2014 May;27(3):216-21. doi: 10.1097/YCO.0000000000000051. PMID: 24662961. Not a study.

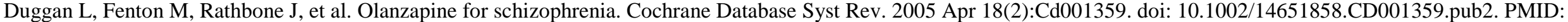
15846619. Excluded due to including a review that was more recent, more comprehensive, or had a more similar scope to this review scope.

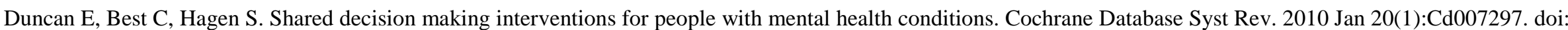
10.1002/14651858.CD007297.pub2. PMID: 20091628. Intervention not included in review.

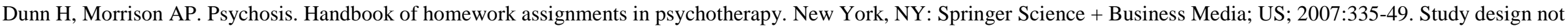
included in review.

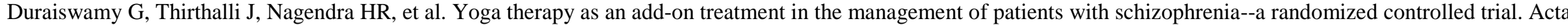
Psychiatr Scand. 2007 Sep;116(3):226-32. PMID: 17655565. Intervention not included in review.

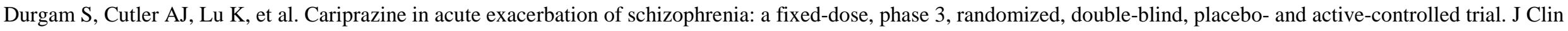
Psychiatry. 2015 Dec;76(12):e1574-82. doi: 10.4088/JCP.15m09997. PMID: 26717533. Inadequate duration (<12 weeks).

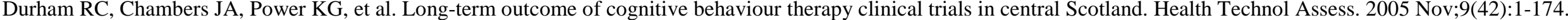
PMID: 16266559. Population ineligible for review.

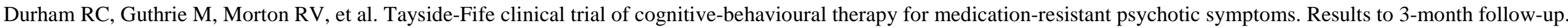
Br J Psychiatry. 2003 Apr;182:303-11. PMID: 12668405. Study covered in a systematic review.

Dyck DG, Hendryx MS, Short RA, et al. Service use among patients with schizophrenia in psychoeducational multiple-family group treatment. Psychiatr Serv. 2002 Jun;53(6):749-54. PMID: 12045314. Study covered in a systematic review.

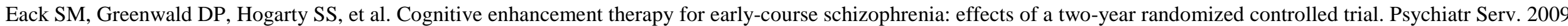
Nov;60(11):1468-76. doi: 10.1176/appi.ps.60.11.1468. PMID: 19880464. Study covered in a systematic review. 
Eack SM, Greenwald DP, Hogarty SS, et al. One-year durability of the effects of cognitive enhancement therapy on functional outcome in early schizophrenia. Schizophr Res. 2010;120(1-3):210-6. PMID: 20472402. Comparator not included in review.

Eack SM, Hogarty GE, Cho RY, et al. Neuroprotective effects of cognitive enhancement therapy against gray matter loss in early schizophrenia: results from a 2-year randomized controlled trial. Arch Gen Psychiatry. 2010 Jul;67(7):674-82. doi: 10.1001/archgenpsychiatry.2010.63. PMID: 20439824. Outcome not included in review.

Eack SM, Hogarty GE, Greenwald DP, et al. Cognitive enhancement therapy improves emotional intelligence in early course schizophrenia: preliminary effects. Schizophr Res. 2007 Jan;89(1-3):308-11. PMID: 17055227. Outcome not included in review.

Eack SM, Hogarty GE, Greenwald DP, et al. Effects of cognitive enhancement therapy on employment outcomes in early schizophrenia: results from a 2-year randomized trial. Res Soc Work Pract. 2011 Jan;21(1):32-42. doi: 10.1177/1049731509355812. PMID: 23885163. Study covered in a systematic review.

Eack SM, Hogarty SS, Greenwald DP, et al. Cognitive enhancement therapy in substance misusing schizophrenia: results of an 18-month feasibility trial. Schizophr Res. 2015 Feb;161(2-3):478-83. doi: 10.1016/j.schres.2014.11.017. PMID: 25510926. Sample size too small ( $<50)$.

Eack SM, Mesholam-Gately RI, Greenwald DP, et al. Negative symptom improvement during cognitive rehabilitation: results from a 2-year trial of cognitive enhancement therapy. Psychiatry Res. 2013 Aug 30;209(1):21-6. doi: 10.1016/j.psychres.2013.03.020. PMID: 23623449. Study covered in a systematic review.

Eack SM, Pogue-Geile MF, Greenwald DP, et al. Mechanisms of functional improvement in a 2-year trial of cognitive enhancement therapy for early schizophrenia. Psychol Med. 2011 Jun;41(6):1253-61. doi: 10.1017/S0033291710001765. PMID: 20860867. Outcome not included in review.

Echeburua E, Gomez M, Freixa M. Cognitive-behavioural treatment of pathological gambling in individuals with chronic schizophrenia: a pilot study. Behav Res Ther. 2011 Nov;49(11):808-14. doi: 10.1016/j.brat.2011.08.009. PMID: 21920501. Outcome not included in review.

Ecker J, Aubry T, Wasylenki D, et al. Predicting alcohol use and drug use among consumers of community mental health programs. J Dual Diagn. 2012 Jul;8(3):188-99. doi: 10.1080/15504263.2012.697861. Study design not included in review.

Eckman TA, Wirshing WC, Marder SR, et al. Technique for training schizophrenic patients in illness self-management: a controlled trial. Am J Psychiatry. 1992 Nov;149(11):1549-55. PMID: 1384364. Comparator not included in review.

Edwards CJ, Cella M, Tarrier N, et al. Investigating the empirical support for therapeutic targets proposed by the temporal experience of pleasure model in schizophrenia: a systematic review. Schizophr Res. 2015 Oct;168(1-2):120-44. doi: 10.1016/j.schres.2015.08.013. PMID: 26342966. Outcome not included in review.

El-Sayeh HG, Morganti C. Aripiprazole for schizophrenia. Cochrane Database Syst Rev. 2006 Apr 19(2):Cd004578. doi: 10.1002/14651858.CD004578.pub3. PMID: 16625607.

Excluded due to including a review that was more recent, more comprehensive, or had a more similar scope to this review scope.

Emmerson LC, Granholm E, Link PC, et al. Insight and treatment outcome with cognitive-behavioral social skills training for older people with schizophrenia. J Rehabil Res Dev. 2009;46(8):1053-8. PMID: 20157862. Not a study.

Engh JA, Andersen E, Holmen TL, et al. Effects of high-intensity aerobic exercise on psychotic symptoms and neurocognition in outpatients with schizophrenia: study protocol for a randomized controlled trial. Trials. 2015;16(1) PMID: 26646670. Intervention not included in review.

England M. Efficacy of cognitive nursing intervention for voice hearing. Perspect Psychiatr Care. 2007 Apr;43(2):69-76. PMID: 17388849. Study covered in a systematic review.

England M. Significance of cognitive intervention for voice hearers. Perspect Psychiatr Care. 2008 Jan;44(1):40-7. doi: 10.1111/j.1744-6163.2008.00146.x. PMID: 18177277.

Study covered in a systematic review.

Erawati E, Keliat BA, Helena N, et al. The influence of metacognitive training on delusion severity and metacognitive ability in schizophrenia. J Psychiatr Ment Health Nurs. 2014;21(9):841-7. doi: 10.1111/jpm.12130. PMID: 24548341. Intervention not included in review.

Essali A, Tarboush M, Awad M. Specialist interventions for homeless people with severe mental illness (Protocol). Cochrane Database Syst Rev. 2012 Dec 12(12):Cd010170. doi: 10.1002/14651858.CD010170. Not a study. 


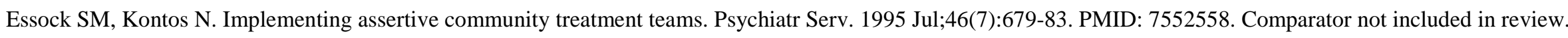

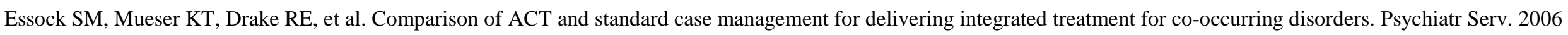
Feb;57(2):185-96. PMID: 16452695. Study covered in a systematic review.

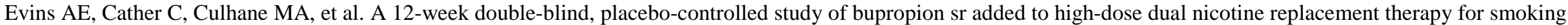
cessation or reduction in schizophrenia. J Clin Psychopharmacol. 2007 Aug;27(4):380-6. PMID: 17632223. Outcome not included in review.

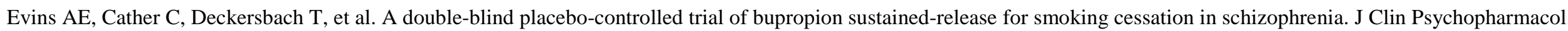
2005 Jun;25(3):218-25. PMID: 15876899. Outcome not included in review.

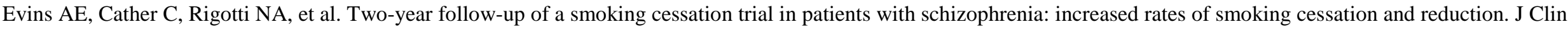
Psychiatry. 2004 Mar;65(3):307-11; quiz 452-3. PMID: 15096068. Intervention not included in review.

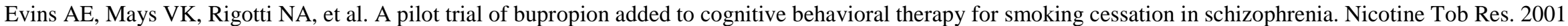
Nov;3(4):397-403. PMID: 11694208. Intervention not included in review.

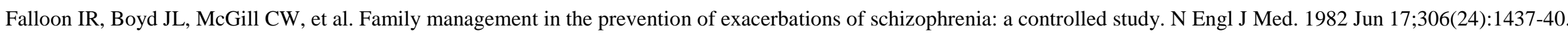
PMID: 6123079. Study covered in a systematic review.

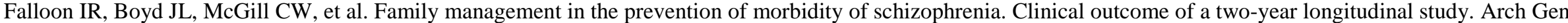
Psychiatry. 1985 Sep;42(9):887-96. PMID: 2864032. Study covered in a systematic review.

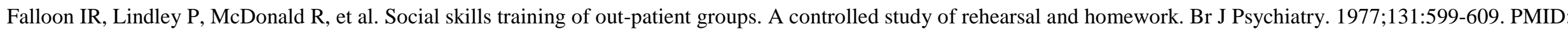
597688. Intervention not included in review.

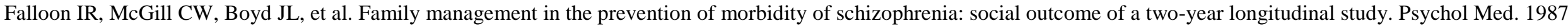
Feb;17(1):59-66. PMID: 3575578. Study covered in a systematic review.

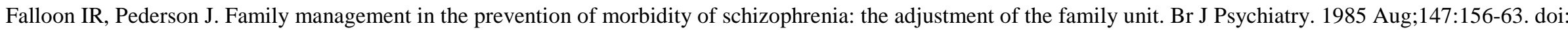
10.1192/bjp.147.2.156. PMID: 4041688. Study covered in a systematic review.

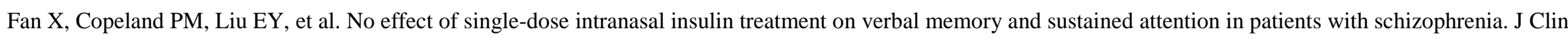
Psychopharmacol. 2011 Apr;31(2):231-4. PMID: 21346605. Intervention not included in review.

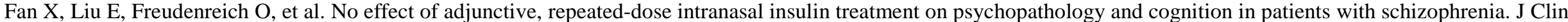
Psychopharmacol. 2013 Apr;33(2):226-30. PMID: 23422397. Intervention not included in review.

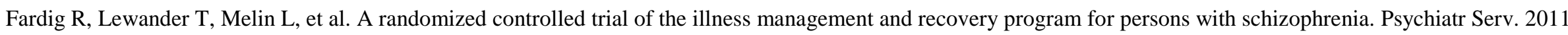
Jun;62(6):606-12. PMID: 21632728. Sample size too small $(<50)$.

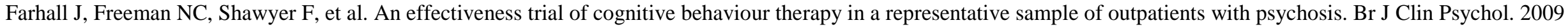
Mar;48(Pt 1):47-62. doi: 10.1348/014466608x360727. PMID: 18851771. Study covered in a systematic review.

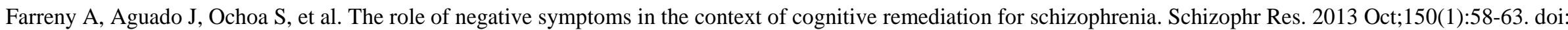
10.1016/j.schres.2013.08.008. PMID: 23993864. Outcome not included in review.

Faulkner G, Cohn T, Remington G. Interventions to reduce weight gain in schizophrenia. Cochrane Database Syst Rev. 2007 Jan 24(1):Cd005148. doi: 10.1002/14651858.CD005148.pub2. PMID: 17253540. Intervention not included in review.

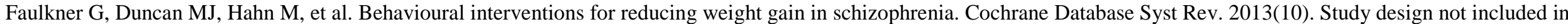
review. 
Favrod J, Rexhaj S, Bardy S, et al. Sustained antipsychotic effect of metacognitive training in psychosis: a randomized-controlled study. Eur Psychiatry. 2014 Jun;29(5):275-81. doi: 10.1016/j.eurpsy.2013.08.003. PMID: 24176646. Outcome not included in review.

Feldmann R, Hornung W, Prein B, et al. Timing of psychoeducational psychotherapeutic interventions in schizophrenic patients. Eur Arch Psychiatry Clin Neurosci. 2002;252(3):115-9. doi: 10.1007/s00406-002-0369-2. PMID: 12192468. Outcome not included in review.

Feldmann R, Hornung WP, Buchkremer G, et al. The influence of familial loading on the course of schizophrenic symptoms and the success of psychoeducational therapy. Psychopathology. 2001 Jul-Aug;34(4):192-7. PMID: 11549929. Not a study.

Fenton FR, Tessier L, Struening EL. A comparative trial of home and hospital psychiatric care. One-year follow-up. Arch Gen Psychiatry. 1979 Sep;36(10):1073-9. PMID: 475542. Comparator not included in review.

Fenton FR, Tessier L, Struening EL, et al. A two-year follow-up of a comparative trial of the cost-effectiveness of home and hospital psychiatric treatment. Can J Psychiatry. 1984 Apr;29(3):205-11. PMID: 6442211. Outcome not included in review.

Fenton WS. Evolving perspectives on individual psychotherapy for schizophrenia. Schizophr Bull. 2000;26(1):47-72. PMID: 10755669. Not a study.

Fenton WS, Dickerson F, Boronow J, et al. A placebo-controlled trial of omega-3 fatty acid (ethyl eicosapentaenoic acid) supplementation for residual symptoms and cognitive impairment in schizophrenia. Am J Psychiatry. 2001 Dec;158(12):2071-4. PMID: 11729030. Intervention not included in review.

Fernandes BS, Massuda R, Torres M, et al. Improvement of schizophrenia with electroconvulsive therapy and serum brain-derived neurotrophic factor levels: lack of association in a pilot study. Psychiatry Clin Neurosci. 2010 Dec;64(6):663-5. doi: 10.1111/j.1440-1819.2010.02153.x. PMID: 21105955. Intervention not included in review.

Fernández ÓV, Giráldez SL, Sáiz AG, et al. Integrated psychological treatment for schizophrenic patients. Psicothema. 1998;10(2):459-74. Intervention not included in review.

Fernandez-Gonzalo S, Turon M, Jodar M, et al. A new computerized cognitive and social cognition training specifically designed for patients with schizophrenia/schizoaffective disorder in early stages of illness: a pilot study. Psychiatry Res. 2015;228(3):501-9. PMID: 26163731. Sample size too small (<50).

Ferreira CD, de Souza MGD, Fernandez-Calvo B, et al. Neurocognitive functions in schizophrenia: a systematic review of the effects of typical and atypical antipsychotic drugs. Psychol Neurosci. 2016 Mar;9(1):12-31. doi: 10.1037/pne0000045. Outcome not included in review.

Fervaha G, Agid O, Takeuchi H, et al. Life satisfaction among individuals with schizophrenia in the Clinical Antipsychotic Trial of Intervention Effectiveness (CATIE) study. Am J Psychiatry. 2013 Sep;170(9):1061-2. doi: 10.1176/appi.ajp.2013.13010060. PMID: 24030617. Not a study.

Fervaha G, Agid O, Takeuchi H, et al. Clinical determinants of life satisfaction in chronic schizophrenia: data from the CATIE study. Schizophr Res. 2013 Dec;151(1-3):203-8. PMID: 24183751. Outcome not included in review.

Field CD, Galletly C, Anderson D, et al. Computer-aided cognitive rehabilitation: possible application to the attentional deficit of schizophrenia, a report of negative results. Percept Mot Skills. 1997 Dec;85(3 Pt 1):995-1002. PMID: 9399309. Inadequate duration ( $<12$ weeks).

Fiorillo A, Bassi M, de Girolamo G, et al. The impact of a psychoeducational intervention on family members' views about schizophrenia: results from the OASIS Italian multicentre study. Int J Soc Psychiatry. 2011 Nov;57(6):596-603. doi: 10.1177/0020764010376607. PMID: 20650976. Outcome not included in review.

Firth J, Cotter J, Elliott R, et al. A systematic review and meta-analysis of exercise interventions in schizophrenia patients. Psychol Med. 2015 May;45(7):1343-61. doi: 10.1017/S0033291714003110. PMID: 25650668. Intervention not included in review.

Fisher M, Holland C, Merzenich MM, et al. Using neuroplasticity-based auditory training to improve verbal memory in schizophrenia. Am J Psychiatry. 2009 Jul;166(7):805-11. PMID: 19448187. Outcome not included in review.

Fisher M, Holland C, Subramaniam K, et al. Neuroplasticity-based cognitive training in schizophrenia: an interim report on the effects 6 months later. Schizophr Bull. 2010 Jul;36(4):869-79. PMID: 19269924. Study covered in a systematic review. 
Fisher M, Loewy R, Carter C, et al. Neuroplasticity-based auditory training via laptop computer improves cognition in young individuals with recent onset schizophrenia. Schizophr Bull. 2015 Jan;41(1):250-8. doi: 10.1093/schbul/sbt232. PMID: 24444862. Inadequate duration (<12 weeks).

Fisher M, Mellon SH, Wolkowitz O, et al. Neuroscience-informed auditory training in schizophrenia: a final report of the effects on cognition and serum brain-derived neurotrophic factor. Schizophr Res Cogn. 2016;3:1-7. PMID: 26705516. Outcome not included in review.

Fiszdon JM, Bryson GJ, Wexler BE, et al. Durability of cognitive remediation training in schizophrenia: performance on two memory tasks at 6-month and 12-month follow-up. Psychiatry Res. 2004 Jan 30;125(1):1-7. PMID: 14967547. Study covered in a systematic review.

Fiszdon JM, Choi J, Bryson GJ, et al. Impact of intellectual status on response to cognitive task training in patients with schizophrenia. Schizophr Res. 2006 Oct;87(1-3):261-9. PMID: 16737798. Study design not included in review.

Fiszdon JM, Choi J, Goulet J, et al. Temporal relationship between change in cognition and change in functioning in schizophrenia. Schizophr Res. 2008 Oct;105(1-3):105-13. doi: 10.1016/j.schres.2008.06.010. PMID: 18657398. Study design not included in review.

Fiszdon JM, Johannesen JK. Comparison of computational methods for the evaluation of learning potential in schizophrenia. J Int Neuropsychol Soc. 2010 Jul;16(4):613-20. doi: 10.1017/S1355617710000317. PMID: 20374673. Study design not included in review.

Fiszdon JM, Kurtz MM, Choi J, et al. Motivational interviewing to increase cognitive rehabilitation adherence in schizophrenia. Schizophr Bull. 2016 Mar;42(2):327-34. doi: 10.1093/schbul/sbv143. PMID: 26420905. Outcome not included in review.

Fiszdon JM, Whelahan H, Bryson GJ, et al. Cognitive training of verbal memory using a dichotic listening paradigm: impact on symptoms and cognition. Acta Psychiatr Scand. 2005 Sep;112(3):187-93. PMID: 16095473. Study covered in a systematic review.

Fitzgerald PB, Benitez J, Daskalakis JZ, et al. A double-blind sham-controlled trial of repetitive transcranial magnetic stimulation in the treatment of refractory auditory hallucinations. J Clin Psychopharmacol. 2005 Aug;25(4):358-62. PMID: 16012279. Intervention not included in review.

Fitzgerald PB, Herring S, Hoy K, et al. A study of the effectiveness of bilateral transcranial magnetic stimulation in the treatment of the negative symptoms of schizophrenia. Brain Stimul. 2008 Jan;1(1):27-32. doi: 10.1016/j.brs.2007.08.001. PMID: 20633367. Intervention not included in review.

Fitzgerald PB, McQueen S, Daskalakis ZJ, et al. A negative pilot study of daily bimodal transcranial direct current stimulation in schizophrenia. Brain Stimul. 2014 NovDec;7(6):813-6. doi: 10.1016/j.brs.2014.08.002. PMID: 25442152. Intervention not included in review.

Ford R, Beadsmoore A, Ryan P, et al. Providing the safety net: case management for people with a serious mental illness. J Ment Health. 1995. Study covered in a systematic review.

Ford R, Ryan P, Beadsmoore A, et al. Intensive case management for people with serious mental illness- Site 2: Clinical and social outcome. J Ment Health. 1997 1997/01/01;6(2):181-90. doi: 10.1080/09638239718941. Study covered in a systematic review.

Forsberg KA, Bjorkman T, Sandman PO, et al. Physical health--a cluster randomized controlled lifestyle intervention among persons with a psychiatric disability and their staff. Nord J Psychiatry. 2008;62(6):486-95. doi: 10.1080/08039480801985179. PMID: 18843564. Intervention not included in review.

Foruzandeh N, Parvin N. Occupational therapy for inpatients with chronic schizophrenia: a pilot randomized controlled trial. Jpn J Nurs Sci. 2013 Jun;10(1):136-41. doi: 10.1111/j.1742-7924.2012.00211.x. PMID: 23735098. Setting ineligible for review (i.e., inpatient only).

Fowler D, Hodgekins J, Painter M, et al. Cognitive behaviour therapy for improving social recovery in psychosis: a report from the ISREP MRC Trial Platform Study (Improving Social Recovery in Early Psychosis). Psychol Med. 2009 Oct;39(10):1627-36. doi: 10.1017/s0033291709005467. PMID: 19335932. Outcome not included in review.

Foxx RM, McMorrow MJ, Bittle RG, et al. Teaching social skills to psychiatric inpatients. Behav Res Ther. 1985;23(5):531-7. PMID: 4051925. Setting ineligible for review (i.e., inpatient only). 
Franck N, Duboc C, Sundby C, et al. Specific vs general cognitive remediation for executive functioning in schizophrenia: a multicenter randomized trial. Schizophr Res. 2013 Jun;147(1):68-74. doi: 10.1016/j.schres.2013.03.009. PMID: 23583327. Comparator not included in review.

Fredrick MM, Mintz J, Roberts DL, et al. Is cognitive adaptation training (CAT) compensatory, restorative, or both? Schizophr Res. 2015 Aug;166(1-3):290-6. doi: 10.1016/j.schres.2015.06.003. PMID: 26126419. Outcome not included in review.

Freeman D, Dunn G, Startup H, et al. The effects of reducing worry in patients with persecutory delusions: study protocol for a randomized controlled trial. Trials 2012;13:223. doi: 10.1186/1745-6215-13-223. PMID: 23171601. Not a study.

Freeman D, Garety P, Fowler D, et al. The London-East Anglia randomized controlled trial of cognitive-behaviour therapy for psychosis. IV: self-esteem and persecutory delusions. Br J Clin Psychol. 1998 Nov;37(Pt 4):415-30. PMID: 9856295. Study design not included in review.

Freeman D, Pugh K, Dunn G, et al. An early Phase II randomised controlled trial testing the effect on persecutory delusions of using CBT to reduce negative cognitions about the self: the potential benefits of enhancing self confidence. Schizophr Res. 2014 Dec;160(1-3):186-92. doi: 10.1016/j.schres.2014.10.038. PMID: 25468186. Outcome not included in review.

Freeman D, Startup H, Myers E, et al. The effects of using cognitive behavioural therapy to improve sleep for patients with delusions and hallucinations (the BEST study): study protocol for a randomized controlled trial. Trials 2013;14:214. doi: 10.1186/1745-6215-14-214. PMID: 23845104. Not a study.

Freeman D, Waite F, Startup H, et al. Efficacy of cognitive behavioural therapy for sleep improvement in patients with persistent delusions and hallucinations (BEST): a prospective, assessor-blind, randomised controlled pilot trial. Lancet Psychiatry. 2015;2(11):975-83. PMID: 26363701. Sample size too small (<50).

Freitas C, Fregni F, Pascual-Leone A. Meta-analysis of the effects of repetitive transcranial magnetic stimulation (rTMS) on negative and positive symptoms in schizophrenia. Schizophr Res. 2009 Mar;108(1-3):11-24. doi: 10.1016/j.schres.2008.11.027. PMID: 19138833. Intervention not included in review.

Fritze J, Forthner B, Schmitt B, et al. Cognitive training adjunctive to pharmacotherapy in schizophrenia and depression: a pilot study on the lateralization hypothesis of schizophrenia and depression and on cognitive therapy as adjunctive to pharmacotherapy. Neuropsychobiology. 1988;19(1):45-50. PMID: 3185895. Outcome not included in review.

Fung KM, Tsang HW, Cheung WM. Randomized controlled trial of the self-stigma reduction program among individuals with schizophrenia. Psychiatry Res. 2011 Sep 30;189(2):208-14. doi: 10.1016/j.psychres.2011.02.013. PMID: 21377738. Interventions evaluated in a single study.

Fusar-Poli P, Kempton MJ, Rosenheck RA. Efficacy and safety of second-generation long-acting injections in schizophrenia: a meta-analysis of randomized-controlled trials. Int Clin Psychopharmacol. 2013 Mar;28(2):57-66. doi: 10.1097/YIC.0b013e32835b091f. PMID: 23165366. Excluded due to including a review that was more recent, more comprehensive, or had a more similar scope to this review scope.

Fusar-Poli P, Papanastasiou E, Stahl D, et al. Treatments of negative symptoms in schizophrenia: meta-analysis of 168 randomized placebo-controlled trials. Schizophr Bull. 2015 Jul;41(4):892-9. doi: 10.1093/schbul/sbu170. PMID: 25528757. Comparator not included in review.

Gaebel W, Riesbeck M, Wolwer W, et al. Rates and predictors of remission in first-episode schizophrenia within 1year of antipsychotic maintenance treatment. Results of a randomized controlled trial within the German research network on schizophrenia. Schizophr Res. 2014;152(2-3):478-86. PMID: 23643327. Outcome not included in review.

Gaebel W, Schreiner A, Bergmans P, et al. Relapse prevention in schizophrenia and schizoaffective disorder with risperidone long-acting injectable vs quetiapine: results of a longterm, open-label, randomized clinical trial. Neuropsychopharmacology. 2010 Nov;35(12):2367-77. doi: 10.1038/npp.2010.111. PMID: 20686456. Study covered in a systematic review.

Galderisi S, Mucci A, Bitter I, et al. Persistent negative symptoms in first episode patients with schizophrenia: results from the European First Episode Schizophrenia Trial. Eur Neuropsychopharmacol. 2013 Mar;23(3):196-204. doi: 10.1016/j.euroneuro.2012.04.019. PMID: 22647933. Study design not included in review.

Galderisi S, Piegari G, Mucci A, et al. Social skills and neurocognitive individualized training in schizophrenia: comparison with structured leisure activities. Eur Arch Psychiatry Clin Neurosci. 2010 Jun;260(4):305-15. doi: 10.1007/s00406-009-0078-1. PMID: 19826855. Comparator not included in review. 
Gallagher AG, Dinan TG, Baker LV. The effects of varying information content and speaking aloud on auditory hallucinations. Br J Med Psychol. 1995 Jun;68(Pt 2):143-55. PMID: 7547611. Study design not included in review.

Garcia S, Fuentes I, Ruiz JC, et al. Application of the IPT in a Spanish sample: evaluation of the "social perception subprogramme". Intern Jour Psych Psychol Ther. 2003 Dec;3(2):299-310. Study design not included in review.

Garety P, Kuipers L, Fowler D, et al. Cognitive behavioural therapy for drug-resistant psychosis. Br J Med Psychol. 1994 Sep;67(3):259-71. doi: 10.1111/j.20448341.1994.tb01795.x. PMID: 7803318. Study design not included in review.

Garrido G, Barrios M, Penades R, et al. Computer-assisted cognitive remediation therapy: cognition, self-esteem and quality of life in schizophrenia. Schizophr Res. 2013 Nov;150(2-3):563-9. doi: 10.1016/j.schres.2013.08.025. PMID: 24035402. Comparator not included in review.

Gater R, Goldberg D, Jackson G, et al. The care of patients with chronic schizophrenia: a comparison between two services. Psychol Med. 1997 Nov;27(6):1325-36. PMID: 9403904. Outcome not included in review.

Gaudiano BA. Is symptomatic improvement in clinical trials of cognitive-behavioral therapy for psychosis clinically significant? J Psychiatr Pract. 2006 Jan;12(1):11-23. PMID: 16432441. Excluded due to including a review that was more recent, more comprehensive, or had a more similar scope to this review scope.

Gaughran F, Stahl D, Ismail K, et al. Improving physical health and reducing substance use in psychosis - randomised control trial (IMPACT RCT): study protocol for a cluster randomised controlled trial. BMC Psychiatry. 2013;13 PMID: 24131496. Not a study.

Gaynes BN, Brown C, Lux LJ, et al. Management Strategies To Reduce Psychiatric Readmissions. Rockville (MD): Agency for Healthcare Research and Quality (US); 2015. Intervention not included in review.

Gearing RE, Alonzo D, Marinelli C. Maternal schizophrenia: psychosocial treatment for mothers and their children. Clin Schizophr Relat Psychoses. 2012 Apr;6(1):27-33. doi: 10.3371/CSRP.6.1.4. PMID: 22453867. Outcome not included in review.

Gelkopf M, Gonen B, Kurs R, et al. The effect of humorous movies on inpatients with chronic schizophrenia. J Nerv Ment Dis. 2006 Nov;194(11):880-3. PMID: 17102716. Setting ineligible for review (i.e., inpatient only).

Gelkopf M, Noam S, Rudinski D, et al. Nonmedication smoking reduction program for inpatients with chronic schizophrenia: a randomized control design study. J Nerv Ment Dis. 2012 Feb;200(2):142-6. doi: 10.1097/NMD.0b013e3182438e92. PMID: 22297311. Intervention not included in review.

Gentile S. Adverse effects associated with second-generation antipsychotic long-acting injection treatment: a comprehensive systematic review. Pharmacotherapy. 2013 Oct;33(10):1087-106. doi: 10.1002/phar.1313. PMID: 23776129. Excluded due to including a review that was more recent, more comprehensive, or had a more similar scope to this review scope.

George TP, Ziedonis DM, Feingold A, et al. Nicotine transdermal patch and atypical antipsychotic medications for smoking cessation in schizophrenia. Am J Psychiatry. 2000 Nov;157(11):1835-42. PMID: 11058482. Intervention not included in review.

Georgiev A, Probst M, De Hert M, et al. Acute effects of progressive muscle relaxation on state anxiety and subjective well-being in chronic Bulgarian patients with schizophrenia. Psychiatr Danub. 2012 Dec;24(4):367-72. PMID: 23132187. Inadequate duration ( $<12$ weeks).

Ghadiri Vasfi M, Moradi-Lakeh M, Esmaeili N, et al. Efficacy of aftercare services for people with severe mental disorders in Iran: a randomized controlled trial. Psychiatr Serv. 2015 Apr 1;66(4):373-80. doi: 10.1176/appi.ps.201400111. PMID: 25828982. Intervention not included in review.

Gharaeipour M, Scott BJ. Effects of cognitive remediation on neurocognitive functions and psychiatric symptoms in schizophrenia inpatients. Schizophr Res. 2012 Dec;142(13):165-70. doi: 10.1016/j.schres.2012.09.018. PMID: 23102691. Setting ineligible for review (i.e., inpatient only).

Gibson CM, Penn DL, Smedley KL, et al. A pilot six-week randomized controlled trial of oxytocin on social cognition and social skills in schizophrenia. Schizophr Res. 2014 Jul;156(2-3):261-5. PMID: 24799299. Intervention not included in review. 
Gil Sanz D, Diego Lorenzo M, Bengochea Seco R, et al. Efficacy of a social cognition training program for schizophrenic patients: a pilot study. Span J Psychol. 2009 May;12(1):184-91. PMID: 19476231. Study design not included in review.

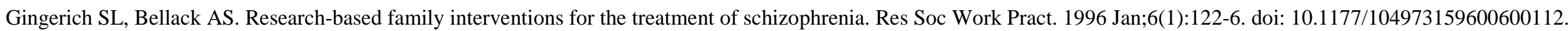
Not a study.

Girgis RR, Merrill DB, Vorel SR, et al. Aripiprazole versus haloperidol treatment in early-stage schizophrenia. J Psychiatr Res. 2011 Jun;45(6):756-62. doi: 10.1016/j.jpsychires.2010.09.003. PMID: 20937506. Study covered in a systematic review.

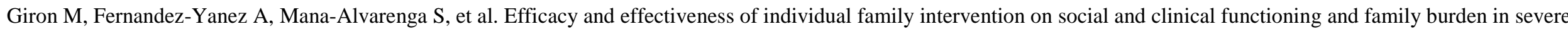

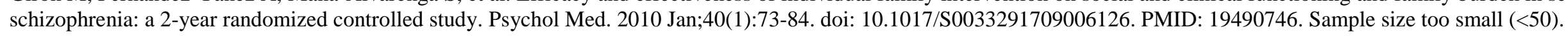

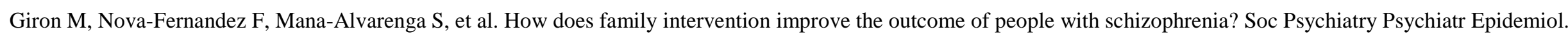
2015 Mar;50(3):379-87. doi: 10.1007/s00127-014-0942-9. PMID: 25087012. Outcome not included in review.

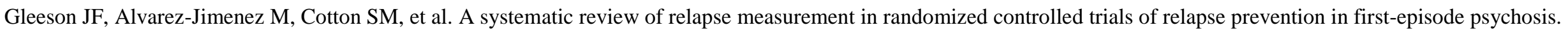

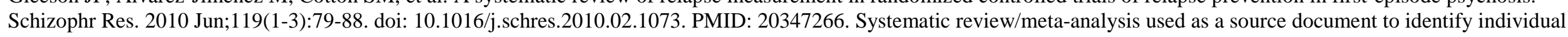
studies.

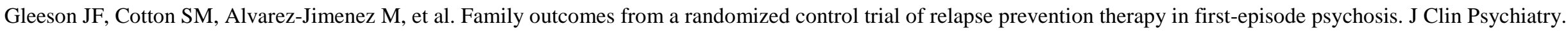
2010 Apr;71(4):475-83. doi: 10.4088/JCP.08m04672yel. PMID: 20021994. Intervention not included in review.

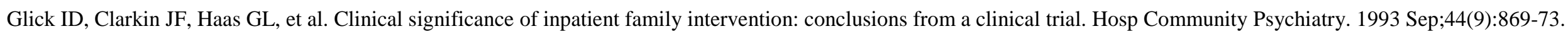
PMID: 8225301. Setting ineligible for review (i.e., inpatient only).

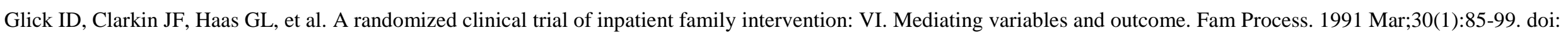
10.1111/j.1545-5300.1991.00085.x. PMID: 2044754. Setting ineligible for review (i.e., inpatient only).

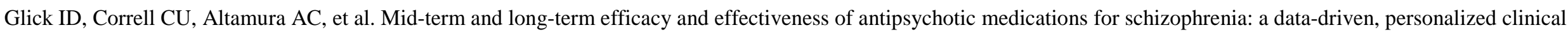
approach. J Clin Psychiatry. 2011 Dec;72(12):1616-27. doi: 10.4088/JCP.11r06927. PMID: 22244023. Excluded due to including a review that was more recent, more comprehensive, or had a more similar scope to this review scope.

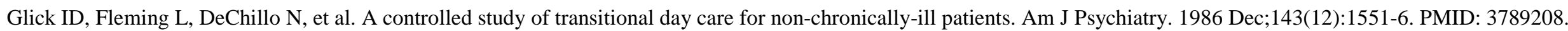
Intervention not included in review.

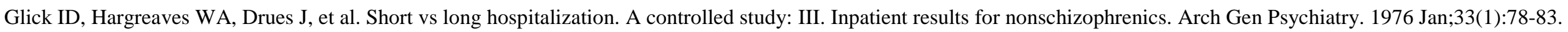
PMID: 813604. Setting ineligible for review (i.e., inpatient only).

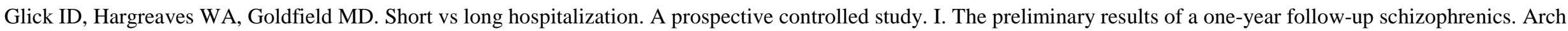
Gen Psychiatry. 1974 Mar;30(3):363-9. PMID: 4813139. Intervention not included in review.

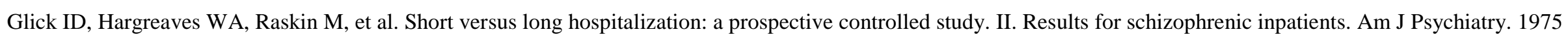
Apr;132(4):385-90. PMID: 1119589. Intervention not included in review.

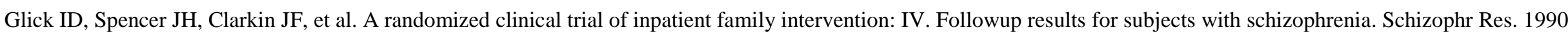
May-Jun;3(3):187-200. doi: 10.1016/0920-9964\%2890\%2990036-7. PMID: 2278982. Setting ineligible for review (i.e., inpatient only).

Glynn SM, Cohen AN, Niv N. New challenges in family interventions for schizophrenia. Expert Rev Neurother. 2007 Jan;7(1):33-43. PMID: 17187495. Not a study.

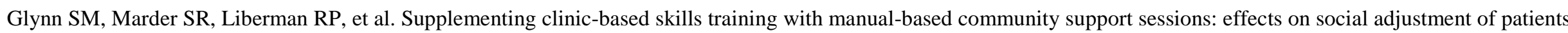
with schizophrenia. Am J Psychiatry. 2002 May;159(5):829-37. PMID: 11986138. Comparator not included in review. 
Gohar SM, Hamdi E, El Ray LA, et al. Adapting and evaluating a social cognitive remediation program for schizophrenia in Arabic. Schizophr Res. 2013 Aug;148(1-3):12-7. doi: 10.1016/j.schres.2013.05.008. PMID: 23756297. Country not eligible.

Gold C, Heldal TO, Dahle T, et al. Music therapy for schizophrenia or schizophrenia-like illnesses. Cochrane Database Syst Rev. 2005 Apr 18(2):Cd004025. doi: 10.1002/14651858.CD004025.pub2. PMID: 15846692. Intervention not included in review.

Gold PB, Meisler N, Santos AB, et al. Randomized trial of supported employment integrated with assertive community treatment for rural adults with severe mental illness. Schizophr Bull. 2006 Apr;32(2):378-95. PMID: 16177278. Study covered in a systematic review.

Goldberg D. Cost-effectiveness in the treatment of patients with schizophrenia. Acta Psychiatr Scand. 1994;89(382, Suppl):89-92. doi: 10.1111/j.1600-0447.1994.tb05873.x. PMID: 8092005. Outcome not included in review.

Goldberg SC, Schooler NR, Hogarty GE, et al. Prediction of relapse in schizophrenic outpatients treated by drug and sociotherapy. Arch Gen Psychiatry. 1977 Feb;34(2):171-84. PMID: 843177. Study design not included in review.

Goldsmith LP, Lewis SW, Dunn G, et al. Psychological treatments for early psychosis can be beneficial or harmful, depending on the therapeutic alliance: an instrumental variable analysis. Psychol Med. 2015 Aug;45(11):2365-73. doi: 10.1017/S003329171500032X. PMID: 25805118. Study design not included in review.

Goldstein MJ. Psychosocial treatment of schizophrenia. Schizophrenia: Scientific progress. New York, NY: Oxford University Press; US; 1989:318-24. Not a study.

Goldstein MJ, Rodnick EH, Evans JR, et al. Drug and family therapy in the aftercare of acute schizophrenics. Arch Gen Psychiatry. 1978 Oct;35(10):1169-77. doi: 10.1001/archpsyc.1978.01770340019001. PMID: 211983. Study covered in a systematic review.

Gomar JJ, Valls E, Radua J, et al. A multisite, randomized controlled clinical trial of computerized cognitive remediation therapy for schizophrenia. Schizophr Bull. 2015 Nov;41(6):1387-96. doi: 10.1093/schbul/sbv059. PMID: 26006264. Outcome not included in review.

Gong W, Xu D, Zhou L, et al. Village doctor-assisted case management of rural patients with schizophrenia: protocol for a cluster randomized control trial. Implement Sci. 2014;9:13. doi: 10.1186/1748-5908-9-13. PMID: 24433461. Not a study.

Gorczynski P, Faulkner G. Exercise therapy for schizophrenia. Cochrane Database Syst Rev. 2010 May 12(5):Cd004412. doi: 10.1002/14651858.CD004412.pub2. PMID: 20464730. Intervention not included in review.

Gottlieb JD, Cather C, Shanahan M, et al. D-cycloserine facilitation of cognitive behavioral therapy for delusions in schizophrenia. Schizophr Res. 2011 Sep;131(1-3):69-74. doi: 10.1016/j.schres.2011.05.029. PMID: 21723096. Intervention not included in review.

Gould RA, Mueser KT, Bolton E, et al. Cognitive therapy for psychosis in schizophrenia: an effect size analysis. Schizophrenia Research. 2001 Mar 30;48(2-3):335-42. PMID: 11295385. Excluded due to including a review that was more recent, more comprehensive, or had a more similar scope to this review scope.

Goyal N, Nizamie SH, Desarkar P. Efficacy of adjuvant high frequency repetitive transcranial magnetic stimulation on negative and positive symptoms of schizophrenia: preliminary results of a double-blind sham-controlled study. J Neuropsychiatry Clin Neurosci. 2007;19(4):464-7. PMID: 18070852. Intervention not included in review.

Graeber DA, Moyers TB, Griffith G, et al. A pilot study comparing motivational interviewing and an educational intervention in patients with schizophrenia and alcohol use disorders. Community Ment Health J. 2003 Jun;39(3):189-202. PMID: 12836801. Study covered in a systematic review.

Graham HL, Copello A, Griffith E, et al. Pilot randomised trial of a brief intervention for comorbid substance misuse in psychiatric in-patient settings. Acta Psychiatr Scand. 2016;133(4):298-309. PMID: 26590876. Setting ineligible for review (i.e., inpatient only).

Granholm E, Ben-Zeev D, Link PC. Social disinterest attitudes and group cognitive-behavioral social skills training for functional disability in schizophrenia. Schizophr Bull. 2009 Sep;35(5):874-83. doi: 10.1093/schbul/sbp072. PMID: 19628761. Comparator not included in review.

Granholm E, Holden J, Link PC, et al. Randomized clinical trial of cognitive behavioral social skills training for schizophrenia: improvement in functioning and experiential negative symptoms. J Consult Clin Psychol. 2014 Dec;82(6):1173-85. doi: 10.1037/a0037098. PMID: 24911420. Comparator not included in review. 
Granholm E, Holden J, Link PC, et al. Randomized controlled trial of cognitive behavioral social skills training for older consumers with schizophrenia: defeatist performance attitudes and functional outcome. Am J Geriatr Psychiatry. 2013 Mar;21(3):251-62. doi: 10.1016/j.jagp.2012.10.014. PMID: 23395192. Comparator not included in review.

Granholm E, Holden JL, Sommerfeld D, et al. Enhancing assertive community treatment with cognitive behavioral social skills training for schizophrenia: study protocol for a randomized controlled trial. Trials. 2015;16(1):438. PMID: 26424639. Not a study.

Granholm E, McQuaid JR, Auslander LA, et al. Group cognitive-behavioral social skills training for older outpatients with chronic schizophrenia. J Cogn Psychother. 2004 Fal;18(3):265-79. doi: 10.1891/jcop.18.3.265.65652. Study design not included in review.

Granholm E, McQuaid JR, Link PC, et al. Neuropsychological predictors of functional outcome in cognitive behavioral social skills training for older people with schizophrenia. Schizophr Res. 2008 Mar;100(1-3):133-43. doi: 10.1016/j.schres.2007.11.032. PMID: 18222648. Outcome not included in review.

Granholm E, McQuaid JR, McClure FS, et al. Randomized controlled trial of cognitive behavioral social skills training for older people with schizophrenia: 12-month follow-up. J Clin Psychiatry. 2007 May;68(5):730-7. PMID: 17503982. Study covered in a systematic review.

Granholm E, McQuaid JR, McClure FS, et al. A randomized controlled pilot study of cognitive behavioral social skills training for older patients with schizophrenia. Schizophr Res. 2002 Jan 1;53(1-2):167-9. PMID: 11728848. Not a study.

Grawe RW, Falloon IR, Widen JH, et al. Two years of continued early treatment for recent-onset schizophrenia: a randomised controlled study. Acta Psychiatr Scand. 2006 Nov;114(5):328-36. PMID: 17022792. Study covered in a systematic review.

Gray R, Leese M, Bindman J, et al. Adherence therapy for people with schizophrenia. European multicentre randomised controlled trial. Br J Psychiatry. 2006;189:508-14. PMID: 17139034. Intervention not included in review.

Greenwood K, Hung CF, Tropeano M, et al. No association between the Catechol-O-Methyltransferase (COMT) val158met polymorphism and cognitive improvement following cognitive remediation therapy (CRT) in schizophrenia.[Erratum appears in Neurosci Lett. 2011 Jul 15;499(1):57]. Neurosci Lett. 2011 Jun 1;496(2):65-9. doi:

10.1016/j.neulet.2011.03.075. PMID: 21458532. Study design not included in review.

Greig TC, Zito W, Wexler BE, et al. Improved cognitive function in schizophrenia after one year of cognitive training and vocational services. Schizophr Res. 2007 Nov;96(13):156-61. PMID: 17669629. Outcome not included in review.

Grinspoon L, Ewalt JR, Shader R. Long-term treatment of chronic schizophrenia. A preliminary report. Int J Psychiatry. 1967 Aug;4(2):116-28. PMID: 4862724. Study design not included in review.

Gromer J. Need-adapted and open-dialogue treatments: empirically supported psychosocial interventions for schizophrenia and other psychotic disorders. Ethical Hum Psychol Psychiatry. 2012;14(3):162-77. doi: 10.1891/1559-4343.14.3.162. Not a study.

Grunder G, Heinze M, Cordes J, et al. Effects of first-generation antipsychotics versus second-generation antipsychotics on quality of life in schizophrenia: A double-blind, randomised study. Lancet Psychiatry. 2016 Aug;3(8):717-29. doi: 10.1016/S2215-0366\%2816\%2900085-7. PMID: 27265548. Study design not included in review.

Grynszpan O, Perbal S, Pelissolo A, et al. Efficacy and specificity of computer-assisted cognitive remediation in schizophrenia: a meta-analytical study. Psychol Med. 2011 Jan;41(1):163-73. doi: 10.1017/S0033291710000607. PMID: 20380784. Excluded due to including a review that was more recent, more comprehensive, or had a more similar scope to this review scope.

Guastella AJ, Ward PB, Hickie IB, et al. A single dose of oxytocin nasal spray improves higher-order social cognition in schizophrenia. Schizophr Res. 2015;168(3):628-33. PMID: 26150070. Intervention not included in review.

Gudeman JE, Dickey B, Rood L, et al. Alternative to the back ward: the quarterway house. Hosp Community Psychiatry. 1981 May;32(5):330-4. PMID: 7239459. Intervention not included in review.

Gumley AI, MacBeth A, Reilly JD, et al. Fear of recurrence: results of a randomized trial of relapse detection in schizophrenia. Br J Clin Psychol. 2015 Mar;54(1):49-62. doi: 10.1111/bjc.12060. PMID: 25040487. Intervention not included in review. 


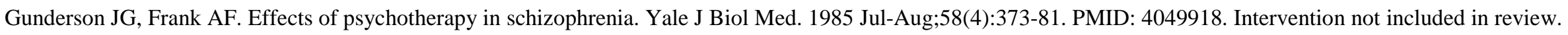

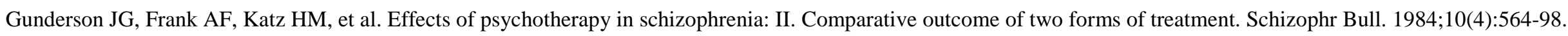
PMID: 6151246. Intervention not included in review.

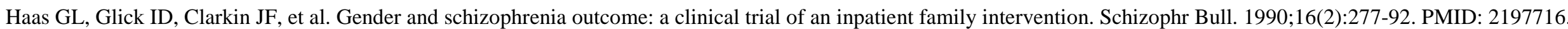
Setting ineligible for review (i.e., inpatient only).

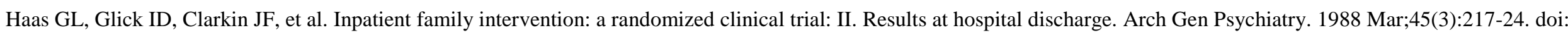
10.1001/archpsyc.1988.01800270025003. PMID: 3277578. Setting ineligible for review (i.e., inpatient only).

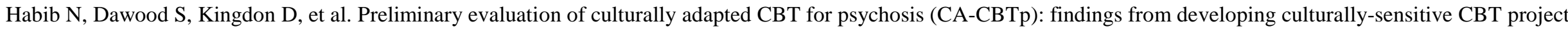
(DCCP). Behav Cogn Psychother. 2015 Mar;43(2):200-8. doi: 10.1017/S1352465813000829. PMID: 24382109. Country not eligible.

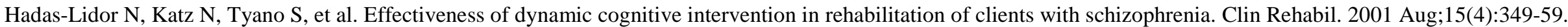
PMID: 11518436. Study covered in a systematic review.

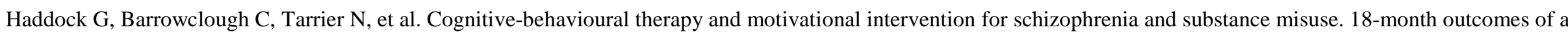
randomised controlled trial. Br J Psychiatry. 2003 Nov;183:418-26. PMID: 14594917. Study covered in a systematic review.

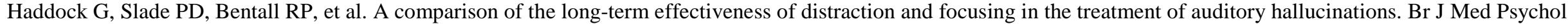
1998 Sep;71(3):339-49. doi: 10.1111/j.2044-8341.1998.tb00996.x. PMID: 9733427. Outcome not included in review.

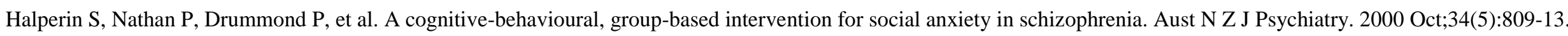
PMID: 11037367. Inadequate duration ( $<12$ weeks).

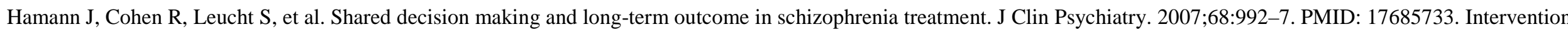
not included in review.

Hamann J, Mendel R, Meier A, et al. "How to speak to your psychiatrist": shared decision-making training for inpatients with schizophrenia. Psychiatr Serv. 2011 Oct;62(10):1218-21. PMID: 21969650. Intervention not included in review.

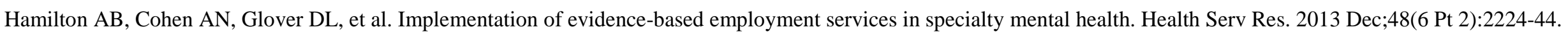
doi: 10.1111/1475-6773.12115. PMID: 24138608. Study design not included in review.

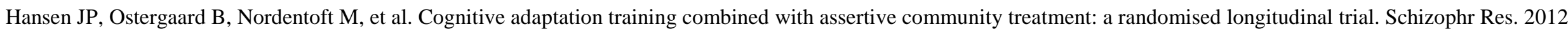
Mar;135(1-3):105-11. doi: 10.1016/j.schres.2011.12.014. PMID: 22264599. Comparator not included in review.

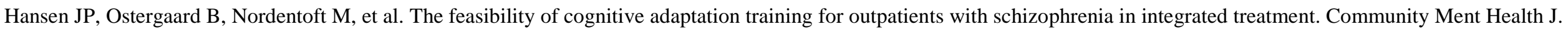
2013 Dec;49(6):630-5. doi: 10.1007/s10597-012-9557-3. PMID: 23064969. Comparator not included in review.

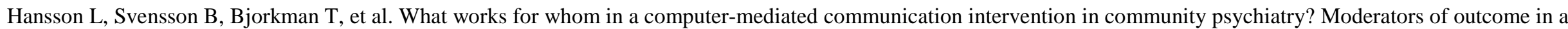
cluster randomized trial. Acta Psychiatr Scand. 2008 Nov;118(5):404-9. doi: 10.1111/j.1600-0447.2008.01258.x. PMID: 18759805. Outcome not included in review.

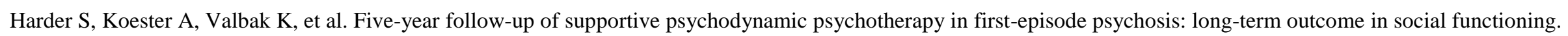
Psychiatry. 2014;77(2):155-68. doi: 10.1521/psyc.2014.77.2.155. PMID: 24865198. Study design not included in review.

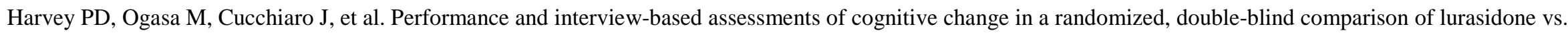
ziprasidone. Schizophr Res. 2011 Apr;127(1-3):188-94. doi: 10.1016/j.schres.2011.01.004. PMID: 21277745. Inadequate duration (<12 weeks).

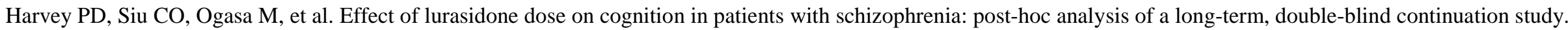
Schizophr Res. 2015 Aug;166(1-3):334-8. doi: 10.1016/j.schres.2015.06.008. PMID: 26117157. Study design not included in review. 


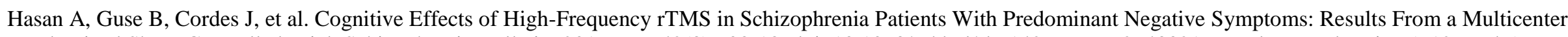

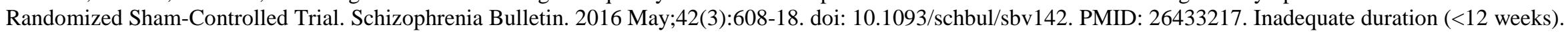

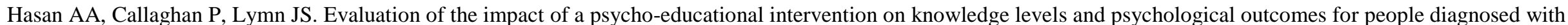

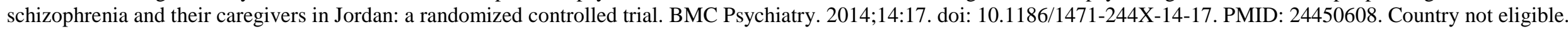

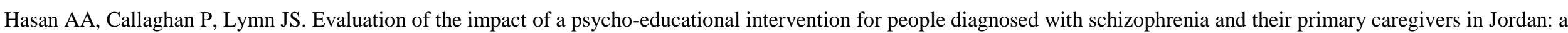
randomized controlled trial. BMC Psychiatry. 2015;15:72. doi: 10.1186/s12888-015-0444-7. PMID: 25885432. Country not eligible.

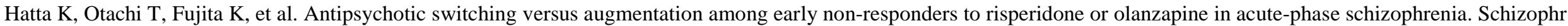
Res. 2014 Sep;158(1-3):213-22. doi: 10.1016/j.schres.2014.07.015. PMID: 25086659. Population ineligible for review.

Hay A, Byers A, Sereno M, et al. Asenapine versus placebo for schizophrenia. Cochrane Database Syst Rev. 2015 Nov 24(11):Cd011458. doi:

10.1002/14651858.CD011458.pub2. PMID: 26599405. Comparator not included in review.

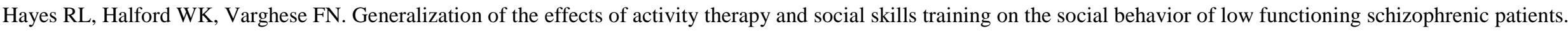
Occup Ther Ment Health. 1991;11(4):3-20. Setting ineligible for review (i.e., inpatient only).

Hayes RL, McGrath JJ. Cognitive rehabilitation for people with schizophrenia and related conditions. Cochrane Database Syst Rev. 2000(3):Cd000968. doi: 10.1002/14651858.cd000968. PMID: 10908479. Intervention not included in review.

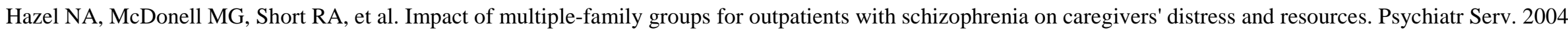
Jan;55(1):35-41. PMID: 14699198. Outcome not included in review.

He Y, Li C. Morita therapy for schizophrenia. Cochrane Database Syst Rev. 2012(9) PMID: 17253588. Intervention not included in review.

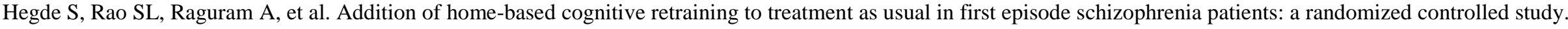
Indian J Psychiatry. 2012;54(1):15-22. PMID: 22556432. Sample size too small (<50).

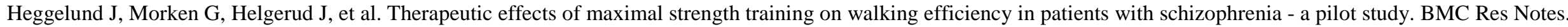
2012;5:344. doi: 10.1186/1756-0500-5-344. PMID: 22759719. Intervention not included in review.

Held T, Falloon IR. Family therapy of schizophrenia. Keio J Med. 1999 Sep;48(3):151-4. PMID: 10535277. Study design not included in review.

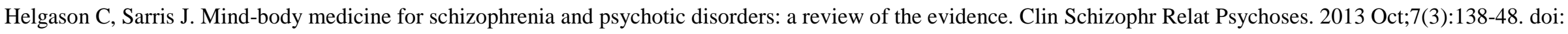
10.3371/CSRP.HESA.020813. PMID: 23428783. Interventions evaluated in a single study.

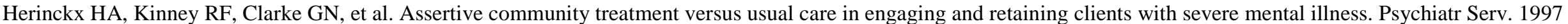
Oct;48(10):1297-306. doi: 10.1176/ps.48.10.1297. PMID: 9323749. Study covered in a systematic review.

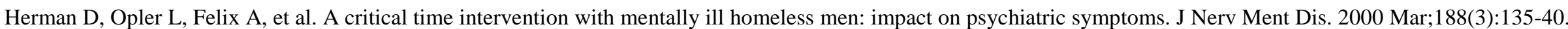
PMID: 10749277. Interventions evaluated in a single study.

Herz MI. Psychosocial treatment. Psychiatr Ann. 1996 Aug;26(8):531-5. Study design not included in review.

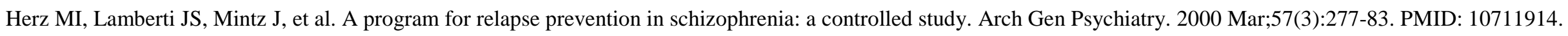
Interventions evaluated in a single study.

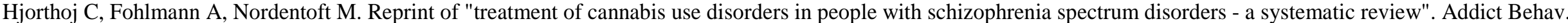
2009 Oct;34(10):846-51. doi: 10.1016/j.addbeh.2009.02.003. PMID: 19604646. Study design not included in review.

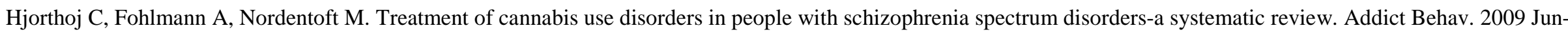
Jul;34(6-7):520-5. doi: 10.1016/j.addbeh.2009.02.001. PMID: 19268481. Study design not included in review. 
Hjorthoj CR, Fohlmann A, Larsen AM, et al. Specialized psychosocial treatment plus treatment as usual (TAU) versus TAU for patients with cannabis use disorder and psychosis: the CapOpus randomized trial. Psychol Med. 2013 Jul;43(7):1499-510. doi: 10.1017/S0033291712002255. PMID: 23040144. Intervention not included in review.

Hjorthoj CR, Orlovska S, Fohlmann A, et al. Psychiatric treatment following participation in the CapOpus randomized trial for patients with comorbid cannabis use disorder and psychosis. Schizophr Res. 2013 Dec;151(1-3):191-6. PMID: 24189290. Study design not included in review.

Ho JS, Moore RC, Davine T, et al. Direct and mediated effects of cognitive function with multidimensional outcome measures in schizophrenia: the role of functional capacity. J Clin Exp Neuropsychol. 2013;35(8):882-95. doi: 10.1080/13803395.2013.828021. PMID: 23984631. Study design not included in review.

Hodge MA, Siciliano D, Withey P, et al. A randomized controlled trial of cognitive remediation in schizophrenia. Schizophr Bull. 2010 Mar;36(2):419-27. doi: 10.1093/schbul/sbn102. PMID: 18718884. Study covered in a systematic review.

Hoffman RE, Boutros NN, Hu S, et al. Transcranial magnetic stimulation and auditory hallucinations in schizophrenia. Lancet. 2000 Mar 25;355(9209):1073-5. PMID: 10744097. Study design not included in review.

Hoffman RE, Hawkins KA, Gueorguieva R, et al. Transcranial magnetic stimulation of left temporoparietal cortex and medication-resistant auditory hallucinations. Arch Gen Psychiatry. 2003 Jan;60(1):49-56. PMID: 12511172. Intervention not included in review.

Hoffmann H, Jackel D, Glauser S, et al. Long-term effectiveness of supported employment: 5-year follow-up of a randomized controlled trial. Am J Psychiatry. 2014 Nov 1;171(11):1183-90. PMID: 25124692. Population ineligible for review.

Hogan M. Updated schizophrenia PORT treatment recommendations: a commentary. Schizophr Bull. 2010;36(1):104-6. Not a study.

Hogarty GE, Anderson CM. Medication, family psychoeducation, and social skills training: first year relapse results of a controlled study. Psychopharmacol Bull. 1986;22(3):8602. PMID: 2879309. Intervention not included in review.

Hogarty GE, Anderson CM, Reiss DJ, et al. Family psychoeducation, social skills training, and maintenance chemotherapy in the aftercare treatment of schizophrenia: I. One-year effects of a controlled study on relapse and expressed emotion. Arch Gen Psychiatry. 1986 Jul;43(7):633-42. doi: 10.1001/archpsyc.1986.01800070019003. PMID: 2872870.

Comparator not included in review.

Hogarty GE, Anderson CM, Reiss DJ, et al. Family psychoeducation, social skills training, and maintenance chemotherapy in the aftercare treatment of schizophrenia: II. Twoyear effects of a controlled study on relapse and adjustment. Arch Gen Psychiatry. 1991 Apr;48(4):340-7. doi: 10.1001/archpsyc.1991.01810280056008. PMID: 1672589. Intervention not included in review.

Hogarty GE, Flesher S, Ulrich R, et al. Cognitive enhancement therapy for schizophrenia: effects of a 2-year randomized trial on cognition and behavior. Arch Gen Psychiatry. 2004 Sep;61(9):866-76. PMID: 15351765. Study covered in a systematic review.

Hogarty GE, Goldberg SC. Drug and sociotherapy in the aftercare of schizophrenic patients. One-year relapse rates. Arch Gen Psychiatry. 1973 Jan;28(1):54-64. PMID: 4345664. Interventions evaluated in a single study.

Hogarty GE, Goldberg SC, Schooler NR. Drug and sociotherapy in the aftercare of schizophrenic patients. III. adjustment of nonrelapsed patients. Arch Gen Psychiatry. 1974 Nov;31(5):609-18. PMID: 4374156. Interventions evaluated in a single study.

Hogarty GE, Goldberg SC, Schooler NR, et al. Drug and sociotherapy in the aftercare of schizophrenic patients. II. two-year relapse rates. Arch Gen Psychiatry. 1974 Nov;31(5):603-8. PMID: 4374155. Interventions evaluated in a single study.

Hogarty GE, Greenwald D, Ulrich RF, et al. Three-year trials of personal therapy among schizophrenic patients living with or independent of family, II: effects on adjustment of patients. Am J Psychiatry. 1997 Nov;154(11):1514-24. PMID: 9356558. Comparator not included in review.

Hogarty GE, Greenwald DP, Eack SM. Durability and mechanism of effects of cognitive enhancement therapy. Psychiatr Serv. 2006 Dec;57(12):1751-7. PMID: 17158490. Study covered in a systematic review. 
Hogarty GE, Kornblith SJ, Greenwald D, et al. Three-year trials of personal therapy among schizophrenic patients living with or independent of family, I: description of study and effects on relapse rates. Am J Psychiatry. 1997 Nov;154(11):1504-13. PMID: 9356557. Comparator not included in review.

Holloway F, Carson J. Intensive case management for the severely mentally ill. Controlled trial. Br J Psychiatry. 1998 Jan;172:19-22. PMID: 9534826. Study covered in a systematic review.

Holzer L, Urben S, Passini CM, et al. A randomized controlled trial of the effectiveness of computer-assisted cognitive remediation (CACR) in adolescents with psychosis or at high risk of psychosis. Behav Cogn Psychother. 2014 Jul;42(4):421-34. doi: 10.1017/s1352465813000313. PMID: 23631951. Population ineligible for review.

Horan WP, Kern RS, Shokat-Fadai K, et al. Social cognitive skills training in schizophrenia: an initial efficacy study of stabilized outpatients. Schizophr Res. 2009 Jan;107(1):4754. doi: 10.1016/j.schres.2008.09.006. PMID: 18930378. Inadequate duration ( $<12$ weeks).

Horan WP, Kern RS, Tripp C, et al. Efficacy and specificity of social cognitive skills training for outpatients with psychotic disorders. J Psychiatr Res. 2011 Aug;45(8):1113-22. doi: 10.1016/j.jpsychires.2011.01.015. PMID: 21377168. Study design not included in review.

Hornung W, Kieserg A, Feldmann R, et al. Psychoeducational training for schizophrenic patients: background, procedure and empirical findings. Patient Educ Couns. 1996 Dec;29(3):257-68. doi: 10.1016/S0738-3991\%2896\%2900918-4. PMID: 9006241. Study covered in a systematic review.

Hornung WP, Feldmann R, Klingberg S, et al. Long-term effects of a psychoeducational psychotherapeutic intervention for schizophrenic outpatients and their key-persons-results of a five-year follow-up. Eur Arch Psychiatry Clin Neurosci. 1999;249(3):162-7. PMID: 10433131. Intervention not included in review.

Hosaini H, Akbari M, Mohamadi J. Effects of cognitive-behavioral therapy on positive symptoms of schizophrenia. J Mazandaran Univ of Med Sci. 2013;23(107):28-37. Not English language.

Hovington CL, McGirr A, Lepage M, et al. Repetitive transcranial magnetic stimulation (rTMS) for treating major depression and schizophrenia: a systematic review of recent meta-analyses. Ann Med. 2013 Jun;45(4):308-21. doi: 10.3109/07853890.2013.783993. PMID: 23687987. Intervention not included in review.

Howard LM, Heslin M, Leese M, et al. Supported employment: randomised controlled trial. Br J Psychiatry. 2010 May;196(5):404-11. doi: 10.1192/bjp.bp.108.061465. PMID: 20435968. Study covered in a systematic review.

Hu S, Yao M, Peterson BS, et al. A randomized, 12-week study of the effects of extended-release paliperidone (paliperidone ER) and olanzapine on metabolic profile, weight, insulin resistance, and beta-cell function in schizophrenic patients. Psychopharmacology (Berl). 2013 Nov;230(1):3-13. doi: 10.1007/s00213-013-3073-1. PMID: 23559220. Setting ineligible for review (i.e., inpatient only).

Hui CL, Chang WC, Chan SK, et al. Early intervention and evaluation for adult-onset psychosis: the JCEP study rationale and design. Early Interv Psychiatry. 2014 Aug;8(3):2618. doi: 10.1111/eip.12034. PMID: 23445124. Not a study.

Hui CL, Wong GH, Tang JY, et al. Predicting 1-year risk for relapse in patients who have discontinued or continued quetiapine after remission from first-episode psychosis. Schizophr Res. 2013 Oct;150(1):297-302. PMID: 23993865 Intervention not included in review.

Hutton P, Wood L, Taylor PJ, et al. Cognitive behavioural therapy for psychosis: rationale and protocol for a systematic review and meta-analysis. Psychosis. 2014 Jul;6(3):22030. doi: 10.1080/17522439.2013.825005. Not a study.

Iancu SC, Hoogendoorn AW, Zweekhorst MB, et al. Farm-based interventions for people with mental disorders: a systematic review of literature. Disabil Rehabil. 2015;37(5):37988. doi: 10.3109/09638288.2014.932441. PMID: 24963943. Intervention not included in review.

Ishigooka J, Nakagome K, Ohmori T, et al. Japan useful medication program for schizophrenia (JUMPs)-long-term study on discontinuation rate, resolution and remission, and improvement in social functioning rate associated with atypical antipsychotic medications in patients with schizophrenia. BMC Psychiatry. 2013;13:243. doi: 10.1186/1471-244X13-243. PMID: 24090047. Not a study.

Issakidis C, Sanderson K, Teesson M, et al. Intensive case management in Australia: a randomized controlled trial. Acta Psychiatr Scand. 1999 May;99(5):360-7. doi: 10.1111/j.1600-0447.1999.tb07242.x. PMID: 10353452. Comparator not included in review. 


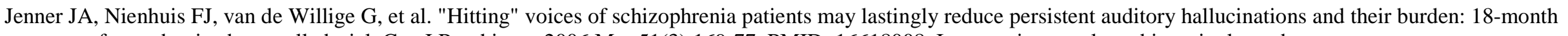
outcome of a randomized controlled trial. Can J Psychiatry. 2006 Mar;51(3):169-77. PMID: 16618008. Interventions evaluated in a single study.

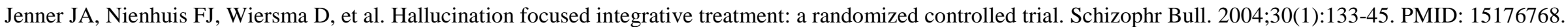
Interventions evaluated in a single study.

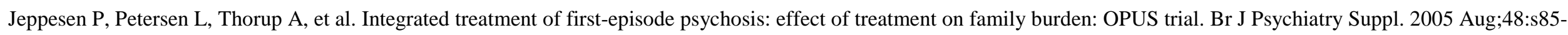
90. PMID: 16055815. Outcome not included in review.

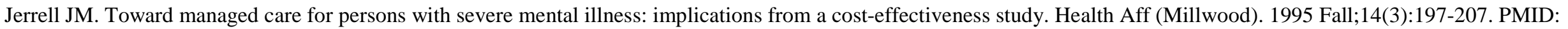
7498892. Study design not included in review.

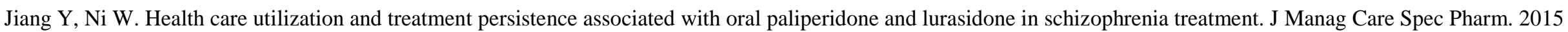
Sep;21(9):780-92. doi: 10.18553/jmcp.2015.21.9.780. PMID: 26308225. Study design not included in review.

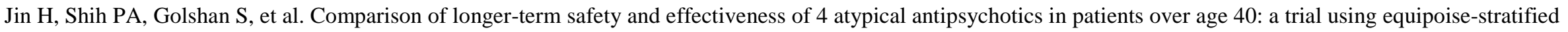
randomization. J Clin Psychiatry. 2013 Jan;74(1):10-8. doi: 10.4088/JCP.12m08001. PMID: 23218100. Population ineligible for review.

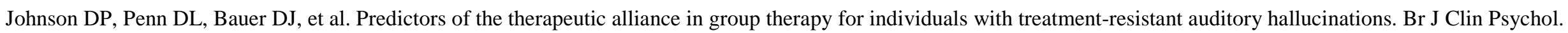
2008 Jun;47(Pt 2):171-83. PMID: 17900393. Study design not included in review.

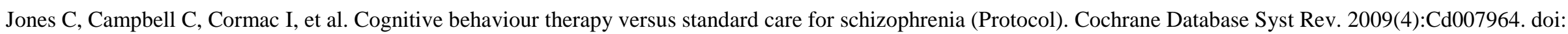
10.1002/14651858.CD007964. Study design not included in review.

Jones C, Cormac I, Silveira da Mota Neto JI, et al. Cognitive behaviour therapy for schizophrenia. Cochrane Database Syst Rev. 2004 Oct 18(4):Cd000524. doi:

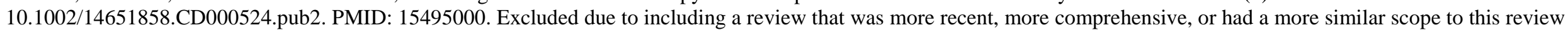
scope.

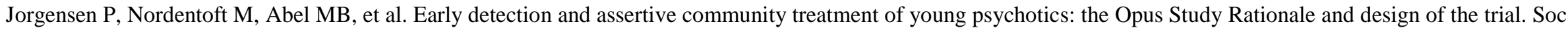
Psychiatry Psychiatr Epidemiol. 2000 Jul;35(7):283-7. PMID: 11016522. Study covered in a systematic review.

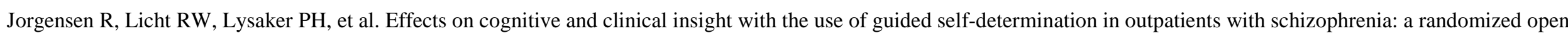
trial. Eur Psychiatry. 2015 Jul;30(5):655-63. doi: 10.1016/j.eurpsy.2014.12.007. PMID: 25601635. Interventions evaluated in a single study.

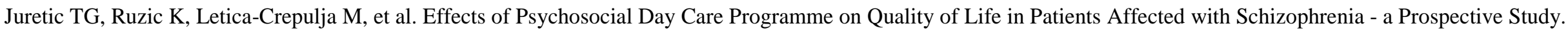
Psychiatria Danubina. 2016 Jun;28(2):111-7. PMID: 27287784. Intervention not included in review.

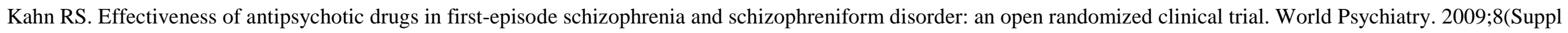
1):44-5. Study covered in a systematic review.

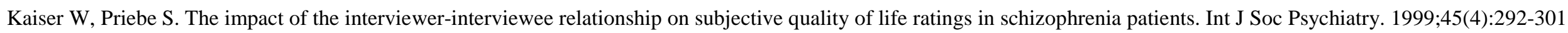
PMID: 10689613. Study design not included in review.

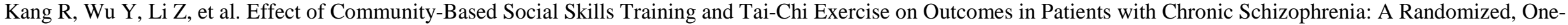
Year Study. Psychopathology. 2016;49(5):345-55. doi: 10.1159/000448195. PMID: 27584836. Country not eligible.

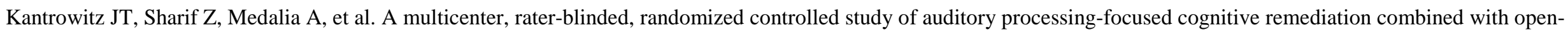

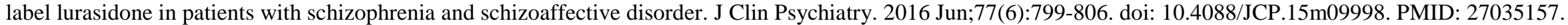
Intervention not included in review.

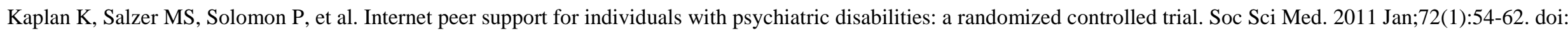
10.1016/j.socscimed.2010.09.037. PMID: 21112682. Interventions evaluated in a single study. 


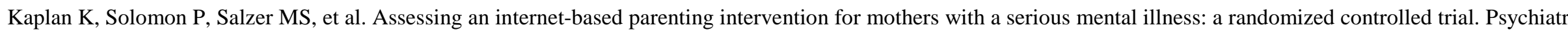
Rehabil J. 2014 Sep;37(3):222-31. doi: 10.1037/prj0000080. PMID: 24978623. Outcome not included in review.

Kasckow J, Felmet K, Appelt C, et al. Telepsychiatry in the assessment and treatment of schizophrenia. Clin Schizophr Relat Psychoses. 2014 Apr;8(1):21-7A. doi: 10.3371/CSRP.KAFE.021513. PMID: 23428781. Intervention not included in review.

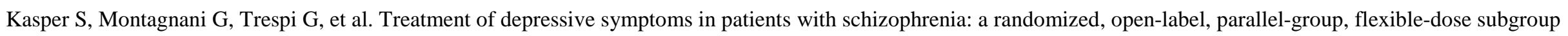

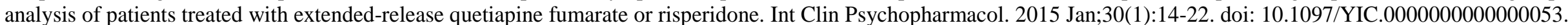
PMID: 25356632. Outcome not included in review.

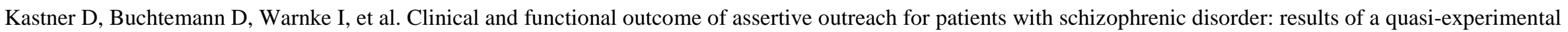
controlled trial. Eur Psychiatry. 2015 Sep;30(6):736-42. doi: 10.1016/j.eurpsy.2015.04.003. PMID: 26003931. Study design not included in review.

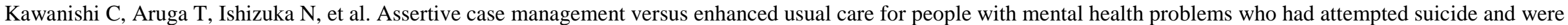

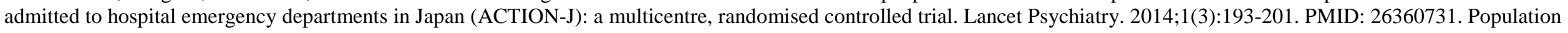
ineligible for review.

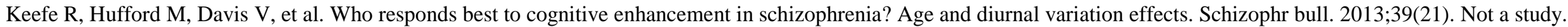

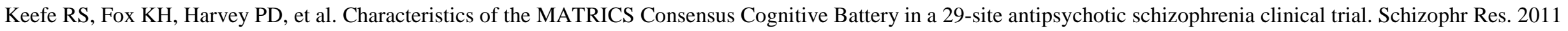
Feb;125(2-3):161-8. doi: 10.1016/j.schres.2010.09.015. PMID: 21075600. Intervention not included in review.

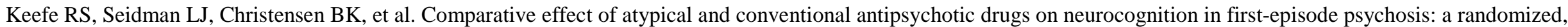
double-blind trial of olanzapine versus low doses of haloperidol. Am J Psychiatry. 2004 Jun;161(6):985-95. PMID: 15169686. Study covered in a systematic review.

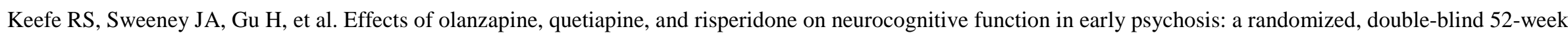
comparison. Am J Psychiatry. 2007 Jul;164(7):1061-71. PMID: 17606658. Study covered in a systematic review.

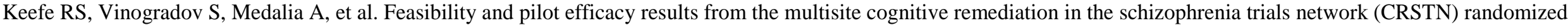
controlled trial. J Clin Psychiatry. 2012 Jul;73(7):1016-22. doi: 10.4088/JCP.11m07100. PMID: 22687548. Sample size too small (<50).

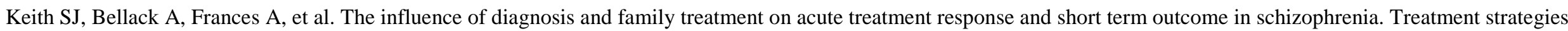
in schizophrenia collaborative study group. Psychopharmacol Bull. 1989;25(3):336-9. PMID: 2697007. Study design not included in review.

Kelly DL, Conley RR. A randomized double-blind 12-week study of quetiapine, risperidone or fluphenazine on sexual functioning in people with schizophrenia. Psychoneuroendocrinology. 2006 Apr;31(3):340-6. PMID: 16198059. Study covered in a systematic review.

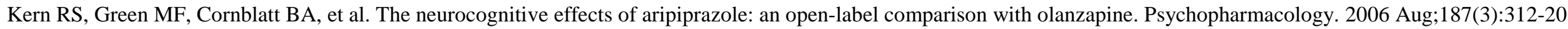
PMID: 16810506. Outcome not included in review.

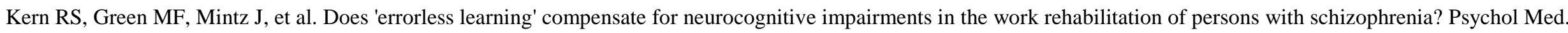
2003 Apr;33(3):433-42. PMID: 12701664. Comparator not included in review.

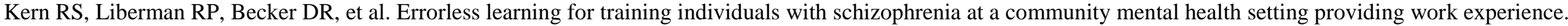
Schizophr Bull. 2009;35(4):807-15. PMID: 18326529. Sample size too small (<50).

Kern RS, Liberman RP, Kopelowicz A, et al. Applications of errorless learning for improving work performance in persons with schizophrenia. Am J Psychiatry. 2002 Nov;159(11):1921-6. PMID: 12411229. Outcome not included in review.

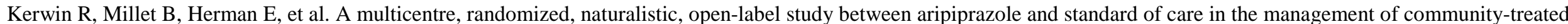

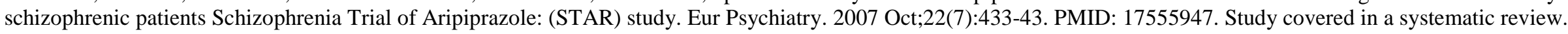




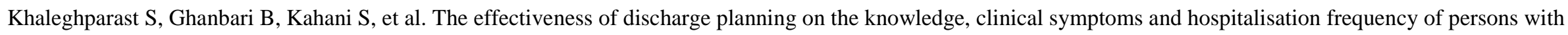

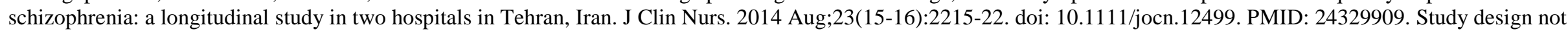
included in review.

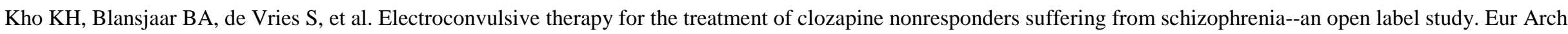
Psychiatry Clin Neurosci. 2004 Dec;254(6):372-9. PMID: 15538604. Study design not included in review.

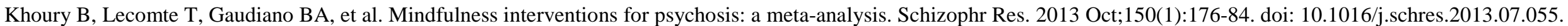
PMID: 23954146. Systematic review/meta-analysis used as a source document to identify individual studies.

Khushu A, Powney MJ. Haloperidol for long-term aggression in psychosis. Cochrane Database of Systematic Reviews. 2016 11 27;11:CD009830. doi:

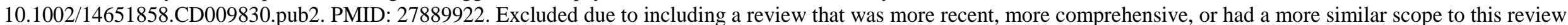
scope.

Kidd SA, Herman Y, Barbic S, et al. Testing a modification of cognitive adaptation training: streamlining the model for broader implementation. Schizophr Res. 2014 Jun;156(1):46-50. doi: 10.1016/j.schres.2014.03.026. PMID: 24794880. Study design not included in review.

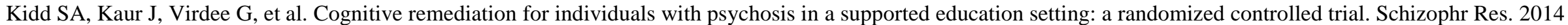
Aug;157(1-3):90-8. doi: 10.1016/j.schres.2014.05.007. PMID: 24893903. Study covered in a systematic review.

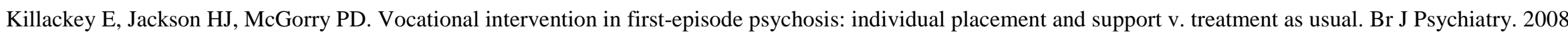
Aug;193(2):114-20. PMID: 18669993. Study covered in a systematic review.

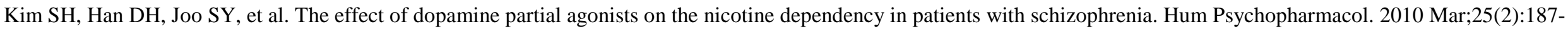
90. doi: 10.1002/hup.1089. PMID: 20033907. Outcome not included in review.

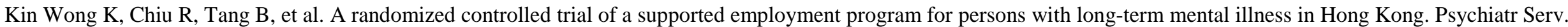
2008 Jan;59(1):84-90. doi: 10.1176/ps.2008.59.1.84. PMID: 18182544. Study covered in a systematic review.

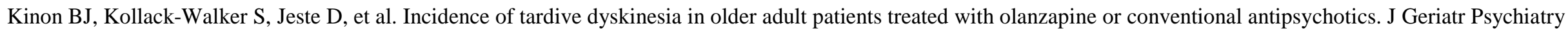
Neurol. 2015 Mar;28(1):67-79. doi: 10.1177/0891988714541867. PMID: 25009161. Population ineligible for review.

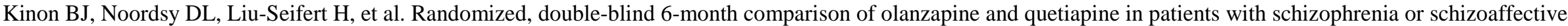

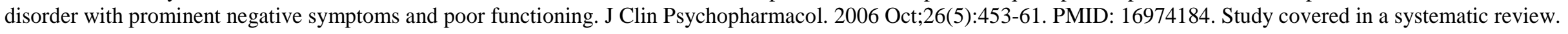

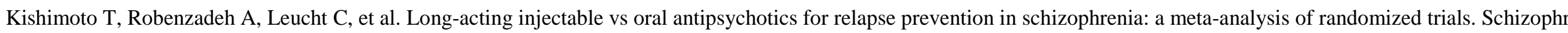

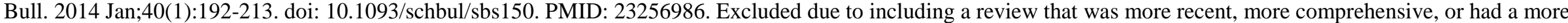
similar scope to this review scope.

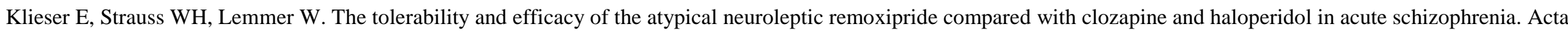
psychiatrica Scandinavica. Supplementum. 1994;380:68-73. PMID: 8048341. Study covered in a systematic review.

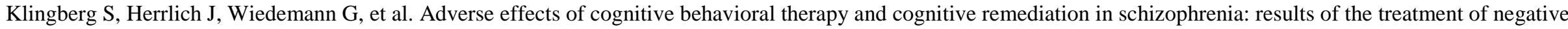
symptoms study. J Nerv Ment Dis. 2012 Jul;200(7):569-76. doi: 10.1097/NMD.0b013e31825bfa1d. PMID: 22759932. Comparator not included in review.

Klingberg S, Wittorf A, Fischer A, et al. Evaluation of a cognitive behaviourally oriented service for relapse prevention in schizophrenia. Acta Psychiatr Scand. 2010 May;121(5):340-50. doi: 10.1111/j.1600-0447.2009.01479.x. PMID: 19895624. Setting ineligible for review (i.e., inpatient only).

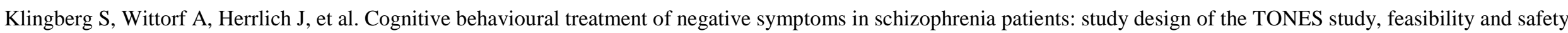

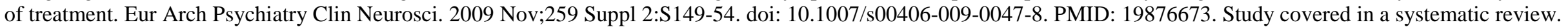


Klingberg S, Wolwer W, Engel C, et al. Negative symptoms of schizophrenia as primary target of cognitive behavioral therapy: results of the randomized clinical TONES study. Schizophr Bull. 2011 Sep;37 Suppl 2:S98-110. doi: 10.1093/schbul/sbr073. PMID: 21860053. Study covered in a systematic review.

Kluwe-Schiavon B, Sanvicente-Vieira B, Kristensen CH, et al. Executive functions rehabilitation for schizophrenia: a critical systematic review. J Psychiatr Res. 2013 Jan;47(1):91-104. doi: 10.1016/j.jpsychires.2012.10.001. PMID: 23122645. Outcome not included in review.

Knapp MR, Marks IM, Wolstenholme J, et al. Home-based versus hospital-based care for serious mental illness: controlled cost-effectiveness study over four years. Br J Psychiatry. 1998 Jun;172(6):506-12. doi: 10.1192/bjp.172.6.506. PMID: 9828991. Comparator not included in review.

Komatsu H, Sekine Y, Okamura N, et al. Effectiveness of information technology aided relapse prevention programme in schizophrenia excluding the effect of user adherence: a randomized controlled trial. Schizophr Res. 2013 Oct;150(1):240-4. doi: 10.1016/j.schres.2013.08.007. PMID: 23998952. Intervention not included in review.

Koolaee AK, Etemadi A. The outcome of family interventions for the mothers of schizophrenia patients in Iran. Int J Soc Psychiatry. 2010 Nov;56(6):634-46. doi: 10.1177/0020764009344144. PMID: 19734180. Country not eligible.

Kopelowicz A. Adapting social skills training for Latinos with schizophrenia. Int Rev Psychiatry. 1998 Feb;10(1):47-50. doi: 10.1080/09540269875087. Not a study.

Kopelowicz A, Zarate R, Gonzalez Smith V, et al. Disease management in Latinos with schizophrenia: a family-assisted, skills training approach. Schizophr Bull. 2003;29(2):21127. PMID: 14552498 . Study covered in a systematic review.

Krabbendam L, Aleman A. Cognitive rehabilitation in schizophrenia: a quantitative analysis of controlled studies. Psychopharmacology (Berl). 2003 Sep;169(3-4):376-82. PMID: 12545330. Excluded due to including a review that was more recent, more comprehensive, or had a more similar scope to this review scope.

Krakowski M, Czobor P. Cholesterol and cognition in schizophrenia: a double-blind study of patients randomized to clozapine, olanzapine and haloperidol. Schizophr Res. 2011 Aug;130(1-3):27-33. PMID: 21549568. Outcome not included in review.

Krakowski MI, Czobor P, Nolan KA. Atypical antipsychotics, neurocognitive deficits, and aggression in schizophrenic patients. J Clin Psychopharmacol. 2008 Oct;28(5):485-93. PMID: 18794642. Outcome not included in review.

Krakvik B, Grawe RW, Hagen R, et al. Cognitive behaviour therapy for psychotic symptoms: a randomized controlled effectiveness trial. Behav Cogn Psychother. 2013 Oct;41(5):511-24. doi: 10.1017/S1352465813000258. PMID: 23635846. Study covered in a systematic review.

Kreyenbuhl J, Buchanan RW, Dickerson FB, et al. The Schizophrenia Patient Outcomes Research Team (PORT): updated treatment recommendations 2009. Schizophr Res. 2010 Jan;36(1):94-103. doi: 10.1093/schbul/sbp130. PMID: 19955388. Excluded due to including a review that was more recent, more comprehensive, or had a more similar scope to this review scope.

Kuipers E, Holloway F, Rabe-Hesketh S, et al. An RCT of early intervention in psychosis: Croydon Outreach and Assertive Support Team (COAST). Soc Psychiatry Psychiatr Epidemiol. 2004 May;39(5):358-63. doi: 10.1007/s00127-004-0754-4. PMID: 15133591. Interventions evaluated in a single study.

Kumar A, Narayan M, Raja H, et al. Asenapine versus typical antipsychotics for schizophrenia (Protocol). Cochrane Database Syst Rev. 2012(11):Cd010230. doi: 10.1002/14651858.CD010230. Not a study.

Kuokkanen R, Lappalainen R, Repo-Tiihonen E, et al. Metacognitive group training for forensic and dangerous non-forensic patients with schizophrenia: a randomised controlled feasibility trial. Crim Behav Ment Health. 2014 Dec;24(5):345-57. doi: 10.1002/cbm.1905. PMID: 24619628. Intervention not included in review.

Kurtz MM, Mueser KT. A meta-analysis of controlled research on social skills training for schizophrenia. J Consult Clin Psychol. 2008 Jun;76(3):491-504. doi: 10.1037/0022006X.76.3.491. PMID: 18540742. Systematic review/meta-analysis used as a source document to identify individual studies.

Kurtz MM, Mueser KT, Thime WR, et al. Social skills training and computer-assisted cognitive remediation in schizophrenia. Schizophr Res. 2015 Mar;162(1-3):35-41. doi: 10.1016/j.schres.2015.01.020. PMID: 25640526. Comparator not included in review. 
Kurtz MM, Richardson CL. Social cognitive training for schizophrenia: a meta-analytic investigation of controlled research. Schizophr Bull. 2012 Sep;38(5):1092-104. doi: 10.1093/schbul/sbr036. PMID: 21525166. Study design not included in review.

Kurtz MM, Seltzer JC, Fujimoto M, et al. Predictors of change in life skills in schizophrenia after cognitive remediation. Schizophr Res. 2009 Feb;107(2-3):267-74. doi: 10.1016/j.schres.2008.10.014. PMID: 19006657. Study design not included in review.

Kurtz MM, Seltzer JC, Shagan DS, et al. Computer-assisted cognitive remediation in schizophrenia: what is the active ingredient? Schizophr Res. 2007 Jan;89(1-3):251-60. PMID: 17070671. Outcome not included in review.

Lally J, Tully J, Robertson D, et al. Augmentation of clozapine with electroconvulsive therapy in treatment resistant schizophrenia: A systematic review and meta-analysis. Schizophr Res. 2016 Mar;171(1-3):215-24. doi: 10.1016/j.schres.2016.01.024. PMID: 26827129. Intervention not included in review.

Lam KCK, Ho CPS, Wa JC, et al. Metacognitive training (MCT) for schizophrenia improves cognitive insight: a randomized controlled trial in a Chinese sample with schizophrenia spectrum disorders. Behav Res Ther. 2015;64:38-42. PMID: 25513971. Intervention not included in review.

Lambert M, Bock T, Schottle D, et al. Assertive community treatment as part of integrated care versus standard care: a 12-month trial in patients with first- and multiple-episode schizophrenia spectrum disorders treated with quetiapine immediate release (ACCESS trial). J Clin Psychiatry. 2010 Oct;71(10):1313-23. doi: 10.4088/JCP.09m05113yel. PMID: 20361911. Study design not included in review.

Laties AM, Flach AJ, Baldycheva I, et al. Cataractogenic potential of quetiapine versus risperidone in the long-term treatment of patients with schizophrenia or schizoaffective disorder: a randomized, open-label, ophthalmologist-masked, flexible-dose, non-inferiority trial. J Psychopharmacol. 2015 Jan;29(1):69-79. doi: 10.1177/0269881114553253. PMID: 25315830. Outcome not included in review.

Latimer EA, Lecomte T, Becker DR, et al. Generalisability of the individual placement and support model of supported employment: results of a Canadian randomised controlled trial. Br J Psychiatry. 2006 Jul;189:65-73. doi: 10.1192/bjp.bp.105.012641. PMID: 16816308. Study covered in a systematic review.

Lawrence R, Bradshaw T, Mairs H. Group cognitive behavioural therapy for schizophrenia: a systematic review of the literature. J Psychiatr Ment Health Nurs. 2006 Dec;13(6):673-81. PMID: 17087669. Excluded due to including a review that was more recent, more comprehensive, or had a more similar scope to this review scope.

Lecardeur L, Stip E, Giguere M, et al. Effects of cognitive remediation therapies on psychotic symptoms and cognitive complaints in patients with schizophrenia and related disorders: a randomized study. Schizophr Res. 2009 Jun;111(1-3):153-8. doi: 10.1016/j.schres.2009.03.029. PMID: 19395240. Study covered in a systematic review.

Lecomte T, Cyr M, Lesage AD, et al. Efficacy of a self-esteem module in the empowerment of individuals with schizophrenia. J Nerv Ment Dis. 1999 Jul;187(7):406-13. PMID: 10426460. Interventions evaluated in a single study.

Lecomte T, Leclerc C, Wykes T. Group CBT for early psychosis--are there still benefits one year later? Int J Group Psychother. 2012 Apr;62(2):309-21. doi: 10.1521/ijgp.2012.62.2.309. PMID: 22468576. Study covered in a systematic review.

Lee MS, Shin BC, Ronan P, et al. Acupuncture for schizophrenia: a systematic review and meta-analysis. Int J Clin Pract. 2009 Nov;63(11):1622-33. doi: 10.1111/j.17421241.2009.02167.x. PMID: 19832819. Intervention not included in review.

Lee RS, Redoblado-Hodge MA, Naismith SL, et al. Cognitive remediation improves memory and psychosocial functioning in first-episode psychiatric out-patients. Psychol Med. 2013 Jun;43(6):1161-73. doi: 10.1017/s0033291712002127. PMID: 23237010. Study covered in a systematic review.

Lee SH, Choi TK, Suh S, et al. Effectiveness of a psychosocial intervention for relapse prevention in patients with schizophrenia receiving risperidone via long-acting injection. Psychiatry Res. 2010 Feb 28;175(3):195-9. doi: 10.1016/j.psychres.2008.06.043. PMID: 20022120. Study design not included in review.

Lee WK. Effectiveness of computerized cognitive rehabilitation training on symptomatological, neuropsychological and work function in patients with schizophrenia. Asia Pac Psychiatry. 2013 Jun;5(2):90-100. doi: 10.1111/appy.12070. PMID: 23857809. Setting ineligible for review (i.e., inpatient only).

Leff J, Berkowitz R, Shavit N, et al. A trial of family therapy v. a relatives group for schizophrenia. Br J Psychiatry. 1989;154:58-66. PMID: 2673479. Study covered in a systematic review. 
Leff J, Berkowitz R, Shavit N, et al. A trial of family therapy versus a relatives' group for schizophrenia. Two-year follow-up. Br J Psychiatry. 1990;157(OCT.):571-7. PMID: 2131140. Study covered in a systematic review.

Leff J, Kuipers L, Berkowitz R, et al. A controlled trial of social intervention in the families of schizophrenic patients. Br J Psychiatry. 1982 Aug;141:121-34. doi: 10.1192/bjp.141.2.121. PMID: 7116051. Study covered in a systematic review.

Leff JP, Kuipers L, Berkowitz R, et al. A controlled trial of social intervention in the families of schizophrenic patients: two year follow-up. Br J Psychiatry. 1985 Jun;146:594600. doi: 10.1192/bjp.146.6.594. PMID: 3893605. Study covered in a systematic review.

Lehman AF, Dixon LB, DeForge BR, et al. Assertive community treatment for homeless persons with schizophrenia and other chronic mental disorders. Schizophr Res. 1995 4//;15(1-2):218-9. doi: 10.1016/0920-9964(95)95671-U. Not a study.

Lehman AF, Dixon LB, Kernan E, et al. A randomized trial of assertive community treatment for homeless persons with severe mental illness. Arch Gen Psychiatry. 1997 Nov;54(11):1038-43. PMID: 9366661. Study covered in a systematic review.

Lehman AF, Goldberg R, Dixon LB, et al. Improving employment outcomes for persons with severe mental illnesses. Archives of General Psychiatry. 2002 Feb;59(2):165-72. PMID: 11825138. Study covered in a systematic review.

Lenior ME, Dingemans PM, Linszen DH, et al. Social functioning and the course of early-onset schizophrenia: five-year follow-up of a psychosocial intervention. Br J Psychiatry. 2001;179:53-8. PMID: 11435269. Study covered in a systematic review.

Lester H, Allan T, Wilson S, et al. A cluster randomised controlled trial of patient-held medical records for people with schizophrenia receiving shared care. Br J Gen Pract. 2003 Mar;53(488):197-203. PMID: 14694695. Interventions evaluated in a single study.

Leucht S, Cipriani A, Spineli L, et al. Comparative efficacy and tolerability of 15 antipsychotic drugs in schizophrenia: a multiple-treatments meta-analysis. Lancet. 2013 Sep 14;382(9896):951-62. doi: 10.1016/S0140-6736(13)60733-3. PMID: 23810019. Study covered in a systematic review.

Levitt AJ, Mueser KT, Degenova J, et al. Randomized controlled trial of illness management and recovery in multiple-unit supportive housing. Psychiatr Serv. 2009 Dec;60(12):1629-36. doi: 10.1176/ps.2009.60.12.1629. PMID: 19952153. Population ineligible for review.

Li F, Wang M. A behavioral training programme for chronic schizophrenic patients: a three-month randomised controlled trial in Beijing. Br J Psychiatry. 1994 Aug;165(Suppl 24):32-7. PMID: 7946229. Setting ineligible for review (i.e., inpatient only).

Li Z, Arthur D. Family education for people with schizophrenia in Beijing, China: randomised controlled trial. Br J Psychiatry. 2005;187:339-45. PMID: 16199793. Country not eligible.

Li ZJ, Guo ZH, Wang N, et al. Cognitive-behavioural therapy for patients with schizophrenia: a multicentre randomized controlled trial in Beijing, China. Psychol Med. 2015 Jul;45(9):1893-905. doi: 10.1017/S0033291714002992. PMID: 25532460. Country not eligible.

Liberman RP, Wallace CJ, Blackwell G, et al. Skills training versus psychosocial occupational therapy for persons with persistent schizophrenia. Am J Psychiatry. 1998 Aug;155(8):1087-91. PMID: 9699698. Comparator not included in review.

Lieberman JA, Tollefson G, Tohen M, et al. Comparative efficacy and safety of atypical and conventional antipsychotic drugs in first-episode psychosis: a randomized, doubleblind trial of olanzapine versus haloperidol. Am J Psychiatry. 2003 Aug;160(8):1396-404. PMID: 12900300. Study covered in a systematic review.

Lieberman JA, Tollefson GD, Charles C, et al. Antipsychotic drug effects on brain morphology in first-episode psychosis. Arch Gen Psychiatry. 2005 Apr;62(4):361-70. PMID: 15809403. Outcome not included in review.

Lincoln T. A comment on Lynch et al. (2009): cognitive behavioural therapy for major psychiatric disorder: does it really work? A meta-analytical review of well-controlled trials. Psychol Med. 2010 May;40(5):877-80. doi: 10.1017/S0033291709991838. PMID: 19917145. Not a study. 


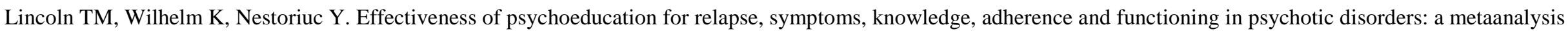

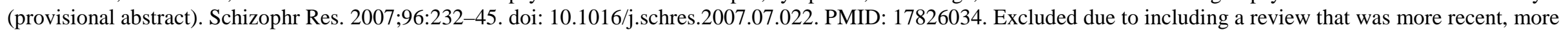
comprehensive, or had a more similar scope to this review scope.

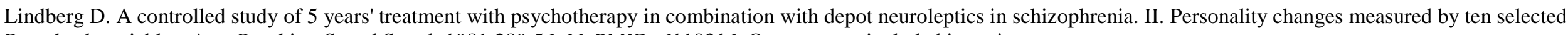
Rorschach variables. Acta Psychiatr Scand Suppl. 1981;289:56-66. PMID: 6110316. Outcome not included in review.

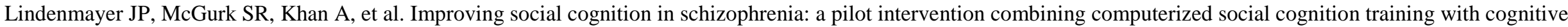
remediation. Schizophr Bull. 2013 May;39(3):507-17. doi: 10.1093/schbul/sbs120. PMID: 23125396. Comparator not included in review.

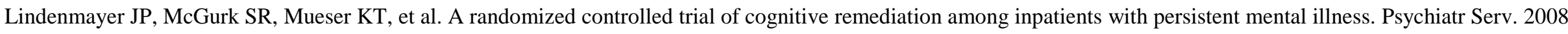
Mar;59(3):241-7. doi: 10.1176/appi.ps.59.3.241. PMID: 18308903. Setting ineligible for review (i.e., inpatient only).

Lindenmayer J-P, Khan A, Lachman H, et al. COMT genotype and response to cognitive remediation in schizophrenia. Schizophr Res. 2015 Oct;168(1-2):279-84. doi: 10.1016/j.schres.2015.07.037. PMID: 26255563. Outcome not included in review.

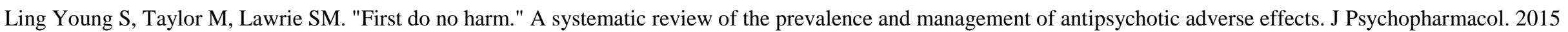
Apr;29(4):353-62. doi: 10.1177/0269881114562090. PMID: 25516373. Study design not included in review.

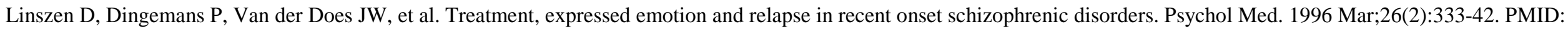
8685289. Setting ineligible for review (i.e., inpatient only).

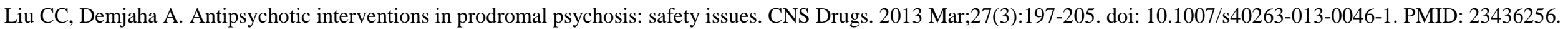
Not a study.

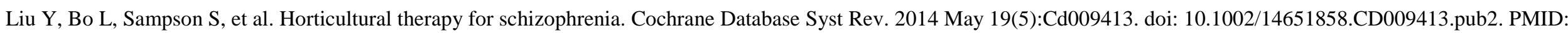
24842458. Inadequate duration ( $<12$ weeks).

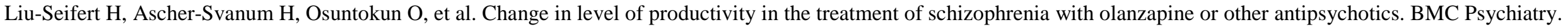
2011;11 PMID: 21586165. Study design not included in review.

Ljubin T, Milas DZ, Mimica N, et al. A preliminary study of the comparative effects of olanzapine and fluphenazine on cognition in schizophrenic patients. Hum Psychopharmacol. 2000;15(7):513-9. PMID: 12404620. Study covered in a systematic review.

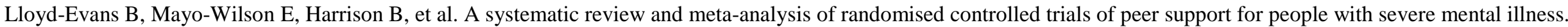
BMC Psychiatry. 2014 Feb;14doi: 10.1186/1471-244X-14-39. PMID: 24528545. Intervention not included in review.

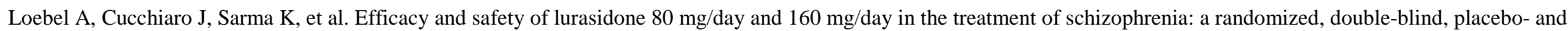
active-controlled trial. Schizophr Res. 2013 Apr;145(1-3):101-9. doi: 10.1016/j.schres.2013.01.009. PMID: 23415311. Inadequate duration (<12 weeks).

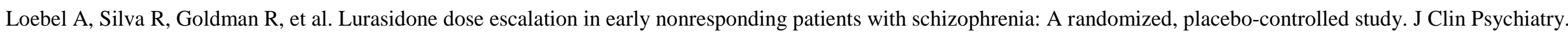
2016 Dec;77(12):1672-80. doi: 10.4088/JCP.16m10698. PMID: 27454547. Inadequate duration (<12 weeks).

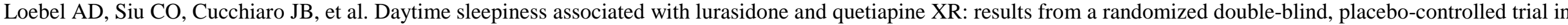
patients with schizophrenia. CNS Spectr. 2014 Apr;19(2):197-205. PMID: 24330860. Study covered in a systematic review.

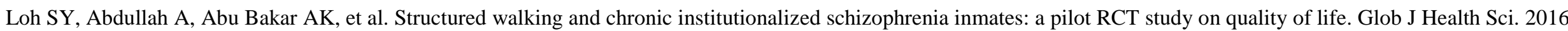
Jan;8(1):238-48. doi: 10.5539/gjhs.v8n1p238. PMID: 26234968. Intervention not included in review.

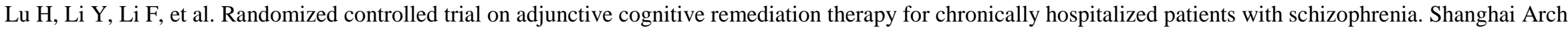
Psychiatry. 2012 Jun;24(3):149-54. PMID: 25324619. Setting ineligible for review (i.e., inpatient only). 


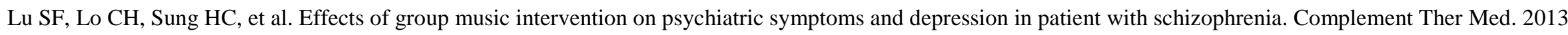
Dec;21(6):682-8. doi: 10.1016/j.ctim.2013.09.002. PMID: 24280478. Intervention not included in review.

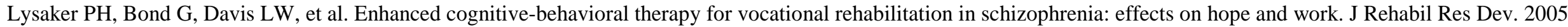
Sep-Oct;42(5):673-82. PMID: 16586193. Study covered in a systematic review.

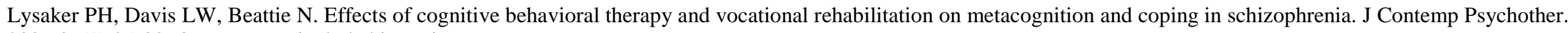
2006;36(1):25-30. Outcome not included in review.

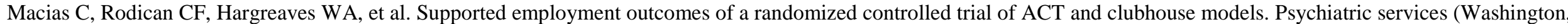
D.C.). 2006 Oct;57(10):1406-15. PMID: CN-00572777 UPDATE. Study covered in a systematic review.

Macpherson R, Edwards TR, Chilvers R, et al. Twenty-four hour care for schizophrenia. Cochrane Database Syst Rev. 2009 Apr 15(2):Cd004409. doi:

10.1002/14651858.CD004409.pub2. PMID: 19370601. Comparator not included in review.

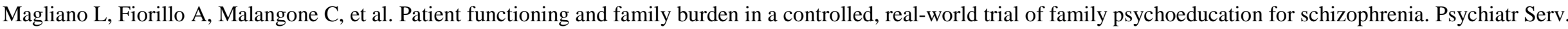
2006 Dec;57(12):1784-91. PMID: 17158495. Study covered in a systematic review.

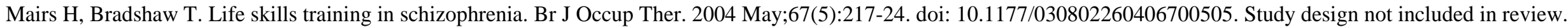

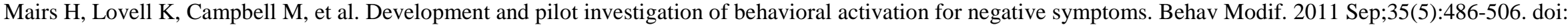
10.1177/0145445511411706. PMID: 21746764. Study design not included in review.

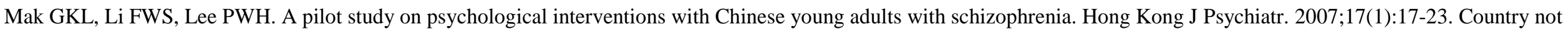
eligible.

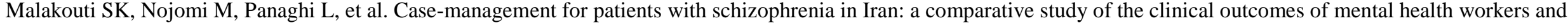

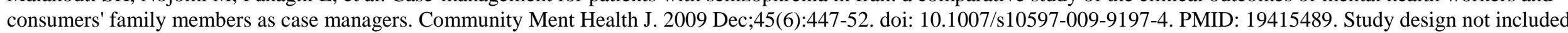
in review.

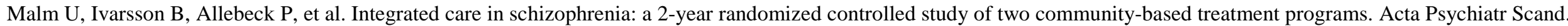
2003 Jun;107(6):415-23. PMID: 12752017. Comparator not included in review.

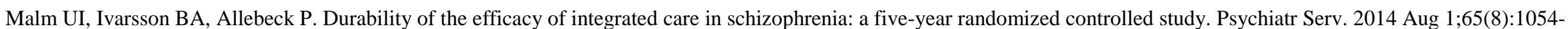
7. doi: 10.1176/appi.ps.201300164. PMID: 24932858. Comparator not included in review.

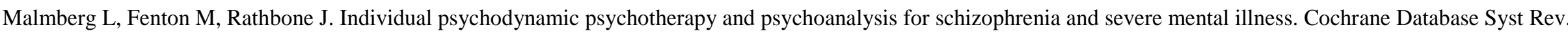
2012(2) PMID: 11686988. Intervention not included in review.

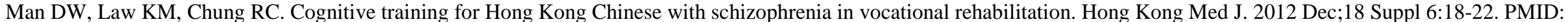
23249847. Comparator not included in review.

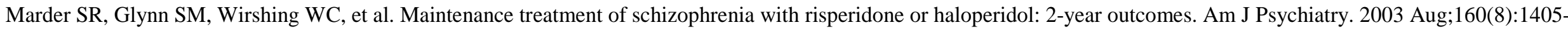
12. PMID: 12900301. Study covered in a systematic review.

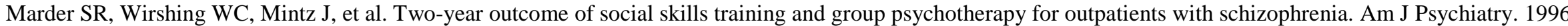
Dec;153(12):1585-92. PMID: 8942455. Comparator not included in review.

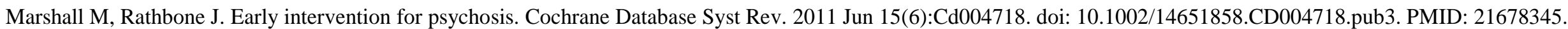

Systematic review/meta-analysis used as a source document to identify individual studies. 
Marshall T, Goldberg RW, Braude L, et al. Supported employment: assessing the evidence. Psychiatr Serv. 2014 Jan 01;65(1):16-23. doi: 10.1176/appi.ps.201300262. PMID: 24247197. Excluded due to including a review that was more recent, more comprehensive, or had a more similar scope to this review scope.

Martin G, Costello H, Leese M, et al. An exploratory study of assertive community treatment for people with intellectual disability and psychiatric disorders: conceptual, clinical, and service issues. J Intellect Disabil Res. 2005 Jul;49(Pt 7):516-24. PMID: 15966959. Population ineligible for review.

Martin-Carrasco M, Fernandez-Catalina P, Dominguez-Panchon AI, et al. A randomized trial to assess the efficacy of a psychoeducational intervention on caregiver burden in schizophrenia. Eur Psychiatry. 2016 Mar;33:9-17. doi: 10.1016/j.eurpsy.2016.01.003. PMID: 26852375. Outcome not included in review.

Maslenikov NV, Mosolov SN, Smirnov NA, et al. Repetitive transcranial magnetic stimulation (rTMS) effects on depression, negative symptoms and cognition in schizophrenia. Brain Stimul. 2015 START: 2015 Mar 2 CONFERENCE END: 2015 Mar 4, 1st International Brain Stimulation Conference Singapore Singapore;8(2):333. Not a study.

Mastroeni A, Bellotti C, Pellegrini E, et al. Clinical and social outcomes five years after closing a mental hospital: a trial of cognitive behavioural interventions. Clin Pract Epidemiol Ment Health. 2005;1(25) PMID: 16305738. Setting ineligible for review (i.e., inpatient only).

Matheson SL, Green MJ, Loo C, et al. Quality assessment and comparison of evidence for electroconvulsive therapy and repetitive transcranial magnetic stimulation for schizophrenia: a systematic meta-review. Schizophr Res. 2010 May;118(1-3):201-10. doi: 10.1016/j.schres.2010.01.002. PMID: 20117918. Intervention not included in review.

Matsuda Y, Sato S, Iwata K, et al. Effects of risperidone and aripiprazole on neurocognitive rehabilitation for schizophrenia. Psychiatry Clin Neurosci. 2014 Jun;68(6):425-31. PMID: 24506576. Outcome not included in review.

Mausbach BT, Cardenas V, McKibbin CL, et al. Reducing emergency medical service use in patients with chronic psychotic disorders: results from the FAST intervention study. Behav Res Ther. 2008 Jan;46(1):145-53. PMID: 17997395. Outcome not included in review.

Mavreas VG, Tomaras V, Karydi V, et al. Expressed emotion in families of chronic schizophrenics and its association with clinical measures. Soc Psychiatry Psychiatr Epidemiol. 1992 Jan;27(1):4-9. PMID: 1557681. Study design not included in review.

May PR, Tuma AH, Dixon WJ, et al. Schizophrenia. A follow-up study of the results of five forms of treatment. Arch Gen Psychiatry. 1981 Jul;38(7):776-84. PMID: 6113821. Intervention not included in review.

McCann E. Brief cognitive-behavioural therapy reduces hospital readmission but does not reduce overall symptoms of schizophrenia. Evid Based Ment Health. $2007 ; 10(2): 55$. PMID: 17459986. Interventions evaluated in a single study.

McCay E, Beanlands H, Leszcz M, et al. A group intervention to promote healthy self-concepts and guide recovery in first episode schizophrenia: a pilot study. Psychiatr Rehabil J. 2006;30(2):105-11. PMID: 17076053. Study design not included in review.

McCay E, Beanlands H, Zipursky R, et al. A randomised controlled trial of a group intervention to reduce engulfment and self-stigmatisation in first episode schizophrenia. AeJAMH. 2007 Nov;6(3):1-9. doi: 10.5172/jamh.6.3.212. Outcome not included in review.

McCreadie RG, Wiles D, Grant S, et al. The Scottish first episode schizophrenia study. VII. Two-year follow-up. Scottish Schizophrenia Research Group. Acta Psychiatr Scand. 1989 Dec;80(6):597-602. PMID: 2694767. Study design not included in review.

McCrone P, Beecham J, Knapp M. Community psychiatric nurse teams: cost-effectiveness of intensive support versus generic care. Br J Psychiatry. 1994 Aug;165(2):218-21. PMID: 7953035. Study design not included in review.

McDonell MG, Short RA, Hazel NA, et al. Multiple-family group treatment of outpatients with schizophrenia: impact on service utilization. Fam Process. 2006 Sep;45(3):359-73. PMID: 16984076. Outcome not included in review.

McEvoy JP, Byerly M, Hamer RM, et al. "Effectiveness of paliperidone palmitate vs haloperidol decanoate for maintenance treatment of schizophrenia: a randomized clinical trial": Erratum. JAMA. 2014 Oct;312(14):1473. Not a study. 
McFarlane WR, Dixon L, Lukens E, et al. Family psychoeducation and schizophrenia: A review of the literature. J Marital Fam Ther. 2003 Apr;29(2):223-45. doi: 10.1111/j.17520606.2003.tb01202.x. PMID: 12728780. Not a study.

McFarlane WR, Dushay RA, Deakins SM, et al. Employment outcomes in family-aided assertive community treatment. Am J Orthopsychiatry. 2000 Apr;70(2):203-14. PMID: 10826032. Not a study.

McFarlane WR, Dushay RA, Stastny P, et al. A comparison of two levels of family-aided assertive community treatment. Psychiatr Serv. 1996 Jul;47(7):744-50. PMID: 8807689. Comparator not included in review.

McFarlane WR, Link B, Dushay R, et al. Psychoeducational multiple family groups: four-year relapse outcome in schizophrenia. Fam Process. 1995 Jun;34(2):127-44. PMID: 7589414. Study covered in a systematic review.

McFarlane WR, Lukens E, Link B, et al. Multiple-family groups and psychoeducation in the treatment of schizophrenia. Arch Gen Psychiatry. 1995 Aug;52(8):679-87. PMID: 7632121. Comparator not included in review.

McGrew JH, Johannesen JK, Griss ME, et al. Performance-based funding of supported employment for persons with severe mental illness: vocational rehabilitation and employment staff perspectives. J Behav Health Serv Res. 2007;34(1):1-16. doi: 10.1007/s11414-006-9045-z. PMID: 17180719. Outcome not included in review.

McGurk SR, Mueser KT. Cognitive functioning, symptoms, and work in supported employment: a review and heuristic model. Schizophr Res 2004 10/1/;70(2-3):147-73. doi: 10.1016/j.schres.2004.01.009. PMID: 15329293. Not a study.

McGurk SR, Mueser KT, DeRosa TJ, et al. Work, recovery, and comorbidity in schizophrenia: a randomized controlled trial of cognitive remediation. Schizophr Bull. 2009 Mar;35(2):319-35. doi: 10.1093/schbul/sbn182. PMID: 19269925. Study covered in a systematic review.

McGurk SR, Mueser KT, Feldman K, et al. Cognitive training for supported employment: 2-3 year outcomes of a randomized controlled trial. Am J Psychiatry. 2007 Mar;164(3):437-41. PMID: 17329468. Study covered in a systematic review.

McGurk SR, Mueser KT, Pascaris A. Cognitive training and supported employment for persons with severe mental illness: one-year results from a randomized controlled trial. Schizophr Bull. 2005 Oct;31(4):898-909. PMID: 16079391. Study covered in a systematic review.

McGurk SR, Mueser KT, Xie H, et al. Cognitive enhancement treatment for people with mental illness who do not respond to supported employment: a randomized controlled trial. Am J Psychiatry. 2015 Sep 1;172(9):852-61. doi: 10.1176/appi.ajp.2015.14030374. PMID: 25998278. Population ineligible for review.

McGurk SR, Twamley EW, Sitzer DI, et al. A meta-analysis of cognitive remediation in schizophrenia. Am J Psychiatry. 2007 Dec;164(12):1791-802. PMID: 18056233. Excluded due to including a review that was more recent, more comprehensive, or had a more similar scope to this review scope.

McHugo GJ, Bebout RR, Harris M, et al. A randomized controlled trial of integrated versus parallel housing services for homeless adults with severe mental illness. Schizophr Bull. 2004;30(4):969-82. PMID: 15957201. Interventions evaluated in a single study.

McHugo GJ, Drake RE, Teague GB, et al. Fidelity to assertive community treatment and client outcomes in the New Hampshire dual disorders study. Psychiatr Serv. 1999 Jun;50(6):818-24. PMID: 10375153. Study covered in a systematic review.

McIntosh AM, Conlon L, Lawrie SM, et al. Compliance therapy for schizophrenia. Cochrane Database Syst Rev. 2009 Jul 19(3):Cd003442. doi: 10.1002/14651858.CD003442.pub2. PMID: 16856009. Intervention not included in review.

McMonagle T, Sultana A. Token economy for schizophrenia. Cochrane Database Syst Rev. 2000(4):Cd001473. doi: 10.1002/14651858.cd001473. PMID: 10908499. Intervention not included in review.

McWilliams S, Hill S, Mannion N, et al. Schizophrenia: a five-year follow-up of patient outcome following psycho-education for caregivers. Eur Psychiatry. 2012 Jan;27(1):56-61. doi: 10.1016/j.eurpsy.2010.08.012. PMID: 21982177. Study design not included in review. 
Medalia A, Richardson R. What predicts a good response to cognitive remediation interventions? Schizophr Bull. 2005 Oct;31(4):942-53. doi: 10.1093/schbul/sbi045. PMID: 16120830. Study design not included in review.

Meltzer H, Lindenmayer J, Kwentus J, et al. A six month randomized controlled trial of long acting injectable risperidone 50 and $100 \mathrm{mg}$ in treatment resistant schizophrenia. Schizophr Res. 2014 Apr;154(1-3):14-22. doi: 10.1016/j.schres.2014.02.015. PMID: 24630262. Comparator not included in review.

Meltzer HY, Bobo WV, Lee MA, et al. A randomized trial comparing clozapine and typical neuroleptic drugs in non-treatment-resistant schizophrenia. Psychiatry Res. 2010 May 30;177(3):286-93. PMID: 20378185. Study covered in a systematic review.

Meltzer HY, Cucchiaro J, Silva R, et al. Lurasidone in the treatment of schizophrenia: a randomized, double-blind, placebo- and olanzapine-controlled study. Am J Psychiatry. 2011 Sep;168(9):957-67. doi: 10.1176/appi.ajp.2011.10060907. PMID: 21676992. Inadequate duration (<12 weeks).

Mendella PD, Burton CZ, Tasca GA, et al. Compensatory cognitive training for people with first-episode schizophrenia: results from a pilot randomized controlled trial. Schizophr Res. 2015 Mar;162(1-3):108-11. doi: 10.1016/j.schres.2015.01.016. PMID: 25631454. Sample size too small (<50).

Mercer G, Finlayson A, Johnstone EC, et al. A study of enhanced management in patients with treatment-resistant schizophrenia. J Psychopharmacol. 1997;11(4):349-56. PMID: 9443524. Excluded due to including a review that was more recent, more comprehensive, or had a more similar scope to this review scope.

Merinder LB, Viuff AG, Laugesen HD, et al. Patient and relative education in community psychiatry: a randomized controlled trial regarding its effectiveness. Soc Psychiatry Psychiatr Epidemiol. 1999 Jun;34(6):287-94. PMID: 10422481. Study covered in a systematic review.

Meyer JM, Mao Y, Pikalov A, et al. Weight change during long-term treatment with lurasidone: pooled analysis of studies in patients with schizophrenia. Int Clin Psychopharmacol. 2015 Nov;30(6):342-50. doi: 10.1097/yic.0000000000000091. PMID: 26196189. Outcome not included in review.

Mintz J, O'B'rien CP, Luborsky L. Predicting the outcome of psychotherapy for schizophrenics. Relative contributions of patient, therapist, and treatment characteristics. Arch Gen Psychiatry. 1976 Oct;33(10):1183-6. PMID: 9919. Outcome not included in review.

Misawa F, Kishimoto T, Hagi K, et al. Safety and tolerability of long-acting injectable versus oral antipsychotics: A meta-analysis of randomized controlled studies comparing the same antipsychotics. Schizophrenia Research. 2016 Oct;176(2-3):220-30. doi: 10.1016/j.schres.2016.07.018. PMID: 27499361. Excluded due to including a review that was more recent, more comprehensive, or had a more similar scope to this review scope.

Misiak B, Frydecka D, Laczmanski L, et al. Effects of second-generation antipsychotics on selected markers of one-carbon metabolism and metabolic syndrome components in first-episode schizophrenia patients. Eur J Clin Pharmacol. 2014;70(12):1433-41. PMID: 25291992. Study design not included in review.

Mohamed S, Rosenheck RA, Lin H, et al. Randomized trial of the effect of four second-generation antipsychotics and one first-generation antipsychotic on cigarette smoking, alcohol, and drug use in chronic schizophrenia. J Nerv Ment Dis. 2015 Jul;203(7):486-92. doi: 10.1097/NMD.0000000000000317. PMID: 26075840. Outcome not included in review.

Mojtabai R, Nicholson RA, Carpenter BN. Role of psychosocial treatments in management of schizophrenia: a meta-analytic review of controlled outcome studies. Schizophr Bull. 1998;24(4):569-87. PMID: 9853790. Excluded due to including a review that was more recent, more comprehensive, or had a more similar scope to this review scope.

Montag C, Haase L, Seidel D, et al. A pilot RCT of psychodynamic group art therapy for patients in acute psychotic episodes: feasibility, impact on symptoms and mentalising capacity. PLoS ONE. 2014;9(11):e112348. doi: 10.1371/journal.pone.0112348. PMID: 25393414. Outcome not included in review.

Montero I, Asencio A, Hernandez I, et al. Two strategies for family intervention in schizophrenia: a randomized trial in a Mediterranean environment. Schizophr Bull. 2001;27(4):661-70. PMID: 11824492. Comparator not included in review.

Montero I, Asencio AP, Ruiz I, et al. Family interventions in schizophrenia: an analysis of non-adherence. Acta Psychiatr Scand. 1999 Aug;100(2):136-41. PMID: 10480199. Outcome not included in review.

Montero I, Hernandez I, Asencio A, et al. Do all people with schizophrenia receive the same benefit from different family intervention programs? Psychiatry Res. 2005 Feb 28;133(2-3):187-95. PMID: 15740994. Intervention not included in review. 
Montero I, Masanet MJ, Bellver F, et al. The long-term outcome of 2 family intervention strategies in schizophrenia. Compr Psychiatry. 2006 Sep-Oct;47(5):362-7. PMID: 16905398. Comparator not included in review.

Moritz S, Andreou C, Schneider BC, et al. Sowing the seeds of doubt: a narrative review on metacognitive training in schizophrenia. Clin Psychol Rev. 2014 Jun;34(4):358-66. doi: 10.1016/j.cpr.2014.04.004. PMID: 24866025. Not a study.

Moritz S, Cludius B, Hottenrott B, et al. Mindfulness and relaxation treatment reduce depressive symptoms in individuals with psychosis. Eur Psychiatry. 2015 Sep;30(6):709-14. doi: 10.1016/j.eurpsy.2015.05.002. PMID: 26163302. Comparator not included in review.

Moritz S, Kerstan A, Veckenstedt R, et al. Further evidence for the efficacy of a metacognitive group training in schizophrenia. Behav Res Ther. 2011 Mar;49(3):151-7. doi: 10.1016/j.brat.2010.11.010. PMID: 21276962. Inadequate duration ( $<12$ weeks).

Moritz S, Veckenstedt R, Andreou C, et al. Sustained and "sleeper" effects of group metacognitive training for schizophrenia: a randomized clinical trial. JAMA Psychiatry. 2014 Oct;71(10):1103-11. doi: 10.1001/jamapsychiatry.2014.1038. PMID: 25103718. Intervention not included in review.

Moritz S, Veckenstedt R, Bohn F, et al. Complementary group metacognitive training (MCT) reduces delusional ideation in schizophrenia. Schizophr Res. 2013 Dec;151(1-3):619. doi: 10.1016/j.schres.2013.10.007. PMID: 24183707. Intervention not included in review.

Morken G, Grawe RW, Widen JH. Effects of integrated treatment on antipsychotic medication adherence in a randomized trial in recent-onset schizophrenia. J Clin Psychiatry. 2007 Apr;68(4):566-71. PMID: 17474812. Outcome not included in review.

Morrison A, Hutton P, Wardle M, et al. Cognitive therapy for people with a schizophrenia spectrum diagnosis not taking antipsychotic medication: an exploratory trial. Psychol Med. 2012 May;42(5):1049-56. doi: 10.1017/S0033291711001899. PMID: 21914252. Study design not included in review.

Morrison A, Renton J, Williams S, et al. Delivering cognitive therapy to people with psychosis in a community mental health setting: an effectiveness study. Acta Psychiatr Scand. 2004 Jul;110(1):36-44. doi: 10.1111/j.1600-0447.2004.00299.x. PMID: 15180778. Study design not included in review.

Morrison AP, Turkington D, Pyle M, et al. Cognitive therapy for people with schizophrenia spectrum disorders not taking antipsychotic drugs: a single-blind randomised controlled trial. Lancet. 2014 Apr 19;383(9926):1395-403. doi: 10.1016/S0140-6736(13)62246-1. PMID: 24508320. Comparator not included in review.

Morriss R, Vinjamuri I, Faizal MA, et al. Training to recognise the early signs of recurrence in schizophrenia. Cochrane Database Syst Rev. 2013 Feb 28(2):Cd005147. doi: 10.1002/14651858.CD005147.pub2. PMID: 23450559. Intervention not included in review.

Mossler K, Chen X, Heldal TO, et al. Music therapy for people with schizophrenia and schizophrenia-like disorders. Cochrane Database Syst Rev. 2011 Dec 07(12):Cd004025. doi: 10.1002/14651858.CD004025.pub3. PMID: 22161383. Intervention not included in review.

Mota NE, Lima MS, Soares BG. Amisulpride for schizophrenia. Cochrane Database Syst Rev. 2002(2):Cd001357. doi: 10.1002/14651858.cd001357. PMID: 12076408. Intervention not included in review.

Mowbray CT, Bybee D, Collins ME. Follow-up client satisfaction in a supported education program. Psychiatr Rehabil J. 2001 Winter;24(3):237-47. PMID: 11315210. Study design not included in review.

Mueller DR, Schmidt SJ, Roder V. Integrated psychological therapy: effectiveness in schizophrenia inpatient settings related to patients' age. Am J Geriatr Psychiatry. 2013 Mar;21(3):231-41. doi: 10.1016/j.jagp.2012.12.011. PMID: 23395190. Setting ineligible for review (i.e., inpatient only).

Mueser KT, Berenbaum H. Psychodynamic treatment of schizophrenia: is there a future? Psychol Med. 1990 May;20(2):253-62. PMID: 2192381. Not a study.

Mueser KT, Corrigan PW, Hilton DW, et al. Illness management and recovery: a review of the research. Psychiatr Serv. 2002;53(10):1272-84. doi: 10.1176/appi.ps.53.10.1272. PMID: 12364675. Not a study.

Mueser KT, Deavers F, Penn DL, et al. Psychosocial treatments for schizophrenia. Annu Rev Clin Psychol. 2013;9:465-97. doi: 10.1146/annurev-clinpsy-050212-185620. PMID: 23330939. Not a study. 
Mueser KT, Drake RE, Sigmon SC, et al. Psychosocial interventions for adults with severe mental illnesses and co-occurring substance use disorders. J Dual Diagn. 2005 2005/04/05;1(2):57-82. doi: 10.1300/J374v01n02_05. Not a study.

Mueser KT, Glynn SM. Have the potential benefits of CBT for severe mental disorders been undersold? World Psychiatry. 2014 Oct;13(3):253-6. doi: 10.1002/wps.20160. PMID: 25273293. Not a study.

Mueser KT, Glynn SM, Cather C, et al. A randomized controlled trial of family intervention for co-occurring substance use and severe psychiatric disorders. Schizophr Bull. 2013 May;39(3):658-72. doi: 10.1093/schbul/sbr203. PMID: 22282453. Comparator not included in review.

Mueser KT, Glynn SM, Cather C, et al. Family intervention for co-occurring substance use and severe psychiatric disorders: participant characteristics and correlates of initial engagement and more extended exposure in a randomized controlled trial. Addict Behav. 2009 Oct;34(10):867-77. doi: 10.1016/j.addbeh.2009.03.025. PMID: 19375870. Outcome not included in review.

Mueser KT, Penn DL, Addington J, et al. The NAVIGATE program for first-episode psychosis: rationale, overview, and description of psychosocial components. Psychiatr Serv. 2015 Jul;66(7):680-90. doi: 10.1176/appi.ps.201400413. PMID: 25772766. Study design not included in review.

Mueser KT, Sengupta A, Schooler NR, et al. Family treatment and medication dosage reduction in schizophrenia: effects on patient social functioning, family attitudes, and burden. J Consult Clin Psychol. 2001 Feb;69(1):3-12. PMID: 11302274. Not a study.

Muijen M, Cooney M, Strathdee G, et al. Community psychiatric nurse teams: intensive support versus generic care. Br J Psychiatry. 1994 Aug;165(2):211-7. PMID: 7953034. Outcome not included in review.

Murphy SM, Irving CB, Adams CE, et al. Crisis intervention for people with severe mental illnesses. Cochrane Database Syst Rev. 2015 Dec 03(12):Cd001087. doi: 10.1002/14651858.CD001087.pub5. PMID: 26633650. Intervention not included in review.

Murray RM, Van Os J. Predictors of outcome in schizophrenia. J Clin Psychopharmacol. 1998 Apr;18(2 Suppl 1):2S-4S. PMID: 9555609. Study design not included in review.

Naeem F, Asmer SM, Khoury B, et al. Acceptance and commitment therapy for schizophrenia and related disorders. Cochrane Database Syst Rev. 2015(6):Cd011734. doi: 10.1002/14651858.CD011734. Not a study.

Naeem F, Farooq S, Kingdon D. Cognitive behavioural therapy (brief versus standard duration) for schizophrenia. Cochrane Database Syst Rev. 2015 Oct 21;10:Cd010646. doi: 10.1002/14651858.CD010646.pub3. PMID: 26488686. Comparator not included in review.

Naeem F, Xiang S, Munshi TA, et al. Self-help and guided self-help interventions for schizophrenia and related disorders. Cochrane Database Syst Rev. 2015(5):Cd011698. doi: 10.1002/14651858.CD011698. Not a study.

Nakajima S, Takeuchi H, Fervaha G, et al. Comparative efficacy between clozapine and other atypical antipsychotics on depressive symptoms in patients with schizophrenia: analysis of the CATIE phase 2E data. Schizophr Res. 2015 Feb;161(2-3):429-33. PMID: 25556080. Outcome not included in review.

Nakamura M, Ogasa M, Guarino J, et al. Lurasidone in the treatment of acute schizophrenia: a double-blind, placebo-controlled trial. J Clin Psychiatry. 2009 Jun;70(6):829-36. doi: 10.4088/JCP.08m04905. PMID: 19497249. Comparator not included in review.

Nasrallah HA, Cucchiaro JB, Mao Y, et al. Lurasidone for the treatment of depressive symptoms in schizophrenia: analysis of 4 pooled, 6-week, placebo-controlled studies. CNS Spectr. 2015 Apr;20(2):140-7. doi: 10.1017/s1092852914000285. PMID: 24955752. Inadequate duration (<12 weeks).

Nasrallah HA, Silva R, Phillips D, et al. Lurasidone for the treatment of acutely psychotic patients with schizophrenia: a 6-week, randomized, placebo-controlled study. J Psychiatr Res. 2013 May;47(5):670-7. Comparator not included in review.

Newton-Howes G, Wood R. Cognitive behavioural therapy and the psychopathology of schizophrenia: systematic review and meta-analysis. Psychol Psychother 2013 Jun;86(2):127-38. doi: 10.1111/j.2044-8341.2011.02048.x. PMID: 23674464. Excluded due to including a review that was more recent, more comprehensive, or had a more similar scope to this review scope. 
Nielssen OB, Malhi GS, McGorry PD, et al. Overview of violence to self and others during the first episode of psychosis. J Clin Psychiatry. 2012 May;73(5):e580-7. doi: 10.4088/JCP.11r07036. PMID: 22697204. Study design not included in review.

Nishio M, Ito J, Oshima I, et al. Preliminary outcome study on assertive community treatment in Japan. Psychiatry Clin Neurosci. 2012 Aug;66(5):383-9. doi: 10.1111/j.14401819.2012.02348.x. PMID: 22834656. Study design not included in review.

Nordentoft M, Jeppesen P, Abel M, et al. OPUS study: suicidal behaviour, suicidal ideation and hopelessness among patients with first-episode psychosis. One-year follow-up of a randomised controlled trial. Br J Psychiatry Suppl. 2002 Sep;43:s98-106. PMID: 12271808. Study covered in a systematic review.

Nordentoft M, Ohlenschlaeger J, Thorup A, et al. Deinstitutionalization revisited: a 5-year follow-up of a randomized clinical trial of hospital-based rehabilitation versus specialized assertive intervention (OPUS) versus standard treatment for patients with first-episode schizophrenia spectrum disorders. Psychol Med. 2010 Oct;40(10):1619-26. doi: 10.1017/S0033291709992182. PMID: 20059798. Intervention not included in review.

Norman RM, Malla AK, McLean TS, et al. An evaluation of a stress management program for individuals with schizophrenia. Schizophr Res. 2002 Dec 1;58(2-3):293-303. PMID: 12409170. Interventions evaluated in a single study.

Novakovic V, Adel T, Peselow E, et al. Long-acting injectable antipsychotics and the development of postinjection delirium/sedation syndrome (PDSS). Clin Neuropharmacol. 2013 Mar-Apr;36(2):59-62. doi: 10.1097/WNF.0b013e3182854f70. PMID: 23503549. Study design not included in review.

O'Brien CP, Hamm KB, Ray BA, et al. Group vs individual psychotherapy with schizophrenics. A controlled outcome study. Arch Gen Psychiatry. 1972 Oct;27(4):474-8. PMID: 4342116. Intervention not included in review.

Ogasa M, Kimura T, Nakamura M, et al. Lurasidone in the treatment of schizophrenia: a 6-week, placebo-controlled study. Psychopharmacology. 2013 Feb;225(3):519-30. PMID: CN-00864694 UPDATE. Comparator not included in review.

Ohlenschlaeger J, Nordentoft M, Thorup A, et al. Effect of integrated treatment on the use of coercive measures in first-episode schizophrenia-spectrum disorder. A randomized clinical trial. Int J Law Psychiatry. 2008 Jan-Feb;31(1):72-6. doi: 10.1016/j.ijlp.2007.11.003. PMID: 18191455. Outcome not included in review.

Ohlenschlaeger J, Thorup A, Petersen L, et al. Intensive treatment models and coercion. Nord J Psychiatry. 2007;61(5):369-78. PMID: 17990199. Intervention not included in review.

Ojeda N, Pena J, Sanchez P, et al. Efficiency of cognitive rehabilitation with REHACOP in chronic treatment resistant Hispanic patients. NeuroRehabilitation. 2012;30(1):65-74. PMID: 22349843. Comparator not included in review.

Oka K, Maeda M, Hirano T, et al. Multicenter study on the effects of day care therapy on schizophrenia: a comparison of day care patients with outpatients. Psychiatry Clin Neurosci. 1999 Aug;53(4):505-10. PMID: 10498233. Intervention not included in review.

Okpokoro U, Adams CE, Sampson S. Family intervention (brief) for schizophrenia. Cochrane Database Syst Rev. 2014;3:CD009802. doi: 10.1002/14651858.CD009802.pub2. PMID: 24595545. Inadequate duration ( $<12$ weeks).

Olbrich R, Mussgay L. Reduction of schizophrenic deficits by cognitive training: an evaluative study. Eur Arch Psychiatry Neurol Sci. 1990;239(6):366-9. PMID: 2144236. Outcome not included in review.

O'Reilly K, Donohoe G, O'Sullivan D, et al. Study protocol: a randomised controlled trial of cognitive remediation for a national cohort of forensic mental health patients with schizophrenia or schizoaffective disorder. BMC Psychiatry. 2016 Dec;16:5. PMID: 26759167. Not a study.

Orfanos S, Banks C, Priebe S. Are group psychotherapeutic treatments effective for patients with schizophrenia? A systematic review and meta-analysis. Psychother Psychosom. 2015;84(4):241-9. doi: 10.1159/000377705. PMID: 26022543. Intervention not included in review.

Ostergaard Christensen T, Vesterager L, Krarup G, et al. Cognitive remediation combined with an early intervention service in first episode psychosis. Acta Psychiatr Scand. 2014 Oct;130(4):300-10. doi: 10.1111/acps.12287. PMID: 24833315. Comparator not included in review. 
Pagsberg A, Jeppesen P, Klauber D, et al. Quetiapine versus aripiprazole in children and adolescents with psychosis-protocol for the randomised, blinded clinical Tolerability and Efficacy of Antipsychotics (TEA) trial. BMC Psychiatry. 2014 Jul;14:199. PMID: 25015535. Not a study.

Palma-Sevillano C, Canete-Crespillo J, Farriols-Hernando N, et al. Randomised controlled trial of cognitive-motivational therapy program for the initial phase of schizophrenia: a 6-month assessment. Eur J Psychiat. 2011 Apr-Jun;25(2):68-80. Sample size too small $(<50)$.

Pandina G, Lane Rd, Gopal S, et al. A double-blind study of paliperidone palmitate and risperidone long-acting injectable in adults with schizophrenia. Prog Neuropsychopharmacol Biol Psychiatry. 2011 Jan 15;35(1):218-26. doi: 10.1016/j.pnpbp.2010.11.008. PMID: 21092748. Study covered in a systematic review.

Parabiaghi A, D'Avanzo B, Tettamanti M, et al. The GiSAS study: rationale and design of a pragmatic randomized controlled trial on aripiprazole, olanzapine and haloperidol in the long-term treatment of schizophrenia. Contemp Clin Trials. 2011 Sep;32(5):675-84. PMID: 21554991. Study covered in a systematic review.

Patterson TL, Bucardo J, McKibbin CL, et al. Development and pilot testing of a new psychosocial intervention for older Latinos with chronic psychosis. Schizophr Bull. 2005 Oct;31(4):922-30. doi: 10.1093/schbul/sbi036. PMID: 16037481. Interventions evaluated in a single study.

Patterson TL, Mausbach BT, McKibbin C, et al. Functional Adaptation Skills Training (FAST): a randomized trial of a psychosocial intervention for middle-aged and older patients with chronic psychotic disorders. Schizophr Res. 2006 Sep;86(1-3):291-9. doi: 10.1016/j.schres.2006.05.017. PMID: 16814526. Study covered in a systematic review.

Patterson TL, McKibbin C, Taylor M, et al. Functional adaptation skills training (FAST): a pilot psychosocial intervention study in middle-aged and older patients with chronic psychotic disorders. Am J Geriatr Psychiatry. 2003 Jan-Feb;11(1):17-23. PMID: 12527536. Sample size too small $(<50)$.

Pawelczyk T, Grancow-Grabka M, Kotlicka-Antczak M, et al. A randomized controlled study of the efficacy of six-month supplementation with concentrated fish oil rich in omega-3 polyunsaturated fatty acids in first episode schizophrenia. J Psychiatr Res. 2016;73:34-44. PMID: 26679763. Intervention not included in review.

Peluso MJ, Lewis SW, Barnes TRE, et al. Extrapyramidal motor side-effects of first- and second-generation antipsychotic drugs. Br J Psychiatry. 2012;200(5):387-92. PMID: 22442101. Comparator not included in review.

Penades R, Catalan R, Salamero M, et al. Cognitive remediation therapy for outpatients with chronic schizophrenia: a controlled and randomized study. Schizophr Res. 2006 Oct;87(1-3):323-31. PMID: 16750611. Comparator not included in review.

Penn DL, Meyer PS, Evans E, et al. A randomized controlled trial of group cognitive-behavioral therapy vs. enhanced supportive therapy for auditory hallucinations. Schizophr Res. 2009 Apr;109(1-3):52-9. doi: 10.1016/j.schres.2008.12.009. PMID: 19176275. Study covered in a systematic review.

Penn DL, Uzenoff SR, Perkins D, et al. A pilot investigation of the Graduated Recovery Intervention Program (GRIP) for first episode psychosis.[Erratum appears in Schizophr Res. 2012 Oct;141(1):106-7]. Schizophr Res. 2011 Feb;125(2-3):247-56. doi: 10.1016/j.schres.2010.08.006. PMID: 20817484. Interventions evaluated in a single study.

Penttila M, Jaaskelainen E, Hirvonen N, et al. Duration of untreated psychosis as predictor of long-term outcome in schizophrenia: systematic review and meta-analysis. Br J Psychiatry. 2014 Aug;205(2):88-94. doi: 10.1192/bjp.bp.113.127753. PMID: 25252316. Outcome not included in review.

Peters E, Landau S, McCrone P, et al. A randomised controlled trial of cognitive behaviour therapy for psychosis in a routine clinical service. Acta Psychiatr Scand. 2010 Oct;122(4):302-18. doi: 10.1111/j.1600-0447.2010.01572.x. PMID: 20491720. Study covered in a systematic review.

Petersen L, Jeppesen P, Thorup A, et al. Substance abuse and first-episode schizophrenia-spectrum disorders. The Danish OPUS trial. Early Interv Psychiatry. 2007 Feb;1(1):8896. doi: 10.1111/j.1751-7893.2007.00015.x. PMID: 21352112. Study covered in a systematic review.

Petretto DR, Preti A, Zuddas C, et al. Study on psychoeducation enhancing results of adherence in patients with schizophrenia (SPERA-S): study protocol for a randomized controlled trial. Trials. 2013;14:323. doi: 10.1186/1745-6215-14-323. PMID: 24099414. Intervention not included in review.

Petrides G, Malur C, Braga RJ, et al. Electroconvulsive therapy augmentation in clozapine-resistant schizophrenia: a prospective, randomized study. Am J Psychiatry. 2015 Jan;172(1):52-8. doi: 10.1176/appi.ajp.2014.13060787. PMID: 25157964. Intervention not included in review. 
Phutane VH, Thirthalli J, Muralidharan K, et al. Double-blind randomized controlled study showing symptomatic and cognitive superiority of bifrontal over bitemporal electrode placement during electroconvulsive therapy for schizophrenia. Brain Stimul. 2013 Mar;6(2):210-7. PMID: 22560048. Intervention not included in review.

Pickett-Schenk SA, Bennett C, Cook JA, et al. Changes in caregiving satisfaction and information needs among relatives of adults with mental illness: results of a randomized evaluation of a family-led education intervention. Am J Orthopsychiatry. 2006 Oct;76(4):545-53. doi: 10.1037/0002-9432.76.4.545. PMID: 17209722. Outcome not included in review.

Pickett-Schenk SA, Cook JA, Steigman P, et al. Psychological well-being and relationship outcomes in a randomized study of family-led education. Arch Gen Psychiatry. 2006 Sep;63(9):1043-50. doi: 10.1001/archpsyc.63.9.1043. PMID: 16953007. Intervention not included in review.

Pickett-Schenk SA, Lippincott RC, Bennett C, et al. Improving knowledge about mental illness through family-led education: the journey of hope. Psychiatr Serv. 2008 Jan;59(1):49-56. doi: 10.1176/ps.2008.59.1.49. PMID: 18182539. Outcome not included in review.

Pilling S, Bebbington P, Kuipers E, et al. Psychological treatments in schizophrenia: I. Meta-analysis of family intervention and cognitive behaviour therapy. Psychol Med. 2002 Jul;32(5):763-82. PMID: 12171372. Excluded due to including a review that was more recent, more comprehensive, or had a more similar scope to this review scope.

Pinninti NR, Rissmiller DJ, Steer RA. Cognitive-behavioral therapy as an adjunct to second-generation antipsychotics in the treatment of schizophrenia. Psychiatr Serv. 2010 Sep;61(9):940-3. doi: 10.1176/appi.ps.61.9.940. PMID: 20810596. Study covered in a systematic review.

Pinto A, La Pia S, Mennella R, et al. Cognitive-behavioral therapy and clozapine for clients with treatment-refractory schizophrenia. Psychiatr Serv. 1999 Jul;50(7):901-4. PMID: 10402608. Study covered in a systematic review.

Pitkanen A, Valimaki M, Kuosmanen L, et al. Patient education methods to support quality of life and functional ability among patients with schizophrenia: a randomised clinical trial. Qual Life Res. 2012 Mar;21(2):247-56. PMID: 21655934. Setting ineligible for review (i.e., inpatient only).

Pitschel-Walz G, Bauml J, Bender W, et al. Psychoeducation and compliance in the treatment of schizophrenia: results of the Munich Psychosis Information Project Study. J Clin Psychiatry. 2006 Mar;67(3):443-52. PMID: 16649832. Outcome not included in review.

Pitschel-Walz G, Leucht S, Bauml J, et al. The effect of family interventions on relapse and rehospitalization in schizophrenia--a meta-analysis. Schizophr Bull. 2001;27(1):73-92. PMID: 11215551. Excluded due to including a review that was more recent, more comprehensive, or had a more similar scope to this review scope.

Poletti S, Anselmetti S, Bechi M, et al. Computer-aided neurocognitive remediation in schizophrenia: durability of rehabilitation outcomes in a follow-up study. Neuropsychol Rehabil. 2010 Oct;20(5):659-74. doi: 10.1080/09602011003683158. PMID: 20661811. Interventions evaluated in a single study.

Pompili M, Lester D, Dominici G, et al. Indications for electroconvulsive treatment in schizophrenia: a systematic review. Schizophr Res. 2013 May;146(1-3):1-9. doi: 10.1016/j.schres.2013.02.005. PMID: 23499244. Intervention not included in review.

Pontes LMM, Martins CB, Napolitano IC, et al. Cognitive training for schizophrenia in developing countries: a pilot trial in Brazil. Schizophr Res Treatment. 2013 PMID: 24288608. Country not eligible.

Posner CM, Wilson KG, Kral MJ, et al. Family psychoeducational support groups in schizophrenia. Am J Orthopsychiatry. 1992 Apr;62(2):206-18. PMID: 1580340. Study covered in a systematic review.

Potkin SG, Kimura T, Guarino J. A 6-week, double-blind, placebo- and haloperidol-controlled, phase II study of lurasidone in patients with acute schizophrenia. Ther Adv Psychopharmacol. 2015;5(6):322-31. PMID: 26834965. Inadequate duration ( $<12$ weeks).

Potkin SG, Phiri P, Szegedi A, et al. Long-term effects of asenapine or olanzapine in patients with persistent negative symptoms of schizophrenia: a pooled analysis. Schizophr Res. 2013 Nov;150(2-3):442-9. doi: 10.1016/j.schres.2013.08.024. PMID: 24075603. Excluded due to including a review that was more recent, more comprehensive, or had a more similar scope to this review scope.

Potkin SG, Preskorn S, Hochfeld M, et al. A thorough QTc study of 3 doses of iloperidone including metabolic inhibition via CYP2D6 and/or CYP3A4 and a comparison to quetiapine and ziprasidone. J Clin Psychopharmacol. 2013 Feb;33(1):3-10. PMID: 23277250. Study design not included in review. 
Potkin SG, Raoufinia A, Mallikaarjun S, et al. Safety and tolerability of once monthly aripiprazole treatment initiation in adults with schizophrenia stabilized on selected atypical oral antipsychotics other than aripiprazole. Curr Med Res Opin. 2013 Oct;29(10):1241-51. doi: 10.1185/03007995.2013.821973. PMID: 23822566. Study design not included in review.

Poulet E, Haesebaert F, Saoud M, et al. Treatment of shizophrenic patients and rTMS. Psychiatr Danub. 2010 Nov;22 Suppl 1:S143-6. PMID: 21057425. Intervention not included in review.

Power P, Iacoponi E, Reynolds N, et al. The Lambeth Early Onset Crisis Assessment Team Study: general practitioner education and access to an early detection team in firstepisode psychosis. Br J Psychiatry. 2007 Dec;51:s133-9. doi: 10.1192/bjp.191.51.s133. PMID: 18055931. Intervention not included in review.

Pratt SI, Mueser KT, Bartels SJ, et al. The impact of skills training on cognitive functioning in older people with serious mental illness. Am J Geriatr Psychiatry. 2013 Mar;21(3):242-50. doi: 10.1097/JGP.0b013e31826682dd. PMID: 23395191. Not a study.

Premkumar P, Peters ER, Fannon D, et al. Coping styles predict responsiveness to cognitive behaviour therapy in psychosis. Psychiatry Res. 2011 May 30;187(3):354-62. doi: 10.1016/j.psychres.2010.12.029. PMID: 21262541. Study design not included in review.

Priebe S. Social outcomes in schizophrenia. Br J Psychiatry. 2007 Aug;50:s15-20. PMID: 18019039. Not a study.

Priebe S, Broker M. Prediction of hospitalizations by schizophrenia patients' assessment of treatment: an expanded study. J Psychiatr Res. 1999 Mar-Apr;33(2):113-9. doi: 10.1016/S0022-3956\%2898\%2900037-5. PMID: 10221743. Outcome not included in review.

Priebe S, Gruyters T. A pilot trial of treatment changes according to schizophrenic patients' wishes. J Nerv Ment Dis. 1999 Jul;187(7):441-3. PMID: 10426465. Intervention not included in review.

Priebe S, Kelley L, Omer S, et al. The effectiveness of a patient-centred assessment with a solution-focused approach (DIALOG+) for patients with psychosis: a pragmatic clusterrandomised controlled trial in community care. Psychother Psychosom. 2015;84(5):304-13. doi: 10.1159/000430991. PMID: 26278784. Interventions evaluated in a single study.

Priebe S, McCabe R, Bullenkamp J, et al. Structured patient-clinician communication and 1-year outcome in community mental healthcare: cluster randomised controlled trial. Br J Psychiatry. 2007 Nov;191:420-6. PMID: 17978322. Interventions evaluated in a single study.

Priebe S, Savill M, Reininghaus U, et al. Effectiveness and cost-effectiveness of body psychotherapy in the treatment of negative symptoms of schizophrenia--a multi-centre randomised controlled trial. BMC Psychiatry. 2013;13:26. doi: 10.1186/1471-244X-13-26. PMID: 23317474. Intervention not included in review.

Prikryl R, Kasparek T, Skotakova S, et al. Treatment of negative symptoms of schizophrenia using repetitive transcranial magnetic stimulation in a double-blind, randomized controlled study.[Erratum appears in Schizophr Res. 2008 Feb;99(1-3):380-1]. Schizophr Res. 2007 Sep;95(1-3):151-7. PMID: 17689931. Inadequate duration (<12 weeks).

Prikryl R, Ustohal L, Prikrylova Kucerova H, et al. A detailed analysis of the effect of repetitive transcranial magnetic stimulation on negative symptoms of schizophrenia: a double-blind trial. Schizophr Res. 2013 Sep;149(1-3):167-73. PMID: 23810122. Intervention not included in review.

Puig O, Penades R, Baeza I, et al. Cognitive remediation therapy in adolescents with early-onset schizophrenia: a randomized controlled trial. J Am Acad Child Adolesc Psychiatry. 2014 Aug;53(8):859-68. doi: 10.1016/j.jaac.2014.05.012. PMID: 25062593. Population ineligible for review.

Pyle M, Norrie J, Schwannauer M, et al. Design and protocol for the Focusing on Clozapine Unresponsive Symptoms (FOCUS) trial: A randomised controlled trial. BMC Psychiatry. 2016 Dec;16 PMID: 27496180. Study design not included in review.

Qiao Y, Yang F, Li C, et al. Add-on effects of a low-dose aripiprazole in resolving hyperprolactinemia induced by risperidone or paliperidone. Psychiatry Res. 2016;237:83-9. PMID: 26921057. Study design not included in review.

Quraishi S, David A. Depot haloperidol decanoate for schizophrenia. Cochrane Database Syst Rev. 2009(4):Cd001361. doi: 10.1002/14651858.cd001361. PMID: 10796438. Study covered in a systematic review. 
Rabany L, Deutsch L, Levkovitz Y. Double-blind, randomized sham controlled study of deep-TMS add-on treatment for negative symptoms and cognitive deficits in schizophrenia. J Psychopharmacol. 2014;28(7):686-90. PMID: 24829210. Inadequate duration (<12 weeks).

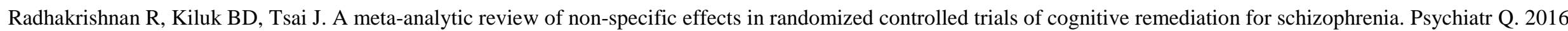
Mar;87(1):57-62. doi: 10.1007/s11126-015-9362-6. PMID: 25952944. Outcome not included in review.

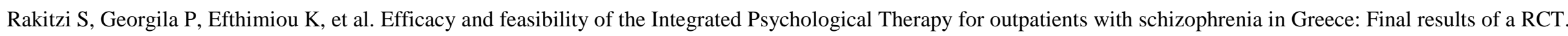
Psychiatry Res. 2016 Aug;242:137-43. doi: 10.1016/j.psychres.2016.05.039. PMID: 27280523. Sample size too small (<50).

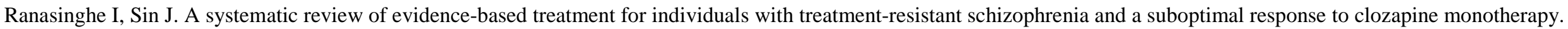

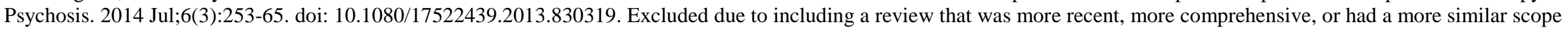
to this review scope.

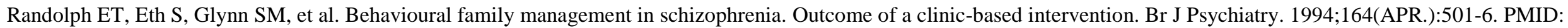
8038939. Study covered in a systematic review.

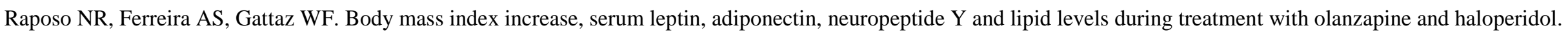
Pharmacopsychiatry. 2011 Jul;44(5):169-72. doi: 10.1055/s-0031-1280793. PMID: 21732272. Study design not included in review.

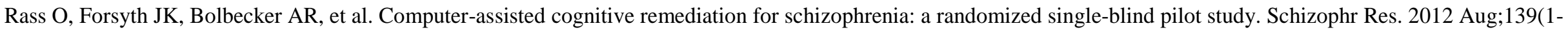
3):92-8. doi: 10.1016/j.schres.2012.05.016. PMID: 22682988. Sample size too small $(<50)$.

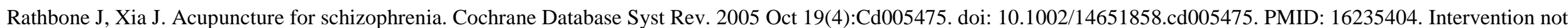
included in review.

Rathod S, Kingdon D, Weiden P, et al. Cognitive-behavioral therapy for medication-resistant schizophrenia: a review. J Psychiatr Pract. 2008 Jan;14(1):22-33. doi: 10.1097/01.pra.0000308492.93003.db. PMID: 18212600. Not a study.

Razali SM, Hasanah CI, Khan UA, et al. Psychosocial interventions for schizophrenia. J Ment Health. 2000;9(3):283-9. Comparator not included in review.

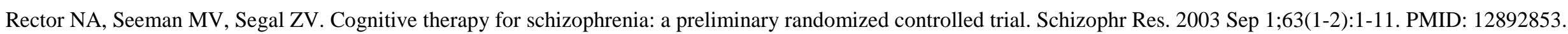
Study covered in a systematic review.

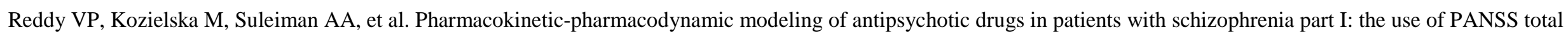
score and clinical utility. Schizophr Res. 2013 May;146(1-3):144-52. doi: 10.1016/j.schres.2013.02.011. PMID: 23473810. Study design not included in review.

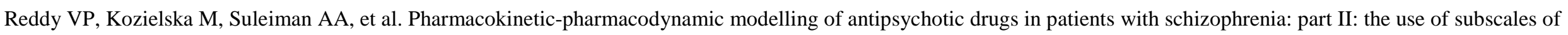
the PANSS score. Schizophr Res. 2013 May;146(1-3):153-61. doi: 10.1016/j.schres.2013.02.010. PMID: 23473811. Study design not included in review.

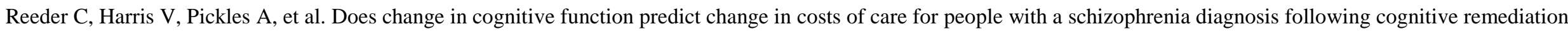
therapy? Schizophr Bull. 2014 Nov;40(6):1472-81. doi: 10.1093/schbul/sbu046. PMID: 24682210. Outcome not included in review.

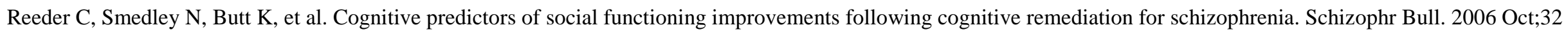
Suppl 1:S123-31. PMID: 16901950. Outcome not included in review.

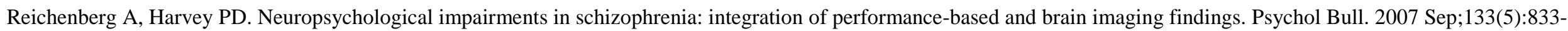
58. doi: 10.1037/0033-2909.133.5.833. PMID: 17723032. Not a study.

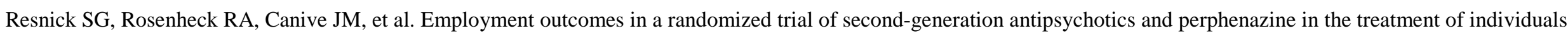
with schizophrenia. J Behav Health Serv Res. 2008 Apr;35(2):215-25. PMID: 18246429. Study covered in a systematic review. 


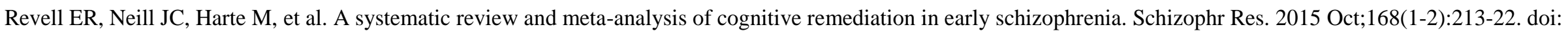
10.1016/j.schres.2015.08.017. PMID: 26305063. Comparator not included in review.

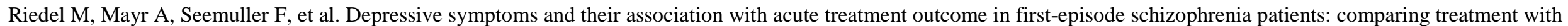

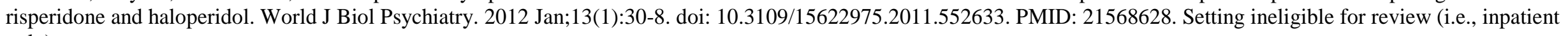
only).

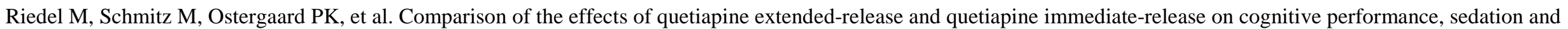

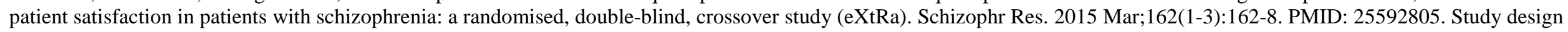
not included in review.

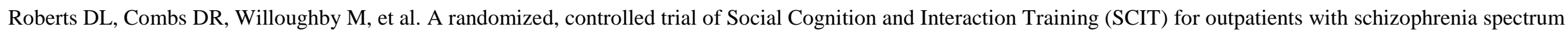
disorders. Br J Clin Psychol. 2014 Sep;53(3):281-98. doi: 10.1111/bjc.12044. PMID: 24417608. Interventions evaluated in a single study.

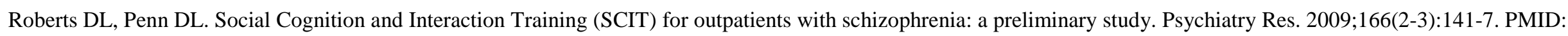
19272654. Study design not included in review.

Rocha NB, Queiros C. Metacognitive and social cognition training (MSCT) in schizophrenia: a preliminary efficacy study. Schizophr Res. 2013 Oct;150(1):64-8. doi: 10.1016/j.schres.2013.07.057. PMID: 23962827. Inadequate duration (<12 weeks).

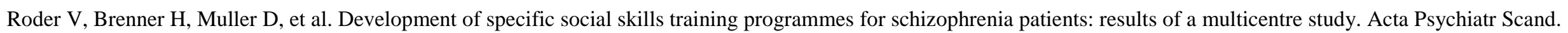
2002 May;105(5):363-71. doi: 10.1034/j.1600-0447.2002.10214.x. PMID: 11942943. Comparator not included in review.

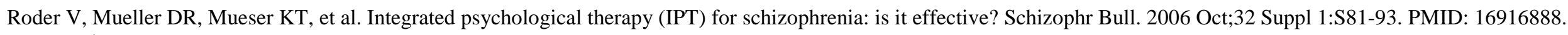
Not a study.

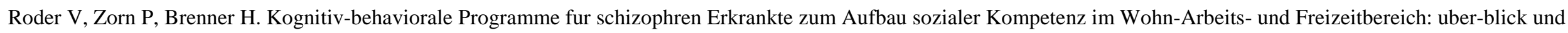
empirische Ergebnisse. Verhaltenstherapie und psychosoziale Praxis 2000;32:195-211. Not English language.

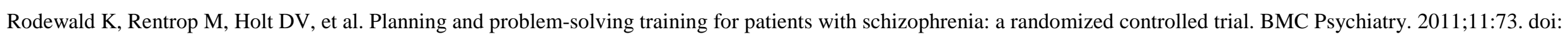
10.1186/1471-244X-11-73. PMID: 21527028. Comparator not included in review.

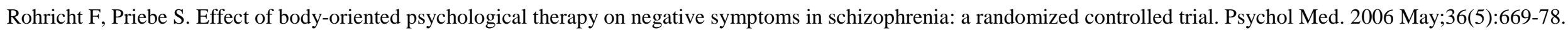
PMID: 16608559. Intervention not included in review.

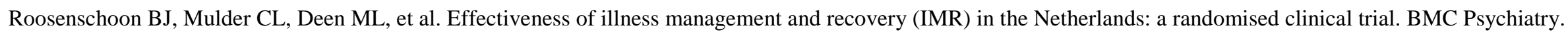
2016;16(1) PMID: 26995361. Systematic review/meta-analysis used as a source document to identify individual studies.

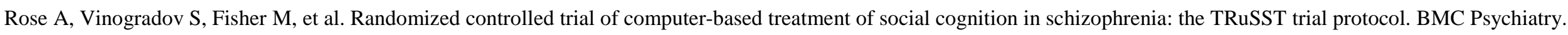
2015;15(142) PMID: 26138715. Study design not included in review.

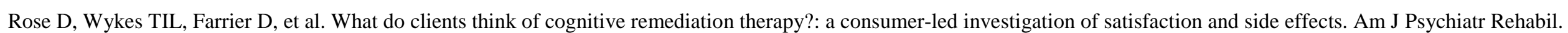
2008 2008/04/10;11(2):181-204. doi: 10.1080/15487760801963694. Study design not included in review.

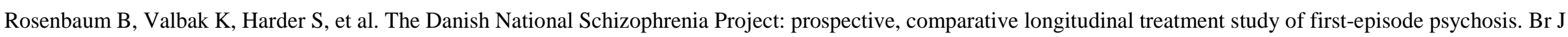
Psychiatry. 2005;186(MAY):394-9. PMID: 15863743. Study design not included in review.

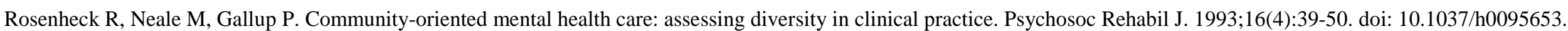
Study design not included in review.

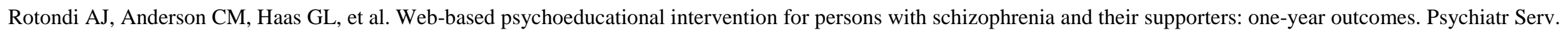
2010 Nov;61(11):1099-105. doi: 10.1176/appi.ps.61.11.1099. PMID: 21041348. Interventions evaluated in a single study. 
Royer A, Grosselin A, Bellot C, et al. Is there any impact of cognitive remediation on an ecological test in schizophrenia? Cogn Neuropsychiatry. 2012;17(1):19-35. doi: 10.1080/13546805.2011.564512. PMID: 21707472. Outcome not included in review.

Ruberg SJ, Chen L, Stauffer V, et al. Identification of early changes in specific symptoms that predict longer-term response to atypical antipsychotics in the treatment of patients with schizophrenia. BMC Psychiatry. 2011;11 PMID: 21306626. Study design not included in review.

Ruddy R, Milnes D. Art therapy for schizophrenia or schizophrenia-like illnesses. Cochrane Database Syst Rev. 2005(4):CD003728. PMID: 16235338. Intervention not included in review.

Rus-Calafell M, Gutierrez-Maldonado J, Ortega-Bravo M, et al. A brief cognitive-behavioural social skills training for stabilised outpatients with schizophrenia: a preliminary study. Schizophr Res. 2013 Feb;143(2-3):327-36. doi: 10.1016/j.schres.2012.11.014. PMID: 23235141. Sample size too small (<50).

Rybakowski JK, Vansteelandt K, Remlinger-Molenda A, et al. Extrapyramidal symptoms during treatment of first schizophrenia episode: results from EUFEST. Eur Neuropsychopharmacol. 2014;24(9):1500-5. PMID: 25085534. Study covered in a systematic review.

Rybakowski JK, Vansteelandt K, Szafranski T, et al. Treatment of depression in first episode of schizophrenia: results from EUFEST. Eur Neuropsychopharmacol. 2012 Dec;22(12):875-82. doi: 10.1016/j.euroneuro.2012.04.001. PMID: 22627166. Outcome not included in review.

Sachs G, Winklbaur B, Jagsch R, et al. Training of affect recognition (TAR) in schizophrenia--impact on functional outcome. Schizophr Res. 2012 Jul;138(2-3):262-7. PMID: 22464728. Inadequate duration ( $<12$ weeks).

Sajatovic M, Forester BP, Tsai J, et al. Efficacy of lurasidone in adults aged 55 years and older with bipolar depression: post hoc analysis of 2 double-blind, placebo-controlled studies. J Clin Psychiatry. 2016 Aug 16doi: 10.4088/JCP.15m10261. PMID: 27529375. Population ineligible for review.

Sajatovic M, Levin J, Ramirez LF, et al. A prospective trial of customized adherence enhancement plus long-acting injectable antipsychotic medication in homeless or recently homeless individuals with schizophrenia or schizoaffective disorder. J Clin Psychiatry. 2013;74(12):1249-55. doi: 10.4088/JCP.12m08331. PMID: 24434094. Study design not included in review.

Sakurai H, Bies RR, Stroup ST, et al. Dopamine D2 receptor occupancy and cognition in schizophrenia: analysis of the CATIE data. Schizophr Bull. 2013 May;39(3):564-74. doi: 10.1093/schbul/sbr189. PMID: 22290266. Study design not included in review.

Salkever D, Gibbons B, Ran X. Do comprehensive, coordinated, recovery-oriented services alter the pattern of use of treatment services? Mental health treatment study impacts on SSDI beneficiaries' use of inpatient, emergency, and crisis services.[Erratum appears in J Behav Health Serv Res. 2014 Oct;41(4):559]. J Behav Health Serv Res. 2014 Oct;41(4):434-46. doi: 10.1007/s11414-013-9388-1. PMID: 24481541. Outcome not included in review.

Salyers MP, McGuire AB, Kukla M, et al. A randomized controlled trial of illness management and recovery with an active control group. Psychiatr Serv. 2014 Aug 1;65(8):100511. PMID: 24733680. Comparator not included in review.

Sampford JR, Sampson S, Li BG, et al. Fluphenazine (oral) versus atypical antipsychotics for schizophrenia. Cochrane Database Syst Rev. 2016 Jul 02;7:CD010832. doi: 10.1002/14651858.CD010832.pub2. PMID: 27370402. Excluded due to including a review that was more recent, more comprehensive, or had a more similar scope to this review scope.

Sampson S, Hosalli P, Furtado VA, et al. Risperidone (depot) for schizophrenia. Cochrane Database Syst Rev. 2016 Apr 14;4:Cd004161. doi: 10.1002/14651858.CD004161.pub2. PMID: 27078222. Study covered in a systematic review.

Sampson S, Mansour M, Maayan N, et al. Intermittent drug techniques for schizophrenia. Cochrane Database Syst Rev. 2013 Jul 20(7):Cd006196. doi: 10.1002/14651858.CD006196.pub2. PMID: 23881657. Study design not included in review.

Saperstein AM, Fiszdon JM, Bell MD. Intrinsic motivation as a predictor of work outcome after vocational rehabilitation in schizophrenia. J Nerv Ment Dis. 2011 Sep;199(9):6727. doi: 10.1097/NMD.0b013e318229d0eb. PMID: 21878781. Study design not included in review. 
Sarin F, Wallin L. Cognitive model and cognitive behavior therapy for schizophrenia: an overview. Nord J Psychiatry. 2014 Apr;68(3):145-53. doi: 10.3109/08039488.2013.789074. PMID: 24627960. Not a study.

Sarin F, Wallin L, Widerlov B. Cognitive behavior therapy for schizophrenia: a meta-analytical review of randomized controlled trials. Nord J Psychiatry. 2011 Jun;65(3):162-74. doi: 10.3109/08039488.2011.577188. PMID: 21563994. Excluded due to including a review that was more recent, more comprehensive, or had a more similar scope to this review scope.

Sato S, Iwata K, Furukawa SI, et al. The effects of the combination of cognitive training and supported employment on improving clinical and working outcomes for people with schizophrenia in Japan. Clin Pract Epidemiol Ment Health. 2014;10(1):18-27. PMID: 24600481. Study design not included in review.

Savitz AJ, Lane R, Nuamah I, et al. Efficacy and safety of paliperidone extended release in adolescents with schizophrenia: a randomized, double-blind study. J Am Acad Child Adolesc Psychiatry. 2015;54(2):126-37.e1. PMID: 25617253. Population ineligible for review.

Schennach-Wolff R, Jager M, Mayr A, et al. Predictors of response and remission in the acute treatment of first-episode schizophrenia patients--is it all about early response? Eur Neuropsychopharmacol. 2011 May;21(5):370-8. doi: 10.1016/j.euroneuro.2010.10.003. PMID: 21255982. Study design not included in review.

Schimmelmann BG, Schmidt SJ, Carbon M, et al. Treatment of adolescents with early-onset schizophrenia spectrum disorders: in search of a rational, evidence-informed approach. Curr Opin Psychiatry. 2013 Mar;26(2):219-30. doi: 10.1097/YCO.0b013e32835dcc2a. PMID: 23364281. Population ineligible for review.

Schmidt HM, Hagen M, Kriston L, et al. Management of sexual dysfunction due to antipsychotic drug therapy. Cochrane Database Syst Rev. 2012 Nov 14;11:Cd003546. doi: 10.1002/14651858.CD003546.pub3. PMID: 23152218. Study design not included in review.

Schmidt-Posner J, Jerrell JM. Qualitative analysis of three case management programs. Community Ment Health J. 1998 Aug;34(4):381-92. PMID: 9693866. Study design not included in review.

Schnell T, Koethe D, Krasnianski A, et al. Ziprasidone versus clozapine in the treatment of dually diagnosed (DD) patients with schizophrenia and cannabis use disorders: a randomized study. Am J Addict. 2014 May-Jun;23(3):308-12. doi: 10.1111/j.1521-0391.2014.12126.x. PMID: 24628830. Sample size too small (<50).

Schottle D, Schimmelmann BG, Karow A, et al. Effectiveness of integrated care including therapeutic assertive community treatment in severe schizophrenia spectrum and bipolar I disorders: the 24-month follow-up ACCESS II study. J Clin Psychiatry. 2014 Dec;75(12):1371-9. PMID: 25188752. Study design not included in review.

Schreiner A, Aadamsoo K, Altamura AC, et al. Paliperidone palmitate versus oral antipsychotics in recently diagnosed schizophrenia. Schizophr Res. 2015;169(1-3):393-9. doi: 10.1016/j.schres.2015.08.015. PMID: 26431793. Comparator not included in review.

Schulz M, Gray R, Spiekermann A, et al. Adherence therapy following an acute episode of schizophrenia: a multi-centre randomised controlled trial. Schizophr Res. 2013 May;146(1-3):59-63. doi: 10.1016/j.schres.2013.01.028. PMID: 23452506. Interventions evaluated in a single study.

Scogin F, Morthland M, Kaufman A, et al. Improving quality of life in diverse rural older adults: a randomized trial of a psychological treatment. Psychol Aging 2007 Dec;22(4):657-65. doi: 10.1037/0882-7974.22.4.657. PMID: 18179286. Population ineligible for review.

Secher RG, Nordentoft M, Austin S, et al. The OPUS trial: Intensive, early, psychosocial intervention versus treatment as usual for people with a first episode within the schizophrenic spectrum. Results from the 10-year follow-up. Early Interv Psychiatry. 2012;6(21). Not a study.

Seeman MV. Clinic versus private treatment of psychosis. J Clin Psychiatry. 1978 Mar;39(3):213-5. PMID: 204624. Intervention not included in review.

Seemuller F, Schennach R, Mayr A, et al. Akathisia and suicidal ideation in first-episode schizophrenia. J Clin Psychopharmacol. 2012 Oct;32(5):694-8. PMID: 22926606. Study design not included in review.

Sengul MCB, Kenar ANI, Hanci E, et al. Practice of acute and maintenance electroconvulsive therapy in the psychiatric clinic of a university hospital from Turkey: between 2007 and 2013. Clin Psychopharmacol Neurosci. 2016;14(1):57-63. PMID: 26792041. Study design not included in review. 


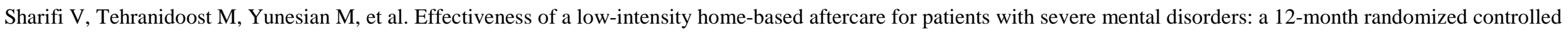
study. Community Ment Health J. 2012 Dec;48(6):766-70. PMID: 22772746. Intervention not included in review.

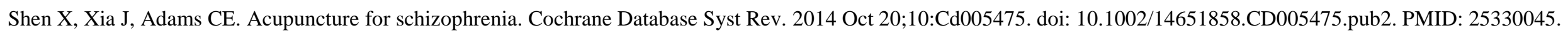
Intervention not included in review.

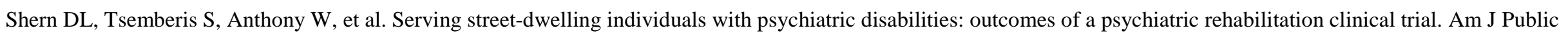
Health. 2000 Dec;90(12):1873-8. PMID: 11111259. Interventions evaluated in a single study.

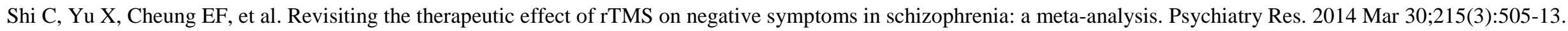
doi: 10.1016/j.psychres.2013.12.019. PMID: 24411074. Intervention not included in review.

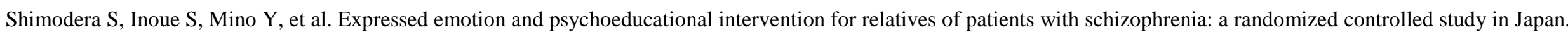
Psychiatry Res. 2000 Oct 30;96(2):141-8. PMID: 11063786. Outcome not included in review.

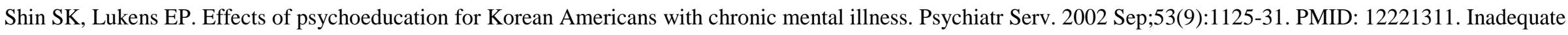
duration (<12 weeks).

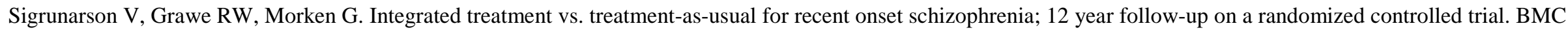
Psychiatry. 2013;13:200. doi: 10.1186/1471-244X-13-200. PMID: 23898805. Sample size too small $(<50)$.

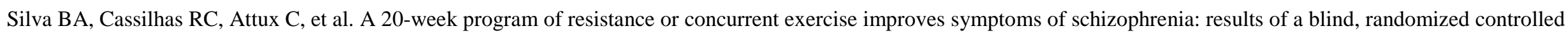
trial. Rev Bras Psiquiatr. 2015 Oct-Dec;37(4):271-9. doi: 10.1590/1516-4446-2014-1595. PMID: 26375919. Intervention not included in review.

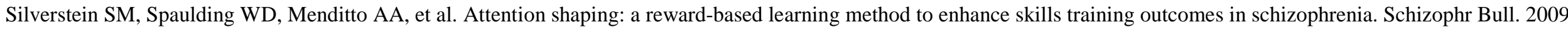
Jan;35(1):222-32. doi: 10.1093/schbul/sbm150. PMID: 18212327. Intervention not included in review.

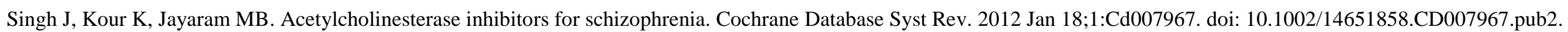
PMID: 22258978. Intervention not included in review.

Sivaraman P, Rattehalli RD, Jayaram MB. Levomepromazine for schizophrenia. Cochrane Database Syst Rev. 2010 Oct 06(10):Cd007779. doi: 10.1002/14651858.CD007779.pub2. PMID: 20927765. Intervention not included in review.

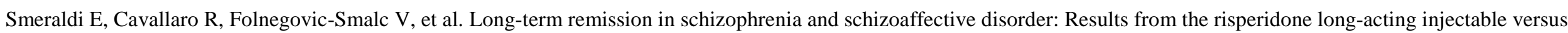
quetiapine relapse prevention trial (ConstaTRE). Ther Adv Psychopharmacol. 2013;3(4):191-9. PMID: 24167692 Outcome not included in review.

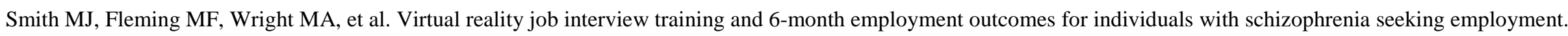
Schizophr Res. 2015 Aug;166(1-3):86-91. doi: 10.1016/j.schres.2015.05.022. PMID: 26032567. Interventions evaluated in a single study.

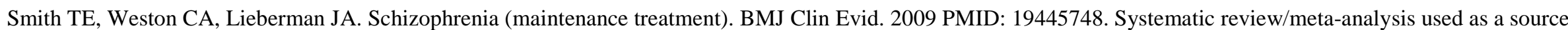
document to identify individual studies.

Soares-Weiser K, Bechard-Evans L, Lawson AH, et al. Time to all-cause treatment discontinuation of olanzapine compared to other antipsychotics in the treatment of

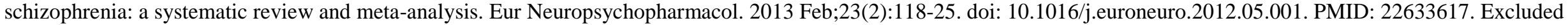
due to including a review that was more recent, more comprehensive, or had a more similar scope to this review scope.

Solomon P. Moving from psychoeducation to family education for families of adults with serious mental illness. Psychiatr Serv. 1996 Dec;47(12):1364-70. doi:

10.1176/ps.47.12.1364. PMID: 9117476. Not a study.

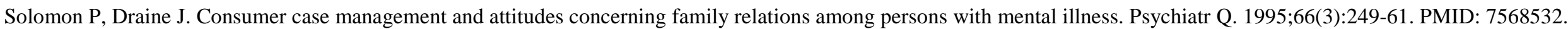
Outcome not included in review. 
Solomon P, Draine J. One-year outcomes of a randomized trial of case management with seriously mentally ill clients leaving jail. Eval Rev. 1995;19(3):256-73. doi: 10.1177/0193841X9501900302. Study covered in a systematic review.

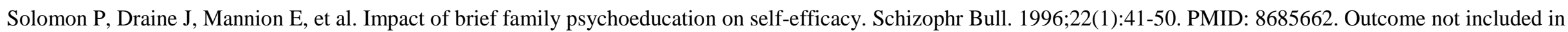
review.

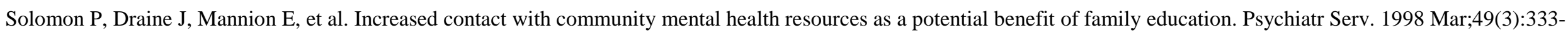
9. PMID: 9525792. Not a study.

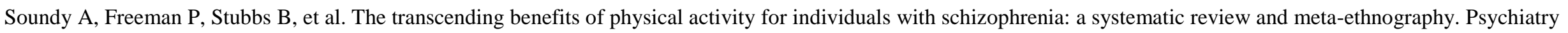
Res. 2014 Dec 15;220(1-2):11-9. doi: 10.1016/j.psychres.2014.07.083. PMID: 25149128. Intervention not included in review.

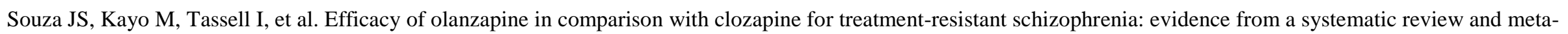

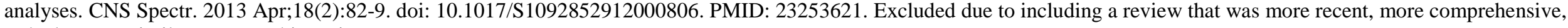
or had a more similar scope to this review scope.

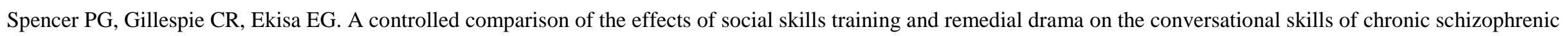
inpatients. Br J Psychiatry. 1983;143:165-72. PMID: 6351955. Setting ineligible for review (i.e., inpatient only).

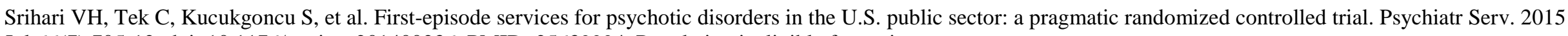
Jul;66(7):705-12. doi: 10.1176/appi.ps.201400236. PMID: 25639994. Population ineligible for review.

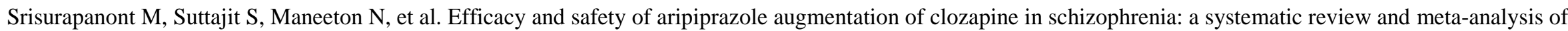
randomized-controlled trials. J Psychiatr Res. 2015 Mar;62:38-47. doi: 10.1016/j.jpsychires.2015.01.004. PMID: 25619176. Comparator not included in review.

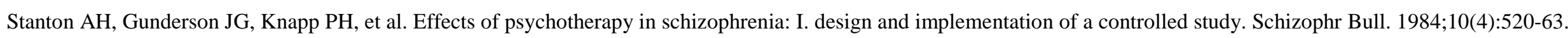
PMID: 6151245. Interventions evaluated in a single study.

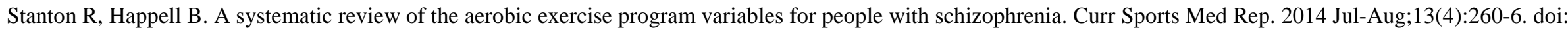
10.1249/JSR.0000000000000069. PMID: 25014392. Intervention not included in review.

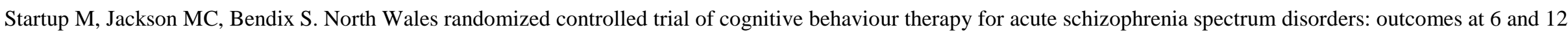
months. Psychol Med. 2004 Apr;34(3):413-22. PMID: 15259826. Population ineligible for review.

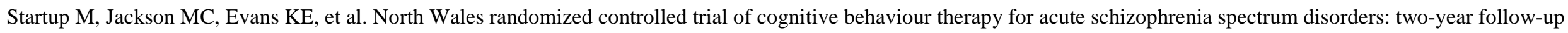
and economic evaluation. Psychol Med. 2005 Sep;35(9):1307-16. PMID: 16168153. Study covered in a systematic review.

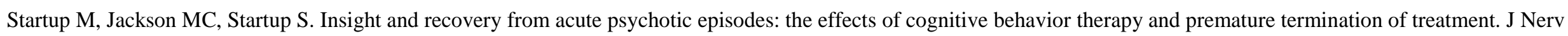
Ment Dis. 2006;194(10):740-5. PMID: 17041285. Outcome not included in review.

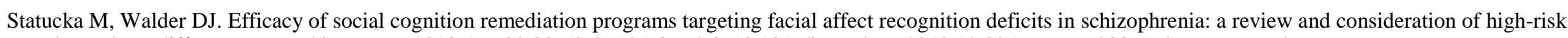
samples and sex differences. Psychiatry Res. 2013 Apr 30;206(2-3):125-39. doi: 10.1016/j.psychres.2012.12.005. PMID: 23375627. Not a study.

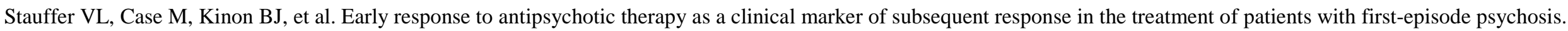
Psychiatry Res. 2011 May 15;187(1-2):42-8. PMID: 21168920. Study design not included in review.

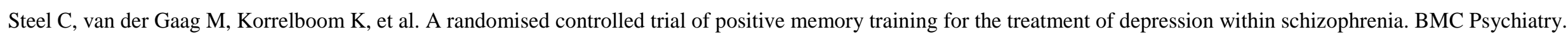
2015;15:85. doi: 10.1186/s12888-015-0453-6. PMID: 25886265. Not a study.

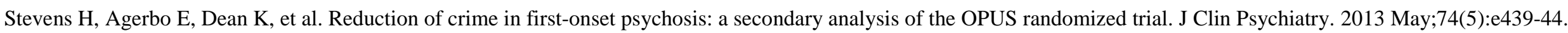
doi: 10.4088/JCP.12m08156. PMID: 23759464. Study design not included in review. 
Stikkelbroek Y, Bodden DHM, Dekovic M, et al. Effectiveness and cost effectiveness of cognitive behavioral therapy (CBT) in clinically depressed adolescents: individual CBT versus treatment as usual (TAU). BMC Psychiatry. 2013;13 PMID: 24261913. Population ineligible for review.

Stip E, Vincent PD, Sablier J, et al. A randomized controlled trial with a canadian electronic pill dispenser used to measure and improve medication adherence in patients with schizophrenia. Front Pharmacol. 2013;4 PMID: 23950746. Intervention not included in review.

Strkalj-Ivezic S, Vrdoljak M, Muzinic L, et al. The impact of a rehabilitation day centre program for persons suffering from schizophrenia on quality of life, social functioning and self-esteem. Psychiatr Danub. 2013 Sep;25 Suppl 2:S194-9. PMID: 23995175. Intervention not included in review.

Stubbs B, Rosenbaum S, Ward PB, et al. No evidence of a control group response in exercise randomised controlled trials in people with schizophrenia: a systematic review and meta-analysis. Psychiatry Res. 2015 Oct 30;229(3):840-3. doi: 10.1016/j.psychres.2015.07.081. PMID: 26254799. Comparator not included in review.

Subramaniam K, Luks T, Aldebot S, et al. Neuroplasticity-based cognitive training improves reality monitoring in schizophrenia patients: behavioral and fmri assessments. Schizophr Res. 2010;117(2-3):471. Not a study.

Subramaniam K, Luks TL, Garrett C, et al. Intensive cognitive training in schizophrenia enhances working memory and associated prefrontal cortical efficiency in a manner that drives long-term functional gains. Neuroimage. 2014 Oct 1;99:281-92. doi: 10.1016/j.neuroimage.2014.05.057. PMID: 24867353. Comparator not included in review.

Sungur M, Soygur H, Guner P, et al. Identifying an optimal treatment for schizophrenia: a 2-year randomized controlled trial comparing integrated care to a high-quality routine treatment. Int J Psychiatry Clin Pract. 2011 Jun;15(2):118-27. doi: 10.3109/13651501.2011.554987. PMID: 22121860. Interventions evaluated in a single study.

Sungur MZ, Guner P, Ustun B, et al. Optimal treatment project for schizophrenia: results from a randomized, controlled, longitudinal study. Seishin Shinkeigaku Zasshi 2003;105(9):1175-80. PMID: 14639941. Interventions evaluated in a single study.

Sunovion. A Twelve Week, Open Label Extension Study in Patients With Schizophrenia [Study completed November 2013; unpublished extension of NCT00044044]. In: ClinicalTrials.gov. Bethesda, MD: National Library of Medicine; 2000-. https://clinicaltrials.gov/ct2/show/study/NCT01566162 Accessed February 6, 2017. NLM Identifier: NCT01566162. Study design not included in review.

Sunovion. A Randomized, Open-Label, Dose-Blinded, Multicenter, 6-Month Study of Safety and Tolerability of 3 Dose Levels of lurasidone in Patients With Schizophrenia [Study completed November 2013; unpublished extension of NCT00044044]. In: ClinicalTrials.gov. Bethesda, MD: National Library of Medicine; 2000-.

https://clinicaltrials.gov/ct2/show/study/NCT00044005. Accessed Accessed February 6, 2017. NLM Identifier: NCT00044005. Study design not included in review.

Suttajit S, Srisurapanont M, Xia J, et al. Quetiapine versus typical antipsychotic medications for schizophrenia. Cochrane Database Syst Rev. 2013 May 31(5):Cd007815. doi: 10.1002/14651858.CD007815.pub2. PMID: 23728667. Excluded due to including a review that was more recent, more comprehensive, or had a more similar scope to this review scope.

Suzuki H, Gen K. The influence of switching from haloperidol decanoate depot to risperidone long-acting injection on the clinical symptoms and cognitive function in schizophrenia. Hum Psychopharmacol. 2012 Sep;27(5):470-5. doi: 10.1002/hup.2249. PMID: 23001955. Study design not included in review.

Suzuki H, Gen K, Inoue Y, et al. The influence of switching from risperidone to paliperidone on the extrapyramidal symptoms and cognitive function in elderly patients with schizophrenia: A preliminary open-label trial. Int J Psychiatry Clin Pract. 2014 Jan;18(1):58-62. doi: 10.3109/13651501.2013.845218. PMID: 24047427. Study design not included in review.

Szmukler GI, Herrman H, Colusa S, et al. A controlled trial of a counselling intervention for caregivers of relatives with schizophrenia. Soc Psychiatry Psychiatr Epidemiol. 1996 Jun;31(3-4):149-55. PMID: 8766460. Intervention not included in review.

Takacs R, Ivanyi Z, Ungvari GS, et al. Safety of the electroconvulsive therapy and amisulpride combination. Psychiatr Danub. 2013;25(1):76-9. PMID: 23470610 Study design not included in review.

Takahashi H, Sassa T, Shibuya T, et al. Effects of sports participation on psychiatric symptoms and brain activations during sports observation in schizophrenia. Transl Psychiatry. 2012;2:e96. doi: 10.1038/tp.2012.22. PMID: 22832861. Study design not included in review. 
Takekita Y, Fabbri C, Kato M, et al. Antagonist and partial agonist at the dopamine D2 receptors in drug-naive and non-drug-naive schizophrenia: a randomized, controlled trial. Eur Arch Psychiatry Clin Neurosci. 2015;265(7):579-88. PMID: 26016467. Comparator not included in review.

Takeuchi H, Fervaha G, Lee J, et al. Effectiveness of different dosing regimens of risperidone and olanzapine in schizophrenia. Eur Neuropsychopharmacol. 2015 Mar;25(3):295302. doi: 10.1016/j.euroneuro.2014.12.008. PMID: 25649680. Outcome not included in review.

Takeuchi H, Fervaha G, Remington G. Effect of antipsychotic dosing regimen on neurocognition in schizophrenia. J Clin Psychopharmacol. 2015 Dec;35(6):728-30. doi: 10.1097/JCP.0000000000000424. PMID: 26479222. Comparator not included in review.

Takeuchi H, Fervaha G, Uchida H, et al. Impact of once- versus twice-daily perphenazine dosing on clinical outcomes: an analysis of the CATIE data. J Clin Psychiatry. 2014;75(5):506-11. PMID: 24569099. Comparator not included in review.

Takeuchi H, Suzuki T, Bies RR, et al. Dose reduction of risperidone and olanzapine and estimated dopamine D2 receptor occupancy in stable patients with schizophrenia: findings from an open-label, randomized, controlled study. J Clin Psychiatry. 2014 Nov;75(11):1209-14. doi: 10.4088/JCP.13m08841. PMID: 25099201. Outcome not included in review.

Takeuchi H, Suzuki T, Remington G, et al. Effects of risperidone and olanzapine dose reduction on cognitive function in stable patients with schizophrenia: an open-label, randomized, controlled, pilot study. Schizophr Bull. 2013 Sep;39(5):993-8. doi: 10.1093/schbul/sbt090. PMID: 23821768. Study design not included in review.

Takeuchi H, Suzuki T, Remington G, et al. Lack of effect of risperidone or olanzapine dose reduction on subjective experiences in stable patients with schizophrenia. Psychiatry Res. 2014 Aug 15;218(1-2):244-6. doi: 10.1016/j.psychres.2014.04.019. PMID: 24768247. Study design not included in review.

Tan BL, King R. The effects of cognitive remediation on functional outcomes among people with schizophrenia: a randomised controlled study. Aust N Z J Psychiatry. 2013 Nov;47(11):1068-80. doi: 10.1177/0004867413493521. PMID: 23785044. Country not eligible.

Tan S, Zou Y, Wykes T, et al. Group cognitive remediation therapy for chronic schizophrenia: A randomized controlled trial. Neurosci Lett. 2016 Jul 28;626:106-11. doi: 10.1016/j.neulet.2015.08.036. PMID: 26314508. Country not eligible.

Tardy M, Huhn M, Engel RR, et al. Fluphenazine versus low-potency first-generation antipsychotic drugs for schizophrenia. Cochrane Database Syst Rev. 2014 Aug 03(8):Cd009230. doi: 10.1002/14651858.CD009230.pub2. PMID: 25087165. Study covered in a systematic review.

Tarrier N, Barrowclough C, Vaughn C, et al. The community management of schizophrenia: a controlled trial of a behavioural intervention with families to reduce relapse. Br J Psychiatry. 1988 Oct;153:532-42. doi: 10.1192/bjp.153.4.532. PMID: 3074860. Study covered in a systematic review.

Tarrier N, Barrowclough C, Vaughn C, et al. Community management of schizophrenia: a two-year follow-up of a behavioral intervention with families. Br J Psychiatry. 1989 May;154:625-8. doi: 10.1192/bjp.154.5.625. PMID: 2597854. Study covered in a systematic review.

Tarrier N, Beckett R, Harwood S, et al. A trial of two cognitive-behavioural methods of treating drug-resistant residual psychotic symptoms in schizophrenic patients: I. Outcome. Br J Psychiatry. 1993 Apr;162:524-32. doi: 10.1192/bjp.162.4.524. PMID: 8481745. Study covered in a systematic review.

Tarrier N, Kinney C, McCarthy E, et al. Two-year follow-up of cognitive--behavioral therapy and supportive counseling in the treatment of persistent symptoms in chronic schizophrenia. J Consult Clin Psychol. 2000 Oct;68(5):917-22. PMID: 11068978. Study covered in a systematic review.

Tarrier N, Kinney C, McCarthy E, et al. Are some types of psychotic symptoms more responsive to cognitive-behavior therapy? Behav Cogn Psychother. 2001 Jan;29(1):45-55. doi: 10.1017/S1352465801001060. Study covered in a systematic review.

Tarrier N, Sharpe L, Beckett R, et al. A trial of two cognitive behavioural methods of treating drug-resistant residual psychotic symptoms in schizophrenic patients. II. Treatmentspecific changes in coping and problem-solving skills. Soc Psychiatry Psychiatr Epidemiol. 1993 Feb;28(1):5-10. PMID: 8096654. Study design not included in review.

Tarrier N, Wykes T. Is there evidence that cognitive behaviour therapy is an effective treatment for schizophrenia? A cautious or cautionary tale? Behav Res Ther. 2004 Dec;42(12):1377-401. PMID: 15500811. Not a study. 
Tas C, Danaci AE, Cubukcuoglu Z, et al. Impact of family involvement on social cognition training in clinically stable outpatients with schizophrenia -- a randomized pilot study. Psychiatry Res. 2012 Jan 30;195(1-2):32-8. doi: 10.1016/j.psychres.2011.07.031. PMID: 21831453. Interventions evaluated in a single study.

Tauber R, Wallace CJ, Lecomte T. Enlisting indigenous community supporters in skills training programs for persons with severe mental illness. Psychiatr Serv. 2000 Nov;51(11):1428-32. doi: 10.1176/appi.ps.51.11.1428. PMID: 11058191. Comparator not included in review.

Telles C, Karno M, Mintz J, et al. Immigrant families coping with schizophrenia. Behavioral family intervention v. case management with a low-income Spanish-speaking population. Br J Psychiatry. 1995 Oct;167(4):473-9. PMID: 8829715 Study covered in a systematic review.

Temple S, Ho BC. Cognitive therapy for persistent psychosis in schizophrenia: a case-controlled clinical trial. Schizophr Res. 2005 May 1;74(2-3):195-9. PMID: 15721999. Study design not included in review.

Terzian E, Tognoni G, Bracco R, et al. Social network intervention in patients with schizophrenia and marked social withdrawal: a randomized controlled study. Can J Psychiatry. 2013 Nov;58(11):622-31. PMID: 24246433. Interventions evaluated in a single study.

Tharyan P, Adams CE. Electroconvulsive therapy for schizophrenia. Cochrane Database Syst Rev. 2009(4) PMID: 15846598. Intervention not included in review.

The University of Texas Health Science Center at San Antonio. Cognitive adaptive training for improving medication adherence, symptoms, and function in people with schizophrenia ClinicalTrials.gov. 2015. Study covered in a systematic review.

Thomas N, Farhall J, Foley F, et al. Randomised controlled trial of a digitally assisted low intensity intervention to promote personal recovery in persisting psychosis: SMARTTherapy study protocol. BMC Psychiatry Vol 16 2016, ArtID 312. 2016 Dec;16 PMID: 27604363. Not a study.

Thomas N, Shawyer F, Castle DJ, et al. A randomised controlled trial of acceptance and commitment therapy (ACT) for psychosis: study protocol. BMC Psychiatry. 2014;14(1) PMID: 25015368. Study design not included in review.

Thorsen AL, Johansson K, Loberg E-M. Neurobiology of cognitive remediation therapy for schizophrenia: a systematic review. Front Psychiatry. 2014 Aug;5:103. PMID: 25177300. Outcome not included in review.

Thorup A, Albert N, Bertelsen M, et al. Gender differences in first-episode psychosis at 5-year follow-up--two different courses of disease? Results from the OPUS study at 5-year follow-up. Eur Psychiatry 2014 Jan;29(1):44-51. PMID: 23394824. Study design not included in review.

Thorup A, Petersen L, Jeppesen P, et al. Integrated treatment ameliorates negative symptoms in first episode psychosis--results from the Danish OPUS trial. Schizophr Res. 2005 Nov 1;79(1):95-105. PMID: 16122909. Study covered in a systematic review.

Thorup A, Petersen L, Jeppesen P, et al. Social network among young adults with first-episode schizophrenia spectrum disorders: results from the Danish OPUS trial. Soc Psychiatry Psychiatr Epidemiol. 2006 Oct;41(10):761-70. PMID: 16900304. Study covered in a systematic review.

Tomaras V, Mavreas V, Economou M, et al. The effect of family intervention on chronic schizophrenics under individual psychosocial treatment: a 3-year study. Soc Psychiatry Psychiatr Epidemiol. 2000 Nov;35(11):487-93. PMID: 11197923. Sample size too small $(<50)$.

Tonin FS, Piazza T, Wiens A, et al. Adverse events and treatment failure leading to discontinuation of recently approved antipsychotic drugs in schizophrenia: a network metaanalysis. Schizophr Res. 2015 Dec;169(1-3):483-5. doi: 10.1016/j.schres.2015.09.019. PMID: 26516102. Study design not included in review.

Trapp W, Landgrebe M, Hoesl K, et al. Cognitive remediation improves cognition and good cognitive performance increases time to relapse - results of a 5 year catamnestic study in schizophrenia patients. BMC Psychiatry. 2013 Jul;13doi: 10.1186/1471-244X-13-184. PMID: 23837673. Comparator not included in review.

Trower P, Birchwood M, Meaden A, et al. Cognitive therapy for command hallucinations: randomised controlled trial. Br J Psychiatry. 2004 Apr;184:312-20. PMID: 15056575. Study covered in a systematic review.

Tsang HW, Chan A, Wong A, et al. Vocational outcomes of an integrated supported employment program for individuals with persistent and severe mental illness. J Behav Ther Exp Psychiatry. 2009 Jun;40(2):292-305. doi: 10.1016/j.jbtep.2008.12.007. PMID: 19154992. Study covered in a systematic review. 


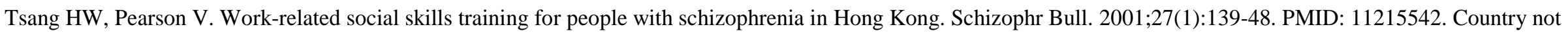
eligible.

Tsapakis EM, Dimopoulou T, Tarazi FI. Clinical management of negative symptoms of schizophrenia: an update. Pharmacol Ther. 2015 Sep;153:135-47. doi: 10.1016/j.pharmthera.2015.06.008. PMID: 26116809. Not a study.

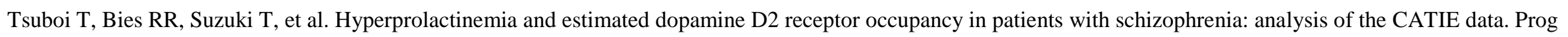
Neuropsychopharmacol Biol Psychiatry. 2013;45:178-82. PMID: 23727135. Study design not included in review.

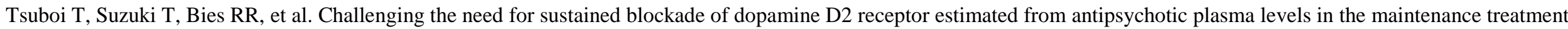

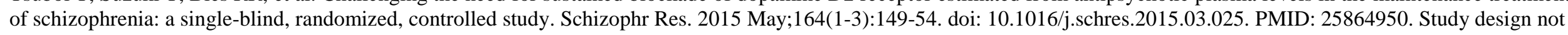
included in review.

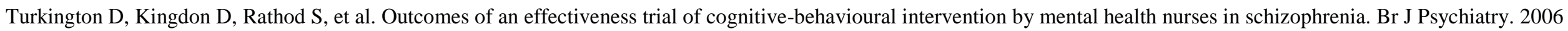
Jul;189:36-40. PMID: 16816304. Not a study.

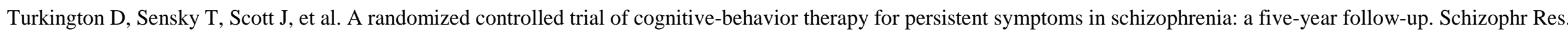
2008 Jan;98(1-3):1-7. PMID: 17936590. Study design not included in review.

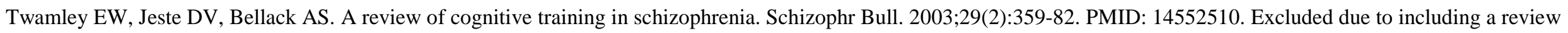
that was more recent, more comprehensive, or had a more similar scope to this review scope.

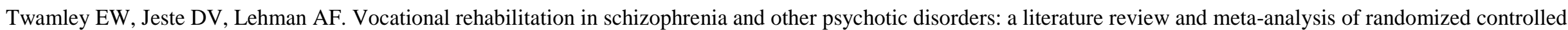

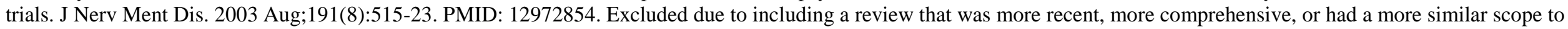
this review scope.

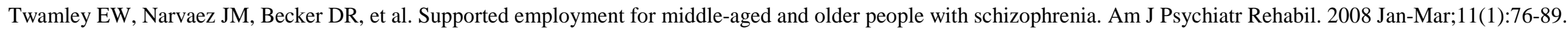
doi: 10.1080/15487760701853326. PMID: 19212460. Study covered in a systematic review.

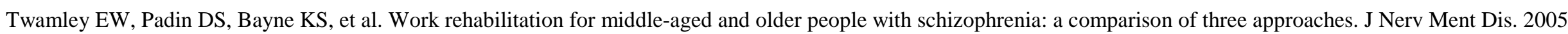
Sep;193(9):596-601. doi: 10.1097/01.nmd.0000177789.53840.e3. PMID: 16131942. Study design not included in review.

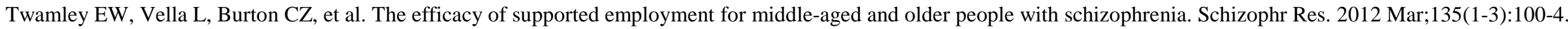
doi: 10.1016/j.schres.2011.11.036. PMID: 22197080. Comparator not included in review.

Ulrich G, Houtmans T, Gold C. The additional therapeutic effect of group music therapy for schizophrenic patients: a randomized study. Acta Psychiatr Scand. 2007 Nov;116(5):362-70. PMID: 17919155. Intervention not included in review.

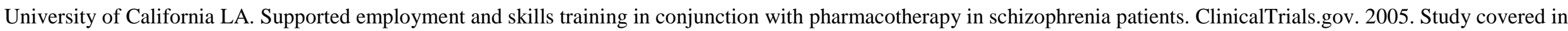
a systematic review.

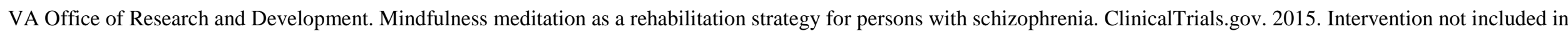
review.

VA Office of Research and Development. Cognitive rehabilitation in schizophrenia ClinicalTrials.gov. 2016. Comparator not included in review.

VA Office of Research and Development. Remediation of working memory in schizophrenia. ClinicalTrials.gov. 2016. Comparator not included in review.

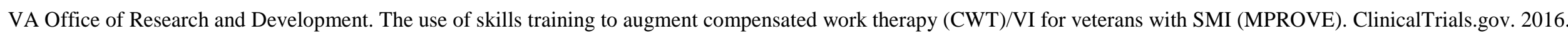
Comparator not included in review. 


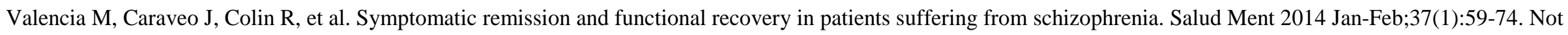
English language.

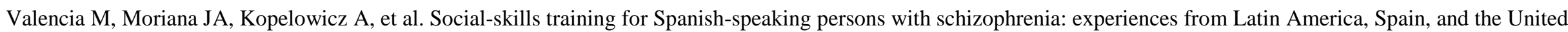
States. Am J Psychiatr Rehabil. 2015 Jul;18(3):209-46. Not a study.

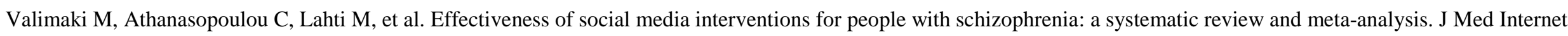
Res. 2016 Apr 22;18(4):e92. doi: 10.2196/jmir.5385. PMID: 27105939. Intervention not included in review.

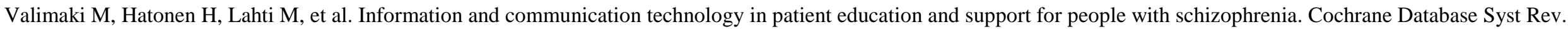
2012 Oct 17;10:Cd007198. doi: 10.1002/14651858.CD007198.pub2. PMID: 23076932. Intervention not included in review.

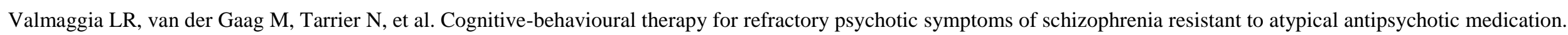
Randomised controlled trial. Br J Psychiatry. 2005 Apr;186:324-30. PMID: 15802690. Study covered in a systematic review.

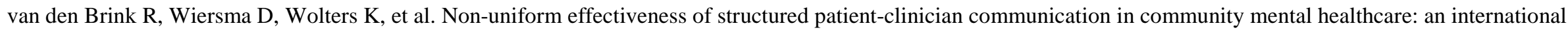
comparison. Soc Psychiatry Psychiatr Epidemiol. 2011 Aug;46(8):685-93. PMID: 20490455. Interventions evaluated in a single study.

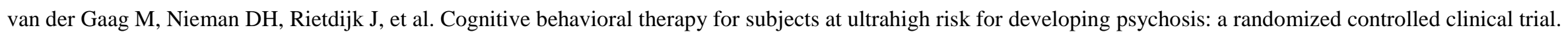
Schizophr Bull. 2012 Nov;38(6):1180-8. doi: 10.1093/schbul/sbs105. PMID: 22941746. Population ineligible for review.

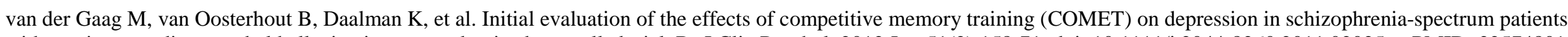

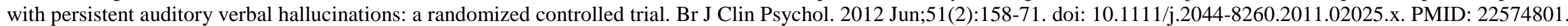
Outcome not included in review.

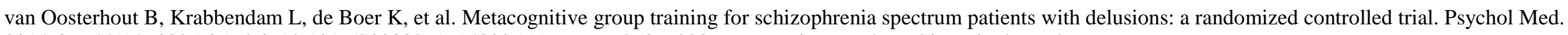
2014 Oct;44(14):3025-35. doi: 10.1017/S0033291714000555. PMID: 25066223. Interventions evaluated in a single study.

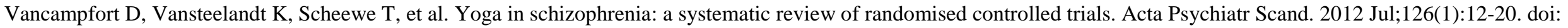
10.1111/j.1600-0447.2012.01865.x. PMID: 22486714. Intervention not included in review.

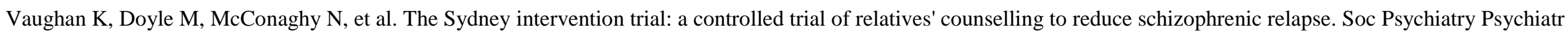
Epidemiol. 1992 Jan;27(1):16-21. doi: 10.1007/BF00788951. PMID: 1557677. Study covered in a systematic review.

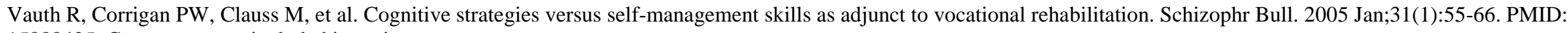
15888425. Comparator not included in review.

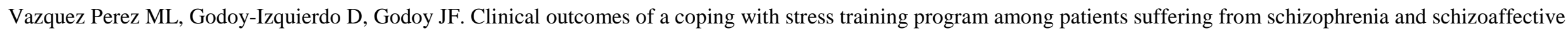
disorder: a pilot study. Anxiety Stress Coping. 2013 Mar;26(2):154-70. PMID: 22300334. Interventions evaluated in a single study.

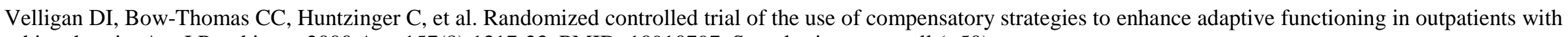
schizophrenia. Am J Psychiatry. 2000 Aug;157(8):1317-23. PMID: 10910797. Sample size too small (<50).

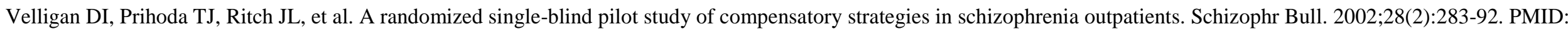
12693434. Study design not included in review.

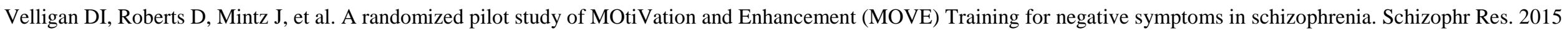
Jul;165(2-3):175-80. doi: 10.1016/j.schres.2015.04.008. PMID: 25937461. Interventions evaluated in a single study.

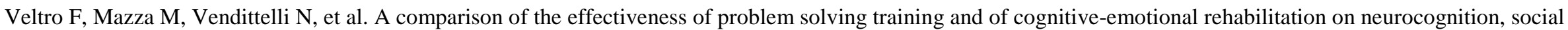

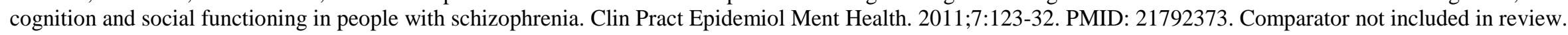


Vera-Garcia E, Mayoral-Cleries F, Vancampfort D, et al. A systematic review of the benefits of physical therapy within a multidisciplinary care approach for people with schizophrenia: an update. Psychiatry Res. 2015 Oct 30;229(3):828-39. doi: 10.1016/j.psychres.2015.07.083. PMID: 26254795. Intervention not included in review.

Vesterager L, Christensen T, Olsen B, et al. Cognitive remediation combined with a comprehensive psychosocial program for patients with first episode schizophrenia: The neuro com trial. Schizophr Bull. 2013;39(21). Not a study.

Vesterager L, Christensen TO, Olsen BB, et al. Cognitive training plus a comprehensive psychosocial programme (OPUS) versus the comprehensive psychosocial programme alone for patients with first-episode schizophrenia (the NEUROCOM trial): a study protocol for a centrally randomised, observer-blinded multi-centre clinical trial. Trials. 2011;12:35. doi: 10.1186/1745-6215-12-35. PMID: 21306612. Not a study.

Victoroff J, Coburn K, Reeve A, et al. Pharmacological management of persistent hostility and aggression in persons with schizophrenia spectrum disorders: a systematic review. J Neuropsychiatry Clin Neurosci. 2014;26(4):283-312. doi: 10.1176/appi.neuropsych.13110335. PMID: 26037853. Excluded due to including a review that was more recent, more comprehensive, or had a more similar scope to this review scope.

Visceglia E, Lewis S. Yoga therapy as an adjunctive treatment for schizophrenia: a randomized, controlled pilot study. J Altern Complement Med. 2011 Jul;17(7):601-7. doi: 10.1089/acm.2010.0075. PMID: 21711202. Inadequate duration (<12 weeks).

Vita A, Cocchi A, Contini A, et al. Applicazione multicentrica del metodo riabilitativo strutturato IPT (Terapia Psicologica Integrata) per pazienti schizofrenici. Psichiatr Oggi. 2002:11-8. Not English language.

Vita A, De Peri L, Barlati S, et al. Psychopathologic, neuropsychological and functional outcome measures during cognitive rehabilitation in schizophrenia: a prospective controlled study in a real-world setting. Eur Psychiatry. 2011 Jul-Aug;26(5):276-83. doi: 10.1016/j.eurpsy.2010.03.008. PMID: 20561768. Study covered in a systematic review.

Volavka J, Czobor P, Citrome L, et al. Effectiveness of antipsychotic drugs against hostility in patients with schizophrenia in the Clinical Antipsychotic Trials of Intervention Effectiveness (CATIE) study.[Erratum appears in CNS Spectr. 2014 Oct;19(5):466]. Cns Spectrums. 2014 Oct;19(5):374-81. doi: 10.1017/S1092852913000849. PMID: 24284234. Outcome not included in review.

Vreeland B, Minsky S, Yanos PT, et al. Efficacy of the team solutions program for educating patients about illness management and treatment. Psychiatric Serv. 2006 Jun;57(6):822-8. PMID: 16754759. Study covered in a systematic review.

Wang L, Zhou J, Yu X, et al. Psychosocial rehabilitation training in the treatment of schizophrenia outpatients: a randomized, psychosocial rehabilitation training-and monomedication-controlled study. Pak J Med Sci. 2013;29(2):597-600. PMID: 24353585. Interventions evaluated in a single study.

Wang L-Q, Chien WT, Yip LK, et al. A randomized controlled trial of a mindfulness-based intervention program for people with schizophrenia: 6-month follow-up. Neuropsychiatr Dis Treat. 2016 Dec;12 PMID: 27994466. Intervention not included in review.

Weiden PJ, Citrome L, Alva G, et al. A trial evaluating gradual- or immediate-switch strategies from risperidone, olanzapine, or aripiprazole to iloperidone in patients with schizophrenia. Schizophr Res. 2014 Mar;153(1-3):160-8. doi: 10.1016/j.schres.2013.11.042. PMID: 24529610. Study design not included in review.

Weisman de Mamani A, Weintraub MJ, Gurak K, et al. A randomized clinical trial to test the efficacy of a family-focused, culturally informed therapy for schizophrenia. J Fam Psychol. 2014 Dec;28(6):800-10. doi: 10.1037/fam0000021. PMID: 25286175. Comparator not included in review.

Widschwendter CG, Karayal ON, Kolluri S, et al. Relating spontaneously reported extrapyramidal adverse events to movement disorder rating scales. Int J Neuropsychopharmacol. 2015;18(12):1-6. PMID: 26116494. Study design not included in review.

Wiedemann G, Hahlweg K, Muller U, et al. Effectiveness of targeted intervention and maintenance pharmacotherapy in conjunction with family intervention in schizophrenia. Eur Arch Psychiatry Clin Neurosci. 2001;251(2):72-84. PMID: 11407442. Study covered in a systematic review.

Wiersma D, Jenner JA, Nienhuis FJ, et al. Hallucination focused integrative treatment improves quality of life in schizophrenia patients. Acta Psychiatr Scand. 2004 Mar;109(3):194-201. PMID: 14984391. Interventions evaluated in a single study. 


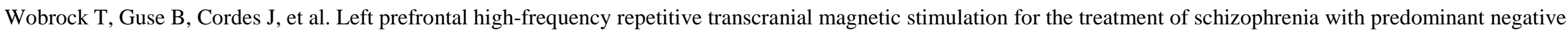

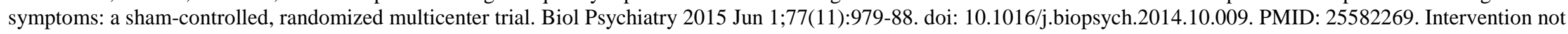
included in review.

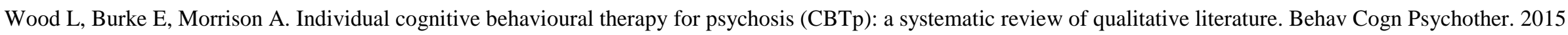
May;43(3):285-97. doi: 10.1017/S1352465813000970. PMID: 24308817. Study design not included in review.

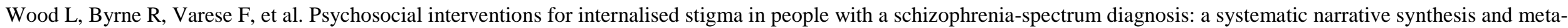
analysis. Schizophr Res. 2016 May:291-303. doi: 10.1016/j.schres.2016.05.001. PMID: 27256518. Outcome not included in review.

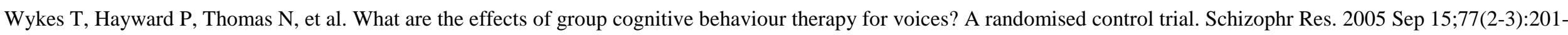
10. PMID: 15885983. Study covered in a systematic review.

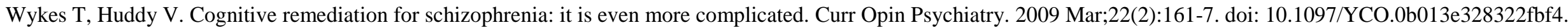
PMID: 19553870. Not a study.

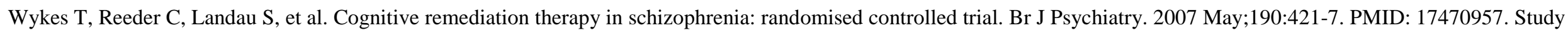
covered in a systematic review.

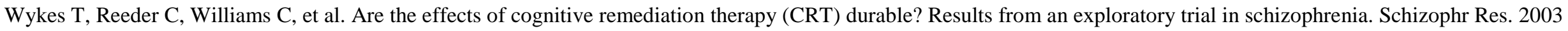
Jun 1;61(2-3):163-74. PMID: 12729868. Study covered in a systematic review.

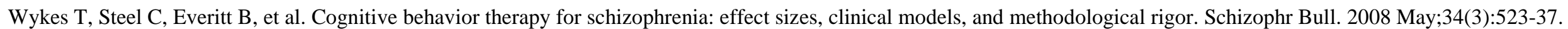
PMID: 17962231. Excluded due to including a review that was more recent, more comprehensive, or had a more similar scope to this review scope.

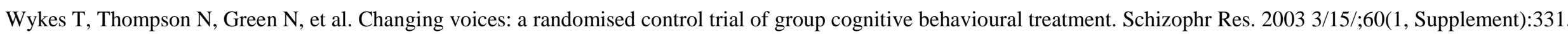
doi: 10.1016/S0920-9964(03)80310-9. Study covered in a systematic review.

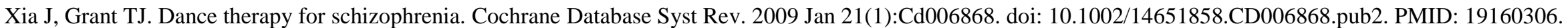
Intervention not included in review.

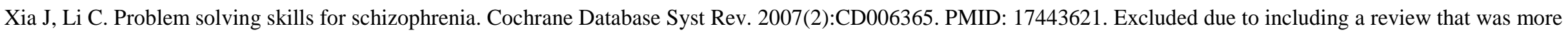
recent, more comprehensive, or had a more similar scope to this review scope.

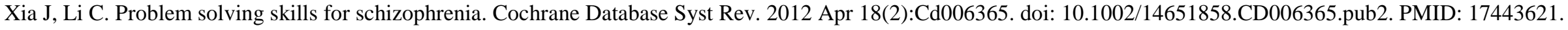
Systematic review/meta-analysis used as a source document to identify individual studies.

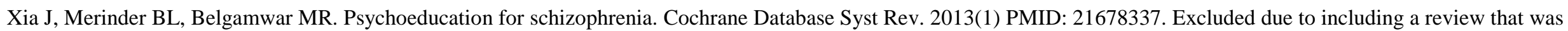
more recent, more comprehensive, or had a more similar scope to this review scope.

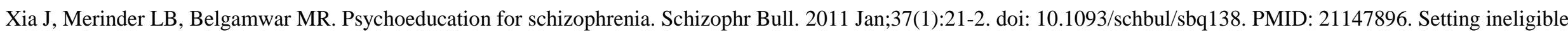
for review (i.e., inpatient only).

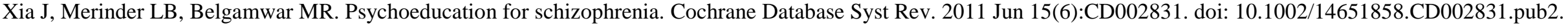
PMID: 21678337. Setting ineligible for review (i.e., inpatient only).

Xiang M, Ran M, Li S. A controlled evaluation of psychoeducational family intervention in a rural Chinese community. Br J Psychiatry. 1994 Oct;165(4):544-8. doi: 10.1192/bjp.165.4.544. PMID: 7804673. Study covered in a systematic review.

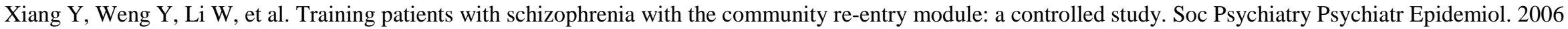
Jun;41(6):464-9. PMID: 16565915. Comparator not included in review. 
Yildiz M, Veznedaroglu B, Eryavuz A, et al. Psychosocial skills training on social functioning and quality of life in the treatment of schizophrenia: a controlled study in Turkey. Int J Psychiatry Clin Pract. 2004 Dec;8(4):219-25. doi: 10.1080/13651500410005595. PMID: 24930550. Intervention not included in review.

Yung AR, Phillips LJ, Nelson B, et al. Randomized controlled trial of interventions for young people at ultra high risk for psychosis: 6-month analysis. J Clin Psychiatry. 2011 Apr;72(4):430-40. doi: 10.4088/JCP.08m04979ora. PMID: 21034687. Population ineligible for review.

Zanello A, Mohr S, Merlo MC, et al. Effectiveness of a brief group cognitive behavioral therapy for auditory verbal hallucinations: a 6-month follow-up study. J Nerv Ment Dis. 2014 Feb;202(2):144-53. doi: 10.1097/NMD.0000000000000084. PMID: 24469527. Not a study.

Zastowny TR, Lehman AF, Cole RE, et al. Family management of schizophrenia: a comparison of behavioral and supportive family treatment. Psychiatr Q. 1992;63(2):159-86. PMID: 1488460. Comparator not included in review.

Zhang J-P, Gallego JA, Robinson DG, et al. Efficacy and safety of individual second-generation vs. first generation antipsychotics in first-episode psychosis: a systematic review and meta-analysis. Int J Neuropsychopharmacol. 2013 Jul;16(6):1205-18. doi: 10.1017/S1461145712001277. PMID: 23199972. Excluded due to including a review that was more recent, more comprehensive, or had a more similar scope to this review scope.

Zhang M, Wang M, Li J, et al. Randomised-control trial of family intervention for 78 first-episode male schizophrenic patients: an 18-month study in Suzhou, Jiangsu. Br J Psychiatry. 1994 Aug;165(Suppl 24):96-102. PMID: 7946238. Study covered in a systematic review.

Zhang S, Lan G. Prospective 8-week trial on the effect of olanzapine, quetiapine, and aripiprazole on blood glucose and lipids among individuals with first-onset schizophrenia. Shanghai Arch Psychiatry. 2014 Dec;26(6):339-46. PMID: 25642108. Study covered in a systematic review.

Zhao J, Song X, Ai X, et al. Adjunctive aripiprazole treatment for risperidone-induced hyperprolactinemia: an 8-week randomized, open-label, comparative clinical trial. PLoS One. 2015;10(10) PMID: 26448615. Study design not included in review.

Zhao S, Sampson S, Xia J, et al. Psychoeducation (brief) for people with serious mental illness. Cochrane Database Syst Rev. 2015 Apr 09(4):Cd010823. doi: 10.1002/14651858.CD010823.pub2. PMID: 25854522. Excluded due to including a review that was more recent, more comprehensive, or had a more similar scope to this review scope.

Zimmermann G, Favrod J, Trieu VH, et al. The effect of cognitive behavioral treatment on the positive symptoms of schizophrenia spectrum disorders: a meta-analysis. Schizophr Res. 2005 Sep 1;77(1):1-9. PMID: 16005380. Excluded due to including a review that was more recent, more comprehensive, or had a more similar scope to this review scope.

Zygmunt A, Olfson M, Boyer CA, et al. Interventions to improve medication adherence in schizophrenia. Am J Psychiatry. 2002 Oct;159(10):1653-64. PMID: 12359668. Outcome not included in review. 


\section{Appendix D. Scale Abbreviations and Scoring}

\begin{tabular}{|c|c|c|c|}
\hline Abbreviation & Measure & Scale & Direction \\
\hline ABS & Agitated Behavior Scale & 15 to 56 & Higher score=more dangerous behavior \\
\hline ACES & Agitation-Calmness Scale & 1 to 9 & Higher score=calmer \\
\hline ADL & Activities of Daily Living & 0 to 6 & Higher score=greater independence in daily living \\
\hline AIMS & Abnormal Involuntary Movement Scale & 0 to 4 & Higher score=greater severity of tardive dyskinesia \\
\hline ASEX & Arizona Sexual Experiences Scale & 5 to 30 & Higher score=greater sexual dysfunction \\
\hline BARS & Barnes Akathisia Rating Scale & 0 to 9 & Higher score=greater severity of akathisia \\
\hline BPRS & Brief Psychiatric Rating Scale & 16 to 112 & Higher score=more severe symptoms \\
\hline C-SSRS & Columbia Suicide Severity Rating Scale & 2 to 25 & Higher score=greater suicide ideation severity \\
\hline CABS & Corrigan Agitated Behavior Scale & 14 to 56 & Higher score=more dangerous behavior \\
\hline CDSS & Calgary Depression Rating Scale for Schizophrenia & 0 to 27 & Higher score=worse depression \\
\hline CES & Coping Efficacy Scale & 1 to 5 & Higher score=more effective coping \\
\hline CGI & Clinical Global Impression & 1 to 7 & Higher score=more severe illness \\
\hline CGI-I & Clinical Global Impression - Improvement & 1 to 7 & $\begin{array}{l}\text { Higher score=higher degree of mental illness relative to other } \\
\text { patients with the same diagnosis }\end{array}$ \\
\hline CGI-S & Clinical Global Impression-Severity & 1 to 7 & Higher score=higher degree of mental illness \\
\hline CHOICE & CHoice of Outcome in Cbt for psychosEs & 0 to 210 & Higher score=more satisfied and better \\
\hline DIEPSS & Drug-Induced Extrapyramidal Symptoms Scale & 0 to 36 & Higher score=more severe symptoms \\
\hline GAF & Global Assessment of Function & 1 to 100 & Higher score=better functioning \\
\hline GAS & Goal Attainment Scaling & -2 to +2 & Higher score=higher goal attainment \\
\hline GPTS & Green Paranoid Thoughts Scale & 32 to 160 & Higher score=more paranoid thoughts \\
\hline Heinrich's & Heinrichs-Carpenter Quality of Life Scale & 0 to 126 & Higher score=greater mental health \\
\hline HoNDS & Health of the Nation Outcomes Scales & 0 to 48 & Higher score=more severe outcomes of mental illness \\
\hline ILSS & Independent Living Skills Survey & 0 to 70 & Higher score=better functioning \\
\hline ISSI & Interview Schedule for Social Interaction & 0 to 30 & Higher score=better social integration and attachment \\
\hline ITAQ & Insight and Treatment Attitudes Questionairre & 0 to 22 & Higher score=more complete insight \\
\hline LOS & Strauss-Carpenter Level of Function Scale & 0 to 36 & Higher score=more functionality \\
\hline LQLP or LQOLP & Lancashire Quality of Life Profile & 105 to 735 & Higher score=higher quality of life \\
\hline LSP & Life Skills Profile & 39 to 156 & Higher score =higher levels of life skills \\
\hline LUNSERS & Liverpool University Neuroleptic Side Effect Rating Scale & 0 to 204 & Higher score=worse neuroleptic side effects \\
\hline
\end{tabular}




\begin{tabular}{|c|c|c|c|}
\hline Abbreviation & Measure & Scale & Direction \\
\hline MANSA & Manchester Short Assessment of Quality of Life & 16 to 112 & Higher score=higher quality of life \\
\hline MASC & Maryland Assessment of Social Competence & 12 to 60 & Higher score=better social skills \\
\hline MCAS & Multnomah Community Ability Scale & 17 to 85 & Higher score=better functioning \\
\hline MOAS & Modified Overt Aggression Scale & 0 to 40 & Higher score=higher frequency of aggressive behaviors \\
\hline PANSS & Positive and Negative Syndromes Scale & 30 to 210 & Higher score=more severe symptoms \\
\hline PSP & Personal and Social Performance Scale & 0 to 100 & Higher score=better personal and social functioning \\
\hline PSR Toolkit & Psychosocial Rehabilitation Toolkit & 0 to 5 & Higher score=better functioning \\
\hline PSWQ & Penn State Worry Questionairre & 16 to 18 & Higher score=more worry \\
\hline PSYRATS & The Psychotic Symptom Rating Scales & 0 to 70 & Higher score=more severe psychotic symptoms \\
\hline PTQ & Perservative Thinking Questionaire & 0 to 60 & Higher score=higher levels of repetitive thought \\
\hline QLS & Quality of Life Scale & 0 to 126 & Higher score=better functioning \\
\hline QOLI & Quality of Life Interview & 143 to 1001 & Higher score=better quality of life \\
\hline R-SES & Revised Self-efficacy Scale & 0 to 100 & Higher score=higher self-efficacy \\
\hline RFS & Role Functioning Scale & 4 to 28 & Higher score=more optimal functioning \\
\hline SADS-C & Schedule for Affective Disorders and Schizophrenia - Change & 75 to 406 & Higher score=more severe symptoms \\
\hline SAI & Scale for the Assessment of Insight Expanded & 0 to 24 & Higher score=better insight \\
\hline SANS & Scale for Assessment of Negative Symptoms & 0 to 130 & Higher score=more severe negative symptoms \\
\hline SAS or SAS-II & Social Adjustment Scale & 0 to 125 & Higher score=better functioning \\
\hline SAPS & Scale for the Assessment of Positive Symptoms & 0 to 175 & Higher score=more severe positive symptoms \\
\hline SBS & Social Behavior Survey & 0 to 84 & Higher score=worse social function \\
\hline SCL or SCL-90-R & Symptom Checklist-90-Revised & 30 to 80 & Higher score=more symptom severity \\
\hline SF-36 & 36-Item Short Form health Survey & 0 to 100 & Higher score=more favorable health state \\
\hline SFS & Social Functioning Scale & 40 to 160 & Higher score=greater social function \\
\hline SOFAS & Social and Occupational Functioning Assessment Scale & 1 to 100 & Higher score=more impairment \\
\hline SWBUNS & Subjective Well-being Under Narcoleptics Scale & 20 to 120 & Higher score=higher wellbeing \\
\hline UPSA & UCSD Performance-based Skills Assessment & 0 to 100 & Higher score=higher levels of functioning \\
\hline WBI & Work Behavior Inventory & 35 to 175 & Higher score=better work behavior \\
\hline WEMWEBS & Warwick-Edinburgh Mental Wellbeing Scale & 14 to 70 & Higher score=higher wellbeing \\
\hline WHOQOL-BREF & $\begin{array}{l}\text { World Health Organization Brief Quality of Life Assessment } \\
\text { Instrument }\end{array}$ & 32 to 160 & Higher score=better quality of life \\
\hline YMRS & Young Mania Rating Scale & 0 to 60 & Higher score=greater severity of manic symptoms \\
\hline
\end{tabular}




\section{Appendix E. Data Abstraction}

Appendix Table E-1. Data abstraction of systematic reviews of pharmacological interventions

\begin{tabular}{|c|c|c|c|c|c|c|}
\hline Author, Year & Aims & $\begin{array}{l}\text { Databases and } \\
\text { Timeperiod } \\
\text { Covered }\end{array}$ & $\begin{array}{l}\text { Number of } \\
\text { Studies } \\
\text { Number of } \\
\text { Patients }\end{array}$ & $\begin{array}{l}\text { Characteristics of } \\
\text { Identified Articles: } \\
\text { Study Designs }\end{array}$ & $\begin{array}{l}\text { Characteristics of } \\
\text { Identified Articles: } \\
\text { Populations }\end{array}$ & $\begin{array}{l}\text { Characteristics of Identified } \\
\text { Articles: Interventions }\end{array}$ \\
\hline $\begin{array}{l}\text { Alberta CER } \\
\text { Abou-Setta } 2012\end{array}$ & $\begin{array}{l}\text { Compare FGAs with } \\
\text { SGAs antipsychotics in } \\
\text { patients with } \\
\text { schizophrenia, } \\
\text { schizophrenia-related } \\
\text { psychosis or bipolar } \\
\text { disorder, with a focus on } \\
\text { core illness symptoms, } \\
\text { functional outcomes, } \\
\text { health care utilization } \\
\text { and adverse events }\end{array}$ & $\begin{array}{l}\text { MEDLINE, Embase, } \\
\text { PsycINFO, } \\
\text { International } \\
\text { Pharmaceuticals } \\
\text { Abstracts, CINAHL, } \\
\text { ProQuest } \\
\text { Dissertations and } \\
\text { Theses Full-Text, } \\
\text { Cochrane Central } \\
\text { Register of } \\
\text { Controlled Trials and } \\
\text { Scopus (1950 to } \\
\text { July 2011) } \\
\text { For adverse events } \\
\text { TOXLINE and } \\
\text { MedEffect (1950- } \\
\text { July 2011) } \\
\text { Grey literature and } \\
\text { hand searches }\end{array}$ & $\begin{array}{l}113 \text { studies (in } \\
\text { people with } \\
\text { schizophrenia or } \\
\text { related psychosis) } \\
\mathrm{N}=118,503 \text {; range } \\
10-95,632, \text { median } \\
86\end{array}$ & $\begin{array}{l}109 \text { RCTs; } 2 \\
\text { nonrandomized } \\
\text { trials; } 2 \text { cohort } \\
\text { RCTs; followup <1 } \\
\text { day to } 22 \text { years, } \\
\text { median } 8 \text { weeks } \\
\text { All had unclear or } \\
\text { high risk of bias }\end{array}$ & $\begin{array}{l}\text { Mean age range } 21-50 \\
\text { years, median } 37 \text { years } \\
50 \% \text { of } \mathrm{RCTs} \\
\text { conducted in inpatient } \\
\text { populations }\end{array}$ & $\begin{array}{l}\text { Fluphenazine vs: } \\
\text {-olanzapine (2 RCTs) } \\
\text {-quetiapine (1 RCT) } \\
\text {-risperidone (1 RCT) } \\
\text { Haloperidol vs: } \\
\text {-aripiprazole (8 RCTs) } \\
\text {-asenapine (1 RCT) } \\
\text {-clozapine (10 RCTs, } 1 \text { cohort } \\
\text { study) } \\
\text {-olanzapine (34 RCTs, } 1 \text { cohort } \\
\text { study) } \\
\text {-quetiapine (10 RCTs, } 1 \text { cohort } \\
\text { study) } \\
\text {-risperidone (37 RCTs, } 1 \\
\text { nonrandomized trial and } 1 \text { cohort } \\
\text { study) } \\
\text {-ziprasidone (8 RCTs, } 1 \\
\text { nonrandomized trial) } \\
\text { Perphenazine vs: } \\
\text {-aripiprazole (1 RCT) } \\
\text {-olanzapine (2 RCTs) } \\
\text {-quetiapine (1 RCT) } \\
\text {-risperidone (2 RCTs) } \\
\text {-ziprasidone (1 RCT) } \\
\text { No included RCTs for any FGA vs: } \\
\text {-Brexpiprazole } \\
\text {-Cariprazine } \\
\text {-Iloperidone } \\
\text {-Lurasidone } \\
\text {-Paliperidone }\end{array}$ \\
\hline
\end{tabular}




\begin{tabular}{|c|c|c|}
\hline Author, Year & Outcomes Reported & Effectiveness Outcomes \\
\hline $\begin{array}{l}\text { Alberta CER } \\
\text { Abou-Setta } 2012\end{array}$ & $\begin{array}{l}\text { Mean between group difference for - } \\
\text { Core illness symptoms: } \\
\text {-negative symptoms } \\
\text {-general psychopathology } \\
\text {-global ratings and total scores } \\
\text { Functional outcomes } \\
\text { Other outcomes: } \\
\text {-response rate } \\
\text {-remission rate } \\
\text {-adherence rate } \\
\text { Health-related quality of life } \\
\text { Adverse events }\end{array}$ & 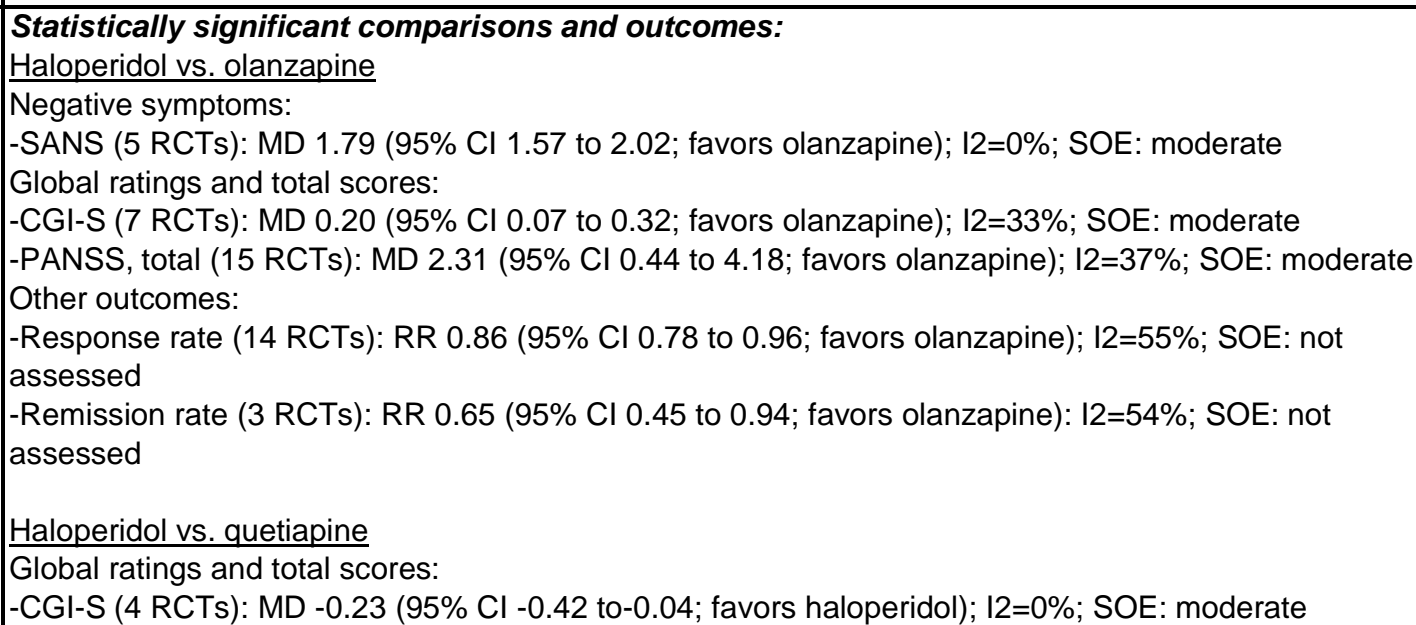 \\
\hline
\end{tabular}




\begin{tabular}{|c|c|c|c|c|}
\hline Author, Year & Harms Outcomes & Subgroups & $\begin{array}{l}\text { Funding/ } \\
\text { Comments }\end{array}$ & $\begin{array}{l}\text { Quality } \\
\text { Rating }\end{array}$ \\
\hline $\begin{array}{l}\text { Alberta CER } \\
\text { Abou-Setta } 2012\end{array}$ & 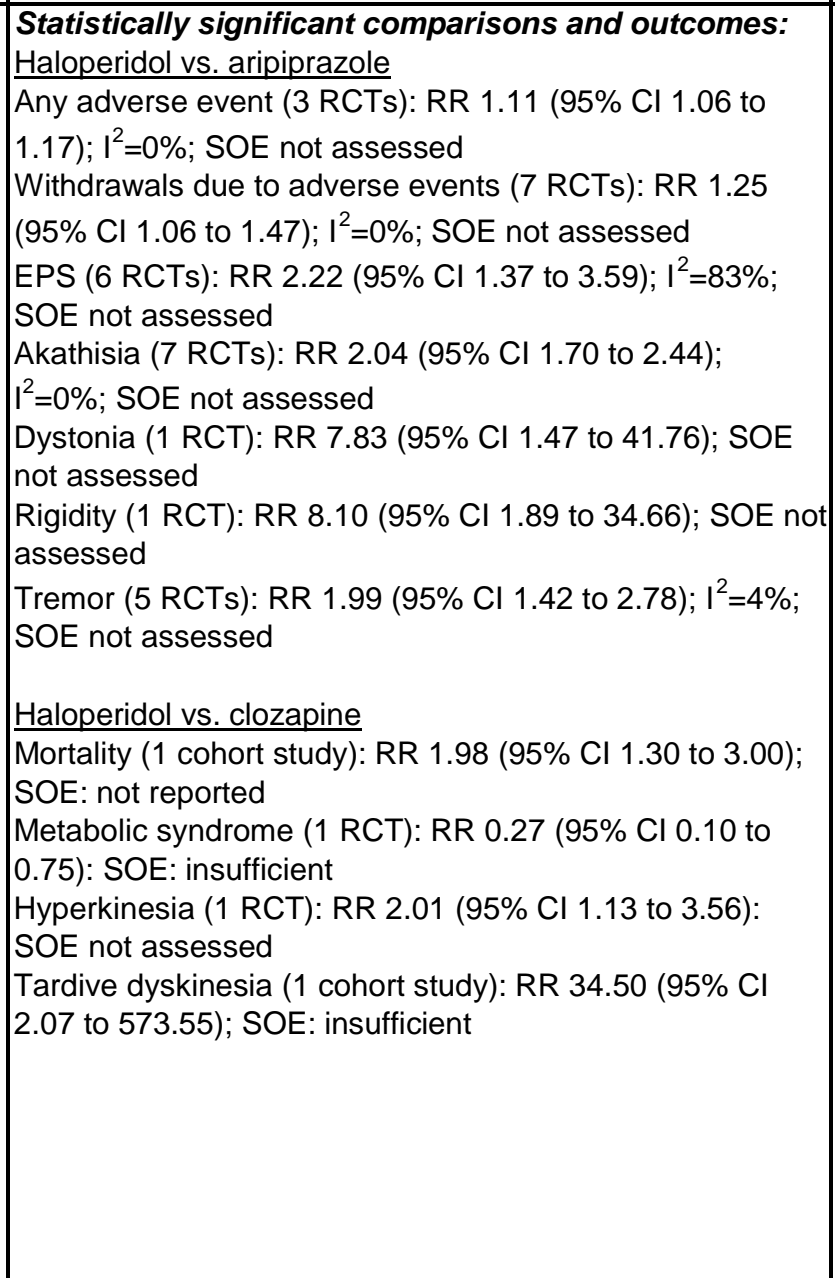 & 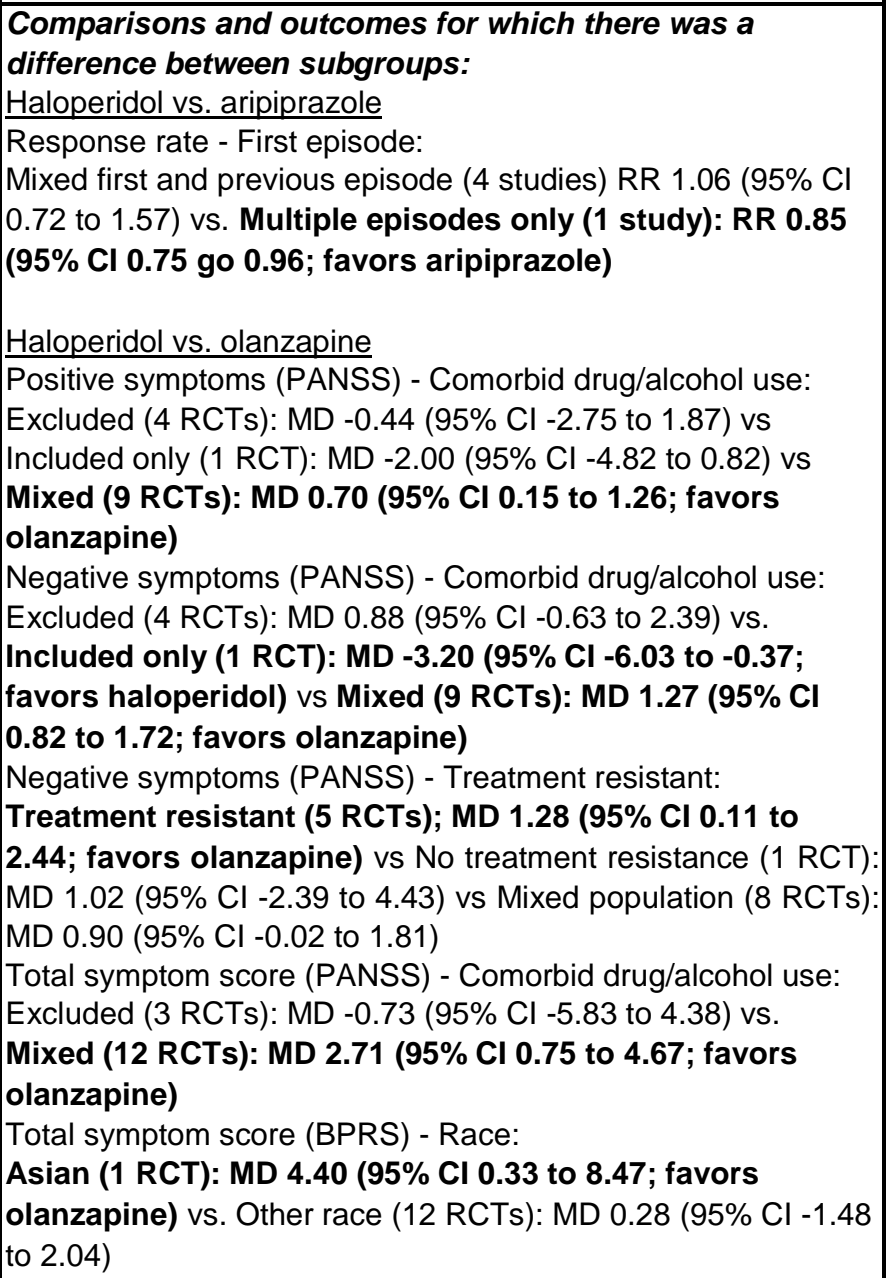 & AHRQ & Good \\
\hline
\end{tabular}




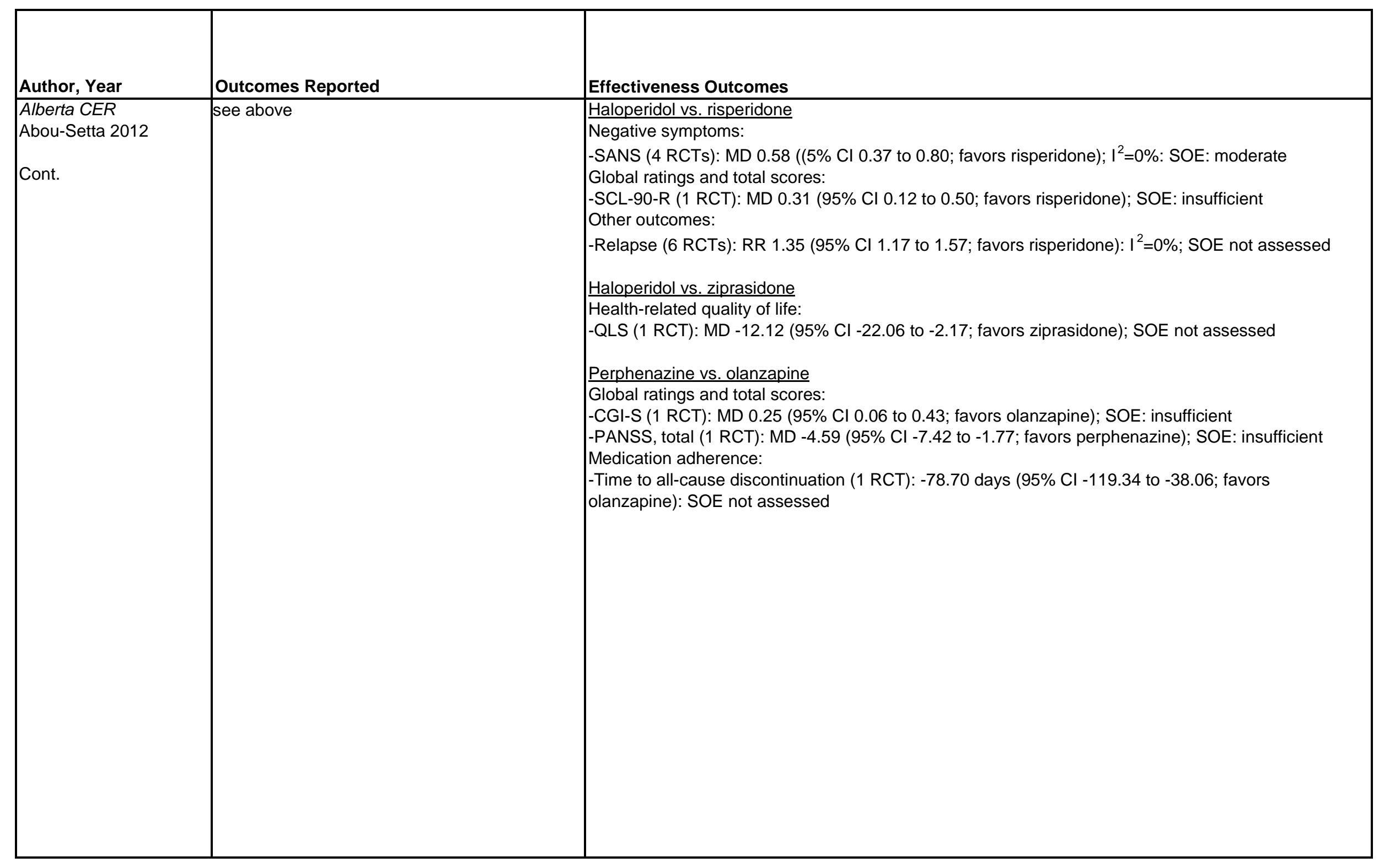




\begin{tabular}{|c|c|c|c|c|}
\hline Author, Year & Harms Outcomes & Subgroups & $\begin{array}{l}\text { Fundingl } \\
\text { Comments }\end{array}$ & $\begin{array}{l}\text { Quality } \\
\text { Rating }\end{array}$ \\
\hline $\begin{array}{l}\text { Alberta CER } \\
\text { Abou-Setta } 2012 \\
\text { Cont. }\end{array}$ & 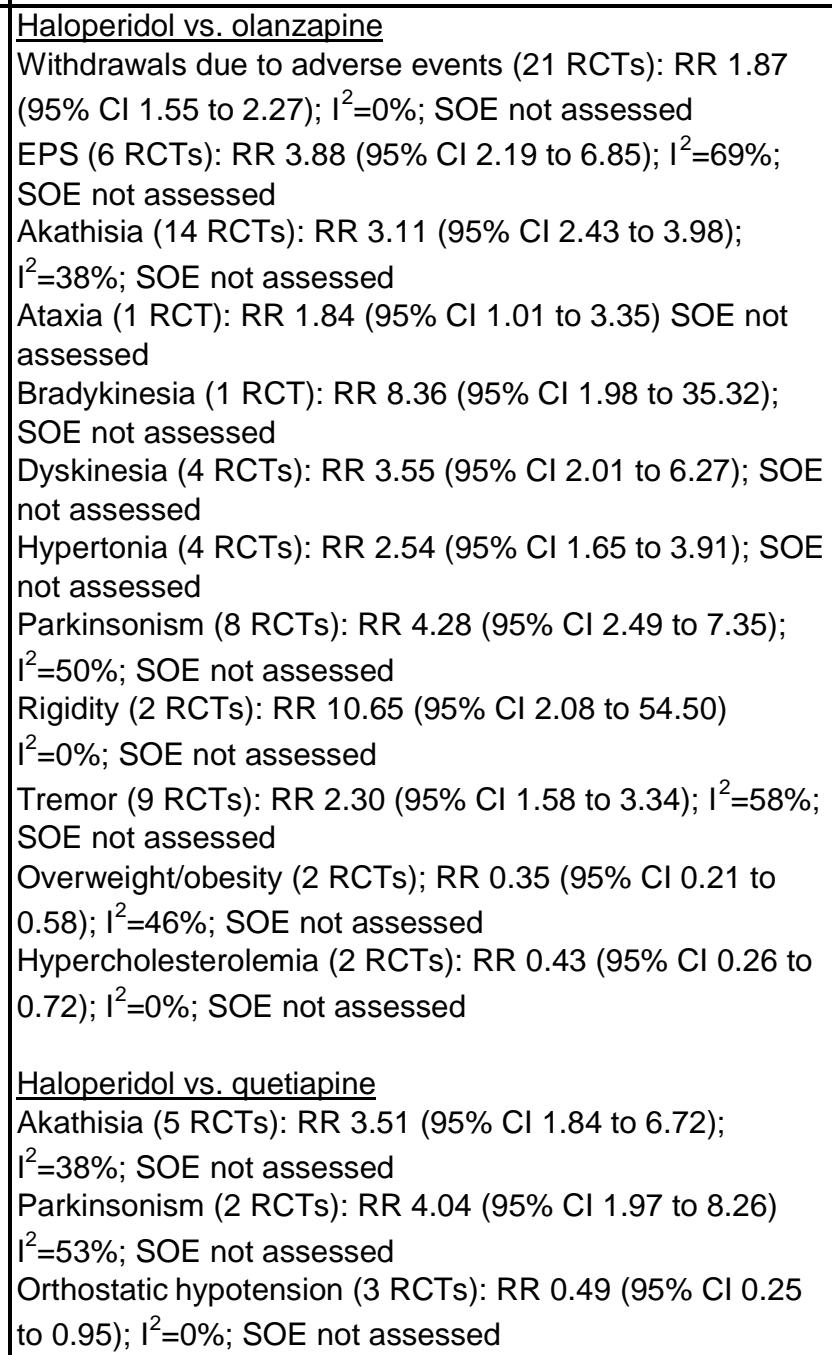 & 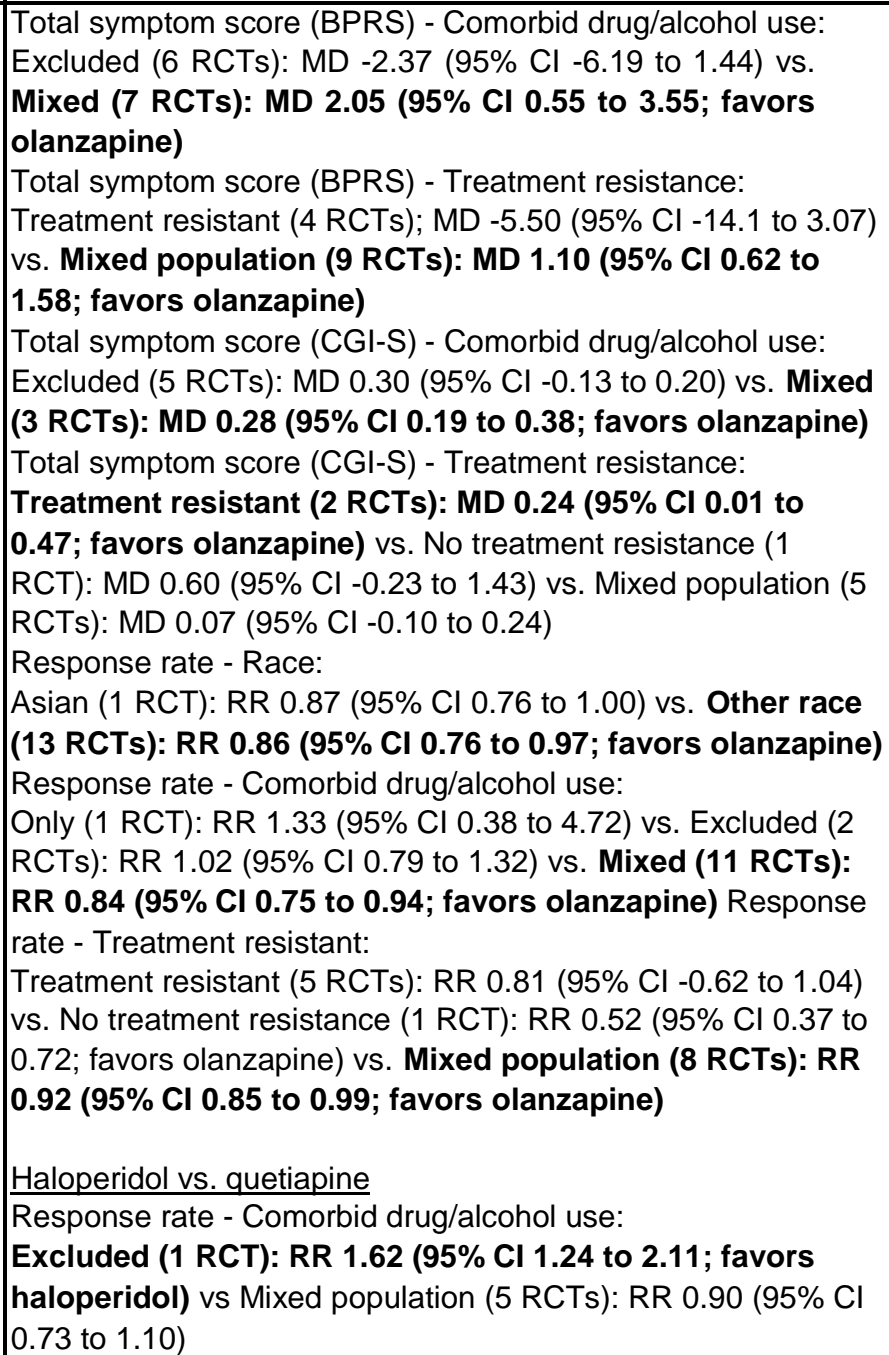 & see above & see above \\
\hline
\end{tabular}




\begin{tabular}{|c|c|c|c|c|}
\hline Author, Year & Harms Outcomes & Subgroups & $\begin{array}{l}\text { Funding/ } \\
\text { Comments }\end{array}$ & $\begin{array}{l}\text { Quality } \\
\text { Rating }\end{array}$ \\
\hline $\begin{array}{l}\text { Alberta CER } \\
\text { Abou-Setta } 2012 \\
\text { Cont. }\end{array}$ & 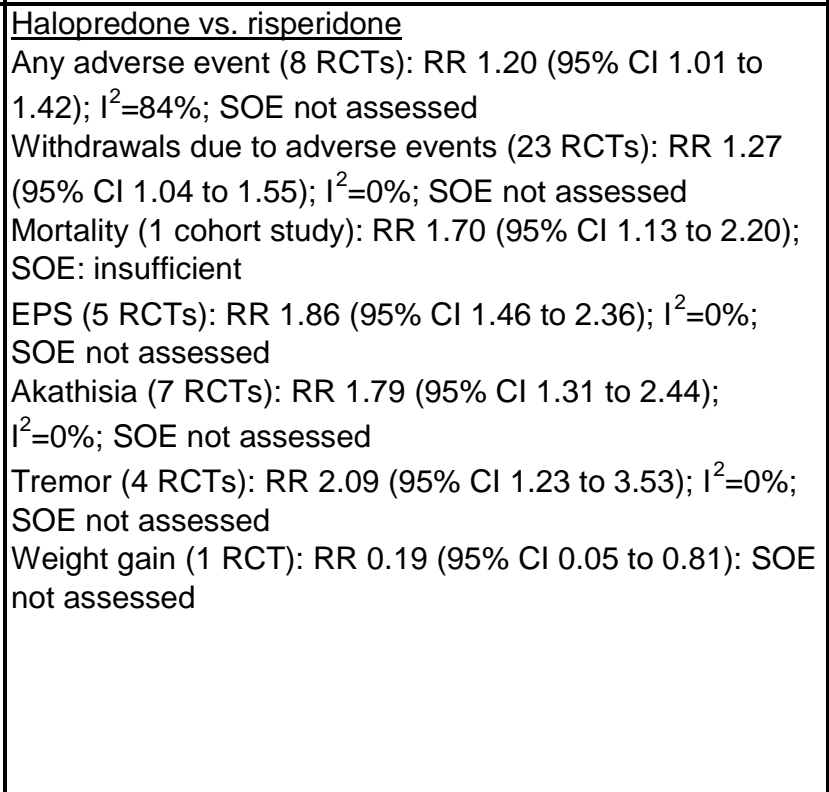 & 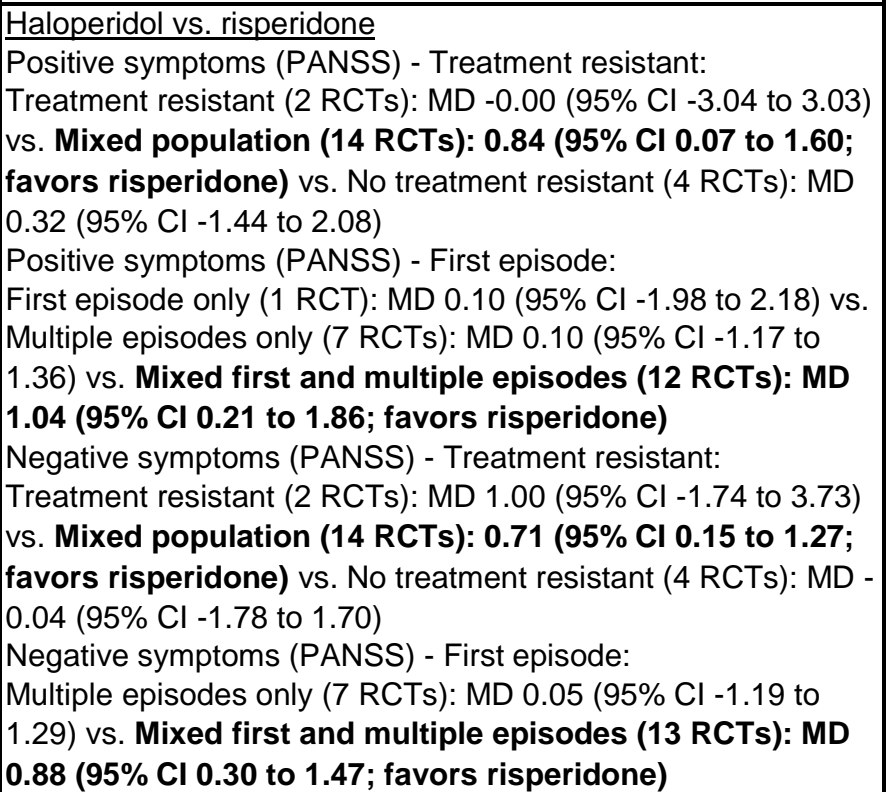 & see above & see above \\
\hline
\end{tabular}




\begin{tabular}{|c|c|c|c|c|}
\hline Author, Year & Harms Outcomes & Subgroups & $\begin{array}{l}\text { Funding/ } \\
\text { Comments }\end{array}$ & $\begin{array}{l}\text { Quality } \\
\text { Rating }\end{array}$ \\
\hline $\begin{array}{l}\text { Alberta CER } \\
\text { Abou-Setta } 2012 \\
\text { Cont. }\end{array}$ & 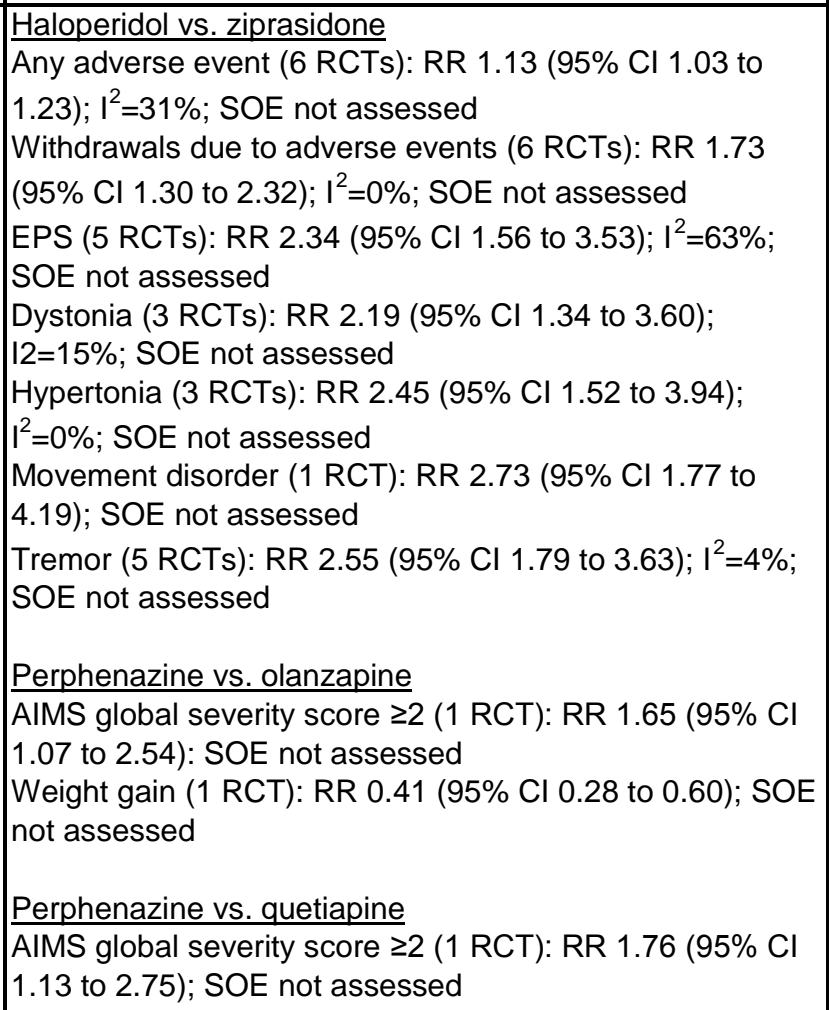 & 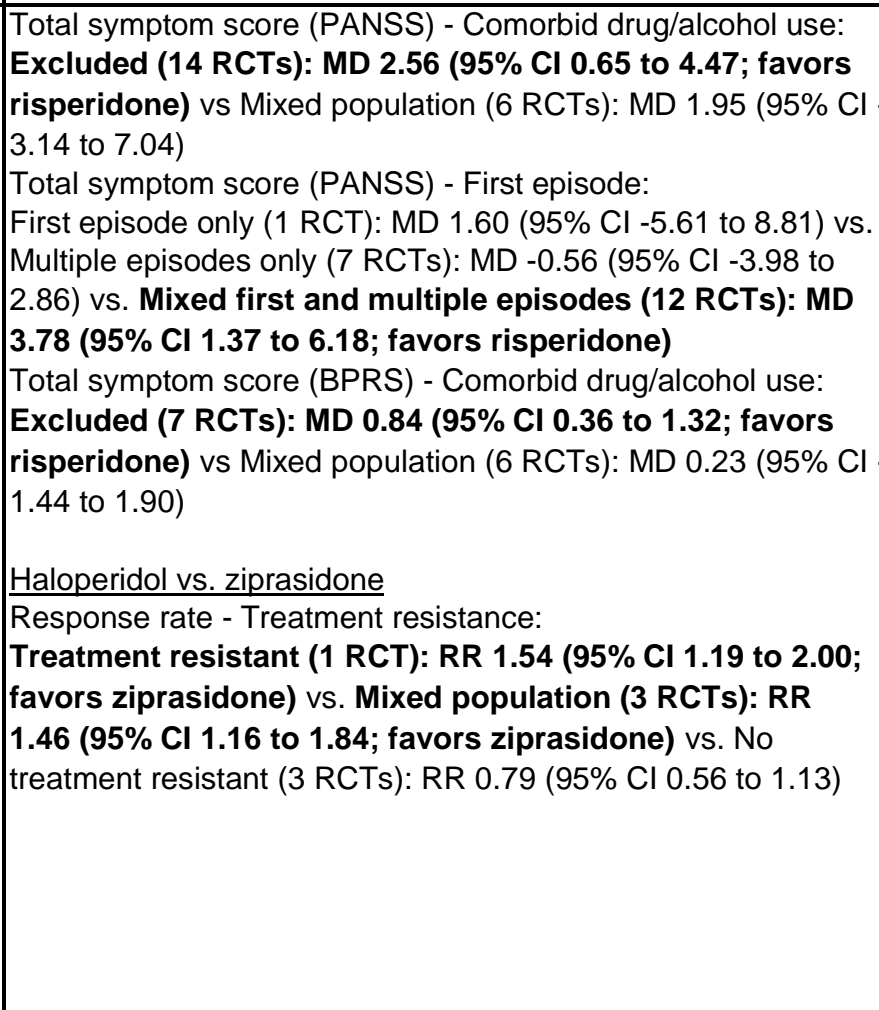 & see above & see above \\
\hline
\end{tabular}




\begin{tabular}{|c|c|c|c|c|c|c|}
\hline Author, Year & Aims & \begin{tabular}{|l} 
Databases and \\
Timeperiod \\
Covered
\end{tabular} & \begin{tabular}{|l} 
Number of \\
Studies \\
Number of \\
Patients \\
\end{tabular} & $\begin{array}{l}\text { Characteristics of } \\
\text { Identified Articles: } \\
\text { Study Designs }\end{array}$ & $\begin{array}{l}\text { Characteristics of } \\
\text { Identified Articles: } \\
\text { Populations }\end{array}$ & $\begin{array}{l}\text { Characteristics of Identified } \\
\text { Articles: Interventions }\end{array}$ \\
\hline $\begin{array}{l}\text { McDonagh } 2013 \\
\text { Drug Effectiveness } \\
\text { Review Project Report }\end{array}$ & $\begin{array}{l}\text { Comparative } \\
\text { effectiveness of } \\
\text { second-generation } \\
\text { antipsychotics in multiple } \\
\text { populations, including } \\
\text { schizophrenia }\end{array}$ & $\begin{array}{l}\text { Medline and } \\
\text { Cochrane from } \\
\text { inception to August } \\
2013\end{array}$ & \begin{tabular}{|l}
$138 \mathrm{RCTs}$ \\
$(\mathrm{N}=47,189)$ \\
31 observational \\
studies $(\mathrm{N}=602$, \\
$547)$
\end{tabular} & \begin{tabular}{|l} 
Head-to-head \\
randomized \\
controlled trials with \\
at least 6 weeks \\
duration
\end{tabular} & $\begin{array}{l}\text { Patients with } \\
\text { schizophrenia or } \\
\text { schizoaffective or } \\
\text { nonaffective functional } \\
\text { psychosis }\end{array}$ & $\begin{array}{l}2 \text { or more of } 14 \text { possible second } \\
\text { generation antipsychotics available } \\
\text { in the United States as of } 2013\end{array}$ \\
\hline
\end{tabular}




\begin{tabular}{|c|c|c|}
\hline Author, Year & Outcomes Reported & Effectiveness Outcomes \\
\hline $\begin{array}{l}\text { McDonagh } 2013 \\
\text { Drug Effectiveness } \\
\text { Review Project Report }\end{array}$ & $\begin{array}{l}\text { Quality of life, mortality, functional capacity, } \\
\text { hospitalization, emergency department visits, } \\
\text { medication persistence, symptom response, } \\
\text { response rates, duration of response, } \\
\text { remission, relapse, speed of response, time to } \\
\text { discontinuation of medication, overall (total) } \\
\text { adverse events, withdrawals due to adverse } \\
\text { events, time to withdrawal due to adverse } \\
\text { events, major adverse events, general adverse } \\
\text { events. }\end{array}$ & $\begin{array}{l}\text { Functioning: Olanzapine, risperidone, immediate-release quetiapine, or ziprasidone were not different } \\
\text { on employment or general function outcomes. Social function was not different between paliperidone } \\
\text { palmitate and long-acting risperidone injections. Global function was superior with olanzapine vs. } \\
\text { ziprasidone in patients with depressive symptoms and with immediate-release quetiapine in patients } \\
\text { with prominent negative symptoms, but similar between immediate-release quetiapine and risperidone } \\
\text { in patients with a first-episode of schizophrenia. } \\
\text { Quality of life. Good-quality trial evidence did not differentiate asenapine, olanzapine, immediate- } \\
\text { release quetiapine, risperidone, or ziprasidone. } \\
\text { Suicide. Clozapine was superior to olanzapine in preventing suicide or suicidality in patients at high } \\
\text { risk of suicide (NNT=12) (InterSePT). } \\
\text { Response: Rates ranged from } 45 \% \text { to } 80 \% \text {, with variation in definition of response, patient } \\
\text { populations and duration of treatment contributing to variability. Limited evidence did not identify } \\
\text { statistically significant differences between risperidone long-acting injection and oral risperidone or } \\
\text { olanzapine or olanzapine and extended-release paliperidone. Evidence was mixed for risperidone } \\
\text { long-acting injection and paliperidone palmitate injection. Evidence was insufficient for iloperidone } \\
\text { and lurasidone. } \\
\text { Relapse. Risk of relapse may be lower with olanzapine and risperidone than immediate-release } \\
\text { quetiapine and with risperidone long-acting injection than oral risperidone (first-episode patients). } \\
\text { Results were mixed with risperidone vs. olanzapine. No differences between risperidone long-acting } \\
\text { injection and aripiprazole, lurasidone and oral risperidone or lurasidone and extended-release } \\
\text { quetiapine } \\
\text { Symptoms Consistent differences in efficacy were not found between clozapine, olanzapine, } \\
\text { quetiapine, risperidone, ziprasidone, aripiprazole, or asenapine in shorter-term trials }\end{array}$ \\
\hline
\end{tabular}




\begin{tabular}{|l|l|l|l|}
\hline Author, Year & Harms Outcomes & $\begin{array}{l}\text { Funding/ } \\
\text { Comments }\end{array}$ & $\begin{array}{l}\text { Quality } \\
\text { Rating }\end{array}$ \\
\hline $\begin{array}{l}\text { McDonagh 2013 } \\
\text { Drug Effectiveness } \\
\text { Review Project Report }\end{array}$ & $\begin{array}{l}\text { Discontinuation due to adverse events. Mixed-treatment } \\
\text { comparisons analysis indicated clozapine resulted in } \\
\text { statistically significantly higher rates than olanzapine, } \\
\text { immediate-release quetiapine, or risperidone. Sensitivity } \\
\text { analyses of studies of greater and less than 6 months } \\
\text { found no statistically significant differences, although the } \\
\text { point estimates were in the same direction as the overall } \\
\text { analysis. Fewer data were available for the lurasidone, } \\
\text { new formulations of olanzapine, asenapine, and } \\
\text { paliperidone palmitate long-acting injection, and no data } \\
\text { for iloperidone. }\end{array}$ & $\begin{array}{l}\text { Drug } \\
\text { Effectiveness } \\
\text { Review Project } \\
\text { (collaboration of } \\
\text { 12 Medicaid } \\
\text { agencies) }\end{array}$ & Good \\
& & & \\
\hline
\end{tabular}

\section{Please see Appendix B. Included Studies for full study references}

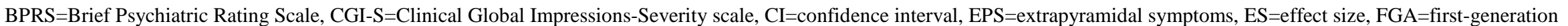

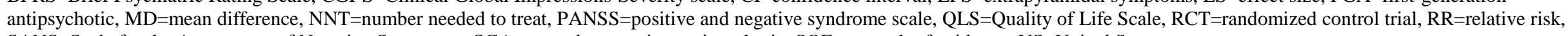
SANS=Scale for the Assessment of Negative Symptoms, SGA=second-generation antipsychotic, SOE=strength of evidence, US=United States 
Appendix Table E-2. Data abstraction of randomized controlled trials of pharmacological interventions

\begin{tabular}{|c|c|c|c|c|c|}
\hline Author, Year & $\begin{array}{l}\text { Setting } \\
\text { Country }\end{array}$ & Inclusion Criteria & $\begin{array}{l}\text { Interventions and Ns per } \\
\text { Group }\end{array}$ & \begin{tabular}{|l} 
Duration \\
(intervention and \\
longest followup)
\end{tabular} & \begin{tabular}{|l} 
Age Gender \\
Race/Ethnicity
\end{tabular} \\
\hline Amr, 2013 & $\begin{array}{l}\text { Amman, Jordan; } \\
10 / 2009 \text { to } \\
9 / 2011\end{array}$ & $\begin{array}{l}\text { Age: 18-60; } \\
\text { Met DSM diagnosis of schizophrenia; } \\
\text { First episode of schizophrenia; } \\
\text { Exclusion: current or past use of } \\
\text { antipsychotics; concurrent DSM Axis } 1 \\
\text { diagnosis; DSM-VI Axis II diagnosis of } \\
\text { borderline personality disorder, } \\
\text { antisocial personality, substance } \\
\text { dependence or abuse, clinically } \\
\text { significant or unstable medical illness. }\end{array}$ & $\begin{array}{l}\text { Initial doses: } \\
\text { haloperidol=5 mg/day } \\
\text { quetiapine=200 mg/day; } \\
\text { Co-admin of psychotropic } \\
\text { medications not allowed, } \\
\text { except lorazepam and } \\
\text { zopiclone and biperiden. } \\
\\
\text { Dose at } 12 \text { weeks: } \\
\text { haloperidol=14.2 } \mathrm{mg} \text {; } \\
\text { quetiapine }=705.8 \mathrm{mg}\end{array}$ & 12 weeks & $\begin{array}{l}\text { Age: haloperidol=30.7; } \\
\text { quetiapine=31.3. } \\
\text { Sex (M/F): H: 21/12; Q: 25/15; } \\
\text { Duration of illness (mos; SD): } \\
\text { haloperidol=4.8 (1.6); } \\
\text { quetiapine=5.0 (2.1); } \\
\text { Marital status (unmarried/married): } \\
\text { haloperidol=19/14; } \\
\text { quetiapine=23.17; } \\
\text { Employment (unemployed/ } \\
\text { employed): haloperidol= 22/11; } \\
\text { quetiapine=28/12; } \\
\text { Education (above/below } \\
\text { secondary): haloperidol=23/10; } \\
\text { quetiapine=31/9; } \\
\text { Income (satisfactory/ } \\
\text { unsatisfactory): haloperidol=7/26; } \\
\text { quetiapine=8/32; } \\
\text { Type of schizophrenia } \\
\text { (paranoid/not paranoid): } \\
\text { haloperidol=24/9; } \\
\text { quetiapine=32/8. }\end{array}$ \\
\hline
\end{tabular}




\begin{tabular}{|c|c|c|c|}
\hline Author, Year & Other Population Characteristics & Total $\mathbf{N}$ & Benefits Outcomes \\
\hline Amr, 2013 & NR & 156 & 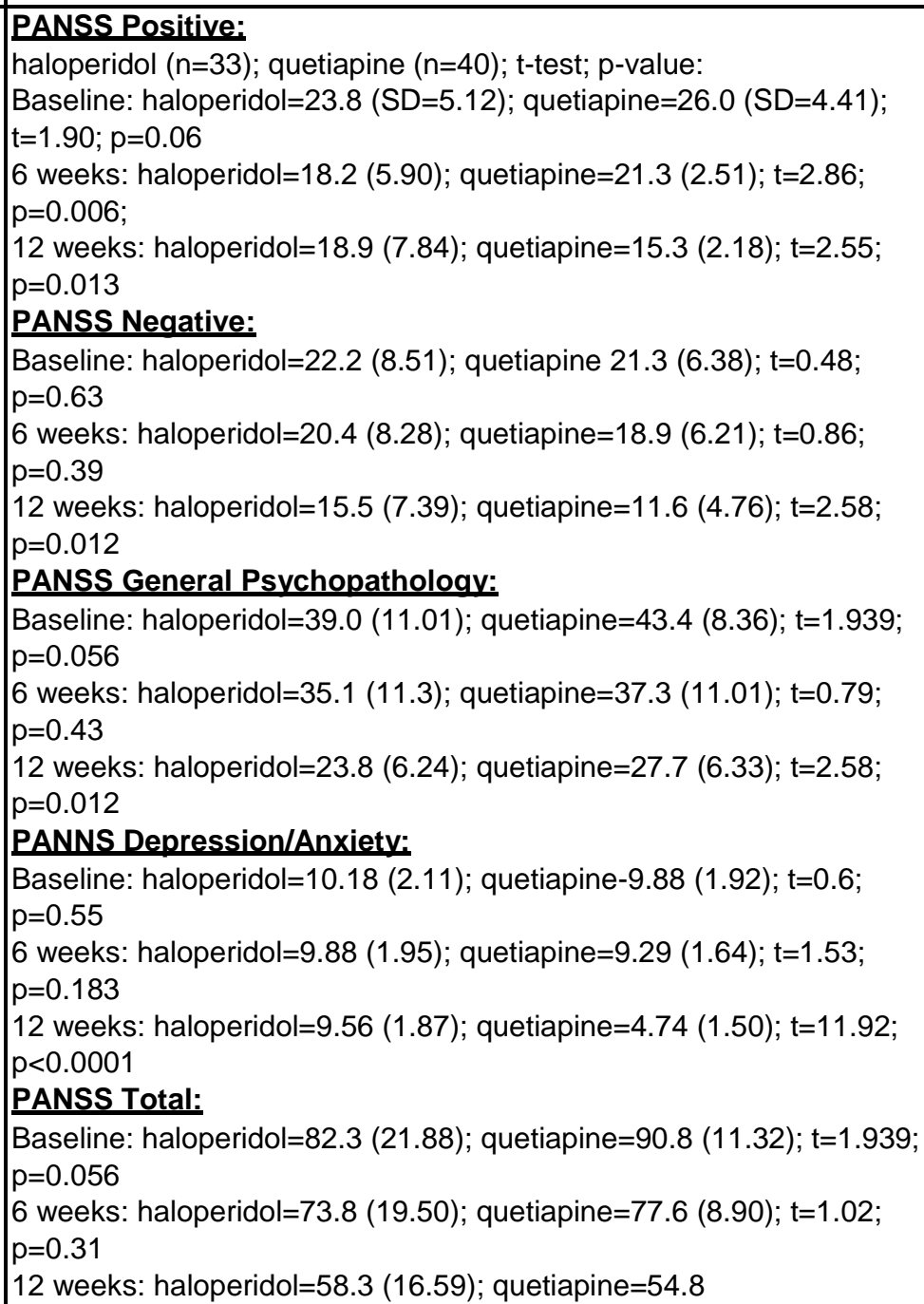 \\
\hline
\end{tabular}




\begin{tabular}{|c|c|c|c|}
\hline Author, Year & Harms Outcomes & Funding & Quality Rating \\
\hline Amr, 2013 & 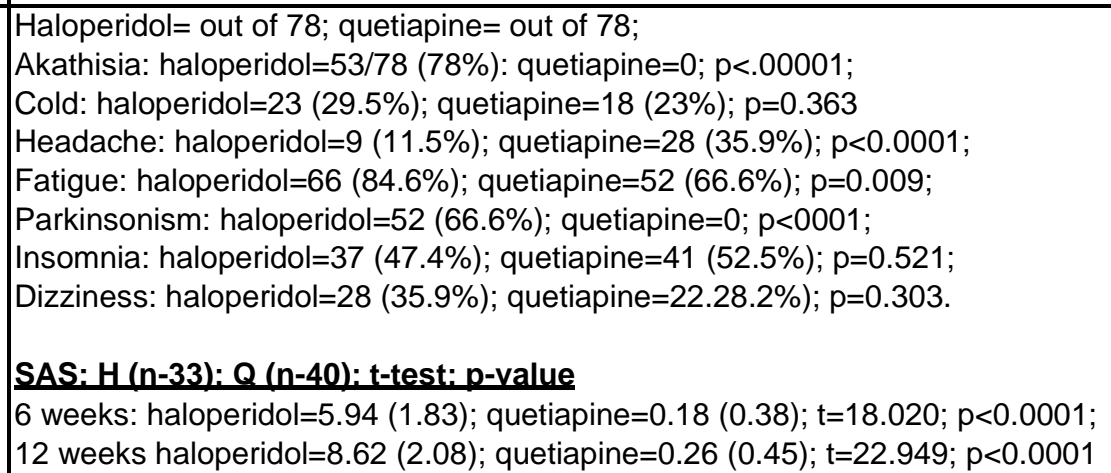 & Not stated & Poor \\
\hline
\end{tabular}




\begin{tabular}{|c|c|c|c|c|c|}
\hline Author, Year & \begin{tabular}{|l} 
Setting \\
Country
\end{tabular} & Inclusion Criteria & $\begin{array}{l}\text { Interventions and Ns per } \\
\text { Group }\end{array}$ & $\begin{array}{l}\text { Duration } \\
\text { (intervention and } \\
\text { longest followup) }\end{array}$ & $\begin{array}{l}\text { Age Gender } \\
\text { Race/Ethnicity }\end{array}$ \\
\hline Citrome, 2016 & US & $\begin{array}{l}\text { Adult patients (18 to } 65 \text { years) with DSM- } \\
\text { IV-TR diagnosis of schizophrenia } \\
\text { confirmed by the MINI International } \\
\text { Neuropsychiatric Interview. }\end{array}$ & $\begin{array}{l}\text { Brexpiprazole } 3 \mathrm{mg} / \text { day } \\
(\mathrm{N}=64) \\
\text { vs. } \\
\text { Aripiprazole } 15 \mathrm{mg} / \text { day } \\
(\mathrm{N}=33)\end{array}$ & 6 weeks & $\begin{array}{l}\text { Age, year: } 42.2 \\
\text { Gender, \% Female: } 29.2 \% \\
\text { Ethnicity, \%: } \\
\text { White: } 23.1 \% \\
\text { African-American: } 73.9 \% \\
\text { Asian: } 0.8 \% \\
\text { Other: } 2.3 \%\end{array}$ \\
\hline
\end{tabular}




\begin{tabular}{|l|l|l|l|}
\hline & & & \\
\hline Author, Year & Other Population Characteristics & Total N & Benefits Outcomes \\
\hline Citrome, 2016 & $\begin{array}{l}\text { PANSS total score baseline, mean: } 93.7 \\
\text { Duration of current episode: } 3.1 \text { weeks }\end{array}$ & $\begin{array}{l}\text { Brexpiprazole vs. Aripiprazole } \\
\text { Change in baseline PANSS total score, LS mean at } 6 \text { w: } \\
-22.9 ; \mathrm{P}<0.0001 \text { vs. }-19.4 ; \mathrm{P}<0.0001 \\
\text { Response rate at } 6 \mathrm{~W}, \%(\mathrm{n} / \mathrm{N})^{\star}: 60.9 \%(39 / 64),(95 \% \mathrm{Cl} 47.9 \text { to } 72.9) \\
\text { vs. 48.5\% }(16 / 33),(95 \% \mathrm{Cl} 30.8 \text { to } 66.5)\end{array}$ \\
\hline
\end{tabular}




\begin{tabular}{|c|c|c|c|}
\hline Author, Year & Harms Outcomes & Funding & Quality Rating \\
\hline Citrome, 2016 & $\begin{array}{l}\text { Brexpiprazole vs. Aripiprazole } \\
\text { Overall AEs, } \%(\mathrm{n} / \mathrm{N}): 57.8 \%(37 / 64) \text { vs. } 63.6 \%(21 / 33) \\
\text { Withdrawal due to AEs, } \%(\mathrm{n} / \mathrm{N}): 4.7 \%(3 / 64) \text { vs. } 3.0 \%(1 / 33) \\
\text { All-cause mortality: } 0 \text { vs. } 0 \\
\text { Clinically relevant weight gain ( } \geq 7 \% \text { increase from baseline) at } 6 \text { weeks, } \%(\mathrm{n} / \mathrm{N}): 35 \%(14 / 40) \\
\text { vs. } 19 \%(4 / 21) \\
\text { Extrapyramidal AEs, } \%(\mathrm{n} / \mathrm{N}): 14.1 \%(9 / 64) \text { vs. } 30.3 \%(10 / 33) \text {. } \\
\text { Simpson Angus, Abnormal Involuntary Movement, and BARS global clinical assessment scales } \\
\text { used but no differences were found between them. }\end{array}$ & $\begin{array}{l}\text { Funding: Otsuka Pharmaceutical } \\
\text { Commercialization and } \\
\text { Development Inc.; H. Lundbeck } \\
\text { A/S } \\
\text { *Reduction of } 30 \% \text { or more from } \\
\text { baseline in PANSS total score, or } \\
\text { CGI-I score of } 1 \text { or } 2 \text {. }\end{array}$ & Fair \\
\hline
\end{tabular}




\begin{tabular}{|c|c|c|c|c|c|}
\hline Author, Year & \begin{tabular}{|l} 
Setting \\
Country
\end{tabular} & Inclusion Criteria & $\begin{array}{l}\text { Interventions and Ns per } \\
\text { Group }\end{array}$ & \begin{tabular}{|l} 
Duration \\
(intervention and \\
longest followup)
\end{tabular} & $\begin{array}{l}\text { Age Gender } \\
\text { Race/Ethnicity }\end{array}$ \\
\hline $\begin{array}{l}\text { Crespo-Facorro, } 2011 \\
\text { Crespo-Facorro, } 2012 \\
\text { Spain }\end{array}$ & Spain & $\begin{array}{l}\text { Age 15-60 years, experiencing first } \\
\text { psychotic episode, <6 weeks lifetime } \\
\text { antipsychotic treatment, meet DSM-IV } \\
\text { criteria for brief psychotic disorder, } \\
\text { schizophreniform disorder, } \\
\text { schizophrenia, schizoaffective disorder. } \\
\text { Excluded DSM-IV criteria for drug } \\
\text { dependence or mental retardation, } \\
\text { history of neurological disease or head } \\
\text { injury. }\end{array}$ & $\begin{array}{l}\text { Haloperidol: } \mathrm{n}, 56 \text {; mean } \\
\text { dose, } 2.9 \text { (1.4) mg/day } \\
\text { Olanzapine: } \mathrm{n}, 55 \text {; mean } \\
\text { dose, } 10.1(3.9) \mathrm{mg} / \text { day } \\
\text { Risperidone: } \mathrm{n}, 63 \text {; mean } \\
\text { dose, } 3.4 \text { (1.8) mg/day }\end{array}$ & 156 weeks & $\begin{array}{l}\text { Age, mean: } 27.4 \\
\text { Gender: } 38 \% \text { female } \\
\text { Ethnicity: NR }\end{array}$ \\
\hline
\end{tabular}




\begin{tabular}{|c|c|c|c|}
\hline Author, Year & Other Population Characteristics & Total $\mathbf{N}$ & Benefits Outcomes \\
\hline $\begin{array}{l}\text { Crespo-Facorro, } 2011 \\
\text { Crespo-Facorro, } 2012 \\
\text { Spain }\end{array}$ & $\begin{array}{l}\text { Age, psychosis onset: } 26 \text { years } \\
\text { Duration of illness: } 25 \text { months } \\
\text { Duration of psychosis: } 11 \text { months } \\
\text { Diagnosis: Schizophrenia, } 60.8 \% \text {; Schizophreniform, } \\
\text { 24.1\%; Schizoaffective, } 2.4 \% \text {, Brief psychotic disorder, } \\
5.4 \% \text {; Unspecified psychotic disorder, } 7.2 \%\end{array}$ & 174 & 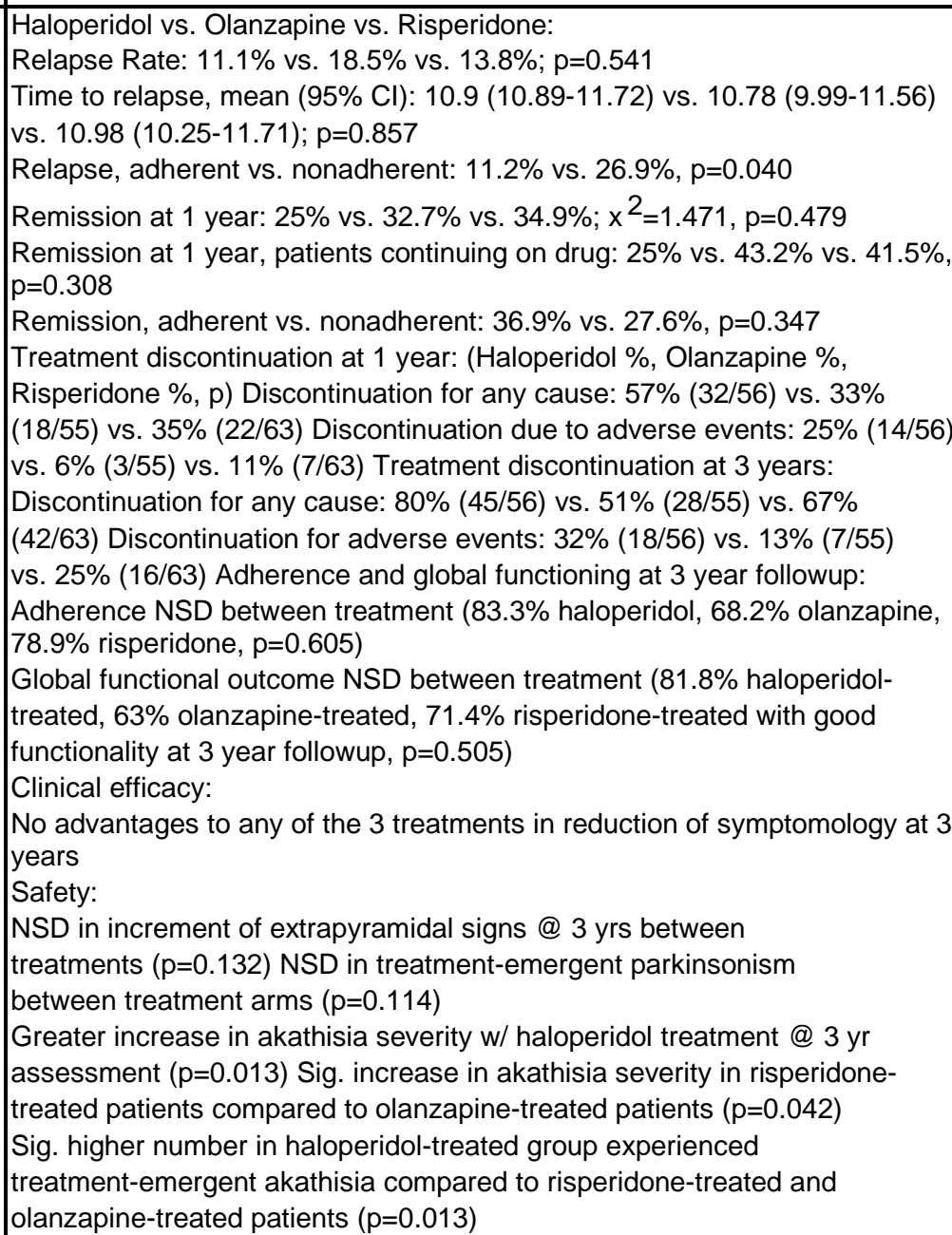 \\
\hline
\end{tabular}




\begin{tabular}{|c|c|c|c|}
\hline Author, Year & Harms Outcomes & Funding & Quality Rating \\
\hline $\begin{array}{l}\text { Crespo-Facorro, } 2011 \\
\text { Crespo-Facorro, } 2012 \\
\text { Spain }\end{array}$ & $\begin{array}{l}\text { Haloperidol \% vs. Olanzapine \% vs. Risperidone } \%, P \\
\text { Concentration difficult: } 9.1 \text { vs. } 7.7 \text { vs. } 0.0,0.419 \\
\text { Asthenia: } 9.1 \text { vs. } 23.1 \text { vs. } 0.0,0.057 \\
\text { Daytime drowsiness: } 0.0 \text { vs. } 34.6 \text { vs. } 10.0,0.022 \\
\text { Increased sleep hours: } 9.1 \text { vs. } 11.5 \text { vs. } 5.0,0.739 \\
\text { Akathisia: } 27.3 \text { vs. } 0.0 \text { vs. } 5.0,0.011 \\
\text { Sialorrhea: } 0.0 \text { vs. } 0.0 \text { vs. } 15.0,0.053 \\
\text { Dry mouth: } 0.0 \text { vs. } 7.7 \text { vs. } 10.0,0.571 \\
\text { Weight gain: } 9.1 \text { vs. } 26.9 \text { s. } 20.0,0.473 \\
\text { Amenorrhea (only females, } n=23): 0.0 \text { vs. } 0.0 \text { vs. } 40.0,0.043 \\
\text { Sexual dysfunctions (only males, } n=34 \text { ): } 14.3 \text { vs. } 5.9 \text { vs. } 40.0,0.078\end{array}$ & NR & Fair \\
\hline
\end{tabular}




\begin{tabular}{|c|c|c|c|c|c|}
\hline Author, Year & $\begin{array}{l}\text { Setting } \\
\text { Country }\end{array}$ & Inclusion Criteria & $\begin{array}{l}\text { Interventions and Ns per } \\
\text { Group }\end{array}$ & \begin{tabular}{|l} 
Duration \\
(intervention and \\
longest followup)
\end{tabular} & $\begin{array}{l}\text { Age Gender } \\
\text { Race/Ethnicity }\end{array}$ \\
\hline Detke, 2014 & $\begin{array}{l}\text { Multisite, USA } \\
\text { and France }\end{array}$ & $\begin{array}{l}\text { Outpatients (18 to } 65 \text { years) who met the } \\
\text { criteria for schizophrenia based on DSM- } \\
\text { IV or the DSM-IV Text Revision. } \\
\text { Required to be "at risk for relapse" (at } \\
\text { least } 2 \text { episodes of clinical worsening of } \\
\text { schizophrenia symptoms in the previous } \\
24 \text { months) }\end{array}$ & $\begin{array}{l}\text { Olanzapine long-acting } \\
\text { injection } 405 \mathrm{mg} / 4 \text { weeks } \\
(\mathrm{n}=264) \\
\text { vs. } \\
\text { Oral olanzapine } 10 \mathrm{mg} / \mathrm{day} \\
(\mathrm{n}=260)\end{array}$ & 2 years & $\begin{array}{l}\text { Age, mean years: } 40.9 \\
\text { Gender, \% female: } 32.8 \\
\text { Ethnicity, \%: } \\
\text { White: } 62.0 \\
\text { African: } 16.8 \\
\text { Hispanic: } 8.0 \\
\text { East Asian: } 8.8 \\
\text { West Asian: } 3.6 \\
\text { Native American: } 0.8\end{array}$ \\
\hline Di Fiorino 2014 & Italy & $\begin{array}{l}\text { Adults (aged } 18 \text { to } 65 \text { years) with a } \\
\text { documented DSM-IV diagnosis of } \\
\text { diagnosis of schizophrenia or } \\
\text { schizoaffective disorder. }\end{array}$ & $\begin{array}{l}\text { Quetiapine extended- } \\
\text { release } 400 \text { to } 800 \mathrm{mg} / \mathrm{day} \\
(\mathrm{n}=109) \\
\text { vs. } \\
\text { Risperidone } 4 \text { to } 6 \mathrm{mg} / \mathrm{day} \\
(\mathrm{n}=107)\end{array}$ & 12 weeks & $\begin{array}{l}\text { Age, years: } 42.3 \\
\text { Gender, \% female: } 43.3 \\
\text { Ethnicity, \%: } \\
\text { White: } 100\end{array}$ \\
\hline Durgam, 2014 & International & $\begin{array}{l}\text { Adults ages } 18 \text { to } 60 \text { years with } \\
\text { schizophrenia (first episode excluded). }\end{array}$ & $\begin{array}{l}\text { Cariprazine } 1.5 \mathrm{mg} / \mathrm{day} \\
\text { (n=145) vs. } \\
\text { Cariprazine } 3.0 \mathrm{mg} / \mathrm{day} \\
(\mathrm{n}=146) \text { vs. } \\
\text { Cariprazine } 4.5 \mathrm{mg} / \mathrm{day} \\
(\mathrm{n}=147) \text { vs. } \\
\text { Risperidone } 4.0 \mathrm{mg} / \mathrm{day} \\
(\mathrm{n}=140) \\
\\
\text { (Placebo arm also } \\
\text { included.) }\end{array}$ & 6 weeks & $\begin{array}{l}\text { Age, mean years: } 36.5 \\
\text { Gender, \% female: } 31.0 \\
\text { Ethnicity, \%: } \\
\text { White: } 50.0 \\
\text { African American: } 24.0 \% \\
\text { Asian: } 25.0 \\
\text { Other: } 0.7 \\
\text { (Placebo arm excluded.) }\end{array}$ \\
\hline
\end{tabular}




\begin{tabular}{|c|c|c|c|}
\hline Author, Year & Other Population Characteristics & Total N & Benefits Outcomes \\
\hline Detke, 2014 & $\begin{array}{l}\text { Age of onset of schizophrenia, mean y (SD): } 26.2(8.9) \\
\text { Previous episodes in last } 24 \text { months, mean (SD): } 2.7(1.6) \\
\text { Length of current episode, mean days (SD): } 175.0(148.0) \\
\text { Poor medication adherence, } \mathrm{n}(\%): 24.0(4.6)\end{array}$ & 524 & $\begin{array}{l}\text { Olanzapine long-acting injection vs. oral olanzapine } \\
\text { All-cause discontinuation rate, \%: } 53.8 \text { vs. } 51.2 ; p=0.600 \\
\text { Time to all-cause discontinuation, median days: } 645 \text { vs. } 678 ; p=0.612 \\
\text { Rate of relapse, } \%: 20.1 \text { vs. } 18.5, p=0.659 \\
\text { Time to relapse/rescue, median days: } 539 \text { vs. } 281 ; p<0.001 \\
\text { Baseline-to-endpoint least-squares mean change on PANSS total } \\
\text { score, (SE): } \\
-0.82 \text { (1.2) vs. }-1.14 \text { (1.2); } p=0.834\end{array}$ \\
\hline Di Fiorino 2014 & $\begin{array}{l}\text { PANSS severity of illness score: } 101.4 \\
\text { Schizoaffective, \%: } 47.7\end{array}$ & 216 & $\begin{array}{l}\text { Quetiapine extended-release } 400 \text { to } 800 \mathrm{mg} / \text { day vs. risperidone } 4 \text { to } 6 \\
\text { mg/day } \\
\text { PANSS total score, LSM (SD): }-30.0 \text { (22.9) vs. }-21.1 \text { (23.8) } \\
\text { Treatment difference: }-8.9, P=0.0002\end{array}$ \\
\hline Durgam, 2014 & $\begin{array}{l}\text { Duration of illness: } 11.5 \text { years } \\
\text { Duration of current illness/psychosis: less than } 2 \text { weeks to } \\
\text { be eligible } \\
\text { Hospitalization data (current): NR } \\
\text { Severity of illness: } 97.3 \text { (PANSS) } \\
\text { Schizoaffective: } 0 \% \text { (excluded) } \\
\text { Substance use: } 0 \% \text { (excluded) } \\
\text { Antipsychotic drug naïve: first episode of psychosis } \\
\text { excluded }\end{array}$ & $\begin{array}{l}578 \\
\text { (active treatment } \\
\text { arms) }\end{array}$ & $\begin{array}{l}\text { Cariprazine } 1.5 \mathrm{mg} / \text { day vs. cariprazine } 3.0 \mathrm{mg} / \text { day vs. cariprazine } 4.5 \\
\text { mg/day vs. risperidone } 4.0 \mathrm{mg} / \text { day } \\
\\
\text { PANSS responders ( } \geq 30 \% \text { improvement from baseline): } \%(\mathrm{n} / \mathrm{N}) \\
31.4 \text { (44/140) vs. } 35.7 \text { (50/140) vs. } 35.9 \text { (52/145) vs. } 43.5(60 / 138) \\
\text { (No p-values comparing active treatments reported.) }\end{array}$ \\
\hline
\end{tabular}




\begin{tabular}{|c|c|c|c|}
\hline Author, Year & Harms Outcomes & Funding & Quality Rating \\
\hline Detke, 2014 & $\begin{array}{l}\text { Olanzapine long-acting injection vs. Oral olanzapine } \\
\text { Any adverse event, n/N (\%): } 182 / 264 \text { (68.9) vs. } 176 / 260(67.7) \\
\text { Discontinuations due to adverse events, n/N (\%): } 26 / 264 \text { (9.8) vs. 25/260 (9.6) } \\
\text { Death, } n / \mathrm{N}(\%): 0 / 264 \text { vs. } 2 / 260(0.8) \\
\text { Weight increased, } \mathrm{n} / \mathrm{N}(\%): 44 / 264(16.7) \text { vs. } 43 / 260(16.5) \\
\text { Weight decreased, n/N (\%): } 15 / 264(5.7) \text { vs. } 14 / 260(5.4) \\
\text { Extrapyramidal symptoms/akathisia, n/N (\%): } 7 / 264(2.7) \text { vs. } 10 / 260(3.8)\end{array}$ & Eli Lilly and Co. & Poor \\
\hline Di Fiorino 2014 & $\begin{array}{l}\text { Quetiapine extended-release } 400 \text { to } 800 \mathrm{mg} / \text { day vs. risperidone } 4 \text { to } 6 \mathrm{mg} / \mathrm{day} \\
\text { Overall } \mathrm{AE}, \mathrm{n} / \mathrm{N}(\%): 40 / 107(37.4) \text { vs. } 36 / 103(35.0) \\
\text { Withdrawals due to } \mathrm{AE}, \mathrm{n} / \mathrm{N}(\%): 10 / 107 \text { (9.4) vs. } 7 / 103(6.8)\end{array}$ & $\begin{array}{l}\text { AstraZeneca Italy } \\
\text { *Included disorientation, } \\
\text { psychotic disorder, delusion, and } \\
\text { extrapyramidal syndrome vs. } \\
\text { fainting, acute psychosis, acute } \\
\text { respiratory failure, social stay } \\
\text { hospitalization, and } \\
\text { cardiocirculatory arrest }\end{array}$ & Fair \\
\hline Durgam, 2014 & $\begin{array}{l}\text { Cariprazine } 1.5 \mathrm{mg} / \text { day vs. cariprazine } 3.0 \mathrm{mg} / \text { day vs. cariprazine } 4.5 \mathrm{mg} \text { /day vs. risperidone } \\
4.0 \mathrm{mg} / \text { day } \\
\text { Treatment-emergent adverse events: } \%(\mathrm{n} / \mathrm{N}) \\
68.3(99 / 145) \text { vs. } 71.2(104 / 146) \text { vs. } 73.5(108 / 147) \text { vs. } 67.9(95 / 140) \\
\text { WAE: } \%(\mathrm{n} / \mathrm{N}) \\
9.7(14 / 145) \text { vs. } 5.5(8 / 146) \text { vs. } 8.2(12 / 147) \text { vs. } 9.3(13 / 140) \\
\text { Extrapyramidal disorder (treatment-emergent): } \\
9.0(13 / 145) \text { vs. } 8.9(13 / 146) \text { vs. } 11.6(17 / 142) \text { vs. } 12.9(18 / 140)\end{array}$ & $\begin{array}{l}\text { Forest Research Institute and } \\
\text { Gedeon Richter Plc. }\end{array}$ & Fair \\
\hline
\end{tabular}




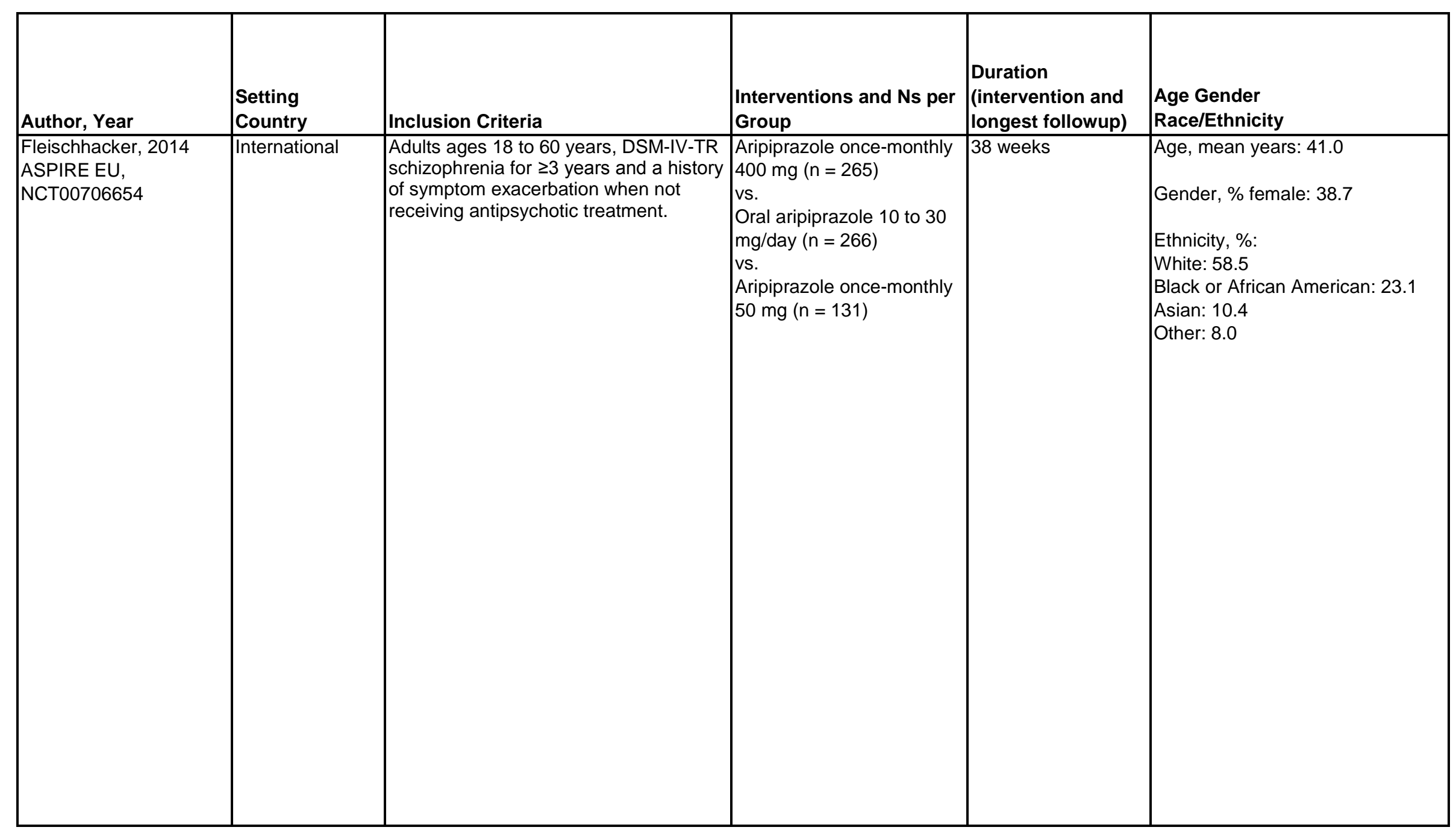




\begin{tabular}{|c|c|c|c|}
\hline Author, Year & Other Population Characteristics & Total N & Benefits Outcomes \\
\hline $\begin{array}{l}\text { Fleischhacker, } 2014 \\
\text { ASPIRE EU, } \\
\text { NCT00706654 }\end{array}$ & \begin{tabular}{|l|} 
PANSS total score, mean: 56.9 \\
CGI-Severity score, mean: 3.07 \\
CGI-Improvement score, mean: 3.2
\end{tabular} & 662 & 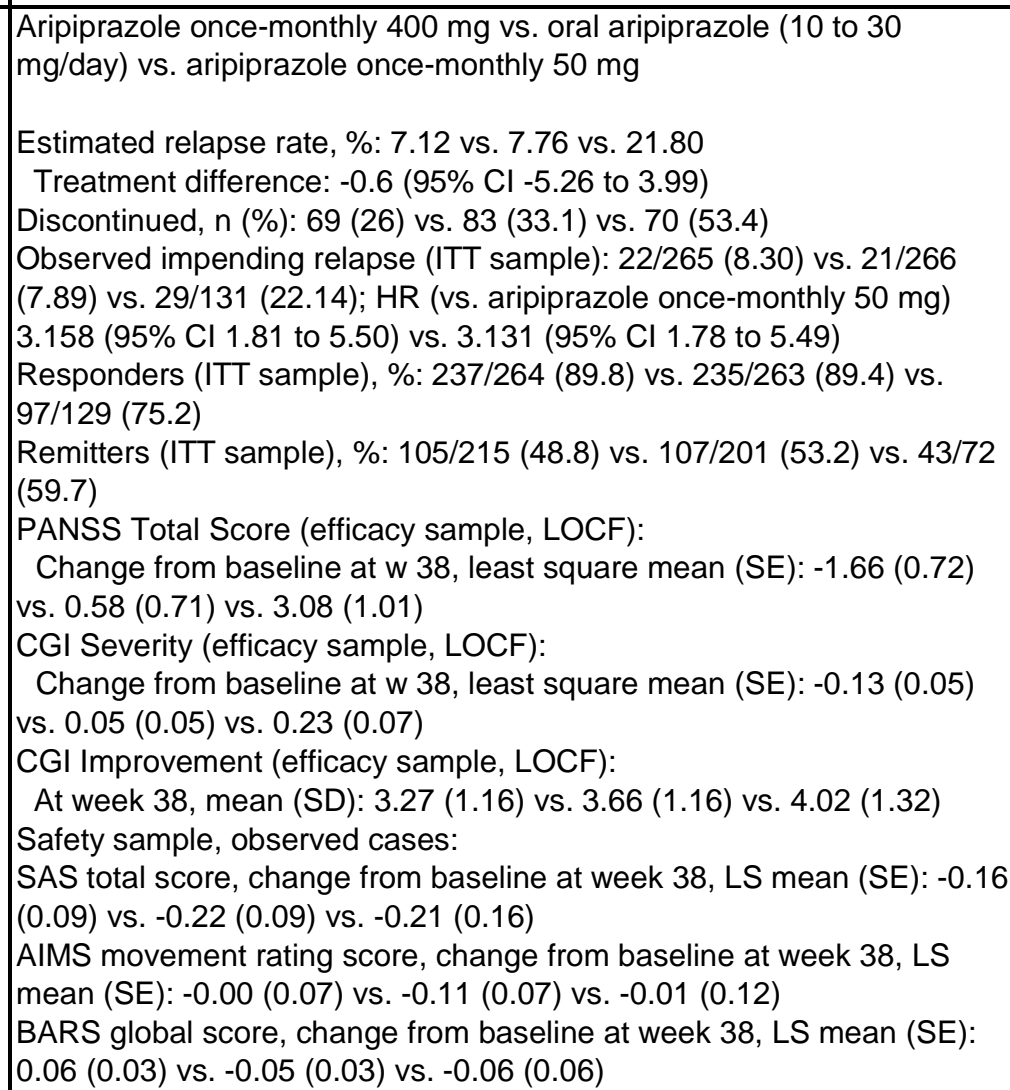 \\
\hline
\end{tabular}




\begin{tabular}{|c|c|c|c|}
\hline Author, Year & Harms Outcomes & Funding & Quality Rating \\
\hline $\begin{array}{l}\text { Fleischhacker, } 2014 \\
\text { ASPIRE EU, } \\
\text { NCT00706654 }\end{array}$ & 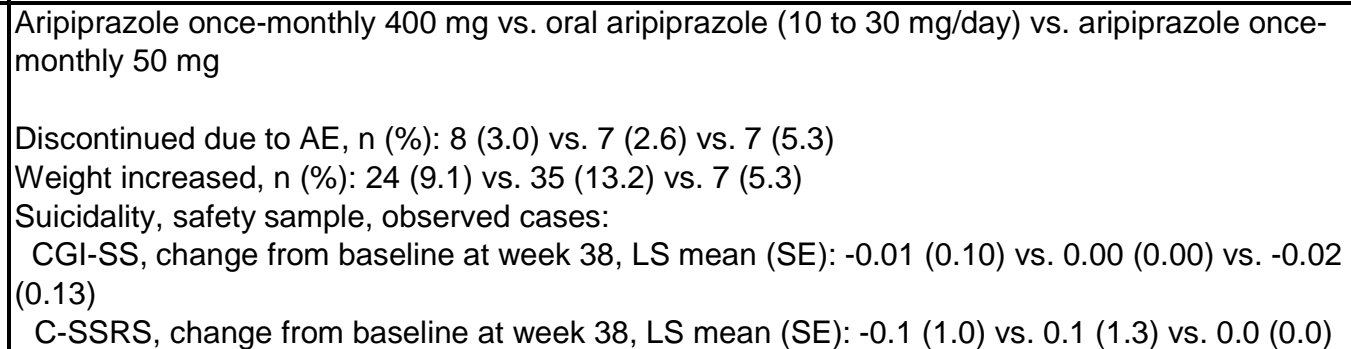 & $\begin{array}{l}\text { Otsuka Pharmaceutical } \\
\text { Commercialization, Inc. }\end{array}$ & Fair \\
\hline
\end{tabular}




\begin{tabular}{|l|l|l|l|l|l|}
\hline Author, Year & $\begin{array}{l}\text { Setting } \\
\text { Country }\end{array}$ & $\begin{array}{l}\text { Inclusion Criteria } \\
\text { clinics (four VA toal) }\end{array}$ & $\begin{array}{l}\text { Age 18-65, with EtOH Use Disorder plus } \\
\text { schizophrenia (48.4\%) or } \\
\text { schizoaffective disorder }\end{array}$ & $\begin{array}{l}\text { LAl risperidone 25-50 mg } \\
\text { every 2 weeks (49) vs. oral } \\
\text { risperidone (up to } \\
\text { Gmg/day) (46) }\end{array}$ \\
\hline Green, 2015 & & $\begin{array}{l}\text { Duration } \\
\text { (intervention and } \\
\text { longest followup) }\end{array}$ & $\begin{array}{l}\text { Age Gender } \\
\text { Race/Ethnicity }\end{array}$ \\
\hline
\end{tabular}




\begin{tabular}{|c|c|c|c|}
\hline Author, Year & Other Population Characteristics & Total N & Benefits Outcomes \\
\hline Green, 2015 & \begin{tabular}{|l|} 
Education 11.0 years \\
Ever employed $97 \%$ \\
Single $51 \%$ \\
Lifetime Hospitalizations 7.5 \\
Cannabis use 1.1 days/week \\
Other drugs 0.3 days/week
\end{tabular} & 95 & $\begin{array}{l}\text { ITT analyses: no significant difference in drinking } \\
\text { Explanatory analyses using weeks } 5-23: \\
\text { Trend significance change in days heavy drinking ( } 5 \text { or more/day) oral } \\
\text { ( } 0.68 \text { days/week) vs. LAl ( }-0.11 \text { days/week) t63.5=-1.96, p=0.054 } \\
\text { Good adherence (exposed to meds } 75 \% \text { of study days): oral } 61 \% \text { vs. } \\
\text { LAl } 88 \% \text {, chi2 } 2=9.08, p=0.003 \text { (oral vs. LAl: } 28 / 46 \text { [61\%] vs. } 43 / 49 \\
\text { [88\%], RR } 3.20 \text { [95\% Cl } 1.39 \text { to } 7.34] \text { ) } \\
\text { No between-group differences in total PANSS, GAF, or CGI }\end{array}$ \\
\hline
\end{tabular}




\begin{tabular}{|l|l|l|l|}
\hline & & & \\
& & & \\
Author, Year & Harms Outcomes & Funding & Janssen \\
\hline Green, 2015 & No differences in side effects between oral and LAl & Fair & \\
& & & \\
\hline
\end{tabular}




\begin{tabular}{|c|c|c|c|c|c|}
\hline Author, Year & $\begin{array}{l}\text { Setting } \\
\text { Country }\end{array}$ & Inclusion Criteria & $\begin{array}{l}\text { Interventions and Ns per } \\
\text { Group }\end{array}$ & \begin{tabular}{|l} 
Duration \\
(intervention and \\
longest followup)
\end{tabular} & $\begin{array}{l}\text { Age Gender } \\
\text { Race/Ethnicity }\end{array}$ \\
\hline Ishigooka, 2015 & Asia & $\begin{array}{l}\text { Asian adults (18 years and older) } \\
\text { diagnosed with schizophrenia according } \\
\text { to DSM-IV-TR criteria. }\end{array}$ & $\begin{array}{l}\text { Aripiprazole } 300 \text { to } 400 \mathrm{mg} \\
\text { once-monthly injection } \\
(\mathrm{n}=228)^{\star} \\
\text { vs. } \\
\text { Aripiprazole } 6 \text { to } 24 \\
\text { mg/day orally }(\mathrm{n}=227)\end{array}$ & $\begin{array}{l}52 \text { weeks (double- } \\
\text { blind phase) }\end{array}$ & $\begin{array}{l}\text { Age, years: } 39.2 \\
\text { Gender, \% female: } 39.2 \\
\text { Ethnicity, \% } \\
\text { Asian: } 100\end{array}$ \\
\hline
\end{tabular}




\begin{tabular}{|c|c|c|c|}
\hline Author, Year & Other Population Characteristics & Total N & Benefits Outcomes \\
\hline Ishigooka, 2015 & $\begin{array}{l}\text { Duration of illness (time since first episode), months } \\
\text { (mean): } 151.6 \\
\text { PANSS severity of illness: } 53.9\end{array}$ & 455 & 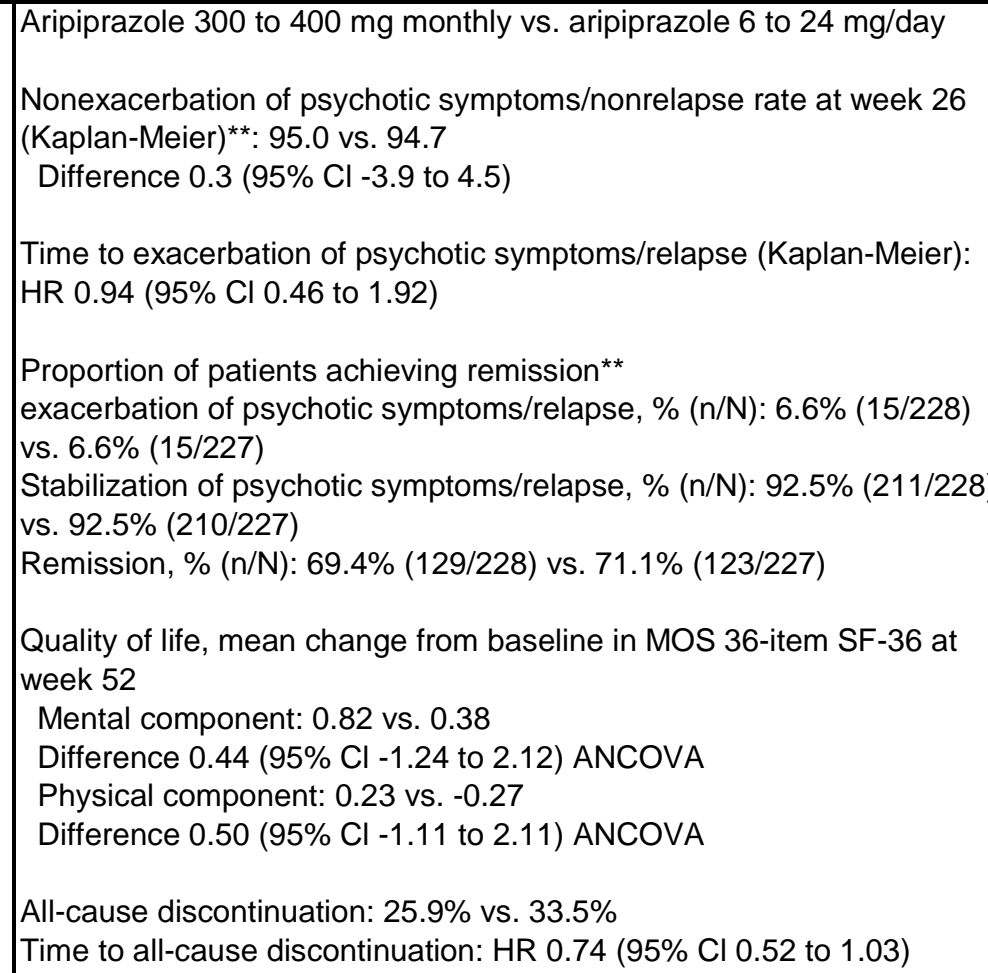 \\
\hline
\end{tabular}




\begin{tabular}{|c|c|c|c|}
\hline Author, Year & Harms Outcomes & Funding & Quality Rating \\
\hline Ishigooka, 2015 & $\begin{array}{l}\text { Aripiprazole } 300 \text { to } 400 \mathrm{mg} \text { monthly vs. aripiprazole } 6 \text { to } 24 \mathrm{mg} / \text { day } \\
\text { Overall AE: \% (n/N): } 77.2 \%(176 / 228) \text { vs. } 79.3 \%(180 / 227) \\
\text { Withdrawal due to AE: } \%(\mathrm{n} / \mathrm{N}): 7.5 \%(17 / 228) \text { vs. } 11.5 \%(25 / 227) \\
\text { Extrapyramidal AE: } \%(\mathrm{n} / \mathrm{N}): 16.2 \%(40 / 228) \text { vs. } 14.1 \%(32 / 227) \\
\text { Tardive dyskinesia: } \%(\mathrm{n} / \mathrm{N}): 0 \text { vs. } 0.4 \%(1 / 227) \\
\text { Akathisia: } \%(\mathrm{n} / \mathrm{N}): 6.6 \%(12 / 228) \text { vs. } 6.2 \%(14 / 227)\end{array}$ & $\begin{array}{l}\text { Otsuka Pharmaceutical Co., Ltd. } \\
\text { *Injection arm patients received } 6 \\
\text { or } 12 \mathrm{mg} / \text { day of oral aripiprazole } \\
\text { for } 2 \text { weeks after start of } \\
\text { randomized period } \\
\text { **Exacerbation/relapse based on } \\
\text { CCG-I and PANSS scores, } \\
\text { hospitalization, violent behavior } \\
\text { resulting in injury }\end{array}$ & Fair \\
\hline
\end{tabular}




\begin{tabular}{|c|c|c|c|c|c|}
\hline Author, Year & $\begin{array}{l}\text { Setting } \\
\text { Country }\end{array}$ & Inclusion Criteria & $\begin{array}{l}\text { Interventions and Ns per } \\
\text { Group }\end{array}$ & \begin{tabular}{|l} 
Duration \\
(intervention and \\
longest followup)
\end{tabular} & $\begin{array}{l}\text { Age Gender } \\
\text { Race/Ethnicity }\end{array}$ \\
\hline $\begin{array}{l}\text { Koshikawa, } 2016 \\
\text { Companion: } \\
\text { Takekita, } 2016\end{array}$ & Japan & $\begin{array}{l}\geq 20 \text { years old, DSM-IV-TR diagnosis of } \\
\text { schizophrenia or schizoaffective } \\
\text { disorder (nonacute phase of the } \\
\text { disease), PANSS total score } \leq 120, \\
\text { received risperidone long- acting for } \geq 2 \\
\text { months. }\end{array}$ & \begin{tabular}{|l} 
Risperidone long-acting \\
injection, adjustable dose \\
(upper limit of $50 \mathrm{mg}$ ) \\
every 2 weeks ( $\mathrm{N}=16)$ \\
vs. \\
Paliperidone palmitate \\
adjustable dose (upper \\
limit of $150 \mathrm{mg}$ ) every 4 \\
weeks ( $\mathrm{N}=14$ )
\end{tabular} & 28 weeks & $\begin{array}{l}\text { Age, year: } 45.0 \\
\text { Gender, } \% \text { female: } 38.0 \\
\text { Ethnicity: Japanese (\% NR) }\end{array}$ \\
\hline
\end{tabular}




\begin{tabular}{|c|c|c|c|}
\hline Author, Year & Other Population Characteristics & Total N & Benefits Outcomes \\
\hline $\begin{array}{l}\text { Koshikawa, } 2016 \\
\text { Companion: } \\
\text { Takekita, } 2016\end{array}$ & $\begin{array}{l}\text { Duration of illness, year*: } 13.8 \\
\text { PANSS total score, mean: } 80.6 \\
\text { Schizoaffective disorder, \%: } 5.0\end{array}$ & 30 & $\begin{array}{l}\text { Risperidone long-acting injection vs. paliperidone palmitate } \\
\text { Koshikawa, 2016: } \\
\text { Social Functioning Scale total score, mean change from baseline (SD): - } \\
1.64 \text { (17.56) vs. } 14.60 \text { (18.75), p=0.038 } \\
\text { No difference in PANSS total score between treatment groups at } 6 \\
\text { months } \\
\text { Takekita, 2016: } \\
\text { PANSS total score, mean change from baseline to } 6 \text { months (SD): - } \\
5.09 \text { (8.18) vs. }-1.70(5.08), p=0.349\end{array}$ \\
\hline
\end{tabular}




\begin{tabular}{|c|c|c|c|}
\hline Author, Year & Harms Outcomes & Funding & Quality Rating \\
\hline $\begin{array}{l}\text { Koshikawa, } 2016 \\
\text { Companion: } \\
\text { Takekita, } 2016\end{array}$ & $\begin{array}{l}\text { Risperidone long-acting injection vs. paliperidone palmitate } \\
\text { Koshikawa, 2016: } \\
\text { Overall AEs, n: } 0 \text { vs. } 2 \\
\text { Takekita, 2016: } \\
\text { DIEPSS }{ }^{\star *} \text { total score, mean change from baseline (SD): }-0.09(0.30) \text { vs. } 0.30(1.06), p=0.220\end{array}$ & $\begin{array}{l}\text { Funding: NR } \\
\text { *Duration of illness calculated } \\
\text { based on average age at onset } \\
\text { and average age at study } \\
\text { enrollment. } \\
\text { **Drug-induced extrapyramidal } \\
\text { symptoms scale. }\end{array}$ & Fair \\
\hline
\end{tabular}




\begin{tabular}{|c|c|c|c|c|c|}
\hline Author, Year & $\begin{array}{l}\text { Setting } \\
\text { Country }\end{array}$ & Inclusion Criteria & $\begin{array}{l}\text { Interventions and Ns per } \\
\text { Group }\end{array}$ & \begin{tabular}{|l} 
Duration \\
(intervention and \\
longest followup)
\end{tabular} & $\begin{array}{l}\text { Age Gender } \\
\text { Race/Ethnicity }\end{array}$ \\
\hline Li, 2014 & China & $\begin{array}{l}\text { Adults (18 to } 65 \text { years) with a DSM-IV } \\
\text { diagnosis of schizophrenia. }\end{array}$ & \begin{tabular}{|l|} 
Aripiprazole 10 to 30 \\
mg/day orally $(n=139)$ \\
vs. \\
Risperidone 2 to $6 \mathrm{mg} /$ day \\
orally $(\mathrm{n}=140)$
\end{tabular} & 6 weeks & $\begin{array}{l}\text { Age, year: } 32.4 \\
\text { Gender, \% female: } 67.0 \\
\text { Ethnicity, \%: } \\
\text { Han Chinese } 100\end{array}$ \\
\hline $\begin{array}{l}\text { Lieberman, } 2005 \\
\text { (CATIE Study) } \\
\text { Rosenheck, } 2014 \\
\text { Fervaha, } 2014 \\
\text { Caroff, } 2011 \\
\text { Arnold, } 2013\end{array}$ & $\begin{array}{l}57 \text { sites } \\
\text { United States }\end{array}$ & $\begin{array}{l}\text { Patients age 18-65, DSM-IV criteria for } \\
\text { schizophrenia, be appropriate } \\
\text { candidates for oral therapy (patient's } \\
\text { assessment in conjunction with clinician) } \\
\text { have adequate decisional capacity to } \\
\text { decide to participate. }\end{array}$ & $\begin{array}{l}\text { Olanzapine } 7.5 \mathrm{mg} \\
\text { Quetiapine } 200 \mathrm{mg} \\
\text { Risperidone } 1.5 \mathrm{mg} \\
\text { Perphenazine } 8 \mathrm{mg} \\
\text { Ziprasidone } 40 \mathrm{mg} \\
\text { The dose of medications } \\
\text { was flexible, ranging from } \\
\text { one to four capsules daily, } \\
\text { and was based on the } \\
\text { study doctor's judgment }\end{array}$ & 78 weeks & $\begin{array}{l}\text { Mean age: } 40.6 \text { years } \\
\text { 26\% female } \\
\text { Ethnicity: white } 60 \% \text {; black 35\%; } \\
\text { Hispanic } 12 \% ; 5 \% \text { other }\end{array}$ \\
\hline Liu, 2014 & China & $\begin{array}{l}\text { Female patients (age } 18 \text { to } 44 \text { years) } \\
\text { with first-episode schizophrenia } \\
\text { diagnosis based on Chinese } \\
\text { Classification of Mental Disorders-3rd } \\
\text { edition. }\end{array}$ & $\begin{array}{l}\text { Risperidone } 3.4 \mathrm{mg} / \text { day } \\
\text { (mean) orally }(\mathrm{n}=40) \\
\text { vs. } \\
\text { Quetiapine } 420 \mathrm{mg} / \text { day } \\
\text { (mean) }(\mathrm{n}=40)\end{array}$ & 52 weeks & $\begin{array}{l}\text { Age, years: } 29.0 \\
\text { Gender, } \% \\
\text { Female: } 100 \\
\text { Ethnicity, \% } \\
\text { Asian: } 100 \text { (Chinese) }\end{array}$ \\
\hline
\end{tabular}




\begin{tabular}{|c|c|c|c|}
\hline Author, Year & Other Population Characteristics & Total $\mathbf{N}$ & Benefits Outcomes \\
\hline Li, 2014 & $\begin{array}{l}\text { Duration of illness: } 7.3 \text { years } \\
\text { PANSS severity of illness: } 87.1 \\
\text { Schizoaffective, \%: } 0 \\
\text { Substance use, \%: } 0\end{array}$ & 279 & $\begin{array}{l}\text { Aripiprazole } 10 \text { to } 30 \mathrm{mg} / \text { day vs. risperidone } 2 \text { to } 6 \mathrm{mg} / \text { day } \\
\text { PANSS responders ( } \geq 30 \% \text { decrease in total score from baseline), } \mathrm{n} / \mathrm{N} \\
\text { (\%): } 99 / 139(71.0) \text { vs. } 107 / 140(76.0) ; p=0.323\end{array}$ \\
\hline $\begin{array}{l}\text { Lieberman, } 2005 \\
\text { (CATIE Study) } \\
\text { Rosenheck, } 2014 \\
\text { Fervaha, } 2014 \\
\text { Caroff, } 2011 \\
\text { Arnold, } 2013\end{array}$ & $\begin{array}{l}\text { Depression } 28 \% \\
\text { Alcohol dependence or alcohol abuse } 25 \% \\
\text { Drug dependence or drug abuse } 29 \% \\
\text { Obsessive-compulsive disorder } 5 \% \\
\text { Other anxiety disorder } 14 \%\end{array}$ & NR/NR/1493 & $\begin{array}{l}\text { Rosenheck 2014 } \\
\text { Olanzapine vs. quetiapine vs. risperidone } \\
\text { PANSS, difference in total score from perphenazine at } 18 \text { months: } 1.79 \\
(95 \% \mathrm{Cl}-0.04 \text { to } 3.54) \text { vs. }-0.30 \text { ( } 95 \% \mathrm{Cl}-2.08 \text { to } 1.49) \text { vs. }-1.92 \text { (95\% } \\
\mathrm{Cl}-3.70 \text { to }-0.14) \\
\text { Fervaha } 2014 \\
\text { Olanzapine vs. quetiapine vs. risperidone } \\
\text { Life satisfaction score, difference in total score from perphenazine at } 12 \\
\text { months: } 0.15 \text { (SD 1.62) vs. } 0.26 \text { (SD 1.30) vs. } 0.32 \text { (SD 1.55); } p=0.93 \\
\text { Caroff 2011: Tardive dyskinesia vs. no tardive dyskinesia } \\
\text { No difference in time to discontinuation ( } p=0.743) \text {, rates of } \\
\text { discontinuation ( } 74 \% \text { vs. } 74 \%) \text {, or change in PANSS total score } \\
\text { ( } p=0.366) \\
\text { Arnold 2013: Ethnicity subgroups } \\
\text { No differences between whites, blacks, and Hispanics in all-cause } \\
\text { discontinuations, discontinuation due to adverse events, change in total } \\
\text { PANSS scores, or quality of life. }\end{array}$ \\
\hline Liu, 2014 & $\begin{array}{l}\text { Duration of illness, mean months: } 4.5 \\
\text { PANSS severity of illness: } 80.4\end{array}$ & 80 & $\begin{array}{l}\text { Risperidone } 3.4 \mathrm{mg} / \mathrm{day} \text { vs. quetiapine } 420 \mathrm{mg} / \mathrm{day} \\
\text { PANSS total score, change at } 12 \text { weeks: }-37.2 \text { vs. }-40.9\end{array}$ \\
\hline
\end{tabular}




\begin{tabular}{|c|c|c|c|}
\hline Author, Year & Harms Outcomes & Funding & Quality Rating \\
\hline Li, 2014 & $\begin{array}{l}\text { Aripiprazole } 10 \text { to } 30 \mathrm{mg} / \text { day vs. risperidone } 2 \text { to } 6 \mathrm{mg} / \text { day } \\
\text { Overall } \mathrm{AE}, \mathrm{n} / \mathrm{N}(\%): 105 / 139(76.0) \text { vs. } 116 / 140(83.0) \\
\text { Withdrawal due to } \mathrm{AE}, \mathrm{n} / \mathrm{N}(\%): 0 \text { vs. } 1 / 140(<1.0) \\
\text { Clinically relevant weight increase ( } \geq 7 \% \text { in body weight), } \mathrm{n} / \mathrm{N}(\%): 4 / 139(3.0) \text { vs.17/140 (12.0) } \\
\text { Extrapyramidal symptoms, } \mathrm{n} / \mathrm{N}(\%): 35 / 139(25.0) \text { vs. } 34 / 140(24.0) \\
\text { Akathisia, } \mathrm{n} / \mathrm{N}(\%): 32 / 139(23.0) \text { vs. } 31 / 140(22.0) \\
\text { Cardiovascular system, } \mathrm{n} / \mathrm{N}(\%): 11 / 139(8.0) \text { vs. } 9 / 140(6.0)\end{array}$ & $\begin{array}{l}\text { Jiangsu Nhwa Pharmaceutical } \\
\text { Co., Ltd and the National Key } \\
\text { Project (2012ZX09303-003), and } \\
\text { the Shanghai municipal } \\
\text { incubation grant for talented } \\
\text { researcher of health care } \\
\text { (XBR2011049) }\end{array}$ & Fair \\
\hline $\begin{array}{l}\text { Lieberman, } 2005 \\
\text { (CATIE Study) } \\
\text { Rosenheck, } 2014 \\
\text { Fervaha, 2014 } \\
\text { Caroff, 2011 } \\
\text { Arnold, } 2013\end{array}$ & NR & NR & Good \\
\hline Liu, 2014 & $\begin{array}{l}\text { Risperidone } 3.4 \mathrm{mg} / \text { day vs. quetiapine } 420 \mathrm{mg} / \text { day } \\
\text { Dropout rate of } 20 \% \text { over 1-year treatment period. }\end{array}$ & $\begin{array}{l}\text { Huzhou Ministry of } \\
\text { Technology }\end{array}$ & Fair \\
\hline
\end{tabular}




\begin{tabular}{|c|c|c|c|c|c|}
\hline Author, Year & $\begin{array}{l}\text { Setting } \\
\text { Country }\end{array}$ & Inclusion Criteria & $\begin{array}{l}\text { Interventions and Ns per } \\
\text { Group }\end{array}$ & $\begin{array}{l}\text { Duration } \\
\text { (intervention and } \\
\text { longest followup) }\end{array}$ & $\begin{array}{l}\text { Age Gender } \\
\text { Race/Ethnicity }\end{array}$ \\
\hline Maat, 2014 & The Netherlands & $\begin{array}{l}\text { Patients ages } 16 \text { to } 50 \text { years with clinical } \\
\text { diagnosis of schizophrenia (DSM- IV-TR } \\
\text { criteria) and an adequate understanding } \\
\text { of Dutch. }\end{array}$ & \begin{tabular}{|l|} 
Aripiprazole 7.5 or $15 \mathrm{mg}$ \\
$(\mathrm{n}=20)$ \\
vs. \\
Risperidone 1 or $2 \mathrm{mg}$ \\
$(\mathrm{n}=28)$ \\
\\
Dosage could be \\
increased to maximum of 6 \\
$\mathrm{mg}$ risperidone or $30 \mathrm{mg}$ of \\
aripiprazole.
\end{tabular} & 8 weeks & $\begin{array}{l}\text { Age, mean years: } 26.2 \\
\text { Gender, \% female: } 20.4 \\
\text { Ethnicity, \%: } \\
\text { Caucasian: } 66.2 \\
\text { Moroccan: } 8.4 \\
\text { Surinamese: } 8.3 \\
\text { Turkish: } 6.2 \\
\text { Other: } 10.9\end{array}$ \\
\hline
\end{tabular}




\begin{tabular}{|c|c|c|c|}
\hline Author, Year & Other Population Characteristics & Total N & Benefits Outcomes \\
\hline Maat, 2014 & $\begin{array}{l}\text { Baseline drug abuse, \%: } \\
\text { Nicotine: } 69.8 \\
\text { Alcohol: } 64.1 \\
\text { Cannabis: } 49.8 \\
\text { Cocaine: } 9.2\end{array}$ & $\begin{array}{l}80 \text { randomized } \\
\text { (48 completed } \\
\text { study) }\end{array}$ & $\begin{array}{l}\text { Aripiprazole vs. risperidone } \\
\text { Mean change in PANSS total score (SD): }-17.24 \text { (15.89) vs. }-12.85 \\
\text { (17.58) } \\
\text { Quality of life, mean (SD): } 4.88 \text { (9.41) vs. } 6.47 \text { (12.73); } p=0.37 \\
\text { Mean change in SFS* (SD): } 4.94 \text { (17.55) vs. }-3.25 \text { (17.14); } p=0.35\end{array}$ \\
\hline
\end{tabular}




\begin{tabular}{|l|l|l|}
\hline & & \\
\hline Author, Year & Harms Outcomes & Funding \\
\hline Maat, 2014 & Aripiprazole vs. risperidone & Bristol-Myers Squibb \\
& Discontinuations due to lack of tolerability, n/N (\%): 6/38 (15.8) vs. 6/42 (14.3) & Quality Rating \\
\hline
\end{tabular}




\begin{tabular}{|c|c|c|c|c|c|}
\hline Author, Year & $\begin{array}{l}\text { Setting } \\
\text { Country }\end{array}$ & Inclusion Criteria & $\begin{array}{l}\text { Interventions and Ns per } \\
\text { Group }\end{array}$ & \begin{tabular}{|l} 
Duration \\
(intervention and \\
longest followup)
\end{tabular} & $\begin{array}{l}\text { Age Gender } \\
\text { Race/Ethnicity }\end{array}$ \\
\hline $\begin{array}{l}\text { McEvoy, } 2014 \\
\text { (ACCLAIMS) }\end{array}$ & $\begin{array}{l}22 \text { US clinical } \\
\text { research sites: } \\
\text { March } 2011 \text { to } \\
\text { July } 2013\end{array}$ & $\begin{array}{l}\text { Inclusion: Adults with schizophrenia or } \\
\text { schizoaffective disorder who were } \\
\text { clinically assessed to be at risk of } \\
\text { relapse or likely to benefit from a long- } \\
\text { acting injectable antipsychotic. }\end{array}$ & $\begin{array}{l}\text { Haloperidol decanoate 25- } \\
200 \text { mg (n-145); } \\
\text { Paliperidone palmitate 39- } \\
234 \text { mg (n-145); }\end{array}$ & $\begin{array}{l}\text { Monthly for as long } \\
\text { as } 24 \text { months }\end{array}$ & $\begin{array}{l}\text { Paliperidone versus haloperidol: } \\
\text { Age, mean (SD): } 43 \text { (12.6); } 45 \\
\text { (12.3); } \\
\text { \% Men: } 106 \text { (73.1\%); } 110 \text { (75.9); } \\
\text { Race, White: } 56 \text { (38.6\%); } 54 \\
\text { (37.2\%); } \\
\text { Race, Black: } 83 \text { (57.2\%); } 83 \\
\text { (57.2\%); } \\
\text { Race, Other: } 6 \text { (4.1\%); } 8 \text { (5.5\%); } \\
\text { Spanish, Hispanic, or Latino: } 6 \\
\text { (4.1\%); } 8 \text { (5.5\%); }\end{array}$ \\
\hline
\end{tabular}




\begin{tabular}{|c|c|c|c|}
\hline Author, Year & Other Population Characteristics & Total N & Benefits Outcomes \\
\hline $\begin{array}{l}\text { McEvoy, } 2014 \\
\text { (ACCLAIMS) }\end{array}$ & $\begin{array}{l}\text { Paliperidone versus haloperidol: } \\
\text { Age at first treatment, mean (SD): } 23 \text { (9.3); } 24 \text { (10.9); } \\
\text { Age at first antipsychotic med, mean (SD): } \\
26 \text { (9.0); } 27 \text { (10.1) }\end{array}$ & 311 & $\begin{array}{l}\text { Adjusted HR for rate of efficacy failure: } \\
\text { Paliperidone compared to haloperidol: HR=0.98 ( } 95 \% \text { Cl: } 0.64 \text { to } 1.47 \text { ); } \\
\text { Paliperidone: } 49 \text { (33.8\%) experienced efficacy failure; } \\
\text { Haloperidol: } 47 \text { (32.4\%) experienced efficacy failure. }\end{array}$ \\
\hline
\end{tabular}




\begin{tabular}{|c|c|c|c|}
\hline Author, Year & Harms Outcomes & Funding & Quality Rating \\
\hline $\begin{array}{l}\text { McEvoy, } 2014 \\
\text { (ACCLAIMS) }\end{array}$ & 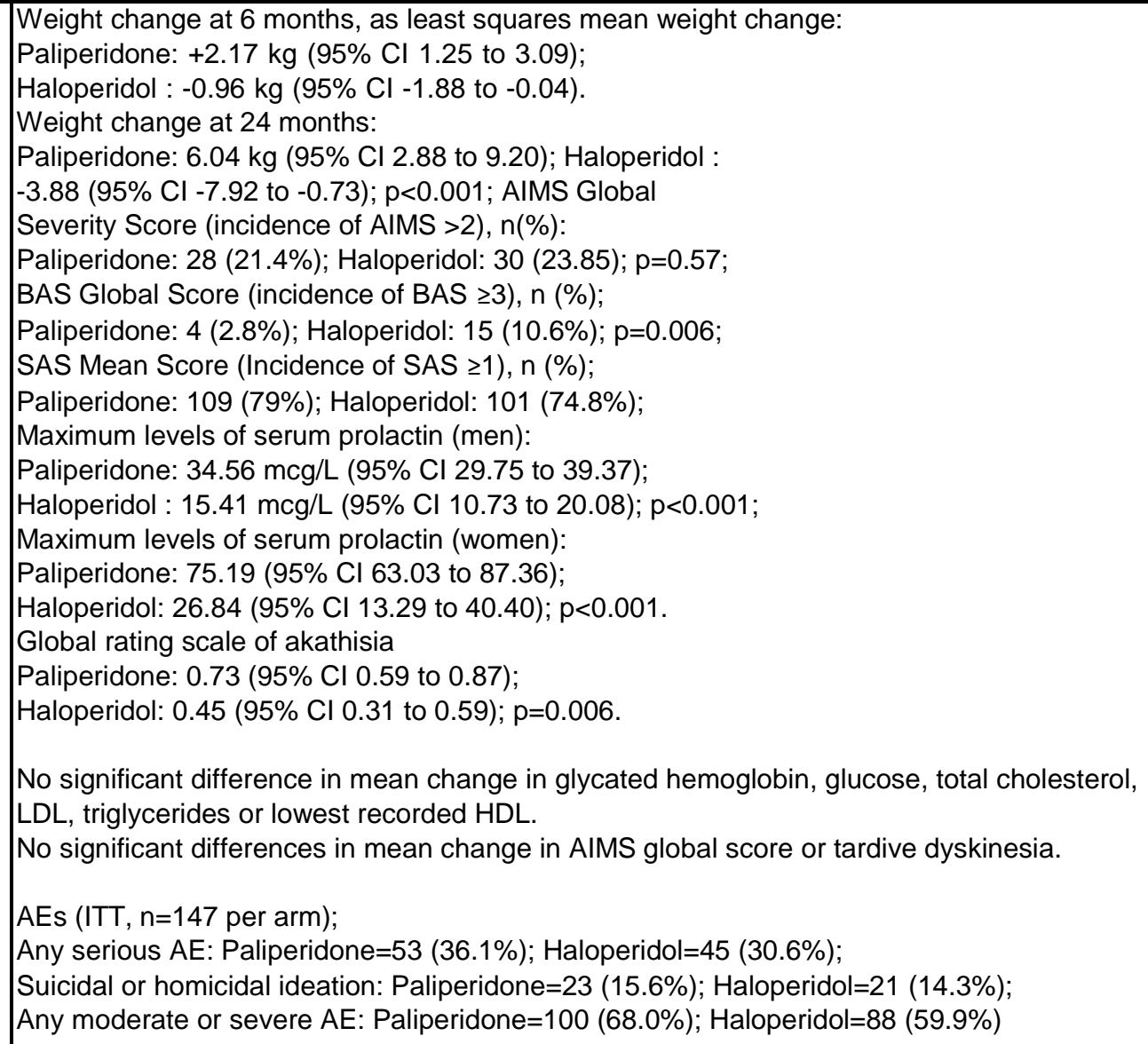 & NIMH & Good \\
\hline
\end{tabular}




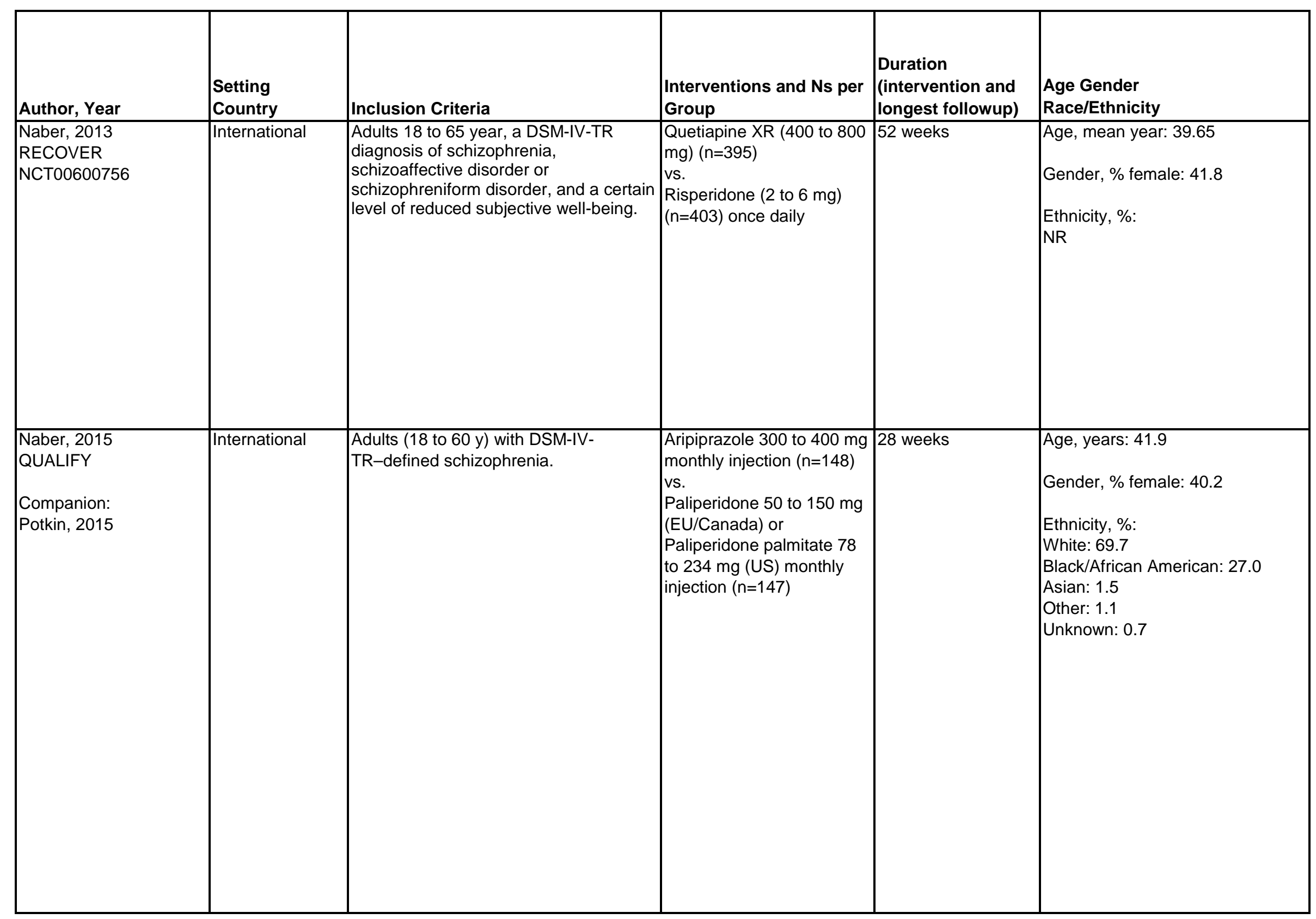




\begin{tabular}{|c|c|c|c|}
\hline Author, Year & Other Population Characteristics & Total N & Benefits Outcomes \\
\hline \begin{tabular}{|l|} 
Naber, 2013 \\
RECOVER \\
NCT00600756
\end{tabular} & $\begin{array}{l}\text { Concurrent substance abuse: } \\
\text { Alcohol use, \%: } 12.1 \\
\text { Cannabis use, \%: } 1.9 \\
\text { DSM-IV schizophrenia subtype diagnosis, \%: } \\
\text { Schizoaffective disorder of bipolar type: } 8.3 \\
\text { Schizoaffective disorder of depressive type: } 7.8 \\
\text { Median duration of present episode, m: } 2.5 \\
\text { Mean years since first known schizophrenia diagnosis: } \\
11.35 \\
\text { Hospitalizations due to } \\
\text { schizophrenia in the previous } 6 \text { months, } \% \text { patients: } 16.1 \\
\text { SWN-K total score, mean: } 64.35\end{array}$ & 798 & 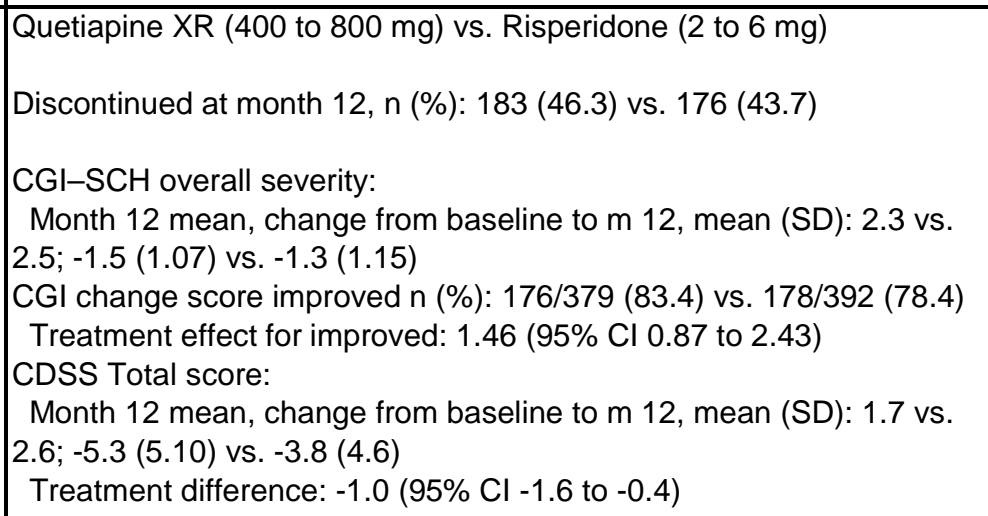 \\
\hline $\begin{array}{l}\text { Naber, } 2015 \\
\text { QUALIFY } \\
\text { Companion: } \\
\text { Potkin, 2015 }\end{array}$ & CGI-S severity of illness score: 4.0 & 295 & 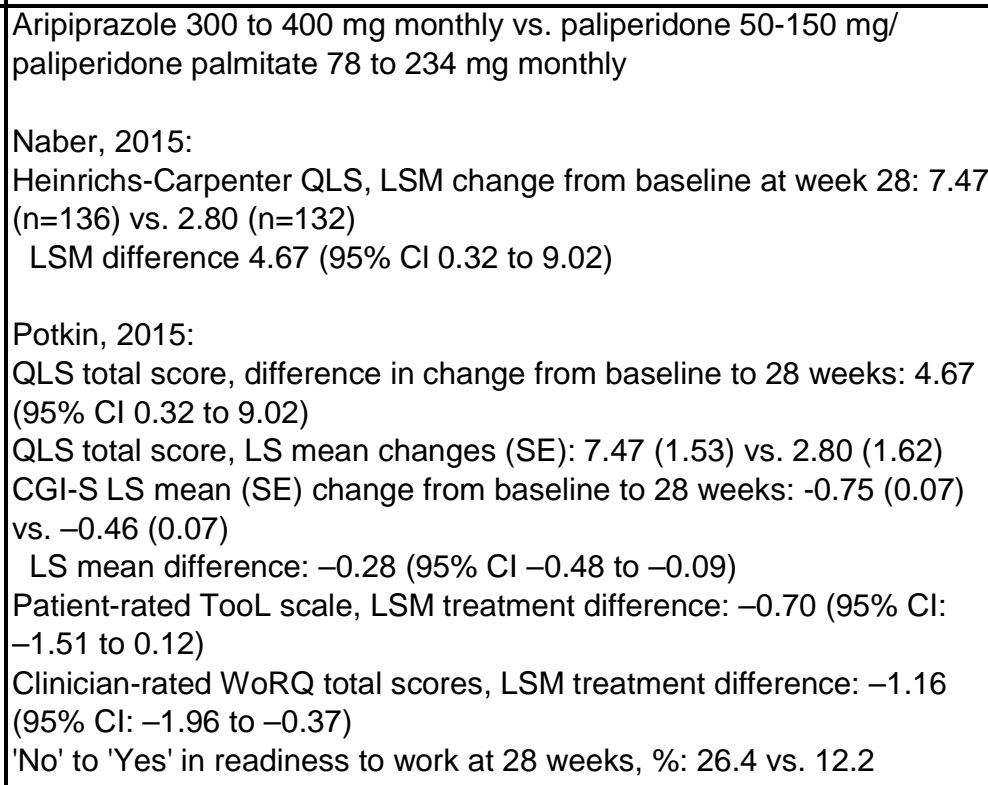 \\
\hline
\end{tabular}




\begin{tabular}{|c|c|c|c|}
\hline Author, Year & Harms Outcomes & Funding & Quality Rating \\
\hline \begin{tabular}{|l|} 
Naber, 2013 \\
RECOVER \\
NCT00600756
\end{tabular} & $\begin{array}{l}\text { Quetiapine XR (400 to } 800 \mathrm{mg} \text { ) vs. Risperidone (2 to } 6 \mathrm{mg} \text { ) } \\
\text { Discontinued due to AE at month 12, n (\%): } 53 \text { (13.4) vs. } 44 \text { (10.9) } \\
\text { n/N (\%); number of events } \\
\text { TEAE: } 238 / 391 \text { (60.9); } 791 \text { vs. } 258 / 402 \text { (64.2); } 834 \\
\text { TEAE leading to discontinuation: } 57 / 391(14.6) ; 72 \text { vs. } 48 / 402 \text { (11.9); } 80 \\
\text { Serious TEAE: } 45 / 391 \text { (11.5); } 49 \text { vs. } 26 / 402(6.5) ; 31 \\
\text { Serious TEAE leading to death: } 0 \text { (0) vs. } 1 / 402(0.2) ; 1 \\
\text { Weight increased: } 18 / 391 \text { (4.6); } 18 \text { vs. } 25 / 402(6.2) ; 25\end{array}$ & AstraZeneca. & Fair \\
\hline $\begin{array}{l}\text { Naber, } 2015 \\
\text { QUALIFY } \\
\text { Companion: } \\
\text { Potkin, 2015 }\end{array}$ & $\begin{array}{l}\text { Aripiprazole } 300 \text { to } 400 \mathrm{mg} \text { monthly vs. paliperidone } 50-150 \mathrm{mg} / \text { paliperidone palmitate } 78 \text { to } \\
234 \text { mg monthly } \\
\text { Naber, } 2015: \\
\text { Overall AE: } \%(\mathrm{n} / \mathrm{N}): 62 / 119(52.1 \%) \text { vs. } 72 / 109(66.1 \%)^{\star} \\
\text { Overall withdrawal due to AE: } \%(\mathrm{n} / \mathrm{N}): 11.1 \%(16 / 148) \text { vs. } 19.7 \%(27 / 147) \\
\text { AE related extrapyramidal symptoms: } \%(\mathrm{n} / \mathrm{N}) \\
\text { Akathisia: } 2.5 \%(2 / 119) \text { vs. } 1.8 \%(2 / 109)^{\star} \\
\text { Dystonia: } 0.8 \%(1 / 119) \text { vs. } 0 \% \%^{*} \\
\text { Extrapyramidal disorder: } 0 \% \text { vs. } 0 \% \text { * } \\
\text { Muscle rigidity: } 0.8 \%(1 / 119) \text { vs. } 0 \\
\text { Muscle spasms: } 0 \text { vs. } 0.9 \%(1 / 109) \\
\text { Tremor: } 1.7 \%(2 / 119) \text { vs.1.8\% (2/109) } \\
\text { Potkin, } 2015: \\
\text { Discontinuation due to AE, } n / \mathrm{N}(\%): 16 / 144(11.1) \text { vs. } 27 / 137(19.7) \\
\text { Weight increased, } \mathrm{n} / \mathrm{N}(\%): 0(0.0) \text { vs. } 2 / 137(1.5) \\
\text { ASEX total score mean (SD) change from baseline to } 28 \text { weeks: }-1.9(6.3) \text { vs. }-0.8(6.1) \\
\text { Decrease in sexual dysfunction at } 28 \text { weeks, } \%: 30 \text { vs. } 4\end{array}$ & $\begin{array}{l}\text { H. Lundbeck A/S and Otsuka } \\
\text { Pharmaceutical Development \& } \\
\text { Commercialization, Inc } \\
\\
\text { ×Treatment continuation period } \\
\text { (main period of interest with } \\
\text { respect to safety evaluation } \\
(n=119 \text { vs. } n=109)\end{array}$ & Fair \\
\hline
\end{tabular}




\begin{tabular}{|c|c|c|c|c|c|}
\hline Author, Year & $\begin{array}{l}\text { Setting } \\
\text { Country }\end{array}$ & Inclusion Criteria & $\begin{array}{l}\text { Interventions and Ns per } \\
\text { Group }\end{array}$ & \begin{tabular}{|l} 
Duration \\
(intervention and \\
longest followup)
\end{tabular} & $\begin{array}{l}\text { Age Gender } \\
\text { Race/Ethnicity }\end{array}$ \\
\hline Nemeth, 2017 & \begin{tabular}{|l|}
66 study centers \\
in 11 European \\
countries \\
(Bulgaria, \\
Croatia, Czech \\
Republic, \\
France, Hungary, \\
Poland, \\
Romania, Serbia, \\
Spain, Russia, \\
and Ukraine) \\
\\
\end{tabular} & $\begin{array}{l}\text { Adults aged 18-65 years who had a } \\
\text { diagnosis of schizophrenia (DSM-IV-TR) } \\
\text { criteria, with onset occurring at least } 2 \\
\text { years before screening. Patients had to } \\
\text { be in a stable condition for at least } 6 \\
\text { months before screening (i.e., no } \\
\text { psychiatric hospital admissions, acute } \\
\text { exacerbations, or imprisonments) and } \\
\text { meet the following clinical criteria: } \\
\text { predominant negative symptoms for at } \\
\text { least } 6 \text { months (based on medical } \\
\text { records/investigator judgment), Positive } \\
\text { and Negative Syndrome Scale factor } \\
\text { score for negative symptoms (PANSS- } \\
\text { FSNS) of } 24 \text { or more, and score of } 4 \text { or } \\
\text { more on at least two of three core } \\
\text { negative PANSS items (blunted affect, } \\
\text { passive or apathetic social withdrawal, } \\
\text { lack of spontaneity, and flow of } \\
\text { conversation) at screening and during a } \\
\text { lead-in period. Additionally, patients } \\
\text { were required to have a PANSS-FSNS } \\
\text { score that diverged less than } 25 \% \text { from } \\
\text { the screening score during a lead-in } \\
\text { period. }\end{array}$ & $\begin{array}{l}\text { Cariprazine } 4.5 \mathrm{mg} \text { (target } \\
\text { dose) daily }(\mathrm{n}=230) \\
\text { Risperidone } 4 \mathrm{mg} \text { (target } \\
\text { dose) daily }(\mathrm{n}=231) \\
\\
\end{array}$ & 26 weeks & $\begin{array}{l}\text { Cariprazine vs. risperidone: } \\
\text { Age, mean years: } 40.2 \text { vs. } 40.7 \\
\text { Gender, \% female: } 46 \text { vs. } 39 \\
\text { Ethnicity, \%: } \\
\text { White: } 96 \text { vs. } 94 \\
\text { (Ethnicity not recorded: } 4 \text { vs. } 6 \text { ) }\end{array}$ \\
\hline
\end{tabular}




\begin{tabular}{|c|c|c|c|}
\hline Author, Year & Other Population Characteristics & Total N & Benefits Outcomes \\
\hline Nemeth, 2017 & $\begin{array}{l}\text { Cariprazine vs. risperidone: } \\
\text { Time from schizophrenia diagnosis to informed consent, } \\
\text { years: } 11.98 \text { vs. } 12.96 \\
\text { Number of acute exacerbations } \\
<5: 64 \%(148 / 230) \text { vs. } 55 \%(126 / 230) \\
5-10: 27 \%(61 / 230) \text { vs. } 34 \%(79 / 230) \\
11-15: 5 \%(11 / 230) \text { vs. } 9 \%(20 / 230) \\
\text { >15: } 4 \%(10 / 230) \text { vs. } 2 \%(5 / 230)\end{array}$ & $\begin{array}{l}461 \text { randomized } \\
460 \text { included in } \\
\text { safety population } \\
456 \text { in modified } \\
\text { ITT }\end{array}$ & 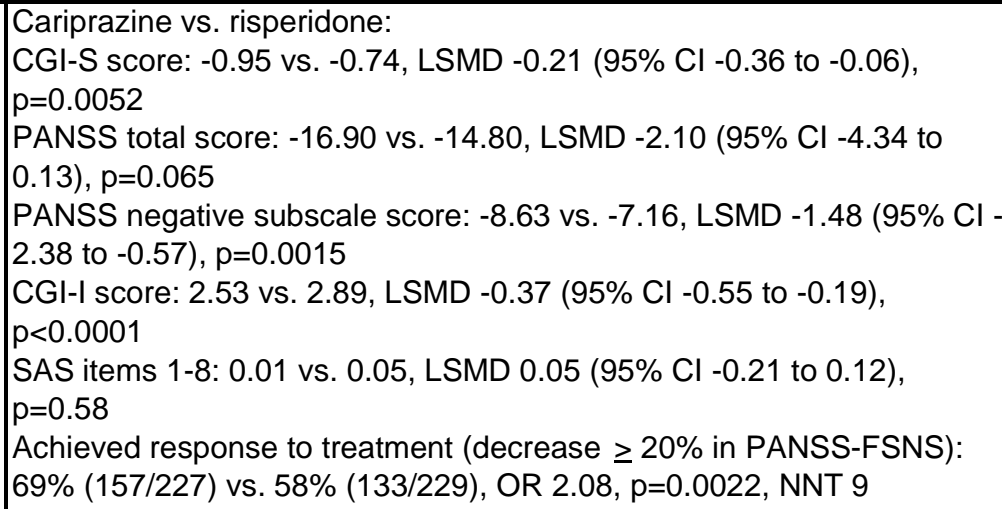 \\
\hline
\end{tabular}




\begin{tabular}{|c|c|c|c|}
\hline Author, Year & Harms Outcomes & Funding & Quality Rating \\
\hline Nemeth, 2017 & $\begin{array}{l}\text { Cariprazine vs. risperidone: } \\
\text { Discontinuations due to adverse events: } 10 \%(22 / 230) \text { vs. } 11 \%(25 / 230) \\
\text { Any serious adverse events: } 3 \%(7 / 230) \text { vs. } 3 \%(7 / 230) \\
\text { Any adverse events: } 53 \%(123 / 230) \text { vs. } 57 \%(131 / 230)\end{array}$ & $\begin{array}{l}\text { Gedeon Richter Plc (Budapest, } \\
\text { Hungary) }\end{array}$ & Good \\
\hline
\end{tabular}




\begin{tabular}{|c|c|c|c|c|c|}
\hline Author, Year & \begin{tabular}{|l} 
Setting \\
Country
\end{tabular} & Inclusion Criteria & $\begin{array}{l}\text { Interventions and Ns per } \\
\text { Group }\end{array}$ & $\begin{array}{l}\text { Duration } \\
\text { (intervention and } \\
\text { longest followup) }\end{array}$ & $\begin{array}{l}\text { Age Gender } \\
\text { Race/Ethnicity }\end{array}$ \\
\hline $\begin{array}{l}\text { Parabiaghi, } 2016 \\
\text { Companion to } \\
\text { Parabiaghi, } 2011\end{array}$ & Italy & $\begin{array}{l}\text { 18 years old, DSM-IV diagnosis of } \\
\text { schizophrenia based on the Mini- } \\
\text { International Neuropsychiatric Interview. }\end{array}$ & $\begin{array}{l}\text { Aripiprazole } 19.7 \mathrm{mg} / \mathrm{day}^{*} \\
(\mathrm{~N}=100) \\
\text { vs. } \\
\text { Olanzapine } 13.7 \mathrm{mg} / \mathrm{day}^{*} \\
(\mathrm{~N}=103) \\
\text { Haloperidol } 4.0 \mathrm{mg} / \mathrm{day} \\
(\mathrm{N}=97)\end{array}$ & 52 weeks & $\begin{array}{l}\text { Age, years: } 42.7 \\
\text { Gender, \% female: } 42.0 \\
\text { Ethnicity: Italian (\% NR) }\end{array}$ \\
\hline Park, 2013 & South Korea & $\begin{array}{l}\text { Age 18-65 years; diagnosed by a } \\
\text { psychiatrist with a brief psychotic } \\
\text { disorder, schizophreniform disorder, } \\
\text { schizophrenia, or schizoaffective } \\
\text { disorder (DSM-IV criteria); no other } \\
\text { active illness. }\end{array}$ & $\begin{array}{l}\text { Ziprasidone } 40 \mathrm{mg} \text { initial } \\
\text { dose (range } 20-160 \mathrm{mg} \text {; } \\
\text { mean } 109 \mathrm{mg})(\mathrm{n}=10) \\
\text { vs. } \\
\text { Olanzapine } 10 \mathrm{mg} \text { initial } \\
\text { dose (range } 5-20 \mathrm{mg} ; \\
\text { mean } 11.6 \mathrm{mg})(\mathrm{n}=10)\end{array}$ & 12 weeks & $\begin{array}{l}\text { Age, mean years: } 33.0 \\
\text { Gender, \% female: } 50.0 \\
\text { Ethnicity: NR }\end{array}$ \\
\hline $\begin{array}{l}\text { Robinson, } 2015 \\
\text { See also: Zhang, } 2015\end{array}$ & US and Canada & $\begin{array}{l}\text { Adults and adolescent (15 to } 40 \text { years) } \\
\text { with DSM-IV-defined diagnosis of } \\
\text { schizophrenia, schizophreniform } \\
\text { disorder, schizoaffective disorder or } \\
\text { psychotic disorder not otherwise } \\
\text { specified. }\end{array}$ & $\begin{array}{l}\text { Aripiprazole } 5 \text { to } 30 \\
\text { mg/day orally }(n=106) \\
\text { vs. } \\
\text { Risperidone } 1 \text { to } 6 \mathrm{mg} / \text { day } \\
\text { orally }(n=103)\end{array}$ & 12 weeks & $\begin{array}{l}\text { Age, years: } 22.1 \\
\text { Gender, \% female: } 29 \\
\text { Ethnicity, \%: } \\
\text { Caucasian: } 24.0 \\
\text { African-American: } 37.0 \\
\text { Hispanic: } 10.0 \\
\text { Other/mixed: } 9.0\end{array}$ \\
\hline
\end{tabular}




\begin{tabular}{|c|c|c|c|}
\hline Author, Year & Other Population Characteristics & Total N & Benefits Outcomes \\
\hline $\begin{array}{l}\text { Parabiaghi, } 2016 \\
\text { Companion to } \\
\text { Parabiaghi, } 2011\end{array}$ & $\begin{array}{l}\text { Duration of illness, year from first psychiatric contact (\%): } \\
0-2 \text { years: } 12.0 \\
\text { 3+ years: } 72.0 \\
\text { Hospitalization, \% in-patient: } 20.0 \\
\text { Current substance abuse or dependence, \%: } 5.0 \\
\text { Antipsychotic drug-naïve, \%: } 6.0\end{array}$ & 300 & NR \\
\hline Park, 2013 & PANSS total score at baseline: 74.8 & 20 & NR \\
\hline $\begin{array}{l}\text { Robinson, } 2015 \\
\text { See also: Zhang, } 2015\end{array}$ & $\begin{array}{l}\text { Duration of current illness/psychosis, weeks: } 125.5^{\star} \\
\text { BPRS-A severity of illness: } 45.1 \\
\text { Schizoaffective, \%: } 3 \\
\text { Substance use, \%: } 0 \\
\text { Antipsychotic drug naïve: lifetime antipsychotic drug } \\
\text { medication treatment } 2 \text { weeks or less }\end{array}$ & 209 & $\begin{array}{l}\text { Aripiprazole } 5-30 \mathrm{mg} / \text { day vs. risperidone } 1-6 \mathrm{mg} / \mathrm{day} \\
\text { Cumulative response rate at week } 12^{\star *}: 62.8 \%(95 \% \mathrm{Cl} 50.8 \text { to } 74.8) \\
\text { vs. } 56.8 \%(95 \% \mathrm{Cl} 43.9 \text { to } 69.9) \\
\text { Mean time to response, w: } 8.0(95 \% \mathrm{Cl} 7.9 \text { to } 8.1) \text { vs. } 8.2(95 \% \mathrm{Cl} 7.3 \text { to } \\
9.2 \text { ) } \\
\text { Discontinuation of controlled treatment before } 12 \text { weeks ( } \mathrm{n} \text {, due to } \\
\text { safety concerns): } 0 \text { vs. } 3 \text { ( } 1 \text { metabolic syndrome, } 1 \text { tardive dyskinesia, } 1 \\
\text { hematologic abnormalities ) } \\
\text { Zhang, } 2015 \\
\text { C/C homozygotes vs. T carriers } \\
\text { BPRS Positive Symptoms Scores at week } 12 \text { (Least Square Estimate, } \\
\text { mean } \pm S E, \text { unadjusted; sample size): } 6.51 \pm 0.5238 \text { vs. } 7.64 \pm 0.5733 \\
p=0.143\end{array}$ \\
\hline
\end{tabular}




\begin{tabular}{|c|c|c|c|}
\hline Author, Year & Harms Outcomes & Funding & Quality Rating \\
\hline $\begin{array}{l}\text { Parabiaghi, } 2016 \\
\text { Companion to } \\
\text { Parabiaghi, } 2011\end{array}$ & 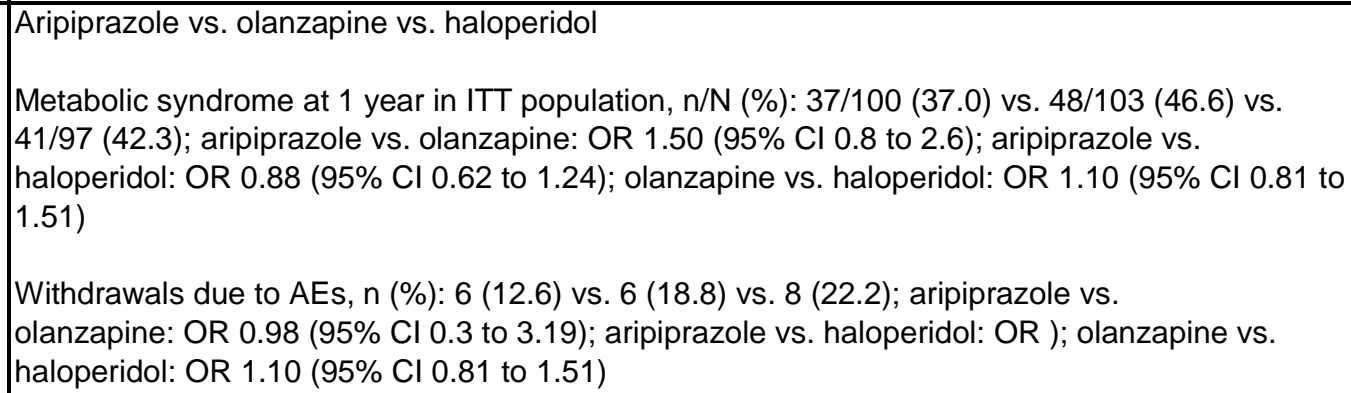 & $\begin{array}{l}\text { Funding: IRCCS-Istituto di } \\
\text { Ricerche Farmacologiche 'Mario } \\
\text { Negri' and Bristol-Myers Squibb } \\
\text { ×Mean dose of treatment. }\end{array}$ & Fair \\
\hline Park, 2013 & $\begin{array}{l}\text { Ziprasidone vs. olanzapine } \\
\text { Body weight, median change in kg (IQR): } 3.43(0.61,9.20) \text { vs. } 10.35(9.27,14.65) ; p=0.016\end{array}$ & Pfizer Pharmaceuticals Korea & Poor \\
\hline $\begin{array}{l}\text { Robinson, } 2015 \\
\text { See also: Zhang, } 2015\end{array}$ & $\begin{array}{l}\text { Aripiprazole 5-30 mg/day vs. risperidone 1-6 mg/day } \\
\text { Sexual dysfunction, } \% \text { (n/N): } 7.8 \% \text { (8/102) vs. } 12.5 \%(12 / 96)\end{array}$ & $\begin{array}{l}\text { National Institutes of } \\
\text { Health and NARSAD Young } \\
\text { Investigator Grant to } \\
\text { J.A.G. from the Brain \& Behavior } \\
\text { Research Foundation } \\
\text { *Report states: "duration of } \\
\text { psychotic symptoms before study } \\
\text { week (weeks)" } \\
\text { **Response criteria based on } \\
\text { BPRS-A and CGI scores }\end{array}$ & Fair \\
\hline
\end{tabular}




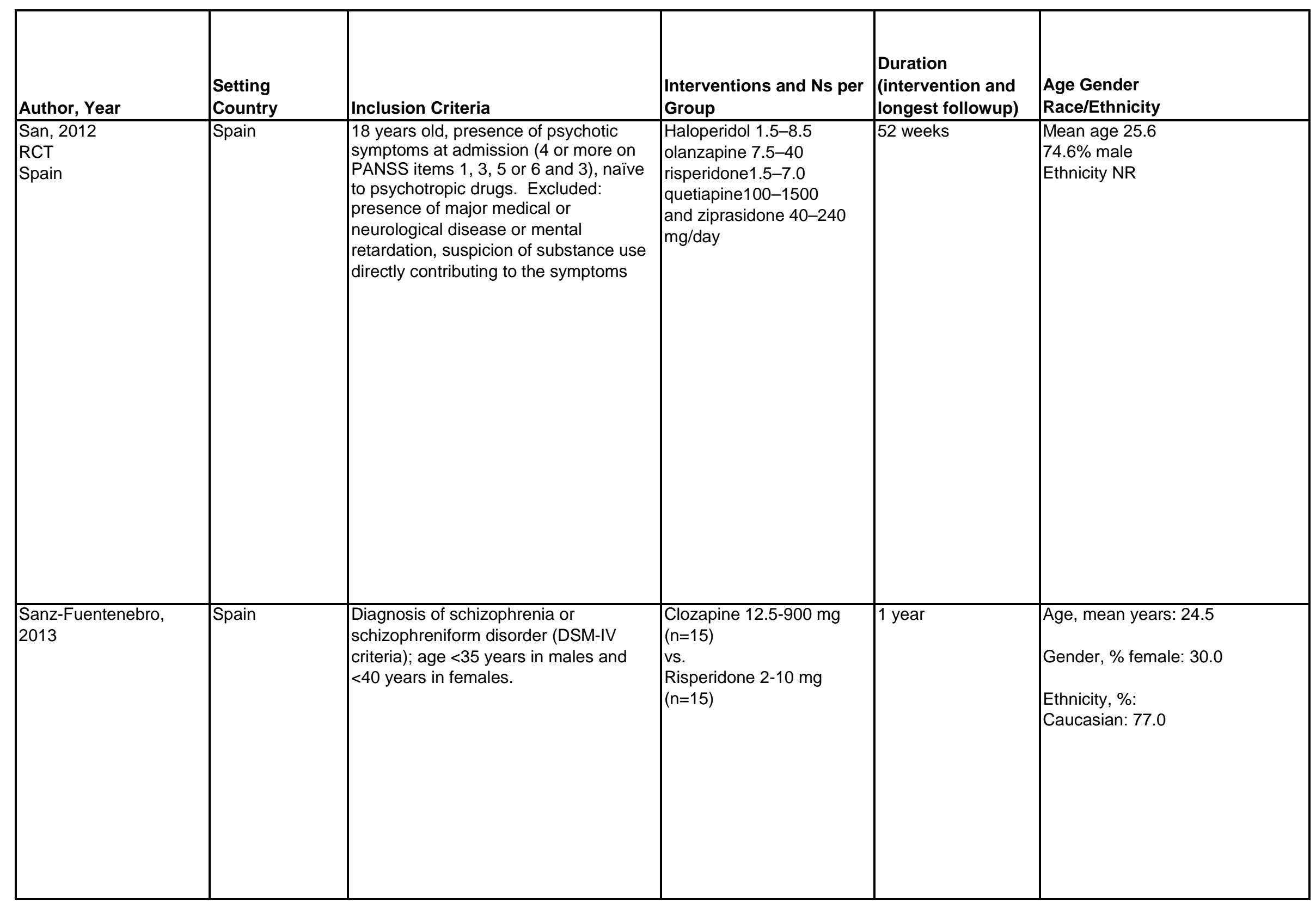




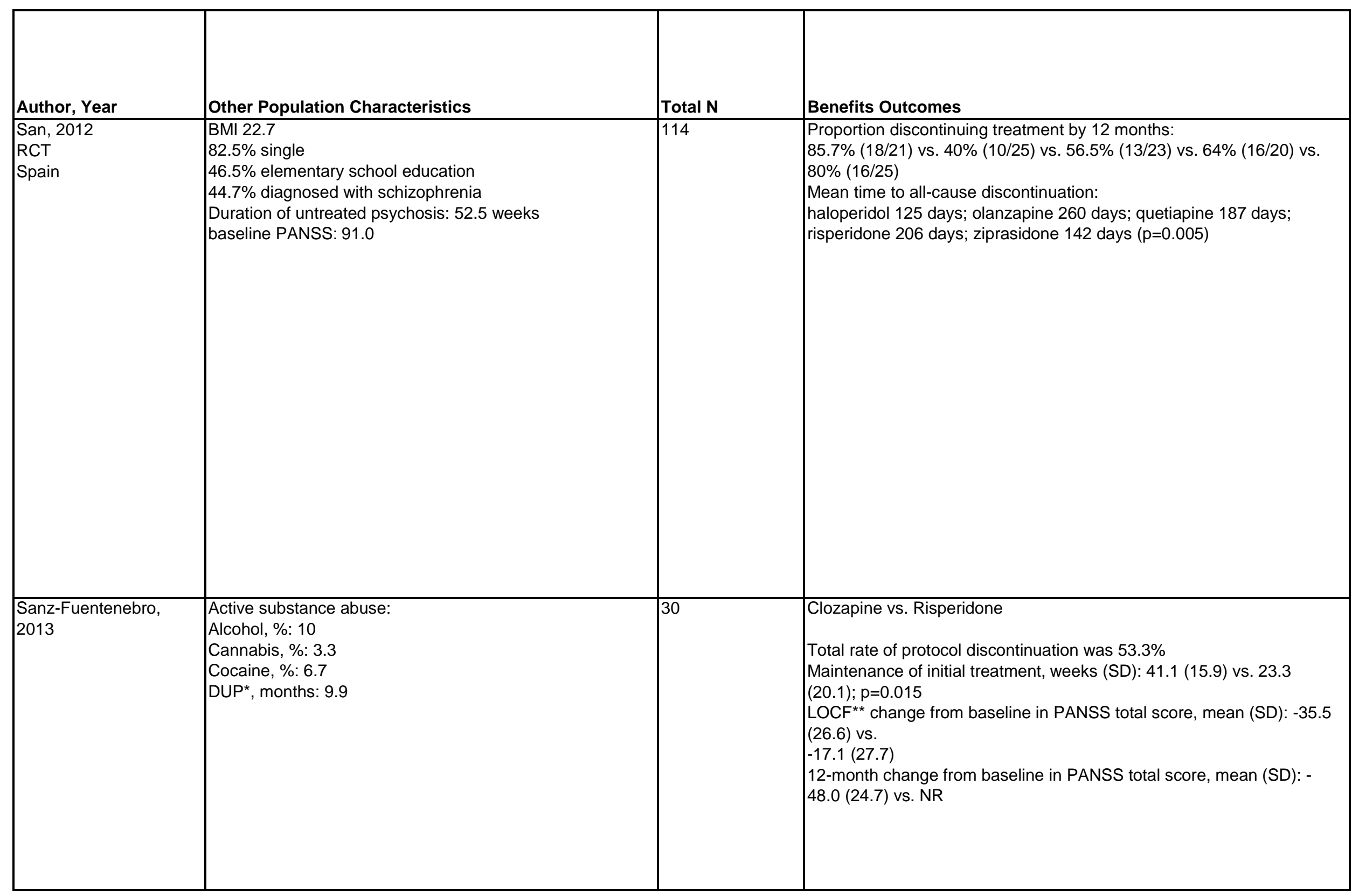




\begin{tabular}{|c|c|c|c|}
\hline Author, Year & Harms Outcomes & Funding & Quality Rating \\
\hline $\begin{array}{l}\text { San, } 2012 \\
\text { RCT } \\
\text { Spain }\end{array}$ & $\begin{array}{l}\text { Discontinuations due to adverse events: } \\
11.1 \% \text { haloperidol; } 20 \% \text { olanzapine, } 7.7 \% \text { quetiapine; } 6.2 \% \text { risperidone; } 25 \% \text { ziprasidone } \\
\text { Time to discontinuation due to adverse events: NR } \\
\text { UKU scores were higher in haloperidol group compared to second-generation drugs, and no } \\
\text { differences were found between the other drugs. } \\
\text { Weight gain ranged from } 3 \mathrm{~kg} \text { with ziprasidone to } 9 \mathrm{~kg} \text { with olanzapine but no statistically } \\
\text { significant differences were found. }\end{array}$ & $\begin{array}{l}\text { La Marato' TV3 Foundation and } \\
\text { Eli Lilly }\end{array}$ & Good \\
\hline 2013 & & $\begin{array}{l}\text { Spanısh Minıstry of Health, } \\
\text { Ayudas } \\
\text { para el fomento de la traslación } \\
\text { de la aplicación terapéutica de } \\
\text { medicamentos } \\
\text { huérfanos y terapias avanzadas } \\
\text { (grant number: TRA-035); and the } \\
\text { Instituto de Salud } \\
\text { Carlos III (grant number: PI- } \\
\text { 060219). }\end{array}$ & Poor \\
\hline
\end{tabular}




\begin{tabular}{|c|c|c|c|c|c|}
\hline Author, Year & \begin{tabular}{|l} 
Setting \\
Country
\end{tabular} & Inclusion Criteria & $\begin{array}{l}\text { Interventions and Ns per } \\
\text { Group }\end{array}$ & \begin{tabular}{|l} 
Duration \\
(intervention and \\
longest followup)
\end{tabular} & $\begin{array}{l}\text { Age Gender } \\
\text { Race/Ethnicity }\end{array}$ \\
\hline Savitz, 2016 & International & $\begin{array}{l}\text { Adult patients age } 18 \text { to } 70 \text { years with a } \\
\text { DSM-IV diagnosis of schizophrenia. }\end{array}$ & $\begin{array}{l}\text { Paliperidone palmitate 3- } \\
\text { month injection ( } \mathrm{N}=504) \\
\text { vs. } \\
\text { Paliperidone palmitate 1- } \\
\text { month injection }(\mathrm{N}=512)\end{array}$ & 48 weeks & $\begin{array}{l}\text { Age, years: } 38.7 \\
\text { Gender, } \% \\
\text { Female: } 47 \% \\
\text { Ethnicity, } \%: \\
\text { White: } 58 \% \\
\text { African American: } 6 \% \\
\text { American Indian: } 35 \% \\
\text { Other: } 1 \%\end{array}$ \\
\hline Shoja Shafti, 2015 & Iran & $\begin{array}{l}\text { Female inpatients diagnosed as having } \\
\text { schizophrenia, according to the DSM-V. }\end{array}$ & $\begin{array}{l}\text { Aripiprazole } 5 \text { to } 25 \\
\text { mg/day orally }(n=25) \\
\text { vs. } \\
\text { Quetiapine } 25 \text { to } 600 \\
\text { mg/day }(n=25)\end{array}$ & 12 weeks & $\begin{array}{l}\text { Age, years: } 36.8 \\
\text { Gender, \% female: } 100 \\
\text { Ethnicity: NR }\end{array}$ \\
\hline
\end{tabular}




\begin{tabular}{|c|c|c|c|}
\hline Author, Year & Other Population Characteristics & Total N & Benefits Outcomes \\
\hline Savitz, 2016 & $\begin{array}{l}\text { Prior hospitalizations, \%: } \\
\text { None: } 41.0 \\
\text { Once: } 37.0 \\
\text { Twice: } 16.0 \\
\text { Three times: } 3.0 \\
\text { Four or more: } 2.0 \\
\text { PANSS Total Score at baseline: } 85.0 \text { (ITT); } 57.8 \text { (double } \\
\text { blind) } \\
\text { Previous antipsychotic use, \%: } 76.0 \text { (new-generation } \\
\text { antipsychotics) }\end{array}$ & 1,016 & $\begin{array}{l}\text { Paliperidone palmitate 3-month injection vs. paliperidone palmitate 1- } \\
\text { month injection } \\
\text { Relapse-free patients, } \%(\mathrm{n} / \mathrm{N})^{*}: 8.0 \%(37 / 504) \text { vs. } 9.0 \%(45 / 512) \\
\text { Clinical response }(\geq 20 \% \text { reduction in PANSS total score), } \%(\mathrm{n} / \mathrm{N}): \\
50.1 \%(241 / 481) \text { vs. } 47.3 \%(237 / 501) \\
\geq 30 \%: 36.4 \%(175 / 481) \text { vs. } 36.1 \%(181 / 501) \\
\geq 40 \%: 26.4 \%(127 / 481) \text { vs. } 27.1 \%(136 / 501) \\
\text { Symptomatic remission (meeting Andreasen remission criteria } 6 \\
\text { months before end of study), } \%: 58.0 \% \text { vs. } 59.0 \% \\
\text { Psychiatric hospitalizations, } \%(\mathrm{n} / \mathrm{N}): 3.0 \%(16 / 504) \text { vs. } 4.0 \%(22 / 512)\end{array}$ \\
\hline Shoja Shafti, 2015 & $\begin{array}{l}\text { Duration of illness, y: } 6.4 \\
\text { Hospitalization, \%: } 100 \\
\text { CGI-S severity of illness: } 3.74 \\
\text { Schizoaffective, \%: } 0\end{array}$ & 50 & NR \\
\hline
\end{tabular}




\begin{tabular}{|c|c|c|c|}
\hline Author, Year & Harms Outcomes & Funding & Quality Rating \\
\hline Savitz, 2016 & $\begin{array}{l}\text { Paliperidone palmitate 3-month injection vs. paliperidone palmitate 1-month injection } \\
\text { Overall AEs, \% (n/N): } 68.0 \%(342 / 504) \text { vs. } 66.0 \%(340 / 512) \\
\text { Withdrawals due to AEs, } \%(\mathrm{n} / \mathrm{N}): 3.0 \%(15 / 504) \text { vs. } 3.0 \%(13 / 512) \\
\text { All-cause mortality, } \mathrm{n}: 1 \text { vs. } 3 \\
\text { Diabetes mellitus/hyperglycemia, } \%(\mathrm{n} / \mathrm{N}): 2.6 \%(13 / 504) \text { vs. } 4.9 \%(25 / 512) \\
\text { Extrapyramidal AEs, } \%(\mathrm{n} / \mathrm{N}): 8.0 \%(42 / 504) \text { vs. } 7.0 \%(38 / 512) \\
\text { Weight change of } \geq 7 \%, \%(\mathrm{n} / \mathrm{N}): 27.0 \%(136 / 504) \text { vs. } 30.0 \%(150 / 512) \\
\text { Tardive dyskinesia, n: } 1 \text { vs. } 1\end{array}$ & \begin{tabular}{|l|} 
Funding: Otsuka, Janssen, \\
Cilag, and Lundbeck \\
\\
*Relapse as $\geq 1$ of following: \\
1)hospitalization for schizophrenia \\
symptoms; 2 2) $25 \%$ increase in \\
PANSS total score for patients \\
scoring $>40$ or a 10-point \\
increase for patients scoring $\leq 40$; \\
3) increase PANSS items; 4 ) \\
clinically significant self-injury or \\
violent behavior resulting in \\
suicide, injury, or damage; 5$)$ \\
suicidal/homicidal ideation
\end{tabular} & Good \\
\hline Shoja Shafti, 2015 & $\begin{array}{l}\text { Aripiprazole } 5 \text { to } 25 \mathrm{mg} / \text { day vs. quetiapine } 25 \text { to } 600 \mathrm{mg} \\
\text { Withdrawal due to } \mathrm{AE}, \mathrm{n} / \mathrm{N}(\%): 0 \text { vs. } 0\end{array}$ & $\begin{array}{l}\text { Research received no specific } \\
\text { grant from any funding agency in } \\
\text { the public, commercial, or not-for- } \\
\text { profit sectors }\end{array}$ & Fair \\
\hline
\end{tabular}




\begin{tabular}{|c|c|c|c|c|c|}
\hline Author, Year & $\begin{array}{l}\text { Setting } \\
\text { Country }\end{array}$ & Inclusion Criteria & $\begin{array}{l}\text { Interventions and Ns per } \\
\text { Group }\end{array}$ & $\begin{array}{l}\text { Duration } \\
\text { (intervention and } \\
\text { longest followup) }\end{array}$ & $\begin{array}{l}\text { Age Gender } \\
\text { Race/Ethnicity }\end{array}$ \\
\hline Subotnik, 2015 & United States & $\begin{array}{l}\text { Adults (18 to } 45 \text { years) with DSM-IV } \\
\text { diagnosis of schizophrenia, } \\
\text { schizoaffective disorder, mainly } \\
\text { depressed type, or schizophreniform } \\
\text { disorder, with an onset of psychosis } \\
\text { within the last } 2 \text { years. }\end{array}$ & $\begin{array}{l}\text { Risperidone modal dosage } \\
25 \mathrm{mg} \text { biweekly (12.5 to } \\
37.5 \mathrm{mg} \text { ) long acting } \\
\text { injectable ( } \mathrm{n}=43 \text { ) } \\
\text { vs. } \\
\text { Risperidone modal dosage } \\
2 \mathrm{mg} \text { daily (1.0 to } 7.5 \mathrm{mg}) \\
\text { oral ( } \mathrm{n}=43 \text { ) } \\
\text { Both arms subsequently } \\
\text { randomized in cognitive } \\
\text { remediation or healthy- } \\
\text { behaviors training. }\end{array}$ & 52 weeks & $\begin{array}{l}\text { Age, years: } 21.5 \\
\text { Gender, \% female: } 22.0 \\
\text { Ethnicity, \%: } \\
\text { White: } 49.0 \\
\text { Asian: } 11.0 \\
\text { Native American: } 5.0 \\
\text { African American: } 28.0 \\
\text { Pacific Islander: } 1.0 \\
\text { Mixed: } 6.0\end{array}$ \\
\hline Tybura, 2013 & Poland & $\begin{array}{l}\text { Caucasian patients of Polish descent } \\
\text { with paranoid schizophrenia (confirmed } \\
\text { with Polish CIDI* and ICD-10 criteria). }\end{array}$ & $\begin{array}{l}\text { Olanzapine } 10-20 \mathrm{mg} \\
(\mathrm{n}=19) \\
\text { vs. } \\
\text { Ziprasidone } 120-160 \mathrm{mg} \\
(\mathrm{n}=20) \\
\text { vs. } \\
\text { Perazine } 300-600 \mathrm{mg} \\
(\mathrm{n}=19)\end{array}$ & 3 months & $\begin{array}{l}\text { Age, mean years (SD): } 36.2(12.0) \\
\text { Gender, \% female: } \\
51.7 \\
\text { Ethnicity, \%: } \\
\text { Caucasian: } 100.0\end{array}$ \\
\hline Tybura, 2014 & Poland & $\begin{array}{l}\text { Caucasian patients of Polish descent } \\
\text { suffering from paranoid schizophrenia. } \\
\text { Diagnosis based on Polish version of the } \\
\text { CIDI and the ICD-10 criteria. } \\
\end{array}$ & $\begin{array}{l}\text { Ziprasidone } 120-160 \\
\text { mg/day orally }(n=59) \\
\text { vs. } \\
\text { Olanzapine } 10-20 \mathrm{mg} / \text { day } \\
\text { orally }(n=72) \\
\text { vs. } \\
\text { Perazine } 300-600 \mathrm{mg} / \text { day } \\
\text { orally }(n=60)\end{array}$ & 12 weeks & $\begin{array}{l}\text { Age, years: } 35.8 \\
\text { Gender, \% female: } 55.1 \\
\text { Ethnicity, \%: } \\
\text { Caucasian: } 100 \text { (Polish descent) }\end{array}$ \\
\hline
\end{tabular}




\begin{tabular}{|c|c|c|c|}
\hline Author, Year & Other Population Characteristics & Total N & Benefits Outcomes \\
\hline Subotnik, 2015 & $\begin{array}{l}\text { Duration of illness, months: } 7.4 \\
\text { (time since psychosis onset) } \\
\text { Severity of illness (BPRS): } \\
\text { Thought disturbance factor at randomization: } 2.1 \\
\text { Withdrawal-retardation factor at randomization: } 1.9 \\
\text { Schizophrenia, \%: } 55.0 \\
\text { Schizophreniform disorder, \%: } 33.0 \\
\text { Schizoaffective, \%: } 12.0 \\
\text { Substance use, \%: } 0\end{array}$ & 86 & $\begin{array}{l}\text { Risperidone } 25 \mathrm{mg} \text { biweekly long acting vs. risperidone } 2 \mathrm{mg} \text { daily } \\
\text { Psychotic exacerbation/relapse, } \mathrm{n} / \mathrm{N}(\%)^{\star}: 2 / 40 \text { (5.0) vs. } 14 / 43(33.0) ; \\
\mathrm{P}<0.001 \\
\text { Hospitalizations due to mental illness, } \mathrm{n} / \mathrm{N}(\%): 2 / 40(5.0) \mathrm{vs.} 8 / 43 \\
\text { (18.6); } \mathrm{P}=0.05 \\
\text { Early discontinuation due to inadequate treatment response, } \mathrm{n} / \mathrm{N}(\%) \text { : } \\
1 / 40(2.5) \text { vs. } 7 / 42(17.0), \mathrm{P}=0.01 \\
\text { Risk of exacerbation and/or relapse over time was significantly lower for } \\
\text { long-acting injectable risperidone than for oral risperidone: } \mathrm{p}<0.004 \\
\text { Mean time to relapse, days: } 298.5 \text { vs. } 218.6 \\
\text { Medication adherence was better for long-acting risperidone vs. oral } \\
\text { risperidone: } p<0.001 \\
\text { Medication adherence was associated with prevention of exacerbation } \\
\text { and/or relapse }(\mathrm{p}=0.003) \text { and control of breakthrough psychotic } \\
\text { symptoms }(\mathrm{p}=0.04) \text {. }\end{array}$ \\
\hline Tybura, 2013 & Mean age (SD) at first psychotic episode, years: 26.9 (6.9) & 58 & $\begin{array}{l}\text { Olanzapine vs. ziprasidone vs. perazine } \\
\text { PANSS total score (SD) after } 3 \text { months: -64.8 (18.9) vs. }-75.2 \text { (27.1) vs. } \\
68.0(28.3)\end{array}$ \\
\hline Tybura, 2014 & $\begin{array}{l}\text { Duration of illness: } 9.9 \text { years* } \\
\text { PANSS severity of illness: } 99.8 \\
\text { Schizoaffective, \%: } 0 \\
\text { Antipsychotic drug naïve, \%: } 0\end{array}$ & 191 & $\begin{array}{l}\text { Ziprasidone } 120-160 \mathrm{mg} / \text { day vs. olanzapine } 10-20 \mathrm{mg} / \text { day vs. perazine } \\
300-600 \mathrm{mg} / \mathrm{day} \\
\text { All-cause discontinuation at week } 12, \mathrm{n} / \mathrm{N}(\%)^{\star \star}: 41 / 60(68.0) \text { vs. } 52 / 72 \\
(76.0) \text { vs. } 40 / 59(68.0)\end{array}$ \\
\hline
\end{tabular}




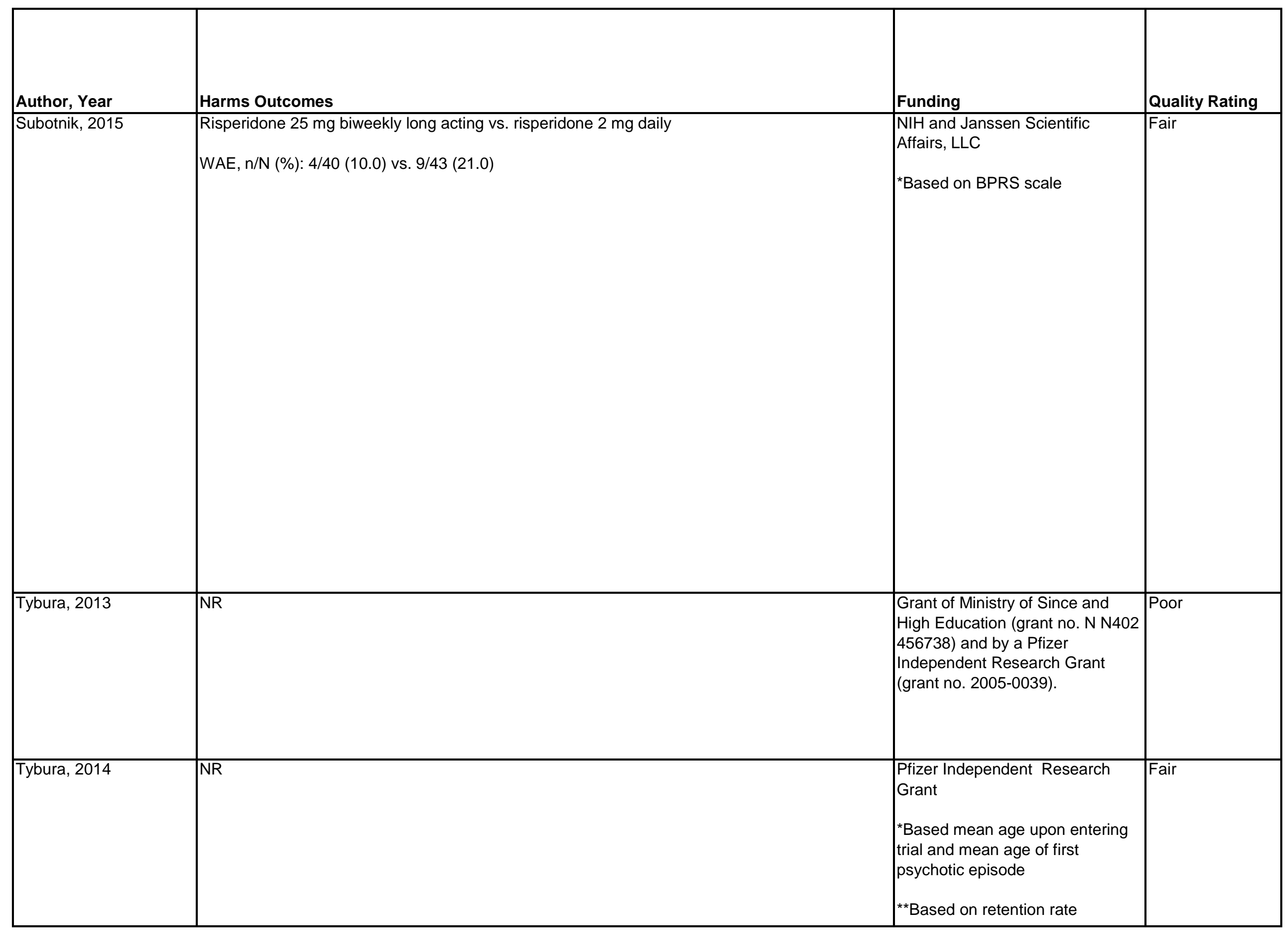




\begin{tabular}{|c|c|c|c|c|c|}
\hline Author, Year & $\begin{array}{l}\text { Setting } \\
\text { Country }\end{array}$ & Inclusion Criteria & $\begin{array}{l}\text { Interventions and Ns per } \\
\text { Group }\end{array}$ & $\begin{array}{l}\text { Duration } \\
\text { (intervention and } \\
\text { longest followup) }\end{array}$ & $\begin{array}{l}\text { Age Gender } \\
\text { Race/Ethnicity }\end{array}$ \\
\hline Wani, 2015 & India & $\begin{array}{l}\text { Adult patients with schizophrenia who } \\
\text { had achieved clinical stability with } \\
\text { olanzapine and who were assessed as } \\
\text { having metabolic syndrome using } \\
\text { modified NCEP ATP-III criteria. } \\
\text { Schizophrenia diagnoses were made } \\
\text { using the DSM IV. }\end{array}$ & $\begin{array}{l}\text { Olanzapine } 10-20 \mathrm{mg} / \text { day } \\
\text { orally }(\mathrm{n}=31) \\
\text { vs. } \\
\text { Aripiprazole } 5-20 \mathrm{mg} / \text { day } \\
\text { orally }(\mathrm{n}=31)^{*}\end{array}$ & 24 weeks & $\begin{array}{l}\text { Age (years): } 29.8 \\
\text { Gender, \% female: } 37.1 \\
\text { Ethnicity: Asian (Indian) }\end{array}$ \\
\hline
\end{tabular}




\begin{tabular}{|l|l|l|l|}
\hline & & & \\
\hline Author, Year & Other Population Characteristics & Total N & Benefits Outcomes \\
\hline Wani, 2015 & $\begin{array}{l}\text { Duration of illness: 4.75 years } \\
\text { PANSS severity of illness: 68.9 } \\
\text { Antipsychotic drug naïve, \%: } 0\end{array}$ & $\begin{array}{l}\text { Olanzapine 10-20 mg/day vs. aripiprazole 5 to 20mg/day } \\
\text { All-cause hospitalization, n/N \%: 2/26 (7.7) vs. 2/21 (9.5) }\end{array}$ \\
\hline
\end{tabular}




\begin{tabular}{|l|l|l|}
\hline Author, Year & Harms Outcomes & Funding \\
\hline Wani, 2015 & $\begin{array}{l}\text { Olanzapine 10-20 mg/day vs. aripiprazole 5 to 20mg/day } \\
\text { Patients meeting modified NCEP ATP-III criteria for the presence of metabolic syndrome, n/N } \\
(\%)^{\star *}: 26 / 26(100) \text { vs. 15/31 (42.8); P<0.001 }\end{array}$ & $\begin{array}{l}* \text { With accompanying reduction of } \\
\text { continuing olanzapine (reduction } \\
\text { from 25\% to 100\% after 3 weeks } \\
* * \text { Based on modified NCEP ATP- } \\
\text { III criteria for the Asian population } \\
\text { (waist circumference, } \\
\text { triglycerides, HDL, Systolic BP, } \\
\text { fasting glucose) }\end{array}$ \\
\hline
\end{tabular}

\section{Please see Appendix B. Included Studies for full study references}

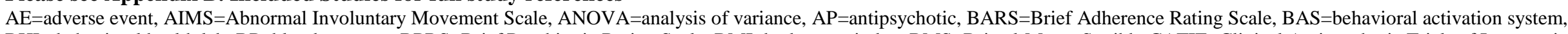

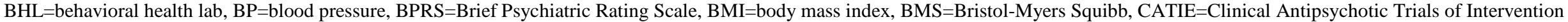

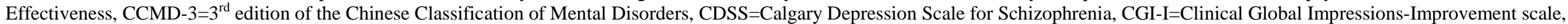

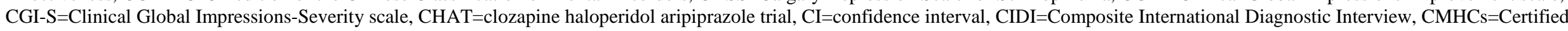

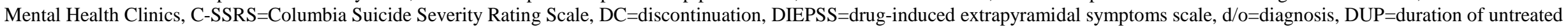

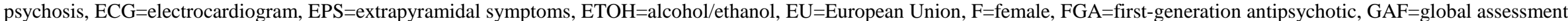

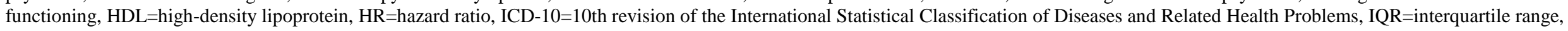
ITT=intention-to-treat, J\&J=Johnson and Johnson, kg=kilogram, LAI=long acting injectable, LDL=low-density lipoprotein, LOCF=last observation carried forward, LOS=living on site,

LUNSERS=Liverpool University Neuroleptic Side Effect Rating Scale, LS=life skills, LSM=life skills mean, M=male, MINI=International Neuropsychiatric Interview, mos=months,

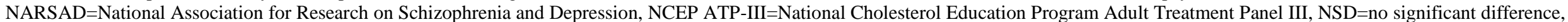

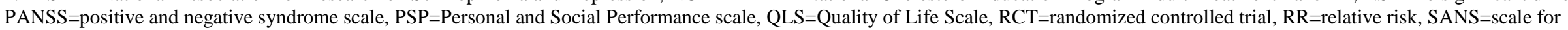

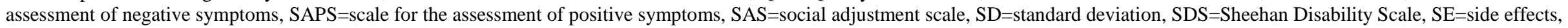

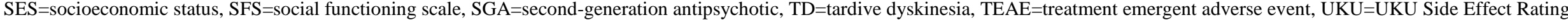

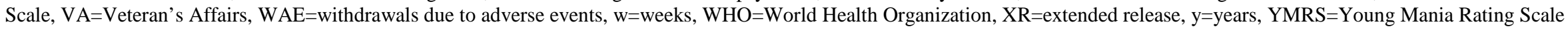


Appendix Table E-3. Data abstraction of systematic reviews of assertive community treatment

\begin{tabular}{|c|c|c|c|c|c|c|}
\hline Author, Year & Aims & $\begin{array}{l}\text { Databases and } \\
\text { Timeperiod Covered } \\
\text { (actual dates) }\end{array}$ & \begin{tabular}{|l} 
Number of \\
Studies \\
Number of \\
Patients
\end{tabular} & $\begin{array}{l}\text { Characteristics of Identified } \\
\text { Articles: Study Designs }\end{array}$ & $\begin{array}{l}\text { Characteristics of } \\
\text { Identified Articles: } \\
\text { Populations }\end{array}$ & $\begin{array}{l}\text { Characteristics of } \\
\text { Identified Articles: } \\
\text { Interventions }\end{array}$ \\
\hline $\begin{array}{l}\text { Marshall } \\
\text { 2000a } \\
\text { (ACT) }\end{array}$ & $\begin{array}{l}\text { Effect of ACT vs } \\
\text { standard community } \\
\text { care in people with } \\
\text { severe mental disorders }\end{array}$ & $\begin{array}{l}\text { MEDLINE (1966-1995); } \\
\text { CINAHL (1982-1997); } \\
\text { EMBASE (1980-1997); } \\
\text { PsycLIT (1974-1997); } \\
\text { SCISEARCH (1997); } \\
\text { Cochrane Schizophrenia } \\
\text { Group Register of Trials } \\
\text { (1997) }\end{array}$ & $\begin{array}{l}14 \text { studies (ACT } \\
\text { vs. standard } \\
\text { care) } \\
n=2,821 \\
\\
\end{array}$ & $\begin{array}{l}\text { Randomized controlled trials } \\
\text { of ACT vs. standard care }\end{array}$ & $\begin{array}{l}\text { Age } 18-65 \text { years with } \\
\text { schizophrenia or } \\
\text { schizophrenia-like } \\
\text { disorders; bipolar } \\
\text { disorder; or depression } \\
\text { with psychotic features. } \\
\text { Proportion with } \\
\text { schizophrenia: <50\%: } 3 \\
\text { studies; >50\%: } 8 \text { studies; } \\
\text { unknown: } 3 \text { studies }\end{array}$ & $\begin{array}{l}\text { ACT: Any intervention } \\
\text { described as Assertive } \\
\text { Community Treatment, } \\
\text { Assertive Case } \\
\text { Management or PACT; } \\
\text { or as being based on } \\
\text { the Madison, } \\
\text { Treatment in } \\
\text { Community Living, } \\
\text { Assertive Community } \\
\text { Treatment or Stein and } \\
\text { Test models. }\end{array}$ \\
\hline
\end{tabular}




\begin{tabular}{|c|c|c|c|c|c|}
\hline Author, Year & Outcomes Reported & Effectiveness Outcomes & Harms Outcomes & $\begin{array}{l}\text { Fundingl } \\
\text { Comments }\end{array}$ & $\begin{array}{l}\text { Quality } \\
\text { rating }\end{array}$ \\
\hline $\begin{array}{l}\text { Marshall } \\
2000 a \\
\text { (ACT) }\end{array}$ & $\begin{array}{l}\text { Symptoms: combined BPRS, } \\
\text { Brief Symptom Inventory, } \\
\text { Colorado Symptom Index } \\
\text { Function: Contact with law } \\
\text { enforcement } \\
\text { Function: Not living } \\
\text { independently } \\
\text { Function: Unemployed } \\
\text { Social function: combined Social } \\
\text { Adjustment Scale, Personality } \\
\text { and Social Network Adjustment } \\
\text { Scale } \\
\text { Mortality }\end{array}$ & 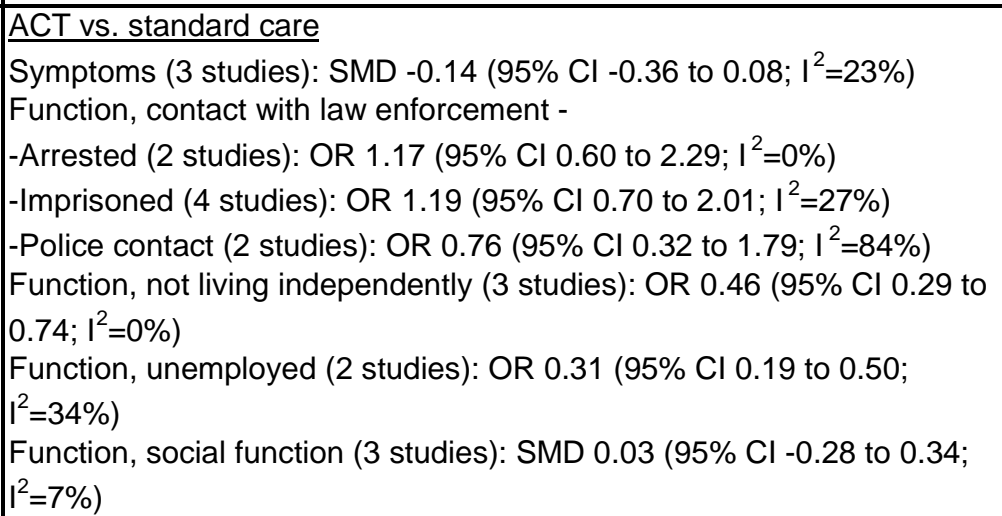 & $\begin{array}{l}\text { ACT vs. standard } \\
\text { care } \\
\text { Mortality (5 studies): } \\
\text { OR } 1.13(95 \% \mathrm{Cl} \\
\left.0.35 \text { to } 3.68 ; I^{2}=0 \%\right)\end{array}$ & \begin{tabular}{|l} 
Manchester \\
University \\
Department of \\
Psychiatry; \\
Nuffield Trust
\end{tabular} & Good \\
\hline
\end{tabular}

Please see Appendix B. Included Studies for full study references

ACT=assertive community treatment, BPRS=Brief Psychiatric Rating Scale, CI=confidence interval, OR=odds ratio, PACT=Program of Assertive Community Treatment, SMD=standard mean difference 
Appendix Table E-4. Data abstraction of randomized controlled trial of assertive community treatment

\begin{tabular}{|c|c|c|c|c|c|c|c|}
\hline $\begin{array}{l}\text { Author, } \\
\text { Year }\end{array}$ & $\begin{array}{l}\text { Setting } \\
\text { Country }\end{array}$ & $\begin{array}{l}\text { Inclusion } \\
\text { Criteria }\end{array}$ & $\begin{array}{l}\text { Interventions } \\
\text { and Ns per } \\
\text { Group }\end{array}$ & Description of Intervention & Description of Comparator & $\begin{array}{l}\text { Duration } \\
\text { (intervention } \\
\text { and longest } \\
\text { followup) }\end{array}$ & $\begin{array}{l}\text { Age Gender } \\
\text { Race/Ethnicity }\end{array}$ \\
\hline $\begin{array}{l}\text { Sytema } \\
2007\end{array}$ & $\begin{array}{l}\text { Winchschoten, } \\
\text { Netherlands; } \\
\text { local mental } \\
\text { health } \\
\text { organization }\end{array}$ & $\begin{array}{l}\text { Long-term } \\
\text { severely mentally } \\
\text { ill patients with } \\
\text { Health of the } \\
\text { Nation Outcomes } \\
\text { Scales total } \\
\text { score of } \geq 15 \text {. }\end{array}$ & $\begin{array}{l}\text { Assertive } \\
\text { community } \\
\text { treatment } \\
(n=59) \\
\text { vs. standard } \\
\text { community } \\
\text { mental health } \\
\text { care }(n=59)\end{array}$ & $\begin{array}{l}\text { Assertive community treatment } \\
\text { teams included the following } \\
\text { characteristics: maximum FTE } \\
\text { caseload of } 10 \text { patients; work } \\
\text { style: shared caseload. All } \\
\text { patients are discussed in } \\
\text { weekly and daily team } \\
\text { meetings Location: Always } \\
\text { there where the patient is } \\
\text { Engagement with client: Assertive; } \\
\text { keep trying to make contact; } \\
\text { no drop-out policy. Working } \\
\text { hours: office hours } \\
\text { 24-h arrangement: The 24-h service } \\
\text { of the institute } \\
\text { Skills: Multidisciplinary team; all } \\
\text { skills are available for each client } \\
\text { because all team members may } \\
\text { have contact with each client } \\
\text { Disciplines available: Psychiatrist, } \\
\text { Psychologist } \\
\text { Psychiatric Nurse, Social Worker, } \\
\text { Client Worker } \\
\text { Dependency Specialist }\end{array}$ & \begin{tabular}{|l|} 
Community mental health \\
teams included the following \\
characteristics: \\
maximum FTE caseload: 40 \\
patients \\
Work style: Individual \\
caseloads \\
Location: Mostly at the office, \\
partly at home of the patient \\
Engagement with client: Not \\
assertive; the client should \\
express a need for care; \\
client will drop out of contact \\
when contact is refuses or \\
when the client does not \\
show up \\
Working hours: Office hours \\
24-hour arrangement: The $24-$ \\
hour service of the institute \\
Skills: Client and practitioner \\
are matched according to the \\
needs of the patient and the \\
skills of the practitioner \\
Disciplines available: \\
Psychiatrist, Psychologist, \\
Psychiatric Nurse, Social \\
Worker
\end{tabular} & \begin{tabular}{|l|} 
April 2004-June \\
1,2005 \\
Followup until \\
August 2006, \\
maximum of 2 \\
years of followup \\
\end{tabular} & $\begin{array}{l}\text { Demographics } \\
\text { (intervention, } \\
\text { control) } \\
\text { Age, mean years: } \\
41.5,37 \\
\text { Gender, \% } \\
\text { female: } \\
56 \%, 63 \% \\
\text { Ethnicity, \% NR }\end{array}$ \\
\hline
\end{tabular}




\begin{tabular}{|c|c|c|c|c|c|c|}
\hline $\begin{array}{l}\text { Author, } \\
\text { Year }\end{array}$ & $\begin{array}{l}\text { Other Population } \\
\text { Characteristics }\end{array}$ & Total N & Benefits Outcomes & \begin{tabular}{|l} 
Harms \\
Outcomes
\end{tabular} & Funding & $\begin{array}{l}\text { Quality } \\
\text { Rating }\end{array}$ \\
\hline $\begin{array}{l}\text { Sytema } \\
2007\end{array}$ & $\begin{array}{l}\text { Years in treatment } \\
\text { (intervention, control) } \\
7.9,8.6\end{array}$ & 118 & $\begin{array}{l}\text { Patients out of contact with mental health services (last } 12 \text { months of observation): } \\
0 \\
\text { vs.13; Peto OR } 0.1095 \% \mathrm{Cl} 0.03 \text { to } 0.33 \\
\text { Homeless patients (end of observation): } 1 \text { vs. } 5 \text {; Peto OR } 0.2495 \% \mathrm{Cl} 0.05 \text { to } \\
1.25 \\
\text { BRPS (intervention, } \\
\text { control) Baseline: } 42,45 \\
\text { After } 12 \text { months: } 38,42 \\
\text { MANSA } \\
\text { Baseline: } 4.7,4.5 \\
\text { After } 12 \text { months: } 4.5,4.3 \\
\text { SFS } \\
\text { Baseline: } 102,103 \\
\text { After } 12 \text { months: } 102,103\end{array}$ & NR & \begin{tabular}{|l|} 
ZonMW, The \\
Netherlands \\
Organization for \\
Health Research and \\
Development
\end{tabular} & Fair \\
\hline
\end{tabular}

Please see Appendix B. Included Studies for full study references

BPRS=Brief Psychiatric Rating Scale, FTE=full time employment, GAF=global assessment functioning, MANSA=Manchester Short Assessment of Quality of Life, SFS=Social Functioning Scale 
Appendix Table E-5. Data abstraction of randomized controlled trials of cognitive adaptation training*

\begin{tabular}{|c|c|c|c|c|}
\hline Author, Year & $\begin{array}{l}\text { Interventions (n) } \\
\text { Duration }\end{array}$ & Population characteristics & Results & Quality Rating \\
\hline $\begin{array}{l}\text { Velligan 2008a } \\
\text { and Velligan } 2009\end{array}$ & $\begin{array}{l}\text { CAT }(n=40) \\
\text { Usual care }(n=40) \\
\text { Duration of intervention and followup: } 24 \\
\text { months }\end{array}$ & $\begin{array}{l}\text { CAT vs. usual care: } \\
\text { Mean age } 41 \text { vs. } 40 \text { years } \\
50 \% \text { vs. } 51 \% \text { female } \\
\text { Race/ethnicity - } \\
42 \% \text { vs. } 36 \% \text { Hispanic } \\
47 \% \text { vs. } 36 \% \text { white } \\
\text { Mean SOFAS } 44.1 \text { vs. } 45.6\end{array}$ & $\begin{array}{l}\text { Function, based on SOFAS score, } \\
\text { improved more with CAT vs. usual care } \\
\text { (effect size 1.10) }\end{array}$ & Fair \\
\hline Velligan 2008b & $\begin{array}{l}\text { CAT }(n=73) \\
\text { Usual care }(n=32) \\
\text { Duration of intervention: } 9 \text { months } \\
\text { Duration of longest followup: } 15 \text { months }\end{array}$ & $\begin{array}{l}\text { CAT vs. usual care: } \\
\text { Mean age } 38 \text { vs. } 39 \text { years } \\
45 \% \text { vs. } 38 \% \text { female } \\
\text { Race/ethnicity - } \\
34 \% \text { vs. } 28 \% \text { Hispanic } \\
34 \% \text { vs. } 45 \% \text { white } \\
\text { Mean SOFAS } 45.8 \text { vs. } 45.6\end{array}$ & $\begin{array}{l}\text { For function, based on SOFAS score, there } \\
\text { was nonsignificant trend favoring CAT over } \\
\text { usual care }(p<0.07) \text {. } \\
\text { Significantly fewer patients in the CAT } \\
\text { groups relapsed compared with usual care. }\end{array}$ & Fair \\
\hline Velligan 2015b & $\begin{array}{l}\text { CAT }(n=68) \\
\text { Usual care }(n=37) \\
\text { Duration of intervention: } 9 \text { months } \\
\text { Duration of longest followup: } 15 \text { months }\end{array}$ & $\begin{array}{l}\text { CAT vs. usual care: } \\
\text { Mean age } 41 \text { vs. } 40 \text { year } \\
46 \% \text { vs. } 46 \% \text { female } \\
\text { Race/ethnicity - } \\
40 \% \text { Hispanic } \\
31 \% \text { white } \\
25 \% \text { Black } \\
\text { Mean MCAS score } 3.7 \text { vs. } 3.8\end{array}$ & $\begin{array}{l}\text { Function, based on MCAS score, improved } \\
\text { more in CAT vs. non-CAT groups (effect } \\
\text { size 0.4). }\end{array}$ & Fair \\
\hline
\end{tabular}

Please see Appendix B. Included Studies for full study references

CAT=cognitive adaption training, MCAS= Multnomah Community Ability Scale, SOFAS=Social and Occupational Assessment Scale

*Overall evidence for this intervention was insufficient 
Appendix Table E-6 Data abstraction of systematic reviews of cognitive-behavioral therapy

\begin{tabular}{|c|c|c|c|c|c|c|}
\hline Author, Year & Aims & $\begin{array}{l}\text { Databases and } \\
\text { Timeperiod Covered }\end{array}$ & \begin{tabular}{|l} 
Number of \\
Studies \\
Number of \\
Patients
\end{tabular} & $\begin{array}{l}\text { Characteristics of Identified } \\
\text { Articles: Study Designs }\end{array}$ & $\begin{array}{l}\text { Characteristics of } \\
\text { Identified Articles: } \\
\text { Populations }\end{array}$ & $\begin{array}{l}\text { Characteristics of } \\
\text { Identified Articles: } \\
\text { Interventions }\end{array}$ \\
\hline Velthorst 2015 & \begin{tabular}{|l|} 
Effect of CBT on \\
negative symptoms; \\
assess which subgroups \\
most likely to respond
\end{tabular} & \begin{tabular}{|l|} 
Databases not reported \\
(1993-July 2013)
\end{tabular} & $\begin{array}{l}30 \text { studies } \\
n=2312\end{array}$ & $\begin{array}{l}\text { Randomized controlled trials } \\
\text { of CBT targeted at psychotic } \\
\text { symptoms, negative } \\
\text { symptoms, social functioning, } \\
\text { self esteem or cannabis use; } \\
\text { duration of followup up to } 12 \\
\text { months }\end{array}$ & $\begin{array}{l}\text { Patients with resent } \\
\text { onset ( } 3 \text { studies) and } \\
\text { mixed chronic and } \\
\text { resent onset ( } 27 \\
\text { studies) schizophrenia; } \\
\text { outpatients (18 studies), } \\
\text { inpatient (4 studies), } \\
\text { mixed inpatient and } \\
\text { outpatient ( } 7 \text { studies) } \\
\text { *table only provides this } \\
\text { information for } 29 \\
\text { studies }\end{array}$ & \begin{tabular}{|l} 
CBT vs. control \\
(usual care, \\
supportive care, \\
befriending, waitlist, \\
psychoeducation)
\end{tabular} \\
\hline Jauhar 2014 & $\begin{array}{l}\text { Effect of CBT on } \\
\text { schizophrenia symptoms }\end{array}$ & $\begin{array}{l}\text { MEDLINE (1993-March } \\
\text { 2013); PsycINFO (1993- } \\
\text { March 2013); EMBASE } \\
\text { (1993-March 2013); } \\
\text { CCRCT (1993-March } \\
\text { 2013) }\end{array}$ & $\begin{array}{l}50 \text { studies } \\
\mathrm{n}=3947\end{array}$ & $\begin{array}{l}\text { Randomized controlled trials } \\
\text { of CBT reporting positive, } \\
\text { negative and overall symptom } \\
\text { outcomes }\end{array}$ & $\begin{array}{l}\text { Patients with } \\
\text { schizophrenia or } \\
\text { schizoaffective or } \\
\text { nonaffective functional } \\
\text { psychosis; } \\
\text { inpatient/outpatient not } \\
\text { reported }\end{array}$ & $\begin{array}{l}\text { CBT vs. control } \\
\text { (waitlist, usual care, or } \\
\text { intervention designed } \\
\text { to control for } \\
\text { nonspecific effects of } \\
\text { psychotherapy) }\end{array}$ \\
\hline
\end{tabular}




\begin{tabular}{|c|c|c|c|c|c|}
\hline Author, Year & Outcomes Reported & Effectiveness Outcomes & \begin{tabular}{|l} 
Harms \\
Outcomes
\end{tabular} & Funding & $\begin{array}{l}\text { Quality } \\
\text { Rating }\end{array}$ \\
\hline Velthorst 2015 & Negative symptoms & 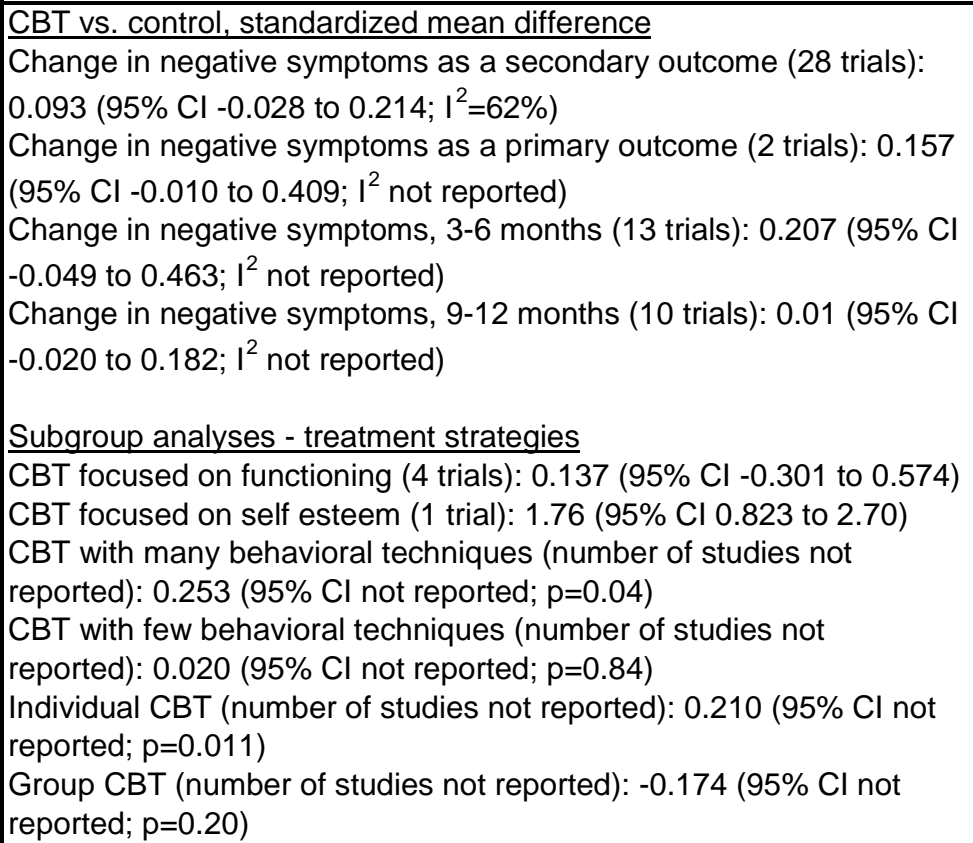 & Not reported & None reported & Good \\
\hline Jauhar 2014 & $\begin{array}{l}\text { Overall symptoms } \\
\text { Positive symptoms } \\
\text { Negative symptoms } \\
\text { Hallucinations }\end{array}$ & \begin{tabular}{|l} 
CBT vs. control, effect size \\
(negative effect size favors CBT) \\
Overall symptoms (34 trials): $-0.33\left(95 \% \mathrm{Cl}-0.47\right.$ to $\left.-0.19 ; \mathrm{I}^{2}=68 \%\right)$ \\
Positive symptoms (33 trials): $-0.25\left(95 \% \mathrm{Cl}-0.37\right.$ to $\left.-0.13 ; \mathrm{I}^{2}=49 \%\right)$ \\
Negative symptoms (34 trials): $-0.13\left(95 \% \mathrm{Cl}-0.25\right.$ to $\left.-0.01 ; \mathrm{I}^{2}=48 \%\right)$ \\
Hallucinations ( 15 trials): $-0.34\left(95 \% \mathrm{Cl}-0.61\right.$ to $\left.-0.06 ; \mathrm{I}^{2}=70 \%\right)$
\end{tabular} & Not reported & $\begin{array}{l}\text { Centro de } \\
\text { Investigacion } \\
\text { Biomedia en } \\
\text { Red de Salud } \\
\text { Mental } \\
\text { (CIBERSAM) }\end{array}$ & Good \\
\hline
\end{tabular}




\begin{tabular}{|c|c|c|c|c|c|c|}
\hline Author, Year & Aims & $\begin{array}{l}\text { Databases and } \\
\text { Timeperiod Covered }\end{array}$ & \begin{tabular}{|l} 
Number of \\
Studies \\
Number of \\
Patients \\
\end{tabular} & $\begin{array}{l}\text { Characteristics of Identified } \\
\text { Articles: Study Designs }\end{array}$ & $\begin{array}{l}\text { Characteristics of } \\
\text { Identified Articles: } \\
\text { Populations }\end{array}$ & $\begin{array}{l}\text { Characteristics of } \\
\text { Identified Articles: } \\
\text { Interventions }\end{array}$ \\
\hline Jones 2010 & $\begin{array}{l}\text { Effect of CBT for people } \\
\text { with schizophrenia }\end{array}$ & $\begin{array}{l}\text { Cochrane Schizophrenia } \\
\text { Group Trials Register } \\
\text { (March 2010; comprises } \\
\text { searches of CINAHL, } \\
\text { EMBASE, MEDLINE } \\
\text { PsycINFO) }\end{array}$ & \begin{tabular}{|l|}
9 comparing \\
CBT with \\
nonactive control \\
(of 20 studies \\
included in \\
review) \\
$n=895$
\end{tabular} & $\begin{array}{l}\text { Randomized controlled trials } \\
\text { of CBT; duration of followup } 8 \\
\text { weeks to } 5 \text { years (mean } 20 \\
\text { months) }\end{array}$ & $\begin{array}{l}\text { Patients with current } \\
\text { diagnosis of } \\
\text { schizophrenia (at least } \\
50 \% \text { of study } \\
\text { participants); } \\
\text { inpatient/outpatient not } \\
\text { reported }\end{array}$ & $\begin{array}{l}\text { CBT vs. nonactive } \\
\text { control (psychosocial } \\
\text { interventions which act } \\
\text { as a control for the } \\
\text { nonspecific effects of } \\
\text { therapy); active-control } \\
\text { comparisons not } \\
\text { abstracted as not akin } \\
\text { to usual care }\end{array}$ \\
\hline
\end{tabular}




\begin{tabular}{|c|c|c|c|c|c|}
\hline Author, Year & Outcomes Reported & Effectiveness Outcomes & \begin{tabular}{|l} 
Harms \\
Outcomes
\end{tabular} & Funding & $\begin{array}{l}\text { Quality } \\
\text { Rating }\end{array}$ \\
\hline Jones 2010 & $\begin{array}{l}\text { Mortality } \\
\text { Global state } \\
\text { Quality of life } \\
\text { Engagement with services } \\
\text { Adverse effects } \\
\text { (Symptoms addressed in more } \\
\text { recent Jauhar } 2014 \text { review) }\end{array}$ & 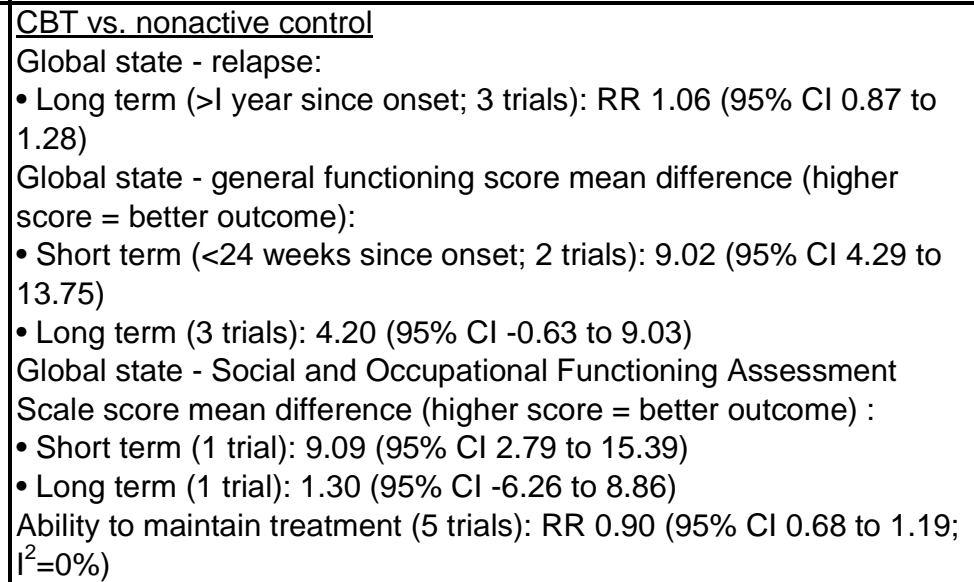 & \begin{tabular}{|l|} 
CBT vS. \\
nonactive control \\
Mortality (1 trial; \\
reported as \\
suicide): $\mathrm{RR}$ \\
$0.68(95 \% \mathrm{Cl}$ \\
0.12 to 3.93$)$ \\
\end{tabular} & None & Good \\
\hline
\end{tabular}

Please see Appendix B. Included Studies for full study references

BPRS=Brief Psychiatric Rating Scale, CBT=cognitive-behavioral therapy, $\mathrm{CI}=$ confidence interval, $\mathrm{RR}=$ relative risk, $\mathrm{SMD}=$ standard mean difference 
Appendix Table E-7. Data abstraction of randomized controlled trials of cognitive-behavioral therapy

\begin{tabular}{|c|c|c|c|c|c|c|}
\hline $\begin{array}{l}\text { Author, Year } \\
\text { Trial Name }\end{array}$ & $\begin{array}{l}\text { Setting } \\
\text { Country }\end{array}$ & Inclusion Criteria & $\begin{array}{l}\text { Interventions } \\
\text { and Ns per } \\
\text { Group }\end{array}$ & Description of Intervention & Description of Comparator & \begin{tabular}{|l|} 
Duration \\
(intervention \\
and longest \\
followup)
\end{tabular} \\
\hline Freeman 2015 & $\begin{array}{l}2 \text { centers } \\
\text { United } \\
\text { Kingdom }\end{array}$ & $\begin{array}{l}\text { Age 18-65 years with a current } \\
\text { persecutory delusion as defined by } \\
\text { Freeman and Garety; score at least } 3 \\
\text { on the conviction scale of the } \\
\text { PSYRATS; delusion had persisted for } \\
\text { at least } 3 \text { months; a clinical diagnosis } \\
\text { of schizophrenia, schizoaffective } \\
\text { disorder, or delusional disorder; score } \\
\text { of more than } 44 \text { on the PSWQ } \\
\text { Exclude: Primary diagnosis of alcohol } \\
\text { or substance dependency or } \\
\text { personality disorder; organic } \\
\text { syndrome or learning disability; } \\
\text { adequate English language skills to } \\
\text { engage in therapy; current CBT }\end{array}$ & $\begin{array}{l}\text { CBT + standard } \\
\text { care }(n-73) \\
\text { Standard care } \\
\text { alone }(n=77)\end{array}$ & $\begin{array}{l}\text { CBT to reduce worry: } \\
\text { Psychoeducation about worry, } \\
\text { identification and reviewing of positive } \\
\text { and negative beliefs about worry, } \\
\text { increasing awareness of the initiation } \\
\text { of worry and individual triggers, use of } \\
\text { worry periods, planning activity at } \\
\text { times of worry (which could include } \\
\text { relaxation), and learning to let go of } \\
\text { worry. Practical application: the use of } \\
\text { worry periods (confining worry to } \\
\text { about a 20-min set period each day) } \\
\text { and planning of activities at peak } \\
\text { worry times. }\end{array}$ & $\begin{array}{l}\text { Standard care: Delivered } \\
\text { according to national and } \\
\text { local service protocols and } \\
\text { guidelines. Generally } \\
\text { consists of prescription } \\
\text { antipsychotic drugs, visits } \\
\text { from a community mental } \\
\text { health worker, and regular } \\
\text { outpatient appointments } \\
\text { with a psychiatrist. }\end{array}$ & $\begin{array}{l}\text { Intervention: } 8 \\
\text { weeks } \\
\text { Followup: } 24 \\
\text { weeks }\end{array}$ \\
\hline Lysaker 2009 & $\begin{array}{l}2 \text { centers } \\
\text { United States }\end{array}$ & $\begin{array}{l}\text { Confirmed diagnosis of schizophrenia } \\
\text { or schizoaffective disorder in post- } \\
\text { acute phase of illness (no } \\
\text { hospitalizations, changes in } \\
\text { psychotropic medication or housing in } \\
\text { the month before entering the study. } \\
\text { Excluded: diagnosis of mental } \\
\text { retardation or another neurological } \\
\text { disorder }\end{array}$ & $\begin{array}{l}\text { IVIP } n=50 \\
\text { Social support } \\
n=50\end{array}$ & $\begin{array}{l}\text { IVIP: weekly group CBT centered on } \\
\text { a rotating curriculum of four } 2 \text {-week } \\
\text { modules focusing on addressing } \\
\text { dysfunctional beliefs about self and } \\
\text { work experiences + individual } \\
\text { sessions }\end{array}$ & $\begin{array}{l}\text { Social support: usual VA work } \\
\text { support including weekly hour } \\
\text { group sessions offering support } \\
\text { and discussion of work-related } \\
\text { issues and concerns }\end{array}$ & 26 weeks \\
\hline
\end{tabular}




\begin{tabular}{|c|c|c|c|c|}
\hline $\begin{array}{l}\text { Author, Year } \\
\text { Trial Name }\end{array}$ & $\begin{array}{l}\text { Age Gender } \\
\text { Race/Ethnicity }\end{array}$ & Other Population Characteristics & Total N & Outcome Measures \\
\hline Freeman 2015 & $\begin{array}{l}\text { CBT vs. standard care } \\
\text { Mean age } 41 \text { vs. } 42 \text { years } \\
42 \% \text { vs. } 43 \% \text { female } \\
\text { Race }- \\
93 \% \text { vs. } 89 \% \text { White } \\
1 \% \text { vs. } 0 \% \text { Black } \\
0 \% \text { vs. } 3 \% \text { Chinese } \\
0 \% \text { vs. } 4 \% \text { Indian } \\
6 \% \text { vs. } 4 \% \text { other }\end{array}$ & $\begin{array}{l}\text { CBT vs. standard care } \\
\text { Age at onset not reported } \\
\text { Time since diagnosis (reported as time in contact with services) - } \\
<1 \text { year: } 8 \% \text { vs. } 9 \% \\
1-5 \text { years: } 16 \% \text { vs. } 22 \% \\
6-10 \text { years: } 22 \% \text { vs. } 16 \% \\
11-20 \text { years: } 25 \% \text { vs. } 34 \% \\
>20 \text { years: } 29 \% \text { vs. } 19 \% \\
\text { Baseline symptom severity - } \\
\text { PANSS mean score: } 82.0 \text { (SD 13.6) vs. } 79.0 \text { (SD 13.5) } \\
\text { CHOICE mean score: } 49.4 \text { (SD } 17.3 \text { ) vs. } 49.5 \text { (SD 18.5) } \\
\text { WEMWEBS mean score: } 36.4 \text { (SD 9.6) vs. } 34.5 \text { (SD 9.2) } \\
\text { Comorbidity not reported } \\
\text { Substance use not reported } \\
\text { Treatment resistance not reported } \\
\text { Socioeconomic status not reported } \\
\text { Pregnancy status not reported }\end{array}$ & 150 & $\begin{array}{l}\text { Symptoms - } \\
\text { Delusions (PSYRATS - Delusion) } \\
\text { Worry (PSWQ) } \\
\text { Distress (PSYRATS - Distress) } \\
\text { Overall symptoms (PANSS) } \\
\text { Paranoia (GPTS) } \\
\text { Rumination (PTQ) } \\
\text { Quality of Life - } \\
\text { Self-confidence, coping skills, sense of } \\
\text { being in control (CHOICE) } \\
\text { Wellbeing (WEMWBS) } \\
\text { Mortality } \\
\text { Suicide attempts } \\
\text { Serious violent incidents }\end{array}$ \\
\hline Lysaker 2009 & $\begin{array}{l}\text { IVIP vs. support } \\
\text { Mean age } 46 \text { vs. } 47 \text { years } \\
16 \% \text { vs. } 14 \% \text { female } \\
\text { Race not stratified by intervention } \\
\text { group - } \\
58 \% \text { African-American } \\
41 \% \text { Caucasian } \\
1 \% \text { Latino }\end{array}$ & $\begin{array}{l}\text { IVIP vS. support } \\
\text { Age at first hospitalization: } 28 \text { vs. } 27 \text { years } \\
\text { Baseline symptom severity - } \\
\text { Total PANSS: } 76.86 \text { vs. } 76.30 \\
\text { SF-36 physical health: } 61.22 \text { vs. } 58.30 \\
\text { SF-36 mental health: } 58.64 \text { vs. } 60.32 \\
\text { Comorbidities not reported } \\
\text { Substance use not reported } \\
\text { Treatment resistant not reported } \\
\text { Socioeconomic status not reported } \\
\text { Pregnancy status not reported }\end{array}$ & 100 & $\begin{array}{l}\text { Function - } \\
\text { Mean total hours worked } \\
\text { Mean hours worked per week } \\
\text { Mean number of weeks at least } 1 \text { hour } \\
\text { worked } \\
\text { Work Behavior Inventory (WBI; scale 35- } \\
175 ; \text { higher score=better outcome) }\end{array}$ \\
\hline
\end{tabular}




\begin{tabular}{|c|c|c|c|c|}
\hline $\begin{array}{l}\text { Author, Year } \\
\text { Trial Name }\end{array}$ & Benefits Outcomes & Harms Outcomes & Funding & $\begin{array}{l}\text { Quality } \\
\text { Rating }\end{array}$ \\
\hline Freeman 2015 & $\begin{array}{l}\text { CBT vs. Standard care } \\
\text { Overall symptoms, mean PANSS: } 71.5 \text { (15.4) vs. } 76.3 \text { (SD 16.7); treatment effect } 6.16 \text { (SE } \\
\text { 1.69; p<0.001) } \\
\text { Quality of life, CHOICE: } 61.6 \text { (SD 21.4) vs. } 52.5 \text { (SD 22.4); treatment effect } 10.45 \text { (SE 2.42; } \\
\text { p<0.001) } \\
\text { Quality of life, WEMWEBS: } 40.2 \text { (SD 10.8) vs. } 36.6 \text { (SD 10.5); treatment effect } 10.45 \text { (SE } \\
\text { 2.42; p<0.001) }\end{array}$ & $\begin{array}{l}\text { Mortality: 0/73 vs. } 0 / 77 \\
\text { Suicide attempts: } 2 / 73 \text { vs. } 4 / 77 \\
\text { Serious violent incidents: } 2 / 73 \\
\text { vs. } 1 / 77\end{array}$ & UK Medical Research Council & Good \\
\hline Lysaker 2009 & $\begin{array}{l}\text { MIP VS. Stpport } \\
\text { Mean toal hours worked: } 360.86 \text { (SD 246.58) vs. } 228.82 \text { (SD 193.36); } p<0.01 \\
\text { Mean number of weeks at least } 1 \text { hour worked: } 18.64 \text { (SD 9.12) vs. } 14.46 \text { (SD 10.40); } \\
\text { p<0.05 } \\
\text { Mean WBI: } 113.34 \text { (SD 13.05) vs. } 105.43 \text { (SD 15.76); } p<0.05\end{array}$ & | Not reported & $\begin{array}{l}\text { VA Renabiltation Research } \\
\text { and Development Service }\end{array}$ & Falr \\
\hline
\end{tabular}




\begin{tabular}{|c|c|c|c|c|c|c|}
\hline $\begin{array}{l}\text { Author, Year } \\
\text { Trial Name }\end{array}$ & $\begin{array}{l}\text { Setting } \\
\text { Country }\end{array}$ & Inclusion Criteria & $\begin{array}{l}\text { Interventions } \\
\text { and Ns per } \\
\text { Group }\end{array}$ & Description of Intervention & Description of Comparator & \begin{tabular}{|l} 
Duration \\
(intervention \\
and longest \\
followup)
\end{tabular} \\
\hline $\begin{array}{l}\text { Malik } 2009 \text { and } \\
\text { Turkington } 2002\end{array}$ & $\begin{array}{l}6 \text { centers } \\
\text { United } \\
\text { Kingdom }\end{array}$ & $\begin{array}{l}\text { ICD-10 diagnosis of schizophrenia } \\
\text { with ongoing positive/negative } \\
\text { symptoms or were at risk of relapse } \\
\text { Excluded: active relapse, primary } \\
\text { diagnosis of substance or alcohol } \\
\text { dependence, organic brain disease, } \\
\text { learning disability severe enough to } \\
\text { interfere with rating. }\end{array}$ & $\begin{array}{l}\text { CBT: } n=257 \text { (76\% } \\
\text { [205/257] included } \\
\text { in 2-year followup } \\
\text { results) } \\
\text { Usual care: } n=165 \\
\text { ([125/165] } \\
\text { included in 2-year } \\
\text { followup results) }\end{array}$ & $\begin{array}{l}\text { CBT: } 6 \text { CBT sessions with a mental } \\
\text { health nurse over 2-3 months. With } \\
\text { patient consent, main caregivers were } \\
\text { offered } 3 \text { sessions of CBT }\end{array}$ & $\begin{array}{l}\text { Usual care: Regular review by a } \\
\text { psychiatrist, free antipsychotic } \\
\text { medication including clozapine, } \\
\text { access to day hospital, social } \\
\text { support in the community }\end{array}$ & 24 months \\
\hline Velligan 2015b & $\begin{array}{l}\text { Community } \\
\text { mental health } \\
\text { center } \\
\text { United States }\end{array}$ & $\begin{array}{l}\text { Age } 18 \text { to } 60 \text { years; diagnosis of } \\
\text { schizophrenia or schizoaffective } \\
\text { disorder; ongoing oral antipsychotic } \\
\text { treatment; persistent positive } \\
\text { symptoms (expanded BPRS score } \\
\geq 4 \text { ); SOFAS }<70\end{array}$ & $\begin{array}{l}\text { CBT: } n=43 \\
\text { Usual care: } \\
n=42 \text { (also } \\
\text { included } \\
\text { CAT individual } \\
\text { arm - see CAT } \\
\text { section) }\end{array}$ & $\begin{array}{l}\text { CBT: Weekly session focused on } \\
\text { patient-identified problems, } \\
\text { particularly those that interfered with } \\
\text { daily functioning or were distressing, } \\
\text { normalizing symptoms, and using } \\
\text { CBT techniques to develop } \\
\text { alternative explanatory models of } \\
\text { events. }\end{array}$ & $\begin{array}{l}\text { Usual care: Case } \\
\text { management and medication } \\
\text { followup appointments } \\
\text { provided by the local } \\
\text { community mental health } \\
\text { center. }\end{array}$ & $\begin{array}{l}\text { Intervention: } 9 \\
\text { months } \\
\text { Followup: } 15 \\
\text { months }\end{array}$ \\
\hline Zimmer 2007 & \begin{tabular}{|l} 
Single center \\
Brazil
\end{tabular} & $\begin{array}{l}\text { 18-65 years; ICD-10 diagnosis of } \\
\text { schizophrenia or schizoaffective } \\
\text { disorder; no organic brain disease; no } \\
\text { history of drug use; not participating in } \\
\text { a rehabilitation program; resident of } \\
\text { Porta Allegre, Brazil; stable } \\
\text { symptomalogy; absence of acute } \\
\text { symptoms for } 6 \text { months preceding } \\
\text { study entry }\end{array}$ & $\begin{array}{l}\text { IPT: } n=23 \\
\text { Usual care: } n=43\end{array}$ & $\begin{array}{l}\text { IPT: one } 60-\text { min session per week for } \\
\text { a period of } 3 \text { months, incorporating } \\
\text { five modules: Cognitive } \\
\text { Differentiation; Social Perception; } \\
\text { Verbal Communication; Social Skills } \\
\text { Training; Interpersonal Problem- } \\
\text { Solving. }\end{array}$ & $\begin{array}{l}\text { Usual care: individual outpatient } \\
\text { consultations, conducted once } \\
\text { every two weeks with psychiatry } \\
\text { residents, according to the usual } \\
\text { standard of care. }\end{array}$ & 12 weeks \\
\hline
\end{tabular}




\begin{tabular}{|c|c|c|c|c|}
\hline $\begin{array}{l}\text { Author, Year } \\
\text { Trial Name }\end{array}$ & $\begin{array}{l}\text { Age Gender } \\
\text { Race/Ethnicity }\end{array}$ & Other Population Characteristics & Total N & Outcome Measures \\
\hline \begin{tabular}{|l|} 
Malik 2009 and \\
Turkington 2002
\end{tabular} & $\begin{array}{l}\text { Not stratified by intervention group } \\
\text { Mean age } 40 \text { years } \\
23 \% \text { female } \\
\text { Race - } \\
89 \% \text { white } \\
8 \% \text { Black } \\
3 \% \text { other }\end{array}$ & $\begin{array}{l}\text { CBT vs usual care } \\
\text { Comprehensive Psychopathological Rating Scale } 23.27 \text { vs. } 24.30\end{array}$ & \begin{tabular}{|l}
$422(78 \%$ \\
{$[330 / 422]$} \\
included at \\
final followup)
\end{tabular} & $\begin{array}{l}\text { Relapse (need for hospitalization) } \\
\text { Function - } \\
\text { Occupational recovery (return to paid or } \\
\text { voluntary work or resumption of education } \\
\text { or training) }\end{array}$ \\
\hline Velligan 2015b & $\begin{array}{l}\text { CBT vs. usual care } \\
\text { Mean age } 30 \text { vs. } 40 \text { years } \\
54 \% \text { vs. } 46 \% \text { female } \\
\text { Race - } \\
54 \% \text { vs. } 49 \% \text { Hispanic } \\
24 \% \text { vs. } 24 \% \text { Black } \\
22 \% \text { vs. } 27 \% \text { white }\end{array}$ & $\begin{array}{l}\text { CBT vs. usual care } \\
\text { Mean MCAS: } 3.8 \text { (SD 0.3) vs. } 3.8 \text { (SD 0.4) }\end{array}$ & $\begin{array}{l}85 \text { (CBT vs. } \\
\text { Usual care; } \\
87 \%[74 / 85] \\
\text { included at } \\
\text { final followup) }\end{array}$ & $\begin{array}{l}\text { Function - } \\
\text { Community function (MCAS) }\end{array}$ \\
\hline Zimmer 2007 & $\begin{array}{l}\text { IPT vs. usual care } \\
\text { Mean age } 36 \text { vs. } 39 \text { years } \\
15 \% \text { vs. } 31 \% \text { female } \\
\text { Race not reported }\end{array}$ & \begin{tabular}{|l} 
IPT vS. Usual care \\
Age at onset: 21 vs. 22 years \\
Mean illness duration: 15 vs. 17 years \\
GAF mean score: 34.70 (SD 4.27) vs. 35.25 (SD 5.46) \\
SOFAS mean score: 34.20 (SD 5.31) vs. 35.81 (SD 5.56) \\
SAS mean score: 2.02 (SD 0.33) vs. 2.15 (SD 0.46) \\
WHOQOL-BREF mean score: 71.82 (SD 18.09) vs. 61.81 (SD 21.33)
\end{tabular} & $\begin{array}{l}66(85 \% \\
{[56 / 66]} \\
\text { included at } \\
\text { final followup) }\end{array}$ & $\begin{array}{l}\text { Function - } \\
\text { Overall function (GAF; SOFAS; SAS) } \\
\text { Quality of life (WHOQOL-BREF) }\end{array}$ \\
\hline
\end{tabular}




\begin{tabular}{|c|c|c|c|c|}
\hline $\begin{array}{l}\text { Author, Year } \\
\text { Trial Name }\end{array}$ & Benefits Outcomes & Harms Outcomes & Funding & $\begin{array}{l}\text { Quality } \\
\text { Rating }\end{array}$ \\
\hline $\begin{array}{l}\text { Malik } 2009 \text { and } \\
\text { Turkington } 2002\end{array}$ & $\begin{array}{l}\text { CBT vs. usual care } \\
\text { Proportion of patients with relapse: } 25 \%(64 / 257) \text { vs. } 35 \% \text { (57/165); RR } 0.72(95 \% \text { Cl } 0.53 \\
\text { to } 0.97) \\
\text { Mean time to relapse: } 356.8 \text { days (SD } 241.9) \text { vs. } 296.1 \text { days (SD } 215.7) ; p=0.03 \\
\text { Proportion of patients with occupational recovery: } 10 \%(21 / 205) \text { vs. } 14 \%(17 / 125) \text {; RR } 0.75 \\
(95 \% \mathrm{Cl} 0.41 \text { to } 1.37)\end{array}$ & Not reported & Pfizer UK & Fair \\
\hline Velligan 2015b & $\begin{array}{l}\text { CBT vs. usual care } \\
\text { Data not shown for CBT vs. usual care; narratively reported a nonsignificant treatment effect } \\
\text { on function with CBT using a regression model that included time and treatment by time } \\
\text { effects. No description of the effect of usual care was reported. }\end{array}$ & Not reported & $\mathrm{NIMH}$ & Fair \\
\hline Zimmer 2007 & $\begin{array}{l}\text { IPT vs. usual care } \\
\text { GAF mean score: } 39.50 \text { (SD 5.36) vs. } 33.81 \text { (SD 5.12); } p=0.00 \\
\text { SOFAS mean score: } 43.25 \text { (SD 6.54) vs. } 34.14 \text { (SD 4.53); } p=0.00 \\
\text { SAS mean score: } 1.86 \text { (SD 0.47) vs. } 2.27 \text { (SD 0.61); } p=0.04 \\
\text { WHOQOL-BREF mean score: } 39.15 \text { (SD 27.82) vs. } 35.63 \text { (SD 24.89); } p=0.03\end{array}$ & Not reported & \begin{tabular}{|l|} 
Conselho Nacional de \\
Desenvolvimento \\
Científico e Tecnológico \\
(CNPq); Coordenação de \\
Aperfeiçoamento \\
de Pessoal de Nível Superior \\
(CAPES); Instituto de \\
Cooperação Científica e \\
Tecnológica Internacional \\
(ICCTI); Fundação de Amparo \\
à Pesquisa do Estado do Rio \\
Grande do Sul (FAPERGS); \\
Brazilian Ministry of Education.
\end{tabular} & Fair \\
\hline
\end{tabular}

\section{Please see Appendix B. Included Studies for full study references}

BPRS=Brief Psychiatric Rating Scale, CAT=cognitive adaptation therapy, CBT=cognitive-behavioral therapy, CHOICE=Choice of Outcome in Cbt for psychosEs, CI=confidence interval, GAF=global assessment functioning, GPTS=Green Paranoid Thoughts Scale, IPT=integrated psychological therapy, IVIP=Indianapolis Vocational Intervention Program, MCAS=Multnomah Community Ability Scale, NS=not significant, PANSS=positive and negative syndrome scale, PSWQ=Penn State Worry Questionnaire, PSYRATS=Psychotic Symptom Rating Scales,

PTQ=Preservative Thinking Questionnaire, RR=relative risk, SAS=Social Adjustment Scale, SD=standard deviation, SE=side effects, SF-36=36 Item Short Form Health Survey, SOFAS=Social and Occupational Functioning Assessment Scale, UK=United Kingdom, VA=Veteran's Affairs, WBI=work behavior inventory, WEMWBS=Warwick-Edinburgh Mental Wellbeing Scale, WHOQOL=World Health Organization Quality of Life 
Appendix Table E-8. Data abstraction of systematic reviews of cognitive remediation therapy

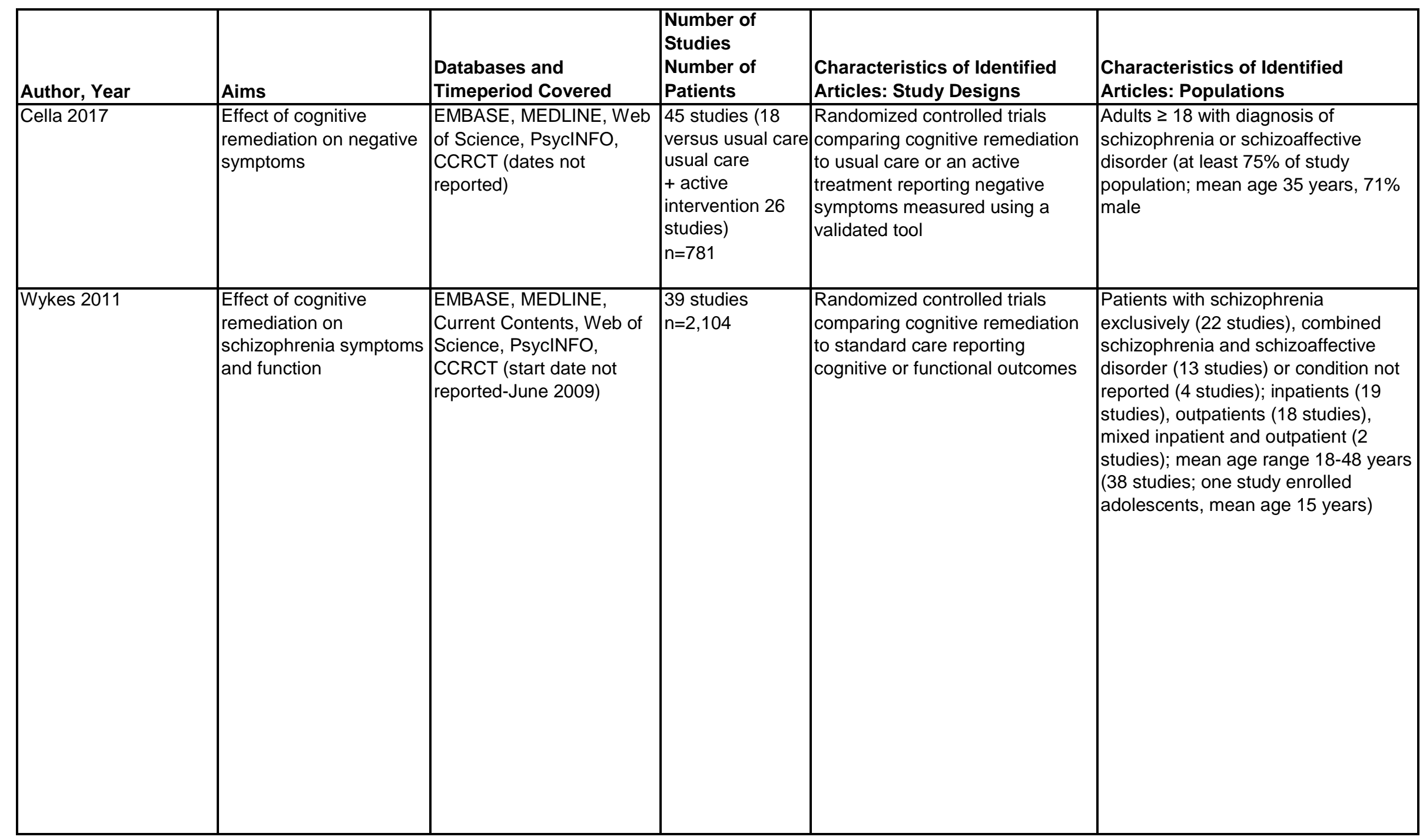




\begin{tabular}{|c|c|c|c|c|c|c|}
\hline Author, Year & \begin{tabular}{|l} 
Characteristics of \\
Identified Articles: \\
Interventions \\
\end{tabular} & Outcomes Reported & Effectiveness Outcomes & \begin{tabular}{|l} 
Harms \\
Outcomes \\
\end{tabular} & Funding & $\begin{array}{l}\text { Quality } \\
\text { Rating } \\
\end{array}$ \\
\hline Cella 2017 & $\begin{array}{l}\text { Cognitive remediation } \\
\text { versus usual care (18 } \\
\text { studies) or active } \\
\text { treatment ( } 26 \text { studies) }\end{array}$ & $\begin{array}{l}\text { Negative symptoms measured by } \\
\text { PANSS (34 studies), BPRS (6 studies), } \\
\text { SANS (5 studies) }\end{array}$ & $\begin{array}{l}\text { Cognitive remediation vs treatment } \\
\text { as usual } \\
\text { Negative symptoms: Random } \\
\text { effects - SMD -0.36 ( } 95 \% \mathrm{Cl}-0.52 \\
\text { to }-0.20) \text {; Fixed effects - SMD - } \\
0.36(95 \% \mathrm{Cl}-0.52 \text { to }-0.20)\end{array}$ & Not reported & None reported & Good \\
\hline Wykes 2011 & \begin{tabular}{|l|} 
Individual (31 studies) or \\
group (9 studies) cognitive \\
remediation (Cognitive \\
Remediation Experts \\
Workshop definition) vs. \\
standard care. \\
Treatment strategy drill and \\
practice (21 studies); drill + \\
strategy (19 studies) \\
Mean treatment time 32 \\
hours (range 4 to 130 \\
hours); mean treatment \\
duration 17 weeks (range 2 \\
to 104 weeks); mean \\
sessions/week 2.2 (range \\
0.6 to 5) \\
\end{tabular} & $\begin{array}{l}\text { Total symptoms (combined measure } \\
\text { including Scale for the Assessment of } \\
\text { Positive Symptoms; Scale for the } \\
\text { Assessment of Negative Symptoms; } \\
\text { Positive and Negative Syndrome Scale; } \\
\text { Brief Psychiatric Rating Scale; Present } \\
\text { State Exam; Paranoid Depression Scale; } \\
\text { Thought, language, and communication; } \\
\text { Holtzman Inkblot Test) Function } \\
\text { (combined measure including Bay Area } \\
\text { Functional Performance Evaluation; } \\
\text { Percent "sick talk"/ incoherence during } \\
\text { the interview; Life skills profile; Global } \\
\text { Assessment Scale; Nurses' Observation } \\
\text { Scale for Inpatient Evaluation; Disability } \\
\text { Assessment Schedule; Employment; } \\
\text { Social Behaviour Schedule; Micro- } \\
\text { Module Learning Test; Assessment of } \\
\text { Interpersonal Problem-Solving Skills; } \\
\text { Social adjustment) }\end{array}$ & \begin{tabular}{|l} 
Cognitive remediation vs. control, \\
effect size (mean) \\
Total symptoms, posttreatment ( 20 \\
studies): 0.177 ( $95 \% \mathrm{Cl} 0.034$ to \\
$0.321)$ \\
Function, posttreatment ( 12 \\
studies): 0.418 ( $95 \% \mathrm{Cl} 0.216$ to \\
$0.620)$ \\
Symptoms, posttreatment followup \\
(8 studies): 0.174 (95\% Cl -0.031 \\
to 0.481) \\
Function, posttreatment followup \\
(12 studies): 0.372 ( $95 \% \mathrm{Cl} 0.110$ \\
to 0.635$)$
\end{tabular} & \begin{tabular}{|l|} 
Not reported \\
\end{tabular} & \begin{tabular}{|l|} 
National \\
Institute for \\
Health \\
Research; \\
Maudsley \\
National Health \\
Service \\
Foundation; \\
Institute of \\
Psychiatry, \\
King's College \\
London
\end{tabular} & Good \\
\hline
\end{tabular}

Please see Appendix B. Included Studies for full study references

$\mathrm{CI}=$ confidence interval 
Appendix Table E-9. Data abstraction of randomized controlled trials of cognitive remediation therapy

\begin{tabular}{|c|c|c|c|c|c|}
\hline $\begin{array}{l}\text { Author, Year } \\
\text { Trial Name }\end{array}$ & \begin{tabular}{|l} 
Setting \\
Country
\end{tabular} & Inclusion Criteria & $\begin{array}{l}\text { Interventions } \\
\text { and Ns per } \\
\text { Group }\end{array}$ & Description of Intervention & Description of Comparator \\
\hline $\begin{array}{l}\text { Deste } 2015 \text { and Vita } \\
2011\end{array}$ & $\begin{array}{l}3 \text { centers } \\
\text { Italy }\end{array}$ & $\begin{array}{l}\text { Age 18-50 years; DSM-IV-TR diagnostic } \\
\text { criteria for schizophrenia; followed in the } \\
\text { rehabilitative centers of the Departments of } \\
\text { Mental Health of Brescia and Cremona } \\
\text { Hospitals (Italy). } \\
\text { Excluded: Concomitant diagnosis of mental } \\
\text { retardation or of substance use disorder; } \\
\text { severe positive symptoms or impulsive } \\
\text { behavior requiring a higher security setting; } \\
\text { significant changes in psychopathologic status } \\
\text { (requiring hospitalization or major change in } \\
\text { pharmacologic treatment) in the last } 3 \text { months. }\end{array}$ & Usual care: $\mathrm{n}=17$ & $\begin{array}{l}\text { CR: Twice weekly sessions for } 6 \text { months including one of } \\
\text { two CR modalities: (1) computerized CR including different } \\
\text { neurocognitive exercises that can be divided into domain- } \\
\text { specific exercises, aimed at training specific cognitive areas } \\
\text { among those known to be impaired in schizophrenia (verbal } \\
\text { memory, verbal fluency, psychomotor speed and } \\
\text { coordination, executive function, working memory, attention) } \\
\text { and nondomain-specific exercises; or (2) neurocognitive and } \\
\text { social cognitive remediation integrated with psychosocial } \\
\text { rehabilitation, made up of five modules, applied in the } \\
\text { following order: cognitive differentiation, social perception, } \\
\text { verbal communication, social skills, and interpersonal } \\
\text { problem solving }\end{array}$ & $\begin{array}{l}\text { Usual care: Noncognitive oriented } \\
\text { rehabilitation interventions, with the } \\
\text { same intensity and } \\
\text { duration of CR }\end{array}$ \\
\hline
\end{tabular}




\begin{tabular}{|c|c|c|c|c|c|}
\hline $\begin{array}{l}\text { Author, Year } \\
\text { Trial Name }\end{array}$ & $\begin{array}{l}\text { Duration } \\
\text { (intervention and } \\
\text { longest followup) }\end{array}$ & $\begin{array}{l}\text { Age Gender } \\
\text { Race/Ethnicity }\end{array}$ & Other Population Characteristics & Total N & Outcome Measures \\
\hline $\begin{array}{l}\text { Deste } 2015 \text { and Vita } \\
2011\end{array}$ & $\begin{array}{l}\text { Intervention: } 6 \\
\text { months } \\
\text { Followup: } 1 \text { year }\end{array}$ & \begin{tabular}{|l} 
Not stratified by \\
intervention group \\
Mean age 40 years \\
$37 \%$ female \\
Race not reported
\end{tabular} & $\begin{array}{l}\text { CR vS. usual care } \\
\text { Mean age at onset of illness: } 25 \text { years } \\
\text { Mean duration of illness: } 16 \text { years } \\
\text { Total PANSS: } 88.06 \text { (SD 18.26) vs. } 79.12 \text { (SD 19.84) } \\
\text { Negative PANSS: } 24.03 \text { (SD 8.55) vs. } 20.35 \text { (SD 7.88) }\end{array}$ & $\begin{array}{l}{\left[\begin{array}{l}\text { 54 (included at final } \\
\text { followup; original trial } \\
\text { [published separately] } \\
\text { included } 86 \text { patients) }\end{array}\right.} \\
\end{array}$ & \begin{tabular}{|l|} 
Symptoms - \\
Overall symptoms (PANSS) \\
Negative symptoms (PANSS)
\end{tabular} \\
\hline
\end{tabular}




\begin{tabular}{|c|c|c|c|c|}
\hline $\begin{array}{l}\text { Author, Year } \\
\text { Trial Name }\end{array}$ & Benefits Outcomes & Harms Outcomes & Funding & $\begin{array}{l}\text { Quality } \\
\text { Rating }\end{array}$ \\
\hline $\begin{array}{l}\text { Deste } 2015 \text { and Vita } \\
2011\end{array}$ & 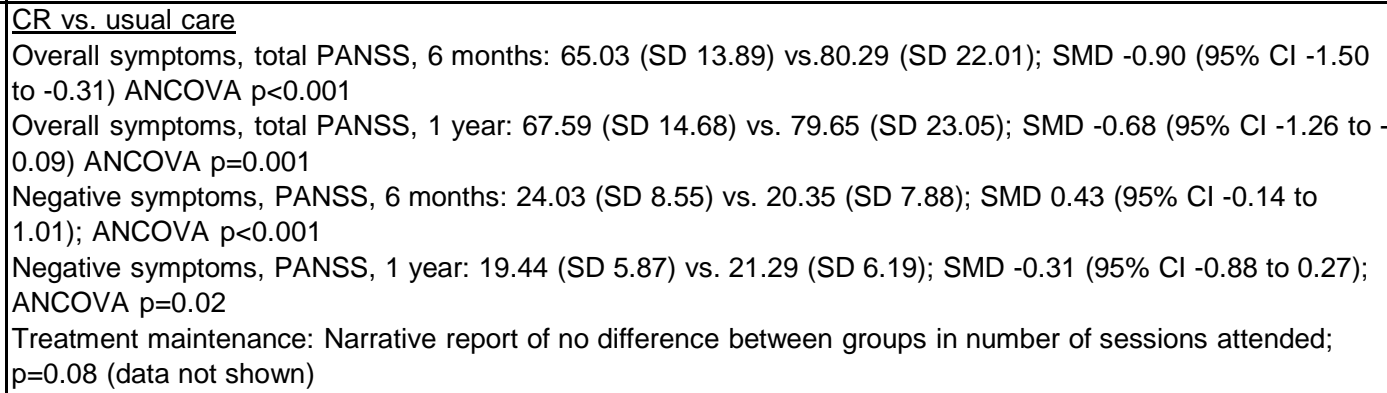 & Not reported & $\begin{array}{l}\text { Health } \\
\text { Authority of the } \\
\text { Lombardia } \\
\text { Region }\end{array}$ & Fair \\
\hline
\end{tabular}




\begin{tabular}{|c|c|c|c|c|c|}
\hline $\begin{array}{l}\text { Author, Year } \\
\text { Trial Name }\end{array}$ & \begin{tabular}{|l} 
Setting \\
Country
\end{tabular} & Inclusion Criteria & $\begin{array}{l}\text { Interventions } \\
\text { and Ns per } \\
\text { Group }\end{array}$ & Description of Intervention & Description of Comparator \\
\hline Farreny 2012 & $\begin{array}{l}\text { Single center } \\
\text { Spain }\end{array}$ & $\begin{array}{l}\text { Diagnosis of schizophrenia or schizoaffective } \\
\text { disorder and more than } 2 \text { years of illness } \\
\text { duration; finished primary studies or they were } \\
\text { able to successfully complete a reading } \\
\text { comprehension task used for } 13 \text {-year-old } \\
\text { students; Mini Mental State Examination score } \\
\text { of } 24 \text { or more and a Global Assessment of } \\
\text { Functioning score between } 40 \text { and } 70 . \\
\text { Excluded: Acute illness exacerbation; } \\
\text { intellectual disability or any neurological } \\
\text { disorder; participating in social skills training, } \\
\text { cognitive remediation, or any other } \\
\text { psychological intervention differing from usual } \\
\text { care; switch of antipsychotic drug the month } \\
\text { before the trial or during the } 40 \text { week study } \\
\text { period; and/or a diagnosis of alcohol or drug } \\
\text { dependence within } 6 \text { months prior to inclusion. }\end{array}$ & $\begin{array}{l}\text { CR: } n=34 \\
\text { Leisure control } \\
n=28\end{array}$ & $\begin{array}{l}\text { CR: Group meetings (4-6 participants), over } 4 \text { months twice } \\
\text { a week and consisting of } 32 \text { sessions lasting } 1 \text { hour each } \\
\text { focusing on problem solving and cognitive flexibility }\end{array}$ & $\begin{array}{l}\text { Control: participation in } 32 \\
\text { stimulating and socializing activities } \\
\text { (e.g., card games, board games, } \\
\text { "coffee \& talk", etc.). }\end{array}$ \\
\hline Mueller 2015 & $\begin{array}{l}8 \text { outpatient } \\
\text { centers } \\
\text { Switzerland, } \\
\text { Germany, and } \\
\text { Austria } \\
\\
\end{array}$ & $\begin{array}{l}\text { Age } 18-50 \text { years; diagnosis of Schizophrenia } \\
\text { or schizoaffective disorder; current enrollment } \\
\text { in outpatient treatment; duration of illness }>2 \\
\text { years; IQ }>80 \\
\text { Excluded: Neurological disorders, substance } \\
\text { dependence and/ } \\
\text { or abuse according to DSM-IV-TR or ICD-10 } \\
\text { within } 6 \text { months before baseline assessments; } \\
\text { hospitalization or changes in medication doses } \\
\text { within } 2 \text { months before baseline assessments. }\end{array}$ & Usual care: $\mathrm{n}=75$ & $\begin{array}{l}\text { CR: } 30 \text { manuallzed, biweekly, } 90-\text { minute group (6-8 } \\
\text { patients) sessions administered by a therapist and a co- } \\
\text { therapist of four therapy modules: (1) speed of processing } \\
\text { and attention and emotion processing; (2) verbal and visual } \\
\text { learning and memory, and social perception and theory of } \\
\text { mind; (3) reasoning and problem solving, and social } \\
\text { schema; and (4) working memory and social attributions } \\
\text { and emotion regulation. Modules progressively increase in } \\
\text { complexity and emotional strain. }\end{array}$ & $\begin{array}{l}\text { Usual care: Standard care including } \\
\text { a broad array of interventions used } \\
\text { in clinical practice for schizophrenia } \\
\text { patients (e.g., medication, individual } \\
\text { therapy, case management). }\end{array}$ \\
\hline
\end{tabular}




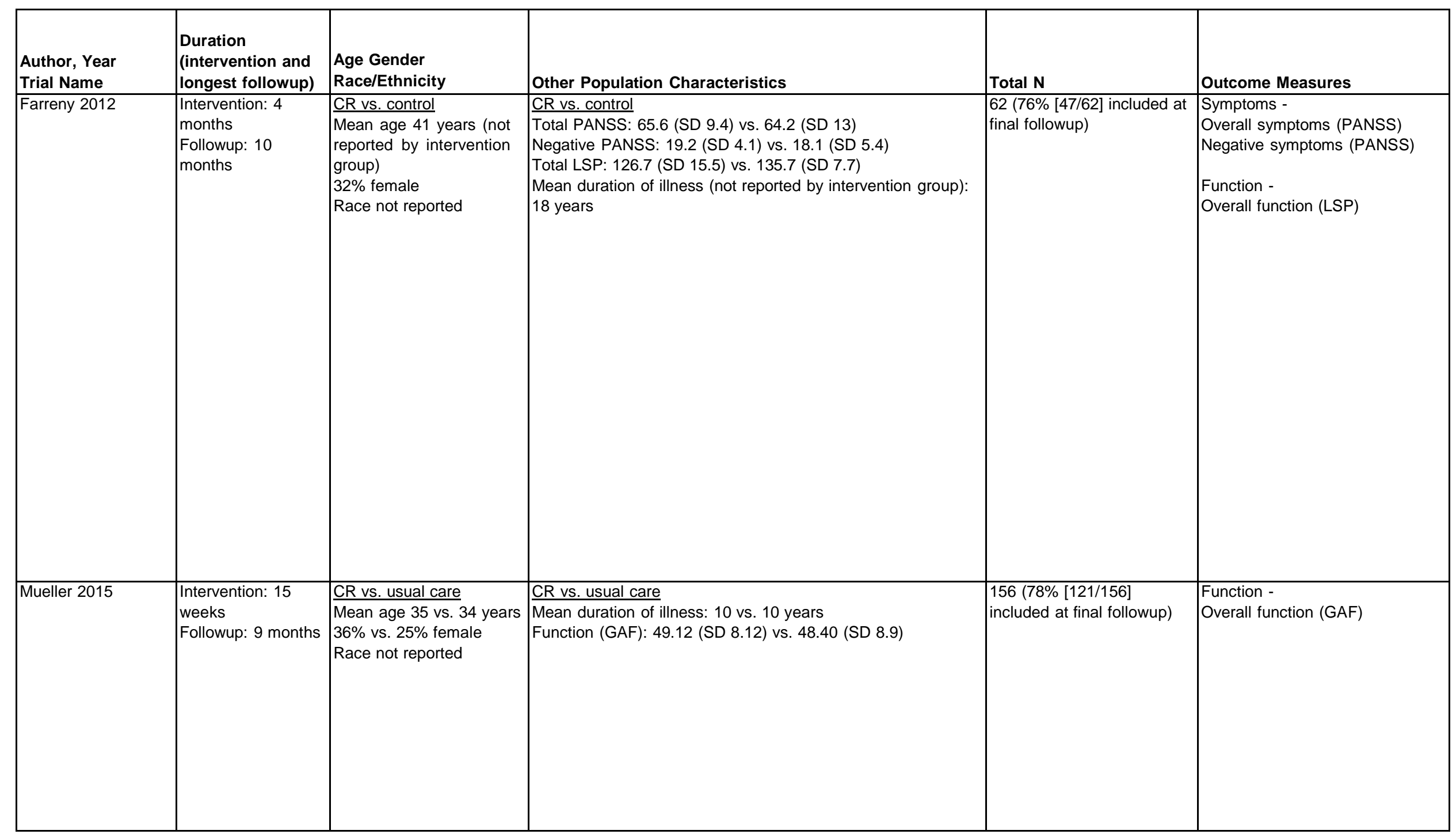




\begin{tabular}{|c|c|c|c|c|}
\hline $\begin{array}{l}\text { Author, Year } \\
\text { Trial Name }\end{array}$ & Benefits Outcomes & Harms Outcomes & Funding & $\begin{array}{l}\text { Quality } \\
\text { Rating }\end{array}$ \\
\hline Farreny 2012 & 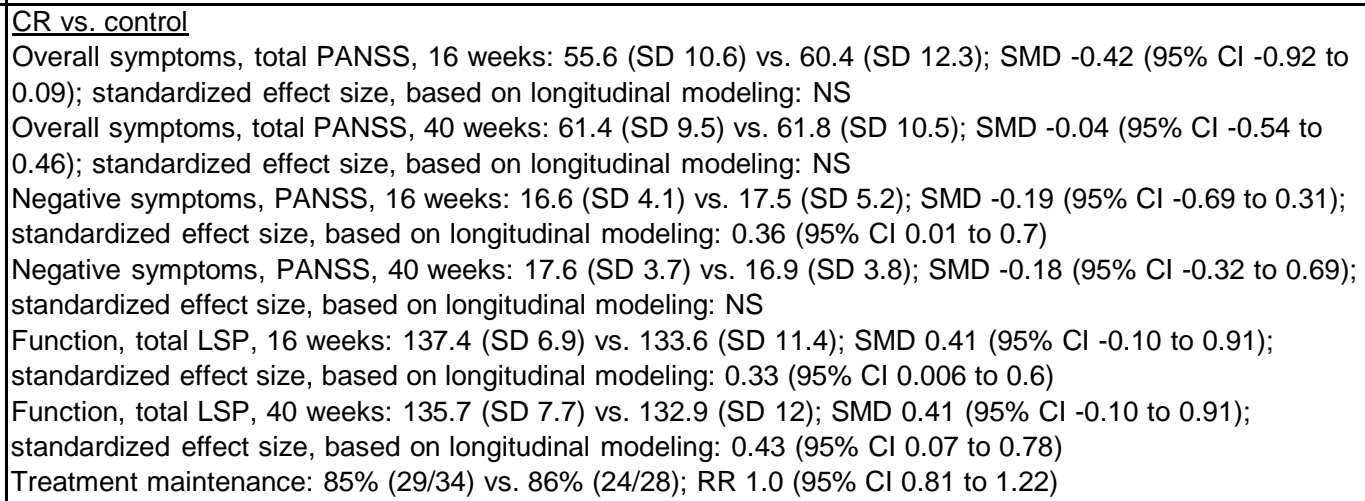 & Not reported & \begin{tabular}{|l} 
Fundació La \\
Caixa and \\
Instituto de \\
Salud Carlos III
\end{tabular} & Fair \\
\hline Mueller 2015 & $\begin{array}{l}\text { CR vS. usual care } \\
\text { Function, GAF: } 55.4 \text { (SD 7.3) VS. } 50.7 \text { (SD 9.4); P<0.01; SMD } 0.56(95 \% \text { Cl } 0.34 \text { to } 0.88) \\
\text { Treatment maintenance: } 90 \% \text { (73/81) vs. 89\% (67/75); RR } 1.01 \text { ( } 95 \% \mathrm{Cl} 0.91 \text { to } 1.12)\end{array}$ & | Not reported & $\begin{array}{l}\text { Swiss } \\
\text { National } \\
\text { Science } \\
\text { Foundation }\end{array}$ & Fair \\
\hline
\end{tabular}




\begin{tabular}{|c|c|c|c|c|c|}
\hline $\begin{array}{l}\text { Author, Year } \\
\text { Trial Name }\end{array}$ & $\begin{array}{l}\text { Setting } \\
\text { Country }\end{array}$ & Inclusion Criteria & $\begin{array}{l}\text { Interventions } \\
\text { and Ns per } \\
\text { Group }\end{array}$ & Description of Intervention & Description of Comparator \\
\hline Twamley 2012 & \begin{tabular}{|l|} 
Two centers \\
United States
\end{tabular} & $\begin{array}{l}\text { Age } \geq 18 \text { years with primary psychotic disorder } \\
\text { (including schizophrenia, schizoaffective } \\
\text { disorder, psychotic mood disorder, or } \\
\text { psychosis NOS; } 97 \% \text { enrolled population } \\
\text { diagnosed with schizophrenia or } \\
\text { schizoaffective disorder) and fluency in } \\
\text { English. } \\
\text { Excluded: Dementia, neurological conditions } \\
\text { affecting cognition, mental retardation, } \\
\text { substance use disorder within the past } \\
\text { month, and participation in other intervention } \\
\text { trials. }\end{array}$ & $\begin{array}{l}\text { CT: } n=38 \\
\text { Usual care: } n=31\end{array}$ & $\begin{array}{l}\text { CT: } 12 \text { weekly 2-hour sessions in groups of } 5 \text { patients and } 2 \\
\text { therapists that included a review of homework, } \\
\text { troubleshooting of strategy use, psychoeducation, and } \\
\text { rationale for the targeted domains (1) prospective memory; } \\
\text { (2) attention and vigilance; (3) learning and memory; and (4) } \\
\text { executive functioning, demonstration and practice of each } \\
\text { strategy, feedback on strategy use, and individualized } \\
\text { discussion regarding implementation of the strategies in } \\
\text { daily life. }\end{array}$ & $\begin{array}{l}\text { Usual care: Standard } \\
\text { pharmacotherapy }\end{array}$ \\
\hline
\end{tabular}




\begin{tabular}{|c|c|c|c|c|c|}
\hline $\begin{array}{l}\text { Author, Year } \\
\text { Trial Name }\end{array}$ & $\begin{array}{l}\text { Duration } \\
\text { (intervention and } \\
\text { longest followup) }\end{array}$ & $\begin{array}{l}\text { Age Gender } \\
\text { Race/Ethnicity }\end{array}$ & Other Population Characteristics & Total $\mathbf{N}$ & Outcome Measures \\
\hline Twamley 2012 & $\begin{array}{l}\text { Intervention: } 12 \\
\text { weeks } \\
\text { Followup: } 6 \text { months }\end{array}$ & \begin{tabular}{|l} 
CT vs. usual care \\
Mean age 38 vs. 31 years \\
$36 \%$ vs. $33 \%$ female \\
Race - \\
$63 \%$ vs. $55 \%$ white; other \\
races not reported
\end{tabular} & $\begin{array}{l}\text { CT vs. usual care } \\
\text { Negative symptoms, PANSS: } 15.66 \text { (SD 6.24) vs. } 14.23 \text { (SD } \\
4.90) \\
\text { Function, UPSA score: } 82.44 \text { (SD 9.90) vs. } 85.47 \text { (SD 8.42) } \\
\text { Quality of life, QOLI: } 4.16 \text { (SD 1.59) vs. } 4.43 \text { (SD 1.38) }\end{array}$ & $\begin{array}{l}69(74 \%[51 / 69] \text { included at } \\
\text { final followup) }\end{array}$ & $\begin{array}{l}\text { Symptoms - } \\
\text { Negative symptoms (PANSS) } \\
\text { Function - } \\
\text { Functional capacity (UPSA) } \\
\\
\text { Quality of life - } \\
\text { Self assessed quality of life } \\
\text { (QOLI) }\end{array}$ \\
\hline
\end{tabular}




\begin{tabular}{|c|c|c|c|c|}
\hline $\begin{array}{l}\text { Author, Year } \\
\text { Trial Name }\end{array}$ & Benefits Outcomes & Harms Outcomes & Funding & $\begin{array}{l}\text { Quality } \\
\text { Rating }\end{array}$ \\
\hline Twamley 2012 & 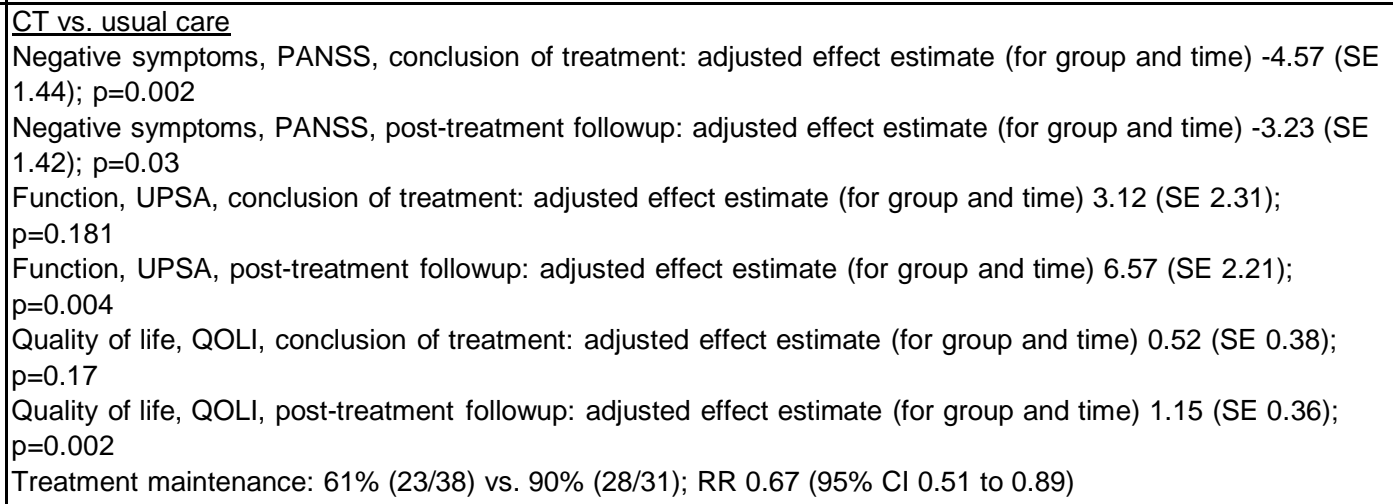 & Not reported & $\begin{array}{l}\text { National } \\
\text { Alliance for } \\
\text { Research on } \\
\text { Schizophrenia } \\
\text { and } \\
\text { Depression; } \\
\text { NIMH }\end{array}$ & Fair \\
\hline
\end{tabular}

Please see Appendix B. Included Studies for full study references

ANCOVA=analysis of covariance, $\mathrm{CI}=$ confidence interval, $\mathrm{CR}=$ cognitive remediation, $\mathrm{CT}=$ cognitive training, $\mathrm{GAF}=$ global assessment functioning, $\mathrm{LSP}=$ life skills profile, $\mathrm{NIMH}=\mathrm{National}$ Institute of Mental Health, PANSS=positive and negative syndrome scale, UPSA=UCSD Performance-based Skills Assessment, QOLI=quality of life interview, RR=relative risk, SD=standard deviation, $\mathrm{SE}=$ side effects, $\mathrm{SMD}=$ standard mean difference 
Appendix Table E-10. Data abstraction of systematic reviews of family interventions

\begin{tabular}{|c|c|c|c|c|c|c|c|}
\hline Author, Year & Aims & \begin{tabular}{|l} 
Databases and \\
Timeperiod \\
Covered \\
\end{tabular} & \begin{tabular}{|l} 
Number of \\
Studies \\
Number of \\
Patients \\
\end{tabular} & $\begin{array}{l}\text { Characteristics } \\
\text { of Identified } \\
\text { Articles: Study } \\
\text { Designs } \\
\end{array}$ & $\begin{array}{l}\text { Characteristics of } \\
\text { Identified Articles: } \\
\text { Populations }\end{array}$ & \begin{tabular}{|l|} 
Characteristics of \\
Identified Articles: \\
Interventions
\end{tabular} & Outcomes Reported \\
\hline Pharoah 2010 & \begin{tabular}{|l|} 
Effect of family \\
psychosocial \\
interventions in \\
community \\
settings for \\
people with \\
schizophrenia or \\
schizophrenia - \\
like conditions \\
compared with \\
standard care on \\
schizophrenia \\
outcomes
\end{tabular} & $\begin{array}{l}\text { Cochrane } \\
\text { Schizophrenia } \\
\text { Group Trials } \\
\text { Register (through } \\
\text { 2008) }\end{array}$ & $\begin{array}{l}53 \text { trials } \\
n=5767\end{array}$ & Trials & $\begin{array}{l}\text { Families of patients with } \\
\text { schizophrenia or } \\
\text { schizoaffective disorder } \\
25 / 53 \text { trials conducted in } \\
\text { China }\end{array}$ & \begin{tabular}{|l|} 
Any family-based \\
psychosocial \\
intervention for \\
schizophrenia with $>5$ \\
sessions (varied from \\
6 weeks to 3 years) \\
\\
\end{tabular} & $\begin{array}{l}\text { Primary outcomes: } \\
\text { Suicide and all sources of } \\
\text { mortality } \\
\text { Clinical global response } \\
\text { Relapse } \\
\text { Secondary outcomes: } \\
\text { Global state } \\
\text { Leaving the study early } \\
\text { Mental state and behavior } \\
\text { (positive and negative } \\
\text { symptoms) } \\
\text { Social functioning } \\
\text { Employment status } \\
\text { Work related activities } \\
\text { Independent living } \\
\text { Imprisonment } \\
\text { Family outcomes } \\
\text { QOL }\end{array}$ \\
\hline
\end{tabular}




\begin{tabular}{|c|c|}
\hline Author, Year & Effectiveness Outcomes \\
\hline Pharoah 2010 & 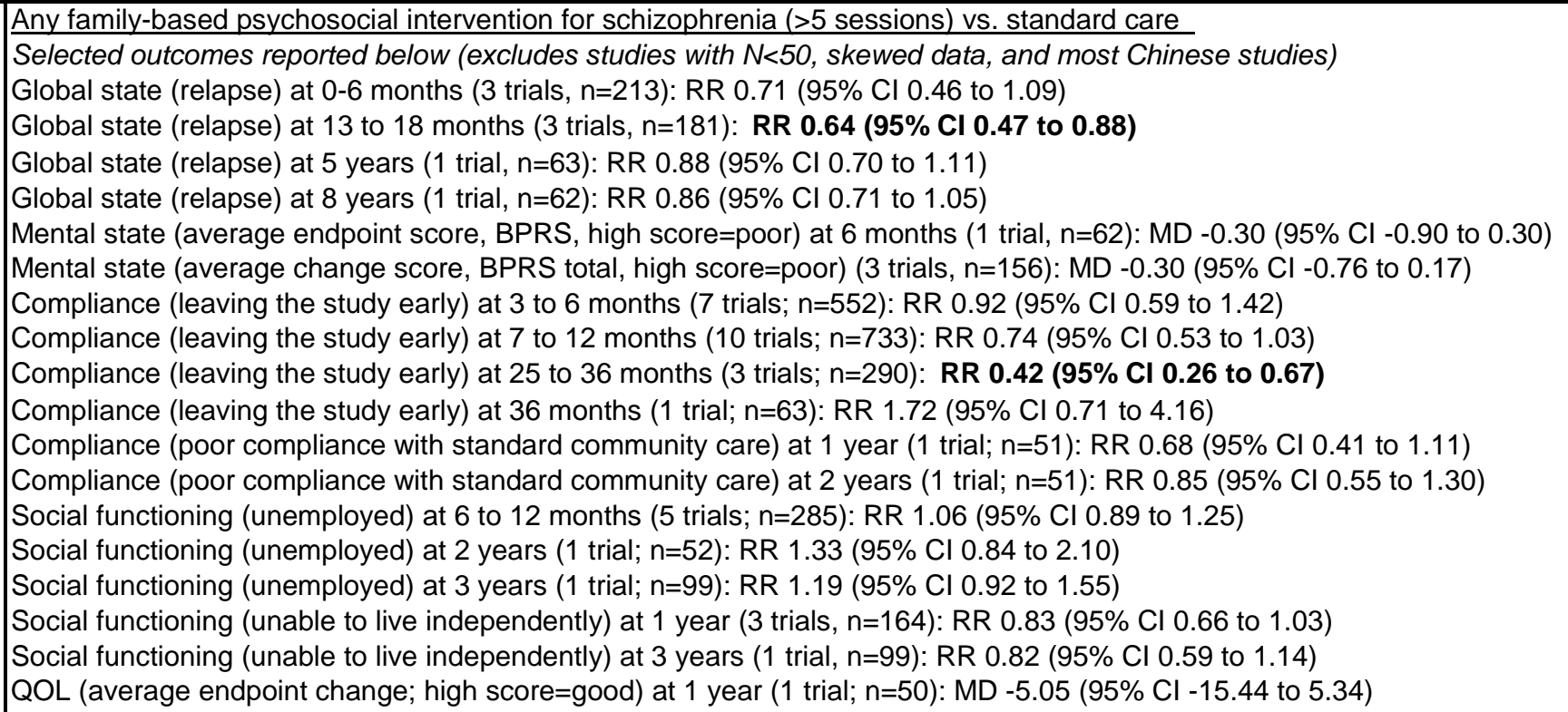 \\
\hline
\end{tabular}




\begin{tabular}{|c|c|c|c|}
\hline Author, Year & Harms Outcomes & $\begin{array}{l}\text { Fundingl } \\
\text { Comments }\end{array}$ & Quality Rating \\
\hline Pharoah 2010 & 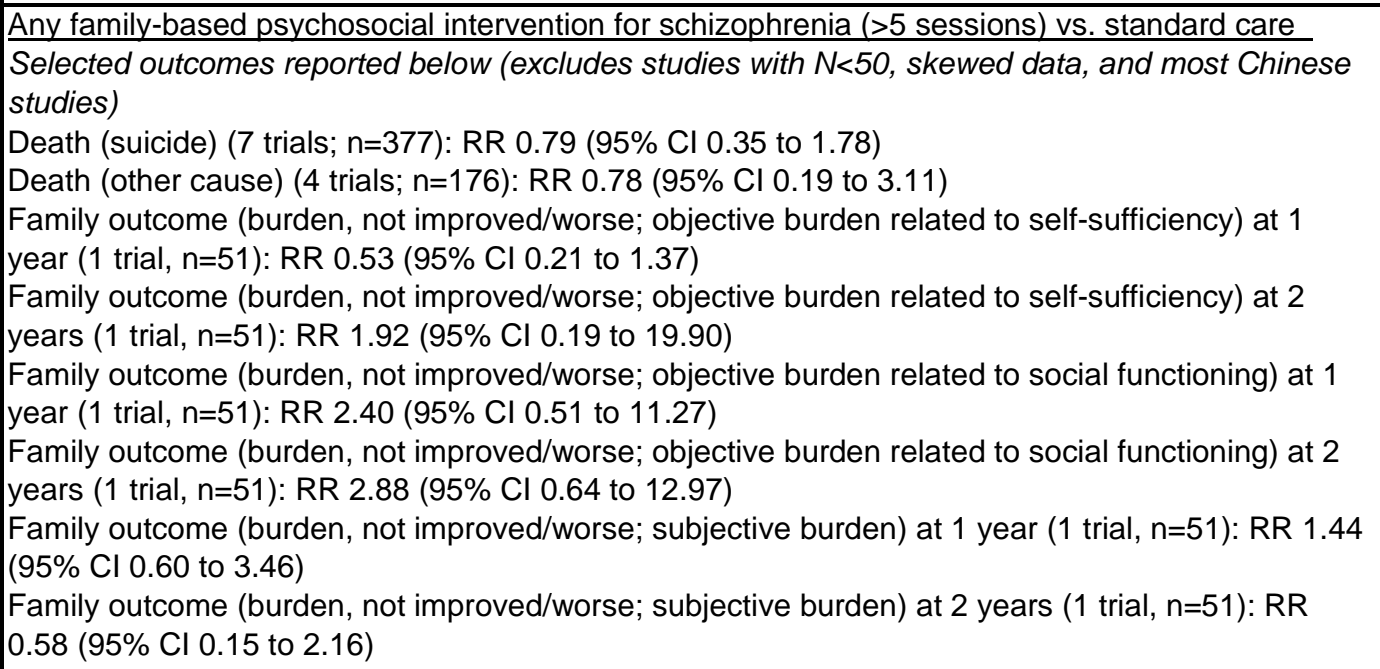 & $\begin{array}{l}\text { McMaster University, Ontario Canada } \\
\text { Universidade Federal de Sao Paulo, Brazil } \\
\text { Hinchingbrook Health Care, } \\
\text { Cambridgeshire, UK } \\
\text { International Clinical Epidemiology Network } \\
\text { (INCLEN), USA }\end{array}$ & Good \\
\hline
\end{tabular}

\section{Please see Appendix B. Included Studies for full study references}

$\mathrm{CI}=$ confidence interval, $\mathrm{MD}=$ mean difference, $\mathrm{QOL}=$ quality of life, $\mathrm{RR}=$ relative risk, UK=United Kingdom, US=United States of America 
Appendix Table E-11. Data abstraction of randomized controlled trials of family interventions

\begin{tabular}{|c|c|c|c|c|c|}
\hline Author, Year & $\begin{array}{l}\text { Setting } \\
\text { Country }\end{array}$ & Inclusion Criteria & $\begin{array}{l}\text { Interventions and } \\
\text { Ns per Group }\end{array}$ & Description of Intervention & Description of Comparator \\
\hline Dyck, 2000 & $\begin{array}{l}\text { Large } \\
\text { community } \\
\text { health center in } \\
\text { Spokane, } \\
\text { Washington }\end{array}$ & \begin{tabular}{|l} 
Diagnosis of schizophrenia or \\
schizoaffective disorder, 18-45 years of \\
age, enrollment in a community outpatient \\
facility in Spokane, residence with family \\
of origin or regular contact with family, \\
patients with either a history of substance \\
abuse or current substance abuse were \\
not excluded
\end{tabular} & $\begin{array}{l}\text { Multifamily group: } \\
\mathrm{n}=64 \\
\text { Standard care: } \mathrm{n}=31\end{array}$ & $\begin{array}{l}\text { Patients assigned to multiple-family group } \\
\text { treatment received standard care plus the } \\
\text { group treatment. Multiple-family group } \\
\text { treatment was intended to improve illness } \\
\text { management, social support, and coping } \\
\text { skills for the patient and family members. } \\
\text { The approach was based on the previous } \\
\text { research reported by McFarlane and } \\
\text { colleagues. Treatment interventions were } \\
\text { designed to educate the family and } \\
\text { patients about the biological } \\
\text { underpinnings of schizophrenia and } \\
\text { engage them in the treatment process by } \\
\text { using a standardized protocol of } \\
\text { videotapes, lecture, and written } \\
\text { guidelines. Treatment compoents } \\
\text { including ongoing support, formal clinical } \\
\text { problem solving, and expansion of social } \\
\text { support networks. }\end{array}$ & \begin{tabular}{|l|} 
Patients assigned to standard care \\
received usual services, including \\
medication management, case \\
management, and, for some patients, \\
therapeutic and rehabilitation \\
services. A treatment team consisting \\
of a case manager, a nurse, a \\
psychiatrist, and a social worker \\
delivered the mental health services. \\
The team provided clinical case \\
management services and out-of- \\
facility services as needed.
\end{tabular} \\
\hline Garety 2008 & $\begin{array}{l}\text { Multicenter trial } \\
\text { in UK }\end{array}$ & $\begin{array}{l}\text { Diagnosis of non-affective psychosis, age } \\
\text { 18-65, psychotic episode starting not } \\
\text { more than } 3 \text { months before entering trial, } \\
\text { rate of at least } 4 \text { on PANSS }\end{array}$ & $\begin{array}{l}\text { Family intervention: } \\
\mathrm{n}=28 \\
\text { Usual care: } 27\end{array}$ & $\begin{array}{l}\text { Family intervention emphasized } \\
\text { improving communication, offering } \\
\text { discussion of up-to-date information } \\
\text { about psychosis, problem-solving, } \\
\text { reducing criticism and conflict, } \\
\text { improving activity, and the emotional } \\
\text { processing of grief, loss and anger. } \\
\text { There was a particular focus on } \\
\text { relapse prevention, including how } \\
\text { family members might understand } \\
\text { warning signs and agree on } \\
\text { appropriated intervention, including } \\
\text { medication }\end{array}$ & Usual care \\
\hline
\end{tabular}




\begin{tabular}{|c|c|c|c|c|c|c|c|c|}
\hline Author, Year & Duration & $\begin{array}{l}\text { Age Gender } \\
\text { Race/Ethnicity }\end{array}$ & $\begin{array}{l}\text { Other Population } \\
\text { Characteristics }\end{array}$ & Total N & Benefits Outcomes & Harms Outcomes & Funding & $\begin{array}{l}\text { Quality } \\
\text { Rating }\end{array}$ \\
\hline Dyck, 2000 & 2 years & $\begin{array}{l}\text { MFG vs. Usual Care } \\
\text { Age, mean years: } 33 \text {, } \\
30 \\
\% \text { male: } 72,74 \\
\text { White, } \%: 94,97\end{array}$ & $\begin{array}{l}\text { MFG vs. Usual care } \\
\text { previous } \\
\text { Lifetime } \\
\text { hospitalizations, } \\
\text { mean: } 5,5 \\
\text { Comorbid SUD \%: } \\
45,50\end{array}$ & 63 & $\begin{array}{l}\text { MFG vs. Usual care: MSANS } \\
\text { baseline } 7.9,8.7 \text { Months 1-3: } \\
7.4,9.1 \\
\text { Months 4-6: 7.2, 8.9 Months } \\
7-9: 7.2,8.9 \text { Months 10-12: } \\
7.2,8.4\end{array}$ & Not reported & Grand NIMH & Poor \\
\hline Garety 2008 & 9 months & $\begin{array}{l}\text { Fl vs. usual care: } \\
\text { Age, mean, years: } \\
35,38.6 \\
\% \text { Male: } 71,68 \\
\% \text { White: } 86,82\end{array}$ & $\begin{array}{l}\text { FAl vs. usual care: } \\
\% \text { Employed: } 11, \\
14 \% \text { Unemployed: } \\
71,71\end{array}$ & \begin{tabular}{|l|}
$56+27$ in \\
CBT \\
group \\
which is \\
not \\
included
\end{tabular} & \begin{tabular}{|l|} 
FI vs. usual care: \\
Mean difference in change \\
scores: \\
Total PANSS at 12 months: - \\
6.44 (-14.12 to 1.24$)$ \\
Total PANSS at 24 months: \\
-6.25 (-14.77 to 2.28$)$ \\
Negative PANSS at 12 \\
months: -2.42 ( -5.18 to 0.35$)$ \\
Negative PANSS at 24 \\
months: -1.32 (-4.42 to 1.78$)$ \\
BAl at 12 months: \\
-0.42 (-6.97 to 6.13$)$ \\
BAl at 24 months: \\
$-2.36(-9.13$ to 4.40$)$ \\
BDI at 12 months: \\
3.35 (-2.46 to 9.34$)$ \\
BDI at 24 months: \\
$-0.11(-6.91$ to 6.68$)$ \\
EuroQol at 24 months: \\
-7.38 (-22.07 to 7.31$)$
\end{tabular} & \begin{tabular}{|l} 
Not reported \\
\end{tabular} & $\begin{array}{l}\text { Welcome } \\
\text { Trust } \\
\text { Programme } \\
\text { Grant }\end{array}$ & Good \\
\hline
\end{tabular}




\begin{tabular}{|c|c|c|c|c|c|}
\hline Author, Year & $\begin{array}{l}\text { Setting } \\
\text { Country }\end{array}$ & Inclusion Criteria & $\begin{array}{l}\text { Interventions and } \\
\text { Ns per Group }\end{array}$ & Description of Intervention & Description of Comparator \\
\hline $\begin{array}{l}\text { Kopeolwicz } \\
2012\end{array}$ & $\begin{array}{l}\text { Two community } \\
\text { mental health } \\
\text { centers in Los } \\
\text { Angeles, } \\
\text { California }\end{array}$ & \begin{tabular}{|l|} 
Diagnosis of schizophrenia or \\
schizoaffective disorder, $18-50$ years of \\
age, of Mexican origin and spoke \\
Spanish fluently, history of nonadherence \\
(had been without antipsychotic \\
medication without medical authorization \\
for 1 continuous week in the month prior \\
to study enrollment), lived with their \\
family of origin, had at least 1 family \\
member willing to participate in the family \\
treatment
\end{tabular} & $\begin{array}{l}\text { MFG-A, } n=64 \\
\text { MFG-S, } n=54 \\
\text { Usual care, } n=60\end{array}$ & $\begin{array}{l}\text { Culturally adapted, multifamily group } \\
\text { therapy based on McFarlane's model that } \\
\text { combines psychoeducation and skills } \\
\text { training. MFG-S consisted of } 3 \text { initial } \\
\text { "joining sessions" conducted separately } \\
\text { with each family, a } 6 \text { hour "survival skills" } \\
\text { educational workshop, and multifamily } \\
\text { group sessions. Modified therapy in the } \\
\text { MFG-A arm was to target improved } \\
\text { adherence using principles of the Theory } \\
\text { of Planned Behavior. MFG arms } \\
\text { convened twice monthly in } 90 \text { minute } \\
\text { sessions. }\end{array}$ & $\begin{array}{l}\text { All study participants received } \\
\text { treatment as usual. Rigid medication } \\
\text { protocols were not used. Patients } \\
\text { received all services as needed from } \\
\text { the Mental Health Department of Los } \\
\text { Angeles County. After inpatient } \\
\text { discharge, patients received a } \\
\text { psychiatric evaluation and } \\
\text { medication, and if clinically stable, } \\
\text { received monthly } 20 \text {-minute sessions. } \\
\text { If patients needed additional services } \\
\text { or rehospitalization, that was } \\
\text { accommodated. }\end{array}$ \\
\hline Mayoral, 2015 & $\begin{array}{l}\text { Four mental } \\
\text { health centers } \\
\text { Spain }\end{array}$ & $\begin{array}{l}\text { Age }>18 \text { years of age; confirmed } \\
\text { diagnosis of schizophrenia according to } \\
\text { the DSM-IV criteria; live with, at least, } \\
\text { one relative; understand and speak } \\
\text { Spanish; no admission within the } \\
6 \text { months prior to the beginning of the } \\
\text { study; treatment with antipsychotic drugs; } \\
\text { and capacity to sign an informed consent }\end{array}$ & \begin{tabular}{|l} 
Family group therapy \\
$(\mathrm{n}=44)$ \\
Treatment as usual \\
$(\mathrm{n}=44)$
\end{tabular} & \begin{tabular}{|l|}
24 weekly $60-$ minute sessions (in home \\
or at a health center) of practical and role- \\
playing exercises, with modules on \\
disease and treatment, assessment of \\
needs and family relations, \\
communication skills training, and \\
problem facing and solving
\end{tabular} & $\begin{array}{l}\text { Usual care in specialized mental } \\
\text { health centers }\end{array}$ \\
\hline
\end{tabular}




\begin{tabular}{|c|c|c|c|c|c|c|c|c|}
\hline Author, Year & Duration & $\begin{array}{l}\text { Age Gender } \\
\text { Race/Ethnicity }\end{array}$ & $\begin{array}{l}\text { Other Population } \\
\text { Characteristics }\end{array}$ & Total N & Benefits Outcomes & Harms Outcomes & Funding & $\begin{array}{l}\text { Quality } \\
\text { Rating }\end{array}$ \\
\hline $\begin{array}{l}\text { Kopeolwicz } \\
2012\end{array}$ & $\begin{array}{l}\text { Intervention and } \\
\text { followup: } 1 \text { year }\end{array}$ & $\begin{array}{l}\text { MFG-A vs. MFG-S vs. } \\
\text { usual care } \\
\text { Age, mean years: } 33, \\
30,33 \\
\% \text { male: } 67,68,61 \\
\text { Ethnicity Mexican } \\
\text { American, \%: } 100, \\
100,100\end{array}$ & \begin{tabular}{|l|} 
MFG-A vs. MFG-S \\
vs. usual care \\
$\%$ inpatient at entry: \\
$88,83,84$ \\
Age at onset, years: \\
$25,23,23$ \\
Lifetime \\
hospitalizations, \\
mean: $5.5,5.6,7.1$ \\
BPRS total score, \\
mean: $87.5,85.8$, \\
81.1
\end{tabular} & 178 & $\begin{array}{l}\text { BPRS: } \\
\text { No differences at baseline } \\
\text { among } 3 \text { groups, } p=0.18 \\
\text { No differences at } 12 \text { month } \\
\text { followup among } 3 \text { groups, } \\
p=0.32 \\
\text { All groups improved } \\
\text { significantly at } 12 \text { month } \\
\text { followup compared to } \\
\text { baseline, } p<0.001\end{array}$ & $\begin{array}{l}\text { Dropped out of treatment } \\
\text { immediately after undergoing } \\
\text { baseline assessments and } \\
\text { before engaging in outpatient } \\
\text { care: } 26 \% \text { ( } 45 / 174 \text { ) overall } \\
\text { Attrition (leaving treatment } \\
\text { before a } 12 \text {-month assessment } \\
\text { could be made): } \\
\text { MFG-A } 27 \% \text { vs. usual care } 51 \% \text {, } \\
\text { p=0.007 } \\
\text { MFG-S } 37 \% \text { vs. usual care } 51 \% \text {, } \\
\text { p=0.11 }\end{array}$ & $\begin{array}{l}\text { National } \\
\text { Institute of } \\
\text { Mental } \\
\text { Health }\end{array}$ & Poor \\
\hline Mayoral, 2015 & $\begin{array}{l}\text { Intervention: } 12 \\
\text { months } \\
\text { Followup: } 18 \\
\text { months }\end{array}$ & $\begin{array}{l}\text { Family therapy vs. } \\
\text { treatment as usual } \\
\text { Age, mean years: } 30 \\
\text { vs. } 30 \text { years } \\
\% \text { male: } 85 \% \text { vs. } 78 \% \\
\text { Ethnicity: NR }\end{array}$ & $\begin{array}{l}\text { Family therapy vs. } \\
\text { usual care } \\
\text { Previous } \\
\text { admissions, mean: } \\
2.83 \text { vs. } 2.17 \\
\text { Suicide attempts, } \\
\text { mean: } 1.3 \text { vs. } 3.0 \\
\text { BPRS total score, } \\
\text { mean: } 2.07 \text { vs. } 2.13\end{array}$ & 88 & $\begin{array}{l}\text { Family therapy vs. usual care } \\
\text { End of treatment (12 months) } \\
\text { BPRS total, mean: } 1.66 \text { vs. } \\
2.14 \text { ( } p=0.0046) \\
\text { Hospitalization: } 0 \% \text { vs. } 21 \% \\
\text { (8/38); RR } 0.06 \text { ( } 95 \% \mathrm{Cl} \\
0.004 \text { to } 1.04) \\
\text { Post-intervention followup (18 } \\
\text { months) } \\
\text { BPRS total, mean: } 1.70 \text { vs. } \\
2.05 \text { ( } p=0.44)\end{array}$ & NR & $\begin{array}{l}\text { Spain's } \\
\text { Ministry of } \\
\text { Health }\end{array}$ & Fair \\
\hline
\end{tabular}




\begin{tabular}{|c|c|c|c|c|c|}
\hline Author, Year & $\begin{array}{l}\text { Setting } \\
\text { Country }\end{array}$ & Inclusion Criteria & $\begin{array}{l}\text { Interventions and } \\
\text { Ns per Group }\end{array}$ & Description of Intervention & Description of Comparator \\
\hline $\begin{array}{l}\text { Sellwood } 2001 \\
\text { Sellwood } 2007 \\
\text { Barrowclough } \\
1999\end{array}$ & \begin{tabular}{|l|}
2 centers \\
United Kingdom
\end{tabular} & $\begin{array}{l}\text { ICD-10 diagnosis of schizophrenia, } \\
\text { schizoaffective disorder or delusional } \\
\text { disorder of at least } 2 \text { years' duration; at } \\
\text { least one relapse of psychotic symptoms } \\
\text { leading to in patient admission in the } 2 \\
\text { years preceding study entry and a } \\
\text { minimum duration of illness of } 2 \text { years; } \\
\text { aged between } 18 \text { and } 65 \text { years; at least } \\
10 \text { hours of face-to-face contact with a } \\
\text { career for each week over the previous } \\
\text { month. }\end{array}$ & $\begin{array}{l}\text { Family CBT: } \mathrm{n}=39 \\
\text { Standard care: } \mathrm{n}=38\end{array}$ & $\begin{array}{l}\text { Family CBT: } 10 \text { to } 20 \text { sessions over } 24 \\
\text { weeks aimed at delivering problem- } \\
\text { solving techniques, cognitive-behavioral } \\
\text { intervention for families, and cognitive- } \\
\text { behavioral interventions with patients to } \\
\text { reduce psychotic symptoms }\end{array}$ & $\begin{array}{l}\text { Standard care: Standard psychiatric } \\
\text { management by the clinical team, } \\
\text { maintenance neuroleptic medication, } \\
\text { monitoring through out-patient and } \\
\text { community followup and the care } \\
\text { programmed approach to case } \\
\text { management. }\end{array}$ \\
\hline
\end{tabular}




\begin{tabular}{|c|c|c|c|c|c|c|c|c|}
\hline Author, Year & Duration & $\begin{array}{l}\text { Age Gender } \\
\text { Race/Ethnicity }\end{array}$ & $\begin{array}{l}\text { Other Population } \\
\text { Characteristics }\end{array}$ & Total N & Benefits Outcomes & Harms Outcomes & Funding & $\begin{array}{l}\text { Quality } \\
\text { Rating }\end{array}$ \\
\hline $\begin{array}{l}\text { Sellwood } 2001 \\
\text { Sellwood } 2007 \\
\text { Barrowlough } \\
1999\end{array}$ & 12 months & \begin{tabular}{|l|} 
Data not stratified by \\
intervention group \\
Mean age 36 (SD 10) \\
years \\
$35 \%$ female \\
Race- \\
$85 \%$ White \\
$9 \%$ Black \\
$6 \%$ Southeast Asian
\end{tabular} & \begin{tabular}{|l} 
Family CBT vs. \\
standard care \\
Mean PANSS: \\
59.10 vs. 53.89 \\
Mean score, Social \\
Functioning scale: \\
99.61 vs. 101.12 \\
Mean score, Global \\
Assessment of \\
Function: 43.00 vs. \\
45.79
\end{tabular} & \begin{tabular}{|l}
$79(80 \%$ \\
{$[63 / 79]$} \\
included \\
at final \\
followup)
\end{tabular} & \begin{tabular}{|l|} 
Family CBT vs. standard \\
care \\
Overall symptoms, PANSS, \\
total score: $62.40(95 \% \mathrm{Cl}$ \\
57.10 to 67.70$)$ vs. 52.32 \\
$(47.92$ to 56.72$) ; \mathrm{p}=0.005 ;$ \\
mean change from baseline \\
1.08 (0.99 to 1.17$)$ vs. 0.98 \\
(0.89 to 1.06$) ; \mathrm{p}=0.09$ \\
Relapse: $16 \%(6 / 38)$ vs. \\
$49 \%(19 / 39)$ \\
Overall function, Social \\
Functioning scale: 102.93 \\
(SD 10.69$)$ vs. 101.03 (SD \\
11.04); p=NS; mean change \\
from baseline 1.29 vs. $2.42 ;$ \\
$p=N S$ \\
Overall function, GAF: 42.67 \\
(SD 10.88$)$ vs. 48.50 (SD \\
$8.81) ; p=0.02 ;$ mean change \\
from baseline 1.50 vs. $1.50 ;$ \\
p=NS
\end{tabular} & Not reported & $\begin{array}{l}\text { National } \\
\text { Health } \\
\text { Service } \\
\text { Tameside } \\
\text { and Glossop } \\
\text { Community } \\
\text { Priority Care } \\
\text { trust }\end{array}$ & Fair \\
\hline
\end{tabular}




\begin{tabular}{|c|c|c|c|c|c|}
\hline Author, Year & $\begin{array}{l}\text { Setting } \\
\text { Country }\end{array}$ & Inclusion Criteria & $\begin{array}{l}\text { Interventions and } \\
\text { Ns per Group }\end{array}$ & Description of Intervention & Description of Comparator \\
\hline Valencia, 2007 & $\begin{array}{l}\text { Single center } \\
\text { Mexico }\end{array}$ & $\begin{array}{l}\text { Outpatients age } 16 \text { to } 50 \text { with DSM-IV } \\
\text { schizophrenia; taking antipsychotic } \\
\text { medication; clinically stable in terms of } \\
\text { psychotic symptoms (PANSS score }<60 \text { ); } \\
\text { completed at least } 6 \text { years of elementary } \\
\text { education; lived with their families and } \\
\text { resided in Mexico City or the metropolitan } \\
\text { area }\end{array}$ & $\begin{array}{l}\text { SST: } n=49 \\
\text { Usual care: } n=49\end{array}$ & $\begin{array}{l}\text { SST: } 48 \text { weekly group sessions (75 } \\
\text { mins/session) composed of seven } \\
\text { sequential treatment areas (each area } \\
\text { includes a specific set of skills) as } \\
\text { follows: (1) symptom management; (2) } \\
\text { medication } \\
\text { management; (3) social relations; (4) } \\
\text { occupational; (5) money management; } \\
\text { (6) couple relations; and (7) family } \\
\text { relations } \\
\text { Additional component of } 8 \text { group and } 4 \\
\text { individual family therapy sessions for } \\
\text { relatives }\end{array}$ & $\begin{array}{l}\text { Usual care: Monthly appointments } \\
\text { (20 mins/session) with clinical } \\
\text { psychiatrist who } \\
\text { controlled the prescription } \\
\text { of their AP medication based upon } \\
\text { the assessment } \\
\text { of their psychotic symptoms, } \\
\text { checked their medication } \\
\text { compliance, recorded their } \\
\text { consultation attendance. }\end{array}$ \\
\hline
\end{tabular}




\begin{tabular}{|c|c|c|c|c|c|c|c|c|}
\hline Author, Year & Duration & $\begin{array}{l}\text { Age Gender } \\
\text { Race/Ethnicity }\end{array}$ & $\begin{array}{l}\text { Other Population } \\
\text { Characteristics }\end{array}$ & Total N & Benefits Outcomes & Harms Outcomes & Funding & $\begin{array}{l}\text { Quality } \\
\text { Rating }\end{array}$ \\
\hline Valencia, 2007 & 1 year & $\begin{array}{l}\text { SST vs usual care } \\
\text { Mean age } 30 \text { vs. } 30 \\
\text { years } \\
30 \% \text { vs. } 15 \% \text { female } \\
\text { Race not reported }\end{array}$ & \begin{tabular}{|l|} 
SST vs. usual care \\
Mean age at onset \\
of illness 21 vs. 21 \\
years \\
Mean duration of \\
illness: 9 vs 9 years \\
Total PANSS: 115.2 \\
(SD 30.5) vs. 107.9 \\
(SD 22.6) \\
Negative PANSS: \\
29.7 (SD 8.5) vs. \\
28.7 (SD 6.3)
\end{tabular} & 98 & 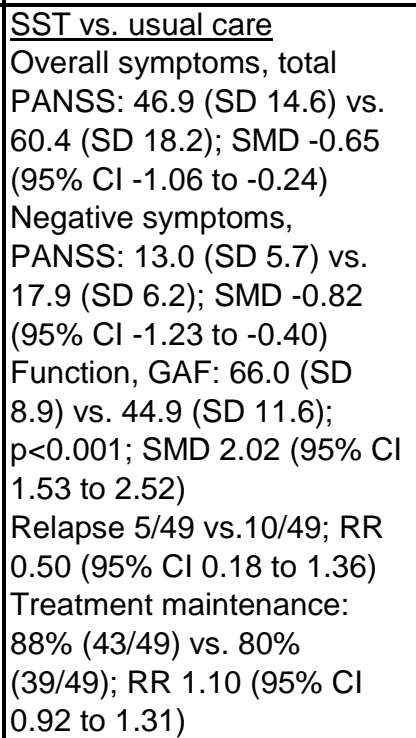 & Not reported & $\begin{array}{l}\text { National } \\
\text { Institute of } \\
\text { Psychiatry } \\
\text { Ramón de la } \\
\text { Fuente; } \\
\text { National } \\
\text { Council on } \\
\text { Science and } \\
\text { Technology }\end{array}$ & Fair \\
\hline
\end{tabular}

\section{Please see Appendix $B$. Included Studies for full study references}

$\mathrm{CI}=$ confidence interval, $\mathrm{MD}=$ mean difference, MFG-A=multifamily group therapy-adherence, MFG-S=multifamily group therapy-standard, $\mathrm{SD}=$ standard deviation 
Appendix Table E-12. Descriptions of family interventions in randomized controlled trials with relapse as an outcome

\begin{tabular}{|c|c|c|}
\hline Study, Year & $\begin{array}{c}\text { N Intervention } \\
\text { N Control }\end{array}$ & Description of Intervention \\
\hline $\begin{array}{l}\text { Barrowclough, 1999, } \\
\text { Sellwood 2001, } \\
2007\end{array}$ & $\begin{array}{l}38 \\
39\end{array}$ & $\begin{array}{l}\text { The planned intervention period was } 24 \text { weeks; sessions took place in the caregiver's homes. All patients in the study were } \\
\text { allocated a family support worker from the volunteer organization Making Space. The services of this support worker included } \\
\text { providing information, giving advice on benefits, advocacy, emotional support, and practical help. The frequency and nature of } \\
\text { contact with the support worker was decided by mutual agreement between caregiver and support worker. The integrated } \\
\text { treatment program attempted to combine three treatment approaches: motivational interviewing, individual cognitive behavior } \\
\text { therapy, and family or caregiver intervention. Patients and carers in the treatment group were offered specific psychosocial } \\
\text { interventions. The focus, content and quantity of the interventions were determined by a systematic assessment of caregiver } \\
\text { needs for psychosocial interventions, measured using the relatives' version of the Cardinal Needs Schedule. Three broad types } \\
\text { of interventions are differentiated: problem-solving techniques; cognitive-behavioral interventions; and individual cognitive } \\
\text { behavioral interventions with patients with psychosis. }\end{array}$ \\
\hline Barrowclough, 2001 & $\begin{array}{l}18 \\
18\end{array}$ & $\begin{array}{l}\text { The planned intervention period was } 9 \text { months; sessions took place in the caregivers' and patients' homes, except when } \\
\text { patients or caregivers expressed a preference for a clinic-based appointment (one individual in the integrated care group } \\
\text { expressed this preference). All patients in the study were allocated a family support worker from the volunteer organization } \\
\text { Making Space. The services of this support worker included providing information, giving advice on benefits, advocacy, } \\
\text { emotional support, and practical help. The frequency and nature of contact with the support worker was decided by mutual } \\
\text { agreement between caregiver and support worker. The integrated treatment program attempted to combine three treatment } \\
\text { approaches: motivational interviewing, individual cognitive behavior therapy, and family or caregiver intervention. }\end{array}$ \\
\hline Bradley, 2006 & $\begin{array}{l}25 \\
25\end{array}$ & $\begin{array}{l}\text { The multiple-family-group procedure was followed with minimal variation. Consumers and caregivers were provided up to three } \\
\text { single-family joining sessions (described below) and then invited to attend two half-day multiple-family psychoeducation } \\
\text { sessions. The family psychoeducation sessions provided information about schizophrenia using the approach described by } \\
\text { Anderson and colleagues. The sessions gave family members the opportunity for informal social networking. Topics included } \\
\text { the nature of the illness, treatment approaches (medication and psychosocial), consumer and family needs, common family } \\
\text { reactions to illness, common problems that consumers and families face, and guidelines about what the family can do to help. } \\
\text { The education was provided to the families by psychiatrists, psychologists, social workers, and occupational therapists. Each } \\
\text { group of six or seven consumer-caregiver pairs was then invited to participate in a multiple-family group with two trained group } \\
\text { leaders; groups met every other week for } 12 \text { months. }\end{array}$ \\
\hline
\end{tabular}




\begin{tabular}{|c|c|c|c|}
\hline Study, Year & Description of Control & $\begin{array}{l}\text { Treatment Duration and Number } \\
\text { of Sessions }\end{array}$ & Target or Primary Outcome \\
\hline $\begin{array}{l}\text { Barrowclough, 1999, } \\
\text { Sellwood 2001, } \\
2007\end{array}$ & Family support worker & $10-20$ sessions over 24 weeks & Relapse \\
\hline Barrowclough, 2001 & $\begin{array}{l}\text { Routine care in the context of the National Health Service of Great Britain } \\
\text { consists of psychiatric management by the clinical team, coordinated through } \\
\text { case management and including maintenance neuroleptic medication, monitoring } \\
\text { through outpatient and community follow-up, and access to community based } \\
\text { rehabilitative activities, such as day centers and drop-in clinics. All of the patients } \\
\text { in the integrated treatment program also received routine care. }\end{array}$ & 29 sessions over 9 months & Global function \\
\hline Bradley, 2006 & $\begin{array}{l}\text { The case management intervention that was provided to all participants and that } \\
\text { constituted the control condition consisted of regular appointments with a case } \\
\text { manager and doctor to assess mental health and to provide medication and } \\
\text { individual psychosocial rehabilitation on the basis of consumers' needs. } \\
\text { Appointment frequency was every } 2 \text { to } 3 \text { weeks on average, and the sessions } \\
\text { lasted from } 30 \text { minutes to } 1 \text { hour. Family contact was provided on an individual } \\
\text { basis as required for all participants in the control and treatment groups. Family } \\
\text { contact consisted of phone or direct contact and focused on providing } \\
\text { psychoeducation, monitoring the consumer's mental state, and giving general } \\
\text { support. Case management for Vietnamese participants in the control group was } \\
\text { provided by a Vietnamese bilingual case manager when possible or with the use of } \\
\text { Vietnamese interpreters. }\end{array}$ & 26 sessions over 12 months & $\begin{array}{l}\text { Relapse, clinical and social } \\
\text { function }\end{array}$ \\
\hline
\end{tabular}




\begin{tabular}{|c|c|c|}
\hline Study, Year & $\begin{array}{c}\text { N Intervention } \\
\mathrm{N} \text { Control }\end{array}$ & Description of Intervention \\
\hline Buchkremer, 1995 & $\begin{array}{l}67 \\
32\end{array}$ & $\begin{array}{l}\text { The relatives' groups met every } 2 \text { weeks and were guided by an experienced psychiatrist/psychologist. They started with a } \\
\text { contact phase (one meeting), followed by psychoeducational training which covered the provision of information on the illness } \\
\text { and treatment plus training in symptom assessment. It comprised two phases: an information phase (two to three meetings) and } \\
\text { a problem-solving phase (about seven meetings). The problem-solving skills were aimed at imparting general competence in } \\
\text { problem solving to make it possible to develop strategies for coping with difficult situations, irrespective of any current problem. } \\
\text { In the last phase (after } 10 \text { meetings), topic-centered personal therapy of the relative was emphasized, but psychoeducation was } \\
\text { continued if requested by the relatives. }\end{array}$ \\
\hline Carra, 2007 & $\begin{array}{l}26 \\
25\end{array}$ & $\begin{array}{l}\text { Weekly meetings with an information group composed of } 16-18 \text { relatives for } 24 \text { sessions ( } 1.75 \text { hours per session) using an } \\
\text { informative approach. Contents and goals are mainly derived from the model of the relatives group (Leff, 1989) but the } \\
\text { preliminary in-home individual family sessions. Curricula include: etiology, positive symptoms, negative symptoms, mood } \\
\text { disorders, problem behaviors, medical and psychiatric treatment, denial and non-compliance, interpersonal and social issues, } \\
\text { relationship with family, education, independence and dependence, resources and benefits. Educational tools include lectures, } \\
\text { videos and leaflets. The second element comprises weekly meetings for } 48 \text { sessions (1.5 hours per session) over } 2 \text { years with a } \\
\text { support group made up of } 8-9 \text { relatives who have previously attend the information group. The first phase involves training on } \\
\text { communication and coping skills, stress identification and management, and multiple family group-based problem solving, } \\
\text { basically derived from the second stage of the psychoeducational multiple family group approach. This usually occurs during the } \\
\text { first year. The second phase emphasized mutual support and consists of deliberate efforts to mould the group into a social } \\
\text { network than can persist for an extended period and satisfy family needs for social contact, support, and ongoing monitoring. } \\
\text { Expansion of the families' social networks occurs through problem solving, direct emotional support, and out-of-group } \\
\text { socializing, all involving members of different families in the group }\end{array}$ \\
\hline Dyck, 2002 & $\begin{array}{l}55 \\
51\end{array}$ & $\begin{array}{l}\text { Patients assigned to multiple-family group treatment received standard care plus the group treatment. Because the clinicians } \\
\text { who provided the group treatment typically were not the case managers for the patients in the group, it was necessary to ensure } \\
\text { that they communicated regularly with the case managers about changes in patients' functional status, medication problems, or } \\
\text { service needs. Multiple-family group treatment was intended to improve illness management, social support, and coping skills } \\
\text { for the patient and family members. The approach was based on the previous research reported by McFarlane and colleagues. } \\
\text { Treatment interventions were designed to educate the family and patients about the biological underpinnings of schizophrenia } \\
\text { and engage them in the treatment process by using a standardized protocol of videotapes, lecture, and written guidelines. } \\
\text { Treatment components including ongoing support, formal clinical problem solving, and expansion of social support networks. }\end{array}$ \\
\hline
\end{tabular}




\begin{tabular}{|c|c|c|c|}
\hline Study, Year & Description of Control & $\begin{array}{l}\text { Treatment Duration and Number } \\
\text { of Sessions }\end{array}$ & Target or Primary Outcome \\
\hline Buchkremer, 1995 & $\begin{array}{l}\text { 2-year group pending therapy. Relatives' groups were then implemented (although } \\
\text { for only } 8 \text { sessions). }\end{array}$ & $\begin{array}{l}26 \text { biweekly meetings over } 1 \text { year } \\
\text { with an additional } 2 \text { year followup }\end{array}$ & Hospitalization \\
\hline Carra, 2007 & Usual care & 72 weekly sessions over $>2$ years & $\begin{array}{l}\text { Hospitalization, relapse, } \\
\text { compliance with community } \\
\text { mental health care, } \\
\text { employment }\end{array}$ \\
\hline Dyck, 2002 & \begin{tabular}{|l|} 
Patients assigned to standard care received usual services, including medication \\
management, case management, and, for some patients, therapeutic and \\
rehabilitation services. A treatment team consisting of a case manager, a nurse, a \\
psychiatrist, and a social worker delivered the mental health services. The team \\
provided clinical case management services and out-of-facility services as needed.
\end{tabular} & Weekly sessions over 2 years & Hospitalization \\
\hline
\end{tabular}




\begin{tabular}{|c|c|c|}
\hline Study, Year & $\begin{array}{c}\text { N Intervention } \\
\text { N Control }\end{array}$ & Description of Intervention \\
\hline $\begin{array}{l}\text { Falloon, } 1981 \\
\text { (1982) }\end{array}$ & $\begin{array}{l}20 \\
19\end{array}$ & $\begin{array}{l}\text { The family-management approach recognizes that effective community after-care of schizophrenia requires both optimal drug } \\
\text { therapy and a supportive milieu. The family-treatment approach was designed to train patients and their parents to reduce } \\
\text { environmental stress effectively. All family-therapy sessions were conducted in the home. This served to enhance generalization } \\
\text { of learning to family life and to minimize failure to keep appointments. The first two session were devoted to educating the } \\
\text { patient and family about the nature, course, and treatment of schizophrenia. Schizophrenia was presented as a major mental } \\
\text { illness with both biologic and psychosocial components. The notion that families somehow "cause" schizophrenia was refuted, } \\
\text { but it was pointed out that families can play an important part in improving the course of the illness. Considerable attention was } \\
\text { given o discussing the rationale for maintenance of neuroleptic medication. Subsequent family sessions were devoted to } \\
\text { reducing existing family tensions and improving the problem solving skills of the family in coping with the causes of stress. The } \\
\text { strength and weakness of the family group were pinpointed, and major deficits became the focus of subsequent sessions. } \\
\text { Specifically, behavioral reversal, modeling, feedback, and social reinforcement were used to enhance skills in the expression of } \\
\text { positive and negative feelings, reflective listening, requests for behavioral change, and reciprocity of conversation. Each family } \\
\text { was taught a structured problem-solving method in which it was encouraged to convene a family meeting whenever an issue } \\
\text { arose, on order to discuss and specify the exact nature of the problem, list and consider alternative solutions, and select and } \\
\text { implement the consensual "best" solution. In most families the therapist merely assisted the family in its structured problem- } \\
\text { solving efforts, but if patients had persisting symptoms of schizophrenia or major discord was observed, additional specific } \\
\text { strategies were employed. These included methods to improve marital relationships, to deal with unacceptable behavior, and to } \\
\text { expand the social contacts of any family member. }\end{array}$ \\
\hline Garety, 2008 & $\begin{array}{l}28 \\
28\end{array}$ & $\begin{array}{l}\text { Family intervention emphasized improving communication, offering discussion of up-to-date information about psychosis, } \\
\text { problem-solving, reducing criticism and conflict, improving activity, and the emotional processing of grief, loss and anger. There } \\
\text { was a particular focus on relapse prevention, including how family member might understand warning signs and agree on } \\
\text { appropriated intervention, including medication }\end{array}$ \\
\hline Glynn, 1992 & $\begin{array}{l}21 \\
20\end{array}$ & $\begin{array}{l}\text { Behavioral family therapy provided patients and their families with education about schizophrenia, communication skills, and } \\
\text { problem-solving training to improve the family's ability to cope with stress. These three components were provided sequentially. } \\
\text { Behavioral family therapy techniques include instruction, role reversal, modeling, social reinforcement, and homework tasks. } \\
\text { The study protocol called for } 25 \text { behavioral family therapy sessions to be held with families over a } 12 \text {-month period on a } \\
\text { declining contact basis. Overall, a mean of } 21 \text { behavioral family therapy sessions were actually held. }\end{array}$ \\
\hline Goldstein, 1978 & $\begin{array}{l}52 \\
52\end{array}$ & $\begin{array}{l}\text { A crisis-oriented six-session family therapy was devised, directed at the following sequence of objectives: (1) the patient and } \\
\text { his family are able to accept the fact that he has had a psychosis; (2) they are willing to identify some of the probable } \\
\text { precipitating stresses in his life at the time the psychosis occurred; (3) they attempt to generalize from that to identification of } \\
\text { future stresses to which the patient and his family are likely to be vulnerable; and (4) they attempt to do some planning on how } \\
\text { to minimize or avoid these future stresses. }\end{array}$ \\
\hline
\end{tabular}




\begin{tabular}{|c|c|c|c|}
\hline Study, Year & Description of Control & $\begin{array}{l}\text { Treatment Duration and Number } \\
\text { of Sessions }\end{array}$ & Target or Primary Outcome \\
\hline $\begin{array}{l}\text { Falloon, } 1981 \\
(1982)\end{array}$ & $\begin{array}{l}\text { The comparison treatment was clinic-base, individual supportive psychotherapy. } \\
\text { It was our intention to provide individual treatment comparable to the best } \\
\text { available at well-staffed community after-care clinics. In addition to receiving } \\
\text { maintenance pharmacotherapy and rehabilitation counseling, individually treated } \\
\text { patients were educated about the nature, course, and treatment of schizophrenia } \\
\text { and assisted in their efforts to cope with problems of everyday living. Although the } \\
\text { issues addressed in treatment were similar to those that arose in the family } \\
\text { treated group, the problems were death with primarily from the patient's } \\
\text { perspective. }\end{array}$ & $\begin{array}{l}\text { Weekly visits for } 3 \text { months the } \\
\text { biweekly visits for } 6 \text { months then } \\
\text { monthly visits thereafter evaluated } \\
\text { at } 9 \text { months }\end{array}$ & Stress management \\
\hline Garety, 2008 & Usual care & 20 sessions over 9 months & Relapse, remission \\
\hline Glynn, 1992 & $\begin{array}{l}\text { Customary care services to all subjects were provided by a special Veteran's } \\
\text { Health Administration outpatient clinic treatment team consisting of } 4 \text { psychiatrists, } \\
2 \text { social workers and } 1 \text { clinical nurse specialist. All members of the team were blind } \\
\text { to treatment assignment. This team provided monthly clinical evaluation and } \\
\text { medication management, vocational and rehabilitation referrals, and crisis } \\
\text { intervention services. Outpatient services available included training in social and } \\
\text { independent living skills, and a variety of recreational and occupational therapy } \\
\text { groups and vocation rehabilitation services. }\end{array}$ & 25 sessions over 12 months & Relapse, work adjustment \\
\hline Goldstein, 1978 & No therapy & 6 weeks & Relapse \\
\hline
\end{tabular}




\begin{tabular}{|c|c|c|}
\hline Study, Year & $\begin{array}{c}\text { N Intervention } \\
\text { N Control }\end{array}$ & Description of Intervention \\
\hline Herz, 2000 & $\begin{array}{l}41 \\
41\end{array}$ & $\begin{array}{l}\text { Program for relapse prevention: (1) education for patients and family members about the process of relapse in schizophrenia } \\
\text { and how to recognize prodromal symptoms and behaviors; (2) active monitoring for prodromal symptoms by treatment team } \\
\text { members, patients, family members and others in frequent contact with patient; (3) clinical intervention within } 24-48 \text { hours of } \\
\text { prodromal episode (4) 1-hour weekly supportive group therapy emphasizing improving coping skills or individual supportive } \\
\text { therapy sessions if patients refused group treatment and (5) 90-minute multifamily psychoeducation groups that family } \\
\text { members were encouraged to attend biweekly for } 6 \text { months and monthly thereafter. }\end{array}$ \\
\hline Hogarty, 1986 & $\begin{array}{l}30 \\
45\end{array}$ & $\begin{array}{l}\text { Our family approach was designed as an education and management strategy intended to lower the emotional climate of the } \\
\text { home while main training reasonable expectations for patient performance. As frequently indicated to us by many relatives, this } \\
\text { strategy should not be formally designated as "family therapy." Rather, through the provision of formal education about the } \\
\text { disorder and strategies for managing more effectively, family members become allies in the treatment process as their anxiety } \\
\text { and distress are decreased. More traditional attempts to promote disclosure, "insight," or direct modification of family systems, } \\
\text { including the resolution of intergenerational and marital issues, were, for the most part, avoided. For ease of communication, we } \\
\text { refer to the process as family treatment. The goal was to reduce both the positive and negative symptoms of schizophrenia that } \\
\text { might be associated with the extremes of stimulation contained in either the therapeutic process or family life. Treatment sought } \\
\text { to increase the stability and predictability of family life by decreasing the family's guilt and anxiety, increasing their self- } \\
\text { confidence, and providing a sense of cognitive mastery through the provision of information concerning the nature and course of } \\
\text { schizophrenia as well as specific management strategies thought to be helpful in coping with schizophrenic symptoms on a day- } \\
\text { to-day basis. }\end{array}$ \\
\hline Hogarty, 1997 & $\begin{array}{l}24 \\
24\end{array}$ & $\begin{array}{l}\text { Family psychoeducation/management. Family therapy was provided by the other two full-time master's-level psychiatric nurse } \\
\text { clinical specialists and by one part-time master's-level psychologist. These included the three broad phases of joining, survival } \\
\text { skills training and reintegration within the home, and reintegration into the community. The principal modification to the family } \\
\text { therapy approach was a change in didactic content that reflected issues of importance to the families of first-episode patients, } \\
\text { such as diagnostic uncertainty and variable prognosis. ( } 27 \% \text { [Number=26] of patients who lived with family in trial } 1 \text { were first- } \\
\text { episode patients.) }\end{array}$ \\
\hline
\end{tabular}




\begin{tabular}{|c|c|c|c|}
\hline Study, Year & Description of Control & $\begin{array}{l}\text { Treatment Duration and Number } \\
\text { of Sessions }\end{array}$ & Target or Primary Outcome \\
\hline Herz, 2000 & Individual supportive therapy and medication management & $\begin{array}{l}6 \text { months biweekly } \\
\text { psychoeducation and monthly } \\
\text { thereafter, weekly group therapy } \\
\text { evaluated at } 18 \text { months }\end{array}$ & Relapse \\
\hline Hogarty, 1986 & Drug-maintained control group & Biweekly then monthly for 2 years & Relapse \\
\hline Hogarty, 1997 & Supportive therapy & $1-2$ visits per month for 3 years & Patient adjustment \\
\hline
\end{tabular}




\begin{tabular}{|c|c|c|}
\hline Study, Year & $\begin{array}{l}\text { N Intervention } \\
\text { N Control }\end{array}$ & Description of Intervention \\
\hline Kopelowicz, 2012 & $\begin{array}{l}64 \\
54 \\
60\end{array}$ & 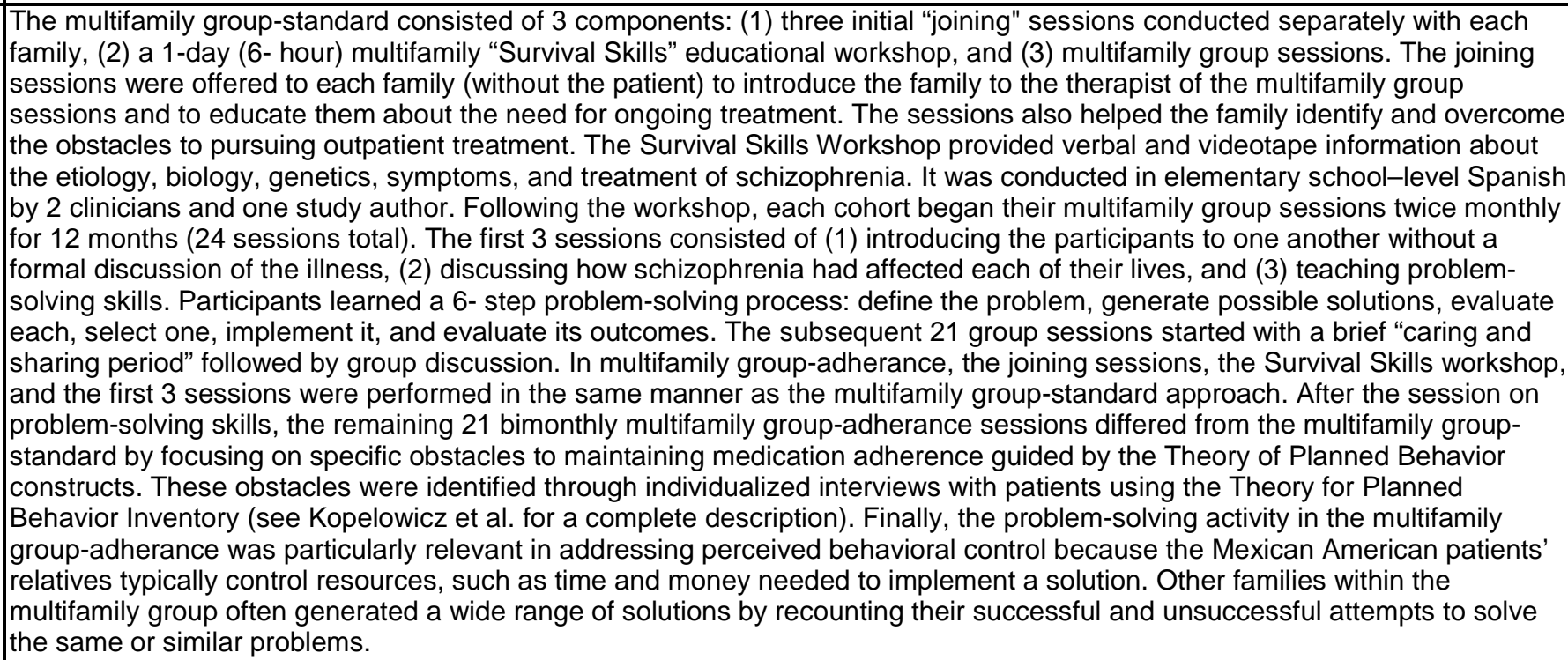 \\
\hline Leff, 1982 & $\begin{array}{l}12 \\
12\end{array}$ & $\begin{array}{l}\text { The package of social interventions. The education program: This consisted of four lectures on the etiology, symptoms, course } \\
\text { and treatment and management of schizophrenia. Initially four visits were made, one for each topic, but after a few relatives had } \\
\text { been instructed in this way we decided it would be preferable to give two lectures at a time. Following each lecture, we allowed } \\
\text { unlimited time for the relative to ask questions. The relatives' group: the group was deliberately set up so that the therapists } \\
\text { acted as facilitators. Both high expressed emotion and low expressed emotion relatives were encouraged to bring their } \\
\text { problems and their solutions to the meeting and share them with others in a similar position. The purpose of this was to enable } \\
\text { them to learn about coping strategies of which they were unaware, and finally to help them try a different approach at home. The } \\
\text { focus of the group was thus on potential or actual difficulties that relatives experienced, and not primarily on interpretations of } \\
\text { the relatives' own behavior. This latter was more useful in discussions between the professionals about the group process that } \\
\text { occurred after each group meeting. Family sessions: Because the relatives' group was not appropriate for dealing with the } \\
\text { whole range of problems or for dynamic work, and because patients were excluded from it, we felt that it needed to be } \\
\text { complemented by sessions with the whole family. }\end{array}$ \\
\hline Leff, 2001 & $\begin{array}{l}16 \\
14\end{array}$ & $\begin{array}{l}\text { Two sessions of education about schizophrenia plus techniques for improving communication within the family, reducing } \\
\text { relatives' criticism and over-involvement, lowering contact between patient and high expressed emotion relatives, increasing } \\
\text { the social networks of family members and setting realistic objectives. The approach includes cognitive and behavioral } \\
\text { elements as well as techniques from strategic and systemic family therapy }\end{array}$ \\
\hline Linszen, 1996 & $\begin{array}{l}37 \\
39\end{array}$ & $\begin{array}{l}\text { Behavioral family intervention including psychoeducation, communication training and the development of problem solving } \\
\text { skills were the main components }\end{array}$ \\
\hline
\end{tabular}




\begin{tabular}{|c|c|c|c|}
\hline Study, Year & Description of Control & $\begin{array}{l}\text { Treatment Duration and Number } \\
\text { of Sessions }\end{array}$ & Target or Primary Outcome \\
\hline Kopelowicz, 2012 & Usual care & 24 sessions over 12 months & $\begin{array}{l}\text { Medication adherence, } \\
\text { hospitalization }\end{array}$ \\
\hline Leff, 1982 & Routine outpatient care & $\begin{array}{l}4 \text { sessions education + biweekly } \\
\text { relatives group for } 9 \text { months }+1 \text { to } \\
25 \text { family sessions }\end{array}$ & Relapse \\
\hline Leff, 2001 & 2 education sessions & $\begin{array}{l}\text { Bi weekly then monthly sessions } \\
\text { over one year }\end{array}$ & Relapse \\
\hline Linszen, 1996 & Psychosocial intervention & 18 sessions over 12 months & Relapse \\
\hline
\end{tabular}




\begin{tabular}{|c|c|c|}
\hline Study, Year & $\begin{array}{c}\text { N Intervention } \\
\mathrm{N} \text { Control }\end{array}$ & Description of Intervention \\
\hline Mayoral, 2015 & $\begin{array}{l}44 \\
44\end{array}$ & $\begin{array}{l}\text { The famly psychoeducation intervention carried out for the group subject to treatment consisted of } 24 \text { sessions, which involved, } \\
\text { at least, the patient and a key relative, apart from other direct relatives who wanted to participate in the sessions. Sessions } \\
\text { lasted approximatedly } 60 \text { minutes and were distributed into weekly sessions during the first quarter, fortnightly sessions during } \\
\text { the } 3 \text { subsequent months and monthsly sessions during the remaining } 6 \text { months. The total intervention period lasted } 12 \text { months } \\
\text { The content of the treatment programme included } 4 \text { modules whose objectives were the following: basic information about the } \\
\text { disease and its treatment; assessment of needs and family relations; training on communication skills; and problem facing and } \\
\text { solving }\end{array}$ \\
\hline Merinder, 1991 & $\begin{array}{l}23 \\
23\end{array}$ & $\begin{array}{l}\text { An 8-session intervention using a mainly didactic interactive method and focusing on the following headings: } \\
\text { 1. Introduction } \\
\text { 2. What is schizophrenia? Diagnosis, prognosis, symptoms } \\
\text { 3. What causes schizophrenia? } \\
\text { 4. Medication: effect and side effects } \\
\text { 5. Psychosocial treatment } \\
\text { 6. Stress and early signs of relapse, emergency plan } \\
\text { 7. What can you and your family do about it? } \\
\text { 8. Laws and regulations } \\
\text { The programme was standardized with a manual for group leaders, overhead presentations and a booklet for participants, to } \\
\text { increase comparability of the intervention between centers. Further, teachers had regular meetings with the aim of increasing } \\
\text { the commitment to the intervention protocol. Patient and relative interventions were conducted separately, with group sizes in } \\
\text { both patient and relative groups of five to eight participants. The programme was the same for both patients and relatives. } \\
\text { Sessions were weekly. }\end{array}$ \\
\hline Tarrier, 1988 & $\begin{array}{l}31 \\
32 \\
\end{array}$ & Education program (2 sessions), stress management (3 sessions), goal setting (8 sessions) \\
\hline Valencia, 2007 & $\begin{array}{l}43 \\
39\end{array}$ & $\begin{array}{l}\text { Psychosocial skills training focusing on (1) symptom management, (2) medication management, (3) social relations, (4) } \\
\text { occupational management, (5) money management, (6) couple relations, (7) family relations (48 sessions); family therapy } \\
\text { consisted of psychoeducation (8 sessions) and problem solving (4 sessions) }\end{array}$ \\
\hline Vaughan, 1992 & $\begin{array}{l}18 \\
18\end{array}$ & $\begin{array}{l}\text { Relatives' counseling. Therapists attempted to (1) form an alliance with relatives (2) increase stability and predictably of family } \\
\text { life by decreasing family guilt and anxiety, increasing self-confidence and providing a sense of mastery through providing } \\
\text { information about schizophrenia. In addition, an attempt was made to improve the relatives' problems solving and } \\
\text { communication skills. }\end{array}$ \\
\hline
\end{tabular}




\begin{tabular}{|c|c|c|c|}
\hline Study, Year & Description of Control & $\begin{array}{l}\text { Treatment Duration and Number } \\
\text { of Sessions }\end{array}$ & Target or Primary Outcome \\
\hline Mayoral, 2015 & Normal standard treatment & 24 sessions over 12 months & Hospitalization \\
\hline Merinder, 1991 & $\begin{array}{l}\text { The usual treatment provided in community psychiatry, i.e., } \\
\text { psychopharmacological treatment, psychosocial rehabilitation efforts and to some } \\
\text { extent supportive psychotherapy }\end{array}$ & 8-sessons & $\begin{array}{l}\text { Relapse, compliance, } \\
\text { knowledge of schizophrenia, } \\
\text { satisfaction with services }\end{array}$ \\
\hline Tarrier, 1988 & Routine care without specialist intervention & 13 sessions over 9 months & Relapse \\
\hline Valencia, 2007 & Usual care & $\begin{array}{l}48 \text { weekly sessions for } \\
\text { psychosocial skills training and } 12 \\
\text { sessions for family therapy }\end{array}$ & $\begin{array}{l}\text { Relapse, hospitalization, } \\
\text { positive and negative } \\
\text { symptom, psychosocial and } \\
\text { global functioning, treatment } \\
\text { adherence }\end{array}$ \\
\hline Vaughan, 1992 & $\begin{array}{l}\text { Standard after-care which consisted of outpatient appointments every } 2 \text { to } 4 \\
\text { weeks for medication and support }\end{array}$ & 10 weekly sessions & Relapse \\
\hline
\end{tabular}

Please see Appendix B. Included Studies for full study references 
Appendix Table E-13. Data abstraction of systematic reviews of intensive case management

\begin{tabular}{|c|c|c|c|c|c|c|}
\hline Author, Year & Aims & $\begin{array}{l}\text { Databases and } \\
\text { Timeperiod Covered }\end{array}$ & $\begin{array}{l}\text { Number of } \\
\text { Studies } \\
\text { Number of } \\
\text { Patients }\end{array}$ & $\begin{array}{l}\text { Characteristics of Identified } \\
\text { Articles: Study Designs }\end{array}$ & $\begin{array}{l}\text { Characteristics of } \\
\text { Identified Articles: } \\
\text { Populations }\end{array}$ & $\begin{array}{l}\text { Characteristics of } \\
\text { Identified Articles: } \\
\text { Interventions }\end{array}$ \\
\hline $\begin{array}{l}\text { Marshall 2000b } \\
\text { (ICM) }\end{array}$ & $\begin{array}{l}\text { Effect of ICM for people } \\
\text { with severe mental } \\
\text { disorders }\end{array}$ & $\begin{array}{l}\text { MEDLINE (1966-1995); } \\
\text { CINAHL (1997); } \\
\text { EMBASE (1980-1995); } \\
\text { PsycLIT (1974-1995); } \\
\text { SCISEARCH (1997); } \\
\text { Cochrane Schizophrenia } \\
\text { Group Register of Trials } \\
\text { (1997) }\end{array}$ & $\begin{array}{l}10 \text { studies } \\
n=1652\end{array}$ & $\begin{array}{l}\text { Randomized controlled trials } \\
\text { of ICM vs. control }\end{array}$ & $\begin{array}{l}\text { Age 18-65 years with } \\
\text { schizophrenia or } \\
\text { schizophrenia-like } \\
\text { disorders; bipolar } \\
\text { disorder; or depression } \\
\text { with psychotic features. } \\
\text { Proportion with } \\
\text { schizophrenia: <50\%: } 2 \\
\text { studies; >50\%: } 5 \text { studies; } \\
\text { unknown: } 2 \text { studies }\end{array}$ & $\begin{array}{l}\text { ICM: Any intervention } \\
\text { described as case } \\
\text { management within a } \\
\text { study; excluded: } \\
\text { Assertive Community } \\
\text { Treatment and Home- } \\
\text { based Care }\end{array}$ \\
\hline
\end{tabular}




\begin{tabular}{|c|c|c|c|c|c|}
\hline Author, Year & Outcomes Reported & Effectiveness Outcomes & Harms Outcomes & $\begin{array}{l}\text { Funding/ } \\
\text { Comments }\end{array}$ & $\begin{array}{l}\text { Quality } \\
\text { Rating }\end{array}$ \\
\hline $\begin{array}{l}\text { Marshall 2000b } \\
\text { (ICM) }\end{array}$ & $\begin{array}{l}\text { Symptoms: BPRS } \\
\text { Function: scales not reported } \\
\text { Quality of life: Quality of Life } \\
\text { interview } \\
\text { Mortality }\end{array}$ & $\begin{array}{l}\text { ICM vS. control } \\
\text { Symptoms, BPRS ( } 2 \text { studies): WMD } 0.46(95 \% \mathrm{Cl}-3.67 \text { to } 4.60) \\
\text { Social function, scales used not reported (3 studies): SMD } 0.46(95 \% \mathrm{Cl} \text { - } \\
0.34 \text { to } 1.26) \\
\text { Quality of life, Quality of Life Interview (2 studies): SMD } 0.09(95 \% \mathrm{Cl} \text { - } \\
0.23 \text { to } 0.42)\end{array}$ & $\begin{array}{l}\text { ICM vs. control } \\
\text { Mortality }(6 \text { studies }): \\
\text { OR } 1.29(95 \% \mathrm{Cl} \\
0.68 \text { to } 2.45 \\
\left.\text { I }^{2}=59 \%\right)\end{array}$ & \begin{tabular}{|l} 
Manchester \\
University \\
Department of \\
Psychiatry; \\
Oxford \\
University \\
Department of \\
Socio-Legal \\
Studies
\end{tabular} & Good \\
\hline
\end{tabular}

Please see Appendix B. Included Studies for full study references

BPRS=Brief Psychiatric Rating Scale, CI=confidence interval, ICM=Intensive Case Management, SMD=standard mean difference, WMD=weighted mean difference 
Appendix Table E-14. Data abstraction of randomized controlled trials of intensive case management

\begin{tabular}{|c|c|c|c|c|c|c|}
\hline Author, Year & $\begin{array}{l}\text { Setting } \\
\text { Country }\end{array}$ & Inclusion Criteria & $\begin{array}{l}\text { Interventions } \\
\text { and Ns per } \\
\text { Group }\end{array}$ & Description of Intervention & Description of Comparator & Duration \\
\hline Bjorkman 2002 & $\begin{array}{l}\text { One case } \\
\text { management } \\
\text { service in } \\
\text { Sweden }\end{array}$ & \begin{tabular}{|l|} 
Ages $18-55$, a \\
diagnosed mental \\
illness and \\
impairment due to \\
the illness with \\
serious and \\
continuous difficulties \\
in functioning in \\
social relationships, \\
housing or work \\
situation for more \\
than 2 years.
\end{tabular} & $\begin{array}{l}\text { Case } \\
\text { management } \\
\text { service }(n=33) \\
\text { vs. standard } \\
\text { care }(n=44) \\
\\
\end{array}$ & $\begin{array}{l}\text { The case management service included } \\
\text { two RNs and two social workers. All } \\
\text { members in the team worked full-time } \\
\text { and all had experience of working in } \\
\text { social services, psychiatric services or } \\
\text { vocational rehabilitation. The caseload } \\
\text { was on average nine clients considering } \\
\text { that four clients not included in the study } \\
\text { were later admitted to the service. Clients } \\
\text { could get in contact with a case manager } \\
\text { after working hours by telephone. A } \\
\text { psychiatrist and psychologist were } \\
\text { available for supervision. The } \\
\text { characteristics of the case management } \\
\text { service were a moderate emphasis on } \\
\text { skills training, low emphasis on } \\
\text { integration of services and a high level of } \\
\text { consumer input, where the client had the } \\
\text { major input, deciding on the planning and } \\
\text { the content of the case management } \\
\text { relationship. The clients in the case } \\
\text { management services had access to all } \\
\text { services in the standard care services. }\end{array}$ & $\begin{array}{l}\text { The psychiatric services were } \\
\text { comprehensive with a joint } \\
\text { management for outpatient, inpatient } \\
\text { and day-care facilities, as well as a } \\
\text { couple of small therapeutic } \\
\text { communities (with a total number of } \\
\text { beds of around } 30 \text { ). One ward was } \\
\text { aimed for patients with dual diagnosis } \\
\text { (12 beds), another ward for } \\
\text { psychiatric patients (not dementia } \\
\text { cases) aged } 65 \text { years or over (15 } \\
\text { beds), and a third ward for general } \\
\text { psychiatric cases (19 beds). The } \\
\text { outpatient care was organized in two } \\
\text { general psychiatric teams and one } \\
\text { team for long-term mentally ill } \\
\text { patients. The total number of staff } \\
\text { mainly working with outpatients was } \\
\text { around } 65 \text {. The local social service } \\
\text { offered sheltered accommodation for } \\
\text { around } 70 \text { people with long-term } \\
\text { mental illness. }\end{array}$ & $\begin{array}{l}\text { 18- and 36- } \\
\text { month followup } \\
\text { with last followup } \\
\text { at } 36 \text { months }\end{array}$ \\
\hline
\end{tabular}




\begin{tabular}{|c|c|c|c|c|c|c|c|}
\hline Author, Year & $\begin{array}{l}\text { Age Gender } \\
\text { Race/Ethnicity }\end{array}$ & $\begin{array}{l}\text { Other Population } \\
\text { Characteristics }\end{array}$ & Total N & Benefits Outcomes & $\begin{array}{l}\text { Harms } \\
\text { Outcomes }\end{array}$ & Funding & $\begin{array}{l}\text { Quality } \\
\text { Rating }\end{array}$ \\
\hline Bjorkman 2002 & \begin{tabular}{|l} 
Demographics \\
(intervention, control) \\
Age, mean years: \\
40,35 \\
Gender, \% female: \\
$51 \%, 54 \%$ \\
Ethnicity, \% NR
\end{tabular} & $\mathrm{NR}$ & 77 & $\begin{array}{l}\text { SCL symptoms, mean (SD) (intervention, control) } \\
\text { Baseline: } 124.8-128.0(70.8-71.5), 101.4-102.0(53.2-55.0) \\
18 \text { months: } 114.9(66.8), 93.7(57.0) \\
36 \text { months: } 102.0(68.5), 81.4(55.1) \\
\\
\text { ISSI social network } \\
\text { Baseline: } 11.1-11.2(6.1-6.3), 15.1-15.2(6.2-6.3) \\
18 \text { months: } 16.7(6.3), 18.1(6.4) \\
36 \text { months: } 14.3(6.4), 17.5(5.9) \\
\text { Strauss Carpenter social functioning } \\
\text { Baseline: } 10.6(2.4), 10.5-10.6(2.7-2.8) \\
18 \text { months: } 11.5(2.9), 10.9(2.9) \\
36 \text { months: } 11.4(2.5), 11.5(2.5) \\
\\
\text { GAF Functioning } \\
\text { Baseline: } 55.7-56.3(11.6-12.3), 46.5-50.2(14.6-15.4) \\
18 \text { months: } 57.0(13.0), 60.3(13.3) \\
36 \text { months: } 52.3(14.6), 55.3(17.0) \\
\\
\text { LQOLP Overall quality of life } \\
\text { Baseline: } 4.3(0.7), 4.5(0.6) \\
18 \text { months: } 4.6(0.7), 40.9(0.7) \\
36 \text { months: } 4.6(0.7), 40.9(0.7)\end{array}$ & NR & NR & Fair \\
\hline
\end{tabular}

\section{Please see Appendix B. Included Studies for full study references}

GAF=global assessment of functioning, ISSI=Interview Schedule for Social Interaction, LQOLP=Lancashire Quality of Life Profile, RN=registered nurse, SCL=Symptom Checklist, SD=standard deviation 
Appendix Table E-15. Data abstraction of systematic reviews of illness self-management and recovery

\begin{tabular}{|c|c|c|c|c|c|c|c|}
\hline Author, Year & Aims & $\begin{array}{l}\text { Databases and } \\
\text { Timeperiod } \\
\text { Covered }\end{array}$ & $\begin{array}{l}\text { Number of Studies } \\
\text { Number of Patients }\end{array}$ & $\begin{array}{l}\text { Characteristics of } \\
\text { Identified Articles: } \\
\text { Study Designs }\end{array}$ & $\begin{array}{l}\text { Description of } \\
\text { Intervention }\end{array}$ & $\begin{array}{l}\text { Characteristics of } \\
\text { Identified Articles: } \\
\text { Populations }\end{array}$ & $\begin{array}{l}\text { Characteristics of } \\
\text { Identified Articles: } \\
\text { Interventions }\end{array}$ \\
\hline Zou, 2012 & $\begin{array}{l}\text { Examine self- } \\
\text { management } \\
\text { education } \\
\text { interventions in } \\
\text { patients with } \\
\text { schizophrenia. }\end{array}$ & $\begin{array}{l}\text { CCRCT; PubMed } \\
\text { MEDLINE; } \\
\text { CINAHL; } \\
\text { EMBASE; } \\
\text { PsycINFO; Web } \\
\text { of Science (all } \\
\text { databases 1996- } \\
\text { 2010) }\end{array}$ & $\begin{array}{l}13 \text { RCTs } \\
N=1404 \\
\text { China }(n=4) \\
\text { USA }(n=3) \\
\text { France }(N=1) \\
\text { Germany }(n=2) \\
\text { Denmark }(n=1) \\
\text { Mexico }(n=1) ; \\
\text { inpatient }(n=3) \\
\text { outpatient }(n=7) \\
\text { community health } \\
\text { centers }(n=3)\end{array}$ & $\begin{array}{l}\text { Randomized } \\
\text { controlled trial of self } \\
\text { management } \\
\text { education } \\
\text { interventions in } \\
\text { persons with } \\
\text { schizophrenia; } \\
\text { Duration of followup: } \\
\text { immediate to } 24 \\
\text { months post } \\
\text { intervention }\end{array}$ & $\begin{array}{l}\text { Self-management } \\
\text { education teaches } \\
\text { problem solving skills to } \\
\text { allow patients to take } \\
\text { appropriate actions to } \\
\text { improve their health by } \\
\text { providing both education } \\
\text { and practical self } \\
\text { management skills to } \\
\text { promote active illness } \\
\text { management }\end{array}$ & $\begin{array}{l}\text { Adults }>18, \text { ICD-10 } \\
\text { diagnosis of } \\
\text { schizophrenia; control } \\
\text { group receiving standard } \\
\text { care or on wait list }\end{array}$ & $\begin{array}{l}\text { Self management } \\
\text { education: } n=726 \\
\text { Standard care: } n=678 \\
\text { (standard care) }\end{array}$ \\
\hline
\end{tabular}




\begin{tabular}{|c|c|c|c|c|c|}
\hline Author, Year & Outcomes Reported & Effectiveness Outcomes & Harms & Funding & Quality \\
\hline Zou, 2012 & $\begin{array}{l}\text { Relapse } \\
\text { Rehospitalization, } \\
\text { Adherence to medication regimen } \\
\text { Psychiatric symptoms } \\
\text { Psychosocial functioning } \\
\text { Symptom scales: PANSS, BPRS }\end{array}$ & $\begin{array}{l}\text { Self management education vs. standard care } \\
\text { Relapse: OR } 0.54(0.36-0.83) \\
\text { Rehospitalization: OR } 0.55(0.39-0.77) \\
\text { Negative PANSS : WMD }-4.01(-5.23 \text { to }-2.79) \\
\text { Total PANSS: WMD-3.39 (-4.5 to }-2.29) \\
\text { BPRS: WMD: -4.19 (-5.84 to }-2.54) \\
\text { Relapse, >10 intervention sessions: OR } 0.41(0.21-0.79) \\
\text { Relapse, <10 intervention sessions: OR } 0.67(0.39-1.15)\end{array}$ & NR & $\begin{array}{l}\text { China } \\
\text { Medical } \\
\text { Board } \\
\text { Grant }\end{array}$ & Fair \\
\hline
\end{tabular}

\section{Please see Appendix B. Included Studies for full study references}

BPRS=brief psychiatric rating scale, RCT=randomized control trial, $\mathrm{OR}=$ odds ratio, PANSS=positive and negative syndrome scale, SC=standard care, $\mathrm{SME}=$ self management education, WMD=weighted means difference, USA=United States of America 
Appendix Table E-16. Data abstraction of randomized controlled trials of illness self-management and recovery

\begin{tabular}{|l|l|l|l|l|l|}
\hline & Setting & & & \\
Author, Year & Country & $\begin{array}{l}\text { Interventions } \\
\text { and Ns per } \\
\text { Group }\end{array}$ & $\begin{array}{l}\text { Duration } \\
\text { (intervention } \\
\text { and longest } \\
\text { followup) }\end{array}$ \\
\hline $\begin{array}{l}\text { Hasson-Ohayon, } \\
2007\end{array}$ & $\begin{array}{l}13 \text { community } \\
\text { rehabilitation } \\
\text { centers } \\
\text { Israel }\end{array}$ & $\begin{array}{l}\text { Severe mental illness; } \\
\text { receiving treatment and } \\
\text { rehabilitation services } \\
\text { at one of the 13 centers; } \\
\text { fluency in Hebrew; } \\
\text { sufficient competence to } \\
\text { provide informed } \\
\text { consent }\end{array}$ & $\begin{array}{l}\text { IMR: } \mathrm{n}=119 \\
\text { Usual care: }\end{array}$ & $\begin{array}{l}\text { IMR: 1-hour, weekly intervention sessions led by two } \\
\text { clinicians, for 8 months using educational handouts } \\
\text { covered sequentially in the same order at all 13 sites }\end{array}$ & $\begin{array}{l}\text { Usual care: } \\
\text { Individual } \\
\text { supportive therapy } \\
\text { and medication } \\
\text { management } \\
\text { biweekly for 15-30 } \\
\text { minutes }\end{array}$ \\
\hline
\end{tabular}




\begin{tabular}{|c|c|c|c|c|c|c|c|c|}
\hline Author, Year & $\begin{array}{l}\text { Age Gender } \\
\text { Race/Ethnicity }\end{array}$ & $\begin{array}{l}\text { Other Population } \\
\text { Characteristics }\end{array}$ & Total N & $\begin{array}{l}\text { Outcomes } \\
\text { Measures }\end{array}$ & Benefits Outcomes & \begin{tabular}{|l} 
Harms \\
Outcomes
\end{tabular} & Funding & $\begin{array}{l}\text { Quality } \\
\text { Rating }\end{array}$ \\
\hline $\begin{array}{l}\text { Hasson-Ohayon, } \\
2007\end{array}$ & \begin{tabular}{|l} 
IMR vs. usual care \\
Mean age 34 vs. 35 \\
years \\
$32 \%$ vs. $38 \%$ female \\
Race not reported
\end{tabular} & $\begin{array}{l}\text { IMR vs. usual care } \\
\text { Schizophrenia: } 80 \% \text { vs. } \\
89 \% \\
\text { Other mental illness: } \\
20 \% \text { vs. } 11 \% \\
\text { Coping Efficacy Scale } \\
2.85 \text { (SD 0.95) vs. } 3.09 \\
\text { (SD 0.98) }\end{array}$ & 210 & $\begin{array}{l}\text { Symptoms - } \\
\text { CES }\end{array}$ & $\begin{array}{l}\text { Scores on scale before and } \\
\text { after the IMR group } \\
\text { intervention } \\
\text { CES, mean score: } 3.25 \text { (SD } \\
0.95 \text { ) vs. } 3.09 \text { (SD 0.87); } p=N S\end{array}$ & NR & $\begin{array}{l}\text { Israel's ministry of } \\
\text { health, national } \\
\text { council for the } \\
\text { rehabilitation of } \\
\text { persons with a } \\
\text { psychiatric disability }\end{array}$ & Fair \\
\hline
\end{tabular}

Please see Appendix B. Included Studies for full study references

CES=Coping Efficacy Scale, CMHC=community mental health center, IMR=Illness management recovery, NS=not significant, PANSS Positive and Negative Syndrome Scale, PRP=program for relapse, QLS=quality of life scale, VA=veteran's affairs 
Appendix Table E-17. Data abstraction of systematic reviews of psychoeducation

\begin{tabular}{|c|c|c|c|c|c|c|c|}
\hline Author, Year & Aims & $\begin{array}{l}\text { Databases and } \\
\text { Timeperiod } \\
\text { Covered }\end{array}$ & \begin{tabular}{|l} 
Number of \\
Studies \\
Number of \\
Patients
\end{tabular} & $\begin{array}{l}\text { Characteristics of } \\
\text { Identified } \\
\text { Articles: Study } \\
\text { Designs }\end{array}$ & $\begin{array}{l}\text { Characteristics of } \\
\text { Identified Articles: } \\
\text { Populations }\end{array}$ & $\begin{array}{l}\text { Characteristics of } \\
\text { Identified Articles: } \\
\text { Interventions }\end{array}$ & $\begin{array}{l}\text { Outcomes } \\
\text { Reported }\end{array}$ \\
\hline Pekkala 2009 & $\begin{array}{l}\text { Assess the effects of } \\
\text { psychoeducational } \\
\text { interventions compared } \\
\text { to the standard levels of } \\
\text { knowledge provision in } \\
\text { schizophrenia patients }\end{array}$ & $\begin{array}{l}\text { CINAHL (1982 to } \\
\text { 1999) } \\
\text { Cochrane Library } \\
\text { CENTRAL (Issue } \\
\text { 1, 1999) Cochran } \\
\text { Schizophrenia } \\
\text { Group Register } \\
\text { (January and May } \\
\text { 2001) } \\
\text { EMBASE (1980 } \\
\text { to June 1999) } \\
\text { MEDLINE } \\
\text { (January 1966 to } \\
\text { January 1999) } \\
\text { PsycLIT (January } \\
\text { 1974 to January } \\
\text { 1999) } \\
\text { SOCIOFILE } \\
\text { (January 1974 to } \\
\text { January 1999) }\end{array}$ & $\begin{array}{l}10 \text { studies } \\
n=1125 \\
\\
\end{array}$ & $\begin{array}{l}\text { Randomized } \\
\text { clinical trials of } \\
\text { psychoeducation } \\
\text { for schizophrenia } \\
\text { and/or related } \\
\text { serious mental } \\
\text { illnesses involving } \\
\text { individuals or } \\
\text { groups }\end{array}$ & $\begin{array}{l}\text { People suffering from } \\
\text { schizophrenia, } \\
\text { schizoaffective } \\
\text { disorder, } \\
\text { schizophreniform } \\
\text { disorder, or } \\
\text { schizotypal } \\
\text { personality disorder. }\end{array}$ & $\begin{array}{l}\text { Group and individual } \\
\text { psychoeducation } \\
\text { interventions compared } \\
\text { to usual care (e.g., } \\
\text { psychosocial } \\
\text { rehabilitation or } \\
\text { supportive } \\
\text { psychotherapy) or } \\
\text { waiting list comparison } \\
\text { groups }\end{array}$ & $\begin{array}{l}\text { Adherence } \\
\text { Knowledge } \\
\text { Symptoms } \\
\text { Function }\end{array}$ \\
\hline
\end{tabular}




\begin{tabular}{|c|c|}
\hline Author, Year & Effectiveness Outcomes \\
\hline Pekkala 2009 & $\begin{array}{l}\text { Any form of psychoeducation vs. standard care } \\
\text { Global functioning (no clinically significant improvement) at discharge (1 study, } 92 \text { participants) } \\
\text { Risk ratio } 0.52,95 \% \mathrm{Cl} 0.24 \text { to } 1.10 \\
\text { Global functioning (no clinically significant improvement) } 6 \text { months ( } 1 \text { study, } 92 \text { participants) } \\
\text { Risk ratio } 0.83,95 \% \mathrm{Cl} 0.50 \text { to } 1.38 \\
\text { Global functioning (no clinically significant improvement) } 18 \text { months (1 study, } 92 \text { participants) } \\
\text { Risk ratio } 0.90,95 \% \mathrm{Cl} 0.58 \text { to } 1.39 \\
\text { Global functioning (average scale score GAF/GAS) at end of intervention (1 study, } 41 \text { participants) } \\
\text { Mean difference }-2.64,95 \% \mathrm{Cl}-12.74 \text { to } 7.46 \\
\text { Global functioning (average scale score GAF/GAS) at } 1 \text { year ( } 3 \text { studies, } 260 \text { participants) } \\
\text { Mean difference }-5.23,95 \% \mathrm{Cl}-8.76 \text { to }-1.71, I^{2}=79 \% \\
\text { Global functioning (average scale score GAF/GAS) at } 2 \text { years ( } 1 \text { study, } 59 \text { participants) } \\
\text { Mean difference }-6.70,95 \% \mathrm{Cl}-13.38 \text { to }-0.02 \\
\text { Global functioning (average scale score GAF/GAS) at } 5 \text { years (1 study, } 60 \text { participants) } \\
\text { Mean difference }-3.80,95 \% \mathrm{Cl}-8.04 \text { to } 0.44, I^{2}=0.0 \% \\
\text { BPRS at } 1 \text { year ( } 1 \text { study, } 159 \text { participants) } \\
\text { Mean difference }-6.0,95 \% \mathrm{Cl}-9.15 \text { to }-2.85 \\
\text { BPRS post treatment (1 study, } 19 \text { participants) } \\
\text { Mean difference }-0.0695 \% \mathrm{Cl}-0.53 \text { to } 0.41, I^{2}=93 \% \\
\text { Social functioning (SAS-II) at end of intervention (1 study, } 19 \text { participants) } \\
\text { Mean difference }-0.10,95 \% \mathrm{Cl}-0.37 \text { to } 0.17 \\
\text { Quality of life (Heinrich's Scale) at end of intervention (1 study, } 114 \text { participants) } \\
\text { Mean difference }-8.20,95 \% \mathrm{Cl}-14.78 \text { to }-1.62\end{array}$ \\
\hline
\end{tabular}




\begin{tabular}{|c|c|}
\hline Author, Year & Effectiveness Outcomes \\
\hline Pekkala 2009 & $\begin{array}{l}\text { Quality of life (Heinrich's Scale) at } 3 \text { months (1 study, } 108 \text { participants) } \\
\text { Mean difference }-9.7095 \% \mathrm{Cl}-17.22 \text { to }-2.18, I^{2}=0.0 \% \\
\text { Standard length group psychoeducation vs. standard care } \\
\text { BPRS Post treatment (1 study, } 19 \text { participants) } \\
\text { Mean difference }-0.0695 \% \mathrm{Cl}-0.53 \text { to } 0.41 \\
\text { Social functioning (SAS-II) at end of intervention (1 study, } 19 \text { participants) } \\
\text { Mean difference }-0.1095 \% \mathrm{Cl}-0.37 \text { to } 0.17 \\
\text { Quality of life (Heinrich's Scale) at end of intervention (1 study, } 114 \text { participants) } \\
\text { Mean difference }-8.2095 \% \mathrm{Cl}-14.78 \text { to }-1.62 \\
\text { Quality of life (Heinrich's Scale) at } 3 \text { months (1 study, } 108 \text { participants) } \\
\text { Mean difference }-9.7095 \% \mathrm{Cl}-17.22 \text { to }-2.18, I^{2}=0.0 \% \\
\text { Brief group psychoeducation vs. standard care } \\
\text { Global functioning (no clinically significant improvement) at discharge (1 study, } 92 \text { participants) } \\
\text { Risk ratio } 0.5295 \% \mathrm{Cl} 0.24 \text { to } 1.10 \\
\text { Global functioning (no clinically significant improvement) } 6 \text { months (1 study, } 92 \text { participants) } \\
\text { Risk ratio } 0.8395 \% \mathrm{Cl} 0.50 \text { to } 1.38 \\
\text { Global functioning (no clinically significant improvement) } 18 \text { Months (1 study, } 92 \text { participants) } \\
\text { Risk ratio } 0.9095 \% \mathrm{Cl} 0.58 \text { to } 1.39 \\
\text { BPRS at } 1 \text { year (1 study, } 159 \text { participants) } \\
\text { Mean difference }-6.095 \% \mathrm{Cl}-9.15 \text { to }-2.85\end{array}$ \\
\hline
\end{tabular}




\begin{tabular}{|c|c|c|c|}
\hline Author, Year & Harms Outcomes & $\begin{array}{l}\text { Fundingl } \\
\text { Comments }\end{array}$ & Quality Rating \\
\hline Pekkala 2009 & $\begin{array}{l}\text { Any form of psychoeducation vs. Standard care } \\
\text { Death ( } 2 \text { studies, } 170 \text { participants) } \\
\text { Risk ratio } 0.53,95 \% \mathrm{Cl} 0.07 \text { to } 3.95, I^{2}=0.0 \% \\
\text { Leaving study any reason (8 studies, } 788 \text { participants) } \\
\text { Risk ratio } 1.13,95 \% \mathrm{Cl} 0.89 \text { to } 1.44, I^{2}=15 \% \\
\text { Standard length group psychoeducation vs. standard care } \\
\text { Leaving study any reason ( } 4 \text { studies, } 280 \text { participants) } \\
\text { Risk ratio } 1.39,95 \% \mathrm{Cl} 0.89 \text { to } 2.18, I^{2}=0.0 \% \\
\text { Brief individual psychoeducation vs. standard care } \\
\text { Leaving study/lost ( } 1 \text { study, } 67 \text { participants) } \\
\text { Risk ratio } 3.06,95 \% \mathrm{Cl} 0.17 \text { to } 56.70, I^{2}=0.0 \% \\
\text { Brief group psychoeducation vs. standard care } \\
\text { Death ( } 2 \text { studies, } 170 \text { participants) } \\
\text { Risk ratio } 0.53,95 \% \mathrm{Cl} 0.07 \text { to } 3.95, I^{2}=0.0 \% \\
\text { Leaving study any reason (4 studies, } 457 \text { participants) } \\
\text { Risk ratio } 0.97,95 \% \mathrm{Cl} 0.73 \text { to } 1.30, I^{2}=59 \%\end{array}$ & $\begin{array}{l}\text { Dept of Psychiatry, Porvoo } \\
\text { Hospital, Finland Dept of } \\
\text { Psychiatric Demography, } \\
\text { Institute of Basic Psychiatric } \\
\text { Research, University } \\
\text { Hospital of Aarhus, } \\
\text { Denmark } \\
\text { Finnish Office for Health } \\
\text { Technology Assessment, } \\
\text { Finland }\end{array}$ & Good \\
\hline
\end{tabular}

Please see Appendix B. Included Studies for full study references

BPRS=Brief Psychiatric Rating Scale, CI=controlled interval, GAS=Global Assessment Scale, GAF=Global Assessment of Functioning, MD=mean difference RR=risk ratio, SAS-II=social adjustment scale II 
Appendix Table E-18. Data abstraction of randomized controlled trials of social skills training

\begin{tabular}{|c|c|c|c|c|c|c|}
\hline $\begin{array}{l}\text { Author, Year } \\
\text { Trial Name }\end{array}$ & $\begin{array}{l}\text { Setting } \\
\text { Country }\end{array}$ & Inclusion Criteria & $\begin{array}{l}\text { Intervention } \\
\text { s and Ns } \\
\text { per Group }\end{array}$ & Description of Intervention & Description of Comparator & $\begin{array}{l}\text { Duration } \\
\text { (interventio } \\
\text { n and } \\
\text { longest } \\
\text { followup) }\end{array}$ \\
\hline $\begin{array}{l}\text { Bartels } 2014 \text { and } \\
\text { Mueser } 2010\end{array}$ & \begin{tabular}{|l|} 
community \\
mental \\
health \\
centers \\
United States \\
\\
\end{tabular} & $\begin{array}{l}\text { Age } \geq 50 \text { years able to provide informed } \\
\text { consent; DSM-IV Axis I disorder } \\
\text { diagnosis of schizophrenia, } \\
\text { schizoaffective disorder, bipolar } \\
\text { disorder, or major depression based on } \\
\text { the Structured Clinical Interview for } \\
\text { DSM- IV in conjunction with } \\
\text { documented persistent impairment in } \\
\text { multiple areas of functioning. Excluded: } \\
\text { Residence in a nursing home or other } \\
\text { institutional setting; primary diagnosis of } \\
\text { dementia or significant cognitive } \\
\text { impairment as indicated by a Mini } \\
\text { Mental Status Exam score<20; physical } \\
\text { illness expected to cause death within } 1 \\
\text { year, or current substance dependence. }\end{array}$ & $\begin{array}{l}\begin{array}{l}\text { Psychosocial } \\
\text { skills training } \\
\text { (HOPES): } \\
n=90\end{array} \\
\text { Usual care: } \\
n=93\end{array}$ & $\begin{array}{l}\text { HOPES: SST: Weekly sessions for } 1 \\
\text { year followed by one year monthly } \\
\text { sessions of social rehabilitation } \\
\text { curriculum, based on social skills } \\
\text { training is manualized and organized } \\
\text { into seven modules: Communicating } \\
\text { Effectively, Making and Keeping } \\
\text { Friends, Making the Most of Leisure } \\
\text { Time, Healthy Living, Using } \\
\text { Medications Effectively, and Making } \\
\text { the Most of a Health Care Visit. }\end{array}$ & $\begin{array}{l}\text { Usual care: Routine mental } \\
\text { health services including } \\
\text { pharmacotherapy, case } \\
\text { management or outreach } \\
\text { by non-nurse clinicians, } \\
\text { individual therapy, and } \\
\text { access to rehabilitation } \\
\text { services, such as groups } \\
\text { and psychoeducation. }\end{array}$ & $\begin{array}{l}\text { Intervention: } \\
2 \text { years } \\
\text { Followup: } 3 \\
\text { years }\end{array}$ \\
\hline Valencia 2007 & \begin{tabular}{|l|} 
Single center \\
Mexico
\end{tabular} & $\begin{array}{l}\text { Outpatients age } 16 \text { to } 50 \text { with DSM- } \\
\text { IV schizophrenia; taking } \\
\text { antipsychotic medication; clinically } \\
\text { stable in terms of psychotic } \\
\text { symptoms (PANSS score <60); } \\
\text { completed at least } 6 \text { years of } \\
\text { elementary education; lived with their } \\
\text { families and resided in Mexico City or } \\
\text { the metropolitan area }\end{array}$ & $\begin{array}{l}\text { SST: } n=49 \\
\text { Usual care: } \\
n=49\end{array}$ & $\begin{array}{l}\text { SST: } 48 \text { weekly group sessions (75 } \\
\text { mins/session) composed of seven } \\
\text { sequential treatment areas (each } \\
\text { area includes a specific set of skills) } \\
\text { as follows: (1) symptom management; } \\
\text { (2) medication management; (3) } \\
\text { social relations; (4) occupational; (5) } \\
\text { money management; (6) couple } \\
\text { relations; and (7) family relations } \\
\text { Additional component of } 8 \text { group and } \\
4 \text { individual family therapy sessions } \\
\text { for relatives }\end{array}$ & $\begin{array}{l}\text { Usual care: Monthly } \\
\text { appointments ( } 20 \\
\text { mins/session) with clinical } \\
\text { psychiatrist who controlled } \\
\text { the prescription } \\
\text { of their AP medication } \\
\text { based upon the } \\
\text { assessment of their } \\
\text { psychotic symptoms, } \\
\text { checked their medication } \\
\text { compliance, recorded } \\
\text { their consultation }\end{array}$ & 1 year \\
\hline
\end{tabular}




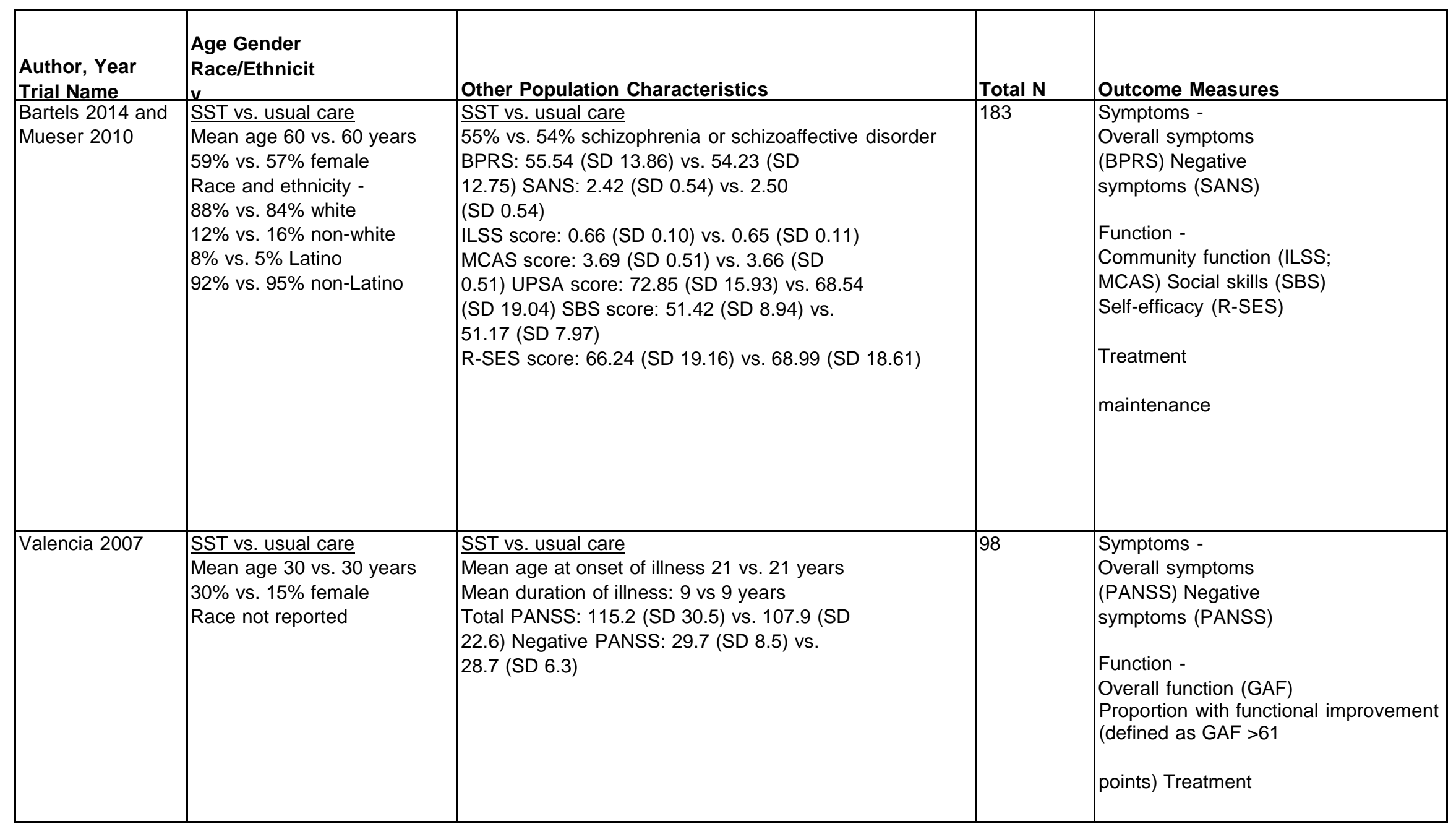




\begin{tabular}{|c|c|c|c|c|c|}
\hline $\begin{array}{l}\text { Author, Year } \\
\text { Trial Name }\end{array}$ & Benefits Outcomes & Harms Outcomes & Funding & $\begin{array}{l}\text { Quality } \\
\text { Rating }\end{array}$ & Comments \\
\hline $\begin{array}{l}\text { Bartels } 2014 \text { and } \\
\text { Mueser } 2010\end{array}$ & 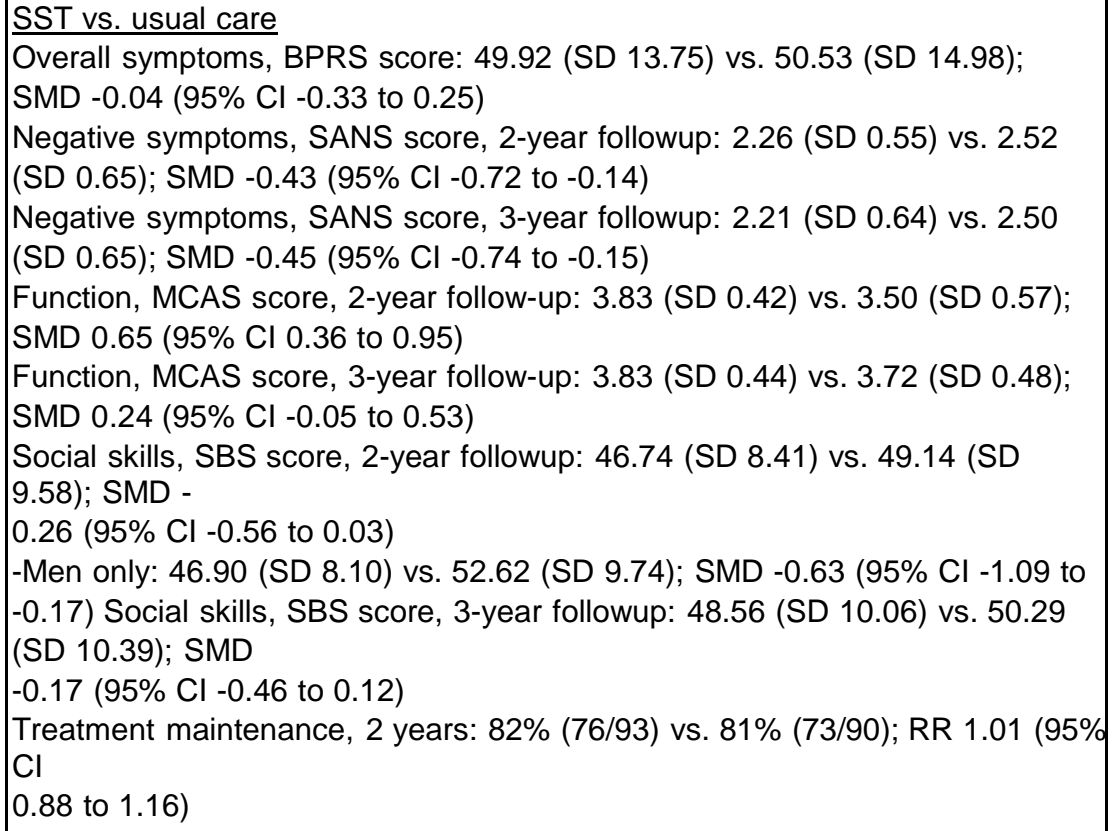 & \begin{tabular}{|l|} 
Not reported \\
$\mid$ \\
\end{tabular} & $\mathrm{NIMH}$ & Fair & $\begin{array}{l}\text { Subgroups: } \\
\text { Subgroup analysis } \\
\text { for 2-year outcomes } \\
\text { (reported in } \\
\text { Mueser) found no } \\
\text { difference in effect } \\
\text { based on age. } \\
\text { Effect sizes for all } \\
\text { outcomes were } \\
\text { consistently higher } \\
\text { in men vs. women. }\end{array}$ \\
\hline Valencia 2007 & $\begin{array}{l}\text { SST vs. usual care } \\
\text { Overall symptoms, total PANSS: } 46.9 \text { (SD 14.6) vs. } 60.4 \text { (SD 18.2); SMD - } \\
0.65(95 \% \mathrm{Cl}-1.06 \text { to }-0.24) \\
\text { Negative symptoms, PANSS: } 13.0 \text { (SD 5.7) vs. } 17.9 \text { (SD 6.2); SMD }-0.82 \\
(95 \% \mathrm{Cl}- \\
1.23 \text { to }-0.40) \\
\text { Function, GAF: } 66.0 \text { (SD 8.9) vs. } 44.9 \text { (SD } 11.6) ; \mathrm{p}<0.001 \text {; SMD } 2.02(95 \% \mathrm{Cl} \\
1.53 \text { to } \\
2.52) \\
\text { Relapse } 5 / 49 \text { vs.10/49; RR } 0.50(95 \% \mathrm{Cl} 0.18 \text { to } 1.36) \\
\text { Treatment maintenance: } 88 \%(43 / 49) \text { vs. } 80 \%(39 / 49) ; \mathrm{RR} 1.10(95 \% \mathrm{Cl} 0.92 \\
\text { to } 1.31)\end{array}$ & Not reported & $\begin{array}{l}\text { National Institute of } \\
\text { Psychiatry } \\
\text { Ramón de la Fuente; } \\
\text { National Council on } \\
\text { Science and } \\
\text { Technology }\end{array}$ & Fair & \\
\hline
\end{tabular}




\begin{tabular}{|l|l|l|l|l|l|}
\hline $\begin{array}{l}\text { Author, Year } \\
\text { Trial Name }\end{array}$ & $\begin{array}{l}\text { Setting } \\
\text { Country }\end{array}$ & Inclusion Criteria & $\begin{array}{l}\text { Intervention } \\
\text { s and Ns } \\
\text { per Group }\end{array}$ & Description of Intervention & Description of Comparator \\
\hline Valencia 2013 & $\begin{array}{l}\text { Single center } \\
\text { Mexico }\end{array}$ & $\begin{array}{l}\text { Age 18-60 years; DSM-IV criteria for } \\
\text { schizophrenia; completed at least } 6 \\
\text { years of elementary education; } \\
\text { furrently living with relatives in Mexico } \\
\text { fity or it's metropolitan area; stable } \\
\text { under antipsychotic medication and on } \\
\text { an ambulatory basis for at least six } \\
\text { months prior to their inclusion in the } \\
\text { study. } \\
\text { Excluded: history of major medical } \\
\text { illness; alcohol or drug dependency; } \\
\text { mental retardation or severe cognitive } \\
\text { impairment as confirmed by the } \\
\text { corresponding clinical chart. }\end{array}$ & $\begin{array}{l}\text { SST: n=68 } \\
\text { Usual care: } \\
\text { n=51 }\end{array}$ & $\begin{array}{l}\text { SST: 24 (weekly) group 60-minute } \\
\text { sessions consisting of four modules } \\
\text { of 1) medication management, 2) } \\
\text { symptom management, 3) social } \\
\text { relations, and 4) family relations plus } \\
\text { "Learning activities" training sessions } \\
\text { that included: introduction } \\
\text { of the skills that would be taught, } \\
\text { skills demonstration by the trainers, } \\
\text { role playing in sessions, and } \\
\text { corrective feedback }\end{array}$ & $\begin{array}{l}\text { Usual care: Regular } \\
\text { treatment }\end{array}$ \\
\hline
\end{tabular}




\begin{tabular}{|l|l|l|l|l|}
\hline $\begin{array}{l}\text { Author, Year } \\
\text { Trial Name }\end{array}$ & $\begin{array}{l}\text { Age Gender } \\
\text { Race/Ethnicit } \\
\text { v }\end{array}$ & Other Population Characteristics & Total N & Outcome Measures \\
\hline Valencia 2013 & $\begin{array}{l}\text { SST vs. USual care } \\
\text { Mean age 30 vs. 26 years } \\
27 \% \text { vs. 24\% female } \\
\text { Race not reported }\end{array}$ & $\begin{array}{l}\text { SST vs. usual care } \\
\text { Mean age at onset of illness 22 vs. 21 years } \\
\text { Mean duration of illness: 8 vs. 8 years } \\
\text { Total PANSS: 92.6 (SD 41.5) vs. 83.5 (33.9) } \\
\text { Negative PANSS: 24.2 (SD 10.4) vs. 22.0 } \\
\text { (SD 8.2) GAF: 43.1 (SD 6.3) vs. 42.9 (SD } \\
6.3)\end{array}$ & $\begin{array}{l}\text { Symptoms - } \\
\text { Overall symptoms } \\
\text { (PANSS) Negative } \\
\text { symptoms (PANSS) } \\
\text { Function - } \\
\text { Overall function (GAF) } \\
\text { Proportion with functional improvement } \\
\text { (defined as GAF >61 } \\
\text { points) Relapse }\end{array}$ \\
\hline
\end{tabular}




\begin{tabular}{|c|c|c|c|c|}
\hline $\begin{array}{l}\text { Author, Year } \\
\text { Trial Name }\end{array}$ & Benefits Outcomes & Harms Outcomes & Funding & $\begin{array}{l}\text { Quality } \\
\text { Rating }\end{array}$ \\
\hline Valencia 2013 & $\begin{array}{l}\text { SST vs. usual care } \\
\text { Overall symptoms, total PANSS: } 43.4 \text { (SD 8.0) vs. } 55.7 \text { (SD 8.3); SMD }-0.63 \\
(95 \% \mathrm{Cl}- \\
1.04 \text { to }-0.23) \\
\text { Negative symptoms, PANSS: } 11.2 \text { (SD 2.6) vs. } 14.9 \text { (SD 3.1); SMD }-1.30 \\
(95 \% \mathrm{Cl}- \\
1.70 \text { to }-0.90) \\
\text { Function, GAF: } 67.0 \text { (SD 14.9) vs. } 43.7 \text { (SD 13.9): SMD } 1.60 \text { (95\% CI } 1.19 \\
\text { to } 2.02) \text { Proportion with functional improvement: } 59 \%(40 / 68) \text { vs. } 2 \% \\
(1 / 51) ; \text { RR } 30(95 \% \mathrm{Cl} \\
4.26 \text { to } 211)\end{array}$ & Not reported & $\begin{array}{l}\text { National Institute of } \\
\text { Psychiatry } \\
\text { Ramón de la Fuente }\end{array}$ & Fair \\
\hline
\end{tabular}

\section{Please see Appendix B. Included Studies for full study references}

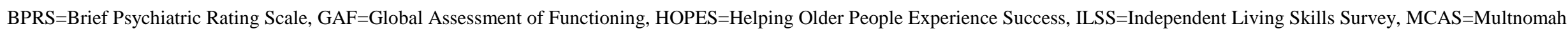

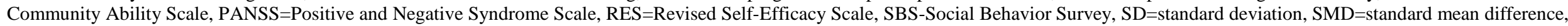

SST=social skills training 
Appendix Table E-19. Data abstraction of randomized controlled trials of supported employment

\begin{tabular}{|c|c|c|c|c|}
\hline Author, Year & $\begin{array}{l}\text { Setting } \\
\text { Country }\end{array}$ & Inclusion Criteria & $\begin{array}{l}\text { Interventions } \\
\text { and Ns per } \\
\text { Group }\end{array}$ & Description of Intervention \\
\hline \begin{tabular}{|l|} 
Cook, 2008, \\
2005
\end{tabular} & \begin{tabular}{|l|}
7 sites \\
Us
\end{tabular} & $\begin{array}{l}\text { Diagnosis, duration, and disability } \\
\text { requirements for severe and } \\
\text { persistent mental illness as } \\
\text { defined by the US Government } \\
\text { Center for Mental Health Services, } \\
\text { age } 18 \text { years or older, willingness } \\
\text { to work, and written informed } \\
\text { consent }\end{array}$ & \begin{tabular}{|l|} 
Different models \\
of supported \\
employment \\
program: 333 \\
Alternative \\
vocational \\
program or usual \\
care: 315
\end{tabular} & $\begin{array}{l}\text { Implementation effectiveness trial in which sites test different "experimental" models of } \\
\text { supported employment. The experimental condition was always a form of enhanced best- } \\
\text { practice supported employment. The Maryland, Connecticut, and South Carolina sites } \\
\text { used Individual Placement and Support, the Massachusetts site used the Assertive } \\
\text { Community Treatment vocational model, and the Texas, Maine, and Arizona sites used } \\
\text { experimental models developed especially for this study. Specifically, Texas included } \\
\text { supported employment services with social network enhancements, Maine used family- } \\
\text { aided Assertive Community Treatment teams working with an employer consortium, and } \\
\text { Arizona's integrated treatment team included psychiatrists, case managers, rehabilitation } \\
\text { counselors, employment specialists, job developers, and benefits specialists emphasizing } \\
\text { rapid job placement and ongoing support. }\end{array}$ \\
\hline Mueser, 2004 & \begin{tabular}{|l|}
1 site \\
US
\end{tabular} & $\begin{array}{l}\text { Clients with severe mental illness } \\
\text { receiving services at the lead } \\
\text { community mental health center } \\
\text { in Hartford, CT with severe mental } \\
\text { illness, as defined by the DSM-IV; } \\
\text { lack of competitive employment; } \\
\text { desire for competitive work; } \\
\text { attendance at two research } \\
\text { introduction meetings; willingness } \\
\text { and capability of giving informed } \\
\text { consent }\end{array}$ & $\begin{array}{l}\text { Individual } \\
\text { placement and } \\
\text { support : } 68 \\
\text { Standard } \\
\text { services: } 69 \\
\\
\end{array}$ & $\begin{array}{l}\text { The individual placement and support model employment specialists serve on treatment } \\
\text { teams, including case managers and psychiatrists, in order to integrate vocational services } \\
\text { with psychiatric treatment. Clients receive a full range of vocational services including } \\
\text { engagement in services, identifying job interests and vocational assessment, job finding, } \\
\text { and job support. Individual placement and support uses assertive outreach to deliver } \\
\text { services in clients' natural settings in the community rather than at mental health agencies. }\end{array}$ \\
\hline
\end{tabular}




\begin{tabular}{|c|c|c|c|c|c|}
\hline Author, Year & \begin{tabular}{|l} 
Description of \\
Comparator
\end{tabular} & $\begin{array}{l}\text { Duration } \\
\text { (intervention and } \\
\text { longest followup) }\end{array}$ & $\begin{array}{l}\text { Age Gender } \\
\text { Race/Ethnicity }\end{array}$ & Other Population Characteristics & Total N \\
\hline $\begin{array}{l}\text { Cook, 2008, } \\
2005\end{array}$ & \begin{tabular}{|l|} 
Either services as usual (4 \\
sites), an "unenhanced" \\
version of the experimental \\
model (2 sites), or the \\
Clubhouse model (1 site)
\end{tabular} & \begin{tabular}{|l|} 
Intervention: \\
Average of 74 hours \\
of vocational \\
services and 125 \\
hours of clinical \\
services per person \\
Followup: 2 years
\end{tabular} & $\begin{array}{l}\text { Age, mean years: } 38 \\
\text { Gender, \% female: } 36 \\
\text { White race, \%: } 41\end{array}$ & $\begin{array}{l}\text { Age at first hospitalization, mean years: } 24 \\
\text { Lifetime hospitalized, mean months: } 19 \\
\text { PANSS Cognitive, mean score: } 16 \\
\text { PANSS Negative, mean score: } 13 \\
\text { PANSS Depressive, mean score: } 15 \\
\text { PANSS Positive, means score: } 11 \\
\text { PANSS Excitement, mean score: } 11\end{array}$ & $\begin{array}{l}648 \text { with } \\
\text { schizophrenia (of a } \\
\text { larger sample of } \\
1,273 \text { that included } \\
\text { those with other } \\
\text { disorders) }\end{array}$ \\
\hline Mueser, 2004 & $\begin{array}{l}\text { Access to all other } \\
\text { vocational services in } \\
\text { Hartford for clients with } \\
\text { severe mental illness: a } \\
\text { supported employment } \\
\text { program located off-site } \\
\text { from the mental health } \\
\text { center (standard- } \\
\text { supported), and a } \\
\text { vocational program in } \\
\text { which clients worked in } \\
\text { jobs paying subminimum } \\
\text { wage or competitive wages } \\
\text { in supervised janitorial } \\
\text { enclaves in the community, } \\
\text { fulfilling contracts obtained } \\
\text { by that program (standard- } \\
\text { enclave). }\end{array}$ & $\begin{array}{l}\text { (includes time from } \\
\text { enrollment through } \\
\text { the 2-year } \\
\text { collection of } \\
\text { employment data } \\
\text { with interviews } \\
\text { conducted at } \\
\text { baseline and every } \\
6 \text { months for } 2 \\
\text { years) }\end{array}$ & $\begin{array}{l}\text { Age, mean years: } 41 . / \text { vs. } \\
40.9 \\
\text { Gender, \% female: } 38.2 \text { vs. } \\
36.2\end{array}$ & $\begin{array}{l}\text { Prımary diagnosıs, Schızophrenıa: } 57 \% \text { vs. } 54 \% \\
\text { Primary diagnosis, schizoaffective: } 19 \% \text { vs. } 22 \% \\
\text { Primary diagnosis, bipolar: } 3 \% \text { vs. } 2 \% \\
\text { Primary diagnosis, major depression: } 16 \% \text { vs. } 21 \% \\
\text { Lifetime hospitalization, mean: } 15.3 \text { vs. } 18.8 \text { months }\end{array}$ & 204 \\
\hline
\end{tabular}




\begin{tabular}{|c|c|c|c|c|}
\hline Author, Year & Benefits Outcomes & Harms Outcomes & Funding & $\begin{array}{l}\text { Quality } \\
\text { Rating }\end{array}$ \\
\hline Cook, 2008, 2005 & $\begin{array}{l}24 \text { month results } \\
\text { Intervention vs. unenhanced/services as usual } \\
\text { Schizophrenia only: } \\
\text { Competitively employed (defined as pays minimum wage or higher, is located in a } \\
\text { mainstream, socially integrated setting, is not set aside for persons with disabilities, and is } \\
\text { held independently/not agency owned), mean proportion estimated from figure: } 22 \% \text { vs. } \\
12 \% \text { (statistics not reported) } \\
\text { All populations (50-50\% schizophrenia spectrum disorders): } \\
\text { Employed } 40 \text { hours or more per month, mean proportion: } 51 \%(330 / 648) \text { vs. } 39 \%(245 / 625) \text {, } \\
\text { p<0.001 } \\
\text { Mean number of dollars earned per month: } \$ 122 / \text { month vs. } \$ 99 / \text { month, } p=0.04 \\
\text { Attrition, } \%: 21 \text { vs. } 24\end{array}$ & Not reported & \begin{tabular}{|l|} 
Center for Mental \\
Health Services/ \\
Substance Abuse and \\
Mental Health \\
Services \\
Administration
\end{tabular} & Fair \\
\hline Mueser, 2004 & $\begin{array}{l}\text { In competitive work: } 75 \%(51 / 68) \text { vs. } 27.5 \% \text { (19/69); RR } 2.72 \text { (95\% Cl } 1.81 \text { to } 4.09) \\
\text { In any employment: vs. } 75 \%(51 / 68) 53.6 \% \text { (37/69); RR } 1.40 \text { (95\% Cl } 1.08 \text { to } 1.82)\end{array}$ & $\begin{array}{l}\text { Treatment } \\
\text { discontinuation at } 2 \\
\text { years: } 9 \%(6 / 68) \text { vs. } \\
83 \%(57 / 69) ; \text { RR } 0.11 \\
(95 \% \mathrm{Cl} 0.05 \text { to } 0.23)\end{array}$ & $\begin{array}{l}\text { USDHHS, SAMHSA, } \\
\text { NIMH }\end{array}$ & Fair \\
\hline
\end{tabular}

Please see Appendix B. Included Studies for full study references

PANSS=positive and negative syndrome scale, QLS=quality of life scale, QOL=quality of life, VA=veteran's affairs, WBI=Work Behavior Inventory 
Appendix Table E-20. Data abstraction of systematic reviews of supported employment

\begin{tabular}{|c|c|c|c|c|c|c|c|}
\hline Author, Year & Aims & $\begin{array}{l}\text { Databases and } \\
\text { Timeperiod } \\
\text { Covered }\end{array}$ & $\begin{array}{l}\text { Number of Studies } \\
\text { Number of Patients }\end{array}$ & $\begin{array}{l}\text { Characteristics of } \\
\text { Identified Articles: } \\
\text { Study Designs }\end{array}$ & $\begin{array}{l}\text { Description of } \\
\text { Intervention }\end{array}$ & $\begin{array}{l}\text { Characteristics of } \\
\text { Identified Articles: } \\
\text { Populations }\end{array}$ & $\begin{array}{l}\text { Characteristics of } \\
\text { Identified Articles: } \\
\text { Interventions }\end{array}$ \\
\hline Kinoshita, 2013 & \begin{tabular}{|l|} 
To review the \\
effectiveness of \\
supported \\
employment \\
compared with \\
other \\
approaches to \\
vocational \\
rehabilitation or \\
treatment as \\
usual.
\end{tabular} & $\begin{array}{l}\text { Cochrane } \\
\text { Schizophrenia } \\
\text { Group Trials } \\
\text { Register } \\
\text { (February 2010). } \\
\\
\end{array}$ & \begin{tabular}{|l|}
14 RCTs \\
$N=2265$
\end{tabular} & $\begin{array}{l}\text { Randomized } \\
\text { controlled trial of } \\
\text { supported } \\
\text { employment in } \\
\text { primarily persons } \\
\text { with schizophrenia; } \\
13 \text { of } 14 \text { trials used } \\
\text { the Individual } \\
\text { Placement and } \\
\text { Support model (IPS). } \\
\text { Duration of followup: } \\
\text { mean of } 18 \text { months }\end{array}$ & $\begin{array}{l}\text { Key principles of Individual } \\
\text { Placement and Support } \\
\text { (IPS): "(a) the goal is } \\
\text { competitive employment in } \\
\text { work settings integrated } \\
\text { into a community's } \\
\text { economy; (b) services are } \\
\text { based on clients' choices; } \\
\text { (c) clients are expected to } \\
\text { obtain jobs directly, rather } \\
\text { than following lengthy pre- } \\
\text { employment training (rapid } \\
\text { job search); (d) attention to } \\
\text { patient preference in the } \\
\text { job search; (e) integration } \\
\text { between employment } \\
\text { services and mental health } \\
\text { treatment teams; (f ) } \\
\text { ongoing individual support; } \\
\text { and (g) systematic benefits } \\
\text { counseling" }\end{array}$ & $\begin{array}{l}\text { Adults, most using DSM } \\
\text { criteria for severe mental } \\
\text { illnesses. } \\
\text { Comparison gourps all } \\
\text { received some form of } \\
\text { vocation al training, with } \\
\text { one also comparing to } \\
\text { usual care. }\end{array}$ & $\begin{array}{l}\text { Mean sample size per } \\
\operatorname{arm}=70\end{array}$ \\
\hline
\end{tabular}




\begin{tabular}{|c|c|c|c|c|c|}
\hline Author, Year & Outcomes Reported & Effectiveness Outcomes & Harms & Funding & Quality \\
\hline Kinoshita, 2013 & $\begin{array}{l}\text { Primary: Employment: days in } \\
\text { competitive employment (long } \\
\text { term) } \\
\text { Secondary: } \\
\text { Other employment outcomes } \\
\text { Education } \\
\text { Leaving study early } \\
\text { Glpba; state } \\
\text { Mental state } \\
\text { Service use } \\
\text { Quality of life } \\
\text { Social/general functioning } \\
\text { Adverse effects } \\
\text { Economic costs }\end{array}$ & $\begin{array}{l}\text { Supported Employment vs. standard vocational training } \\
\text { Days in competitive employment (primary outcome) - long term } \\
\text { Mean Difference }(95 \% \mathrm{Cl}) 70.63[43.22,98.04] \\
\text { Days in any form of paid employment - long term } \\
\text { Mean Difference }(95 \% \mathrm{Cl}) 84.94[51.99,117.89] \\
\text { Job tenure for competitive employment (weeks) - long term } \\
\text { Mean Difference }(95 \% \mathrm{Cl}) 9.86[5.36,14.36] \\
\text { Job tenure for any paid employment (weeks) - long term } \\
\text { Mean Difference }(95 \% \mathrm{Cl}) 3.86[-5.66,13.38] \\
\text { Obtained any job during the study (high=better) } \\
\text { Risk Ratio }(95 \% \mathrm{Cl}) 3.24[2.17,4.82] \\
\text { Days to first competitive employment (long-term) } \\
\text { Mean Difference }(95 \% \mathrm{Cl})-161.60 \text { [-225.73, }-97.47] \\
\text { Leaving the study early for any reason } \\
\text { Risk Ratio }(95 \% \mathrm{Cl}) 0.76[0.57,1.01] \\
\text { PANSS negative symptoms } \\
\text { Mean Difference }(95 \% \mathrm{Cl})-2.12[-3.20,-1.05] \\
\text { Quality of Life: (high = better) } \\
\text { Mean Difference }(95 \% \mathrm{Cl}) 0.04[-0.10,0.18]\end{array}$ & \begin{tabular}{|l|} 
Death - natural \\
and suicide long \\
term \\
Risk Ratio (95\% \\
CI) \\
$1.5[0.25,8.85]$
\end{tabular} & University & Good \\
\hline
\end{tabular}

Please see Appendix B. Included Studies for full study references

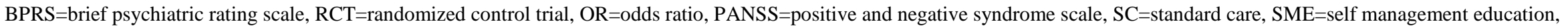
$\mathrm{WMD}=$ weighted means difference, USA=United States of America 
Appendix Table E-21. Data abstraction of systematic reviews in trials of supportive therapy

\begin{tabular}{|c|c|c|c|c|c|c|}
\hline Author, Year & Aims & $\begin{array}{l}\text { Databases and } \\
\text { Timeperiod } \\
\text { Covered }\end{array}$ & \begin{tabular}{|l|} 
Number of \\
Studies \\
Number of \\
Patients \\
\end{tabular} & \begin{tabular}{|l|} 
Characteristics of \\
Identified \\
Articles: Study \\
Designs
\end{tabular} & $\begin{array}{l}\text { Characteristics of } \\
\text { Identified Articles: } \\
\text { Populations }\end{array}$ & $\begin{array}{l}\text { Characteristics of Identified Articles: } \\
\text { Interventions }\end{array}$ \\
\hline Buckley 2015 & $\begin{array}{l}\text { To review the effects of } \\
\text { supportive therapy } \\
\text { compared with usual } \\
\text { care in patients with } \\
\text { schizophrenia }\end{array}$ & $\begin{array}{l}\text { Cochrane } \\
\text { Schizophrenia } \\
\text { Group Trials } \\
\text { Register (28 } \\
\text { November 2012) }\end{array}$ & $\begin{array}{l}5 \text { studies vs. } \\
\text { standard care } \\
(\mathrm{N}=822)\end{array}$ & \begin{tabular}{l|} 
Randomized \\
controlled trials of \\
supportive therapy \\
compared with \\
usual care \\
reporting relapse, \\
hospitalization, or \\
general functioning \\
as primary \\
outcomes with \\
multiple secondary \\
outcomes
\end{tabular} & $\begin{array}{l}\text { Schizophrenia or } \\
\text { schizophrenia-like illnesses } \\
\text { using any criteria including } \\
\text { trials where it was implied } \\
\text { that the majority of } \\
\text { participants had a severe } \\
\text { mental illness that was } \\
\text { likely to be schizophrenia. }\end{array}$ & $\begin{array}{l}\text { Supportive therapy and supportive care } \\
\text { (provided by a single person with the main } \\
\text { purpose of maintaining current functioning } \\
\text { or assisting pre-existing coping abilities in } \\
\text { people who have a diagnosis of } \\
\text { schizophrenia or schizophrenia-like illness. } \\
\text { The therapies can be aimed at individuals or } \\
\text { groups of people) vs. standard care (health } \\
\text { care a person would normally receive had } \\
\text { they not been included in the research trial, } \\
\text { including interventions such as medication, } \\
\text { hospitalization, community psychiatric } \\
\text { nursing input and/or day hospital). }\end{array}$ \\
\hline
\end{tabular}




\begin{tabular}{|c|c|c|c|c|c|}
\hline Author, Year & Outcomes Reported & Effectiveness Outcomes & Harms Outcomes & $\begin{array}{l}\text { Fundingl } \\
\text { Comments }\end{array}$ & $\begin{array}{l}\text { Quality } \\
\text { Rating }\end{array}$ \\
\hline Buckley 2015 & \begin{tabular}{|l} 
Change in general \\
functioning quality \\
of life Overall \\
symptoms \\
Discontinuation of \\
treatment \\
Relapse
\end{tabular} & $\begin{array}{l}\text { Supportive therapy or care vs. standard care } \\
\text { Change in general functioning (GAS): Mean general } \\
\text { functioning in the intervention groups was } 1.4 \text { higher ( } 95 \% \mathrm{Cl} \\
5.09 \text { lower to } 7.89 \text { higher) } \\
\text { Quality of life: Mean quality of life in the intervention groups } \\
\text { was } 2.73 \text { lower ( } 95 \% \mathrm{Cl} 6.04 \text { lower to } 0.58 \text { higher) } \\
\text { Overall symptoms: Mental state: No clinically important } \\
\text { improvement (followup } 1 \text { to } 2 \text { years) (RR } 0.95,95 \% \mathrm{Cl} 0.82 \text { to } \\
1.11 \text { ) } \\
\text { Discontinuation of treatment: (RR } 0.86,95 \% \mathrm{Cl} 0.53 \text { to } 1.4 \text { ) } \\
\text { Relapse: RR } 0.96,95 \% \mathrm{Cl} 0.44 \text { to } 2.11\end{array}$ & Not reported & $\begin{array}{l}\text { Affinity Healthcare, Cheadle } \\
\text { Royal Hospital, UK. Leeds } \\
\text { Community and Mental } \\
\text { Health Services, NHS } \\
\text { Teaching Trust, UK. } \\
\text { Northumberland Tyne and } \\
\text { Wear NHS Trust, UK. }\end{array}$ & Good \\
\hline
\end{tabular}

Please see Appendix B. Included Studies for full study references

$\mathrm{CI}=$ confidence interval, GAS=Global Assessment Scale, NHS=National Health Service, RR=relative risk, UK=United Kingdom 
Appendix Table E-22. Data abstraction of randomized controlled trials of early interventions for patients with first-episode psychosis

\begin{tabular}{|c|c|c|c|c|}
\hline Author, Year & $\begin{array}{l}\text { Setting } \\
\text { Country }\end{array}$ & Inclusion Criteria & $\begin{array}{l}\text { Interventions and Ns } \\
\text { per Group }\end{array} \mid$ & Description of Intervention \\
\hline $\begin{array}{l}\text { Early } \\
\text { Treatment Program } \\
\text { (ETP)-RAISE } \\
\text { Kane 2015, } 2016\end{array}$ & U.S. & \begin{tabular}{|l|} 
1) Ages $15-40$ \\
2) ability to participate in research \\
assessments in English \\
3) ability to full consent (or assent if under \\
18) \\
4) presence of definite psychotic symptoms \\
and evidence of one of following diagnoses: \\
schizophrenia, schizoaffective disorder, \\
schizophreniform, psychotic disorder NOS, or \\
brief psychotic disorder (DSM-IV) \\
5) up to 6 months of cumulative exposure to \\
antipsychotic medications. \\
First-episode schizophrenia spectrum (89\% \\
for NAVIGATE, 90\% for standard care) \\
Schizophrenia-only: NAVIGATE (51\%), \\
standard care (56\%)
\end{tabular} & \begin{tabular}{|l|} 
By participant: \\
NAVIGATE ( $\mathrm{n}=223)$, \\
Community Care \\
$(\mathrm{n}=181)$ \\
Cluster randomization \\
by site: \\
NAVIGATE ( $\mathrm{n}=17)$ \\
Community Care \\
$(\mathrm{n}=17)$
\end{tabular} & $\begin{array}{l}\text { NAVIGATE model includes four core interventions delivered } \\
\text { by a multidisciplinary team: personalized medication } \\
\text { management via a secure web-based decision support } \\
\text { system, family psychoeducation, individual resiliency } \\
\text { therapy, and supported employment and education. }\end{array}$ \\
\hline
\end{tabular}




\begin{tabular}{|c|c|c|c|c|c|}
\hline Author, Year & Description of Comparator & Duration & $\begin{array}{l}\text { Age Gender } \\
\text { Race/Ethnicity }\end{array}$ & Other Population Characteristics & Total N \\
\hline $\begin{array}{l}\text { Early } \\
\text { Treatment Program } \\
\text { (ETP)-RAISE } \\
\text { Kane 2015, } 2016\end{array}$ & $\begin{array}{l}\text { Called "Community Care;" } \\
\text { Standard care for psychosis } \\
\text { treatment }\end{array}$ & 2 years & $\begin{array}{l}\text { Demographics (intervention, control) } \\
\text { Mean age: } 23.18,23.08 \\
\text { Gender/males: } 78 \%, 66 \% \text { (significantly } \\
\text { more males in NAVIGATE) } \\
\text { Race/ethnicity: } \\
\text { White }=62 \%, 44 \% \\
\text { African American=28\%, 49\% } \\
\text { Other }=10 \%, 7 \% \\
\text { Hispanic=25\%, } 10 \%\end{array}$ & $\begin{array}{l}\text { (intervention, control) } \\
\text { Weeks of duration of untreated psychosis: } \\
178.91,211.43 \\
\text { Heinrich-Carpenter QLS total: } 18.44,18.99 \\
\text { PANSS total: } 14.95,14.87 \text { (significantly worse } \\
\text { for NAVIGATE) } \\
\text { Calgary Depression Scale: } 4.27,4.30 \\
\text { CGI: } 0.80,0.83 \\
\text { Duration of time on antipsychotics (days): } \\
42.88,48.98 \\
\text { Patient's education: } \\
\text { College or higher: } 32 \%, 30 \% \\
\text { Completed high school: } 34 \%, 32 \% \\
\text { Some high school: } 30 \%, 32 \% \\
\text { Lifetime alcohol use (did not meet criteria): } \\
60 \%, 68 \% \\
\text { Lifetime cannabis use (did not meet criteria): } \\
61 \%, 68 \%\end{array}$ & 404 \\
\hline
\end{tabular}




\begin{tabular}{|c|c|c|c|c|}
\hline Author, Year & Benefits Outcomes & Harms Outcomes & Funding & $\begin{array}{l}\text { Quality } \\
\text { Rating }\end{array}$ \\
\hline $\begin{array}{l}\text { Early } \\
\text { Treatment Program } \\
\text { (ETP)-RAISE } \\
\text { Kane 2015, } 2016\end{array}$ & 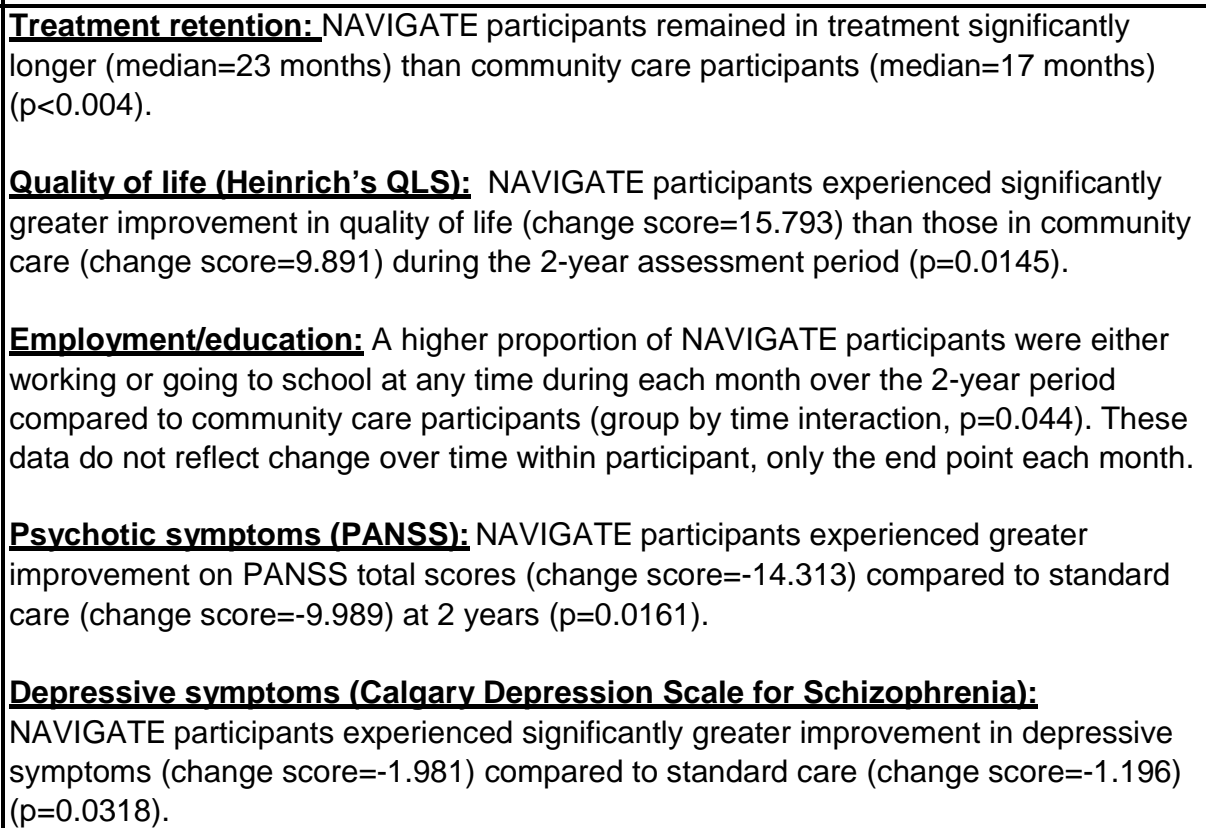 & None reported. & NIMH & Poor \\
\hline
\end{tabular}




\begin{tabular}{|c|c|c|c|c|}
\hline Author, Year & $\begin{array}{l}\text { Setting } \\
\text { Country }\end{array}$ & Inclusion Criteria & $\begin{array}{l}\text { Interventions and Ns } \\
\text { per Group }\end{array}$ & Description of Intervention \\
\hline Guo 2007, 2010 & $\begin{array}{l}10 \text { clinical } \\
\text { sites in China }\end{array}$ & \begin{tabular}{|l|} 
1) Ages $16-50$ \\
2) DSM-IV criteria for schizophrenia, or \\
schizophreniform disorder for not more than \\
5 years \\
3) being confirmed to be clinically \\
stable by the investigator (the total score $\leq 60$ \\
on the PANSS or a decrease of $50 \%$ from \\
acute period in the total score on PANSS \\
4) taking maintenance therapy with any one \\
of the following seven oral antipsychotics: \\
chlorpromazine, sulpiride, clozapine, \\
risperidone, olanzapine, quetiapine, and \\
aripiprazole. First episode schizophrenia \\
$84.6 \%$, schizophreniform $15.4 \%$
\end{tabular} & \begin{tabular}{|l|} 
Combined Treatment \\
$(n=604)$, Medication \\
Treatment $(n=635)$
\end{tabular} & $\begin{array}{l}\text { Antipsychotic medication plus group psychoeducation, } \\
\text { family intervention, skills training, and CBT (Once a month x } \\
12 \text { months, } 48 \text { sessions total). }\end{array}$ \\
\hline
\end{tabular}




\begin{tabular}{|c|c|c|c|c|c|}
\hline Author, Year & Description of Comparator & Duration & $\begin{array}{l}\text { Age Gender } \\
\text { Race/Ethnicity }\end{array}$ & Other Population Characteristics & Total N \\
\hline Guo 2007,2010 & $\begin{array}{l}\text { Antipsychotic medication } \\
\text { only }\end{array}$ & $\begin{array}{l}12 \text { month } \\
\text { intervention only }\end{array}$ & $\begin{array}{l}\text { Demographics (intervention, control) } \\
\text { Age, mean years: } 26.1,26.4 \\
\text { Gender, \% Male: } 54.3 \%, 55.7 \% \\
\text { Race/ethnicity: NR }\end{array}$ & $\begin{array}{l}\text { (Intervention, control) } \\
\text { Age at onset: } 23.8,24.2 \\
\text { Duration of schizophrenia: } 24.6 \text { months, } 23.3 \\
\text { months } \\
\text { PANSS total score: } 44.7,45.6 \\
\text { CGI severity score: } 2.5,2.6\end{array}$ & 1268 \\
\hline
\end{tabular}




\begin{tabular}{|c|c|c|c|c|}
\hline Author, Year & Benefits Outcomes & Harms Outcomes & Funding & $\begin{array}{l}\text { Quality } \\
\text { Rating }\end{array}$ \\
\hline Guo 2007, 2010 & 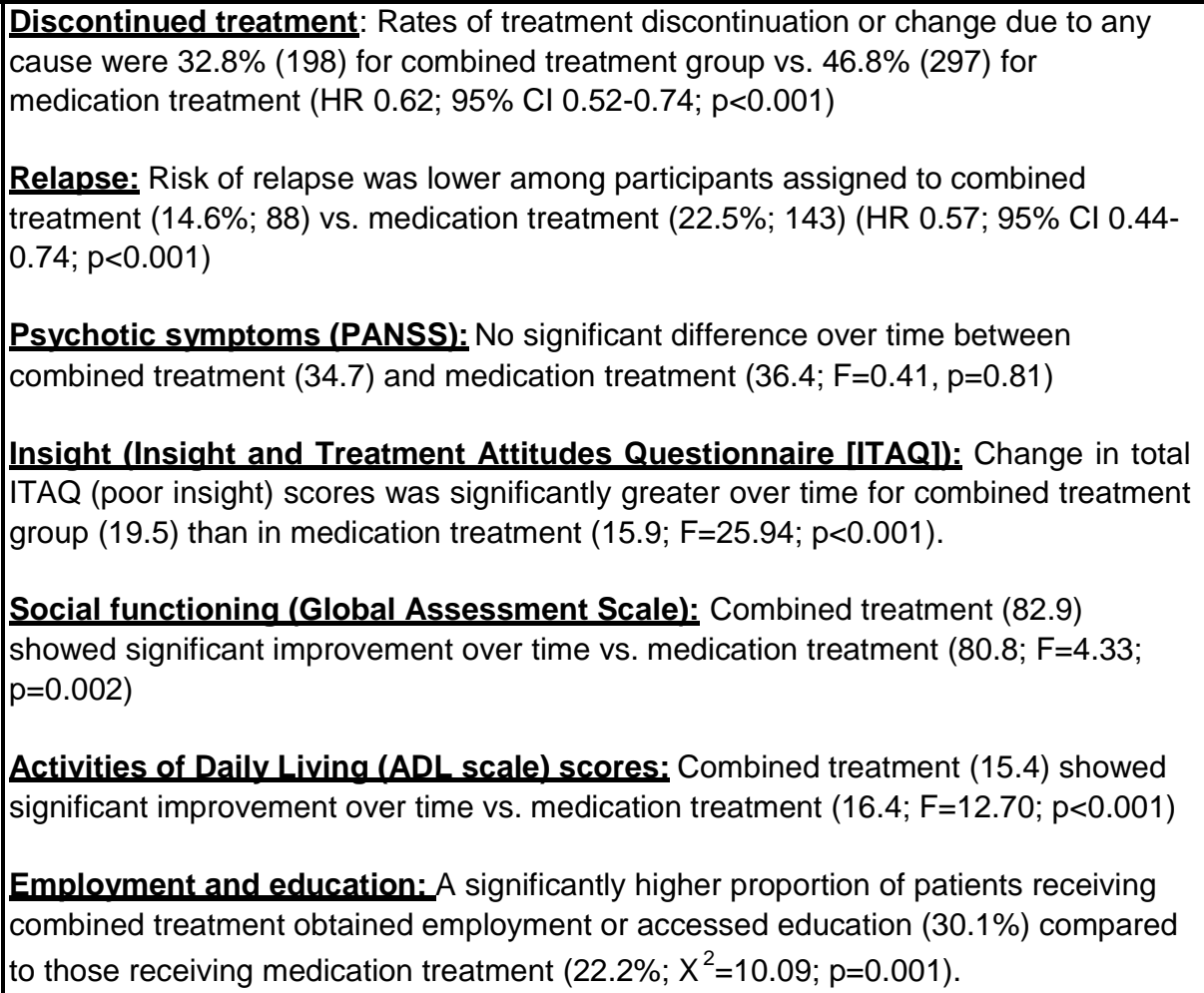 & $\begin{array}{l}\text { Extrapyramidal symptoms: No } \\
\text { significant differences between } \\
\text { combined treatment }(135) \text { and } \\
\text { medication treatment (142; } \\
\left.x^{2}=0.20, p=0.66\right) \text {. } \\
\text { Weight gain }>7 \% \text { from baseline to } \\
\text { last observation: No significant } \\
\text { differences between combined } \\
\text { treatment }(149) \text { and medication } \\
\text { treatment }\left(132 ; x^{2}=1.39, p=0.24\right) .\end{array}$ & \begin{tabular}{|l|} 
Grants from National \\
Key Technologies \\
R\&D Program, \\
National Natural \\
Science Foundation \\
of China, and the \\
National Basic \\
Research Program of \\
China
\end{tabular} & Fair \\
\hline
\end{tabular}




\begin{tabular}{|c|c|c|c|c|}
\hline Author, Year & $\begin{array}{l}\text { Setting } \\
\text { Country }\end{array}$ & Inclusion Criteria & $\begin{array}{l}\text { Interventions and Ns } \\
\text { per Group }\end{array}$ & Description of Intervention \\
\hline $\begin{array}{l}\text { Lambeth Early Onset } \\
\text { (LEO) trial } \\
\text { Craig } 2004\end{array}$ & England & $\begin{array}{l}\text { 16-40 years living in the London borough of } \\
\text { Lambeth and presenting to mental health } \\
\text { services for the first time with nonaffective } \\
\text { psychosis (schizophrenia, schizotypal, and } \\
\text { delusional disorders). Also considered people } \\
\text { who had presented once but had } \\
\text { subsequently disengaged without treatment } \\
\text { from routine community services. } \\
\\
\text { Schizophrenia: } \\
\text { Specialized care = } 51(72 \%) \\
\text { Standard care = } 49(67 \%)\end{array}$ & \begin{tabular}{|l|} 
Early intervention \\
specialized care \\
$(\mathrm{n}=71) ;$ Standard care \\
$(\mathrm{n}=73)$
\end{tabular} & $\begin{array}{l}\text { Community team of } 10 \text { staff (team leader, } 0.5 \text { consultant } \\
\text { psychiatrist, trainee psychiatrist, } 0.5 \text { clinical psychologist, } \\
\text { OT, four psychiatric nurses, two healthcare assistants). } \\
\text { Established on the principles of assertive outreach, } \\
\text { providing an extended hours service by including weekends } \\
\text { and public holidays. Evidence-based interventions adapted } \\
\text { to the needs of people with early psychosis included low- } \\
\text { dose atypical antipsychotic regimens, CBT based on } \\
\text { manualized protocols, and family counseling and vocational } \\
\text { strategies based on established protocols. }\end{array}$ \\
\hline
\end{tabular}




\begin{tabular}{|l|l|l|l|l|}
\hline & & $\begin{array}{l}\text { Age Gender } \\
\text { Race/Ethnicity }\end{array}$ & Other Population Characteristics \\
\hline $\begin{array}{l}\text { Author, Year } \\
\text { (LEO) trial }\end{array}$ & Description of Comparator & Duration & (intervention, control) \\
Craig 2004 & $\begin{array}{l}\text { Standard care delivered by } \\
\text { the community mental health } \\
\text { teams. Teams received no } \\
\text { additional training in the } \\
\text { management of early } \\
\text { psychosis, although they } \\
\text { were encouraged to follow } \\
\text { available guidelines }\end{array}$ & $\begin{array}{l}\text { intervention, control) } \\
\text { Teanths }\end{array}$ & $\begin{array}{l}\text { Mean age: 26 (6.0), 26.6 (6.4) } \\
\text { Gender: Male, 39 (55\%), 54 (74\%) } \\
\text { Race/ethnicity: } \\
\text { White 27 (38\%) 18 (25\%) } \\
\text { Black British 10 (14\%) 6 (8\%) } \\
\text { Black Caribbean 9 (13\%) 13(18\%) } \\
\text { Black African 16 (23\%) 25(34\%) } \\
\text { Mixed 6 (8\%) 6 (8\%) } \\
\text { Other 3 (4\%) 5 (6\%) }\end{array}$ & First episode: 61 (86\%) 52 (71\%) \\
\hline
\end{tabular}




\begin{tabular}{|c|c|c|c|c|}
\hline Author, Year & Benefits Outcomes & Harms Outcomes & Funding & \begin{tabular}{|l} 
Quality \\
Rating
\end{tabular} \\
\hline $\begin{array}{l}\text { Lambeth Early Onset } \\
\text { (LEO) trial } \\
\text { Craig } 2004\end{array}$ & $\begin{array}{l}\text { Relapse: Participants in the specialized care group were less likely to relapse }(30 \%, \\
18 \text { out of } 61) \text { than those in standard care }(48 \%, 29 \text { out of } 61) \text { (odds ratio } 0.46,95 \% \mathrm{Cl} \\
0.22 \text { to } 0.97 ; p=0.042) \text {. When rates were adjusted for baseline differences in sex, } \\
\text { previous psychotic episode, and ethnicity, the difference in relapse was no longer } \\
\text { significant (odds ratio } 0.55,95 \% \mathrm{Cl} 0.24 \text { to } 1.26, p=0.157) \text {. } \\
\text { Retention in treatment/dropout: At } 18 \text { months, } 53(86 \%) \text { patients in the specialized } \\
\text { group and } 44(68 \%) \text { in standard care were in regular contact with the clinical team } \\
\text { (lost to care: odds ratio } 0.35,95 \% \mathrm{Cl} 0.15 \text { to } 0.81) \text {. When rates were adjusted for } \\
\text { baseline differences in sex, previous psychotic episode, and ethnicity, drop-out rates } \\
\text { remained significant }(0.28,95 \% \mathrm{Cl} 0.12 \text { to } 0.73) \text {. }\end{array}$ & None reported. & $\begin{array}{l}\text { Directorate of Health } \\
\text { and Social Care for } \\
\text { London R\&D } \\
\text { Organisation and } \\
\text { Management } \\
\text { Programme }\end{array}$ & Good \\
\hline
\end{tabular}




\begin{tabular}{|l|l|l|l|l|}
\hline Author, Year & $\begin{array}{l}\text { Setting } \\
\text { Country }\end{array}$ & Inclusion Criteria & $\begin{array}{l}\text { Interventions and Ns } \\
\text { per Group }\end{array}$ & Description of Intervention \\
\hline $\begin{array}{l}\text { Lambeth Early Onset } \\
\text { (LEO) Trial }\end{array}$ & England & $\begin{array}{l}\text { 16-40 years with an address in Lambeth for } \\
\text { the first time with a nonaffective psychosis. } \\
\text { Patients who met these demographic and } \\
\text { diagnostic criteria who had presented once } \\
\text { previously but had immediately disengaged } \\
\text { and were not known to any of the existing } \\
\text { mental health services were also deemed } \\
\text { eligible. } \\
\text { Majority met ICD-10 diagnostic criteria } \\
\text { for schizophrenia (69\%) }\end{array}$ & $\begin{array}{l}\text { A multidisciplinard care } \\
\text { group (n=44) }\end{array}$ & $\begin{array}{l}\text { single point of access, and extended service hours. The } \\
\text { interventions provided were specially adapted for a group } \\
\text { with early psychosis and followed protocols and manuals } \\
\text { from the Early Psychosis Prevention and Intervention Centre } \\
\text { and, for CBT, pilot work conducted locally. A mix of } \\
\text { medication management, CBT, vocational input and family } \\
\text { interventions was provided according to individual need. }\end{array}$ \\
\hline
\end{tabular}




\begin{tabular}{|c|c|c|c|c|c|}
\hline Author, Year & Description of Comparator & Duration & $\begin{array}{l}\text { Age Gender } \\
\text { Race/Ethnicity }\end{array}$ & Other Population Characteristics & Total N \\
\hline $\begin{array}{l}\text { Lambeth Early Onset } \\
\text { (LEO) Trial } \\
\text { Garety } 2006\end{array}$ & $\begin{array}{l}\text { Standard care by community } \\
\text { mental health teams. }\end{array}$ & 18 months & $\begin{array}{l}\text { Average age: } 26 \text { years } \\
\text { Gender: Male }(65 \%) \\
\text { More than } 50 \% \text { were from a minority ethnic, } \\
\text { predominantly } \\
\text { of African or Caribbean parentage. }\end{array}$ & $\begin{array}{l}\text { (intervention, control) } \\
\text { First episode: } 61(86 \%) 52(71 \%) \\
\text { PANSS Total: } 67.4,73.3 \\
\text { GAF: } 46.5,42.2 \\
\text { Calgary Depression Scale: } 4.1,3.3\end{array}$ & 99 \\
\hline
\end{tabular}




\begin{tabular}{|c|c|c|c|c|}
\hline Author, Year & Benefits Outcomes & Harms Outcomes & Funding & $\begin{array}{l}\text { Quality } \\
\text { Rating }\end{array}$ \\
\hline $\begin{array}{l}\text { Lambeth Early Onset } \\
\text { (LEO) Trial } \\
\text { Garety } 2006\end{array}$ & 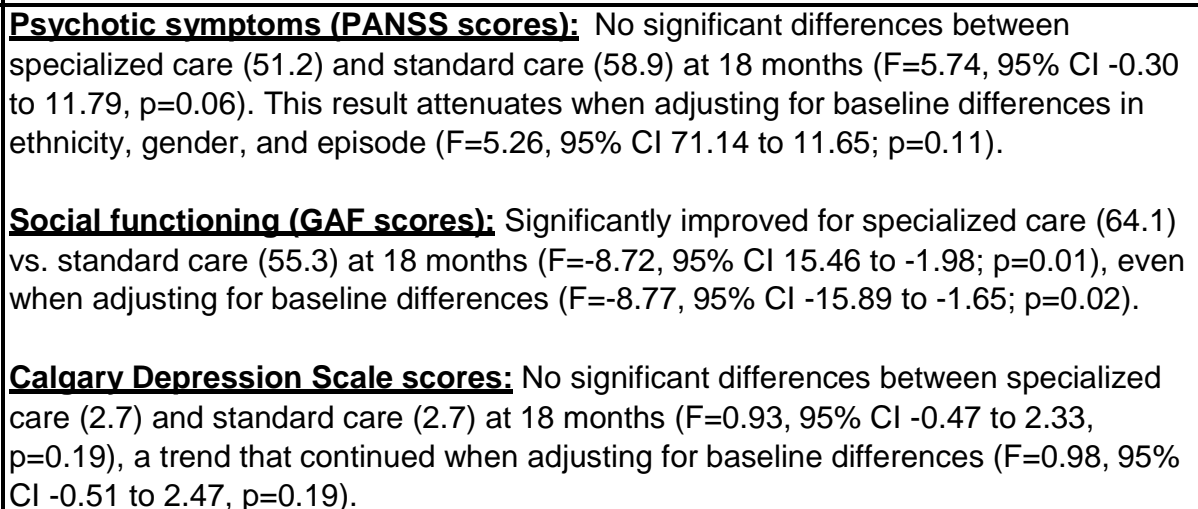 & $\begin{array}{l}\text { Reported on deaths, prison, self- } \\
\text { harm, violence to others and } \\
\text { homelessness, but no statistical } \\
\text { tests conducted. }\end{array}$ & $\begin{array}{l}\text { Directorate of Health } \\
\text { and Social Care for } \\
\text { London R\&D } \\
\text { Organisation and } \\
\text { Management } \\
\text { Programme }\end{array}$ & Good \\
\hline
\end{tabular}




\begin{tabular}{|c|c|c|c|c|}
\hline Author, Year & Benefits Outcomes & Harms Outcomes & Funding & $\begin{array}{l}\text { Quality } \\
\text { Rating }\end{array}$ \\
\hline $\begin{array}{l}\text { Lambeth Early Onset } \\
\text { (LEO) Trial } \\
\text { Garety } 2006 \\
\text { Continued }\end{array}$ & 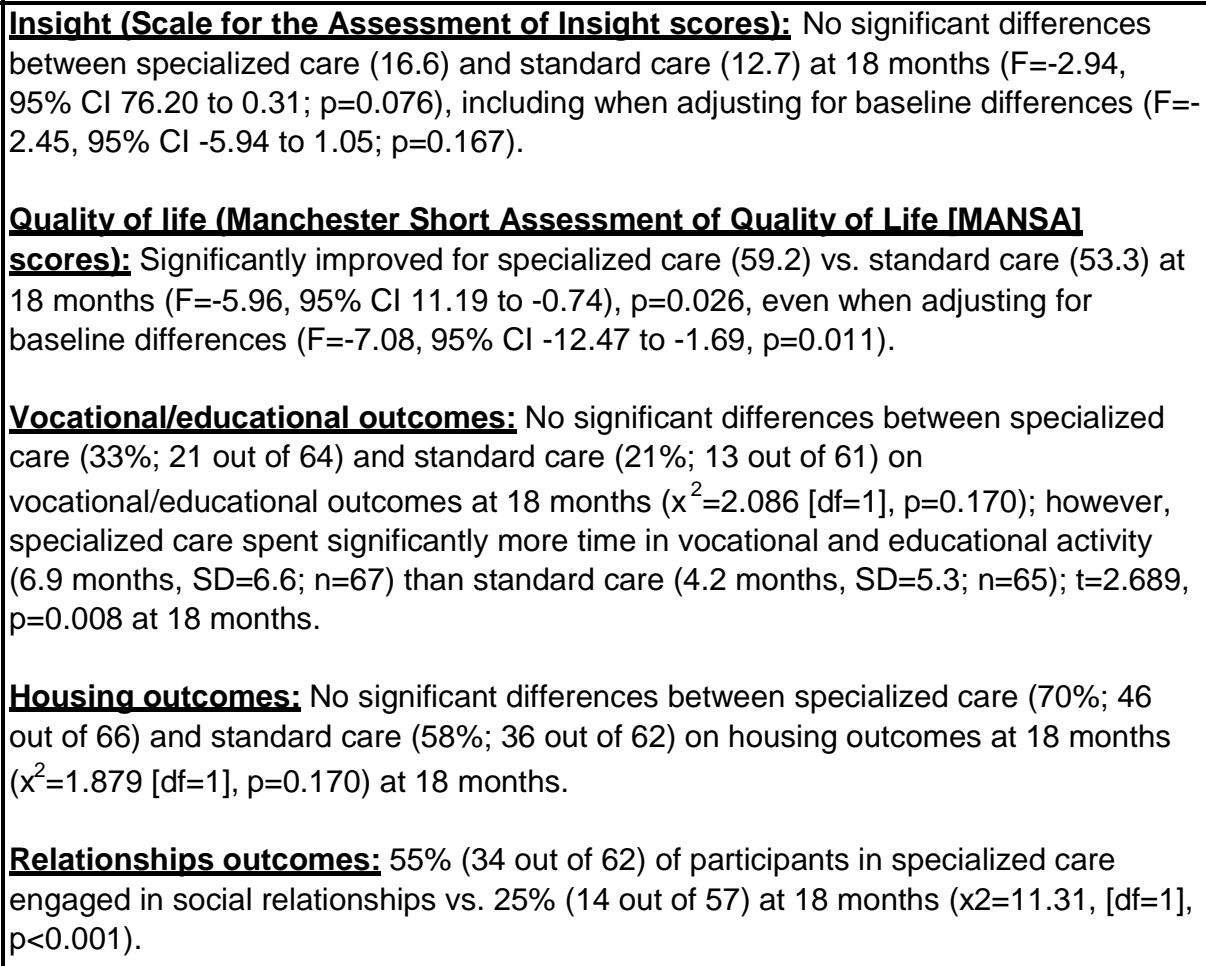 & see above & see above & see above \\
\hline
\end{tabular}




\begin{tabular}{|c|c|c|c|c|}
\hline Author, Year & $\begin{array}{l}\text { Setting } \\
\text { Country }\end{array}$ & Inclusion Criteria & $\begin{array}{l}\text { Interventions and Ns } \\
\text { per Group }\end{array}$ & Description of Intervention \\
\hline $\begin{array}{l}\text { Secondary analysis } \\
\text { of subset of } \\
\text { participants from the } \\
\text { Lambeth Early Onset } \\
\text { (LEO) Trial } \\
\text { Tempier } 2012\end{array}$ & England & $\begin{array}{l}\text { Residents of the London borough } \\
\text { of Lambeth, ages } 16-40 \text {, presented } \\
\text { to mental health services with } \\
\text { a first episode of nonaffective psychosis } \\
\text { between January } 2000 \text { and October } \\
2001 .\end{array}$ & \begin{tabular}{|l|} 
Specialized care \\
$(\mathrm{n}=57)$, standard care \\
$(\mathrm{n}=50)$
\end{tabular} & $\begin{array}{l}\text { Specialized early intervention following the ACT model, } \\
\text { including an interdisciplinary team, low patient-to-staff ratios } \\
\text { and high availability of individualized care. } \\
\text { Team adhered to Maudsley Prescribing Guidelines, and } \\
\text { had access to good practice guidelines for the wider } \\
\text { psychosocial management of first-episode psychosis, } \\
\text { psychological advice, and CBT. }\end{array}$ \\
\hline $\begin{array}{l}\text { OPUS } \\
\text { Bertelsen, } 2009\end{array}$ & $\begin{array}{l}\text { Multicenter } \\
\text { Denmark }\end{array}$ & $\begin{array}{l}\text { Age } 18-45 \text { years, first use of mental health } \\
\text { services, diagnosis within the schizophrenia } \\
\text { spectrum, and no prior use of antipsychotics } \\
\text { for more than } 12 \text { weeks }\end{array}$ & $\begin{array}{l}\text { Intensive early } \\
\text { intervention program } \\
(\mathrm{n}=275) \\
\text { Standard care } \\
(\mathrm{n}=272)\end{array}$ & $\begin{array}{l}\text { Intensive intervention, including assertive community } \\
\text { treatment, family treamtent, social skills training, and } \\
\text { antipsychotics }\end{array}$ \\
\hline
\end{tabular}




\begin{tabular}{|c|c|c|c|c|c|}
\hline Author, Year & Description of Comparator & Duration & $\begin{array}{l}\text { Age Gender } \\
\text { Race/Ethnicity }\end{array}$ & Other Population Characteristics & Total N \\
\hline $\begin{array}{l}\text { Secondary analysis } \\
\text { of subset of } \\
\text { participants from the } \\
\text { Lambeth Early Onset } \\
\text { (LEO) Trial } \\
\text { Tempier } 2012\end{array}$ & $\begin{array}{l}\text { Standard care provided by a } \\
\text { generic community mental } \\
\text { health team. Team adhered } \\
\text { to Maudsley Prescribing } \\
\text { Guidelines, and had access } \\
\text { to good practice guidelines for } \\
\text { the wider psychosocial } \\
\text { management of first-episode } \\
\text { psychosis, psychological } \\
\text { advice, and CBT. }\end{array}$ & $\begin{array}{l}\text { 6- and 18-month } \\
\text { followup }\end{array}$ & $\begin{array}{l}\text { Demographics (intervention, control) } \\
\text { Mean age: } 25.7,26.0 \\
\text { Gender/males: } 53 \%, 78 \% \text { (significantly } \\
\text { more males in standard care) } \\
\text { Race/ethnicity: } \\
\text { White }=42 \%, 24 \% \text { (difference not statistically } \\
\text { significant) } \\
\text { Black=42\%, } 58 \% \\
\text { Other=16\%, } 18 \%\end{array}$ & None reported. & 107 \\
\hline $\begin{array}{l}\text { OPUS } \\
\text { Bertelsen, } 2009\end{array}$ & $\begin{array}{l}\text { Standard treatment at a } \\
\text { community mental health } \\
\text { center, including } \\
\text { antipsychotics }\end{array}$ & $\begin{array}{l}\text { Intervention: } 2 \\
\text { years } \\
\text { Follow-up: } 5 \\
\text { years }\end{array}$ & $\begin{array}{l}\text { Demographics (intervention, control) } \\
\text { Mean age: } 26.6 \text { years, } 26.6 \text { years } \\
\text { Female sex: } 42 \%, 40 \% \\
\text { Race/ethnicity: NR }\end{array}$ & $\begin{array}{l}\text { (Intervention, control) } \\
\text { Inpatient at randomization: } 43 \%, 47 \% \\
\text { Median duration of untreated psychosis: } 46 \\
\text { weeks, } 53 \text { weeks } \\
\text { Schizophrenia diagnosis: } 67 \%, 65 \% \\
\text { Substance abuse: } 27 \%, 27 \%\end{array}$ & 547 \\
\hline
\end{tabular}




\begin{tabular}{|c|c|c|c|c|}
\hline Author, Year & Benefits Outcomes & Harms Outcomes & Funding & \begin{tabular}{|l} 
Quality \\
Rating
\end{tabular} \\
\hline $\begin{array}{l}\text { Secondary analysis } \\
\text { of subset of } \\
\text { participants from the } \\
\text { Lambeth Early Onset } \\
\text { (LEO) Trial } \\
\text { Tempier } 2012\end{array}$ & $\begin{array}{l}\text { Psychotic symptoms (PANSS Total score): Participants in specialized care } \\
\text { experienced significant improvement in symptoms }(51.60 \pm 15.41) \text { compared to those } \\
\text { in standard care }(59.70 \pm 14.12), t=2.51, d f=85, p=0.01) \text { at } 18 \text { months. } \\
\\
\text { Functioning (GAF): Participants in specialized care experienced significant } \\
\text { improvement in functioning }(64.20 \pm 15.23) \text { compared to those in standard care } \\
(55.89 \pm 14.04), t=2.59, d f=85, p=0.01) \text { at } 18 \text { months. } \\
\\
\text { Social networks: Significantly larger social networks for those in specialized care } \\
(2.40+1.2) \text { vs. standard care }(1.71+1.06) t=2.77, d f=84, p=0.01) \text { at } 18 \text { months. }\end{array}$ & None reported. & $\begin{array}{l}\text { Directorate of Health } \\
\text { and Social Care for } \\
\text { London R\&D } \\
\text { Organisation and } \\
\text { Management } \\
\text { Programme }\end{array}$ & Fair \\
\hline $\begin{array}{l}\text { OPUS } \\
\text { Bertelsen, } 2009\end{array}$ & $\begin{array}{l}\text { End of intervention (2 years) } \\
\text { GAF, symptoms: } 51.2 \text { vs. } 48.7 \text {; mean difference } 2.45(95 \% \mathrm{Cl}-0.32 \text { to } 5.22) \\
\text { GAF, function: } 55.2 \text { vs. } 51.1 \text {; mean difference } 3.12(95 \% \mathrm{Cl} 0.37 \text { to } 5.88) \\
\text { End of follow-up (5 years) } \\
\text { GAF, symptoms: } 53.5 \text { vs. } 53.8 \text {; mean difference }-0.16(95 \% \mathrm{Cl}-3.97 \text { to } 3.37) \\
\text { GAF, function: } 55.4 \text { vs. } 54.2 \text {; mean difference } 1.34(95 \% \mathrm{Cl}-2.65 \text { to } 5.34) \\
\text { Not living independently: } 4 \% \text { vs. } 10 \% \text {; OR } 2.3(95 \% \mathrm{Cl} 1.1 \text { to } 4.8) \mathrm{p}=0.02 \\
\text { Unemployed: } 57 \% \text { vs. } 54 \% \text {; OR } 1.1 \text { ( } 95 \% \mathrm{Cl} 0.8 \text { to } 1.6) \mathrm{p}=0.57 \\
\text { Suicide attempts: } 9 \% \text { vs. } 9 \% \text {; OR } 0.9 \text { ( } 95 \% \mathrm{Cl} 0.4 \text { to } 2.1) \mathrm{p}=0.86\end{array}$ & NR & $\begin{array}{l}\text { Danish Ministry of } \\
\text { Health; Danish } \\
\text { Ministry of Social } \\
\text { Affairs; University of } \\
\text { Copenhagen; } \\
\text { Copenhagen Hospital } \\
\text { Cooperation; Danish } \\
\text { Medical Research } \\
\text { Council; } \\
\text { Slagtermester } \\
\text { Worners Foundation; } \\
\text { and the Stanley } \\
\text { Wada Research } \\
\text { Foundation }\end{array}$ & Good \\
\hline
\end{tabular}




\begin{tabular}{|c|c|c|c|c|}
\hline Author, Year & $\begin{array}{l}\text { Setting } \\
\text { Country }\end{array}$ & Inclusion Criteria & $\begin{array}{l}\text { Interventions and Ns } \\
\text { per Group }\end{array}$ & Description of Intervention \\
\hline $\begin{array}{l}\text { OPUS } \\
\text { Secher } 2015 \\
\text { (Note: Referred to } \\
\text { description of OPUS } \\
\text { trial in Peterson, } \\
\text { 2005; did not include } \\
\text { Peterson due to its } \\
\text { inclusion in SR) }\end{array}$ & Denmark & $\begin{array}{l}18-45 \text { years of age, recent first diagnosis } \\
\text { within the schizophrenic spectrum (F2X.X in } \\
\text { ICD-10), and at most } 12 \text { consecutive weeks of } \\
\text { antipsychotic medication. In the } 10-y e a r \\
\text { followup study reported here, interviewed } \\
68 \% \text { of the participants who were alive and } \\
\text { lived in Denmark. } \\
\text { First episode psychosis, including: } \\
\text { Schizophrenia (67\% OPUS, } 65 \% \text { usual care), } \\
\text { Schizotypal (15\% OPUS, } 14 \% \text { usual care), } \\
\text { Brief Psychosis (7\% OPUS, } 10 \% \text { usual care) }\end{array}$ & $\begin{array}{l}\text { OPUS }(n=181) \text {, usual } \\
\text { care }(n=166) \text { Included } \\
\text { in ITT: OPUS } \\
(n=275), \text { usual care } \\
(n=272) \\
\end{array}$ & $\begin{array}{l}\text { Enhanced ACT, multi-family group psychoeducation, and } \\
\text { social skills training. Also offered CBT and supportive } \\
\text { therapy if needed. Staff-to-client ratio = 1:10. Antipsychotic } \\
\text { medication based on same principles for both groups. }\end{array}$ \\
\hline
\end{tabular}




\begin{tabular}{|c|c|c|c|c|c|}
\hline Author, Year & Description of Comparator & Duration & $\begin{array}{l}\text { Age Gender } \\
\text { Race/Ethnicity }\end{array}$ & Other Population Characteristics & Total N \\
\hline $\begin{array}{l}\text { OPUS } \\
\text { Secher } 2015 \\
\text { (Note: Referred to } \\
\text { description of OPUS } \\
\text { trial in Peterson, } \\
\text { 2005; did not include } \\
\text { Peterson due to its } \\
\text { inclusion in SR) }\end{array}$ & $\begin{array}{l}\text { Usual care in community } \\
\text { mental health, staff-to-client } \\
\text { ratio = 1:30. Antipsychotic } \\
\text { medication based on same } \\
\text { principles for both groups. }\end{array}$ & $\begin{array}{l}\text { Intervention } \\
\text { length: } 2 \text { years } \\
\text { Followup at } 10 \\
\text { years }\end{array}$ & $\begin{array}{l}\text { Demographics: (intervention, control) } \\
\text { Mean age: } 26.6 \text { for both conditions } \\
\text { Males: } 58 \% \text { for OPUS, } 60 \% \text { for usual care } \\
\text { Race/ethnicity: Not reported }\end{array}$ & $\begin{array}{l}\text { (intervention, control) } \\
\text { Median duration of untreated psychosis } \\
\text { (duration of untreated psychosis; weeks): } 46 \text {, } \\
53 \\
\text { Psychopathology scores (SAPS/SANS) } \\
\text { summarized into three dimensions: } \\
\text { Psychotic dimension: } 2.8,2.6 \\
\text { Negative dimension: } 2.2 \text { for both } \\
\text { Disorganized dimension: } 1.0 \text { for both } \\
\\
\text { Substance Abuse Diagnosis: } \\
27 \% \text { for both } \\
\text { Education: } \\
\text { None: } 60 \%, 59 \% \\
\text { Currently being educated: } 14 \%, 12 \% \\
\text { Short education/skilled: } 20 \%, 20 \% \\
\text { Longer education: } 6 \%, 9 \%\end{array}$ & 347 \\
\hline
\end{tabular}




\begin{tabular}{|c|c|c|c|c|}
\hline Author, Year & Benefits Outcomes & Harms Outcomes & Funding & $\begin{array}{l}\text { Quality } \\
\text { Rating }\end{array}$ \\
\hline \begin{tabular}{|l} 
OPUS \\
Secher 2015 \\
(Note: Referred to \\
description of OPUS \\
trial in Peterson, \\
2005; did not include \\
Peterson due to its \\
inclusion in SR)
\end{tabular} & 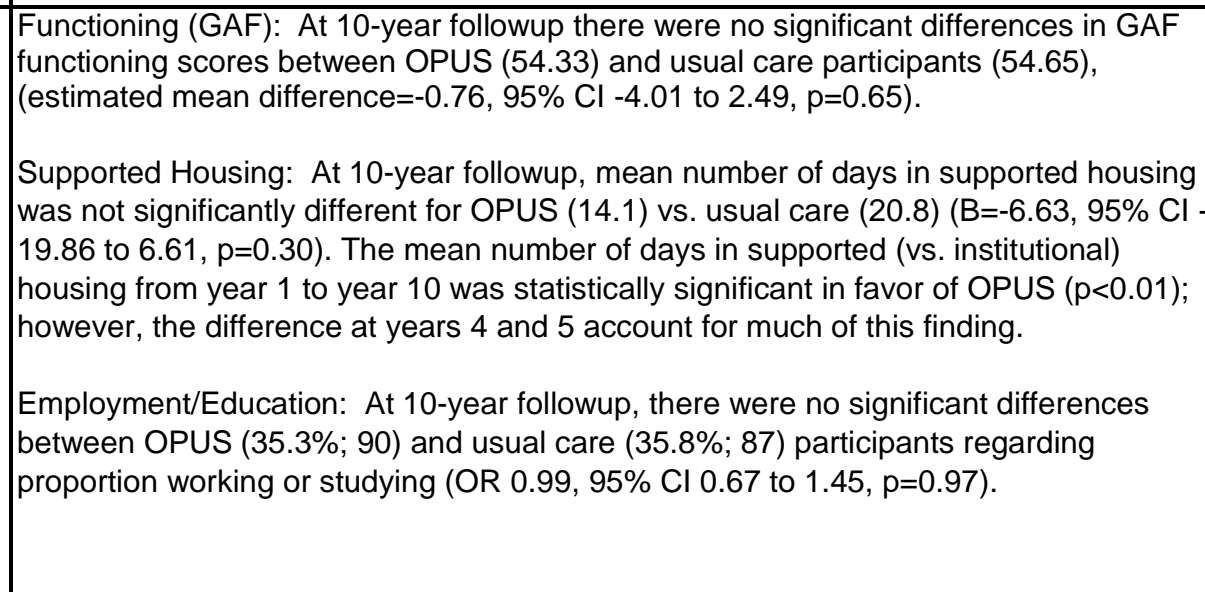 & $\begin{array}{l}\text { Deaths: After } 10 \text { years, } 14 \\
\text { OPUS participants (5.1\%) were } \\
\text { deceased vs. } 15 \text { usual care } \\
\text { participants (5.5\%), } p=0.83 \text {. } \\
\text { Suicidal ideation: The proportion } \\
\text { of participants who, at } 10 \text {-year } \\
\text { followup, had experienced } \\
\text { suicidal ideation within the } \\
\text { preceding two years was similar } \\
\text { to OPUS }(39.4 \%) \text { and in usual } \\
\text { care }(379 \%), p=0.77 \text {. }\end{array}$ & $\begin{array}{l}\text { Danish Council for } \\
\text { Independent } \\
\text { Research; } \\
\text { Trygfonden; The } \\
\text { Mental Health } \\
\text { Services of the } \\
\text { Capital Region of } \\
\text { Denmark; the Danish } \\
\text { Ministry of Health; the } \\
\text { Danish Ministry of } \\
\text { Social Affairs; the } \\
\text { Psychiatry and Social } \\
\text { Service Dept in } \\
\text { Central Denmark } \\
\text { Region }\end{array}$ & Good \\
\hline
\end{tabular}

\section{Please see Appendix B. Included Studies for full study references}

ACT=assertive community treatment, $\mathrm{CBT}=$ cognitive behavioral therapy, $\mathrm{CGI}=$ clinical global impression, $\mathrm{CI}=$ confidence interval, df=degrees of freedom, ETP=early treatment program, $\mathrm{F}=\mathrm{fixation}$ index, GAF=global assessment of functioning, HR=hazard ratio, ITT=intention to treat, LEO=Lambeth Early Onset, NIMH=National Institute of Mental Health, NOS=not otherwise specified, OPUS=Specialized Early Intervention Trail, OR=odds ratio, OT=occupational therapy, PANSS=positive and negative syndrome scale, QLS=quality of life scale, R\&D=research and development, SAPS=Scale for the Assessment of Positive Symptoms, SANS=Scale for Assessment of Negative Symptoms, SD=standard deviation, SR=systematic review, U.S.=United States 
Appendix Table E-23. Data abstraction of systematic reviews of co-occurring substance use and schizophrenia

\begin{tabular}{|c|c|c|c|c|c|c|c|}
\hline Author, Year & Aims & $\begin{array}{l}\text { Databases and } \\
\text { Timeperiod } \\
\text { Covered }\end{array}$ & $\begin{array}{l}\text { Number of } \\
\text { Studies } \\
\text { Number of } \\
\text { Patients }\end{array}$ & $\begin{array}{l}\text { Characteristics } \\
\text { of Identified } \\
\text { Articles: Study } \\
\text { Designs }\end{array}$ & $\begin{array}{l}\text { Characteristics of } \\
\text { Identified Articles: } \\
\text { Populations }\end{array}$ & $\begin{array}{l}\text { Characteristics of } \\
\text { Identified Articles: } \\
\text { Interventions }\end{array}$ & Outcomes Reported \\
\hline Hunt, 2013 & \begin{tabular}{|l|} 
Effect of various \\
psychosocial \\
interventions compared \\
with standard care for \\
people with both severe \\
mental illness and \\
substance misuse on \\
substance use and \\
schizophrenia outcomes
\end{tabular} & \begin{tabular}{|l|} 
Cochrane \\
Schizophrenia \\
Group Trials \\
Register (2008- \\
July 2012) \\
CDSR, \\
MEDLINE, \\
PsycINFO (2008- \\
January 2013) \\
Web of Science, \\
Scopus (2008- \\
February 2013) \\
\end{tabular} & $\begin{array}{l}32 \text { trials } \\
n=3,165\end{array}$ & RCTs & $\begin{array}{l}\text { Patients with both severe } \\
\text { mental illness (majority } \\
\text { schizophrenia, } \\
\text { schizoaffective disorder, or } \\
\text { psychosis) and substance } \\
\text { misuse disorder } \\
\text { Settings: } 3 \text { hospital-based } \\
\text { studies, } 19 \text { community- } \\
\text { based studies, } 10 \text { mixed } \\
\text { setting studies including } 2 \\
\text { with jail populations } \\
\text { Countries: } 19 \text { US, } 6 \\
\text { Australia, } 3 \text { UK, } 1 \text { each from } \\
\text { Denmark, Germany, Ireland } \\
\text { and Switzerland }\end{array}$ & $\begin{array}{l}\text { Various psychosocial } \\
\text { interventions } \\
\text { (Assertive Community } \\
\text { Treatment, Intensive } \\
\text { Case Management, } \\
\text { Cognitive Behavioral } \\
\text { Therapy, Motivational } \\
\text { Interviewing, } \\
\text { Contingency } \\
\text { Management, Social } \\
\text { Skills Training) } \\
\text { compared with usual } \\
\text { care }\end{array}$ & $\begin{array}{l}\text { Primary outcomes: } \\
\text { Loss to treatment } \\
\text { Change in substance } \\
\text { use } \\
\text { Symptoms } \\
\text { Secondary outcomes: } \\
\text { Death } \\
\text { Substance use } \\
\text { Mental state } \\
\text { Global function } \\
\text { Social function } \\
\text { QOL } \\
\text { Homelessness }\end{array}$ \\
\hline
\end{tabular}




\begin{tabular}{|c|c|}
\hline Author, Year & Effectiveness Outcomes \\
\hline Hunt, 2013 & 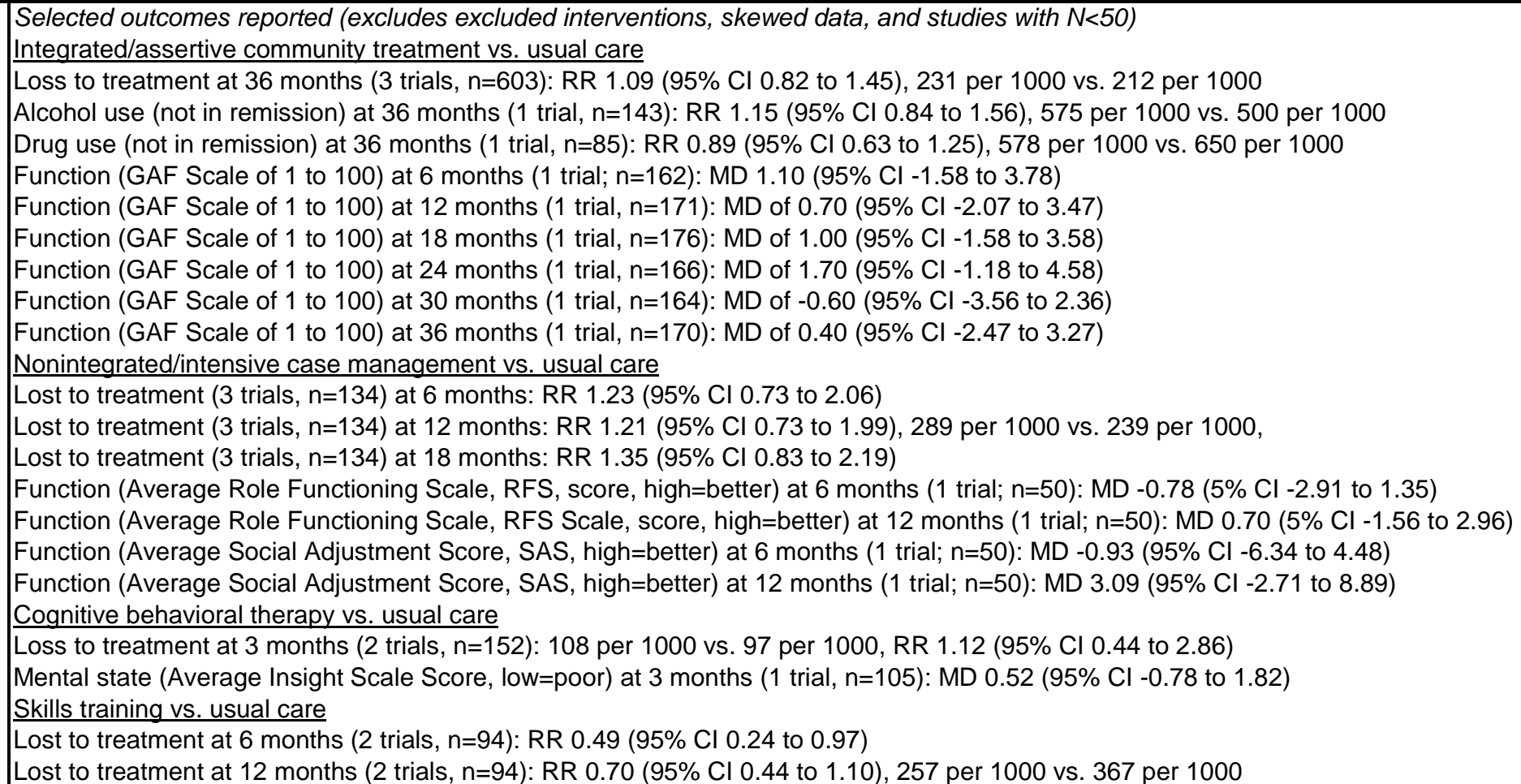 \\
\hline
\end{tabular}




\begin{tabular}{|c|c|c|c|}
\hline Author, Year & Harms Outcomes & Funding & Quality Rating \\
\hline Hunt, 2013 & $\begin{array}{l}\text { Selected outcomes reported (excludes excluded interventions, skewed data, and studies with } N<50) \\
\text { Integrated/assertive community treatment vs. usual care } \\
\text { Death at } 36 \text { months ( } 2 \text { trials, } n=421) \text { : RR } 1.18(95 \% \mathrm{Cl} 0.39 \text { to } 3.57) 33 \text { per } 1000 \text { vs. } 28 \text { per } 1000\end{array}$ & Not reported & Good \\
\hline
\end{tabular}

Please see Appendix B. Included Studies for full study references

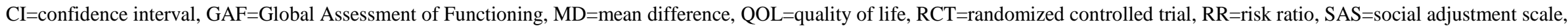
UK=United Kingdom, US=United States 


\section{Appendix F. Quality Assessment}

Appendix Table F-1. Quality assessment of systematic reviews of pharmacological treatments

\begin{tabular}{|c|c|c|c|c|c|c|}
\hline Author, Year & $\begin{array}{l}\text { 1. Was an "a priori" } \\
\text { design provided? }\end{array}$ & $\begin{array}{l}\text { 2. Was there duplicate } \\
\text { study selection and } \\
\text { data extraction? }\end{array}$ & $\begin{array}{l}\text { 3. Was a } \\
\text { comprehensive } \\
\text { literature search } \\
\text { performed? }\end{array}$ & $\begin{array}{l}\text { 4. Was the status of } \\
\text { publication (i.e. grey } \\
\text { literature) used as an } \\
\text { inclusion criterion? }\end{array}$ & $\begin{array}{l}\text { 5. Was a list of } \\
\text { studies (included } \\
\text { and excluded) } \\
\text { provided? }\end{array}$ & $\begin{array}{l}\text { 6. Were the } \\
\text { characteristics of the } \\
\text { included studies } \\
\text { provided? }\end{array}$ \\
\hline Abou-Setta 2012 & Yes & Yes & Yes & Yes & Yes & Yes \\
\hline $\begin{array}{l}\text { DERP SGA systematic } \\
\text { review, } 2013 \text { (Update 4) }\end{array}$ & Yes & $\begin{array}{l}\text { Yes } \\
\text { Yes }\end{array}$ & Yes & Yes & Yes & Yes \\
\hline
\end{tabular}




\begin{tabular}{|c|c|c|c|c|c|c|}
\hline Author, Year & $\begin{array}{l}\text { 7. Was the scientific } \\
\text { quality of the included } \\
\text { studies assessed and } \\
\text { documented? }\end{array}$ & $\begin{array}{l}\text { 8. Was the scientific quality of } \\
\text { the included studies used } \\
\text { appropriately in formulating } \\
\text { conclusions? }\end{array}$ & $\begin{array}{l}\text { 9. Were the methods } \\
\text { used to combine the } \\
\text { findings of studies } \\
\text { appropriate? }\end{array}$ & $\begin{array}{l}\text { 10. Was the } \\
\text { likelihood of } \\
\text { publication bias } \\
\text { assessed? }\end{array}$ & $\begin{array}{l}\text { 11. Was the } \\
\text { conflict of } \\
\text { interest } \\
\text { included? }\end{array}$ & $\begin{array}{l}\text { Quality } \\
\text { Rating }\end{array}$ \\
\hline Abou-Setta 2012 & Yes & Yes & Yes & Yes & Yes & Good \\
\hline $\begin{array}{l}\text { DERP SGA systematic } \\
\text { review, } 2013 \text { (Update 4) }\end{array}$ & Yes & Yes & Yes & $\begin{array}{l}\text { Not applicable due } \\
\text { to number of } \\
\text { poolable studies }\end{array}$ & $\begin{array}{l}\text { Yes for report } \\
\text { authors } \\
\text { No for individual } \\
\text { studies in this } \\
\text { update, but they } \\
\text { are likely mostly } \\
\text { industry funded }\end{array}$ & Good \\
\hline
\end{tabular}

Please see Appendix B. Included Studies for full study references 
Appendix Table F-2. Quality assessment of randomized controlled trials of pharmacological treatments

\begin{tabular}{|c|c|c|c|c|c|c|c|c|c|c|}
\hline $\begin{array}{l}\text { Author, Year } \\
\text { Study Name }\end{array}$ & $\begin{array}{l}\text { Random- } \\
\text { ization } \\
\text { adequate? }\end{array}$ & $\begin{array}{l}\text { Allocation } \\
\text { concealment } \\
\text { adequate? }\end{array}$ & $\begin{array}{l}\text { Groups similar at } \\
\text { baseline? }\end{array}$ & $\begin{array}{l}\text { Outcome } \\
\text { assessors } \\
\text { blinded? }\end{array}$ & $\begin{array}{l}\text { Clinician } \\
\text { blinded? }\end{array}$ & $\begin{array}{l}\text { Patient } \\
\text { blinded? }\end{array}$ & $\begin{array}{l}\text { Intention to } \\
\text { treat? }\end{array}$ & $\begin{array}{l}\text { Acceptable } \\
\text { level of } \\
\text { overall } \\
\text { attrition } \\
(\leq 30 \%) ?\end{array}$ & $\begin{array}{l}\text { Acceptable } \\
\text { level of } \\
\text { differential } \\
\text { attrition } \\
(<10 \%) ?\end{array}$ & $\begin{array}{l}\text { Overall } \\
\text { Quality }\end{array}$ \\
\hline Amr, 2013 & Yes & Yes & Yes & $\begin{array}{l}\text { No (clinicians } \\
\text { were one of } \\
\text { the raters) }\end{array}$ & No & Yes & No & $\begin{array}{l}\text { No } \\
73 / 156=47 \%\end{array}$ & \begin{tabular}{|l} 
No \\
$55 \%$ \\
quetiapine, \\
$39 \%$ \\
haloperidol
\end{tabular} & Poor \\
\hline Citrome, 2016 & Unclear & $\begin{array}{l}\text { Yes } \\
\text { Interactive } \\
\text { response } \\
\text { system }\end{array}$ & $\begin{array}{l}\text { Yes, though } \\
\text { comorbidity NR }\end{array}$ & $\begin{array}{l}\text { No } \\
\text { (raters aware) }\end{array}$ & $\begin{array}{l}\text { No } \\
\text { (open-label) }\end{array}$ & No & $\begin{array}{l}\text { Yes } \\
\text { (none excluded) }\end{array}$ & $\begin{array}{l}\text { No } \\
37 \%\end{array}$ & $\begin{array}{l}\text { Yes } \\
(38 \% \text { vs. 36\%) }\end{array}$ & Fair \\
\hline $\begin{array}{l}\text { Crespo-Faccoro } \\
2011\end{array}$ & Yes & Unclear & $\begin{array}{l}\text { No } \\
\text { Not schizophrenia } \\
\text { diagnosis }\end{array}$ & No & No & No & Yes & Yes & Yes & Fair \\
\hline Detke, 2014 & Unclear & Unclear & $\begin{array}{l}\text { Yes } \\
\text { Age, sex, age at } \\
\text { onset, length of } \\
\text { current episode, } \\
\text { and baseline } \\
\text { severity all similar }\end{array}$ & Unclear & $\begin{array}{l}\text { No } \\
\text { (open-label) }\end{array}$ & No & Yes & $\begin{array}{l}\text { No } \\
52.5 \%\end{array}$ & Yes & Poor \\
\hline Di Fiorino, 2014 & Yes & Yes & Unclear & No & No & No & Yes & $\begin{array}{l}\text { Yes } \\
(25 \%)\end{array}$ & $\begin{array}{l}\text { Yes } \\
17 \% \text { vs. } 25 \%\end{array}$ & Fair \\
\hline
\end{tabular}




\begin{tabular}{|c|c|c|c|c|c|c|c|c|c|c|}
\hline $\begin{array}{l}\text { Author, Year } \\
\text { Study Name }\end{array}$ & $\begin{array}{l}\text { Random- } \\
\text { ization } \\
\text { adequate? }\end{array}$ & $\begin{array}{l}\text { Allocation } \\
\text { concealment } \\
\text { adequate? }\end{array}$ & $\begin{array}{l}\text { Groups similar at } \\
\text { baseline? }\end{array}$ & $\begin{array}{l}\text { Outcome } \\
\text { assessors } \\
\text { blinded? }\end{array}$ & $\begin{array}{l}\text { Clinician } \\
\text { blinded? }\end{array}$ & $\begin{array}{l}\text { Patient } \\
\text { blinded? }\end{array}$ & $\begin{array}{l}\text { Intention to } \\
\text { treat? }\end{array}$ & $\begin{array}{l}\text { Acceptable } \\
\text { level of } \\
\text { overall } \\
\text { attrition } \\
(\leq 30 \%) ?\end{array}$ & $\begin{array}{l}\text { Acceptable } \\
\text { level of } \\
\text { differential } \\
\text { attrition } \\
(<10 \%) ?\end{array}$ & $\begin{array}{l}\text { Overall } \\
\text { Quality }\end{array}$ \\
\hline Durgam, 2014 & Unclear & Unclear & \begin{tabular}{|l|} 
Yes \\
\end{tabular} & Unclear & Unclear & Unclear & Yes & $\begin{array}{l}\text { No } \\
\text { Excluding } \\
\text { placebo arm, } \\
193 / 578=33 \% \\
\text { discontinued }\end{array}$ & $\begin{array}{l}\text { Yes Excluding } \\
\text { placebo, range } \\
27.9 \text { to } 37.9 \%\end{array}$ & Fair \\
\hline $\begin{array}{l}\text { Fleischhacker, } \\
2014 \\
\text { ASPIRE EU }\end{array}$ & Unclear & Unclear & Yes & $\begin{array}{l}\text { Unclear } \\
\text { Dosing } \\
\text { adjustments } \\
\text { allowed - no } \\
\text { explanation } \\
\text { given for how } \\
\text { conducted to } \\
\text { maintain } \\
\text { blinding }\end{array}$ & $\begin{array}{l}\text { Unclear } \\
\text { Dosing } \\
\text { adjustments } \\
\text { allowed - no } \\
\text { explanation } \\
\text { given for how } \\
\text { conducted to } \\
\text { maintain } \\
\text { blinding }\end{array}$ & Yes & Yes & $\begin{array}{l}\text { No } \\
157 / 531=30 \%\end{array}$ & $\begin{array}{l}\text { Yes } \\
26 \% \text { vs. } 33 \%\end{array}$ & Fair \\
\hline Green, 2015 & Yes & Unclear & Yes & Yes & Yes & No & Yes & Yes & Yes & Fair \\
\hline $\begin{array}{l}\text { Ishigooka, } 2015 \\
\text { ALPHA }\end{array}$ & Yes & Yes & $\begin{array}{l}\text { Unclear } \\
\text { Gender, age, } \\
\text { baseline severity } \\
\text { similar, but } \\
\text { duration of illness } \\
163 \text { vs. } 140 \\
\text { months }\end{array}$ & $\begin{array}{l}\text { Unclear } \\
\text { Dosing } \\
\text { adjustments } \\
\text { allowed - no } \\
\text { explanation } \\
\text { given for how } \\
\text { conducted to } \\
\text { maintain } \\
\text { blinding }\end{array}$ & $\begin{array}{l}\text { Unclear } \\
\text { Dosing } \\
\text { adjustments } \\
\text { allowed - no } \\
\text { explanation } \\
\text { given for how } \\
\text { conducted to } \\
\text { maintain } \\
\text { blinding }\end{array}$ & Yes & Yes & $\begin{array}{l}\text { No } \\
135 / 455=30 \%\end{array}$ & $\begin{array}{l}\text { Yes } \\
26 \% \text { to } 33 \%\end{array}$ & Fair \\
\hline
\end{tabular}




\begin{tabular}{|c|c|c|c|c|c|c|c|c|c|c|}
\hline $\begin{array}{l}\text { Author, Year } \\
\text { Study Name }\end{array}$ & $\begin{array}{l}\text { Random- } \\
\text { ization } \\
\text { adequate? }\end{array}$ & $\begin{array}{l}\text { Allocation } \\
\text { concealment } \\
\text { adequate? }\end{array}$ & $\begin{array}{l}\text { Groups similar at } \\
\text { baseline? }\end{array}$ & $\begin{array}{l}\text { Outcome } \\
\text { assessors } \\
\text { blinded? }\end{array}$ & $\begin{array}{l}\text { Clinician } \\
\text { blinded? }\end{array}$ & $\begin{array}{l}\text { Patient } \\
\text { blinded? }\end{array}$ & $\begin{array}{l}\text { Intention to } \\
\text { treat? }\end{array}$ & $\begin{array}{l}\text { Acceptable } \\
\text { level of } \\
\text { overall } \\
\text { attrition } \\
(\leq 30 \%) ?\end{array}$ & $\begin{array}{l}\text { Acceptable } \\
\text { level of } \\
\text { differential } \\
\text { attrition } \\
(<10 \%) ?\end{array}$ & $\begin{array}{l}\text { Overall } \\
\text { Quality }\end{array}$ \\
\hline Koshikawa, 2016 & $\begin{array}{l}\text { Yes } \\
\text { Computer- } \\
\text { generated }\end{array}$ & Unclear & \begin{tabular}{|l|} 
Unclear \\
Reported only for \\
the $70 \%$ of \\
participants \\
completing the \\
study
\end{tabular} & $\begin{array}{l}\text { No } \\
\text { Open-label }\end{array}$ & $\begin{array}{l}\text { No } \\
\text { Open-label }\end{array}$ & $\begin{array}{l}\text { No } \\
\text { Open-label }\end{array}$ & $\begin{array}{l}\text { No } \\
9 / 30(30 \%) \\
\text { excluded }\end{array}$ & $\begin{array}{l}\text { Yes } \\
30 \%\end{array}$ & $\begin{array}{l}\text { Yes } \\
29 \% \text { vs. 31\% }\end{array}$ & Fair \\
\hline Li, 2014 & $\begin{array}{l}\text { Unclear } \\
\text { Only } \\
\text { described as } \\
\text { randomized }\end{array}$ & $\begin{array}{l}\text { Unclear } \\
\text { "Assigned } \\
\text { sequentially in } \\
\text { ascending } \\
\text { order" }\end{array}$ & Yes & $\begin{array}{l}\text { Yes } \\
\text { Double- } \\
\text { dummy }\end{array}$ & $\begin{array}{l}\text { Yes } \\
\text { Double- } \\
\text { dummy }\end{array}$ & $\begin{array}{l}\text { Yes } \\
\text { Double- } \\
\text { dummy }\end{array}$ & $\begin{array}{l}\text { Yes } \\
\text { None excluded }\end{array}$ & $\begin{array}{l}\text { Yes } \\
41 / 279=15 \%\end{array}$ & $\begin{array}{l}\text { Yes } \\
17 \% \text { vs. } 12 \%\end{array}$ & Fair \\
\hline $\begin{array}{l}\text { Lieberman } 2005 \\
\text { (CATIE Study) }\end{array}$ & Yes & $\begin{array}{l}\text { Yes } \\
\text { "Done under DB } \\
\text { conditions" }\end{array}$ & $\begin{array}{l}\text { Few minor } \\
\text { differences }\end{array}$ & Yes & Yes & Yes & Yes & No & No & Good \\
\hline Liu, 2014 & Yes & Unclear & \begin{tabular}{|l|} 
Unclear \\
Age and baseline \\
PANSS similar but \\
duration of illness \\
4.5 vs. 5.5 months
\end{tabular} & No & No & No & Yes & Yes & Yes & Fair \\
\hline Maat, 2014 & $\begin{array}{l}\text { Unclear } \\
\text { Only } \\
\text { described as } \\
\text { randomized }\end{array}$ & Unclear & Yes & $\begin{array}{l}\text { No } \\
\text { Open-label }\end{array}$ & $\begin{array}{l}\text { No } \\
\text { Open-label }\end{array}$ & $\begin{array}{l}\text { No } \\
\text { Open-label }\end{array}$ & $\begin{array}{l}\text { No } \\
36 / 80=45 \% \\
\text { excluded }\end{array}$ & $\begin{array}{l}\text { No } \\
40 \% \\
\text { discontinued }\end{array}$ & $\begin{array}{l}\text { No } \\
47.4 \% \text { vs. } \\
33.3 \%\end{array}$ & Poor \\
\hline McEvoy, 2014 & Yes & Yes & Yes & Yes & Yes & Yes & Yes & \begin{tabular}{|l|} 
No \\
$130 / 311=42 \%$
\end{tabular} & Yes & Good \\
\hline $\begin{array}{l}\text { Naber, } 2013 \\
\text { RECOVER }\end{array}$ & Unclear & \begin{tabular}{|l|} 
Yes \\
IVRS
\end{tabular} & Mostly yes & No & No & No & $\begin{array}{l}\text { Yes } \\
\text { With LOCF }\end{array}$ & $\begin{array}{l}\text { No } \\
45 \%\end{array}$ & Yes & Fair \\
\hline
\end{tabular}




\begin{tabular}{|c|c|c|c|c|c|c|c|c|c|c|}
\hline $\begin{array}{l}\text { Author, Year } \\
\text { Study Name }\end{array}$ & $\begin{array}{l}\text { Random- } \\
\text { ization } \\
\text { adequate? }\end{array}$ & $\begin{array}{l}\text { Allocation } \\
\text { concealment } \\
\text { adequate? }\end{array}$ & $\begin{array}{l}\text { Groups similar at } \\
\text { baseline? }\end{array}$ & $\begin{array}{l}\text { Outcome } \\
\text { assessors } \\
\text { blinded? }\end{array}$ & $\begin{array}{l}\text { Clinician } \\
\text { blinded? }\end{array}$ & $\begin{array}{l}\text { Patient } \\
\text { blinded? }\end{array}$ & $\begin{array}{l}\text { Intention to } \\
\text { treat? }\end{array}$ & $\begin{array}{l}\text { Acceptable } \\
\text { level of } \\
\text { overall } \\
\text { attrition } \\
(\leq 30 \%) ?\end{array}$ & $\begin{array}{l}\text { Acceptable } \\
\text { level of } \\
\text { differential } \\
\text { attrition } \\
(<10 \%) ?\end{array}$ & $\begin{array}{l}\text { Overall } \\
\text { Quality }\end{array}$ \\
\hline $\begin{array}{l}\text { Naber, } 2015 \\
\text { QUALIFY }\end{array}$ & $\begin{array}{l}\text { Yes } \\
\text { Stratified } \\
\text { random- } \\
\text { ization }\end{array}$ & Unclear & $\begin{array}{l}\text { Unclear } \\
\text { Age, age at onset, } \\
\text { and gender } \\
\text { similar, but } \\
\text { baseline severity } \\
\text { not reported for all } \\
\text { patients } \\
\text { randomized ( } 9 \% \\
\text { excluded) }\end{array}$ & $\begin{array}{l}\text { Yes for QLS } \\
\text { and IAQ but } \\
\text { not for other } \\
\text { assessments }\end{array}$ & No & No & No & $\begin{array}{l}\text { No } \\
112 / 295=38 \%\end{array}$ & $\begin{array}{l}\text { No } \\
32 \% \text { vs. } 44 \%\end{array}$ & Fair \\
\hline Nemeth, 2017 & Yes & Yes & Yes & Yes & Unclear & Yes & $\begin{array}{l}\text { Yes (99\% in } \\
\text { each group) }\end{array}$ & $\begin{array}{l}\text { Yes (23\% in } \\
\text { each group) }\end{array}$ & Yes & Good \\
\hline $\begin{array}{l}\text { Parabiaghi, } 2016 \\
\text { GiSAS }\end{array}$ & $\begin{array}{l}\text { Yes } \\
\text { Computer- } \\
\text { generated, } \\
\text { stratified, } \\
\text { block }\end{array}$ & $\begin{array}{l}\text { Yes } \\
\text { Central with } \\
\text { IVRS }\end{array}$ & $\begin{array}{l}\text { Yes } \\
\text { Mostly similar } \\
\text { though some } \\
\text { baseline data } \\
\text { incomplete }\end{array}$ & $\begin{array}{l}\text { Yes } \\
\text { Outcome } \\
\text { assessment } \\
\text { and data } \\
\text { analysis } \\
\text { blinded }\end{array}$ & $\begin{array}{l}\text { No } \\
\text { Open-label }\end{array}$ & $\begin{array}{l}\text { No } \\
\text { Open-label }\end{array}$ & $\begin{array}{l}\text { Yes } \\
\text { None excluded, } \\
\text { both LOCF and } \\
\text { multiple } \\
\text { imputation used }\end{array}$ & $\begin{array}{l}\text { No } \\
86 / 200=43 \%\end{array}$ & $\begin{array}{l}\text { No } \\
53 \% \text { vs. } 33 \%\end{array}$ & Fair \\
\hline Park, 2013 & $\begin{array}{l}\text { Yes } \\
\text { Stratified } \\
\text { random- } \\
\text { ization }\end{array}$ & Unclear & $\begin{array}{l}\text { Unclear } \\
\text { PANSS } 67.5 \text { vs. } \\
82.0 \text { (small } \\
\text { sample, } N=20 \text { ) }\end{array}$ & $\begin{array}{l}\text { No } \\
\text { Open-label }\end{array}$ & $\begin{array}{l}\text { No } \\
\text { Open-label }\end{array}$ & $\begin{array}{l}\text { No } \\
\text { Open-label }\end{array}$ & $\begin{array}{l}\text { Unclear } \\
\text { Followup and } \\
\text { N's analyzed } \\
\text { NR }\end{array}$ & $\begin{array}{l}\text { Unclear } \\
\text { Followup NR }\end{array}$ & Unclear & Poor \\
\hline Robinson, 2015 & Unclear & Unclear & Yes & Yes & Yes & Yes & $\begin{array}{l}\text { Yes } \\
\text { Mixed models }\end{array}$ & $\begin{array}{l}\text { No } \\
93 / 209=44 \%\end{array}$ & Yes & Fair \\
\hline San, 2012 & Yes & Yes & Yes & No & No & No & Yes & Yes & Yes & Good \\
\hline $\begin{array}{l}\text { Sanz-Fuentenebro, } \\
2013\end{array}$ & $\begin{array}{l}\text { No } \\
\text { (alternating } \\
\text { assignment) }\end{array}$ & No & $\begin{array}{l}\text { Some differences: } \\
60 \% \text { vs. } 80 \% \\
\text { male and duration } \\
\text { of active } \\
\text { psychosis } 7.5 \\
\text { months vs. } 12.3 \\
\text { months }\end{array}$ & Unclear & No & No & Yes with LOCF & \begin{tabular}{|l} 
No \\
$(53.3 \%)$
\end{tabular} & $\begin{array}{l}\text { No } \\
\text { (20\% vs. } 47 \%)\end{array}$ & Poor \\
\hline
\end{tabular}




\begin{tabular}{|c|c|c|c|c|c|c|c|c|c|c|}
\hline $\begin{array}{l}\text { Author, Year } \\
\text { Study Name }\end{array}$ & $\begin{array}{l}\text { Random- } \\
\text { ization } \\
\text { adequate? }\end{array}$ & $\begin{array}{l}\text { Allocation } \\
\text { concealment } \\
\text { adequate? }\end{array}$ & $\begin{array}{l}\text { Groups similar at } \\
\text { baseline? }\end{array}$ & $\begin{array}{l}\text { Outcome } \\
\text { assessors } \\
\text { blinded? }\end{array}$ & $\begin{array}{l}\text { Clinician } \\
\text { blinded? }\end{array}$ & $\begin{array}{l}\text { Patient } \\
\text { blinded? }\end{array}$ & $\begin{array}{l}\text { Intention to } \\
\text { treat? }\end{array}$ & $\begin{array}{l}\text { Acceptable } \\
\text { level of } \\
\text { overall } \\
\text { attrition } \\
(\leq 30 \%) ?\end{array}$ & $\begin{array}{l}\text { Acceptable } \\
\text { level of } \\
\text { differential } \\
\text { attrition } \\
(<10 \%) ?\end{array}$ & $\begin{array}{l}\text { Overall } \\
\text { Quality }\end{array}$ \\
\hline Savitz, 2016 & $\begin{array}{l}\text { Yes } \\
\text { (computer- } \\
\text { generated) }\end{array}$ & $\begin{array}{l}\text { Yes } \\
\text { (IWRS) }\end{array}$ & $\begin{array}{l}\text { Yes, though } \\
\text { comorbidity NR }\end{array}$ & Unclear & $\begin{array}{l}\text { Yes } \\
\text { (double } \\
\text { dummy) }\end{array}$ & $\begin{array}{l}\text { Yes } \\
\text { (double } \\
\text { dummy) }\end{array}$ & $\begin{array}{l}\text { Yes } \\
(2.1 \% \text { excluded } \\
\text { from mITT set } \\
\text { in double-blind } \\
\text { phase) }\end{array}$ & $\begin{array}{l}\text { Yes } \\
(17 \%)\end{array}$ & $\begin{array}{l}\text { Yes } \\
(16 \% \text { vs. 18\%) }\end{array}$ & Good \\
\hline Shoja Shafti, 2015 & $\begin{array}{l}\text { Unclear } \\
\text { Only } \\
\text { described as } \\
\text { randomized }\end{array}$ & Unclear & Yes & Yes & Yes & Yes & $\begin{array}{l}\text { Unclear } \\
\text { Followup and } \\
\text { N's analyzed } \\
\text { NR }\end{array}$ & $\begin{array}{l}\text { Unclear } \\
\text { Overall } \\
\text { withdrawals } \\
\text { NR }\end{array}$ & Unclear & Fair \\
\hline Subotnik, 2015 & Unclear & Unclear & $\begin{array}{l}\text { Unclear } \\
\text { Age, sex, \% } \\
\text { schizoaffective } \\
\text { and baseline } \\
\text { symptoms similar } \\
\text { but time since } \\
\text { onset } 7.9 \text { vs. } 6.9 \\
\text { months }\end{array}$ & No & No & No & Yes & $31 \%$ & $\begin{array}{l}\text { No } \\
25 \% \text { vs 37\% }\end{array}$ & Fair \\
\hline Tybura, 2013 & Unclear & Unclear & $\begin{array}{l}\text { Unclear } \\
\text { Age and baseline } \\
\text { PANSS similar, } \\
\text { but no other } \\
\text { baseline } \\
\text { characteristics } \\
\text { reported }\end{array}$ & $\begin{array}{l}\text { No } \\
\text { Open-label }\end{array}$ & $\begin{array}{l}\text { No } \\
\text { Open-label }\end{array}$ & $\begin{array}{l}\text { No } \\
\text { Open-label }\end{array}$ & $\begin{array}{l}\text { Yes } \\
\text { Tables show } \\
\text { N's at baseline } \\
\text { and } 3 \text { months } \\
\text { as the same }\end{array}$ & $\begin{array}{l}\text { Yes } \\
\text { Tables show } \\
\text { N's at baseline } \\
\text { and } 3 \text { months } \\
\text { as the same }\end{array}$ & $\begin{array}{l}\text { Yes } \\
\text { Tables show } \\
\text { N's at baseline } \\
\text { and } 3 \text { months } \\
\text { as the same }\end{array}$ & Poor \\
\hline Tybura, 2014 & Yes & Yes & Yes & Unclear & Unclear & Unclear & Yes & $\begin{array}{l}\text { Yes } \\
36 / 131=27 \% \\
\text { discontinued } \\
\text { (SGAs only) }\end{array}$ & $\begin{array}{l}\text { Yes } \\
32 \% \text { vs. } 24 \%\end{array}$ & Fair \\
\hline
\end{tabular}




\begin{tabular}{|c|c|c|c|c|c|c|c|c|c|c|}
\hline $\begin{array}{l}\text { Author, Year } \\
\text { Study Name }\end{array}$ & $\begin{array}{l}\text { Random- } \\
\text { ization } \\
\text { adequate? }\end{array}$ & \begin{tabular}{|l} 
Allocation \\
concealment \\
adequate?
\end{tabular} & $\begin{array}{l}\text { Groups similar at } \\
\text { baseline? }\end{array}$ & $\begin{array}{l}\text { Outcome } \\
\text { assessors } \\
\text { blinded? }\end{array}$ & $\begin{array}{l}\text { Clinician } \\
\text { blinded? }\end{array}$ & $\begin{array}{l}\text { Patient } \\
\text { blinded? }\end{array}$ & $\begin{array}{l}\text { Intention to } \\
\text { treat? }\end{array}$ & \begin{tabular}{|l|} 
Acceptable \\
level of \\
overall \\
attrition \\
$(\leq 30 \%) ?$
\end{tabular} & \begin{tabular}{|l|} 
Acceptable \\
level of \\
differential \\
attrition \\
$(<10 \%) ?$
\end{tabular} & $\begin{array}{l}\text { Overall } \\
\text { Quality }\end{array}$ \\
\hline Wani, 2015 & $\begin{array}{l}\text { Unclear } \\
\text { Only } \\
\text { described as } \\
\text { randomized }\end{array}$ & Unclear & Yes & $\begin{array}{l}\text { No } \\
\text { Open-label }\end{array}$ & $\begin{array}{l}\text { No } \\
\text { Open-label }\end{array}$ & $\begin{array}{l}\text { No } \\
\text { Open-label }\end{array}$ & \begin{tabular}{|l|} 
Unclear \\
States that \\
LOCF but gives \\
N's analyzed at \\
24 weeks as \\
those \\
continuing \\
treatment (Fig \\
1 ; excludes \\
$24 \%)$
\end{tabular} & $\begin{array}{l}\text { Yes } \\
15 / 62=24 \% \\
\\
\end{array}$ & \begin{tabular}{|l|} 
No \\
$16 \%$ vs. $32 \%$
\end{tabular} & Fair \\
\hline
\end{tabular}

Please see Appendix B. Included Studies for full study references

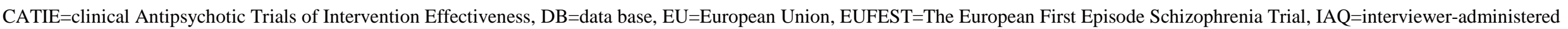

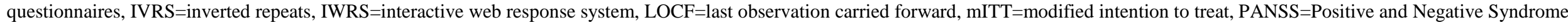
Scale, QLS=quality of life scale, SGA=second-generation antipsychotics 
Appendix Table F-3. Quality assessment of systematic reviews of psychosocial and nonpharmacological treatments

\begin{tabular}{|c|c|c|c|c|c|c|}
\hline Author, Year & $\begin{array}{l}\text { 1. Was an "a } \\
\text { priori" design } \\
\text { provided? }\end{array}$ & $\begin{array}{l}\text { 2. Was there duplicate } \\
\text { study selection and data } \\
\text { extraction? }\end{array}$ & $\begin{array}{l}\text { 3. Was a } \\
\text { comprehensive } \\
\text { literature search } \\
\text { performed? }\end{array}$ & $\begin{array}{l}\text { 4. Was the status of } \\
\text { publication used as an } \\
\text { inclusion criterion (i.e. was } \\
\text { grey literature included)? }\end{array}$ & $\begin{array}{l}\text { 5. Was a list of studies } \\
\text { (included and } \\
\text { excluded) provided? }\end{array}$ & $\begin{array}{l}\text { 6. Were the } \\
\text { characteristics of the } \\
\text { included studies } \\
\text { provided? }\end{array}$ \\
\hline \multicolumn{7}{|c|}{$\begin{array}{l}\text { Assertive community } \\
\text { treatment }\end{array}$} \\
\hline Marshall 2000a & Yes & Yes & Yes & Unclear & Yes & Yes \\
\hline \multicolumn{7}{|c|}{$\begin{array}{l}\text { Cognitive-behavioral } \\
\text { therapy }\end{array}$} \\
\hline Jauhar 2014 & Yes & Yes & Yes & Yes & Yes & Yes \\
\hline Jones 2010 & Yes & Yes & Yes & Yes & Yes & Yes \\
\hline Velthorst 2015 & Yes & Unclear & Yes & No & No & Yes \\
\hline \multicolumn{7}{|l|}{$\begin{array}{l}\text { Cognitive } \\
\text { remediation }\end{array}$} \\
\hline Cella 2017 & Yes & Yes & Yes & No & No & No \\
\hline Wykes 2011 & Yes & Yes & Yes & No & No & Yes \\
\hline \multicolumn{7}{|c|}{ Family interventions } \\
\hline Pharoah 2010 & Yes & \begin{tabular}{|l|} 
Yes \\
Yes
\end{tabular} & Yes & Yes & Yes & Yes \\
\hline \multicolumn{7}{|l|}{$\begin{array}{l}\text { Intensive case } \\
\text { management }\end{array}$} \\
\hline Marshall 2000b & Yes & Yes & Yes & Unclear & Yes & Yes \\
\hline \multicolumn{7}{|l|}{$\begin{array}{l}\text { IIIness self- } \\
\text { management }\end{array}$} \\
\hline Zou, 2012 & Yes & Yes & Yes & Unclear & No & Yes \\
\hline \multicolumn{7}{|c|}{ Psychoeducation } \\
\hline Pekkala 2009 & Yes & Yes & Yes & No & Yes & Yes \\
\hline
\end{tabular}




\begin{tabular}{|c|c|c|c|c|c|c|}
\hline Author, Year & $\begin{array}{l}\text { 7. Was the scientific } \\
\text { quality of the included } \\
\text { studies assessed and } \\
\text { documented? }\end{array}$ & $\begin{array}{l}\text { 8. Was the scientific quality of } \\
\text { the included studies used } \\
\text { appropriately in formulating } \\
\text { conclusions? }\end{array}$ & $\begin{array}{l}\text { 9. Were the methods } \\
\text { used to combine the } \\
\text { findings of studies } \\
\text { appropriate? }\end{array}$ & $\begin{array}{l}\text { 10. Was the likelihood } \\
\text { of publication bias } \\
\text { assessed? }\end{array}$ & $\begin{array}{l}\text { 11. Was the } \\
\text { conflict of interest } \\
\text { included? }\end{array}$ & Quality Rating \\
\hline \multicolumn{7}{|c|}{$\begin{array}{l}\text { Assertive community } \\
\text { treatment }\end{array}$} \\
\hline Marshall 2000a & Yes & Yes & Yes & No & Yes & Good \\
\hline \multicolumn{7}{|c|}{$\begin{array}{l}\text { Cognitive-behavioral } \\
\text { therapy }\end{array}$} \\
\hline Jauhar 2014 & Yes & Yes & Yes & Yes & Yes & Good \\
\hline Jones 2010 & Yes & Yes & Yes & Yes & Yes & Good \\
\hline Velthorst 2015 & Yes & Yes & Yes & Yes & Yes & Good \\
\hline \multicolumn{7}{|l|}{$\begin{array}{l}\text { Cognitive } \\
\text { remediation }\end{array}$} \\
\hline Cella 2017 & Yes & Yes & Yes & Yes & Yes & Good \\
\hline Wykes 2011 & Yes & Yes & Yes & Yes & Yes & Good \\
\hline \multicolumn{7}{|c|}{ Family interventions } \\
\hline Pharoah 2010 & Yes & Yes & Yes & Yes & Yes & Good \\
\hline \multicolumn{7}{|l|}{$\begin{array}{l}\text { Intensive case } \\
\text { management }\end{array}$} \\
\hline Marshall 2000b & Yes & Yes & Yes & No & Yes & Good \\
\hline \multicolumn{7}{|l|}{$\begin{array}{l}\text { IIIness self- } \\
\text { management }\end{array}$} \\
\hline Zou, 2012 & Yes & Yes & Yes & Yes & Yes & Fair \\
\hline \multicolumn{7}{|c|}{ Psychoeducation } \\
\hline Pekkala 2009 & Yes & Yes & Yes & Yes & No & Good \\
\hline
\end{tabular}




\begin{tabular}{|c|c|c|c|c|c|c|}
\hline Author, Year & $\begin{array}{l}\text { 1. Was an "a } \\
\text { priori" design } \\
\text { provided? }\end{array}$ & $\begin{array}{l}\text { 2. Was there duplicate } \\
\text { study selection and data } \\
\text { extraction? }\end{array}$ & $\begin{array}{l}\text { 3. Was a } \\
\text { comprehensive } \\
\text { literature search } \\
\text { performed? }\end{array}$ & $\begin{array}{l}\text { 4. Was the status of } \\
\text { publication used as an } \\
\text { inclusion criterion (i.e. was } \\
\text { grey literature included)? }\end{array}$ & $\begin{array}{l}\text { 5. Was a list of studies } \\
\text { (included and } \\
\text { excluded) provided? }\end{array}$ & $\begin{array}{l}\text { 6. Were the } \\
\text { characteristics of the } \\
\text { included studies } \\
\text { provided? }\end{array}$ \\
\hline \multicolumn{7}{|c|}{$\begin{array}{l}\text { Substance use and } \\
\text { schizophrenia }\end{array}$} \\
\hline Hunt, 2013 & Yes & \begin{tabular}{|l|} 
Yes \\
Yes
\end{tabular} & Yes & Yes & Yes & Yes \\
\hline \multicolumn{7}{|l|}{$\begin{array}{l}\text { Supported } \\
\text { employment }\end{array}$} \\
\hline Kinoshita, 2013 & Yes & Yes & Yes & Yes & Yes & Yes \\
\hline \multicolumn{7}{|c|}{ Supportive therapy } \\
\hline Buckley, 2015 & Yes & $\begin{array}{l}\text { Yes } \\
\text { Yes }\end{array}$ & Yes & No & Yes & Yes \\
\hline
\end{tabular}




\begin{tabular}{|l|l|l|l|l|l|}
\hline & $\begin{array}{l}\text { 7. Was the scientific } \\
\text { quality of the included } \\
\text { studies assessed and } \\
\text { documented? }\end{array}$ & $\begin{array}{l}\text { 8. Was the scientific quality of } \\
\text { the included studies used } \\
\text { appropriately in formulating } \\
\text { conclusions? }\end{array}$ & $\begin{array}{l}\text { 9. Were the methods } \\
\text { used to combine the } \\
\text { findings of studies } \\
\text { appropriate? }\end{array}$ & $\begin{array}{l}\text { 10. Was the likelihood } \\
\text { of publication bias } \\
\text { assessed? }\end{array}$ & $\begin{array}{l}\text { 11. Was the } \\
\text { conflict of interest } \\
\text { included? }\end{array}$ \\
\hline $\begin{array}{l}\text { Author, Year } \\
\text { Substance use and } \\
\text { schizophrenia }\end{array}$ & & & & & \\
\hline Qunt, 2013 & Yesuality Rating \\
\hline $\begin{array}{l}\text { Suppoorted } \\
\text { employment }\end{array}$ & & Yes & Yes & No & No \\
\hline Kinoshita, 2013 & Yes & Yes & & Good \\
\hline Supportive therapy & & & Yes & Yes & Yes \\
\hline Buckley, 2015 & Yes & Unclear & Yes & No & Ges \\
\hline
\end{tabular}

Please see Appendix B. Included Studies for full study references

ACT=assertive community treatment, ICM=intensive case management 
Appendix Table F-4. Quality assessment of randomized controlled trials of psychosocial and nonpharmacological treatments

\begin{tabular}{|c|c|c|c|c|c|c|c|c|}
\hline $\begin{array}{l}\text { Author, Year } \\
\text { Study name }\end{array}$ & $\begin{array}{l}\text { Randomization } \\
\text { adequate? }\end{array}$ & $\begin{array}{l}\text { Allocation } \\
\text { concealment } \\
\text { adequate? }\end{array}$ & $\begin{array}{l}\text { Groups similar } \\
\text { at baseline? }\end{array}$ & $\begin{array}{l}\text { Outcome } \\
\text { assessors } \\
\text { blinded? }\end{array}$ & $\begin{array}{l}\text { Intention to } \\
\text { treat? }\end{array}$ & $\begin{array}{l}\text { Acceptable } \\
\text { level of overall } \\
\text { attrition } \\
(\leq 30 \%) ?\end{array}$ & $\begin{array}{l}\text { Acceptable level } \\
\text { of differential } \\
\text { attrition }(<10 \%) ?\end{array}$ & $\begin{array}{l}\text { Overall } \\
\text { Quality }\end{array}$ \\
\hline \multicolumn{9}{|l|}{$\begin{array}{l}\text { Assertive community } \\
\text { Treatment }\end{array}$} \\
\hline Sytema, 2007 & Yes & Unclear & Yes & Unclear & Yes & No & No & Fair \\
\hline \multicolumn{9}{|l|}{$\begin{array}{l}\text { Cognitive-behavioral } \\
\text { Therapy }\end{array}$} \\
\hline Freeman 2015 & Yes & Yes & Yes & Yes & Yes & Yes & Yes & Good \\
\hline Lysaker 2009 & Unclear & Unclear & Yes & Yes & Unclear & Unclear & Unclear & Fair \\
\hline Malik 2009 & Yes & Unclear & Unclear & Yes & No & Yes & Yes & Fair \\
\hline Velligan 2015b & Yes & Unclear & Yes & Yes & Unclear & Yes & Unclear & Fair \\
\hline Zimmer 2007 & Yes & Unclear & Yes & Yes & No & Yes & Yes & Fair \\
\hline \multicolumn{9}{|c|}{ Cognitive remediation } \\
\hline Farreny 2012 & Yes & Unclear & Yes & Unclear & No & Yes & Yes & Fair \\
\hline Mueller 2015 & Yes & Unclear & Yes & Yes & No & Yes & Yes & Fair \\
\hline Twamley 2012 & Unclear & Unclear & No & Yes & Yes & Yes & Yes & Fair \\
\hline $\begin{array}{l}\text { Vita } 2011 \text { and Deste } \\
2015\end{array}$ & Yes & Unclear & Yes & Yes & Yes & Yes & Yes & Good \\
\hline
\end{tabular}




\begin{tabular}{|c|c|c|c|c|c|c|c|c|}
\hline $\begin{array}{l}\text { Author, Year } \\
\text { Study name }\end{array}$ & $\begin{array}{l}\text { Randomization } \\
\text { adequate? }\end{array}$ & $\begin{array}{l}\text { Allocation } \\
\text { concealment } \\
\text { adequate? } \\
\end{array}$ & $\begin{array}{l}\text { Groups similar } \\
\text { at baseline? }\end{array}$ & $\begin{array}{l}\text { Outcome } \\
\text { assessors } \\
\text { blinded? }\end{array}$ & $\begin{array}{l}\text { Intention to } \\
\text { treat? }\end{array}$ & $\begin{array}{l}\text { Acceptable } \\
\text { level of overall } \\
\text { attrition } \\
(\leq 30 \%) ?\end{array}$ & $\begin{array}{l}\text { Acceptable level } \\
\text { of differential } \\
\text { attrition }(<10 \%) ?\end{array}$ & $\begin{array}{l}\text { Overall } \\
\text { Quality }\end{array}$ \\
\hline \multicolumn{9}{|l|}{$\begin{array}{l}\text { Early intervention for } \\
\text { patients with } \\
\text { first-episode } \\
\text { psychosis }\end{array}$} \\
\hline $\begin{array}{l}\text { ETP-RAISE trial } \\
\text { Kane } 2015 \text { and Kane } \\
2016\end{array}$ & Unclear & Unclear & No & Yes & Unclear & Unclear & Unclear & Poor \\
\hline Guo 2007 and Guo 2010 & Unclear & Unclear & Yes & Yes & Yes & No & No & Fair \\
\hline \begin{tabular}{|l|} 
LEO trial \\
Craig 2004, Garety 2006
\end{tabular} & Yes & Yes & No & Yes & Yes & Yes & Yes & Good \\
\hline $\begin{array}{l}\text { LEO trial } \\
\text { Tempier, } 2012\end{array}$ & Yes & Yes & No & Yes & Yes & No & No & Fair \\
\hline $\begin{array}{l}\text { OPUS trial } \\
\text { Secher 2015, Bertelsen } \\
2009\end{array}$ & Yes & Yes & Yes & Yes & Yes & No & Yes & Good \\
\hline \multicolumn{9}{|l|}{ Family interventions } \\
\hline Dyck 2000 & Unclear & Unclear & Yes & Unclear & No & No & Yes & Poor \\
\hline Garety 2008 & Yes & Yes & Yes & Yes & Yes & Yes & Yes & Good \\
\hline Kopelowicz 2012 & Yes & Unclear & Yes & Unclear & Yes & No & $\begin{array}{l}\text { No, } 27 \%, 37 \% \text {, and } \\
51 \%\end{array}$ & Poor \\
\hline Mayoral, 2015 & Unclear & Unclear & Yes & Yes & $\begin{array}{l}\text { Unclear; likely } \\
\text { no }\end{array}$ & Yes & Yes & Fair \\
\hline Sellwood 2001 & Yes & Unclear & Unclear & Yes & Unclear & Yes & Yes & Fair \\
\hline Valencia 2007 & Unclear & Unclear & Yes & Yes & Yes & Yes & Yes & Fair \\
\hline \multicolumn{9}{|l|}{$\begin{array}{l}\text { Intensive case } \\
\text { Management }\end{array}$} \\
\hline Bjorkman, 2002 & Yes & Unclear & No & Unclear & Yes & Yes & No & Fair \\
\hline
\end{tabular}




\begin{tabular}{|c|c|c|c|c|c|c|c|c|}
\hline $\begin{array}{l}\text { Author, Year } \\
\text { Study name }\end{array}$ & $\begin{array}{l}\text { Randomization } \\
\text { adequate? }\end{array}$ & $\begin{array}{l}\text { Allocation } \\
\text { concealment } \\
\text { adequate? }\end{array}$ & $\begin{array}{l}\text { Groups similar } \\
\text { at baseline? }\end{array}$ & \begin{tabular}{|l} 
Outcome \\
assessors \\
blinded?
\end{tabular} & $\begin{array}{l}\text { Intention to } \\
\text { treat? }\end{array}$ & $\begin{array}{l}\text { Acceptable } \\
\text { level of overall } \\
\text { attrition } \\
(\leq 30 \%) ?\end{array}$ & $\begin{array}{l}\text { Acceptable level } \\
\text { of differential } \\
\text { attrition }(<10 \%) \text { ? }\end{array}$ & $\begin{array}{l}\text { Overall } \\
\text { Quality }\end{array}$ \\
\hline \multicolumn{9}{|l|}{$\begin{array}{l}\text { IIIness self- } \\
\text { management and } \\
\text { recovery }\end{array}$} \\
\hline Hasson-Ohayon, 2007 & Yes & Unclear & Yes & Unclear & Unclear & Yes & Unclear & Fair \\
\hline \multicolumn{9}{|l|}{ Social skills training } \\
\hline $\begin{array}{l}\text { Mueser } 2010 \text { and Bartels } \\
2014\end{array}$ & Yes & Unclear & No & Yes & No & Yes & Yes & Fair \\
\hline Valencia 2007 & Unclear & Unclear & Yes & Yes & No & Yes & Yes & Fair \\
\hline Valencia 2013 & Unclear & Unclear & Yes & Yes & Yes & Yes & Yes & Fair \\
\hline \multicolumn{9}{|l|}{ Supported employment } \\
\hline Cook, 2008, 2005 & $\begin{array}{l}\text { Yes, for entire study } \\
\text { population; data not } \\
\text { reported for } \\
\text { schizophrenia } \\
\text { subgroup }\end{array}$ & Unclear & \begin{tabular}{|l|} 
Yes, for entire \\
study population; \\
data not reported \\
for schizophrenia \\
subgroup \\
(Numerous \\
baseline \\
differences were \\
found between \\
those with \\
schizophrenia \\
and those with \\
other diagnoses)
\end{tabular} & Unclear & Unclear & \begin{tabular}{|l|} 
Yes, for entire \\
study population; \\
data not reported \\
for schizophrenia \\
subgroup
\end{tabular} & $\begin{array}{l}\text { Yes, for entire study } \\
\text { population; data not } \\
\text { reported for } \\
\text { schizophrenia } \\
\text { subgroup }\end{array}$ & Fair \\
\hline Mueser, 2004 & Unclear & Unclear & Yes & No & Yes & Yes & Yes & Fair \\
\hline
\end{tabular}

Please see Appendix B. Included Studies for full study references 


\section{Appendix G. Forest Plots for Pooled Analyses and Matrixes of Results for Network Meta-Analyses}

Appendix Table G-1a. SGA versus SGA network analysis of response (odds ratio, 95\% confidence interval)

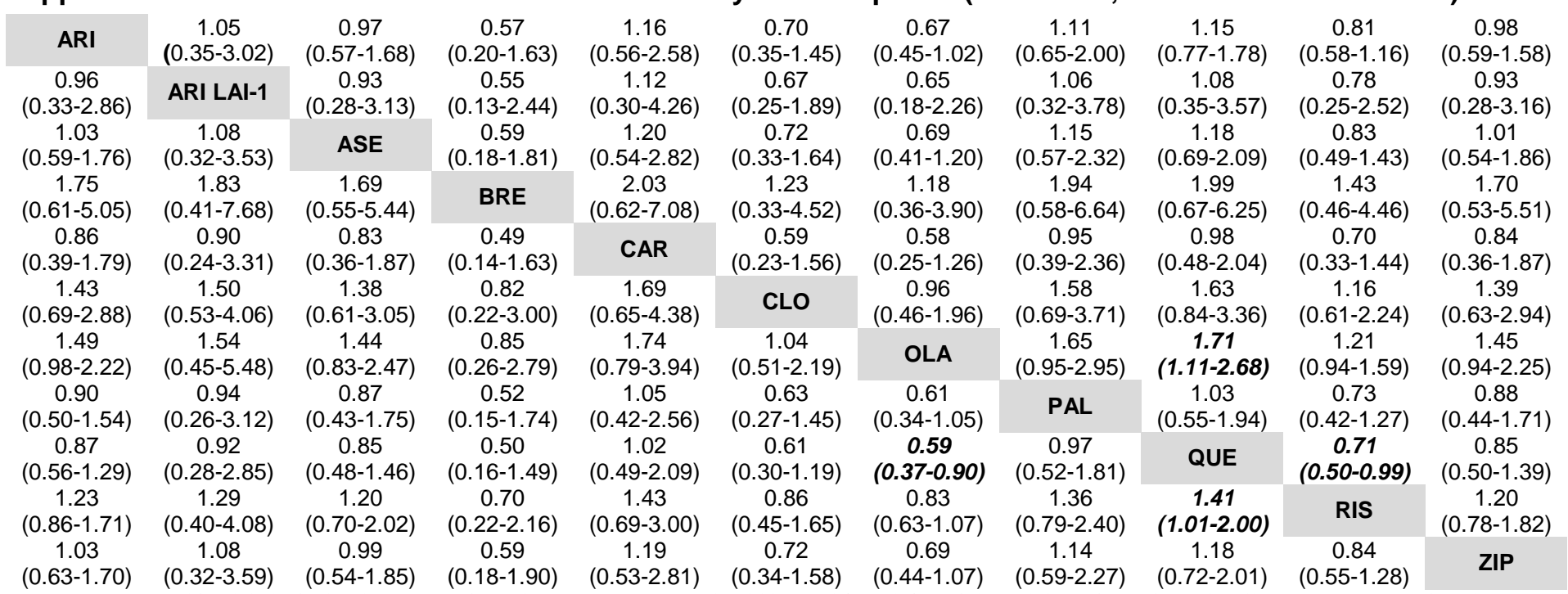

Drugs are reported in alphabetical order. Comparisons between treatments should be read from left to right and the estimate in each cell represents comparison between the row-defining treatment and the column-defining treatment. Odds ratios (ORs) higher than 1 favor the column-defining treatment, meaning less withdrawal in the column-defining treatment; and ORs less than 1 favor the rowdefining treatment, meaning less withdrawal in the row-defining treatment. For example, the OR for comparing QUE (row-defining treatment) vs. RIS (column-defining treatment) is 0.71 (95\% CrI 0.50 to 0.99 ), which means that QUE has lower odds of response and the comparison favors RIS, which has greater response. Each comparison is represented twice in the table, for example, for two treatments A and B, once with A versus B and once with B versus A (i.e. the reciprocal OR). For the example above, the OR for comparing RIS (now the row-defining treatment) vs. QUE (now the column-defining treatment) is 0.1.41 (95\% CrI 1.01 to 2.00), which means that RIS has a higher odds of response and again, it means that the comparison favors RIS, which has greater response. Significant results are in bold.

$\mathrm{ARI}=$ aripiprazole- ASE = asenapine- BRE = brepiprazole- CAR = cariprazine- CLO=clozapine- ILO = iloperidone- LUR = lurasidone- OLA = olanzapine. PAL=paliperidone, QUE=quetiapine, RIS=risperidone- ZIP=ziprasidone. LAI = long-acting injection; 1 = once monthly- 3 - every 3 months. 


\section{Appendix Table G1b. Numbers of studies per comparison contributing to network meta-analysis of response for SGAs versus SGAs}

\begin{tabular}{|l|l|}
\hline Comparison & No. Studies \\
\hline Aripiprazole : Brexipiprazole & 1 \\
\hline Aripiprazole : Olanzapine & 3 \\
\hline Aripiprazole : Quetiapine IR & 1 \\
\hline Aripiprazole : Risperidone & 3 \\
\hline Aripiprazole Monthly LAI : Aripiprazole & 1 \\
\hline Asenapine : Olanzapine & 3 \\
\hline Cariprazine : Risperidone & 1 \\
\hline Clozapine : Olanzapine & 3 \\
\hline Clozapine : Risperidone & 4 \\
\hline Paliperidone : Aripiprazole & 1 \\
\hline Paliperidone : Olanzapine & 1 \\
\hline Paliperidone Monthly LAI : Paliperidone 3-month LAI & 1 \\
\hline Paliperidone Monthly LAI : Risperidone LAI & 3 \\
\hline Quetiapine IR : Olanzapine & 5 \\
\hline Quetiapine IR : Risperidone & 9 \\
\hline Risperidone : Olanzapine & 16 \\
\hline Ziprasidone : Aripiprazole & 1 \\
\hline Ziprasidone : Olanzapine & 3 \\
\hline Ziprasidone : Quetiapine IR & 1 \\
\hline Ziprasidone : Risperidone & 1 \\
\hline
\end{tabular}

Appendix Table G-1c. SGAs versus SGAs meta-regression results for response

\begin{tabular}{|l|c|}
\hline Meta-regression variables & OR (95\% Cl) \\
\hline OR for duration & $1.15(0.57$ to 2.07$)$ \\
\hline Drug dose: Low versus Medium & $1.00(0.53$ to 1.78$)$ \\
\hline Drug dose: High versus Medium & $1.02(0.99$ to 1.04$)$ \\
\hline Response definition category B, as compared to category A & $0.94(0.67$ to 1.27$)$ \\
\hline Response definition category C, as compared to category A & $0.94(0.63$ to 1.34$)$ \\
\hline Treatment Resistant & $0.84(0.44$ to 1.49$)$ \\
\hline First Episode & $1.29(0.82$ to 2.0$)$ \\
\hline
\end{tabular}




\section{Appendix Table G-2a. SGA versus SGA network meta-analysis of withdrawals from study due to adverse events (odds ratio, $95 \%$}

confidence interval)

\begin{tabular}{|c|c|c|c|c|c|c|c|c|c|c|c|c|c|c|c|c|c|}
\hline ARI & $\begin{array}{c}0.90 \\
(0.31- \\
2.69)\end{array}$ & $\begin{array}{c}0.83 \\
(0.54- \\
1.25)\end{array}$ & $\begin{array}{c}0.48 \\
(0.02- \\
5.34)\end{array}$ & $\begin{array}{l}1.08 \\
(0.56- \\
2.12)\end{array}$ & $\begin{array}{c}0.43 \\
(0.21- \\
0.88)\end{array}$ & $\begin{array}{l}1.25 \\
(0.58- \\
2.76)\end{array}$ & $\begin{array}{c}0.62 \\
(0.37- \\
1.08)\end{array}$ & $\begin{array}{c}1.11 \\
(0.82- \\
1.48)\end{array}$ & $\begin{array}{c}0.69 \\
(0.21- \\
2.40)\end{array}$ & $\begin{array}{c}0.72 \\
(0.38- \\
1.30)\end{array}$ & $\begin{array}{c}0.80 \\
(0.35- \\
1.99)\end{array}$ & $\begin{array}{c}0.73 \\
(0.21- \\
2.78)\end{array}$ & $\begin{array}{c}0.32 \\
(0.04- \\
1.75)\end{array}$ & $\begin{array}{c}0.68 \\
(0.48- \\
1.01)\end{array}$ & $\begin{array}{c}0.80 \\
(0.57- \\
1.13)\end{array}$ & $\begin{array}{c}1.60 \\
(0.85- \\
3.05)\end{array}$ & $\begin{array}{c}0.64 \\
(0.44- \\
0.94)\end{array}$ \\
\hline $\begin{array}{l}1.12 \\
(0.37- \\
3.19)\end{array}$ & $\begin{array}{l}\text { ARI } \\
\text { LAI-1 }\end{array}$ & $\begin{array}{c}0.92 \\
(0.28- \\
2.80)\end{array}$ & $\begin{array}{c}0.52 \\
(0.02- \\
7.79)\end{array}$ & $\begin{array}{l}1.21 \\
(0.34- \\
3.98)\end{array}$ & $\begin{array}{c}0.49 \\
(0.19- \\
1.16)\end{array}$ & $\begin{array}{l}1.43 \\
(0.38- \\
5.04)\end{array}$ & $\begin{array}{c}0.71 \\
(0.21- \\
2.19)\end{array}$ & $\begin{array}{c}1.24 \\
(0.37- \\
3.76)\end{array}$ & $\begin{array}{c}0.78 \\
(0.15- \\
3.92)\end{array}$ & $\begin{array}{c}0.81 \\
(0.21- \\
2.94)\end{array}$ & $\begin{array}{c}0.89 \\
(0.42- \\
1.94)\end{array}$ & $\begin{array}{c}0.82 \\
(0.23- \\
2.86)\end{array}$ & $\begin{array}{c}0.36 \\
(0.03- \\
2.76)\end{array}$ & $\begin{array}{c}0.77 \\
(0.25- \\
2.28)\end{array}$ & $\begin{array}{c}0.89 \\
(0.28- \\
2.59)\end{array}$ & $\begin{array}{c}1.77 \\
(0.57- \\
5.56)\end{array}$ & $\begin{array}{c}0.72 \\
(0.23- \\
2.16)\end{array}$ \\
\hline $\begin{array}{l}1.21 \\
(0.80-\end{array}$ & $\begin{array}{c}1.09 \\
(0.36-\end{array}$ & ASE & $\begin{array}{r}0.58 \\
(0.02-\end{array}$ & $\begin{array}{c}1.32 \\
(0.65-\end{array}$ & $\begin{array}{c}0.52 \\
(0.25-\end{array}$ & $\begin{array}{c}1.51 \\
(0.67-\end{array}$ & $\begin{array}{c}0.75 \\
(0.43-\end{array}$ & $\begin{array}{c}1.34 \\
(0.95-\end{array}$ & $\begin{array}{c}0.85 \\
(0.25-\end{array}$ & $\begin{array}{c}0.88 \\
(0.44-\end{array}$ & $\begin{array}{c}0.96 \\
(0.40-\end{array}$ & $\begin{array}{c}0.90 \\
(0.24-\end{array}$ & $\begin{array}{c}0.39 \\
(0.04-\end{array}$ & $\begin{array}{c}0.83 \\
(0.54-\end{array}$ & $\begin{array}{c}0.97 \\
(0.65-\end{array}$ & $\begin{array}{c}1.93 \\
(0.96-\end{array}$ & $\begin{array}{c}0.78 \\
(0.51-\end{array}$ \\
\hline $\begin{array}{l}(0.80- \\
1.85)\end{array}$ & $\begin{array}{l}(0.36- \\
3.59)\end{array}$ & ASE & $\begin{array}{l}(0.02- \\
6.68)\end{array}$ & $\begin{array}{l}(0.65- \\
2.62)\end{array}$ & $\begin{array}{l}(0.25- \\
1.10)\end{array}$ & $\begin{array}{l}(0.67- \\
3.49)\end{array}$ & $\begin{array}{l}(0.43- \\
1.36)\end{array}$ & $\begin{array}{l}(0.95- \\
1.87)\end{array}$ & $\begin{array}{l}(0.25- \\
2.88)\end{array}$ & $\begin{array}{l}(0.44- \\
1.63)\end{array}$ & 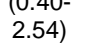 & $\begin{array}{l}(0.24- \\
3.64)\end{array}$ & $\begin{array}{l}(0.04-- \\
2.22)\end{array}$ & $\begin{array}{l}(0.54- \\
1.28)\end{array}$ & $\begin{array}{l}(0.65- \\
1.47)\end{array}$ & $\begin{array}{l}(0.90- \\
4.08)\end{array}$ & $\begin{array}{l}(0.51- \\
1.24)\end{array}$ \\
\hline 2.08 & 1.92 & 1.72 & & 2.34 & 0.89 & 2.57 & 1.27 & 2.29 & 1.50 & 1.52 & 1.71 & 1.60 & 0.68 & 1.42 & 1.65 & 3.32 & 1.33 \\
\hline $\begin{array}{l}(0.19- \\
57.77)\end{array}$ & $\begin{array}{l}(0.13- \\
62.00)\end{array}$ & $\begin{array}{l}(0.15- \\
48.12)\end{array}$ & BRE & $\begin{array}{l}(0.19- \\
62.11)\end{array}$ & $\begin{array}{l}(0.07- \\
26.93)\end{array}$ & $\begin{array}{l}(0.21- \\
78.40)\end{array}$ & $\begin{array}{l}(0.11- \\
36.14)\end{array}$ & $\begin{array}{l}(0.20- \\
66.19)\end{array}$ & $\begin{array}{l}(0.09- \\
51.64)\end{array}$ & $\begin{array}{l}(0.13- \\
44.95)\end{array}$ & $\begin{array}{l}(0.13- \\
53.38)\end{array}$ & $\begin{array}{l}(0.10- \\
58.93)\end{array}$ & $\begin{array}{l}(0.03- \\
30.54)\end{array}$ & $\begin{array}{l}(0.12- \\
39.94)\end{array}$ & $\begin{array}{l}(0.15- \\
46.54)\end{array}$ & $\begin{array}{c}(0.28- \\
100.40)\end{array}$ & $\begin{array}{l}(0.12- \\
37.67)\end{array}$ \\
\hline 0.92 & 0.83 & 0.76 & 0.43 & & 0.40 & 1.15 & 0.58 & 1.02 & 0.65 & 0.66 & 0.73 & 0.67 & 0.30 & 0.63 & 0.74 & 1.47 & 0.60 \\
\hline $\begin{array}{l}(0.47- \\
1.79)\end{array}$ & $\begin{array}{l}(0.25- \\
2.96)\end{array}$ & $\begin{array}{l}(0.38- \\
1.54)\end{array}$ & $\begin{array}{l}(0.02- \\
5.42)\end{array}$ & CAR & $\begin{array}{c}(0.17- \\
0.95)\end{array}$ & $\begin{array}{l}(0.46- \\
2.88)\end{array}$ & $\begin{array}{l}(0.28- \\
1.18)\end{array}$ & $\begin{array}{l}(0.51- \\
1.99)\end{array}$ & $\begin{array}{l}(0.18- \\
2.32)\end{array}$ & $\begin{array}{l}(0.28- \\
1.50)\end{array}$ & $\begin{array}{l}(0.29- \\
2.06)\end{array}$ & $\begin{array}{l}(0.18- \\
2.75)\end{array}$ & $\begin{array}{l}(0.03- \\
1.73)\end{array}$ & $\begin{array}{l}(0.35- \\
1.15)\end{array}$ & $\begin{array}{l}(0.41- \\
1.33)\end{array}$ & $\begin{array}{l}(0.66- \\
3.32)\end{array}$ & $\begin{array}{l}(0.31- \\
1.16)\end{array}$ \\
\hline 2.32 & 2.05 & 1.91 & 1.12 & 2.52 & & 2.93 & 1.46 & 2.54 & 1.60 & 1.66 & 1.85 & 1.71 & 0.75 & 1.58 & 1.84 & 3.66 & 1.50 \\
\hline $\begin{array}{l}(1.13- \\
4.71)\end{array}$ & $\begin{array}{l}(0.87- \\
5.26)\end{array}$ & $\begin{array}{l}(0.91- \\
3.96)\end{array}$ & $\begin{array}{l}(0.04- \\
13.61)\end{array}$ & $\begin{array}{l}(1.06- \\
5.90)\end{array}$ & CLO & $\begin{array}{l}(1.10- \\
7.54)\end{array}$ & $\begin{array}{l}(0.67- \\
3.18)\end{array}$ & $\begin{array}{l}(1.27- \\
5.21)\end{array}$ & $\begin{array}{l}(0.41- \\
6.43)\end{array}$ & $\begin{array}{c}(0.64- \\
4.20)\end{array}$ & $\begin{array}{l}(0.80- \\
4.37)\end{array}$ & $\begin{array}{l}(0.46- \\
6.38)\end{array}$ & $\begin{array}{c}(0.07- \\
4.66)\end{array}$ & $\begin{array}{l}(0.81- \\
3.08)\end{array}$ & $\begin{array}{l}(0.96- \\
3.58)\end{array}$ & $\begin{array}{l}(1.42- \\
10.05)\end{array}$ & $\begin{array}{l}(0.76- \\
2.91)\end{array}$ \\
\hline 0.80 & 0.70 & 0.66 & 0.39 & 0.87 & 0.34 & & 0.50 & 0.89 & 0.55 & 0.57 & 0.63 & 0.58 & 0.25 & 0.54 & 0.64 & 1.27 & 0.51 \\
\hline (0.36- & $(0.20-$ & $(0.29-$ & (0.01- & (0.35- & (0.13- & ILO & $(0.22-$ & (0.41- & (0.14- & $(0.22-$ & (0.21- & (0.13- & (0.02- & (0.26- & (0.31- & $(0.47-$ & (0.24- \\
\hline 1.61 & 1.42 & 1.33 & 0.78 & 1.73 & 0.68 & 2.01 & & 1.77 & 1.11 & 1.15 & 1.27 & 1.17 & 0.52 & 1.09 & 1.28 & 2.55 & 1.03 \\
\hline $\begin{array}{l}(0.93- \\
2.69)\end{array}$ & $\begin{array}{l}(0.46- \\
4.87)\end{array}$ & $\begin{array}{l}(0.74- \\
2.34)\end{array}$ & $\begin{array}{l}(0.03- \\
8.97)\end{array}$ & $\begin{array}{l}(0.85- \\
3.54)\end{array}$ & $\begin{array}{l}(0.31- \\
1.50)\end{array}$ & $\begin{array}{c}(0.86- \\
4.57)\end{array}$ & LUR & $\begin{array}{l}(1.06- \\
2.90)\end{array}$ & $\begin{array}{l}(0.32- \\
3.93)\end{array}$ & $\begin{array}{l}(0.53- \\
2.39)\end{array}$ & $\begin{array}{l}(0.51- \\
3.50)\end{array}$ & $\begin{array}{l}(0.30- \\
4.71)\end{array}$ & $\begin{array}{l}(0.06- \\
3.02)\end{array}$ & $\begin{array}{l}(0.67- \\
1.79)\end{array}$ & $\begin{array}{l}(0.79- \\
2.03)\end{array}$ & $\begin{array}{l}(1.19- \\
5.62)\end{array}$ & $\begin{array}{l}(0.63- \\
1.68)\end{array}$ \\
\hline 0.90 & 0.81 & 0.75 & 0.44 & 0.98 & 0.39 & 1.13 & 0.57 & & 0.63 & 0.65 & 0.72 & 0.66 & 0.29 & 0.62 & 0.72 & 1.44 & 0.58 \\
\hline $\begin{array}{l}(0.68- \\
1.23)\end{array}$ & $\begin{array}{l}(0.27- \\
2.71)\end{array}$ & $\begin{array}{l}(0.54- \\
1.05)\end{array}$ & $\begin{array}{c}(0.02- \\
4.92)\end{array}$ & $\begin{array}{l}(0.50- \\
1.95)\end{array}$ & $\begin{array}{c}(0.19- \\
0.79)\end{array}$ & $\begin{array}{l}(0.53- \\
2.48)\end{array}$ & $\begin{array}{c}(0.34- \\
0.94)\end{array}$ & OLA & $\begin{array}{l}(0.19- \\
2.18)\end{array}$ & $\begin{array}{c}(0.36- \\
1.14)\end{array}$ & $\begin{array}{l}(0.29- \\
1.95)\end{array}$ & $\begin{array}{l}(0.18- \\
2.74)\end{array}$ & $\begin{array}{l}(0.03- \\
1.61)\end{array}$ & $\begin{array}{c}(0.44- \\
0.87)\end{array}$ & $\begin{array}{c}(0.55- \\
0.96)\end{array}$ & $\begin{array}{l}(0.72- \\
2.98)\end{array}$ & $\begin{array}{c}(0.41- \\
0.82)\end{array}$ \\
\hline 1.44 & 1.28 & 1.19 & 0.67 & 1.54 & 0.63 & 1.81 & 0.90 & 1.59 & & 1.03 & 1.14 & 1.04 & 0.46 & 0.98 & 1.15 & 2.28 & 0.93 \\
\hline $\begin{array}{l}(0.42- \\
4.92)\end{array}$ & $\begin{array}{l}(0.26- \\
6.56)\end{array}$ & $\begin{array}{l}(0.35- \\
4.04)\end{array}$ & $\begin{array}{l}(0.02- \\
10.97)\end{array}$ & $\begin{array}{l}(0.43- \\
5.67)\end{array}$ & $\begin{array}{l}(0.16- \\
2.43)\end{array}$ & $\begin{array}{l}(0.45- \\
7.35)\end{array}$ & $\begin{array}{l}(0.26- \\
3.15)\end{array}$ & $\begin{array}{l}(0.46- \\
5.34)\end{array}$ & $\begin{array}{l}\text { OLA } \\
\text { LAI }\end{array}$ & $\begin{array}{l}(0.27- \\
3.81)\end{array}$ & $\begin{array}{l}(0.28- \\
4.90)\end{array}$ & $\begin{array}{l}(0.20- \\
6.14)\end{array}$ & $\begin{array}{l}(0.04- \\
3.89)\end{array}$ & $\begin{array}{l}(0.29- \\
3.38)\end{array}$ & $\begin{array}{l}(0.33- \\
4.00)\end{array}$ & $\begin{array}{l}(0.66- \\
8.33)\end{array}$ & $\begin{array}{l}(0.27- \\
3.15)\end{array}$ \\
\hline 1.38 & 1.24 & 1.14 & 0.66 & 1.51 & 0.60 & 1.74 & 0.87 & 1.54 & 0.97 & & 1.11 & 1.01 & 0.46 & 0.95 & 1.11 & 2.20 & 0.89 \\
\hline $\begin{array}{l}(0.77- \\
2.65)\end{array}$ & $\begin{array}{l}(0.34- \\
4.78)\end{array}$ & $\begin{array}{l}(0.62- \\
2.26)\end{array}$ & $\begin{array}{l}(0.02- \\
8.01)\end{array}$ & $\begin{array}{l}(0.67- \\
3.62)\end{array}$ & $\begin{array}{l}(0.24- \\
1.57)\end{array}$ & $\begin{array}{c}(0.70- \\
4.56)\end{array}$ & $\begin{array}{l}(0.42- \\
1.88)\end{array}$ & $\begin{array}{l}(0.88- \\
2.77)\end{array}$ & $\begin{array}{l}(0.26- \\
3.65)\end{array}$ & PAL & $\begin{array}{l}(0.38- \\
3.40)\end{array}$ & $\begin{array}{l}(0.24- \\
4.60)\end{array}$ & $\begin{array}{l}(0.05- \\
2.79)\end{array}$ & $\begin{array}{l}(0.51- \\
1.82)\end{array}$ & $\begin{array}{l}(0.60- \\
2.09)\end{array}$ & $\begin{array}{l}(0.96- \\
5.38)\end{array}$ & $\begin{array}{l}(0.48- \\
1.73)\end{array}$ \\
\hline 1.25 & 1.13 & 1.04 & 0.59 & 1.37 & 0.54 & 1.59 & 0.79 & 1.39 & 0.88 & 0.91 & & 0.92 & 0.41 & 0.86 & 1.01 & 1.99 & 0.81 \\
\hline $\begin{array}{l}(0.50- \\
2.90)\end{array}$ & $\begin{array}{l}(0.52- \\
2.41)\end{array}$ & $\begin{array}{l}(0.40- \\
2.50)\end{array}$ & $\begin{array}{l}(0.02- \\
7.96)\end{array}$ & $\begin{array}{l}(0.49- \\
3.41)\end{array}$ & $\begin{array}{l}(0.23- \\
1.25)\end{array}$ & $\begin{array}{l}(0.49- \\
4.76)\end{array}$ & $\begin{array}{l}(0.29- \\
1.99)\end{array}$ & $\begin{array}{l}(0.51- \\
3.43)\end{array}$ & $\begin{array}{l}(0.21- \\
3.54)\end{array}$ & $\begin{array}{l}(0.30- \\
2.62)\end{array}$ & $\begin{array}{l}\text { PAL } \\
\text { LAI }\end{array}$ & $\begin{array}{l}(0.36- \\
2.28)\end{array}$ & $\begin{array}{l}(0.04- \\
2.57)\end{array}$ & $\begin{array}{c}(0.36- \\
1.95)\end{array}$ & $\begin{array}{l}(0.40- \\
2.32)\end{array}$ & $\begin{array}{l}(1.00- \\
3.89)\end{array}$ & $\begin{array}{l}(0.32- \\
1.92)\end{array}$ \\
\hline 1.37 & 1.22 & 1.12 & 0.63 & 1.50 & 0.59 & 1.73 & 0.85 & 1.51 & $\begin{array}{l}0.04) \\
0.96\end{array}$ & $\begin{array}{l}2.02) \\
0.99\end{array}$ & 1.09 & & 0.43 & 0.93 & $\begin{array}{l}2.02) \\
1.09\end{array}$ & 2.18 & 0.88 \\
\hline $\begin{array}{l}(0.36- \\
4.80)\end{array}$ & $\begin{array}{c}(0.35- \\
4.31)\end{array}$ & $\begin{array}{l}(0.28- \\
4.19)\end{array}$ & $\begin{array}{l}(0.02- \\
10.31)\end{array}$ & $\begin{array}{l}(0.36- \\
5.65)\end{array}$ & $\begin{array}{l}(0.16- \\
2.16)\end{array}$ & $\begin{array}{l}(0.35- \\
7.58)\end{array}$ & $\begin{array}{l}(0.21- \\
3.32)\end{array}$ & $\begin{array}{l}(0.37- \\
5.71)\end{array}$ & $\begin{array}{l}(0.16- \\
5.01)\end{array}$ & $\begin{array}{c}(0.22- \\
4.26)\end{array}$ & $\begin{array}{l}(0.44- \\
2.77)\end{array}$ & $\begin{array}{l}\text { PAL } \\
\text { LAl-3 }\end{array}$ & $\begin{array}{l}(0.03- \\
3.66)\end{array}$ & $\begin{array}{l}(0.25- \\
3.35)\end{array}$ & $\begin{array}{l}(0.27- \\
4.05)\end{array}$ & $\begin{array}{l}(0.69- \\
6.83)\end{array}$ & $\begin{array}{l}(0.23- \\
3.20)\end{array}$ \\
\hline 3.13 & 2.80 & 2.56 & 1.48 & 3.32 & 1.34 & 3.93 & 1.94 & 3.40 & 2.17 & 2.19 & 2.46 & 2.30 & & 2.10 & 2.43 & 4.83 & 1.97 \\
\hline (0.57- & (0.36- & $\begin{array}{l}(0.45- \\
2378)\end{array}$ & (0.03- & $(0.58-$ & $(0.22-$ & $(0.63-$ & $(0.33-$ & $(0.62-)$ & $\begin{array}{l}(0.26- \\
24.49)\end{array}$ & (0.36- & $(0.39-$ & (0.27- & $\begin{array}{c}\text { QUE } \\
\text { ER }\end{array}$ & $(0.39-$ & $\begin{array}{l}(0.47- \\
23.59)\end{array}$ & $\begin{array}{l}(0.82- \\
47-58)\end{array}$ & $\begin{array}{l}(0.36- \\
18.35)\end{array}$ \\
\hline $\begin{array}{c}28.00) \\
1.47\end{array}$ & $\begin{array}{c}34.90) \\
1.30\end{array}$ & $\begin{array}{c}23 .(8) \\
1.21\end{array}$ & $\begin{array}{c}36.92) \\
0.70\end{array}$ & $\begin{array}{c}32.31) \\
1.59\end{array}$ & $\begin{array}{c}13.96) \\
0.63\end{array}$ & $\begin{array}{c}41.7() \\
1.84\end{array}$ & $\begin{array}{c}18.04) \\
0.92\end{array}$ & $\begin{array}{c}32.84) \\
1.62\end{array}$ & $\begin{array}{c}\text { c4.49) } \\
1.02\end{array}$ & $\begin{array}{c}22.09) \\
1.05\end{array}$ & $\begin{array}{c}28.03) \\
1.17\end{array}$ & $\begin{array}{c}1.13) \\
1.07\end{array}$ & 0.48 & & 1.17 & 2.33 & $\begin{array}{c}0.94 \\
0.94\end{array}$ \\
\hline $\begin{array}{l}(0.99- \\
2.10)\end{array}$ & $\begin{array}{c}(0.44- \\
4.04)\end{array}$ & $\begin{array}{l}(0.78- \\
1.85)\end{array}$ & $\begin{array}{c}(0.03- \\
8.16)\end{array}$ & $\begin{array}{l}(0.87- \\
2.89)\end{array}$ & $\begin{array}{l}(0.33- \\
1.23)\end{array}$ & $\begin{array}{l}(0.87- \\
3.82)\end{array}$ & $\begin{array}{l}(0.56- \\
1.50)\end{array}$ & $\begin{array}{l}(1.15- \\
2.26)\end{array}$ & $\begin{array}{l}(0.30- \\
3.44)\end{array}$ & $\begin{array}{l}(0.55- \\
1.97)\end{array}$ & $\begin{array}{l}(0.51- \\
2.81)\end{array}$ & $\begin{array}{l}(0.30- \\
4.06)\end{array}$ & $\begin{array}{l}(0.05- \\
2.61)\end{array}$ & QUE & $\begin{array}{l}(0.89- \\
1.49)\end{array}$ & $\begin{array}{l}(1.24- \\
4.46)\end{array}$ & $\begin{array}{l}(0.66- \\
1.33)\end{array}$ \\
\hline 1.26 & 1.12 & 1.04 & 0.61 & 1.35 & 0.54 & 1.57 & 0.78 & 1.39 & 0.87 & 0.91 & 0.99 & 0.92 & 0.41 & 0.85 & & 1.99 & 0.81 \\
\hline $\begin{array}{l}(0.89- \\
1.77)\end{array}$ & $\begin{array}{l}(0.39- \\
3.53)\end{array}$ & $\begin{array}{l}(0.68- \\
1.54)\end{array}$ & $\begin{array}{c}(0.02- \\
6.63)\end{array}$ & $\begin{array}{l}(0.75- \\
2.45)\end{array}$ & $\begin{array}{l}(0.28- \\
1.04)\end{array}$ & $\begin{array}{l}(0.79- \\
3.24)\end{array}$ & $\begin{array}{l}(0.49- \\
1.26)\end{array}$ & $\begin{array}{l}(1.04- \\
1.81)\end{array}$ & $\begin{array}{l}(0.25- \\
3.08)\end{array}$ & $\begin{array}{l}(0.48- \\
1.68)\end{array}$ & $\begin{array}{l}(0.43- \\
2.52)\end{array}$ & $\begin{array}{l}(0.25- \\
3.67)\end{array}$ & $\begin{array}{l}(0.04- \\
2.16)\end{array}$ & $\begin{array}{l}(0.67- \\
1.13)\end{array}$ & RIS & $\begin{array}{l}(1.01- \\
4.07)\end{array}$ & $\begin{array}{l}(0.58- \\
1.11)\end{array}$ \\
\hline 0.63 & 0.57 & 0.52 & 0.30 & 0.68 & 0.27 & 0.79 & 0.39 & 0.70 & 0.44 & 0.45 & 0.50 & 0.46 & 0.21 & 0.43 & 0.50 & & 0.40 \\
\hline $\begin{array}{l}(0.33- \\
1.17)\end{array}$ & $\begin{array}{l}(0.18- \\
1.74)\end{array}$ & $\begin{array}{l}(0.25- \\
1.04)\end{array}$ & $\begin{array}{l}(0.01- \\
3.54)\end{array}$ & $\begin{array}{l}(0.30- \\
1.52)\end{array}$ & $\begin{array}{c}(0.10- \\
0.71)\end{array}$ & $\begin{array}{l}(0.29- \\
2.12)\end{array}$ & $\begin{array}{c}(0.18- \\
0.84)\end{array}$ & $\begin{array}{l}(0.34- \\
1.40)\end{array}$ & $\begin{array}{l}(0.12- \\
1.53)\end{array}$ & $\begin{array}{l}(0.19- \\
1.04)\end{array}$ & $\begin{array}{l}(0.26- \\
1.00)\end{array}$ & $\begin{array}{l}(0.15- \\
1.45)\end{array}$ & $\begin{array}{l}(0.02- \\
1.22)\end{array}$ & $\begin{array}{c}(0.22- \\
0.81)\end{array}$ & $\begin{array}{c}(0.25- \\
0.99)\end{array}$ & RIS LAI & $\begin{array}{c}(0.20- \\
0.82)\end{array}$ \\
\hline 1.55 & 1.39 & 1.28 & 0.75 & 1.68 & 0.67 & 1.95 & 0.97 & 1.72 & 1.08 & 1.12 & 1.24 & 1.14 & 0.51 & 1.06 & 1.24 & 2.47 & \\
\hline $\begin{array}{l}(1.06- \\
2.26)\end{array}$ & $\begin{array}{c}(0.46- \\
4.44)\end{array}$ & $\begin{array}{l}(0.81- \\
1.98)\end{array}$ & $\begin{array}{c}(0.03- \\
8.36)\end{array}$ & $\begin{array}{l}(0.86- \\
3.19)\end{array}$ & $\begin{array}{l}(0.34- \\
1.31)\end{array}$ & $\begin{array}{l}(0.89- \\
4.18)\end{array}$ & $\begin{array}{l}(0.59- \\
1.59)\end{array}$ & $\begin{array}{l}(1.22- \\
2.42)\end{array}$ & $\begin{array}{l}(0.32- \\
3.77)\end{array}$ & $\begin{array}{l}(0.58- \\
2.09)\end{array}$ & $\begin{array}{l}(0.52- \\
3.10)\end{array}$ & $\begin{array}{l}(0.31- \\
4.44)\end{array}$ & $\begin{array}{l}(0.05- \\
2.80)\end{array}$ & $\begin{array}{l}(0.75- \\
1.52)\end{array}$ & $\begin{array}{l}(0.90- \\
1.73)\end{array}$ & $\begin{array}{l}(1.23- \\
5.08)\end{array}$ & ZIP \\
\hline
\end{tabular}


Drugs are reported in alphabetical order. Comparisons between treatments should be read from left to right and the estimate in each cell represents comparison between the row-defining treatment and the column-defining treatment. Odds ratios (ORs) higher than 1 favor the column-defining treatment, meaning less withdrawal in the column-defining treatment; and ORs less than 1 favor the row-

defining treatment, meaning less withdrawal in the row-defining treatment. For example, the OR for comparing LUR (row-defining treatment) vs. OLA (column-defining treatment) is 1.77 (95\% CrI 1.06 to 2.90), which means that LUR has higher odds of withdrawal and the comparison favors OLA, which has less withdrawal. Each comparison is represented twice in the table, for example, for two treatments A and B, once with A versus B and once with B versus A (i.e. the reciprocal OR). For the example above, the OR for comparing OLA (now the row-defining treatment) vs. LUR (now the column-defining treatment) is 0.57 (95\% CrI 0.34 to 0.94), which means that OLA has a lower odds of withdrawal and again, it means that the comparison favors OLA, which has less withdrawal. Significant results are in bold.

$\mathrm{ARI}=$ aripiprazole- $\mathrm{ASE}=$ asenapine- $\mathrm{BRE}=$ brepiprazole- $\mathrm{CAR}=$ cariprazine- $\mathrm{CLO}=$ clozapine- $\mathrm{ILO}=$ iloperidone- $\mathrm{LUR}=$ lurasidone- $\mathrm{OLA}=$ olanzapine. $\mathrm{PAL}=$ paliperidone, $\mathrm{QUE}=\mathrm{quetiapine}$, RIS=risperidone- ZIP=ziprasidone. LAI = long-acting injection; 1 = once monthly- 3 - every 3 months.

\section{Appendix Table G-2b. SGA versus SGA: numbers of studies per comparison contributing to network meta-analysis of withdrawals due} to adverse events

\begin{tabular}{|l|l|}
\hline Comparison & No. of studies \\
\hline Aripiprazole : Brexipiprazole & 1 \\
\hline Aripiprazole : Olanzapine & 9 \\
\hline Aripiprazole : Quetiapine IR & 3 \\
\hline Aripiprazole : Risperidone & 6 \\
\hline Aripiprazole : Risperidone LAI & 1 \\
\hline Aripiprazole : Ziprasidone & 3 \\
\hline Aripiprazole Monthly LAI : Aripiprazole & 2 \\
\hline Asenapine : Olanzapine & 6 \\
\hline Asenapine : Risperidone & 1 \\
\hline Cariprazine : Risperidone & 2 \\
\hline Clozapine : Olanzapine & 9 \\
\hline Clozapine : Risperidone & 9 \\
\hline Iloperidone : Risperidone & 2 \\
\hline Lurasidone : Olanzapine & 1 \\
\hline Lurasidone : Risperidone & 1 \\
\hline Lurasidone : Ziprasidone & 1 \\
\hline Olanzapine LAI : Olanzapine & 1 \\
\hline Paliperidone : Aripiprazole & 2 \\
\hline Paliperidone : Olanzapine & 3 \\
\hline Paliperidone : Risperidone & 1 \\
\hline Paliperidone : Ziprasidone & 1 \\
\hline Paliperidone Monthly LAI : Aripiprazole & 1 \\
\hline Paliperidone Monthly LAI : Paliperidone 3-month LAI & 1 \\
\hline Paliperidone Monthly LAI : Risperidone LAI & 4 \\
\hline Quetiapine ER : Risperidone & 1 \\
\hline Quetiapine IR : Clozapine & 2 \\
\hline Quetiapine IR : Olanzapine & 9 \\
\hline Quetiapine IR : Risperidone & 14 \\
\hline Quetiapine IR : Risperidone LAI & 1 \\
\hline Risperidone : Olanzapine & 16 \\
\hline Risperidone LAl : Risperidone & 1 \\
\hline Ziprasidone : Clozapine & 1 \\
\hline & \\
\hline
\end{tabular}




\begin{tabular}{|l|l|}
\hline Comparison & No. of studies \\
\hline Ziprasidone : Olanzapine & 9 \\
\hline Ziprasidone : Quetiapine IR & 5 \\
\hline Ziprasidone : Risperidone & 5 \\
\hline
\end{tabular}

Appendix Table G-2c. Meta-regression results for withdrawals due to adverse events

\begin{tabular}{|l|c|}
\hline Meta-regression variables & OR (95\% Cl) \\
\hline Duration & $0.69(0.36$ to 1.19$)$ \\
\hline Drug dose: Low versus Medium & $1.67(0.96$ to 2.60$)$ \\
\hline Drug dose: High versus Medium & $1.01(0.99$ to 1.03$)$ \\
\hline Treatment Resistant & $1.63(0.72$ to 3.26$)$ \\
\hline First Episode & $0.54(0.25$ to 1.03$)$ \\
\hline
\end{tabular}


Appendix Table G-3a. SGA versus SGA network meta-analysis of discontinuation from study for any cause (odds ratio, $95 \%$ confidence interval)

\begin{tabular}{|c|c|c|c|c|c|c|c|c|c|c|c|c|c|c|c|c|c|}
\hline ARI & $\begin{array}{l}1.18 \\
(0.73- \\
1.95)\end{array}$ & $\begin{array}{c}0.84 \\
(0.64- \\
1.09)\end{array}$ & $\begin{array}{c}0.94 \\
(0.37- \\
2.38)\end{array}$ & $\begin{array}{c}0.84 \\
(0.55- \\
1.29)\end{array}$ & $\begin{array}{l}1.45 \\
(0.97- \\
2.19)\end{array}$ & $\begin{array}{c}0.61 \\
(0.40- \\
0.94)\end{array}$ & $\begin{array}{c}0.76 \\
(0.54- \\
1.10)\end{array}$ & $\begin{array}{c}1.45 \\
(1.20- \\
1.76)\end{array}$ & $\begin{array}{c}0.66 \\
(0.37- \\
1.20)\end{array}$ & $\begin{array}{c}0.97 \\
(0.71- \\
1.32)\end{array}$ & $\begin{array}{c}0.81 \\
(0.51- \\
1.34)\end{array}$ & $\begin{array}{c}0.88 \\
(0.45- \\
1.82)\end{array}$ & $\begin{array}{c}2.16 \\
(0.77- \\
6.15)\end{array}$ & $\begin{array}{c}0.76 \\
(0.61- \\
0.95)\end{array}$ & $\begin{array}{c}0.99 \\
(0.82- \\
1.20)\end{array}$ & $\begin{array}{l}1.02 \\
(0.70- \\
1.50)\end{array}$ & $\begin{array}{c}0.84 \\
(0.67- \\
1.07)\end{array}$ \\
\hline $\begin{array}{c}0.85 \\
(0.51-\end{array}$ & ARI LAI- & $\begin{array}{c}0.71 \\
(0.41-\end{array}$ & $\begin{array}{c}0.80 \\
(0.27-\end{array}$ & $\begin{array}{c}0.71 \\
(0.38-\end{array}$ & $\begin{array}{l}1.23 \\
(0.79 \\
\end{array}$ & $\begin{array}{c}0.52 \\
(0.28-\end{array}$ & $\begin{array}{c}0.65 \\
(0.36-\end{array}$ & $\begin{array}{l}1.23 \\
(0.72-\end{array}$ & $\begin{array}{c}0.56 \\
(0.26-\end{array}$ & $\begin{array}{c}0.82 \\
(0.45-\end{array}$ & $\begin{array}{l}0.69 \\
(0.43-\end{array}$ & $\begin{array}{c}0.75 \\
(0.37-\end{array}$ & $\begin{array}{c}1.83 \\
(0.61-\end{array}$ & $\begin{array}{c}0.65 \\
0.38-\end{array}$ & $\begin{array}{c}0.84 \\
(0.50-\end{array}$ & $\begin{array}{l}0.87 \\
(0.47-\end{array}$ & $\begin{array}{c}0.72 \\
0.42-\end{array}$ \\
\hline 1.37$)$ & 1 & 1.23) & $2.25)$ & 1.31) & 1.93) & (0.98) & 1.17) & 2.10) & $1.17)$ & 1.47) & $\begin{array}{l}1.45- \\
1.09)\end{array}$ & $1.44)$ & $5.84)$ & 1.08) & 1.39) & $1.55)$ & $1.21)$ \\
\hline 1.19 & 1.41 & & 1.12 & 1.00 & 1.73 & 0.73 & 0.91 & 1.73 & 0.79 & 1.15 & 0.97 & 1.06 & 2.56 & 0.91 & 1.18 & 1.23 & 1.00 \\
\hline $\begin{array}{l}(0.92- \\
1.57)\end{array}$ & $\begin{array}{l}(0.81- \\
2.44)\end{array}$ & ASE & $\begin{array}{l}(0.42- \\
2.94)\end{array}$ & $\begin{array}{l}(0.64- \\
1.56)\end{array}$ & $\begin{array}{l}(1.15- \\
2.63)\end{array}$ & $\begin{array}{l}(0.46- \\
1.14)\end{array}$ & $\begin{array}{l}(0.62- \\
1.32)\end{array}$ & $\begin{array}{l}(1.37- \\
2.19)\end{array}$ & $\begin{array}{l}(0.43- \\
1.43)\end{array}$ & (0.81- & $\begin{array}{l}(0.57- \\
1.63)\end{array}$ & $\begin{array}{l}(0.51- \\
2.17)\end{array}$ & $\begin{array}{l}(0.90- \\
7.47)\end{array}$ & $\begin{array}{l}(0.69- \\
1.19)\end{array}$ & $\begin{array}{l}(0.92- \\
151)\end{array}$ & (0.78- & (0.76- \\
\hline 1.06 & $\begin{array}{l}.44) \\
1.25\end{array}$ & 0.89 & & $\begin{array}{l}1.50) \\
0.90\end{array}$ & $\begin{array}{l}2.03) \\
1.55\end{array}$ & $\begin{array}{l}1.14) \\
0.65\end{array}$ & 0.81 & 1.54 & $\begin{array}{l}1.43) \\
0.71\end{array}$ & $\begin{array}{l}1.04) \\
1.03\end{array}$ & $\begin{array}{l}1.03) \\
0.86\end{array}$ & 0.94 & $\begin{array}{l}1.47) \\
2.29\end{array}$ & 0.81 & $\begin{array}{l}1.51) \\
1.05\end{array}$ & $\begin{array}{l}1.85) \\
1.08\end{array}$ & $\begin{array}{l}1.33) \\
0.89\end{array}$ \\
\hline$(0.42-$ & $(0.44-$ & (0.34- & BRE & (0.32- & $(0.57-$ & $(0.23-$ & $(0.30-$ & $(0.60-$ & $(0.23-$ & (0.40- & $(0.31-$ & $(0.31-$ & $(0.57-$ & (0.31- & (0.41- & (0.40- & $(0.34-$ \\
\hline 2.74) & 3.77) & 2.41) & & 2.57) & 4.38) & 1.80) & 2.27) & 4.02) & 2.14) & 2.75) & 2.52) & $3.10)$ & $9.56)$ & 2.11) & 2.73) & 3.03) & 2.37) \\
\hline 1.19 & 1.42 & 1.00 & 1.12 & & 1.72 & 0.73 & 0.91 & 1.73 & 0.79 & 1.15 & 0.97 & 1.06 & 2.56 & 0.91 & 1.18 & 1.22 & 1.00 \\
\hline$(0.77-$ & $(0.76-$ & (0.64- & $(0.39-$ & CAR & (1.01- & $(0.42-$ & (0.56- & (1.13- & $(0.40-$ & $(0.71-$ & $(0.57-$ & (0.50- & (0.92- & $(0.60-$ & $(0.80-$ & $(0.75-$ & (0.65- \\
\hline 1.83) & 2.63) & 1.57) & 3.12) & & 2.93) & 1.25) & 1.48) & 2.69) & 1.53) & 1.90) & 1.71) & 2.24) & 7.72) & 1.37) & 1.75) & 2.01) & 1.58) \\
\hline 0.69 & 0.82 & 0.58 & 0.65 & 0.58 & & 0.42 & 0.53 & 1.00 & 0.46 & 0.67 & 0.56 & 0.61 & 1.49 & 0.53 & 0.69 & 0.71 & 0.58 \\
\hline (0.46- & (0.52- & (0.38- & (0.23- & (0.34- & CLO & (0.25- & (0.32- & $(0.68-$ & (0.23- & $(0.41-$ & (0.33- & $(0.29-$ & (0.54- & (0.35- & (0.47- & (0.40- & (0.39- \\
\hline 1.03) & 1.26) & 0.87 ) & $1.76)$ & 0.99 ) & & $0.70)$ & 0.84 ) & $1.45)$ & $0.90)$ & $1.07)$ & $0.92)$ & $1.24)$ & 4.52) & 0.78 ) & 0.98 ) & 1.24) & $0.84)$ \\
\hline $\begin{array}{c}1.65 \\
(1.06-\end{array}$ & $\begin{array}{c}1.93 \\
(1.02-\end{array}$ & $\begin{array}{l}1.37 \\
(0.87-\end{array}$ & $\begin{array}{l}1.53 \\
(0.56-\end{array}$ & $\begin{array}{l}1.38 \\
(0.80-\end{array}$ & $\begin{array}{r}2.37 \\
(1.42\end{array}$ & ILO & $\begin{array}{c}1.25 \\
(0.76-\end{array}$ & $\begin{array}{r}2.38 \\
(1.57-\end{array}$ & $\begin{array}{l}1.09 \\
(0.53-\end{array}$ & $\begin{array}{l}1.59 \\
10.97-\end{array}$ & $\begin{array}{l}1.34 \\
(0.73-\end{array}$ & $\begin{array}{c}1.44 \\
(0.64-\end{array}$ & $\begin{array}{r}3.53 \\
1.19\end{array}$ & 1.25 & 1.63 & 1.68 & $\begin{array}{l}1.38 \\
0.89-\end{array}$ \\
\hline 2.48) & 3.62) & 2.19) & (..28) & $2.36)$ & 4.03) & & 2.04) & (1.60) & 2.20) & 2.61) & 2.46) & $3.25)$ & 10.58) & 1.88) & 2.34) & $2.90)$ & 2.13) \\
\hline 1.32 & 1.55 & 1.10 & 1.23 & 1.10 & 1.89 & 0.80 & & 1.91 & 0.86 & 1.27 & 1.06 & 1.16 & 2.81 & 1.00 & 1.30 & 1.34 & 1.10 \\
\hline$(0.91-$ & (0.86- & (0.76- & (0.44- & (0.68- & (1.19- & (0.49- & LUR & (1.35- & (0.46- & $(0.83-$ & (0.62- & (0.54- & (1.00- & (0.71- & (0.93- & (0.84- & (0.78- \\
\hline 1.87) & 2.82) & 1.60) & 3.37) & 1.78) & 3.10) & 1.31) & & 2.67) & 1.67) & 1.94) & 1.89) & 2.53) & 8.57) & $1.40)$ & 1.79) & 2.15) & 1.54) \\
\hline 0.69 & 0.81 & 0.58 & 0.65 & 0.58 & 1.00 & 0.42 & 0.52 & & 0.46 & 0.67 & 0.56 & 0.60 & 1.49 & 0.53 & 0.68 & 0.71 & 0.58 \\
\hline (0.57- & (0.48- & $(0.46-$ & $(0.25-$ & (0.37- & $(0.69-$ & (0.28- & (0.37- & OLA & (0.25- & $(0.50-$ & $(0.33-$ & $(0.29-$ & (0.54- & $(0.42-$ & (0.59- & $(0.45-$ & $(0.48-$ \\
\hline 0.84$)$ & 1.40) & $0.73)$ & 1.68) & 0.89 ) & 1.48) & $0.64)$ & $0.74)$ & L & $0.84)$ & 0.89 ) & 0.96 ) & 1.31) & 4.29) & $0.65)$ & $0.79)$ & $1.09)$ & $0.71)$ \\
\hline 1.52 & 1.78 & 1.27 & 1.41 & 1.27 & 2.18 & 0.92 & 1.16 & 2.19 & & 1.47 & 1.23 & 1.34 & 3.28 & 1.16 & 1.50 & 1.55 & 1.27 \\
\hline (0.84- & $(0.85-$ & $(0.70-$ & $(0.47-$ & (0.65- & (1.12- & $(0.46-$ & $(0.60-$ & (1.19- & OLA LAI & $(0.77-$ & $(0.62-$ & $(0.58-$ & (1.02- & $(0.64-$ & $(0.82-$ & $(0.83-$ & $(0.69-$ \\
\hline 2.73) & 3.81 ) & 2.32) & 4.28) & 2.52) & 4.32) & $1.89)$ & $2.20)$ & 3.99) & & $2.75)$ & 2.47) & 3.11) & $10.80)$ & $2.04)$ & $2.66)$ & $2.91)$ & 2.31) \\
\hline 1.03 & 1.22 & 0.87 & 0.97 & 0.87 & 1.50 & 0.63 & 0.79 & 1.50 & 0.68 & & 0.84 & 0.91 & 2.24 & 0.79 & 1.02 & 1.06 & 0.87 \\
\hline$(0.76-$ & (0.68- & (0.61- & $(0.37-$ & (0.53- & $(0.93-$ & $(0.38-$ & (0.52- & (1.13- & $(0.36-$ & PAL & (0.48- & (0.43- & $(0.77-$ & $(0.57-$ & $(0.75-$ & $(0.66-$ & (0.63- \\
\hline 1.41) & $2.20)$ & $1.24)$ & $2.52)$ & $1.40)$ & $2.43)$ & 1.03) & $1.21)$ & $2.00)$ & $1.30)$ & & 1.48) & 1.99) & 6.63) & $1.08)$ & $1.39)$ & 1.67) & $1.20)$ \\
\hline 1.23 & 1.44 & 1.03 & 1.16 & 1.03 & 1.77 & 0.75 & 0.94 & 1.78 & 0.81 & 1.19 & PAL LAI- & 1.09 & 2.66 & 0.94 & 1.22 & 1.26 & 1.04 \\
\hline (0.75- & $(0.92-$ & (0.62- & (0.40- & (0.58- & (1.09- & $(0.41-$ & (0.53- & (1.04- & $(0.41-$ & (0.68- & 1 & (0.68- & $(0.90-$ & (0.59- & $(0.74-$ & (0.86- & (0.62- \\
\hline 1.97) & 2.31) & 1.76) & 3.27) & 1.77) & 3.01) & 1.36) & 1.62) & 3.03) & 1.61) & 2.10) & & 1.72) & 8.31) & 1.50) & 1.99) & 1.80) & 1.73) \\
\hline 1.14 & 1.33 & 0.95 & 1.06 & 0.94 & 1.63 & 0.70 & 0.86 & 1.65 & 0.75 & 1.10 & 0.92 & PAL LAI- & 2.45 & 0.87 & 1.13 & 1.16 & 0.95 \\
\hline (0.55- & $(0.70-$ & $(0.46-$ & (0.32- & (0.45- & (0.81- & (0.31- & $(0.40-$ & $(0.77-$ & $(0.32-$ & $(0.50-$ & (0.58- & 3 & $(0.72-$ & (0.43- & $(0.54-$ & (0.65- & (0.46- \\
\hline 2.25) & 2.71) & 1.99) & 3.27) & 2.02) & 3.43) & 1.56) & 1.84) & 3.42) & 1.72) & 2.35) & 1.48) & 3 & $8.27)$ & 1.72) & 2.27) & 2.06) & $1.95)$ \\
\hline 0.46 & 0.55 & 0.39 & 0.44 & 0.39 & 0.67 & 0.28 & 0.36 & 0.67 & 0.31 & 0.45 & 0.38 & 0.41 & & 0.35 & 0.46 & 0.47 & 0.39 \\
\hline$(0.16-$ & (0.17- & (0.13- & (0.11- & (0.13- & $(0.22-$ & $(0.09-$ & (0.12- & $(0.23-$ & $(0.09-$ & $(0.15-$ & $(0.12-$ & $(0.12-$ & QUE ER & (0.12- & $(0.16-$ & $(0.16-$ & $(0.14-$ \\
\hline 1.30) & 1.65) & 1.12) & $1.76)$ & 1.09) & 1.87) & $0.84)$ & $1.00)$ & $1.86)$ & 0.99) & $1.30)$ & 1.11) & 1.39) & & $0.98)$ & $1.26)$ & $1.38)$ & $1.06)$ \\
\hline 1.31 & 1.55 & 1.09 & 1.23 & 1.10 & 1.90 & 0.80 & 0.99 & 1.90 & 0.86 & 1.27 & 1.06 & 1.15 & 2.82 & & 1.30 & 1.34 & 1.10 \\
\hline (1.05- & (0.93- & (0.84- & $(0.48-$ & $(0.73-$ & (1.28- & (0.53- & $(0.71-$ & (1.54- & $(0.49-$ & $(0.92-$ & $(0.67-$ & (0.58- & (1.02- & QUE & (1.10- & $(0.92-$ & $(0.88-$ \\
\hline 1.64) & 2.63) & 1.45) & 3.20) & $1.66)$ & 2.85) & $1.20)$ & 1.41) & 2.36 ) & 1.58) & 1.76) & 1.70) & $2.35)$ & $8.21)$ & & 1.52) & $1.91)$ & 1.40) \\
\hline 1.01 & 1.19 & 0.85 & 0.95 & 0.85 & 1.45 & 0.62 & 0.77 & 1.47 & 0.67 & 0.98 & 0.82 & 0.89 & 2.17 & 0.77 & & 1.03 & 0.85 \\
\hline (0.83- & $(0.72-$ & (0.66- & $(0.37-$ & (0.57- & (1.02- & $(0.43-$ & (0.56- & (1.26- & (0.38- & $(0.72-$ & $(0.50-$ & $(0.44-$ & $(0.80-$ & (0.66- & RIS & $(0.69-$ & $(0.70-$ \\
\hline 1.22$)$ & 2.01) & $1.09)$ & 2.43) & 1.25) & 2.12) & $0.90)$ & 1.08) & $1.70)$ & 1.23) & $1.33)$ & 1.35) & $1.86)$ & $6.36)$ & $0.91)$ & & 1.54) & 1.05) \\
\hline 0.98 & 1.15 & 0.82 & 0.93 & 0.82 & 1.42 & 0.60 & 0.74 & 1.42 & 0.65 & 0.95 & 0.80 & 0.87 & 2.12 & 0.75 & & & 0.82 \\
\hline (0.67- & (0.65- & $(0.54-$ & $(0.33-$ & $(0.50-$ & (0.81- & $(0.34-$ & (0.47- & $(0.92-$ & $(0.34-$ & $(0.60-$ & $(0.56-$ & $(0.49-$ & $(0.72-$ & (0.53- & (0.65- & RIS LAI & $(0.54-$ \\
\hline 1.43) & 2.14) & $1.28)$ & 2.50) & $1.34)$ & 2.49) & 1.03) & 1.19) & $2.21)$ & $1.21)$ & 1.51) & $1.17)$ & $1.54)$ & $6.34)$ & $1.08)$ & $1.45)$ & & 1.27) \\
\hline 1.19 & 1.40 & 1.00 & 1.12 & 1.00 & 1.72 & 0.72 & 0.91 & 1.72 & 0.79 & 1.16 & 0.96 & 1.05 & 2.55 & 0.91 & 1.18 & 1.22 & \\
\hline (0.94- & (0.83- & (0.76- & $(0.42-$ & (0.64- & (1.19- & $(0.47-$ & (0.65- & (1.41- & (0.43- & (0.83- & (0.58- & (0.51- & $(0.94-$ & (0.72- & (0.95- & $(0.79-$ & ZIP \\
\hline 1.50) & 2.38) & 1.32) & 2.92) & 1.53) & 2.56) & 1.12) & 1.28) & 2.10) & 1.46) & 1.58) & 1.63) & 2.20) & 7.38) & 1.14) & 1.43) & 1.87) & \\
\hline
\end{tabular}

Drugs are reported in alphabetical order. Comparisons between treatments should be read from left to right and the estimate in each cell represents comparison between the row-defining treatment and the column-defining treatment. Odds ratios (ORs) higher than 1 favor the column-defining treatment, meaning less withdrawal in the column-defining treatment; and ORs less than 1 favor the row1.35 to 2.67), which means that LUR has higher odds of withdrawal and the comparison favors OLA, which has less withdrawal. Each comparison is represented twice in the table, for example, for two treatments A and B, once with A versus B and once with B versus A (i.e. the reciprocal OR). For the example above, the OR for comparing OLA (now the row-defining treatment) vs. LUR (now the column-defining treatment) is 0.52 (95\% CrI 0.37 to 0.74 ), which means that OLA has a lower odds of withdrawal and again, it means that the comparison favors OLA, which has less withdrawal. 
Significant results are in bold. ARI = aripiprazole- ASE $=$ asenapine- BRE $=$ brepiprazole- CAR $=$ cariprazine- CLO=clozapine- ILO = iloperidone- LUR $=$ lurasidone- OLA $=$ olanzapine $\mathrm{PAL}=$ paliperidone, QUE=quetiapine, RIS=risperidone- ZIP=ziprasidone. LAI = long-acting injection; 1 = once monthly- 3 - every 3 months.

\section{Appendix Table G-3b. SGA versus SGA: numbers of studies per comparison contributing to network meta-analysis of discontinuation} for any cause

\begin{tabular}{|c|c|}
\hline Comparison & No. of studies \\
\hline Aripiprazole : Brexipiprazole & 1 \\
\hline Aripiprazole : Olanzapine & 9 \\
\hline Aripiprazole : Quetiapine IR & 3 \\
\hline Aripiprazole : Risperidone & 6 \\
\hline Aripiprazole : Risperidone LAI & 1 \\
\hline Aripiprazole : Ziprasidone & 3 \\
\hline $\begin{array}{l}\text { Aripiprazole Monthly LAI : } \\
\text { Aripiprazole }\end{array}$ & 2 \\
\hline A--senapine : Olanzapine & 6 \\
\hline Asenapine : Risperidone & 1 \\
\hline Cariprazine : Risperidone & 1 \\
\hline Clozapine : Olanzapine & 10 \\
\hline Clozapine : Risperidone & 9 \\
\hline Iloperidone : Risperidone & 2 \\
\hline Lurasidone : Olanzapine & 1 \\
\hline Lurasidone : Risperidone & 1 \\
\hline Lurasidone : Ziprasidone & 1 \\
\hline Olanzapine LAI : Olanzapine & 1 \\
\hline Paliperidone : Aripiprazole & 2 \\
\hline Paliperidone : Olanzapine & 3 \\
\hline Paliperidone : Risperidone & 1 \\
\hline Paliperidone : Ziprasidone & 1 \\
\hline $\begin{array}{l}\text { Paliperidone Monthly LAI : } \\
\text { Aripiprazole }\end{array}$ & 1 \\
\hline $\begin{array}{l}\text { Paliperidone Monthly LAI : } \\
\text { Paliperidone }\end{array}$ & 1 \\
\hline $\begin{array}{l}\text { Paliperidone Monthly LAI : } \\
\text { Risperidone }\end{array}$ & 4 \\
\hline Quetiapine ER : Risperidone & 1 \\
\hline Quetiapine IR : Clozapine & 2 \\
\hline Quetiapine IR : Olanzapine & 17 \\
\hline Quetiapine IR : Risperidone & 19 \\
\hline Quetiapine IR : Risperidone LAI & 1 \\
\hline Risperidone : Olanzapine & 31 \\
\hline Risperidone LAl : Risperidone & 1 \\
\hline Ziprasidone : Clozapine & 1 \\
\hline --Ziprasidone : Olanzapine & 10 \\
\hline Ziprasidone : Quetiapine IR & 5 \\
\hline Ziprasidone : Risperidone & 5 \\
\hline
\end{tabular}


Appendix Table G-3c. SGA versus SGA: meta-regression results for discontinuations for any cause

\begin{tabular}{|l|c|}
\hline Meta-regression variables & OR (95\% Cl) \\
\hline Duration & $1.14(0.82$ to 1.55$)$ \\
\hline Drug dose: Low versus Medium & $1.13(0.84$ to 1.48$)$ \\
\hline Drug dose: High versus Medium & $1.00(0.99$ to 1.01$)$ \\
\hline Treatment Resistant & $1.47(0.93$ to 2.25$)$ \\
\hline First Episode & $0.78(0.58$ to 1.02$)$ \\
\hline
\end{tabular}


Appendix Figure G-4. Haloperidol versus aripiprazole withdrawals due to adverse events

\begin{tabular}{|c|c|c|c|c|c|c|c|c|c|c|c|}
\hline \multirow[b]{2}{*}{ Study or Subgroup } & \multicolumn{2}{|c|}{ Haloperidol } & \multicolumn{2}{|c|}{ Aripiprazole } & \multirow[b]{2}{*}{ Weight } & \multicolumn{2}{|l|}{ Risk Ratio } & \multirow{2}{*}{\multicolumn{4}{|c|}{$\begin{array}{c}\text { Risk Ratio } \\
\mathrm{M}-\mathrm{H}, \text { Random, } 95 \% \mathrm{Cl}\end{array}$}} \\
\hline & Events & Total & Events & Total & & M-H, Random, $95 \% \mathrm{Cl}$ & Year & & & & \\
\hline Kane 2002 & 11 & 104 & 17 & 204 & $4.8 \%$ & $1.27[0.62,2.61]$ & 2002 & & & 7 & \\
\hline Kasper 2003 & 138 & 433 & 213 & 861 & $76.7 \%$ & $1.29[1.08,1.54]$ & 2003 & & & & \\
\hline Vieta 2005 & 24 & 172 & 15 & 175 & $6.7 \%$ & $1.63[0.88,3.00]$ & 2005 & & & & \\
\hline Andrezina 2006 & 2 & 185 & 1 & 175 & $0.4 \%$ & $1.89[0.17,20.68]$ & 2006 & & & & \\
\hline Tran-Johnson 2007 & 0 & 60 & 2 & 235 & $0.3 \%$ & $0.77[0.04,15.91]$ & 2007 & & & & \\
\hline de Olivera 2009 & 2 & 33 & 5 & 66 & $1.0 \%$ & $0.80[0.16,3.91]$ & 2009 & & & & \\
\hline Young 2009 & 18 & 165 & 24 & 167 & $7.6 \%$ & $0.76[0.43,1.34]$ & 2009 & & - & - & \\
\hline Parabiaghi 2016 & 8 & 97 & 6 & 100 & $2.4 \%$ & $1.37[0.50,3.82]$ & 2016 & & & $\pi$ & \\
\hline Total $(95 \% \mathrm{Cl})$ & & 1249 & & 1983 & $100.0 \%$ & $1.25[1.07,1.47]$ & & & & 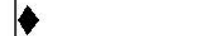 & \\
\hline Total events & & \multicolumn{6}{|c|}{$\begin{array}{l}\text { Total events } \quad 203 \quad 283 \\
\text { Heterogeneity: } \mathrm{Tau}^{2}=0.00 ; \mathrm{Ch}^{2}=4.31, \mathrm{df}=7(\mathrm{P}=0.74) ; \mathrm{F}^{2}=0 \%\end{array}$} & & & & \\
\hline \multicolumn{8}{|c|}{ Test for overall effect: $Z=2.79(P=0.005)$} & 0.01 & $\begin{array}{c}0.1 \\
\text { Favors haloperidol }\end{array}$ & $\begin{array}{l}1 \\
\text { Favors aripiprazole }\end{array}$ & $\overrightarrow{100}$ \\
\hline
\end{tabular}


Appendix Figure G-5. Haloperidol versus olanzapine withdrawals due to adverse events

\begin{tabular}{|c|c|c|c|c|c|c|c|c|c|c|c|}
\hline \multirow[b]{2}{*}{ Study or Subgroup } & \multicolumn{2}{|c|}{ Haloperidol } & \multicolumn{2}{|c|}{ Olanzapine } & \multirow[b]{2}{*}{ Weight } & \multirow{2}{*}{$\begin{array}{c}\text { Risk Ratio } \\
\mathrm{MH}, \text { Random, } 95 \% \mathrm{Cl}\end{array}$} & \multirow[b]{2}{*}{ Year } & \multirow{2}{*}{\multicolumn{4}{|c|}{$\begin{array}{c}\text { Risk Ratio } \\
\text { M-H, Random, } 95 \% \mathrm{Cl} \\
\end{array}$}} \\
\hline & Events & Total & Events & Total & & & & & & & \\
\hline Beasley 1996 & 10 & 69 & 17 & 198 & $6.4 \%$ & $1.69[0.81,3.51]$ & 1996 & & & 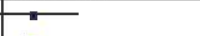 & \\
\hline Tollefson 1997 & 48 & 660 & 60 & 1336 & $25.1 \%$ & $1.62[1.12,2.34]$ & 1997 & & & $\rightarrow$ & \\
\hline Beasley 1997 & 12 & 81 & 38 & 350 & $9.4 \%$ & $1.36[0.75,2.49]$ & 1997 & & & $=-$ & \\
\hline Purdon 2000 & 7 & 23 & 2 & 21 & $1.6 \%$ & $3.20[0.75,13.70]$ & 2000 & & & & \\
\hline Avasthi 2001 & 1 & 10 & 0 & 17 & $0.4 \%$ & $4.91[0.22,110.23]$ & 2001 & & & & \\
\hline Ishigooka 2001 & 22 & 89 & 8 & 93 & $6.0 \%$ & $2.87[1.35,6.12]$ & 2001 & & & - & \\
\hline Wight 2001 & 2 & 126 & 2 & 131 & $0.9 \%$ & $1.04[0.15,7.27]$ & 2001 & & & & \\
\hline Altamura 2002 & 1 & 15 & 0 & 13 & $0.3 \%$ & $2.63[0.12,59.40]$ & 2002 & & & & \\
\hline Volavka 2002 & 6 & 37 & 4 & 39 & $2.4 \%$ & $1.58[0.48,5.16]$ & 2002 & & & 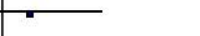 & \\
\hline De Haan 2003 & 1 & 12 & 1 & 12 & $0.5 \%$ & $1.00[0.07,14.21]$ & 2003 & & & & \\
\hline Tohen 2003 & 25 & 219 & 19 & 234 & $10.6 \%$ & $1.41[0.80,2.48]$ & 2003 & & & $\pi$ & \\
\hline Rosenheck 2003 & 15 & 150 & 6 & 159 & $4.0 \%$ & $2.65[1.06,6.65]$ & 2003 & & & & \\
\hline Lieberman 2003a & 19 & 132 & 7 & 131 & $4.9 \%$ & $2.69[1.17,6.19]$ & 2003 & & & $\longrightarrow$ & \\
\hline Goldman 2004 & 3 & 5 & 0 & 5 & $0.5 \%$ & $7.00[0.45,108.26]$ & 2004 & & & & \\
\hline Crespo-Facorro 2006 & 17 & 56 & 6 & 55 & $4.7 \%$ & $2.78[1.19,6.53]$ & 2006 & & & $\longrightarrow$ & \\
\hline Kongsakon 2006 & 13 & 132 & 5 & 144 & $3.4 \%$ & $2.84[1.04,7.74]$ & 2006 & & & & \\
\hline Keefe 2006 & 14 & 97 & 15 & 159 & $7.3 \%$ & $1.53[0.77,3.03]$ & 2006 & & & $=$ & \\
\hline Krakowski 2006 & 4 & 36 & 1 & 37 & $0.7 \%$ & $4.11[0.48,35.04]$ & 2006 & & & & \\
\hline Boulay 2007 & 4 & 11 & 0 & 14 & $0.4 \%$ & $11.25[0.67,189.01]$ & 2007 & & & & \\
\hline Lindenmayer 2007 & 2 & 19 & 1 & 16 & $0.6 \%$ & $1.68[0.17,16.91]$ & 2007 & & & & \\
\hline Kahn 2008 & 12 & 103 & 5 & 105 & $3.4 \%$ & $2.45[0.89,6.70]$ & 2008 & & & & \\
\hline Crespo-Facorro 2011 & 14 & 56 & 3 & 55 & $2.4 \%$ & $4.58[1.39,15.07]$ & 2011 & & & & \\
\hline San 2012 & 2 & 21 & 2 & 25 & $1.0 \%$ & $1.19[0.18,7.74]$ & 2012 & & & & \\
\hline Parabiaghi 2016 & 8 & 97 & 6 & 103 & $3.3 \%$ & $1.42[0.51,3.93]$ & 2016 & & & & \\
\hline Total $(95 \% \mathrm{Cl})$ & & 2256 & & 3452 & $100.0 \%$ & $1.89[1.57,2.27]$ & & & & $\bullet$ & \\
\hline Total events & 262 & & 208 & & & & & & & & \\
\hline $\begin{array}{l}\text { Heterogeneity: } \text { Tau }^{2}= \\
\text { Test for overall effect: }\end{array}$ & $\begin{array}{l}\mathrm{Chi}^{2}=14 \\
76(\mathrm{P}<0\end{array}$ & $\begin{array}{l}70, d f= \\
00001)\end{array}$ & $23(P=0.9$ & $0) 1^{2}=0$ & & & & 0.01 & $\begin{array}{c}0.1 \\
\text { Favors haloperidol }\end{array}$ & $\begin{array}{c}10 \\
\text { Favors olanzapine }\end{array}$ & 100 \\
\hline
\end{tabular}


Appendix Figure G-6. Haloperidol versus risperidone withdrawals due to adverse events

\begin{tabular}{|c|c|c|c|c|c|c|c|c|c|c|c|c|}
\hline \multirow{3}{*}{$\begin{array}{l}\text { Study or Subgroup } \\
\text { Claus } 1992\end{array}$} & \multicolumn{2}{|c|}{ Haloperidol } & \multicolumn{2}{|c|}{ Risperidone } & \multirow{3}{*}{$\frac{\text { Weight }}{0 . \%}$} & \multirow{3}{*}{$\begin{array}{c}\begin{array}{c}\text { Risk Ratio } \\
\text { M-H, Random, } 95 \% ~ C l\end{array} \\
3.00[0.13,69.70]\end{array}$} & \multirow{3}{*}{$\begin{array}{l}\text { Year } \\
1992\end{array}$} & \multirow{2}{*}{\multicolumn{5}{|c|}{$\begin{array}{c}\text { Risk Ratio } \\
\text { M-H, Random, } 95 \% \mathrm{Cl}\end{array}$}} \\
\hline & Events & Total & Events & Total & & & & & & & & \\
\hline & 1 & 21 & 0 & 21 & & & & & & & & \\
\hline Ceskova 1993 & 1 & 31 & 0 & 31 & $0.4 \%$ & $3.00[0.13,70.92]$ & 1993 & & & & & \\
\hline Chouinard 1993 & 1 & 21 & 3 & 92 & $0.8 \%$ & $1.46[0.16,13.35]$ & 1993 & & & & - & \\
\hline Peuskens 1995 & 23 & 226 & 103 & 1136 & $20.6 \%$ & $1.12[0.73,1.72]$ & 1995 & & & & $=$ & \\
\hline Blin 1996 & 0 & 20 & 0 & 21 & & Not estimable & 1996 & & & & & \\
\hline Wirshing 1999 & 0 & 33 & 3 & 34 & $0.4 \%$ & $0.15[0.01,2.74]$ & 1999 & $\leftrightarrow$ & & & & \\
\hline Emsley 1999 & 15 & 84 & 6 & 99 & $4.7 \%$ & $2.95[1.20,7.25]$ & 1999 & & & & & \\
\hline Heck 2000 & 6 & 37 & 5 & 40 & $3.1 \%$ & $1.30[0.43,3.89]$ & 2000 & & & &. & \\
\hline Purdon 2000 & 7 & 23 & 3 & 21 & $2.6 \%$ & $2.13[0.63,7.19]$ & 2000 & & & 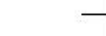 & & \\
\hline Zhang 2001 & 2 & 37 & 0 & 41 & $0.4 \%$ & $5.53[0.27,111.50]$ & 2001 & & & & & \\
\hline Janicak 2001 & 6 & 32 & 0 & 30 & $0.5 \%$ & $12.21[0.72,207.84]$ & 2001 & & & & & \\
\hline Cavallaro 2001 & 3 & 16 & 4 & 17 & $2.1 \%$ & $0.80[0.21,3.02]$ & 2001 & & & & & \\
\hline Csernansky 2002 & 30 & 188 & 23 & 177 & $14.9 \%$ & $1.23[0.74,2.03]$ & 2002 & & & & - & \\
\hline Sachs 2002 & 1 & 53 & 2 & 52 & $0.7 \%$ & $0.49[0.05,5.25]$ & 2002 & & & & & \\
\hline Volavka 2002 & 6 & 37 & 4 & 41 & $2.7 \%$ & $1.66[0.51,5.43]$ & 2002 & & & - & $\longrightarrow$ & \\
\hline Yen 2004 & 2 & 20 & 1 & 21 & $0.7 \%$ & $2.10[0.21,21.39]$ & 2004 & & & & & \\
\hline Smulevich 2005 & 7 & 144 & 11 & 154 & $4.5 \%$ & $0.68[0.27,1.71]$ & 2005 & & & & - & \\
\hline Schooler 2005 & 17 & 277 & 15 & 278 & $8.3 \%$ & $1.14[0.58,2.23]$ & 2005 & & & & $=$ & \\
\hline Keefe 2006 & 14 & 97 & 24 & 158 & $10.2 \%$ & $0.95[0.52,1.75]$ & 2006 & & & & - & \\
\hline Crespo-Facorro 2006 & 17 & 56 & 8 & 61 & $6.6 \%$ & $2.31[1.08,4.94]$ & 2006 & & & & & \\
\hline Moller 2008 & 17 & 146 & 14 & 143 & $8.5 \%$ & $1.19[0.61,2.32]$ & 2008 & & & - & $=$ & \\
\hline Fakra 2008 & 1 & 15 & 1 & 15 & $0.5 \%$ & $1.00[0.07,14.55]$ & 2008 & & & & & \\
\hline $\operatorname{Lim} 2010$ & 2 & 62 & 1 & 62 & $0.7 \%$ & $2.00[0.19,21.49]$ & 2010 & & & & & \\
\hline Crespo-Facorro 2011 & 14 & 56 & 7 & 63 & $5.5 \%$ & $2.25[0.98,5.17]$ & 2011 & & & & & \\
\hline San 2012 & 2 & 21 & 0 & 20 & $0.4 \%$ & $4.77[0.24,93.67]$ & 2012 & & & & & \\
\hline Total $(95 \% \mathrm{CD})$ & & 1753 & & 2828 & $100.0 \%$ & $1.32[1.09,1.60]$ & & & & & $\bullet$ & \\
\hline & & & & & \\
\hline \multicolumn{8}{|c|}{$\begin{array}{l}\text { Heterogeneity: } \text { Tau }^{2}=0.00 ; \mathrm{Ch}^{2}=19.71, \mathrm{df}=23(P=0.66) ; l^{2}=0 \% \\
\text { Test for overall effect. } Z=2.79(P=0.005)\end{array}$} & 0.01 & $\begin{array}{c}0.1 \\
\text { Favors halc }\end{array}$ & loperidol & $\begin{array}{c}10 \\
\text { Favors risperidone }\end{array}$ & 100 \\
\hline
\end{tabular}


Appendix Figure G-7. Haloperidol versus ziprasidone withdrawals due to adverse events

\begin{tabular}{|c|c|c|c|c|c|c|c|c|c|c|c|}
\hline \multirow[b]{2}{*}{ Study or Subgroup } & \multicolumn{2}{|c|}{ Haloperidol } & \multicolumn{3}{|c|}{ Ziprasidone } & \multirow{2}{*}{$\begin{array}{c}\text { Risk Ratio } \\
\text { M-H, Random, } 95 \% \mathrm{Cl}\end{array}$} & \multirow[b]{2}{*}{ Year } & \multirow{2}{*}{\multicolumn{3}{|c|}{$\begin{array}{c}\text { Risk Ratio } \\
\mathrm{M}-\mathrm{H}, \text { Random, } 95 \% \mathrm{Cl} \\
\end{array}$}} & \\
\hline & Events & Total & Events & Total & Weight & & & & & & \\
\hline Goff 1998 & 1 & 17 & 2 & 73 & $1.5 \%$ & $2.15[0.21,22.33]$ & 1998 & & & & \\
\hline Hirsch 2002 & 24 & 153 & 12 & 148 & $18.8 \%$ & $1.93[1.00,3.72]$ & 2002 & & & $\longrightarrow$ & \\
\hline Brook 2005 & 19 & 138 & 43 & 429 & $31.7 \%$ & $1.37[0.83,2.28]$ & 2005 & & & & \\
\hline Kahn 2008 & 12 & 103 & 7 & 82 & $10.3 \%$ & $1.36[0.56,3.31]$ & 2008 & & & $=$ & \\
\hline Vieta 2010 & 36 & 172 & 17 & 178 & $28.0 \%$ & $2.19[1,28,3.75]$ & 2010 & & & $\rightarrow-$ & \\
\hline Miceli 2010 & 7 & 27 & 4 & 31 & $6.5 \%$ & $2.01[0.66,6.13]$ & 2010 & & & + & \\
\hline San 2012 & 2 & 21 & 4 & 25 & $3.2 \%$ & $0.60[0.12,2.93]$ & 2012 & & & & \\
\hline Total $(95 \% \mathrm{Cl})$ & & 631 & & 966 & $100.0 \%$ & $1.68[1.26,2.23]$ & & & & & \\
\hline Total events & 101 & & 89 & & & & & & & & \\
\hline $\begin{array}{l}\text { Heterogeneity: } \operatorname{Tau}^{2}= \\
\text { Test for overall effect: }\end{array}$ & $\begin{array}{l}0: \mathrm{Chi}^{2}=3 \\
3.56(\mathrm{P}=\end{array}$ & $\begin{array}{l}71, d f= \\
.0004)\end{array}$ & $6(P=0.72$ & $z^{2}=0 \%$ & & & & 0.01 & $\begin{array}{c}0.1 \\
\text { Favors haloperidol }\end{array}$ & $\begin{array}{l}10 \\
\text { Favors ziprasidone }\end{array}$ & 100 \\
\hline
\end{tabular}


Appendix Figure G-8. Haloperidol versus quetiapine withdrawals due to adverse events

\begin{tabular}{|c|c|c|c|c|c|c|c|c|c|}
\hline Study or Subgroup & \multicolumn{2}{|c|}{ Haloperidol } & \multicolumn{2}{|c|}{ Quetiapine } & \multirow{2}{*}{$\begin{array}{r}\text { Weight } \\
6.8 \%\end{array}$} & $\begin{array}{c}\text { Risk Ratio } \\
\text { M-H, Random, } 95 \% \mathrm{Cl}\end{array}$ & Year & \multicolumn{2}{|c|}{$\begin{array}{l}\text { Risk Ratio } \\
\text { M-H, Random, } 95 \% \mathrm{Cl}\end{array}$} \\
\hline Anvantis 1997 & 4 & 52 & 1 & 258 & & $19.85[2.26,173.98]$ & 1997 & & \\
\hline Emsley 2000 & 5 & 145 & 12 & 143 & $13.5 \%$ & $0.41[0.15,1.14]$ & 2000 & & \\
\hline Copolov 2000 & 18 & 227 & 4 & 221 & $13.1 \%$ & $4.38[1.51,12.74]$ & 2000 & & \\
\hline Purdon 2001 & 2 & 12 & 2 & 13 & $8.5 \%$ & $1.08[0.18,6.53]$ & 2001 & & \\
\hline Atmaca 2002 & 0 & 17 & 0 & 18 & & Not estimable & 2002 & & \\
\hline Emsley 2005 & 5 & 23 & 7 & 22 & $13.7 \%$ & $0.68[0.25,1.83]$ & 2005 & & - \\
\hline McIntyre 2005 & 10 & 99 & 5 & 102 & $13.4 \%$ & $2.06[0.73,5.81]$ & 2005 & & \\
\hline Kahn 2008 & 12 & 103 & 2 & 104 & $10.4 \%$ & $6.06[1.39,26.40]$ & 2008 & & \\
\hline $\operatorname{San} 2012$ & 2 & 21 & 1 & 23 & $6.3 \%$ & $2.19[0.21,22.43]$ & 2012 & & \\
\hline Amr 2013 & 13 & 78 & 6 & 78 & $14.3 \%$ & $2.17[0.87,5.41]$ & 2013 & & \\
\hline Total $(95 \% \mathrm{Cl})$ & & 777 & & 982 & $100.0 \%$ & $1.97[0.96,4.01]$ & & & \\
\hline Total events & 71 & & 40 & & & & & & \\
\hline $\begin{array}{l}\text { Heterogeneity. } \mathrm{Tau}^{2}= \\
\text { Test for overall effect }\end{array}$ & $\begin{array}{l}0.71 ; \mathrm{Ch} \\
z=1.86\end{array}$ & $\begin{array}{l}l^{2}=22 . \\
(P=0 .\end{array}$ & $\begin{array}{l}46, d f= \\
06)\end{array}$ & $8(P=$ & $0.004 \mathrm{j}^{2} \mathrm{I}^{2}$ & $2=64 \%$ & & 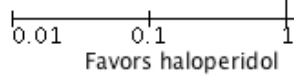 & $\begin{array}{c}10 \\
\text { Favors quetiapine }\end{array}$ \\
\hline
\end{tabular}


Appendix Figure G-9. Assertive community treatment: unable to live independently

\begin{tabular}{|c|c|c|c|c|c|c|c|c|c|c|}
\hline \multirow[b]{2}{*}{ Study or Subgroup } & \multicolumn{2}{|c|}{ АCT } & \multicolumn{2}{|c|}{ Usual care } & \multirow[b]{2}{*}{ Weight } & \multicolumn{2}{|l|}{ Odds Ratio } & \multirow{2}{*}{\multicolumn{3}{|c|}{$\begin{array}{c}\text { Odds Ratio } \\
\text { M-H, Random, } 95 \% \mathrm{Cl}\end{array}$}} \\
\hline & Events & Total & Events & Total & & M-H, Random, 95\% Cl & & & & \\
\hline Bond 1990 & 33 & 45 & 37 & 43 & $14.3 \%$ & $0.45[0.15,1.32]$ & & $\because$ & & \\
\hline Lehman 1995 & 15 & 77 & 20 & 75 & $29.2 \%$ & $0.67[0.31,1.42]$ & & & & \\
\hline Petersen 2005 & 13 & 243 & 14 & 193 & $27.8 \%$ & $0.72[0.33,1.58]$ & & $\rightarrow-$ & - & \\
\hline Test 1991 & 20 & 75 & 25 & 47 & $28.7 \%$ & $0.32[0.15,0.69]$ & & $=$ & & \\
\hline Total $(95 \% \mathrm{Cl})$ & & 440 & & 358 & $100.0 \%$ & $0.52[0.35,0.79]$ & & & & \\
\hline Total events & 81 & & 96 & & & & & & & \\
\hline \multicolumn{6}{|c|}{$\begin{array}{l}\text { Heterogeneity: } \mathrm{Tau}^{2}=0.00 ; \mathrm{Chi}^{2}=2.70, \mathrm{df}=3(\mathrm{P}=0.44) ; \mathrm{I}^{2}=0 \% \\
\text { Test for overall effect: } Z=3.11(\mathrm{P}=0.002)\end{array}$} & & 0.01 & $\begin{array}{ll}0.1 & 1 \\
\text { Favors ACT } & \end{array}$ & $\begin{array}{c}10 \\
\text { Favors usual care }\end{array}$ & 100 \\
\hline
\end{tabular}


Appendix Figure G-10. Assertive community treatment versus usual care: homelessness

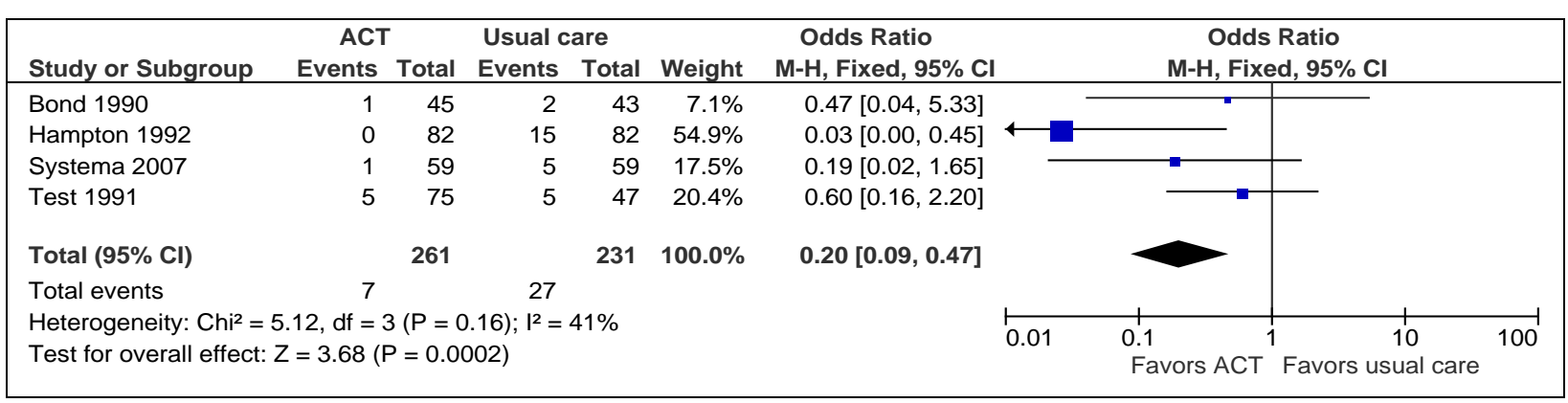


Appendix Figure G-11. Assertive community treatment: unemployment

\begin{tabular}{|c|c|c|c|c|c|c|c|c|c|c|}
\hline \multirow[b]{2}{*}{ Study or Subgroup } & \multicolumn{2}{|l|}{ ACT } & \multicolumn{2}{|c|}{ Usual care } & \multirow[b]{2}{*}{ Weight } & \multirow{2}{*}{$\begin{array}{c}\text { Odds Ratio } \\
\text { M-H, Random, } 95 \% \mathrm{Cl}\end{array}$} & \multirow{2}{*}{\multicolumn{4}{|c|}{$\begin{array}{l}\text { Odds Ratio } \\
\text { M-H, Random, } 95 \% \mathrm{Cl}\end{array}$}} \\
\hline & Events & Total & Events & Total & & & & & & \\
\hline Bond 1990 & 43 & 45 & 41 & 43 & $11.5 \%$ & $1.05[0.14,7.80]$ & & & & \\
\hline Chandler 1996 & 194 & 252 & 245 & 264 & $42.1 \%$ & $0.26[0.15,0.45]$ & & $\rightarrow$ & & \\
\hline Petersen 2005 & 61 & 243 & 67 & 193 & $46.4 \%$ & $0.63[0.42,0.95]$ & & 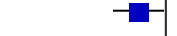 & & \\
\hline Total $(95 \% \mathrm{Cl})$ & & 540 & & 500 & $100.0 \%$ & $0.46[0.21,0.99]$ & & & & \\
\hline Total events & 298 & & 353 & & & & & & & \\
\hline \multicolumn{6}{|c|}{$\begin{array}{l}\text { Heterogeneity: } \mathrm{Tau}^{2}=0.29 ; \mathrm{Chi}^{2}=7.03, \mathrm{df}=2(\mathrm{P}=0.03) ; \mathrm{I}^{2}=72 \% \\
\text { Test for overall effect: } Z=1.98(P=0.05)\end{array}$} & & 0.01 & $\begin{array}{l}0.1 \\
\text { Favors ACT }\end{array}$ & 10 & 100 \\
\hline
\end{tabular}


Appendix Figure G-12. Assertive community treatment: loss to followup

\begin{tabular}{|c|c|c|c|c|c|c|c|c|c|}
\hline \multirow[b]{2}{*}{ Study or Subgroup } & \multicolumn{2}{|c|}{ ACT } & \multicolumn{2}{|c|}{ Usual care } & \multirow[b]{2}{*}{ Weight } & \multicolumn{2}{|l|}{ Odds Ratio } & \multirow{2}{*}{\multicolumn{2}{|c|}{$\begin{array}{c}\text { Odds Ratio } \\
\text { M-H, Random, } 95 \% \mathrm{Cl}\end{array}$}} \\
\hline & Events & Total & Events & Total & & M-H, Random, 95\% Cl & & & \\
\hline Aberg-Wistedt 1995 & 2 & 20 & 3 & 20 & $1.2 \%$ & $0.63[0.09,4.24]$ & & & \\
\hline Audini 1994 & 3 & 33 & 4 & 32 & $1.7 \%$ & $0.70[0.14,3.41]$ & & & \\
\hline Bond 1988 & 11 & 44 & 19 & 43 & $5.2 \%$ & $0.42[0.17,1.05]$ & & & \\
\hline Bond 1990 & 18 & 84 & 25 & 83 & $8.8 \%$ & $0.63[0.31,1.28]$ & & $\longrightarrow$ & \\
\hline Chandler 1996 & 44 & 252 & 72 & 264 & $24.1 \%$ & $0.56[0.37,0.86]$ & & $\rightarrow-$ & \\
\hline Hampton 1992 & 12 & 80 & 15 & 82 & $6.2 \%$ & $0.79[0.34,1.81]$ & & & \\
\hline Herinckx 1997 & 26 & 116 & 29 & 58 & $9.5 \%$ & $0.29[0.15,0.57]$ & & & \\
\hline Lehman 1995 & 10 & 77 & 17 & 75 & $5.9 \%$ & $0.51[0.22,1.20]$ & & & \\
\hline Morse 1992 & 15 & 52 & 29 & 64 & $7.2 \%$ & $0.49[0.23,1.06]$ & & & \\
\hline Petersen 2005 & 14 & 59 & 23 & 59 & $6.8 \%$ & $0.49[0.22,1.08]$ & & & \\
\hline Systema 2007 & 39 & 275 & 67 & 275 & $22.6 \%$ & $0.51[0.33,0.79]$ & & - & \\
\hline Test 1991 & 1 & 73 & 4 & 45 & $0.9 \%$ & $0.14[0.02,1.32]$ & & & \\
\hline Total $(95 \% \mathrm{Cl})$ & & 1165 & & 1100 & $100.0 \%$ & $0.51[0.41,0.63]$ & & $\diamond$ & \\
\hline Total events & 195 & & 307 & & & & & & \\
\hline \multirow{2}{*}{\multicolumn{6}{|c|}{$\begin{array}{l}\text { Heterogeneity: } \mathrm{Tau}^{2}=0.00 ; \mathrm{Chi}^{2}=6.02, \mathrm{df}=11(P=0.87) ; \mathrm{I}^{2}=0 \% \\
\text { Test for overall effect: } Z=6.37(\mathrm{P}<0.00001)\end{array}$}} & & 0.01 & $0.1 \quad 1$ & $\begin{array}{ll}1 & 100 \\
10 & 100\end{array}$ \\
\hline & & & & & & & & \multicolumn{2}{|c|}{$\begin{array}{l}1 \\
\text { Favors ACT }\end{array}$} \\
\hline
\end{tabular}




\section{Appendix Figure G-13. Cognitive behavioral therapy: short-term function}

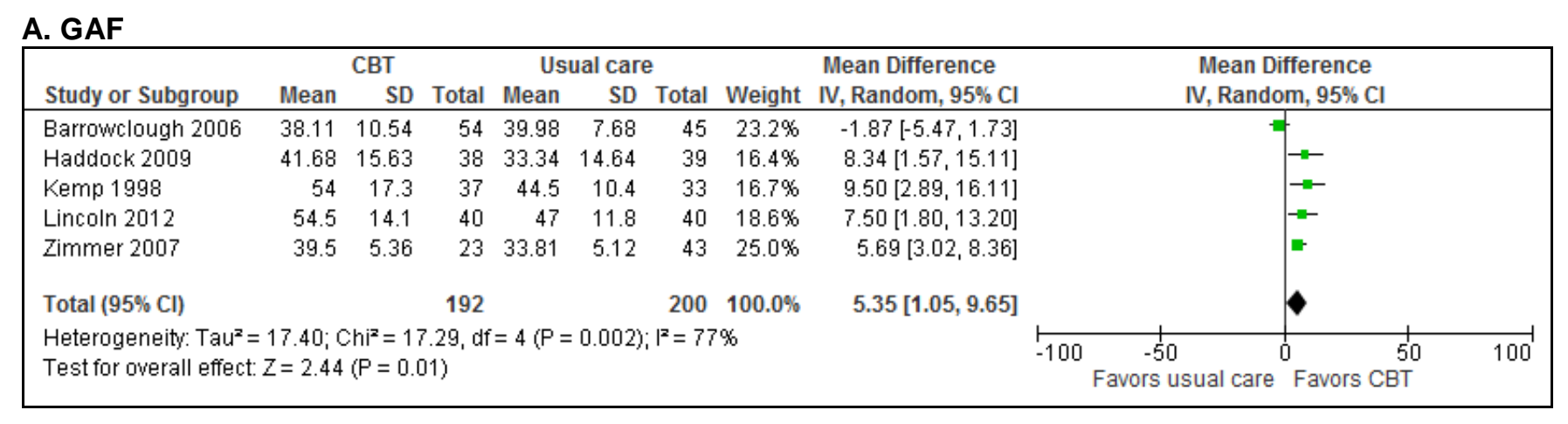

\section{B. SOFAS}

\begin{tabular}{|c|c|c|c|c|c|c|c|c|c|c|c|c|}
\hline \multirow[b]{2}{*}{ Study or Subgroup } & \multicolumn{3}{|c|}{ usual care } & \multicolumn{3}{|c|}{ CBT } & \multicolumn{2}{|r|}{ Mean Difference } & \multirow{2}{*}{\multicolumn{3}{|c|}{$\begin{array}{c}\text { Mean Difference } \\
\text { IV, Random, } 95 \% \mathrm{Cl}\end{array}$}} & \\
\hline & Mean & SD & Total & Mean & SD & Total & Weight & IV, Random, $95 \% \mathrm{Cl}$ & & & & \\
\hline Jackson 2008 & 66.69 & 13.81 & 31 & 57.6 & 11.37 & 31 & $19.8 \%$ & $9.09[2.79,15.39]$ & & & $=$ & \\
\hline Zimmer 2007 & 43.25 & 6.54 & 23 & 34.14 & 5.43 & 43 & $80.2 \%$ & $9.11[5.98,12.24]$ & & & m & \\
\hline Total $(95 \% \mathrm{Cl})$ & & & 54 & & & 74 & $100.0 \%$ & $9.11[6.31,11.91]$ & & & 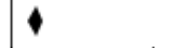 & \\
\hline \multicolumn{7}{|c|}{$\begin{array}{l}\text { Heterogeneity: } \operatorname{Tau}^{2}=0.00 ; \mathrm{Chi}^{2}=0.00, \mathrm{df}=1(\mathrm{P}=1.00) ; \mathrm{I}^{2}=0 \% \\
\text { Test for overall effect: } Z=6.37(\mathrm{P}=0.00001)\end{array}$} & & & -100 & $\begin{array}{r}-50 \\
\text { ors ut }\end{array}$ & $\begin{array}{lr}0 & 50 \\
\text { Favors CBT }\end{array}$ & 100 \\
\hline
\end{tabular}


Appendix Figure G-14. Cognitive behavioral therapy: treatment maintenance

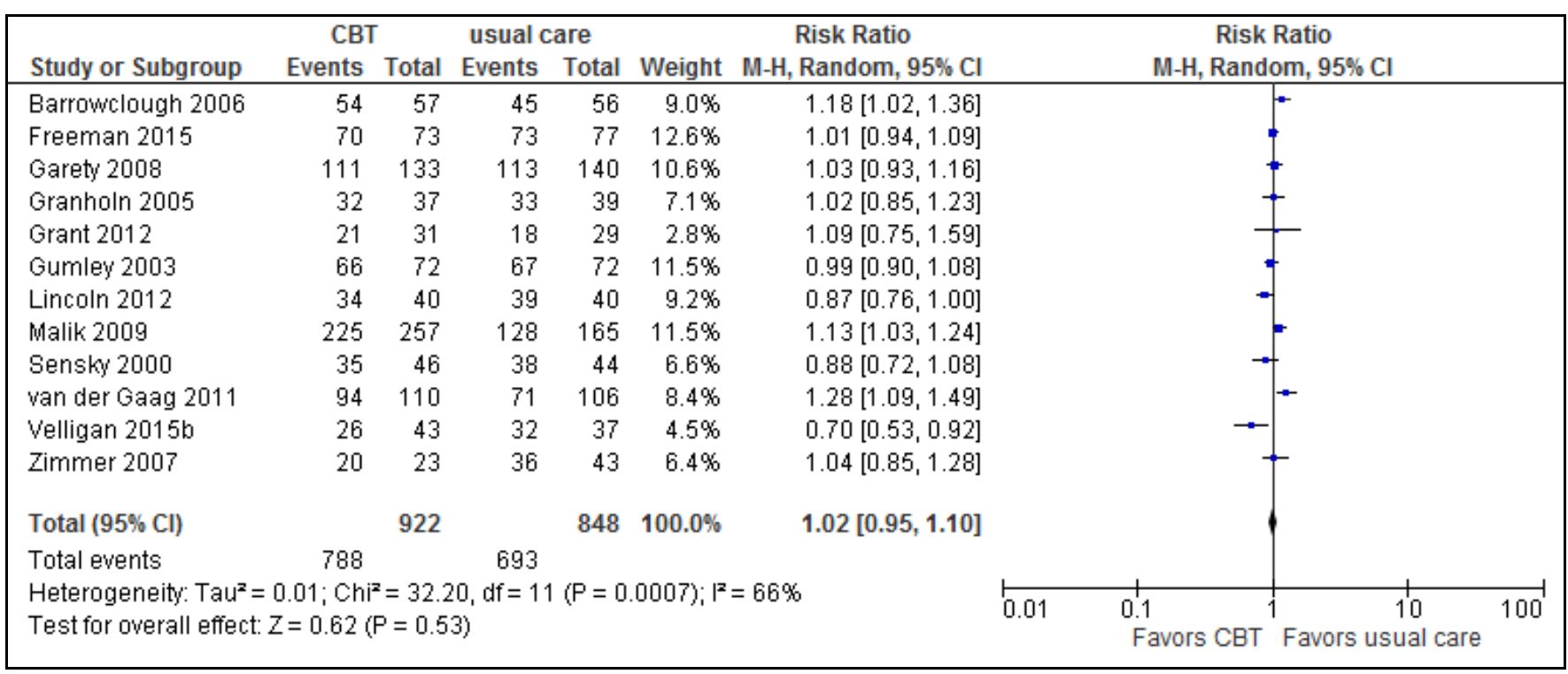


Appendix Figure G-15. Cognitive behavioral therapy: relapse

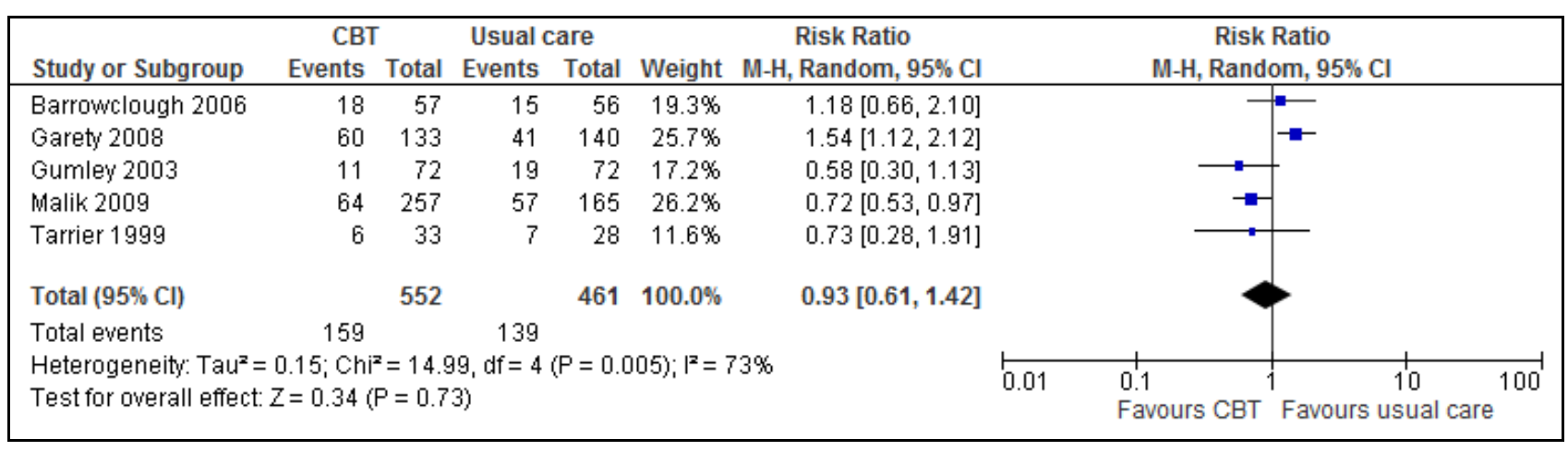


Appendix Figure G-16. Family interventions: unemployment

\begin{tabular}{|c|c|c|c|}
\hline \multicolumn{4}{|c|}{ Unemployed } \\
\hline \multirow[b]{2}{*}{ Study } & \multirow[b]{2}{*}{ Treatment } & \multirow[b]{2}{*}{ Control } & \multirow{2}{*}{$\begin{array}{l}\text { Relative } \\
\text { Risk (95\% Cl) }\end{array}$} \\
\hline & & & \\
\hline \multicolumn{2}{|c|}{ Buchkremer, 1995 55/67 } & $23 / 32$ & $1.14(0.89,1.46)$ \\
\hline Cara, 2007 & $18 / 26$ & $13 / 25$ & $1.33(0.84,2.10)$ \\
\hline Fallon, 1981 & $12 / 20$ & $11 / 19$ & $1.04(0.61,1.75)$ \\
\hline Flynn, 1992 & $15 / 21$ & $16 / 20$ & $0.89(0.63,1.26)$ \\
\hline \multicolumn{3}{|c|}{ Overall $(\mathrm{I}$-squared $=0.0 \%, p=0.536)$} & $1.09(0.91,1.29)$ \\
\hline \multicolumn{3}{|c|}{ NOTE: Weights are from random effects analysis } & \\
\hline & & $\begin{array}{l}.6 \\
\text { Favors Treatment }\end{array}$ & \\
\hline
\end{tabular}


Appendix Figure G-17. Family interventions: suicide

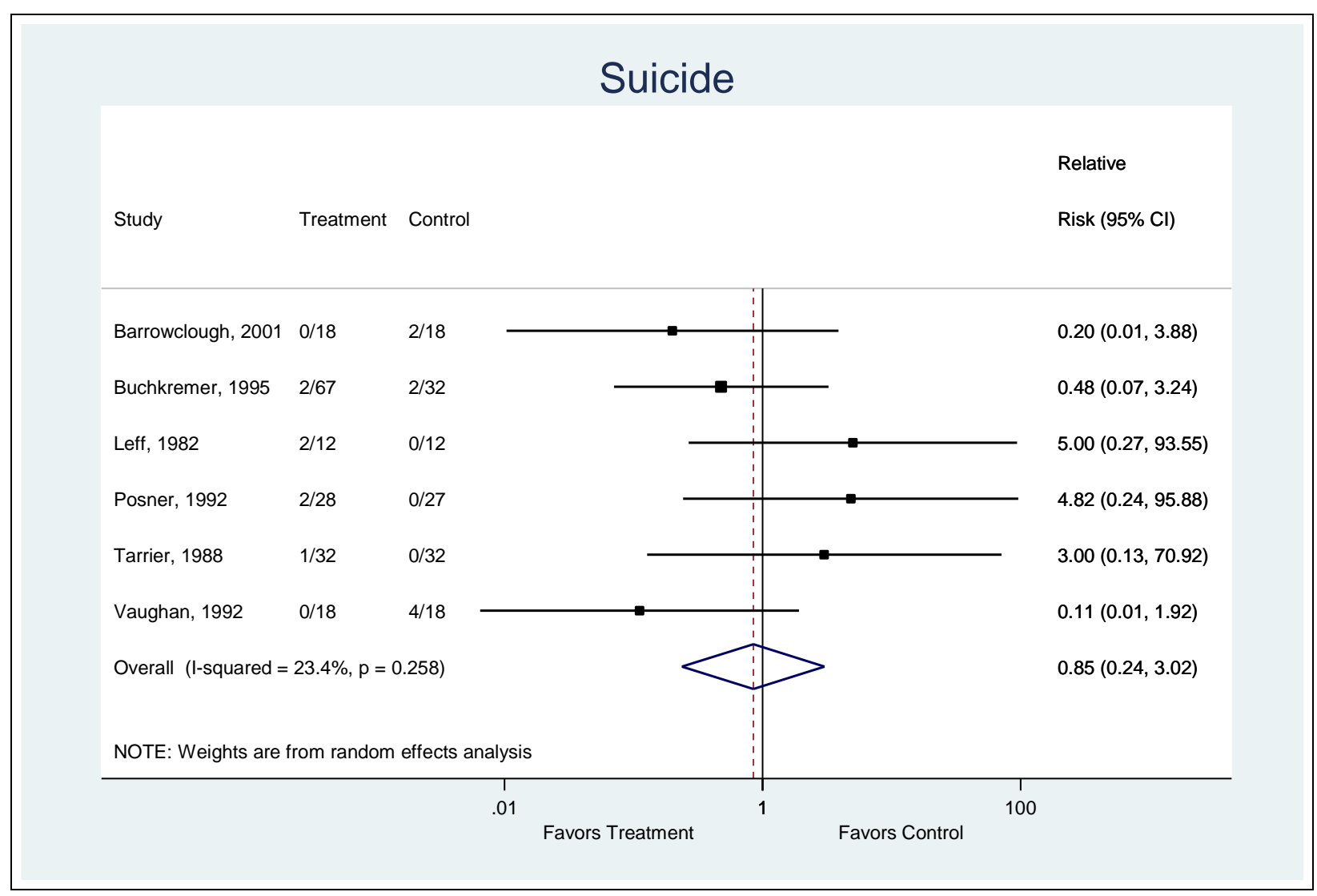


Appendix Figure G-18. Family interventions: core illness symptoms

\section{Core Illness Symptoms}

BPRS and PANSS

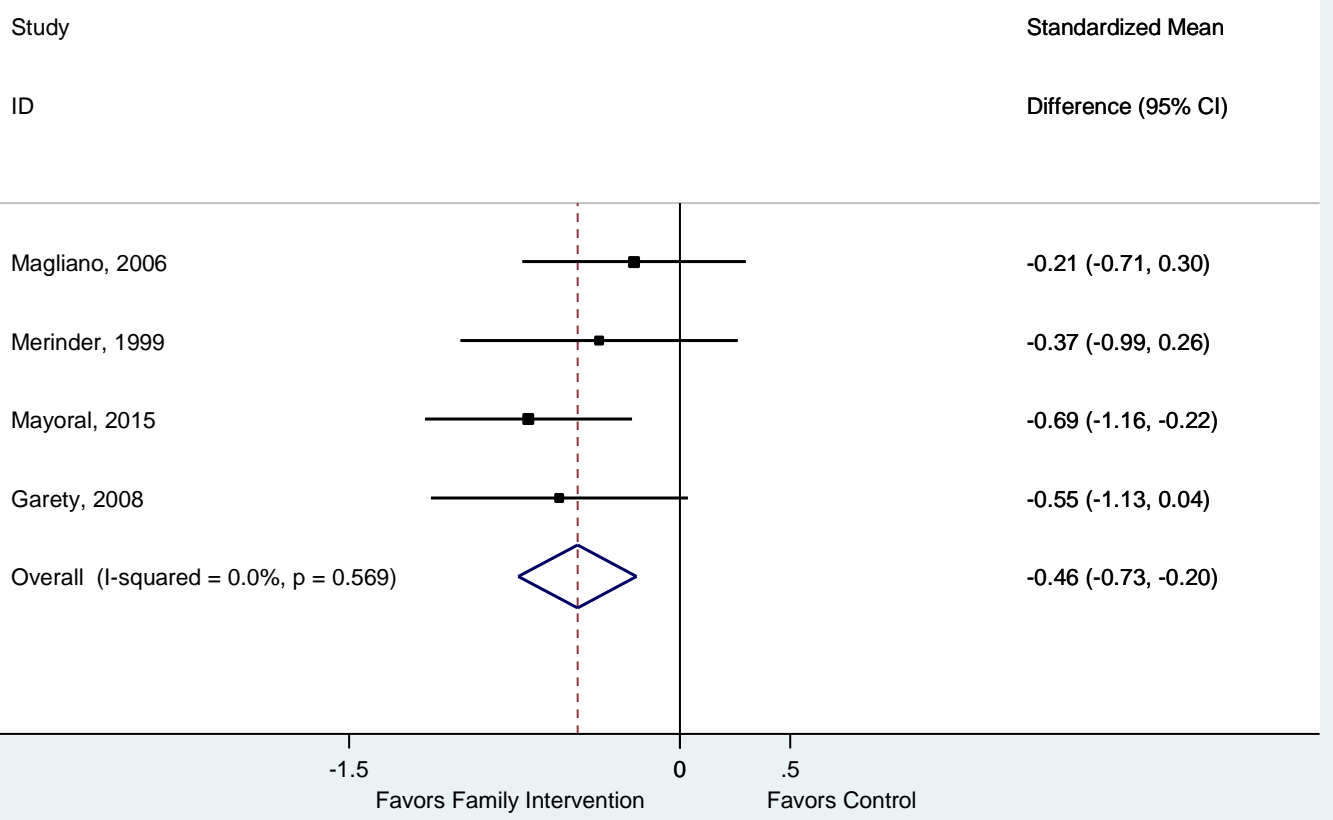


Appendix Figure G-19. Family interventions: negative symptoms

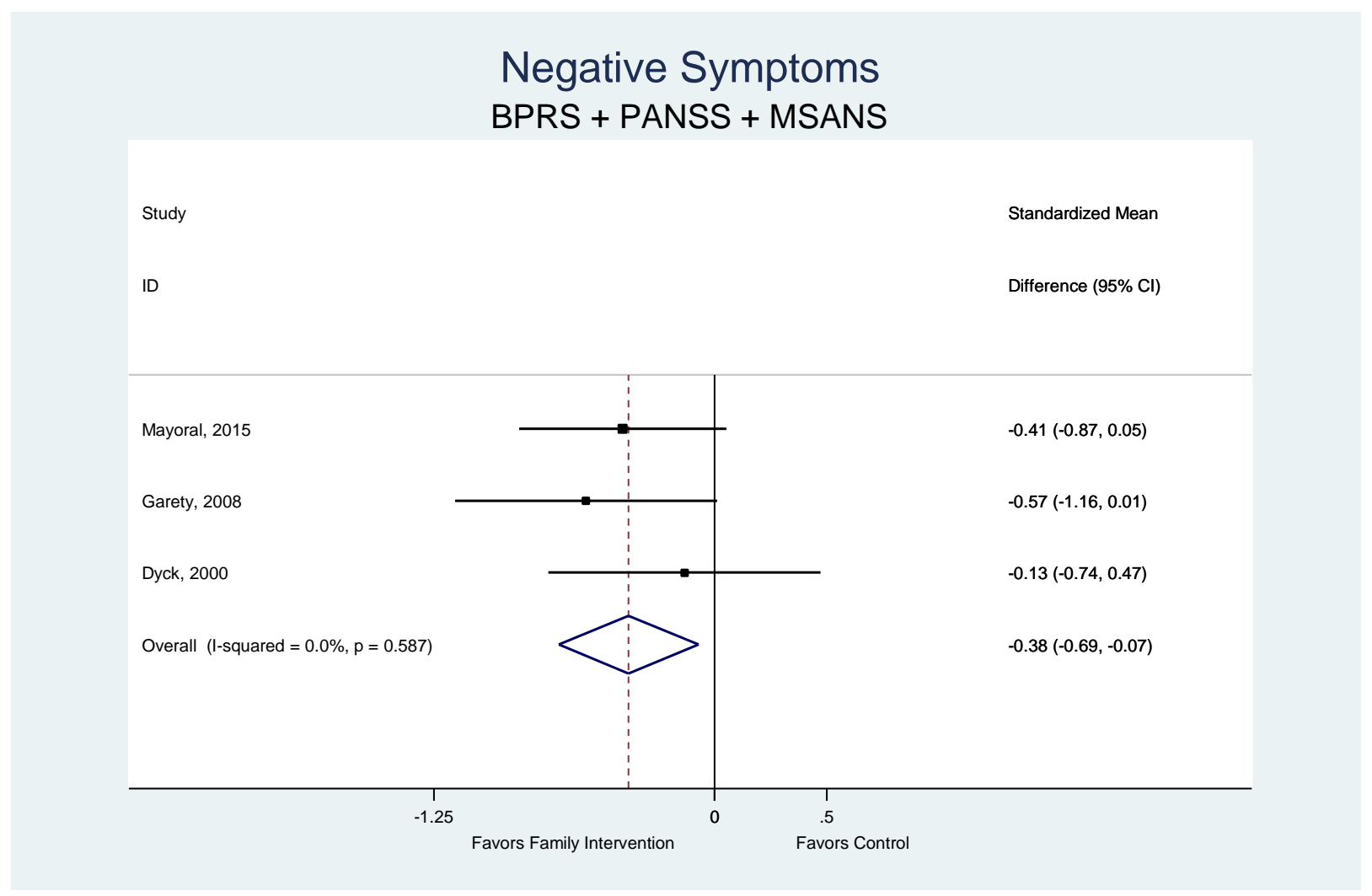


Appendix Figure G-20. Family interventions: treatment maintenance at 3-6 months

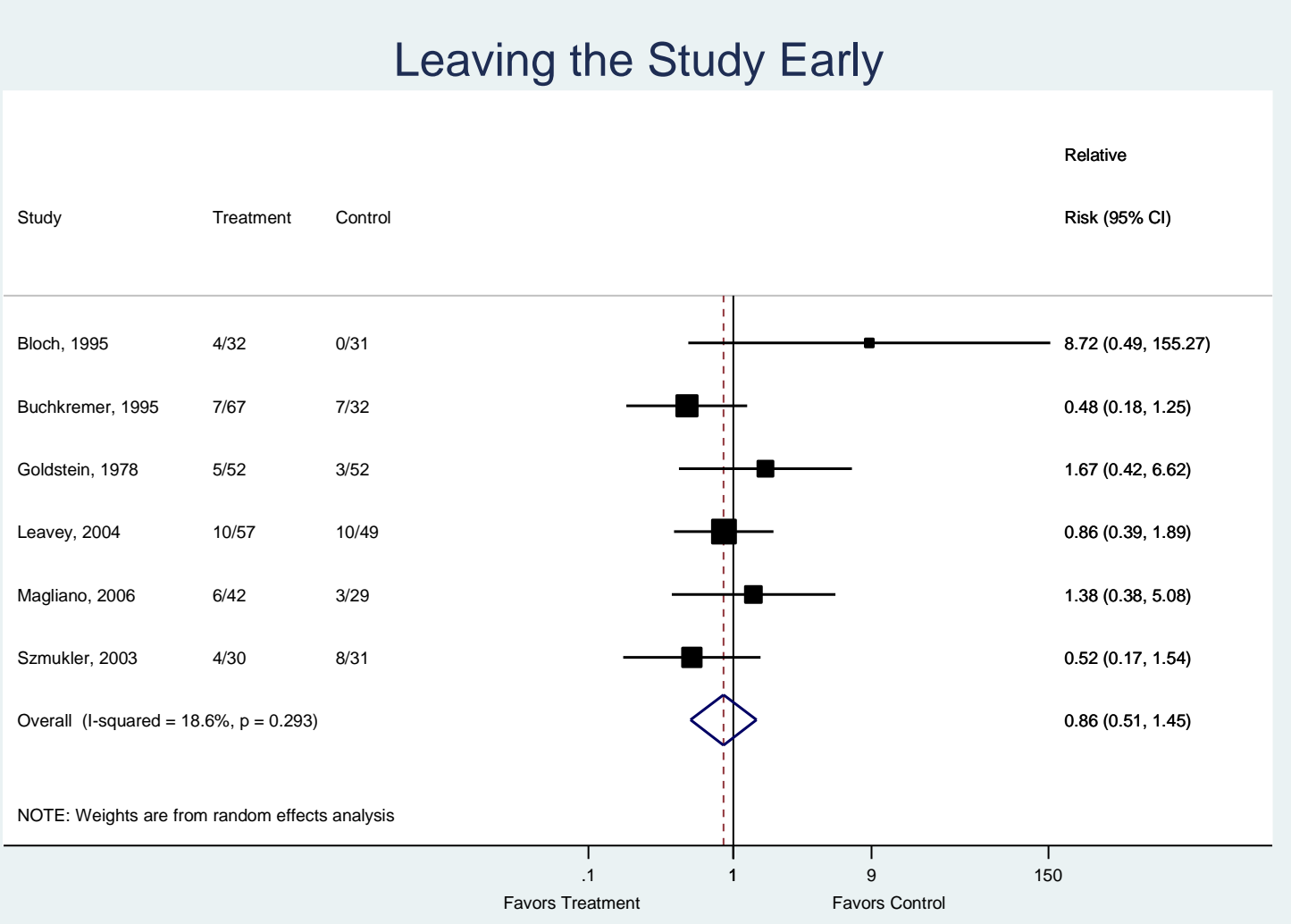




\section{Appendix Figure G-21. Family interventions: treatment maintenance at 7-12 months}

\section{Leaving Study Early}

7-12 Months

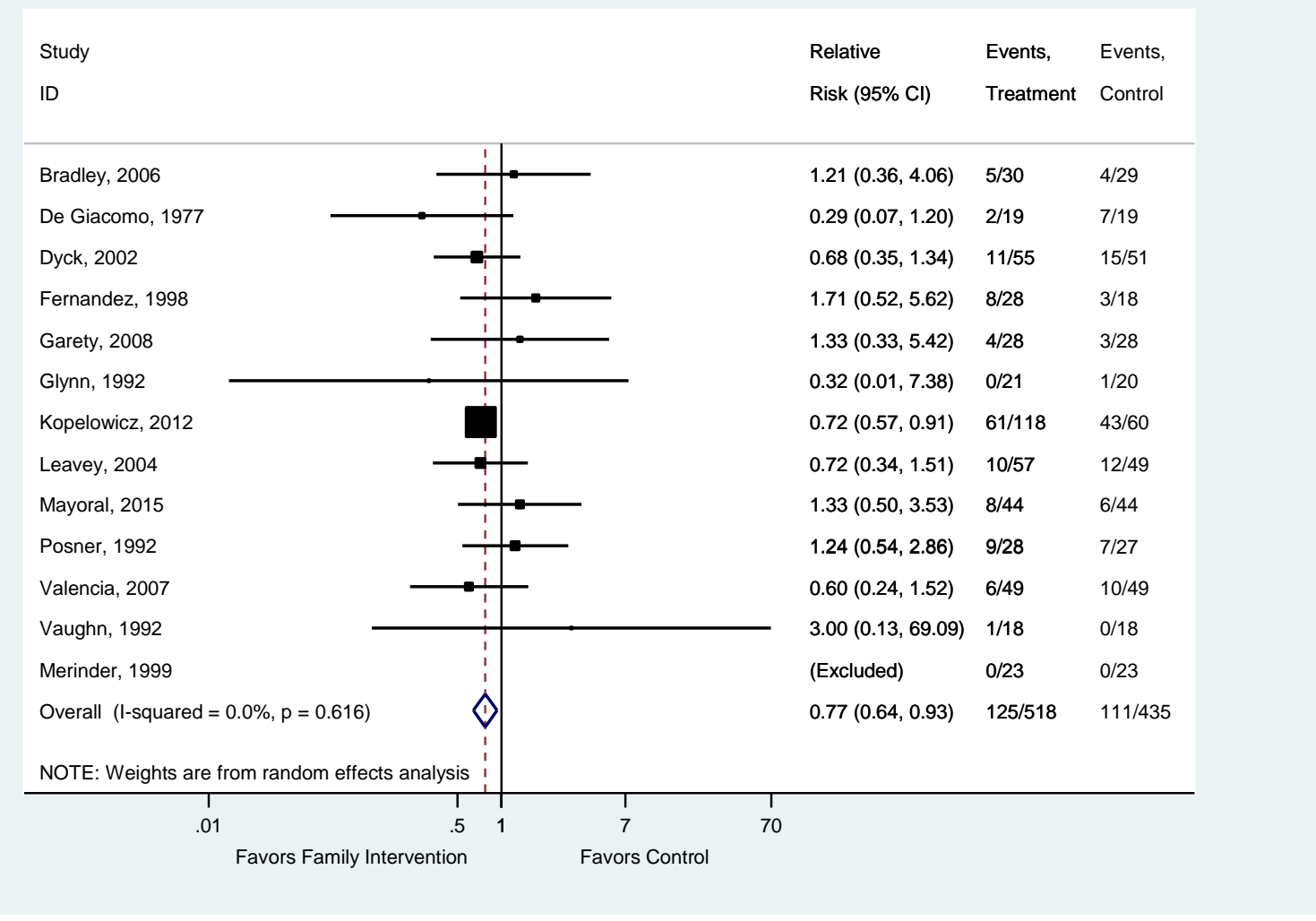


Appendix Figure G-22. Family Interventions: relapse at 0-6 months by number of sessions

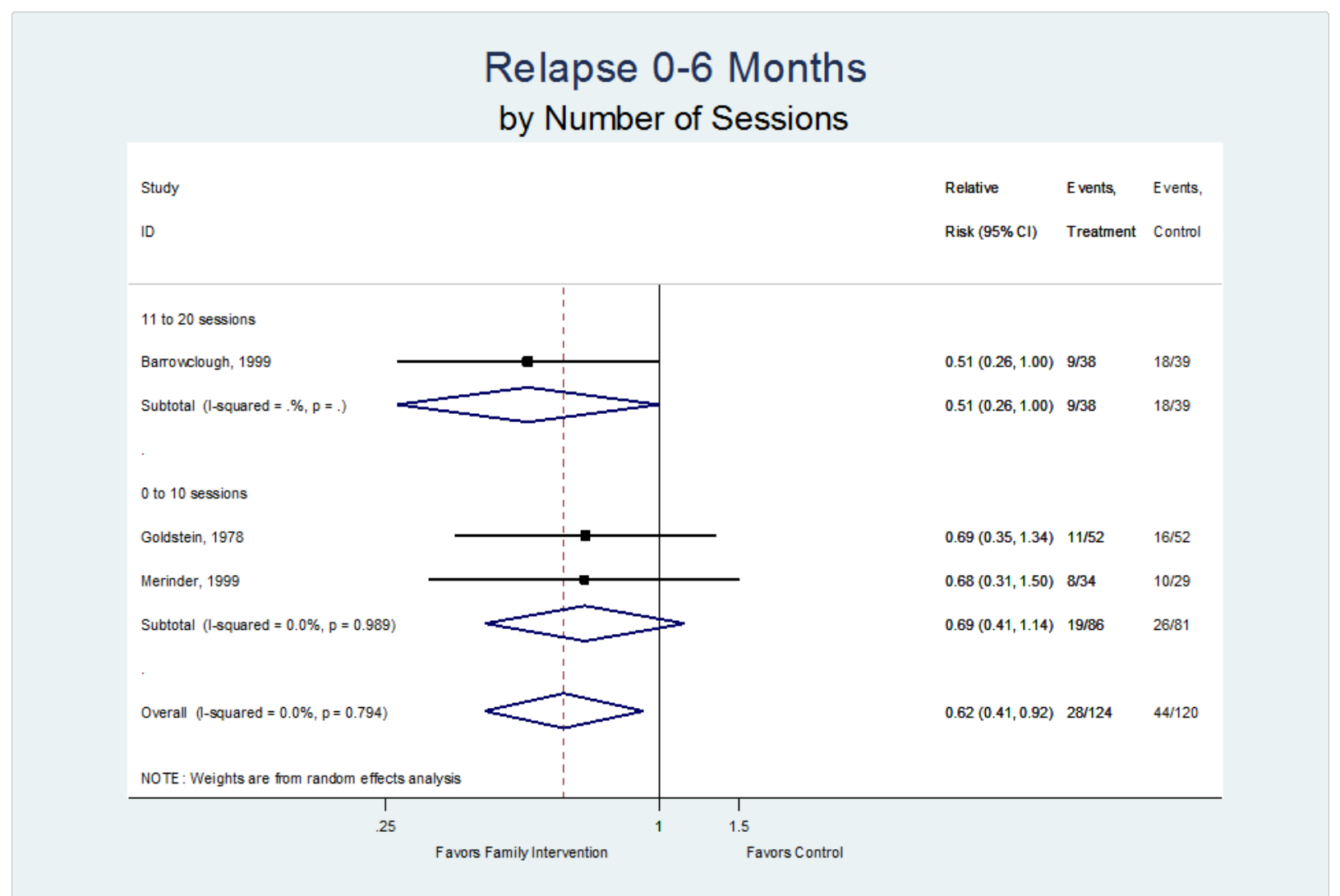




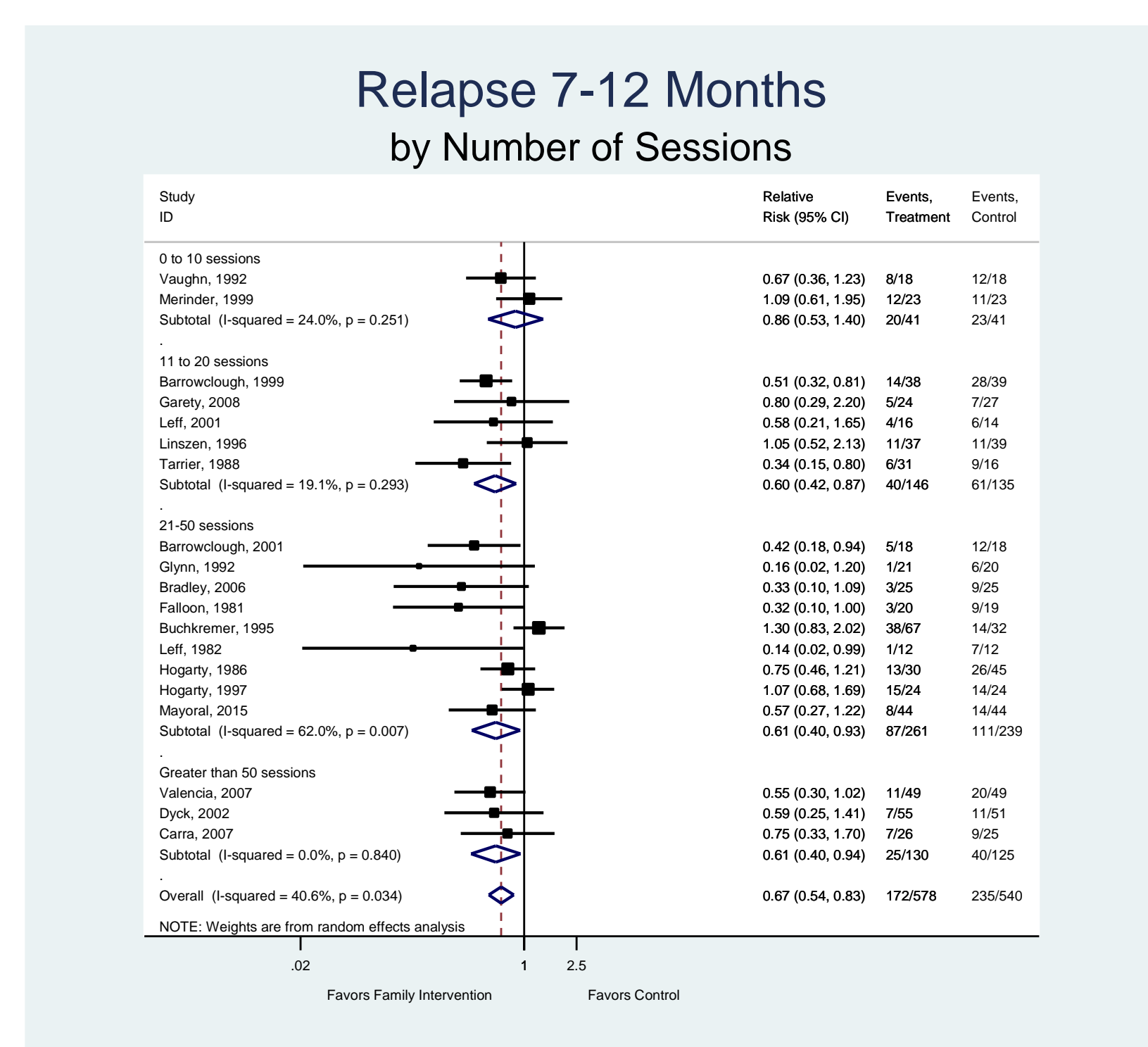




\section{Relapse 7-12 Months by Duration of Intervention}

Study
ID


Appendix Figure G-25. Family interventions: relapse at 7-12 months by psychoeducation

\section{Relapse 7-12 Months \\ by Psychoeducation}

Study
ID


Appendix Figure G-26. Family interventions: relapse at 13-18 months by number of sessions

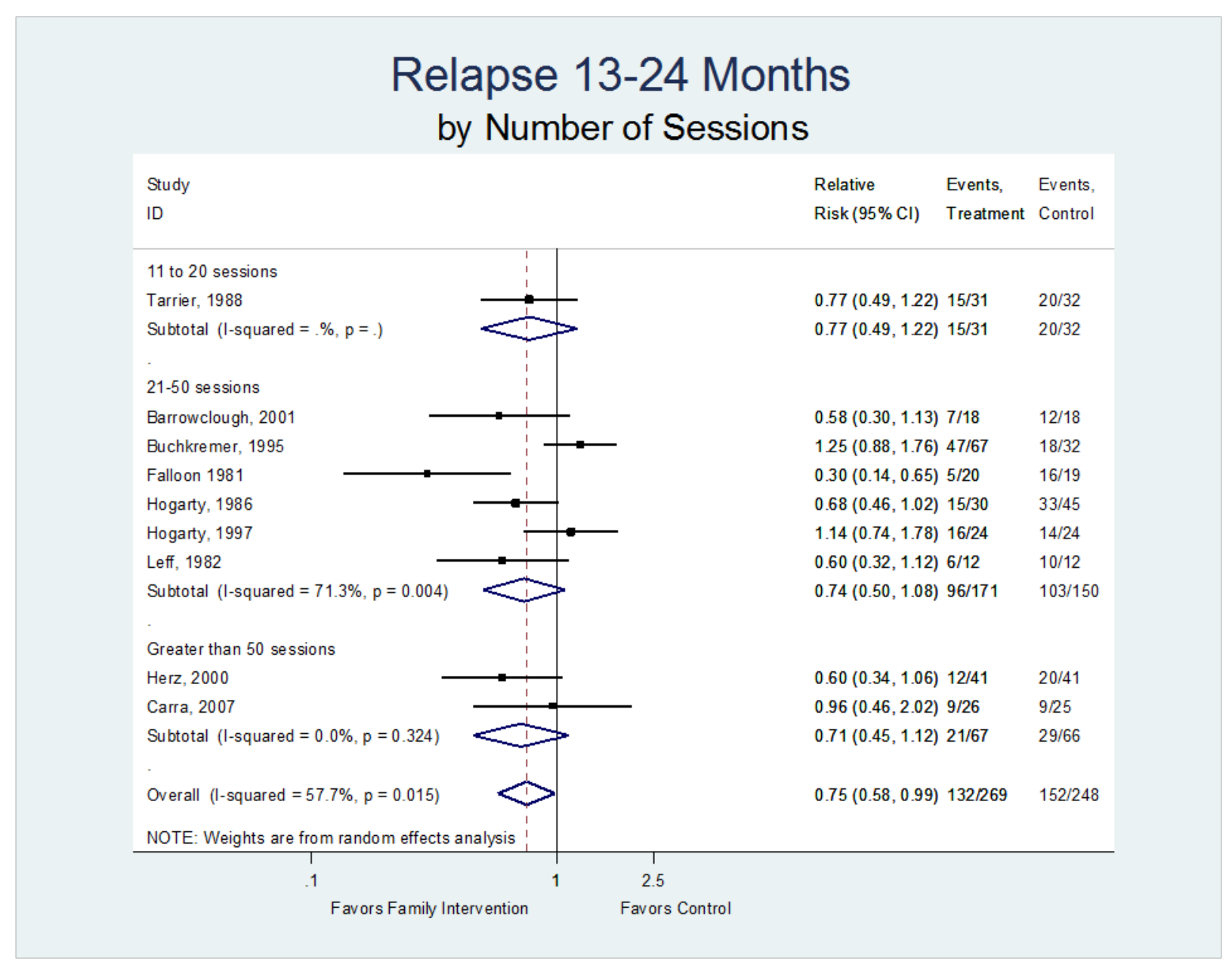


Appendix Figure G-27. Family interventions; relapse at 13-24 months by duration of intervention

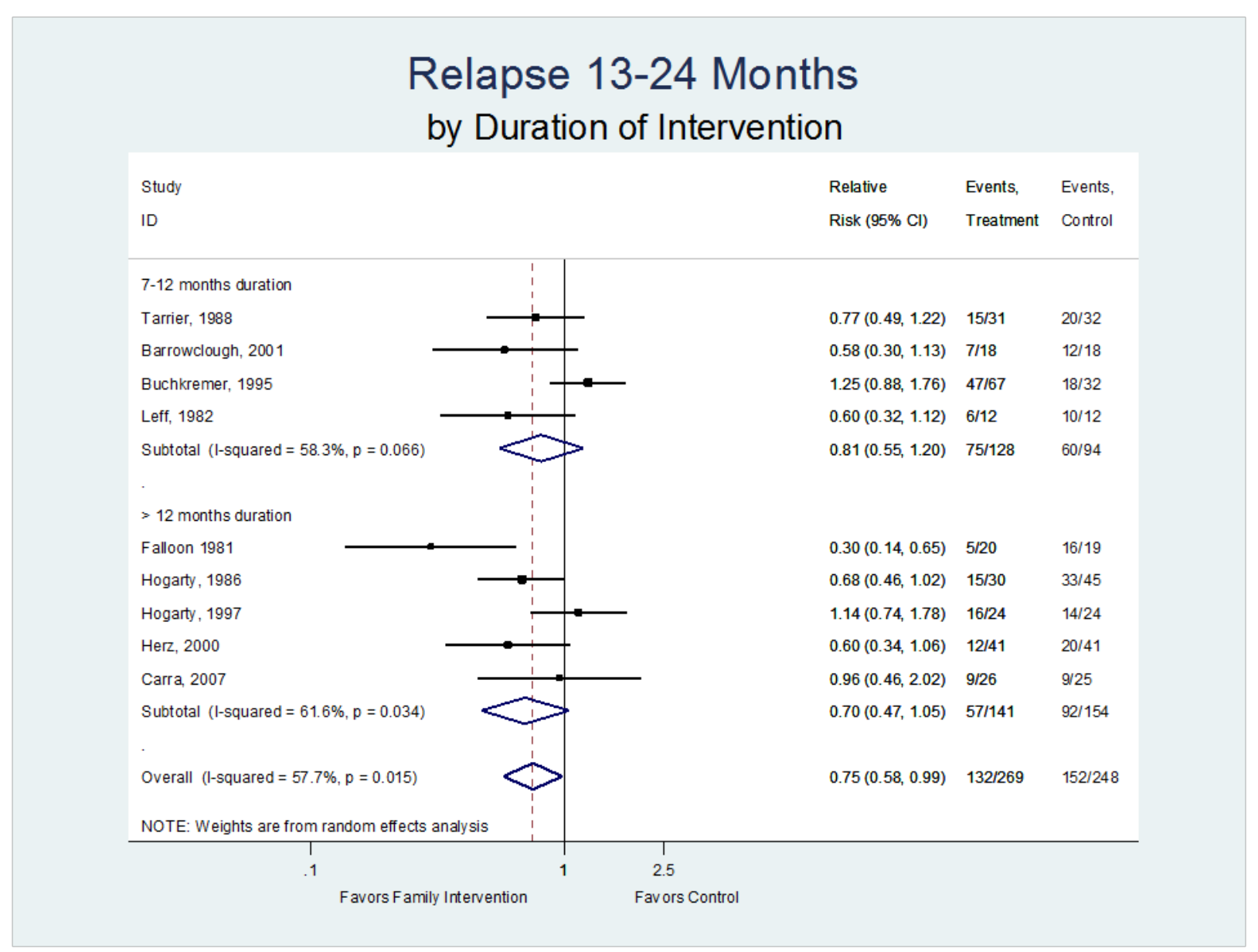


Appendix Figure G-28. Family interventions: relapse at 25-36 months by duration of intervention

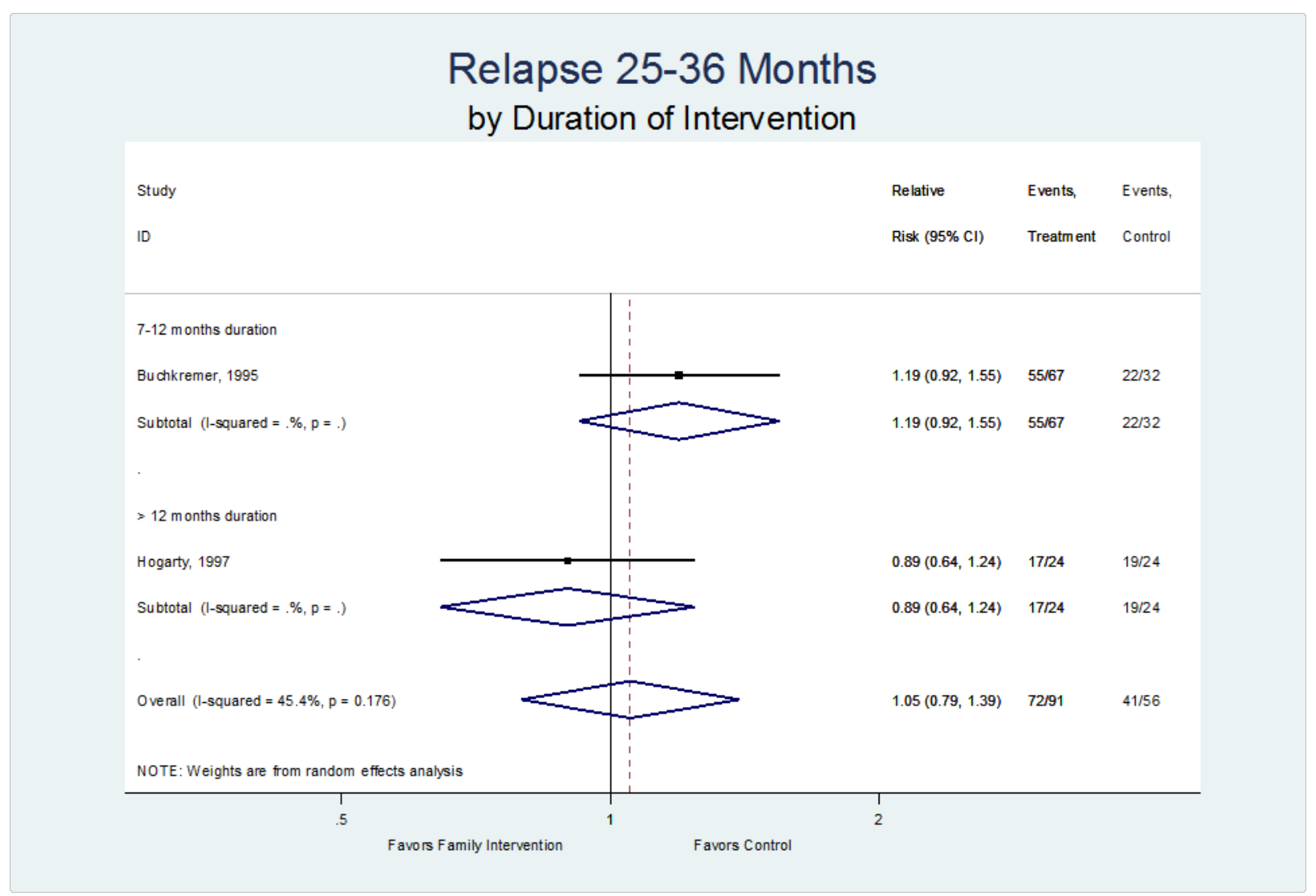


Appendix Figure G-29. Family interventions: relapse at 5 years by duration of intervention

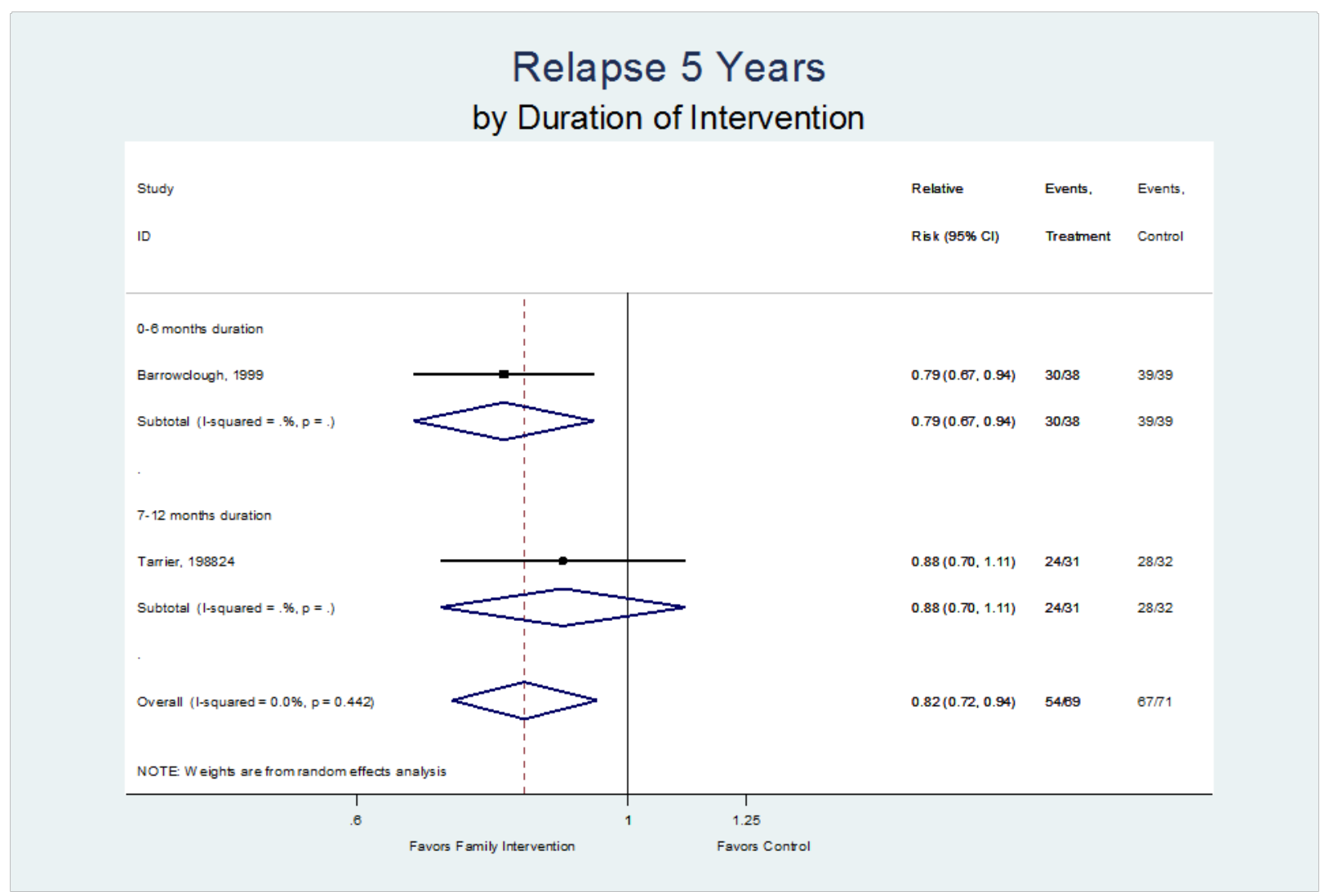


Appendix Figure G-30. Family interventions: nonsuicide mortality

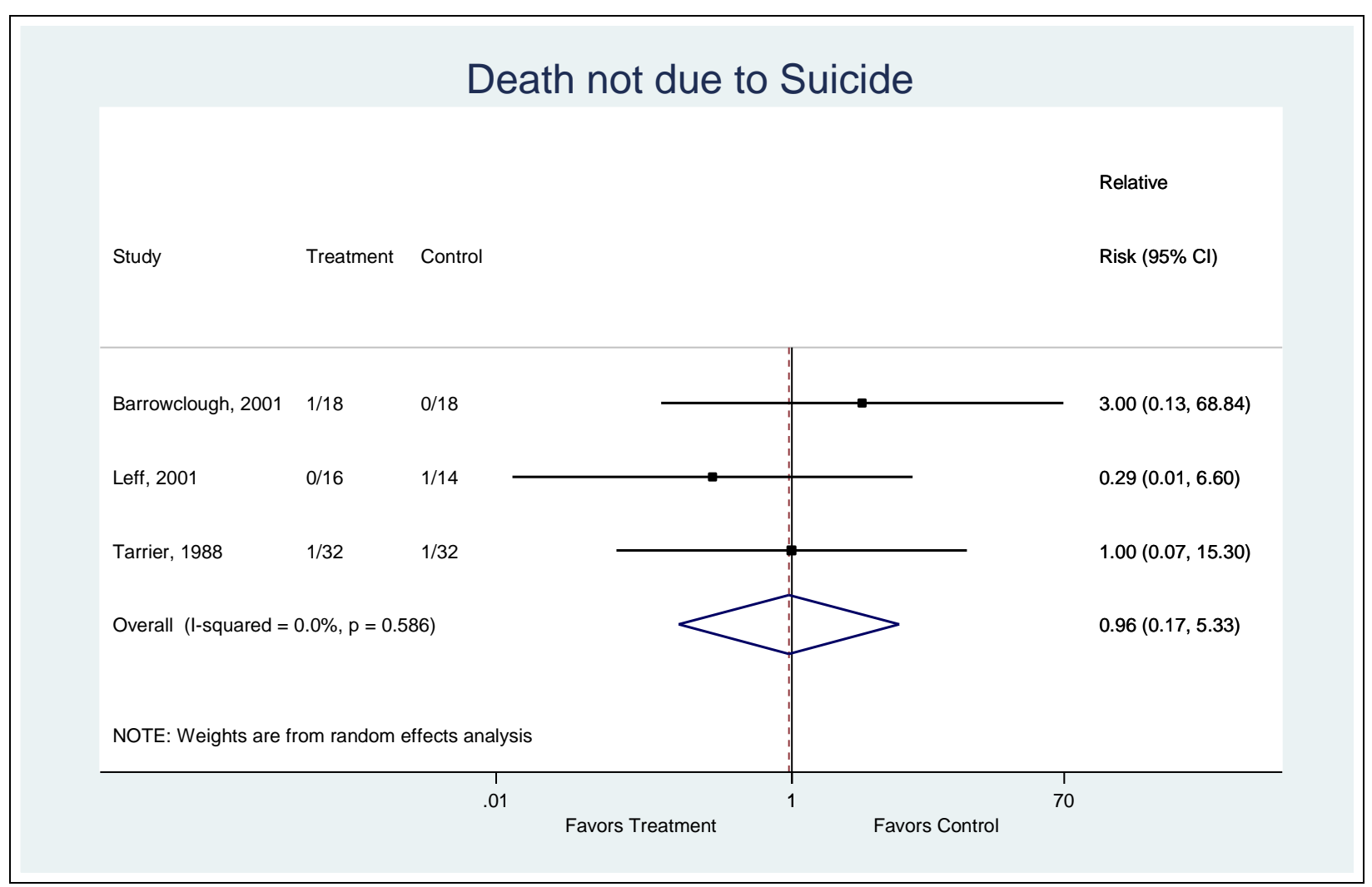


Appendix Figure G-31. Family interventions: poor compliance with medication

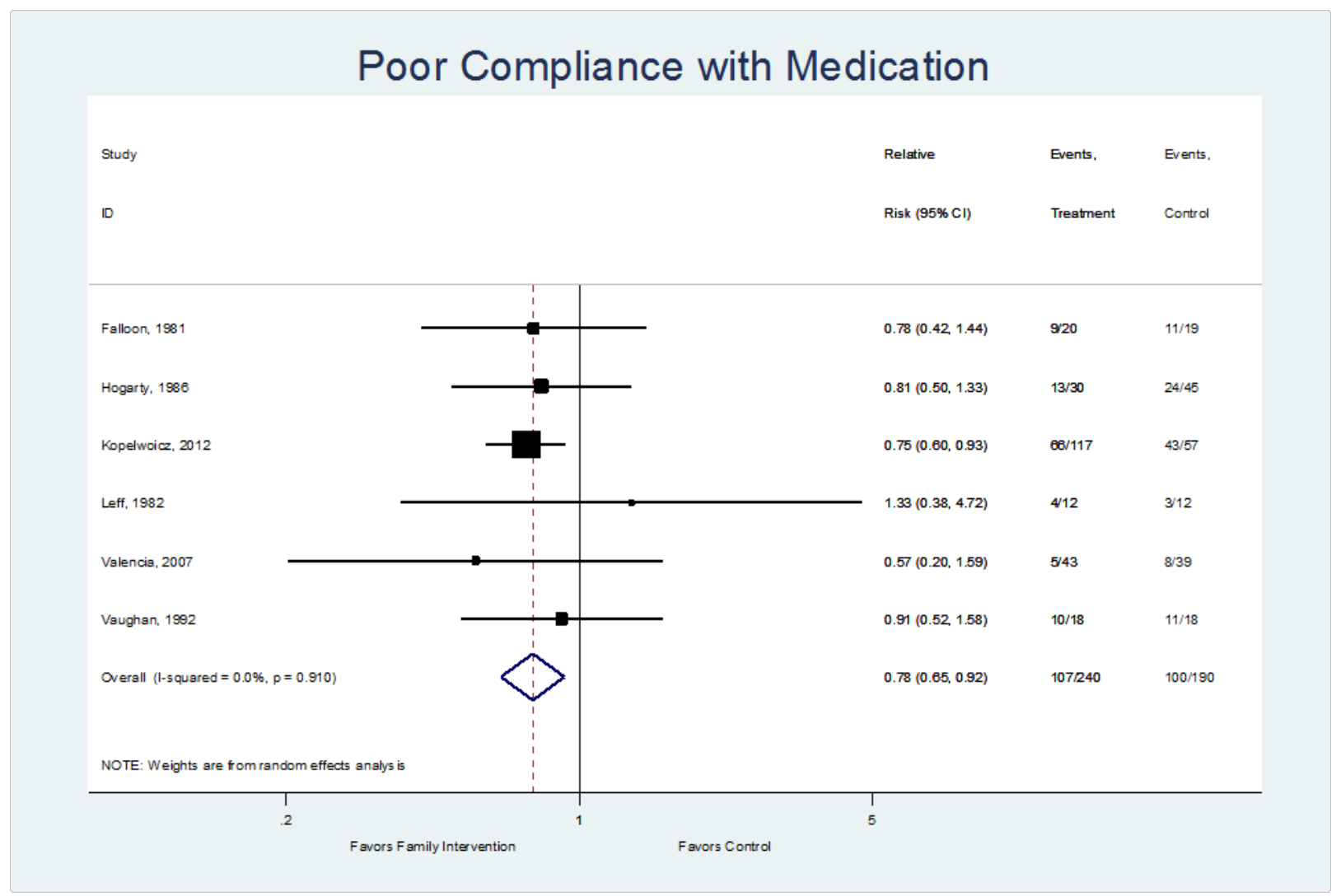


Appendix Figure G-32. Intensive case management: loss to followup

\begin{tabular}{|c|c|c|c|c|c|c|c|c|c|c|}
\hline \multirow[b]{2}{*}{ Study or Subgroup } & \multicolumn{2}{|c|}{ Intensive case management } & \multicolumn{2}{|c|}{ Usual care } & \multirow[b]{2}{*}{ Weight } & \multirow{2}{*}{$\begin{array}{l}\text { Odds Ratio } \\
\text { M-H, Random, } 95 \% \mathrm{Cl}\end{array}$} & \multirow{2}{*}{\multicolumn{4}{|c|}{$\begin{array}{l}\text { Odds Ratio } \\
\text { M-H, Random, } 95 \% \mathrm{Cl} \\
\end{array}$}} \\
\hline & Events & Total & Events & Total & & & & & & \\
\hline Bjorkman 2002 & 5 & 33 & 8 & 44 & $5.3 \%$ & $0.80[0.24,2.73]$ & & & & \\
\hline Ford 1995 & 0 & 35 & 6 & 38 & $1.0 \%$ & $0.07[0.00,1.30]$ & & & 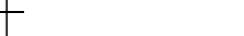 & \\
\hline Franklin 1987 & 75 & 213 & 74 & 200 & $34.8 \%$ & $0.93[0.62,1.38]$ & & - & - & \\
\hline Macias 1994 & 2 & 20 & 5 & 21 & $2.6 \%$ & $0.36[0.06,2.09]$ & & & & \\
\hline Marshall 1995 & 9 & 40 & 8 & 38 & $6.7 \%$ & $1.09[0.37,3.19]$ & & & & \\
\hline Muijen 1994 & 10 & 41 & 14 & 41 & $8.3 \%$ & $0.62[0.24,1.63]$ & & & - & \\
\hline Solomon 1994 & 17 & 60 & 25 & 80 & $13.5 \%$ & $0.87[0.42,1.81]$ & & . & & \\
\hline Tyrer 1995 & 37 & 190 & 63 & 193 & $28.0 \%$ & $0.50[0.31,0.80]$ & & $\rightarrow$ & & \\
\hline Total $(95 \% \mathrm{Cl})$ & & 632 & & 655 & $100.0 \%$ & $0.71[0.54,0.95]$ & & 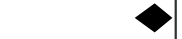 & & \\
\hline Total events & 155 & & 203 & & & & & & & \\
\hline \multirow{2}{*}{\multicolumn{7}{|c|}{$\begin{array}{l}\text { Heterogeneity: } \text { Tau }^{2}=0.02 ; \mathrm{Chi}^{2}=7.88, \mathrm{df}=7(\mathrm{P}=0.34) ; \mathrm{I}^{2}=11 \% \\
\text { Test for overall effect: } Z=2.30(P=0.02)\end{array}$}} & 0.01 & 0.1 & 10 & 100 \\
\hline & & & & & & & & Favors ICM & Favors usual care & \\
\hline
\end{tabular}




\section{Appendix H. Strength of Evidence}

Appendix Table H-1. Strength of evidence: second-generation antipsychotic versus second-generation antipsychotic

\begin{tabular}{|c|c|c|c|c|c|c|c|c|}
\hline Outcome & Comparators & $\begin{array}{l}\text { Number of Studies } \\
\text { Number of Subjects }\end{array}$ & $\begin{array}{l}\text { Study } \\
\text { Limitations }\end{array}$ & Consistency & : Directness & Precision & $\begin{array}{l}\text { Magnitude of Effect: } \\
\text { Summary Effect Size } \\
(95 \% \mathrm{Cl})\end{array}$ & $\begin{array}{l}\text { Strength of } \\
\text { Evidence } \\
\text { (High, } \\
\text { Moderate, } \\
\text { Low, } \\
\text { Insufficient) }\end{array}$ \\
\hline $\begin{array}{l}\text { Social } \\
\text { Functioning }\end{array}$ & $\begin{array}{l}\text { Olanzapine, } \\
\text { risperidone, } \\
\text { quetiapine } \\
\text { immediate-release }\end{array}$ & $\begin{array}{l}1 \mathrm{SR}^{1}(2 \mathrm{RCTs} ; \mathrm{N}=358 ;) \\
(1 \text { observational study; } \mathrm{N} \\
=9028)\end{array}$ & $\begin{array}{l}\text { Moderate } \\
\text { Observational } \\
\text { evidence: } \\
\text { Moderate }\end{array}$ & $\begin{array}{l}\text { Inconsistent } \\
\text { Observational } \\
\text { evidence: } \\
\text { Unknown }\end{array}$ & $\begin{array}{l}\text { Direct } \\
\text { Observational } \\
\text { evidence: } \\
\text { Direct }\end{array}$ & $\begin{array}{l}\text { Imprecise } \\
\text { Observa- } \\
\text { tional } \\
\text { evidence: } \\
\text { Precise }\end{array}$ & $\begin{array}{l}\text { Inconclusive: } \\
\text { RCT 1: no significant } \\
\text { differences on RFS or the } \\
\text { SAS-SMI } \\
\text { RCT 2: Change on SFS } \\
\text { greater with App H } \\
\text { olanzapine (+7.75) than } \\
\text { risperidone (-0.92; } \\
\mathrm{P}=0.0028)(\text { Socially active: } \\
\text { OR 1.27, 95\% CI } 1.05 \text { to } \\
1.54 \text { olanzapine } 84.6 \% \text { vs. } \\
\text { risperidone } 82.4 \%) \text {. }\end{array}$ & Insufficient \\
\hline $\begin{array}{l}\text { Social } \\
\text { Functioning }\end{array}$ & $\begin{array}{l}\text { Paliperidone monthly } \\
\text { LAI vs. risperidone } q \\
2 \text { wks LAI }\end{array}$ & $1 \mathrm{SR}^{1}(2 \mathrm{RCTs} ; \mathrm{N}=452)$ & Moderate & Inconsistent & Direct & Precise & $\begin{array}{l}\text { No statistically significant } \\
\text { differences in PSP scale: } \\
\text { mean change from baseline } \\
16.8 \text { paliperidone and } 18.6 \\
\text { risperidone; (LSM difference } \\
0.5,95 \% \mathrm{Cl}-2.14 \text { to } 3.12 \text { ) }\end{array}$ & Low \\
\hline $\begin{array}{l}\text { Social } \\
\text { Functioning }\end{array}$ & $\begin{array}{l}\text { Paliperidone } \\
\text { extended release vs. } \\
\text { olanzapine }\end{array}$ & $\begin{array}{l}1 \text { Meta-analysis of } \\
\text { selected studies }^{2}\end{array}$ & High & Unknown & Direct & Precise & $\begin{array}{l}\text { No significant difference in } \\
\text { PSP scale (change } 7.8 \text { to } \\
12.2 \text { in paliperidone dose u } \\
\text { vs. } 8.7 \text { in olanzapine group). }\end{array}$ & Insufficient \\
\hline $\begin{array}{l}\text { Social } \\
\text { Functioning }\end{array}$ & $\begin{array}{l}\text { Risperidone LAI vs. } \\
\text { Quetiapine } \\
\text { immediate release }\end{array}$ & $1 \mathrm{RCT}^{3}(\mathrm{~N}=666)$ & Moderate & Unknown & Direct & Precise & $\begin{array}{l}\text { Risperidone LAI resulted in } \\
\text { greater improvements in } \\
\text { SOFA at } 6 \text { months ( } 6.1 \text { vs. } \\
2.7 ; P=0.02), 12 \text { months } \\
\text { ( } 9.5 \text { vs. } 6.1 ; P=0.009) \text {, and } \\
\text { endpoint ( } 6.6 \text { vs. } 1.1 ; P< \\
0.0001) .\end{array}$ & Low \\
\hline $\begin{array}{l}\text { Employment } \\
\text { Outcomes }\end{array}$ & $\begin{array}{l}\text { Older SGAs } \\
\text { (olanzapine, } \\
\text { risperidone, } \\
\text { quetiapine, } \\
\text { ziprasidone) }\end{array}$ & $\begin{array}{l}1 \mathrm{SR}^{1}(2 \mathrm{RCTs}, 3 \\
\text { observational } \\
\mathrm{N}=1,379)\end{array}$ & $\begin{array}{l}\text { Low } \\
\text { Observational } \\
\text { evidence: } \\
\text { Moderate }\end{array}$ & $\begin{array}{l}\text { Inconsistent } \\
\text { Observational } \\
\text { evidence: } \\
\text { Consistent }\end{array}$ & $\begin{array}{l}\text { Direct } \\
\text { Observational } \\
\text { evidence: } \\
\text { Direct }\end{array}$ & $\begin{array}{l}\text { Imprecise } \\
\text { Observa- } \\
\text { tional } \\
\text { evidence: } \\
\text { Imprecise }\end{array}$ & $\begin{array}{l}\text { No significant differences in } \\
\text { rates of employment (mean } \\
18 \% \text { in CATIE Phase I) }\end{array}$ & Low \\
\hline
\end{tabular}




\begin{tabular}{|c|c|c|c|c|c|c|c|c|}
\hline Outcome & Comparators & $\begin{array}{l}\text { Number of Studies } \\
\text { Number of Subjects }\end{array}$ & $\begin{array}{l}\text { Study } \\
\text { Limitations }\end{array}$ & Consistency & : Directness & Precision & $\begin{array}{l}\text { Magnitude of Effect: } \\
\text { Summary Effect Size } \\
(95 \% \mathrm{Cl})\end{array}$ & $\begin{array}{l}\text { Strength of } \\
\text { Evidence } \\
\text { (High, } \\
\text { Moderate, } \\
\text { Low, } \\
\text { Insufficient) } \\
\end{array}$ \\
\hline $\begin{array}{l}\text { Occupation } \\
\text { and } \\
\text { Residential } \\
\text { Status }\end{array}$ & $\begin{array}{l}\text { Older SGAS } \\
\text { (olanzapine, } \\
\text { risperidone, } \\
\text { quetiapine, } \\
\text { ziprasidone) }\end{array}$ & $1 \mathrm{SR}^{1}(21 \mathrm{RCT}, \mathrm{N}=771)$ & Moderate & Unknown & Direct & Imprecise & $\begin{array}{l}\text { Inconclusive: } \\
75.5 \% \text { and } 75.3 \% \text { had stable } \\
\text { status, } 3.8 \% \text { and } 3.1 \% \\
\text { improved status (NS) }\end{array}$ & Insufficient \\
\hline $\begin{array}{l}\text { Global } \\
\text { Functioning } \\
\text { (GAF) }\end{array}$ & $\begin{array}{l}\text { Olanzapine vs. } \\
\text { Risperidone }\end{array}$ & $\begin{array}{l}1 \mathrm{SR}^{1}(4 \text { cohort studies; } \mathrm{N} \\
=3211)\end{array}$ & High & Inconsistent & Direct & Precise & $\begin{array}{l}\text { No difference: } \\
\text { Pooled WMD } 0.61,95 \% \mathrm{Cl} \text {, - } \\
1.78-2.99 ; \mathrm{I}^{2}=43 \%\end{array}$ & Low \\
\hline $\begin{array}{l}\text { Global } \\
\text { Functioning } \\
\text { (GAF) }\end{array}$ & $\begin{array}{l}\text { Olanzapine vs. } \\
\text { Quetiapine }\end{array}$ & $1 \mathrm{SR}^{1}$ (2 RCTs; N = 363) & Moderate & Consistent & Direct & Imprecise & $\begin{array}{l}\text { Pooled WMD } 1.14,95 \% \mathrm{Cl}- \\
4.75 \text { to } 7.02 ; \mathrm{Q}=3.99, \mathrm{df}= \\
1 ; \mathrm{P}=0.045\end{array}$ & Low \\
\hline $\begin{array}{l}\text { Quality of } \\
\text { Life }\end{array}$ & $\begin{array}{l}\text { Olanzapine vs. } \\
\text { risperidone }\end{array}$ & $1 \mathrm{SR}^{1}$ (2 RCTs; $\mathrm{N}=492$ ) & Moderate & Consistent & Direct & Precise & $\begin{array}{l}\text { QLS Change }=12 \text { months } \\
0.19 \vee 0.26(P=0.53) ; 7 \\
\text { months } 13.4 \text { vs. } 8.8(P \\
>0.074)\end{array}$ & Moderate \\
\hline Quality of Life & $\begin{array}{l}\text { Olanzapine vs. } \\
\text { ziprasidone }\end{array}$ & $1 \mathrm{SR}^{1}$ (2 RCTs; N = 740) & Moderate & Consistent & Direct & Precise & $\begin{array}{l}\text { QLS Change = } 12 \text { months } \\
0.19 \text { v } 0.26(P \text { NR); 6-7 } \\
\text { months Endpoint } 61.3 \text { vs. } \\
58.9 \text { ( } P=0.36 \text { using mixed- } \\
\text { effect modeling) }\end{array}$ & Moderate \\
\hline Quality of Life & $\begin{array}{l}\text { Olanzapine vs. } \\
\text { quetiapine } \\
\text { immediate release }\end{array}$ & $1 \mathrm{SR}^{1}(1 \mathrm{RCT} ; \mathrm{N}=227)$ & Low & Unknown & Direct & Imprecise & $\begin{array}{l}\text { QLS Change }=12 \text { months } \\
0.19 \vee 0.09(P>0.05)\end{array}$ & Low \\
\hline Quality of Life & $\begin{array}{l}\text { Olanzapine vs. } \\
\text { asenapine }\end{array}$ & $1 \mathrm{SR}^{1}(1 \mathrm{RCT} ; \mathrm{N}=464)$ & Moderate & Unknown & Direct & Imprecise & $\begin{array}{l}\text { QLS Change =12 months } \\
11.7 \text { vs. } 11.8 \text { and } 11.1 \text { vs. } \\
7.1 \text { (multi-country study } \\
\text { reported by hemisphere) P = } \\
\text { NS }\end{array}$ & Insufficient \\
\hline Quality of Life & $\begin{array}{l}\text { Olanzapine vs. } \\
\text { clozapine }\end{array}$ & $1 \mathrm{SR}^{1}(1 \mathrm{RCT} ; \mathrm{N}=114)$ & Moderate & Unknown & Direct & Imprecise & $\begin{array}{l}\text { SWN: } 26 \text { weeks O found } \\
\text { non-inferior to } \mathrm{C} \text { (difference } \\
3.2,95 \% \mathrm{Cl}: 4.2 \text { to } 10.5 \text { ) }\end{array}$ & Insufficient \\
\hline Quality of Life & $\begin{array}{l}\text { Risperidone vs. } \\
\text { ziprasidone }\end{array}$ & $1 \mathrm{SR}^{1}(\mathrm{~N}=154)$ & Low & Unknown & Direct & Imprecise & $\begin{array}{l}\text { QLS Change }=12 \text { months } \\
0.19 \vee 0.26(P>0.05)\end{array}$ & Low \\
\hline Quality of Life & $\begin{array}{l}\text { Risperidone vs. } \\
\text { Quetiapine }\end{array}$ & $1 \mathrm{SR}^{1}(1 \mathrm{RCT} ; \mathrm{N}=189)$ & Low & Unknown & Direct & Imprecise & $\begin{array}{l}\text { QLS Change = } 12 \text { months } \\
0.26 \text { v } 0.26(P>0.05)\end{array}$ & Low \\
\hline Quality of Life & $\begin{array}{l}\text { Quetiapine-ER vs. } \\
\text { Risperidone }\end{array}$ & $1 \mathrm{RCT} ; \mathrm{N}=798^{4}$ & Moderate & Unknown & Direct & Imprecise & $\begin{array}{l}\text { SWN } 20 \% \text { Response } 12 \\
\text { months: } 65 \% \text { vs. } 68 \% \text {, } \\
\text { adjusted difference }(-5.7, \\
95 \% \mathrm{Cl}-15.1 \text { to } 3.7) \text { but not } \\
\text { meet non-inferiority criteria }\end{array}$ & Insufficient \\
\hline
\end{tabular}




\begin{tabular}{|c|c|c|c|c|c|c|c|c|}
\hline Outcome & Comparators & $\begin{array}{l}\text { Number of Studies } \\
\text { Number of Subjects }\end{array}$ & $\begin{array}{l}\text { Study } \\
\text { Limitations }\end{array}$ & Consistency & : Directness & Precision & $\begin{array}{l}\text { Magnitude of Effect: } \\
\text { Summary Effect Size } \\
(95 \% \mathrm{Cl})\end{array}$ & $\begin{array}{l}\text { Strength of } \\
\text { Evidence } \\
\text { (High, } \\
\text { Moderate, } \\
\text { Low, } \\
\text { Insufficient) }\end{array}$ \\
\hline Quality of Life & $\begin{array}{l}\text { Aripiprazole oral vs. } \\
\text { aripiprazole LAI } \\
\text { (monthly) }\end{array}$ & $1 \mathrm{RCT} ; \mathrm{N}=724^{5}$ & Moderate & Unknown & Direct & Precise & $\begin{array}{l}\text { SF-36 } 12 \text { months: mental } \\
\text { component scores ( } 0.82 \text { vs. } \\
0.38 \text {; difference } 0.44,95 \% \\
\mathrm{Cl}-1.24 \text { to } 2.12) \text { and } \\
\text { physical component scores } \\
(0.23 \text { vs. }-0.27 \text {; difference } \\
0.50,95 \% \mathrm{Cl}-1.11 \text { to } 2.11)\end{array}$ & Low \\
\hline Quality of Life & $\begin{array}{l}\text { Aripiprazole LAI vs. } \\
\text { paliperidone } \\
\text { palmitate LAI } \\
\text { (monthly) }\end{array}$ & $1 \mathrm{RCT} ; \mathrm{N}=295^{6}$ & Moderate & Unknown & Direct & Imprecise & $\begin{array}{l}\text { QLS: } 28 \text { weeks change }= \\
7.47 \text { vs. } 2.80 \text { (least squares } \\
\text { mean difference } 4.67,95 \% \\
\text { Cl } 0.32 \text { to } 9.02 \text { ). Meets non- } \\
\text { inferiority criteria; not meet } \\
\text { MCID }\end{array}$ & Insufficient \\
\hline Quality of Life & $\begin{array}{l}\text { Risperidone LAI vs. } \\
\text { quetiapine }\end{array}$ & $1 \mathrm{RCT} ; \mathrm{N}=666^{3}$ & Moderate & Unknown & Direct & Precise & $\begin{array}{l}\text { SF-12 physical and mental } \\
\text { component scores and } \\
\text { SQLS-R4 scores improved } \\
\text { from baseline in both groups } \\
\text { but were not significantly } \\
\text { different at endpoint ( } 24 \\
\text { months; SF-12 physical P= } \\
\text { 0.09; SF-12 mental and } \\
\text { SQLS-R4 P = NR). }\end{array}$ & Low \\
\hline Response & & $\begin{array}{l}\text { Network meta-analysis of } \\
\text { olanzapine, risperidone, } \\
\text { quetiapine IR, } \\
\text { aripiprazole, clozapine, } \\
\text { ziprasidone> asenapine, } \\
\text { paliperidone, aripiprazole } \\
\text { LAI monthly, } \\
\text { carpipramine, } \\
\text { brexpiprazole, lurasidone } \\
46 \text { RCTs }(\mathrm{N}=12,536)\end{array}$ & Moderate & Consistent & Indirect & Precise & $\begin{array}{l}\text { There were } 2 \text { statistically } \\
\text { significant differences } \\
\text { between the drugs; both } \\
\text { olanzapine (OR 1.71. 95\% } \\
\mathrm{Cl} 1.11,2.68 \text { ) and } \\
\text { risperidone (OR } 1.41,95 \% \\
\mathrm{Cl} 1.01,2.00) \text { were } \\
\text { significantly more likely to } \\
\text { result in response than } \\
\text { quetiapine IR. }\end{array}$ & Low \\
\hline $\begin{array}{l}\text { Mortality } \\
\text { (All-Cause) }\end{array}$ & $\begin{array}{l}\text { Olanzapine vs. } \\
\text { risperidone vs. } \\
\text { quetiapine }\end{array}$ & $\begin{array}{l}1 \mathrm{SR}^{1} \text { (1 retrospective } \\
\text { cohort study; } \mathrm{N}=48,595)\end{array}$ & Low & Unknown & Direct & Precise & $\begin{array}{l}\text { No difference in all-cause } \\
\text { mortality between } \\
\text { risperidone and olanzapine } \\
\text { (HR } 1.09,95 \% \mathrm{Cl} 0.79 \text { to } \\
1.49 \text { ) or quetiapine (HR } \\
0.75,95 \% \mathrm{Cl} 0.53 \text { to } 1.07) \text {. }\end{array}$ & Low \\
\hline
\end{tabular}




\begin{tabular}{|c|c|c|c|c|c|c|c|c|}
\hline Outcome & Comparators & $\begin{array}{l}\text { Number of Studies } \\
\text { Number of Subjects } \\
\end{array}$ & \begin{tabular}{|l} 
Study \\
Limitations \\
\end{tabular} & Consistency & : Directness & Precision & $\begin{array}{l}\text { Magnitude of Effect: } \\
\text { Summary Effect Size } \\
(95 \% \mathrm{Cl})\end{array}$ & $\begin{array}{l}\text { Strength of } \\
\text { Evidence } \\
\text { (High, } \\
\text { Moderate, } \\
\text { Low, } \\
\text { Insufficient) } \\
\end{array}$ \\
\hline $\begin{array}{l}\text { Mortality (All- } \\
\text { Cause) }\end{array}$ & $\begin{array}{l}\text { Clozapine, } \\
\text { risperidone, } \\
\text { olanzapine and } \\
\text { quetiapine vs. no } \\
\text { treatment }\end{array}$ & $\begin{array}{l}1 \mathrm{SR}^{1} \text { (1retrospective } \\
\text { cohort study; } \mathrm{N}=6,987 \text { ) }\end{array}$ & Low & Unknown & Direct & Imprecise & $\begin{array}{l}\text { Clozapine and quetiapine } \\
\text { had significantly lower risk of } \\
\text { all-cause mortality (adjusted } \\
\text { ORs } 0.35,95 \% \mathrm{Cl} 0.21 \text { to } \\
0.58 \text { and } 0.46,95 \% \mathrm{Cl} 0.30 \\
\text { to } 0.72 \text { ), risperidone and } \\
\text { olanzapine not statistically } \\
\text { significantly different from } \\
\text { control }\end{array}$ & Insufficient \\
\hline $\begin{array}{l}\text { Mortality (All- } \\
\text { Cause) }\end{array}$ & $\begin{array}{l}\text { Asenapine vs. } \\
\text { Olanzapine }\end{array}$ & $2 \mathrm{RCTs}^{7,8}$ & Moderate & Consistent & Direct & Imprecise & $\begin{array}{l}\text { Inconclusive: } \\
0.41 \% \text { vs. } 0.42 \%, 0 \% \text { vs. } \\
0 \%, 0.77 \% \text { vs. } 0.32 \% ; \text { RR } \\
2.49(95 \% \mathrm{Cl} 0.54 \text { to } 11.5)\end{array}$ & Low \\
\hline $\begin{array}{l}\text { Mortality (All- } \\
\text { Cause) }\end{array}$ & $\begin{array}{l}\text { Paliperidone } \\
\text { palmitate LAI } \\
\text { (monthly) vs. } \\
\text { Risperidone LAI } \\
\end{array}$ & $2 \mathrm{RCTs}^{9,10}$ & Moderate & Consistent & Direct & Imprecise & $\begin{array}{l}\text { Inconclusive: } \\
0.79 \% \text { vs. } 0.27 \%, 0 \% \text { vs. } \\
0.45 \% ; \text { RR } 1.26(95 \% \mathrm{Cl} \\
0.21 \text { to } 7.49)\end{array}$ & Low \\
\hline $\begin{array}{l}\text { Mortality (All- } \\
\text { Cause) }\end{array}$ & $\begin{array}{l}\text { Quetiapine vs. } \\
\text { Risperidone }\end{array}$ & $2 \mathrm{RCTs}^{11,12}$ & Moderate & Consistent & Direct & Imprecise & $\begin{array}{l}\text { Inconclusive: } \\
1.17 \% \text { vs. } 0.40 \% \text { and } 0.72 \% \\
\text { vs. } 0 \% \text {; RR } 3.24(95 \% \mathrm{Cl} \\
0.72 \text { to } 14.6) \\
\end{array}$ & Low \\
\hline $\begin{array}{l}\text { Cardio- } \\
\text { vascular } \\
\text { Mortality }\end{array}$ & $\begin{array}{l}\text { Olanzapine vs. } \\
\text { risperidone vs. } \\
\text { quetiapine }\end{array}$ & $\begin{array}{l}1 \mathrm{SR}^{1} \text { (2 retrospective } \\
\text { cohort study; } N=55,582 \text { ) }\end{array}$ & Low & Consistent & Direct & Precise & $\begin{array}{l}\text { No significant differences } \\
\text { between the drugs (HRs } \\
0.99,95 \% \mathrm{Cl} 0.37 \text { to } 2.67 \\
\text { and } 0.76,95 \% \mathrm{Cl} 0.25 \text { to } \\
2.28 \text {, respectively). }\end{array}$ & Low \\
\hline $\begin{array}{l}\text { Cardio- } \\
\text { vascular } \\
\text { Mortality }\end{array}$ & $\begin{array}{l}\text { Clozapine vs. } \\
\text { risperidone }\end{array}$ & $\begin{array}{l}1 \mathrm{SR}^{1} \text { (2 retrospective } \\
\text { cohort studies; } \mathrm{N}=1,686 \text { ) }\end{array}$ & Moderate & Unknown & Direct & Imprecise & $\begin{array}{l}\text { Inconclusive: } \\
\text { No significant differences } \\
\text { between drugs } 4.8 \% \text { and } \\
2.5 \% \text {; RR } 1.39,95 \% \mathrm{Cl} 0.61 \\
\text { to } 2.53\end{array}$ & Insufficient \\
\hline $\begin{array}{l}\text { Self-Harm: } \\
\text { Suicidal } \\
\text { Behavior, } \\
\text { Suicide } \\
\end{array}$ & $\begin{array}{l}\text { Clozapine versus } \\
\text { olanzapine in high- } \\
\text { risk patients }\end{array}$ & $1 \mathrm{SR}^{1}(1 \mathrm{RCT} ; \mathrm{N}=980)$ & Low & Unknown & Direct & Imprecise & $\begin{array}{l}\text { Suicidal behavior: HR } 0.76 \text {, } \\
95 \% \mathrm{Cl} 0.58 \text { to } 0.97\end{array}$ & Low \\
\hline $\begin{array}{l}\text { Self-Harm: } \\
\text { Suicidal } \\
\text { Behavior, } \\
\text { Suicide } \\
\end{array}$ & $\begin{array}{l}\text { Clozapine vs. } \\
\text { olanzapine in high- } \\
\text { risk patients }\end{array}$ & $1 \mathrm{SR}^{1}(1 \mathrm{RCT} ; \mathrm{N}=980)$ & Low & Unknown & Direct & Precise & $\begin{array}{l}\text { Worsening on CGI-Suicide } \\
\text { Severity: HR } 0.78,95 \% \mathrm{Cl} \\
0.61 \text { to } 0.99\end{array}$ & Moderate \\
\hline $\begin{array}{l}\text { Self-Harm: } \\
\text { Suicidal } \\
\text { Behavior, } \\
\text { Suicide } \\
\end{array}$ & $\begin{array}{l}\text { Clozapine vs. } \\
\text { olanzapine in high- } \\
\text { risk patients }\end{array}$ & $1 \mathrm{SR}^{1}$ (1 RCT; $\left.\mathrm{N}=980\right)$ & Low & Unknown & Direct & Imprecise & $\begin{array}{l}\text { Suicide deaths: No } \\
\text { significant differences ( } 5 \\
\text { clozapine, } 3 \text { olanzapine) }\end{array}$ & Low \\
\hline
\end{tabular}




\begin{tabular}{|c|c|c|c|c|c|c|c|c|}
\hline Outcome & Comparators & $\begin{array}{l}\text { Number of Studies } \\
\text { Number of Subjects }\end{array}$ & $\begin{array}{l}\text { Study } \\
\text { Limitations }\end{array}$ & Consistency & : Directness & Precision & $\begin{array}{l}\text { Magnitude of Effect: } \\
\text { Summary Effect Size } \\
(95 \% \mathrm{Cl})\end{array}$ & $\begin{array}{l}\text { Strength of } \\
\text { Evidence } \\
\text { (High, } \\
\text { Moderate, } \\
\text { Low, } \\
\text { Insufficient) } \\
\end{array}$ \\
\hline $\begin{array}{l}\text { Self-Harm: } \\
\text { Suicidal } \\
\text { Behavior, } \\
\text { Suicide }\end{array}$ & $\begin{array}{l}\text { Clozapine, } \\
\text { risperidone, } \\
\text { olanzapine, } \\
\text { quetiapine, } \\
\text { ziprasidone, } \\
\text { aripiprazole }\end{array}$ & $\begin{array}{l}1 \mathrm{SR}^{1}(2 \text { retrospective } \\
\text { cohorts; } \mathrm{N}=16,584)\end{array}$ & Moderate & Consistent & Direct & Precise & $\begin{array}{l}\text { Death by suicide lower with } \\
\text { clozapine: OR } 0.29(0.14 \text { to } \\
0.63) \text { compared with no } \\
\text { treatment at } 6 \text { months and } \\
\text { clozapine }(1.1 \%) \text { lower than } \\
\text { baseline }(2.2 \%) \text { or other } \\
\text { drugs (range } 2.1 \% \text { to } 3.7 \%) \\
\text { at } 1 \text { year. }\end{array}$ & Low \\
\hline $\begin{array}{l}\text { Self-Harm: } \\
\text { Suicidal } \\
\text { Behavior, } \\
\text { Suicide }\end{array}$ & $\begin{array}{l}\text { Clozapine, } \\
\text { risperidone, } \\
\text { olanzapine, } \\
\text { quetiapine, } \\
\text { ziprasidone, } \\
\text { aripiprazole }\end{array}$ & $\begin{array}{l}1 \mathrm{SR}^{1} \text { (1 prospective } \\
\text { cohort; } \mathrm{N}=10,204)\end{array}$ & High & Unknown & Direct & Precise & $\begin{array}{l}\text { Suicide attempts ( } 6 \text { months: } \\
\text { No statistically significant } \\
\text { difference between drugs }\end{array}$ & Insufficient \\
\hline $\begin{array}{l}\text { Self-Harm: } \\
\text { Suicidal } \\
\text { Behavior, } \\
\text { Suicide }\end{array}$ & $\begin{array}{l}\text { Clozapine, } \\
\text { risperidone, } \\
\text { olanzapine, } \\
\text { quetiapine, } \\
\text { ziprasidone, } \\
\text { aripiprazole }\end{array}$ & $\begin{array}{l}1 \mathrm{SR}^{1} \text { (1 prospective } \\
\text { cohort; } \mathrm{N}=20,489 \text { ) }\end{array}$ & High & Unknown & Direct & Precise & $\begin{array}{l}\text { Inconclusive: } \\
\text { Suicide attempts or death by } \\
\text { suicide: aripiprazole vs. } \\
\text { others combined HR } 0.69 \text {, } \\
95 \% \mathrm{Cl} 0.42 \text { to } 1.14\end{array}$ & Insufficient \\
\hline $\begin{array}{l}\text { Core Illness } \\
\text { Symptoms }\end{array}$ & $\begin{array}{l}\text { Oral SGAs (except } \\
\text { carpipramine) }\end{array}$ & $(212 \text { RCTs })^{13}$ & Moderate & Consistent & Indirect & Precise & $\begin{array}{l}\text { Clozapine had significantly } \\
\text { better improvement than the } \\
\text { other drugs except } \\
\text { olanzapine (SMDs on } \\
\text { PANSS or BPRS -0.32 to - } \\
0.55 \text {. Olanzapine and } \\
\text { risperidone superior to the } \\
\text { other drugs, except for each } \\
\text { other and paliperidone } \\
\text { (SMDs - } 0.13 \text { to }-0.26) \text {. } \\
\text { Paliperidone superior to } \\
\text { lurasidone and iloperidone } \\
\text { (SMD -0.17). All drugs } \\
\text { superior to placebo (SMDs - } \\
0.33 \text { to -0.88). }\end{array}$ & Low \\
\hline $\begin{array}{l}\text { Core Illness } \\
\text { Symptoms }\end{array}$ & $\begin{array}{l}\text { Treatment resistant } \\
\text { patients: clozapine, } \\
\text { risperidone, } \\
\text { olanzapine, } \\
\text { quetiapine, and } \\
\text { ziprasidone }\end{array}$ & $(40 \text { RCTs })^{14}$ & Moderate & Consistent & Indirect & Precise & $\begin{array}{l}\text { The only significant } \\
\text { difference was that the mean } \\
\text { change in the PANSS } \\
\text { greater with olanzapine than } \\
\text { quetiapine (SMD }-0.29,95 \% \\
\mathrm{Cl}-0.56 \text { to }-0.13) \text {. }\end{array}$ & Low \\
\hline
\end{tabular}




\begin{tabular}{|c|c|c|c|c|c|c|c|c|}
\hline Outcome & Comparators & $\begin{array}{l}\text { Number of Studies } \\
\text { Number of Subjects }\end{array}$ & $\begin{array}{l}\text { Study } \\
\text { Limitations }\end{array}$ & Consistency & : Directness & Precision & $\begin{array}{l}\text { Magnitude of Effect: } \\
\text { Summary Effect Size } \\
(95 \% \mathrm{Cl})\end{array}$ & $\begin{array}{l}\text { Strength of } \\
\text { Evidence } \\
\text { (High, } \\
\text { Moderate, } \\
\text { Low, } \\
\text { Insufficient) } \\
\end{array}$ \\
\hline $\begin{array}{l}\text { Core Illness } \\
\text { Symptoms }\end{array}$ & $\begin{array}{l}\text { Brexpiprazole vs. } \\
\text { aripiprazole }\end{array}$ & $(\mathrm{N}=97)^{15}$ & Moderate & Unknown & Indirect & Imprecise & $\begin{array}{l}\text { Inconclusive: } \\
\text { PANSS scale -22.9 vs. }-19.4 \\
\text { from baseline; direct } \\
\text { comparison not reported. }\end{array}$ & Insufficient \\
\hline \multicolumn{9}{|l|}{$\begin{array}{l}\text { Overall/Any } \\
\text { Adverse } \\
\text { Events }\end{array}$} \\
\hline $\begin{array}{l}\text { Overall/Any } \\
\text { Adverse } \\
\text { Events }\end{array}$ & $\begin{array}{l}\text { Asenapine vs. } \\
\text { olanzapine }\end{array}$ & $\begin{array}{l}5 \text { RCTs (4 publications); N } \\
=2189)^{7,8,16,17}\end{array}$ & Moderate & Consistent & Direct & Precise & $\begin{array}{l}\text { Pooled RR } 1.00(95 \% \mathrm{Cl} \\
0.96-1.05) ; I^{2} 9 \%\end{array}$ & Moderate \\
\hline $\begin{array}{l}\text { Overall/Any } \\
\text { Adverse } \\
\text { Events }\end{array}$ & $\begin{array}{l}\text { Quetiapine vs. } \\
\text { risperidone }\end{array}$ & 7 RCTs $(\mathrm{N}=3254)^{11,12,18-22}$ & Moderate & Consistent & Direct & Precise & $\begin{array}{l}\text { Pooled } 1.04(95 \% \mathrm{Cl} 0.97 \text { to } \\
1.12) ; I^{2} 56 \%\end{array}$ & Moderate \\
\hline $\begin{array}{l}\text { Overall/Any } \\
\text { Adverse } \\
\text { Events } \\
\end{array}$ & $\begin{array}{l}\text { Clozapine vs. } \\
\text { olanzapine }\end{array}$ & 2 RCTs $(N=182)^{6,20}$ & Moderate & Consistent & Direct & Imprecise & $\begin{array}{l}\text { Pooled RR } 1.15(95 \% \mathrm{Cl}= \\
1.00 \text { to } 1.33) ; I^{2} 0 \%\end{array}$ & Low \\
\hline $\begin{array}{l}\text { Overall/Any } \\
\text { Adverse } \\
\text { Events }\end{array}$ & $\begin{array}{l}\text { Risperidone vs. } \\
\text { olanzapine }\end{array}$ & 5 RCTs $(N=873)^{20-24}$ & Moderate & Inconsistent & Direct & Precise & $\begin{array}{l}\text { Pooled = RR } 1.02(95 \% \mathrm{Cl}= \\
0.81 \text { to } 1.29) ; I^{2}=77 \%\end{array}$ & Low \\
\hline $\begin{array}{l}\text { Overall/Any } \\
\text { Adverse } \\
\text { Events }\end{array}$ & $\begin{array}{l}\text { Olanzapine vs. } \\
\text { ziprasidone }\end{array}$ & $\begin{array}{l}5 \text { RCTs }(\mathrm{N}=1097 ; 6 \\
\text { weeks to } 6 \text { months } \\
\text { durations) }^{25-29}\end{array}$ & Moderate & Inconsistent & Direct & Precise & $\begin{array}{l}\text { Pooled RR } 1.00(95 \% \mathrm{Cl}= \\
0.86 \text { to } 1.16) ; I^{2}=80 \%\end{array}$ & Low \\
\hline $\begin{array}{l}\text { Overall/Any } \\
\text { Adverse } \\
\text { Events }\end{array}$ & $\begin{array}{l}\text { Olanzapine vs. } \\
\text { quetiapine }\end{array}$ & 3 RCTs $(N=448)^{20-22}$ & Moderate & Consistent & Direct & Imprecise & $\begin{array}{l}\text { Pooled RR } 0.90(95 \% \mathrm{Cl} \\
0.74 \text { to } 1.11) ; I^{2}=30 \%\end{array}$ & Low \\
\hline
\end{tabular}




\begin{tabular}{|c|c|c|c|c|c|c|c|c|}
\hline Outcome & Comparators & $\begin{array}{l}\text { Number of Studies } \\
\text { Number of Subjects }\end{array}$ & $\begin{array}{l}\text { Study } \\
\text { Limitations }\end{array}$ & Consistency & : Directness & Precision & $\begin{array}{l}\text { Magnitude of Effect: } \\
\text { Summary Effect Size } \\
(95 \% \mathrm{Cl})\end{array}$ & $\begin{array}{l}\text { Strength of } \\
\text { Evidence } \\
\text { (High, } \\
\text { Moderate, } \\
\text { Low, } \\
\text { Insufficient) }\end{array}$ \\
\hline $\begin{array}{l}\text { Overall/Any } \\
\text { Adverse } \\
\text { Events }\end{array}$ & $\begin{array}{l}\text { Quetiapine ER } \\
\text { versus quetiapine IR } \\
\text { and risperidone; } \\
\text { risperidone versus } \\
\text { clozapine and } \\
\text { aripiprazole; } \\
\text { olanzapine versus } \\
\text { paliperidone; } \\
\text { risperidone LAl } \\
\text { versus paliperidone } \\
\text { and paliperidone } \\
\text { palmitate monthly } \\
\text { LAl; and aripiprazole } \\
\text { versus aripiprazole } \\
\text { monthly LAl. } \\
\text { Additionally there } \\
\text { were six trials } \\
\text { comparing } \\
\text { asenapine and } \\
\text { olanzapine }\end{array}$ & $1 \mathrm{SR}(28 \mathrm{RCTs} ; \mathrm{N}=7810)$ & Moderate & Consistent & Direct & Imprecise & $\begin{array}{l}\text { No statistically significant } \\
\text { differences were found in } \\
\text { each comparison. }\end{array}$ & Low \\
\hline
\end{tabular}




\begin{tabular}{|c|c|c|c|c|c|c|c|c|}
\hline Outcome & Comparators & $\begin{array}{l}\text { Number of Studies } \\
\text { Number of Subjects }\end{array}$ & $\begin{array}{l}\text { Study } \\
\text { Limitations }\end{array}$ & Consistency & : Directness & Precision & $\begin{array}{l}\text { Magnitude of Effect: } \\
\text { Summary Effect Size } \\
(95 \% \mathrm{Cl})\end{array}$ & $\begin{array}{l}\text { Strength of } \\
\text { Evidence } \\
\text { (High, } \\
\text { Moderate, } \\
\text { Low, } \\
\text { Insufficient) }\end{array}$ \\
\hline $\begin{array}{l}\text { Overall/Any } \\
\text { Adverse } \\
\text { Events }\end{array}$ & $\begin{array}{l}\text { Oral aripiprazole } \\
\text { versus } \\
\text { brexpiprazole, } \\
\text { olanzapine, } \\
\text { paliperidone, and } \\
\text { risperidone LAl; } \\
\text { ziprasidone versus } \\
\text { clozapine, } \\
\text { risperidone, } \\
\text { iloperidone and } \\
\text { lurasidone; } \\
\text { risperidone versus } \\
\text { asenapine, } \\
\text { carpipramine and } \\
\text { risperidone LAl; } \\
\text { clozapine versus } \\
\text { quetiapine, } \\
\text { quetiapine versus } \\
\text { risperidone LAl; } \\
\text { olanzapine versus } \\
\text { olanzapine LAl and } \\
\text { lurasidone; } \\
\text { aripiprazole monthly } \\
\text { LAl versus } \\
\text { paliperidone; and } \\
\text { paliperidone } \\
\text { palmitate monthly } \\
\text { LAl versus 3- } \\
\text { monthly LAl. }\end{array}$ & 1 SR (31 RCTs; N = 6700) & Moderate & Unknown & Direct & Imprecise & $\begin{array}{l}\text { No statistically significant } \\
\text { differences were found in } \\
\text { single studies of each } \\
\text { comparison. }\end{array}$ & Insufficient \\
\hline
\end{tabular}




\begin{tabular}{|c|c|c|c|c|c|c|c|c|}
\hline Outcome & Comparators & $\begin{array}{l}\text { Number of Studies } \\
\text { Number of Subjects }\end{array}$ & $\begin{array}{l}\text { Study } \\
\text { Limitations }\end{array}$ & Consistency & : Directness & Precision & $\begin{array}{l}\text { Magnitude of Effect: } \\
\text { Summary Effect Size } \\
(95 \% \mathrm{Cl})\end{array}$ & $\begin{array}{l}\text { Strength of } \\
\text { Evidence } \\
\text { (High, } \\
\text { Moderate, } \\
\text { Low, } \\
\text { Insufficient) }\end{array}$ \\
\hline $\begin{array}{l}\text { Discontinu- } \\
\text { ation Due to } \\
\text { Adverse } \\
\text { Events }\end{array}$ & $\begin{array}{l}\text { Network meta- } \\
\text { analysis of } \\
\text { aripiprazole, } \\
\text { aripiprazole monthly } \\
\text { LAl, } \\
\text { asenapine, } \\
\text { brexipiprazole, } \\
\text { cariprazine, } \\
\text { clozapine, } \\
\text { iloperidone, } \\
\text { lurasidone10, } \\
\text { olanzapine, } \\
\text { olanzapine LAI, } \\
\text { paliperidone 3- } \\
\text { month LAI, } \\
\text { paliperidone, } \\
\text { paliperidone Monthly } \\
\text { LAl, } \\
\text { quetiapine ER, } \\
\text { quetiapine IR, } \\
\text { risperidone, } \\
\text { risperidone LAI, } \\
\text { ziprasidone }\end{array}$ & 89 RCTs $(\mathrm{N}=29,678)$ & Moderate & Consistent & Indirect & Precise & $\begin{array}{l}\text { LAl risperidone had } \\
\text { statistically significantly } \\
\text { lower risk of withdrawals due } \\
\text { to adverse events than } \\
\text { asenapine (OR 0.50, 95\% Cl } \\
0.23,0.97) ; \text { clozapine (OR } \\
0.26,95 \% \mathrm{Cl} 0.10,0.67) ; \\
\text { lurasidone (OR 0.38, 95\% Cl } \\
0.17,0.79) ; \text { paliperidone (OR } \\
0.43,95 \% \mathrm{Cl} 0.17,0.98) ; \\
\text { paliperidone LAl monthly } \\
\text { (OR 0.51, 95\% Cl } \\
\text { O.26,0.98); quetiapine ER } \\
\text { (OR 0.42, 95\% Cl } \\
0.21,0.78) ; \text { risperidone (OR } \\
0.48,95 \% \mathrm{Cl} 0.23,0.92) ; \text { and } \\
\text { ziprasidone (OR 0.39, 95\% } \\
\mathrm{Cl} 0.18,0.76) \text {. Olanzapine } \\
\text { had lower risk than } \\
\text { clozapine (OR 0.40, 95\% Cl } \\
0.21,0.79) ; \text { lurasidone (OR } \\
0.58,95 \% \mathrm{Cl} 0.36,0.98) ; \\
\text { quetiapine IR (OR 0.64, 95\% } \\
\mathrm{Cl} 0.45,0.93) \text {; risperidone } \\
\text { (OR 0.74, 95\% Cl } \\
\text { 0.55,0.98); and ziprasidone } \\
\text { (OR 0.59, 95\% Cl } \\
0.43,0.84) \text {. Aripiprazole had } \\
\text { lower risk than ziprasidone } \\
\text { (OR 0.65, 95\% Cl 0.44,0.95) } \\
\text { and iloperidone had lower } \\
\text { risk than clozapine (OR } \\
0.35,95 \% \mathrm{Cl} 0.13,0.91) \text {. }\end{array}$ & Low \\
\hline
\end{tabular}

BPRS=Brief Psychiatric Rating Scale, CATIE=clinical Antipsychotic Trials of Intervention Effectiveness, CGI-S=Clinical Global Impression - Severity, CI=confidence interval, ER=efficacy ratio, GAF=global assessment of functioning, HR=hazard ratio, LAI=long acting injectable, NR=normal range, NS=not significant, NSD=no significant difference, OR=odds ratio, PSP=, Q=Cochran's Q test, QLS=quality of life scale, RCT=randomized controlled trial, RR=relative risk, SF=short form, SFS=Social Functioning Scale, SGA=second-generation antipsychotic, SMD=standard mean difference, SOFA=Social and Occupational Functioning Assessment, SQLS=Schizophrenia Quality of Life Scale, SR=systematic review, WMD=weighted mean difference 
Appendix Table H-2. Strength of evidence: first-generation antipsychotic versus second-generation antipsychotic

\begin{tabular}{|c|c|c|c|c|c|c|c|c|}
\hline Outcome & Comparators & $\begin{array}{l}\text { Number of Studies } \\
\text { Number of Subjects }\end{array}$ & $\begin{array}{l}\text { Study } \\
\text { Limitations }\end{array}$ & Consistency & Directness & Precision & $\begin{array}{l}\text { Magnitude of Effect: } \\
\text { Summary Effect Size } \\
(95 \% \mathrm{Cl})\end{array}$ & $\begin{array}{l}\text { Strength of } \\
\text { Evidence } \\
\text { (High, } \\
\text { Moderate, Low, } \\
\text { Insufficient) } \\
\end{array}$ \\
\hline $\begin{array}{l}\text { Function: } \\
\text { General }\end{array}$ & $\begin{array}{l}\text { Haloperidol vs. } \\
\text { olanzapine }\end{array}$ & $\begin{array}{l}1 \mathrm{SR}(1 \mathrm{RCT})^{30} \\
\mathrm{~N}=208\end{array}$ & Moderate & Unknown & Direct & Imprecise & $\begin{array}{l}\text { Inconclusive: } \\
\text { GAF effect estimate: }-4.00 \text {; } \\
95 \% \mathrm{Cl}-13.70 \text { to } 5.70\end{array}$ & Insufficient \\
\hline $\begin{array}{l}\text { Function: } \\
\text { Encounters } \\
\text { with Legal } \\
\text { System } \\
\end{array}$ & $\begin{array}{l}\text { Haloperidol vs. } \\
\text { olanzapine }\end{array}$ & $\begin{array}{l}1 \mathrm{SR}(1 \mathrm{RCTs})^{30} \\
\mathrm{~N}=31\end{array}$ & Moderate & Unknown & Direct & Imprecise & $\begin{array}{l}\text { Inconclusive: } \\
\text { Encounters with legal } \\
\text { system: RR } 3.20,95 \% \mathrm{Cl} \\
0.76 \text { to } 13.46 \\
\end{array}$ & Insufficient \\
\hline $\begin{array}{l}\text { Function: } \\
\text { Employment }\end{array}$ & $\begin{array}{l}\text { Haloperidol vs. } \\
\text { risperidone }\end{array}$ & $\begin{array}{l}1 \mathrm{SR}^{30}(1 \mathrm{RCT}) \\
\mathrm{N}=100\end{array}$ & Moderate & Unknown & Direct & Imprecise & $\begin{array}{l}\text { Inconclusive: } \\
\text { Proportion of patients with } \\
\text { economic independence: } \\
\text { RR 0.94; } 95 \% \text { CI } 0.68 \text { to } \\
1.29\end{array}$ & Insufficient \\
\hline $\begin{array}{l}\text { Function: } \\
\text { Employment }\end{array}$ & $\begin{array}{l}\text { Perphenazine vs. } \\
\text { olanzapine }\end{array}$ & $\begin{array}{l}1 \mathrm{SR}^{30}(1 \mathrm{RCT}) \\
\mathrm{N}=597\end{array}$ & Moderate & Unknown & Direct & Imprecise & $\begin{array}{l}\text { Inconclusive: } \\
\text { Proportion with paid } \\
\text { employment: RR } 1.29 \\
95 \% \mathrm{Cl} 0.70 \text { to } 2.38 \\
\end{array}$ & Insufficient \\
\hline $\begin{array}{l}\text { Function: } \\
\text { Employment }\end{array}$ & $\begin{array}{l}\text { Perphenazine vs. } \\
\text { quetiapine }\end{array}$ & $\begin{array}{l}1 \mathrm{SR}^{30}(1 \mathrm{RCT}) \\
\mathrm{N}=598\end{array}$ & Moderate & Unknown & Direct & Imprecise & $\begin{array}{l}\text { Inconclusive: } \\
\text { Proportion with paid } \\
\text { employment: RR } 1.75 \text {; } \\
95 \% \mathrm{Cl} 0.90 \text { to } 3.43\end{array}$ & Insufficient \\
\hline $\begin{array}{l}\text { Function: } \\
\text { Employment }\end{array}$ & $\begin{array}{l}\text { Perphenazine vs. } \\
\text { risperidone }\end{array}$ & $\begin{array}{l}1 \mathrm{SR}^{30}(1 \mathrm{RCT}) \\
\mathrm{N}=602\end{array}$ & Moderate & Unknown & Direct & Imprecise & $\begin{array}{l}\text { Inconclusive: } \\
\text { Proportion with paid } \\
\text { employment: RR } 1.38 \text {; } \\
95 \% \mathrm{Cl} 0.74 \text { to } 2.57\end{array}$ & Insufficient \\
\hline $\begin{array}{l}\text { Function: } \\
\text { Employment }\end{array}$ & $\begin{array}{l}\text { Perphenazine vs. } \\
\text { ziprasidone }\end{array}$ & $\begin{array}{l}1 \mathrm{SR}^{30}(1 \mathrm{RCT}) \\
\mathrm{N}=446\end{array}$ & Moderate & Unknown & Direct & Imprecise & $\begin{array}{l}\text { Inconclusive: } \\
\text { Proportion with paid } \\
\text { employment: RR } 1.22 ; \\
95 \% \mathrm{Cl} 0.60 \text { to } 2.51 \\
\end{array}$ & Insufficient \\
\hline $\begin{array}{l}\text { Quality of } \\
\text { Life }\end{array}$ & $\begin{array}{l}\text { Haloperidol vs. } \\
\text { olanzapine }\end{array}$ & $\begin{array}{l}1 \mathrm{SR}^{30} \text { (5 RCTs) } \\
\mathrm{N}=816\end{array}$ & Moderate & Consistent & Direct & Precise & $\begin{array}{l}\text { Inconclusive: } \\
\text { Effect sizes ranged from - } \\
3.62 \text { to } 0 \text { using different } \\
\text { measures; Cls were not } \\
\text { significant }\end{array}$ & Moderate \\
\hline Quality of Life & $\begin{array}{l}\text { Haloperidol vs. } \\
\text { quetiapine }\end{array}$ & $\begin{array}{l}1 \mathrm{SR}^{30}(1 \mathrm{RCT}) \\
\mathrm{N}=207\end{array}$ & Moderate & Unknown & Direct & Imprecise & $\begin{array}{l}\text { Inconclusive: } \\
\text { Effect estimate } 0.00 ; 95 \% \\
\mathrm{Cl}-1.38 \text { to } 1.38 \\
\end{array}$ & Insufficient \\
\hline Quality of Life & $\begin{array}{l}\text { Haloperidol vs. } \\
\text { risperidone }\end{array}$ & $\begin{array}{l}1 \mathrm{SR}^{30}(2 \mathrm{RCTs}) \\
\mathrm{N}=352\end{array}$ & Moderate & Inconsistent & Direct & Imprecise & $\begin{array}{l}\text { Inconclusive: } \\
\text { Effect estimates ranged } \\
\text { from }-0.10 \text { to } 0.10 ; \mathrm{Cls} \\
\text { were not significant }\end{array}$ & Insufficient \\
\hline
\end{tabular}




\begin{tabular}{|c|c|c|c|c|c|c|c|c|}
\hline Outcome & Comparators & $\begin{array}{l}\text { Number of Studies } \\
\text { Number of Subjects }\end{array}$ & $\begin{array}{l}\text { Study } \\
\text { Limitations }\end{array}$ & Consistency & Directness & Precision & $\begin{array}{l}\text { Magnitude of Effect: } \\
\text { Summary Effect Size } \\
(95 \% \mathrm{Cl})\end{array}$ & $\begin{array}{l}\text { Strength of } \\
\text { Evidence } \\
\text { (High, } \\
\text { Moderate, Low, } \\
\text { Insufficient) }\end{array}$ \\
\hline Quality of Life & $\begin{array}{l}\text { Haloperidol vs. } \\
\text { ziprasidone }\end{array}$ & $\begin{array}{l}1 \mathrm{SR}^{30}(2 \mathrm{RCTs}) \\
\mathrm{N}=784\end{array}$ & High & Inconsistent & Direct & Imprecise & $\begin{array}{l}\text { Studies favored } \\
\text { ziprasidone in quality of life } \\
\text { measures. One trial found } \\
\text { effect favoring ziprasidone } \\
\text { based on QLS (effect } \\
\text { estimate } 12.12 ; 95 \% \mathrm{Cl} \text { - } \\
22.06 \text { to }-2.17 \text { ); there was } \\
\text { no difference in another } \\
\text { trial in MANSA (effect } \\
\text { estimate }-0.10 ; 95 \% \mathrm{Cl} \text { - } \\
1.48 \text { to } 1.28 \text { ) } \\
\end{array}$ & Low \\
\hline Quality of Life & $\begin{array}{l}\text { Perphenazine vs. } \\
\text { aripiprazole }\end{array}$ & $\begin{array}{l}1 \mathrm{SR}^{30}(1 \mathrm{RCT}) \\
\mathrm{N}=300\end{array}$ & Moderate & Unknown & Direct & Imprecise & $\begin{array}{l}\text { Inconclusive: } \\
\text { Proportion with } 20 \% \\
\text { improvement: RR } 4.74 ; \\
95 \% \text { Cl } 2.58 \text { to } 8.69 \\
\end{array}$ & Insufficient \\
\hline Quality of Life & $\begin{array}{l}\text { Perphenazine vs. } \\
\text { olanzapine }\end{array}$ & $\begin{array}{l}1 \mathrm{SR}^{30}(1 \mathrm{RCT}) \\
\mathrm{N}=597\end{array}$ & Moderate & Unknown & Direct & Precise & $\begin{array}{l}\text { No difference: } \\
\text { Effect estimate } 0.00 ; 95 \% \\
\mathrm{Cl}-0.16 \text { to } 0.16\end{array}$ & Low \\
\hline Quality of Life & $\begin{array}{l}\text { Perphenazine vs. } \\
\text { quetiapine }\end{array}$ & $\begin{array}{l}1 \mathrm{SR}^{30}(1 \mathrm{RCT}) \\
\mathrm{N}=598\end{array}$ & Moderate & Unknown & Direct & Precise & $\begin{array}{l}\text { No difference: } \\
\text { Effect estimate } 0.10 ; 95 \% \\
\mathrm{Cl}-0.07 \text { to } 0.27\end{array}$ & Low \\
\hline Quality of Life & $\begin{array}{l}\text { Perphenazine vs. } \\
\text { risperidone }\end{array}$ & $\begin{array}{l}1 \mathrm{SR}^{30}(1 \mathrm{RCT}) \\
\mathrm{N}=602\end{array}$ & Moderate & Unknown & Direct & Precise & $\begin{array}{l}\text { No difference: } \\
\text { Effect estimate }-0.07 ; 95 \% \\
\mathrm{Cl}-0.24 \text { to } 0.10\end{array}$ & Low \\
\hline Quality of Life & $\begin{array}{l}\text { Perphenazine vs. } \\
\text { ziprasidone }\end{array}$ & $\begin{array}{l}1 \mathrm{SR}^{30}(1 \mathrm{RCT}) \\
\mathrm{N}=446\end{array}$ & Moderate & Unknown & Direct & Precise & $\begin{array}{l}\text { No difference: } \\
\text { Effect estimate }-0.07 ; 95 \% \\
\mathrm{Cl}-0.27 \text { to } 0.13 \\
\end{array}$ & Low \\
\hline Response & $\begin{array}{l}\text { Fluphenazine vs. } \\
\text { olanzapine }\end{array}$ & $\begin{array}{l}1 \mathrm{SR}^{30}(1 \mathrm{RCT}) \\
\mathrm{N}=60\end{array}$ & Moderate & Unknown & Direct & Imprecise & $\begin{array}{l}\text { Inconclusive: } \\
\text { RR } 0.74 \text { (95\% Cl } 0.51 \text { to } \\
1.07)\end{array}$ & Insufficient \\
\hline Response & $\begin{array}{l}\text { Fluphenazine vs. } \\
\text { quetiapine }\end{array}$ & $\begin{array}{l}1 \mathrm{SR}^{30}(1 \mathrm{RCT}) \\
\mathrm{N}=25\end{array}$ & Moderate & Unknown & Direct & Imprecise & $\begin{array}{l}\text { Inconclusive: } \\
\text { RR } 0.62(95 \% \mathrm{Cl} 0.12 \text { to } \\
3.07)\end{array}$ & Insufficient \\
\hline Response & $\begin{array}{l}\text { Fluphenazine vs. } \\
\text { risperidone }\end{array}$ & $\begin{array}{l}1 \mathrm{SR}^{30}(1 \mathrm{RCT}) \\
\mathrm{N}=26\end{array}$ & Moderate & Unknown & Direct & Imprecise & $\begin{array}{l}\text { Inconclusive: } \\
\text { RR } 0.67(95 \% \mathrm{Cl} 0.13 \text { to } \\
\text { 3.35) }\end{array}$ & Insufficient \\
\hline Response & $\begin{array}{l}\text { Haloperidol vs. } \\
\text { aripiprazole }\end{array}$ & $\begin{array}{l}1 \mathrm{SR}^{30} \text { (5 RCTs) } \\
\mathrm{N}=2,185\end{array}$ & Moderate & Inconsistent & Direct & Precise & $\begin{array}{l}\text { No difference: } \\
\text { RR } 1.01(95 \% \text { Cl } 0.76 \text { to } \\
\left.1.34 ; I^{2}=83 \%\right)\end{array}$ & Low \\
\hline Response & $\begin{array}{l}\text { Haloperidol vs. } \\
\text { asenapine }\end{array}$ & $\begin{array}{l}1 \mathrm{SR}^{30}(1 \mathrm{RCT}) \\
\mathrm{N}=335\end{array}$ & Moderate & Unknown & Direct & Imprecise & $\begin{array}{l}\text { Inconclusive: } \\
\text { RR } 0.82(95 \% \mathrm{Cl} 0.64 \text { to } \\
1.04)\end{array}$ & Insufficient \\
\hline
\end{tabular}




\begin{tabular}{|c|c|c|c|c|c|c|c|c|}
\hline Outcome & Comparators & $\begin{array}{l}\text { Number of Studies } \\
\text { Number of Subjects }\end{array}$ & $\begin{array}{l}\text { Study } \\
\text { Limitations }\end{array}$ & Consistency & Directness & Precision & $\begin{array}{l}\text { Magnitude of Effect: } \\
\text { Summary Effect Size } \\
(95 \% \mathrm{Cl})\end{array}$ & $\begin{array}{l}\text { Strength of } \\
\text { Evidence } \\
\text { (High, } \\
\text { Moderate, Low, } \\
\text { Insufficient) }\end{array}$ \\
\hline Response & $\begin{array}{l}\text { Haloperidol vs. } \\
\text { clozapine }\end{array}$ & $\begin{array}{l}1 \mathrm{SR}^{30}(2 \mathrm{RCTs}) \\
\mathrm{N}=144\end{array}$ & Moderate & Inconsistent & Direct & Imprecise & $\begin{array}{l}\text { Inconclusive: } \\
\text { RR } 0.64(95 \% \mathrm{Cl} 0.28 \text { to } \\
\left.1.47 ; I^{2}=72 \%\right)\end{array}$ & Insufficient \\
\hline Response & $\begin{array}{l}\text { Haloperidol vs. } \\
\text { olanzapine }\end{array}$ & $\begin{array}{l}1 \mathrm{SR}^{30} \text { (14 RCTs) } \\
\mathrm{N}=4,099\end{array}$ & Moderate & Inconsistent & Direct & Precise & $\begin{array}{l}\mathrm{RR} 0.86(95 \% \mathrm{Cl} 0.78 \text { to } \\
\left.0.96 ; \mathrm{I}^{2}=55 \%\right) ; \text { favors } \\
\text { olanzapine }\end{array}$ & Low \\
\hline Response & $\begin{array}{l}\text { Haloperidol vs. } \\
\text { quetiapine }\end{array}$ & $\begin{array}{l}1 \mathrm{SR}^{30} \text { (6 RCTs) } \\
\mathrm{N}=1,421\end{array}$ & Moderate & Inconsistent & Direct & Precise & $\begin{array}{l}\text { No difference: } \\
\text { RR } 0.99(95 \% \mathrm{Cl} 0.76 \text { to } \\
\left.1.30 ; I^{2}=77 \%\right)\end{array}$ & Low \\
\hline Response & $\begin{array}{l}\text { Haloperidol vs. } \\
\text { risperidone }\end{array}$ & $\begin{array}{l}1 \mathrm{SR}^{30}(16 \mathrm{RCTs}) \\
\mathrm{N}=3,452)\end{array}$ & Moderate & Consistent & Direct & Precise & $\begin{array}{l}\text { No difference: } \\
\text { RR } 0.94(95 \% \mathrm{Cl} 0.87 \text { to } \\
\left.1.02 ;\left.\right|^{2}=29 \%\right)\end{array}$ & Moderate \\
\hline Response & $\begin{array}{l}\text { Haloperidol vs. } \\
\text { ziprasidone }\end{array}$ & $\begin{array}{l}1 \mathrm{SR}^{30} \text { (6 RCTs) } \\
\mathrm{N}=1,283\end{array}$ & Moderate & Inconsistent & Direct & Imprecise & $\begin{array}{l}\text { Inconclusive: } \\
\text { RR } 0.98(95 \% \mathrm{Cl} 0.74 \text { to } \\
\left.1.30 ; I^{2}=80 \%\right)\end{array}$ & Low \\
\hline Response & $\begin{array}{l}\text { Perphenazine vs. } \\
\text { aripiprazole }\end{array}$ & $\begin{array}{l}1 \mathrm{SR}^{30}(1 \mathrm{RCT}) \\
\mathrm{N}=300\end{array}$ & Moderate & Unknown & Direct & Imprecise & $\begin{array}{l}\text { Inconclusive: } \\
\text { RR } 0.95(95 \% \mathrm{Cl} 0.64 \text { to } \\
1.40)\end{array}$ & Insufficient \\
\hline Remission & $\begin{array}{l}\text { Haloperidol vs. } \\
\text { clozapine }\end{array}$ & $\begin{array}{l}1 \mathrm{SR}^{30}(1 \mathrm{RCT}) \\
\mathrm{N}=71\end{array}$ & Moderate & Unknown & Direct & Imprecise & $\begin{array}{l}\text { Inconclusive: } \\
\text { RR } 0.16(95 \% \mathrm{Cl} 0.02 \text { to } \\
1.20)\end{array}$ & Insufficient \\
\hline Remission & $\begin{array}{l}\text { Haloperidol vs. } \\
\text { olanzapine }\end{array}$ & $\begin{array}{l}1 \mathrm{SR}^{30}(3 \mathrm{RCTS}) \\
\mathrm{N}=582\end{array}$ & Moderate & Consistent & Direct & Imprecise & $\begin{array}{l}\text { RR } 0.65(95 \% \mathrm{Cl} 0.45 \text { to } \\
\left.0.94 ; I^{2}=54 \%\right) ; \text { favors } \\
\text { olanzapine }\end{array}$ & Low \\
\hline Remission & $\begin{array}{l}\text { Haloperidol vs. } \\
\text { quetiapine }\end{array}$ & $\begin{array}{l}1 \mathrm{SR}^{30}(1 \mathrm{RCT}) \\
\mathrm{N}=207\end{array}$ & High & Unknown & Direct & Imprecise & $\begin{array}{l}\text { Inconclusive: } \\
\text { RR } 0.72(95 \% \mathrm{Cl} 0.41 \text { to } \\
1.25)\end{array}$ & Insufficient \\
\hline Remission & $\begin{array}{l}\text { Haloperidol vs. } \\
\text { risperidone }\end{array}$ & $\begin{array}{l}1 \mathrm{SR}^{30} \text { (2 RCTs) } \\
\mathrm{N}=179\end{array}$ & Moderate & Consistent & Direct & Imprecise & $\begin{array}{l}\text { Inconclusive: } \\
\text { RR } 0.84(95 \% \mathrm{Cl} 0.56 \text { to } \\
\left.1.24 ; I^{2}=0 \%\right)\end{array}$ & Low \\
\hline Remission & $\begin{array}{l}\text { Haloperidol vs. } \\
\text { ziprasidone }\end{array}$ & $\begin{array}{l}1 \mathrm{SR}^{30}(3 \mathrm{RCTs}) \\
\mathrm{N}=1,085\end{array}$ & High & Consistent & Direct & Precise & $\begin{array}{l}\text { No difference: RR } 0.89 \\
\left(95 \% \mathrm{Cl} 0.71 \text { to } 1.12 ; I^{2}=\right. \\
12 \%)\end{array}$ & Low \\
\hline $\begin{array}{l}\text { Reduction in } \\
\text { Self Harm }\end{array}$ & $\begin{array}{l}\text { Haloperidol vs. } \\
\text { olanzapine }\end{array}$ & $\begin{array}{l}1 \mathrm{SR}^{30}(1 \mathrm{RCT}) \\
\mathrm{N}=182\end{array}$ & Moderate & Unknown & Indirect & Imprecise & $\begin{array}{l}\text { Inconclusive: } \\
\text { Attempted suicide: RR } \\
3.13 ; 95 \% \mathrm{Cl} 0.13 \text { to } 76 \\
\text { and } 0.64 \\
\text { Completed suicide: } \mathrm{RR} \\
3.13 ; 95 \% \mathrm{Cl} 0.13 \text { to } 76 \\
\text { and } 3.86\end{array}$ & Insufficient \\
\hline
\end{tabular}




\begin{tabular}{|c|c|c|c|c|c|c|c|c|}
\hline Outcome & Comparators & $\begin{array}{l}\text { Number of Studies } \\
\text { Number of Subjects }\end{array}$ & $\begin{array}{l}\text { Study } \\
\text { Limitations }\end{array}$ & Consistency & Directness & Precision & $\begin{array}{l}\text { Magnitude of Effect: } \\
\text { Summary Effect Size } \\
(95 \% \mathrm{Cl})\end{array}$ & $\begin{array}{l}\text { Strength of } \\
\text { Evidence } \\
\text { (High, } \\
\text { Moderate, Low, } \\
\text { Insufficient) } \\
\end{array}$ \\
\hline $\begin{array}{l}\text { Reduction in } \\
\text { Self Harm }\end{array}$ & $\begin{array}{l}\text { Perphenazine and } \\
\text { olanzapine }\end{array}$ & $\begin{array}{l}1 \mathrm{SR}^{30}(1 \mathrm{RCT}) \\
\mathrm{N}=597\end{array}$ & Moderate & Unknown & Indirect & Imprecise & $\begin{array}{l}\text { Inconclusive: } \\
\text { Attempted suicide: RR } \\
0.64 ; 95 \% \text { Cl } 0.06 \text { to } 7.06 \\
\text { Completed suicide: RR } \\
\text { 3.86; } 95 \% \text { Cl } 0.40 \text { to } 37\end{array}$ & Insufficient \\
\hline $\begin{array}{l}\text { Overall } \\
\text { Adverse } \\
\text { Events }\end{array}$ & $\begin{array}{l}\text { Haloperidol vs. } \\
\text { aripiprazole }\end{array}$ & $\begin{array}{l}1 \mathrm{SR}^{30}(3 \mathrm{RCTS}) \\
\mathrm{N}=1,713\end{array}$ & Moderate & Consistent & Direct & Precise & $\begin{array}{l}\text { RR } 1.11 ; 95 \% \mathrm{Cl} 1.06 \text { to } \\
1.17 ; \text { I }^{2}=0 \% \text {; less with } \\
\text { aripiprazole }\end{array}$ & Moderate \\
\hline $\begin{array}{l}\text { Overall } \\
\text { Adverse } \\
\text { Events }\end{array}$ & $\begin{array}{l}\text { Haloperidol vs. } \\
\text { risperidone }\end{array}$ & $\begin{array}{l}1 \mathrm{SR}^{30} \text { (8 RCTs) } \\
\mathrm{N}=1,313\end{array}$ & Moderate & Consistent & Direct & Precise & $\begin{array}{l}\text { RR } 1.20 ; 95 \% \mathrm{Cl} 1.01 \text { to } \\
1.42 ; I^{2}=84 \% \text {; less with } \\
\text { risperidone }\end{array}$ & Moderate \\
\hline $\begin{array}{l}\text { Overall } \\
\text { Adverse } \\
\text { Events }\end{array}$ & $\begin{array}{l}\text { Haloperidol vs. } \\
\text { ziprasidone }\end{array}$ & $\begin{array}{l}1 \mathrm{SR}^{30} \text { (6 RCTs) } \\
\mathrm{N}=1,448\end{array}$ & Moderate & Consistent & Direct & Precise & $\begin{array}{l}\text { RR } 1.13 ; 95 \% \mathrm{Cl} 1.03 \text { to } \\
1.23 ; I^{2}=31 \% \text {; less with } \\
\text { ziprasidone }\end{array}$ & Moderate \\
\hline \multirow{2}{*}{$\begin{array}{l}\text { Withdrawal } \\
\text { Due to } \\
\text { Adverse } \\
\text { Events }\end{array}$} & $\begin{array}{l}\text { Fluphenazine vs. } \\
\text { olanzapine }\end{array}$ & $\begin{array}{l}1 \mathrm{SR}^{30}(1 \mathrm{RCT}) \\
\mathrm{N}=60\end{array}$ & Moderate & Unknown & Indirect & Imprecise & $\begin{array}{l}\text { Inconclusive: } \\
\text { RR } 0.74 ; 95 \% \mathrm{Cl} 0.51 \text { to } \\
1.07\end{array}$ & Insufficient \\
\hline & $\begin{array}{l}\text { Fluphenazine vs. } \\
\text { quetiapine }\end{array}$ & $\begin{array}{l}1 \mathrm{SR}^{30}(1 \mathrm{RCT}) \\
\mathrm{N}=25\end{array}$ & Moderate & Unknown & Indirect & Imprecise & $\begin{array}{l}\text { Inconclusive: } \\
\text { RR 0.19; } 95 \% \text { Cl } 0.01 \text { to } \\
3.52\end{array}$ & Insufficient \\
\hline $\begin{array}{l}\text { Withdrawal } \\
\text { Due to } \\
\text { Adverse } \\
\text { Events }\end{array}$ & $\begin{array}{l}\text { Haloperidol vs. } \\
\text { asenapine }\end{array}$ & $\begin{array}{l}1 \mathrm{SR}^{30}(1 \mathrm{RCT}) \\
\mathrm{N}=335\end{array}$ & Moderate & Unknown & Indirect & Imprecise & $\begin{array}{l}\text { Inconclusive: } \\
\text { RR 1.53; 95\% } 0.74 \text { to } 3.16\end{array}$ & Insufficient \\
\hline $\begin{array}{l}\text { Withdrawal } \\
\text { Due to } \\
\text { Adverse } \\
\text { Events }\end{array}$ & $\begin{array}{l}\text { Haloperidol vs. } \\
\text { aripiprazole }\end{array}$ & $\begin{array}{l}1 \mathrm{SR}^{30}(7 \mathrm{RCTs})+1 \\
\text { additional } \mathrm{RCT} \\
\mathrm{N}=3,232\end{array}$ & Moderate & Consistent & Direct & Precise & $\begin{array}{l}\text { RR } 1.25 ; 1.07 \text { to } 1.47 ; \\
I^{2}=0 \%\end{array}$ & Moderate \\
\hline $\begin{array}{l}\text { Withdrawal } \\
\text { Due to } \\
\text { Adverse } \\
\text { Events }\end{array}$ & $\begin{array}{l}\text { Haloperidol vs. } \\
\text { clozapine }\end{array}$ & $\begin{array}{l}1 \mathrm{SR}^{30}(5 \mathrm{RCTs}) \\
\mathrm{N}=719\end{array}$ & Moderate & Consistent & Direct & Imprecise & $\begin{array}{l}\text { Inconclusive: } \\
\text { RR } 1.00 ; 95 \% \mathrm{Cl} 0.66 \text { to } \\
1.50 ; I^{2}=0 \%\end{array}$ & Low \\
\hline $\begin{array}{l}\text { Withdrawal } \\
\text { Due to } \\
\text { Adverse } \\
\text { Events }\end{array}$ & $\begin{array}{l}\text { Haloperidol vs. } \\
\text { olanzapine }\end{array}$ & $\begin{array}{l}1 \mathrm{SR}^{30}(21 \mathrm{RCTs})+3 \\
\mathrm{RCTs} \\
\mathrm{N}=5,708\end{array}$ & Moderate & Consistent & Direct & Precise & $\begin{array}{l}\text { RR } 1.89 ; 95 \% \mathrm{Cl} 1.57 \text { to } \\
2.27 ; \mathrm{I}^{2}=0 \%\end{array}$ & Moderate \\
\hline $\begin{array}{l}\text { Withdrawal } \\
\text { Due to } \\
\text { Adverse } \\
\text { Events }\end{array}$ & $\begin{array}{l}\text { Haloperidol vs. } \\
\text { quetiapine }\end{array}$ & $\begin{array}{l}1 \mathrm{SR}^{30}(8 \mathrm{RCTs})+2 \\
\mathrm{RCTs} \\
\mathrm{N}=1,759\end{array}$ & Moderate & Consistent & Direct & Imprecise & $\begin{array}{l}\text { Inconclusive: } \\
\text { RR } 1.97 ; 95 \% \mathrm{Cl} 0.96 \text { to } \\
4.01 ; I^{2}=62 \%\end{array}$ & Low \\
\hline
\end{tabular}




\begin{tabular}{|c|c|c|c|c|c|c|c|c|}
\hline Outcome & Comparators & $\begin{array}{l}\text { Number of Studies } \\
\text { Number of Subjects }\end{array}$ & $\begin{array}{l}\text { Study } \\
\text { Limitations }\end{array}$ & Consistency & Directness & Precision & $\begin{array}{l}\text { Magnitude of Effect: } \\
\text { Summary Effect Size } \\
(95 \% \mathrm{Cl})\end{array}$ & $\begin{array}{l}\text { Strength of } \\
\text { Evidence } \\
\text { (High, } \\
\text { Moderate, Low, } \\
\text { Insufficient) } \\
\end{array}$ \\
\hline $\begin{array}{l}\text { Withdrawal } \\
\text { Due to } \\
\text { Adverse } \\
\text { Events }\end{array}$ & $\begin{array}{l}\text { Haloperidol vs. } \\
\text { risperidone }\end{array}$ & $\begin{array}{l}1 \mathrm{SR}^{30}(23 \mathrm{RCTs})+2 \\
\mathrm{RCTs} \\
\mathrm{N}=4,581\end{array}$ & Moderate & Consistent & Direct & Precise & $\begin{array}{l}\text { RR } 1.32 ; 95 \% \text { Cl } 1.09 \text { to } \\
1.60 ; I^{2}=0 \%\end{array}$ & Moderate \\
\hline $\begin{array}{l}\text { Withdrawal } \\
\text { Due to } \\
\text { Adverse } \\
\text { Events }\end{array}$ & $\begin{array}{l}\text { Haloperidol vs. } \\
\text { ziprasidone }\end{array}$ & $\begin{array}{l}1 \mathrm{SR}^{30}(6 \mathrm{RCTs})+1 \mathrm{RCT} \\
\mathrm{N}=1597\end{array}$ & Moderate & Consistent & Direct & Precise & $\begin{array}{l}\text { RR } 1.68 ; 95 \% \mathrm{Cl} 1.26 \text { to } \\
2.23 ; 1^{2}=0 \%\end{array}$ & Moderate \\
\hline $\begin{array}{l}\text { Withdrawal } \\
\text { Due to } \\
\text { Adverse } \\
\text { Events }\end{array}$ & $\begin{array}{l}\text { Perphenazine vs. } \\
\text { aripiprazole }\end{array}$ & $\begin{array}{l}1 \mathrm{SR}^{30}(1 \mathrm{RCT}) \\
\mathrm{N}=300\end{array}$ & Moderate & Unknown & Direct & Imprecise & $\begin{array}{l}\text { Inconclusive: } \\
\text { RR } 0.53 ; 95 \% \mathrm{Cl} 0.27 \text { to } \\
1.05\end{array}$ & Insufficient \\
\hline $\begin{array}{l}\text { Withdrawal } \\
\text { Due to } \\
\text { Adverse } \\
\text { Events }\end{array}$ & $\begin{array}{l}\text { Perphenazine vs. } \\
\text { olanzapine }\end{array}$ & $\begin{array}{l}1 \mathrm{SR}^{30}(1 \mathrm{RCT}) \\
\mathrm{N}=597\end{array}$ & Moderate & Unknown & Direct & Imprecise & $\begin{array}{l}\text { Inconclusive: } \\
\text { RR } 0.83 ; 95 \% \mathrm{Cl} 0.58 \text { to } \\
1.19\end{array}$ & Insufficient \\
\hline $\begin{array}{l}\text { Withdrawal } \\
\text { Due to } \\
\text { Adverse } \\
\text { Events }\end{array}$ & $\begin{array}{l}\text { Perphenazine vs. } \\
\text { quetiapine }\end{array}$ & $\begin{array}{l}1 \mathrm{SR}^{30}(1 \mathrm{RCT}) \\
\mathrm{N}=598\end{array}$ & Moderate & Unknown & Direct & Imprecise & $\begin{array}{l}\text { Inconclusive: } \\
\text { RR } 1.05 ; 95 \% \mathrm{Cl} 0.72 \text { to } \\
1.55\end{array}$ & Insufficient \\
\hline $\begin{array}{l}\text { Withdrawal } \\
\text { Due to } \\
\text { Adverse } \\
\text { Events }\end{array}$ & $\begin{array}{l}\text { Perphenazine vs. } \\
\text { risperidone }\end{array}$ & $\begin{array}{l}1 \mathrm{SR}^{30}(1 \mathrm{RCT}) \\
\mathrm{N}=602\end{array}$ & Moderate & Unknown & Direct & Imprecise & $\begin{array}{l}\text { Inconclusive: } \\
\text { RR } 1.54 ; 95 \% \mathrm{Cl} 1.00 \text { to } \\
2.36\end{array}$ & Insufficient \\
\hline $\begin{array}{l}\text { Withdrawal } \\
\text { Due to } \\
\text { Adverse } \\
\text { Events }\end{array}$ & $\begin{array}{l}\text { Perphenazine vs. } \\
\text { ziprasidone }\end{array}$ & $\begin{array}{l}1 \mathrm{SR}^{30}(1 \mathrm{RCT}) \\
\mathrm{N}=446\end{array}$ & Moderate & Unknown & Direct & Imprecise & $\begin{array}{l}\text { Inconclusive: } \\
\text { RR 1.01; 95\% Cl } 0.65 \\
\text { to1.58 }\end{array}$ & Insufficient \\
\hline
\end{tabular}

$\mathrm{CI}=$ confidence interval, $\mathrm{OR}=$ odds ratio, $\mathrm{RCT}=$ randomized controlled trial, $\mathrm{SR}=$ systematic review 
Appendix Table H-3. Strength of evidence: assertive community treatment

\begin{tabular}{|c|c|c|c|c|c|c|c|c|}
\hline Outcome & Comparators & $\begin{array}{l}\text { Number of Studies } \\
\text { Number of Subjects }\end{array}$ & $\begin{array}{l}\text { Strength of } \\
\text { Evidence } \\
\text { Domain: } \\
\text { Study } \\
\text { Limitations }\end{array}$ & $\begin{array}{l}\text { Strength of } \\
\text { Evidence } \\
\text { Domain: } \\
\text { Consistency }\end{array}$ & $\begin{array}{l}\text { Strength of } \\
\text { Evidence } \\
\text { Domain: } \\
\text { Directness }\end{array}$ & $\begin{array}{l}\text { Strength of } \\
\text { Evidence } \\
\text { Domain: } \\
\text { Precision }\end{array}$ & $\begin{array}{l}\text { Magnitude of Effect: } \\
\text { Summary Effect Size } \\
(95 \% \mathrm{Cl})\end{array}$ & $\begin{array}{l}\text { Strength of } \\
\text { Evidence } \\
\text { (High, } \\
\text { Moderate, Low, } \\
\text { Insufficient) } \\
\end{array}$ \\
\hline Function & $\begin{array}{l}\text { Assertive community } \\
\text { treatment vs. usual } \\
\text { care }\end{array}$ & $\begin{array}{l}1 \mathrm{SR}^{31} \text { (3 RCTs) } \\
1 \mathrm{RCT}^{32} \\
\mathrm{~N}=118\end{array}$ & Moderate & Consistent & Direct & Imprecise & $\begin{array}{l}\text { No difference in social } \\
\text { function compared with } \\
\text { usual care. } \\
\text { Social function, mean } \\
\text { difference } 0.03(95 \% \mathrm{Cl} \text { - } \\
0.28 \text { to } 0.34)\end{array}$ & Low \\
\hline $\begin{array}{l}\text { Trouble With } \\
\text { Police }\end{array}$ & $\begin{array}{l}\text { Assertive community } \\
\text { treatment vs. usual } \\
\text { care }\end{array}$ & $1 \mathrm{SR}^{31}$ (4 RCTs) & Moderate & Consistent & Direct & Imprecise & $\begin{array}{l}\text { No differences in arrests (2 } \\
\text { trials; OR } 1.17,95 \% \mathrm{Cl} \\
0.60 \text { to } 2.29) \text {, imprisonment } \\
\text { (4 trials; OR } 1.19,95 \% \mathrm{Cl} \\
0.70 \text { to } 2.01) \text {, or police } \\
\text { contacts (2 trials; OR } 0.76 \text {, } \\
95 \% \mathrm{Cl} 0.32 \text { to } 1.79) \\
\end{array}$ & Low \\
\hline $\begin{array}{l}\text { Housing and } \\
\text { Independent } \\
\text { Living }\end{array}$ & $\begin{array}{l}\text { Assertive community } \\
\text { treatment vs. usual } \\
\text { care }\end{array}$ & $\begin{array}{l}1 \mathrm{SR}^{31}(3 \mathrm{RCTS}) \\
1 \mathrm{RCT}^{32} \\
\mathrm{~N}=118\end{array}$ & Moderate & Consistent & Direct & Precise & $\begin{array}{l}\text { Less likely to be not living } \\
\text { independently ( } 4 \text { trials; OR } \\
0.52,95 \% \mathrm{Cl} 0.35 \text { to } 0.79 \text { ), } \\
\text { to be homeless ( } 4 \text { trials; } \\
\text { OR } 0.20,95 \% \text { Cl } 0.09 \text { to } \\
0.47 \text { ). } \\
\text { Less likely to be homeless } \\
\text { (4 trials, OR } 0.24,95 \% \mathrm{Cl} \\
0.12 \text { to } 0.48 \text { ). }\end{array}$ & Moderate \\
\hline Employment & $\begin{array}{l}\text { Assertive community } \\
\text { treatment vs. usual } \\
\text { care }\end{array}$ & $1 \mathrm{SR}^{31}$ (3 RCTs) & Moderate & Consistent & Direct & Precise & $\begin{array}{l}\text { Less likely to be } \\
\text { unemployed ( } 3 \text { trials; OR } \\
0.46,95 \% \mathrm{Cl} 0.21 \text { to } 0.99 \text { ) } \\
\end{array}$ & Moderate \\
\hline $\begin{array}{l}\text { Quality of } \\
\text { Life }\end{array}$ & $\begin{array}{l}\text { Assertive community } \\
\text { treatment vs. usual } \\
\text { care }\end{array}$ & $\begin{array}{l}1 \mathrm{SR}^{31}(1 \mathrm{RCT}) \\
\mathrm{N}=125 \\
1 \mathrm{RCT}^{32} \\
\mathrm{~N}=118\end{array}$ & Moderate & Inconsistent & Direct & Imprecise & $\begin{array}{l}\text { Quality of life was slightly } \\
\text { better with assertive } \\
\text { community treatment } \\
\text { (mean difference, }-0.52, \\
95 \% \mathrm{Cl}-0.99 \text { to }-0.05 \text { ) in } \\
\text { one trial, but no differences } \\
\text { found in the other trial. }\end{array}$ & Insufficient \\
\hline
\end{tabular}




\begin{tabular}{|c|c|c|c|c|c|c|c|c|}
\hline Outcome & Comparators & $\begin{array}{l}\text { Number of Studies } \\
\text { Number of Subjects }\end{array}$ & $\begin{array}{l}\text { Strength of } \\
\text { Evidence } \\
\text { Domain: } \\
\text { Study } \\
\text { Limitations }\end{array}$ & $\begin{array}{l}\text { Strength of } \\
\text { Evidence } \\
\text { Domain: } \\
\text { Consistency }\end{array}$ & $\begin{array}{l}\text { Strength of } \\
\text { Evidence } \\
\text { Domain: } \\
\text { Directness }\end{array}$ & $\begin{array}{l}\text { Strength of } \\
\text { Evidence } \\
\text { Domain: } \\
\text { Precision }\end{array}$ & $\begin{array}{l}\text { Magnitude of Effect: } \\
\text { Summary Effect Size } \\
(95 \% \mathrm{Cl})\end{array}$ & $\begin{array}{l}\text { Strength of } \\
\text { Evidence } \\
\text { (High, } \\
\text { Moderate, Low, } \\
\text { Insufficient) }\end{array}$ \\
\hline $\begin{array}{l}\text { Overall } \\
\text { Symptoms }\end{array}$ & $\begin{array}{l}\text { Assertive community } \\
\text { treatment vs. usual } \\
\text { care }\end{array}$ & $\begin{array}{l}1 \mathrm{SR}^{31} \text { (3 RCTs) } \\
1 \mathrm{RCT}^{32} \\
\mathrm{~N}=118\end{array}$ & Moderate & Consistent & Direct & Precise & $\begin{array}{l}\text { No differences were found } \\
\text { in } 4 \text { trials. Mean difference, } \\
-0.14(95 \% \mathrm{Cl}-0.36 \text { to } \\
0.08) \text {. }\end{array}$ & Moderate \\
\hline $\begin{array}{l}\text { Treatment } \\
\text { Maintenance } \\
\text { (Loss to } \\
\text { Followup) }\end{array}$ & $\begin{array}{l}\text { Assertive community } \\
\text { treatment vs. usual } \\
\text { care }\end{array}$ & $\begin{array}{l}1 \mathrm{SR}^{31}(10 \mathrm{RCTS}) \\
1 \mathrm{RCT}^{32} \\
\mathrm{~N}=118\end{array}$ & Moderate & Consistent & Direct & Precise & $\begin{array}{l}\text { Significantly less loss to } \\
\text { followup with assertive } \\
\text { community treatment (OR } \\
0.51,95 \% \mathrm{Cl} 0.40 \text { to } 0.65 \text { ) } \\
\text { based on } 10 \text { trials in SR; } \\
\text { and significantly fewer } \\
\text { patients "out-of-care" in the } \\
\text { other trial (OR 0.10, } 95 \% \\
\mathrm{Cl} 0.03-0.33 \text { ) }\end{array}$ & Moderate \\
\hline
\end{tabular}

$\mathrm{CI}=$ confidence interval, $\mathrm{N}=$ number, $\mathrm{OR}=$ odds ratio, $\mathrm{RCT}=$ randomized controlled trial, $\mathrm{SR}=$ systematic review 
Appendix Table H-4. Strength of evidence: cognitive behavioral therapy

\begin{tabular}{|c|c|c|c|c|c|c|c|c|}
\hline Outcome & Comparators & $\begin{array}{l}\text { Number of Studies } \\
\text { Number of Subjects }\end{array}$ & $\begin{array}{l}\text { Strength of } \\
\text { Evidence } \\
\text { Domain: } \\
\text { Study } \\
\text { Limitations }\end{array}$ & $\begin{array}{l}\text { Strength of } \\
\text { Evidence } \\
\text { Domain: } \\
\text { Consistency }\end{array}$ & $\begin{array}{l}\text { Strength of } \\
\text { Evidence } \\
\text { Domain: } \\
\text { Directness }\end{array}$ & $\begin{array}{l}\text { Strength of } \\
\text { Evidence } \\
\text { Domain: } \\
\text { Precision }\end{array}$ & $\begin{array}{l}\text { Magnitude of Effect: } \\
\text { Summary Effect Size } \\
(95 \% \mathrm{Cl})\end{array}$ & $\begin{array}{l}\text { Strength of } \\
\text { Evidence } \\
\text { (High, } \\
\text { Moderate, } \\
\text { Low, } \\
\text { Insufficient) }\end{array}$ \\
\hline $\begin{array}{l}\text { Function: } \\
\text { Global } \\
\text { Function - } \\
\text { Short term } \\
\text { ( } \leq 6 \text { months } \\
\text { since CBT } \\
\text { initiation) }\end{array}$ & CBT vs. usual care & $\begin{array}{l}1 \mathrm{SR}^{33}(3 \mathrm{RCTs}) \\
5 \mathrm{RCTS}^{34-38} \\
\mathrm{~N}=701\end{array}$ & Moderate & Consistent & Direct & Precise & $\begin{array}{l}\text { GAF (6 RCTs): mean } \\
\text { difference } 5.49 \text { (95\% Cl } 1.85 \\
\text { to } 9.14 ; I^{2}=75 \% \text { ); excluding } \\
\text { one outlier: } 6.62 \text { (95\% Cl } 4.68 \\
\left.\text { to } 8.56 ; I^{2}=0 \%\right) \\
\text { SOFAS (2 RCTs): mean } \\
\text { difference } 9.11 \text { (95\% Cl } 6.31 \\
\text { to } 11.91 \text { ) } \\
\text { Proportion with normal } \\
\text { function (1 RCT): RR } 2.21 \\
\text { (95\% Cl } 1.25 \text { to } 3.93 \text { ) }\end{array}$ & Moderate \\
\hline $\begin{array}{l}\text { Function: } \\
\text { Global } \\
\text { Function - } \\
\text { Medium } \\
\text { term (>6 } \\
\text { months to } 1 \\
\text { year since } \\
\text { CBT } \\
\text { initiation) }\end{array}$ & CBT vs. usual care & $\begin{array}{l}3 \mathrm{RCTs}^{34,36,39} \\
\mathrm{~N}=465\end{array}$ & Moderate & Inconsistent & Direct & Imprecise & $\begin{array}{l}\text { Inconclusive: } \\
\text { GAF: One trial found with } 6 \\
\text { months posttreatment } \\
\text { followup found no difference; } \\
\text { another trial found effect } \\
\text { favoring CBT } \\
\text { SOFAS, SFS: No difference } \\
\text { between groups }\end{array}$ & Insufficient \\
\hline $\begin{array}{l}\text { Function: } \\
\text { Global } \\
\text { Function - } \\
\text { Long term } \\
\text { (>1 year } \\
\text { since CBT } \\
\text { initiation) }\end{array}$ & CBT vs. usual care & $\begin{array}{l}1 \mathrm{SR}^{33} \text { (4 RCTs) } \\
4 \mathrm{RCTS}^{37,39-41} \\
n=851\end{array}$ & Moderate & Consistent & Direct & Imprecise & $\begin{array}{l}\text { Inconclusive: } \\
\text { GAF: } 1 \text { SR found mean } \\
\text { difference } 4.20 \text { ( } 95 \% \mathrm{CI}-0.63 \\
\text { to } 9.03) \text {. One other RCT } \\
\text { found positive effect of CBT. } \\
3 \text { RCTs found no difference in } \\
\text { SOFAS, global function (scale } \\
\text { not reported) and proportion } \\
\text { of patients with normal } \\
\text { function. }\end{array}$ & Low \\
\hline $\begin{array}{l}\text { Function: } \\
\text { Basic Living } \\
\text { Skills } \\
\end{array}$ & CBT vs. usual care & $\begin{array}{l}1 \mathrm{RCT}^{42} \\
\mathrm{~N}=76\end{array}$ & Moderate & Unknown & Direct & Imprecise & $\begin{array}{l}\text { No difference between } \\
\text { groups. }\end{array}$ & Insufficient \\
\hline
\end{tabular}




\begin{tabular}{|c|c|c|c|c|c|c|c|c|}
\hline Outcome & Comparators & $\begin{array}{l}\text { Number of Studies } \\
\text { Number of Subjects }\end{array}$ & $\begin{array}{l}\text { Strength of } \\
\text { Evidence } \\
\text { Domain: } \\
\text { Study } \\
\text { Limitations }\end{array}$ & $\begin{array}{l}\text { Strength of } \\
\text { Evidence } \\
\text { Domain: } \\
\text { Consistency }\end{array}$ & $\begin{array}{l}\text { Strength of } \\
\text { Evidence } \\
\text { Domain: } \\
\text { Directness }\end{array}$ & $\begin{array}{l}\text { Strength of } \\
\text { Evidence } \\
\text { Domain: } \\
\text { Precision }\end{array}$ & $\begin{array}{l}\text { Magnitude of Effect: } \\
\text { Summary Effect Size } \\
(95 \% \mathrm{Cl})\end{array}$ & $\begin{array}{l}\text { Strength of } \\
\text { Evidence } \\
\text { (High, } \\
\text { Moderate, } \\
\text { Low, } \\
\text { Insufficient) }\end{array}$ \\
\hline $\begin{array}{l}\text { Function: } \\
\text { Employment } \\
\text { Outcomes }\end{array}$ & CBT vs. usual care & $\begin{array}{l}2 \mathrm{RCTS}^{43,44} \\
\mathrm{~N}=522\end{array}$ & Moderate & Inconsistent & Direct & Imprecise & $\begin{array}{l}\text { Inconclusive: } \\
\text { One RCT of vocational- } \\
\text { focused CBT favored CBT for } \\
\text { hours worked and WBI score; } \\
\text { another trial found no } \\
\text { difference in proportion of } \\
\text { patients with occupational } \\
\text { recovery }\end{array}$ & Insufficient \\
\hline $\begin{array}{l}\text { Quality of } \\
\text { Life }\end{array}$ & CBT vs. usual care & $\begin{array}{l}\text { 12-24 weeks followup; } 2 \\
\text { RCTs } \\
N=216\end{array}$ & Moderate & Consistent & Direct & Imprecise & $\begin{array}{l}\text { CBT led to improved quality } \\
\text { of life } 0 \text { and } 16 \text { weeks after } \\
\text { cessation of treatment based } \\
\text { on CHOICE, WEMWEBS and } \\
\text { WHOQoL-BREF scales. }\end{array}$ & Low \\
\hline $\begin{array}{l}\text { Quality of } \\
\text { Life }\end{array}$ & CBT vs. usual care & $\begin{array}{l}18 \text { to } 24 \text { months followup; } \\
2 \text { RCTs }{ }^{37,39} \\
\mathrm{~N}=489\end{array}$ & Moderate & Consistent & Direct & Imprecise & $\begin{array}{l}\text { CBT not different from usual } \\
\text { care based on WHOQoL and } \\
\text { EROQOL scales }\end{array}$ & Low \\
\hline $\begin{array}{l}\text { Suicide and } \\
\text { Suicidality }\end{array}$ & CBT vs. usual care & $\begin{array}{l}2 \mathrm{RCTs}^{45,46} \\
\mathrm{~N}=307\end{array}$ & Moderate & Consistent & Direct & Imprecise & $\begin{array}{l}\text { Inconclusive: } \\
\text { RR } 0.68(95 \% \mathrm{Cl} 0.12 \text { to } \\
3.93) \text { and } \mathrm{RR} 0.53(95 \% \mathrm{Cl} \\
0.12 \text { to } 2.79)\end{array}$ & Insufficient \\
\hline $\begin{array}{l}\text { Core Illness } \\
\text { Symptoms }\end{array}$ & CBT vs. usual care & $\begin{array}{l}1 \mathrm{SR}(34 \mathrm{RCTs})^{47} \\
\mathrm{~N}=2,989\end{array}$ & Moderate & Consistent & Direct & Precise & $\begin{array}{l}\text { SMD }-0.33,95 \% \mathrm{Cl}-0.47 \text { to }- \\
0.19) \text {; subgroup with outcome } \\
\text { assessment blinding SMD - } \\
0.15,95 \% \mathrm{Cl}-0.27 \text { to }-0.03) \text {, }\end{array}$ & Moderate \\
\hline $\begin{array}{l}\text { Negative } \\
\text { Symptoms }\end{array}$ & CBT vs. usual care & $\begin{array}{l}2 \text { SRs (34 RCTs) } \\
\mathrm{N}=3,393\end{array}$ & Moderate & Inconsistent & Direct & Precise & $\begin{array}{l}\text { Standardized mean difference } \\
-0.13,95 \% \mathrm{Cl}-0.25 \text { to }-0.01 ; \\
\mathrm{I}^{2}=48 \% \text { [in this review a } \\
\text { negative estimate favors } \\
\mathrm{CBT} \text { ]; and SMD } 0.09,95 \% \mathrm{Cl} \\
-0.03 \text { to } 0.21 ; \mathrm{I}^{2}=63 \% \text { [in this } \\
\text { review, a positive estimate } \\
\text { favors CBT]) }\end{array}$ & Low \\
\hline $\begin{array}{l}\text { Ability to } \\
\text { Maintain } \\
\text { Treatment }\end{array}$ & CBT vs. usual care & $\begin{array}{l}13 \text { RCTs }^{34-42,44,45,49,50} \\
\mathrm{~N}=1,847\end{array}$ & Moderate & Inconsistent & Direct & Precise & $\begin{array}{l}\text { No difference: } \\
\text { Relative risk } 1.03,95 \% \mathrm{Cl} \\
0.96 \text { to } 1.10 ; I^{2}=64 \%\end{array}$ & Low \\
\hline Relapse & CBT vs. usual care & $\begin{array}{l}6 \text { RCTs }^{34,36,39,44,49,51} \\
\mathrm{~N}=1,090\end{array}$ & Moderate & Inconsistent & Direct & Imprecise & $\begin{array}{l}\text { Inconclusive: } \\
\text { Relative Risk: } 0.80,95 \% \mathrm{Cl} \\
\left.0.51 \text { to } 1.25 ; I^{2}=77 \%\right) \\
\text { Subanalysis limited to relapse } \\
\text { defined as "hospitalization" (3 } \\
\text { RCTs): } 0.70,95 \% \mathrm{Cl} 0.54 \text { to } \\
0.91 ; I^{2}=0 \%\end{array}$ & Insufficient \\
\hline Harms & CBT vs. usual care & $1 \mathrm{RCT}^{45}$ & & & & & & Insufficient \\
\hline
\end{tabular}


$\mathrm{CBT}=$ cognitive behavioral therapy, $\mathrm{CI}=$ confidence interval, EROQOL= European Quality of Life scale, GAF=global assessment of functioning, OR=odds ratio, RCT=randomized controlled trial, RR=relative risk, SMD=standard mean difference, SOFAS=Social and Occupational Functioning Assessment Scale, SR=systematic review, WHOQOL= World Health Organization Quality of Life 
Appendix Table H-5. Strength of evidence: cognitive remediation

\begin{tabular}{|c|c|c|c|c|c|c|c|c|}
\hline Outcome & Comparators & \begin{tabular}{|l|} 
Number of Studies \\
Number of Subjects
\end{tabular} & \begin{tabular}{|l} 
Strength of \\
Evidence \\
Domain: Study \\
Limitations \\
\end{tabular} & $\begin{array}{l}\text { Strength of } \\
\text { Evidence } \\
\text { Domain: } \\
\text { Consistency } \\
\end{array}$ & \begin{tabular}{|l} 
Strength of \\
Evidence \\
Domain: \\
Directness \\
\end{tabular} & $\begin{array}{l}\text { Strength of } \\
\text { Evidence } \\
\text { Domain: } \\
\text { Precision } \\
\end{array}$ & $\begin{array}{l}\text { Magnitude of Effect: } \\
\text { Summary Effect Size } \\
(95 \% \mathrm{Cl})\end{array}$ & $\begin{array}{l}\text { Strength of } \\
\text { Evidence } \\
\text { (High, } \\
\text { Moderate, Low, } \\
\text { Insufficient) } \\
\end{array}$ \\
\hline Function & $\begin{array}{l}\text { Cognitive } \\
\text { remediation vs. } \\
\text { usual care }\end{array}$ & $\begin{array}{l}1 \mathrm{SR}^{52}(19 \mathrm{RCTs}) \\
3 \mathrm{RCTs}{ }^{53-55} \\
\mathrm{~N}=1,323\end{array}$ & Moderate & Consistent & Direct & Imprecise & $\begin{array}{l}\text { In studies comparing with } \\
\text { usual care, cognitive } \\
\text { remediation resulted in a } \\
\text { small positive effect on } \\
\text { function that was not } \\
\text { consistently statistically } \\
\text { significant. (effect size } \\
0.16,95 \% \mathrm{Cl}-0.16 \text { to } 0.49 \text {; } \\
\text { SMD } 0.56,95 \% \mathrm{Cl} 0.34 \text { to } \\
0.88 \text {, and SMD } 0.41,95 \% \\
\mathrm{Cl}-.10 \text { to } 0.91 \text { ). } \\
\end{array}$ & Low \\
\hline Quality of life & $\begin{array}{l}\text { Cognitive } \\
\text { remediation vs. } \\
\text { usual care }\end{array}$ & $\begin{array}{l}1 \mathrm{RCT}^{55} \\
\mathrm{~N}=69\end{array}$ & Moderate & Unknown & Direct & Imprecise & $\begin{array}{l}\text { Quality of life was only } \\
\text { reported in one trial, with } \\
\text { no difference between } \\
\text { cognitive remediation and } \\
\text { usual care. }\end{array}$ & Insufficient \\
\hline $\begin{array}{l}\text { Overall } \\
\text { symptoms }\end{array}$ & $\begin{array}{l}\text { Cognitive } \\
\text { remediation vs. } \\
\text { usual care }\end{array}$ & $\begin{array}{l}2 \text { RCTs }^{55,56} \\
N=153\end{array}$ & Moderate & Consistent & Direct & Imprecise & $\begin{array}{l}\text { Cognitive remediation } \\
\text { improved total symptoms, } \\
\text { based on } 2 \text { trials ( } \mathrm{N}=153, \\
\mathrm{SMD}-0.62(95 \% \mathrm{Cl}-1.01 \\
\text { to }-0.24) . \text { Four trials } \\
\text { included in the Wykes } \\
\text { review reported effect sizes } \\
\text { ranging from } 0.05 \text { to } 0.45 \\
(95 \% \mathrm{Cls} \text { were not } \\
\text { reported). }\end{array}$ & Moderate \\
\hline $\begin{array}{l}\text { Negative } \\
\text { symptoms }\end{array}$ & $\begin{array}{l}\text { Cognitive } \\
\text { remediation vs. } \\
\text { usual care }\end{array}$ & $\begin{array}{l}1 \mathrm{SR}^{57} \text { (18 RCTs; } \\
\mathrm{N}=781)\end{array}$ & Moderate & Consistent & Direct & Precise & $\begin{array}{l}\text { Negative symptoms } \\
\text { improved more in CR } \\
\text { groups (effect size }-0.36 \\
(95 \% \mathrm{Cl}-0.52 \text { to }-0.20 ; \text { a } \\
\text { negative effect size } \\
\text { favors cognitive } \\
\text { remediation). }\end{array}$ & Moderate \\
\hline $\begin{array}{l}\text { Ability to } \\
\text { maintain } \\
\text { treatment }\end{array}$ & $\begin{array}{l}\text { Cognitive } \\
\text { remediation vs. } \\
\text { usual care }\end{array}$ & $\begin{array}{l}3 \mathrm{RCTs}^{53,54,56,58} \\
\mathrm{~N}=302\end{array}$ & Moderate & Consistent & Direct & Imprecise & $\begin{array}{l}\text { There was no difference in } \\
\text { ability to maintain treatment } \\
\text { in three RCTs of cognitive } \\
\text { remediation }\end{array}$ & Low \\
\hline
\end{tabular}

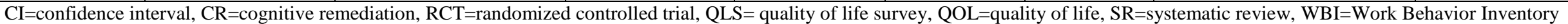


Appendix Table H-6. Strength of evidence: family interventions

\begin{tabular}{|c|c|c|c|c|c|c|c|c|}
\hline Outcome & Comparators & $\begin{array}{l}\text { Number of Studies } \\
\text { Number of Subjects }\end{array}$ & $\begin{array}{l}\text { Strength of } \\
\text { Evidence } \\
\text { Domain: Study } \\
\text { Limitations }\end{array}$ & $\begin{array}{l}\text { Strength of } \\
\text { Evidence } \\
\text { Domain: } \\
\text { Consistency }\end{array}$ & $\begin{array}{l}\text { Strength of } \\
\text { Evidence } \\
\text { Domain: } \\
\text { Directness }\end{array}$ & $\begin{array}{l}\text { Strength of } \\
\text { Evidence } \\
\text { Domain: } \\
\text { Precision }\end{array}$ & $\begin{array}{l}\text { Magnitude of Effect: } \\
\text { Summary Effect Size } \\
\text { (95\% Cl) }\end{array}$ & $\begin{array}{l}\text { Strength of } \\
\text { Evidence } \\
\text { (High, } \\
\text { Moderate, } \\
\text { Low, } \\
\text { Insufficient) }\end{array}$ \\
\hline $\begin{array}{l}\text { Function: } \\
\text { Occupational } \\
\text { (Unemployed) - } \\
1 \text { year }\end{array}$ & $\begin{array}{l}\text { Family intervention } \\
\text { vs. usual care }\end{array}$ & $1 \mathrm{SR}^{59}$ (4 RCTs; $\left.\mathrm{N}=230\right)$ & Moderate & Consistent & Direct & Imprecise & RR 1.09 (0.92 to 1.29$)$ & Low \\
\hline $\begin{array}{l}\text { Function: } \\
\text { Occupational } \\
\text { (Unemployed) } \\
-2 \text { years }\end{array}$ & $\begin{array}{l}\text { Family intervention } \\
\text { vs. usual care }\end{array}$ & $1 \mathrm{SR}^{59}$ (1 RCT; N=51) & Moderate & Unknown & Direct & Imprecise & RR 1.33 (0.84 to 2.10$)$ & Insufficient \\
\hline $\begin{array}{l}\text { Function: } \\
\text { Occupational } \\
\text { (Unemployed) } \\
-3 \text { years }\end{array}$ & $\begin{array}{l}\text { Family intervention } \\
\text { vs. usual care }\end{array}$ & $1 \mathrm{SR}^{59}(1 \mathrm{RCT} ; \mathrm{N}=99)$ & Moderate & Unknown & Direct & Imprecise & RR 1.19 (0.92 to 1.55) & Insufficient \\
\hline $\begin{array}{l}\text { Function: } \\
\text { Living } \\
\text { situation } \\
\text { (cannot live } \\
\text { independently) } \\
\text { - } 1 \text { year } \\
\end{array}$ & $\begin{array}{l}\text { Family intervention } \\
\text { vs. usual care }\end{array}$ & $1 \mathrm{SR}^{59}$ (3 RCTs; N=164) & Moderate & Consistent & Direct & Imprecise & RR 0.83 (0.66 to 1.03) & Low \\
\hline $\begin{array}{l}\text { Function: } \\
\text { Living } \\
\text { situation } \\
\text { (cannot live } \\
\text { independently) } \\
-3 \text { years }\end{array}$ & $\begin{array}{l}\text { Family intervention } \\
\text { vs. usual care }\end{array}$ & $1 \mathrm{SR}^{59}(1 \mathrm{RCT} ; \mathrm{N}=99)$ & Moderate & Unknown & Direct & Imprecise & RR 0.82 (0.59 to 1.14) & Insufficient \\
\hline $\begin{array}{l}\text { Function: } \\
\text { Living } \\
\text { situation } \\
\text { (cannot live } \\
\text { independently, } \\
\text { months in } \\
\text { psychiatric } \\
\text { facility) - } 5 \\
\text { years }\end{array}$ & $\begin{array}{l}\text { Family intervention } \\
\text { vs. usual care }\end{array}$ & $\left.1 \mathrm{RCT}^{60} ; \mathrm{N}=63\right)$ & Moderate & Unknown & Direct & Imprecise & $\begin{array}{l}10.87 \text { vs. } 21.18 \text { months, } \\
p=0.04)\end{array}$ & Insufficient \\
\hline $\begin{array}{l}\text { Social } \\
\text { Functioning }\end{array}$ & $\begin{array}{l}\text { Family intervention } \\
\text { vs. usual care }\end{array}$ & $1 \mathrm{RCT}^{36} \mathrm{~N}=69$ & Moderate & Unknown & Direct & Imprecise & & Insufficient \\
\hline Quality of Life & $\begin{array}{l}\text { Family intervention } \\
\text { vs. usual care }\end{array}$ & $\begin{array}{l}1 \mathrm{SR}^{59}(1 \mathrm{RCT} ; \mathrm{N}=50) \\
\text { plus one RCT not in } \\
\mathrm{SR} ;{ }^{39} \mathrm{~N}=55\end{array}$ & Moderate & Unknown & Direct & Imprecise & $\begin{array}{l}\text { Heinrichs scale: MD }-5.05 \\
(-15.44 \text { to } 5.34) \\
\text { EuroQol: MD }-7.38(-22.07 \\
\text { to } 7.31)\end{array}$ & Insufficient \\
\hline
\end{tabular}




\begin{tabular}{|c|c|c|c|c|c|c|c|c|}
\hline Outcome & Comparators & $\begin{array}{l}\text { Number of Studies } \\
\text { Number of Subjects }\end{array}$ & $\begin{array}{l}\text { Strength of } \\
\text { Evidence } \\
\text { Domain: Study } \\
\text { Limitations } \\
\end{array}$ & $\begin{array}{l}\text { Strength of } \\
\text { Evidence } \\
\text { Domain: } \\
\text { Consistency }\end{array}$ & $\begin{array}{l}\text { Strength of } \\
\text { Evidence } \\
\text { Domain: } \\
\text { Directness } \\
\end{array}$ & $\begin{array}{l}\text { Strength of } \\
\text { Evidence } \\
\text { Domain: } \\
\text { Precision } \\
\end{array}$ & $\begin{array}{l}\text { Magnitude of Effect: } \\
\text { Summary Effect Size } \\
(95 \% \mathrm{Cl})\end{array}$ & $\begin{array}{l}\text { Strength of } \\
\text { Evidence } \\
\text { (High, } \\
\text { Moderate, } \\
\text { Low, } \\
\text { Insufficient) } \\
\end{array}$ \\
\hline Depression & $\begin{array}{l}\text { Family intervention } \\
\text { vs. usual care }\end{array}$ & 2 RCTs; $; 6,39 \mathrm{~N}=124$ & Moderate & Consistent & Direct & Imprecise & $\begin{array}{l}1 \text { RCT, } 6 \text { months: }-1.0 \text { (-12, } \\
\text { 22) vs. } 0(-15,17) \\
1 \text { RCTs } 12 \text { months: } 3.0 \text { (- } \\
15,17) \text { vs. } 0 \text { (-14 to } 17) \\
1 \text { RCT } 12 \text { months; } 3.35 \text { (- } \\
2.64 \text { to } 9.34) \\
1 \text { RCT } 24 \text { months: }-0.11 \text { (- } \\
6.91 \text { to } 6.68)\end{array}$ & Low \\
\hline Anxiety & $\begin{array}{l}\text { Family intervention } \\
\text { vs. usual care }\end{array}$ & $1 \mathrm{RCT} ; 3^{39} \mathrm{~N}=55$ & Low & Unknown & Direct & Imprecise & $\begin{array}{l}12 \text { months: }-0.42 \text { ( }-6.97 \text { to } \\
6.13) \\
24 \text { months: }-2.36 \text { ( }-9.13 \text { to } \\
4.40)\end{array}$ & Insufficient \\
\hline Suicide & $\begin{array}{l}\text { Family intervention } \\
\text { vs. usual care }\end{array}$ & $1 \mathrm{SR}^{59}$ (6 RCTs; N=314) & Moderate & Consistent & Direct & Imprecise & RR 0.85 (0.24 to 3.02) & Low \\
\hline $\begin{array}{l}\text { Core Illness } \\
\text { Symptoms: }\end{array}$ & $\begin{array}{l}\text { Family intervention } \\
\text { vs. usual care }\end{array}$ & $\begin{array}{l}1 \mathrm{SR}^{59}\left(2 \mathrm{RCTs} ;{ }^{39,61}\right. \\
\mathrm{N}=223)\end{array}$ & Moderate & Consistent & Direct & Imprecise & SMD $-0.46(-0.73$ to -0.20$)$ & Low \\
\hline $\begin{array}{l}\text { Negative } \\
\text { Symptoms }\end{array}$ & $\begin{array}{l}\text { Family intervention } \\
\text { vs. usual care }\end{array}$ & 3 RCTs; ${ }^{36,39,61} \mathrm{~N}=163$ & Moderate & Consistent & Direct & Imprecise & $\begin{array}{l}\text { SMD }-0.38,95 \% \mathrm{Cl}-0.69 \\
\text { to }-0.07\end{array}$ & Low \\
\hline $\begin{array}{l}\text { Leaving the } \\
\text { study early (3- } \\
6 \text { months) } \\
\end{array}$ & $\begin{array}{l}\text { Family intervention } \\
\text { vs. usual care }\end{array}$ & $1 \mathrm{SR}^{59}$ (6 RCTs; N=504) & Moderate & Consistent & Indirect & Imprecise & RR 0.86 (0.50 to 1.47) & Low \\
\hline $\begin{array}{l}\text { Leaving the } \\
\text { study early ( } 7- \\
12 \text { months) } \\
\end{array}$ & $\begin{array}{l}\text { Family intervention } \\
\text { vs. usual care }\end{array}$ & $\begin{array}{l}\left.1 \mathrm{SR}^{59} \text { (9 RCTs; } \mathrm{N}=487\right) \\
\text { plus } 4 \text { RCTs; } ; 9,61-63 \\
\mathrm{~N}=466\end{array}$ & Moderate & Consistent & Indirect & Imprecise & RR 0.77 (0.64 to 0.93) & Low \\
\hline $\begin{array}{l}\text { Leaving the } \\
\text { study early } \\
\text { (13-24 months) }\end{array}$ & $\begin{array}{l}\text { Family intervention } \\
\text { vs. usual care }\end{array}$ & $1 \mathrm{SR}^{59}$ (6 RCTs; N=362) & Moderate & Consistent & Indirect & Imprecise & RR 0.82 (0.57 to 1.16) & Low \\
\hline $\begin{array}{l}\text { Leaving the } \\
\text { study early } \\
\text { (25-36 months) }\end{array}$ & $\begin{array}{l}\text { Family intervention } \\
\text { vs. usual care }\end{array}$ & $1 \mathrm{SR}^{59}$ (2 RCTs; N=90) & High & Consistent & Indirect & Imprecise & RR 0.59 (0.24 to 1.49) & Insufficient \\
\hline $\begin{array}{l}\text { Leaving the } \\
\text { study early } \\
\text { after } 3 \text { years } \\
\end{array}$ & $\begin{array}{l}\text { Family intervention } \\
\text { vs. usual care }\end{array}$ & $1 \mathrm{SR}^{59}$ (1 RCT; N=63) & Moderate & Unknown & Indirect & Imprecise & RR 1.72 (0.71 to 4.16) & Insufficient \\
\hline $\begin{array}{l}\text { Poor } \\
\text { compliance } \\
\text { with } \\
\text { medication }\end{array}$ & $\begin{array}{l}\text { Family intervention } \\
\text { vs. usual care }\end{array}$ & $\begin{array}{l}1 \mathrm{SR}^{59}(4 \mathrm{RCTS} ; \mathrm{N}=174) \\
\text { plus } 2 \mathrm{RCTs}^{62,63} \mathrm{~N}=256\end{array}$ & Moderate & Consistent & Indirect & Imprecise & RR 0.78 (0.65 to 0.92) & Low \\
\hline $\begin{array}{l}\text { Relapse 0-6 } \\
\text { months }\end{array}$ & $\begin{array}{l}\text { Family intervention } \\
\text { vs. usual care }\end{array}$ & $1 \mathrm{SR}^{59}$ (2 RCTs; N=167) & Moderate & Consistent & Direct & Imprecise & RR 0.62 (0.41 to 0.92) & Low \\
\hline $\begin{array}{l}\text { Relapse (7-12 } \\
\text { months) }\end{array}$ & $\begin{array}{l}\text { Family intervention } \\
\text { vs. usual care }\end{array}$ & $\begin{array}{l}1 \mathrm{SR}^{59} \text { (16 RCTs; } \\
\mathrm{N}=861 \text { ) plus } 4 \\
\text { RCTs; }{ }^{39,61,63,64} \mathrm{~N}=314\end{array}$ & Moderate & Consistent & Direct & Imprecise & RR 0.67 (0.54 to 0.83) & Moderate \\
\hline
\end{tabular}




\begin{tabular}{|c|c|c|c|c|c|c|c|c|}
\hline Outcome & Comparators & $\begin{array}{l}\text { Number of Studies } \\
\text { Number of Subjects }\end{array}$ & $\begin{array}{l}\text { Strength of } \\
\text { Evidence } \\
\text { Domain: Study } \\
\text { Limitations } \\
\end{array}$ & $\begin{array}{l}\text { Strength of } \\
\text { Evidence } \\
\text { Domain: } \\
\text { Consistency }\end{array}$ & $\begin{array}{l}\text { Strength of } \\
\text { Evidence } \\
\text { Domain: } \\
\text { Directness } \\
\end{array}$ & $\begin{array}{l}\text { Strength of } \\
\text { Evidence } \\
\text { Domain: } \\
\text { Precision }\end{array}$ & $\begin{array}{l}\text { Magnitude of Effect: } \\
\text { Summary Effect Size } \\
(95 \% \mathrm{Cl})\end{array}$ & \begin{tabular}{|l} 
Strength of \\
Evidence \\
(High, \\
Moderate, \\
Low, \\
Insufficient) \\
\end{tabular} \\
\hline $\begin{array}{l}\text { Relapse (13-24 } \\
\text { months) }\end{array}$ & $\begin{array}{l}\text { Family intervention } \\
\text { vs. usual care }\end{array}$ & $1 \mathrm{SR}^{59}$ (9 RCTs; N=517) & Moderate & Consistent & Direct & Imprecise & RR 0.75 (0.58 to 0.99) & Low \\
\hline $\begin{array}{l}\text { Relapse (25-36 } \\
\text { months) }\end{array}$ & $\begin{array}{l}\text { Family intervention } \\
\text { vs. usual care }\end{array}$ & $1 \mathrm{SR}^{59}$ (2 RCTs; N=147) & Moderate & Inconsistent & Direct & Imprecise & RR 1.05 (0.80 to 1.39) & Low \\
\hline $\begin{array}{l}\text { Relapse (5 } \\
\text { years) }\end{array}$ & $\begin{array}{l}\text { Family intervention } \\
\text { vs. usual care }\end{array}$ & $\begin{array}{l}1 \mathrm{SR}^{59}(1 \mathrm{RCT} ; \mathrm{N}=63) \\
\text { plus } 1 \mathrm{RCT} ;{ }^{\prime} \mathrm{N}=77\end{array}$ & Moderate & Consistent & Direct & Imprecise & RR 0.82 (0.72 to 0.94) & Low \\
\hline $\begin{array}{l}\text { Relapse (8 } \\
\text { years) }\end{array}$ & $\begin{array}{l}\text { Family intervention } \\
\text { vs. usual care }\end{array}$ & $1 \mathrm{SR}^{59}$ (1 RCT; N=62) & Moderate & Unknown & Direct & Imprecise & RR 0.86 (0.71 to 1.05) & Insufficient \\
\hline $\begin{array}{l}\text { Family Burden } \\
\text { Not Improved } \\
\text { or Worse }\end{array}$ & $\begin{array}{l}\text { Family intervention } \\
\text { vs. usual care }\end{array}$ & $1 \mathrm{SR}^{59}(1 \mathrm{RCT} ; \mathrm{N}=51)$ & Moderate & Unknown & Direct & Imprecise & $\begin{array}{l}\text { Social functioning: } \\
\text { RR } 2.40 \text { (0.51 to } 11.27) \text { at } \\
1 \text { year } \\
\text { RR } 2.88 \text { (0.64 to } 12.97) \text { at } \\
2 \text { years } \\
\text { Subjective burden: } \\
\text { RR } 1.44 \text { (0.60 to } 3.46) \text { at } 1 \\
\text { year } \\
\text { RR } 0.58(0.15 \text { to } 2.16) \text { at } 2 \\
\text { years }\end{array}$ & Insufficient \\
\hline $\begin{array}{l}\text { Nonsuicide } \\
\text { mortality }\end{array}$ & $\begin{array}{l}\text { Family intervention } \\
\text { vs. usual care }\end{array}$ & $1 \mathrm{SR}^{59}$ (3 RCTs; N=113) & Moderate & Consistent & Direct & Imprecise & RR 0.96 (0.17 to 5.33) & Insufficient \\
\hline
\end{tabular}

BPRS=Brief Psychiatric Rating Scale, MD=mean difference, RR=relative risk, RCT=randomized controlled trial, SR=systematic review 
Appendix Table H-7. Strength of evidence: intensive case management

\begin{tabular}{|c|c|c|c|c|c|c|c|c|}
\hline Outcome & Comparators & $\begin{array}{l}\text { Number of Studies } \\
\text { Number of Subjects }\end{array}$ & $\begin{array}{l}\text { Strength of } \\
\text { Evidence } \\
\text { Domain: Study } \\
\text { Limitations }\end{array}$ & $\begin{array}{l}\text { Strength of } \\
\text { Evidence } \\
\text { Domain: } \\
\text { Consistency }\end{array}$ & $\begin{array}{l}\text { Strength of } \\
\text { Evidence } \\
\text { Domain: } \\
\text { Directness }\end{array}$ & $\begin{array}{l}\text { Strength of } \\
\text { Evidence } \\
\text { Domain: } \\
\text { Precision }\end{array}$ & $\begin{array}{l}\text { Magnitude of Effect: } \\
\text { Summary Effect Size } \\
(95 \% \mathrm{Cl})\end{array}$ & $\begin{array}{l}\text { Strength of } \\
\text { Evidence } \\
\text { (High, } \\
\text { Moderate, } \\
\text { Low, } \\
\text { Insufficient) }\end{array}$ \\
\hline Function & $\begin{array}{l}\text { Intensive case } \\
\text { management vs. } \\
\text { usual care }\end{array}$ & $\begin{array}{l}1 \mathrm{SR}^{65}(3 \mathrm{RCTs}) \\
1 \mathrm{RCT}^{66}(\mathrm{n}=77)\end{array}$ & Moderate & Consistent & Direct & Imprecise & $\begin{array}{l}\text { Inconclusive: } \\
\text { Pooled mean difference, } \\
0.46(95 \% \mathrm{Cl}-0.34 \text { to } \\
0.1 .26) ; \text { one subsequent } \\
\text { trial also found no } \\
\text { difference using a different } \\
\text { scale }\end{array}$ & Low \\
\hline Quality of Life & $\begin{array}{l}\text { Intensive case } \\
\text { management vs. } \\
\text { usual care }\end{array}$ & $\begin{array}{l}1 \mathrm{SR}^{65}(2 \mathrm{RCTs}) \\
1 \mathrm{RCT}^{66}(\mathrm{n}=77)\end{array}$ & Moderate & Consistent & Direct & Imprecise & $\begin{array}{l}\text { Inconclusive: } \\
\text { Pooled mean difference, } \\
0.09 \text { ( } 95 \% \mathrm{Cl}-0.23 \text { to } \\
0.42) \text {; One subsequent trial } \\
\text { also found no difference } \\
\text { between groups in quality } \\
\text { of life using a different } \\
\text { scale. }\end{array}$ & Insufficient \\
\hline $\begin{array}{l}\text { Overall } \\
\text { Symptoms }\end{array}$ & $\begin{array}{l}\text { Intensive case } \\
\text { management vs. } \\
\text { usual care }\end{array}$ & $\begin{array}{l}1 \mathrm{SR}^{65} \text { (2 RCTs) } \\
1 \mathrm{RCT}^{66}(\mathrm{n}=77)\end{array}$ & Moderate & Consistent & Direct & Imprecise & $\begin{array}{l}\text { Inconclusive: } \\
\text { Pooled mean difference, } \\
0.46(95 \% \mathrm{Cl}-3.67 \text { to } \\
4.60) \text {; one subsequent trial } \\
\text { also reported no difference. }\end{array}$ & Low \\
\hline $\begin{array}{l}\text { Loss to } \\
\text { Followup }\end{array}$ & $\begin{array}{l}\text { Intensive case } \\
\text { management vs. } \\
\text { usual care }\end{array}$ & $\begin{array}{l}1 \mathrm{SR}^{65} \text { (7 RCTs) } \\
1 \mathrm{RCT}^{66}(\mathrm{n}=77)\end{array}$ & Moderate & Consistent & Direct & Precise & $\begin{array}{l}\text { Less loss to followup with } \\
\text { intensive case } \\
\text { management compared to } \\
\text { usual care (OR } 0.70,95 \% \\
\text { Cl } 0.54 \text { to } 0.90)\end{array}$ & Moderate \\
\hline Imprisonment & $\begin{array}{l}\text { Intensive case } \\
\text { management vs. } \\
\text { usual care }\end{array}$ & $1 \mathrm{SR}^{65}$ (5 RCTs) & Moderate & Consistent & Direct & Imprecise & $\begin{array}{l}\text { No significant differences } \\
\text { in imprisonment (OR 0.90, } \\
95 \% \mathrm{Cl} 0.45 \text { to } 1.82 \text { ) }\end{array}$ & Low \\
\hline
\end{tabular}

$\mathrm{CI}=$ confidence interval, $\mathrm{RCT}=$ randomized controlled trial, $\mathrm{OR}=$ odds ratio, $\mathrm{SR}=$ systematic review 
Appendix Table H-8. Strength of evidence: illness management and recovery

\begin{tabular}{|c|c|c|c|c|c|c|c|c|}
\hline Outcome & Comparators & $\begin{array}{l}\text { Number of Studies } \\
\text { Number of Subjects }\end{array}$ & $\begin{array}{l}\text { Strength of } \\
\text { Evidence } \\
\text { Domain: Study } \\
\text { Limitations } \\
\end{array}$ & $\begin{array}{l}\text { Strength of } \\
\text { Evidence } \\
\text { Domain: } \\
\text { Consistency }\end{array}$ & $\begin{array}{l}\text { Strength of } \\
\text { Evidence } \\
\text { Domain: } \\
\text { Directness } \\
\end{array}$ & $\begin{array}{l}\text { Strength of } \\
\text { Evidence } \\
\text { Domain: } \\
\text { Precision }\end{array}$ & $\begin{array}{l}\text { Magnitude of Effect: } \\
\text { Summary Effect Size } \\
(95 \% \mathrm{Cl})\end{array}$ & \begin{tabular}{|l} 
Strength of \\
Evidence \\
(High, \\
Moderate, \\
Low, \\
Insufficient) \\
\end{tabular} \\
\hline Functioning & $\begin{array}{l}\text { Illness self- } \\
\text { management/ self- } \\
\text { management } \\
\text { education } \\
\text { intervention vs. } \\
\text { usual care }\end{array}$ & $\begin{array}{l}1 \mathrm{SR} \text { of } 10 \mathrm{RCTS}(\mathrm{N}= \\
409)^{67}+1 \mathrm{RCT} ; \mathrm{N}=210\end{array}$ & Moderate & Inconsistent & Direct & Imprecise & $\begin{array}{l}\text { Inconclusive: } \\
\text { Heterogeneous methods } \\
\text { for measuring various } \\
\text { types of functioning were } \\
\text { used, with } 5 \text { finding benefit } \\
\text { ad } 6 \text { not. }\end{array}$ & Insufficient \\
\hline Symptoms & $\begin{array}{l}\text { Illness self- } \\
\text { management/ self- } \\
\text { management } \\
\text { education } \\
\text { intervention vs. } \\
\text { usual care }\end{array}$ & $1 \mathrm{SR}^{67} 5$ RCTs $\mathrm{n}=409$ & Moderate & Consistent & Direct & Precise & $\begin{array}{l}\text { BPRS }(n=409), \text { WMD: - } \\
4.19(-5.84 \text { to }-2.54)\end{array}$ & Moderate \\
\hline $\begin{array}{l}\text { Negative } \\
\text { Symptoms }\end{array}$ & $\begin{array}{l}\text { Illness self- } \\
\text { management/ self- } \\
\text { management } \\
\text { education } \\
\text { intervention vs. } \\
\text { usual care } \\
\end{array}$ & $1 \mathrm{SR},{ }^{67} 3 \mathrm{RCTs} ; \mathrm{N}=257$ & Moderate & Consistent & Direct & Imprecise & $\begin{array}{l}\text { PANSS negative } \\
-4.01(-5.23 \text { to }-2.79)\end{array}$ & Low \\
\hline Relapse & $\begin{array}{l}\text { Illness self- } \\
\text { management/ self- } \\
\text { management } \\
\text { education } \\
\text { intervention vs. } \\
\text { usual care }\end{array}$ & $\begin{array}{l}1 \mathrm{SR},{ }^{67} 3 \mathrm{RCTs} \\
\mathrm{N}=534\end{array}$ & Moderate & Consistent & Direct & Imprecise & $\begin{array}{l}\text { Relapse }(>10 \\
\text { interventions }), n=233 \\
O R=0.41(0.21-0.79) \\
p=0.008 \\
\text { Relapse }(<10 \\
\text { interventions), } n=269 \\
\text { OR=0.67 (0.39-1.15), } \\
p=0.014\end{array}$ & Low \\
\hline
\end{tabular}

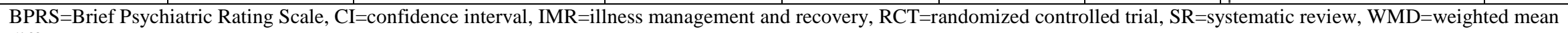
difference 
Appendix Table H-9. Strength of evidence: psychoeducation

\begin{tabular}{|c|c|c|c|c|c|c|c|c|}
\hline Outcome & Comparators & $\begin{array}{l}\text { Number of Studies } \\
\text { Number of Subjects }\end{array}$ & $\begin{array}{l}\text { Strength of } \\
\text { Evidence } \\
\text { Domain: Study } \\
\text { Limitations }\end{array}$ & $\begin{array}{l}\text { Strength of } \\
\text { Evidence Domain: } \\
\text { Consistency }\end{array}$ & $\begin{array}{l}\text { Strength of } \\
\text { Evidence } \\
\text { Domain: } \\
\text { Directness }\end{array}$ & $\begin{array}{l}\text { Strength } \\
\text { of } \\
\text { Evidence } \\
\text { Domain: } \\
\text { Precision } \\
\end{array}$ & $\begin{array}{l}\text { Magnitude } \\
\text { of Effect: } \\
\text { Summary } \\
\text { Effect Size } \\
(95 \% \mathrm{Cl})\end{array}$ & \begin{tabular}{|l} 
Strength of \\
Evidence \\
(High, \\
Moderate, \\
Low, \\
Insufficient) \\
\end{tabular} \\
\hline $\begin{array}{l}\text { Global Functioning } \\
\text { (GAFIGAS) at end of } \\
\text { intervention }\end{array}$ & $\begin{array}{l}\text { Psychoeducation vs. } \\
\text { standard care }\end{array}$ & $1 \mathrm{SR}^{68}(1 \mathrm{RCT} ; \mathrm{N}=41)$ & Medium & Unknown & Direct & Imprecise & $\begin{array}{l}\text { Inconclusive: } \\
\text { Mean } \\
\text { Difference - } \\
2.64 \mathrm{Cl} \text { - } \\
12.74 \text { to } \\
7.46\end{array}$ & Insufficient \\
\hline $\begin{array}{l}\text { Global Functioning } \\
\text { (GAS) at } 6 \text { Months }\end{array}$ & $\begin{array}{l}\text { Psychoeducation vs. } \\
\text { standard care }\end{array}$ & $1 \mathrm{SR}^{68}(1 \mathrm{RCT} ; \mathrm{N}=92)$ & Medium & Unknown & Direct & Imprecise & $\begin{array}{l}\text { Inconclusive: } \\
\text { Risk } \\
\text { Ratio } 0.83 \\
\mathrm{Cl} 0.50 \text { to } \\
1.38\end{array}$ & Insufficient \\
\hline $\begin{array}{l}\text { Global Functioning } \\
\text { (GAF/GAS) at } 1 \text { Year }\end{array}$ & $\begin{array}{l}\text { Psychoeducation vs. } \\
\text { standard care }\end{array}$ & $\begin{array}{l}1 \mathrm{SR}^{68} \text { (3 RCTs; N = } \\
260)\end{array}$ & Medium & Consistent & Direct & Imprecise & $\begin{array}{l}\text { Mean } \\
\text { Difference - } \\
5.23 \mathrm{Cl}-8.76 \\
\text { to }-1.71 \\
\mathrm{I}^{2} 79 \%\end{array}$ & Low \\
\hline $\begin{array}{l}\text { Global Functioning } \\
\text { (GAS) at } 18 \text { Months }\end{array}$ & $\begin{array}{l}\text { Psychoeducation vs. } \\
\text { standard care }\end{array}$ & $1 \mathrm{SR}^{68}(1 \mathrm{RCT} ; \mathrm{N}=92)$ & Medium & Unknown & Direct & Imprecise & $\begin{array}{l}\text { Inconclusive: } \\
\text { Risk } \\
\text { Ratio } 0.90 \\
\text { Cl } 0.58 \text { to } \\
1.39\end{array}$ & Insufficient \\
\hline $\begin{array}{l}\text { Global Functioning } \\
\text { (GAF/GAS) at } 2 \text { Years }\end{array}$ & $\begin{array}{l}\text { Psychoeducation vs. } \\
\text { standard care }\end{array}$ & $1 \mathrm{SR}^{68}(1 \mathrm{RCT} ; \mathrm{N}=59)$ & Medium & Unknown & Direct & Imprecise & $\begin{array}{l}\text { Mean } \\
\text { Difference - } \\
6.70 \mathrm{Cl} \text { - } \\
13.38 \text { to - } \\
0.02\end{array}$ & Insufficient \\
\hline $\begin{array}{l}\text { Global Functioning } \\
\text { (GAFIGAS) at } 5 \text { Years }\end{array}$ & $\begin{array}{l}\text { Psychoeducation vs. } \\
\text { standard care }\end{array}$ & $1 \mathrm{SR}^{68}(1 \mathrm{RCT} ; \mathrm{N}=60)$ & Medium & Unknown & Direct & Imprecise & $\begin{array}{l}\text { Inconclusive: } \\
\text { Mean } \\
\text { Difference - } \\
3.80 \mathrm{Cl}-8.04 \\
\text { to } 0.44\end{array}$ & Insufficient \\
\hline $\begin{array}{l}\text { Social Functioning } \\
\text { (SAS-II) at End of } \\
\text { Intervention }\end{array}$ & $\begin{array}{l}\text { Psychoeducation vs. } \\
\text { standard care }\end{array}$ & $1 \mathrm{SR}^{68}(1 \mathrm{RCT} ; \mathrm{N}=19)$ & Medium & Unknown & Direct & Imprecise & $\begin{array}{l}\text { Inconclusive: } \\
\text { Mean } \\
\text { Difference - } \\
0.10 \mathrm{Cl}-0.37 \\
\text { to } 0.17\end{array}$ & Insufficient \\
\hline
\end{tabular}




\begin{tabular}{|c|c|c|c|c|c|c|c|c|}
\hline Outcome & Comparators & $\begin{array}{l}\text { Number of Studies } \\
\text { Number of Subjects }\end{array}$ & $\begin{array}{l}\text { Strength of } \\
\text { Evidence } \\
\text { Domain: Study } \\
\text { Limitations } \\
\end{array}$ & $\begin{array}{l}\text { Strength of } \\
\text { Evidence Domain: } \\
\text { Consistency }\end{array}$ & $\begin{array}{l}\text { Strength of } \\
\text { Evidence } \\
\text { Domain: } \\
\text { Directness }\end{array}$ & $\begin{array}{l}\text { Strength } \\
\text { of } \\
\text { Evidence } \\
\text { Domain: } \\
\text { Precision } \\
\end{array}$ & $\begin{array}{l}\text { Magnitude } \\
\text { of Effect: } \\
\text { Summary } \\
\text { Effect Size } \\
(95 \% \mathrm{Cl})\end{array}$ & $\begin{array}{l}\text { Strength of } \\
\text { Evidence } \\
\text { (High, } \\
\text { Moderate, } \\
\text { Low, } \\
\text { Insufficient) }\end{array}$ \\
\hline $\begin{array}{l}\text { Quality of Life } \\
\text { (Heinrich's Scale) at } \\
\text { End of Intervention }\end{array}$ & $\begin{array}{l}\text { Psychoeducation vs. } \\
\text { standard care }\end{array}$ & $\begin{array}{l}1 \mathrm{SR}^{68}(1 \mathrm{RCT} ; \mathrm{N}= \\
114)\end{array}$ & Medium & Unknown & Direct & Imprecise & $\begin{array}{l}\text { Mean } \\
\text { Difference - } \\
8.20 \mathrm{Cl} \text { - } \\
14.78 \text { to - } \\
1.62\end{array}$ & Insufficient \\
\hline $\begin{array}{l}\text { Quality of Life } \\
\text { (Heinrich's Scale) at } \\
3 \text { Months }\end{array}$ & $\begin{array}{l}\text { Psychoeducation vs. } \\
\text { standard care }\end{array}$ & $\begin{array}{l}1 \mathrm{SR}^{68}(1 \mathrm{RCT} ; \mathrm{N}= \\
108)\end{array}$ & Medium & Unknown & Direct & Imprecise & $\begin{array}{l}\text { Mean } \\
\text { Difference - } \\
9.70 \mathrm{Cl} \text { - } \\
17.22 \text { to - } \\
2.18\end{array}$ & Insufficient \\
\hline BPRS at 3 Months & $\begin{array}{l}\text { Psychoeducation vs. } \\
\text { standard care }\end{array}$ & $1 \mathrm{SR}^{68}(1 \mathrm{RCT} ; \mathrm{N}=19)$ & Medium & Unknown & Direct & Imprecise & $\begin{array}{l}\text { Inconclusive: } \\
\text { Mean } \\
\text { Difference - } \\
0.06 \mathrm{Cl}-0.53 \\
\text { to } 0.41\end{array}$ & \begin{tabular}{|l|} 
Insufficient \\
\end{tabular} \\
\hline BPRS at 1 Year & $\begin{array}{l}\text { Psychoeducation vs. } \\
\text { standard care }\end{array}$ & $\begin{array}{l}1 \mathrm{SR}^{68}(1 \mathrm{RCT} ; \mathrm{N}= \\
159)\end{array}$ & Medium & Unknown & Direct & Imprecise & $\begin{array}{l}\text { Mean } \\
\text { Difference - } \\
6.0 \mathrm{Cl}-9.15 \\
\text { to }-2.85\end{array}$ & Insufficient \\
\hline $\begin{array}{l}\text { Relapse With or } \\
\text { Without } \\
\text { Readmission: } 9 \text { to } 18 \\
\text { Months }\end{array}$ & & $\begin{array}{l}1 \mathrm{SR}^{68} \text { (6 RCTs; } \mathrm{N}= \\
720)\end{array}$ & Medium & Consistent & Direct & Precise & $\begin{array}{l}\text { Risk Ratio } \\
0.80 \\
\mathrm{Cl} 0.70 \text { to } \\
0.92 \\
\mathrm{I}^{2} 54 \%\end{array}$ & Moderate \\
\hline $\begin{array}{l}\text { Relapse Without } \\
\text { Readmission: Total }\end{array}$ & $\begin{array}{l}\text { Psychoeducation vs. } \\
\text { standard care }\end{array}$ & $\begin{array}{l}1 \mathrm{SR}^{68} \text { (3 RCTs; } \mathrm{N}= \\
385)\end{array}$ & Medium & Consistent & Direct & Imprecise & $\begin{array}{l}\text { Inconclusive: } \\
\text { Risk Ratio } \\
1.05 \\
\mathrm{Cl} 0.84 \text { to } \\
1.31 \\
\mathrm{I}^{2} 60 \%\end{array}$ & Low \\
\hline $\begin{array}{l}\text { Relapse Without } \\
\text { Readmission: } 1 \text { Year }\end{array}$ & $\begin{array}{l}\text { Psychoeducation vs. } \\
\text { standard care }\end{array}$ & $\begin{array}{l}1 \mathrm{SR}^{68}(2 \mathrm{RCTs} ; \mathrm{N}= \\
303)\end{array}$ & Medium & Consistent & Direct & Imprecise & $\begin{array}{l}\text { Inconclusive: } \\
\text { Risk Ratio } \\
1.16 \\
\mathrm{Cl} 0.92 \text { to } \\
1.46 \\
\mathrm{I}^{2} 0.0 \%\end{array}$ & Low \\
\hline
\end{tabular}




\begin{tabular}{|c|c|c|c|c|c|c|c|c|}
\hline Outcome & Comparators & \begin{tabular}{|l|} 
Number of Studies \\
Number of Subjects \\
\end{tabular} & $\begin{array}{l}\text { Strength of } \\
\text { Evidence } \\
\text { Domain: Study } \\
\text { Limitations } \\
\end{array}$ & $\begin{array}{l}\text { Strength of } \\
\text { Evidence Domain: } \\
\text { Consistency }\end{array}$ & $\begin{array}{l}\text { Strength of } \\
\text { Evidence } \\
\text { Domain: } \\
\text { Directness } \\
\end{array}$ & $\begin{array}{l}\text { Strength } \\
\text { of } \\
\text { Evidence } \\
\text { Domain: } \\
\text { Precision }\end{array}$ & $\begin{array}{l}\text { Magnitude } \\
\text { of Effect: } \\
\text { Summary } \\
\text { Effect Size } \\
(95 \% \mathrm{Cl})\end{array}$ & \begin{tabular}{|l} 
Strength of \\
Evidence \\
(High, \\
Moderate, \\
Low, \\
Insufficient) \\
\end{tabular} \\
\hline $\begin{array}{l}\text { Relapse Without } \\
\text { Readmission: } 18 \\
\text { Months }\end{array}$ & $\begin{array}{l}\text { Psychoeducation vs. } \\
\text { standard care }\end{array}$ & $\begin{array}{l}1 \mathrm{SR}^{68}(1 \mathrm{RCT} ; \mathrm{N}= \\
382)\end{array}$ & Medium & Unknown & Direct & Imprecise & $\begin{array}{l}\text { Inconclusive: } \\
\text { Risk Ratio } \\
0.5 \\
\mathrm{Cl} 0.23 \text { to } \\
1.11\end{array}$ & Insufficient \\
\hline Harms: Mortality & $\begin{array}{l}\text { Psychoeducation vs. } \\
\text { standard care }\end{array}$ & $\begin{array}{l}1 \mathrm{SR}^{68}(2 \mathrm{RCTs} ; \mathrm{N}= \\
170)\end{array}$ & Medium & Consistent & Direct & Imprecise & $\begin{array}{l}\text { Inconclusive: } \\
\text { Risk Ratio } \\
0.53 \\
\mathrm{Cl} 0.07 \text { to } \\
3.95 \\
\mathrm{I}^{2} 0.0 \%\end{array}$ & Low \\
\hline
\end{tabular}

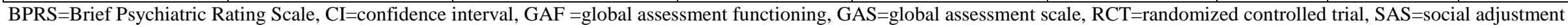
score, $\mathrm{SR}=$ systematic review 
Appendix Table H-10. Strength of evidence: social skills training

\begin{tabular}{|c|c|c|c|c|c|c|c|c|}
\hline Outcome & Comparators & $\begin{array}{l}\text { Number of Studies } \\
\text { Number of Subjects }\end{array}$ & $\begin{array}{l}\text { Strength of } \\
\text { Evidence } \\
\text { Domain: Study } \\
\text { Limitations } \\
\end{array}$ & $\begin{array}{l}\text { Strength of } \\
\text { Evidence } \\
\text { Domain: } \\
\text { Consistency }\end{array}$ & $\begin{array}{l}\text { Strength of } \\
\text { Evidence } \\
\text { Domain: } \\
\text { Directness } \\
\end{array}$ & $\begin{array}{l}\text { Strength of } \\
\text { Evidence } \\
\text { Domain: } \\
\text { Precision } \\
\end{array}$ & $\begin{array}{l}\text { Magnitude of Effect: } \\
\text { Summary Effect Size } \\
(95 \% \mathrm{Cl})\end{array}$ & \begin{tabular}{|l} 
Strength of \\
Evidence \\
(High, \\
Moderate, \\
Low, \\
Insufficient) \\
\end{tabular} \\
\hline Function & $\begin{array}{l}\text { Social skills } \\
\text { training vs. usual } \\
\text { care }\end{array}$ & $\begin{array}{l}\text { 3 RCTs (4 publications) } \\
63,69-71 \\
\mathrm{~N}=384\end{array}$ & Moderate & Consistent & Direct & Imprecise & $\begin{array}{l}\text { Significant improvement in } \\
\text { scale scores during } \\
\text { treatment for } 6 \text { months to } 2 \\
\text { years (SMD } 0.65 \text { to } 1.60 \text { ) }\end{array}$ & Low \\
\hline Function & $\begin{array}{l}\text { Social skills } \\
\text { training vs. usual } \\
\text { care }\end{array}$ & $\begin{array}{l}1 \mathrm{RCT}^{69,70} \\
\mathrm{~N}=183\end{array}$ & Moderate & Unknown & Direct & Imprecise & $\begin{array}{l}\text { Social function not different } \\
\text { from control after treatment } \\
\text { cessation ( } 1 \text { study; SMD } \\
0.24 ; 95 \% \mathrm{Cl}-0.05 \text { to } 0.53 \text { ) }\end{array}$ & Insufficient \\
\hline $\begin{array}{l}\text { Overall } \\
\text { Symptoms }\end{array}$ & $\begin{array}{l}\text { Social skills } \\
\text { training vs. usual } \\
\text { care }\end{array}$ & $\begin{array}{l}2 \text { RCTs }^{63,71} \\
N=201\end{array}$ & Moderate & Consistent & Direct & Imprecise & $\begin{array}{l}\text { Inconclusive: } \\
\text { PANSS: SMD }-1.50(95 \% \\
\mathrm{Cl}-1.92 \text { to }-1.09 \text { and }-0.81 \\
\text { ( } 95 \% \mathrm{Cl}-1.22 \text { to }-0.40) \\
\text { BPRS (mixed population): } \\
\text { SMD }-0.04 \text { ( } 95 \% \mathrm{Cl}-0.33 \text { to } \\
0.25 \text { ) }\end{array}$ & Low \\
\hline $\begin{array}{l}\text { Overall } \\
\text { Symptoms }\end{array}$ & $\begin{array}{l}\text { Social skills } \\
\text { training vs. usual } \\
\text { care }\end{array}$ & $\begin{array}{l}1 \mathrm{RCT}^{69,70} \\
\mathrm{~N}=183\end{array}$ & Moderate & Unknown & Direct & Imprecise & $\begin{array}{l}\text { Inconclusive: } \\
\text { Mixed population (55\% } \\
\text { schizophrenia): no } \\
\text { significant effect on } \\
\text { symptoms (BPRS) SMD - } \\
0.04 ; 95 \% \mathrm{Cl}-0.33 \text { to } 0.25 \text {. }\end{array}$ & Insufficient \\
\hline $\begin{array}{l}\text { Negative } \\
\text { Symptoms }\end{array}$ & $\begin{array}{l}\text { Social skills } \\
\text { training vs. usual } \\
\text { care }\end{array}$ & $\begin{array}{l}\text { 3 RCTs (4 publications) } \\
63,69-71 \\
N=384\end{array}$ & Moderate & Consistent & Direct & Imprecise & $\begin{array}{l}\text { Negative symptoms } \\
\text { improved with SST versus } \\
\text { usual care based on } \\
\text { PANSS-negative and } \\
\text { SANS SMD range }-0.45 \text { to - } \\
1.30 \text { ) at } 6 \text { months to } 2 \\
\text { years }\end{array}$ & Low \\
\hline $\begin{array}{l}\text { Negative } \\
\text { Symptoms }\end{array}$ & $\begin{array}{l}\text { Social skills } \\
\text { training vs. usual } \\
\text { care }\end{array}$ & $\begin{array}{l}1 \mathrm{RCT}^{69,70} \\
\mathrm{~N}=183\end{array}$ & Moderate & Unknown & Direct & Imprecise & $\begin{array}{l}\text { Negative symptoms were } \\
\text { better with SST than usual } \\
\text { care } 1 \text { year after treatment } \\
\text { discontinuation (SMD }-0.45 \\
95 \% \mathrm{Cl}-0.74 \text { to }-0.15\end{array}$ & Insufficient \\
\hline $\begin{array}{l}\text { Ability to } \\
\text { Maintain } \\
\text { Treatment }\end{array}$ & $\begin{array}{l}\text { Social skills } \\
\text { training vs. usual } \\
\text { care }\end{array}$ & $\begin{array}{l}2 \text { RCTs }^{63,70} \\
N=384\end{array}$ & Moderate & Consistent & Direct & Imprecise & $\begin{array}{l}\text { No difference: } \\
\text { One year: RR } 1.10(95 \% \mathrm{Cl} \\
0.92 \text { to } 1.31) \\
\text { Two-year: RR } 1.01 ; 95 \% \mathrm{Cl} \\
0.88 \text { to } 1.16 \\
\end{array}$ & Low \\
\hline
\end{tabular}




\begin{tabular}{|c|c|c|c|c|c|c|c|c|}
\hline Outcome & Comparators & $\begin{array}{l}\text { Number of Studies } \\
\text { Number of Subjects }\end{array}$ & $\begin{array}{l}\text { Strength of } \\
\text { Evidence } \\
\text { Domain: Study } \\
\text { Limitations }\end{array}$ & $\begin{array}{l}\text { Strength of } \\
\text { Evidence } \\
\text { Domain: } \\
\text { Consistency }\end{array}$ & $\begin{array}{l}\text { Strength of } \\
\text { Evidence } \\
\text { Domain: } \\
\text { Directness }\end{array}$ & $\begin{array}{l}\text { Strength of } \\
\text { Evidence } \\
\text { Domain: } \\
\text { Precision }\end{array}$ & $\begin{array}{l}\text { Magnitude of Effect: } \\
\text { Summary Effect Size } \\
(95 \% \mathrm{Cl})\end{array}$ & $\begin{array}{l}\text { Strength of } \\
\text { Evidence } \\
\text { (High, } \\
\text { Moderate, } \\
\text { Low, } \\
\text { Insufficient) }\end{array}$ \\
\hline Relapse & $\begin{array}{l}\text { Social skills } \\
\text { training vs. usual } \\
\text { care }\end{array}$ & $\begin{array}{l}1 \mathrm{RCT}^{63} \\
\mathrm{~N}=82\end{array}$ & Moderate & Unknown & Direct & Imprecise & $\begin{array}{l}\text { Inconclusive: } \\
\text { RR } 0.50 ; 95 \% \mathrm{Cl} 0.18 \text { to } \\
1.36\end{array}$ & Insufficient \\
\hline
\end{tabular}

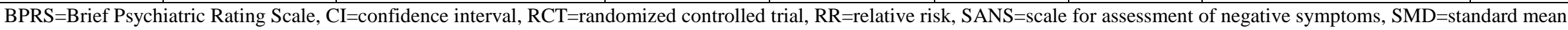
difference, SST=social skills training 
Appendix Table H-11. Strength of evidence: supported employment

\begin{tabular}{|c|c|c|c|c|c|c|c|c|}
\hline Outcome & Comparators & $\begin{array}{l}\text { Number of Studies } \\
\text { Number of Subjects }\end{array}$ & $\begin{array}{l}\text { Strength of } \\
\text { Evidence } \\
\text { Domain: Study } \\
\text { Limitations }\end{array}$ & $\begin{array}{l}\text { Strength of } \\
\text { Evidence } \\
\text { Domain: } \\
\text { Consistency }\end{array}$ & $\begin{array}{l}\text { Strength of } \\
\text { Evidence } \\
\text { Domain: } \\
\text { Directness }\end{array}$ & $\begin{array}{l}\text { Strength of } \\
\text { Evidence } \\
\text { Domain: } \\
\text { Precision }\end{array}$ & $\begin{array}{l}\text { Magnitude of Effect: } \\
\text { Summary Effect Size } \\
(95 \% \mathrm{Cl})\end{array}$ & $\begin{array}{l}\text { Strength of } \\
\text { Evidence } \\
\text { (High, } \\
\text { Moderate, } \\
\text { Low, } \\
\text { Insufficient) } \\
\end{array}$ \\
\hline \multirow[t]{2}{*}{$\begin{array}{l}\text { Functional } \\
\text { (occupational) - } \\
\text { \# in competitive } \\
\text { employment }\end{array}$} & $\begin{array}{l}\text { Individual } \\
\text { placement and } \\
\text { support (IPS) vs. } \\
\text { standard services }\end{array}$ & $\begin{array}{l}1 \text { trial }^{72} \\
\mathrm{~N}=204\end{array}$ & Moderate & Unknown & Direct & Imprecise & $\begin{array}{l}\text { IPS vs. standard services: } \\
75 \% \text { vs } 27.5 \%(p<0.001)\end{array}$ & Low \\
\hline & $\begin{array}{l}\text { Supported } \\
\text { Employment } \\
\text { (primarily IPS) vs. } \\
\text { vocational training } \\
\text { or usual care }\end{array}$ & $1 \mathrm{RCT}^{73,74}(\mathrm{~N}=1,273)$ & Moderate & Consistent & $\begin{array}{l}\text { Indirect for } \\
\text { this review } \\
\text { question }\end{array}$ & Precise & $\begin{array}{l}\text { IPS vs. vocational training } \\
\text { or usual care } \\
55 \% \text { vs } 34 \%(P<0.001) \\
\text { Subgroup analysis of sonly } \\
\text { patients with } \\
\text { schizophrenia: } \\
22 \% \text { vs. } 12 \% \text { (from figure) } \\
P<0.001 \text { with mixed } \\
\text { effects logistic regression }\end{array}$ & Moderate \\
\hline Combined SOE & & & & & & & & Moderate \\
\hline $\begin{array}{l}\text { Functional } \\
\text { (occupational) - } \\
\text { Days to first } \\
\text { competitive } \\
\text { employment }\end{array}$ & $\begin{array}{l}\text { Individual } \\
\text { placement and } \\
\text { support (IPS) vs. } \\
\text { standard services }\end{array}$ & $\begin{array}{l}1 \text { trial }^{72} \\
\mathrm{~N}=204\end{array}$ & Moderate & Unknown & Direct & Imprecise & $\begin{array}{l}\text { IPS vs. standard services: } \\
\text { Days to first job, } 196.63 \text { vs. } \\
218.84 ; P=0.019\end{array}$ & Low \\
\hline \multirow[t]{2}{*}{$\begin{array}{l}\text { Functional } \\
\text { (occupational) } \\
\text { - Worked more } \\
\text { than } 20 \text { hours } \\
\text { per week }\end{array}$} & $\begin{array}{l}\text { Individual } \\
\text { placement and } \\
\text { support (IPS) vs. } \\
\text { standard services }\end{array}$ & $\begin{array}{l}1 \text { trial }^{72} \\
\mathrm{~N}=204\end{array}$ & Moderate & Unknown & Direct & Imprecise & $\begin{array}{l}\text { IPS vs. standard services: } \\
\text { Worked }>20 \text { hrs per } \\
\text { week33.8\% vs 13\%; } \\
P=0.001\end{array}$ & Low \\
\hline & $\begin{array}{l}\text { Supported } \\
\text { Employment } \\
\text { (primarily IPS) vs. } \\
\text { vocational training } \\
\text { or usual care }\end{array}$ & $1 \mathrm{RCT}^{73,74}(\mathrm{~N}=1,273)$ & Moderate & Consistent & $\begin{array}{l}\text { Indirect for } \\
\text { this review } \\
\text { question }\end{array}$ & Precise & $\begin{array}{l}\text { IPS vs. vocational training } \\
\text { or usual care } \\
\text { \# working } \geq 40 \text { hours per } \\
\text { month } \\
51 \% \text { vs } 39 \% \text {; P }<0.001\end{array}$ & Moderate \\
\hline Combined SOE & & & & & & & & Moderate \\
\hline \multirow[t]{2}{*}{$\begin{array}{l}\text { Functional } \\
\text { (occupational) } \\
\text { - Wages } \\
\text { earned }\end{array}$} & $\begin{array}{l}\text { Individual } \\
\text { placement and } \\
\text { support (IPS) vs. } \\
\text { standard services } \\
\end{array}$ & $\begin{array}{l}1 \text { trial }^{72} \\
\mathrm{~N}=204\end{array}$ & Moderate & Unknown & Direct & Imprecise & $\begin{array}{l}\text { IPS vs. standard services: } \\
\$ 2,078 / \mathrm{mo} \text { vs } \$ 617.59 / \mathrm{mo} \\
\mathrm{P}<0.001\end{array}$ & Low \\
\hline & $\begin{array}{l}\text { Supported } \\
\text { Employment } \\
\text { (primarily IPS) vs. } \\
\text { vocational training } \\
\text { or usual care } \\
\end{array}$ & $1 \mathrm{RCT}^{73,74}(\mathrm{~N}=1,273)$ & Moderate & Consistent & $\begin{array}{l}\text { Indirect for } \\
\text { this review } \\
\text { question }\end{array}$ & Precise & $\begin{array}{l}\text { IPS vs. vocational training } \\
\text { or usual care } \\
\$ 122 / \mathrm{mo} \text { vs } \$ 99 / \mathrm{mo} ; \mathrm{P}=.04\end{array}$ & Moderate \\
\hline Combined SOE & & & & & & & & Moderate \\
\hline
\end{tabular}




\begin{tabular}{|c|c|c|c|c|c|c|c|c|}
\hline Outcome & Comparators & $\begin{array}{l}\text { Number of Studies } \\
\text { Number of Subjects }\end{array}$ & $\begin{array}{l}\text { Strength of } \\
\text { Evidence } \\
\text { Domain: Study } \\
\text { Limitations }\end{array}$ & $\begin{array}{l}\text { Strength of } \\
\text { Evidence } \\
\text { Domain: } \\
\text { Consistency }\end{array}$ & $\begin{array}{l}\text { Strength of } \\
\text { Evidence } \\
\text { Domain: } \\
\text { Directness }\end{array}$ & $\begin{array}{l}\text { Strength of } \\
\text { Evidence } \\
\text { Domain: } \\
\text { Precision }\end{array}$ & $\begin{array}{l}\text { Magnitude of Effect: } \\
\text { Summary Effect Size } \\
(95 \% \mathrm{Cl})\end{array}$ & $\begin{array}{l}\text { Strength of } \\
\text { Evidence } \\
\text { (High, } \\
\text { Moderate, } \\
\text { Low, } \\
\text { Insufficient) }\end{array}$ \\
\hline \multirow[t]{2}{*}{$\begin{array}{l}\text { Functional } \\
\text { (occupational) } \\
\text { - Weeks } \\
\text { worked (mean) }\end{array}$} & $\begin{array}{l}\text { Individual } \\
\text { placement and } \\
\text { support (IPS) vs. } \\
\text { standard services }\end{array}$ & $\begin{array}{l}1 \text { trial }^{72} \\
\mathrm{~N}=204\end{array}$ & Moderate & Unknown & Direct & Imprecise & $\begin{array}{l}\text { IPS vs. standard services: } \\
\text { Total weeks worked } \\
29.72 \text { vs } 5.45 ; P<0.001\end{array}$ & Low \\
\hline & $\begin{array}{l}\text { Supported } \\
\text { Employment } \\
\text { (primarily IPS) vs. } \\
\text { vocational training }\end{array}$ & $1 \mathrm{SR}^{75}(\mathrm{~N}=2,265)$ & Moderate & Consistent & $\begin{array}{l}\text { Indirect for } \\
\text { this review } \\
\text { question }\end{array}$ & Precise & $\begin{array}{l}\text { Supported Employment vs. } \\
\text { vocational training Days } \\
\text { employed } \\
\text { Mean Difference } 70.63 \\
(95 \% \text { Cl } 43.22,98.04)\end{array}$ & Moderate \\
\hline Combined SOE & & & & & & & & Moderate \\
\hline
\end{tabular}

BPRS=Brief Psychiatric Rating Scale, CI=confidence interval, NNT=number needed to treat, OR=odds ratio, PANSS=positive and negative syndrome scale, QOL=quality of life,

$\mathrm{RCT}=$ randomized controlled trial, $\mathrm{RR}=$ relative risk, $\mathrm{SR}=$ systematic review, WBI=Work Behavior Inventory 
Appendix Table H-12. Strength of evidence: supportive therapy

\begin{tabular}{|c|c|c|c|c|c|c|c|c|}
\hline Outcome & Comparators & $\begin{array}{l}\text { Number of Studies } \\
\text { Number of Subjects }\end{array}$ & $\begin{array}{l}\text { Strength of } \\
\text { Evidence } \\
\text { Domain: Study } \\
\text { Limitations }\end{array}$ & $\begin{array}{l}\text { Strength of } \\
\text { Evidence } \\
\text { Domain: } \\
\text { Consistency }\end{array}$ & $\begin{array}{l}\text { Strength of } \\
\text { Evidence } \\
\text { Domain: } \\
\text { Directness }\end{array}$ & $\begin{array}{l}\text { Strength of } \\
\text { Evidence } \\
\text { Domain: } \\
\text { Precision }\end{array}$ & $\begin{array}{l}\text { Magnitude of Effect: } \\
\text { Summary Effect Size } \\
(95 \% \mathrm{Cl})\end{array}$ & $\begin{array}{l}\text { Strength of } \\
\text { Evidence } \\
\text { (High, } \\
\text { Moderate, } \\
\text { Low, } \\
\text { Insufficient) } \\
\end{array}$ \\
\hline $\begin{array}{l}\text { Global } \\
\text { Functioning }\end{array}$ & $\begin{array}{l}\text { Supportive } \\
\text { therapy vs. } \\
\text { standard care }\end{array}$ & $1 \mathrm{SR}^{76}$ (2 RCTs; N =289) & Moderate & Consistent & Direct & Imprecise & $\begin{array}{l}\text { Inconclusive: } \\
\text { GAF-M, } \mathrm{n}=29, \text { mean } \\
\text { difference } 1.40 \mathrm{Cl}-5.09 \text { to } \\
\text { 7.89; GAS, } \mathrm{n}=260 \text {, mean } \\
\text { difference }-2.66 \mathrm{Cl}-6.20 \\
\text { to } 0.88 \text {. }\end{array}$ & Low \\
\hline $\begin{array}{l}\text { Social } \\
\text { Functioning }\end{array}$ & $\begin{array}{l}\text { Supportive } \\
\text { therapy vs. } \\
\text { standard care }\end{array}$ & $1 \mathrm{SR}^{76}(1 \mathrm{RCT} ; \mathrm{N}=260)$ & Moderate & Unknown & Direct & Imprecise & $\begin{array}{l}\text { Inconclusive: } \\
\text { SFS: } n=260, \text { mean } \\
\text { difference }-0.67 \mathrm{Cl}-7.05 \\
\text { to } 5.71 .\end{array}$ & Insufficient \\
\hline Quality of Life & $\begin{array}{l}\text { Supportive } \\
\text { therapy vs. } \\
\text { standard care }\end{array}$ & $1 \mathrm{SR}^{76}(1 \mathrm{RCT} ; \mathrm{N}=260)$ & Moderate & Unknown & Direct & Imprecise & $\begin{array}{l}\text { Inconclusive: } \\
\text { Rosenberg Self-Esteem } \\
\text { Scale (RSES) } \mathrm{n}=260, \\
\mathrm{MD}-1.21 \mathrm{Cl}-2.85 \text { to } 0.43 \text {. } \\
\text { Well-being scale (WBS) } \\
\text { score }=260, \mathrm{MD}-2.73 \mathrm{Cl} \text { - } \\
6.04 \text { to } 0.58 . \\
\text { Global health quotient } \\
(\mathrm{GHQ}) \mathrm{n}=260, \mathrm{MD}-2.45 \\
\mathrm{Cl}-2.41 \text { to } 7.31 .\end{array}$ & Insufficient \\
\hline Relapse & $\begin{array}{l}\text { Supportive } \\
\text { therapy vs. } \\
\text { standard care }\end{array}$ & $1 \mathrm{SR}^{76}(1 \mathrm{RCT}, \mathrm{N}=54)$ & Moderate & Unknown & Direct & Imprecise & $\begin{array}{l}\text { Inconclusive: } \\
\text { Medium term followup (13 } \\
\text { to } 26 \text { weeks, } \mathrm{N}=54, \mathrm{RR} \\
0.12 \mathrm{Cl} 0.01 \text { to } 2.11 \mathrm{~s} \text { ), or } \\
\text { long term followup (more } \\
\text { than } 26 \text { weeks, } \mathrm{n}=54, \mathrm{RR} \\
0.96 \mathrm{Cl} 0.44 \text { to } 2.11 \text { ) } \\
\end{array}$ & Insufficient \\
\hline $\begin{array}{l}\text { Core } \\
\text { Symptoms }\end{array}$ & $\begin{array}{l}\text { Supportive } \\
\text { therapy vs. } \\
\text { standard care }\end{array}$ & $1 \mathrm{SR}^{76}(2 \mathrm{RCTs}, \mathrm{N}=167)$ & Moderate & Unknown & Direct & Imprecise & $\begin{array}{l}\text { Inconclusive: } \\
\text { PANSS: Short term (13 to } \\
26 \text { weeks; } \mathrm{N}=131 \mathrm{Mean} \\
\text { Difference }-4.42(95 \% \mathrm{Cl} \text { - } \\
10.13,1.29) . \\
\text { Long term (more than } 26 \\
\text { weeks, } \mathrm{n}=36, \text { Mean } \\
\text { Difference } 4.70(95 \% \mathrm{Cl} \text { - } \\
6.71,16.11) . \\
\end{array}$ & Insufficient \\
\hline
\end{tabular}




\begin{tabular}{|c|c|c|c|c|c|c|c|c|}
\hline Outcome & Comparators & $\begin{array}{l}\text { Number of Studies } \\
\text { Number of Subjects }\end{array}$ & $\begin{array}{l}\text { Strength of } \\
\text { Evidence } \\
\text { Domain: Study } \\
\text { Limitations }\end{array}$ & $\begin{array}{l}\text { Strength of } \\
\text { Evidence } \\
\text { Domain: } \\
\text { Consistency }\end{array}$ & $\begin{array}{l}\text { Strength of } \\
\text { Evidence } \\
\text { Domain: } \\
\text { Directness }\end{array}$ & $\begin{array}{l}\text { Strength of } \\
\text { Evidence } \\
\text { Domain: } \\
\text { Precision }\end{array}$ & $\begin{array}{l}\text { Magnitude of Effect: } \\
\text { Summary Effect Size } \\
(95 \% \mathrm{Cl})\end{array}$ & $\begin{array}{l}\text { Strength of } \\
\text { Evidence } \\
\text { (High, } \\
\text { Moderate, } \\
\text { Low, } \\
\text { Insufficient) }\end{array}$ \\
\hline $\begin{array}{l}\text { Negative } \\
\text { Symptoms }\end{array}$ & $\begin{array}{l}\text { Supportive } \\
\text { therapy vs. } \\
\text { standard care }\end{array}$ & $1 \mathrm{SR}^{76}(1 \mathrm{RCT}, \mathrm{N}=47)$ & Moderate & Unknown & Direct & Imprecise & $\begin{array}{l}\text { Inconclusive: } \\
\text { Short term: } 10.19 \text { and } \\
\text { 10.73; long-term: } 9.90 \text { and } \\
11.46 \text { (no statistical } \\
\text { analysis because of } \\
\text { skewed data). }\end{array}$ & Insufficient \\
\hline $\begin{array}{l}\text { Discontinuing } \\
\text { Treatment }\end{array}$ & $\begin{array}{l}\text { Supportive } \\
\text { therapy vs. } \\
\text { standard care }\end{array}$ & $1 \mathrm{SR}^{76}(4 \mathrm{RCTS}, \mathrm{N}=354)$ & Moderate & Consistent & Direct & Imprecise & $\begin{array}{l}\text { Inconclusive: } \\
\text { Relative Risk } 0.86(95 \% \\
\text { Cl } 0.53,1.40)\end{array}$ & Low \\
\hline
\end{tabular}

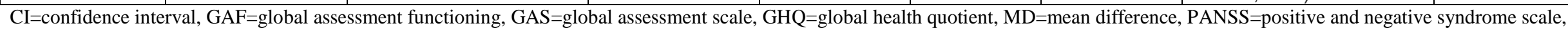
RCT=randomized controlled trial, RR=relative risk, RSES=Rosenberg Self-Esteem Scale, SR=systematic review 
Appendix Table H-13. Strength of evidence: early interventions for patients with first-episode psychosis

\begin{tabular}{|c|c|c|c|c|c|c|c|}
\hline Outcome & $\begin{array}{l}\text { Number of Studies } \\
\text { Number of Subjects }\end{array}$ & \begin{tabular}{|l} 
Strength of \\
Evidence \\
Domain: Study \\
Limitations \\
\end{tabular} & $\begin{array}{l}\text { Strength of } \\
\text { Evidence } \\
\text { Domain: } \\
\text { Consistency }\end{array}$ & \begin{tabular}{|l|} 
Strength of \\
Evidence \\
Domain: \\
Directness \\
\end{tabular} & $\begin{array}{l}\text { Strength of } \\
\text { Evidence } \\
\text { Domain: } \\
\text { Precision } \\
\end{array}$ & $\begin{array}{l}\text { Magnitude of Effect: Summary } \\
\text { Effect Size } \\
(95 \% \mathrm{Cl})\end{array}$ & $\begin{array}{l}\text { Strength of } \\
\text { Evidence } \\
\text { (High, Moderate, } \\
\text { Low, Insufficient) }\end{array}$ \\
\hline $\begin{array}{l}\text { Functional: } \\
\text { Global (GAS, } \\
\text { GAF) }\end{array}$ & $\begin{array}{l}1 \mathrm{SR}^{77}, 1 \mathrm{RCT}^{78} \mathrm{~N}=369 \\
\text { (two-year data only) } \\
2 \mathrm{RCTs} \\
\mathrm{N}=744^{79} \\
\mathrm{~N}=98^{80}\end{array}$ & Moderate & Consistent & Direct & Precise & $\begin{array}{l}\text { GAS and GAF results only } \\
\text { Team-based CSC result in higher } \\
\text { functioning scores. } \\
\text { Pooled WMD: } 3.88(95 \% \mathrm{Cl}=0.91 \\
\text { to } 6.85) \mathrm{I}^{2}=64 \%\end{array}$ & Moderate \\
\hline $\begin{array}{l}\text { Functional: } \\
\text { Working or } \\
\text { School }\end{array}$ & $\begin{array}{l}1 \mathrm{SR}^{77}, 1 \mathrm{RCT}^{78} \text { (OPUS- } \\
\text { Scandinavia) } \mathrm{N}=547 \text { ) } \\
2 \mathrm{RCTs} \\
\mathrm{N}=125^{80} \\
\mathrm{~N}=744^{79}\end{array}$ & Moderate & Consistent & Direct & Precise & $\begin{array}{l}\text { Significantly more people }(22 \%) \text { are } \\
\text { working or in school with team- } \\
\text { based CSC } \\
\text { Pooled RR } 1.22(95 \% \mathrm{Cl}=1.01 \text { to } \\
1.47)\end{array}$ & Moderate \\
\hline $\begin{array}{l}\text { Functional: } \\
\text { Housing Status }\end{array}$ & \begin{tabular}{|l|}
$1 \mathrm{SR}^{77}$ \\
$1 \mathrm{RCT}$ \\
$\left.\mathrm{N}=547^{78}\right)$ \\
\\
$1 \mathrm{RCT}$ \\
$\mathrm{N}=128^{80}$ \\
\end{tabular} & Moderate & Consistent & Direct & Imprecise & $\begin{array}{l}\text { No significant difference between } \\
\text { groups } \\
\text { Pooled RR } 1.06(95 \% \mathrm{Cl}=0.86 \text { to } \\
1.30)\end{array}$ & Low \\
\hline $\begin{array}{l}\text { Health-Related } \\
\text { Quality of Life }\end{array}$ & $\begin{array}{l}2 \text { RCTs } \\
\mathrm{N}=92^{80} \\
\mathrm{~N}=403^{81}\end{array}$ & Moderate & Consistent & Direct & Precise & $\begin{array}{l}\text { Team-based CSC results in greater } \\
\text { quality of life ratings as endpoint. } \\
\text { Pooled effect size } 0.84(95 \% \mathrm{Cl}= \\
0.14 \text { to } 1.55) ; \mathrm{P}=0.02 \text {. } \\
\text { Cochrane } \mathrm{Q} \text { for heterogeneity }= \\
7.43, \mathrm{p}=0.0064 \text { (significant } \\
\text { heterogeneity) }\end{array}$ & Moderate \\
\hline
\end{tabular}




\begin{tabular}{|c|c|c|c|c|c|c|c|}
\hline Outcome & $\begin{array}{l}\text { Number of Studies } \\
\text { Number of Subjects }\end{array}$ & $\begin{array}{l}\text { Strength of } \\
\text { Evidence } \\
\text { Domain: Study } \\
\text { Limitations }\end{array}$ & $\begin{array}{l}\text { Strength of } \\
\text { Evidence } \\
\text { Domain: } \\
\text { Consistency }\end{array}$ & $\begin{array}{l}\text { Strength of } \\
\text { Evidence } \\
\text { Domain: } \\
\text { Directness }\end{array}$ & $\begin{array}{l}\text { Strength of } \\
\text { Evidence } \\
\text { Domain: } \\
\text { Precision }\end{array}$ & $\begin{array}{l}\text { Magnitude of Effect: Summary } \\
\text { Effect Size } \\
(95 \% \mathrm{Cl})\end{array}$ & $\begin{array}{l}\text { Strength of } \\
\text { Evidence } \\
\text { (High, Moderate, } \\
\text { Low, Insufficient) }\end{array}$ \\
\hline $\begin{array}{l}\text { Core Illness } \\
\text { Symptoms } \\
\text { (PANSS) }\end{array}$ & $\begin{array}{l}3 \text { RCTs } \\
N=99^{80} \\
N=403^{81} \\
N=1,184^{79}\end{array}$ & Moderate & Inconsistent & Direct & Precise & $\begin{array}{l}\text { No clinically important difference } \\
\text { between groups in endpoint scores. } \\
\text { Pooled WMD of all } 3 \mathrm{RCTs}=-2.53 \\
\left.\text { ( } 95 \% \mathrm{Cl}-5.45 \text { to } 0.39 ; \mathrm{I}^{2}=55 \%\right) \text {. } \\
\text { Sensitivity analysis removing a study } \\
\text { with a } 5.9 \text {-point difference at } \\
\text { baseline results in a very small but } \\
\text { statistically significant difference and } \\
\text { no heterogeneity. Pooled WMD of } 2 \\
\text { RCTs = }-1.40(95 \% \mathrm{Cl}-2.25 \text { to }-0.55 \text {; } \\
\text { Cochran Q for heterogeneity = } \\
0.0014(\mathrm{df}=1) \mathrm{P}=0.97)\end{array}$ & Low \\
\hline $\begin{array}{l}\text { Core IIIness } \\
\text { Symptoms } \\
\text { (Calgary } \\
\text { Depression } \\
\text { Scale) }\end{array}$ & $\begin{array}{l}2 \text { RCTs } \\
\mathrm{N}=99^{80} \\
\mathrm{~N}=205^{82}\end{array}$ & Moderate & Consistent & Direct & Precise & $\begin{array}{l}\text { No significant difference between } \\
\text { groups in endpoint scores. Pooled } \\
\text { WMD -0.44 ( } 95 \% \mathrm{Cl}-1.08 \text { to } 0.20) \text {. } \\
\text { Heterogeneity: Cochran } \mathrm{Q}= \\
0.528157(\mathrm{df}=1) \mathrm{P}=0.4674\end{array}$ & Moderate \\
\hline $\begin{array}{l}\text { Discontinuation } \\
\text { of Treatment }\end{array}$ & $\begin{array}{l}2 \text { RCTs } \\
\mathrm{N}=1239^{79} \\
\mathrm{~N}=136^{83}\end{array}$ & Moderate & Consistent & Direct & Precise & $\begin{array}{l}\text { Team-based CSC had a significantly } \\
\text { greater rate of treatment retention } \\
\text { compared to standard care Pooled } \\
\text { relative risk }=1.27(95 \% \mathrm{Cl} 1.16 \text { to } \\
1.38) \text {; Cochran } \mathrm{Q}=0.03(\mathrm{df}=1) \mathrm{P}= \\
0.86\end{array}$ & High \\
\hline $\begin{array}{l}\text { Rates of } \\
\text { Relapse }\end{array}$ & $\begin{array}{l}2 \text { RCTs } \\
N=1239^{79} \\
N=122^{83}\end{array}$ & Moderate & Consistent & Direct & Imprecise & $\begin{array}{l}\text { Participants in team-based CSC } \\
\text { were significantly less likely to } \\
\text { relapse than those in standard care. } \\
\text { Pooled relative risk }=0.64(95 \% \mathrm{Cl} \\
0.52 \text { to } 0.79 \text { ) Cochran } \mathrm{Q}=0.024 \text { (df } \\
=1 \text { ) } \mathrm{P}=0.88\end{array}$ & Moderate \\
\hline
\end{tabular}

$\mathrm{CI}=$ confidence interval, FEP=first episode psychosis, GAF=global assessment functioning, GAS=global assessment scale, GAF=Global Assessment of Functioning, GAS=Global Assessment Scale, PANSS=Positive and Negative Syndrome Scale, RCT=randomized controlled trial, RR=relative risk, SR=systematic review, WMD=weighted mean difference 
Appendix Table H-14. Strength of evidence: co-occurring substance use and schizophrenia

\begin{tabular}{|c|c|c|c|c|c|c|c|}
\hline Outcome & $\begin{array}{l}\text { Number of Studies } \\
\text { Number of Subjects }\end{array}$ & $\begin{array}{l}\text { Strength of } \\
\text { Evidence } \\
\text { Domain: Study } \\
\text { Limitations }\end{array}$ & $\begin{array}{l}\text { Strength of } \\
\text { Evidence } \\
\text { Domain: } \\
\text { Consistency }\end{array}$ & $\begin{array}{l}\text { Strength of } \\
\text { Evidence } \\
\text { Domain: } \\
\text { Directness }\end{array}$ & $\begin{array}{l}\text { Strength of } \\
\text { Evidence } \\
\text { Domain: } \\
\text { Precision }\end{array}$ & $\begin{array}{l}\text { Magnitude of Effect: } \\
\text { Summary Effect Size } \\
(95 \% \mathrm{Cl})\end{array}$ & $\begin{array}{l}\text { Strength of } \\
\text { Evidence } \\
\text { (High, Moderate, } \\
\text { Low, Insufficient) }\end{array}$ \\
\hline $\begin{array}{l}\text { Function: } \\
\text { Global Function } \\
\text { (Integrated: } \\
\text { GAF; } 6 \text { months) }\end{array}$ & $1 \mathrm{SR}^{84}(1 \mathrm{RCT} ; \mathrm{N}=162)$ & Moderate & Unknown & Direct & Imprecise & $\begin{array}{l}\text { Inconclusive: } \\
\text { MD } 1.10 \text { (-1.58 to } 3.78)\end{array}$ & Low \\
\hline $\begin{array}{l}\text { Function: } \\
\text { Global Function } \\
\text { (Integrated: } \\
\text { GAF; } 18 \\
\text { months) }\end{array}$ & $1 \mathrm{SR}^{84}(1 \mathrm{RCT} ; \mathrm{N}=176)$ & Moderate & Unknown & Direct & Imprecise & $\begin{array}{l}\text { Inconclusive: } \\
\text { MD } 1.00 \text { (-1.58 to } 3.58)\end{array}$ & Low \\
\hline $\begin{array}{l}\text { Function: } \\
\text { Global Function } \\
\text { (Integrated: } \\
\text { GAF; } 24 \\
\text { months) }\end{array}$ & $1 \mathrm{SR}^{84}(1 \mathrm{RCT} ; \mathrm{N}=166)$ & Moderate & Unknown & Direct & Imprecise & $\begin{array}{l}\text { Inconclusive: } \\
\text { MD } 1.70 \text { (-1.18 to } 4.58)\end{array}$ & Low \\
\hline $\begin{array}{l}\text { Function: } \\
\text { Global Function } \\
\text { (Integrated: } \\
\text { GAF: } \mathbf{3 0} \\
\text { months) } \\
\end{array}$ & $1 \mathrm{SR}^{84}(1 \mathrm{RCT} ; \mathrm{N}=164)$ & Moderate & Unknown & Direct & Imprecise & $\begin{array}{l}\text { Inconclusive: } \\
\text { MD -0.60 (-3.56 to } 2.36)\end{array}$ & Low \\
\hline $\begin{array}{l}\text { Function: } \\
\text { Global Function } \\
\text { (Integrated: } \\
\text { GAF: } 36 \\
\text { months) } \\
\end{array}$ & $1 \mathrm{SR}^{84}(1 \mathrm{RCT} ; \mathrm{N}=170)$ & Moderate & Unknown & Direct & Imprecise & $\begin{array}{l}\text { Inconclusive: } \\
\text { MD } 0.40 \text { (-2.47 to } 3.27)\end{array}$ & Low \\
\hline $\begin{array}{l}\text { Function: } \\
\text { Global Function } \\
\text { (Non-Integrated: } \\
\text { mean RFS } \\
\text { score; } 6 \\
\text { months) }\end{array}$ & $1 \mathrm{SR}^{84}(1 \mathrm{RCT} ; \mathrm{N}=50)$ & Moderate & Unknown & Direct & Imprecise & $\begin{array}{l}\text { Inconclusive: } \\
\text { MD }-0.78(-2.91 \text { to } 1.35)\end{array}$ & Insufficient \\
\hline $\begin{array}{l}\text { Function: } \\
\text { Global Function } \\
\text { (Non-Integrated: } \\
\text { mean RFS } \\
\text { score; } 6 \\
\text { months) }\end{array}$ & $1 \mathrm{SR}^{84}$ (1 RCT; N=29) & Moderate & Unknown & Direct & Imprecise & MD $-2.67(-5.28$ to -0.06$)$ & Insufficient \\
\hline $\begin{array}{l}\text { Ability to } \\
\text { maintain } \\
\text { treatment (6 } \\
\text { months) }\end{array}$ & $1 \mathrm{SR}^{84}$ (3 RCTs; N=134) & Moderate & Consistent & Direct & Imprecise & $\begin{array}{l}\text { Inconclusive: } \\
\text { RR } 1.23 \text { (0.73 to } 2.06)\end{array}$ & Insufficient \\
\hline
\end{tabular}




\begin{tabular}{|c|c|c|c|c|c|c|c|}
\hline Outcome & $\begin{array}{l}\text { Number of Studies } \\
\text { Number of Subjects }\end{array}$ & $\begin{array}{l}\text { Strength of } \\
\text { Evidence } \\
\text { Domain: Study } \\
\text { Limitations } \\
\end{array}$ & $\begin{array}{l}\text { Strength of } \\
\text { Evidence } \\
\text { Domain: } \\
\text { Consistency }\end{array}$ & $\begin{array}{l}\text { Strength of } \\
\text { Evidence } \\
\text { Domain: } \\
\text { Directness }\end{array}$ & $\begin{array}{l}\text { Strength of } \\
\text { Evidence } \\
\text { Domain: } \\
\text { Precision }\end{array}$ & $\begin{array}{l}\text { Magnitude of Effect: } \\
\text { Summary Effect Size } \\
(95 \% \mathrm{Cl})\end{array}$ & \begin{tabular}{|l} 
Strength of \\
Evidence \\
(High, Moderate, \\
Low, Insufficient)
\end{tabular} \\
\hline $\begin{array}{l}\text { Ability to } \\
\text { maintain } \\
\text { treatment (18 } \\
\text { months) }\end{array}$ & $1 \mathrm{SR}^{84}$ (3 RCTs; N=134) & Moderate & Consistent & Direct & Imprecise & $\begin{array}{l}\text { Inconclusive: } \\
\text { RR } 1.35 \text { (0.83 to } 2.19)\end{array}$ & Insufficient \\
\hline
\end{tabular}

GAF=global assessment functioning, MD=mean difference, RCT=randomized controlled trial, RFS=role functioning score, SR=systematic review 


\section{Appendix H References}

1. McDonagh M, Peterson K, Fu R, et al. Second Generation Antipsychotic Drugs. Final Update 4 Report prepared by the Pacific Northwest Evidence-based Practice Center for the Drug Effectiveness Review Project. Oregon Health \& Science University, Portland, Oregon, November 2013. 2013. Available with membership in the Drug Effectiveness Review Project. http://www.ohsu.edu/xd/research/centersinstitutes/evidence-based-practicecenter/drug-effectiveness-reviewproject/upload/SGA final-update-4report_Nov-2013_version-2.pdf. Accessed May 23, 2017.

2. Meltzer HY, Bobo WV, Nuamah IF, et al. Efficacy and tolerability of oral paliperidone extended-release tablets in the treatment of acute schizophrenia: pooled data from three 6-week, placebo-controlled studies. J Clin Psychiatry. 2008 May;69(5):817-29. PMID: 18466043.

3. Rouillon F, Eriksson L, Burba B, et al. Functional recovery results from the risperidone long-acting injectable versus quetiapine relapse prevention trial (ConstaTRE). Acta Neuropsychiatr. 2013;25(5):297-306. doi: 10.1017/neu.2013.7 PMID: 25287730.

4. Naber D, Peuskens J, Schwarzmann N, et al. Subjective well-being in schizophrenia: a randomised controlled open-label 12-month non-inferiority study comparing quetiapine XR with risperidone (RECOVER). Eur Neuropsychopharmacol. 2013 Oct;23(10):1257-69. doi: 10.1016/j.euroneuro.2013.07.006. PMID: 23953270 .

5. Ishigooka J, Nakamura J, Fujii Y, et al. Efficacy and safety of aripiprazole oncemonthly in Asian patients with schizophrenia: a multicenter, randomized, double-blind, non-inferiority study versus oral aripiprazole. Schizophr Res. 2015 Feb;161(2-3):421-8. doi: 10.1016/j.schres.2014.12.013. PMID: 25556976.
6. Naber D, Hansen K, Forray C, et al. Qualify: a randomized head-to-head study of aripiprazole once-monthly and paliperidone palmitate in the treatment of schizophrenia. Schizophr Res. 2015;168(1-2):498-504. PMID: 26232241

7. Schoemaker J, Naber D, Vrijland P, et al. Long-term assessment of asenapine vs. olanzapine in patients with schizophrenia or schizoaffective disorder.

Pharmacopsychiatry. 2010 Jun;43(4):13846. doi: 10.1055/s-0030-1248313. PMID: 20205074.

8. Buchanan RW, Panagides J, Zhao J, et al. Asenapine versus olanzapine in people with persistent negative symptoms of schizophrenia. J Clin Psychopharmacol. 2012 Feb;32(1):36-45. doi: 10.1097/JCP.0b013e31823f880a. PMID: 22198451.

9. Fleischhacker W, Gopal S, Lane R, et al. A randomized trial of paliperidone palmitate and risperidone long-acting injectable in schizophrenia. Int $\mathrm{J}$ Neuropsychopharmacology. 2012 Feb;15(1):107-18. doi: 10.1017/S1461145711001076. PMID: 21777507.

10. Li H, Rui Q, Ning X, et al. A comparative study of paliperidone palmitate and risperidone long-acting injectable therapy in schizophrenia. Prog Neuropsychopharmacol Biol Psychiatry. 2011 Jun 1;35(4):1002-8. doi: 10.1016/j.pnpbp.2011.02.001. PMID: 21315787.

11. AstraZeneca. A multicenter, open-label, flexible-dose, parallel-group evaluation of the cataractogenic potential of quetiapine fumarate (Seroquel) and risperidone (Risperdal) in the Long-term treatment of patients with schizophrenia or schizoaffective disorder [CLEARS]. Clinical Study Report Synopsis. 2010. 
12. Mullen J, Jibson MD, Sweitzer D. A comparison of the relative safety, efficacy, and tolerability of quetiapine and risperidone in outpatients with schizophrenia and other psychotic disorders: the quetiapine experience with safety and tolerability (QUEST) study. Clin Ther. 2001;23(11):1839-54. PMID: 11768836.

13. Leucht S, Cipriani A, Spineli L, et al. Comparative efficacy and tolerability of 15 antipsychotic drugs in schizophrenia: a multiple-treatments meta-analysis. Lancet. 2013 Sep 14;382(9896):951-62. doi: 10.1016/S0140-6736(13)60733-3. PMID: 23810019.

14. Samara MT, Dold M, Gianatsi M, et al. Efficacy, acceptability, and tolerability of antipsychotics in treatment-resistant schizophrenia: a network meta-analysis. JAMA Psychiatry. 2016 Mar;73(3):199-210. doi: 10.1001/jamapsychiatry.2015.2955. PMID: 26842482.

15. Citrome L, Ota A, Nagamizu K, et al. The effect of brexpiprazole (OPC-34712) and aripiprazole in adult patients with acute schizophrenia: results from a randomized, exploratory study. Int Clin Psychopharmacol. 2016 Jul;31(4):192-201. doi: 10.1097/yic.0000000000000123. PMID: 26963842.

16. Schering Plough. (Study 041022) A multicenter, randomized, double-blind, flexible-dose, 6-week trial of the efficacy and safety of asenapine compared with placebo using olanzapine positive control in subjects with an acute exacerbation of schizophrenia. 2006.

17. Schoemaker J, Stet L, Vrijland P, et al. Long-term efficacy and safety of asenapine or olanzapine in patients with schizophrenia or schizoaffective disorder: an extension study. Pharmacopsychiatry. 2012 Jul;45(5):196-203. doi: 10.1055/s-00311301310. PMID: 22454251.

18. Potkin SG, Gharabawi GM, Greenspan AJ, et al. A double-blind comparison of risperidone, quetiapine and placebo in patients with schizophrenia experiencing an acute exacerbation requiring hospitalization. Schizophr Res. 2006 Jul;85(1-3):254-65. PMID: 16797162.
19. Zhong KX, Sweitzer DE, Hamer RM, et al. Comparison of quetiapine and risperidone in the treatment of schizophrenia: a randomized, double-blind, flexible-dose, 8week study. J Clin Psychiatry. 2006 Jul;67(7):1093-103. PMID: 16889453.

20. McEvoy JP, Lieberman JA, Perkins DO, et al. Efficacy and tolerability of olanzapine, quetiapine, and risperidone in the treatment of early psychosis: a randomized, doubleblind 52-week comparison. Am J Psychiatry. 2007 Jul;164(7):1050-60. PMID: 17606657.

21. Newcomer JW, Ratner RE, Eriksson JW, et al. A 24-week, multicenter, open-label, randomized study to compare changes in glucose metabolism in patients with schizophrenia receiving treatment with olanzapine, quetiapine, or risperidone. J Clin Psychiatry. 2009 Apr;70(4):487-99. PMID: 19358783.

22. Stroup TS, Lieberman JA, McEvoy JP, et al. Effectiveness of olanzapine, quetiapine, and risperidone in patients with chronic schizophrenia after discontinuing perphenazine: a CATIE study. Am J Psychiatry. 2007 Mar;164(3):415-27. PMID: 17329466.

23. Jeste DV, Barak Y, Madhusoodanan S, et al. International multisite double-blind trial of the atypical antipsychotics risperidone and olanzapine in 175 elderly patients with chronic schizophrenia. Am J Geriatr Psychiatry. 2003;11(6):638-47. PMID: 14609804.

24. Alvarez E, Ciudad A, Olivares JM, et al. A randomized, 1-year follow-up study of olanzapine and risperidone in the treatment of negative symptoms in outpatients with schizophrenia. J Clin Psychopharmacol. 2006 Jun;26(3):238-49. PMID: 16702888.

25. Li YM, Zhao JP, Ou JJ, et al. Efficacy and tolerability of ziprasidone vs. olanzapine in naive first-episode schizophrenia: a 6-week, randomized, open-label, flexible-dose study. Pharmacopsychiatry. 2012 Jul;45(5):177-81. doi: 10.1055/s-0031-1299769. PMID: 22290206. 
26. Simpson GM, Glick ID, Weiden PJ, et al. Randomized, controlled, double-blind multicenter comparison of the efficacy and tolerability of ziprasidone and olanzapine in acutely ill inpatients with schizophrenia or schizoaffective disorder. Am J Psychiatry. 2004 Oct;161(10):1837-47. doi: 10.1176/ajp.161.10.1837 PMID: 15465981.

27. Grootens KP, van Veelen NMJ, Peuskens J, et al. Ziprasidone vs olanzapine in recentonset schizophrenia and schizoaffective disorder: results of an 8-week double-blind randomized controlled trial. Schizophr Res. 2011 Mar;37(2):352-61. doi: 10.1093/schbul/sbp037. PMID: 19542525.

28. Breier A, al e. Olanzapine versus ziprasidone: results of a 28-week doubleblind study in patients with schizophrenia. Am J Psychiatry. 2005 Oct;162:1879-87. doi: 10.1176/appi.ajp.162.10.1879 PMID: 16199834.

29. Simpson G, al. E. Six-month, blinded, multicenter continuation study of ziprasidone versus olanzapine in schizophrenia. Am J Psychiatry 2005 Aug;162:1535-8. doi: 10.1176/appi.ajp.162.8.1535 PMID: 16055779.

30. Abou-Setta AM, Mousavi SS, Spooner C, et al. First-generation versus second-generation antipsychotics in adults: comparative effectiveness. Comparative effectiveness review No. 63. (Prepared by the University of Alberta Evidence-based Practice Center under Contract No. 290-200-10021.) AHRQ Publication No. 12-EHC054-EF. Rockville, MD: Agency for Healthcare Research and Quality: 2012. www.effectivehealthcare.ahrq.gov/reports/fi nal.cfm Accessed April 22, 2017. PMID: 23035275.

31. Marshall M, Lockwood A. Assertive community treatment for people with severe mental disorders. Cochrane Database Syst Rev. 2000a(2):Cd001089. doi: 10.1002/14651858.cd001089. PMID: 10796415.

32. Sytema S, Wunderink L, Bloemers W, et al. Assertive community treatment in the Netherlands: a randomized controlled trial. Acta Psychiatr Scand. 2007 Aug;116(2):105-12. PMID: 17650271.
33. Jones C, Hacker D, Cormac I, et al. Cognitive behavioural therapy versus other psychosocial treatments for schizophrenia. Cochrane Database Syst Rev. 2012 Apr 18(4):Cd008712. doi: 10.1002/14651858.CD008712.pub2. PMID: 22513966.

34. Barrowclough C, Haddock G, Lobban F, et al. Group cognitive-behavioural therapy for schizophrenia. Randomised controlled trial. Br J Psychiatry. 2006 Dec;189:527-32. PMID: 17139037.

35. Lincoln TM, Ziegler M, Mehl S, et al. Moving from efficacy to effectiveness in cognitive behavioral therapy for psychosis: a randomized clinical practice trial. J Consult Clin Psychol. 2012 Aug;80(4):674-86. doi: 10.1037/a0028665. PMID: 22663901.

36. Sellwood W, Barrowclough C, Tarrier N, et al. Needs-based cognitive-behavioural family intervention for carers of patients suffering from schizophrenia: 12-month follow-up. Acta Psychiatr Scand. 2001 Nov;104(5):346-55. PMID: 11722315.

37. van der Gaag M, Stant AD, Wolters KJ, et al. Cognitive-behavioural therapy for persistent and recurrent psychosis in people with schizophrenia-spectrum disorder: costeffectiveness analysis. Br J Psychiatry. 2011 Jan;198(1):59-65, sup 1. doi: 10.1192/bjp.bp.109.071522. PMID: 21200078.

38. Zimmer M, Duncan AV, Laitano D, et al. A twelve-week randomized controlled study of the cognitive-behavioral integrated psychological therapy program: positive effect on the social functioning of schizophrenic patients. Rev Bras Psiquiatr. 2007 Jun;29(2):140-7. PMID: 17650536.

39. Garety PA, Fowler DG, Freeman D, et al. Cognitive--behavioural therapy and family intervention for relapse prevention and symptom reduction in psychosis: randomised controlled trial. Br J Psychiatry. 2008 Jun;192(6):412-23. doi: 10.1192/bjp.bp.107.043570. PMID: 18515890. 
40. Grant PM, Huh GA, Perivoliotis D, et al. Randomized trial to evaluate the efficacy of cognitive therapy for low-functioning patients with schizophrenia. Arch Gen Psychiatry. 2012 Feb;69(2):121-7. doi: 10.1001/archgenpsychiatry.2011.129. PMID: 21969420.

41. Velligan DI, Tai S, Roberts DL, et al. A randomized controlled trial comparing cognitive behavior therapy, cognitive adaptation training, their combination and treatment as usual in chronic schizophrenia. Schizophr Bull. 2015 May;41(3):597-603. doi: 10.1093/schbul/sbu127. PMID: 25193976.

42. Granholm E, McQuaid JR, McClure FS, et al. A randomized, controlled trial of cognitive behavioral social skills training for middle-aged and older outpatients with chronic schizophrenia. Am J Psychiatry. 2005 Mar;162(3):520-9. PMID: 15741469.

43. Lysaker PH, Davis LW, Bryson GJ, et al. Effects of cognitive behavioral therapy on work outcomes in vocational rehabilitation for participants with schizophrenia spectrum disorders. Schizophr Res. 2009 Feb;107(23):186-91. doi: 10.1016/j.schres.2008.10.018. PMID: 19046856.

44. Malik N, Kingdon D, Pelton J, et al. Effectiveness of brief cognitive-behavioral therapy for schizophrenia delivered by mental health nurses: relapse and recovery at 24 months. J Clin Psychiatry. 2009 Feb;70(2):201-7. PMID: 19210949.

45. Freeman D, Dunn G, Startup H, et al. Effects of cognitive behaviour therapy for worry on persecutory delusions in patients with psychosis (WIT): a parallel, singleblind, randomised controlled trial with a mediation analysis. Lancet Psychiatry. 2015;2(4):305-13. PMID: 26360083.

46. Lewis S, Tarrier N, Haddock G, et al. Randomised controlled trial of cognitivebehavioural therapy in early schizophrenia: acute-phase outcomes. Br J Psychiatry Suppl. 2002 Sep;43:s91-7. PMID: 12271807.
47. Jauhar S, McKenna PJ, Radua J, et al. Cognitive-behavioural therapy for the symptoms of schizophrenia: systematic review and meta-analysis with examination of potential bias. Br J Psychiatry. 2014 Jan;204(1):20-9. doi: 10.1192/bjp.bp.112.116285. PMID: 24385461.

48. Velthorst E, Koeter M, van der Gaag M, et al. Adapted cognitive-behavioural therapy required for targeting negative symptoms in schizophrenia: meta-analysis and metaregression. Psychol Med. 2015 Feb;45(3):453-65. doi: 10.1017/S0033291714001147. PMID: 24993642.

49. Gumley A, O'Grady M, McNay L, et al. Early intervention for relapse in schizophrenia: results of a 12-month randomized controlled trial of cognitive behavioural therapy. Psychol Med. 2003 Apr;33(3):419-31. PMID: 12701663.

50. Sensky T, Turkington D, Kingdon D, et al. A randomized controlled trial of cognitivebehavioral therapy for persistent symptoms in schizophrenia resistant to medication. Arch Gen Psychiatry. 2000 Feb;57(2):16572. PMID: 10665619.

51. Tarrier N, Wittkowski A, Kinney C, et al. Durability of the effects of cognitivebehavioural therapy in the treatment of chronic schizophrenia: 12-month follow-up. Br J Psychiatry. 1999 Jun;174:500-4. doi: 10.1192/bjp.174.6.500. PMID: 10616627.

52. Wykes T, Huddy V, Cellard C, et al. A meta-analysis of cognitive remediation for schizophrenia: methodology and effect sizes. Am J Psychiatry. 2011 May;168(5):472-85. doi: 10.1176/appi.ajp.2010.10060855. PMID: 21406461.

53. Farreny A, Aguado J, Ochoa S, et al. REPYFLEC cognitive remediation group training in schizophrenia: looking for an integrative approach. Schizophr Res. 2012 Dec;142(1-3):137-44. doi: 10.1016/j.schres.2012.08.035. PMID: 23017827. 
54. Mueller DR, Schmidt SJ, Roder V. One-year randomized controlled trial and follow-up of integrated neurocognitive therapy for schizophrenia outpatients. Schizophr Bull. 2015 May;41(3):604-16. doi: 10.1093/schbul/sbu223. PMID: 25713462.

55. Twamley EW, Vella L, Burton CZ, et al. Compensatory cognitive training for psychosis: effects in a randomized controlled trial. J Clin Psychiatry. 2012 Sep;73(9):1212-9. doi: 10.4088/JCP.12m07686. PMID: 22939029.

56. Deste G, Barlati S, Cacciani P, et al. Persistence of effectiveness of cognitive remediation interventions in schizophrenia: a 1-year follow-up study. Schizophr Res. 2015 Feb;161(2-3):403-6. doi: 10.1016/j.schres.2014.12.004. PMID: 25533593.

57. Cella M, Preti A, Edwards C, et al. Cognitive remediation for negative symptoms of schizophrenia: A network meta-analysis. Clin Psychol Rev. 2017 Mar;52:43-51. doi: 10.1016/j.cpr.2016.11.009. PMID: 27930934.

58. Vita A, De Peri L, Barlati S, et al. Effectiveness of different modalities of cognitive remediation on symptomatological, neuropsychological, and functional outcome domains in schizophrenia: a prospective study in a realworld setting. Schizophr Res. 2011

Dec;133(1-3):223-31. doi: 10.1016/j.schres.2011.08.010. PMID: 21907544.

59. Pharoah F, Mari J, Rathbone J, et al. Family intervention for schizophrenia. Cochrane Database Syst Rev. 2010 Dec 08(12):CD000088. doi: 10.1002/14651858.CD000088.pub2. PMID: 21154340.

60. Lenior ME, Dingemans PM, Linszen DH, et al. Social functioning and the course of early-onset schizophrenia: five-year followup of a psychosocial intervention. Br J Psychiatry. 2001;179:53-8. PMID: 11435269.
61. Mayoral F, Berrozpe A, de la Higuera J, et al. Efficacy of a family intervention program for prevention of hospitalization in patients with schizophrenia. A naturalistic multicenter controlled and randomized study in Spain. Rev Psiquiatr Salud Ment. 2015 Apr-Jun;8(2):83-91. doi: 10.1016/j.rpsm.2013.11.001. PMID: 25017624.

62. Kopelowicz A, Zarate R, Wallace CJ, et al. The ability of multifamily groups to improve treatment adherence in Mexican Americans with schizophrenia. Arch Gen Psychiatry. 2012 Mar;69(3):265-73. doi: 10.1001/archgenpsychiatry.2011.135. PMID: 22393219.

63. Valencia M, Rascon ML, Juarez F, et al. A psychosocial skills training approach in Mexican out-patients with schizophrenia. Psychol Med. 2007 Oct;37(10):1393-402. PMID: 17472761.

64. Barrowclough C, Tarrier N, Lewis S, et al. Randomised controlled effectiveness trial of a needs-based psychosocial intervention service for carers of people with schizophrenia. Br J Psychiatry. 1999 Jun;174:505-11. PMID: 10616628.

65. Marshall M, Gray A, Lockwood A, et al. Case management for people with severe mental disorders. Cochrane Database Syst Rev. 2000b(2):Cd000050. doi: 10.1002/14651858.cd000050. PMID: 10796288.

66. Bjorkman T, Hansson L, Sandlund M. Outcome of case management based on the strengths model compared to standard care. A randomised controlled trial. Soc Psychiatry Psychiatr Epidemiol. 2002 Apr;37(4):147-52. PMID: 12027240.

67. Zou H, Li Z, Nolan MT, et al. Selfmanagement education interventions for persons with schizophrenia: a meta-analysis. Int J Ment Health Nurs. 2013 Jun;22(3):25671. doi: $10.1111 /$ j.1447-0349.2012.00863.x. PMID: 22882803.

68. Pekkala E, Merinder L. Psychoeducation for schizophrenia. Cochrane Database Syst Rev. 2002(2):Cd002831. doi: 10.1002/14651858.cd002831. PMID: 12076455. 
69. Bartels SJ, Pratt SI, Mueser KT, et al. Longterm outcomes of a randomized trial of integrated skills training and preventive healthcare for older adults with serious mental illness. Am J Geriatr Psychiatry. 2014 Nov;22(11):1251-61. doi: 10.1016/j.jagp.2013.04.013. PMID: 23954039.

70. Mueser KT, Pratt SI, Bartels SJ, et al. Randomized trial of social rehabilitation and integrated health care for older people with severe mental illness. J Consult Clin Psychol. 2010 Aug;78(4):561-73. doi: 10.1037/a0019629. PMID: 20658812.

71. Valencia M, Fresan A, Juarez F, et al. The beneficial effects of combining pharmacological and psychosocial treatment on remission and functional outcome in outpatients with schizophrenia. J Psychiatr Res. 2013 Dec;47(12):1886-92. doi: 10.1016/j.jpsychires.2013.09.006. PMID: 24112947.

72. Mueser KT, Clark RE, Haines M, et al. The Hartford study of supported employment for persons with severe mental illness. J Consult Clin Psychol. 2004 Jun;72(3):479-90. doi: 10.1037/0022-006x.72.3.479. PMID: 15279531.

73. Cook JA, Leff HS, Blyler CR, et al. Results of a multisite randomized trial of supported employment interventions for individuals with severe mental illness. Arch Gen Psychiatry. 2005 May;62(5):505-12. PMID: 15867103.

74. Cook JA, Blyler CR, Burke-Miller JK, et al. Effectiveness of supported employment for individuals with schizophrenia: results of a multi-site, randomized trial. Clin Schizophr Relat Psychoses. 2008;2(1):37-46.

75. Kinoshita Y, Furukawa TA, Kinoshita K, et al. Supported employment for adults with severe mental illness. Cochrane Database Syst Rev. 2013 Sep 13(9):Cd008297. doi: 10.1002/14651858.CD008297.pub2. PMID: 24030739.

76. Buckley LA, Maayan N, Soares-Weiser K, et al. Supportive therapy for schizophrenia. Cochrane Database Syst Rev. 2015 Apr 14(4):Cd004716. doi: 10.1002/14651858.CD004716.pub4. PMID: 25871462 .
77. Marshall M, Rathbone J. Early intervention for psychosis. Cochrane Database Syst Rev. 2011 Jun 15(6):Cd004718. doi: 10.1002/14651858.CD004718.pub3. PMID: 21678345.

78. Bertelsen M, Jeppesen P, Petersen L, et al. Course of illness in a sample of 265 patients with first-episode psychosis - five-year follow-up of the Danish OPUS trial. Schizophr Res. 2009;107(2-3):173-8. doi: 10.1016/j.schres.2008.09.018. PMID: 18945593.

79. Guo X, Zhai J, Liu Z, et al. Effect of antipsychotic medication alone vs combined with psychosocial intervention on outcomes of early-stage schizophrenia: a randomized, 1-year study. Arch Gen Psychiatry. 2010 Sep;67(9):895-904. doi: 10.1001/archgenpsychiatry.2010.105. PMID: 20819983.

80. Garety PA, Craig TK, Dunn G, et al. Specialised care for early psychosis: symptoms, social functioning and patient satisfaction: randomised controlled trial. $\mathrm{Br}$ J Psychiatry. 2006 Jan;188:37-45. doi: 10.1192/bjp.bp.104.007286. PMID: 16388068.

81. Kane JM, Schooler NR, Marcy P, et al. The RAISE early treatment program for firstepisode psychosis: background, rationale, and study design. J Clin Psychiatry. 2015 Mar;76(3):240-6. doi: 10.4088/JCP.14m09289. PMID: 25830446.

82. Kane JM, Robinson DG, Schooler NR, et al. Comprehensive versus usual community care for first-episode psychosis: 2-year outcomes from the NIMH RAISE early treatment program. Am J Psychiatry. 2016 Apr 1;173(4):362-72. doi: 10.1176/appi.ajp.2015.15050632. PMID: 26481174.

83. Craig TK, Garety P, Power P, et al. The Lambeth Early Onset (LEO) team: randomised controlled trial of the effectiveness of specialised care for early psychosis. BMJ. 2004 Nov 6;329(7474):1067. doi: 10.1136/bmj.38246.594873.7C. PMID: 15485934. 
84. Hunt GE, Siegfried N, Morley K, et al. Psychosocial interventions for people with both severe mental illness and substance misuse. Cochrane Database Syst Rev. 2013
Oct 03(10):Cd001088. doi: 10.1002/14651858.CD001088.pub3. PMID: 24092525. 


\section{Appendix I. Strength of Evidence-Drug Comparisons}

Appendix Table I-1. Summary of findings for aripiprazole LAI-1 versus other SGAs

\begin{tabular}{|c|c|c|c|c|c|c|c|c|c|}
\hline Drug & Comparator & Function & QOL & Response & Mortality & Self-harm & Symptoms & Any AE & W/D AEs \\
\hline $\begin{array}{c}\text { Aripiprazole } \\
\text { LAI-1 }\end{array}$ & Aripiprazole & - & No difference* & No difference* & - & - & - & No difference* & $\begin{array}{c}\text { No } \\
\text { difference* }\end{array}$ \\
\hline $\begin{array}{c}\text { Aripiprazole } \\
\text { LAI-1 }\end{array}$ & Aripiprazole LAI-6w & - & - & - & - & - & - & - & - \\
\hline $\begin{array}{c}\text { Aripiprazole } \\
\text { LAI-1 }\end{array}$ & Asenapine & - & - & No difference* & - & - & - & - & $\begin{array}{c}\text { No } \\
\text { difference* }\end{array}$ \\
\hline $\begin{array}{c}\text { Aripiprazole } \\
\text { LAI-1 }\end{array}$ & Brexpiprazole & - & - & No difference* & - & - & - & - & $\begin{array}{c}\text { No } \\
\text { difference* }\end{array}$ \\
\hline $\begin{array}{c}\text { Aripiprazole } \\
\text { LAI-1 }\end{array}$ & Cariprazine & - & - & No difference* & - & - & - & - & $\begin{array}{c}\text { No } \\
\text { difference* }\end{array}$ \\
\hline $\begin{array}{c}\text { Aripiprazole } \\
\text { LAI-1 }\end{array}$ & Clozapine & - & - & No difference* & - & - & - & - & $\begin{array}{c}\text { No } \\
\text { difference* }\end{array}$ \\
\hline $\begin{array}{c}\text { Aripiprazole } \\
\text { LAI-1 }\end{array}$ & lloperidone & - & - & - & - & - & - & - & Moderate $(2)^{\star}$ \\
\hline $\begin{array}{c}\text { Aripiprazole } \\
\text { LAI-1 }\end{array}$ & Lurasidone & - & - & - & - & - & - & - & $\begin{array}{c}\text { No } \\
\text { difference* }\end{array}$ \\
\hline $\begin{array}{c}\text { Aripiprazole } \\
\text { LAI-1 }\end{array}$ & Olanzapine & - & - & No difference* & - & - & - & - & $\begin{array}{c}\text { No } \\
\text { difference* }\end{array}$ \\
\hline $\begin{array}{c}\text { Aripiprazole } \\
\text { LAI-1 }\end{array}$ & Olanzapine LAI & - & - & - & - & - & - & - & $\begin{array}{c}\text { No } \\
\text { difference* }\end{array}$ \\
\hline $\begin{array}{c}\text { Aripiprazole } \\
\text { LAI-1 } \\
\end{array}$ & Paliperidone & - & - & No difference* & - & - & - & Insufficient & $\begin{array}{c}\text { No } \\
\text { difference* }\end{array}$ \\
\hline $\begin{array}{c}\text { Aripiprazole } \\
\text { LAI-1 } \\
\end{array}$ & Paliperidone LAI-1 & - & Insufficient & - & - & - & - & - & $\begin{array}{c}\text { No } \\
\text { difference* }\end{array}$ \\
\hline $\begin{array}{c}\text { Aripiprazole } \\
\text { LAl-1 }\end{array}$ & Paliperidone LAI-3 & - & - & - & - & - & - & - & $\begin{array}{c}\text { No } \\
\text { difference* }\end{array}$ \\
\hline $\begin{array}{c}\text { Aripiprazole } \\
\text { LAI-1 }\end{array}$ & Quetiapine & - & - & No difference* & - & - & - & - & $\begin{array}{c}\text { No } \\
\text { difference* }\end{array}$ \\
\hline $\begin{array}{c}\text { Aripiprazole } \\
\text { LAI-1 }\end{array}$ & Quetiapine ER & - & - & - & - & - & - & - & $\begin{array}{c}\text { No } \\
\text { difference* }\end{array}$ \\
\hline $\begin{array}{c}\text { Aripiprazole } \\
\text { LAI-1 }\end{array}$ & Risperidone & - & - & No difference* & - & - & - & - & $\begin{array}{c}\text { No } \\
\text { difference* }\end{array}$ \\
\hline $\begin{array}{c}\text { Aripiprazole } \\
\text { LAI-1 }\end{array}$ & Risperidone LAI & - & - & - & - & - & - & - & $\begin{array}{c}\text { No } \\
\text { difference* }\end{array}$ \\
\hline $\begin{array}{c}\text { Aripiprazole } \\
\text { LAl-1 }\end{array}$ & Ziprasidone & - & - & No difference* & - & - & - & - & $\begin{array}{c}\text { No } \\
\text { difference* }\end{array}$ \\
\hline
\end{tabular}

* = Low SOE, ** = Moderate SOE, *** = High SOE, - = No data, (1) = Estimate favors Drug 1, (2) = Estimate favors Drug 2 
Appendix Table I-2. Summary of findings for aripiprazole LAI 4-6w versus other SGAs

\begin{tabular}{|c|c|c|c|c|c|c|c|c|c|}
\hline Drug & Comparator & Function & QOL & Response & Mortality & Self-harm & Symptoms & Any AE & WID AES \\
\hline $\begin{array}{c}\text { Aripiprazole } \\
\text { LAl-6w }\end{array}$ & Aripiprazole & - & - & - & - & - & - & - & - \\
\hline $\begin{array}{c}\text { Aripiprazole } \\
\text { LAl-6w }\end{array}$ & Aripiprazole LAI-1 & - & - & - & - & - & - & - & - \\
\hline $\begin{array}{c}\text { Aripiprazole } \\
\text { LAl-6w }\end{array}$ & Asenapine & - & - & - & - & - & - & - & - \\
\hline $\begin{array}{c}\text { Aripiprazole } \\
\text { LAl-6w }\end{array}$ & Brexpiprazole & - & - & - & - & - & - & - & - \\
\hline $\begin{array}{c}\text { Aripiprazole } \\
\text { LAl-6w }\end{array}$ & Cariprazine & - & - & - & - & - & - & - & - \\
\hline $\begin{array}{c}\text { Aripiprazole } \\
\text { LAl-6w }\end{array}$ & Clozapine & - & - & - & - & - & - & - & - \\
\hline $\begin{array}{c}\text { Aripiprazole } \\
\text { LAl-6w }\end{array}$ & Iloperidone & - & - & - & - & - & - & - & - \\
\hline $\begin{array}{c}\text { Aripiprazole } \\
\text { LAl-6w }\end{array}$ & Lurasidone & - & - & - & - & - & - & - & - \\
\hline $\begin{array}{c}\text { Aripiprazole } \\
\text { LAl-6w }\end{array}$ & Olanzapine & - & - & - & - & - & - & - & - \\
\hline $\begin{array}{c}\text { Aripiprazole } \\
\text { LAl-6w }\end{array}$ & Olanzapine LAI & - & - & - & - & - & - & - & - \\
\hline $\begin{array}{c}\text { Aripiprazole } \\
\text { LAI-6w }\end{array}$ & Paliperidone & - & - & - & - & - & - & - & - \\
\hline $\begin{array}{c}\text { Aripiprazole } \\
\text { LAl-6w }\end{array}$ & Paliperidone LAI-1 & - & - & - & - & - & - & - & - \\
\hline $\begin{array}{c}\text { Aripiprazole } \\
\text { LAl-6w }\end{array}$ & Paliperidone LAI-3 & - & - & - & - & - & - & - & - \\
\hline $\begin{array}{c}\text { Aripiprazole } \\
\text { LAI-6w }\end{array}$ & Quetiapine & - & - & - & - & - & - & - & - \\
\hline $\begin{array}{c}\text { Aripiprazole } \\
\text { LAl-6w }\end{array}$ & Quetiapine ER & - & - & - & - & - & - & - & - \\
\hline $\begin{array}{c}\text { Aripiprazole } \\
\text { LAI-6w }\end{array}$ & Risperidone & - & - & - & - & - & - & - & - \\
\hline $\begin{array}{c}\text { Aripiprazole } \\
\text { LAl-6w }\end{array}$ & Risperidone LAI & - & - & - & - & - & - & - & - \\
\hline $\begin{array}{c}\text { Aripiprazole } \\
\text { LAl-6w }\end{array}$ & Ziprasidone & - & - & - & - & - & - & - & - \\
\hline
\end{tabular}

* = Low SOE, ** = Moderate SOE, *** = High SOE, - = No data, (1) = Estimate favors Drug 1, (2) = Estimate favors Drug 2 
Appendix Table I-3. Summary of findings for aripiprazole versus other SGAs

\begin{tabular}{|c|c|c|c|c|c|c|c|c|c|}
\hline Drug & Comparator & Function & QOL & Response & Mortality & Self-harm & Symptoms & Any AE & WID AEs \\
\hline Aripiprazole & Aripiprazole LAI-1 & - & No difference* & No difference* & - & - & - & No difference* & No difference \\
\hline Aripiprazole & Aripiprazole LAI-6w & - & - & - & - & - & - & - & - \\
\hline Aripiprazole & Asenapine & - & - & No difference* & - & - & - & - & No difference \\
\hline Aripiprazole & Brexpiprazole & - & - & No difference* & Insufficient & - & Insufficient & Insufficient & No difference ${ }^{x}$ \\
\hline Aripiprazole & Cariprazine & - & - & No difference* & - & - & - & - & No difference \\
\hline Aripiprazole & Clozapine & - & - & No difference* & - & Insufficient & - & - & No difference* \\
\hline Aripiprazole & Iloperidone & - & - & - & - & - & - & - & No difference \\
\hline Aripiprazole & Lurasidone & - & - & - & - & - & - & - & No differencex \\
\hline Aripiprazole & Olanzapine & - & - & No difference* & - & Insufficient & - & - & No difference \\
\hline Aripiprazole & Olanzapine LAI & - & - & No difference* & - & - & - & - & No differencex \\
\hline Aripiprazole & Paliperidone & - & - & No difference* & - & - & - & - & No difference ${ }^{x}$ \\
\hline Aripiprazole & Paliperidone LAI-1 & - & Insufficient & - & - & - & - & - & No difference ${ }^{x}$ \\
\hline Aripiprazole & Paliperidone LAI-3 & - & - & - & - & - & - & - & No difference \\
\hline Aripiprazole & Quetiapine & - & - & No difference* & - & Insufficient & - & - & No difference* \\
\hline Aripiprazole & Quetiapine ER & - & - & No difference* & - & - & - & - & No difference* \\
\hline Aripiprazole & Risperidone & - & - & No difference* & - & Insufficient & - & No difference* & No difference \\
\hline Aripiprazole & Risperidone LAI & - & - & - & - & Insufficient & - & - & No difference \\
\hline Aripiprazole & Ziprasidone & - & - & No difference* & - & Insufficient & - & - & Medium* (1) \\
\hline & & & & & & & $\begin{array}{l}\text { Possibly data } \\
\text { out there }\end{array}$ & & \\
\hline
\end{tabular}

* = Low SOE, ${ }^{* *}=$ Moderate SOE, ${ }^{* * *}=$ High SOE, - = No data, $(1)=$ Estimate favors Drug 1, (2) = Estimate favors Drug 2 
Appendix Table I-4. Summary of findings for asenapine versus other SGAs

\begin{tabular}{|c|c|c|c|c|c|c|c|c|c|}
\hline Drug & Comparator & Function & QOL & Response & Mortality & Self-harm & Symptoms & Any AE & W/D AEs \\
\hline Asenapine & Aripiprazole & - & - & $\begin{array}{c}\text { No } \\
\text { difference* }\end{array}$ & - & - & - & - & $\begin{array}{c}\text { No } \\
\text { difference* }\end{array}$ \\
\hline Asenapine & Aripiprazole LAI-1 & - & - & $\begin{array}{c}\text { No } \\
\text { difference* }\end{array}$ & - & - & - & - & $\begin{array}{c}\text { No } \\
\text { difference* }\end{array}$ \\
\hline Asenapine & $\begin{array}{c}\text { Aripiprazole LAI- } \\
6 \mathrm{w} \\
\end{array}$ & - & - & - & - & - & - & - & - \\
\hline Asenapine & Brexpiprazole & - & - & $\begin{array}{c}\text { No } \\
\text { difference* }\end{array}$ & - & - & - & - & $\begin{array}{c}\text { No } \\
\text { difference* }\end{array}$ \\
\hline Asenapine & Cariprazine & - & - & $\begin{array}{c}\text { No } \\
\text { difference* }\end{array}$ & - & - & - & - & $\begin{array}{c}\text { No } \\
\text { difference* }\end{array}$ \\
\hline Asenapine & Clozapine & - & - & $\begin{array}{c}\text { No } \\
\text { difference* }\end{array}$ & - & - & - & - & $\begin{array}{c}\text { No } \\
\text { difference* }\end{array}$ \\
\hline Asenapine & Iloperidone & - & - & - & - & - & - & - & $\begin{array}{c}\text { No } \\
\text { difference* }\end{array}$ \\
\hline Asenapine & Lurasidone & - & - & - & - & - & - & - & $\begin{array}{c}\text { No } \\
\text { difference* }\end{array}$ \\
\hline Asenapine & Olanzapine & - & - & $\begin{array}{c}\text { No } \\
\text { difference* }\end{array}$ & $\begin{array}{c}\text { No } \\
\text { difference* }\end{array}$ & Insufficient & - & $\begin{array}{c}\text { No } \\
\text { difference }{ }^{\star *} \\
\end{array}$ & $\begin{array}{c}\text { No } \\
\text { difference* }\end{array}$ \\
\hline Asenapine & Olanzapine LAI & - & - & - & - & - & - & - & - \\
\hline Asenapine & Paliperidone & - & - & $\begin{array}{c}\text { No } \\
\text { difference* }\end{array}$ & - & - & - & - & $\begin{array}{c}\text { No } \\
\text { difference* }\end{array}$ \\
\hline Asenapine & Paliperidone LAI-1 & - & - & - & - & - & - & - & $\begin{array}{c}\text { No } \\
\text { difference* }\end{array}$ \\
\hline Asenapine & Paliperidone LAI-3 & - & - & - & - & - & - & - & $\begin{array}{c}\text { No } \\
\text { difference* }\end{array}$ \\
\hline Asenapine & Quetiapine & - & - & $\begin{array}{c}\text { No } \\
\text { difference* }\end{array}$ & - & - & - & - & $\begin{array}{c}\text { No } \\
\text { difference* }\end{array}$ \\
\hline Asenapine & Quetiapine ER & - & - & - & - & - & - & - & $\begin{array}{c}\text { No } \\
\text { difference* }\end{array}$ \\
\hline Asenapine & Risperidone & - & - & $\begin{array}{c}\text { No } \\
\text { difference* }\end{array}$ & - & - & - & Insufficient & $\begin{array}{c}\text { No } \\
\text { difference* }\end{array}$ \\
\hline Asenapine & Risperidone LAI & - & - & - & - & - & - & - & $\begin{array}{c}\text { No } \\
\text { difference* }\end{array}$ \\
\hline Asenapine & Ziprasidone & - & - & $\begin{array}{c}\text { No } \\
\text { difference* }\end{array}$ & - & - & - & - & $\begin{array}{c}\text { No } \\
\text { difference* }\end{array}$ \\
\hline
\end{tabular}

* = Low SOE, ** = Moderate SOE, *** = High SOE, - = No data, (1) = Estimate favors Drug 1, (2) = Estimate favors Drug 2 
Appendix Table I-5. Summary of findings for brexpiprazole versus other SGAs

\begin{tabular}{|c|c|c|c|c|c|c|c|c|c|}
\hline Drug & Comparator & Function & QOL & Response & Mortality & Self-harm & Symptoms & Any AE & W/D AEs \\
\hline Brexpiprazole & Aripiprazole & - & - & No difference* & Insufficient & - & Insufficient & Insufficient & $\begin{array}{c}\text { No } \\
\text { difference* }\end{array}$ \\
\hline Brexpiprazole & Aripiprazole LAI-1 & - & - & No difference* & - & - & - & - & $\begin{array}{c}\text { No } \\
\text { difference* }\end{array}$ \\
\hline Brexpiprazole & $\begin{array}{c}\text { Aripiprazole LAl- } \\
6 \mathrm{w} \\
\end{array}$ & - & - & - & - & - & - & - & - \\
\hline Brexpiprazole & Asenapine & - & - & No difference* & - & - & - & - & $\begin{array}{c}\text { No } \\
\text { difference* }\end{array}$ \\
\hline Brexpiprazole & Cariprazine & - & - & No difference* & - & - & - & - & $\begin{array}{c}\text { No } \\
\text { difference* }\end{array}$ \\
\hline Brexpiprazole & Clozapine & - & - & No difference* & - & - & - & - & $\begin{array}{c}\text { No } \\
\text { difference* }\end{array}$ \\
\hline Brexpiprazole & Iloperidone & - & - & - & - & - & - & - & $\begin{array}{c}\text { No } \\
\text { difference* }\end{array}$ \\
\hline Brexpiprazole & Lurasidone & - & - & - & - & - & - & - & $\begin{array}{c}\text { No } \\
\text { difference* }\end{array}$ \\
\hline Brexpiprazole & Olanzapine & - & - & No difference* & - & - & - & - & $\begin{array}{c}\text { No } \\
\text { difference* }\end{array}$ \\
\hline Brexpiprazole & Olanzapine LAI & - & - & - & - & - & - & - & $\begin{array}{c}\text { No } \\
\text { difference* }\end{array}$ \\
\hline Brexpiprazole & Paliperidone & - & - & No difference* & - & - & - & - & $\begin{array}{c}\text { No } \\
\text { difference* }\end{array}$ \\
\hline Brexpiprazole & Paliperidone LAI-1 & - & - & - & - & - & - & - & $\begin{array}{c}\text { No } \\
\text { difference* }\end{array}$ \\
\hline Brexpiprazole & Paliperidone LAI-3 & - & - & - & - & - & - & - & $\begin{array}{c}\text { No } \\
\text { difference* }\end{array}$ \\
\hline Brexpiprazole & Quetiapine & - & - & No difference* & - & - & - & - & $\begin{array}{c}\text { No } \\
\text { difference* }\end{array}$ \\
\hline Brexpiprazole & Quetiapine ER & - & - & - & - & - & - & - & $\begin{array}{c}\text { No } \\
\text { difference* }\end{array}$ \\
\hline Brexpiprazole & Risperidone & - & - & No difference* & - & - & - & - & $\begin{array}{c}\text { No } \\
\text { difference* }\end{array}$ \\
\hline Brexpiprazole & Risperidone LAI & - & - & - & - & - & - & - & $\begin{array}{c}\text { No } \\
\text { difference* }\end{array}$ \\
\hline Brexpiprazole & Ziprasidone & - & - & No difference* & - & - & - & - & $\begin{array}{c}\text { No } \\
\text { difference* }\end{array}$ \\
\hline
\end{tabular}

* = Low SOE, $* *=$ Moderate SOE, $* * *$ = High SOE, - = No data, (1) = Estimate favors Drug 1, (2) = Estimate favors Drug 2 
Appendix Table I-6. Summary of findings for cariprazine versus other SGAS

\begin{tabular}{|c|c|c|c|c|c|c|c|c|c|}
\hline Drug & Comparator & Function & QOL & Response & Mortality & Self-harm & Symptoms & Any AE & W/D AEs \\
\hline Cariprazine & Aripiprazole & - & - & $\begin{array}{c}\text { No } \\
\text { difference* }\end{array}$ & - & - & - & - & $\begin{array}{c}\text { No } \\
\text { difference* }\end{array}$ \\
\hline Cariprazine & Aripiprazole LAI-1 & - & - & $\begin{array}{c}\text { No } \\
\text { difference* }\end{array}$ & - & - & - & - & $\begin{array}{c}\text { No } \\
\text { difference* }\end{array}$ \\
\hline Cariprazine & $\begin{array}{c}\text { Aripiprazole LAI- } \\
6 w \\
\end{array}$ & - & - & - & - & - & - & - & - \\
\hline Cariprazine & Asenapine & - & - & $\begin{array}{c}\text { No } \\
\text { difference* }\end{array}$ & - & - & - & - & $\begin{array}{c}\text { No } \\
\text { difference* }\end{array}$ \\
\hline Cariprazine & Brexpiprazole & - & - & $\begin{array}{c}\text { No } \\
\text { difference* }\end{array}$ & - & - & - & - & $\begin{array}{c}\text { No } \\
\text { difference* }\end{array}$ \\
\hline Cariprazine & Clozapine & - & - & $\begin{array}{c}\text { No } \\
\text { difference* }\end{array}$ & - & - & - & - & $\begin{array}{c}\text { Moderate } \\
(2)^{\star}\end{array}$ \\
\hline Cariprazine & Iloperidone & - & - & - & - & - & - & - & $\begin{array}{c}\text { No } \\
\text { difference* }\end{array}$ \\
\hline Cariprazine & Lurasidone & - & - & - & - & - & - & - & $\begin{array}{c}\text { No } \\
\text { difference* }\end{array}$ \\
\hline Cariprazine & Olanzapine & - & - & $\begin{array}{c}\text { No } \\
\text { difference* }\end{array}$ & - & - & - & - & $\begin{array}{c}\text { No } \\
\text { difference* }\end{array}$ \\
\hline Cariprazine & Olanzapine LAI & - & - & - & - & - & - & - & $\begin{array}{c}\text { No } \\
\text { difference* }\end{array}$ \\
\hline Cariprazine & Paliperidone & - & - & $\begin{array}{c}\text { No } \\
\text { difference* }\end{array}$ & - & - & - & - & $\begin{array}{c}\text { No } \\
\text { difference* }\end{array}$ \\
\hline Cariprazine & Paliperidone LAI-1 & - & - & - & - & - & - & - & $\begin{array}{c}\text { No } \\
\text { difference* }\end{array}$ \\
\hline Cariprazine & Paliperidone LAI-3 & - & - & - & - & - & - & - & $\begin{array}{c}\text { No } \\
\text { difference* }\end{array}$ \\
\hline Cariprazine & Quetiapine & - & - & $\begin{array}{c}\text { No } \\
\text { difference* }\end{array}$ & - & - & - & - & $\begin{array}{c}\text { No } \\
\text { difference* }\end{array}$ \\
\hline Cariprazine & Quetiapine ER & - & - & - & - & - & - & - & $\begin{array}{c}\text { No } \\
\text { difference* }\end{array}$ \\
\hline Cariprazine & Risperidone & - & - & $\begin{array}{c}\text { No } \\
\text { difference* }\end{array}$ & - & - & - & - & $\begin{array}{c}\text { No } \\
\text { difference* }\end{array}$ \\
\hline Cariprazine & Risperidone LAI & - & - & - & - & - & - & Insufficient & $\begin{array}{c}\text { No } \\
\text { difference* }^{*}\end{array}$ \\
\hline Cariprazine & Ziprasidone & - & - & $\begin{array}{c}\text { No } \\
\text { difference* }\end{array}$ & - & - & - & - & $\begin{array}{c}\text { No } \\
\text { difference* }\end{array}$ \\
\hline
\end{tabular}

* = Low SOE, ** = Moderate SOE, *** = High SOE, - = No data, (1) = Estimate favors Drug 1, (2) = Estimate favors Drug 2 
Appendix Table 1-7. Summary of findings for clozapine versus other SGAs

\begin{tabular}{|c|c|c|c|c|c|c|c|c|c|}
\hline Drug & Comparator & Function & QOL & Response & Mortality & Self-harm & Symptoms & Any AE & W/D AEs \\
\hline Clozapine & Aripiprazole & - & - & No difference* & - & - & Small $(1)^{*}$ & $\begin{array}{c}\text { No } \\
\text { difference* }\end{array}$ & Moderate $(2)^{\star}$ \\
\hline Clozapine & Aripiprazole LAI-1 & - & - & No difference* & - & - & Small $(1)^{\star}$ & - & $\begin{array}{c}\text { No } \\
\text { difference* }\end{array}$ \\
\hline Clozapine & Aripiprazole LAI-6w & - & - & - & - & - & Small $(1)^{\star}$ & - & - \\
\hline Clozapine & Asenapine & - & - & No difference* & - & - & Small $(1)^{*}$ & - & $\begin{array}{c}\text { No } \\
\text { difference* }\end{array}$ \\
\hline Clozapine & Brexpiprazole & - & - & No difference* & - & - & Small $(1)^{\star}$ & - & $\begin{array}{c}\text { No } \\
\text { difference* }\end{array}$ \\
\hline Clozapine & Cariprazine & - & - & No difference* & - & - & Small $(1)^{*}$ & - & Moderate $(2)^{\star}$ \\
\hline Clozapine & Iloperidone & - & - & - & - & - & Small $(1)^{\star}$ & Insufficient & Large $(2)^{\star}$ \\
\hline Clozapine & Lurasidone & - & - & - & - & - & Small $(1)^{\star}$ & Insufficient & $\begin{array}{c}\text { No } \\
\text { difference* } \\
\end{array}$ \\
\hline Clozapine & Olanzapine & Insufficient & - & No difference* & Insufficient & Small $(1)^{\star}$ & No difference* & - & Moderate $(2)^{\star}$ \\
\hline Clozapine & Olanzapine LAI & - & - & - & - & - & Small $(1)^{\star}$ & - & $\begin{array}{c}\text { No } \\
\text { difference* } \\
\end{array}$ \\
\hline Clozapine & Paliperidone & - & - & No difference* & - & - & Small $(1)^{\star}$ & - & $\begin{array}{c}\text { No } \\
\text { difference* }\end{array}$ \\
\hline Clozapine & Paliperidone LAI-1 & - & - & - & - & - & Small $(1)^{\star}$ & - & $\begin{array}{c}\text { No } \\
\text { difference* }\end{array}$ \\
\hline Clozapine & Paliperidone LAI-3 & - & - & - & - & - & Small $(1)^{\star}$ & - & $\begin{array}{c}\text { No } \\
\text { difference* }\end{array}$ \\
\hline Clozapine & Quetiapine & - & - & No difference* & Insufficient & Insufficient & Small $(1)^{\star}$ & Insufficient & $\begin{array}{c}\text { No } \\
\text { difference* }\end{array}$ \\
\hline Clozapine & Quetiapine ER & - & - & - & - & - & Small $(1)^{\star}$ & - & $\begin{array}{c}\text { No } \\
\text { difference* }\end{array}$ \\
\hline Clozapine & Risperidone & No difference* & - & No difference* & Insufficient & Insufficient & Small $(1)^{\star}$ & $\begin{array}{c}\text { No } \\
\text { difference* }\end{array}$ & $\begin{array}{c}\text { No } \\
\text { difference* }\end{array}$ \\
\hline Clozapine & Risperidone LAI & - & - & - & - & - & Small $(1)^{\star}$ & - & Large $(2)^{\star}$ \\
\hline Clozapine & Ziprasidone & - & - & No difference* & - & - & Small $(1)^{\star}$ & Insufficient & $\begin{array}{c}\text { No } \\
\text { difference* }\end{array}$ \\
\hline
\end{tabular}

* = Low SOE, ${ }^{*}=$ Moderate SOE, $* * *=$ High SOE, $-=$ No data, $(1)=$ Estimate favors Drug 1, (2) = Estimate favors Drug 2 
Appendix Table I-8. Summary of findings for iloperidone versus other SGAs

\begin{tabular}{|c|c|c|c|c|c|c|c|c|c|}
\hline Drug & Comparator & Function & QOL & Response & Mortality & Self-harm & Symptoms & Any AE & W/D AEs \\
\hline Iloperidone & Aripiprazole & - & - & - & - & - & - & - & $\begin{array}{c}\text { No } \\
\text { difference* }\end{array}$ \\
\hline lloperidone & Aripiprazole LAI-1 & - & - & - & - & - & - & - & $\begin{array}{c}\text { No } \\
\text { difference* }\end{array}$ \\
\hline Iloperidone & $\begin{array}{c}\text { Aripiprazole LAI- } \\
6 w\end{array}$ & - & - & - & - & - & - & - & - \\
\hline Iloperidone & Asenapine & - & - & - & - & - & - & - & $\begin{array}{c}\text { No } \\
\text { difference* }\end{array}$ \\
\hline Iloperidone & Brexpiprazole & - & - & - & - & - & - & - & $\begin{array}{c}\text { No } \\
\text { difference* }\end{array}$ \\
\hline Iloperidone & Cariprazine & - & - & - & - & - & - & - & $\begin{array}{c}\text { No } \\
\text { difference* }\end{array}$ \\
\hline Iloperidone & Clozapine & - & - & - & - & - & - & Insufficient & $\begin{array}{c}\text { Moderate } \\
(1)^{\star}\end{array}$ \\
\hline Iloperidone & Lurasidone & - & - & - & - & - & - & Insufficient & $\begin{array}{c}\text { No } \\
\text { difference* }\end{array}$ \\
\hline Iloperidone & Olanzapine & - & - & - & - & - & - & - & $\begin{array}{c}\text { No } \\
\text { difference* }\end{array}$ \\
\hline Iloperidone & Olanzapine LAI & - & - & - & - & - & - & - & $\begin{array}{c}\text { No } \\
\text { difference* }\end{array}$ \\
\hline Iloperidone & Paliperidone & - & - & - & - & - & Small $(2)^{\star}$ & - & $\begin{array}{c}\text { No } \\
\text { difference* }\end{array}$ \\
\hline Iloperidone & Paliperidone LAI-1 & - & - & - & - & - & - & - & $\begin{array}{c}\text { No } \\
\text { difference* }\end{array}$ \\
\hline Iloperidone & Paliperidone LAI-3 & - & - & - & - & - & - & - & $\begin{array}{c}\text { No } \\
\text { difference* }\end{array}$ \\
\hline Iloperidone & Quetiapine & - & - & - & - & - & - & - & $\begin{array}{c}\text { No } \\
\text { difference* }\end{array}$ \\
\hline Iloperidone & Quetiapine ER & - & - & - & - & - & - & - & $\begin{array}{c}\text { No } \\
\text { difference* }\end{array}$ \\
\hline Iloperidone & Risperidone & - & - & - & - & - & - & Insufficient & $\begin{array}{c}\text { No } \\
\text { difference* }\end{array}$ \\
\hline Iloperidone & Risperidone LAI & - & - & - & - & - & - & - & $\begin{array}{c}\text { No } \\
\text { difference* }\end{array}$ \\
\hline Iloperidone & Ziprasidone & - & - & - & - & - & - & Insufficient & $\begin{array}{c}\text { No } \\
\text { difference* }\end{array}$ \\
\hline
\end{tabular}

* = Low SOE, $* *=$ Moderate SOE, $* * *$ = High SOE, $-=$ No data, (1) = Estimate favors Drug 1, (2) = Estimate favors Drug 2 
Appendix Table I-9. Summary of findings for lurasidone versus other SGAs

\begin{tabular}{|c|c|c|c|c|c|c|c|c|c|}
\hline Drug & Comparator & Function & QOL & Response & Mortality & Self-harm & Symptoms & Any AE & WID AEs \\
\hline Lurasidone & Aripiprazole & - & - & - & - & - & - & - & $\begin{array}{c}\text { No } \\
\text { difference* }\end{array}$ \\
\hline Lurasidone & Aripiprazole LAI-1 & - & - & - & - & - & - & - & $\begin{array}{c}\text { No } \\
\text { difference* }\end{array}$ \\
\hline Lurasidone & $\begin{array}{c}\text { Aripiprazole LAl- } \\
6 w\end{array}$ & - & - & - & - & - & - & - & - \\
\hline Lurasidone & Asenapine & - & - & - & - & - & - & - & $\begin{array}{c}\text { No } \\
\text { difference* }\end{array}$ \\
\hline Lurasidone & Brexpiprazole & - & - & - & - & - & - & - & $\begin{array}{c}\text { No } \\
\text { difference* }\end{array}$ \\
\hline Lurasidone & Cariprazine & - & - & - & - & - & - & - & $\begin{array}{c}\text { No } \\
\text { difference* }\end{array}$ \\
\hline Lurasidone & Clozapine & - & - & - & - & - & - & Insufficient & $\begin{array}{c}\text { No } \\
\text { difference* }\end{array}$ \\
\hline Lurasidone & Iloperidone & - & - & - & - & - & - & Insufficient & $\begin{array}{c}\text { No } \\
\text { difference* }\end{array}$ \\
\hline Lurasidone & Olanzapine & - & - & - & - & - & - & Insufficient & $\begin{array}{c}\text { Moderate } \\
(2)^{\star}\end{array}$ \\
\hline Lurasidone & Olanzapine LAI & - & - & - & - & - & - & Insufficient & $\begin{array}{c}\text { No } \\
\text { difference* }\end{array}$ \\
\hline Lurasidone & Paliperidone & - & - & - & - & - & - & - & Small (2)* \\
\hline Lurasidone & Paliperidone LAI-1 & - & - & - & - & - & - & - & $\begin{array}{c}\text { No } \\
\text { difference* }\end{array}$ \\
\hline Lurasidone & Paliperidone LAI-3 & - & - & - & - & - & $\begin{array}{c}\text { Moderate } \\
(2)^{\star}\end{array}$ & - & $\begin{array}{c}\text { No } \\
\text { difference* }\end{array}$ \\
\hline Lurasidone & Quetiapine & - & - & - & - & - & - & - & $\begin{array}{c}\text { No } \\
\text { difference* }\end{array}$ \\
\hline Lurasidone & Quetiapine ER & - & - & - & - & - & - & - & $\begin{array}{c}\text { No } \\
\text { difference* }\end{array}$ \\
\hline Lurasidone & Risperidone & - & - & - & - & - & - & Insufficient & $\begin{array}{c}\text { No } \\
\text { difference* }\end{array}$ \\
\hline Lurasidone & Risperidone LAI & - & - & - & - & - & - & - & $\begin{array}{c}\text { Moderate } \\
(2)^{\star}\end{array}$ \\
\hline Lurasidone & Ziprasidone & - & - & - & - & - & - & Insufficient & $\begin{array}{c}\text { No } \\
\text { difference* }\end{array}$ \\
\hline
\end{tabular}

* = Low SOE, ** = Moderate SOE, *** = High SOE, - = No data, (1) = Estimate favors Drug 1, (2) = Estimate favors Drug 2 
Appendix Table I-10. Summary of findings for olanzapine LAI versus other SGAs

\begin{tabular}{|c|c|c|c|c|c|c|c|c|c|}
\hline Drug & Comparator & Function & QOL & Response & Mortality & Self-harm & Symptoms & Any AE & W/D AEs \\
\hline $\begin{array}{c}\text { Olanzapine } \\
\text { LAI }\end{array}$ & Aripiprazole & - & - & - & - & - & - & - & $\begin{array}{c}\text { No } \\
\text { difference* }\end{array}$ \\
\hline $\begin{array}{c}\text { Olanzapine } \\
\text { LAI }\end{array}$ & Aripiprazole LAI-1 & - & - & - & - & - & - & - & $\begin{array}{c}\text { No } \\
\text { difference* }\end{array}$ \\
\hline $\begin{array}{c}\text { Olanzapine } \\
\text { LAI }\end{array}$ & $\begin{array}{c}\text { Aripiprazole LAI- } \\
6 \mathrm{w} \\
\end{array}$ & - & - & - & - & - & - & - & - \\
\hline $\begin{array}{c}\text { Olanzapine } \\
\text { LAI }\end{array}$ & Asenapine & - & - & - & - & - & - & - & $\begin{array}{c}\text { No } \\
\text { difference* }\end{array}$ \\
\hline $\begin{array}{c}\text { Olanzapine } \\
\text { LAI }\end{array}$ & Brexpiprazole & - & - & - & - & - & - & - & $\begin{array}{c}\text { No } \\
\text { difference* }\end{array}$ \\
\hline $\begin{array}{c}\text { Olanzapine } \\
\text { LAI }\end{array}$ & Cariprazine & - & - & - & - & - & - & - & $\begin{array}{c}\text { No } \\
\text { difference* }\end{array}$ \\
\hline $\begin{array}{c}\text { Olanzapine } \\
\text { LAI }\end{array}$ & Clozapine & - & - & - & - & - & - & - & $\begin{array}{c}\text { No } \\
\text { difference* }\end{array}$ \\
\hline $\begin{array}{c}\text { Olanzapine } \\
\text { LAI }\end{array}$ & lloperidone & - & - & - & - & - & - & - & $\begin{array}{c}\text { No } \\
\text { difference* }\end{array}$ \\
\hline $\begin{array}{c}\text { Olanzapine } \\
\text { LAI }\end{array}$ & Lurasidone & - & - & - & - & - & - & Insufficient & $\begin{array}{c}\text { No } \\
\text { difference* }\end{array}$ \\
\hline $\begin{array}{c}\text { Olanzapine } \\
\text { LAI }\end{array}$ & Olanzapine & - & - & - & - & - & - & Insufficient & $\begin{array}{c}\text { No } \\
\text { difference* }\end{array}$ \\
\hline $\begin{array}{c}\text { Olanzapine } \\
\text { LAI }\end{array}$ & Paliperidone & - & - & - & - & - & - & - & $\begin{array}{c}\text { No } \\
\text { difference* }\end{array}$ \\
\hline $\begin{array}{c}\text { Olanzapine } \\
\text { LAI }\end{array}$ & Paliperidone LAI-1 & - & - & - & - & - & - & - & $\begin{array}{c}\text { No } \\
\text { difference* }\end{array}$ \\
\hline $\begin{array}{c}\text { Olanzapine } \\
\text { LAI }\end{array}$ & Paliperidone LAI-3 & - & - & - & - & - & - & - & $\begin{array}{c}\text { No } \\
\text { difference* }\end{array}$ \\
\hline $\begin{array}{c}\text { Olanzapine } \\
\text { LAI }\end{array}$ & Quetiapine & - & - & - & - & - & - & - & $\begin{array}{c}\text { No } \\
\text { difference* }\end{array}$ \\
\hline $\begin{array}{c}\text { Olanzapine } \\
\text { LAI }\end{array}$ & Quetiapine ER & - & - & - & - & - & - & - & $\begin{array}{c}\text { No } \\
\text { difference* }\end{array}$ \\
\hline $\begin{array}{c}\text { Olanzapine } \\
\text { LAI }\end{array}$ & Risperidone & - & - & - & - & - & - & - & $\begin{array}{c}\text { No } \\
\text { difference* }\end{array}$ \\
\hline $\begin{array}{c}\text { Olanzapine } \\
\text { LAI }\end{array}$ & Risperidone LAI & - & - & - & - & - & - & - & $\begin{array}{c}\text { No } \\
\text { difference* }^{*}\end{array}$ \\
\hline $\begin{array}{c}\text { Olanzapine } \\
\text { LAI }\end{array}$ & Ziprasidone & - & - & - & - & - & - & - & $\begin{array}{c}\text { No } \\
\text { difference* }\end{array}$ \\
\hline
\end{tabular}

* = Low SOE, ${ }^{* *}=$ Moderate SOE, ${ }^{* * *}=$ High SOE, - = No data, (1) = Estimate favors Drug 1, (2) = Estimate favors Drug 2 
Appendix Table I-11. Summary of findings for olanzapine versus other SGAs

\begin{tabular}{|c|c|c|c|c|c|c|c|c|c|}
\hline Drug & Comparator & Function & QOL & Response & Mortality & Self-harm & Symptoms & Any AE & W/D AEs \\
\hline Olanzapine & Aripiprazole & - & - & $\begin{array}{c}\text { No } \\
\text { difference* }\end{array}$ & - & - & Small $(1)^{\star}$ & - & $\begin{array}{c}\text { No } \\
\text { difference* }\end{array}$ \\
\hline Olanzapine & Aripiprazole LAI-1 & - & - & $\begin{array}{c}\text { No } \\
\text { difference* }\end{array}$ & - & - & - & - & $\begin{array}{c}\text { No } \\
\text { difference* }\end{array}$ \\
\hline Olanzapine & $\begin{array}{c}\text { Aripiprazole LAl- } \\
6 \mathrm{w}\end{array}$ & - & - & 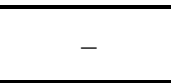 & - & - & - & - & 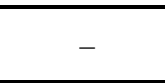 \\
\hline Olanzapine & Asenapine & - & - & $\begin{array}{c}\text { No } \\
\text { difference* }\end{array}$ & $\begin{array}{c}\text { No } \\
\text { difference* }\end{array}$ & Insufficient & Small $(1)^{\star}$ & $\begin{array}{c}\text { No } \\
\text { difference }\end{array}$ & $\begin{array}{c}\text { No } \\
\text { difference* }\end{array}$ \\
\hline Olanzapine & Brexpiprazole & - & - & $\begin{array}{c}\text { No } \\
\text { difference* }\end{array}$ & - & - & Small $(1)^{\star}$ & - & $\begin{array}{c}\text { No } \\
\text { difference* }\end{array}$ \\
\hline Olanzapine & Cariprazine & - & - & $\begin{array}{c}\text { No } \\
\text { difference* }\end{array}$ & - & - & Small $(1)^{\star}$ & - & $\begin{array}{c}\text { No } \\
\text { difference* }\end{array}$ \\
\hline Olanzapine & Clozapine & $\begin{array}{c}\text { No } \\
\text { difference* }\end{array}$ & $\begin{array}{c}\text { No } \\
\text { difference* }\end{array}$ & $\begin{array}{c}\text { No } \\
\text { difference* }\end{array}$ & - & Small $(2)^{\star}$ & $\begin{array}{c}\text { No } \\
\text { difference* }\end{array}$ & - & $\begin{array}{l}\text { Moderate } \\
(1)^{\star}\end{array}$ \\
\hline Olanzapine & Iloperidone & - & - & - & - & - & Small $(1)^{\star}$ & - & $\begin{array}{c}\text { No } \\
\text { difference* }\end{array}$ \\
\hline Olanzapine & Lurasidone & - & - & - & - & - & Small $(1)^{\star}$ & Insufficient & $\begin{array}{c}\text { Moderate } \\
(1)^{\star}\end{array}$ \\
\hline Olanzapine & Olanzapine LAI & - & - & - & - & - & - & Insufficient & $\begin{array}{c}\text { No } \\
\text { difference* }\end{array}$ \\
\hline Olanzapine & Paliperidone & Insufficient & - & $\begin{array}{c}\text { No } \\
\text { difference* }\end{array}$ & - & - & $\begin{array}{c}\text { No } \\
\text { difference* }\end{array}$ & $\begin{array}{c}\text { No } \\
\text { difference* }\end{array}$ & $\begin{array}{c}\text { No } \\
\text { difference* }\end{array}$ \\
\hline Olanzapine & Paliperidone LAI-1 & - & - & - & - & - & - & - & $\begin{array}{c}\text { No } \\
\text { difference* }\end{array}$ \\
\hline Olanzapine & Paliperidone LAI-3 & - & - & - & - & - & - & - & $\begin{array}{c}\text { No } \\
\text { difference* }\end{array}$ \\
\hline Olanzapine & Quetiapine & $\begin{array}{c}\text { No } \\
\text { difference* }\end{array}$ & $\begin{array}{c}\text { No } \\
\text { difference** }\end{array}$ & $\begin{array}{c}\text { Moderate } \\
(1)^{\star}\end{array}$ & $\begin{array}{c}\text { No } \\
\text { difference* }\end{array}$ & - & Small $(1)^{\star}$ & - & $\begin{array}{c}\text { Moderate } \\
(1)^{\star}\end{array}$ \\
\hline Olanzapine & Quetiapine ER & - & - & - & - & - & - & - & $\begin{array}{c}\text { No } \\
\text { difference* }\end{array}$ \\
\hline Olanzapine & Risperidone & $\begin{array}{c}\text { No } \\
\text { difference* }\end{array}$ & $\begin{array}{c}\text { No } \\
\text { difference** }\end{array}$ & $\begin{array}{c}\text { No } \\
\text { difference* }\end{array}$ & $\begin{array}{c}\text { No } \\
\text { difference* }\end{array}$ & - & $\begin{array}{c}\text { No } \\
\text { difference* }\end{array}$ & - & $\begin{array}{c}\text { Moderate } \\
(1)^{\star}\end{array}$ \\
\hline Olanzapine & Risperidone LAI & - & $\begin{array}{c}\text { No } \\
\text { difference* }\end{array}$ & - & - & - & - & - & $\begin{array}{c}\text { No } \\
\text { difference* }\end{array}$ \\
\hline Olanzapine & Ziprasidone & - & $\begin{array}{c}\text { No } \\
\text { difference }^{\star *}\end{array}$ & $\begin{array}{c}\text { No } \\
\text { difference* }\end{array}$ & - & - & Small $(1)^{\star}$ & - & $\begin{array}{c}\text { Moderate } \\
(1)^{\star}\end{array}$ \\
\hline
\end{tabular}

* = Low SOE, ${ }^{* *}=$ Moderate SOE, ${ }^{* * *}=$ High SOE, - = No data, (1) = Estimate favors Drug 1, (2) = Estimate favors Drug 2 
Appendix Table I-12. Summary of findings for paliperidone LAI-1 versus other SGAs

\begin{tabular}{|c|c|c|c|c|c|c|c|c|c|}
\hline Drug & Comparator & Function & QOL & Response & Mortality & Self-harm & Symptoms & Any AE & W/D AEs \\
\hline $\begin{array}{c}\text { Paliperidone } \\
\text { LAI-1 }\end{array}$ & Aripiprazole & - & - & - & - & - & - & - & $\begin{array}{c}\text { No } \\
\text { difference* }\end{array}$ \\
\hline $\begin{array}{c}\text { Paliperidone } \\
\text { LAI-1 }\end{array}$ & Aripiprazole LAI-1 & - & Insufficient & - & - & - & - & - & $\begin{array}{c}\text { No } \\
\text { difference* }\end{array}$ \\
\hline $\begin{array}{c}\text { Paliperidone } \\
\text { LAI-1 }\end{array}$ & $\begin{array}{c}\text { Aripiprazole LAI- } \\
6 \mathrm{w} \\
\end{array}$ & - & - & - & - & - & - & - & - \\
\hline $\begin{array}{c}\text { Paliperidone } \\
\text { LAI-1 }\end{array}$ & Asenapine & - & - & - & - & - & - & - & $\begin{array}{c}\text { No } \\
\text { difference* }\end{array}$ \\
\hline $\begin{array}{c}\text { Paliperidone } \\
\text { LAI-1 }\end{array}$ & Brexpiprazole & - & - & - & - & - & - & - & $\begin{array}{c}\text { No } \\
\text { difference* }\end{array}$ \\
\hline $\begin{array}{c}\text { Paliperidone } \\
\text { LAI-1 }\end{array}$ & Cariprazine & - & - & - & - & - & - & - & $\begin{array}{c}\text { No } \\
\text { difference* }\end{array}$ \\
\hline $\begin{array}{l}\text { Paliperidone } \\
\text { LAI-1 }\end{array}$ & Clozapine & - & - & - & - & - & - & - & $\begin{array}{c}\text { No } \\
\text { difference* }\end{array}$ \\
\hline $\begin{array}{c}\text { Paliperidone } \\
\text { LAI-1 }\end{array}$ & lloperidone & - & - & - & - & - & - & - & $\begin{array}{c}\text { No } \\
\text { difference* }\end{array}$ \\
\hline $\begin{array}{c}\text { Paliperidone } \\
\text { LAI-1 }\end{array}$ & Lurasidone & - & - & - & - & - & - & - & $\begin{array}{c}\text { No } \\
\text { difference* }\end{array}$ \\
\hline $\begin{array}{c}\text { Paliperidone } \\
\text { LAI-1 }\end{array}$ & Olanzapine & - & - & - & - & - & - & - & $\begin{array}{c}\text { No } \\
\text { difference* }\end{array}$ \\
\hline $\begin{array}{l}\text { Paliperidone } \\
\text { LAI-1 }\end{array}$ & Olanzapine LAI & - & - & - & - & - & - & - & $\begin{array}{c}\text { No } \\
\text { difference* }\end{array}$ \\
\hline $\begin{array}{c}\text { Paliperidone } \\
\text { LAI-1 }\end{array}$ & Paliperidone & - & - & - & Insufficient & - & - & - & $\begin{array}{c}\text { No } \\
\text { difference* }\end{array}$ \\
\hline $\begin{array}{c}\text { Paliperidone } \\
\text { LAI-1 }\end{array}$ & Paliperidone LAI-3 & - & - & - & Insufficient & - & - & Insufficient & $\begin{array}{c}\text { No } \\
\text { difference* }\end{array}$ \\
\hline $\begin{array}{l}\text { Paliperidone } \\
\text { LAI-1 }\end{array}$ & Quetiapine & - & - & - & - & - & - & - & $\begin{array}{c}\text { No } \\
\text { difference* }\end{array}$ \\
\hline $\begin{array}{l}\text { Paliperidone } \\
\text { LAI-1 }\end{array}$ & Quetiapine ER & - & - & - & - & - & - & - & $\begin{array}{c}\text { No } \\
\text { difference* }\end{array}$ \\
\hline $\begin{array}{l}\text { Paliperidone } \\
\text { LAI-1 }\end{array}$ & Risperidone & - & - & - & - & - & - & - & $\begin{array}{c}\text { No } \\
\text { difference* }\end{array}$ \\
\hline $\begin{array}{c}\text { Paliperidone } \\
\text { LAI-1 }\end{array}$ & Risperidone LAI & $\begin{array}{c}\text { No } \\
\text { difference* }\end{array}$ & - & - & $\begin{array}{c}\text { No } \\
\text { difference* }\end{array}$ & Insufficient & - & $\begin{array}{c}\text { No } \\
\text { difference* }\end{array}$ & $\begin{array}{c}\text { Moderate } \\
(2)^{\star}\end{array}$ \\
\hline $\begin{array}{c}\text { Paliperidone } \\
\text { LAI-1 }\end{array}$ & Ziprasidone & - & - & - & - & - & - & - & $\begin{array}{c}\text { No } \\
\text { difference* }\end{array}$ \\
\hline
\end{tabular}

* = Low SOE, ** = Moderate SOE, ${ }^{* * *}=$ High SOE, - = No data, (1) = Estimate favors Drug 1, (2) = Estimate favors Drug 2 
Appendix Table I-13. Summary of findings for paliperidone LAI-3 versus other SGAs

\begin{tabular}{|c|c|c|c|c|c|c|c|c|c|}
\hline Drug & Comparator & Function & QOL & Response & Mortality & Self-harm & Symptoms & Any AE & W/D AEs \\
\hline $\begin{array}{c}\text { Paliperidone } \\
\text { LAI-3 }\end{array}$ & Aripiprazole & - & - & - & - & - & - & - & $\begin{array}{c}\text { No } \\
\text { difference* }\end{array}$ \\
\hline $\begin{array}{c}\text { Paliperidone } \\
\text { LAI-3 }\end{array}$ & Aripiprazole LAI-1 & - & - & - & - & - & - & - & $\begin{array}{c}\text { No } \\
\text { difference* }\end{array}$ \\
\hline $\begin{array}{c}\text { Paliperidone } \\
\text { LAI-3 }\end{array}$ & $\begin{array}{c}\text { Aripiprazole LAI- } \\
6 \mathrm{w} \\
\end{array}$ & - & - & - & - & - & - & - & - \\
\hline $\begin{array}{c}\text { Paliperidone } \\
\text { LAI-3 }\end{array}$ & Asenapine & - & - & - & - & - & - & - & $\begin{array}{c}\text { No } \\
\text { difference* }\end{array}$ \\
\hline $\begin{array}{l}\text { Paliperidone } \\
\text { LAI-3 }\end{array}$ & Brexpiprazole & - & - & - & - & - & - & - & $\begin{array}{c}\text { No } \\
\text { difference* }\end{array}$ \\
\hline $\begin{array}{c}\text { Paliperidone } \\
\text { LAI-3 }\end{array}$ & Cariprazine & - & - & - & - & - & - & - & $\begin{array}{c}\text { No } \\
\text { difference* }\end{array}$ \\
\hline $\begin{array}{l}\text { Paliperidone } \\
\text { LAI-3 }\end{array}$ & Clozapine & - & - & - & - & - & - & - & $\begin{array}{c}\text { No } \\
\text { difference* }\end{array}$ \\
\hline $\begin{array}{c}\text { Paliperidone } \\
\text { LAI-3 }\end{array}$ & lloperidone & - & - & - & - & - & - & - & $\begin{array}{c}\text { No } \\
\text { difference* }\end{array}$ \\
\hline $\begin{array}{c}\text { Paliperidone } \\
\text { LAI-3 }\end{array}$ & Lurasidone & - & - & - & - & - & - & - & $\begin{array}{c}\text { No } \\
\text { difference* }\end{array}$ \\
\hline $\begin{array}{c}\text { Paliperidone } \\
\text { LAI-3 }\end{array}$ & Olanzapine & - & - & - & - & - & - & - & $\begin{array}{c}\text { No } \\
\text { difference* }\end{array}$ \\
\hline $\begin{array}{l}\text { Paliperidone } \\
\text { LAI-3 }\end{array}$ & Olanzapine LAI & - & - & - & - & - & - & - & $\begin{array}{c}\text { No } \\
\text { difference* }\end{array}$ \\
\hline $\begin{array}{l}\text { Paliperidone } \\
\text { LAI-3 }\end{array}$ & Paliperidone & - & - & - & - & - & - & - & $\begin{array}{c}\text { No } \\
\text { difference* }\end{array}$ \\
\hline $\begin{array}{c}\text { Paliperidone } \\
\text { LAI-3 }\end{array}$ & Paliperidone LAI-1 & - & - & - & Insufficient & - & - & Insufficient & $\begin{array}{c}\text { No } \\
\text { difference* }\end{array}$ \\
\hline $\begin{array}{l}\text { Paliperidone } \\
\text { LAI-3 }\end{array}$ & Quetiapine & - & - & - & - & - & - & - & $\begin{array}{c}\text { No } \\
\text { difference* }\end{array}$ \\
\hline $\begin{array}{c}\text { Paliperidone } \\
\text { LAI-3 }\end{array}$ & Quetiapine ER & - & - & - & - & - & - & - & $\begin{array}{c}\text { No } \\
\text { difference* }\end{array}$ \\
\hline $\begin{array}{l}\text { Paliperidone } \\
\text { LAI-3 }\end{array}$ & Risperidone & - & - & - & - & - & - & - & $\begin{array}{c}\text { No } \\
\text { difference* }\end{array}$ \\
\hline $\begin{array}{c}\text { Paliperidone } \\
\text { LAI-3 }\end{array}$ & Risperidone LAI & - & - & - & - & - & - & - & $\begin{array}{c}\text { No } \\
\text { difference* }^{*}\end{array}$ \\
\hline $\begin{array}{c}\text { Paliperidone } \\
\text { LAI-3 }\end{array}$ & Ziprasidone & - & - & - & - & - & - & - & $\begin{array}{c}\text { No } \\
\text { difference* }\end{array}$ \\
\hline
\end{tabular}

* = Low SOE, ** = Moderate SOE, ${ }^{* * *}=$ High SOE, - = No data, (1) = Estimate favors Drug 1, (2) = Estimate favors Drug 2 
Appendix Table I-14. Summary of findings for paliperidone versus other SGAs

\begin{tabular}{|c|c|c|c|c|c|c|c|c|c|}
\hline Drug & Comparator & Function & QOL & Response & Mortality & Self-harm & Symptoms & Any AE & W/D AEs \\
\hline Paliperidone & Aripiprazole & - & - & $\begin{array}{c}\text { No } \\
\text { difference* }\end{array}$ & - & - & - & Insufficient & $\begin{array}{c}\text { No } \\
\text { difference* }\end{array}$ \\
\hline Paliperidone & Aripiprazole LAI-1 & - & - & $\begin{array}{c}\text { No } \\
\text { difference* }\end{array}$ & - & - & - & Insufficient & $\begin{array}{c}\text { No } \\
\text { difference* }\end{array}$ \\
\hline Paliperidone & $\begin{array}{c}\text { Aripiprazole LAI- } \\
6 w\end{array}$ & - & - & - & - & - & - & - & - \\
\hline Paliperidone & Asenapine & - & - & $\begin{array}{c}\text { No } \\
\text { difference* }\end{array}$ & - & - & - & - & $\begin{array}{c}\text { No } \\
\text { difference* }\end{array}$ \\
\hline Paliperidone & Brexpiprazole & - & - & $\begin{array}{c}\text { No } \\
\text { difference* }\end{array}$ & - & - & - & Insufficient & $\begin{array}{c}\text { No } \\
\text { difference* }\end{array}$ \\
\hline Paliperidone & Cariprazine & - & - & $\begin{array}{c}\text { No } \\
\text { difference* }\end{array}$ & - & - & - & - & $\begin{array}{c}\text { No } \\
\text { difference* }\end{array}$ \\
\hline Paliperidone & Clozapine & - & - & $\begin{array}{c}\text { No } \\
\text { difference* }\end{array}$ & - & - & - & - & $\begin{array}{c}\text { No } \\
\text { difference* }\end{array}$ \\
\hline Paliperidone & Iloperidone & - & - & - & - & - & Small $(1)^{\star}$ & - & $\begin{array}{c}\text { No } \\
\text { difference* }\end{array}$ \\
\hline Paliperidone & Lurasidone & - & - & - & - & - & Small $(1)^{\star}$ & - & $\begin{array}{c}\text { No } \\
\text { difference* }\end{array}$ \\
\hline Paliperidone & Olanzapine & Insufficient & - & $\begin{array}{c}\text { No } \\
\text { difference* }\end{array}$ & - & - & $\begin{array}{c}\text { No } \\
\text { difference* }\end{array}$ & $\begin{array}{c}\text { No } \\
\text { difference* }\end{array}$ & $\begin{array}{c}\text { No } \\
\text { difference* }\end{array}$ \\
\hline Paliperidone & Olanzapine LAI & - & - & - & - & - & - & - & $\begin{array}{c}\text { No } \\
\text { difference* }\end{array}$ \\
\hline Paliperidone & Paliperidone LAI-1 & - & - & - & Insufficient & - & - & - & $\begin{array}{c}\text { No } \\
\text { difference* }\end{array}$ \\
\hline Paliperidone & Paliperidone LAI-3 & - & - & - & - & - & - & - & $\begin{array}{c}\text { No } \\
\text { difference* }\end{array}$ \\
\hline Paliperidone & Quetiapine & - & - & $\begin{array}{c}\text { No } \\
\text { difference* }\end{array}$ & - & - & - & - & $\begin{array}{c}\text { No } \\
\text { difference* }\end{array}$ \\
\hline Paliperidone & Quetiapine ER & - & - & - & - & - & - & - & $\begin{array}{c}\text { No } \\
\text { difference* }\end{array}$ \\
\hline Paliperidone & Risperidone & - & - & $\begin{array}{c}\text { No } \\
\text { difference* }\end{array}$ & - & - & - & - & $\begin{array}{c}\text { No } \\
\text { difference* }\end{array}$ \\
\hline Paliperidone & Risperidone LAI & - & - & - & - & - & - & Insufficient & $\begin{array}{c}\text { No } \\
\text { difference* }^{*}\end{array}$ \\
\hline Paliperidone & Ziprasidone & - & - & $\begin{array}{c}\text { No } \\
\text { difference* }\end{array}$ & - & - & - & - & $\begin{array}{c}\text { No } \\
\text { difference* }\end{array}$ \\
\hline
\end{tabular}

* = Low SOE, ** = Moderate SOE, ${ }^{* * *}=$ High SOE, - = No data, (1) = Estimate favors Drug 1, (2) = Estimate favors Drug 2 
Appendix Table I-15. Summary of findings for quetiapine ER versus other SGAs

\begin{tabular}{|c|c|c|c|c|c|c|c|c|c|}
\hline Drug & Comparator & Function & QOL & Response & Mortality & Self-harm & Symptoms & Any AE & W/D AEs \\
\hline $\begin{array}{c}\text { Quetiapine } \\
\text { ER }\end{array}$ & Aripiprazole & - & - & - & - & - & - & - & $\begin{array}{c}\text { No } \\
\text { difference* }\end{array}$ \\
\hline $\begin{array}{c}\text { Quetiapine } \\
\text { ER }\end{array}$ & Aripiprazole LAI-1 & - & - & - & - & - & - & - & $\begin{array}{c}\text { No } \\
\text { difference* }\end{array}$ \\
\hline $\begin{array}{c}\text { Quetiapine } \\
\text { ER }\end{array}$ & $\begin{array}{c}\text { Aripiprazole LAI- } \\
6 \mathrm{w} \\
\end{array}$ & - & - & - & - & - & - & - & \\
\hline $\begin{array}{c}\text { Quetiapine } \\
\text { ER }\end{array}$ & Asenapine & - & - & - & - & - & - & - & $\begin{array}{c}\text { No } \\
\text { difference* }\end{array}$ \\
\hline $\begin{array}{c}\text { Quetiapine } \\
\text { ER }\end{array}$ & Brexpiprazole & - & - & - & - & - & - & - & $\begin{array}{c}\text { No } \\
\text { difference* }\end{array}$ \\
\hline $\begin{array}{c}\text { Quetiapine } \\
\text { ER }\end{array}$ & Cariprazine & - & - & - & - & - & - & - & $\begin{array}{c}\text { No } \\
\text { difference* }\end{array}$ \\
\hline $\begin{array}{c}\text { Quetiapine } \\
\text { ER }\end{array}$ & Clozapine & - & - & - & - & - & - & - & $\begin{array}{c}\text { No } \\
\text { difference* }\end{array}$ \\
\hline $\begin{array}{c}\text { Quetiapine } \\
\text { ER }\end{array}$ & Iloperidone & - & - & - & - & - & - & - & $\begin{array}{c}\text { No } \\
\text { difference* }\end{array}$ \\
\hline $\begin{array}{c}\text { Quetiapine } \\
\text { ER }\end{array}$ & Lurasidone & - & - & - & - & - & - & - & $\begin{array}{c}\text { No } \\
\text { difference* }\end{array}$ \\
\hline $\begin{array}{c}\text { Quetiapine } \\
\text { ER }\end{array}$ & Olanzapine & - & - & - & - & - & - & - & $\begin{array}{c}\text { No } \\
\text { difference* }\end{array}$ \\
\hline $\begin{array}{c}\text { Quetiapine } \\
\text { ER }\end{array}$ & Olanzapine LAI & - & - & - & - & - & - & - & $\begin{array}{c}\text { No } \\
\text { difference* }\end{array}$ \\
\hline $\begin{array}{c}\text { Quetiapine } \\
\text { ER }\end{array}$ & Paliperidone & - & - & - & - & - & - & - & $\begin{array}{c}\text { No } \\
\text { difference* }\end{array}$ \\
\hline $\begin{array}{c}\text { Quetiapine } \\
\text { ER }\end{array}$ & Paliperidone LAI-1 & - & - & - & - & - & - & - & $\begin{array}{c}\text { No } \\
\text { difference* }\end{array}$ \\
\hline $\begin{array}{c}\text { Quetiapine } \\
\text { ER }\end{array}$ & Paliperidone LAI-3 & - & - & - & - & - & - & - & $\begin{array}{c}\text { No } \\
\text { difference* }\end{array}$ \\
\hline $\begin{array}{c}\text { Quetiapine } \\
\text { ER }\end{array}$ & Quetiapine & - & - & - & Insufficient & - & - & $\begin{array}{c}\text { No } \\
\text { difference* }\end{array}$ & $\begin{array}{c}\text { No } \\
\text { difference* }\end{array}$ \\
\hline $\begin{array}{c}\text { Quetiapine } \\
\text { ER }\end{array}$ & Risperidone & - & Insufficient & - & - & - & - & $\begin{array}{c}\text { No } \\
\text { difference* }\end{array}$ & $\begin{array}{c}\text { No } \\
\text { difference* }\end{array}$ \\
\hline $\begin{array}{c}\text { Quetiapine } \\
\text { ER }\end{array}$ & Risperidone LAI & - & - & - & - & - & - & - & $\begin{array}{c}\text { No } \\
\text { difference* }\end{array}$ \\
\hline $\begin{array}{c}\text { Quetiapine } \\
\text { ER }\end{array}$ & Ziprasidone & - & - & - & - & - & - & - & $\begin{array}{c}\text { No } \\
\text { difference* }\end{array}$ \\
\hline
\end{tabular}

* = Low SOE, ${ }^{* *}=$ Moderate SOE, ${ }^{* * *}=$ High SOE, - = No data, (1) = Estimate favors Drug 1, (2) = Estimate favors Drug 2 
Appendix Table I-16. Summary of findings for quetiapine versus other SGAs

\begin{tabular}{|c|c|c|c|c|c|c|c|c|c|}
\hline Drug & Comparator & Function & QOL & Response & Mortality & Self-harm & Symptoms & Any AE & W/D AEs \\
\hline Quetiapine & Aripiprazole & - & - & $\begin{array}{c}\text { No } \\
\text { difference* }\end{array}$ & - & - & - & - & $\begin{array}{c}\text { No } \\
\text { difference* }\end{array}$ \\
\hline Quetiapine & Aripiprazole LAI-1 & - & - & $\begin{array}{c}\text { No } \\
\text { difference* }\end{array}$ & - & - & - & - & $\begin{array}{c}\text { No } \\
\text { difference* }\end{array}$ \\
\hline Quetiapine & $\begin{array}{c}\text { Aripiprazole LAI- } \\
6 \mathrm{w} \\
\end{array}$ & - & - & - & - & - & - & - & - \\
\hline Quetiapine & Asenapine & - & - & $\begin{array}{c}\text { No } \\
\text { difference* }\end{array}$ & - & - & - & - & $\begin{array}{c}\text { No } \\
\text { difference* }\end{array}$ \\
\hline Quetiapine & Brexpiprazole & - & - & $\begin{array}{c}\text { No } \\
\text { difference* }\end{array}$ & - & - & - & - & $\begin{array}{c}\text { No } \\
\text { difference* }\end{array}$ \\
\hline Quetiapine & Cariprazine & - & - & $\begin{array}{c}\text { No } \\
\text { difference* }\end{array}$ & - & - & - & - & $\begin{array}{c}\text { No } \\
\text { difference* }\end{array}$ \\
\hline Quetiapine & Clozapine & - & $\begin{array}{c}\text { No } \\
\text { difference* }\end{array}$ & $\begin{array}{c}\text { No } \\
\text { difference* }\end{array}$ & - & - & - & Insufficient & $\begin{array}{c}\text { No } \\
\text { difference* }\end{array}$ \\
\hline Quetiapine & Iloperidone & - & - & - & - & - & - & - & $\begin{array}{c}\text { No } \\
\text { difference* }\end{array}$ \\
\hline Quetiapine & Lurasidone & - & - & - & - & - & - & - & $\begin{array}{c}\text { No } \\
\text { difference* }\end{array}$ \\
\hline Quetiapine & Olanzapine & $\begin{array}{c}\text { No } \\
\text { difference* }\end{array}$ & $\begin{array}{c}\text { No } \\
\text { difference* }\end{array}$ & $\begin{array}{c}\text { Moderate } \\
(2)^{\star}\end{array}$ & - & - & - & - & $\begin{array}{c}\text { Moderate } \\
(1)^{\star}\end{array}$ \\
\hline Quetiapine & Olanzapine LAI & - & - & - & - & - & - & - & $\begin{array}{c}\text { No } \\
\text { difference* }\end{array}$ \\
\hline Quetiapine & Paliperidone & - & - & $\begin{array}{c}\text { No } \\
\text { difference* }\end{array}$ & - & - & - & - & $\begin{array}{c}\text { No } \\
\text { difference* }\end{array}$ \\
\hline Quetiapine & Paliperidone LAI-1 & - & - & - & - & - & - & - & $\begin{array}{c}\text { No } \\
\text { difference* }\end{array}$ \\
\hline Quetiapine & Paliperidone LAI-3 & - & - & - & - & - & - & - & $\begin{array}{c}\text { No } \\
\text { difference* }\end{array}$ \\
\hline Quetiapine & Quetiapine ER & - & - & - & Insufficient & - & - & $\begin{array}{c}\text { No } \\
\text { difference* }\end{array}$ & $\begin{array}{c}\text { No } \\
\text { difference* }\end{array}$ \\
\hline Quetiapine & Risperidone & Insufficient & $\begin{array}{c}\text { No } \\
\text { difference* }\end{array}$ & $\begin{array}{c}\text { Moderate } \\
(2)^{\star}\end{array}$ & $\begin{array}{c}\text { No } \\
\text { difference* }\end{array}$ & Insufficient & - & $\begin{array}{c}\text { No } \\
\text { difference }\end{array}$ & $\begin{array}{c}\text { No } \\
\text { difference* }\end{array}$ \\
\hline Quetiapine & Risperidone LAI & Small $(2)^{\star}$ & $\begin{array}{c}\text { No } \\
\text { difference* }\end{array}$ & - & - & - & - & Insufficient & $\begin{array}{l}\text { Moderate } \\
(2)^{\star}\end{array}$ \\
\hline Quetiapine & Ziprasidone & - & $\begin{array}{c}\text { No } \\
\text { difference* }\end{array}$ & $\begin{array}{c}\text { No } \\
\text { difference* }\end{array}$ & - & - & - & - & $\begin{array}{c}\text { No } \\
\text { difference* }\end{array}$ \\
\hline
\end{tabular}

* = Low SOE, ${ }^{* *}=$ Moderate SOE, ${ }^{* * *}=$ High SOE, $-=$ No data, (1) = Estimate favors Drug 1, (2) = Estimate favors Drug 2 
Appendix Table I-17. Summary of findings for risperidone LAI versus other SGAs

\begin{tabular}{|c|c|c|c|c|c|c|c|c|c|}
\hline Drug & Comparator & Function & QOL & Response & Mortality & Self-harm & Symptoms & Any AE & W/D AEs \\
\hline $\begin{array}{c}\text { Risperidone } \\
\text { LAI }\end{array}$ & Aripiprazole & - & - & - & - & - & - & Insufficient & $\begin{array}{c}\text { No } \\
\text { difference* }\end{array}$ \\
\hline $\begin{array}{c}\text { Risperidone } \\
\text { LAI }\end{array}$ & Aripiprazole LAI-1 & - & - & - & - & - & - & - & $\begin{array}{c}\text { No } \\
\text { difference* }\end{array}$ \\
\hline $\begin{array}{c}\text { Risperidone } \\
\text { LAI } \\
\end{array}$ & $\begin{array}{c}\text { Aripiprazole LAI- } \\
6 \mathrm{w} \\
\end{array}$ & - & - & - & - & - & - & - & - \\
\hline $\begin{array}{c}\text { Risperidone } \\
\text { LAI }\end{array}$ & Asenapine & - & - & - & - & - & - & - & $\begin{array}{c}\text { Moderate } \\
(1)^{\star}\end{array}$ \\
\hline $\begin{array}{c}\text { Risperidone } \\
\text { LAI }\end{array}$ & Brexpiprazole & - & - & - & - & - & - & - & $\begin{array}{c}\text { No } \\
\text { difference* }\end{array}$ \\
\hline $\begin{array}{c}\text { Risperidone } \\
\text { LAI }\end{array}$ & Cariprazine & - & - & - & - & - & - & - & $\begin{array}{c}\text { No } \\
\text { difference* }\end{array}$ \\
\hline $\begin{array}{c}\text { Risperidone } \\
\text { LAI }\end{array}$ & Clozapine & - & - & - & - & - & - & Insufficient & Large $(1)^{\star}$ \\
\hline $\begin{array}{c}\text { Risperidone } \\
\text { LAI }\end{array}$ & Iloperidone & - & - & - & - & - & - & - & $\begin{array}{c}\text { No } \\
\text { difference* }\end{array}$ \\
\hline $\begin{array}{c}\text { Risperidone } \\
\text { LAI }\end{array}$ & Lurasidone & - & - & - & - & - & - & - & $\begin{array}{l}\text { Moderate } \\
(1)^{\star}\end{array}$ \\
\hline $\begin{array}{c}\text { Risperidone } \\
\text { LAI }\end{array}$ & Olanzapine & - & - & - & - & - & - & - & $\begin{array}{c}\text { No } \\
\text { difference* }\end{array}$ \\
\hline $\begin{array}{c}\text { Risperidone } \\
\text { LAI }\end{array}$ & Olanzapine LAI & - & - & - & - & - & - & - & $\begin{array}{c}\text { No } \\
\text { difference* }\end{array}$ \\
\hline $\begin{array}{l}\text { Risperidone } \\
\text { LAI }\end{array}$ & Paliperidone & $\begin{array}{c}\text { No } \\
\text { difference* }\end{array}$ & - & - & - & - & - & $\begin{array}{c}\text { No } \\
\text { difference* }\end{array}$ & $\begin{array}{c}\text { Moderate } \\
(1)^{\star}\end{array}$ \\
\hline $\begin{array}{c}\text { Risperidone } \\
\text { LAI }\end{array}$ & Paliperidone LAI-1 & $\begin{array}{c}\text { No } \\
\text { difference* }\end{array}$ & - & - & $\begin{array}{c}\text { No } \\
\text { difference* }\end{array}$ & - & - & $\begin{array}{c}\text { No } \\
\text { difference* }\end{array}$ & $\begin{array}{c}\text { Moderate } \\
(1)^{\star}\end{array}$ \\
\hline $\begin{array}{c}\text { Risperidone } \\
\text { LAI }\end{array}$ & Paliperidone LAI-3 & - & - & - & - & - & - & - & $\begin{array}{c}\text { No } \\
\text { difference* }\end{array}$ \\
\hline $\begin{array}{c}\text { Risperidone } \\
\text { LAI }\end{array}$ & Quetiapine & Small $(1)^{*}$ & $\begin{array}{c}\text { No } \\
\text { difference* }\end{array}$ & - & - & - & - & - & $\begin{array}{c}\text { No } \\
\text { difference* }\end{array}$ \\
\hline $\begin{array}{c}\text { Risperidone } \\
\text { LAI }\end{array}$ & Quetiapine ER & - & - & - & - & - & - & - & $\begin{array}{l}\text { Moderate } \\
(1)^{\star}\end{array}$ \\
\hline $\begin{array}{c}\text { Risperidone } \\
\text { LAI }\end{array}$ & Risperidone & - & - & - & - & - & - & Insufficient & $\begin{array}{c}\text { Moderate } \\
(1)^{\star}\end{array}$ \\
\hline $\begin{array}{c}\text { Risperidone } \\
\text { LAI }\end{array}$ & Ziprasidone & - & - & - & - & - & - & - & $\begin{array}{c}\text { Moderate } \\
(1)^{\star}\end{array}$ \\
\hline
\end{tabular}

* = Low SOE, ** = Moderate SOE, ${ }^{* * *}=$ High SOE, - = No data, $(1)=$ Estimate favors Drug 1, (2) = Estimate favors Drug 2 
Appendix Table I-18. Summary of findings for risperidone versus other SGAS

\begin{tabular}{|c|c|c|c|c|c|c|c|c|c|}
\hline Drug & Comparator & Function & QOL & Response & Mortality & Self-harm & Symptoms & Any AE & W/D AEs \\
\hline Risperidone & Aripiprazole & - & - & $\begin{array}{c}\text { No } \\
\text { difference* }\end{array}$ & - & - & Small $(1)^{\star}$ & $\begin{array}{c}\text { No } \\
\text { difference* }\end{array}$ & $\begin{array}{c}\text { No } \\
\text { difference* }\end{array}$ \\
\hline Risperidone & Aripiprazole LAI-1 & - & - & $\begin{array}{c}\text { No } \\
\text { difference* }\end{array}$ & - & - & - & - & $\begin{array}{c}\text { No } \\
\text { difference* }\end{array}$ \\
\hline Risperidone & $\begin{array}{c}\text { Aripiprazole LAl- } \\
6 w \\
\end{array}$ & - & - & - & - & - & - & - & - \\
\hline Risperidone & Asenapine & - & - & $\begin{array}{c}\text { No } \\
\text { difference* }\end{array}$ & $\begin{array}{c}\text { No } \\
\text { difference* }\end{array}$ & - & Small $(1)^{\star}$ & Insufficient & $\begin{array}{c}\text { No } \\
\text { difference* }\end{array}$ \\
\hline Risperidone & Brexpiprazole & - & - & $\begin{array}{c}\text { No } \\
\text { difference* }\end{array}$ & - & - & Small $(1)^{\star}$ & - & $\begin{array}{c}\text { No } \\
\text { difference* }\end{array}$ \\
\hline Risperidone & Cariprazine & - & - & $\begin{array}{c}\text { No } \\
\text { difference* }\end{array}$ & - & - & - & Insufficient & $\begin{array}{c}\text { No } \\
\text { difference* }\end{array}$ \\
\hline Risperidone & Clozapine & - & - & $\begin{array}{c}\text { No } \\
\text { difference* }\end{array}$ & - & - & - & $\begin{array}{c}\text { No } \\
\text { difference* }\end{array}$ & $\begin{array}{c}\text { No } \\
\text { difference* }\end{array}$ \\
\hline Risperidone & Iloperidone & - & - & - & - & - & Small $(1)^{\star}$ & - & $\begin{array}{c}\text { No } \\
\text { difference* }\end{array}$ \\
\hline Risperidone & Lurasidone & - & - & - & - & - & Small $(1)^{\star}$ & - & $\begin{array}{c}\text { No } \\
\text { difference* }\end{array}$ \\
\hline Risperidone & Olanzapine & $\begin{array}{c}\text { No } \\
\text { difference }^{\star \star}\end{array}$ & $\begin{array}{c}\text { No } \\
\text { difference** }\end{array}$ & $\begin{array}{c}\text { No } \\
\text { difference* }\end{array}$ & - & - & $\begin{array}{c}\text { No } \\
\text { difference* }\end{array}$ & - & Small $(2)^{\star}$ \\
\hline Risperidone & Olanzapine LAI & - & - & - & - & - & - & - & $\begin{array}{c}\text { No } \\
\text { difference* }\end{array}$ \\
\hline Risperidone & Paliperidone & - & - & $\begin{array}{c}\text { No } \\
\text { difference* }\end{array}$ & - & - & - & - & $\begin{array}{c}\text { No } \\
\text { difference* }\end{array}$ \\
\hline Risperidone & Paliperidone LAI-1 & - & - & - & - & - & - & - & $\begin{array}{c}\text { No } \\
\text { difference* }\end{array}$ \\
\hline Risperidone & Paliperidone LAI-3 & - & - & - & - & - & - & - & $\begin{array}{c}\text { No } \\
\text { difference* }\end{array}$ \\
\hline Risperidone & Quetiapine & Insufficient & $\begin{array}{c}\text { No } \\
\text { difference* }\end{array}$ & $\begin{array}{c}\text { Moderate } \\
(1)^{\star}\end{array}$ & $\begin{array}{c}\text { No } \\
\text { difference* }\end{array}$ & - & - & $\begin{array}{c}\text { No } \\
\text { difference } \\
\text { d* }\end{array}$ & $\begin{array}{c}\text { No } \\
\text { difference* }\end{array}$ \\
\hline Risperidone & Quetiapine ER & - & - & - & Insufficient & - & - & $\begin{array}{c}\text { No } \\
\text { difference* }\end{array}$ & $\begin{array}{c}\text { No } \\
\text { difference* }\end{array}$ \\
\hline Risperidone & Risperidone LAI & - & - & - & - & - & - & Insufficient & $\begin{array}{c}\text { Moderate } \\
(2)^{\star}\end{array}$ \\
\hline Risperidone & Ziprasidone & - & $\begin{array}{c}\text { No } \\
\text { difference* }\end{array}$ & $\begin{array}{c}\text { No } \\
\text { difference* }\end{array}$ & - & - & - & Insufficient & $\begin{array}{c}\text { No } \\
\text { difference* }\end{array}$ \\
\hline
\end{tabular}

* = Low SOE, ${ }^{* *}=$ Moderate SOE, $* * *=$ High SOE, - = No data, (1) = Estimate favors Drug 1, (2) = Estimate favors Drug 2 
Appendix Table I-19. Summary of findings for ziprasidone versus other SGAS

\begin{tabular}{|c|c|c|c|c|c|c|c|c|c|}
\hline Drug & Comparator & Function & QOL & Response & Mortality & Self-harm & Symptoms & Any AE & W/D AEs \\
\hline Ziprasidone & Aripiprazole & - & - & $\begin{array}{c}\text { No } \\
\text { difference* }\end{array}$ & - & - & - & - & $\begin{array}{c}\text { Moderate } \\
(2)^{\star}\end{array}$ \\
\hline Ziprasidone & Aripiprazole LAI-1 & - & - & $\begin{array}{c}\text { No } \\
\text { difference* }\end{array}$ & - & - & - & - & $\begin{array}{c}\text { No } \\
\text { difference* }\end{array}$ \\
\hline Ziprasidone & $\begin{array}{c}\text { Aripiprazole LAI- } \\
6 \mathrm{w} \\
\end{array}$ & - & - & - & - & - & - & - & - \\
\hline Ziprasidone & Asenapine & - & - & $\begin{array}{c}\text { No } \\
\text { difference* }\end{array}$ & - & - & - & - & $\begin{array}{c}\text { No } \\
\text { difference* }\end{array}$ \\
\hline Ziprasidone & Brexpiprazole & - & - & $\begin{array}{c}\text { No } \\
\text { difference* }\end{array}$ & - & - & - & - & $\begin{array}{c}\text { No } \\
\text { difference* }\end{array}$ \\
\hline Ziprasidone & Cariprazine & - & - & $\begin{array}{c}\text { No } \\
\text { difference* }\end{array}$ & - & - & - & - & $\begin{array}{c}\text { No } \\
\text { difference* }\end{array}$ \\
\hline Ziprasidone & Clozapine & - & $\begin{array}{c}\text { No } \\
\text { difference* }\end{array}$ & $\begin{array}{c}\text { No } \\
\text { difference* }\end{array}$ & - & - & - & Insufficient & $\begin{array}{c}\text { No } \\
\text { difference* }\end{array}$ \\
\hline Ziprasidone & Iloperidone & - & - & - & - & - & - & Insufficient & $\begin{array}{c}\text { No } \\
\text { difference* }\end{array}$ \\
\hline Ziprasidone & Lurasidone & - & - & - & - & - & - & Insufficient & $\begin{array}{c}\text { No } \\
\text { difference* }\end{array}$ \\
\hline Ziprasidone & Olanzapine & Insufficient & $\begin{array}{c}\text { No } \\
\text { difference } \\
\text { d* }\end{array}$ & $\begin{array}{c}\text { No } \\
\text { difference* }\end{array}$ & - & - & - & - & $\begin{array}{c}\text { Moderate } \\
(2)^{\star}\end{array}$ \\
\hline Ziprasidone & Olanzapine LAI & - & - & - & - & - & - & - & $\begin{array}{c}\text { No } \\
\text { difference* }\end{array}$ \\
\hline Ziprasidone & Paliperidone & - & - & $\begin{array}{c}\text { No } \\
\text { difference* }\end{array}$ & - & - & - & - & $\begin{array}{c}\text { No } \\
\text { difference* }\end{array}$ \\
\hline Ziprasidone & Paliperidone LAI-1 & - & - & - & - & - & - & - & $\begin{array}{c}\text { No } \\
\text { difference* }\end{array}$ \\
\hline Ziprasidone & Paliperidone LAI-3 & - & - & - & - & - & - & - & $\begin{array}{c}\text { No } \\
\text { difference* }\end{array}$ \\
\hline Ziprasidone & Quetiapine & - & $\begin{array}{c}\text { No } \\
\text { difference* }\end{array}$ & $\begin{array}{c}\text { No } \\
\text { difference* }\end{array}$ & - & - & - & - & $\begin{array}{c}\text { No } \\
\text { difference* }\end{array}$ \\
\hline Ziprasidone & Quetiapine ER & - & - & - & - & - & - & - & $\begin{array}{c}\text { No } \\
\text { difference* }\end{array}$ \\
\hline Ziprasidone & Risperidone & - & $\begin{array}{c}\text { No } \\
\text { difference* }\end{array}$ & $\begin{array}{c}\text { No } \\
\text { difference* }\end{array}$ & - & - & - & Insufficient & $\begin{array}{c}\text { No } \\
\text { difference* }\end{array}$ \\
\hline Ziprasidone & Risperidone LAI & - & - & - & - & - & - & - & $\begin{array}{c}\text { Moderate } \\
(2)^{\star}\end{array}$ \\
\hline
\end{tabular}

* = Low SOE, ${ }^{* *}=$ Moderate SOE, ${ }^{* * *}=$ High SOE, - = No data, (1) = Estimate favors Drug 1, (2) = Estimate favors Drug 2 